Supporting Information

\title{
How Solvents Control the Stereospecificity of Ni-Catalyzed Miyaura Borylation of Allylic Pivalates
}

Pan-Pan Chen, ${ }^{+}$Haiyan Zhang, ${ }^{\ddagger}$ Biao Cheng, ${ }^{\dagger} \mathrm{Xu}$ Chen, ${ }^{+}$Fengchang Cheng, ${ }^{\ddagger}$ Shuo-Qing Zhang, ${ }^{\dagger}$ Zhan Lu, ${ }^{*,+}$ Fanke Meng ${ }^{*}, \neq$ and Xin Hong ${ }^{*}$,

${ }^{+}$Department of Chemistry, Zhejiang University, Hangzhou, 310027, China

${ }^{\ddagger}$ State Key Laboratory of Organometallic Chemistry, Center for Excellence in Molecular Synthesis, Shanghai Institute of Organic Chemistry, 345 Lingling Road, Shanghai, 200032, China

Email: luzhan@zju.edu.cn,mengf@sioc.ac.cn, hxchem@zju.edu.cn

\section{Contents}

1. DFT-optimized structures of selected intermediates and transition states for the $[\mathrm{Ni}(t \mathrm{Bu}-\mathrm{XantPhos})]$-catalyzed Miyaura borylation with allylic pivalate $(R)-1$ in toluene leading to the stereoretention product...........................S5

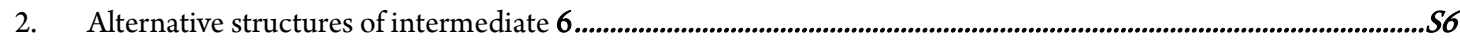

3. Explorations of ligand coordination types of $(t \mathrm{Bu}-\mathrm{XantPhos}) \mathrm{Ni}$ (substrate $)$ complex in various solvents ..........S6

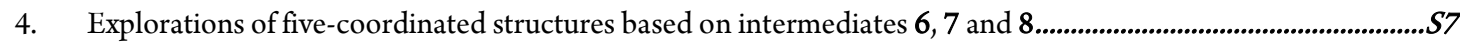

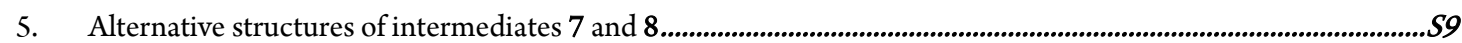

6. Comparisons of the stabilities of monomer, dimer and tetramer of potassium pivalate. ..........................................S9

7. Explorations of alternative transmetalation transition states.......................................................................................... S10

8. Explorations of alternative reductive elimination transition states..........................................................................S S11

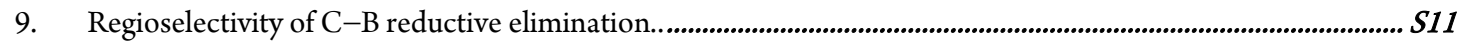

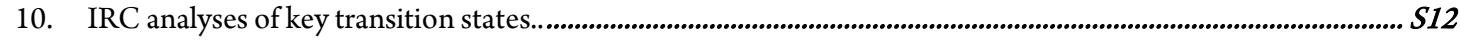

11. Explorations of the possible epimerization of allyl-nickel species.. ........................................................................... S13

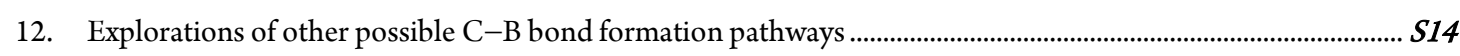

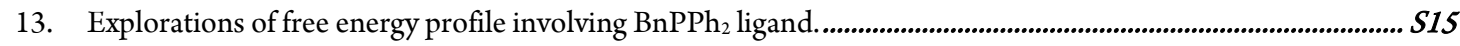

14. Explorations of free energy profile involving $\mathrm{P}(o-\mathrm{Tol})_{3}$ ligand.............................................................................S16

15. Explorations of free energy profile involving $t \mathrm{Bu}-\mathrm{XPh}$ os ligand ........................................................................S S17

16. Explorations of free energy profile involving dppb ligand ..................................................................................... S18

17. Explorations of ligand coordination types of stereo-invertive $\mathrm{C}-\mathrm{O}$ activation transition state in various solvents 
18. Origins of ligand effects on the stereospecificity in toluene

19. Explorations of ligand coordination of related metal/ $t \mathrm{Bu}$-XantPhos complexes with $\mathrm{X}$-ray crystal structure $S 21$

20. Explorations of the alternative $\mathrm{C}-\mathrm{O}$ bond activation transition states S22

21. Calculations of stereospecificity $\left(\Delta \Delta G_{\text {exp }}\right.$ in Table 1) in a series of solvents based on Watson's experimetnal results. $S 25$

22. Computations of selectivities with additional functionals. ... 526

23. Dipole moment calculations in a series of solvents. .. $S 26$

24. Computed stereospecificity in additional solvents S27

25. Alternative stereo-invertive $\mathrm{C}-\mathrm{O}$ bond activation transition states with the coordination of acetonitrile ........ S27

26. Explorations of solvent coordinations in C-O bond activation for other potentially coordinating solvents ... $S 28$

27. DFT-computed Gibbs free energy changes of the Ni/acetonitrile-catalyzed Miyaura borylation with allylic pivalate $(R)$-1 leading to the stereoinversion product . .. S30

28. Solvent concentration corrections. S3O

29. Counterpoise corrections for selected intermediates .. S31

30. General experimental procedures. S32

31. Experimental verifications of ligandless $\mathrm{Ni}$-catalyzed allylic $\mathrm{C}-\mathrm{O}$ bond activation in acetonitrile S32

32. Experimental verifications of ligandless $\mathrm{Ni}$-catalyzed allylic $\mathrm{C}-\mathrm{O}$ bond activation in hexane with catalytic amount of acetonitrile . S33

33. References S35

34. NMR spectra S36

35. HPLC spectra S37

36. Zero-point vibrational energy $(Z P V E)$, thermal correction to enthalpy $(T C H)$, thermal correction to Gibbs free energy $(T C G)$, energies $(E)$, enthalpies $(H)$, and Gibbs free energies $(G)$ (in Hartree) of the structures calculated at the M06-L/def2-TZVPP-SMD(toluene)//B3LYP-D3(BJ)/def2-SVP-SMD(toluene) level of theory...

37. Zero-point vibrational energy ( $Z P V E)$, thermal correction to enthalpy $(T C H)$, thermal correction to Gibbs free energy $(T C G)$, energies $(E)$, enthalpies $(H)$, and Gibbs free energies $(G)$ (in Hartree) of the structures calculated at the M06-L/def2-TZVPP-SMD(acetonitrile)//B3LYP-D3(BJ)/def2-SVP-SMD(acetonitrile) level of theory ... 445

38. Zero-point vibrational energy ( $Z P V E)$, thermal correction to enthalpy $(T C H)$, thermal correction to Gibbs free energy (TCG), energies $(E)$, enthalpies $(H)$, and Gibbs free energies $(G)$ (in Hartree) of the structures calculated at the M06-L/def2-TZVPP-SMD $(N, N$-dimethylformamide $) / / B 3 L Y P-D 3(B J) /$ def2-SVP$\operatorname{SMD}(N, N$-dimethylformamide $)$ level of theory $S 46$

39. Zero-point vibrational energy ( $Z P V E)$, thermal correction to enthalpy $(T C H)$, thermal correction to Gibbs free energy $(T C G)$, energies $(E)$, enthalpies $(H)$, and Gibbs free energies $(G)$ (in Hartree) of the structures 
calculated at the M06-L/def2-TZVPP-SMD(EtOAc)//B3LYP-D3(BJ)/def2-SVP-SMD(EtOAc) level of theory.

40. Zero-point vibrational energy ( $Z P V E)$, thermal correction to enthalpy $(T C H)$, thermal correction to Gibbs free energy ( $T C G$ ), energies $(E)$, enthalpies $(H)$, and Gibbs free energies $(G)$ (in Hartree) of the structures calculated at the M06-L/def2-TZVPP-SMD(THF)//B3LYP-D3(BJ)/def2-SVP-SMD(THF) level of theory.

41. Zero-point vibrational energy $(Z P V E)$, thermal correction to enthalpy $(T C H)$, thermal correction to Gibbs free energy (TCG), energies $(E)$, enthalpies $(H)$, and Gibbs free energies $(G)$ (in Hartree) of the structures calculated at the M06-L/def2-TZVPP-SMD(hexane)//B3LYP-D3(BJ)/def2-SVP-SMD(hexane) level of

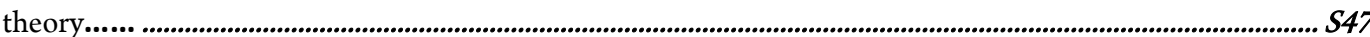

42. Zero-point vibrational energy $(Z P V E)$, thermal correction to enthalpy $(T C H)$, thermal correction to Gibbs free energy $(T C G)$, energies $(E)$, enthalpies $(H)$, and Gibbs free energies $(G)$ (in Hartree) of the structures calculated at the M06-L/def2-TZVPP-SMD(benzene)//B3LYP-D3(BJ)/def2-SVP-SMD(benzene) level of theory...

43. Zero-point vibrational energy $(Z P V E)$, thermal correction to enthalpy $(T C H)$, thermal correction to Gibbs free energy ( $T C G$ ), energies $(E)$, enthalpies $(H)$, and Gibbs free energies $(G)$ (in Hartree) of the structures calculated at the M06-L/def2-TZVPP-SMD(DCM)//B3LYP-D3(BJ)/def2-SVP-SMD(DCM) level of theory............ $\boldsymbol{S 4 8}$

44. Zero-point vibrational energy ( $Z P V E)$, thermal correction to enthalpy $(T C H)$, thermal correction to Gibbs free energy ( TCG), energies $(E)$, enthalpies $(H)$, and Gibbs free energies $(G)$ (in Hartree) of the structures calculated at the M06/def2-TZVPP-SMD(solvent)//B3LYP-D3(BJ)/def2-SVP-SMD(solvent) level of theory 548

45. Zero-point vibrational energy $(Z P V E)$, thermal correction to enthalpy $(T C H)$, thermal correction to Gibbs free energy $(T C G)$, energies $(E)$, enthalpies $(H)$, and Gibbs free energies $(G)$ (in Hartree) of the structures calculated at the B3LYP-D3(BJ)/def2-TZVPP-SMD(solvent)//B3LYP-D3(BJ)/def2-SVP-SMD(solvent) level of theory

46. Zero-point vibrational energy ( $Z P V E)$, thermal correction to enthalpy $(T C H)$, thermal correction to Gibbs free energy ( TCG), energies $(E)$, enthalpies $(H)$, and Gibbs free energies $(G)$ (in Hartree) of the structures calculated at the M06-L/def2-TZVPP-SMD(heptane)//B3LYP-D3(BJ)/def2-SVP-SMD(heptane) level of theory...... $\boldsymbol{S 4 9}$

47. Zero-point vibrational energy ( $Z P V E)$, thermal correction to enthalpy $(T C H)$, thermal correction to Gibbs free energy ( $T C G$ ), energies $(E)$, enthalpies $(H)$, and Gibbs free energies $(G)$ (in Hartree) of the structures calculated at the M06-L/def2-TZVPP-SMD(1,2-dichloroethane)//B3LYP-D3(BJ)/def2-SVP-SMD(1,2-dichloroethane) level of theory S5O

48. Zero-point vibrational energy $(Z P V E)$, thermal correction to enthalpy $(T C H)$, thermal correction to Gibbs free energy ( TCG), energies $(E)$, enthalpies $(H)$, and Gibbs free energies $(G)$ (in Hartree) of the structures calculated at the M06-L/def2-TZVPP-SMD $(N, N$-dimethylacetamide $) / / B 3 L Y P-D 3(B J) /$ def2-SVP-SMD $(N, N$ dimethylacetamide) level of theory

49. Cartesian coordinates of the structures (optimization in toluene)... S51

50. Cartesian coordinates of the structures (optimization in acetonitrile) ...................................................................S303

51. Cartesian coordinates of the structures (optimization in DMF) .......................................................................S355

52. Cartesian coordinates of the structures (optimization in EtOAc). $S 383$ 
53. Cartesian coordinates of the structures (optimization in THF).

54. Cartesian coordinates of the structures (optimization in hexane)...

55. Cartesian coordinates of the structures (optimization in benzene)

56. Cartesian coordinates of the structures (optimization in DCM).

57. Cartesian coordinates of the structures (optimization in heptane) $S 481$

58. Cartesian coordinates of the structures (optimization in 1,2-dichloroethane)...

59. Cartesian coordinates of the structures (optimization in $N, N$-dimethylacetamide). 
1. DFT-optimized structures of selected intermediates and transition states for the $[\mathrm{Ni}(\mathrm{ABu}$-XantPhos $)]$-catalyzed Miyaura borylation with allylic pivalate $(R)-1$ in toluene leading to the stereoretention product.
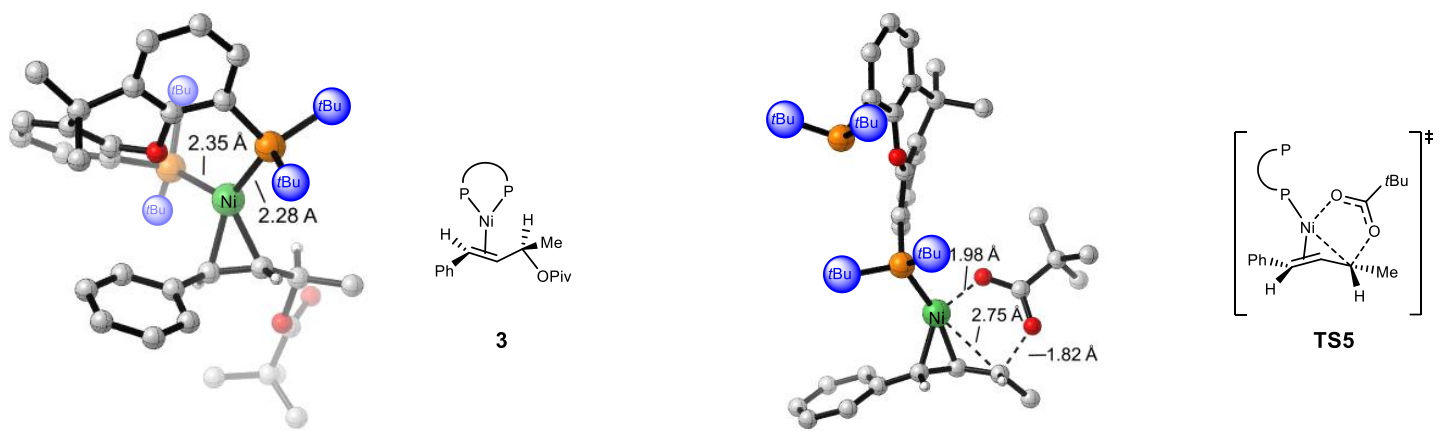

3
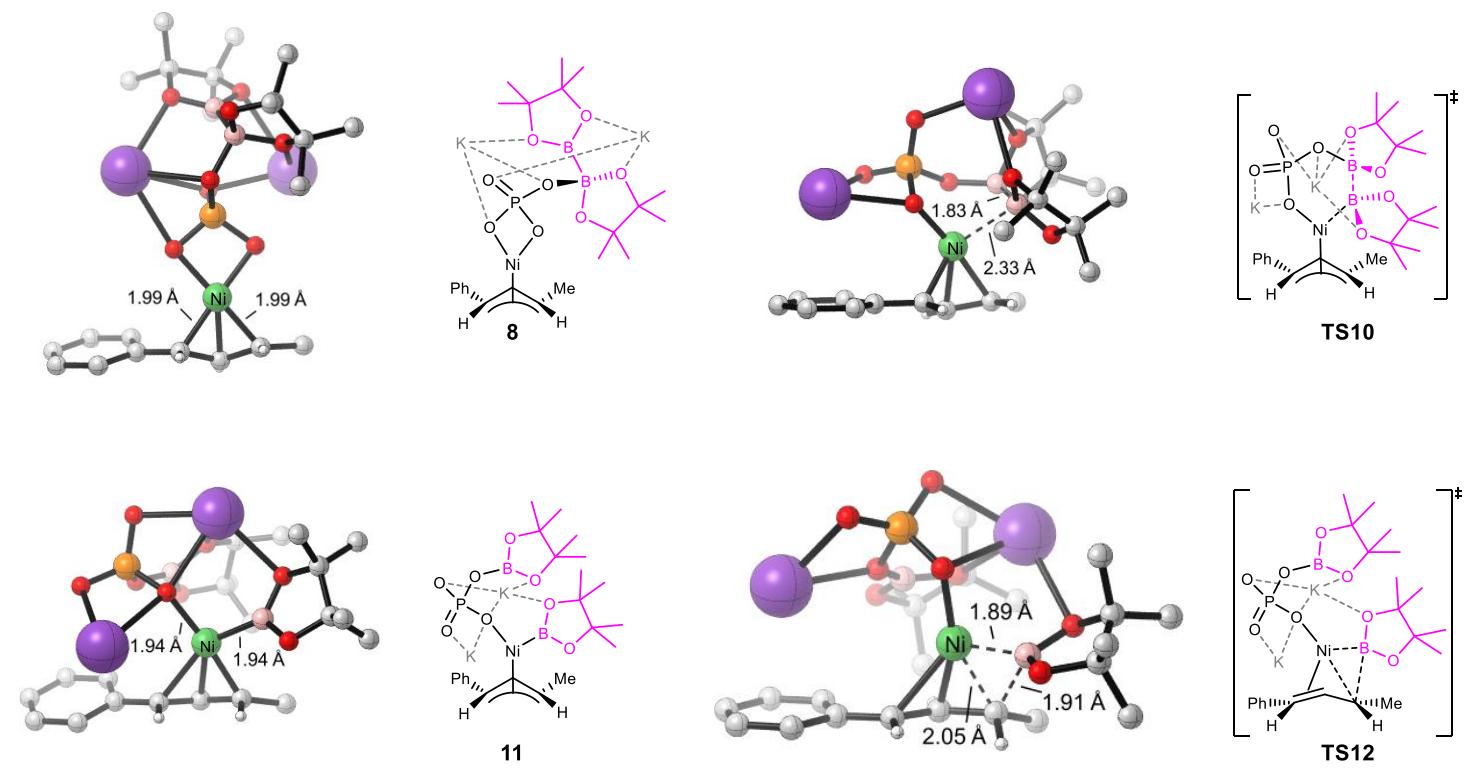

Figure S1. DFT-optimized structures of selected intermediates and transition states in toluene for the stereo-retentive borylation. 


\section{Alternative structures of intermediate 6 .}

In addition to intermediate 6, we also located 6-iso with pivalate at the position trans to the carbon bound with methyl group, but this species is less favorable compared to 6 in terms of Gibbs free energy.
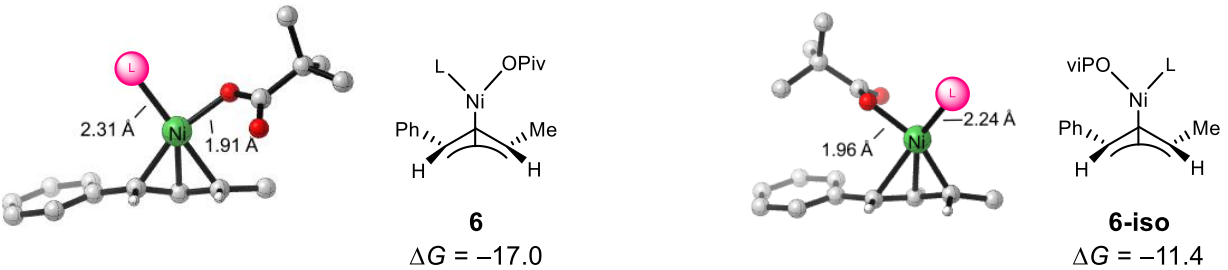

Figure S2. Free energies of conformers of intermediate 6. Free energies in toluene are in $\mathrm{kcal} / \mathrm{mol} . \mathrm{L}=t \mathrm{Bu}-\mathrm{XantPhos}$.

\section{Explorations of ligand coordination types of ( $t \mathrm{Bu}$-XantPhos) $\mathrm{Ni}$ (substrate) complex in various solvents.}

We found that the ( $t \mathrm{Bu}$-XantPhos) $\mathrm{Ni}$ (substrate) complex prefers to have two phosphine coordinations of $t \mathrm{Bu}$ XantPhos ligand (Conformation-A) in most solvents, except DMF and acetonitrile which favor Conformation-B slightly.

Table S1. Explorations of Ligand Coordination Types of ( $t \mathrm{Bu}$-XantPhos)Ni(substrate) Complex ${ }^{a}$

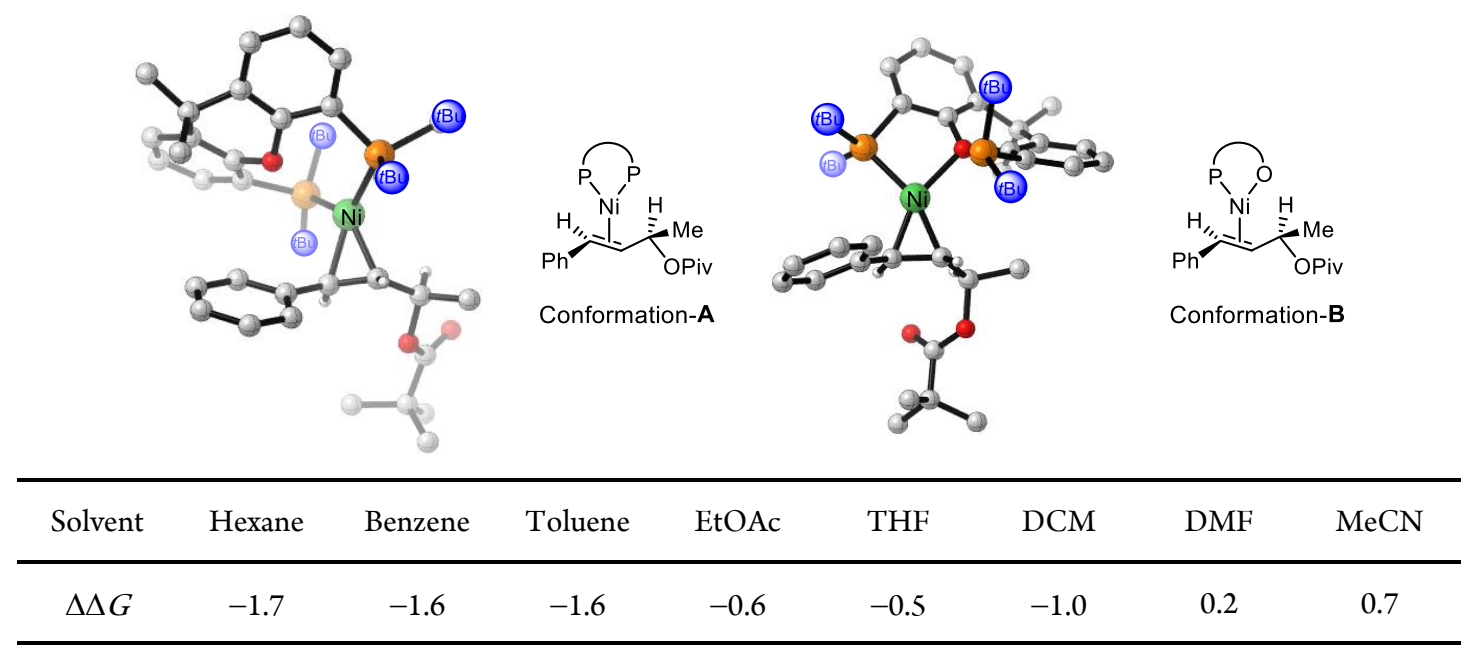

${ }^{a} \Delta \Delta G=\Delta G($ Conformation-A $)-\Delta G($ Conformation-B $)$ in kcal $/ \mathrm{mol}$. 


\section{Explorations of five-coordinated structures based on intermediates 6, 7 and 8 .}

Based on intermediates 6,7 and 8, we performed additional computations to address the possibility of five-coordinated conformers for intermediates 6, 7 and 8 . These unfavorable conformers were optimized to be the more stable fourcoordinated conformers. Even starting with the five-coodinated structure under bond constraints, these structures will dissociate one of the coordinations once the constraint is removed (Figures S3-S5). Therefore, the five-coordinated species are not stable for the studied nickel complex.

(a)

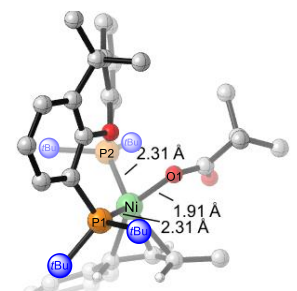

optimization with the constrained bonds: $\mathrm{Ni}-\mathrm{P} 1=\mathbf{2 . 3 1}$ $\begin{aligned} \mathrm{Ni}-\mathrm{P} 2 & =2.31 \\ \mathrm{Ni}-\mathrm{O} 1 & =1.91\end{aligned}$

(b)

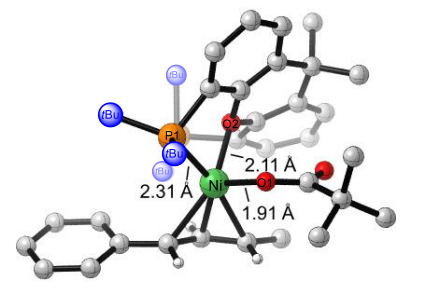

optimization with the constrained bonds: $\mathrm{Ni}-\mathrm{P} 1=\mathbf{2} .31$

$\mathrm{Ni}-01=1.91$
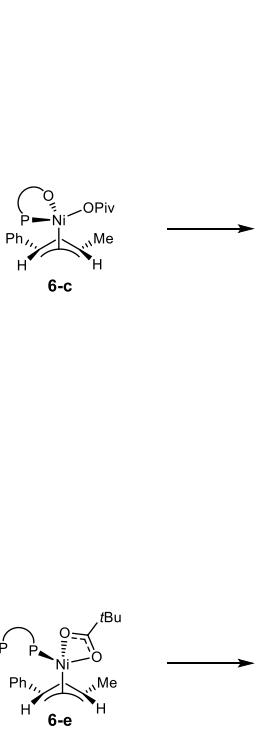

6-c
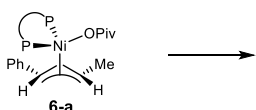

optimization without the constraints: $\mathrm{Ni}-\mathrm{P} 1=3.94$ $\mathrm{Ni}-\mathrm{P2}=2.26$
$\mathrm{Ni}-01=1.91$

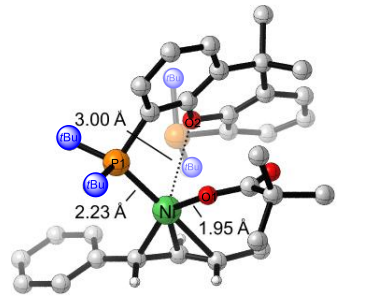

optimization without the constraints: $\mathrm{Ni}-\mathrm{P} \mathbf{1}=\mathbf{2 . 2 3}$

$\mathrm{Ni}-\mathrm{O} 1=1.95$

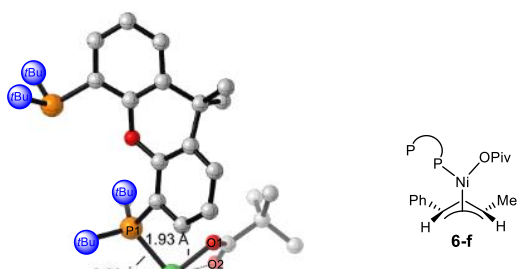

optimization with the constrained bonds: $\mathrm{Ni}-\mathrm{P} 1=\mathbf{2 . 3 1}$ $\mathrm{Ni}-\mathrm{O} 1=1.91$ $\mathrm{Ni}-\mathrm{O} 2=1.91$

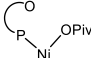

Ph,

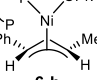

(c)

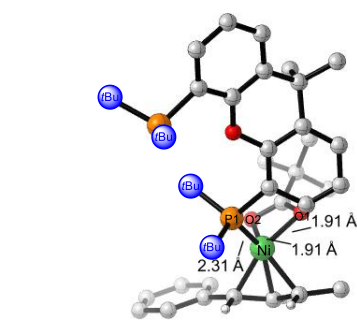

Figure S3. Explorations of possible five-coordinated conformers of intermediate 6. 
(a)

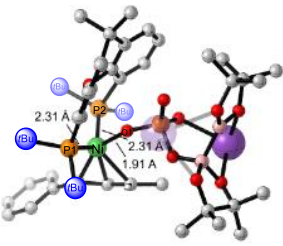

optimization with the constrained bonds: Ni-P1 $=2.31$ $\mathrm{Ni}-01=1.91$

(b)

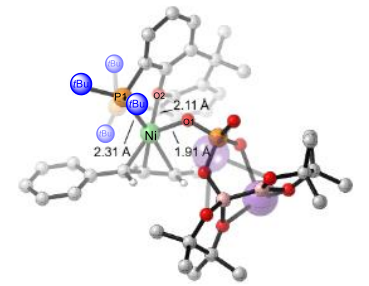

optimization with the constrained bonds: Ni-P1 $=231$

$\mathrm{Ni}-\mathrm{O} 1=1.91$
$\mathrm{Ni}-\mathrm{O} 2=2.11$

optimization with the constrained bonds: Ni-P1 $=2.31$

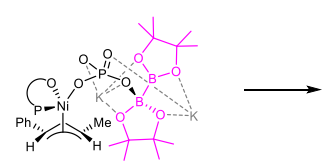

$7-\mathrm{c}$
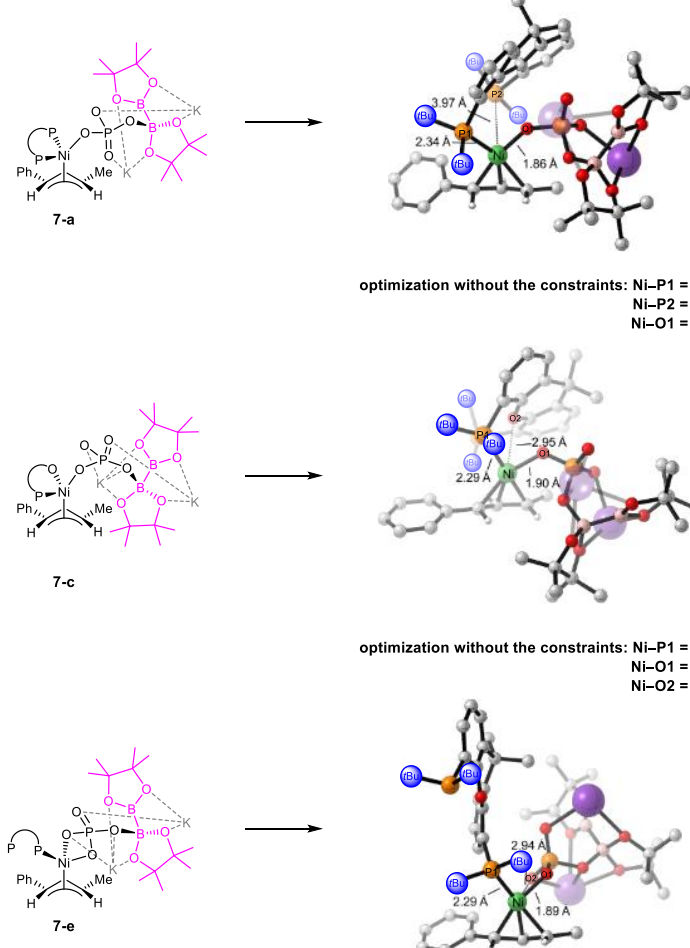

optimization without the constraints: $\mathrm{Ni}-\mathrm{P} 1=2.34$ $\mathrm{Ni}-\mathrm{O} 1=1.86$

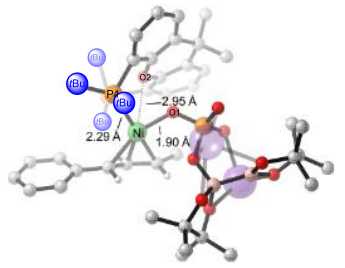

optimization without the constraints: $\begin{aligned} \mathrm{Ni}-\mathrm{P} 1 & =2.29 \\ \mathrm{Ni}-\mathrm{O} 1 & =1.90\end{aligned}$

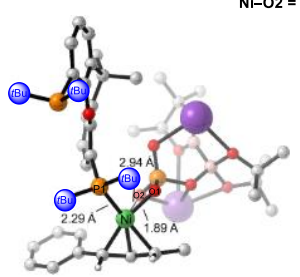

optimization without the constraints: $\mathrm{Ni}-\mathrm{P} 1=2.29$
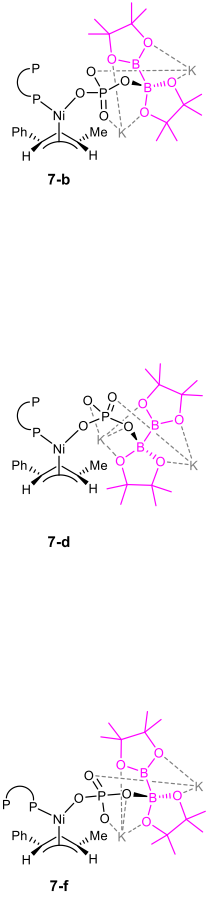
Ni-O $02=1.91$

Figure S4. Explorations of possible five-coordinated conformers of intermediate 7.

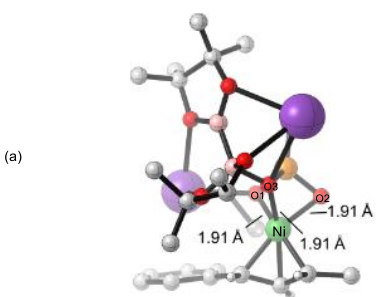

optimization with the constrained bonds: $\mathrm{Ni}-\mathrm{O} 1=1.91$

$\begin{aligned} \mathrm{Ni}-\mathrm{O} 2 & =1.91 \\ \mathrm{Ni}-\mathrm{O} 3 & =1.91\end{aligned}$

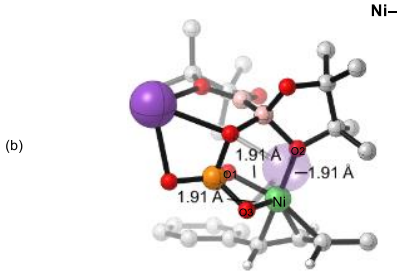

optimization with the constrained bonds: $\mathrm{Ni}-01=1.91$ $\mathrm{Ni}-\mathrm{O} 2=1.91$
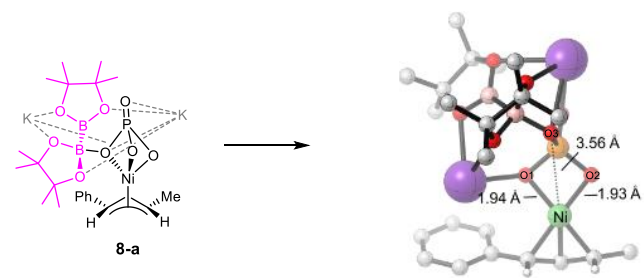

optimization without the constraints: $\mathrm{Ni-O}=1.94$

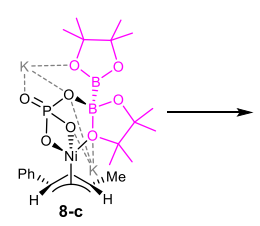
$\mathrm{Ni}-\mathrm{O} 2=1.99$ $\begin{aligned} \mathrm{Ni}-\mathrm{O} 2 & =1.93 \\ \mathrm{Ni}-\mathrm{O} 3 & =3.56\end{aligned}$
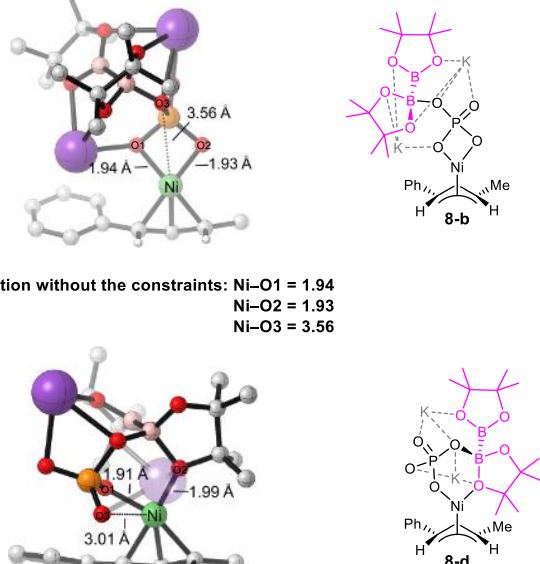

8-d

Figure S5. Explorations of possible five-coordinated conformers of intermediate 8. 


\section{Alternative structures of intermediates 7 and 8 .}

We performed additional calculations to examine if two boron atoms can interact with two oxygen atoms of phosphate. The located intermediates 7-iso and 8-iso are less favorable as compared to the intermediates $\mathbf{7}$ and $\mathbf{8}$ in terms of Gibbs free energy.

(a)

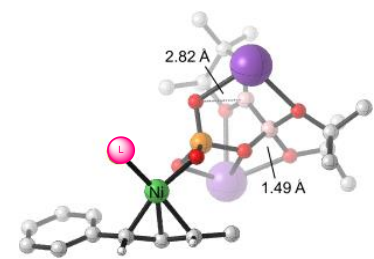

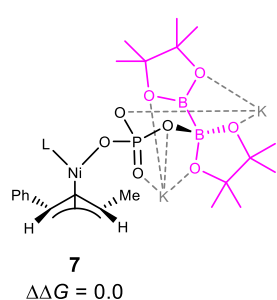

$\Delta \Delta G=0.0$

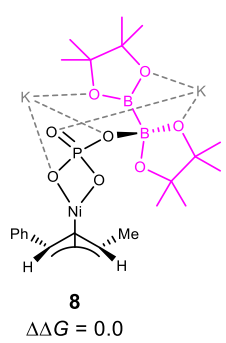

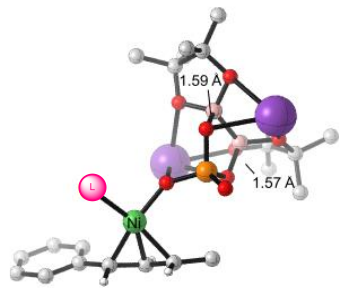

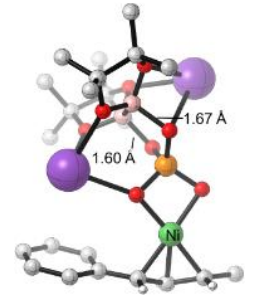

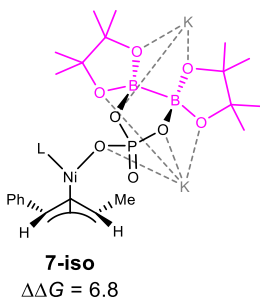

$\Delta \Delta G=6.8$

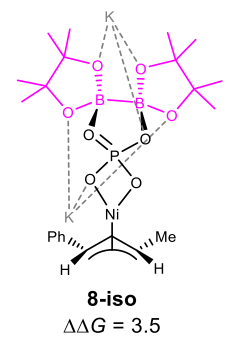

Figure S6. Conformers of intermediates 7 and 8 . Free energies in toluene are in $\mathrm{kcal} / \mathrm{mol} . \mathrm{L}=t \mathrm{Bu}-\mathrm{XantPhos}$.

\section{Comparisons of the stabilities of monomer, dimer and tetramer of potassium pivalate.}

We calculated the relative stabilities of the monomer, dimer and tetramer of potassium pivalate. The tetramer is significantly more stable than the corresponding monomer and dimer. Therefore, the reference structure of potassium pivalate is the tetramer $\mathbf{S 3}$.

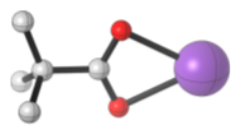

monomer<smiles>CC(C)(C)[O+]1[Al]2C[I+]1O2</smiles>

S1

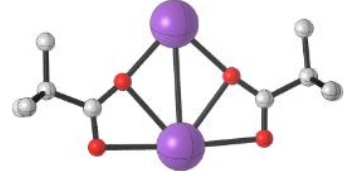

dimer

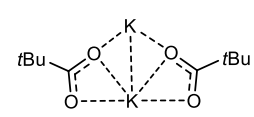

S2

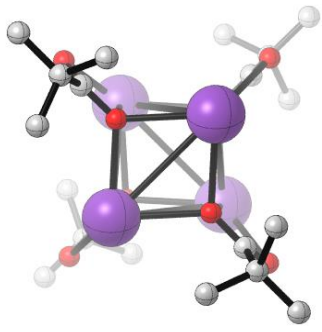

tetramer

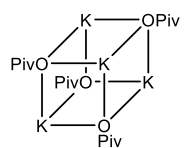

S3

$(\Delta \Delta G$ in toluene $=11.8)$

$(\Delta \Delta G$ in toluene $=3.7)$

$(\Delta \Delta G$ in toluene $=0.0)$

Figure S7. Optimized structures and relative free energies of the monomer, dimer and tetramer of potassium pivalate. Free energies in toluene are in $\mathrm{kcal} / \mathrm{mol}$. 


\section{Explorations of alternative transmetalation transition states.}

In addition to the transmetalation via TS10, we also explored a number of alternative transmetalations. The transmetalation can occur via TS-S4. In TS-S4, pivalate acts as an intramlecular base and nickel has a $\eta^{1}$-coordination of allylic moiety. In addition, the complex of $\mathrm{B}_{2} \mathrm{pin}_{2}$ and potassium phosphate can exchange with the pivalate, and the transmetalation proceeds via TS-S5. TS-S6 is the transmetalation transition state with pivalate, the complex of $\mathrm{B}_{2} \operatorname{pin}_{2}$ and potassium phosphate, as well as $t \mathrm{Bu}$-XantPhos ligand. These three possible transmetalation transition states are all much higher in free energy as compared to the favorable TS10.

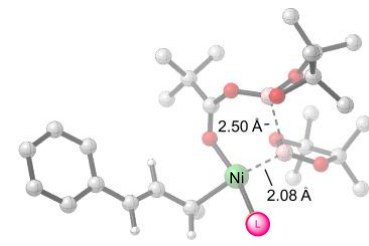

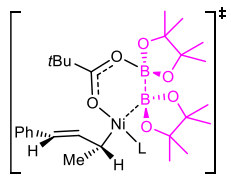
$\left(\Delta G^{\ddagger}\right.$ in toluene $\left.=33.5\right)$
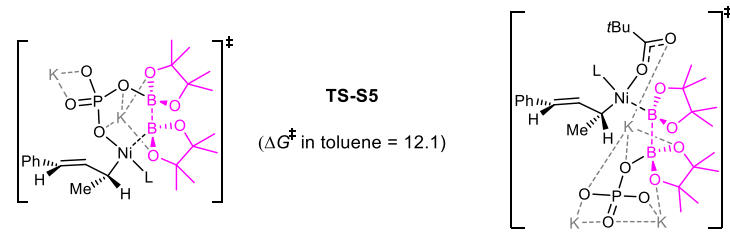

TS-S6

$\left(\Delta G^{\ddagger}\right.$ in toluene $\left.=28.9\right)$

Figure S8. Optimized structures and free energies of alternative transmetalation transition states. Free energies in toluene are in $\mathrm{kcal} / \mathrm{mol}$ compared to intermediate $3 . \mathrm{L}=\boldsymbol{t} \mathrm{Bu}$-XantPhos. 


\section{Explorations of alternative reductive elimination transition states.}

In addition to TS12, the C-B reductive elimination can also occur via the alternative transition states, TS-S7 and TS-

S8. TS-S7 is the reductive elimination with $t \mathrm{Bu}$-XantPhos ligand while without the presence of the $\mathrm{Bpin}-\mathrm{K}_{2} \mathrm{PO}_{4}$ complex. TS-S8 is the same C-B bond formation transition state with an extra Bpin- $\mathrm{K}_{2} \mathrm{PO}_{4}$ complex. Both transition states are much higher in free energy comparing with TS12.
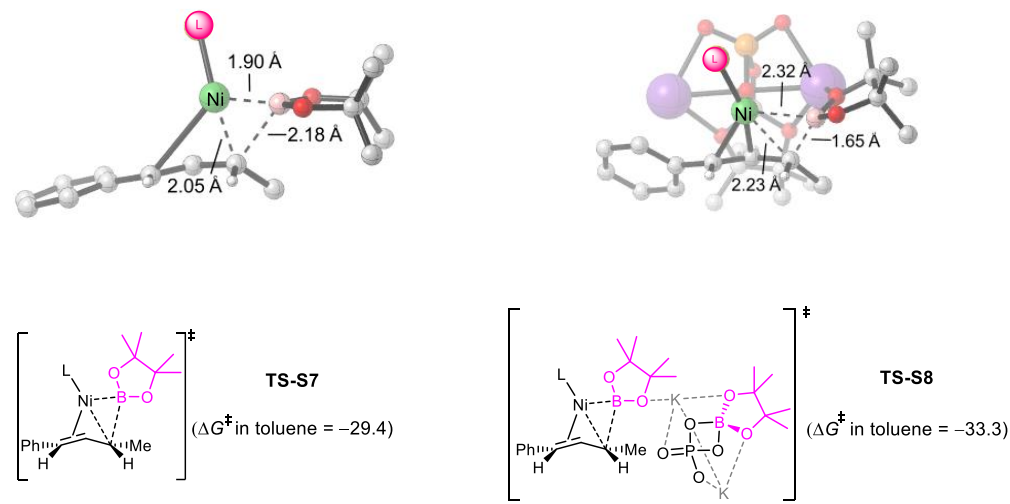

Figure S9. Optimized structures and free energies of alternative reductive elimination transition states. Free energies in toluene are in $\mathrm{kcal} / \mathrm{mol}$ compared to intermediate $3 . \mathrm{L}=t \mathrm{Bu}$-XantPhos.

\section{Regioselectivity of $\mathrm{C}-\mathrm{B}$ reductive elimination.}

Based on the favorable $\mathrm{C}-\mathrm{B}$ reductive elimination transition state $\mathrm{TS} 12$, we calculated the regioselectivity of $\mathrm{C}-\mathrm{B}$ bond formation. TS12 is the C-B bond formation involving the allylic carbon, while TS-S9 is the same type of bond formation involving the benzylic carbon. TS-S9 is $10.2 \mathrm{kcal} / \mathrm{mol}$ less favorable than TS12 in toluene, which is consistent with the experimental facts that the allylic borylation is favored. ${ }^{1}$

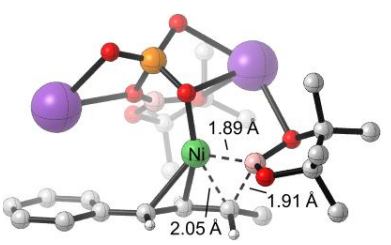

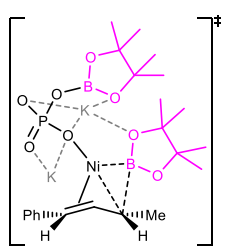

TS12

$\left(\Delta G^{\ddagger}\right.$ in toluene $\left.=0.0\right)$

favored
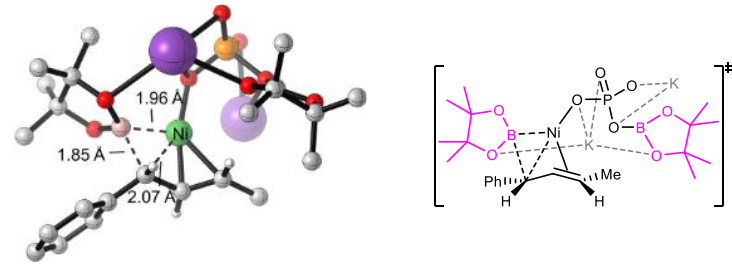

TS-S9

$\left(\Delta G^{\ddagger}\right.$ in toluene $\left.=10.2\right)$

disfavored

Figure S10. Optimized structures and free energies of regioisomeric $\mathrm{C}-\mathrm{B}$ reductive elimination transition states. Free energies in toluene are in $\mathrm{kcal} / \mathrm{mol}$. 


\section{IRC analyses of key transition states.}

We have performed IRC analyses from both directions, for the transition states that are involved in Ni-catalyzed Miyaura borylation. The computations further verified the key transition states (TS5, TS10 and TS12).

(a) IRC analysis of transmetalation transition state TS5
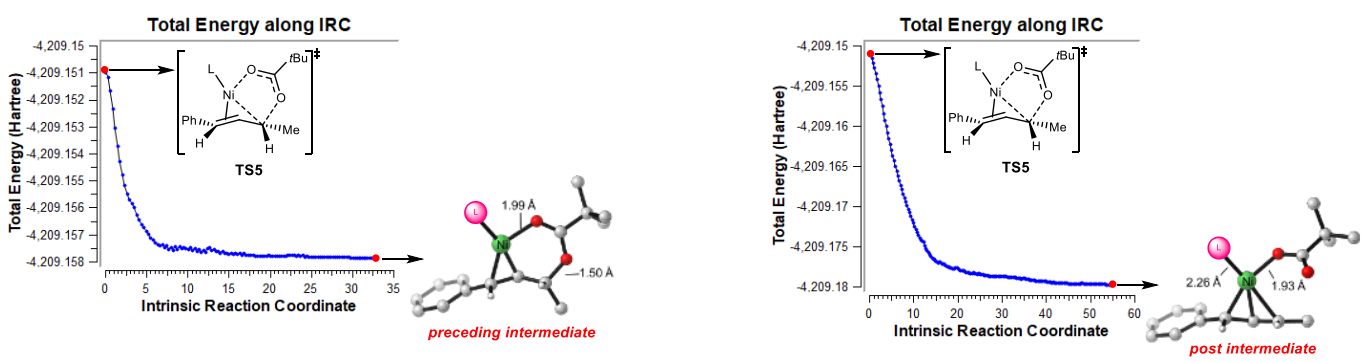

(b) IRC analysis of transmetalation transition state TS10
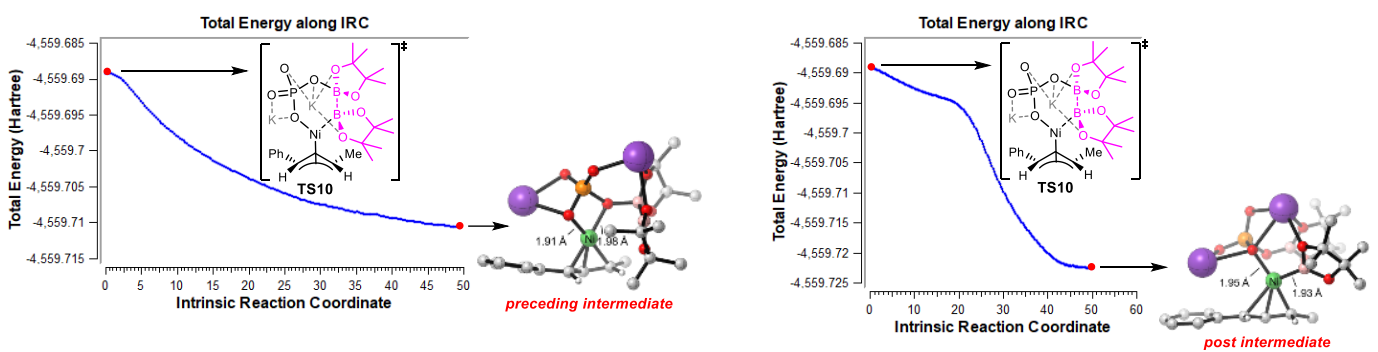

(c) IRC analysis of transmetalation transition state TS12
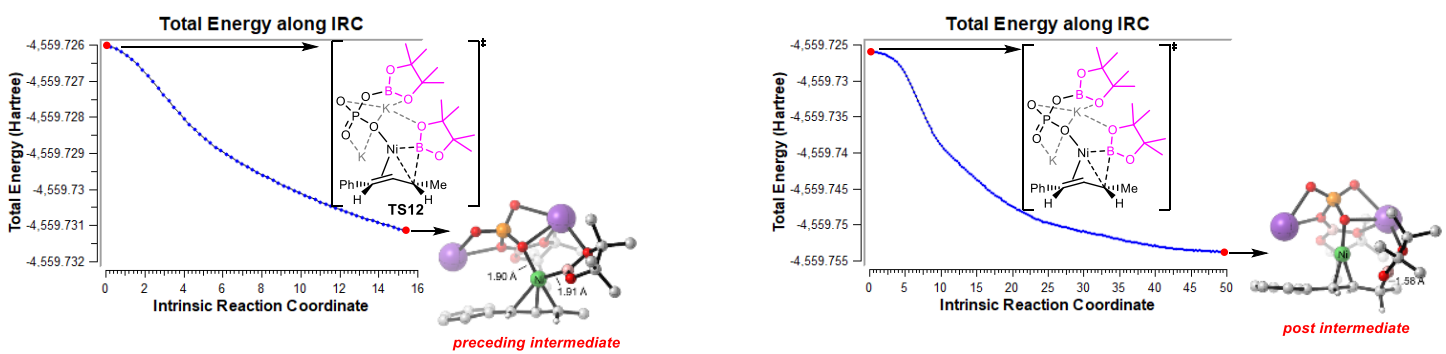

Figure S11. IRC analyses of the key transition states (a) TS5; (b) TS10; (c) TS12. L = tBu-XantPhos. 


\section{Explorations of the possible epimerization of allyl-nickel species.}

We explored the possible epimerization of allyl-nickel species 6, 8 and 11 in the reaction mechanism. As shown in Figure S12, these processes all require unsurmountable barriers.

(a)

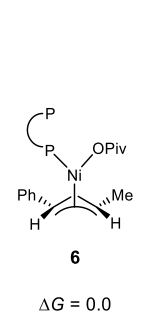

(b)
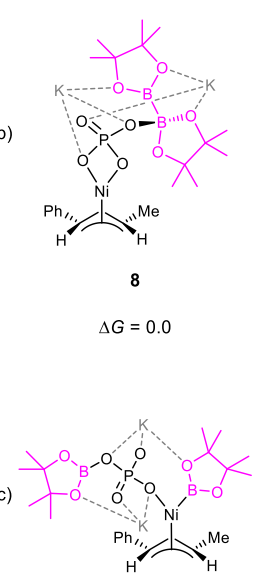

11

$\Delta G=0.0$
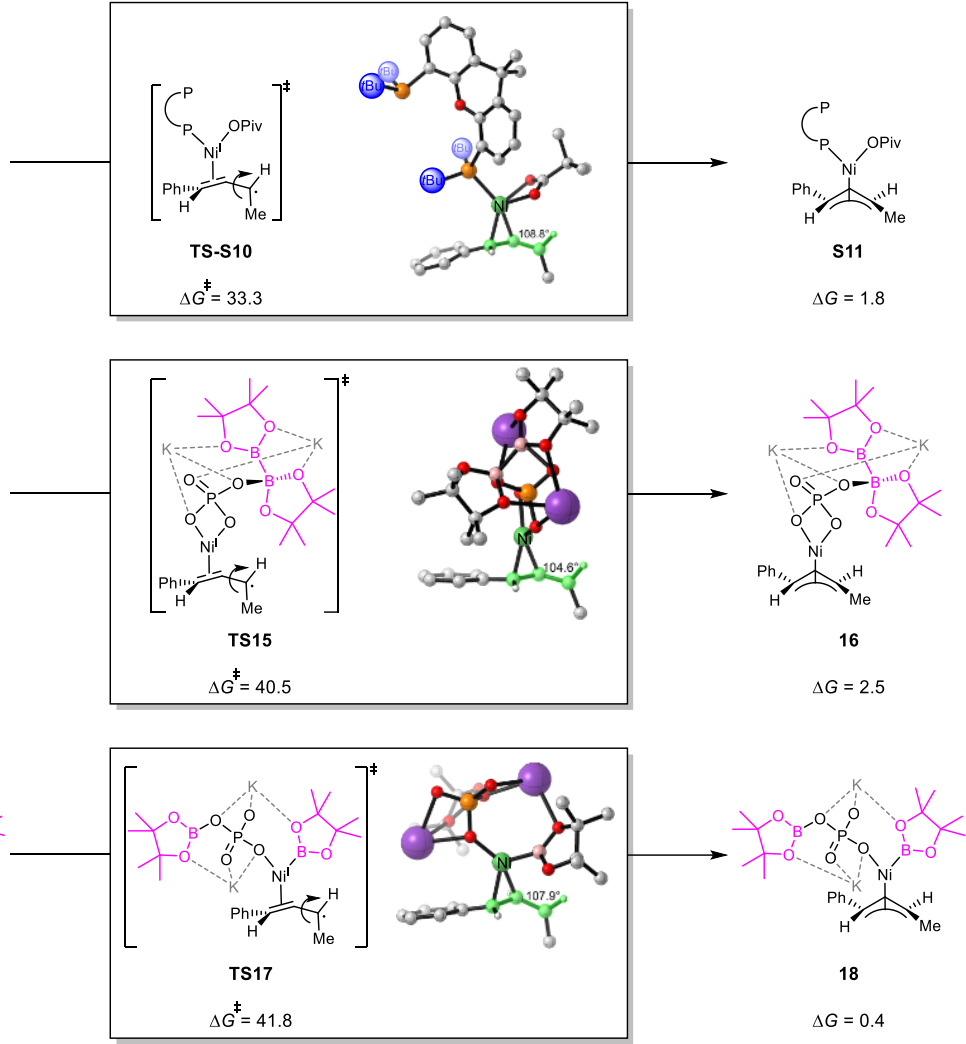

Figure S12. Computed barriers of allylic $\mathrm{C}-\mathrm{C}$ bond rotations. Free energies in toluene are in $\mathrm{kcal} / \mathrm{mol}$. 
12. Explorations of other possible $\mathrm{C}-\mathrm{B}$ bond formation pathways.

From intermediate 8, direct $\mathrm{C}-\mathrm{B}$ bond formation leading to borylation product can occur via TS-S12. This process requires an unsurmountable barrier $(71.2 \mathrm{kcal} / \mathrm{mol})$. The $\mathrm{C}-\mathrm{B}$ bond formation may also involve an additional equivalent of the $\mathrm{B}_{2} \mathrm{pin}_{2}-\mathrm{K}_{3} \mathrm{PO}_{4}$ complex. TS-S13 is the stereo-retentive transition state, and TS-S15 is the stereoinvertive transition state. Both transition states are over $30 \mathrm{kcal} / \mathrm{mol}$ higher than the preceding intermediate 8 . Therefore, these hypothetical processes are not operative.

(a)

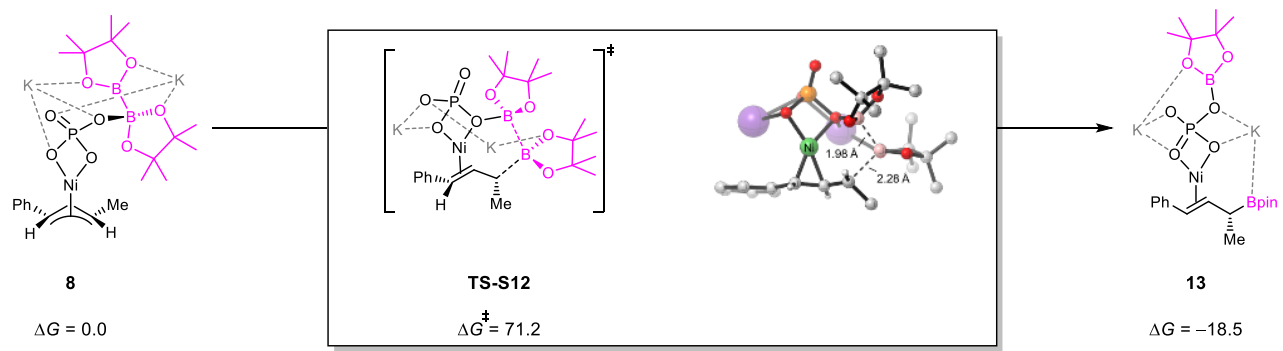

(b)
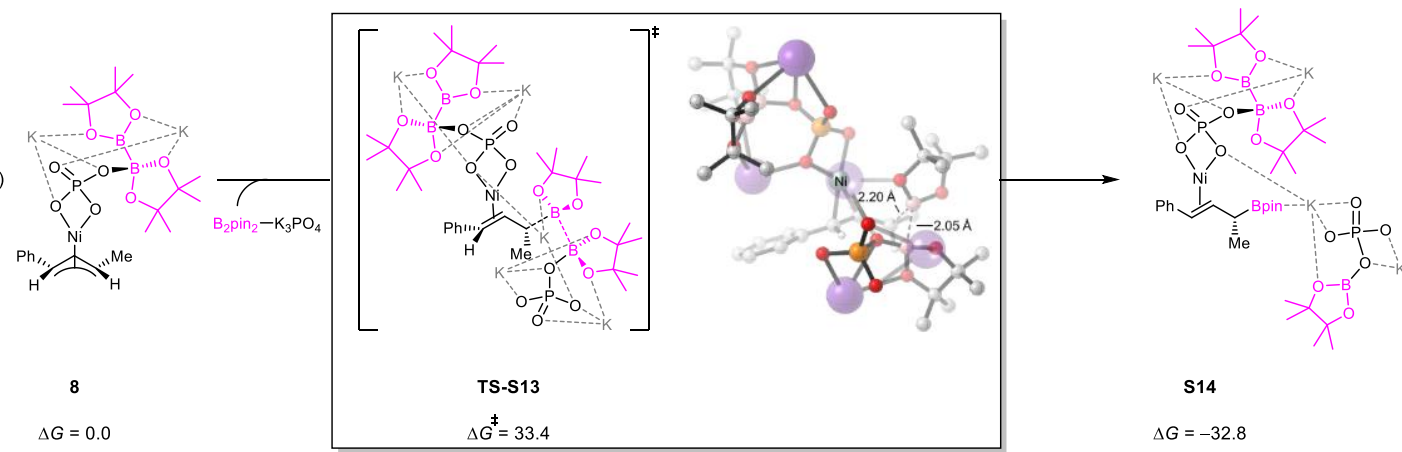

S14

$\Delta G=0.0$

$\Delta G^{\ddagger}=33.4$

$\Delta G=-32.8$

(c)
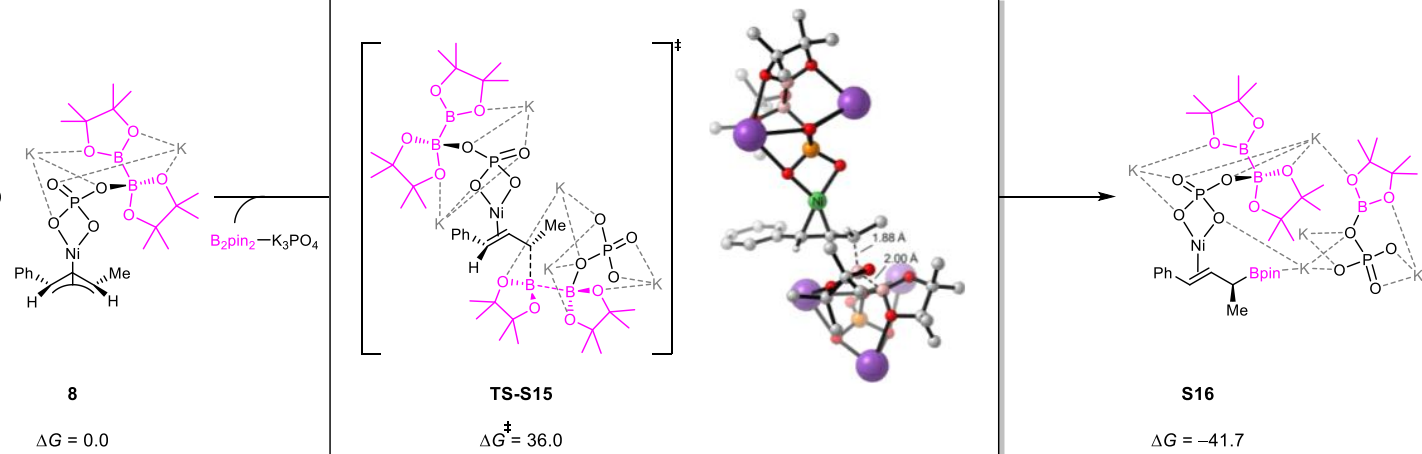

Figure S13. Computed barriers of additional C-B bond formation pathways from intermediate 8. Free energies in toluene are in $\mathrm{kcal} / \mathrm{mol}$. 
13. Explorations of free energy profile involving $\mathrm{BnPPh}_{2}$ ligand.

The computations of the free energy profile of borylation involving $\mathrm{BnPPh}_{2}$ ligand suggests that the transmetalation is the rate-determining step ( 8 to TS10) with an overall barrier of $23.4 \mathrm{kcal} / \mathrm{mol}$.

(a)
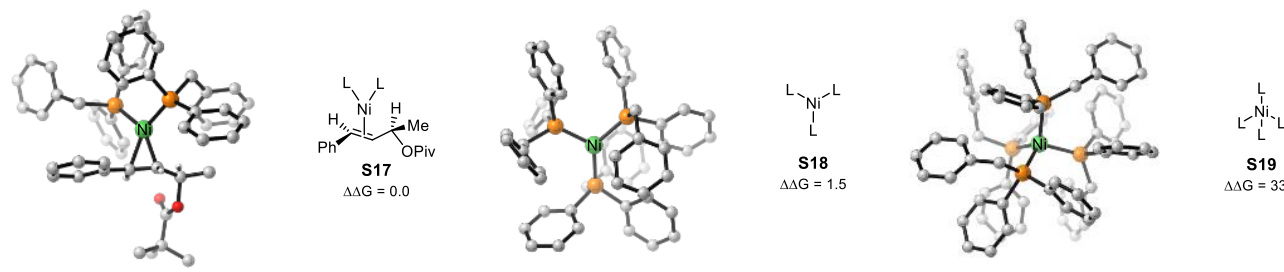

$\mathbf{S} 19$
$\Delta \Delta \mathrm{G}=33.9$

(b)
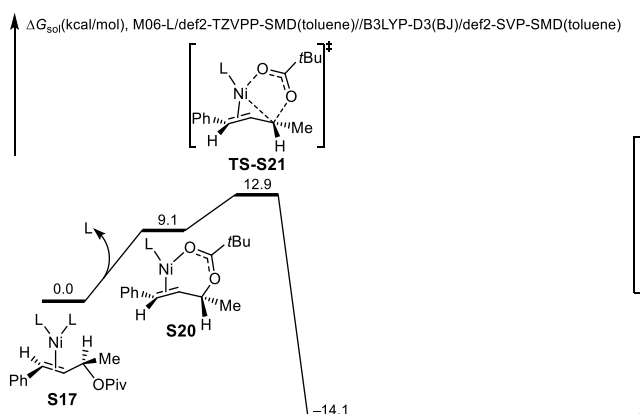

S17 OP

Transmetalation Reduc

Figure S14. (a) Possible Ni(0) complexes with $\mathrm{BnPPh}_{2}$ ligand. (b) DFT-computed Gibbs free energy changes of the $\mathrm{Ni} / \mathrm{BnPPh}_{2}$-catalyzed Miyaura borylation with allylic pivalate $(R)-1$ in toluene leading to the stereoretention product. 


\section{Explorations of free energy profile involving $\mathrm{P}(o-\mathrm{Tol})_{3}$ ligand.}

We found that the $\mathrm{Ni}\left(\mathrm{P}(o-\mathrm{Tol})_{3}\right)_{4}$ species $\mathbf{S 2 6}$ is not a stable intermediate, it will automatically dissociates one phosphorus coordination to form S27. The computations of the free energy profile of borylation involving $\mathrm{P}(o-\mathrm{Tol})_{3}$ ligand suggests that the transmetalation is the rate-determining step ( 8 to TS10) with an overall barrier of 23.4 $\mathrm{kcal} / \mathrm{mol}$.

(a)
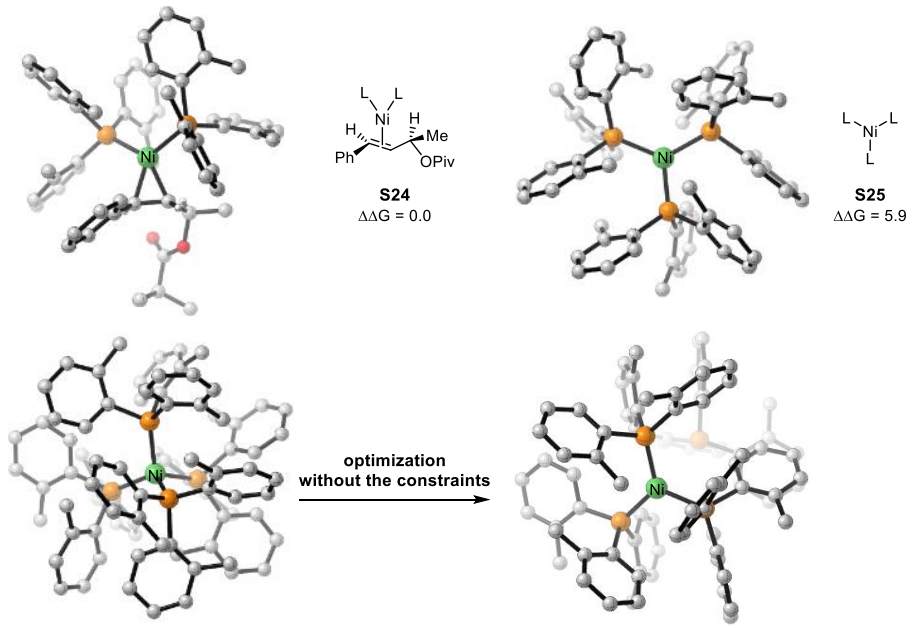

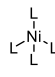
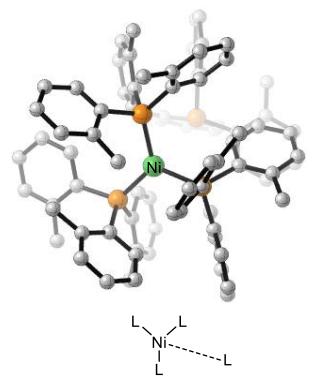

optimization with the constrained four $\mathrm{Ni}-\mathrm{P}$ bond

one phosphorus coordination automatically dissociates

(b) $\Delta \Delta G_{\text {sol }}(\mathrm{kcal} / \mathrm{mol}$ ), M06-L/def2-TZVPP-SMD(toluene)//B3LYP-D3(BJ)/def2-SVP-SMD(toluene)

L, ${ }^{\prime}$

Ph

S24 OPiv
0.0

$\left[\begin{array}{ccc}\mathrm{tBu} \\ 1\end{array}\right]^{\mathrm{L}}$

$\lambda$

TS-S29

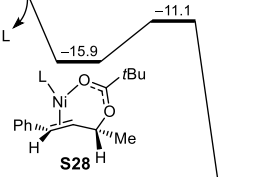

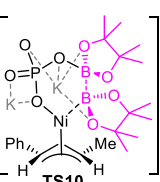

OPiv $\mathrm{B}_{2} \mathrm{Pin}_{2}-\mathrm{K}_{3} \mathrm{PO}_{4}$

Ph,
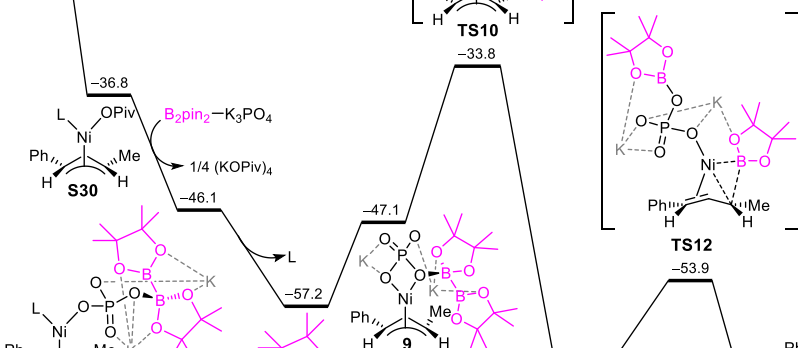

Ph,. 采, Me

s31

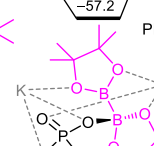

(1)

$=\mathrm{P}(\mathrm{o}-\mathrm{Tol})_{3}$

Oxidative

13

$\stackrel{\text { Reductive Elimination }}{\longrightarrow} \mid \stackrel{\text { Product Liberation }}{\longrightarrow}$

Figure S15. (a) Possible Ni $(0)$ complexes with $\mathrm{P}(o \text {-Tol })_{3}$ ligand. (b) DFT-computed Gibbs free energy changes of the $\mathrm{Ni} / \mathrm{P}(o-\mathrm{Tol})_{3}$-catalyzed Miyaura borylation with allylic pivalate $(R)-1$ in toluene leading to the stereoretention product. 


\section{Explorations of free energy profile involving $\mathrm{ABu}-\mathrm{XPhos}$ ligand.}

The computations of the free energy profile of borylation involving $t \mathrm{Bu}-\mathrm{XPhos}$ ligand suggests that the transmetalation is the rate-determining step ( 8 to TS10) with an overall barrier of $23.4 \mathrm{kcal} / \mathrm{mol}$.

(a)
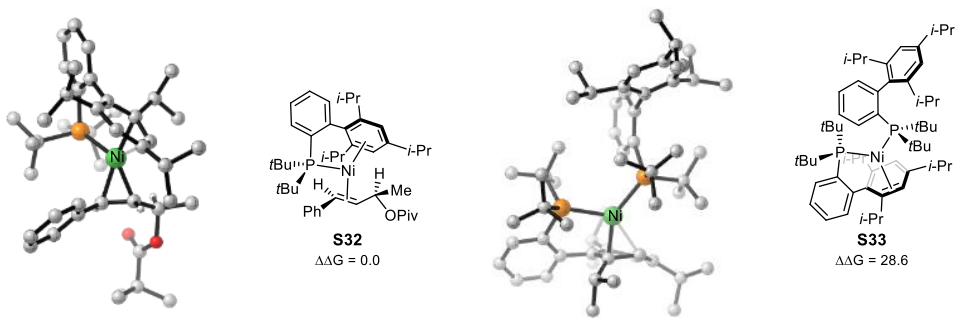

(b)
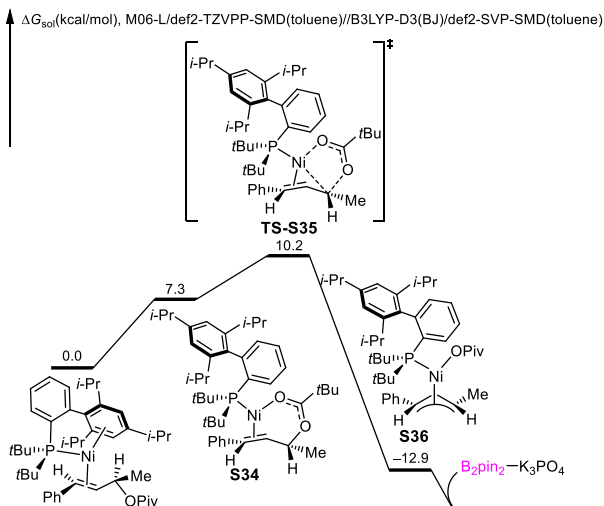

10.2
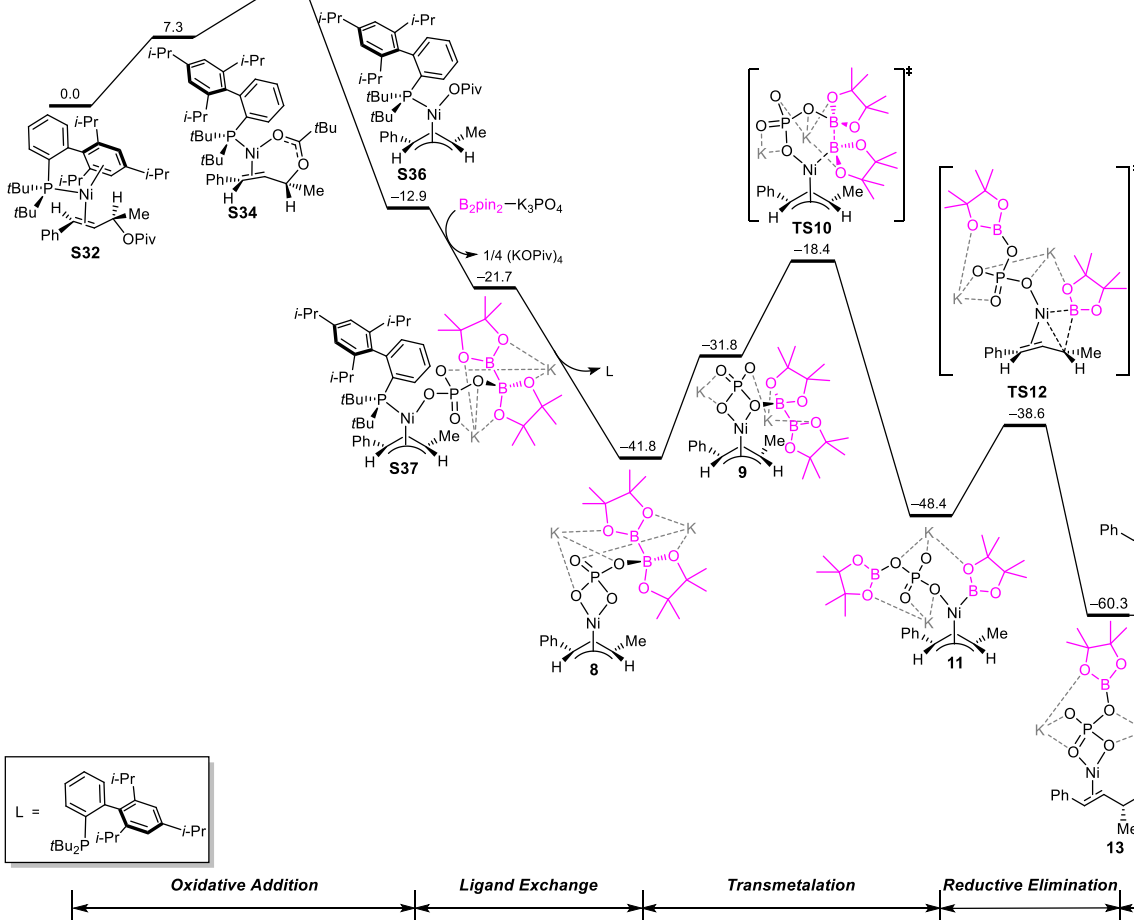

${ }_{34}^{\prime \prime \prime} M$

$\mathrm{H} / \mathrm{H}$
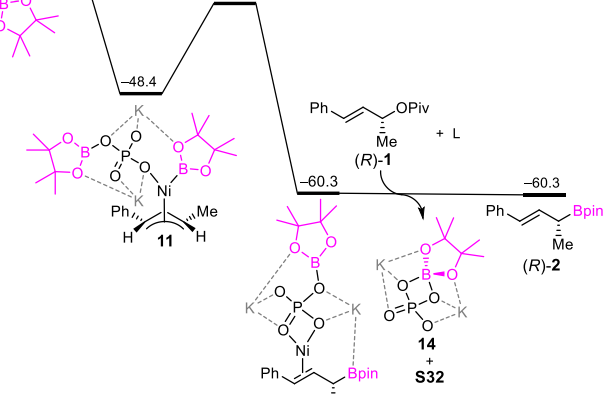

${ }_{13}^{\text {Me }}$

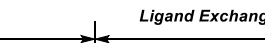

Transmetalation

Reductive Elimination Product Liberation

Figure S16. (a) Possible Ni(0) complexes with $t \mathrm{Bu}$-XPhos ligand. (b) DFT-computed Gibbs free energy changes of the $\mathrm{Ni} / \mathrm{tBu}$-XPhos-catalyzed Miyaura borylation with allylic pivalate $(R)-1$ in toluene leading to the stereoretention product. 


\section{Explorations of free energy profile involving dppb ligand.}

With dppb ligand, the $\mathrm{Ni}(\mathrm{dppb})_{2}$ complex $\mathbf{S 3 8}$ is $7.0 \mathrm{kcal} / \mathrm{mol}$ more stable than the Ni(dppb)(substrate) comlex S39. This stable Ni(0) species S38 significantly increases the overall barrier required for oxidative addition (S38 to TS-S41), which alters the rate-determining step. Therefore, for the borylation involving dppb ligand, the $\mathrm{Ni}(\mathrm{dppb})_{2}$ complex S38 is the resting state of catalytic cycle, and the rate-determining step is the $\mathrm{C}-\mathrm{O}$ bond activation with an overall barrier of $27.2 \mathrm{kcal} / \mathrm{mol}$ (S38 to TS-S41). This $27.2 \mathrm{kcal} / \mathrm{mol}$ barrier is higher than the $23.4 \mathrm{kcal} / \mathrm{mol}$ transmetalation barrier for the other studied phosphorus ligands (Figure 1 and Figures S14-S16), which explains the lower productivity with dppb ligand in experiments. ${ }^{1}$

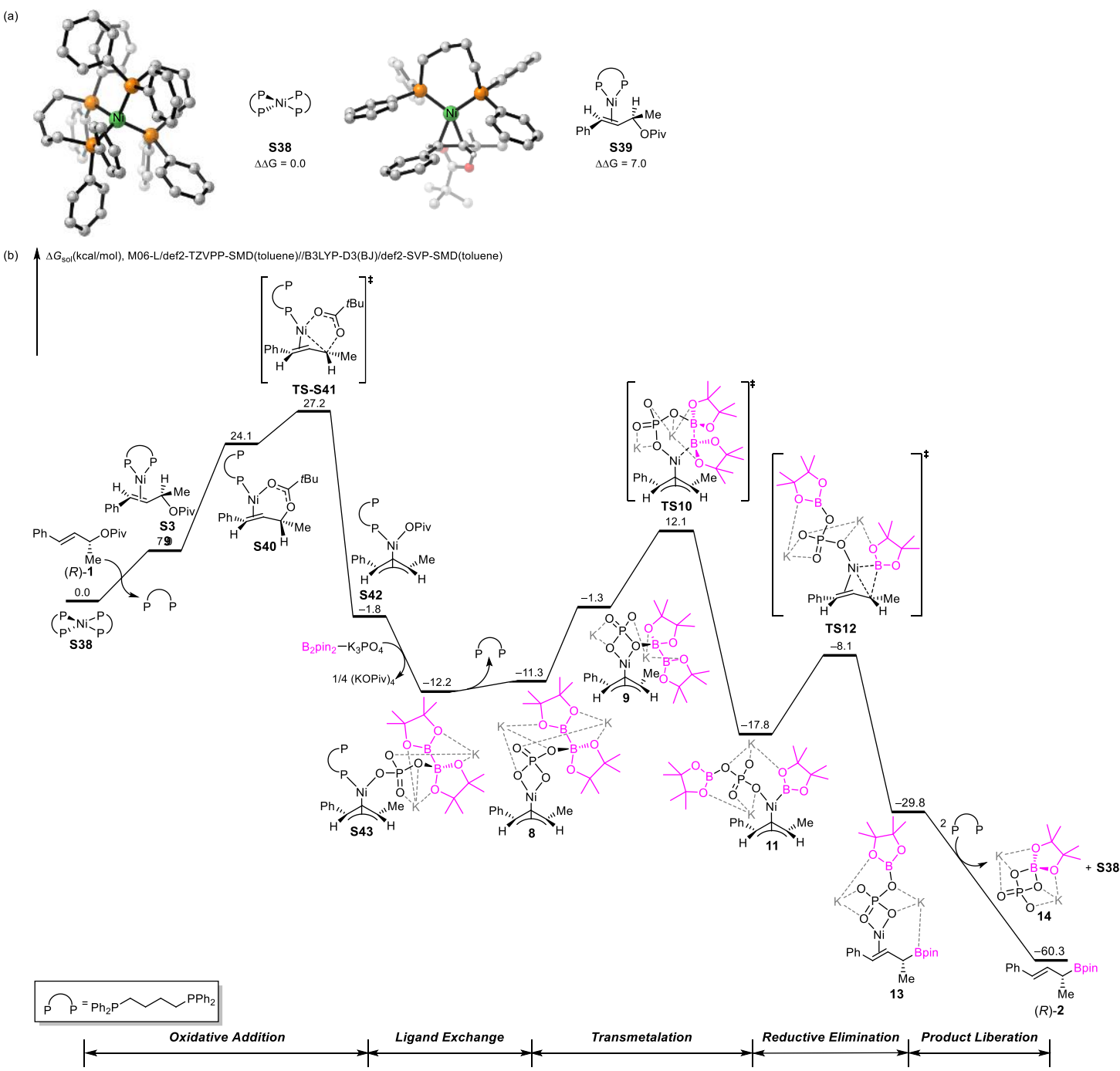

Figure S17. (a) Possible Ni(0) complexes with dppb ligand. (b) DFT-computed Gibbs free energy changes of the $\mathrm{Ni} /$ dppb-catalyzed Miyaura borylation with allylic pivalate $(R)-1$ in toluene leading to the stereoretention product. 
17. Explorations of ligand coordination types of stereo-invertive $\mathrm{C}-\mathrm{O}$ activation transition state in various solvents. We found that the stereo-invertive $\mathrm{C}-\mathrm{O}$ activation transition state prefers to have one phosphine coordination and one oxygen coordination of $t \mathrm{Bu}$-XantPhos ligand (TS-Conformation-B) in most solvents, except DCM and DMF which favor TS-Conformation-A slightly.

Table S2. Explorations of Ligand Coordination Types of Stereo-invertive C-O Activation Transition State ${ }^{a}$

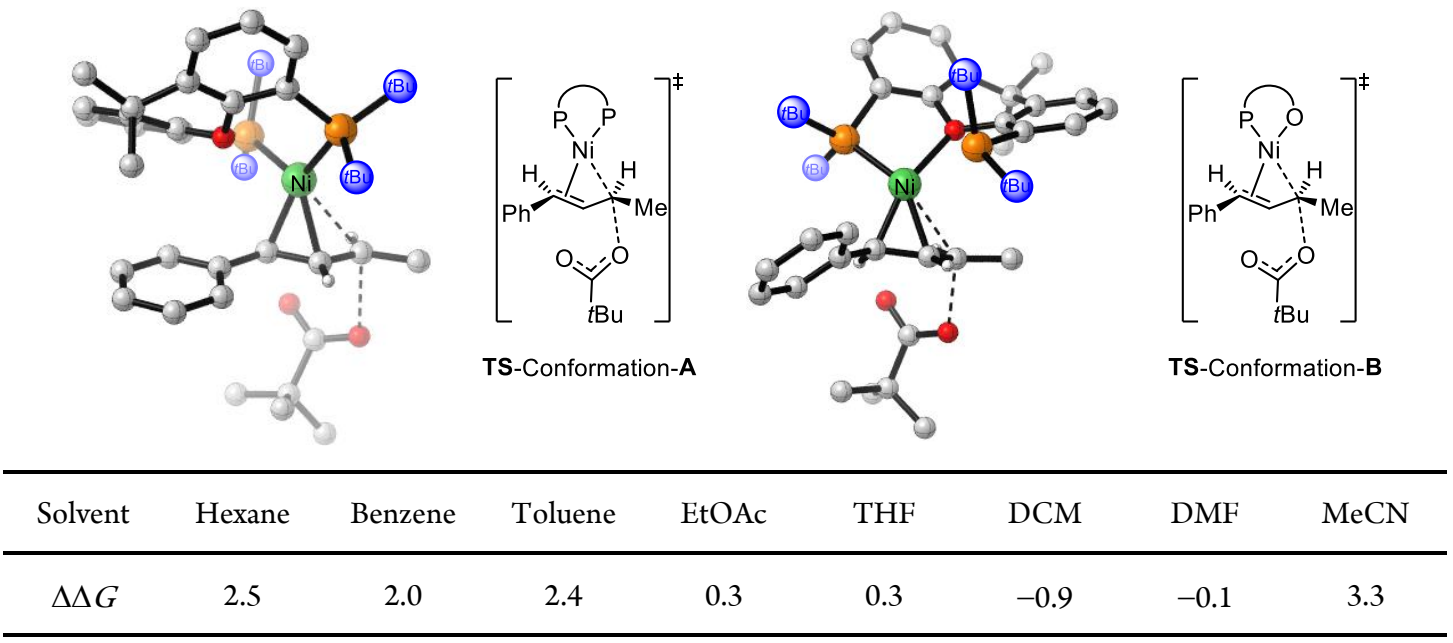

${ }^{a} \Delta \Delta G=\Delta G^{\ddagger}($ TS-Conformation-A $)-\Delta G^{\ddagger}($ TS-Conformation-B $)$ in kcal $/ \mathrm{mol}$.

\section{Origins of ligand effects on the stereospecificity in toluene.}

As shown in Scheme S1, the ligand has certain influence on the degree of stereoretention in toluene. ${ }^{1}$ To understand the ligand effects on the degree of stereoretention in toluene (Scheme S1), we compared the competing C-O bond activation transition states with the $t \mathrm{Bu}$-XantPhos and $\mathrm{BnPPh}_{2}$ ligands. The results are summarized in Figure $\mathrm{S} 18$. In the stereo-invertive transition states (TS19 and TS-S44), the nickel is bisligated to increase the nucleophilicity for the $\mathrm{S}_{\mathrm{N}}$ 2-type $\mathrm{C}-\mathrm{O}$ bond cleavage. While in the stereo-retentive transition states (TS5 and TS-S21), nickel is monoligated to accommodate the forming nickel-oxygen bond. Thus, the dissociation of one ligation is necessary changing from the stereo-invertive $\mathrm{C}-\mathrm{O}$ bond activation to the corresponding stereo-retentive process. This is the leading cause for the ligand effects on the stereospecificity in toluene. The $\mathrm{Ni} / t \mathrm{Bu}$-XantPhos catalyst dissociates the weak coordination of oxygen, while the $\mathrm{Ni} / \mathrm{BnPPh}_{2}$ catalyst must dissociate the strong coordination of phosphine (Figure $\mathrm{S} 18$ ).

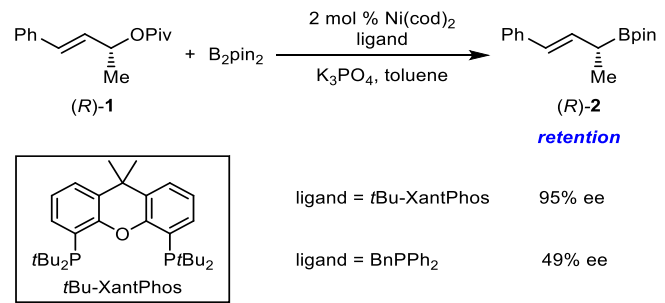

Scheme S1. Effects of Ligand on the Stereospecificity of Ni-Catalyzed Miyaura Borylation of Allylic Pivalates 


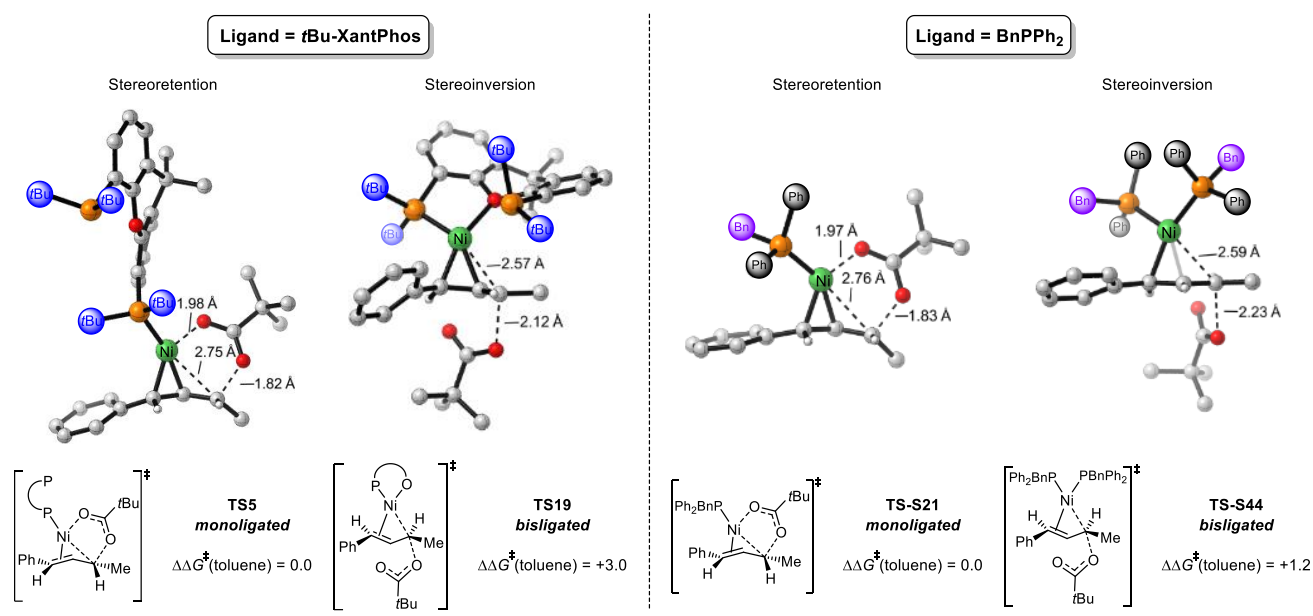

Figure S18. Relative Gibbs free energies and optimized structures of the Ni-mediated stereo-retentive and invertive allylic $\mathrm{C}-\mathrm{O}$ bond activation transition states with $t \mathrm{Bu}$-XantPhos and $\mathrm{BnPPh}_{2}$ ligands. Free energies in toluene are in $\mathrm{kcal} / \mathrm{mol}$.

To confirm that the binding energies of oxygen and phosphine are different in this catalytic system, we calculated the free energy required for the ligand dissociation from the $\mathrm{L}_{\mathrm{n}} \mathrm{Ni}$ (pivalate) complex (Scheme S2). The dissociation of the oxygen coordination from 3 is endergonic by $7.2 \mathrm{kcal} / \mathrm{mol}$ (Scheme S2a), while the dissociation of the $\mathrm{BnPPh}_{2}$ ligand from S17 is $19.1 \mathrm{kcal} / \mathrm{mol}$ endergonic (Scheme S2b). This difference supports the rationalization on the ligand affinity, and is consistent with the change of the stereospecificity between the two ligands.

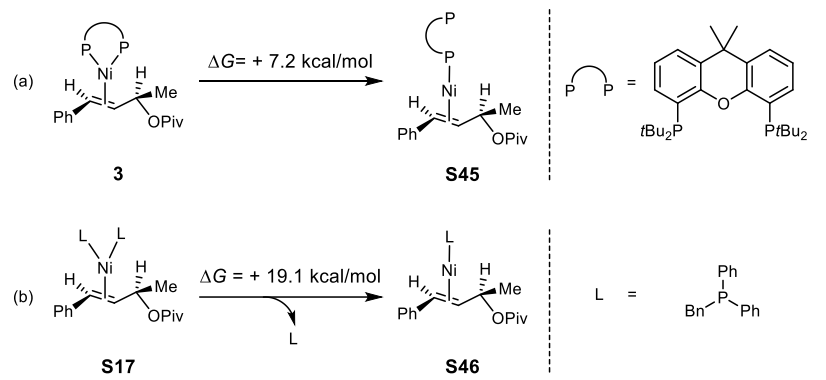

Scheme S2. Free Energy Changes in Toluene of Ligand Dissociation of $\mathrm{L}_{n} \mathrm{Ni}$ (Pivalate) Complexes

In order to provide direct confirmation for the proposed differences of ligand dissociation energies, we performed the distortion/interaction analysis ${ }^{2,3}$ on the transition states TS5 and TS-S21 as shown in Figure S19. Each transition state was separated into two fragments (the distorted catalyst and substrate), followed by single point energy calculations at the M06-L level of theory with def2-TZVPP basis set without the inclusion of solvation energy corrections on each distorted fragment. The energy differences between the distorted structures and optimized ground-state structures are the distortion energy of catalyst $\left(\Delta E_{\text {dist-cat }}\right)$ and substrate $\left(\Delta E_{\text {dist-sub }}\right)$, respectively. The interaction energy $\left(\Delta E_{\text {int }}\right)$ is the difference between the activation energy $\left(\Delta E_{\text {act }}\right)$ and the total distortion energy $\left(\Delta E_{\text {dist-cat }}+\Delta E_{\text {dist-sub }}\right)$. Comparing the two C-O bond activation transition states (TS5 and TS-S21), $\Delta E_{\text {dist-cat }}$ is the leading cause for the stereoselectivity (Figure S19). $\Delta E_{\text {dist-cat }}$ is $57.0 \mathrm{kcal} / \mathrm{mol}$ in TS-S21 and $49.8 \mathrm{kcal} / \mathrm{mol}$ in TS5, which is due to the dissociation of a strong phosphine coordination in TS-S21. The distortion/interaction analysis confirms the proposed differences of ligand dissociation. 


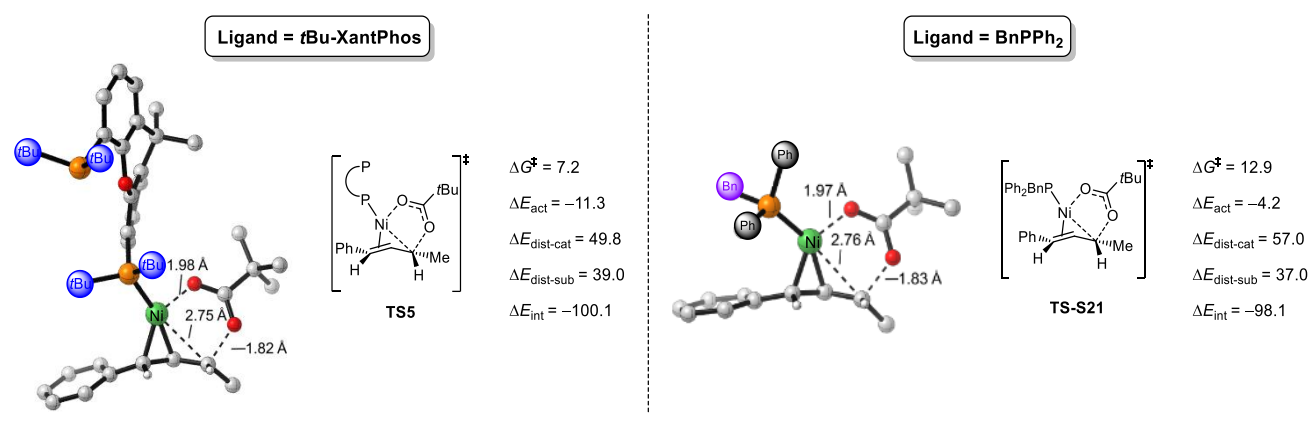

Figure S19. Distortion/interaction analysis of TS5 and TS-S21. Energies are in kcal/mol.

19. Explorations of ligand coordination of related metal/ $t \mathrm{Bu}$-XantPhos complexes with $\mathrm{X}$-ray crystal structure.

To prove the reliability of our computational approach for describing the coordinations of $t \mathrm{Bu}$-XantPhos ligand, we optimized several metal/ $t \mathrm{Bu}$-XantPhos complexes with X-ray crystal structure. The computed structures nicely converged with the experimental structures, which validate our computational approach.
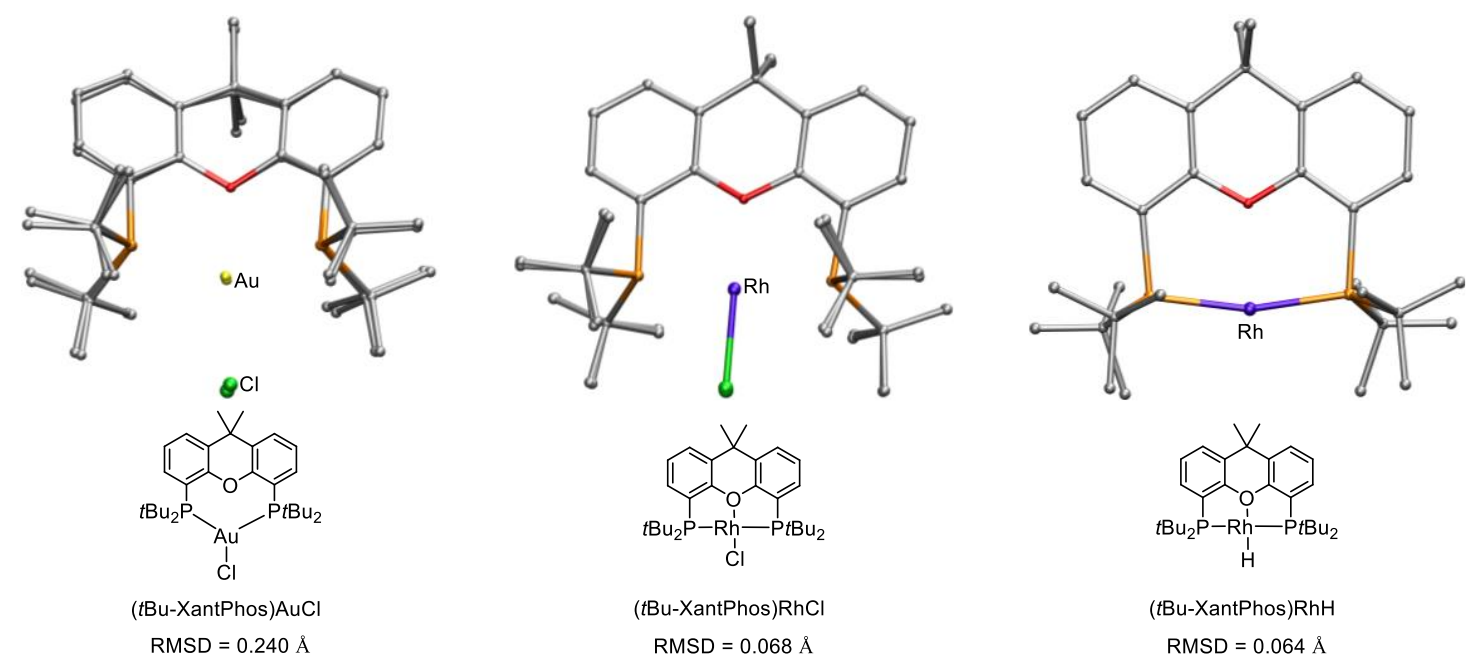

Figure S20. Comparsion between computed and X-ray crystal ${ }^{4,5}$ structures for selected metal/ $t \mathrm{Bu}$-XantPhos complexes. 


\section{Explorations of the alternative $\mathrm{C}-\mathrm{O}$ bond activation transition states.}

To ensure the favorable $\mathrm{C}-\mathrm{O}$ bond activation transition states are located, we also explored a number of alternative transition states. These high-energy transition states are not operative for the $\mathrm{Ni} / t \mathrm{Bu}$-XantPhos-catalyzed $\mathrm{C}-\mathrm{O}$ bond activation of allylic pivalates.

\section{(a) Retentive $\mathrm{C}-\mathrm{O}$ bond activation transition states involving bidentate coordinations of $t \mathrm{Bu}-\mathrm{X}$ antPhos ligand.}

We have explored the possible bidentate coordinations of $t \mathrm{Bu}$-XantPhos ligand for the retentive $\mathrm{C}-\mathrm{O}$ bond activation transition states. For the retentive $\mathrm{C}-\mathrm{O}$ bond activation transition states, the bidentate coordination of $t \mathrm{Bu}-\mathrm{XantPhos}$ ligand are not feasible, because the cyclic pre-intermediates of the retentive $\mathrm{C}-\mathrm{O}$ bond activations do not exist. With constraints on two $\mathrm{Ni}-\mathrm{P}$ bonds, the cyclic $\mathrm{LNi}$ (substrate) intermediate with the bidentate coordination of the $t \mathrm{Bu}$ XantPhos ligand is optimized to be S47. Once the constraints are removed, one phosphine coordination dissociates, and S47 is optimized to be the monoligated species S48. Therefore, the bisligated cyclic intermediate S47 is not a saddle point of the potential energy surface, and the corresponding $\mathrm{C}-\mathrm{O}$ bond activation transition state does not exist. Similarly, the attempts to locate the cyclic intermediate with the coordinations of one phosphine and one oxygen of the ligand give the similar results, $\mathbf{S 4 9}$ is optimized to be $\mathbf{S 5 0 .}$

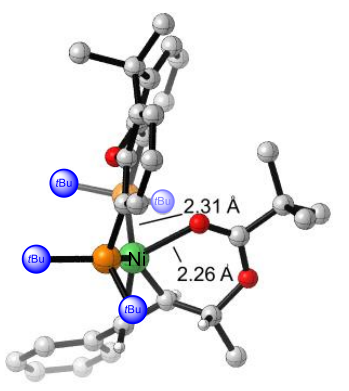

optimization with the constrained two $\mathrm{Ni}-\mathrm{P}$ bonds

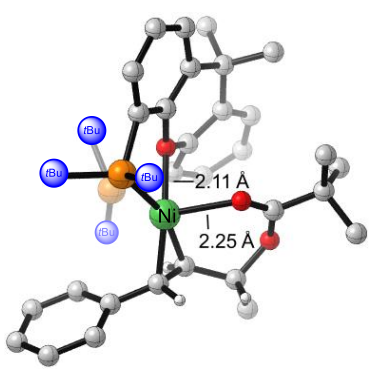

optimization with the constrained $\mathrm{Ni}-\mathrm{P}$ and $\mathrm{Ni}-\mathrm{O}$ bonds
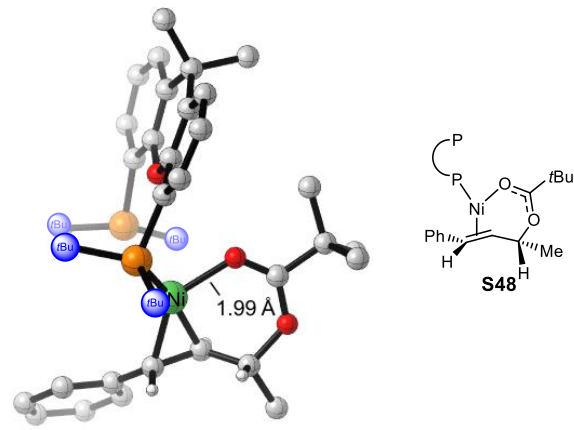

S47

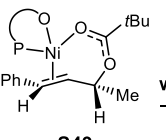

optimization without the constraints

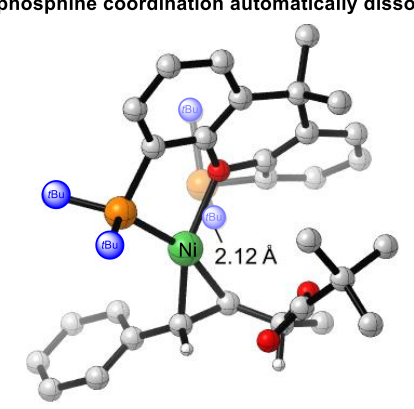

oxygen coordination automatically dissociates

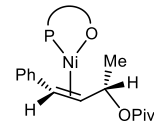

S50

Figure S21. Explorations of possible bidentate coordinations of retentive C-O bond activation transition states. 
(b) Invertive $\mathrm{C}-\mathrm{O}$ bond activation transition states assisted by potassium phosphate.

We hypothesized that the involvement of a potassium cation can stablize the dissociating pivalate anion in TS19. The addition of the $\mathrm{B}_{2} \mathrm{pin}_{2}-\mathrm{K}_{3} \mathrm{PO}_{4}$ complex leads to TS-S51. TS-S51 is indeed $15.4 \mathrm{kcal} / \mathrm{mol}$ more favorable than TS19 in terms of enthalpy, due to the favorable electrostatic interaction between potassium and the dissociating pivalate anion. However, the involvement of the extra $\mathrm{B}_{2} \mathrm{pin}_{2}-\mathrm{K}_{3} \mathrm{PO}_{4}$ complex is significantly unfavorable in terms of entropy $(\Delta \mathrm{S}=$ $-50.6 \mathrm{cal} /(\mathrm{mol} \cdot \mathrm{K})$ for TS-S51, while $\Delta \mathrm{S}=10.9 \mathrm{cal} /(\mathrm{mol} \cdot \mathrm{K})$ for TS19). This significant entropy penalty makes TSS51 $2.9 \mathrm{kcal} / \mathrm{mol}$ less favorable than TS19 in terms of Gibbs free energy.

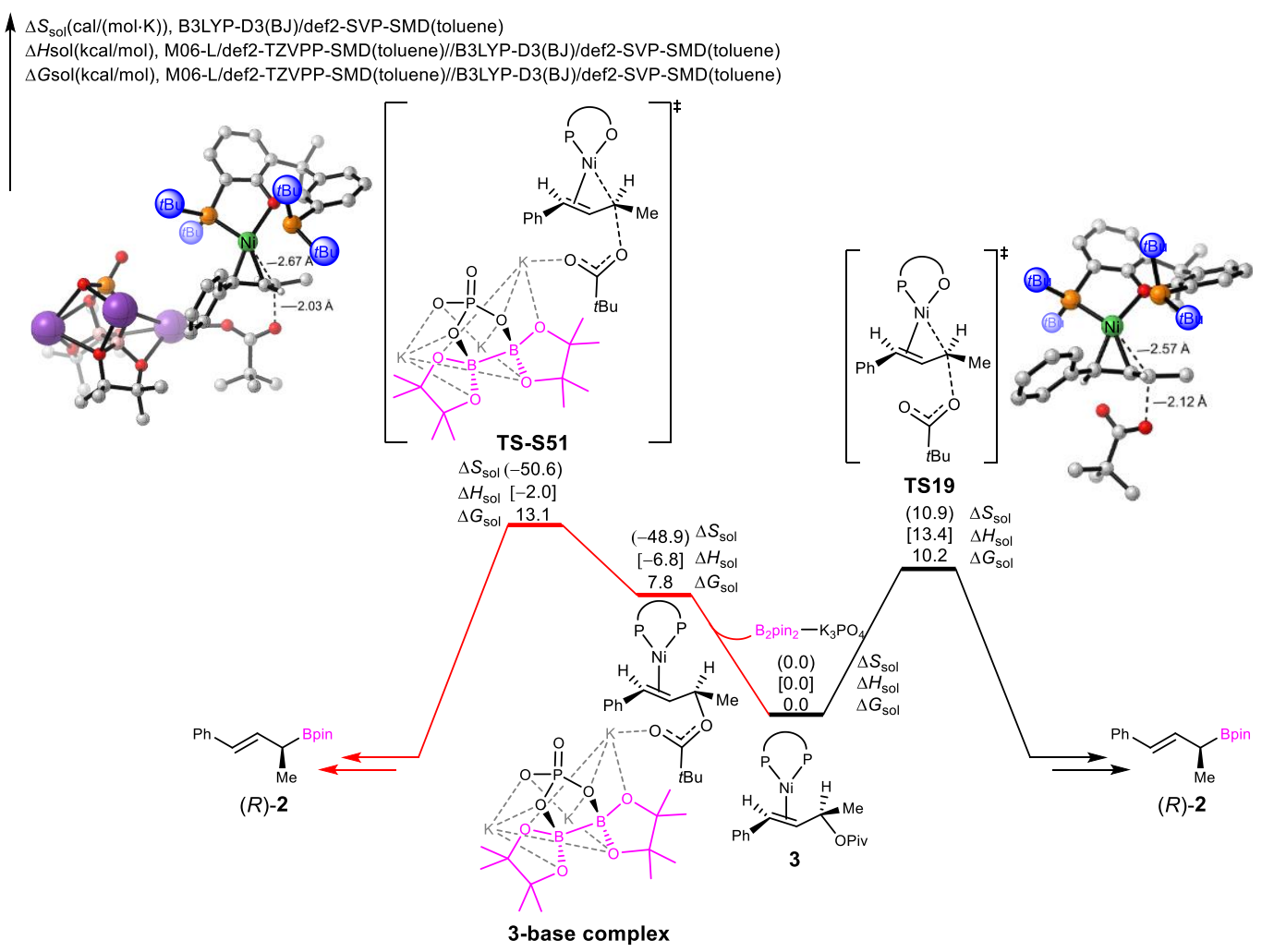

Figure S22. Invertive C-O bond activation with (red path, via TS-S51) or without (black path, via TS19) the assistance of $\mathrm{B}_{2} \mathrm{pin}_{2}-\mathrm{K}_{3} \mathrm{PO}_{4}$ complex. Gibbs free energies and enthalpies in toluene are in $\mathrm{kcal} / \mathrm{mol}$, entropy in toluene is in $\mathrm{cal} /(\mathrm{mol} \cdot \mathrm{K})$. 
(c) The alternative $\mathrm{Ni} / t \mathrm{Bu}$-XantPhos-catalyzed $\mathrm{C}-\mathrm{O}$ bond activation transition states.

With the same active nickel catalyst, the favorable transition states TS5 and TS19 can have isomeric transition states (TS5 vs. TS-S52, TS19 vs. TS-S53). These additional transition states are less favorable.
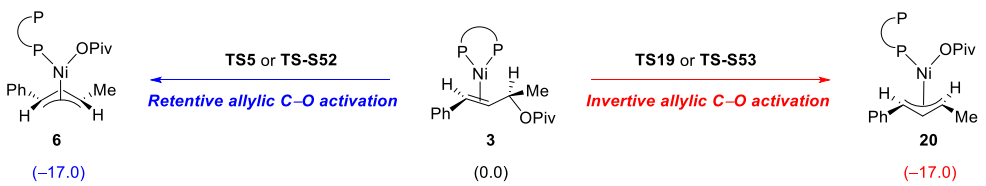

Retentive $\mathrm{C}-\mathrm{O}$ activation transition states
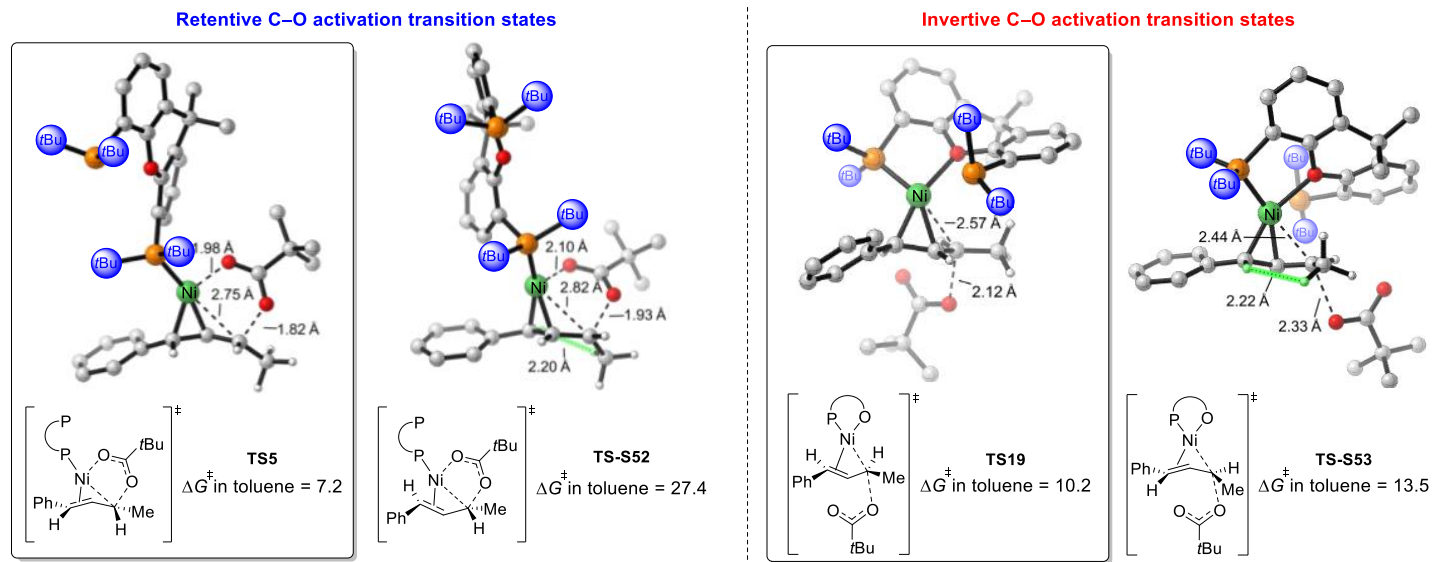

Figure S23. Alternative $\mathrm{Ni} / \mathrm{tBu}$-XantPhos-catalyzed $\mathrm{C}-\mathrm{O}$ bond activation transition states. Free energies in toluene are in $\mathrm{kcal} / \mathrm{mol}$. 
21. Calculations of stereospecificity $\left(\Delta \Delta G_{\text {exp }}\right.$ in Table 1$)$ in a series of solvents based on Watson's experimetnal results.

For substrate $(R)-1$ with X\% ee, we can get $(50+\mathrm{X} / 2) \%(R)-1$ and $(50-\mathrm{X} / 2) \%(S)-1$, both $(R)-1$ and $(S)-1$ can undergo stereo-retentive or stereo-invertive borylation. From $(R)-1, \mathrm{C}-\mathrm{O}$ bond activation with stereoretention delivers $\mathbf{A}$, while stereo-invertive $\mathrm{C}-\mathrm{O}$ bond activation produces $\mathbf{B}$. From $(S)$-1, stereo-retentive $\mathrm{C}-\mathrm{O}$ bond activation gives $\mathbf{C}, \mathbf{D}$ is produced with stereoinversion. Both $\mathbf{A}$ and $\mathbf{D}$ are $(R)-\mathbf{2}, \mathbf{B}$ and $\mathbf{C}$ are $(S)-\mathbf{2}$ (Scheme S3), delivering product $(R)-2$ with Y\% ee.
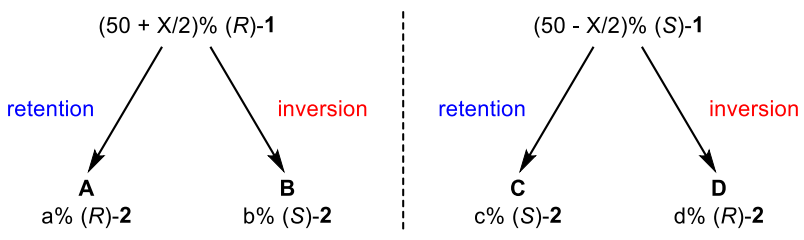

\section{Scheme S3. Stereo-retentive or Stereo-invertive C-O Bond Activation From $(R)$-1 or $(S)$-1}

Enantioselectivity (E) equals to $r_{\text {ret }} / r_{\text {inv }}, r_{\text {ret }}$ is the rate delivering stereoretention product, $r_{\text {inv }}$ is the rate producing product with stereoinversion. From above analysis, we can get:

$(\mathrm{a}+\mathrm{b})-(\mathrm{c}+\mathrm{d})=\mathrm{X}$

eqn 1

$(\mathrm{a}+\mathrm{d})-(\mathrm{b}+\mathrm{c})=\mathrm{Y}$

eqn 2

$\mathrm{E}=\mathrm{r}_{\text {ret }} / \mathrm{r}_{\text {inv }}=\mathrm{a} / \mathrm{b}=\mathrm{c} / \mathrm{d}=(\mathrm{a}-\mathrm{c}) /(\mathrm{b}-\mathrm{d})=2 *(\mathrm{a}-\mathrm{c}) / 2 *(\mathrm{~b}-\mathrm{d})=($ eqn $1+$ eqn 2$) /($ eqn $1-$ eqn 2$)=(\mathrm{X}+\mathrm{Y}) /(\mathrm{X}-$

$\mathrm{Y})=\left(\mathrm{ee}_{(R)-1}+\mathrm{ee}_{(R)-2}\right) /\left(\mathrm{ee}_{(R)-1}-\mathrm{ee}_{(R)-2}\right)$

eqn 3

$\Delta \Delta G_{\text {exp }}=\Delta G_{\mathrm{Inv}}-\Delta G_{\mathrm{ret}}=R T \ln \left(\mathrm{r}_{\mathrm{ret}} / \mathrm{r}_{\mathrm{inv}}\right)=R \operatorname{Tln}\left(\left(\mathrm{ee}_{(R)-1}+\mathrm{ee}_{(R)-2}\right) /\left(\mathrm{ee}_{(R)-1}-\mathrm{ee}_{(R)-2}\right)\right)$

eqn 4

$R$ is the gas constant $(8.314 \mathrm{~J} /(\mathrm{mol} \cdot \mathrm{K})), \mathrm{T}=298.15 \mathrm{~K}, \Delta G_{\mathrm{inv}}$ is the Gibbs free energy change leading to product with stereoinversion, $\Delta G_{\text {ret }}$ is the Gibbs free energy change leading to product with stereoretention.

(a) For Hexane: $(R)-1=98 \%$ ee, $(R)-2=97 \%$ ee, thus, $\Delta \Delta G_{\text {exp }}=R \operatorname{Tln}\left(\left(\mathrm{ee}_{(R)-1}+\mathrm{ee}_{(R)-2}\right) /\left(\mathrm{ee}_{(R)-1}-\mathrm{ee}_{(R)-2}\right)\right)=3.1$ $\mathrm{kcal} / \mathrm{mol}$.

(b) For Benzene: $(R)-\mathbf{1}=98 \%$ ee, $(R)-2=95 \%$ ee, thus, $\Delta \Delta G_{\exp }=R \ln \left(\left(\mathrm{ee}_{(R)-1}+\mathrm{ee}_{(R)-2}\right) /\left(\mathrm{ee}_{(R)-1}-\mathrm{ee}_{(R)-2}\right)\right)=2.5$ $\mathrm{kcal} / \mathrm{mol}$.

(c) For Toluene: $(R)-1=98 \%$ ee, $(R)-2=91 \%$ ee, thus, $\Delta \Delta G_{\exp }=R \ln \left(\left(\mathrm{ee}_{(R)-1}+\mathrm{ee}_{(R)-2}\right) /\left(\mathrm{ee}_{(R)-1}-\mathrm{ee}_{(R)-2}\right)\right)=2.0$ $\mathrm{kcal} / \mathrm{mol}$.

(d) For EtOAc: $(R)-\mathbf{1}=98 \%$ ee, $(R)-2=92 \%$ ee, thus, $\Delta \Delta G_{\text {exp }}=R \ln \left(\left(\mathrm{ee}_{(R)-1}+\mathrm{ee}_{(R)-2}\right) /\left(\mathrm{ee}_{(R)-1}-\mathrm{ee}_{(R)-2}\right)\right)=2.0$ $\mathrm{kcal} / \mathrm{mol}$.

(e) For THF: $(R)-1=98 \%$ ee, $(R)-2=88 \%$ ee, thus, $\Delta \Delta G_{\text {exp }}=R \ln \left(\left(\mathrm{ee}_{(R)-1}+\mathrm{ee}_{(R)-2}\right) /\left(\mathrm{ee}_{(R)-1}-\mathrm{ee}_{(R)-2}\right)\right)=1.7 \mathrm{kcal} / \mathrm{mol}$.

(f) For DCM: $(R)-1=98 \%$ ee, $(R)-2=39 \%$ ee, thus, $\Delta \Delta G_{\text {exp }}=R \ln \left(\left(\mathrm{ee}_{(R)-1}+\mathrm{ee}_{(R)-2}\right) /\left(\mathrm{ee}_{(R)-1}-\mathrm{ee}_{(R)-2}\right)\right)=0.5$ $\mathrm{kcal} / \mathrm{mol}$.

(g) For DMF: $(R)-1=98 \%$ ee, $(R)-2=26 \%$ ee, thus, $\Delta \Delta G_{\text {exp }}=R \operatorname{Tln}\left(\left(\mathrm{ee}_{(R)-1}+\mathrm{ee}_{(R)-2}\right) /\left(\mathrm{ee}_{(R)-1}-\mathrm{ee}_{(R)-2}\right)\right)=0.3$ $\mathrm{kcal} / \mathrm{mol}$.

(h) For MeCN: $(R)-1=98 \%$ ee, $(R)-2=-85 \%$ ee, thus, $\Delta \Delta G_{\text {exp }}=R \ln \left(\left(\mathrm{ee}_{(R)-1}+\mathrm{ee}_{(R)-2}\right) /\left(\mathrm{ee}_{(R)-1}-\mathrm{ee}_{(R)-2}\right)\right)=-1.6$ $\mathrm{kcal} / \mathrm{mol}$. 
22. Computations of selectivities with additional functionals.

Table S3. Computations of Selectivities with M06- $\mathrm{L}^{6}$ and Additional Functionals (M06 ${ }^{7}$ B3LYP $\left.{ }^{8}-\mathrm{D} 3(\mathrm{BJ})^{9}\right)$. Free Energies Are in $\mathrm{kcal} / \mathrm{mol}$

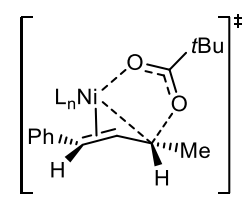

TS22

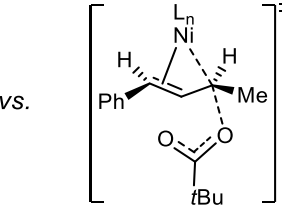

TS23

\begin{tabular}{c|cccccccc}
\multicolumn{8}{c}{ Retentive C-O activation } & Invertive C-O activation \\
\hline Solvent & Hexane $^{a}$ & Benzene $^{a}$ & Toluene $^{a}$ & EtOAc $^{a}$ & THF $^{a}$ & DCM $^{a}$ & DMF $^{a}$ & MeCN $^{b}$ \\
\hline$\Delta \Delta G_{\text {cal }}($ Method A) & 4.3 & 3.5 & 3.0 & 2.7 & 2.3 & 1.9 & 1.8 & -3.0 \\
$\Delta \Delta G_{\text {cal }}($ Method B) & 8.5 & 7.8 & 7.3 & 6.7 & 6.4 & 5.1 & 5.6 & -1.8 \\
$\Delta \Delta G_{\text {cal }}($ Method C) & 3.4 & 2.4 & 1.9 & 1.0 & 0.6 & 0.0 & 0.0 & -2.5 \\
\hline
\end{tabular}

${ }^{a} \mathrm{~L}=t \mathrm{Bu}$-XantPhos, $\mathrm{n}=1 . \Delta \Delta G_{\text {cal }}=\Delta G^{\ddagger}(\mathrm{TS} 23)-\Delta G^{\ddagger}(\mathrm{TS} 22)$.

${ }^{b} \mathrm{~L}=\mathrm{MeCN}, \mathrm{n}=1$ for TS22, $\mathrm{n}=2$ for TS23.

Method A: M06-L/def2-TZVPP ${ }^{10,12}$-SMD(solvent)//B3LYP-D3(BJ)/def2-SVP ${ }^{10}$ SMD $^{11}$ (solvent).

Method B: M06/def2-TZVPP-SMD(solvent)//B3LYP-D3(BJ)/def2-SVP-SMD(solvent).

Method C: B3LYP-D3(BJ)/def2-TZVPP-SMD(solvent)//B3LYP-D3(BJ)/def2-SVP-SMD(solvent).

\section{Dipole moment calculations in a series of solvents.}

We have performed dipole moment calculations on reactant and transition states (both retention and inversion) in a series of solvents (Table S4). For all the solvents studied, the stereo-retentive $\mathrm{C}-\mathrm{O}$ bond activation transition state (TS22) has limited dipole moment, while the dipole moment of the stereo-invertive $\mathrm{C}-\mathrm{O}$ bond activation transition state (TS23) is significantly large. From $\mathrm{L}_{n} \mathrm{Ni}$ (pivalate) to the stereo-retentive transition state, the dipole moment decreases. This suggests that the retentive $\mathrm{C}-\mathrm{O}$ bond activation would be favorable in low-polar solvents, which is consistent with experimental observations. For the stereo-invertive $\mathrm{C}-\mathrm{O}$ bond activation, the dipole moment increases and the process is favored in high-polar solvents.

Table S4. DFT-Calculated Dipole Moment of $\mathrm{L}_{\mathrm{n}} \mathrm{Ni}$ (substrate) Species and C-O Bond Activation Transition States in A Series of Solvents.

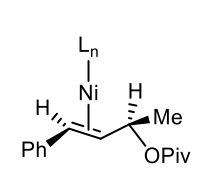

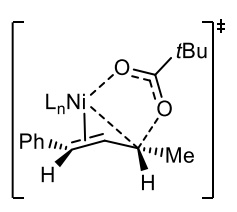

TS22

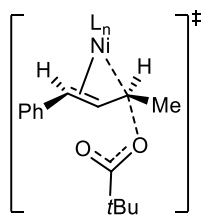

TS23

\begin{tabular}{c|ccccccccc}
\hline & solvent & Hexane $^{a}$ & Benzene $^{a}$ & Toluene $^{a}$ & EtOAc $^{a}$ & THF $^{a}$ & DCM $^{a}$ & DMF $^{a}$ & MeCN $^{b}$ \\
\hline \multirow{3}{*}{$\mu / \mathrm{D}$} & $\mathrm{L}_{\mathbf{n}} \mathrm{Ni}$ (pivalate) & 5.76 & 5.94 & 5.98 & 6.61 & 6.71 & 6.81 & 9.95 & 11.53 \\
& $\mathrm{TS22}$ & 4.87 & 5.05 & 5.10 & 5.84 & 5.98 & 6.21 & 6.57 & 4.78 \\
& $\mathrm{TS23}$ & 13.05 & 13.25 & 13.29 & 14.38 & 14.53 & 17.00 & 17.44 & 19.86 \\
\hline
\end{tabular}

${ }^{a} \mathrm{~L}=t \mathrm{Bu}$-XantPhos, $\mathrm{n}=1$.

${ }^{b} \mathrm{~L}=\mathrm{MeCN}, \mathrm{n}=2$ for $\mathrm{L}_{\mathrm{n}} \mathrm{Ni}$ (pivalate) and $\mathrm{TS} 23, \mathrm{n}=1$ for $\mathrm{TS} 22$. 
24. Computed stereospecificity in additional solvents.

Table S5. Explorations of Ligand Coordination Types of Stereo-invertive C-O Activation Transition State ${ }^{a}$

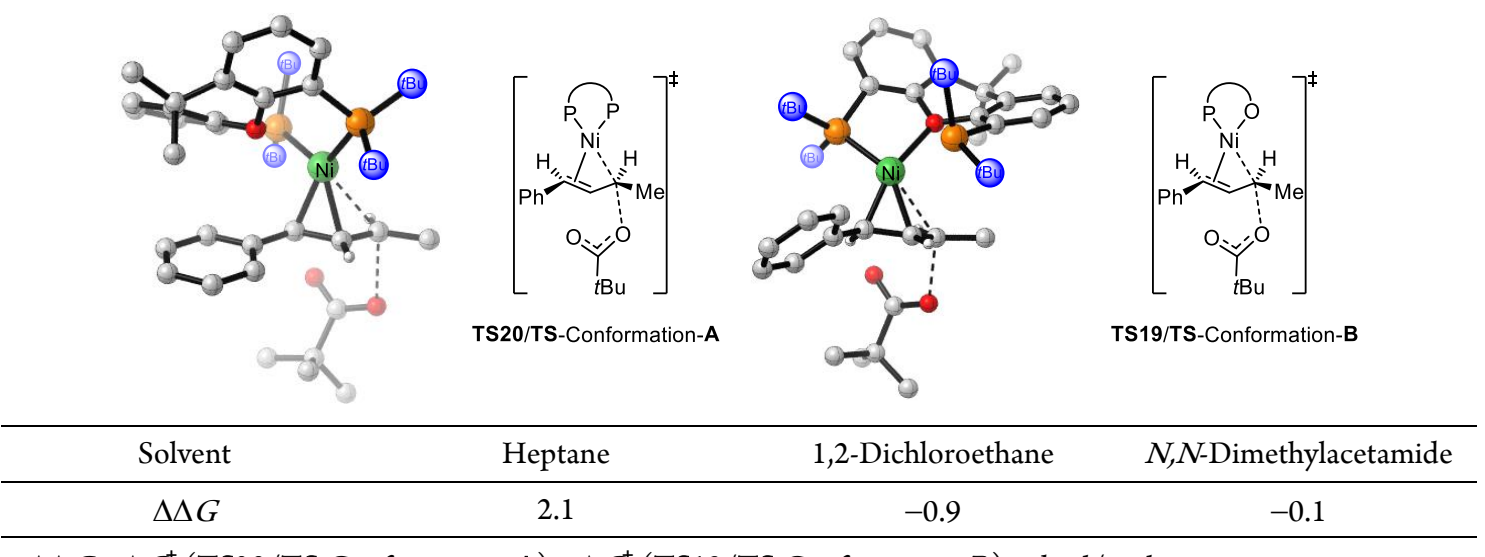

${ }^{a} \Delta \Delta G=\Delta G^{\ddagger}($ TS20/TS-Conformation-A $)-\Delta G^{\ddagger}($ TS19/TS-Conformation-B $)$ in kcal $/ \mathrm{mol}$.

Table S6. Computed Stereospecificity in Additional Solvents. Free Energies Are in kcal/mol

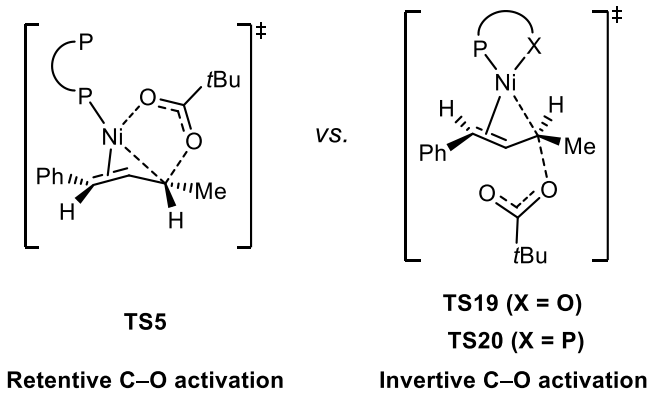

\begin{tabular}{cccc}
\hline Solvent & Heptane & 1,2-Dichloroethane & $N, N$-Dimethylacetamide \\
\hline $\mathcal{E}_{\mathrm{r}}^{a}$ & 1.92 & 10.36 & 37.78 \\
$\Delta \Delta G_{\text {cal }}$ & $4.2^{b}$ & $2.7^{c}$ & $1.8^{c}$ \\
\hline
\end{tabular}

$$
\begin{aligned}
& { }^{a} \text { dielectric constant at } 25^{\circ} \mathrm{C} .{ }^{13} \\
& { }^{b} \mathrm{X}=\mathrm{O}, \Delta \Delta G_{\text {cal }}=\Delta G^{\ddagger}(\mathrm{TS} 19)-\Delta G^{\ddagger}(\mathrm{TS5}) . \\
& { }^{\mathrm{C}}=\mathrm{P}, \Delta \Delta G_{\text {cal }}=\Delta G^{\ddagger}(\mathrm{TS} 20)-\Delta G^{\ddagger}(\mathrm{TS5}) .
\end{aligned}
$$

25. Alternative stereo-invertive $\mathrm{C}-\mathrm{O}$ bond activation transition states with the coordination of acetonitrile.

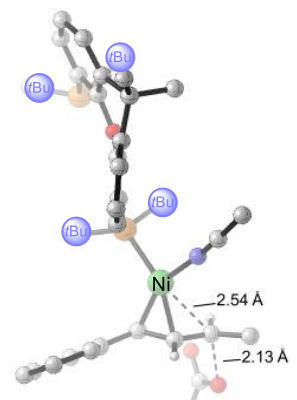

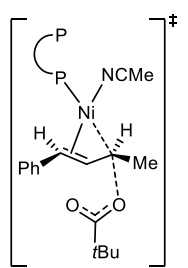

TS-S54

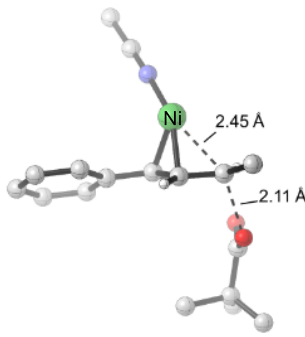

$\left(\Delta G^{\ddagger}\right.$ in acetonitrile $\left.=7.6\right)$

Figure S24. Alternative stereo-invertive $\mathrm{C}-\mathrm{O}$ bond activation transition states with the coordination of acetonitrile. Free energies in $\mathrm{kcal} / \mathrm{mol}$ are compared to intermediate 24 . 
26. Explorations of solvent coordinations in $\mathrm{C}-\mathrm{O}$ bond activation for other potentially coordinating solvents.

In addition to acetonitrile, we also carefully examined the coordination of other potentially coordinating solvents in $\mathrm{C}-\mathrm{O}$ bond activation transition states. Among the experimentally studied solvents, ${ }^{1} \mathrm{DMF}, \mathrm{EtOAc}$ and THF solvents can coordinate to nickel with oxygen atom. The computations of these solvent-coordinated $\mathrm{C}-\mathrm{O}$ bond activation transition states are summarized in Figures S25-S27. Unlike acetonitrile, none of these solvents can favorably replace the $t \mathrm{Bu}$-Xantphos ligand coordination in the stereo-invertive $\mathrm{C}-\mathrm{O}$ bond activation transition states. These calculations highlight the strong coordinating nature of acetonitrile solvent, which makes acetonitrile distinct from the other studied solvents.

(a) Solvent $=\mathrm{DMF}$

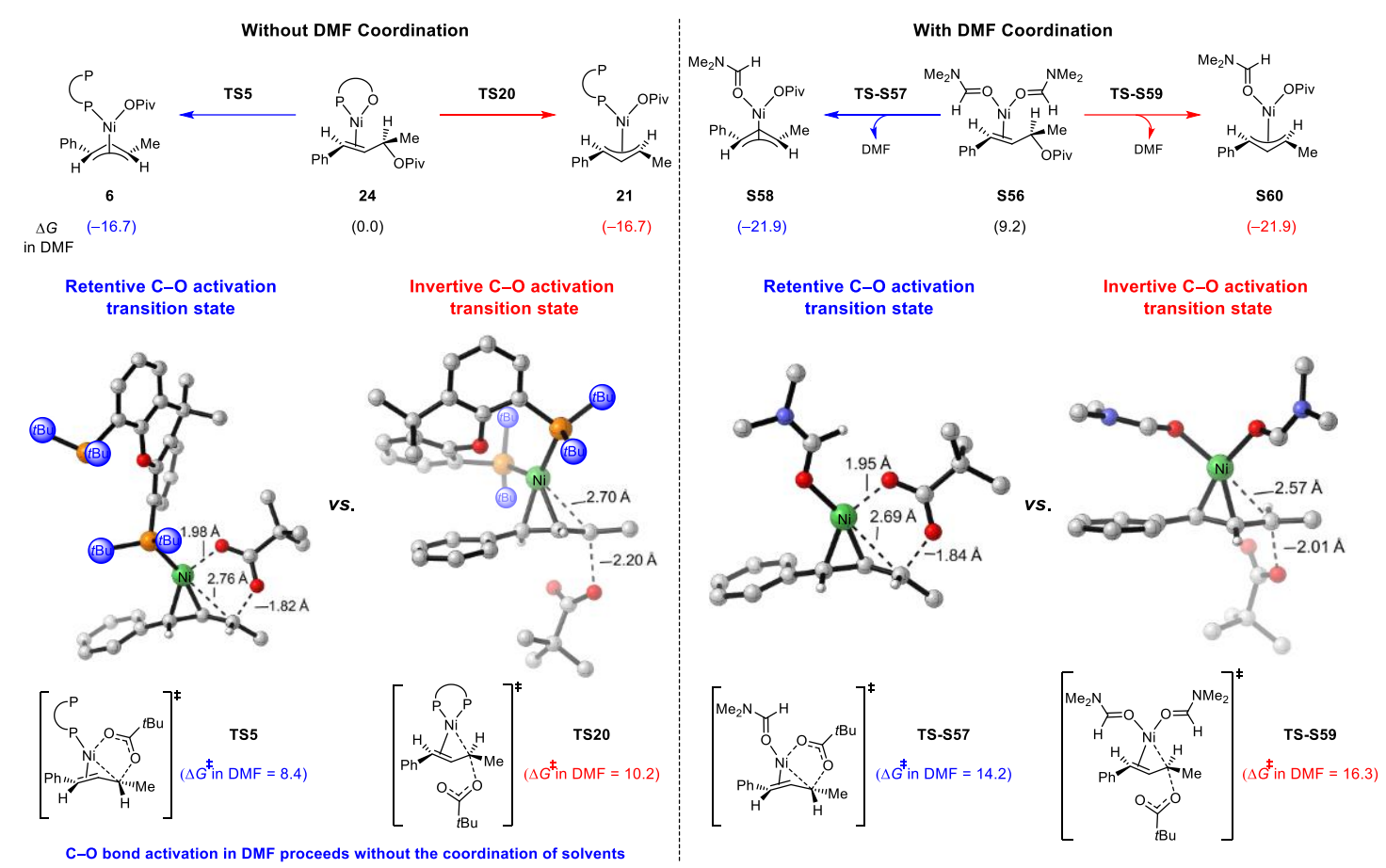

Figure S25. DFT-computed Gibbs free energies and optimized structures of the stereo-retentive and invertive allylic $\mathrm{C}-\mathrm{O}$ bond activation transition states with allylic pivalate $(R)-1$ in DMF. Free energies are in $\mathrm{kcal} / \mathrm{mol}$. 
(b) Solvent $=$ EtOAc

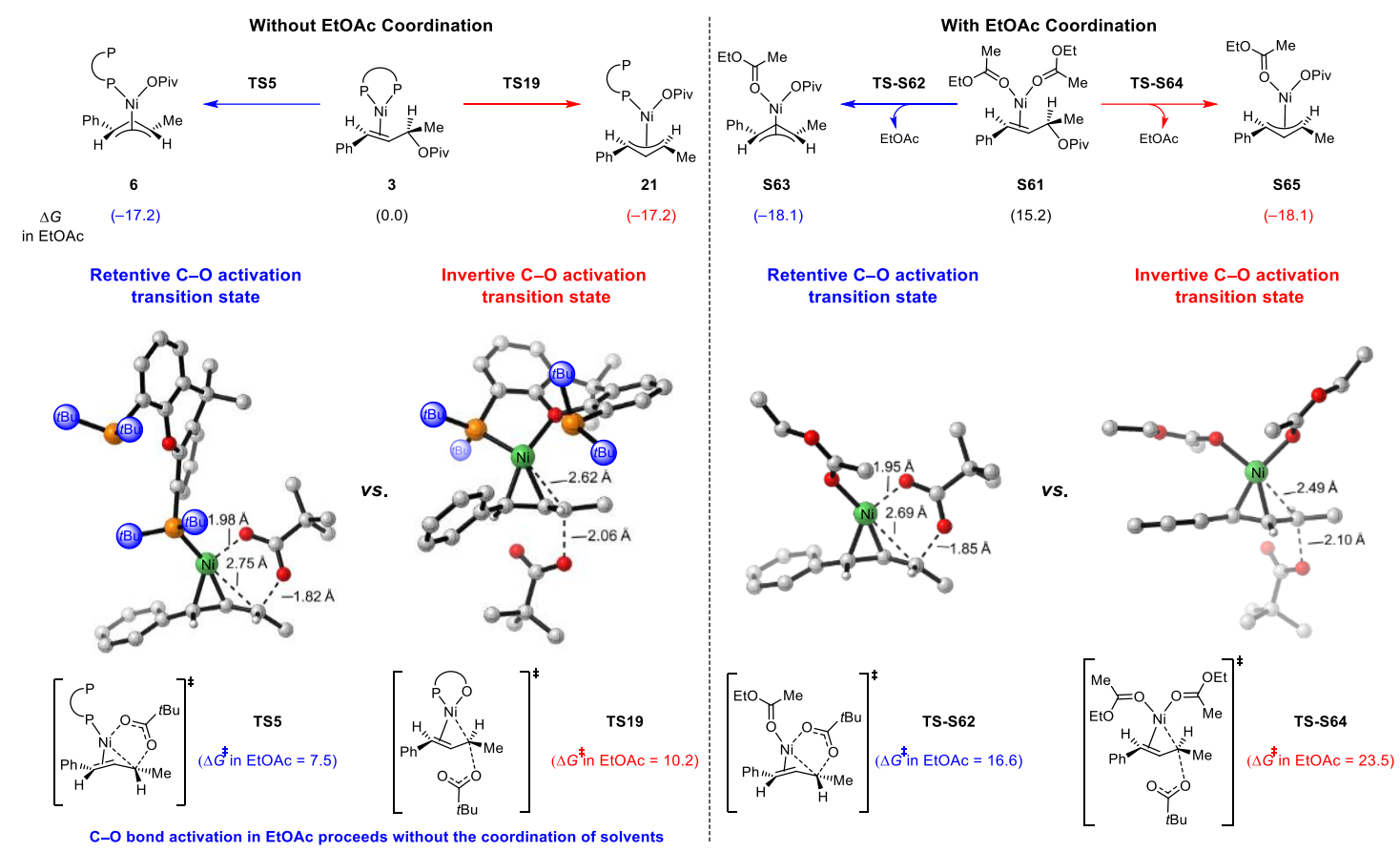

Figure S26. DFT-computed Gibbs free energies and optimized structures of the stereo-retentive and invertive allylic $\mathrm{C}-\mathrm{O}$ bond activation transition states with allylic pivalate $(R)-1$ in EtOAc. Free energies are in $\mathrm{kcal} / \mathrm{mol}$.

(c) Solvent $=\mathrm{THF}$

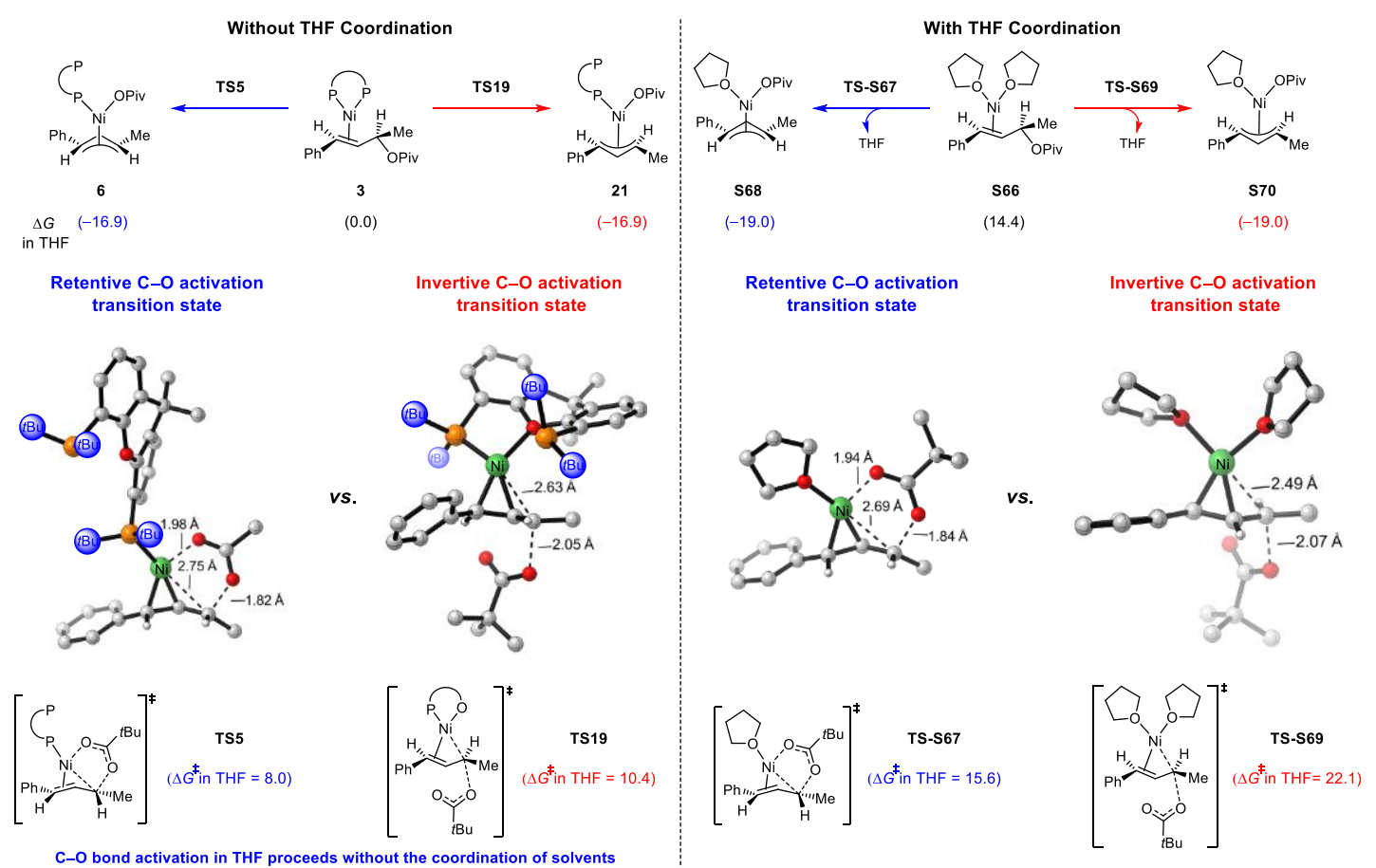

Figure S27. DFT-computed Gibbs free energies and optimized structures of the stereo-retentive and invertive allylic $\mathrm{C}-\mathrm{O}$ bond activation transition states with allylic pivalate $(R)-1$ in THF. Free energies are in $\mathrm{kcal} / \mathrm{mol}$. 
27. DFT-computed Gibbs free energy changes of the Ni/acetonitrile-catalyzed Miyaura borylation with allylic pivalate $(R)$-1 leading to the stereoinversion product.

Based on the mechanistic understandings of acetonitrile-coordinated stereo-invertive $\mathrm{C}-\mathrm{O}$ bond activation, we envision that the entire Miyaura borylation can proceed without the involvement of phosphine ligand. The computed free energy changes of the corresponding catalytic cycle are included in Figure S28. The resting state of the predicted borylation is the $\mathrm{Ni}$ (II)allyl species S73, and the rate-determining step is the transmetalation step via TS-S75. The calculations indeed showed a surmountable overall barrier of $24.2 \mathrm{kcal} / \mathrm{mol}$ (S73 to TS-S75). These computational predictions motivated us to further experimentally prove the ligandless $\mathrm{Ni}$-catalyzed allylic $\mathrm{C}-\mathrm{O}$ bond activation.

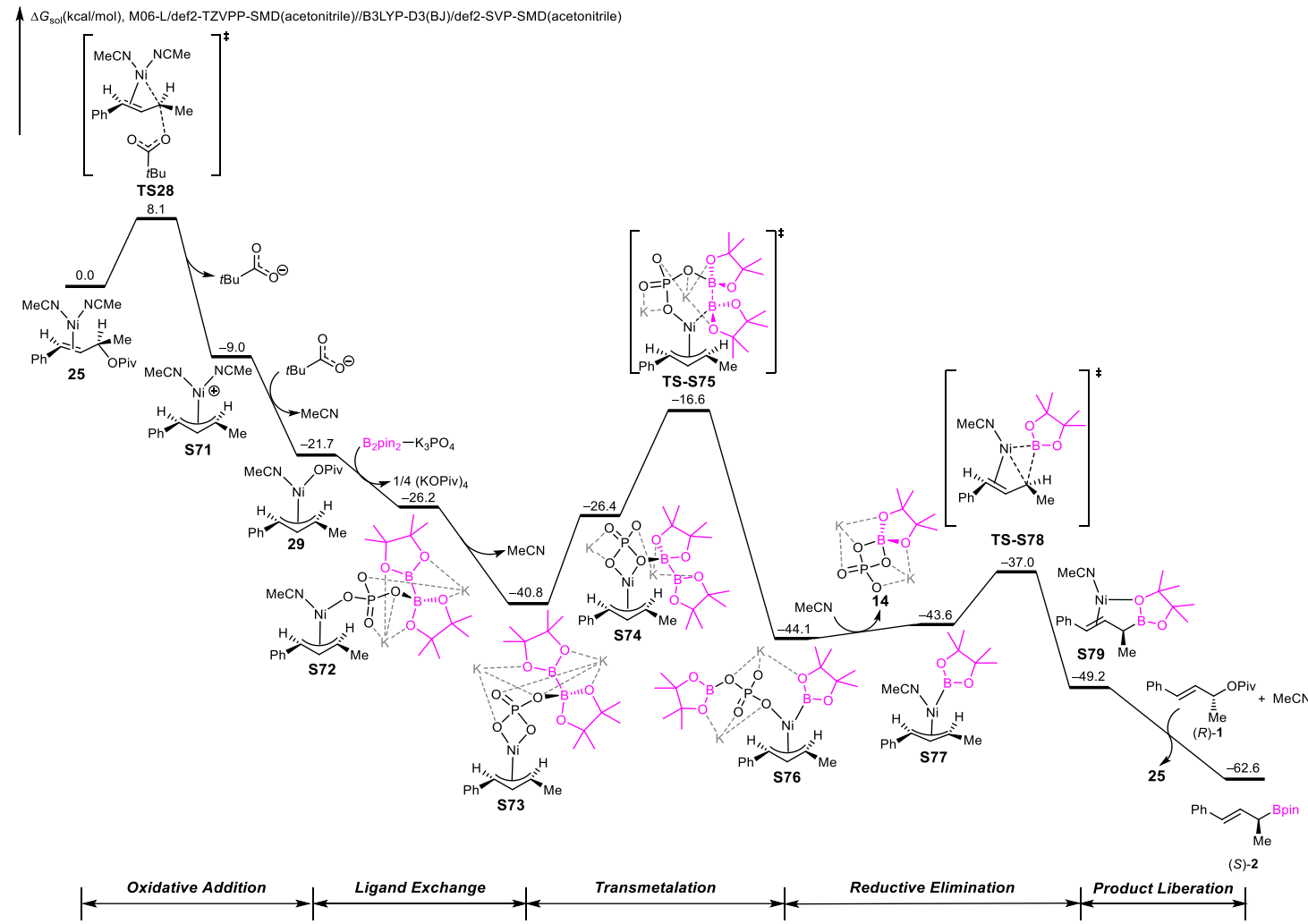

Figure S28. DFT-computed Gibbs free energy changes of the $\left[\mathrm{Ni}(\mathrm{MeCN})_{2}\right]$-catalyzed Miyaura borylation with allylic pivalate $(R)$-1 leading to the stereoinversion product.

\section{Solvent concentration corrections.}

In order to adjust the Gibbs free energies of solvent molecules from $1 \mathrm{~mol} / \mathrm{L}$ standard state to the actual solvent concentration, a correction of $R T \ln \left(c_{a} / c_{s}\right)$ is added to energies of all solvents. ${ }^{14} c_{a}$ is the actual molar concentration of solvent $\left(c_{a}=\rho / M\right), c_{s}$ is the standard molar concentration in solution $(1 \mathrm{~mol} / \mathrm{L})$, and $R$ is the gas constant $(8.314$ $\mathrm{J} /(\mathrm{mol} \cdot \mathrm{K})), \mathrm{T}=298.15 \mathrm{~K}, \Delta \Delta G=\Delta G_{\mathrm{a}}-\Delta G_{\mathrm{s}}=R \ln \left(\mathrm{c}_{\mathrm{a}} / \mathrm{c}_{\mathrm{s}}\right), \Delta G_{\mathrm{a}}$ is the actual Gibbs free energy change, $\Delta G_{\mathrm{s}}$ is the standard Gibbs free energy change.

(i) For EtOAc: $\rho=0.902 \mathrm{~g} / \mathrm{ml}, \mathrm{M}=88.11 \mathrm{~g} / \mathrm{mol}$, thus, $\Delta \Delta G=R T \ln \left(\mathrm{c}_{\mathrm{a}} / \mathrm{c}_{\mathrm{s}}\right)=1.38 \mathrm{kcal} / \mathrm{mol}$.

(j) For THF: $\rho=0.889 \mathrm{~g} / \mathrm{ml}, \mathrm{M}=72.10 \mathrm{~g} / \mathrm{mol}$, thus, $\Delta \Delta G=R T \ln \left(\mathrm{c}_{\mathrm{a}} / \mathrm{c}_{\mathrm{s}}\right)=1.49 \mathrm{kcal} / \mathrm{mol}$.

(k) For DMF: $\rho=0.945 \mathrm{~g} / \mathrm{ml}, \mathrm{M}=73.09 \mathrm{~g} / \mathrm{mol}$, thus, $\Delta \Delta G=R T \ln \left(\mathrm{c}_{\mathrm{a}} / \mathrm{c}_{\mathrm{s}}\right)=1.52 \mathrm{kcal} / \mathrm{mol}$.

(1) For $\mathrm{MeCN}: \rho=0.790 \mathrm{~g} / \mathrm{ml}, \mathrm{M}=41.05 \mathrm{~g} / \mathrm{mol}$, thus, $\Delta \Delta G=R T \ln \left(\mathrm{c}_{\mathrm{a}} / \mathrm{c}_{\mathrm{s}}\right)=1.75 \mathrm{kcal} / \mathrm{mol}$. 


\section{Counterpoise corrections for selected intermediates.}

Considering that the following processes shown in Scheme S4 involve ligand dissociation that may lead to basis set superposition error (BSSE), thus, counterpoise corrections were performed for intermediates 7, 24, 25 and 31 (Table S7). The results in Table S7 indicate that the BSSE is relatively small $(0.6-2.4 \mathrm{kcal} / \mathrm{mol})$ with the def2-TZVPP basis set.
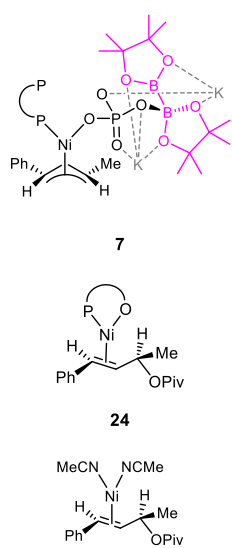

25
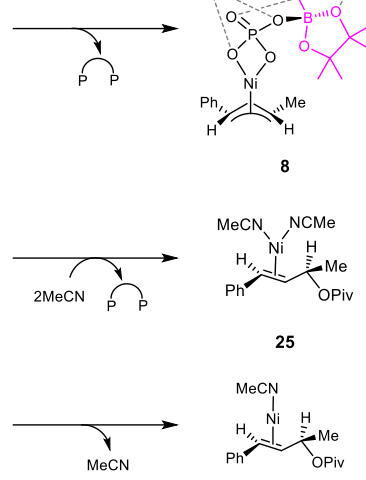

Scheme S4. Processes Involving Ligand Dissociation That May Lead to BSSE

Table S7. Counterpoise Corrections for Intermediates 7, 24, 25 and 31. Energies Are in kcal/mol

\begin{tabular}{cccc}
\hline Intermediate & BSSE Energy & $\begin{array}{c}\text { Complexation Energy } \\
\text { (Uncorrected) }\end{array}$ & $\begin{array}{c}\text { Complexation Energy } \\
\text { (Corrected) }\end{array}$ \\
\hline 7 & 2.06 & -52.67 & -50.61 \\
24 & 2.35 & -69.52 & -67.17 \\
25 & 0.64 & -28.98 & -28.34 \\
31 & 0.65 & -38.23 & -37.58 \\
\hline
\end{tabular}




\section{General experimental procedures.}

${ }^{1} \mathrm{H}$ NMR spectra were recorded on an Agilent $400 \mathrm{MHz}$ spectrometers. Chemical shifts are reported in ppm from tetramethylsilane with the solvent resonance as the internal standard $\left(\mathrm{CDCl}_{3}: \delta 7.26 \mathrm{ppm}\right)$. Data are reported as follows: chemical shift, integration, multiplicity $(s=$ singlet, $d=$ doublet, $\mathrm{t}=$ triplet, $\mathrm{q}=$ quartet, $\mathrm{m}=$ multiplet $)$, and coupling constant $(\mathrm{Hz})$. Enantiomeric ratios were determined by high-performance liquid chromatography (HPLC) with a Shimadzu LC-20AT chromatograph (Chiralcel IB $(4.6 \times 250 \mathrm{~mm})$ ) in comparison with authentic racemic materials. Specific rotations were measured on a Rudolph Research Analytical Autopol VI Polarimeter. ( $R, \mathrm{E})-4-$ phenylbut-3-en-2-yl pivalate substrate with different ee values (97\% ee and $88 \%$ ee) were used in the experimental verifications due to the ee value change of allylic alcohol starting material $(R, \mathrm{E})$-4-phenylbut-3-en-2-ol. This ee value change of pivalate does not affect the stereospecificity determination (es value) of borylation.

\section{Experimental verifications of ligandless $\mathrm{Ni}$-catalyzed allylic $\mathrm{C}-\mathrm{O}$ bond activation in acetonitrile.}

\subsection{Procedures for preparation of $(R, E)-4$-phenylbut-3-en-2-yl pivalate (1).}<smiles>[C]C(O)C=Cc1ccccc1</smiles>

$97 \%$ ee

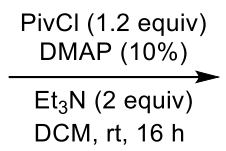

DCM, rt, $16 \mathrm{~h}$

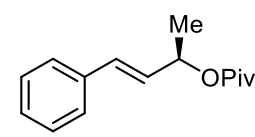

$(R)-1$

$88 \%$ yield, $97 \%$ ee

$(R, \mathrm{E})$-4-phenylbut-3-en-2-yl pivalate $(\mathbf{1})$ was prepared according to the previously reported procedures. ${ }^{15}(R, \mathrm{E})-4$ phenylbut-3-en-2-ol (0.72 g, $4.83 \mathrm{mmol}, 97 \% \mathrm{ee})$ and DMAP $(65.6 \mathrm{mg}, 0.54 \mathrm{mmol})$ were dissolved in DCM $(10 \mathrm{~mL})$. $\mathrm{Et}_{3} \mathrm{~N}(1.39 \mathrm{~mL}, 10 \mathrm{mmol})$ and $\mathrm{PivCl}(0.74 \mathrm{~g}, 6 \mathrm{mmol})$ were then added. The reaction mixture was stirred for $16 \mathrm{~h}$ at room temperature, before $\mathrm{H}_{2} \mathrm{O}(8 \mathrm{~mL})$ was added. The organic layer was extracted with DCM $(2 \times 16 \mathrm{~mL})$. The combined organic layers were washed with aq. $\mathrm{KOH}(2.0 \mathrm{M}, 16 \mathrm{~mL})$, dried $\left(\mathrm{Na}_{2} \mathrm{SO}_{4}\right)$, concentrated. The resulting residue was purified by flash column chromatography using PE/EtOAc (30/1) as the eluent to give $0.9868 \mathrm{~g}(4.2$ mmol, $88 \%$ yield) of the title compound as a colorless oil. Specific rotation: $[\alpha]^{25} \mathrm{D}=+88.4$ (c 1.18, $\left.\mathrm{CHCl}_{3}\right) .97 \%$ ee, determined by HPLC, HPLC conditions: Chiralcel IC, $n$-hexane $/ i$-PrOH $=99 / 1,0.8 \mathrm{~mL} / \mathrm{min}, \mathrm{n}=254 \mathrm{~nm}$, tr 6.4 (major), 7.1 (minor). ${ }^{1} \mathrm{H}$ NMR $\left(\mathrm{CDCl}_{3}, 400 \mathrm{MHz}\right): \delta 7.42-7.35(\mathrm{~m}, 2 \mathrm{H}), 7.35-7.28(\mathrm{~m}, 2 \mathrm{H}), 7.27-7.20(\mathrm{~m}, 1 \mathrm{H})$, $6.59(\mathrm{~d}, J=16.0 \mathrm{~Hz}, 1 \mathrm{H}), 6.19(\mathrm{dd}, J=6.4,16.0 \mathrm{~Hz}, 1 \mathrm{H}), 5.55-5.45(\mathrm{~m}, 1 \mathrm{H}), 1.39(\mathrm{~d}, J=6.4 \mathrm{~Hz}, 3 \mathrm{H}), 1.22(\mathrm{~s}, 9 \mathrm{H})$; These spectroscopic data are corresponding to the previously reported data. ${ }^{15}$

\subsection{Experimental results that verify the proposed ligandless Ni-catalyzed Miyaura borylation in acetonitrile.}

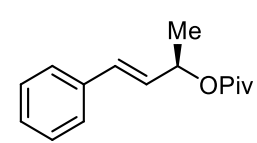

$(R)-1$ $97 \%$ ee

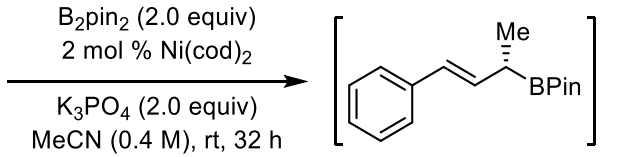

$(S)-2$

INVERSION

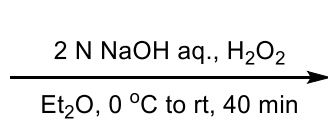

$\mathrm{Et}_{2} \mathrm{O}, 0^{\circ} \mathrm{C}$ to rt, $40 \mathrm{~min}$<smiles>C[C@@H](O)/C=C/c1ccccc1</smiles>

(S)-3

$65 \%$ yield, $88 \%$ ee

In a $\mathrm{N}_{2}$-atmosphere glovebox, $\mathrm{Ni}(\mathrm{cod})_{2}(4.9 \mathrm{mg}, 0.0178 \mathrm{mmol}, 2 \mathrm{~mol} \%), \mathrm{K}_{3} \mathrm{PO}_{4}(337.7 \mathrm{mg}, 1.59 \mathrm{mmol}, 2.0$ equiv), $\mathrm{B}_{2} \operatorname{pin}_{2}$ (404.6 mg, $1.59 \mathrm{mmol}, 2.0$ equiv), allylic pivalate ( $185.0 \mathrm{mg}, 0.80 \mathrm{mmol}, 1.0$ equiv) and $\mathrm{MeCN}(2.0 \mathrm{~mL}, 0.4 \mathrm{M})$ were added sequentially into a vial equipped with a magnetic stirrer. The mixture was stirred at room temperature for $32 \mathrm{~h}$ before removed from the glovebox. The reaction mixture quickly filtered through a pad of silica gel, washed with $\mathrm{Et}_{2} \mathrm{O}$. The solution of the crude reaction mixture and $\mathrm{Et}_{2} \mathrm{O}(1.5 \mathrm{~mL})$ was cooled to $0^{\circ} \mathrm{C}$. Aqueous $\mathrm{NaOH}(2 \mathrm{~N}, 0.45$ mol) was added, followed by $\mathrm{H}_{2} \mathrm{O}_{2}(30 \%, 0.19 \mathrm{~mL})$. The mixture was stirred at $0^{\circ} \mathrm{C}$ for $10 \mathrm{~min}$ and then at room temperature for an additional $30 \mathrm{~min}$. The reaction mixture was diluted with $\mathrm{H}_{2} \mathrm{O}$, extracted with $\mathrm{Et}_{2} \mathrm{O}$, dried with $\mathrm{Na}_{2} \mathrm{SO}_{4}$, concentrated. The resulting residue was purified by flash column chromatography using PE/EtOAc (5:1) as the eluent to give $0.0767 \mathrm{~g}$ (65\% yield) of the allylic alcohol as a white solid. Specific rotation: $[\alpha]^{20} \mathrm{D}=-32.9$ (c 1.00, $\mathrm{CHCl}_{3}$ ). Enantiomeric purity of allylic alcohol 3 was determined by HPLC analysis in comparison with authentic 
racemic material, HPLC Condition: Chiralcel IB, $n$-hexane $/ i-\operatorname{PrOH}=97 / 3,1.0 \mathrm{~mL} / \mathrm{min}, \mathrm{n}=254 \mathrm{~nm}$, tr 14.1 (minor), 20.9 (major). ${ }^{1} \mathrm{H}$ NMR (400 MHz, $\left.\mathrm{CDCl}_{3}\right): \delta 7.39$ (d, $\left.J=7.4 \mathrm{~Hz}, 2 \mathrm{H}\right), 7.33(\mathrm{t}, J=7.5 \mathrm{~Hz}, 2 \mathrm{H}), 7.27-7.23(\mathrm{~m}, 1 \mathrm{H})$, $6.57(\mathrm{~d}, J=15.8 \mathrm{~Hz}, 1 \mathrm{H}), 6.27(\mathrm{dd}, J=15.8,6.4 \mathrm{~Hz}, 1 \mathrm{H}), 4.49(\mathrm{p}, J=6.2 \mathrm{~Hz}, 1 \mathrm{H}), 2.15(\mathrm{br}, 1 \mathrm{H}), 1.38(\mathrm{~d}, J=6.4 \mathrm{~Hz}$, $3 \mathrm{H})$.

32. Experimental verifications of ligandless Ni-catalyzed allylic $\mathrm{C}-\mathrm{O}$ bond activation in hexane with catalytic amount of acetonitrile.

32.1. Procedures for preparation of $(R, \mathrm{E})-4$-phenylbut-3-en-2-yl pivalate (1).<smiles>[N+]=[W]C(O)/C=C/c1ccccc1</smiles>

$88 \%$ ee

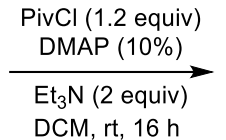

DCM, rt, $16 \mathrm{~h}$

$85 \%$ yield, $88 \%$ ee

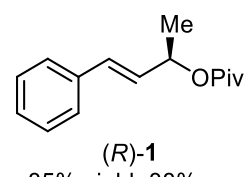

$(R, \mathrm{E})$-4-phenylbut-3-en-2-yl pivalate $(\mathbf{1})$ was prepared according to the previously reported procedures. ${ }^{15}(R, \mathrm{E})-4$ phenylbut-3-en-2-ol ( $0.85 \mathrm{~g}, 5.70 \mathrm{mmol}, 88 \% \mathrm{ee})$ and DMAP $(70.5 \mathrm{mg}, 0.58 \mathrm{mmol})$ were dissolved in DCM $(10 \mathrm{~mL})$. $\mathrm{Et}_{3} \mathrm{~N}(1.58 \mathrm{~mL}, 1137 \mathrm{mmol})$ and $\mathrm{PivCl}(0.84 \mathrm{~g}, 6.81 \mathrm{mmol})$ were then added. The reaction mixture was stirred for 16 $\mathrm{h}$ at room temperature, before $\mathrm{H}_{2} \mathrm{O}(8 \mathrm{~mL})$ was added. The organic layer was extracted with $\mathrm{DCM}(2 \times 16 \mathrm{~mL})$. The combined organic layers were washed with aq. $\mathrm{KOH}(2.0 \mathrm{M}, 16 \mathrm{~mL})$, dried $\left(\mathrm{Na}_{2} \mathrm{SO}_{4}\right)$, concentrated. The resulting residue was purified by flash column chromatography using PE/EtOAc (30/1) as the eluent to give $1.1305 \mathrm{~g}(4.8$ mmol, $85 \%$ yield $)$ of the title compound as a colorless oil. Specific rotation: $[\alpha]^{25} \mathrm{D}=+88.2\left(\mathrm{c} 1.18, \mathrm{CHCl}_{3}\right) .88 \%$ ee, determined by HPLC, HPLC conditions: Chiralcel IC, $n$-hexane $/ i-\mathrm{PrOH}=99 / 1,0.8 \mathrm{~mL} / \mathrm{min}, \mathrm{n}=220 \mathrm{~nm}$, tr 5.9 (major), 6.7 (minor). ${ }^{1} \mathrm{H}$ NMR $\left(\mathrm{CDCl}_{3}, 400 \mathrm{MHz}\right): \delta 7.42-7.35(\mathrm{~m}, 2 \mathrm{H}), 7.35-7.28(\mathrm{~m}, 2 \mathrm{H}), 7.27-7.20(\mathrm{~m}, 1 \mathrm{H})$, $6.59(\mathrm{~d}, J=16.0 \mathrm{~Hz}, 1 \mathrm{H}), 6.19(\mathrm{dd}, J=6.4,16.0 \mathrm{~Hz}, 1 \mathrm{H}), 5.55-5.45(\mathrm{~m}, 1 \mathrm{H}), 1.39(\mathrm{~d}, J=6.4 \mathrm{~Hz}, 3 \mathrm{H}), 1.22(\mathrm{~s}, 9 \mathrm{H})$; These spectroscopic data are corresponding to the previously reported data. ${ }^{15}$

32.2. Experimental results that verify the proposed ligandless Ni-catalyzed Miyaura borylation in hexane with catalytic amount of acetonitrile.

(a) With $t$ Bu-XantPhos Ligand

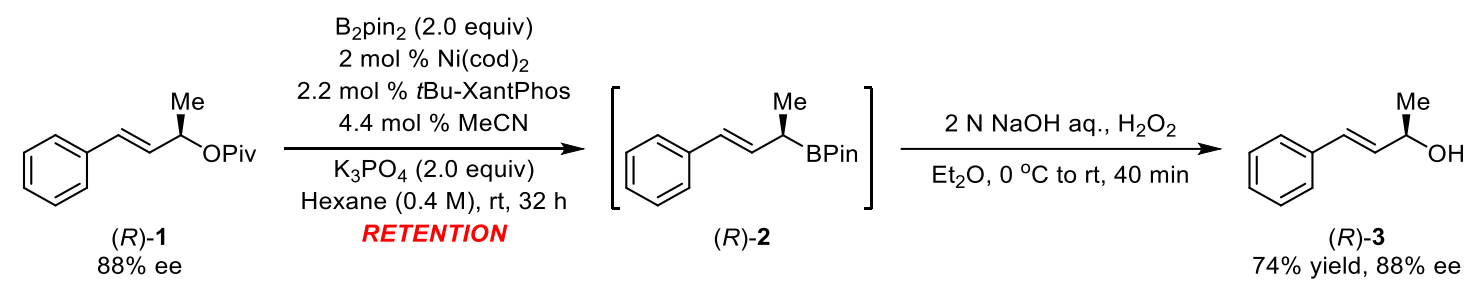

In a $\mathrm{N}_{2}$-atmosphere glovebox, $\mathrm{Ni}(\mathrm{cod})_{2}(4.9 \mathrm{mg}, 0.0178 \mathrm{mmol}, 2 \mathrm{~mol} \%)$, $t \mathrm{Bu}$-XantPhos Ligand $(9.7 \mathrm{mg}, 0.0196 \mathrm{mmol}$, $2.2 \mathrm{~mol} \%) \mathrm{K}_{3} \mathrm{PO}_{4}\left(337.7 \mathrm{mg}, 1.59 \mathrm{mmol}, 2.0\right.$ equiv), $\mathrm{B}_{2} \operatorname{pin}_{2}$ (404.6 mg, $1.59 \mathrm{mmol}, 2.0$ equiv), allylic pivalate (185.0 $\mathrm{mg}, 0.80 \mathrm{mmol}, 1.0$ equiv), MeCN (1.44 mg, 0.0352mmol, $4.4 \mathrm{~mol} \%)$ and Hexane (2.0 $\mathrm{mL}, 0.4 \mathrm{M})$ were added sequentially into a vial equipped with a magnetic stirrer. The mixture was stirred at room temperature for $32 \mathrm{~h}$ before removed from the glovebox. The reaction mixture quickly filtered through a pad of silica gel, washed with $\mathrm{Et}_{2} \mathrm{O}$. The solution of the crude reaction mixture and $\mathrm{Et}_{2} \mathrm{O}(1.5 \mathrm{~mL})$ was cooled to $0^{\circ} \mathrm{C}$. Aqueous $\mathrm{NaOH}(2 \mathrm{~N}, 0.45 \mathrm{~mol})$ was added, followed by $\mathrm{H}_{2} \mathrm{O}_{2}(30 \%, 0.19 \mathrm{~mL})$. The mixture was stirred at $0^{\circ} \mathrm{C}$ for $10 \mathrm{~min}$ and then at room temperature for an additional $30 \mathrm{~min}$. The reaction mixture was diluted with $\mathrm{H}_{2} \mathrm{O}$, extracted with $\mathrm{Et}_{2} \mathrm{O}$, dried with $\mathrm{Na}_{2} \mathrm{SO}_{4}$, concentrated. The resulting residue was purified by flash column chromatography using PE/EtOAc (5:1) as the eluent to give $0.0880 \mathrm{~g}$ (74\% yield) of the allylic alcohol as a colorless oil. Specific rotation: $[\alpha]^{20} \mathrm{D}=+31.8\left(\mathrm{c} 1.00, \mathrm{CHCl}_{3}\right)$. Enantiomeric purity of allylic alcohol 3 was determined by HPLC analysis in comparison with authentic racemic material, HPLC Condition: Chiralcel IB, $n$-hexane $/ i$-PrOH = 97/3, $1.0 \mathrm{~mL} / \mathrm{min}, \mathrm{n}=220 \mathrm{~nm}$, tr 12.8 (major), 18.5 
(minor). ${ }^{1} \mathrm{H}$ NMR (400 MHz, $\left.\mathrm{CDCl}_{3}\right): \delta 7.39(\mathrm{~d}, J=7.4 \mathrm{~Hz}, 2 \mathrm{H}), 7.33(\mathrm{t}, J=7.5 \mathrm{~Hz}, 2 \mathrm{H}), 7.27-7.23(\mathrm{~m}, 1 \mathrm{H}), 6.57$ $(\mathrm{d}, J=15.8 \mathrm{~Hz}, 1 \mathrm{H}), 6.27(\mathrm{dd}, J=15.8,6.4 \mathrm{~Hz}, 1 \mathrm{H}), 4.49(\mathrm{p}, J=6.2 \mathrm{~Hz}, 1 \mathrm{H}), 2.15(\mathrm{br}, 1 \mathrm{H}), 1.38(\mathrm{~d}, J=6.4 \mathrm{~Hz}, 3 \mathrm{H})$.

(b) Without $t \mathrm{Bu}-\mathrm{XantPhos}$ Ligand

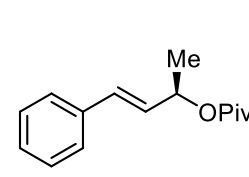
$(R)-1$
$88 \%$ ee

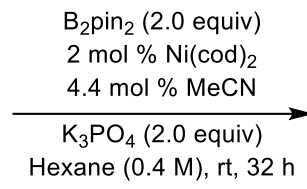
Hexane $(0.4 \mathrm{M}), \mathrm{rt}, 32 \mathrm{~h}$ RETENTION

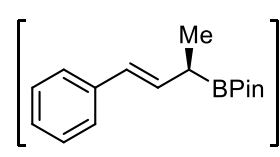

$(R)-2$

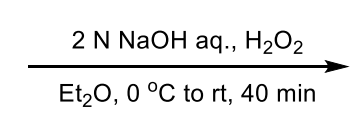

$\mathrm{Et}_{2} \mathrm{O}, 0^{\circ} \mathrm{C}$ to rt, $40 \mathrm{~min}$<smiles>C[C@@H](O)/C=C/c1ccccc1</smiles>

$(R)-3$

$76 \%$ yield, $88 \%$ ee

In a $\mathrm{N}_{2}$-atmosphere glovebox, $\mathrm{Ni}(\mathrm{cod})_{2}(4.9 \mathrm{mg}, 0.0178 \mathrm{mmol}, 2 \mathrm{~mol} \%), \mathrm{K}_{3} \mathrm{PO}_{4}(337.7 \mathrm{mg}, 1.59 \mathrm{mmol}, 2.0$ equiv), $\mathrm{B}_{2} \operatorname{pin}_{2}$ (404.6 mg, $1.59 \mathrm{mmol}, 2.0$ equiv), allylic pivalate (185.0 mg, $0.80 \mathrm{mmol}, 1.0$ equiv), MeCN (1.44 mg, $0.0352 \mathrm{mmol}, 4.4 \mathrm{~mol} \%)$ and Hexane $(2.0 \mathrm{~mL}, 0.4 \mathrm{M})$ were added sequentially into a vial equipped with a magnetic stirrer. The mixture was stirred at room temperature for $32 \mathrm{~h}$ before removed from the glovebox. The reaction mixture quickly filtered through a pad of silica gel, washed with $\mathrm{Et}_{2} \mathrm{O}$. The solution of the crude reaction mixture and $\mathrm{Et}_{2} \mathrm{O}(1.5$ $\mathrm{mL})$ was cooled to $0^{\circ} \mathrm{C}$. Aqueous $\mathrm{NaOH}(2 \mathrm{~N}, 0.45 \mathrm{~mol})$ was added, followed by $\mathrm{H}_{2} \mathrm{O}_{2}(30 \%, 0.19 \mathrm{~mL})$. The mixture was stirred at $0^{\circ} \mathrm{C}$ for $10 \mathrm{~min}$ and then at room temperature for an additional $30 \mathrm{~min}$. The reaction mixture was diluted with $\mathrm{H}_{2} \mathrm{O}$, extracted with $\mathrm{Et}_{2} \mathrm{O}$, dried with $\mathrm{Na}_{2} \mathrm{SO}_{4}$, concentrated. The resulting residue was purified by flash column chromatography using PE/EtOAc (5:1) as the eluent to give $0.0900 \mathrm{~g}$ (76\% yield) of the allylic alcohol as a colorless oil. Specific rotation: $[\alpha]^{20} \mathrm{D}=+31.8\left(\mathrm{c} 1.00, \mathrm{CHCl}_{3}\right)$. Enantiomeric purity of allylic alcohol 3 was determined by HPLC analysis in comparison with authentic racemic material, HPLC Condition: Chiralcel IB, $n$-hexane $/ i$-PrOH $=$ 97/3, $1.0 \mathrm{~mL} / \mathrm{min}, \mathrm{n}=220 \mathrm{~nm}$, tr 13.3 (major), 19.4 (minor). ${ }^{1} \mathrm{H} \mathrm{NMR}\left(400 \mathrm{MHz}, \mathrm{CDCl}_{3}\right): \delta 7.39(\mathrm{~d}, J=7.4 \mathrm{~Hz}, 2 \mathrm{H})$, $7.33(\mathrm{t}, J=7.5 \mathrm{~Hz}, 2 \mathrm{H}), 7.27-7.23(\mathrm{~m}, 1 \mathrm{H}), 6.57(\mathrm{~d}, J=15.8 \mathrm{~Hz}, 1 \mathrm{H}), 6.27(\mathrm{dd}, J=15.8,6.4 \mathrm{~Hz}, 1 \mathrm{H}), 4.49(\mathrm{p}, J=6.2$ $\mathrm{Hz}, 1 \mathrm{H}), 2.15(\mathrm{br}, 1 \mathrm{H}), 1.38(\mathrm{~d}, J=6.4 \mathrm{~Hz}, 3 \mathrm{H})$. 


\section{References.}

(1) Zhou, Q.; Srinivas, H. D.; Zhang, S.; Watson, M. P. Accessing Both Retention and Inversion Pathways in Stereospecific, Nickel-Catalyzed Miyaura Borylations of Allylic Pivalates. J. Am. Chem. Soc. 2016, 138, 11989-11995. (2) For early studies relate to distortion/interaction analysis, see: (a) Kitaura, K.; Morokuma, K. A New Energy Decomposition Scheme for Molecular Interactions within the Hartree-Fock Approximation. Int. J. Quantum Chem. 1976, 10, 325-340. (b) Ziegler, T.; Rauk, A. A Theoretical Study of the Ethylene-Metal Bond in Complexes Between Copper(1+), Silver(1+), Gold(1+), Platinum(0) or Platinum(2+) and Ethylene, Based on the Hartree-Fock-Slater Transition-State Method. Inorg. Chem. 1979, 18, 1558-1565.

(3) For reviews of distortion/interaction analysis, see: (a) van Leeuwen, P. W. N. M.; Kamer, P. C. J.; Reek, J. N. H.; Dierkes, P. Ligand Bite Angle Effects in Metal-Catalyzed C-C Bond Formation. Chem. Rev. 2000, 100, 2741-2770. (b) Van Zeist, W.-J.; Bickelhaupt, F. M. The Activation Strain Model of Chemical Reactivity. Org. Biomol. Chem. 2010, 8,3118-3127. (c) Fernandez, I.; Bickelhaupt, F. M. The Activation Strain Model and Molecular Orbital Theory: Understanding and Designing Chemical Reactions. Chem. Soc. Rev. 2014, 43, 4953-4967. (d) Bickelhaupt, F. M.; Houk, K. N. Analyzing Reaction Rates with the Distortion/Interaction-Activation Strain Model. Angew. Chem., Int. Ed. 2017, 56, 10070-10086.

(4) Hu, J.-Y.; Zhang, J.; Wang, G.-X.; Sun, H.-L.; Zhang, J.-L. Constructing A Catalytic Cycle for C-F to C-X (X = O, S, N) Bond Transformation Based on Gold-Mediated Ligand Nucleophilic Attack. Inorg. Chem. 2016, 55, 2274-2283.

(5) Adams, G. M.; Colebatch, A. L.; Skornia, J. T.; McKay, A. I.; Johnson, H. C.; Lloyd-Jones, G. C.; Macgregor, S. A.; Beattie, N. A.; Weller, A. S. Dehydropolymerization of $\mathrm{H}_{3} \mathrm{~B} \cdot \mathrm{NMeH}_{2}$ to Form Polyaminoboranes Using [Rh(Xantphosalkyl)] Catalysts. J. Am. Chem. Soc. 2018, 140, 1481-1495.

(6) Zhao, Y.; Truhlar, D. G. A New Local Density Functional for Main Group Thermochemistry, Transition Metal Bonding, Thermochemical Kinetics, and Noncovalent Interactions. J. Chem. Phys. 2006, 125, 194101-1-194101-18. (7) (a) Zhao, Y.; Truhlar, D. G. The M06 Suite of Density Functionals for Main Group Thermochemistry, Thermochemical Kinetics, Noncovalent Interactions, Excited States, and Transition Elements: Two New Functionals and Systematic Testing of Four M06-Class Functionals and 12 Other Functionals. Theor. Chem. Acc. 2008, 120, 215-241. (b) Zhao, Y.; Truhlar, D. G. Density Functionals with Broad Applicability in Chemistry. Acc. Chem. Res. 2008, 41, 157-167.

(8) (a) Becke, A. D. Density-Functional Thermochemistry. III. The Role of Exact Exchange. J. Chem. Phys. 1993, 98, 5648-5652. (b) Lee, C.; Yang, W.; Parr, R. G. Development of the Colle-Salvetti Correlation-Energy Formula into A Functional of the Electron Density. Phys. Rev. B. 1988, 37, 785-789.

(9) Grimme, S.; Antony, J.; Ehrlich, S.; Krieg, H. A Consistent and Accurate Ab Initio Parametrization of Density Functional Dispersion Correction (DFT-D) for the 94 Elements H-Pu. J. Chem. Phys. 2010, 132, 154104.

(10) Weigend, F.; Ahlrichs, R. Balanced Basis Sets of Split Valence, Triple Zeta Valence and Quadruple Zeta Valence Quality for H to Rn: Design and Assessment of Accuracy. PhSCys. Chem. Chem. Phys. 2005, 7, 3297-3305.

(11) Marenich, A. V.; Cramer, C. J.; Truhlar, D. G. Universal Solvation Model Based on Solute Electron Density and on A Continuum Model of the Solvent Defined by the Bulk Dielectric Constant and Atomic Surface Tensions. J. Phys. Chem. B. 2009, 113, 6378-6396.

(12) Weigend, F. Accurate Coulomb-Fitting Basis Sets for H to Rn. Phys. Chem. Chem. Phys. 2006, 8, 1057-1065.

(13) Reichardt, C. Solvents and Solvent Effects in Organic Chemistry, 3rd ed.; Wiley-VCH Verlag GmbH \& Co. KGaA: Weinheim, Germany, 2003.

(14) Sparta, M.; Riplinger, C.; Neese, F. Mechanism of Olefin Asymmetric Hydrogenation Catalyzed by Iridium Phosphino-Oxazoline: A Pair Natural Orbital Coupled Cluster Study. J. Chem. Theory Comput. 2014, 10, 1099-1108. (15) Srinivas, H. D.; Zhou, Q.;Watson, M. P. Enantiospecific, Nickel-Catalyzed Cross-Couplings of Allylic Pivalates and Arylboroxines. Org. Lett. 2014, 16, 3596-3599. 
34. NMR spectra.

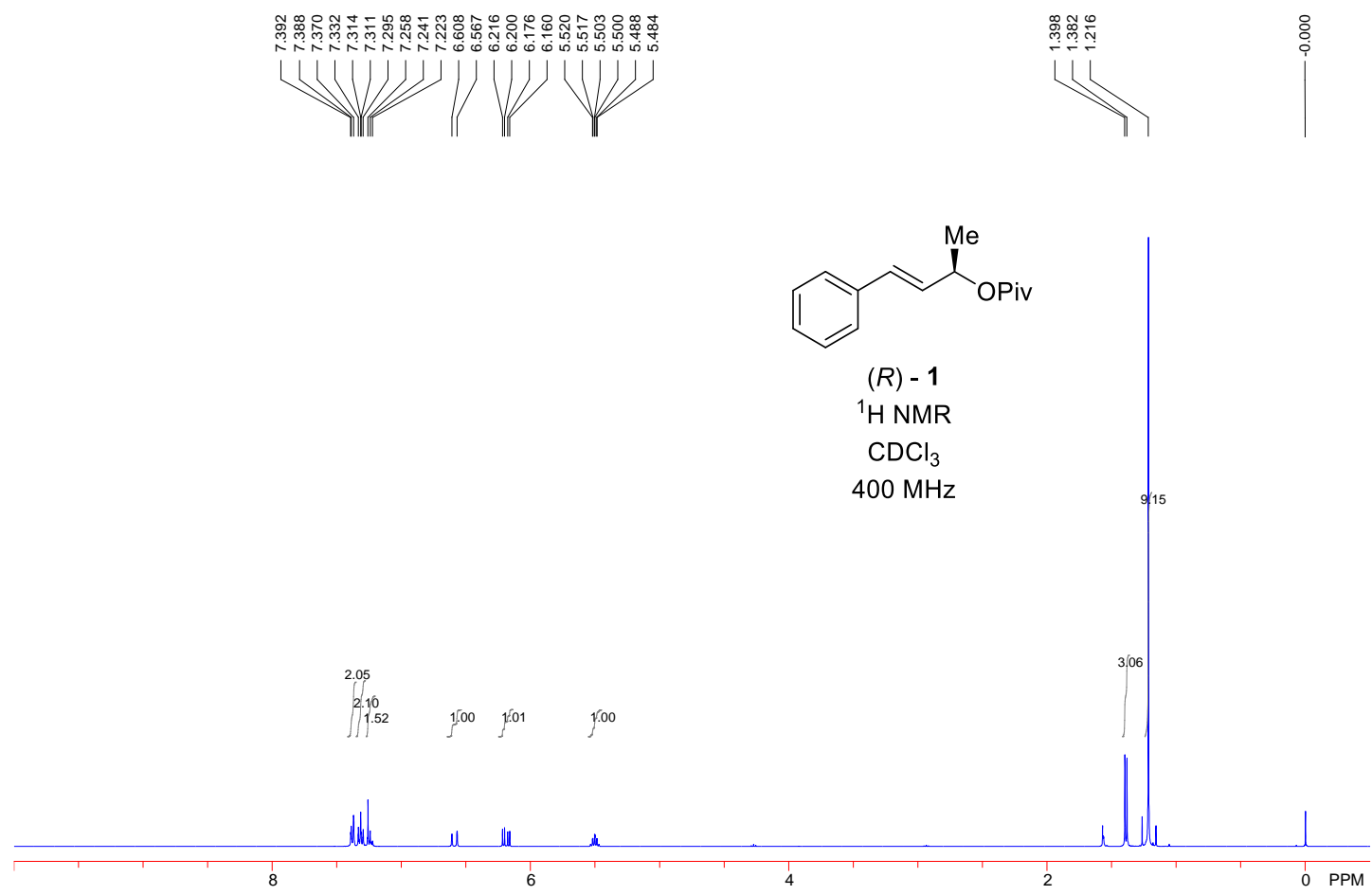

Figure S29. ${ }^{1} \mathrm{H}$ NMR spectrum of $(R)-1$.

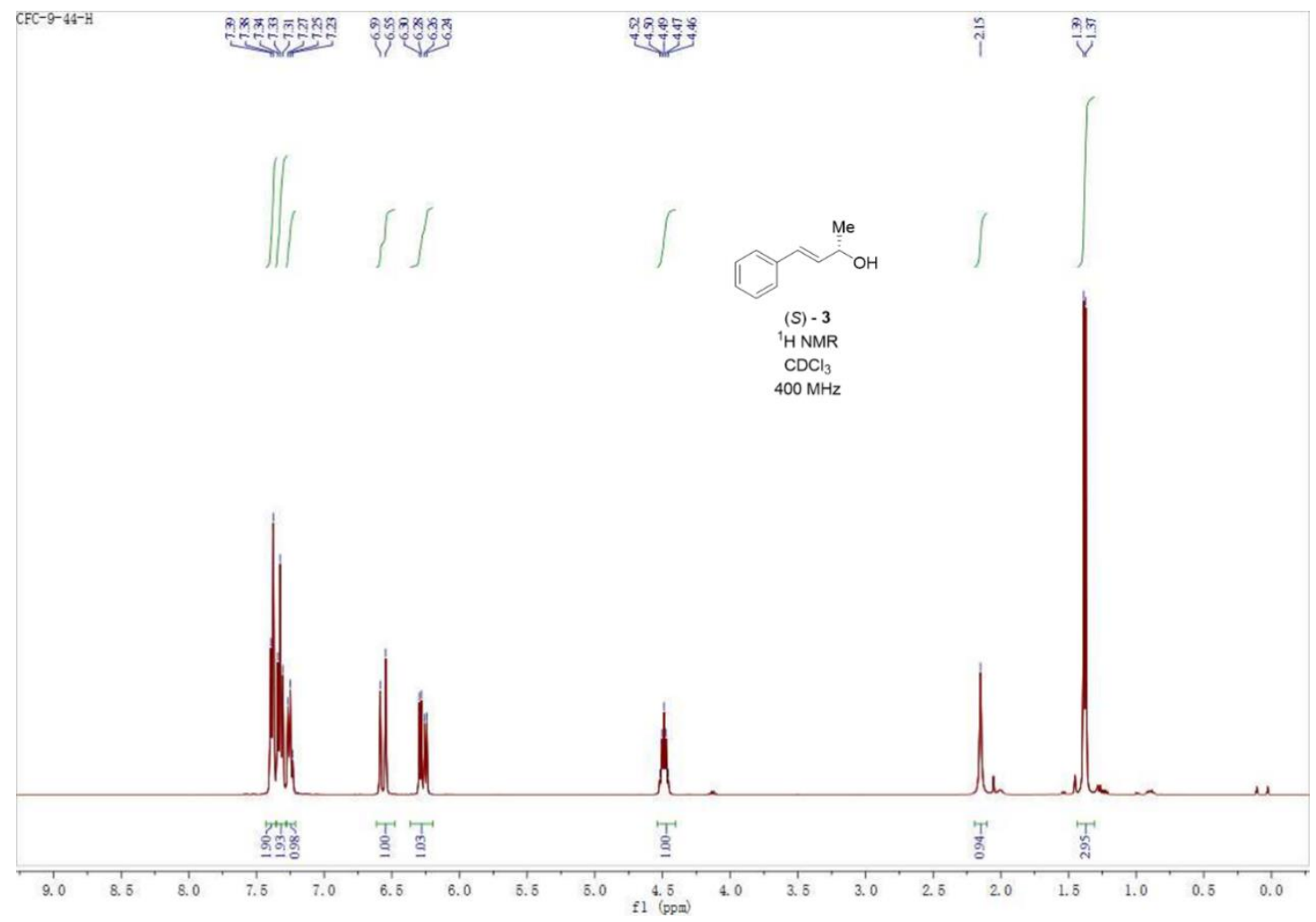

Figure S30. ${ }^{1} \mathrm{H}$ NMR spectrum of $(S)$-3. 


\section{HPLC spectra.}

35.1. HPLC spectra of substrate and product in ligandless Ni-catalyzed allylic $\mathrm{C}-\mathrm{O}$ bond activation in acetonitrile.<smiles>CC(O)C=Cc1ccccc1</smiles>

$97 \%$ ee

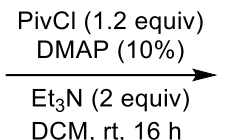

DCM, rt, $16 \mathrm{~h}$

(1)

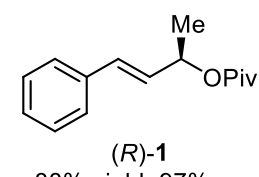

$88 \%$ yield, $97 \%$ ee

HPLC Condition: IC, $n$-hexane $/ i-\mathrm{PrOH}=99 / 1,0.8 \mathrm{~mL} / \mathrm{min}, 254 \mathrm{~nm}$

Racemic 1

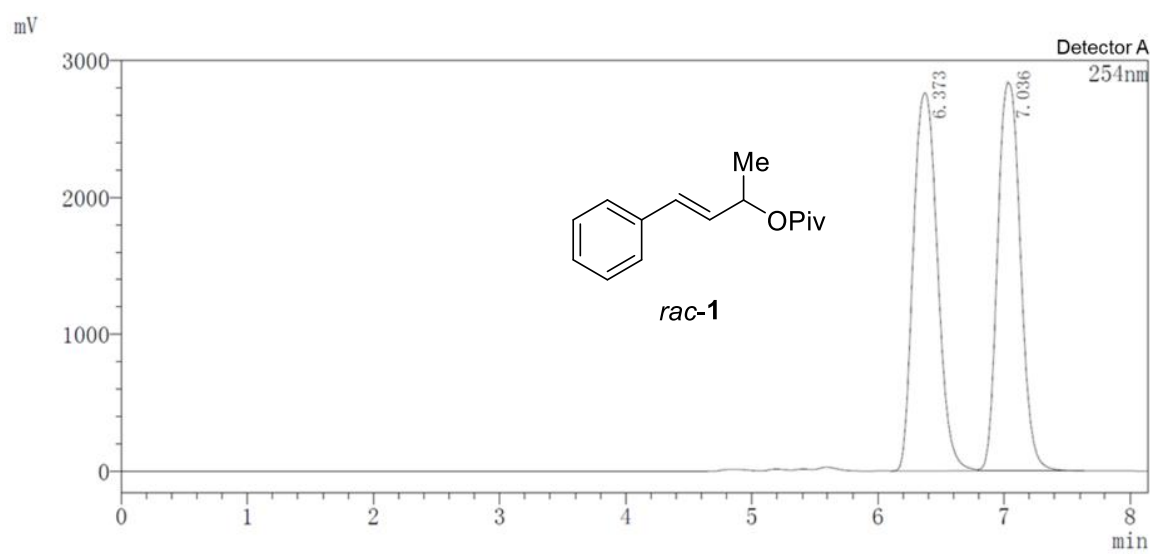

Detector A 254nm

Area Percent Report

\begin{tabular}{|c|c|c|c|c|}
\hline Peak & Remaining Time & Area & Height & Area \% \\
\hline 1 & 6.373 & 36799493 & 2763410 & 50.690 \\
\hline 2 & 7.036 & 35798302 & 2838234 & 49.310 \\
\hline Total & & 72597795 & 5601644 & 100.000 \\
\hline
\end{tabular}

Figure S31. HPLC spectrum of racemic 1 used in ligandless Ni-catalyzed allylic $\mathrm{C}-\mathrm{O}$ bond activation in acetonitrile. HPLC Condition: IC, $n$-hexane $/ i$-PrOH $=99 / 1,0.8 \mathrm{~mL} / \mathrm{min}, 254 \mathrm{~nm}$

Enantioenriched, $(R)-\mathbf{1}, 97 \%$ ee

$\mathrm{mV}$

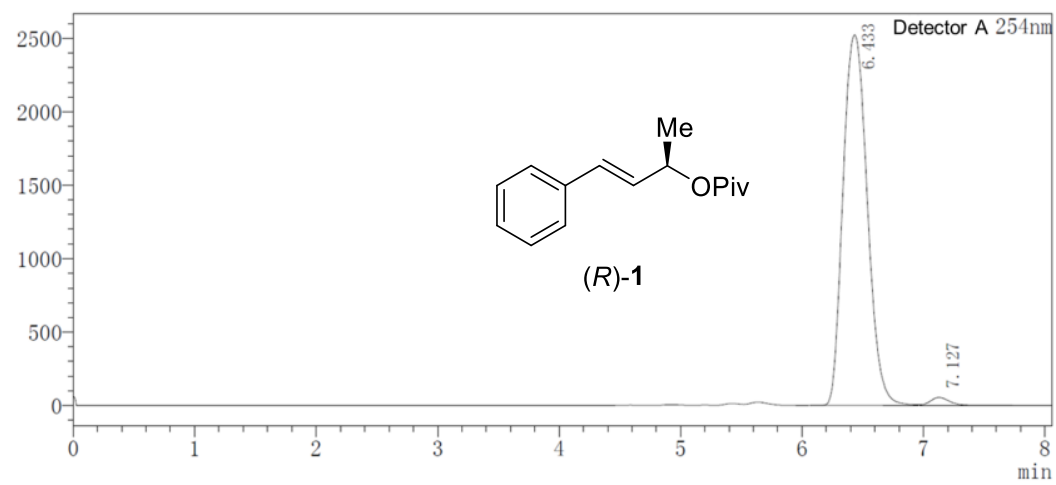

Detector A 254nm

Area Percent Report

\begin{tabular}{|c|c|c|c|c|}
\hline Peak & Remaining Time & Area & Height & Area \% \\
\hline 1 & 6.433 & 35085978 & 2525436 & 98.351 \\
\hline 2 & 7.127 & 588434 & 54510 & 1.649 \\
\hline Total & & 35674412 & 2579946 & 100.000 \\
\hline
\end{tabular}

Figure S32. HPLC spectrum of enantioenriched $(R)-1$ used in ligandless Ni-catalyzed allylic C $-\mathrm{O}$ bond activation in acetonitrile. 


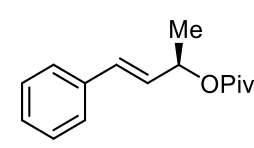

(R)-1

$97 \%$ ee

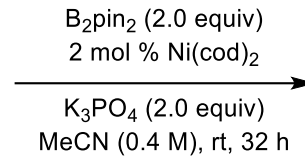

INVERSION

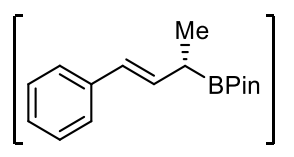

(S)-2

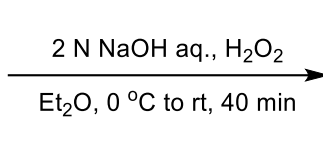

$\mathrm{Et}_{2} \mathrm{O}, 0^{\circ} \mathrm{C}$ to rt, $40 \mathrm{~min}$

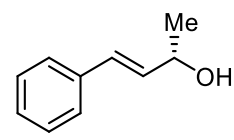

(S)-3

$65 \%$ yield, $88 \%$ ee

HPLC Condition: IB, $n$-hexane $/ i-\mathrm{PrOH}=97 / 3,1.0 \mathrm{~mL} / \mathrm{min}, 254 \mathrm{~nm}$

Racemic 3

\section{$<$ Chromatogram >}

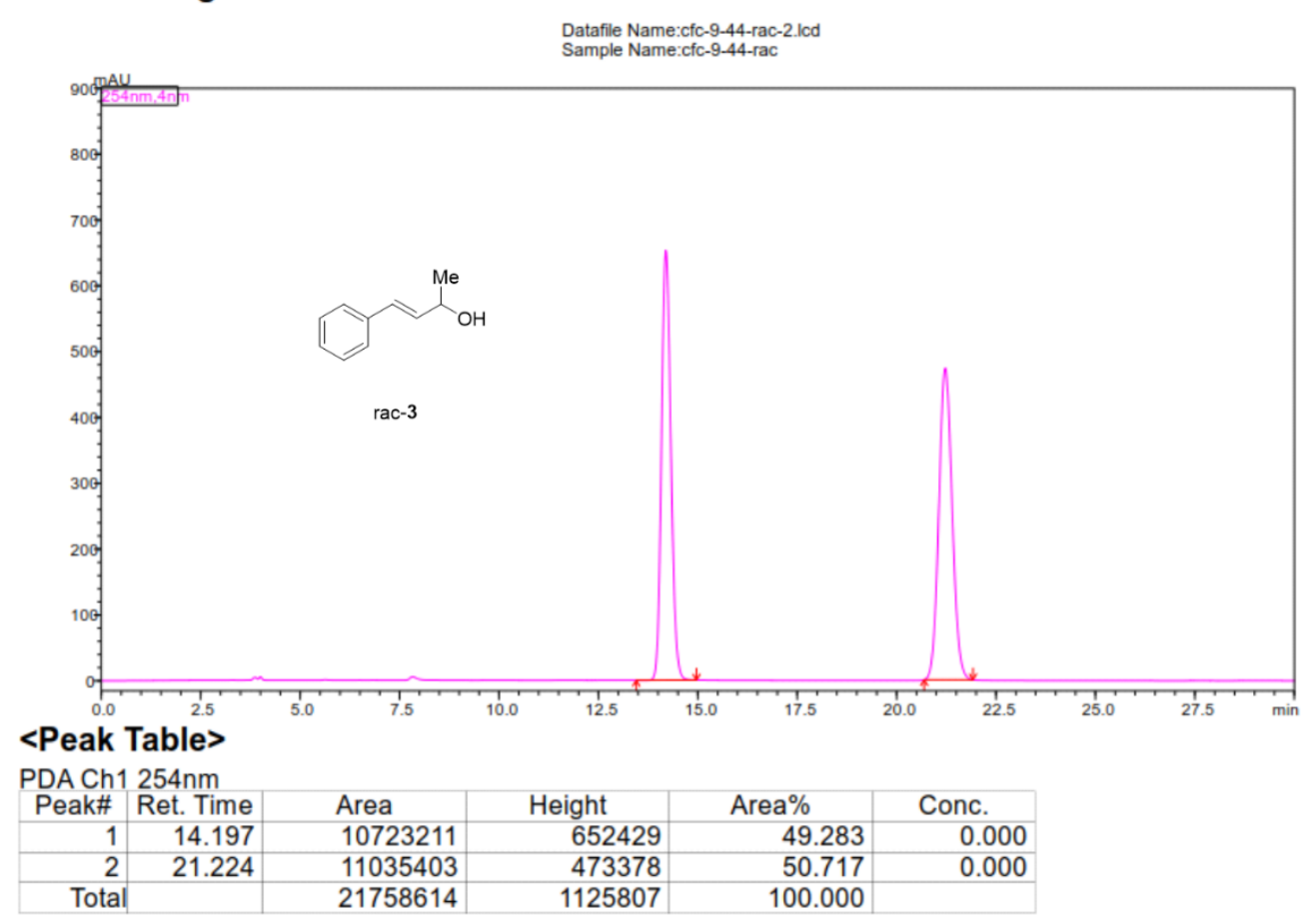

Figure S33. HPLC spectrum of racemic 3 formed in ligandless Ni-catalyzed allylic C-O bond activation in acetonitrile. 
HPLC Condition: IB, $n$-hexane $/ i-\mathrm{PrOH}=97 / 3,1.0 \mathrm{~mL} / \mathrm{min}, 254 \mathrm{~nm}$

Enantioenriched, $(S)-3,88 \%$ ee

\section{<Chromatogram>}

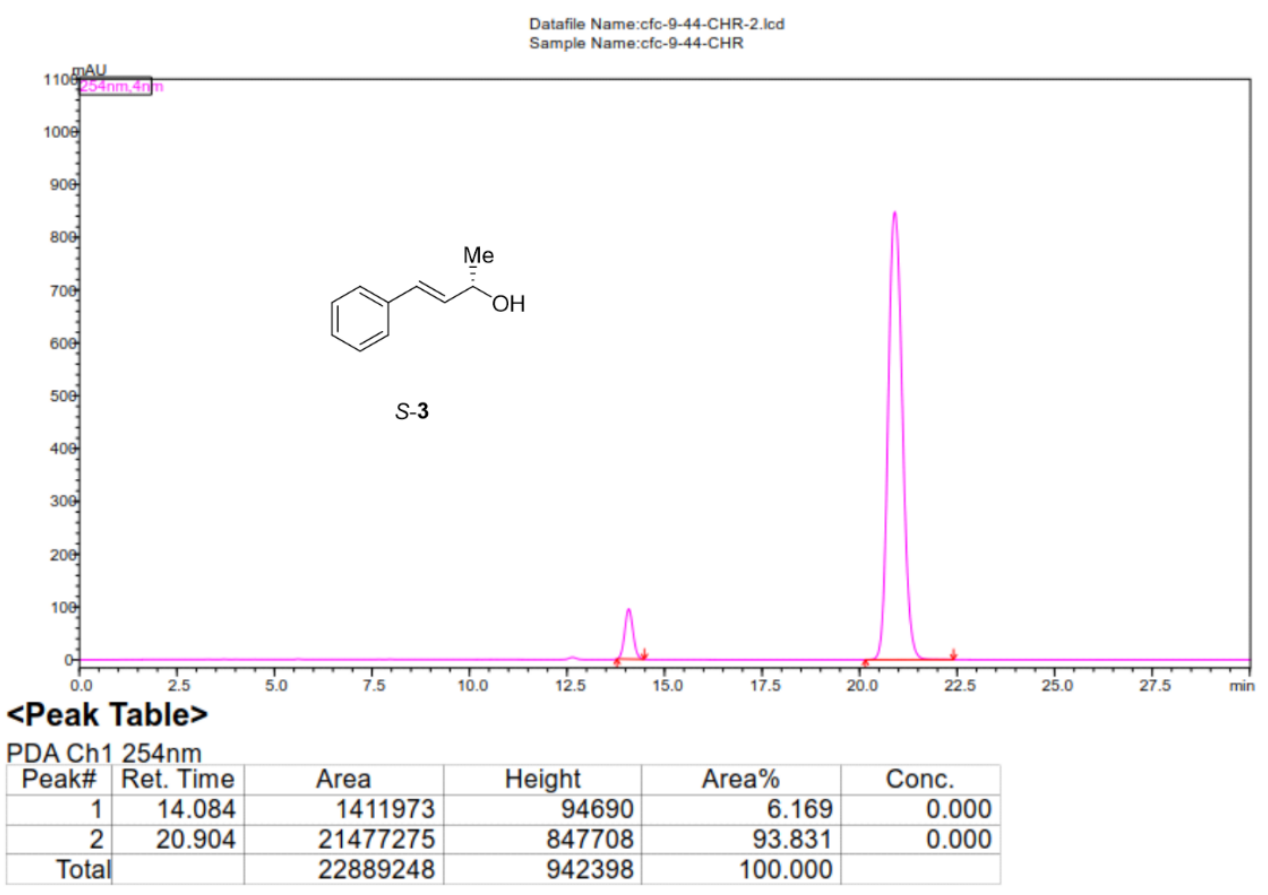

Figure S34. HPLC spectrum of enantioenriched $(S)-3$ formed in ligandless Ni-catalyzed allylic C-O bond activation in acetonitrile. 
35.2. HPLC spectra of substrate and product in ligandless Ni-catalyzed allylic C-O bond activation in hexane with catalytic amount of acetonitrile.

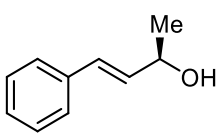

$88 \%$ ee

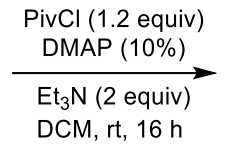

DCM, rt, $16 \mathrm{~h}$

HPLC Condition: IC, $n$-hexane $/ i$-PrOH $=99 / 1,0.8 \mathrm{~mL} / \mathrm{min}, 220 \mathrm{~nm}$

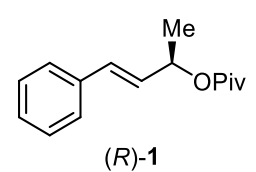

$85 \%$ yield, $88 \%$ ee

Enantioenriched, $(R)-1,88 \%$ ee

\section{<Chromatogram>}

Datafile Name:hy-11-82-0-chir.lcd

Sample Name:hy-11-82-0-chir

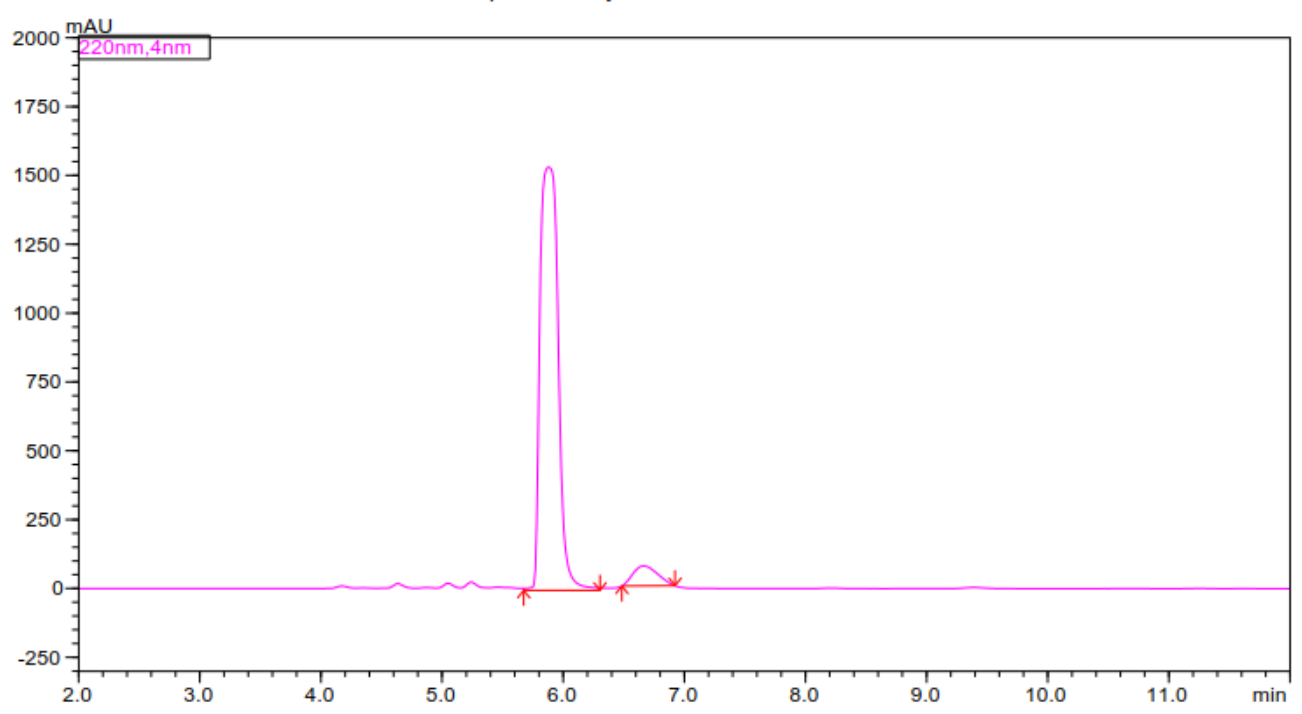

<Peak Table>

\section{PDA Ch1 220nm}

\begin{tabular}{|r|r|r|r|r|r|}
\hline Peak\# & Ret. Time & Area & Height & Area\% & \multicolumn{1}{c|}{ Conc. } \\
\hline 1 & 5.881 & 16106535 & 1536295 & 94.031 & 0.000 \\
\hline 2 & 6.664 & 1022508 & 72336 & 5.969 & 0.000 \\
\hline Total & & 17129043 & 1608631 & 100.000 & \\
\hline
\end{tabular}

Figure S35. HPLC spectrum of enantioenriched $(R)-1$ used in ligandless Ni-catalyzed allylic C-O bond activation in hexane with catalytic amount of acetonitrile. 


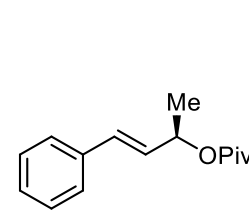

$(R)-1$
$88 \%$ ee

$$
\begin{gathered}
\mathrm{B}_{2} \mathrm{pin}_{2}(2.0 \text { equiv) } \\
2 \mathrm{~mol} \% \mathrm{Ni} \text { (cod) })_{2} \\
2.2 \mathrm{~mol} \% \mathrm{tBu}-\text { XantPhos } \\
4.4 \mathrm{~mol} \% \mathrm{MeCN} \\
\underset{\mathrm{K}_{3} \mathrm{PO}_{4}(2.0 \text { equiv })}{\longrightarrow} \\
\text { Hexane }(0.4 \mathrm{M}), \mathrm{rt}, 32 \mathrm{~h}
\end{gathered}
$$

RETENTION

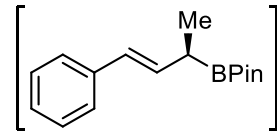

$(R)-2$

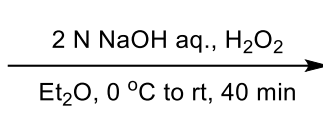

$\mathrm{Et}_{2} \mathrm{O}, 0^{\circ} \mathrm{C}$ to $\mathrm{rt}, 40 \mathrm{~min}$

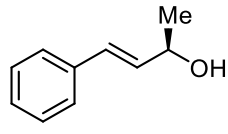

$(R)-3$

$74 \%$ yield, $88 \%$ ee

HPLC Condition: IB, $n$-hexane $/ i-\mathrm{PrOH}=97 / 3,1.0 \mathrm{~mL} / \mathrm{min}, 220 \mathrm{~nm}$

Enantioenriched, $(R)-3,88 \%$ ee

\section{$<$ Chromatogram>}

ame:hy-11-82-2.Icd

Sample Name:hy-11-82-2

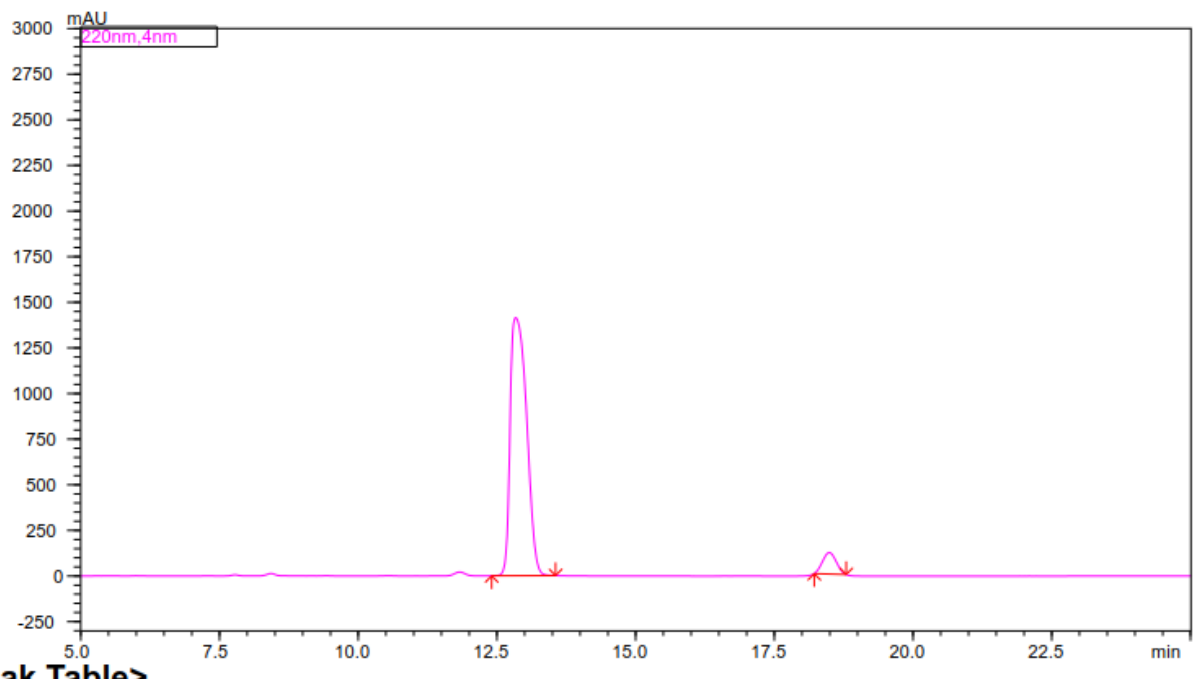

<Peak Table>

PDA Ch1 220nm

\begin{tabular}{|r|r|r|r|r|r|}
\hline Peak\# & Ret. Time & \multicolumn{1}{|c|}{ Area } & Height & Area $\%$ & \multicolumn{1}{c|}{ Conc. } \\
\hline 1 & 12.838 & 29166194 & 1413671 & 93.552 & 0.000 \\
\hline 2 & 18.490 & 2010394 & 117258 & 6.448 & 0.000 \\
\hline Total & & 31176588 & 1530929 & 100.000 & \\
\hline
\end{tabular}

Figure S36. HPLC spectrum of enantioenriched $(R)-3$ formed in ligandless Ni-catalyzed allylic C-O bond activation in hexane with catalytic amount of acetonitrile in the presence of $t \mathrm{Bu}$-XantPhos ligand. 
without $t \mathrm{Bu}-\mathrm{XantPhos}$ ligand

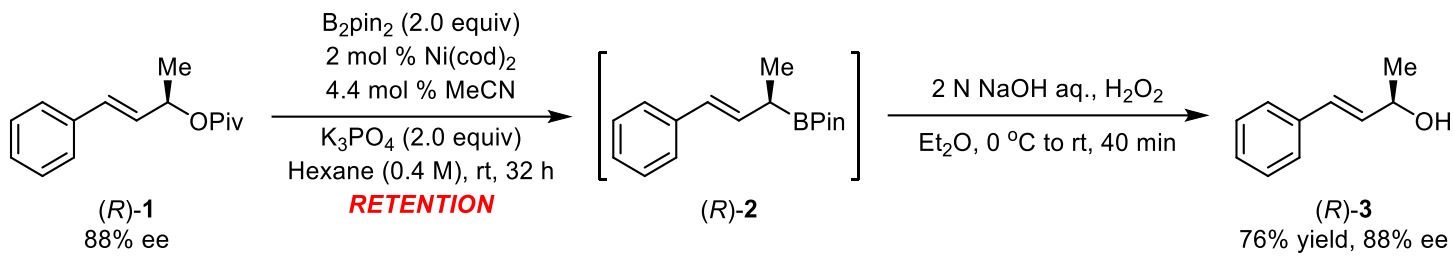

HPLC Condition: IB, $n$-hexane $/ i-\mathrm{PrOH}=97 / 3,1.0 \mathrm{~mL} / \mathrm{min}, 220 \mathrm{~nm}$

Enantioenriched, $(R)-3,88 \%$ ee

\section{$<$ Chromatogram $>$}

Datafile Name:hy-11-82-1.Icd

Sample Name:hy-11-82-1

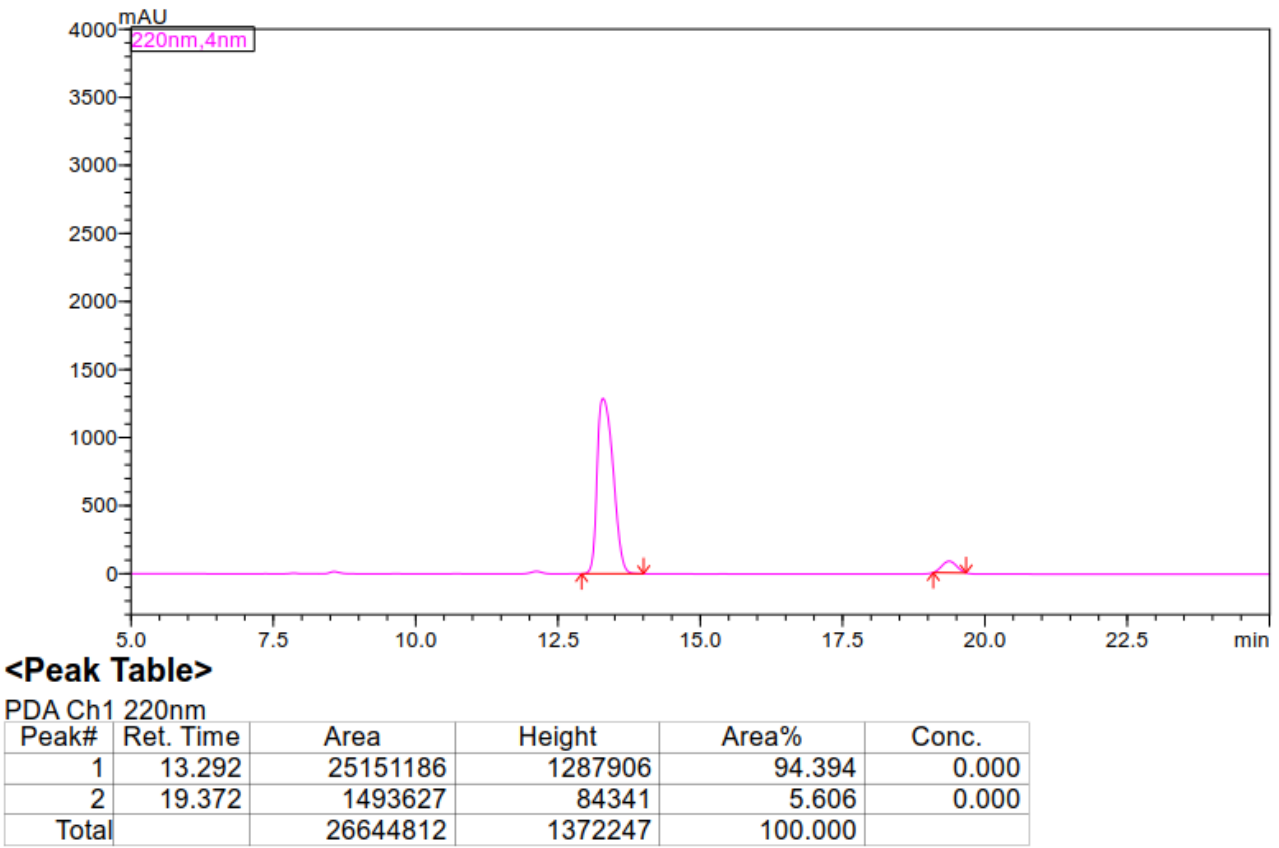

Figure S37. HPLC spectrum of enantioenriched $(R)$-3 formed in ligandless Ni-catalyzed allylic C-O bond activation in hexane with catalytic amount of acetonitrile in the absence of $t \mathrm{Bu}$-XantPhos ligand. 
36. Zero-point vibrational energy ( $Z P V E)$, thermal correction to enthalpy ( $T C H)$, thermal correction to Gibbs free energy (TCG), energies $(E)$, enthalpies $(H)$, and Gibbs free energies $(G)$ (in Hartree) of the structures calculated at the M06-L/def2-TZVPP-SMD(toluene)//B3LYP-D3(BJ)/def2-SVP-SMD(toluene) level of theory

Table S8. Energies in Figure 1, Scheme 3, Figure 2, Table 1, Figure S2, Table S1, Figure S6, Figure S7, Figure S8,

Figure S9, Figure S10, Figure S12, Figure S13, Figure S14, Figure S15, Figure S16, Figure S17, Figure S18,

Scheme S2, Figure S22, Figure S23

\begin{tabular}{|c|c|c|c|c|c|c|c|}
\hline Structures & $Z P V E$ & $T C H$ & $T C G$ & $E$ & $H$ & $G$ & Imaginary Frequency \\
\hline$(R)-1$ & 0.316541 & 0.335393 & 0.268262 & -734.287333 & -733.319994 & -733.387125 & \\
\hline$(R)-2$ & 0.361219 & 0.381570 & 0.312324 & -799.159351 & -798.103477 & -798.172723 & \\
\hline 3/Conformation-A & 1.042322 & 1.101351 & 0.956462 & -4211.134954 & -4208.072985 & -4208.217874 & \\
\hline 4 & 1.038499 & 1.098371 & 0.947432 & -4211.120679 & -4208.059366 & -4208.210306 & \\
\hline TS5/TS22 & 1.037656 & 1.097038 & 0.948343 & -4211.115301 & -4208.053960 & -4208.202654 & $329.5 i$ \\
\hline $6 / 21$ & 1.038141 & 1.098432 & 0.949719 & -4211.155273 & -4208.087556 & -4208.236269 & \\
\hline 7 & 1.284003 & 1.365146 & 1.169120 & -6529.908994 & -6525.653223 & -6525.849248 & \\
\hline 8 & 0.560941 & 0.602304 & 0.485708 & -4561.420948 & -4559.111466 & -4559.228062 & \\
\hline 9 & 0.561856 & 0.602726 & 0.490696 & -4561.409983 & -4559.110616 & -4559.222647 & \\
\hline TS10 & 0.560528 & 0.601226 & 0.489398 & -4561.387354 & -4559.088138 & -4559.1999666 & $85.2 i$ \\
\hline 11 & 0.562988 & 0.604304 & 0.491099 & -4561.428393 & -4559.125047 & -4559.238252 & \\
\hline TS12 & 0.561503 & 0.602478 & 0.489629 & -4561.425413 & -4559.123639 & -4559.236487 & $78.4 i$ \\
\hline 13 & 0.563512 & 0.604682 & 0.491182 & -4561.457154 & -4559.154861 & -4559.268361 & \\
\hline 14 & 0.199331 & 0.218117 & 0.152976 & -2253.935230 & -2252.800035 & -2252.865175 & \\
\hline $\mathrm{B}_{2} \mathrm{pin}_{2}-\mathrm{K}_{3} \mathrm{PO}_{4}$ & 0.380241 & 0.411082 & 0.322149 & -3265.270347 & -3263.553641 & -3263.642574 & \\
\hline$(\mathrm{KOPiv})_{4} / \mathbf{S 3}$ & 0.540837 & 0.586797 & 0.455246 & -3786.191723 & -3784.150719 & -3784.282271 & \\
\hline tBu-XantPhos & 0.718411 & 0.758253 & 0.651256 & -1968.474533 & -1966.500194 & -1966.607190 & \\
\hline TS15 & 0.556709 & 0.598717 & 0.479601 & -4561.350358 & -4559.052222 & -4559.171338 & $246.7 i$ \\
\hline 16 & 0.561519 & 0.602776 & 0.487184 & -4561.418378 & -4559.108733 & -4559.224326 & \\
\hline TS17 & 0.556872 & 0.599260 & 0.478947 & -4561.358000 & -4559.061463 & -4559.181776 & $287.6 i$ \\
\hline 18 & 0.561719 & 0.603570 & 0.486504 & -4561.431571 & -4559.130482 & -4559.247547 & \\
\hline TS19/TS23/TS-Conformation-B & 1.038684 & 1.098290 & 0.948212 & -4211.110501 & -4208.047415 & -4208.197493 & $145.8 i$ \\
\hline TS20/TS-Conformation-A & 1.039379 & 1.099035 & 0.951954 & -4211.110432 & -4208.047056 & -4208.194137 & $59.4 i$ \\
\hline 6-iso & 1.039346 & 1.099568 & 0.949851 & -4211.146447 & -4208.082815 & -4208.232533 & \\
\hline Conformation-B & 1.040031 & 1.099756 & 0.950042 & -4211.126058 & -4208.064869 & -4208.214583 & \\
\hline 7-iso & 1.284119 & 1.364750 & 1.172644 & -6529.901751 & -6525.653358 & -6525.845465 & \\
\hline 8-iso & 0.561174 & 0.601693 & 0.490759 & -4561.420462 & -4559.123150 & -4559.234084 & \\
\hline S1 & 0.134174 & 0.145103 & 0.097684 & -946.512963 & -945.988226 & -946.035645 & \\
\hline S2 & 0.269261 & 0.292325 & 0.211302 & -1893.067704 & -1892.03238 & -1892.113404 & \\
\hline TS-S4 & 1.402640 & 1.482966 & 1.292090 & -5033.854807 & -5029.709140 & -5029.900015 & $166.7 i$ \\
\hline TS-S5 & 1.285738 & 1.365782 & 1.176600 & -6529.849842 & -6525.593148 & -6525.782330 & $217.9 i$ \\
\hline TS-S6 & 1.423501 & 1.514054 & 1.303153 & -7476.383776 & -7471.621442 & -7471.832342 & $137.4 i$ \\
\hline $\mathrm{B}_{2} \mathrm{pin}_{2}$ & 0.362341 & 0.382860 & 0.315497 & -822.753177 & -821.650147 & -821.717510 & \\
\hline TS-S7 & 1.085734 & 1.146000 & 0.999825 & -4275.957057 & -4272.808921 & -4272.955096 & $47.6 i$ \\
\hline TS-S8 & 1.285490 & 1.365586 & 1.175536 & -6529.921097 & -6525.660997 & -6525.851047 & $144.4 i$ \\
\hline TS-S9 & 0.561315 & 0.602316 & 0.489533 & -4561.409067 & -4559.107394 & -4559.220177 & $159.1 i$ \\
\hline
\end{tabular}




\begin{tabular}{|c|c|c|c|c|c|c|c|}
\hline TS-S10 & 1.033925 & 1.094871 & 0.941541 & -4211.093953 & -4208.039804 & -4208.193134 & $268.8 i$ \\
\hline S11 & 1.037820 & 1.098517 & 0.947338 & -4211.150061 & -4208.083437 & -4208.234616 & \\
\hline TS-S12 & 0.560560 & 0.600755 & 0.491099 & -4561.312813 & -4559.018823 & -4559.128479 & $500.0 i$ \\
\hline TS-S13 & 0.943116 & 1.015809 & 0.836112 & -7826.666363 & -7822.677556 & -7822.857253 & $525.2 i$ \\
\hline S14 & 0.945680 & 1.019148 & 0.835537 & -7826.771232 & -7822.771588 & -7822.955198 & \\
\hline TS-S15 & 0.942838 & 1.015423 & 0.832166 & -7826.658182 & -7822.635366 & -7822.818622 & $463.0 i$ \\
\hline S16 & 0.945755 & 1.018982 & 0.836546 & -7826.786390 & -7822.797758 & -7822.980194 & \\
\hline $\mathrm{BnPPh}_{2}$ & 0.302380 & 0.320323 & 0.254959 & -1075.797888 & -1074.792612 & -1074.857975 & \\
\hline S17 & 0.927899 & 0.984457 & 0.832282 & -4394.297763 & -4391.213645 & -4391.365820 & \\
\hline S18 & 0.913960 & 0.969876 & 0.821842 & -4735.808853 & -4732.687607 & -4732.835642 & \\
\hline S19 & 1.222706 & 1.295639 & 1.116048 & -5811.594227 & -5807.485712 & -5807.665303 & \\
\hline S20 & 0.621379 & 0.659769 & 0.547410 & -3318.455421 & -3316.364465 & -3316.476824 & \\
\hline TS-S21 & 0.620077 & 0.658134 & 0.546513 & -3318.448494 & -3316.357580 & -3316.469202 & $326.2 i$ \\
\hline S22 & 0.620351 & 0.659330 & 0.548016 & -3318.493052 & -3316.398444 & -3316.509758 & \\
\hline S23 & 0.866052 & 0.925686 & 0.769820 & -5637.245392 & -5633.962902 & -5634.118767 & \\
\hline $\mathrm{P}(o-\mathrm{Tol})_{3}$ & 0.356515 & 0.378000 & 0.306539 & -1154.452378 & -1153.328007 & -1153.399468 & \\
\hline S24 & 1.038991 & 1.101555 & 0.940454 & -4551.562218 & -4548.237360 & -4548.398460 & \\
\hline S25 & 1.080801 & 1.145391 & 0.985958 & -4971.725055 & -4968.243005 & -4968.402439 & \\
\hline S28 & 0.676470 & 0.717700 & 0.602827 & -3397.104059 & -3394.893462 & -3395.008335 & \\
\hline TS-S29 & 0.675312 & 0.716159 & 0.603139 & -3397.096831 & -3394.886647 & -3394.999666 & $327.4 i$ \\
\hline S30 & 0.675933 & 0.717689 & 0.604034 & -3397.138628 & -3394.921653 & -3395.035309 & \\
\hline S31 & 0.921077 & 0.983866 & 0.822921 & -5715.886330 & -5712.480969 & -5712.641915 & \\
\hline tBu-XPhos & 0.669057 & 0.704836 & 0.605819 & -1473.844384 & -1472.149157 & -1472.248174 & \\
\hline S32 & 0.990543 & 1.046086 & 0.905531 & -3716.498653 & -3713.716571 & -3713.857126 & \\
\hline S33 & 1.345468 & 1.417635 & 1.246292 & -4456.013280 & -4452.501719 & -4452.673062 & \\
\hline S34 & 0.989418 & 1.045180 & 0.902390 & -3716.483958 & -3713.702681 & -3713.845471 & \\
\hline TS-S35 & 0.987860 & 1.043452 & 0.900876 & -3716.477757 & -3713.696782 & -3713.839358 & $331.1 i$ \\
\hline S36 & 0.988997 & 1.045331 & 0.903127 & -3716.516769 & -3713.727060 & -3713.869265 & \\
\hline S37 & 1.233772 & 1.311200 & 1.121834 & -6035.263610 & -6031.287368 & -6031.476735 & \\
\hline dppb & 0.480285 & 0.508856 & 0.416197 & -1766.823520 & -1765.263207 & -1765.355865 & \\
\hline S38 & 0.968448 & 1.025594 & 0.878848 & -5042.077616 & -5038.850504 & -5038.997250 & \\
\hline S39 & 0.801638 & 0.849862 & 0.716071 & -4009.515417 & -4006.871915 & -4007.005706 & \\
\hline S40 & 0.799164 & 0.848402 & 0.706656 & -4009.478729 & -4006.827911 & -4006.969657 & \\
\hline TS-S41 & 0.798384 & 0.847029 & 0.708707 & -4009.475825 & -4006.827339 & -4006.965661 & $321.3 i$ \\
\hline S42 & 0.798155 & 0.847953 & 0.707852 & -4009.521230 & -4006.868649 & -4007.008750 & \\
\hline S43 & 1.044063 & 1.114290 & 0.932817 & -6328.276828 & -6324.437360 & -6324.618833 & \\
\hline TS-S44 & 0.925936 & 0.982504 & 0.831875 & -4394.274814 & -4391.189011 & -4391.339640 & $133.1 i$ \\
\hline S45 & 1.037748 & 1.098053 & 0.944017 & -4211.110973 & -4208.045387 & -4208.199423 & \\
\hline S46 & 0.622239 & 0.660560 & 0.547459 & -3318.439624 & -3316.344707 & -3316.457808 & \\
\hline $\mathrm{BnPPh}_{2}$ & 0.302380 & 0.320323 & 0.254959 & -1075.797888 & -1074.792612 & -1074.857975 & \\
\hline 3-base complex & 1.426061 & 1.516210 & 1.305631 & -7476.419939 & -7471.670285 & -7471.880863 & \\
\hline TS-S51 & 1.423118 & 1.513249 & 1.303470 & -7476.409233 & -7471.658324 & -7471.868104 & $138.0 i$ \\
\hline TS-S52 & 1.035484 & 1.095637 & 0.943194 & -4211.078075 & -4208.017724 & -4208.170166 & $304.8 i$ \\
\hline TS-S53 & 1.040014 & 1.099177 & 0.952913 & -4211.10986 & -4208.041662 & -4208.187926 & $34.1 i$ \\
\hline
\end{tabular}


37. Zero-point vibrational energy ( $Z P V E)$, thermal correction to enthalpy ( $T C H)$, thermal correction to Gibbs free energy ( $T C G$ ), energies $(E)$, enthalpies $(H)$, and Gibbs free energies $(G)$ (in Hartree) of the structures calculated at the M06-L/def2-TZVPP-SMD(acetonitrile)//B3LYP-D3(BJ)/def2-SVP-SMD(acetonitrile) level of theory

Table S9. Energies in Table 1, Figure 4, Scheme 4, Table S1, Table S2, Figure S24, Figure S28

\begin{tabular}{|c|c|c|c|c|c|c|c|}
\hline Structures & $Z P V E$ & $\mathrm{TCH}$ & $T C G$ & E & $H$ & $G$ & Imaginary Frequency \\
\hline TS5/TS22 & 1.034073 & 1.094162 & 0.941509 & -4211.116940 & -4208.057311 & -4208.209965 & $338.5 i$ \\
\hline TS19/TS23/TS-Conformation-B & 1.034761 & 1.095038 & 0.944084 & -4211.117092 & -4208.056714 & -4208.207668 & $209.7 i$ \\
\hline $6 / 21$ & 1.035649 & 1.096175 & 0.946074 & -4211.157607 & -4208.091807 & -4208.241908 & \\
\hline 24/Conformation-B & 1.037051 & 1.097201 & 0.946265 & -4211.130765 & -4208.071380 & -4208.222315 & \\
\hline 25 & 0.410204 & 0.440022 & 0.346109 & -2508.260193 & -2506.804620 & -2506.898533 & \\
\hline TS26 & 0.361769 & 0.386112 & 0.306739 & -2375.428257 & -2374.151985 & -2374.231358 & $209.6 i$ \\
\hline $27 / 29$ & 0.361268 & 0.387003 & 0.302934 & -2375.476671 & -2374.195402 & -2374.279471 & \\
\hline TS28 & 0.407248 & 0.437512 & 0.341892 & -2508.243071 & -2506.784030 & -2506.879651 & $190.4 i$ \\
\hline tBu-XantPhos & 0.716541 & 0.756630 & 0.646758 & -1968.476590 & -1966.503230 & -1966.613102 & \\
\hline 30 & 1.035345 & 1.095783 & 0.941087 & -4211.112163 & -4208.048986 & -4208.203681 & \\
\hline 31 & 0.362761 & 0.387999 & 0.304032 & -2375.423489 & -2374.141367 & -2374.225334 & \\
\hline $\mathrm{MeCN}$ & 0.044951 & 0.049490 & 0.021979 & -132.799690 & -132.619208 & -132.646718 & \\
\hline Conformation-A & 1.040358 & 1.099516 & 0.954112 & -4211.137472 & -4208.077311 & -4208.222715 & \\
\hline TS-Conformation-A & 1.038267 & 1.097616 & 0.951688 & -4211.119500 & -4208.058108 & -4208.204036 & $177.6 i$ \\
\hline TS-S54 & 1.081067 & 1.146328 & 0.982444 & -4343.929801 & -4340.685777 & -4340.849660 & $219.1 i$ \\
\hline TS-S55 & 0.359839 & 0.385422 & 0.300980 & -2375.406818 & -2374.121936 & -2374.206377 & $221.6 i$ \\
\hline$(R)-1$ & 0.316073 & 0.334895 & 0.268244 & -734.287916 & -733.321184 & -733.387834 & \\
\hline$(S)-2$ & 0.360546 & 0.380910 & 0.311386 & -799.160292 & -798.105116 & -798.174641 & \\
\hline 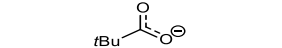 & 0.132319 & 0.141259 & 0.100449 & -346.640572 & -346.168758 & -346.209569 & \\
\hline S71 & 0.274195 & 0.295093 & 0.223068 & -2161.611418 & -2160.612899 & -2160.684925 & \\
\hline $\mathrm{B}_{2} \mathrm{pin}_{2}-\mathrm{K}_{3} \mathrm{PO}_{4}$ & 0.379025 & 0.410083 & 0.320726 & -3265.289305 & -3263.572011 & -3263.661368 & \\
\hline$(\mathrm{KOPiv})_{4}$ & 0.539511 & 0.585657 & 0.452927 & -3786.203059 & -3784.158396 & -3784.291126 & \\
\hline S72 & 0.606342 & 0.652938 & 0.524607 & -4694.236516 & -4691.760948 & -4691.889279 & \\
\hline S73 & 0.560247 & 0.601561 & 0.486898 & -4561.447251 & -4559.141914 & -4559.256577 & \\
\hline S74 & 0.560402 & 0.601655 & 0.487834 & -4561.425236 & -4559.125652 & -4559.239473 & \\
\hline TS-S75 & 0.560070 & 0.600625 & 0.489038 & -4561.410826 & -4559.108256 & -4559.219844 & $87.4 i$ \\
\hline S76 & 0.560576 & 0.602393 & 0.487046 & -4561.452643 & -4559.148106 & -4559.263453 & \\
\hline 14 & 0.198704 & 0.217624 & 0.152053 & -2253.953606 & -2252.815324 & -2252.880895 & \\
\hline S77 & 0.406499 & 0.433494 & 0.347942 & -2440.286022 & -2438.920735 & -2439.006287 & \\
\hline TS-S78 & 0.406284 & 0.432395 & 0.349845 & -2440.277415 & -2438.910649 & -2438.993200 & $102.7 i$ \\
\hline S79 & 0.407498 & 0.433865 & 0.350442 & -2440.297576 & -2438.933535 & -2439.016957 & \\
\hline
\end{tabular}


38. Zero-point vibrational energy ( $Z P V E)$, thermal correction to enthalpy ( $T C H)$, thermal correction to Gibbs free energy (TCG), energies $(E)$, enthalpies $(H)$, and Gibbs free energies $(G)$ (in Hartree) of the structures calculated at the M06-L/def2-TZVPP-SMD $(N, N$-dimethylformamide $) / /$ B3LYP-D3(BJ $) /$ def2-SVP-SMD $(N, N$ dimethylformamide) level of theory

Table S10. Energies in Table 1, Table S1, Table S2, Figure S25

\begin{tabular}{cccccccc}
\hline Structures & ZPVE & $T C H$ & $T C G$ & $E$ & $H$ & $G$ & Imaginary Frequency \\
\hline TS5/TS22 & 1.035290 & 1.094922 & 0.945441 & -4211.110868 & -4208.051399 & -4208.200879 & $335.7 i$ \\
TS20/TS23/TS-Conformation-A & 1.038404 & 1.097734 & 0.951975 & -4211.11446 & -4208.052834 & -4208.198594 & $164.0 i$ \\
TS-Conformation-B & 1.037392 & 1.096750 & 0.948878 & -4211.111240 & -4208.050729 & -4208.198601 & $195.4 i$ \\
Conformation-A & 1.040561 & 1.099691 & 0.954491 & -4211.132985 & -4208.072637 & -4208.217837 \\
24/Conformation-B & 1.037379 & 1.097440 & 0.947576 & -4211.126361 & -4208.066660 & -4208.216525 \\
6/21 & 1.035955 & 1.096412 & 0.947103 & -4211.152561 & -4208.086495 & -4208.235804 \\
tBu-XantPhos & 0.717286 & 0.757107 & 0.648357 & -1968.473029 & -1966.498869 & -1966.607620 & -248.280576 \\
DMF & 0.102011 & 0.109011 & 0.072978 & -248.584767 & -248.244544 & -2738.149982 \\
S56 & 0.523968 & 0.559352 & 0.451945 & -2739.810077 & -2738.042575 & -2738 \\
TS-S57 & 0.418625 & 0.445861 & 0.358664 & -2491.199529 & -2489.768649 & -2489.855846 \\
S58/S60 & 0.418829 & 0.446973 & 0.359264 & -2491.257572 & -2489.821220 & -2489.908928
\end{tabular}

39. Zero-point vibrational energy ( $Z P V E)$, thermal correction to enthalpy $(T C H)$, thermal correction to Gibbs free energy (TCG), energies $(E)$, enthalpies $(H)$, and Gibbs free energies $(G)$ (in Hartree) of the structures calculated at the M06-L/def2-TZVPP-SMD(EtOAc)//B3LYP-D3(BJ)/def2-SVP-SMD(EtOAc) level of theory

Table S11. Energies in Table 1, Table S1, Table S2, Figure S26

\begin{tabular}{|c|c|c|c|c|c|c|c|}
\hline Structures & $Z P V E$ & $T C H$ & $T C G$ & $E$ & $H$ & $G$ & Imaginary Frequency \\
\hline TS5/TS22 & 1.036318 & 1.095820 & 0.946626 & -4211.114237 & -4208.054048 & -4208.203243 & $333.3 i$ \\
\hline TS19/TS23/TS-Conformation-B & 1.038209 & 1.097588 & 0.948684 & -4211.112022 & -4208.050590 & -4208.199493 & $176.6 i$ \\
\hline TS-Conformation-A & 1.038795 & 1.098241 & 0.952215 & -4211.115021 & -4208.052164 & -4208.198191 & $123.2 i$ \\
\hline 3/Conformation-A & 1.041391 & 1.100432 & 0.955647 & -4211.135237 & -4208.074205 & -4208.218991 & \\
\hline Conformation-B & 1.038831 & 1.098616 & 0.948554 & -4211.127197 & -4208.067096 & -4208.217157 & \\
\hline $6 / 21$ & 1.036957 & 1.097306 & 0.948105 & -4211.155149 & -4208.088471 & -4208.237672 & \\
\hline tBu-XantPhos & 0.717843 & 0.757582 & 0.651407 & -1968.474201 & -1966.500204 & -1966.606379 & \\
\hline EtOAc & 0.117419 & 0.125791 & 0.085179 & -307.796169 & -307.378037 & -307.418649 & \\
\hline S61 & 0.555072 & 0.592884 & 0.479851 & -2858.229948 & -2856.305975 & -2856.419009 & \\
\hline TS-S62 & 0.434061 & 0.462531 & 0.371360 & -2550.410550 & -2548.900547 & -2548.991718 & $301.7 i$ \\
\hline S63/S65 & 0.434409 & 0.463783 & 0.372045 & -2550.466512 & -2548.951732 & -2549.043470 & \\
\hline TS-S64 & 0.553165 & 0.590945 & 0.478614 & -2858.215589 & -2856.287902 & -2856.400232 & $190.2 i$ \\
\hline
\end{tabular}


40. Zero-point vibrational energy ( $Z P V E)$, thermal correction to enthalpy ( $T C H)$, thermal correction to Gibbs free energy (TCG), energies $(E)$, enthalpies $(H)$, and Gibbs free energies $(G)$ (in Hartree) of the structures calculated at the M06-L/def2-TZVPP-SMD(THF)//B3LYP-D3(BJ)/def2-SVP-SMD(THF) level of theory

Table S12. Energies in Table 1, Table S1, Table S2, Figure S27

\begin{tabular}{|c|c|c|c|c|c|c|c|}
\hline Structures & $Z P V E$ & $T C H$ & $T C G$ & $E$ & $H$ & $G$ & Imaginary Frequency \\
\hline TS5/TS22 & 1.036134 & 1.095658 & 0.946318 & -4211.113214 & -4208.053146 & -4208.202486 & $334.0 i$ \\
\hline TS19/TS23/TS-Conformation-B & 1.038128 & 1.097498 & 0.948421 & -4211.111592 & -4208.050227 & -4208.199304 & $179.8 i$ \\
\hline TS-Conformation-A & 1.038622 & 1.098140 & 0.951581 & -4211.114286 & -4208.052059 & -4208.198618 & $135.7 i$ \\
\hline 3/Conformation-A & 1.041255 & 1.100308 & 0.955473 & -4211.135164 & -4208.073523 & -4208.218357 & \\
\hline Conformation-B & 1.038589 & 1.098424 & 0.947653 & -4211.126510 & -4208.066563 & -4208.217334 & \\
\hline $6 / 21$ & 1.036786 & 1.097153 & 0.947697 & -4211.154285 & -4208.087713 & -4208.237169 & \\
\hline$t$ Bu-XantPhos & 0.717098 & 0.757112 & 0.649413 & -1968.473510 & -1966.499926 & -1966.607625 & \\
\hline THF & 0.116254 & 0.122134 & 0.087331 & -232.507537 & -232.176978 & -232.211781 & \\
\hline S66 & 0.553274 & 0.585970 & 0.485152 & -2707.653534 & -2705.911367 & -2706.012185 & \\
\hline TS-S67 & 0.433134 & 0.459139 & 0.374863 & -2475.123429 & -2473.705313 & -2473.789589 & $307.2 i$ \\
\hline S68/S70 & 0.433801 & 0.460706 & 0.375495 & -2475.179211 & -2473.753597 & -2473.838807 & \\
\hline TS-S69 & 0.551233 & 0.583933 & 0.484066 & -2707.640080 & -2705.894312 & -2705.994179 & $196.4 i$ \\
\hline
\end{tabular}

41. Zero-point vibrational energy $(Z P V E)$, thermal correction to enthalpy $(T C H)$, thermal correction to Gibbs free energy ( $T C G$ ), energies $(E)$, enthalpies $(H)$, and Gibbs free energies $(G)$ (in Hartree) of the structures calculated at the M06-L/def2-TZVPP-SMD(hexane)//B3LYP-D3(BJ)/def2-SVP-SMD(hexane) level of theory

Table S13. Energies in Table 1, Table S1, Table S2

\begin{tabular}{ccccccccc}
\hline Structures & ZPVE & $T C H$ & $T C G$ & $E$ & $H$ & $G$ & Imaginary Frequency \\
\hline TS22 & 1.037926 & 1.097292 & 0.948599 & -4211.114321 & -4208.052759 & -4208.201452 & $328.1 i$ \\
TS23/TS-Conformation-B & 1.039028 & 1.098628 & 0.949460 & -4211.108278 & -4208.044746 & -4208.193913 & $127.7 i$ \\
TS-Conformation-A & 1.039665 & 1.099254 & 0.952612 & -4211.107369 & -4208.043993 & -4208.190635 & $19.5 i$ \\
Conformation-A & 1.042455 & 1.101515 & 0.956375 & -4211.133566 & -4208.071451 & -4208.216592 & -4208.213012 \\
Conformation-B & 1.040270 & 1.100006 & 0.950041 & -4211.124508 & -4208.063046 & -42 \\
\hline
\end{tabular}

42. Zero-point vibrational energy ( $Z P V E)$, thermal correction to enthalpy ( $T C H)$, thermal correction to Gibbs free energy (TCG), energies $(E)$, enthalpies $(H)$, and Gibbs free energies $(G)$ (in Hartree) of the structures calculated at the M06-L/def2-TZVPP-SMD(benzene)//B3LYP-D3(BJ)/def2-SVP-SMD(benzene) level of theory

Table S14. Energies in Table 1, Table S1, Table S2

\begin{tabular}{ccccccccc}
\hline Structures & ZPVE & $T C H$ & $T C G$ & $E$ & $H$ & $G$ & Imaginary Frequency \\
\hline TS22 & 1.037716 & 1.097096 & 0.948396 & -4211.116277 & -4208.054889 & -4208.203589 & $329.3 i$ \\
TS23/TS-Conformation-B & 1.038747 & 1.098352 & 0.948829 & -4211.111196 & -4208.048013 & -4208.197537 & $142.7 i$ \\
TS-Conformation-A & 1.039330 & 1.099029 & 0.951713 & -4211.110956 & -4208.047613 & -4208.194929 & $70.4 i$ \\
Conformation-A & 1.042367 & 1.101398 & 0.956482 & -4211.135802 & -4208.073786 & -4208.218702 & \\
Conformation-B & 1.040105 & 1.099825 & 0.950144 & -4211.126905 & -4208.065639 & -4208.215320 & \\
\hline
\end{tabular}


43. Zero-point vibrational energy ( $Z P V E)$, thermal correction to enthalpy ( $T C H)$, thermal correction to Gibbs free energy (TCG), energies $(E)$, enthalpies $(H)$, and Gibbs free energies $(G)$ (in Hartree) of the structures calculated at the M06-L/def2-TZVPP-SMD(DCM)//B3LYP-D3(BJ)/def2-SVP-SMD(DCM) level of theory

Table S15. Energies in Table 1, Table S1, Table S2

\begin{tabular}{ccccccccc}
\hline Structures & ZPVE & $T C H$ & $T C G$ & $E$ & $H$ & $G$ & Imaginary Frequency \\
\hline TS22 & 1.038245 & 1.097058 & 0.953315 & -4211.127016 & -4208.067315 & -4208.211059 & $320.4 i$ \\
TS23/TS-Conformation-A & 1.038729 & 1.098060 & 0.952398 & -4211.123042 & -4208.061004 & -4208.206666 & $152.8 i$ \\
TS-Conformation-B & 1.038300 & 1.097473 & 0.950434 & -4211.119638 & -4208.059250 & -4208.206289 & $191.1 i$ \\
Conformation-A & 1.040917 & 1.100033 & 0.954809 & -4211.142020 & -4208.081367 & -4208.226591 & -4208.224098
\end{tabular}

44. Zero-point vibrational energy ( $Z P V E)$, thermal correction to enthalpy $(T C H)$, thermal correction to Gibbs free energy (TCG), energies $(E)$, enthalpies $(H)$, and Gibbs free energies $(G)$ (in Hartree) of the structures calculated at the M06/def2-TZVPP-SMD(solvent)//B3LYP-D3(BJ)/def2-SVP-SMD(solvent) level of theory

Table S16. Energies in Figure S22, Table S3

\begin{tabular}{|c|c|c|c|c|c|c|c|c|}
\hline Solvent & Structures & $Z P V E$ & $T C H$ & $T C G$ & E & $H$ & $G$ & Imaginary Frequency \\
\hline & TS22 & 1.037926 & 1.097292 & 0.948599 & -4209.842628 & -4208.052759 & -4208.201452 & $328.1 i$ \\
\hline \multirow[t]{3}{*}{ Hexane } & TS-Conformation-A & 1.039665 & 1.099254 & 0.952612 & -4209.825930 & -4208.043993 & -4208.190635 & $19.5 i$ \\
\hline & TS23/TS-Conformation-B & 1.039028 & 1.098628 & 0.949460 & -4209.829872 & -4208.044746 & -4208.193913 & $127.7 i$ \\
\hline & TS22 & 1.037716 & 1.097096 & 0.948396 & -4209.844796 & -4208.054889 & -4208.203589 & $329.3 i$ \\
\hline \multirow[t]{5}{*}{ Benzene } & TS-Conformation-A & 1.039330 & 1.099029 & 0.951713 & -4209.829413 & -4208.047613 & -4208.194929 & $70.4 i$ \\
\hline & TS23/TS-Conformation-B & 1.038747 & 1.098352 & 0.948829 & -4209.832814 & -4208.048013 & -4208.197537 & $142.7 i$ \\
\hline & 3 & 1.042322 & 1.101351 & 0.956462 & -4209.862948 & -4208.072985 & -4208.217874 & \\
\hline & TS19 & 1.038684 & 1.098290 & 0.948212 & -4209.832164 & -4208.047415 & -4208.197493 & $145.8 i$ \\
\hline & B2pin2- $\mathrm{K}_{3} \mathrm{PO}_{4}$ & 0.380241 & 0.411082 & 0.322149 & -3264.726878 & -3263.553641 & -3263.642574 & \\
\hline \multirow{5}{*}{ Toluene } & TS-S51 & 1.423118 & 1.513249 & 1.303470 & -7474.586453 & -7471.658324 & -7471.868104 & $138.0 i$ \\
\hline & TS22 & 1.037656 & 1.097038 & 0.948343 & -4209.843865 & -4208.053960 & -4208.202654 & $329.5 i$ \\
\hline & TS-Conformation-A & 1.039379 & 1.099035 & 0.951954 & -4209.828911 & -4208.047056 & -4208.194137 & $59.4 i$ \\
\hline & TS23/TS-Conformation-B & 1.038684 & 1.098290 & 0.948212 & -4209.832164 & -4208.047415 & -4208.197493 & $145.8 i$ \\
\hline & TS22 & 1.036318 & 1.095820 & 0.946626 & -4209.843540 & -4208.054048 & -4208.203243 & $333.3 i$ \\
\hline \multirow[t]{3}{*}{ EtOAc } & TS-Conformation-A & 1.038795 & 1.098241 & 0.952215 & -4209.834916 & -4208.052164 & -4208.198191 & $123.2 i$ \\
\hline & TS23/TS-Conformation-B & 1.038209 & 1.097588 & 0.948684 & -4209.834886 & -4208.050590 & -4208.199493 & $176.6 i$ \\
\hline & TS22 & 1.036134 & 1.095658 & 0.946318 & -4209.842642 & -4208.053146 & -4208.202486 & $334.0 i$ \\
\hline \multirow[t]{3}{*}{ THF } & TS-Conformation-A & 1.038622 & 1.098140 & 0.951581 & -4209.834551 & -4208.052059 & -4208.198618 & $135.7 i$ \\
\hline & TS23/TS-Conformation-B & 1.038128 & 1.097498 & 0.948421 & -4209.834473 & -4208.050227 & -4208.199304 & $179.8 i$ \\
\hline & TS22 & 1.038245 & 1.097058 & 0.953315 & -4209.852882 & -4208.067315 & -4208.211059 & $320.4 i$ \\
\hline \multirow[t]{3}{*}{ DCM } & TS23/TS-Conformation-A & 1.038729 & 1.098060 & 0.952398 & -4209.843607 & -4208.061004 & -4208.206666 & $152.8 i$ \\
\hline & TS-Conformation-B & 1.038300 & 1.097473 & 0.950434 & -4209.843610 & -4208.059250 & -4208.206289 & $191.1 i$ \\
\hline & TS22 & 1.035290 & 1.094922 & 0.945441 & -4209.840847 & -4208.051399 & -4208.200879 & $335.7 i$ \\
\hline \multirow[t]{3}{*}{ DMF } & TS-Conformation-A & 1.038404 & 1.097734 & 0.951975 & -4209.835788 & -4208.052834 & -4208.198594 & $164.0 i$ \\
\hline & TS23/TS-Conformation-B & 1.037392 & 1.096750 & 0.948878 & -4209.835299 & -4208.050729 & -4208.198601 & $195.4 i$ \\
\hline & TS22 & 0.361769 & 0.386112 & 0.306739 & -2374.907219 & -2374.151985 & -2374.231358 & $290.6 i$ \\
\hline \multirow[t]{2}{*}{$\mathrm{MeCN}$} & TS23 & 0.407248 & 0.437512 & 0.341892 & -2507.641091 & -2506.784030 & -2506.879651 & $190.4 i$ \\
\hline & $\mathrm{MeCN}$ & 0.044951 & 0.049490 & 0.021979 & -132.720666 & -132.619208 & -132.646718 & \\
\hline
\end{tabular}


45. Zero-point vibrational energy ( $Z P V E)$, thermal correction to enthalpy ( $T C H)$, thermal correction to Gibbs free energy ( $T C G$ ), energies $(E)$, enthalpies $(H)$, and Gibbs free energies $(G)$ (in Hartree) of the structures calculated at the B3LYP-D3(BJ)/def2-TZVPP-SMD(solvent)//B3LYP-D3(BJ)/def2-SVP-SMD(solvent) level of theory

Table S17. Energies in Table S3

\begin{tabular}{|c|c|c|c|c|c|c|c|c|}
\hline Solvent & Structures & $Z P V E$ & $T C H$ & $T C G$ & E & $H$ & $G$ & Imaginary Frequency \\
\hline \multirow{3}{*}{ Hexane } & TS22 & 1.037926 & 1.097292 & 0.948599 & -4211.821757 & -4208.052759 & -4208.201452 & $328.1 i$ \\
\hline & TS-Conformation-A & 1.039665 & 1.099254 & 0.952612 & -4211.816676 & -4208.043993 & -4208.190635 & $19.5 i$ \\
\hline & TS23/TS-Conformation-B & 1.039028 & 1.098628 & 0.949460 & -4211.817243 & -4208.044746 & -4208.193913 & $127.7 i$ \\
\hline \multirow{3}{*}{ Benzene } & TS22 & 1.037716 & 1.097096 & 0.948396 & -4211.823945 & -4208.054889 & -4208.203589 & $329.3 i$ \\
\hline & TS-Conformation-A & 1.039330 & 1.099029 & 0.951713 & -4211.820326 & -4208.047613 & -4208.194929 & $70.4 i$ \\
\hline & TS23/TS-Conformation-B & 1.038747 & 1.098352 & 0.948829 & -4211.820502 & -4208.048013 & -4208.197537 & $142.7 i$ \\
\hline \multirow{3}{*}{ Toluene } & TS22 & 1.037656 & 1.097038 & 0.948343 & -4211.823020 & -4208.053960 & -4208.202654 & $329.5 i$ \\
\hline & TS-Conformation-A & 1.039379 & 1.099035 & 0.951954 & -4211.819840 & -4208.047056 & -4208.194137 & $59.4 i$ \\
\hline & TS23/TS-Conformation-B & 1.038684 & 1.098290 & 0.948212 & -4211.819906 & -4208.047415 & -4208.197493 & $145.8 i$ \\
\hline \multirow{3}{*}{ EtOAc } & TS22 & 1.036318 & 1.095820 & 0.946626 & -4211.822848 & -4208.054048 & -4208.203243 & $333.3 i$ \\
\hline & TS-Conformation-A & 1.038795 & 1.098241 & 0.952215 & -4211.825559 & -4208.052164 & -4208.198191 & $123.2 i$ \\
\hline & TS23/TS-Conformation-B & 1.038209 & 1.097588 & 0.948684 & -4211.823277 & -4208.050590 & -4208.199493 & $176.6 i$ \\
\hline \multirow{3}{*}{ THF } & TS22 & 1.036134 & 1.095658 & 0.946318 & -4211.821948 & -4208.053146 & -4208.202486 & $334.0 i$ \\
\hline & TS-Conformation-A & 1.038622 & 1.098140 & 0.951581 & -4211.825486 & -4208.052059 & -4208.198618 & $135.7 i$ \\
\hline & TS23/TS-Conformation-B & 1.038128 & 1.097498 & 0.948421 & -4211.823020 & -4208.050227 & -4208.199304 & $179.8 i$ \\
\hline \multirow{3}{*}{ DCM } & TS22 & 1.038245 & 1.097058 & 0.953315 & -4211.835541 & -4208.067315 & -4208.211059 & $320.4 i$ \\
\hline & TS23/TS-Conformation-A & 1.038729 & 1.098060 & 0.952398 & -4211.834622 & -4208.061004 & -4208.206666 & $152.8 i$ \\
\hline & TS-Conformation-B & 1.038300 & 1.097473 & 0.950434 & -4211.831976 & -4208.059250 & -4208.206289 & $191.1 i$ \\
\hline \multirow{3}{*}{ DMF } & TS22 & 1.035290 & 1.094922 & 0.945441 & -4211.820098 & -4208.051399 & -4208.200879 & $335.7 i$ \\
\hline & TS-Conformation-A & 1.038404 & 1.097734 & 0.951975 & -4211.826571 & -4208.052834 & -4208.198594 & $164.0 i$ \\
\hline & TS23/TS-Conformation-B & 1.037392 & 1.096750 & 0.948878 & -4211.823631 & -4208.050729 & -4208.198601 & $195.4 i$ \\
\hline \multirow{3}{*}{$\mathrm{MeCN}$} & TS22 & 0.361769 & 0.386112 & 0.306739 & -2375.724344 & -2374.151985 & -2374.231358 & $290.6 i$ \\
\hline & TS23 & 0.407248 & 0.437512 & 0.341892 & -2508.565064 & -2506.784030 & -2506.879651 & $190.4 i$ \\
\hline & $\mathrm{MeCN}$ & 0.044951 & 0.049490 & 0.021979 & -132.826382 & -132.619208 & -132.646718 & \\
\hline
\end{tabular}

46. Zero-point vibrational energy $(Z P V E)$, thermal correction to enthalpy $(T C H)$, thermal correction to Gibbs free energy (TCG), energies $(E)$, enthalpies $(H)$, and Gibbs free energies $(G)$ (in Hartree) of the structures calculated at the M06-L/def2-TZVPP-SMD(heptane)//B3LYP-D3(BJ)/def2-SVP-SMD(heptane) level of theory

Table S18. Energies in Table S5, Table S6

\begin{tabular}{ccccccccc}
\hline Structures & ZPVE & $T C H$ & $T C G$ & $\boldsymbol{E}$ & $\boldsymbol{H}$ & Imaginary Frequency \\
\hline TSS & 1.037922 & 1.097287 & 0.948605 & -4211.113649 & -4208.052082 & -4208.200764 & $328.2 i$ \\
TS20/TS-Conformation-A & 1.039551 & 1.099209 & 0.952052 & -4211.107053 & -4208.043522 & -4208.190679 & $20.2 i$ \\
TS19/TS-Conformation-B & 1.039015 & 1.098615 & 0.949442 & -4211.107724 & -4208.044208 & -4208.193382 & $129.5 i$ \\
\hline
\end{tabular}


47. Zero-point vibrational energy ( $Z P V E)$, thermal correction to enthalpy ( $T C H)$, thermal correction to Gibbs free energy (TCG), energies $(E)$, enthalpies $(H)$, and Gibbs free energies $(G)$ (in Hartree) of the structures calculated at the M06-L/def2-TZVPP-SMD(1,2-dichloroethane)//B3LYP-D3(BJ)/def2-SVP-SMD(1,2-dichloroethane) level of theory

Table S19. Energies in Table S5, Table S6

\begin{tabular}{ccccccccc}
\hline Structures & ZPVE & $T C H$ & $T C G$ & $E$ & $H$ & $G$ & Imaginary Frequency \\
\hline TSS & 1.036006 & 1.095448 & 0.946473 & -4211.117459 & -4208.057563 & -4208.206537 & $337.2 i$ \\
TS20/TS-Conformation-A & 1.038773 & 1.098071 & 0.952650 & -4211.119334 & -4208.057353 & -4208.202774 & $158.2 i$ \\
TS19/TS-Conformation-B & 1.038315 & 1.097466 & 0.950560 & -4211.115788 & -4208.055416 & -4208.202321 & $193.1 i$ & \\
\hline
\end{tabular}

48. Zero-point vibrational energy ( $Z P V E)$, thermal correction to enthalpy ( $T C H)$, thermal correction to Gibbs free energy (TCG), energies $(E)$, enthalpies $(H)$, and Gibbs free energies $(G)$ (in Hartree) of the structures calculated at the M06-L/def2-TZVPP-SMD $(N, N$-dimethylacetamide $) / / B 3 L Y P-D 3(B J) /$ def2-SVP-SMD $(N, N$ dimethylacetamide) level of theory

Table S20. Energies in Table S5, Table S6

\begin{tabular}{cccccccc}
\hline Structures & ZPVE & $T C H$ & $T C G$ & $E$ & $H$ & $G$ & Imaginary Frequency \\
\hline TS5 & 1.035293 & 1.094924 & 0.945444 & -4211.111502 & -4208.052033 & -4208.201514 & $335.7 i$ \\
TS20/TS-Conformation-A & 1.038399 & 1.097732 & 0.951962 & -4211.115096 & -4208.053475 & -4208.199245 & $164.2 i$ \\
TS19/TS-Conformation-B & 1.037472 & 1.096854 & 0.948436 & -4211.111433 & -4208.051234 & -4208.199652 & $198.0 i$ \\
\hline
\end{tabular}




\section{Cartesian coordinates of the structures (optimization in toluene)}

$(R)-1$

C

C

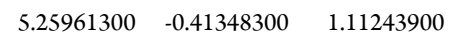




\begin{tabular}{|c|c|c|c|}
\hline $\mathrm{C}$ & 0.39808400 & 2.04022800 & -0.59156000 \\
\hline $\mathrm{H}$ & -0.99570100 & 1.53771500 & 1.04585200 \\
\hline $\mathrm{C}$ & -3.35691400 & 0.07327400 & 1.09760800 \\
\hline $\mathrm{C}$ & -3.96850100 & -0.32979200 & -1.20147100 \\
\hline $\mathrm{C}$ & 0.77310300 & 3.38055700 & 0.05598100 \\
\hline $\mathrm{H}$ & 0.29549500 & 2.15859800 & -1.68346100 \\
\hline $\mathrm{C}$ & -4.53424300 & -0.54384800 & 1.51533900 \\
\hline $\mathrm{H}$ & -2.66295100 & 0.45977100 & 1.84694500 \\
\hline $\mathrm{C}$ & -5.14778200 & -0.94927600 & -0.78387900 \\
\hline $\mathrm{H}$ & -3.74804400 & -0.24792600 & -2.26934300 \\
\hline $\mathrm{H}$ & -0.00648600 & 4.14198500 & -0.11209200 \\
\hline $\mathrm{H}$ & 1.71931800 & 3.77051700 & -0.35279200 \\
\hline $\mathrm{H}$ & 0.90743700 & 3.27198900 & 1.14441800 \\
\hline $\mathrm{C}$ & -5.43815600 & -1.05889600 & 0.57811300 \\
\hline $\mathrm{H}$ & -4.74936000 & -0.62798600 & 2.58383700 \\
\hline $\mathrm{H}$ & -5.84301000 & -1.34867600 & -1.52683200 \\
\hline $\mathrm{H}$ & -6.35942800 & -1.54397800 & 0.90975300 \\
\hline B & 1.46277900 & 0.90315800 & -0.33348000 \\
\hline $\mathrm{O}$ & 1.84059500 & -0.00804500 & -1.28069800 \\
\hline $\mathrm{O}$ & 2.03557000 & 0.67696900 & 0.88814200 \\
\hline $\mathrm{C}$ & 2.53098600 & -1.08745400 & -0.59583800 \\
\hline $\mathrm{C}$ & 3.01859900 & -0.38043800 & 0.72841000 \\
\hline $\mathrm{C}$ & 3.64473300 & -1.60865000 & -1.49132800 \\
\hline $\mathrm{C}$ & 1.49181200 & -2.18092200 & -0.34395700 \\
\hline $\mathrm{C}$ & 2.99784100 & -1.26254500 & 1.96856500 \\
\hline $\mathrm{C}$ & 4.38055800 & 0.29952800 & 0.58287000 \\
\hline $\mathrm{H}$ & 3.21180500 & -2.07570800 & -2.38892700 \\
\hline $\mathrm{H}$ & 4.24235600 & -2.37054500 & -0.96732500 \\
\hline $\mathrm{H}$ & 4.31330500 & -0.80256800 & -1.81997400 \\
\hline $\mathrm{H}$ & 1.04740600 & -2.47794700 & -1.30547300 \\
\hline $\mathrm{H}$ & 1.94118300 & -3.07167300 & 0.11887800 \\
\hline $\mathrm{H}$ & 0.67899100 & -1.82017400 & 0.30325300 \\
\hline $\mathrm{H}$ & 3.34649700 & -0.68737400 & 2.83974200 \\
\hline $\mathrm{H}$ & 3.66748600 & -2.12736400 & 1.84279900 \\
\hline $\mathrm{H}$ & 1.98722600 & -1.62982100 & 2.18976600 \\
\hline $\mathrm{H}$ & 5.19816200 & -0.43424700 & 0.52955400 \\
\hline $\mathrm{H}$ & 4.55266200 & 0.94398400 & 1.45783800 \\
\hline $\mathrm{H}$ & 4.42073300 & 0.93253200 & -0.31601500 \\
\hline
\end{tabular}

3/Conformation-A

$\begin{array}{llll}\text { C } & 1.91500400 & 1.20953500 & 0.96497700 \\ \text { C } & 1.65616100 & -0.15812300 & 1.24962600 \\ \text { C } & 0.99121600 & -0.59126400 & 2.50872400 \\ \text { C } & 1.08271400 & 0.16432300 & 3.69519200 \\ \mathrm{H} & 0.13669200 & -2.38883300 & 1.66928500\end{array}$


$2.36282900 \quad-0.89072400 \quad 0.85744200$

$\begin{array}{lll}0.24251900 & -1.78397700 & 2.56702300\end{array}$

$0.43254300 \quad-0.23203300 \quad 4.86595800$

$\begin{array}{lll}-0.31947200 & -1.40975400 \quad 4.89512700\end{array}$

$\begin{array}{lll}-0.40168400 & -2.18850700 \quad 3.73587300\end{array}$

$\begin{array}{lll}0.52182600 & 0.38246100 & 5.76625500\end{array}$

$\begin{array}{lll}-0.83053100 & -1.71953500 & 5.81019700\end{array}$

$\begin{array}{lll}-0.98474200 & -3.11338200 & 3.73476600\end{array}$

$3.13886000 \quad 1.59401100 \quad 0.16090100$

$5.24248200 \quad 0.45199600 \quad-0.17344500$

$5.27060000 \quad 0.76032800 \quad-1.34567600$

$6.30347100 \quad-0.40213200 \quad 0.53276600$

$\begin{array}{lll}7.33452800 & -0.87392500 & -0.49668100\end{array}$

$\begin{array}{lll}6.98254700 & 0.46977500 & 1.60670100\end{array}$

$5.61781000 \quad-1.61007500 \quad 1.19739900$

$6.86548000 \quad-1.48983000 \quad-1.27878800$

$7.82100900 \quad-0.02262400 \quad-0.99511500$

$\begin{array}{lll}8.11230200 & -1.47809300 & -0.00316100\end{array}$

$\begin{array}{lll}6.25464800 & 0.81663100 & 2.35413200\end{array}$

$7.76332400 \quad-0.10915800 \quad 2.12589000$

$\begin{array}{lll}7.46096700 & 1.35453100 & 1.15594400\end{array}$

$\begin{array}{lll}6.36977100 & -2.24056500 & 1.69865600\end{array}$

$\begin{array}{lll}4.87956900 & -1.28966500 & 1.94568400\end{array}$

$\begin{array}{lll}5.09794100 & -2.23268400 & 0.45256300\end{array}$

$\begin{array}{lll}4.27607900 & 0.81881900 & 0.67243400\end{array}$

$\begin{array}{lll}-1.33633100 & -3.90420300 & -0.73678000\end{array}$

$\begin{array}{lll}-1.15184800 & -2.52234800 & -0.56651000\end{array}$

$-2.15083200 \quad-1.83775000 \quad 0.14600500$

$\begin{array}{lll}-3.34502000 & -2.43254000 & 0.57200300\end{array}$

$\begin{array}{lll}-3.49421100 & -3.80775000 & 0.36672700\end{array}$

$-2.48110000 \quad-4.54319000 \quad-0.25540400$

$\begin{array}{lll}-4.33382800 & -0.18831100 & 0.42835200\end{array}$

$\begin{array}{lll}-3.05928900 & 0.31795800 & 0.13217300\end{array}$

$\begin{array}{lll}-2.82749200 & 1.58913900 & -0.41817200\end{array}$

$\begin{array}{lll}-3.96796000 & 2.31925500 & -0.79083000\end{array}$

$\begin{array}{lll}-5.25259500 & 1.81056300 \quad-0.59097700\end{array}$

$\begin{array}{lll}-5.43775900 & 0.57613900 & 0.04080700\end{array}$

$\begin{array}{lll}-0.59467100 & -4.48816700 & -1.27931000\end{array}$

$\begin{array}{lll}-4.40458300 & -4.31400000 & 0.68925500\end{array}$

$\begin{array}{lll}-2.60106000 & -5.61945700 & -0.39877900\end{array}$

$\begin{array}{lll}-3.85544500 & 3.30739900 & -1.23286700\end{array}$

$\begin{array}{lll}-6.12059800 & 2.39649200 \quad-0.90171600\end{array}$

$\begin{array}{lll}-6.44944400 & 0.21812800 & 0.23449100\end{array}$

$-4.38605600 \quad-1.50858800 \quad 1.21095500$ 


\begin{tabular}{|c|c|c|}
\hline-5.78557300 & -2.12647400 & 1.20723000 \\
\hline-6.51119700 & -1.44451100 & 1.67377500 \\
\hline-6.13105700 & -2.35505600 & 0.18752100 \\
\hline-5.80285200 & -3.05597700 & 1.79447500 \\
\hline-3.95855000 & -1.22041800 & 2.67383300 \\
\hline-3.97325000 & -2.15025700 & 3.26342300 \\
\hline-2.94103700 & -0.80852900 & 2.72214300 \\
\hline-4.64871500 & -0.49918700 & 3.13902300 \\
\hline-1.97567400 & -0.49153800 & 0.37511400 \\
\hline 0.13848900 & -1.47643700 & -1.39326100 \\
\hline-1.07438700 & 2.22529400 & -0.42427600 \\
\hline 1.71613500 & -2.57764500 & -1.56824500 \\
\hline 1.97314300 & -3.33889300 & -0.25572500 \\
\hline 2.00240200 & -2.68646800 & 0.62155500 \\
\hline 1.22206300 & -4.11757000 & -0.06756100 \\
\hline 2.95389800 & -3.83918600 & -0.32475400 \\
\hline 2.87360600 & -1.59372900 & -1.81240200 \\
\hline 2.76342600 & -1.04614700 & -2.75848500 \\
\hline 2.94057000 & -0.85141500 & -1.01452400 \\
\hline 3.82925400 & -2.14097500 & -1.85838500 \\
\hline 1.73090200 & -3.60542400 & -2.70865600 \\
\hline 1.71246000 & -3.13116400 & -3.69762300 \\
\hline 2.67403900 & -4.17524200 & -2.64621900 \\
\hline 0.91019800 & -4.33283900 & -2.65654900 \\
\hline-0.67962000 & -1.35169300 & -3.14147600 \\
\hline-1.99383200 & -0.59710700 & -2.89992700 \\
\hline-2.74644600 & -1.24596600 & -2.42946700 \\
\hline-1.84558500 & 0.26957700 & -2.25415900 \\
\hline-2.40996700 & -0.24769500 & -3.85884400 \\
\hline 0.26788000 & -0.51217500 & -4.01167000 \\
\hline 1.15763600 & -1.08339100 & -4.31397200 \\
\hline-0.24583600 & -0.19322300 & -4.93441900 \\
\hline 0.61259400 & 0.38950900 & -3.48352000 \\
\hline-1.05250000 & -2.65103400 & -3.86852600 \\
\hline-1.61989100 & -3.33670300 & -3.22325700 \\
\hline-1.70886900 & -2.39657400 & -4.71903800 \\
\hline-0.19399900 & -3.18873300 & -4.28115000 \\
\hline-0.91010200 & 3.43628000 & -1.91346700 \\
\hline-1.44995700 & 2.74984400 & -3.17621400 \\
\hline-0.92336400 & 1.81406800 & -3.38572300 \\
\hline-2.52560900 & 2.53378900 & -3.11602700 \\
\hline-1.28981400 & 3.41695100 & -4.04024600 \\
\hline-1.57155100 & 4.81706000 & -1.79861700 \\
\hline .36008900 & 5.38354900 & -2.72209000 \\
\hline
\end{tabular}




\begin{tabular}{|c|c|c|c|}
\hline $\mathrm{H}$ & -2.66402400 & 4.76559000 & -1.70099600 \\
\hline $\mathrm{H}$ & -1.17814700 & 5.40968600 & -0.96401100 \\
\hline $\mathrm{C}$ & 0.60543200 & 3.63931400 & -2.09374800 \\
\hline $\mathrm{H}$ & 0.79742100 & 4.20052400 & -3.02392700 \\
\hline $\mathrm{H}$ & 1.04117600 & 4.21369100 & -1.26587700 \\
\hline $\mathrm{H}$ & 1.13821700 & 2.67848700 & -2.15416100 \\
\hline $\mathrm{C}$ & -1.23057900 & 3.28485300 & 1.19422100 \\
\hline $\mathrm{C}$ & -2.49057700 & 4.15270200 & 1.32459800 \\
\hline $\mathrm{H}$ & -2.43287900 & 4.70132200 & 2.28095900 \\
\hline $\mathrm{H}$ & -2.59613300 & 4.89992600 & 0.53051800 \\
\hline $\mathrm{H}$ & -3.40674900 & 3.54909800 & 1.35606600 \\
\hline $\mathrm{C}$ & 0.00920300 & 4.17907400 & 1.33792200 \\
\hline $\mathrm{H}$ & -0.01839300 & 5.03888700 & 0.65418600 \\
\hline $\mathrm{H}$ & 0.05441300 & 4.58178000 & 2.36416300 \\
\hline $\mathrm{H}$ & 0.93682700 & 3.62887500 & 1.15534300 \\
\hline $\mathrm{C}$ & -1.26891000 & 2.25705300 & 2.33545300 \\
\hline $\mathrm{H}$ & -2.20843000 & 1.68522200 & 2.33236200 \\
\hline $\mathrm{H}$ & -0.44802800 & 1.53887400 & 2.27506000 \\
\hline $\mathrm{H}$ & -1.20241000 & 2.77510200 & 3.30697800 \\
\hline $\mathrm{Ni}$ & 0.39471200 & 0.50385600 & -0.15710000 \\
\hline $\mathrm{H}$ & 1.68014200 & 1.07783800 & $3.6982320 C$ \\
\hline $\mathrm{H}$ & 1.64848900 & 1.94364900 & 1.7295700 \\
\hline $\mathrm{H}$ & 3.03866200 & 1.31105800 & -0.89638300 \\
\hline $\mathrm{C}$ & 3.50921900 & 3.06430900 & 0.24973900 \\
\hline $\mathrm{H}$ & 3.68169000 & 3.35699500 & 1.29756600 \\
\hline $\mathrm{H}$ & 4.42009700 & 3.27054900 & -0.33116900 \\
\hline $\mathrm{H}$ & 2.70142200 & 3.68687000 & -0.15519100 \\
\hline \multicolumn{4}{|c|}{4} \\
\hline $\mathrm{C}$ & -1.11264500 & 0.74675000 & 2.06651600 \\
\hline $\mathrm{C}$ & -0.15637400 & 0.41867700 & 1.08325500 \\
\hline $\mathrm{C}$ & 1.04488400 & 1.16050000 & 1.09639300 \\
\hline $\mathrm{C}$ & 1.16483500 & 2.33255100 & 1.87379200 \\
\hline $\mathrm{C}$ & 0.19657500 & 2.59630400 & 2.84590000 \\
\hline $\mathrm{C}$ & -0.91859200 & 1.77112100 & 2.98478100 \\
\hline $\mathrm{C}$ & 3.47638500 & 2.61100500 & 1.02975900 \\
\hline $\mathrm{C}$ & 3.34939600 & 1.32825400 & 0.46193300 \\
\hline $\mathrm{C}$ & 4.48336100 & 0.60503800 & 0.01969400 \\
\hline $\mathrm{C}$ & 5.72719600 & 1.25587400 & 0.07169900 \\
\hline $\mathrm{C}$ & 5.86762100 & 2.53769900 & 0.59431200 \\
\hline $\mathrm{C}$ & 4.74592300 & 3.19455900 & 1.09357600 \\
\hline $\mathrm{H}$ & -2.05400100 & 0.19101900 & 2.06659900 \\
\hline $\mathrm{H}$ & 0.29270900 & 3.47653400 & $3.4808250 \mathrm{C}$ \\
\hline $\mathrm{H}$ & -1.66976200 & 1.97566200 & 3.75062200 \\
\hline $\mathrm{H}$ & 6.61012000 & 0.73893400 & -0.29563500 \\
\hline
\end{tabular}


$\begin{array}{lll}4.85873300 & 4.18960800 & 1.52274100\end{array}$

$2.22726400 \quad 3.35728500$

1.48198300

$2.52972500 \quad 4.34415000$

2.61807300

$3.25534400 \quad 5.10363300$

2.29696200

$2.93031600 \quad 3.83323400$

3.50667500

$\begin{array}{lll}1.62352100 & 4.89260600 & 2.90930800\end{array}$

$\begin{array}{lll}1.67011200 & 4.15845400 & 0.27443600\end{array}$

$0.74640000 \quad 4.68635600 \quad 0.55895100$

$\begin{array}{lll}1.43458400 & 3.49593000 & -0.57115700\end{array}$

$2.40960200 \quad 4.89913800 \quad-0.06938900$

$2.10154900 \quad 0.75540800 \quad 0.30959600$

$\begin{array}{lll}-0.35548800 & -2.48502100 & 0.48711700\end{array}$

$\begin{array}{lll}1.13772900 & -2.58133300 & 0.79011500\end{array}$

$\begin{array}{lll}1.43621800 & -1.81841800 \quad 1.52305800\end{array}$

$\begin{array}{lll}1.77141000 & -2.44660200 & -0.09313800\end{array}$

$1.36577800 \quad-3.57091600 \quad 1.22439300$

$\begin{array}{lll}-1.14958700 & -2.70033100 & 1.78787200\end{array}$

$\begin{array}{lll}-0.97533800 & -3.72481400 & 2.15735500\end{array}$

$\begin{array}{lll}-2.22786700 & -2.57757300 \quad 1.62646000\end{array}$

$\begin{array}{lll}-0.83429800 & -2.00340700 & 2.57893400\end{array}$

$\begin{array}{lll}-0.79407100 & -3.54357300 & -0.53294400\end{array}$

$\begin{array}{lll}-1.85778100 & -3.43053400 & -0.78356300\end{array}$

$\begin{array}{lll}-0.66059400 & -4.54921300 & -0.09986600\end{array}$

$-0.20478500 \quad-3.50156500 \quad-1.45940300$

$\begin{array}{lll}-0.21438100 & -0.41678900 & -1.89609000\end{array}$

$\begin{array}{lll}-1.29650400 & -0.93457300 & -2.86499000\end{array}$

$\begin{array}{lll}-1.46159800 & -2.01700900 & -2.77272400\end{array}$

$\begin{array}{lll}-0.98288000 & -0.72886300 & -3.90302700\end{array}$

$\begin{array}{lll}-2.26000300 & -0.43434600 & -2.68609300\end{array}$

$1.13307200 \quad-1.03219400 \quad-2.26855900$

$1.92520900 \quad-0.74723700 \quad-1.56934500$

$1.42127000 \quad-0.68760300 \quad-3.27720300$

$\begin{array}{lll}1.09309400 & -2.12997400 & -2.30367700\end{array}$

$\begin{array}{lll}-0.14223900 & 1.11333400 & -2.04031800\end{array}$

$\begin{array}{lll}-1.05547900 \quad 1.59900400 & -1.66789500\end{array}$

$\begin{array}{lll}-0.02816300 & 1.37231200 & -3.10684000\end{array}$

$0.71903600 \quad 1.52954800 \quad-1.50385800$

$5.37016500 \quad-2.06624700 \quad 0.82174500$

$\begin{array}{lll}4.51005600 & -1.96139400 & 2.09461700\end{array}$

$3.52465200 \quad-2.42660200 \quad 1.96274100$

$\begin{array}{lll}4.35523600 & -0.91411400 & 2.39881600\end{array}$

$\begin{array}{lll}5.01775000 & -2.47746700 & 2.92791200\end{array}$

$6.77449900 \quad-1.55090900 \quad 1.15970500$ 
$\begin{array}{lll}7.22932800 & -2.22037900 & 1.91141800\end{array}$

$\begin{array}{lll}6.74529400 & -0.54645400 & 1.60353400\end{array}$

$\begin{array}{lll}7.44948000 & -1.52847000 & 0.29547300\end{array}$

$5.44885500 \quad-3.54208000 \quad 0.39775700$

$\begin{array}{lll}4.46005200 & -3.93775300 & 0.11661000\end{array}$

$5.82728800 \quad-4.15151700 \quad 1.23661400$

$6.13287100 \quad-3.69337200 \quad-0.45050600$

$\begin{array}{lll}5.25389600 & -1.20916500 & -2.21848200\end{array}$

$4.79812000 \quad 0.03269500 \quad-3.00395800$

$\begin{array}{lll}5.08713900 & -0.07513300 & -4.06393500\end{array}$

$\begin{array}{lll}5.25848900 & 0.95488100 & -2.62250500\end{array}$

$\begin{array}{lll}3.70614200 & 0.16396000 & -2.96890500\end{array}$

$\begin{array}{lll}6.78527300 & -1.27889700 & -2.22873300\end{array}$

$\begin{array}{lll}7.25585100 & -0.40815000 & -1.75232200\end{array}$

$7.13959400 \quad-1.30294800 \quad-3.27472400$

$\begin{array}{lll}7.16382400 & -2.18707900 & -1.73934100\end{array}$

$4.70250300 \quad-2.46256700 \quad-2.92716400$

$\begin{array}{lll}3.60576300 & -2.43244200 & -3.00471100\end{array}$

$\begin{array}{lll}4.97462900 & -3.38905900 & -2.40039900\end{array}$

$5.11518800 \quad-2.52896200 \quad-3.94908500$

$\begin{array}{lll}4.34037900 & -1.16314300 & -0.52444200\end{array}$

$-0.88107100 \quad-0.78137900 \quad-0.14781100$

$\begin{array}{lll}-4.93813900 & -0.36843800 & -0.38573400\end{array}$

$\begin{array}{lll}-4.38698200 & -1.69079500 & -0.56503600\end{array}$

$\begin{array}{lll}-4.56917000 & -2.77743900 & 0.41269100\end{array}$

$\begin{array}{lll}-4.87124300 & -2.54320300 & 1.77292600\end{array}$

$\begin{array}{lll}-4.15085000 & -4.33515400 & -1.02549900\end{array}$

$-4.27542000 \quad-2.05753100 \quad-1.59546900$

$\begin{array}{lll}-4.37572000 & -4.11901300 & 0.02259600\end{array}$

$\begin{array}{lll}-4.96119000 & -3.59091300 & 2.68781500\end{array}$

$\begin{array}{lll}-4.74789900 & -4.91408100 \quad 2.28238300\end{array}$

$\begin{array}{lll}-4.45592400 & -5.16846400 & 0.93923100\end{array}$

$\begin{array}{lll}-5.19039500 & -3.37208700 & 3.73462600\end{array}$

$\begin{array}{lll}-4.81050600 & -5.73294300 & 3.00317400\end{array}$

$\begin{array}{lll}-4.29184700 & -6.19515200 & 0.59984600\end{array}$

$\begin{array}{lll}-5.29571400 & 0.48317300 & -1.56019400\end{array}$

$\begin{array}{lll}-4.72079100 & 0.17312400 & -2.44768000\end{array}$

$\begin{array}{lll}-3.87102400 & 2.36948500 & -0.72958400\end{array}$

$\begin{array}{lll}-3.00652200 & 1.67780000 & -0.19292500\end{array}$

$\begin{array}{lll}-3.76304800 & 3.89523200 & -0.73293100\end{array}$

$\begin{array}{lll}-3.55073600 & 4.34445500 & -2.19293100\end{array}$

$\begin{array}{lll}-2.56593100 & 4.31703700 \quad 0.12798400\end{array}$

$\begin{array}{lll}-5.06530400 & 4.49843500 & -0.17750400\end{array}$

$-4.40145800 \quad 4.05570300 \quad-2.82723800$ 


\begin{tabular}{|c|c|c|c|}
\hline $\mathrm{H}$ & -2.63656400 & 3.89832500 & -2.61675800 \\
\hline $\mathrm{H}$ & -3.44335400 & 5.43981500 & -2.23527200 \\
\hline $\mathrm{H}$ & -2.68799100 & 3.99395900 & 1.17204200 \\
\hline $\mathrm{H}$ & -2.46498000 & 5.41345200 & 0.11281900 \\
\hline $\mathrm{H}$ & -1.63006700 & 3.87474500 & -0.24139000 \\
\hline $\mathrm{H}$ & -4.99324100 & 5.59743000 & -0.16674200 \\
\hline $\mathrm{H}$ & -5.24983200 & 4.16088600 & 0.85503600 \\
\hline $\mathrm{H}$ & -5.93182900 & 4.21435900 & -0.79104400 \\
\hline $\mathrm{O}$ & -4.93442800 & 1.92684000 & -1.36079300 \\
\hline $\mathrm{H}$ & -5.55275300 & -0.16577700 & 0.50363100 \\
\hline $\mathrm{H}$ & -5.01103300 & -1.51801100 & 2.12215000 \\
\hline $\mathrm{C}$ & -6.77480100 & 0.53970600 & -1.90050100 \\
\hline $\mathrm{H}$ & -7.11952900 & -0.47119900 & -2.16539500 \\
\hline $\mathrm{H}$ & -6.97210700 & 1.21453400 & -2.74849600 \\
\hline $\mathrm{H}$ & -7.35730800 & 0.88529800 & -1.03187300 \\
\hline $\mathrm{Ni}$ & -3.04245700 & -0.31300700 & -0.17844400 \\
\hline
\end{tabular}

\section{TS5/TS22}

\begin{tabular}{|c|c|c|c|}
\hline $\mathrm{C}$ & -1.14949700 & 0.66630800 & 2.16888700 \\
\hline $\mathrm{C}$ & -0.20166200 & 0.38174400 & 1.16477100 \\
\hline $\mathrm{C}$ & 1.02069600 & 1.08779200 & 1.22481400 \\
\hline $\mathrm{C}$ & 1.17287700 & 2.19481300 & 2.08700600 \\
\hline $\mathrm{C}$ & 0.21188600 & 2.41547100 & 3.07687500 \\
\hline $\mathrm{C}$ & -0.92698800 & 1.61635300 & 3.15735100 \\
\hline $\mathrm{C}$ & 3.50142300 & 2.44959600 & 1.29409300 \\
\hline $\mathrm{C}$ & 3.33498100 & 1.22651300 & 0.61532800 \\
\hline $\mathrm{C}$ & 4.44812500 & 0.50896000 & 0.11484000 \\
\hline $\mathrm{C}$ & 5.71417000 & 1.10501300 & 0.24265200 \\
\hline $\mathrm{C}$ & 5.89546600 & 2.32231600 & 0.89068800 \\
\hline $\mathrm{C}$ & 4.79053200 & 2.97394400 & 1.43222700 \\
\hline $\mathrm{H}$ & -2.10474800 & 0.13763700 & 2.13370700 \\
\hline $\mathrm{H}$ & 0.33329100 & 3.24308300 & 3.77496300 \\
\hline $\mathrm{H}$ & -1.67319100 & 1.78816500 & 3.93580700 \\
\hline $\mathrm{H}$ & 6.58085000 & 0.59356500 & -0.16764900 \\
\hline $\mathrm{H}$ & 6.89322700 & 2.75749700 & 0.98124000 \\
\hline $\mathrm{H}$ & 4.93258800 & 3.92095900 & 1.95176200 \\
\hline $\mathrm{C}$ & 2.27108200 & 3.20798100 & 1.77474200 \\
\hline $\mathrm{C}$ & 2.58421000 & 4.11516900 & 2.97198300 \\
\hline $\mathrm{H}$ & 3.33752100 & 4.86918600 & 2.70676300 \\
\hline $\mathrm{H}$ & 2.95490900 & 3.54116600 & 3.83470900 \\
\hline $\mathrm{H}$ & 1.69042200 & 4.67373400 & 3.28162200 \\
\hline $\mathrm{C}$ & 1.76214100 & 4.09792300 & 0.60841500 \\
\hline $\mathrm{H}$ & 0.84664300 & 4.63267900 & 0.90551400 \\
\hline $\mathrm{H}$ & 1.52959500 & 3.49696600 & -0.28268700 \\
\hline $\mathrm{H}$ & 2.52977900 & 4.83702900 & 0.32912500 \\
\hline
\end{tabular}


$1.41963800 \quad-1.89552900 \quad 1.36935500$

$\begin{array}{lll}1.68412900 & -2.43832300 & -0.28966000\end{array}$

$1.29831900 \quad-3.62599100 \quad 0.97665800$

$\begin{array}{lll}-1.16764800 & -2.75881700 & 1.67882200\end{array}$

$\begin{array}{lll}-1.00334300 & -3.80969300 & 1.97011400\end{array}$

$-2.24759000 \quad-2.60164400 \quad 1.57551600$

$\begin{array}{lll}-0.79995500 & -2.12435500 & 2.49899100\end{array}$

$\begin{array}{lll}-0.90572800 & -3.45659100 & -0.70960300\end{array}$

$\begin{array}{lll}-1.97544900 & -3.32449200 & -0.91765400\end{array}$

$\begin{array}{lll}-0.76201600 & -4.48968600 & -0.35101700\end{array}$

$\begin{array}{lll}-0.34887800 & -3.35287700 & -1.65113900\end{array}$

$-0.29731500 \quad-0.23034900 \quad-1.86376400$

$\begin{array}{lll}-1.41913900 & -0.61760900 & -2.84813200\end{array}$

$\begin{array}{lll}-1.66246300 & -1.68870200 & -2.80677900\end{array}$

$\begin{array}{lll}-1.09366400 & -0.38524300 & -3.87675400\end{array}$

$\begin{array}{lll}-2.34048400 & -0.05164600 & -2.64457700\end{array}$

$1.01331400 \quad-0.87359400 \quad-2.31124500$

$1.82812400 \quad-0.69061200 \quad-1.60372100$

$\begin{array}{lll}1.30421600 & -0.44920300 & -3.28780700\end{array}$

$0.92000300 \quad-1.95986500 \quad-2.44837500$

$\begin{array}{lll}-0.15130600 & 1.30095100 & -1.88285000\end{array}$

$\begin{array}{lll}-1.01487100 & 1.79589400 & -1.42032300\end{array}$

$\begin{array}{lll}-0.07819700 & 1.64418200 & -2.92872500\end{array}$

$0.75753000 \quad 1.62484200 \quad-1.36194600$

$5.29623100 \quad-2.26473300 \quad 0.56912200$

$\begin{array}{lll}4.47005800 & -2.30000900 & 1.86804000\end{array}$

$3.47577000 \quad-2.73671500 \quad 1.70991300$

$4.33729300 \quad-1.29296000 \quad 2.29436700$

$\begin{array}{lll}4.99158000 & -2.91417100 \quad 2.62249000\end{array}$

$6.72181500 \quad-1.82946000 \quad 0.93252400$

$\begin{array}{lll}7.17873400 & -2.60431200 & 1.57351700\end{array}$

$\begin{array}{lll}6.72962400 & -0.89403200 & 1.50818200\end{array}$

$\begin{array}{lll}7.37345800 & -1.70510300 & 0.05926300\end{array}$

$\begin{array}{lll}5.32885900 & -3.67750600 & -0.03761300\end{array}$

$4.32591400 \quad-4.01133400 \quad-0.34752500$

$\begin{array}{lll}5.70446300 & -4.39653400 & 0.71080600\end{array}$

$5.99537900 \quad-3.73831100 \quad-0.91097200$

$5.12604000 \quad-1.03443200 \quad-2.33948300$

$\begin{array}{lll}4.67030000 & 0.30103900 & -2.95161300\end{array}$

$\begin{array}{lll}4.93376400 & 0.32527000 & -4.02334600\end{array}$

$5.15151600 \quad 1.16180600 \quad-2.46640800$ 
$3.58115500 \quad 0.43813500 \quad-2.87377400$

$6.65522500 \quad-1.12256100 \quad-2.40067200$

$\begin{array}{lll}7.15292900 & -0.31828200 & -1.84164800\end{array}$

$6.98070900 \quad-1.02948400 \quad-3.45221700$

$\begin{array}{lll}7.03168100 & -2.08662200 & -2.03138100\end{array}$

$\begin{array}{lll}4.53523700 & -2.18329400 & -3.18108200\end{array}$

$\begin{array}{lll}3.43798800 & -2.12580700 & -3.22765900\end{array}$

$\begin{array}{lll}4.80112200 & -3.17130000 & -2.77767600\end{array}$

$4.92339500 \quad-2.13149600 \quad-4.21334100$

$\begin{array}{lll}4.25633000 & -1.17982700 & -0.62722300\end{array}$

$\begin{array}{lll}-0.94412500 & -0.72331500 & -0.13777100\end{array}$

$\begin{array}{lll}-5.04211300 & -0.42373800 & -0.45957600\end{array}$

$\begin{array}{lll}-4.33666900 & -1.70182800 & -0.55916700\end{array}$

$\begin{array}{lll}-4.54977200 & -2.76407200 & 0.44355200\end{array}$

$\begin{array}{lll}-4.83700500 & -2.47965000 & 1.79659100\end{array}$

$\begin{array}{lll}-4.18817600 & -4.37198000 & -0.95389500\end{array}$

$\begin{array}{lll}-4.20614800 & -2.10003900 & -1.57596100\end{array}$

$\begin{array}{lll}-4.40053200 & -4.11949900 & 0.08836900\end{array}$

$\begin{array}{lll}-4.96001000 & -3.49727800 & 2.74117100\end{array}$

$\begin{array}{lll}-4.79164700 & -4.83672700 & 2.37046500\end{array}$

$\begin{array}{lll}-4.51161800 & -5.13952400 & 1.03506800\end{array}$

$\begin{array}{lll}-5.17735100 & -3.24259900 \quad 3.78218100\end{array}$

$-4.87917400 \quad-5.63287200 \quad 3.11378400$

$\begin{array}{lll}-4.38237100 & -6.18033800 & 0.72549400\end{array}$

$\begin{array}{lll}-5.36178900 & 0.36325900 & -1.61758500\end{array}$

$\begin{array}{lll}-4.98306500 & -0.03772700 & -2.56370000\end{array}$

$\begin{array}{lll}-3.64269300 & 2.38829900 & -0.88735200\end{array}$

$\begin{array}{lll}-3.01855200 & 1.71271600 & -0.04243600\end{array}$

$\begin{array}{lll}-3.45356800 & 3.91619600 & -0.90146800\end{array}$

$\begin{array}{lll}-3.03712800 & 4.34701700 & -2.31890500\end{array}$

$\begin{array}{lll}-2.37676700 \quad 4.32088800 & 0.11186600\end{array}$

$\begin{array}{lll}-4.80469100 & 4.55580000 & -0.53313900\end{array}$

$\begin{array}{lll}-3.79764000 \quad 4.05884500 & -3.05843000\end{array}$

$\begin{array}{lll}-2.08295200 & 3.87991000 \quad-2.61077100\end{array}$

$\begin{array}{lll}-2.90578500 & 5.44028300 & -2.35991800\end{array}$

$-2.65577300 \quad 4.02916900 \quad 1.13425300$

$\begin{array}{lll}-2.22988800 & 5.41281000 & 0.08762100\end{array}$

$\begin{array}{lll}-1.41510300 & 3.83742200 & -0.11210700\end{array}$

$\begin{array}{lll}-4.71700000 & 5.65429500 & -0.52704500\end{array}$

$\begin{array}{lll}-5.13489300 & 4.23757200 & 0.46917200\end{array}$

$\begin{array}{lll}-5.58441600 & 4.27491600 & -1.25606100\end{array}$

$\begin{array}{lll}-4.44371200 & 1.92640800 & -1.77334900\end{array}$

$\begin{array}{lll}-5.65510900 & -0.22247800 & 0.42792800\end{array}$

$\begin{array}{lll}-4.93756200 & -1.44017300 \quad 2.11566400\end{array}$ 


$\begin{array}{lll}-6.72698500 & 0.98411600 & -1.71835500 \\ -7.46104500 & 0.17131700 & -1.85546100 \\ -6.81070100 & 1.67684000 & -2.56666900 \\ -6.98775700 & 1.51691200 & -0.79100600 \\ -3.10506400 & -0.26489100 & -0.17237500\end{array}$

6

\begin{tabular}{|c|c|c|}
\hline 5.24759600 & -0.79528700 & -0.36124100 \\
\hline 4.55190400 & -1.53995400 & 0.62919300 \\
\hline 4.37996700 & -3.00066200 & 0.51797400 \\
\hline 4.28909900 & -3.66042700 & -0.72407400 \\
\hline 4.39332200 & -3.30120600 & 2.65769100 \\
\hline 4.66347900 & -1.18722300 & 1.66307400 \\
\hline 4.31066200 & -3.78760500 & 1.68281600 \\
\hline 4.11268000 & -5.04184700 & -0.79404700 \\
\hline 4.02592100 & -5.80585500 & 0.37506600 \\
\hline 4.13325900 & -5.17005600 & 1.61476600 \\
\hline 4.03429300 & -5.52669800 & -1.77057200 \\
\hline 3.88119000 & -6.88732800 & 0.31851000 \\
\hline 4.07765600 & -5.75433400 & 2.53688300 \\
\hline 5.42907700 & 0.57254400 & -0.20686400 \\
\hline 5.43450600 & 0.99825900 & 0.80203400 \\
\hline 3.40304900 & 0.01859100 & -0.07827800 \\
\hline 3.22409700 & 2.81677500 & -0.33474500 \\
\hline 2.88654800 & 1.68500400 & -0.86264200 \\
\hline 2.79615000 & 4.05337400 & -1.16643900 \\
\hline 3.46097100 & 5.31191500 & -0.59962300 \\
\hline 1.26678800 & 4.18955200 & -1.06351300 \\
\hline 3.20060200 & 3.85937200 & -2.63630700 \\
\hline 4.55885300 & 5.24223600 & -0.64529200 \\
\hline 3.18753400 & 5.46139000 & 0.45455100 \\
\hline 3.15004200 & 6.20168500 & -1.17226400 \\
\hline 0.75701900 & 3.30879100 & -1.47633900 \\
\hline 0.92308500 & 5.07821900 & -1.61899700 \\
\hline 0.95225600 & 4.29913100 & -0.01657500 \\
\hline 2.72911500 & 2.96286500 & -3.06258900 \\
\hline 4.29183900 & 3.74817100 & -2.74236900 \\
\hline 2.89366100 & 4.73169800 & -3.23754300 \\
\hline 3.81942900 & 2.95059600 & 0.73456900 \\
\hline 4.33433200 & -3.08264600 & -1.64862100 \\
\hline 5.45217700 & -1.25157500 & -1.33674900 \\
\hline 5.99663700 & 1.44146600 & -1.28712700 \\
\hline 7.09981600 & 1.46203900 & -1.22124400 \\
\hline 5.65312400 & 2.47829500 & -1.17958200 \\
\hline 5.72437600 & 1.08283300 & -2.29150400 \\
\hline
\end{tabular}


$-4.15732700$

0.32763800

$-0.44919300$

$-3.16329000$

1.21707200

0.03448500

$-3.46305800$

2.56902100

0.27272400

C

$-4.69912400$

3.06606400

$-0.16040900$

$-5.64050100$

2.24406600

$-0.77015200$

$-1.23794300$

2.73924200

1.41387700

$-0.93061300$

1.47258300

0.88558400

0.38320300

0.94547500

0.99660800

$\begin{array}{lll}1.29490500 & 1.64445900 & 1.80430900\end{array}$

0.96254000

2.83172800

2.44749300

$-0.28687100$

3.38799200

2.21255800

$\begin{array}{lll}-6.14572800 & 0.22146800 & -1.26678700\end{array}$

$-4.93016700$

$-6.59140300$

4.12120500

$-0.00443300$

2.31476400

2.65209300

$-1.11945300$

1.70373900

1.27166100

1.88981000

$-0.52703700$

.34714100

3.05952500

\section{$-2.52939100$}

$-3.25944900$

$-2.63449600$

$-3.51521200$

$-4.19068000$

$-2.17006400$

$-3.07078100$

$-1.67497600$

$-1.49017600$

$-1.89940300$

$-4.65036900$

$-6.16625400$

$-6.73652200$

$-6.54693200$

$-6.40072500$

$-4.18041600$

$-4.73579900$

$-4.33603700$

$-3.11133800$

$-3.96530400$

$-2.86961200$

$-4.28110800$

$-4.22998600$

$-5.07949100$

$-4.37630200$

$-3.35852300$

$-1.67406900$
2.63467700

1.06552000

2.35965800

2.96661600

2.97214400

2.12627600

0.22849900

$-0.02199100$

$-0.71014800$

0.78311400

0.26649000

$-1.96743000$

$\begin{array}{ll}-2.42359000 & -2.07670200 \\ -1.48902900 & -1.98907500\end{array}$

$-3.13014500-1.32639400$

$-2.84886300 \quad-3.06869400$

$-1.30613800-3.11453100$

$-0.35907900-3.14714400$

$-1.82013000 \quad-4.07897200$

$-1.06193100 \quad-3.03643700$

$-3.59096400 \quad-2.12648200$

$-3.49784700 \quad-2.14252300$

$-4.05967200 \quad-3.07470600$

$-4.28170200 \quad-1.31198400$

$-1.90811100 \quad 1.14997100$

2.36930700

2.50170700 


\begin{tabular}{|c|c|c|c|}
\hline $\mathrm{H}$ & -4.94857600 & -1.51599600 & 3.28407700 \\
\hline $\mathrm{H}$ & -4.31516800 & -0.18725300 & 2.28826700 \\
\hline $\mathrm{C}$ & -5.08317600 & -3.43685200 & 1.31021300 \\
\hline $\mathrm{H}$ & -5.64158800 & -3.93755600 & 0.50564300 \\
\hline $\mathrm{H}$ & -5.56597500 & -3.71169000 & 2.26396300 \\
\hline $\mathrm{H}$ & -4.06017900 & -3.84512600 & 1.32330000 \\
\hline $\mathrm{C}$ & -6.51805000 & -1.38115600 & 1.11132200 \\
\hline $\mathrm{H}$ & -7.09477700 & -1.76525100 & 0.26152800 \\
\hline $\mathrm{H}$ & -6.54924300 & -0.28316100 & 1.08324800 \\
\hline $\mathrm{H}$ & -7.04246600 & -1.69599300 & 2.03109800 \\
\hline $\mathrm{C}$ & 0.57466400 & -0.80010900 & -1.57422800 \\
\hline $\mathrm{C}$ & 1.76107100 & -1.45242600 & -2.30874100 \\
\hline $\mathrm{H}$ & 2.04395100 & -2.41492700 & -1.85947800 \\
\hline $\mathrm{H}$ & 2.64509600 & -0.79615200 & -2.31157500 \\
\hline $\mathrm{H}$ & 1.47681400 & -1.64333800 & -3.35746100 \\
\hline $\mathrm{C}$ & 0.29836800 & 0.57693300 & -2.19835000 \\
\hline $\mathrm{H}$ & 0.14870800 & 0.44700200 & -3.28384700 \\
\hline $\mathrm{H}$ & 1.14079700 & 1.26001600 & -2.03873700 \\
\hline $\mathrm{H}$ & -0.61533300 & 1.02792200 & -1.79021600 \\
\hline $\mathrm{C}$ & -0.65962500 & -1.68084500 & -1.73901300 \\
\hline $\mathrm{H}$ & -0.94364800 & -1.68457300 & -2.80471700 \\
\hline $\mathrm{H}$ & -1.51329300 & -1.31242500 & -1.16309700 \\
\hline $\mathrm{H}$ & -0.47846500 & -2.72401900 & -1.45327100 \\
\hline $\mathrm{C}$ & 0.67836900 & -1.93666700 & 1.39827100 \\
\hline $\mathrm{C}$ & 0.99468100 & -3.31045400 & 0.78969800 \\
\hline $\mathrm{H}$ & 0.98955800 & -4.06970500 & 1.58818400 \\
\hline $\mathrm{H}$ & 1.97865300 & -3.34691000 & 0.31436900 \\
\hline $\mathrm{H}$ & 0.24011000 & -3.61234100 & 0.05308800 \\
\hline $\mathrm{C}$ & 1.52092400 & -1.71908000 & 2.66762200 \\
\hline $\mathrm{H}$ & 1.30928800 & -2.52933800 & 3.38500500 \\
\hline $\mathrm{H}$ & 1.27325000 & -0.76745000 & 3.16066200 \\
\hline $\mathrm{H}$ & 2.59628500 & -1.72406900 & 2.45654300 \\
\hline $\mathrm{C}$ & -0.80195600 & -1.87593800 & 1.78406700 \\
\hline $\mathrm{H}$ & -1.47509600 & -1.96539200 & 0.92306100 \\
\hline $\mathrm{H}$ & -1.04696300 & -0.94315100 & 2.31045300 \\
\hline $\mathrm{H}$ & -1.02136300 & -2.71188200 & 2.47125100 \\
\hline $\mathrm{P}$ & 1.18316900 & -0.53512500 & 0.21457900 \\
\hline $\mathrm{P}$ & -3.92304700 & -1.50773600 & -0.33158200 \\
\hline \multicolumn{4}{|c|}{7} \\
\hline $\mathrm{C}$ & 1.29640200 & -3.81617300 & 0.27371400 \\
\hline $\mathrm{C}$ & -0.10186800 & -3.72048700 & -0.03348100 \\
\hline $\mathrm{C}$ & -0.56553600 & -3.85683800 & -1.42514200 \\
\hline $\mathrm{C}$ & 0.20733400 & -3.38397900 & -2.50741900 \\
\hline $\mathrm{H}$ & -2.44276700 & -4.77184300 & -0.87432400 \\
\hline
\end{tabular}


$\begin{array}{lll}-0.80478200 & -4.10816600 & 0.71042400\end{array}$

$\begin{array}{lll}-1.82562800 & -4.41787900 & -1.70402700\end{array}$

$-0.26680000$

$-3.47997700 \quad-3.81495700$

$-1.52295500$

$-2.30068300$

0.34113500

$-1.89572600$

$-3.28696800$

1.76029300

1.06613400

1.16249800

2.02431400

3.20724500

3.48657500

3.42812000

3.85144700

$-7.76783700$

$-6.41085900$

$-5.90727400$

$-6.75718900$

$-8.10887800$

$-8.61288500$

$-4.93377400$

$-4.06741200$

$-2.71169100$

$-2.36600200$

$-3.27321500$

$-4.53695700$

$-8.17640500$

$-8.77768500$

$-9.66435300$

$-1.33224900$

$-2.97143700$

$-5.20866500$

$-6.17312900$

$-5.70521900$

$-5.26108300$

$-4.94713600$

$-6.55853500$

$-7.20780400$

$-8.06408600$

$-7.58205200$

$-6.77638400$

$-4.54932400$
$-4.03955200 \quad-4.07800700$

$-4.50625900 \quad-3.01395300$

$-3.09346600 \quad-4.63746600$

$-4.10270200 \quad-5.10336800$

$-4.93762800 \quad-3.20492400$

$-3.49336200 \quad 1.53733700$

$-3.55818600 \quad 2.38601800$

$-2.88998000 \quad-2.30865200$

$-3.87962800-0.54120100$

$-3.34975600 \quad 1.88845600$

$-4.04081200 \quad 2.70216100$

$-2.32116900 \quad 2.21136700$

$-3.56468500 \quad 1.02268800$

$1.33830800-0.49489200$

$1.19394100 \quad-0.16031600$

$-0.12334300-0.03900300$

$-1.24263400 \quad-0.12049200$

$-1.03823100 \quad-0.41618900$

$0.24149700-0.63466200$

$\begin{array}{ll}-2.53686500 & 0.93839400\end{array}$

$-1.43837100 \quad 0.74539200$

$-1.50921600 \quad 1.13763600$

$-2.56739000 \quad 2.00136700$

$-3.54640400 \quad 2.38882600$

$-3.56265100 \quad 1.79869000$

$2.33611800 \quad-0.63646800$

$-1.89486900-0.49574700$

$0.38395400 \quad-0.89393400$

$-2.63010900 \quad 2.34106500$

$-4.33483000 \quad 3.08142800$

$-4.39827900 \quad 1.99248000$

$-2.63955700 \quad 0.05136400$

$-3.15553200-1.33575300$

$-4.15835900 \quad-1.23908500$

$-2.49335700 \quad-1.77752400$

$-3.21247500 \quad-2.03017400$

$-3.62398800 \quad 0.61385000$

$-3.72638200-0.06649600$

$-3.30440200 \quad 1.59825400$

$-4.62950900 \quad 0.71335600$

$-0.29544200 \quad 0.15172100$ 
$-8.04348300$

$-8.03607700$

$-5.90655900$

$-6.34723100$

$-6.33831200$

$-4.82760400$

$-5.48904000$

$-4.39478700$

$-5.79090100$

$-5.73830900$

$-5.59333300$

$-5.54695800$

$-4.69990600$

$-5.43037200$

$-6.46628400$

$-4.35087900$

$-4.30703800$

$-4.37391600$

$-3.42018700$

$-6.84452800$

$-6.88563100$

$-7.77858200$

$-6.82858600$

$-1.05756100$

$-0.07571800$

$-0.50331400$

0.86100100

0.14908000

$-0.48904600$

$-0.43416700$

0.52102300

$-1.13649100$

$-2.42512400$

$-2.29671600$

$-3.10245300$

$-2.92588000$

$-1.48333900$

$-2.22441800$

$-2.37697800$

$-1.65224500$

$-3.20484500$
$4.28472400 \quad 0.96079700$

$4.04686500 \quad 2.71698100$

$2.33629400 \quad 2.88395300$

$1.35339400 \quad 2.65374700$

$2.67718600 \quad 3.84092600$

$2.19870900 \quad 3.03210900$

$4.68384700 \quad 2.12336600$

$4.55809300 \quad 2.12731400$

$5.02600800 \quad 3.12845400$

$5.48839000 \quad 1.41563000$

$3.83971300 \quad-1.28097300$

$2.96570600 \quad-2.54578300$

$2.26399600 \quad-2.52994700$

$3.60948400-3.43487700$

$2.37745600 \quad-2.67419600$

$4.75337600 \quad-1.27205400$

$5.38226700-0.37042200$

$5.42757900 \quad-2.14604900$

$4.16956600-1.31823500$

$4.72527800 \quad-1.32320700$

$5.42805800 \quad-0.47908600$

$4.14737400 \quad-1.33656100$

$5.33047500 \quad-2.24712900$

$0.81267000 \quad 1.91384900$

$1.90808100 \quad 1.46995700$

$2.56336400 \quad 0.70069100$

$1.48052500 \quad 1.09410300$

$2.54422100 \quad 2.34551100$

$0.13356300 \quad 3.17338700$

$0.88372800 \quad 3.98168400$

$-0.25235800 \quad 2.98519100$

$-0.67871300 \quad 3.53617200$

$1.41270800 \quad 2.24632800$

$2.20281800 \quad 3.00729500$

$0.65555500 \quad 2.66771600$

$1.86166200 \quad 1.38047700$

$0.18114700-1.16047100$

$1.51577700 \quad-1.23102900$

$1.76811100 \quad-2.29441500$

$2.34015500 \quad-0.78758500$

$1.47887900 \quad-0.74831900$ 


\begin{tabular}{|c|c|c|}
\hline-0.07221400 & 0.35441500 & -1.74755800 \\
\hline-0.16463300 & 0.74544100 & -2.77575300 \\
\hline 0.48740900 & -0.58846900 & -1.79508900 \\
\hline 0.55025700 & 1.05474500 & -1.17605900 \\
\hline-2.25160800 & -0.85661200 & -1.99268200 \\
\hline-3.31939100 & -0.86428900 & -1.74537500 \\
\hline-1.85309000 & -1.86679600 & -1.86727800 \\
\hline-2.15679100 & -0.59531500 & -3.05922800 \\
\hline-1.21525100 & -0.54706700 & 0.57906700 \\
\hline-5.34422200 & 2.66333500 & 0.22046900 \\
\hline 4.30213600 & -0.43461700 & 0.85499100 \\
\hline 1.80114900 & -0.60298900 & 1.22511000 \\
\hline 2.85718900 & -0.25188200 & 0.10219100 \\
\hline 5.55517900 & 0.35610000 & 0.66140000 \\
\hline 3.86286000 & 2.79420200 & 1.32421200 \\
\hline 5.30779800 & -1.89745800 & -1.24024200 \\
\hline 2.81560900 & -1.28000700 & -1.04761200 \\
\hline 2.73924800 & 1.23328200 & -0.29859400 \\
\hline 6.67392800 & -0.64072400 & 0.65100000 \\
\hline 5.50835200 & 1.28088000 & -0.80719300 \\
\hline 5.84704800 & 1.20295400 & 1.85470800 \\
\hline 7.65235100 & -0.24581500 & 1.59744400 \\
\hline 5.61293600 & 0.69707700 & -2.06342000 \\
\hline 5.40300400 & 2.65776200 & -0.92557100 \\
\hline 6.79321700 & 0.52356300 & 2.67046200 \\
\hline 8.36779600 & -1.48493900 & 2.12876300 \\
\hline 8.66294200 & 0.68218000 & 0.90379100 \\
\hline 5.22486400 & 1.66586700 & -3.07131900 \\
\hline 5.46498700 & 3.03744400 & -2.32286500 \\
\hline 7.57203300 & 1.55002700 & 3.48823500 \\
\hline 6.05548000 & -0.44143900 & 3.60976900 \\
\hline 9.05799500 & -1.23128800 & 2.94964700 \\
\hline 8.96109600 & -1.94969400 & 1.32433000 \\
\hline 7.64966100 & -2.23226100 & 2.49262000 \\
\hline 9.51527600 & 0.94164400 & 1.55130500 \\
\hline 9.05514500 & 0.17185900 & 0.01013200 \\
\hline 8.17554300 & 1.61264800 & 0.57721500 \\
\hline 6.09412400 & 1.45551700 & -4.30466500 \\
\hline 3.75486300 & 1.39822300 & -3.39957400 \\
\hline 4.39283700 & 4.08878500 & -2.58062600 \\
\hline 6.85666400 & 3.62631600 & -2.55545900 \\
\hline 6.90733800 & 2.02018800 & 4.23143900 \\
\hline 8.40481500 & 1.08118400 & 4.03702800 \\
\hline 7.97810000 & 2.34610000 & 2.84910800 \\
\hline
\end{tabular}




\begin{tabular}{|c|c|c|c|}
\hline $\mathrm{H}$ & 5.30163100 & 0.12366900 & 4.18048100 \\
\hline $\mathrm{H}$ & 6.73626100 & -0.92467500 & 4.32853500 \\
\hline $\mathrm{H}$ & 5.52678000 & -1.21226900 & 3.03416300 \\
\hline $\mathrm{H}$ & 5.86973500 & 0.47311700 & -4.74855200 \\
\hline $\mathrm{H}$ & 5.88570000 & 2.22105700 & -5.06833100 \\
\hline $\mathrm{H}$ & 7.16552600 & 1.48166800 & -4.06504800 \\
\hline $\mathrm{H}$ & 3.37979200 & 2.08768000 & -4.17118700 \\
\hline $\mathrm{H}$ & 3.65837600 & 0.37220600 & -3.78594400 \\
\hline $\mathrm{H}$ & 3.13644100 & 1.46297400 & -2.49254400 \\
\hline $\mathrm{H}$ & 4.62424200 & 5.00332100 & -2.01159000 \\
\hline $\mathrm{H}$ & 4.35275900 & 4.36069000 & -3.64689000 \\
\hline $\mathrm{H}$ & 3.40168500 & 3.72972500 & -2.27445500 \\
\hline $\mathrm{H}$ & 7.64242500 & 2.88338600 & -2.35557600 \\
\hline $\mathrm{H}$ & 6.97916900 & 4.00030000 & -3.58296800 \\
\hline $\mathrm{H}$ & 7.00835100 & 4.46888400 & -1.86356600 \\
\hline $\mathrm{Ni}$ & 0.58553000 & -1.94660000 & 0.64882800 \\
\hline \multicolumn{4}{|l|}{8} \\
\hline $\mathrm{Ni}$ & -2.80062600 & -1.14724100 & -0.37780100 \\
\hline B & 2.59238900 & 0.85334200 & 0.14452100 \\
\hline $\mathrm{O}$ & -1.84738200 & 0.25652200 & 0.58498100 \\
\hline $\mathrm{O}$ & 3.29969200 & 1.00572400 & -1.04260100 \\
\hline $\mathrm{O}$ & 2.46404100 & 2.09282900 & 0.77644400 \\
\hline $\mathrm{P}$ & -0.50844300 & -0.17806800 & -0.12958000 \\
\hline $\mathrm{K}$ & 1.52631500 & -0.76266700 & -2.45711400 \\
\hline $\mathrm{C}$ & 3.47972900 & 2.41379400 & -1.33032200 \\
\hline $\mathrm{C}$ & 3.27205600 & 3.07624300 & 0.08566600 \\
\hline $\mathrm{O}$ & 0.50619000 & -0.54540400 & 1.04992400 \\
\hline $\mathrm{K}$ & -0.07874500 & 2.13240700 & 1.64566900 \\
\hline $\mathrm{O}$ & 0.13051400 & 0.93867100 & -0.97362400 \\
\hline $\mathrm{O}$ & -0.95618000 & -1.42539000 & -0.98283000 \\
\hline $\mathrm{O}$ & 2.26353300 & -1.74775800 & -0.22048800 \\
\hline $\mathrm{C}$ & 4.86764800 & 2.61237000 & -1.92685200 \\
\hline $\mathrm{C}$ & 2.40599600 & 2.81158200 & -2.34677100 \\
\hline $\mathrm{C}$ & 2.51807900 & 4.40089500 & 0.06501800 \\
\hline $\mathrm{C}$ & 4.56789900 & 3.22208700 & 0.88471300 \\
\hline B & 2.02348700 & -0.66099900 & 0.76914000 \\
\hline $\mathrm{C}$ & 2.55358900 & -2.93674400 & 0.50772800 \\
\hline $\mathrm{H}$ & 5.07835700 & 3.68018300 & -2.09490600 \\
\hline $\mathrm{H}$ & 4.92688500 & 2.10156100 & -2.90039100 \\
\hline $\mathrm{H}$ & 5.65279400 & 2.19571300 & -1.28286200 \\
\hline $\mathrm{H}$ & 2.54995300 & 2.22123300 & -3.26720100 \\
\hline $\mathrm{H}$ & 2.48400600 & 3.87171300 & -2.62988200 \\
\hline $\mathrm{H}$ & 1.39989900 & 2.60138700 & -1.95926400 \\
\hline $\mathrm{H}$ & 2.40003000 & 4.77906500 & 1.09326400 \\
\hline
\end{tabular}


$3.07248900 \quad 5.16372200 \quad-0.50307100$

$\begin{array}{lll}1.52174800 & 4.29881400 & -0.38778100\end{array}$

$\begin{array}{lll}5.22601900 & 3.99752100 & 0.46527000\end{array}$

$5.12131100 \quad 2.27278800 \quad 0.92280800$

$\begin{array}{lll}4.31936800 & 3.50376400 & 1.91905100\end{array}$

$2.65201000 \quad-1.12882300 \quad 1.99653200$

$\begin{array}{lll}1.23993300 & -3.62795500 & 0.90263100\end{array}$

$\begin{array}{lll}3.37611700 & -3.86803300 & -0.37920300\end{array}$

$3.32431700 \quad-2.35334800 \quad 1.74989000$

$\begin{array}{lll}1.41478600 & -4.61945900 & 1.35012800\end{array}$

$\begin{array}{lll}0.62010000 & -3.75148400 & 0.00175500\end{array}$

$0.66598100 \quad-3.00946800 \quad 1.60417600$

$2.75160700 \quad-4.24626100 \quad-1.20594000$

$3.74142900 \quad-4.74132900 \quad 0.18466400$

$\begin{array}{lll}4.24054200 & -3.34869300 & -0.81583300\end{array}$

$\begin{array}{lll}4.79103400 & -2.03795700 & 1.41277700\end{array}$

$3.25782300 \quad-3.22731400 \quad 2.99974700$

$\begin{array}{lll}5.21323300 & -1.42618400 & 2.22517900\end{array}$

$\begin{array}{lll}5.41087300 & -2.94246900 & 1.30560100\end{array}$

$4.85347600 \quad-1.45421100 \quad 0.48219700$

$3.82526200 \quad-2.75339900 \quad 3.81670500$

$3.69283100 \quad-4.22410300 \quad 2.81948800$

$2.22181700 \quad-3.35125800 \quad 3.34247600$

$\begin{array}{lll}-4.59148000 & -1.50181800 & -1.07852900\end{array}$

$\begin{array}{lll}-4.68996600 & -0.93707800 & 0.22421900\end{array}$

$\begin{array}{lll}-5.17225700 & 0.42970600 & 0.48364400\end{array}$

$\begin{array}{lll}-4.92874400 & 1.48358800 & -0.41966400\end{array}$

$\begin{array}{lll}-6.04381400 & -0.07514800 \quad 2.39477400\end{array}$

$\begin{array}{lll}-4.77468300 & -1.62713400 & 1.07429400\end{array}$

$\begin{array}{lll}-5.85882900 & 0.72654800 & 1.67472900\end{array}$

$\begin{array}{lll}-5.38122900 & 2.77468600 & -0.15313500\end{array}$

$\begin{array}{lll}-6.07633900 & 3.05150300 & 1.03018100\end{array}$

$\begin{array}{lll}-6.30796100 & 2.02084000 & 1.94542800\end{array}$

$\begin{array}{lll}-5.17965900 & 3.57635500 & -0.86877500\end{array}$

$\begin{array}{lll}-6.42774200 & 4.06502300 \quad 1.23944900\end{array}$

$\begin{array}{lll}-6.84370800 & 2.22491400 \quad 2.87633400\end{array}$

$\begin{array}{lll}-3.78778700 & -2.65126700 & -1.23414700\end{array}$

$-3.76877000 \quad-3.38074500 \quad-0.41118200$

$\begin{array}{lll}-4.34900600 & 1.29031500 & -1.32447800\end{array}$

$\begin{array}{lll}-4.88630900 & -0.91654400 & -1.95851000\end{array}$

$\begin{array}{lll}-3.36476700 & -3.17828300 & -2.57450300\end{array}$

$\begin{array}{lll}-4.01876100 & -4.00135900 & -2.91575700\end{array}$

$\begin{array}{lll}-2.33776600 & -3.57385900 & -2.52389700\end{array}$

$\begin{array}{lll}-3.38211500 & -2.38920600 & -3.34278400\end{array}$ 


\begin{tabular}{|c|c|c|}
\hline 1.23798800 & -0.19869300 & -0.80890100 \\
\hline-1.78491400 & 1.13723200 & -0.30279900 \\
\hline 1.64279700 & 0.11217900 & 1.02955700 \\
\hline-2.58940100 & 2.03133500 & 0.40651400 \\
\hline-0.83671300 & 1.83186800 & -1.02539900 \\
\hline 0.34817400 & -0.62645600 & 1.63669300 \\
\hline-3.00514500 & 0.51293500 & 2.61882800 \\
\hline-1.97298800 & 3.34831300 & 0.37199300 \\
\hline-1.05769900 & 3.25207500 & -0.90784100 \\
\hline-0.32304400 & -0.97488400 & 0.13405800 \\
\hline 3.29354600 & -1.65584700 & 1.94311500 \\
\hline-0.55721900 & 0.31774500 & 2.41560300 \\
\hline 0.78804400 & -1.92201700 & 2.31538400 \\
\hline-2.72117500 & -1.17429400 & 0.75785800 \\
\hline-3.07184700 & 4.39946300 & 0.29582100 \\
\hline-1.17300500 & 3.50319100 & 1.66809300 \\
\hline 0.29848500 & 3.93552300 & -0.77605000 \\
\hline-1.75912600 & 3.70640500 & -2.18906500 \\
\hline-1.80183500 & -0.59099000 & -0.24096600 \\
\hline-3.14254600 & -2.44930800 & 0.27883600 \\
\hline-2.64417000 & 5.40812600 & 0.18456600 \\
\hline-3.66427500 & 4.38860300 & 1.22432200 \\
\hline-3.75653500 & 4.21495400 & -0.54212800 \\
\hline-1.86323800 & 3.46115700 & 2.52738600 \\
\hline-0.66078000 & 4.47536700 & 1.71525900 \\
\hline-0.44432000 & 2.68920400 & 1.78816200 \\
\hline 0.86054800 & 3.82137000 & -1.71574100 \\
\hline 0.18560800 & 5.01347900 & -0.58115300 \\
\hline 0.89220000 & 3.48679300 & 0.03094100 \\
\hline-1.91334800 & 4.79577000 & -2.20941800 \\
\hline-2.73359600 & 3.21081500 & -2.30867700 \\
\hline-1.13589100 & 3.43197700 & -3.05337300 \\
\hline-2.12694000 & -1.28733300 & -1.48027800 \\
\hline-2.09875000 & -3.50708000 & 0.66401700 \\
\hline-4.48247200 & .79469100 & 0.92408100 \\
\hline-3.19863400 & -2.19308300 & -1.27087400 \\
\hline-2.43836900 & -4.52627100 & 0.41992900 \\
\hline-1.91476600 & -3.44921200 & 1.74723600 \\
\hline-1.13948400 & -3.31216400 & 0.16960900 \\
\hline-4.34276500 & -2.97069900 & 2.00377200 \\
\hline-4.91179200 & -3.71384900 & 0.49418000 \\
\hline-5.21325300 & -1.98258300 & 0.80163200 \\
\hline & & 8100 \\
\hline
\end{tabular}




\begin{tabular}{|c|c|c|}
\hline-2.98411000 & -3.44197200 & -2.123 \\
\hline-4.38890800 & -1.14370300 & -2.73133400 \\
\hline-5.37680500 & -2.16289500 & -1.65046200 \\
\hline-4.69279900 & -0.61720500 & -1.06062400 \\
\hline-3.06099200 & -3.18600100 & -3.19211400 \\
\hline-3.74356900 & -4.21110200 & -1.90697800 \\
\hline-1.98868700 & -3.87334200 & -1.95361900 \\
\hline 2.27163600 & -0.39680400 & -2.50189900 \\
\hline 2.87045900 & 0.55980900 & -1.63355600 \\
\hline 4.12564300 & 0.33272600 & -0.89875200 \\
\hline 4.92022200 & -0.82420000 & -1.05884700 \\
\hline 3.90393000 & 2.15283700 & 0.24381400 \\
\hline 2.57823000 & 1.61170600 & -1.73735900 \\
\hline 4.51520700 & 1.26092700 & 0.09101100 \\
\hline 6.04075200 & -1.04991200 & -0.25143700 \\
\hline 6.40031900 & -0.12609200 & 0.73933200 \\
\hline 5.63108700 & 1.03551700 & 0.89871000 \\
\hline 6.64196400 & -1.95057600 & -0.40378600 \\
\hline 7.27707300 & -0.30107700 & 1.36765000 \\
\hline 5.90134300 & 1.76892200 & 1.66301900 \\
\hline 0.92566400 & -0.25340000 & -2.85590300 \\
\hline 0.49512900 & 0.75442200 & -2.89392500 \\
\hline 4.66268100 & -1.55942100 & -1.82375400 \\
\hline 2.75112400 & -1.36797900 & -2.67024400 \\
\hline 0.16583900 & -1.31919500 & -3.58708800 \\
\hline 0.11194800 & -1.09177200 & -4.66801200 \\
\hline-0.85739800 & -1.38424400 & -3.19038400 \\
\hline 64196 & -2.30660600 & -3.47153200 \\
\hline
\end{tabular}

TS10

$\begin{array}{lrrr}\mathrm{Ni} & 0.84710700 & 0.30529400 & -0.82908800 \\ \mathrm{~B} & -1.22758500 & 0.91228500 & 0.03943200 \\ \mathrm{O} & 1.47682800 & -0.30348500 & 0.84897800 \\ \mathrm{O} & -1.15478800 & 1.28698100 & 1.38933300 \\ \mathrm{O} & -1.68504300 & 1.98604800 & -0.70106100 \\ \mathrm{P} & 0.86004100 & -1.79779400 & 0.94608300 \\ \mathrm{~K} & -1.79038600 & -0.72445600 & 2.98167000 \\ \mathrm{C} & -1.23848300 & 2.72342200 & 1.47937600 \\ \mathrm{C} & -2.04666000 & 3.07300800 & 0.17978600 \\ \mathrm{O} & -0.22782400 & -1.75111900 & -0.27712500 \\ \mathrm{~K} & 3.69909200 & -1.39973600 & 1.79267200 \\ \mathrm{O} & 0.19721900 & -1.98411900 & 2.31463800 \\ \mathrm{O} & 1.95369600 & -2.80707700 & 0.60403400 \\ \mathrm{O} & -2.49665400 & -1.15212200 & 0.55637000 \\ \mathrm{C} & -1.92104600 & 3.10169200 & 2.78801400\end{array}$


$0.19623200 \quad 3.25573400 \quad 1.46230900$

$-1.65984600$

$4.38872500 \quad-0.48207900$

$-3.56114300$

$3.00689800 \quad 0.38380000$

$-1.39806000$

$-3.44548800$

$-2.07780700$

$-1.28474800$

$-2.89752600$

0.76342000

0.23345100

0.69753700

$-2.25647700$

$-1.85344100$

$-0.59968100$

$-3.92915500$

$-3.85510500$

$-4.05721900$

$-2.00154300$

$-2.98746100$

$-4.80704000$

$-3.36172300$

$-3.72338700$

$-2.85688500$

$-2.01723600$

$-4.78325900$

$-5.59507400$

$-5.09142500$

$-4.23908900$

$-3.68772900$

4.18959300

$2.80921800 \quad 3.63873000$

$2.61007700 \quad 2.90011300$

2.77221200

2.27098700

$4.34595900 \quad 1.60531900$

$3.00118500 \quad 0.51850700$

$4.53375500 \quad-1.39541100$

$5.24011000 \quad 0.18932900$

$4.40435100 \quad-0.76730600$

$3.84698500 \quad 0.99179500$

$2.06475600 \quad 0.86852600$

$3.04983000-0.59652900$

$-1.08129400 \quad-1.71317100$

$-3.44558500 \quad 0.02194000$

$-1.81395200 \quad 0.55918300$

$-1.45535600 \quad-1.58748700$

$-4.14971900 \quad-0.39702700$

$-3.68342100 \quad 1.08967000$

$-3.59794200 \quad-0.46799000$

$-2.23429200 \quad 1.57890300$

$-2.34736800 \quad 0.00407700$

$-0.75526300 \quad 0.63340600$

$-0.21393300-1.80805400$

$\begin{array}{lll}-3.99316300 & 0.22496300 & -2.78693700\end{array}$

$\begin{array}{lll}-5.31461400 & -0.45133200 & -1.80201700\end{array}$

$\begin{array}{lll}-4.03618000 & 0.54763000 & -1.04509900\end{array}$

$\begin{array}{lll}-3.61375800 & -2.05104900 & -3.65044400\end{array}$

$\begin{array}{lll}-4.71089300 & -2.89702600 & -2.53045900\end{array}$

$\begin{array}{lll}-2.97925100 & -3.34186500 & -2.61011000\end{array}$

$1.74406200 \quad 0.77057100 \quad-2.55153400$

$\begin{array}{lll}2.49555400 & 1.24582900 & -1.43728100\end{array}$

$\begin{array}{lll}3.81539000 & 0.72271800 & -1.05506600\end{array}$

$4.30164300 \quad-0.52492600 \quad-1.50463500$

$\begin{array}{lll}4.26707100 & 2.43135400 & 0.19086800\end{array}$

$2.31105800 \quad 2.27712100 \quad-1.11074000$

$4.62983200 \quad 1.46767400 \quad-0.17641900$

$\begin{array}{lll}5.55668000 & -0.99258500 & -1.10771000\end{array}$

$6.35768500 \quad-0.23640700 \quad-0.23787500$ 
$5.90815900 \quad-1.96149600 \quad-1.47190500$

$\begin{array}{lll}7.34096000 & -0.60307100 & 0.06729100\end{array}$

$6.49563700 \quad 1.59920400 \quad 0.90536300$

$\begin{array}{lll}0.42728000 & 1.20361300 & -2.69952700\end{array}$

$\begin{array}{lll}0.15311900 & 2.17997200 & -2.28922700\end{array}$

$\begin{array}{lll}3.67969700 & -1.15163000 & -2.14567300\end{array}$

$2.09757300 \quad-0.08487600 \quad-3.13722600$

$\begin{array}{lll}-0.51497200 & 0.64769200 & -3.71978100\end{array}$

$\begin{array}{lll}-0.59306900 & 1.33233300 & -4.58455000\end{array}$

$\begin{array}{lll}-1.51272200 & 0.51633600 & -3.28206700\end{array}$

$\begin{array}{lll}-0.18707300 & -0.33853900 & -4.08090200\end{array}$ 


\begin{tabular}{|c|c|c|}
\hline-4.42051400 & -2.42394100 & -2.64941400 \\
\hline 1.41647700 & 2.88180200 & -0.75926900 \\
\hline-0.52096300 & 4.83146800 & 0.74120700 \\
\hline-2.21146500 & 3.31524700 & -0.33316400 \\
\hline 0.20422700 & 3.43994300 & -1.31943600 \\
\hline-0.93297500 & 5.70605000 & 0.21643000 \\
\hline-1.03063200 & 4.74173500 & 1.71277000 \\
\hline 0.54620300 & 5.01056500 & 0.93758000 \\
\hline-2.79416600 & 3.46159500 & 0.59126100 \\
\hline-2.58954000 & 4.03637200 & -1.07382600 \\
\hline-2.39910600 & 2.29588700 & -0.69395600 \\
\hline-0.31985200 & 2.42545600 & -2.33353400 \\
\hline 0.52745300 & 4.76566700 & -1.98950400 \\
\hline 0.45771700 & 2.23818400 & -3.08838200 \\
\hline-1.22147900 & 2.78450200 & -2.85044800 \\
\hline-0.55148200 & 1.47254800 & -1.83613300 \\
\hline 1.18072400 & 4.59213100 & -2.85805500 \\
\hline-0.39067400 & 5.25676600 & -2.34775800 \\
\hline 1.04961400 & 5.44876500 & -1.30686200 \\
\hline 1.05875100 & -1.08709700 & -2.26508600 \\
\hline 1.99163100 & -1.76533300 & -1.48225000 \\
\hline 3.35904000 & -1.33617200 & -1.18242400 \\
\hline 3.74021500 & 0.02021600 & -1.12821400 \\
\hline 4.08457700 & -3.36964000 & -0.96305800 \\
\hline 1.81062300 & -2.84430800 & -1.36299700 \\
\hline 4.34323000 & -2.30665000 & -0.88845800 \\
\hline 5.04271300 & 0.38130200 & -0.79512800 \\
\hline 6.00381200 & -0.59166400 & -0.49439800 \\
\hline 5.64688600 & -1.94115600 & -0.54358200 \\
\hline 5.30427700 & 1.44109600 & -0.74208600 \\
\hline 7.01902700 & -0.29824100 & -0.21701700 \\
\hline 6.38704300 & -2.71467500 & -0.32151400 \\
\hline-0.27439200 & -1.57547800 & -2.41165500 \\
\hline-0.38979300 & -2.66636600 & -2.32695100 \\
\hline 2.99790000 & 0.79834700 & -1.30274700 \\
\hline 1.30874000 & -0.09203300 & -2.65115000 \\
\hline-1.20864200 & -0.96928200 & -3.43131600 \\
\hline-0.93537800 & -1.28538200 & -4.45553300 \\
\hline-2.24332500 & -1.29316100 & -3.25985400 \\
\hline-1.19202700 & 0.12969100 & -3.41439400 \\
\hline
\end{tabular}

$\begin{array}{lll}-0.63700400 & -0.93143300 & -0.10150300\end{array}$

$\begin{array}{lll}-2.46806000 & -0.74860300 & -0.54920800\end{array}$

$\begin{array}{lll}-0.62176000 & -0.50379700 & 1.73514900\end{array}$ 
$-3.34852600-1.81755000$

$-0.39154500$

$-1.62759100 \quad 1.94561800$

$-4.51456800 \quad 0.16844300$

$-4.70087000$

1.77559900

$-1.33090200$

0.34523000

$3.19633200 \quad-1.78979800$

0.25984600

1.46783300

$1.33190300 \quad-0.92531900$

0.74101600

$-5.49745600$

2.19981000

1.14992000

$-4.50355700$

$-5.51943000$

$-5.25245700$

1.81377300

1.28104000

$-6.53272400$

$-5.28372900$

$-5.42899500$

$-4.29951800$

$-5.48078600$

$-3.74140800$

$-5.59654700$

$-6.54057500$

$-5.04676400$

$-6.31673200$

$-4.69132000$

$-5.14849800$

2.96024000

1.49166400

0.27993800

2.63594700

1.86169400

0.53309900

2.19792300

$-0.62776500$

0.70061300

$-0.01866800$

2.46772100

3.78308000

3.38602200

2.29055600

1.63135600

0.33212600

$-2.18481100$

$-1.46936800$

1.37888900

3.37149000

0.90721600

2.17133800

1.15388700

1.38380900

0.09228800

$-0.30856900$

$-3.20924000$

$-1.78863700$

$-2.24412700$

$-1.19602100$

$-0.84687400$

$-2.51784200$

1.74977600

4.43359100

3.85802400

2.81523400

5.37831100

4.64004500

4.08845200

4.23671200

4.68616300

3.05523300

2.19753000

3.81631900

$1.65429700 \quad-2.84297300$

$2.96499600 \quad-3.34360900$

$1.48570500 \quad-2.60159700$ 


\begin{tabular}{|c|c|c|c|}
\hline $\mathrm{H}$ & 4.68164900 & 3.34286400 & -1.61950800 \\
\hline $\mathrm{H}$ & 3.53794700 & 4.69251100 & -1.81555200 \\
\hline $\mathrm{H}$ & 4.02994300 & 4.15855100 & -0.18232500 \\
\hline $\mathrm{C}$ & 0.14985100 & -1.19530500 & -1.85657300 \\
\hline $\mathrm{C}$ & 0.75460600 & -2.36035800 & -1.38075900 \\
\hline $\mathrm{C}$ & 2.18352700 & -2.56994800 & -1.21900100 \\
\hline $\mathrm{C}$ & 3.15537500 & -1.55248800 & -1.42237000 \\
\hline $\mathrm{H}$ & 1.95085300 & -4.64514200 & -0.63024800 \\
\hline $\mathrm{H}$ & 0.13042800 & -3.24988800 & -1.24398000 \\
\hline $\mathrm{C}$ & 2.66929500 & -3.83771600 & -0.79790300 \\
\hline $\mathrm{C}$ & 4.51448000 & -1.79078700 & -1.21928400 \\
\hline $\mathrm{C}$ & 4.97211300 & -3.05065800 & -0.79701800 \\
\hline $\mathrm{C}$ & 4.03092800 & -4.07177700 & -0.59090400 \\
\hline $\mathrm{H}$ & 5.22606200 & -0.97601500 & -1.38079400 \\
\hline $\mathrm{H}$ & 6.03802900 & -3.23474900 & -0.64340500 \\
\hline $\mathrm{H}$ & 4.36287300 & -5.06369000 & -0.27074400 \\
\hline $\mathrm{C}$ & -1.30197500 & -0.99826900 & -2.03849400 \\
\hline $\mathrm{H}$ & -1.80779800 & -1.92397600 & -2.34824900 \\
\hline $\mathrm{H}$ & 2.83836800 & -0.55214400 & -1.71275400 \\
\hline $\mathrm{H}$ & 0.79646300 & -0.36345700 & -2.16080200 \\
\hline $\mathrm{C}$ & -1.63984900 & 0.17056900 & -2.96302000 \\
\hline $\mathrm{H}$ & -1.26285000 & -0.01256200 & -3.98472300 \\
\hline $\mathrm{H}$ & -2.72164800 & 0.35407900 & -3.02907800 \\
\hline $\mathrm{H}$ & -1.18050300 & 1.10173600 & -2.59721600 \\
\hline 13 & & & \\
\hline $\mathrm{Ni}$ & 0.50035200 & 0.73990000 & -0.20693900 \\
\hline B & 2.65321500 & -0.26603400 & -1.52004600 \\
\hline $\mathrm{O}$ & 0.59163900 & -0.08517000 & 1.44367400 \\
\hline $\mathrm{O}$ & 2.78476800 & -1.37561800 & -0.69393700 \\
\hline $\mathrm{O}$ & 3.65431900 & 0.64542600 & -1.31255500 \\
\hline $\mathrm{P}$ & -0.44724000 & -0.40641900 & 2.60089800 \\
\hline K & 0.72564400 & -2.61846900 & 0.66463800 \\
\hline $\mathrm{C}$ & 4.01739400 & -1.23524500 & 0.07094000 \\
\hline $\mathrm{C}$ & 4.35434000 & 0.30106800 & -0.09312400 \\
\hline $\mathrm{O}$ & -1.94267400 & -0.13764300 & 1.75053000 \\
\hline K & -1.40760100 & 2.52299600 & 2.08279900 \\
\hline $\mathrm{O}$ & -0.38229000 & -1.87596500 & 2.95655700 \\
\hline $\mathrm{O}$ & -0.50043000 & 0.69444200 & 3.63924900 \\
\hline $\mathrm{O}$ & -1.84016800 & -1.97737300 & 0.15240200 \\
\hline $\mathrm{C}$ & 5.03854400 & -2.16067400 & -0.58795700 \\
\hline $\mathrm{C}$ & 3.75990600 & -1.66135200 & 1.51214400 \\
\hline $\mathrm{C}$ & 3.77719200 & 1.16859900 & 1.02642400 \\
\hline $\mathrm{C}$ & 5.83426300 & 0.60662300 & -0.28489200 \\
\hline B & -2.45538700 & -0.88284200 & 0.75247300 \\
\hline
\end{tabular}




\begin{tabular}{|c|c|c|}
\hline-2.85850200 & -2.67105300 & -0.61517100 \\
\hline 5.99356500 & -2.16869300 & -0.04246700 \\
\hline 4.64116600 & -3.18740600 & -0.59539700 \\
\hline 5.23207000 & -1.86732100 & -1.62989500 \\
\hline 3.58005500 & -2.74873800 & 1.56413500 \\
\hline 4.64232900 & -1.46115100 & 2.13795300 \\
\hline 2.90241000 & -1.12866600 & 1.94422600 \\
\hline 3.86481300 & 2.22552300 & 0.73250400 \\
\hline 4.32596200 & 1.02875100 & 1.96934400 \\
\hline 2.71567200 & 0.94611200 & 1.20055400 \\
\hline 6.41858400 & 0.28273900 & 0.59006800 \\
\hline 6.24190400 & 0.12072000 & -1.18106600 \\
\hline 5.97100900 & 1.69251000 & -0.39929400 \\
\hline-3.69153100 & -0.62989600 & 0.20923300 \\
\hline-3.44763200 & -3.74801800 & 0.29599300 \\
\hline-2.21469000 & -3.30459200 & -1.83909500 \\
\hline-3.87159700 & -1.50370100 & -0.92874100 \\
\hline-4.19631500 & -4.35907500 & -0.22890000 \\
\hline-2.64082700 & -4.41368900 & 0.63890200 \\
\hline-3.91693300 & -3.30631200 & 1.18715600 \\
\hline-1.51710300 & -4.09998300 & -1.52974400 \\
\hline-2.97813900 & -3.77032600 & -2.48066600 \\
\hline-1.65765400 & -2.57219800 & -2.43502500 \\
\hline-3.48436800 & -0.71277000 & -2.17803800 \\
\hline-5.33116600 & -1.92870300 & -0.99629800 \\
\hline-4.08488000 & 0.20764300 & -2.21867400 \\
\hline-3.66978900 & -1.28430800 & -3.09915600 \\
\hline-2.42311500 & -0.42985600 & -2.14912200 \\
\hline-5.96085800 & -1.05359700 & -1.21769800 \\
\hline-5.48809300 & -2.66974400 & -1.79539900 \\
\hline-5.67366000 & -2.35699100 & -0.04521200 \\
\hline 0.42866500 & 0.72620000 & -2.11686600 \\
\hline 0.66287500 & 2.04367300 & -1.62099500 \\
\hline-0.39017800 & 3.01723700 & -1.30944000 \\
\hline-1.77518700 & 2.73749800 & -1.44042400 \\
\hline 1.01769900 & 4.52514700 & -0.64875700 \\
\hline 1.67624500 & 2.46145200 & -1.66949500 \\
\hline-0.04165800 & 4.27432100 & -0.75372900 \\
\hline-2.74507100 & 3.64275800 & -1.00713300 \\
\hline-2.37771300 & 4.87064700 & -0.4318400 \\
\hline-1.01398400 & 5.17945200 & -0.31849000 \\
\hline-3.80261300 & 3.38561800 & -1.11563300 \\
\hline-3.13936100 & 5.57908700 & -0.09743500 \\
\hline .70494000 & 6.13805600 & $0.108210 c$ \\
\hline
\end{tabular}




\begin{tabular}{|c|c|c|c|}
\hline $\mathrm{C}$ & 1.57419900 & -0.13284700 & -2.66746900 \\
\hline $\mathrm{H}$ & 2.05034800 & 0.38169800 & -3.52612300 \\
\hline $\mathrm{H}$ & -2.09208000 & 1.78431900 & -1.86164200 \\
\hline $\mathrm{H}$ & -0.55732900 & 0.48633300 & -2.54221600 \\
\hline C & 1.06223700 & -1.49665500 & -3.13827300 \\
\hline $\mathrm{H}$ & 0.32763000 & -1.39480000 & -3.95505000 \\
\hline $\mathrm{H}$ & 1.87779000 & -2.14388100 & -3.49884700 \\
\hline $\mathrm{H}$ & 0.55494300 & -2.03192800 & -2.31912400 \\
\hline \multicolumn{4}{|l|}{14} \\
\hline $\mathrm{O}$ & -0.83616400 & -1.13950000 & -0.23578300 \\
\hline $\mathrm{C}$ & -2.16439200 & -0.65951900 & -0.43252700 \\
\hline B & 0.04799400 & -0.00001200 & 0.00001900 \\
\hline $\mathrm{C}$ & -3.15518300 & -1.72204300 & 0.03075100 \\
\hline $\mathrm{C}$ & -2.36281700 & -0.37386300 & -1.92612700 \\
\hline $\mathrm{C}$ & -2.16440100 & 0.65948700 & 0.43254100 \\
\hline $\mathrm{O}$ & 1.05605900 & 0.31437900 & -1.06985100 \\
\hline $\mathrm{O}$ & -0.83617200 & 1.13947100 & 0.23581900 \\
\hline $\mathrm{H}$ & -3.10066000 & -2.60060400 & -0.63223900 \\
\hline $\mathrm{H}$ & -4.19001400 & -1.34605100 & -0.00166000 \\
\hline $\mathrm{H}$ & -2.93784600 & -2.05561400 & 1.05470100 \\
\hline $\mathrm{H}$ & -3.39612600 & -0.07524600 & -2.16038100 \\
\hline $\mathrm{H}$ & -2.13252500 & -1.28722000 & -2.49607100 \\
\hline $\mathrm{H}$ & -1.67792500 & 0.41445800 & -2.26945700 \\
\hline $\mathrm{C}$ & -2.36284900 & 0.37383900 & 1.92614100 \\
\hline $\mathrm{C}$ & -3.15518500 & 1.72200400 & -0.03076000 \\
\hline $\mathrm{P}$ & 2.29599800 & 0.00002000 & 0.00002800 \\
\hline $\mathrm{K}$ & 1.06197800 & -2.88847800 & 0.18717200 \\
\hline $\mathrm{H}$ & -1.67798300 & -0.41450100 & 2.26947700 \\
\hline $\mathrm{H}$ & -3.39616900 & 0.07525100 & 2.16038500 \\
\hline $\mathrm{H}$ & -2.13253600 & 1.28719000 & 2.49608600 \\
\hline $\mathrm{H}$ & -3.10066900 & 2.60057600 & 0.63221800 \\
\hline $\mathrm{H}$ & -4.19001800 & 1.34601600 & 0.00164600 \\
\hline $\mathrm{H}$ & -2.93783900 & 2.05556000 & -1.05471300 \\
\hline $\mathrm{O}$ & 3.03173600 & -1.28737800 & -0.30954500 \\
\hline $\mathrm{K}$ & 1.06190500 & 2.88849300 & -0.18726000 \\
\hline $\mathrm{O}$ & 3.03167400 & 1.28745300 & 0.30960300 \\
\hline $\mathrm{O}$ & 1.05606100 & -0.31439400 & 1.06988900 \\
\hline \multicolumn{4}{|c|}{$\mathrm{B}_{2} \mathrm{pin}_{2}-\mathrm{K}_{3} \mathrm{PO}_{4}$} \\
\hline $\mathrm{K}$ & 0.03976100 & -0.66248100 & -2.71347700 \\
\hline $\mathrm{O}$ & -0.98929100 & -1.64561100 & -0.48408800 \\
\hline $\mathrm{O}$ & 1.15864500 & -2.80388800 & -1.23401600 \\
\hline $\mathrm{P}$ & 0.32392900 & -2.44237300 & 0.01671100 \\
\hline $\mathrm{K}$ & -0.55113500 & -1.19137600 & 2.76556300 \\
\hline B & -1.02801700 & -0.12721700 & -0.08084600 \\
\hline
\end{tabular}


$1.15499200 \quad-1.21748900 \quad 0.76915400$

$\begin{array}{lll}0.00282300 & -3.47592600 & 1.07350800\end{array}$

$\begin{array}{lll}-1.67103200 & 0.57256000 & -1.22702800\end{array}$

$\begin{array}{lll}-2.00161500 & 0.04972800 & 1.02416000\end{array}$

$\begin{array}{lll}0.65135400 & 0.17743000 & 0.33305400\end{array}$

$\begin{array}{lll}-2.90727200 & 1.14446900 & -0.82251600\end{array}$

$\begin{array}{lll}-3.28180100 & 0.31319000 & 0.46429900\end{array}$

$\begin{array}{lll}1.62640300 & 0.60232100 & -0.77463600\end{array}$

$0.90027700 \quad 1.15320600 \quad 1.40041800$

$\begin{array}{lll}-2.66816700 & 2.62453800 & -0.49261500\end{array}$

$\begin{array}{lll}-3.91370800 & 1.02837100 & -1.96541500\end{array}$

$\begin{array}{lll}-4.11891800 & 1.07591900 & 1.48932600\end{array}$

$\begin{array}{lll}-3.98092800 & -1.01181300 & 0.12265900\end{array}$

$2.09435300 \quad 1.91044200 \quad-0.47054500$

$2.03544300 \quad 1.94352800 \quad 1.10977000$

$\begin{array}{lll}-1.97151200 & 2.71873400 & 0.35227900\end{array}$

$\begin{array}{lll}-2.21659900 & 3.11709500 & -1.36738200\end{array}$

$\begin{array}{lll}-3.59937300 & 3.15796000 & -0.24555800\end{array}$

$\begin{array}{lll}-4.91153100 & 1.38849200 & -1.66606800\end{array}$

$\begin{array}{lll}-3.58112300 & 1.64150600 \quad-2.81924700\end{array}$

$\begin{array}{lll}-4.00770000 & -0.01039500 & -2.31049300\end{array}$

$\begin{array}{lll}-4.35280400 & 0.41767000 & 2.34217300\end{array}$

$\begin{array}{lll}-3.57941400 & 1.94994900 & 1.87824400\end{array}$

$\begin{array}{lll}-5.07612600 & 1.41418800 \quad 1.06035900\end{array}$

$-3.40947600 \quad-1.57872700 \quad-0.62264900$

$\begin{array}{lll}-4.03312600 & -1.63013600 & 1.03331500\end{array}$

$\begin{array}{lll}-5.00950800 & -0.85537700 & -0.23983500\end{array}$

$3.49471500 \quad 2.10257700 \quad-1.05275900$

$\begin{array}{lll}1.13800500 & 2.92601000 & -1.10819700\end{array}$

$\begin{array}{lll}3.27628100 & 1.31273100 & 1.76602200\end{array}$

$1.83143300 \quad 3.34177800 \quad 1.69277100$

$\begin{array}{lll}3.45906500 & 2.03448300 & -2.15230300\end{array}$

$3.90271900 \quad 3.09234500 \quad-0.79394400$

$\begin{array}{lll}4.21016600 & 1.34938900 & -0.68818900\end{array}$

$\begin{array}{lll}1.10110100 & 2.74725800 & -2.19537800\end{array}$

$\begin{array}{lll}0.12344700 & 2.79091800 & -0.71528600\end{array}$

$\begin{array}{lll}1.45446000 & 3.96813900 & -0.95021600\end{array}$

$\begin{array}{lll}3.08399300 & 1.20473300 & 2.84441600\end{array}$

$3.46879800 \quad 0.30161500 \quad 1.38070600$

$\begin{array}{lll}4.18696700 & 1.91973600 & 1.64241000\end{array}$

$1.81301100 \quad 3.28640400 \quad 2.79292000$

$2.64327700 \quad 4.02904700 \quad 1.40341300$

$\begin{array}{lll}0.87379600 & 3.76833900 & 1.36703700\end{array}$

$3.30114400 \quad-1.51395800 \quad-0.66361300$ 
(KOPiv) $)_{4} / \mathbf{S 3}$

\begin{tabular}{|c|c|c|c|}
\hline $\mathrm{C}$ & -1.45532900 & 2.11871500 & -2.15616300 \\
\hline $\mathrm{O}$ & -1.35155200 & 1.22167300 & -1.24794500 \\
\hline C & -2.90074200 & 2.51815700 & -2.55251500 \\
\hline C & -3.62024800 & 3.04572600 & -1.29793100 \\
\hline $\mathrm{C}$ & -2.88230700 & 3.59637800 & -3.63903300 \\
\hline C & -3.62666900 & 1.26223900 & -3.06803400 \\
\hline $\mathrm{H}$ & -3.71758300 & 2.25632300 & -0.53576200 \\
\hline $\mathrm{H}$ & -3.07812900 & 3.89726600 & -0.85369600 \\
\hline $\mathrm{H}$ & -4.63731400 & 3.39111400 & -1.54654000 \\
\hline $\mathrm{H}$ & -2.36256800 & 3.24047200 & -4.54066100 \\
\hline $\mathrm{H}$ & -3.90954100 & 3.88240600 & -3.92020100 \\
\hline $\mathrm{H}$ & -2.35417800 & 4.49720400 & -3.29347500 \\
\hline $\mathrm{H}$ & -4.64447100 & 1.51156000 & -3.41081000 \\
\hline $\mathrm{H}$ & -3.08918700 & 0.81069800 & -3.91861400 \\
\hline $\mathrm{H}$ & -3.72273800 & 0.50547000 & -2.27328700 \\
\hline $\mathrm{O}$ & -0.48566700 & 2.66435400 & -2.71464600 \\
\hline K & 1.41523500 & 1.35840900 & -1.38661300 \\
\hline C & 1.45695000 & -2.15946400 & -2.11463400 \\
\hline $\mathrm{O}$ & 0.48780800 & -2.72051800 & -2.65855800 \\
\hline $\mathrm{C}$ & 2.90270500 & -2.55706400 & -2.51142200 \\
\hline $\mathrm{C}$ & 3.62677300 & -3.07007800 & -1.25333100 \\
\hline C & 2.88538100 & -3.64615700 & -3.58707500 \\
\hline $\mathrm{C}$ & 3.62350400 & -1.30403400 & -3.04086300 \\
\hline $\mathrm{H}$ & 3.72337500 & -2.27335700 & -0.49867400 \\
\hline $\mathrm{H}$ & 3.08788700 & -3.91882200 & -0.79995100 \\
\hline $\mathrm{H}$ & 4.64436200 & -3.41483000 & -1.50079000 \\
\hline $\mathrm{H}$ & 3.91294700 & -3.92821900 & -3.87100700 \\
\hline $\mathrm{H}$ & 2.36503200 & -4.54681700 & -3.22961800 \\
\hline $\mathrm{H}$ & 2.35847500 & -3.30268200 & -4.48941800 \\
\hline $\mathrm{H}$ & 4.64084900 & -1.55398000 & -3.38443800 \\
\hline $\mathrm{H}$ & 3.08262400 & -0.86137100 & -3.89403300 \\
\hline $\mathrm{H}$ & 3.72023100 & -0.54015400 & -2.25307000 \\
\hline $\mathrm{O}$ & 1.35230800 & -1.24784800 & -1.22115300 \\
\hline K & -1.41430600 & -1.38620200 & -1.35994000 \\
\hline $\mathrm{C}$ & -1.45885800 & -2.11697900 & 2.15560900 \\
\hline $\mathrm{O}$ & -0.49012600 & -2.66150700 & 2.71675600 \\
\hline C & -2.90496700 & -2.51569200 & 2.55017900 \\
\hline $\mathrm{C}$ & -3.63142500 & -1.25898100 & 3.06308100 \\
\hline $\mathrm{C}$ & -3.62253200 & -3.04480400 & 1.29510100 \\
\hline $\mathrm{C}$ & -2.88862000 & -3.59246300 & 3.63811800 \\
\hline $\mathrm{H}$ & -3.09445300 & -0.80582600 & 3.91312700 \\
\hline $\mathrm{H}$ & -3.72751600 & -0.50369300 & 2.26694500 \\
\hline $\mathrm{H}$ & -4.64931300 & -1.50791900 & 3.40590500 \\
\hline
\end{tabular}


H

$\mathrm{H}$

$\mathrm{H}$

$\mathrm{H}$

$\mathrm{H}$

$\mathrm{H}$

O

K

C

O

C

\begin{tabular}{|c|c|c|}
\hline-3.07966800 & -3.89691900 & 0.85284700 \\
\hline-4.64008100 & -3.38975200 & 1.54241600 \\
\hline-3.71851700 & -2.25648200 & 0.53166700 \\
\hline-3.91643000 & -3.87783200 & 3.91781800 \\
\hline-2.36000900 & -4.49383600 & 3.29474400 \\
\hline-2.37048300 & -3.23534700 & 4.54020100 \\
\hline-1.35356900 & -1.22159500 & 1.24587600 \\
\hline 1.41312700 & -1.35939100 & 1.38765600 \\
\hline 1.45719700 & 2.15776100 & 2.11569000 \\
\hline 0.48803500 & 2.71826700 & 2.66012500 \\
\hline 2.90295500 & 2.55493800 & 2.51306800 \\
\hline 3.62165100 & 1.30215900 & 3.04606200 \\
\hline 3.62921500 & 3.06468400 & 1.25499400 \\
\hline 2.88548900 & 3.64616200 & 3.58651100 \\
\hline 3.07908500 & 0.86199300 & 3.89942600 \\
\hline 3.71849300 & 0.53644000 & 2.26008600 \\
\hline 4.63885400 & 1.55160400 & 3.39042800 \\
\hline 3.09217900 & 3.91349500 & 0.79955100 \\
\hline 4.64705700 & 3.40828700 & 1.50302700 \\
\hline 3.72542700 & 2.26659200 & 0.50179700 \\
\hline 3.91301900 & 3.92783800 & 3.87098800 \\
\hline 2.36645800 & 4.54665200 & 3.22666800 \\
\hline 2.35723300 & 3.30498200 & 4.48890700 \\
\hline 1.35252000 & 1.24661700 & 1.22165900 \\
\hline .41406900 & 1.38690600 & 1.35921900 \\
\hline-2.02959100 & -1.10531800 & -0.02745000 \\
\hline 0.01244800 & 0.95710300 & 0.40115400 \\
\hline 2.06941500 & -1.13147500 & 0.17154000 \\
\hline-1.18642600 & 1.56368000 & 0.12315200 \\
\hline-2.31504500 & 0.73078500 & -0.01094500 \\
\hline-3.54907600 & 1.37126200 & -0.21294100 \\
\hline-3.63623900 & 2.75766300 & -0.34344400 \\
\hline-2.48532300 & 3.54480200 & -0.26175000 \\
\hline 3988000 & 2.95986300 & -0.01169400 \\
\hline 1.16386500 & 1.51098100 & -0.09685400 \\
\hline 2.2458 & .64650 & \\
\hline 3. & $1.2144 \varsigma$ & -0.97474700 \\
\hline 3.42022700 & 2.57300200 & -1.28922800 \\
\hline 35419800 & 3.41121000 & -0.95161900 \\
\hline 1.21067100 & 2.89589800 & -0.33458800 \\
\hline 849 & 74765 & \\
\hline-4.45794500 & 0.77728700 & -0.28648600 \\
\hline & & \\
\hline
\end{tabular}




\begin{tabular}{|c|c|c|}
\hline-2.56665700 & 4.62626300 & -0.37476300 \\
\hline 4.21901100 & 0.58319400 & -1.23010000 \\
\hline 4.30218500 & 2.98616800 & -1.78426300 \\
\hline 2.42388800 & 4.47801700 & -1.16653800 \\
\hline 0.00054700 & 5.12953200 & -0.46024300 \\
\hline 0.26461800 & 3.92801400 & 1.72497200 \\
\hline-0.15460100 & 5.05785500 & -1.54748900 \\
\hline-0.81259200 & 5.73338400 & -0.03326200 \\
\hline 0.93080700 & 5.68610100 & -0.27764900 \\
\hline-0.56382200 & 4.51030300 & 2.15857400 \\
\hline 0.30362900 & 2.95628300 & 2.23914400 \\
\hline 1.20994400 & 4.45741800 & 1.92333900 \\
\hline-2.44988800 & -1.55375100 & 1.78782800 \\
\hline-3.36062600 & -1.81880600 & -1.21485300 \\
\hline 2.26419700 & -2.08734400 & -1.47788300 \\
\hline 3.58935800 & -1.38576500 & 1.31133000 \\
\hline-2.84796300 & -3.23354000 & -1.56020400 \\
\hline-2.82087500 & -3.89698300 & -0.68449800 \\
\hline-3.51496100 & -3.69831400 & -2.30711800 \\
\hline-1.83356800 & -3.20139500 & -1.98610600 \\
\hline-4.81352800 & -1.93370900 & -0.73647000 \\
\hline-5.25362900 & -0.96960100 & -0.44804600 \\
\hline-5.43675600 & -2.34272700 & -1.55185300 \\
\hline-4.91301500 & -2.61805700 & 0.11791800 \\
\hline-3.29556800 & -0.98094200 & -2.50493200 \\
\hline-3.82172300 & -1.51313600 & -3.31632200 \\
\hline-3.76389700 & 0.00611700 & -2.39401900 \\
\hline-2.25524200 & -0.82169600 & -2.83269300 \\
\hline-1.25651700 & -1.02677000 & 2.60712900 \\
\hline-1.19940500 & 0.07153800 & 2.58732800 \\
\hline-1.37084300 & -1.33522300 & 3.66122000 \\
\hline-0.29980900 & -1.41562300 & 2.22809300 \\
\hline-3.74034100 & -0.94190700 & 2.34463600 \\
\hline-3.72758500 & 0.15599000 & 2.28208100 \\
\hline-4.63973900 & -1.30302600 & 1.82895300 \\
\hline-3.84487800 & -1.20878400 & 3.41169800 \\
\hline-2.48902500 & -3.08341300 & 1.91668500 \\
\hline-3.36408000 & -3.52342300 & 1.41701400 \\
\hline-1.58214900 & -3.54790900 & 1.49871300 \\
\hline-2.54587200 & -3.36410000 & 2.98277200 \\
\hline 3.66937200 & -2.88937100 & 1.62197700 \\
\hline 4.35607000 & -3.05938500 & 2.46938100 \\
\hline 2.68674500 & -3.30267600 & 1.89992000 \\
\hline 4.05482600 & -3.46604700 & 0.76786500 \\
\hline
\end{tabular}




\begin{tabular}{|c|c|c|}
\hline 4.96861400 & -0.88576300 & 0.86412800 \\
\hline 5.00109800 & 0.20926400 & 0.78698100 \\
\hline 5.72177000 & -1.17730800 & 1.61812800 \\
\hline 5.28792800 & -1.31232000 & -0.09586000 \\
\hline 3.19547600 & -0.63244900 & 2.59703500 \\
\hline 3.98856200 & -0.74382400 & 3.35720200 \\
\hline 3.06276500 & 0.44514700 & 2.40919000 \\
\hline 2.25698200 & -1.01721800 & 3.02514200 \\
\hline 3.66727200 & -2.19616200 & -2.08181300 \\
\hline 4.10481200 & -1.21278100 & -2.30558600 \\
\hline 4.35968000 & -2.74339500 & -1.42558600 \\
\hline 3.61717500 & -2.75109300 & -3.03613900 \\
\hline 1.32207300 & -1.38451800 & -2.47188700 \\
\hline 0.32149200 & -1.23557900 & -2.03518700 \\
\hline 1.70887500 & -0.40371800 & -2.78413000 \\
\hline 1.20817800 & -2.00688600 & -3.37666900 \\
\hline 1.70920400 & -3.49948000 & -1.21278200 \\
\hline 0.69253300 & -3.45174200 & -0.79441000 \\
\hline 1.66387800 & -4.06823700 & -2.15817200 \\
\hline 2.33692900 & -4.07144000 & -0.51460200 \\
\hline & & \\
\hline 4.31660400 & -2.05771100 & -0.66651500 \\
\hline 4.26718400 & -0.65494200 & -0.74483200 \\
\hline 4.87205200 & 0.29551400 & 0.20758300 \\
\hline 5.69024100 & -0.10314100 & 1.28399700 \\
\hline 3.94207500 & 1.99491900 & -0.75236000 \\
\hline 3.88573600 & -0.20757700 & -1.66868100 \\
\hline 4.58251600 & 1.66855600 & 0.07056000 \\
\hline 6.18686600 & 0.83365700 & 2.19004600 \\
\hline 5.88534300 & 2.19185800 & 2.04318300 \\
\hline 5.08173300 & 2.60417900 & 0.97559900 \\
\hline 6.82044700 & 0.50095800 & 3.01638200 \\
\hline 6.27610400 & 2.92315600 & 2.75482300 \\
\hline 4.83726500 & 3.66189200 & 0.84926300 \\
\hline 4.01285300 & -2.93131700 & -1.81881700 \\
\hline 3.05421600 & -3.45991300 & -1.85210100 \\
\hline 5.94675800 & -1.15694300 & 1.41104300 \\
\hline 4.93976200 & -2.50730100 & 0.12701900 \\
\hline 5.07968000 & -3.29468200 & -2.79681100 \\
\hline 5.61686800 & -2.39944700 & -3.16157400 \\
\hline 4.67646100 & -3.83614300 & -3.66704500 \\
\hline 5.85637200 & -3.94369900 & -2.33766800 \\
\hline 2.68870000 & -1.32699500 & 0.26386200 \\
\hline 1.17107000 & -1.45498200 & 1.51491500 \\
\hline
\end{tabular}


$1.05471400 \quad-0.95836100 \quad-0.92443600$

0.17105400

$-1.02528700$

0.37140800

$-1.44419700$

$-0.96477200$

2.85674900

$-1.29125800$

$-0.95573000$

$-2.35189100$

$-0.35158300$

$0.42836300 \quad 0.82457500$

$-1.06251800$

$-1.93453700$

0.22809400

$-3.54848000$

$-0.25318800$

1.36272100

$-1.13489500$

1.39578000

$-1.32299700$

$-1.50251300$

1.14736700

0.09617600

$-2.93089700$

0.16381000

0.18276400

$-4.82522700$

$-0.86422200$

1.05514400

$-0.54597100$

2.68985900

$-1.38914900$

$-1.61063800$

$-3.64857200$

2.47510600

0.69149500

$-4.66394300$

$-5.03206000$

$-0.91086500$

$-5.89530400$$$
-2.05227000 \quad 1.98709700
$$

$0.19830300 \quad 1.30900600$

$\begin{array}{lll}0.94551800 & 2.59381900 & -1.02817400\end{array}$

$\begin{array}{lll}-0.69177800 & 3.22365900 & -2.81189900\end{array}$

$\begin{array}{lll}-1.38198700 & 3.46267600 & -0.30120500\end{array}$

$\begin{array}{lll}-5.91638100 & -1.03215800 & -1.30925400\end{array}$

$\begin{array}{lll}-4.10762300 & -2.64949400 & -0.68589400\end{array}$

$\begin{array}{lll}-5.10800600 & -1.69949000 \quad 3.02818500\end{array}$

$\begin{array}{lll}-5.96619800 & -2.58489500 & 1.75057100\end{array}$

$-4.20437800 \quad-2.77227000 \quad 1.92153300$

$\begin{array}{lll}-6.91092600 & -0.19506500 & 1.15377900\end{array}$

$\begin{array}{lll}-5.75131700 & 1.07117400 & 0.65605400\end{array}$

$\begin{array}{lll}-5.81533600 & 0.54447800 & 2.35055700\end{array}$

$\begin{array}{lll}1.47264100 & 3.54659000 & -1.19801700\end{array}$

$\begin{array}{lll}1.40589600 & 1.81509800 & -1.65396300\end{array}$

$\begin{array}{lll}1.07900500 & 2.28579000 & 0.01610500\end{array}$

$\begin{array}{lll}-0.05521100 & 2.63911100 & -3.49701400\end{array}$

$\begin{array}{lll}-0.37383600 & 4.27644400 & -2.88244200\end{array}$

$\begin{array}{lll}-1.72917900 & 3.14803600 & -3.16612100\end{array}$

$\begin{array}{lll}-2.74511500 & 3.91758300 & -0.84736800\end{array}$

$\begin{array}{lll}-0.65398100 & 4.65010300 \quad 0.32428600\end{array}$

$\begin{array}{lll}-6.74157000 & -1.66854700 & -0.95308400\end{array}$

$\begin{array}{lll}-5.70886500 & -1.30596600 & -2.35534000\end{array}$

$\begin{array}{lll}-6.24855800 & 0.01383000 & -1.29725500\end{array}$

$\begin{array}{lll}-3.91721800 & -2.79528000 & -1.76239500\end{array}$

$\begin{array}{lll}-4.82668600 & -3.42353500 & -0.37949600\end{array}$

$-3.15821100 \quad-2.78577200 \quad-0.14984200$

$\begin{array}{lll}-3.37339200 & 4.23446700 & -0.00030000\end{array}$

$\begin{array}{lll}-2.66608700 & 4.76095700 & -1.55196900\end{array}$ 


\begin{tabular}{|c|c|c|c|}
\hline $\mathrm{H}$ & -3.25348200 & 3.08220900 & -1.35181100 \\
\hline $\mathrm{H}$ & -1.30480700 & 5.13613300 & 1.06880900 \\
\hline $\mathrm{H}$ & -0.38519100 & 5.40428600 & -0.43364400 \\
\hline $\mathrm{H}$ & 0.26003100 & 4.32705600 & 0.84045300 \\
\hline \multicolumn{4}{|l|}{16} \\
\hline $\mathrm{Ni}$ & 2.80655400 & 1.04136200 & -0.57846200 \\
\hline B & -2.61047700 & -0.81477300 & 0.18547300 \\
\hline $\mathrm{O}$ & 1.85164700 & -0.28976600 & 0.47686300 \\
\hline $\mathrm{O}$ & -3.34857600 & -1.03602100 & -0.97194400 \\
\hline $\mathrm{O}$ & -2.50083300 & -2.00663200 & 0.90656800 \\
\hline $\mathrm{P}$ & 0.50480000 & 0.11868000 & -0.23794500 \\
\hline K & -1.58070100 & 0.59319500 & -2.53839700 \\
\hline $\mathrm{C}$ & -3.57360000 & -2.45677000 & -1.14645000 \\
\hline $\mathrm{C}$ & -3.35713100 & -3.01228900 & 0.31333200 \\
\hline $\mathrm{O}$ & -0.47128900 & 0.59928900 & 0.93452700 \\
\hline K & 0.05722700 & -2.03752000 & 1.71994600 \\
\hline $\mathrm{O}$ & -0.18035000 & -1.04391000 & -0.97670900 \\
\hline $\mathrm{O}$ & 0.95432300 & 1.28866800 & -1.19129600 \\
\hline $\mathrm{O}$ & -2.24550700 & 1.75112400 & -0.36142500 \\
\hline $\mathrm{C}$ & -4.97800700 & -2.66102800 & -1.70046800 \\
\hline $\mathrm{C}$ & -2.53176800 & -2.96497500 & -2.14672800 \\
\hline $\mathrm{C}$ & -2.64948000 & -4.36030900 & 0.38387400 \\
\hline $\mathrm{C}$ & -4.63966800 & -3.04571400 & 1.14588700 \\
\hline B & -1.99221600 & 0.72541400 & 0.68919700 \\
\hline $\mathrm{C}$ & -2.50516200 & 2.98733600 & 0.29584100 \\
\hline $\mathrm{H}$ & -5.22134400 & -3.73250400 & -1.77526700 \\
\hline $\mathrm{H}$ & -5.04284500 & -2.23069400 & -2.71186700 \\
\hline $\mathrm{H}$ & -5.73806400 & -2.17006700 & -1.07914200 \\
\hline $\mathrm{H}$ & -2.67647600 & -2.44621400 & -3.10913200 \\
\hline $\mathrm{H}$ & -2.64589200 & -4.04152800 & -2.34237700 \\
\hline $\mathrm{H}$ & -1.51239000 & -2.75472600 & -1.79544700 \\
\hline $\mathrm{H}$ & -2.52132700 & -4.66105500 & 1.43626100 \\
\hline $\mathrm{H}$ & -3.24146700 & -5.14474500 & -0.11227600 \\
\hline $\mathrm{H}$ & -1.66096700 & -4.32842200 & -0.09508000 \\
\hline $\mathrm{H}$ & -5.33272200 & -3.82901700 & 0.80460200 \\
\hline $\mathrm{H}$ & -5.15832600 & -2.07681400 & 1.11372700 \\
\hline $\mathrm{H}$ & -4.37859600 & -3.24962300 & 2.19527000 \\
\hline $\mathrm{O}$ & -2.57568500 & 1.28205800 & 1.90226100 \\
\hline $\mathrm{C}$ & -1.17530700 & 3.68894100 & 0.60893800 \\
\hline $\mathrm{C}$ & -3.34542400 & 3.86853300 & -0.62477900 \\
\hline $\mathrm{C}$ & -3.24502100 & 2.49390600 & 1.59488000 \\
\hline $\mathrm{H}$ & -1.32986700 & 4.70119000 & 1.01545700 \\
\hline $\mathrm{H}$ & -0.59000700 & 3.76859900 & -0.31969900 \\
\hline $\mathrm{H}$ & -0.57928200 & 3.10005800 & 1.31756700 \\
\hline
\end{tabular}


$\begin{array}{lll}-2.74325900 & 4.18603100 & -1.49256600\end{array}$

$-3.68557100$

$4.78009600 \quad-0.10757600$

$\begin{array}{lll}-4.22728600 & 3.33163700 & -1.00101000\end{array}$

$\begin{array}{lll}-3.13581500 & 3.44649700 & 2.78272700\end{array}$

TS17

$\begin{array}{lll}-4.81747700 & 1.52879300 & 0.43278600\end{array}$

$\begin{array}{lll}-3.68520700 \quad 3.03323200 & 3.64384000\end{array}$

$\begin{array}{lll}-3.56557100 & 4.43456100 & 2.54954000\end{array}$

$\begin{array}{lll}-2.08941400 \quad 3.58013200 & 3.08833900\end{array}$

$\begin{array}{lll}4.60710900 & 1.38640300 & -1.25087700\end{array}$

$\begin{array}{lll}4.69123800 & 0.85530500 & 0.06585700\end{array}$

$\begin{array}{lll}5.17472600 & -0.50682200 & 0.35307000\end{array}$

$\begin{array}{lll}4.90825000 & -1.58402900 & -0.51542900\end{array}$

$\begin{array}{lll}6.09065200 & 0.04639200 & 2.22985100\end{array}$

$4.76630900 \quad 1.55222700 \quad 0.90895200$

$\begin{array}{lll}5.88757800 & -0.77358200 & 1.53568200\end{array}$

$\begin{array}{lll}5.36491500 & -2.86862300 & -0.22548300\end{array}$

$6.08753100 \quad-3.11518500 \quad 0.94784800$

$\begin{array}{lll}6.34166400 & -2.06113500 \quad 1.82985900\end{array}$

$\begin{array}{lll}5.14431200 & -3.68865700 & -0.91417400\end{array}$

$6.44227400 \quad-4.12352400 \quad 1.17570200$

$\begin{array}{lll}6.89873500 & -2.24159900 & 2.75307600\end{array}$

$3.79619400 \quad 2.52237600 \quad-1.48071300$

$\begin{array}{lll}4.30498500 & -1.41364400 & -1.40935600\end{array}$

$\begin{array}{lll}4.90265500 & 0.76412100 & -2.10339700\end{array}$

$\begin{array}{lll}3.65309300 & 3.71762200 & -0.57233600\end{array}$

$\begin{array}{lll}2.60278400 & 4.04894000 & -0.53101800\end{array}$

$\begin{array}{lll}4.24813600 & 4.56956600 & -0.95106800\end{array}$

$3.97988400 \quad 3.52005300 \quad 0.45861000$

$\begin{array}{lll}3.54628000 & 2.72110000 & -2.52981900\end{array}$

$\begin{array}{rrr}-0.94269500 & 2.05420000 & -1.53605500 \\ -0.65432000 & 2.81210700 & -0.35096800 \\ 0.65737400 & 3.25500100 & 0.10171100 \\ 1.84090400 & 3.12884000 & -0.67148800 \\ -0.09607400 & 3.96648400 & 2.00695000 \\ -1.50297600 & 3.27737700 & 0.16292000 \\ 0.79650800 & 3.84900600 & 1.38659000 \\ 3.08152900 & 3.54176500 & -0.18193900 \\ 3.19858900 & 4.11174300 & 1.09585500 \\ 2.03534400 & 4.26793700 & 1.87062200 \\ 3.96842200 & 3.43789100 & -0.81400400\end{array}$


$4.16787200 \quad 4.45067900 \quad 1.46950200$

$\begin{array}{lll}2.09832500 & 4.72595500 & 2.86213500\end{array}$

$\begin{array}{lll}-2.13556400 & 2.34151900 & -2.36417200\end{array}$

$\begin{array}{lll}-2.98942400 & 1.66371000 & -2.37515700\end{array}$

$\begin{array}{lll}1.77446100 & 2.71619300 & -1.67930600\end{array}$

$\begin{array}{lll}-0.05469500 & 1.80628500 & -2.15254400\end{array}$

$\begin{array}{lll}-2.18237500 & 3.58857000 & -3.18342800\end{array}$

$\begin{array}{lll}-2.22265100 & 4.49692100 & -2.54698100\end{array}$

$\begin{array}{lll}-3.05620200 & 3.61534400 & -3.85395300\end{array}$

$\begin{array}{lll}-1.27216200 & 3.70780700 & -3.80513500\end{array}$

$\begin{array}{lll}0.36759300 & -1.37699100 & 1.90289800\end{array}$

$\begin{array}{lll}-1.45880800 & -3.20865300 & -0.06705200\end{array}$

$\begin{array}{lll}2.57316500 & 0.83241100 & 2.09699200\end{array}$

$2.54446400 \quad-2.72776600 \quad-1.19715400$

$0.87228800 \quad-2.08100400 \quad 0.37357500$

$\begin{array}{lll}-3.10087200 & -1.21739900 & -0.55637300\end{array}$

$\begin{array}{lll}-0.88262900 & -2.18671400 & 2.18002600\end{array}$

$\begin{array}{lll}1.58998400 & -1.46513400 \quad 2.79871700\end{array}$

$\begin{array}{lll}0.11663000 & 0.12201000 \quad 1.48966200\end{array}$

$3.91715300 \quad-2.34752000 \quad-1.45192300$

$\begin{array}{lll}2.04447000 & -1.86429800 & -0.24987400\end{array}$

$\begin{array}{lll}-4.47157100 & -1.32657900 & -0.06875300\end{array}$

$\begin{array}{lll}-2.60868900 & 0.06438700 & -0.23944400\end{array}$

$\begin{array}{lll}4.25581000 & -2.64596100 & -2.90471600\end{array}$

$4.79526600 \quad-3.17881000 \quad-0.51445400$

$3.90672100 \quad-0.81778300 \quad-1.07820200$

$\begin{array}{lll}2.88010000 & -0.77129600 & -0.05178700\end{array}$

$-5.26361200 \quad-2.20899200 \quad-1.02366200$

$\begin{array}{lll}-4.41501100 & -1.96551200 & 1.32162100\end{array}$

$\begin{array}{lll}-4.91780100 & 0.17802600 & -0.03029900\end{array}$

$\begin{array}{lll}-3.66088800 & 0.86306000 & 0.16371500\end{array}$

$\begin{array}{lll}-0.78907100 & 0.79513600 & -0.05097500\end{array}$

$5.26224800 \quad-2.27732500 \quad-3.15598800$

$\begin{array}{lll}4.24224400 & -3.73355500 & -3.07231700\end{array}$

$\begin{array}{lll}3.53184000 & -2.18947500 & -3.59233200\end{array}$

$4.58600900 \quad-4.24543300 \quad-0.68375800$

$\begin{array}{lll}5.86618900 & -3.00505900 & -0.69612000\end{array}$

$\begin{array}{lll}4.57638900 & -2.95668600 & 0.54085500\end{array}$

$5.20675800 \quad-0.28682500 \quad-0.49115700$

$\begin{array}{lll}3.43688400 & 0.07099800 & -2.22806800\end{array}$

$\begin{array}{lll}-6.32717200 & -2.23752500 & -0.73938900\end{array}$

$\begin{array}{lll}-4.88644300 & -3.24421200 & -0.98609900\end{array}$

$\begin{array}{lll}-5.18832800 & -1.86009500 & -2.06155800\end{array}$

$\begin{array}{lll}-5.41622600 & -2.04710100 \quad 1.76876300\end{array}$ 
H

H

C

\begin{tabular}{|c|c|c|}
\hline-4.01636300 & -2.99084100 & 1.25316300 \\
\hline-3.75926300 & -1.40106500 & 1.99851700 \\
\hline-5.50629500 & 0.66064100 & -1.35815600 \\
\hline-5.85032700 & 0.54211600 & 1.11772600 \\
\hline 5.10687300 & 0.78845500 & -0.27248700 \\
\hline 6.03539000 & -0.40084800 & -1.20660700 \\
\hline 5.47644000 & -0.80721700 & 0.43761200 \\
\hline 4.18771500 & 0.13093100 & $-3.0291500 c$ \\
\hline 3.26099200 & 1.08383100 & $-1.8437640 c$ \\
\hline 2.49363900 & -0.29726800 & -2.65805800 \\
\hline-5.56709600 & 1.75868900 & -1.34113300 \\
\hline-6.51762500 & 0.26051400 & -1.52666400 \\
\hline-4.87185700 & 0.37065700 & -2.20795400 \\
\hline-6.10013600 & 1.61262000 & 1.06230200 \\
\hline-6.79075100 & -0.02903400 & 1.06389500 \\
\hline-5.37943900 & 0.35861800 & 2.09227200 \\
\hline-0.19596000 & -1.09197600 & -1.12761100 \\
\hline 1.10518100 & -2.05894600 & 1.64807100 \\
\hline-2.20888300 & 1.15234000 & -1.85366100 \\
\hline-2.93860600 & 2.55751300 & 0.82474800 \\
\hline-1.03625800 & -1.12859300 & 0.42700300 \\
\hline 3.36662200 & -0.85744700 & 0.97038000 \\
\hline 0.91216400 & -2.10215300 & -0.90984300 \\
\hline-1.31460200 & -1.33797700 & -2.13084100 \\
\hline 0.24916700 & 0.41328000 & -1.17881300 \\
\hline-4.28328900 & -2.59083800 & 0.28559700 \\
\hline-2.36851100 & -1.38147100 & 0.40310800 \\
\hline 4.49268800 & -1.64677800 & 0.49042100 \\
\hline 3.00467600 & 0.07710700 & -0.01954500 \\
\hline-5.18295000 & 3.37075200 & 1.23172600 \\
\hline-4.18463600 & 3.28908800 & -1.07301400 \\
\hline-4.62843200 & -1.05506100 & 0.15900400 \\
\hline-3.31868300 & -0.46844500 & -0.02979200 \\
\hline 5.41934300 & -1.92671100 & 1.66669200 \\
\hline 3.93734700 & -2.95843000 & -0.06965000 \\
\hline 5.09766400 & -0.71138400 & -0.62280600 \\
\hline 3.96136600 & 0.08850800 & -1.01396300 \\
\hline 1.50511400 & 1.27207500 & -0.04858500 \\
\hline-6.22841100 & -3.34514000 & 0.88731500 \\
\hline-4.86304900 & -4.42330000 & 1.26105300 \\
\hline-5.14069300 & -2.97667000 & 2.25582000 \\
\hline-3.77650400 & -4.29943800 & -0.92025600 \\
\hline-5.1671090 & -3.38588900 & -1.5581170 \\
\hline
\end{tabular}




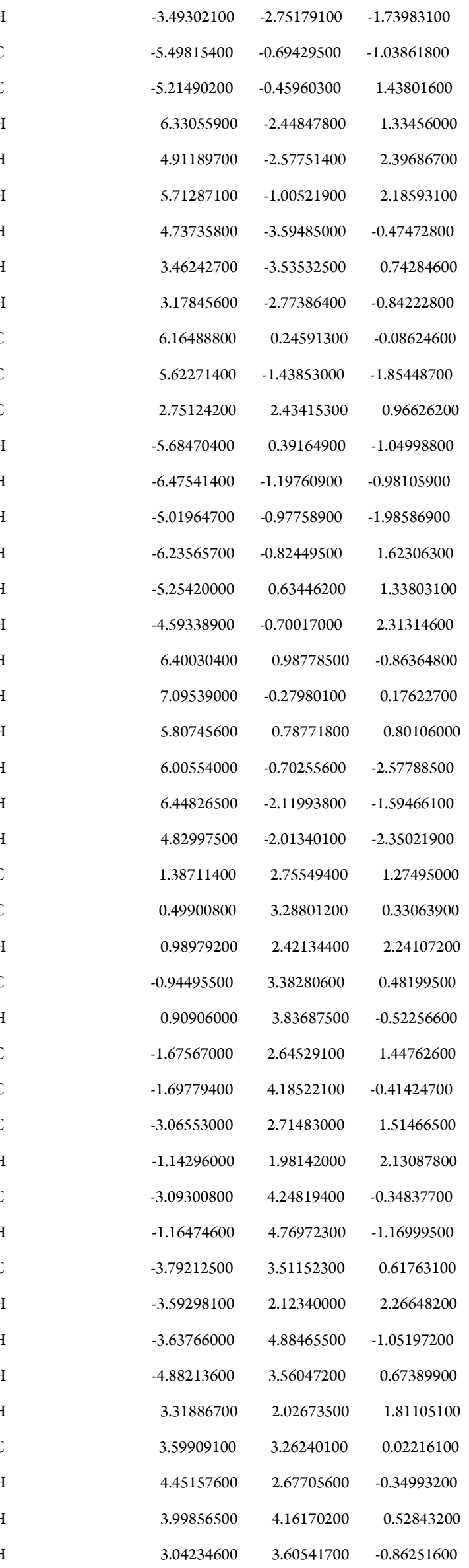


TS19/TS23/TS-Conformation-B$$
-1.80626800
$$$$
-1.31808800
$$$$
-1.87229700
$$$$
-2.68521800
$$$$
-3.13851900
$$$$
-2.73882300
$$$$
-3.19478800
$$$$
-2.49761700
$$$$
-2.73906400
$$$$
-3.67659200
$$$$
-4.34774500
$$$$
-4.12205900
$$

$-1.43464000$

$-3.79386300$

$-3.11155600$

$-3.90490400$

$-5.06876000$

$-4.67443800$

$-2.88821400$

$-1.54762700$

$-1.64638900$

$-0.72934100$

$-1.26517900$

$-3.98294900$

$-3.72631100$

$-4.96119600$

$-4.08164900$

$-1.56016800$

0.24966600

$-0.38632700$

$-1.46100600$

0.09421100

$-0.28659800$

$-0.53841600$

$-0.46539500$

$-0.13880400$

$-1.60121800$

1.69966400

2.19149500

1.68989700

2.31248300

1.51974300

1.50033000
$-3.25338100 \quad 2.36191800$

$-2.17843600 \quad 1.59915700$

$-2.02170800 \quad 0.31578100$

$-2.98711900 \quad-0.29118200$

$-4.04448400 \quad 0.50335700$

$-4.14868700 \quad 1.83783600$

$-1.40646700 \quad-2.08720000$

$-0.43778100-1.35137800$

$0.94139900 \quad-1.47960200$

$1.32910700 \quad-2.45211200$

$0.39159600 \quad-3.23474300$

$-0.97053900 \quad-3.03838900$

$-3.41204000 \quad 3.37048500$

$-4.80432900 \quad 0.07730600$

$-4.96818200 \quad 2.45611300$

$2.38377700 \quad-2.58592400$

$0.72422500-3.98459500$

$-1.69875500 \quad-3.63183900$

$-2.87303000 \quad-1.80276100$

$-3.24009500 \quad-2.49375600$

$-3.14107500 \quad-3.58568900$

$-2.58316100 \quad-2.16786600$

$-4.27755100 \quad-2.25790500$

$-3.81181400 \quad-2.31398000$

$-4.85792400 \quad-2.09363400$

$-3.59055300-1.86097600$

$-3.73647000 \quad-3.40603800$

$-0.87471300 \quad-0.41195100$

$-0.57877900 \quad 3.71842000$

$-1.49658200 \quad 4.77010200$

$-1.63865100 \quad 4.58975700$

$-2.48051000 \quad 4.83781200$

$-1.01748600 \quad 5.75924200$

$0.73838000 \quad 3.69752700$

$1.22354900 \quad 4.68543500$

$1.43239900 \quad 2.94745300$

$0.56833300 \quad 3.49042000$

$-0.25327800 \quad 4.11270600$

$0.39146600 \quad 3.37189500$

$0.29638000 \quad 5.06850400$

$-1.15268300 \quad 4.25830300$

$-2.66627500 \quad 1.73510700$

$-3.66511200 \quad 2.89963700$ 
$2.17044000 \quad-4.50541300 \quad 2.65062600$

$\begin{array}{lll}1.87155500 & -3.22518300 \quad 3.83557100\end{array}$

$0.50603500 \quad-4.09317900 \quad 3.08024700$

$\begin{array}{lll}1.16236400 & -3.40281100 & 0.43159000\end{array}$

$\begin{array}{lll}1.05191200 & -2.69965300 & -0.40665000\end{array}$

$1.98888200 \quad-4.08297300 \quad 0.17008000$

$0.24521000 \quad-4.00115600 \quad 0.52046600$

$\begin{array}{lll}2.94865800 & -2.10467400 & 1.57200400\end{array}$

$\begin{array}{lll}3.17675100 & -1.27367600 & 2.24857500\end{array}$

$\begin{array}{lll}3.66565600 & -2.91327500 & 1.79214300\end{array}$

$\begin{array}{lll}3.14263100 & -1.79285500 & 0.53866500\end{array}$

$\begin{array}{lll}-1.77088000 & 3.78174700 & -1.22240000\end{array}$

$\begin{array}{lll}-3.02234000 & 4.66415300 & -1.33008000\end{array}$

$\begin{array}{lll}-3.87257800 \quad 4.16810000 & -1.81573600\end{array}$

$\begin{array}{lll}-3.35480700 & 5.02537800 & -0.34745400\end{array}$

$\begin{array}{lll}-2.77546700 & 5.55413700 & -1.93474600\end{array}$

$\begin{array}{lll}-0.69859900 & 4.57559600 & -0.45119800\end{array}$

$\begin{array}{lll}-0.96689100 & 4.73363900 & 0.60384400\end{array}$

$0.27689900 \quad 4.07416000 \quad-0.48240800$

$\begin{array}{lll}-0.57604400 & 5.56969900 & -0.91348200\end{array}$

$\begin{array}{lll}-1.21787100 & 3.50272800 & -2.62990500\end{array}$

$\begin{array}{lll}-0.87825400 & 4.45210500 & -3.07745800\end{array}$

$\begin{array}{lll}-0.35662300 & 2.82494800 \quad-2.60380500\end{array}$

$\begin{array}{lll}-1.96402700 & 3.06590700 & -3.30580500\end{array}$

$\begin{array}{lll}-3.34357400 & 2.20935400 & 1.01478500\end{array}$

$\begin{array}{lll}-4.73695000 & 2.62522800 & 0.52800500\end{array}$

$\begin{array}{lll}-5.46412400 & 2.44995000 & 1.34042700\end{array}$

$\begin{array}{lll}-4.80153800 & 3.68610000 & 0.26179400\end{array}$

$\begin{array}{lll}-5.06670800 & 2.02760100 & -0.33412900\end{array}$

$\begin{array}{lll}-2.86366900 & 3.16606500 \quad 2.11717200\end{array}$

$\begin{array}{lll}-2.83371900 \quad 4.20840700 & 1.76637100\end{array}$

$\begin{array}{lll}-3.55592400 & 3.12840800 \quad 2.97571300\end{array}$

$\begin{array}{lll}-1.86127100 \quad 2.90046100 & 2.48261800\end{array}$

$\begin{array}{lll}-3.45162100 & 0.77748100 & 1.56748400\end{array}$

$\begin{array}{lll}-3.99316600 & 0.11844600 & 0.87353000\end{array}$

$\begin{array}{lll}-2.46678100 & 0.33446600 & 1.74767100\end{array}$

$\begin{array}{lll}-4.00808900 & 0.78304700 & 2.51948300\end{array}$

$1.40735900 \quad 1.13054400 \quad-1.14904700$

$\begin{array}{lll}2.04737300 & 0.79366200 & 0.10444900\end{array}$

$\begin{array}{lll}2.50902100 & 1.80130800 \quad 1.08211700\end{array}$

$\begin{array}{lll}1.66518800 & 2.81983700 \quad 1.56103600\end{array}$

$\begin{array}{lll}4.48056500 & 0.93768200 & 1.25602900\end{array}$

$\begin{array}{lll}2.72624300 & -0.05575400 & 0.02392600\end{array}$

$3.81021000 \quad 1.72247400 \quad 1.61382800$ 
$3.39860600 \quad 3.63830900 \quad 3.04160300$

TS20/TS-Conformation-A

C

C

C

C

$\mathrm{H}$

$\mathrm{H}$

C

C

C

C

$\begin{array}{lll}1.42154300 \quad 4.50679500 & 2.88233300\end{array}$

$\begin{array}{lll}3.74158500 & 4.34648000 \quad 3.80003800\end{array}$

$5.26601600 \quad 2.54783900 \quad 2.97669500$

$\begin{array}{lll}1.27101000 & 0.13314000 & -2.12548500\end{array}$

$\begin{array}{lll}3.14959700 & 0.05754800 & -3.11295500\end{array}$

$3.92353400 \quad-0.73630500 \quad-2.49153900$

$\begin{array}{lll}5.44961300 & -0.54937900 & -2.75328600\end{array}$

$3.57946100 \quad-1.60511300 \quad-1.66572300$

$5.70608500 \quad 0.32106900 \quad-3.98687900$

$6.01540400 \quad 0.14184400 \quad-1.49855500$

$\begin{array}{lll}6.10323200 & -1.92643700 \quad-2.92496400\end{array}$

$5.28265200 \quad-0.13946800 \quad-4.89349900$

$\begin{array}{lll}5.23999300 & 1.31042200 & -3.87630700\end{array}$

$6.78955800 \quad 0.45888700 \quad-4.14753600$

$\begin{array}{lll}5.85870100 & -0.48475500 & -0.60721500\end{array}$

$\begin{array}{lll}7.09817500 & 0.32753400 & -1.60479300\end{array}$

$5.52021400 \quad 1.11146400 \quad-1.32614900$

$\begin{array}{lll}7.19864000 & -1.83490700 & -3.02407000\end{array}$

$5.87749600 \quad-2.56677400 \quad-2.06072100$

$5.72484300 \quad-2.43541200 \quad-3.82737800$

$\begin{array}{lll}0.64352900 & 2.86557800 \quad 1.18776900\end{array}$

$\begin{array}{lll}0.23405000 & 0.14826900 & 0.22424800\end{array}$

$0.24754400 \quad-1.22917900 \quad 1.92114500$

$\begin{array}{lll}-1.93384100 & 2.10681700 & -0.28771300\end{array}$

$\begin{array}{lll}1.17807100 & 2.16970700 \quad-1.39714100\end{array}$

$\begin{array}{lll}0.47703700 & 0.30072700 & -3.38085000\end{array}$

$0.70464200 \quad 1.25844500 \quad-3.87102200$

$\begin{array}{lll}-0.60301000 & 0.28342700 & -3.16323800\end{array}$

$\begin{array}{lll}0.70050300 & -0.50791200 & -4.08875700\end{array}$

$1.52245300 \quad-0.88341600 \quad-1.82381400$

\begin{tabular}{rrr}
-1.83407300 & 0.00356300 & -0.56024700 \\
-1.59091300 & -0.93794300 & -1.67262200 \\
-1.46959300 & -0.52766500 & -3.01642600 \\
-1.62630300 & -2.67283200 & -0.39086600 \\
-2.49326300 & -0.38660700 & 0.21255000 \\
-1.50851100 & -2.32099900 & -1.41169500 \\
-1.24638500 & -1.44988200 & -4.03976000 \\
-1.14207400 & -2.81417300 & -3.75352000 \\
\hline-1.28197700 & -3.24412900 & -2.43057600
\end{tabular}


H

H

$\mathrm{H}$

C

C

O

\begin{tabular}{|c|c|c|}
\hline-1.16404200 & -1.09867800 & -5.07160300 \\
\hline-0.96513300 & -3.53520500 & -4.55529800 \\
\hline 1.21570500 & -4.30737900 & -2.18686000 \\
\hline 2.36223800 & 2.22144600 & 0.29414200 \\
\hline 5.06185900 & 0.73939000 & 0.07165500 \\
\hline-4.56509700 & -0.07074400 & 0.89000000 \\
\hline-6.06350800 & 0.18389200 & -0.99497700 \\
\hline-6.32219400 & -1.31019300 & -0.78814100 \\
\hline-7.37716100 & 0.97248900 & -0.88813900 \\
\hline-5.43122800 & 0.41811100 & -2.37716800 \\
\hline-5.39205200 & -1.88958000 & -0.88796400 \\
\hline-6.72202400 & -1.50865400 & 0.21780400 \\
\hline-7.04628400 & -1.68975400 & -1.53095700 \\
\hline-7.18219900 & 2.05026700 & -0.98831600 \\
\hline-8.09312000 & 0.66779200 & -1.67193900 \\
\hline-7.86031300 & 0.80819500 & 0.09016800 \\
\hline-6.09883400 & 0.07149600 & -3.18573700 \\
\hline-5.22420000 & 1.48808700 & -2.52575100 \\
\hline-4.47763400 & -0.12694900 & -2.47235200 \\
\hline-4.80942500 & 1.96958400 & -0.01011100 \\
\hline 0.50365100 & -3.69381600 & 1.44079400 \\
\hline 0.61517800 & -2.34629200 & 1.06469100 \\
\hline 1.46092300 & -2.05935800 & -0.02096000 \\
\hline 2.26450800 & -3.01776100 & -0.64815800 \\
\hline 2.12829300 & -4.34736800 & -0.23555300 \\
\hline 1.23118500 & -4.68595800 & 0.78080000 \\
\hline 3.75047500 & -1.15861700 & -1.27284700 \\
\hline 2.81309500 & -0.26918400 & -0.72924800 \\
\hline 3.09176400 & 1.07858200 & -0.44322300 \\
\hline 4.42262600 & 1.49475100 & -0.61059300 \\
\hline 5.40155300 & 0.60945700 & -1.06515800 \\
\hline 5.06302100 & -0.70024100 & -1.42064700 \\
\hline-0.13971800 & -3.97272200 & 2.27362900 \\
\hline 2.72496000 & -5.12887300 & -0.70667500 \\
\hline 1.12427500 & -5.72928400 & 1.08571500 \\
\hline 4.69918200 & 2.52509900 & -0.38999100 \\
\hline 6.43272100 & 0.95232800 & -1.17419000 \\
\hline 5.83110300 & -1.36096500 & -1.82350500 \\
\hline 3.22475500 & -2.52461500 & -1.73391200 \\
\hline 4.35672300 & -3.52028600 & -1.99640800 \\
\hline 5.02607400 & -3.14880000 & -2.78569300 \\
\hline 4.95585000 & -3.70982000 & -1.09277500 \\
\hline 3.95460500 & -4.47951200 & -2.35199200 \\
\hline 2 & -2.31036400 & -3.0428180 \\
\hline
\end{tabular}


$2.00998600 \quad-3.26756100 \quad-3.39628400$

$\begin{array}{lll}1.57894000 & -1.62237300 & -2.89181600\end{array}$

$3.07374600-1.89584500 \quad-3.82737900$

$1.55381900 \quad-0.74722100 \quad-0.44769300$

$\begin{array}{lll}-0.07201900 & -0.90486600 & 1.99610200\end{array}$

$\begin{array}{lll}1.65991100 & 2.20036900 & -0.08681900\end{array}$

$\begin{array}{lll}-1.67685800 & -1.45842500 & 2.91604800\end{array}$

$\begin{array}{lll}-2.58042200 & -2.33890500 & 2.03460900\end{array}$

$-2.96179200 \quad-1.82089000 \quad 1.15125100$

$\begin{array}{lll}-2.10028200 & -3.28114800 & 1.73845000\end{array}$

$-3.47512900 \quad-2.59667600 \quad 2.62434300$

$\begin{array}{lll}-2.43958800 & -0.15812300 \quad 3.25125900\end{array}$

$\begin{array}{lll}-1.78483700 & 0.63414800 & 3.64236700\end{array}$

$\begin{array}{lll}-3.00811700 & 0.20812700 \quad 2.38486600\end{array}$

$\begin{array}{lll}-3.18881900 & -0.37758700 & 4.03027600\end{array}$

$\begin{array}{lll}-1.43014700 & -2.22930600 \quad 4.22312200\end{array}$

$-0.95364600 \quad-1.61104900 \quad 4.99306300$

$\begin{array}{lll}-2.41046500 & -2.53691600 & 4.62285700\end{array}$

$\begin{array}{lll}-0.83351000 & -3.14111100 \quad 4.08793200\end{array}$

$1.32858500 \quad-0.72122600 \quad 3.32368000$

$\begin{array}{lll}2.60070200 & -0.39734300 & 2.53311200\end{array}$

$\begin{array}{lll}2.98406700 & -1.28057800 & 2.00399300\end{array}$

$\begin{array}{lll}2.43234200 & 0.39130200 & 1.80095000\end{array}$

$\begin{array}{lll}3.38972700 & -0.05940800 & 3.22372400\end{array}$

$0.95264200 \quad 0.46818000 \quad 4.21947000$

$\begin{array}{lll}0.09856800 & 0.24088100 & 4.87256000\end{array}$

$\begin{array}{lll}1.80385000 & 0.73130200 \quad 4.86992000\end{array}$

$\begin{array}{lll}0.69297000 \quad 1.35719300 & 3.62556800\end{array}$

$\begin{array}{lll}1.66460500 & -1.95384200 & 4.17925700\end{array}$

$\begin{array}{lll}1.76695700 & -2.86305700 & 3.56964300\end{array}$

$2.64220700 \quad-1.78056700 \quad 4.66109500$

$\begin{array}{lll}0.94636200 & -2.14792800 & 4.97986800\end{array}$

$2.23645400 \quad 3.57733700 \quad 1.11493200$

$\begin{array}{lll}3.06214700 & 2.96440700 & 2.25543400\end{array}$

$\begin{array}{lll}2.48967100 & 2.22702700 \quad 2.82517700\end{array}$

$3.98627300 \quad 2.48618100 \quad 1.90275800$

$\begin{array}{lll}3.34598500 & 3.76862900 & 2.95457400\end{array}$

$3.04273100 \quad 4.72912100 \quad 0.50024700$

$3.30272100 \quad 5.43933000 \quad 1.30326300$

$\begin{array}{lll}3.98491600 & 4.39658000 & 0.04462800\end{array}$

$2.47252700 \quad 5.28908900 \quad-0.25139400$

$0.93899600 \quad 4.15294300 \quad 1.71226300$

$\begin{array}{lll}1.18888900 & 4.91024100 & 2.47391900\end{array}$

$0.31952200 \quad 4.64221300 \quad 0.94849700$ 
$\begin{array}{lll}0.32975100 & 3.37281500 \quad 2.19326800\end{array}$

$\begin{array}{lll}1.47807200 & 2.93280400 & -1.86509700\end{array}$

$\begin{array}{lll}2.73629900 & 3.53491400 & -2.50555200\end{array}$

$\begin{array}{lll}2.49333100 & 3.78779600 & -3.55203900\end{array}$

$\begin{array}{lll}3.07688300 & 4.45730800 & -2.02405000\end{array}$ 
$3.20060200 \quad 3.85937200 \quad 2.63630700$

$\begin{array}{lll}4.55885300 & 5.24223600 & 0.64529200\end{array}$

$3.15004200 \quad 6.20168500 \quad 1.17226400$

$\begin{array}{lll}0.75701900 \quad 3.30879100 & 1.47633900\end{array}$

$0.92308500 \quad 5.07821900 \quad 1.61899700$

$0.95225600 \quad 4.29913100 \quad 0.01657500$

$\begin{array}{lll}2.72911500 & 2.96286500 \quad 3.06258900\end{array}$

$\begin{array}{lll}4.29183900 & 3.74817100 \quad 2.74236900\end{array}$

$\begin{array}{lll}2.89366100 & 4.73169800 \quad 3.23754300\end{array}$

$\begin{array}{lll}3.81942900 & 2.95059600 & -0.73456900\end{array}$

$4.33433200 \quad-3.08264600 \quad 1.64862100$

$\begin{array}{lll}5.45217700 & -1.25157500 & 1.33674900\end{array}$

$\begin{array}{lll}5.99663700 & 1.44146600 \quad 1.28712700\end{array}$

$\begin{array}{lll}7.09981600 & 1.46203900 & 1.22124400\end{array}$

$\begin{array}{lll}5.65312400 & 2.47829500 & 1.17958200\end{array}$

$5.72437600 \quad 1.08283300 \quad 2.29150400$

$\begin{array}{lll}-5.37608000 & 0.88025900 & 0.87063300\end{array}$

$\begin{array}{lll}-4.15732700 & 0.32763800 & 0.44919300\end{array}$

$\begin{array}{lll}-3.16329000 & 1.21707200 & -0.03448500\end{array}$

$\begin{array}{lll}-3.46305800 & 2.56902100 & -0.27272400\end{array}$

$-4.69912400 \quad 3.06606400 \quad 0.16040900$

$\begin{array}{lll}-5.64050100 & 2.24406600 & 0.77015200\end{array}$

$\begin{array}{lll}-1.23794300 & 2.73924200 & -1.41387700\end{array}$

$\begin{array}{lll}-0.93061300 & 1.47258300 & -0.88558400\end{array}$

$\begin{array}{lll}0.38320300 & 0.94547500 & -0.99660800\end{array}$

$\begin{array}{lll}1.29490500 & 1.64445900 & -1.80430900\end{array}$

$\begin{array}{lll}0.96254000 & 2.83172800 & -2.44749300\end{array}$

$\begin{array}{lll}-0.28687100 & 3.38799200 & -2.21255800\end{array}$

$\begin{array}{lll}-6.14572800 & 0.22146800 & 1.26678700\end{array}$

$\begin{array}{lll}-4.93016700 \quad 4.12120500 & 0.00443300\end{array}$

$\begin{array}{lll}-6.59140300 & 2.65209300 \quad 1.11945300\end{array}$

$2.31476400 \quad 1.27166100 \quad-1.88981000$

$\begin{array}{lll}1.70373900 & 3.34714100 \quad-3.05952500\end{array}$

$\begin{array}{lll}-0.52703700 & 4.36503400 & -2.63467700\end{array}$

$\begin{array}{lll}-2.52939100 \quad 3.47469300 & -1.06552000\end{array}$

$\begin{array}{lll}-3.25944900 & 3.90522200 & -2.35965800\end{array}$

$\begin{array}{lll}-2.63449600 & 4.57665500 & -2.96661600\end{array}$

$\begin{array}{lll}-3.51521200 & 3.02713600 & -2.97214400\end{array}$

$\begin{array}{lll}-4.19068000 & 4.44227200 & -2.12627600\end{array}$

$\begin{array}{lll}-2.17006400 & 4.72545700 \quad-0.22849900\end{array}$

$\begin{array}{lll}-3.07078100 & 5.30597500 \quad 0.02199100\end{array}$

$\begin{array}{lll}-1.67497600 & 4.43782300 & 0.71014800\end{array}$

$\begin{array}{lll}-1.49017600 & 5.38879700 & -0.78311400\end{array}$ 
6-iso

C

C

$$
\begin{array}{rrr}
0.24011000 & -3.61234100 & -0.05308800 \\
1.52092400 & -1.71908000 & -2.66762200 \\
1.30928800 & -2.52933800 & -3.38500500 \\
1.27325000 & -0.76745000 & -3.16066200 \\
2.59628500 & -1.72406900 & -2.45654300 \\
-0.80195600 & -1.87593800 & -1.78406700 \\
-1.47509600 & -1.96539200 & -0.92306100 \\
-1.04696300 & -0.94315100 & -2.31045300 \\
-1.02136300 & -2.71188200 & -2.47125100 \\
1.18316900 & -0.53512500 & -0.21457900 \\
-3.92304700 & -1.50773600 & 0.33158200
\end{array}
$$




\begin{tabular}{|c|c|c|c|}
\hline $\mathrm{H}$ & 6.30407500 & -0.19508700 & 1.10136200 \\
\hline $\mathrm{H}$ & 5.21035500 & -2.04562100 & 1.58525600 \\
\hline $\mathrm{C}$ & 3.32740100 & -3.81990900 & 2.36667400 \\
\hline $\mathrm{H}$ & 4.04089900 & -4.65965500 & 2.46427800 \\
\hline $\mathrm{H}$ & 2.32835800 & -4.23065300 & 2.56111400 \\
\hline $\mathrm{H}$ & 3.55837500 & -3.10473400 & 3.17261000 \\
\hline $\mathrm{C}$ & -5.33637600 & 1.70939400 & -1.20043100 \\
\hline $\mathrm{C}$ & -4.33279400 & 0.89081700 & -0.66054900 \\
\hline $\mathrm{C}$ & -3.17131500 & 1.52546500 & -0.14910700 \\
\hline $\mathrm{C}$ & -3.11012000 & 2.92361700 & -0.02197900 \\
\hline $\mathrm{C}$ & -4.14353400 & 3.69091800 & -0.57499100 \\
\hline $\mathrm{C}$ & -5.23639400 & 3.09849900 & -1.19728400 \\
\hline $\mathrm{C}$ & -1.01786600 & 2.59228300 & 1.31539100 \\
\hline $\mathrm{C}$ & -1.02579300 & 1.24896000 & 0.89651900 \\
\hline $\mathrm{C}$ & 0.07863000 & 0.40398400 & 1.17374800 \\
\hline $\mathrm{C}$ & 1.06990500 & 0.89258300 & 2.04189600 \\
\hline $\mathrm{C}$ & 1.01158100 & 2.16881800 & 2.58933300 \\
\hline $\mathrm{C}$ & -0.00674800 & 3.02197800 & 2.18378500 \\
\hline $\mathrm{H}$ & -6.22837700 & 1.24788500 & -1.61759800 \\
\hline $\mathrm{H}$ & -4.08906000 & 4.77875900 & -0.50916100 \\
\hline $\mathrm{H}$ & -6.02452800 & 3.71209700 & -1.63889400 \\
\hline $\mathrm{H}$ & 1.94021900 & 0.27141500 & 2.25007600 \\
\hline $\mathrm{H}$ & 1.80083300 & 2.51262800 & 3.25956000 \\
\hline $\mathrm{H}$ & -0.00329700 & 4.05851300 & 2.52346000 \\
\hline $\mathrm{C}$ & -1.98902000 & 3.61915000 & 0.74033500 \\
\hline $\mathrm{C}$ & -2.61082700 & 4.46717100 & 1.87336800 \\
\hline $\mathrm{H}$ & -1.83864900 & 5.01499900 & 2.43237500 \\
\hline $\mathrm{H}$ & -3.16162500 & 3.82978100 & 2.58167800 \\
\hline $\mathrm{H}$ & -3.30944400 & 5.21512400 & 1.47166100 \\
\hline $\mathrm{C}$ & -1.19670400 & 4.53704400 & -0.22243600 \\
\hline $\mathrm{H}$ & -1.85260000 & 5.30085600 & -0.66801300 \\
\hline $\mathrm{H}$ & -0.74778500 & 3.95126900 & -1.03796600 \\
\hline $\mathrm{H}$ & -0.38528000 & 5.05594700 & 0.30957900 \\
\hline $\mathrm{O}$ & -2.10391100 & 0.73005000 & 0.21661300 \\
\hline $\mathrm{C}$ & -5.36112100 & -1.52433300 & -2.12172200 \\
\hline $\mathrm{C}$ & -6.86846100 & -1.34023700 & -2.33691700 \\
\hline $\mathrm{H}$ & -7.17989600 & -0.28718100 & -2.31998300 \\
\hline $\mathrm{H}$ & -7.46396900 & -1.88432800 & -1.59064300 \\
\hline $\mathrm{H}$ & -7.14440500 & -1.74210200 & -3.32796800 \\
\hline $\mathrm{C}$ & -4.59481700 & -0.83140200 & -3.26166900 \\
\hline $\mathrm{H}$ & -4.87776800 & 0.22417100 & -3.37319100 \\
\hline $\mathrm{H}$ & -4.81617400 & -1.34098200 & -4.21552400 \\
\hline $\mathrm{H}$ & -3.50639500 & -0.86947200 & -3.10888400 \\
\hline $\mathrm{C}$ & -5.04507100 & -3.03332400 & -2.17304900 \\
\hline
\end{tabular}




\begin{tabular}{|c|c|c|c|}
\hline $\mathrm{H}$ & -3.96379500 & -3.22091000 & -2.09996900 \\
\hline $\mathrm{H}$ & -5.40186200 & -3.45999200 & -3.12667200 \\
\hline $\mathrm{H}$ & -5.53590400 & -3.58637200 & -1.35847100 \\
\hline $\mathrm{C}$ & -5.90221100 & -0.95602900 & 0.93639600 \\
\hline C & -5.14206500 & -0.49008000 & 2.19155300 \\
\hline $\mathrm{H}$ & -4.27041500 & -1.12400500 & 2.40239800 \\
\hline $\mathrm{H}$ & -5.81256300 & -0.53494600 & 3.06709900 \\
\hline $\mathrm{H}$ & -4.79437900 & 0.55045400 & 2.09638100 \\
\hline C & -6.32459000 & -2.42153700 & 1.13110600 \\
\hline $\mathrm{H}$ & -6.94016000 & -2.79169200 & 0.29790600 \\
\hline $\mathrm{H}$ & -6.92686600 & -2.51743200 & 2.05105100 \\
\hline $\mathrm{H}$ & -5.45172300 & -3.08590200 & 1.23320700 \\
\hline C & -7.14322000 & -0.07000100 & 0.77920600 \\
\hline $\mathrm{H}$ & -7.74989900 & -0.32448400 & -0.09863800 \\
\hline $\mathrm{H}$ & -6.87809700 & 0.99468700 & 0.72027200 \\
\hline $\mathrm{H}$ & -7.78824400 & -0.19319700 & 1.66746300 \\
\hline $\mathrm{C}$ & 0.04183500 & -1.44444100 & -1.32995500 \\
\hline $\mathrm{C}$ & 1.06885700 & -2.40981500 & -1.95378800 \\
\hline $\mathrm{H}$ & 1.06929000 & -3.39465300 & -1.46396300 \\
\hline $\mathrm{H}$ & 2.08864300 & -1.99994700 & -1.90772800 \\
\hline $\mathrm{H}$ & 0.81464800 & -2.56524200 & -3.01588700 \\
\hline $\mathrm{C}$ & 0.18585700 & -0.06986100 & -2.00536400 \\
\hline $\mathrm{H}$ & 0.10141500 & -0.20559700 & -3.09713300 \\
\hline $\mathrm{H}$ & 1.15919200 & 0.38623600 & -1.78274300 \\
\hline $\mathrm{H}$ & -0.61239700 & 0.61654600 & -1.69570500 \\
\hline $\mathrm{C}$ & -1.36207500 & -1.99000800 & -1.57553800 \\
\hline $\mathrm{H}$ & -1.54686500 & -2.00135500 & -2.66282500 \\
\hline $\mathrm{H}$ & -2.13519400 & -1.37504200 & -1.10568300 \\
\hline $\mathrm{H}$ & -1.48470600 & -3.02201000 & -1.21915700 \\
\hline $\mathrm{C}$ & -0.36453800 & -2.46596100 & 1.64763200 \\
\hline $\mathrm{C}$ & -0.13317800 & -3.90235700 & 1.15653600 \\
\hline $\mathrm{H}$ & -0.48706900 & -4.61335800 & 1.92178700 \\
\hline $\mathrm{H}$ & 0.92721700 & -4.11081200 & 0.96905900 \\
\hline $\mathrm{H}$ & -0.68964700 & -4.11335100 & 0.23354200 \\
\hline $\mathrm{C}$ & 0.27481200 & -2.26212400 & 3.03405400 \\
\hline $\mathrm{H}$ & -0.07147700 & -3.05644600 & 3.71618100 \\
\hline $\mathrm{H}$ & -0.02007200 & -1.29698700 & 3.46992300 \\
\hline $\mathrm{H}$ & 1.36959700 & -2.29479900 & 2.99555200 \\
\hline $\mathrm{C}$ & -1.86211500 & -2.17635200 & 1.76825400 \\
\hline $\mathrm{H}$ & -2.41300800 & -2.30221100 & 0.83056300 \\
\hline $\mathrm{H}$ & -2.04010100 & -1.15029400 & 2.11675100 \\
\hline $\mathrm{H}$ & -2.30066300 & -2.86255800 & 2.51377700 \\
\hline $\mathrm{P}$ & 0.54253800 & -1.26116500 & 0.50393000 \\
\hline $\mathrm{P}$ & -4.58508200 & -0.93499400 & -0.46127700 \\
\hline
\end{tabular}




\begin{tabular}{|c|c|c|}
\hline 3.06970700 & 3.20832000 & -1.09572800 \\
\hline 2.14681100 & 2.23111700 & -0.68846400 \\
\hline 2.21650200 & 0.99628700 & -1.35678700 \\
\hline 2.93051700 & 0.79872500 & -2.54275400 \\
\hline 3.82362700 & 1.80646100 & -2.92375200 \\
\hline 3.93427700 & 2.97887100 & -2.16879500 \\
\hline 2.35503500 & -1.59643000 & -2.46335600 \\
\hline 1.86936300 & -1.35955900 & -1.17058000 \\
\hline 1.80431000 & -2.35633600 & -0.17827200 \\
\hline 2.07061200 & -3.66933500 & -0.59903500 \\
\hline 2.45162200 & -3.95472400 & -1.91026100 \\
\hline 2.63662900 & -2.91810200 & -2.82482300 \\
\hline 3.09062000 & 4.17344200 & -0.59300900 \\
\hline 4.42260600 & 1.69039200 & -3.82746200 \\
\hline 4.65054000 & 3.74893000 & -2.46365400 \\
\hline 2.01629100 & -4.48494000 & 0.11851500 \\
\hline 2.64906900 & -4.98721600 & -2.20694000 \\
\hline 2.99657800 & -3.14227600 & -3.82887700 \\
\hline 2.52156800 & -0.40228500 & -3.40062500 \\
\hline 1.13813700 & -0.07191900 & -4.02321000 \\
\hline 0.78288100 & -0.91789000 & -4.63145800 \\
\hline 0.38656900 & 0.12642000 & -3.24579600 \\
\hline 1.20797700 & 0.82036900 & -4.66447800 \\
\hline 3.52425300 & -0.67822600 & -4.52345500 \\
\hline 3.62305800 & 0.20150900 & -5.17522700 \\
\hline 4.51984100 & -0.93427400 & -4.13030300 \\
\hline 3.18110800 & -1.50384400 & -5.16271100 \\
\hline 1.47674200 & -0.06164700 & -0.84296900 \\
\hline 0.99040100 & 3.14137900 & 1.98097700 \\
\hline 2.15294800 & 4.13944700 & 2.02716800 \\
\hline 3.09158100 & 3.68198100 & 1.68229400 \\
\hline 1.96617800 & 5.04293200 & 1.43207000 \\
\hline 2.31163200 & 4.46127300 & 3.07119200 \\
\hline 1.37368500 & 1.89779900 & 2.79704500 \\
\hline 1.61863600 & 2.19711800 & 3.83061700 \\
\hline 0.55523800 & 1.16758900 & 2.83347200 \\
\hline 2.25448400 & 1.39749200 & 2.37724800 \\
\hline-0.27265000 & 3.75415900 & 2.60415600 \\
\hline-1.13801100 & 3.08034400 & 2.52308800 \\
\hline-0.09315300 & 3.93203500 & 3.67776100 \\
\hline-0.53420400 & 4.72006700 & 2.15027100 \\
\hline-0.27256200 & 3.84754400 & -0.81618700 \\
\hline .33 & 5.25124100 & 0.7174 \\
\hline
\end{tabular}




\begin{tabular}{|c|c|c|}
\hline-0.20768600 & 5.92479200 & -1.40453700 \\
\hline 0.23590000 & 5.67505600 & 0.29188200 \\
\hline 1.39175000 & 5.27987600 & -1.00910400 \\
\hline-0.19621800 & 3.34783300 & -2.27012100 \\
\hline-0.56266200 & 2.31254800 & -2.35125200 \\
\hline-0.83794900 & 3.98069200 & -2.90548200 \\
\hline 0.82397400 & 3.38643500 & -2.67630400 \\
\hline-1.75913200 & 3.91051900 & -0.42555300 \\
\hline-1.91931200 & 4.25099600 & 0.60429800 \\
\hline-2.27502300 & 4.61947300 & -1.09501500 \\
\hline-2.23613500 & 2.93117300 & -0.53911700 \\
\hline 0.93072100 & -3.32500100 & 2.56884900 \\
\hline 1.84189100 & -4.52783900 & 2.84799200 \\
\hline 2.25056300 & -4.99447100 & 1.94248400 \\
\hline 2.68308400 & -4.26156500 & 3.50190900 \\
\hline 1.25738000 & -5.30172500 & 3.37593000 \\
\hline 0.49168800 & -2.74690100 & 3.93099800 \\
\hline 1.34247900 & -2.39888200 & 4.53264900 \\
\hline-0.20048300 & -1.90336000 & 3.81159400 \\
\hline-0.02695000 & -3.52848900 & 4.51218500 \\
\hline-0.33453500 & -3.79547000 & 1.83214300 \\
\hline-0.85329700 & -4.55145100 & 2.44585700 \\
\hline-1.03920300 & -2.96921300 & 1.66810200 \\
\hline-0.11737200 & -4.24936400 & 0.85569900 \\
\hline 3.51453400 & -1.63538900 & 1.98282100 \\
\hline 4.41485900 & -2.83336500 & 1.65815500 \\
\hline 5.46794900 & -2.54765500 & 1.83008000 \\
\hline 4.21132700 & -3.70822700 & 2.28515700 \\
\hline 4.33063500 & -3.13576100 & 0.60473000 \\
\hline 3.63908300 & -1.27680100 & 3.47198600 \\
\hline 3.40370600 & -2.13257000 & 4.12090000 \\
\hline 4.67642800 & -0.97409500 & 3.69615900 \\
\hline 2.97806000 & -0.44235300 & 3.75217700 \\
\hline 4.00941900 & -0.44947500 & 1.13547100 \\
\hline 4.08495900 & -0.71411100 & 0.07150600 \\
\hline 3.36317800 & 0.43280000 & 1.21569300 \\
\hline 5.01997700 & -0.15790400 & 1.46861900 \\
\hline-1.73819700 & -0.82496100 & -0.25603600 \\
\hline-2.24141300 & 0.42816100 & 0.25309100 \\
\hline-2.57424100 & 0.68528400 & 1.66386600 \\
\hline-1.94675400 & -0.00454900 & 2.72277700 \\
\hline-4.01298800 & 2.23318300 & 1.21007200 \\
\hline 2.82892400 & 1.03411400 & -0.44558500 \\
\hline .50693900 & 1.68510300 & 2.00910700 \\
\hline
\end{tabular}




\begin{tabular}{|c|c|c|}
\hline-2.23065000 & 0.29267900 & 4.05375000 \\
\hline-3.15325000 & 1.29571000 & 4.37746200 \\
\hline-3.79062900 & 1.98761400 & 3.34322600 \\
\hline-1.71816200 & -0.25504500 & 4.84987200 \\
\hline-3.37170500 & 1.53301700 & 5.42159000 \\
\hline-4.51735600 & 2.77096900 & 3.57637400 \\
\hline-2.15599500 & -1.26517400 & -1.63024300 \\
\hline-3.56431600 & -1.71588100 & -1.58141200 \\
\hline-4.53192100 & -0.85038600 & -1.88239200 \\
\hline-5.91543000 & -1.40742400 & -1.51945200 \\
\hline-4.34409700 & 0.25023100 & -2.35861000 \\
\hline-6.09052800 & -2.81074500 & -2.12422500 \\
\hline-5.98596900 & -1.48597600 & 0.01921800 \\
\hline-6.99623200 & -0.46055600 & -2.04970800 \\
\hline-6.01477800 & -2.78356300 & -3.22358900 \\
\hline-5.32625900 & -3.50447000 & -1.74716200 \\
\hline-7.08325900 & -3.21243500 & -1.86398200 \\
\hline-5.84396700 & -0.49391400 & 0.47612700 \\
\hline-6.97093600 & -1.86835600 & 0.33299700 \\
\hline-5.20946000 & -2.15405800 & 0.41879800 \\
\hline-7.99533300 & -0.83586900 & -1.77644600 \\
\hline-6.87915500 & 0.55027900 & -1.63308700 \\
\hline-6.94954000 & -0.37402000 & -3.14621000 \\
\hline-1.19715300 & -0.75811200 & 2.48190500 \\
\hline-0.34375300 & 0.50662100 & -0.03888200 \\
\hline 0.56071700 & 2.48735300 & 0.25047500 \\
\hline 1.62765400 & -1.82087200 & 1.59194100 \\
\hline-1.66981200 & -1.66764900 & 0.44303800 \\
\hline-2.13067000 & -0.42148400 & -2.33267900 \\
\hline-1.37680700 & -2.44520000 & -2.17821600 \\
\hline-1.76815300 & -2.75572500 & -3.15959000 \\
\hline-1.44011000 & -3.30487400 & -1.49269700 \\
\hline-0.31792900 & -2.18305900 & -2.29148000 \\
\hline-1.86142300 & -0.13631300 & 0.41834700 \\
\hline-2.47120600 & 0.90705400 & 1.03675500 \\
\hline-2.10605400 & -1.39393000 & 0.87467600 \\
\hline-3.73721900 & 0.71587800 & 1.60656400 \\
\hline-1.77844000 & 2.12633200 & 1.00391000 \\
\hline-3.39083400 & -1.71479200 & 1.33735800 \\
\hline-1.00860800 & -2.26170200 & 0.93061400 \\
\hline-4.44872500 & -0.60925800 & 1.30403600 \\
\hline-4.30081300 & 1.79586900 & 2.29140200 \\
\hline 4020 & 3.18539100 & 1.68325800 \\
\hline
\end{tabular}




\begin{tabular}{|c|c|c|}
\hline-0.30286700 & 2.22269100 & -0.17267100 \\
\hline-3.60891000 & -3.01869800 & 1.78891300 \\
\hline-1.27838800 & -3.55946900 & 1.39763200 \\
\hline 0.70617600 & -1.54536500 & 0.71768400 \\
\hline-5.59435700 & -0.88379200 & 2.28253100 \\
\hline-5.02262200 & -0.52153200 & -0.13356400 \\
\hline-3.62346000 & 3.01569600 & 2.34281300 \\
\hline-5.27842600 & 1.69933100 & 2.76514800 \\
\hline-1.95530000 & 4.16979800 & 1.69645000 \\
\hline 0.84284300 & 0.28570300 & -0.67828600 \\
\hline-2.56079000 & -3.94155800 & 1.79403200 \\
\hline-4.59382600 & -3.31806000 & 2.14868900 \\
\hline-0.47619900 & -4.28119800 & 1.50381200 \\
\hline-6.34809800 & -0.08496900 & 2.23261100 \\
\hline-5.23628400 & -0.95882600 & 3.32058900 \\
\hline-6.11180900 & -1.81914200 & 2.02557700 \\
\hline-5.51667800 & -1.46731100 & -0.40631500 \\
\hline-5.75801000 & 0.29502500 & -0.20544300 \\
\hline-4.22821600 & -0.32995500 & -0.86756700 \\
\hline-4.06683900 & 3.86184100 & 2.87286100 \\
\hline-2.73288500 & -4.96033200 & 2.14829200 \\
\hline 0.75781800 & 3.69790200 & 0.56187200 \\
\hline 1.53340400 & 3.07901100 & 1.73723600 \\
\hline 0.12281700 & 4.98157100 & 1.14172900 \\
\hline 1.69848300 & 4.18547500 & -0.56042500 \\
\hline 0.86639000 & 2.92850400 & 2.59761300 \\
\hline 2.33833800 & 3.76203000 & 2.05816900 \\
\hline 1.97204300 & 2.10976600 & 1.49432600 \\
\hline-0.30424400 & 4.82267300 & 2.13847800 \\
\hline 0.93563100 & 5.71593800 & 1.27704500 \\
\hline-0.62661900 & 5.45111100 & 0.49944600 \\
\hline 1.16226600 & 4.84134700 & -1.26083200 \\
\hline 2.51821900 & 4.77769900 & -0.12004600 \\
\hline 2.16224300 & 3.39190900 & -1.15287800 \\
\hline 1.76507300 & -3.16292600 & 0.34101700 \\
\hline 3.05365700 & -2.66465300 & 0.30762100 \\
\hline 1.07300700 & -4.08471400 & -0.67828800 \\
\hline 2.17180000 & -4.00634500 & 1.56007300 \\
\hline 3.57777000 & -1.94850700 & 0.33194900 \\
\hline 3.73539300 & -3.51455800 & -0.48050300 \\
\hline 2.83001400 & -2.20447100 & -1.27405800 \\
\hline 0.17540000 & -4.58252000 & -0.29752800 \\
\hline 1.79269100 & -4.87752600 & -0.94764700 \\
\hline 0.82604800 & -3.54504800 & -1.59689100 \\
\hline
\end{tabular}




\begin{tabular}{|c|c|c|c|}
\hline $\mathrm{H}$ & 2.79470100 & -3.44945200 & 2.27064200 \\
\hline $\mathrm{H}$ & 2.78266500 & -4.84690300 & 1.18955400 \\
\hline $\mathrm{H}$ & 1.32840600 & -4.44280200 & 2.10776800 \\
\hline $\mathrm{C}$ & -1.38519400 & 2.98573800 & -1.63714700 \\
\hline $\mathrm{C}$ & -0.63772000 & 2.92660100 & -2.97156500 \\
\hline $\mathrm{C}$ & -1.84791200 & 4.42915600 & -1.41674900 \\
\hline $\mathrm{C}$ & -2.63288300 & 2.09727900 & -1.78353500 \\
\hline $\mathrm{H}$ & 0.30589100 & 3.48872100 & -2.96885900 \\
\hline $\mathrm{H}$ & -1.27623500 & 3.37299000 & -3.75267800 \\
\hline $\mathrm{H}$ & -0.44131600 & 1.89129100 & -3.25685300 \\
\hline $\mathrm{H}$ & -1.02178900 & 5.15098000 & -1.47024400 \\
\hline $\mathrm{H}$ & -2.55323100 & 4.68883600 & -2.22557800 \\
\hline $\mathrm{H}$ & -2.38660100 & 4.56764200 & -0.47070100 \\
\hline $\mathrm{H}$ & -3.38276900 & 2.30379800 & -1.00999700 \\
\hline $\mathrm{H}$ & -3.10351900 & 2.30239900 & -2.75968200 \\
\hline $\mathrm{H}$ & -2.36246300 & 1.03750500 & -1.75409900 \\
\hline $\mathrm{C}$ & 0.97933600 & -1.11635000 & 2.60514900 \\
\hline $\mathrm{C}$ & 2.42984600 & -0.66362300 & 2.78073300 \\
\hline $\mathrm{C}$ & 0.05721900 & 0.07050300 & 2.91098800 \\
\hline $\mathrm{C}$ & 0.63724100 & -2.19933100 & 3.63936800 \\
\hline $\mathrm{H}$ & 3.16215200 & -1.44942000 & 2.55156500 \\
\hline $\mathrm{H}$ & 2.59875400 & -0.34863700 & 3.82458000 \\
\hline $\mathrm{H}$ & 2.65131000 & 0.19012500 & 2.13783600 \\
\hline $\mathrm{H}$ & -0.99507100 & -0.23531000 & 2.98181400 \\
\hline $\mathrm{H}$ & 0.33620100 & 0.50458600 & 3.88549900 \\
\hline $\mathrm{H}$ & 0.13570300 & 0.85640000 & 2.15833400 \\
\hline $\mathrm{H}$ & -0.38375300 & -2.58476900 & 3.52068400 \\
\hline $\mathrm{H}$ & 0.68988000 & -1.73488200 & 4.63923000 \\
\hline $\mathrm{H}$ & 1.33192500 & -3.04307500 & 3.64416900 \\
\hline $\mathrm{C}$ & 2.73115400 & 0.39960100 & -1.74947100 \\
\hline $\mathrm{C}$ & 1.85590700 & 1.04669000 & -2.59724800 \\
\hline $\mathrm{C}$ & 3.04300700 & 0.92275000 & -0.47355200 \\
\hline $\mathrm{H}$ & 3.15050000 & -0.55116300 & -2.07267800 \\
\hline $\mathrm{C}$ & 1.59623700 & 0.59441700 & -4.00098000 \\
\hline $\mathrm{H}$ & 1.64777400 & 2.09284200 & -2.39820500 \\
\hline $\mathrm{C}$ & 4.30074900 & 0.58716800 & 0.22684600 \\
\hline $\mathrm{H}$ & 2.76592600 & 1.95654800 & -0.30738600 \\
\hline $\mathrm{H}$ & 0.52389200 & 0.50934800 & -4.23171600 \\
\hline $\mathrm{H}$ & 2.00679200 & 1.33976100 & -4.70607200 \\
\hline $\mathrm{H}$ & 2.04681300 & -0.38421000 & -4.20126800 \\
\hline $\mathrm{C}$ & 5.22828500 & -0.35351900 & -0.27167600 \\
\hline $\mathrm{C}$ & 4.68579600 & 1.30548300 & 1.38029500 \\
\hline $\mathrm{C}$ & 6.43657700 & -0.60372800 & 0.37830400 \\
\hline $\mathrm{H}$ & 5.01325300 & -0.89067000 & -1.19475900 \\
\hline
\end{tabular}




\begin{tabular}{rrr}
5.89110900 & 1.05615900 & 2.03415500 \\
4.02280200 & 2.07507400 & 1.77373300 \\
6.77345100 & 0.08835100 & 1.54507800 \\
7.12624700 & -1.34198600 & -0.03924300 \\
\hline 6.14542100 & 1.62813300 & 2.93035900 \\
7.71958900 & -0.10950800 & 2.05457300 \\
-0.25556900 & -1.40437200 & -2.63466700 \\
-1.46599200 & -1.95693300 & -3.43800000 \\
-0.50486400 & -0.42967700 & -1.83190400 \\
\hline 0.84402200 & -1.94822700 & -2.77047400 \\
\hline-2.54159700 & -0.89541500 & -3.68080700 \\
\hline-0.96337900 & -2.49972100 & -4.78280800 \\
\hline-2.06811800 & -3.10661500 & -2.60640600 \\
\hline-2.13168400 & -0.00462200 & -4.18118300 \\
\hline-3.33967000 & -1.30616700 & -4.32142400 \\
-3.00030000 & -0.57016400 & -2.73981100 \\
-0.54556800 & -1.69237900 & -5.40638500 \\
-1.79085700 & -2.96498000 & -5.34361600 \\
-0.17210600 & -3.24622600 & -4.63367400 \\
\hline-3.39310900 & -2.75439700 & -1.61617500 \\
\hline & -3.53243500 & -3.12253500 \\
\hline & -3.91580600 & -2.45479100 \\
\hline
\end{tabular}

6-b

\begin{tabular}{lrr}
-1.55858600 & -0.15445400 & 0.67520100 \\
-2.55222700 & 0.74008100 & 1.02191300 \\
-1.55738700 & -1.40599900 & 1.25568600 \\
-3.76692500 & 0.25471300 & 1.54412800 \\
-2.34403800 & 2.12280900 & 0.81252500 \\
-2.77945700 & -1.98032100 & 1.65987800 \\
-0.33063400 & -2.07982800 & 1.46118000 \\
-4.06728000 & -1.22755600 & 1.35435500 \\
-4.67827100 & 1.16483900 & 2.08587200 \\
\hline-3.29819800 & 2.99691000 & 1.36210400 \\
-1.02370800 & 2.69017600 & -0.36236400 \\
\hline-2.77941000 & -3.23785800 & 2.26877100 \\
\hline-0.39035900 & -3.34173000 & 2.07176400 \\
\hline 1.32292400 & -1.34649700 & 1.06231900 \\
\hline-5.24619800 & -1.69745900 & 2.21206500 \\
-4.42253000 & -1.45865800 & -0.13714500 \\
-4.42430700 & 2.53509500 & 2.03835900 \\
\hline-5.60682200 & 0.80519600 & 2.52859300 \\
-3.17085500 & 4.06850700 & 1.23510900 \\
1.30190200 & -0.42083000 & -1.00351000 \\
-1.58820400 & -3.92474100 & 2.47055900 \\
\hline & & \\
\hline
\end{tabular}


$\begin{array}{lll}0.52580000 & -3.88630100 & 2.26223200\end{array}$

$-6.15592100 \quad-1.13715000 \quad 1.95417100$

$\begin{array}{lll}-5.05176300 \quad-1.57050900 & 3.28785800\end{array}$

$\begin{array}{lll}-5.47082000 & -2.75606900 & 2.02209300\end{array}$

$\begin{array}{lll}-4.63230600 & -2.52392700 & -0.31906800\end{array}$

$\begin{array}{lll}-5.30983300 & -0.86943900 & -0.41755700\end{array}$

$\begin{array}{lll}-3.59326400 & -1.16640200 & -0.79311400\end{array}$

$\begin{array}{lll}-5.13355600 \quad 3.24364300 & 2.47189000\end{array}$

$\begin{array}{lll}-1.58687900 & -4.90944400 & 2.94256200\end{array}$

$\begin{array}{lll}-0.54894700 & 4.48190100 & 0.16025800\end{array}$

$\begin{array}{lll}-0.23990700 & 4.44293500 & 1.66611500\end{array}$

$\begin{array}{lll}-1.51173600 & 5.63750400 & -0.14664100\end{array}$

$0.77331900 \quad 4.77143800 \quad-0.58133500$

$\begin{array}{lll}-1.13802400 & 4.29527200 & 2.28124300\end{array}$

$\begin{array}{lll}0.22442000 & 5.39648300 & 1.97163000\end{array}$

$\begin{array}{lll}0.46761600 \quad 3.63752400 & 1.90974500\end{array}$

$\begin{array}{lll}-2.49301300 & 5.53759900 & 0.33444000\end{array}$

$\begin{array}{lll}-1.06836200 & 6.57983800 & 0.22071500\end{array}$

$-1.67479900 \quad 5.76001400 \quad-1.22593400$

$\begin{array}{lll}0.64523400 & 4.80228700 & -1.67220100\end{array}$

$\begin{array}{lll}1.16609400 & 5.75367500 & -0.26698400\end{array}$

$\begin{array}{lll}1.54243900 & 4.01914600 & -0.35996900\end{array}$

$\begin{array}{lll}2.59306000 & -2.79404800 & 0.97915600\end{array}$

$\begin{array}{lll}3.84764500 & -2.18498900 & 0.32655700\end{array}$

$\begin{array}{lll}2.06889600 & -3.86467700 & 0.00628700\end{array}$

$\begin{array}{lll}3.01759700 & -3.44741000 & 2.30581400\end{array}$

$\begin{array}{lll}4.33506400 & -1.43055900 & 0.95326700\end{array}$

$\begin{array}{lll}4.57947300 & -2.98726400 & 0.13544800\end{array}$

$3.59591600 \quad-1.72034500 \quad-0.63572300$

$\begin{array}{lll}1.12407100 & -4.32393800 & 0.32041600\end{array}$

$\begin{array}{lll}2.82165700 & -4.66866000 & -0.06023800\end{array}$

$\begin{array}{lll}1.93419700 & -3.45817100 & -1.00313500\end{array}$

$\begin{array}{lll}3.45390300 & -2.73145400 \quad 3.01171900\end{array}$

$\begin{array}{lll}3.79665200 & -4.19577000 & 2.08242500\end{array}$

$2.21169700 \quad-3.97914000 \quad 2.82683100$

$\begin{array}{lll}-2.10331900 & 2.75464100 & -1.96058900\end{array}$

$\begin{array}{lll}-1.27353400 & 3.41100500 & -3.07587000\end{array}$

$-3.45949200 \quad 3.46310600 \quad-1.85831100$

$\begin{array}{lll}-2.36871000 & 1.27545900 & -2.30789700\end{array}$

$\begin{array}{lll}-1.16381600 & 4.49353600 & -2.91992600\end{array}$

$\begin{array}{lll}-1.78107000 & 3.26972000 & -4.04541300\end{array}$

$\begin{array}{lll}-0.26664500 & 2.97860600 & -3.16356400\end{array}$

$\begin{array}{lll}-3.37007000 \quad 4.53600400 & -1.64754800\end{array}$ 


\begin{tabular}{|c|c|c|c|}
\hline $\mathrm{H}$ & -3.98955000 & 3.36228800 & -2.82218600 \\
\hline $\mathrm{H}$ & -4.09953200 & 3.01233900 & -1.08778300 \\
\hline $\mathrm{H}$ & -3.12965800 & 0.84813800 & -1.64035600 \\
\hline $\mathrm{H}$ & -2.75848300 & 1.19790000 & -3.33705700 \\
\hline $\mathrm{H}$ & -1.47670800 & 0.64096700 & -2.22839300 \\
\hline $\mathrm{C}$ & 1.63845100 & -0.31860700 & 2.64782500 \\
\hline $\mathrm{C}$ & 3.13174000 & -0.00395800 & 2.81470400 \\
\hline $\mathrm{C}$ & 0.87944400 & 1.00223000 & 2.45284300 \\
\hline $\mathrm{C}$ & 1.11430200 & -1.00731500 & 3.91609500 \\
\hline $\mathrm{H}$ & 3.71259600 & -0.87084100 & 3.15218700 \\
\hline $\mathrm{H}$ & 3.24672700 & 0.78086000 & 3.58052300 \\
\hline $\mathrm{H}$ & 3.58045500 & 0.37700700 & 1.89389000 \\
\hline $\mathrm{H}$ & -0.20740700 & 0.85878800 & 2.43087400 \\
\hline $\mathrm{H}$ & 1.10828000 & 1.67924400 & 3.29254200 \\
\hline $\mathrm{H}$ & 1.16630300 & 1.49668000 & 1.51721700 \\
\hline $\mathrm{H}$ & 0.02311900 & -1.13276800 & 3.89727400 \\
\hline $\mathrm{H}$ & 1.36235900 & -0.37714600 & 4.78755200 \\
\hline $\mathrm{H}$ & 1.56719300 & -1.99356700 & 4.08413500 \\
\hline $\mathrm{C}$ & 2.47775200 & 0.69263500 & -2.25465200 \\
\hline $\mathrm{C}$ & 1.21516900 & 0.58652300 & -2.82704800 \\
\hline $\mathrm{C}$ & 2.58672100 & 1.20444100 & -0.93429400 \\
\hline $\mathrm{H}$ & 3.33400500 & 0.20913800 & -2.73849000 \\
\hline $\mathrm{C}$ & 0.93714300 & -0.00062400 & -4.17441400 \\
\hline $\mathrm{H}$ & 0.44917700 & 1.25649300 & -2.42895000 \\
\hline $\mathrm{C}$ & 3.88257200 & 1.46773600 & -0.28447300 \\
\hline $\mathrm{H}$ & 1.80067200 & 1.90803300 & -0.64087200 \\
\hline $\mathrm{H}$ & -0.07291300 & -0.43248300 & -4.21703200 \\
\hline $\mathrm{H}$ & 0.97936100 & 0.78922000 & -4.94713000 \\
\hline $\mathrm{H}$ & 1.65118500 & -0.79270500 & -4.43301500 \\
\hline $\mathrm{C}$ & 5.06324100 & 0.76835100 & -0.60253500 \\
\hline $\mathrm{C}$ & 3.97131400 & 2.49222600 & 0.67912400 \\
\hline $\mathrm{C}$ & 6.26905700 & 1.06565600 & 0.03234100 \\
\hline $\mathrm{H}$ & 5.03730100 & -0.02877000 & -1.34687300 \\
\hline $\mathrm{C}$ & 5.17675200 & 2.79425000 & 1.31233600 \\
\hline $\mathrm{H}$ & 3.07053100 & 3.05143000 & 0.94016500 \\
\hline $\mathrm{C}$ & 6.33406500 & 2.07652100 & 0.99777400 \\
\hline $\mathrm{H}$ & 7.16800800 & 0.50080500 & -0.22804700 \\
\hline $\mathrm{H}$ & 5.21133700 & 3.59273100 & 2.05795100 \\
\hline $\mathrm{H}$ & 7.27998300 & 2.30570200 & 1.49436000 \\
\hline $\mathrm{C}$ & 0.09567000 & -2.53280400 & -2.40245100 \\
\hline $\mathrm{C}$ & -1.18200200 & -3.33043700 & -2.76731100 \\
\hline $\mathrm{O}$ & -0.09859700 & -1.61159200 & -1.51954600 \\
\hline $\mathrm{O}$ & 1.18294000 & -2.80938600 & -2.91338800 \\
\hline $\mathrm{C}$ & -2.25382400 & -2.35236400 & -3.27365400 \\
\hline
\end{tabular}


$\begin{array}{lll}-1.93611700 & -1.85936700 & -4.20673400\end{array}$

$-3.19893300 \quad-2.88200300 \quad-3.48027300$

$\begin{array}{lll}-2.44642000 & -1.57138600 & -2.52791300\end{array}$

$-0.48419600 \quad-3.88615300 \quad-4.76161800$

$\begin{array}{lll}-1.76519900 & -4.94451500 & -4.10704200\end{array}$

$\begin{array}{lll}-0.08716500 & -5.07009200 & -3.50563300\end{array}$

$\begin{array}{lll}-1.89741600 & -3.30284400 & -0.70101400\end{array}$

$\begin{array}{lll}-2.60721100 & -4.60012800 & -1.70236800\end{array}$

$-0.93467100 \quad-4.73956800 \quad-1.10782300$

$\begin{array}{lll}-0.77527100 & 0.99833900 & 2.44510200\end{array}$

$\begin{array}{lll}-0.51081900 & 2.32716500 & 2.04029100\end{array}$

$\begin{array}{lll}-1.52296900 \quad 3.26826800 & 1.56116500\end{array}$

$\begin{array}{lll}-2.73083000 & 2.85937800 & 0.96023300\end{array}$

$\begin{array}{lll}-0.36943500 & 4.99284600 & 2.17188200\end{array}$

$\begin{array}{lll}0.37792000 & 2.80127900 & 2.47017000\end{array}$

$\begin{array}{lll}-1.29935300 & 4.65322500 & 1.70842700\end{array}$

$\begin{array}{lll}-3.66744200 & 3.79340000 & 0.52418300\end{array}$

$\begin{array}{lll}-3.42882500 & 5.16480200 & 0.67430400\end{array}$

$\begin{array}{lll}-2.23816400 & 5.58855200 & 1.27218100\end{array}$

$\begin{array}{lll}-4.59042000 & 3.44826800 & 0.05072400\end{array}$

$\begin{array}{lll}-4.16365800 & 5.89492100 & 0.32687200\end{array}$

$\begin{array}{lll}-2.03761400 & 6.65574600 & 1.39760200\end{array}$

$\begin{array}{lll}0.18740100 & 0.17140900 \quad 3.02672100\end{array}$

$\begin{array}{lll}1.10977700 & 0.64484200 & 3.37958100\end{array}$

$2.93292900 \quad-0.81726200 \quad 1.64294000$

$\begin{array}{lll}2.44440900 & 0.24077700 & 1.10725700\end{array}$

$\begin{array}{lll}4.38289300 & -0.68323000 & 2.18699500\end{array}$

$\begin{array}{lll}4.61836600 & -1.73381000 & 3.27956800\end{array}$

$\begin{array}{lll}4.62385600 & 0.71983900 & 2.76065800\end{array}$

$\begin{array}{lll}5.34163700 & -0.94484000 & 1.00920200\end{array}$

$\begin{array}{lll}4.44608300 & -2.74903500 & 2.89710100\end{array}$

$\begin{array}{lll}3.93414700 & -1.58260100 & 4.13009400\end{array}$

$5.65211900 \quad-1.67122000 \quad 3.65913600$

$\begin{array}{lll}4.49482600 & 1.49742900 & 1.99709800\end{array}$

$\begin{array}{lll}5.64722200 & 0.80012700 \quad 3.16480500\end{array}$

$\begin{array}{lll}3.92210200 & 0.93912800 & 3.58204800\end{array}$

$\begin{array}{lll}5.20266400 & -0.20817700 & 0.20443000\end{array}$

$\begin{array}{lll}5.17732100 & -1.94680800 & 0.58192100\end{array}$

$\begin{array}{lll}6.39143200 & -0.88802300 & 1.34400700\end{array}$

$\begin{array}{lll}2.35682900 & -1.90665300 & 1.72198700\end{array}$

$\begin{array}{lll}-2.91634700 & 1.79679300 & 0.80280300\end{array}$ 


\begin{tabular}{|c|c|c|c|}
\hline $\mathrm{H}$ & -1.75589800 & 0.57004800 & 2.23458200 \\
\hline $\mathrm{C}$ & -0.08603700 & -1.20891200 & 3.53260600 \\
\hline $\mathrm{H}$ & -0.05862700 & -1.21619300 & 4.63841100 \\
\hline $\mathrm{H}$ & 0.67669200 & -1.91409600 & 3.17344400 \\
\hline $\mathrm{H}$ & -1.07102300 & -1.57220700 & 3.21502100 \\
\hline $\mathrm{Ni}$ & 0.61476600 & 0.73373900 & 0.84588400 \\
\hline $\mathrm{C}$ & 2.51872800 & 0.26350200 & -3.04185500 \\
\hline $\mathrm{C}$ & 1.61466300 & 0.28681700 & -1.97469100 \\
\hline $\mathrm{C}$ & 1.21863900 & -0.94391900 & -1.39671900 \\
\hline $\mathrm{C}$ & 1.82729100 & -2.14891700 & -1.76443100 \\
\hline $\mathrm{C}$ & 2.75190600 & -2.11712500 & -2.82086700 \\
\hline $\mathrm{C}$ & 3.07628600 & -0.93684600 & -3.48130600 \\
\hline $\mathrm{C}$ & 0.42581400 & -3.27832800 & -0.02519800 \\
\hline $\mathrm{C}$ & -0.33351800 & -2.09942000 & 0.03043700 \\
\hline $\mathrm{C}$ & -1.65769700 & -2.11809100 & 0.53958200 \\
\hline $\mathrm{C}$ & -2.01754400 & -3.22986900 & 1.31932800 \\
\hline $\mathrm{C}$ & -1.16094100 & -4.31270800 & 1.49655500 \\
\hline $\mathrm{C}$ & 0.01556800 & -4.36413300 & 0.75555100 \\
\hline $\mathrm{H}$ & 2.81288900 & 1.19883700 & -3.51707000 \\
\hline $\mathrm{H}$ & 3.22903300 & -3.04825800 & -3.13081000 \\
\hline $\mathrm{H}$ & 3.78527700 & -0.94560000 & -4.31175600 \\
\hline $\mathrm{H}$ & -3.00713300 & -3.27359200 & 1.76409300 \\
\hline $\mathrm{H}$ & -1.45315400 & -5.14990300 & 2.13418900 \\
\hline $\mathrm{H}$ & 0.62636100 & -5.26806300 & 0.77037100 \\
\hline $\mathrm{C}$ & 1.53218000 & -3.46777700 & -1.05941100 \\
\hline $\mathrm{C}$ & 2.82052800 & -4.03652800 & -0.43028000 \\
\hline $\mathrm{H}$ & 2.63988400 & -5.04306900 & -0.02298600 \\
\hline $\mathrm{H}$ & 3.15442000 & -3.38730400 & 0.38718800 \\
\hline $\mathrm{H}$ & 3.61584100 & -4.12959700 & -1.18479000 \\
\hline $\mathrm{C}$ & 0.99028100 & -4.47687800 & -2.10712900 \\
\hline $\mathrm{H}$ & 1.74208300 & -4.67806000 & -2.88511600 \\
\hline $\mathrm{H}$ & 0.08384500 & -4.09101300 & -2.59728000 \\
\hline $\mathrm{H}$ & 0.73814100 & -5.43483900 & -1.62914300 \\
\hline $\mathrm{O}$ & 0.20970400 & -0.89273300 & -0.43576100 \\
\hline $\mathrm{C}$ & -0.21693100 & 2.59665800 & -2.24428600 \\
\hline $\mathrm{C}$ & 0.15627800 & 2.62005400 & -3.73231600 \\
\hline $\mathrm{H}$ & 0.38373600 & 1.61425000 & -4.11298500 \\
\hline $\mathrm{H}$ & 1.00330900 & 3.27943600 & -3.95752500 \\
\hline $\mathrm{H}$ & -0.71054000 & 2.99634700 & -4.30270000 \\
\hline $\mathrm{C}$ & -1.41273100 & 1.66220800 & -2.05702300 \\
\hline $\mathrm{H}$ & -2.28511800 & 2.03636700 & -2.61904700 \\
\hline $\mathrm{H}$ & -1.69599400 & 1.56476000 & -1.00564600 \\
\hline $\mathrm{H}$ & -1.17795100 & 0.65736500 & -2.42334300 \\
\hline $\mathrm{C}$ & -0.59406000 & 4.00481300 & -1.76520800 \\
\hline
\end{tabular}




\begin{tabular}{|c|c|c|c|}
\hline $\mathrm{H}$ & -0.82181400 & 4.02602700 & -0.69513300 \\
\hline $\mathrm{H}$ & -1.50080000 & 4.33226100 & -2.29982800 \\
\hline $\mathrm{H}$ & 0.18874500 & 4.74761900 & -1.96716100 \\
\hline $\mathrm{C}$ & 2.63548900 & 2.98149000 & -1.02658600 \\
\hline $\mathrm{C}$ & 2.87901200 & 3.87951600 & -2.24653500 \\
\hline $\mathrm{H}$ & 3.78274900 & 4.48258500 & -2.05386800 \\
\hline $\mathrm{H}$ & 2.05935600 & 4.58043200 & -2.44554000 \\
\hline $\mathrm{H}$ & 3.07053700 & 3.30091500 & -3.16063000 \\
\hline $\mathrm{C}$ & 3.90085800 & 2.14620500 & -0.78070200 \\
\hline $\mathrm{H}$ & 3.72599800 & 1.39922600 & -0.00009200 \\
\hline $\mathrm{H}$ & 4.71232100 & 2.82191600 & -0.46097800 \\
\hline $\mathrm{H}$ & 4.23920400 & 1.63009000 & -1.68896000 \\
\hline $\mathrm{C}$ & 2.37465700 & 3.85326800 & 0.21713200 \\
\hline $\mathrm{H}$ & 1.44481000 & 4.43504000 & 0.13589200 \\
\hline $\mathrm{H}$ & 3.20682800 & 4.56529100 & 0.34830700 \\
\hline $\mathrm{H}$ & 2.30881500 & 3.22918700 & 1.11900500 \\
\hline $\mathrm{C}$ & -4.50185100 & -1.05375900 & 0.98382200 \\
\hline $\mathrm{C}$ & -5.35757700 & -2.33060900 & 1.01060700 \\
\hline $\mathrm{H}$ & -4.82306600 & -3.21540800 & 1.37790900 \\
\hline $\mathrm{H}$ & -5.77709000 & -2.57429600 & 0.02661900 \\
\hline $\mathrm{H}$ & -6.21148200 & -2.16735900 & 1.69111600 \\
\hline $\mathrm{C}$ & -5.41143100 & 0.10092300 & 0.50970500 \\
\hline $\mathrm{H}$ & -5.82470200 & -0.06897600 & -0.49247000 \\
\hline $\mathrm{H}$ & -4.87940300 & 1.06167200 & 0.49776000 \\
\hline $\mathrm{H}$ & -6.26292400 & 0.20156800 & 1.20435300 \\
\hline $\mathrm{C}$ & -4.06223900 & -0.71379300 & 2.41888700 \\
\hline $\mathrm{H}$ & -4.94883700 & -0.70575700 & 3.07550400 \\
\hline $\mathrm{H}$ & -3.61780000 & 0.28877800 & 2.47880500 \\
\hline $\mathrm{H}$ & -3.34586100 & -1.43194200 & 2.83834200 \\
\hline $\mathrm{C}$ & -3.35149500 & -2.03521400 & -1.74559400 \\
\hline $\mathrm{C}$ & -3.46721900 & -3.54634400 & -1.50080200 \\
\hline $\mathrm{H}$ & -3.78278200 & -4.03939400 & -2.43740300 \\
\hline $\mathrm{H}$ & -4.19914400 & -3.80900000 & -0.72911800 \\
\hline $\mathrm{H}$ & -2.50191500 & -3.98267800 & -1.21011700 \\
\hline $\mathrm{C}$ & -4.63756600 & -1.49627700 & -2.39136100 \\
\hline $\mathrm{H}$ & -5.54044400 & -1.75404800 & -1.82203100 \\
\hline $\mathrm{H}$ & -4.75287200 & -1.93690600 & -3.39671000 \\
\hline $\mathrm{H}$ & -4.60609000 & -0.40202000 & -2.51364100 \\
\hline $\mathrm{C}$ & -2.20672300 & -1.81983800 & -2.75003800 \\
\hline $\mathrm{H}$ & -1.22177600 & -2.05960500 & -2.32836800 \\
\hline $\mathrm{H}$ & -2.18038900 & -0.79110500 & -3.12899600 \\
\hline $\mathrm{H}$ & -2.35771200 & -2.48705300 & -3.61575800 \\
\hline $\mathrm{P}$ & 1.11066400 & 1.83095700 & -1.12145400 \\
\hline $\mathrm{P}$ & -2.94358600 & -0.97071900 & -0.16787000 \\
\hline
\end{tabular}




\begin{tabular}{|c|c|c|}
\hline 0.83806600 & -1.35520400 & 2.61476400 \\
\hline 0.60716600 & -2.60067100 & 1.94058600 \\
\hline 1.70732200 & -3.40103900 & 1.37581200 \\
\hline 2.87484200 & -2.81989100 & 0.84602600 \\
\hline 0.70854600 & -5.27793600 & 1.76401700 \\
\hline-0.18237300 & -3.22768200 & 2.38048800 \\
\hline 1.60379100 & -4.80540100 & 1.35138200 \\
\hline 3.88635600 & -3.60835100 & 0.30079900 \\
\hline 3.76384900 & -5.00218300 & 0.27428000 \\
\hline 2.61701200 & -5.59695300 & 0.80805300 \\
\hline 4.77572300 & -3.12896300 & -0.11656200 \\
\hline 4.55648100 & -5.61831100 & -0.15696300 \\
\hline 2.50895800 & -6.68463400 & 0.79952800 \\
\hline-0.14141100 & -0.69504100 & 3.33068800 \\
\hline-1.01536300 & -1.26420300 & 3.67147400 \\
\hline-2.81842900 & 0.73884200 & 1.65106900 \\
\hline-2.43322600 & -0.39907300 & 1.18951800 \\
\hline-4.32095900 & 0.78472800 & 2.04182900 \\
\hline-4.60534000 & 2.02891500 & 2.89064600 \\
\hline-4.68990000 & -0.47668800 & 2.83862700 \\
\hline-5.14313200 & 0.84009900 & 0.74062200 \\
\hline-4.37107400 & 2.95248000 & 2.34348600 \\
\hline-4.00214700 & 2.03106400 & 3.81192700 \\
\hline-5.66965500 & 2.06039600 & 3.17823200 \\
\hline-4.52744600 & -1.38741900 & 2.24665500 \\
\hline-5.75046800 & -0.44573100 & 3.13990200 \\
\hline-4.08383500 & -0.55837900 & 3.75617800 \\
\hline-4.94889500 & -0.03860900 & 0.11087500 \\
\hline-4.89494900 & 1.73584800 & 0.15149400 \\
\hline-6.22243800 & 0.87121400 & 0.96730400 \\
\hline-2.11116800 & 1.74009300 & 1.76689400 \\
\hline 2.97647800 & -1.73415900 & 0.83315900 \\
\hline 1.75731600 & -0.80936500 & 2.39108100 \\
\hline 0.01367400 & 0.69785300 & 3.85280700 \\
\hline-0.02598700 & 0.70523800 & 4.95689300 \\
\hline-0.78956900 & 1.34808500 & 3.47574600 \\
\hline 0.96936200 & 1.13806500 & 3.53341000 \\
\hline-0.69465600 & -1.27557600 & 1.07691600 \\
\hline-2.80506600 & -0.37291300 & -2.68404000 \\
\hline-1.73276300 & -0.30412400 & -1.78519300 \\
\hline-1.30386100 & 0.98506700 & -1.36271700 \\
\hline-2.06269100 & 2.12638600 & -1.66703200 \\
\hline 3.15295500 & 1.99369000 & -2.53661200 \\
\hline
\end{tabular}




\begin{tabular}{|c|c|c|}
\hline-3.50269500 & 0.76479000 & -3.08347500 \\
\hline-0.56031900 & 3.39491000 & -0.11123800 \\
\hline 0.28545500 & 2.27596400 & -0.10841800 \\
\hline 1.58901400 & 2.35070000 & 0.44828100 \\
\hline 1.86908900 & 3.45973300 & 1.26228900 \\
\hline 0.95521800 & 4.49565200 & 1.43599100 \\
\hline-0.22161300 & 4.48650200 & 0.69438500 \\
\hline-3.11562700 & -1.33971800 & -3.07107100 \\
\hline-3.73306900 & 2.88043300 & -2.79676800 \\
\hline-4.33397500 & 0.68404600 & -3.78677700 \\
\hline 2.84219400 & 3.54491800 & 1.73656400 \\
\hline 1.19398600 & 5.33830700 & 2.08864000 \\
\hline-0.89025100 & 5.34697600 & 0.73643300 \\
\hline-1.74345300 & 3.49345300 & -1.06765100 \\
\hline-2.98724600 & 4.04219900 & -0.33710900 \\
\hline-2.82229600 & 5.07701500 & -0.00269200 \\
\hline-3.19896700 & 3.42049100 & 0.54093200 \\
\hline-3.86486900 & 4.05621000 & -0.99894600 \\
\hline-1.33982000 & 4.46292300 & -2.20704200 \\
\hline-2.16319400 & 4.59013400 & -2.92727000 \\
\hline-0.46235700 & 4.08408300 & -2.75315700 \\
\hline-1.08597500 & 5.45429300 & -1.80158800 \\
\hline-0.11657000 & 1.08458200 & -0.68340500 \\
\hline 0.28350000 & -2.39815700 & -2.26473100 \\
\hline-0.14624500 & -2.24742500 & -3.73058700 \\
\hline-0.42673000 & -1.21202000 & -3.96921500 \\
\hline-0.97949200 & -2.90428300 & -4.00906600 \\
\hline 0.71028900 & -2.51392300 & -4.37332300 \\
\hline 1.43060200 & -1.42513700 & -1.99127100 \\
\hline 2.29106500 & -1.66513800 & -2.63851600 \\
\hline 1.76519200 & -1.46939200 & -0.95191400 \\
\hline 1.12983100 & -0.39221600 & -2.18682100 \\
\hline 0.76444500 & -3.82863600 & -1.99508300 \\
\hline 1.03054600 & -3.97787900 & -0.94454600 \\
\hline 1.67240200 & -4.01501600 & -2.59192900 \\
\hline 0.02691100 & 4.59058800 & -2.27957700 \\
\hline-2.47113700 & -3.18951200 & -1.03773300 \\
\hline-2.73076300 & -3.92677700 & -2.36034300 \\
\hline-3.55402600 & -4.64332900 & -2.19917900 \\
\hline-1.86464700 & -4.50283200 & -2.7077850 \\
\hline-3.04498100 & -3.25971200 & -3.17410100 \\
\hline-3.78636300 & -2.56549500 & -0.54404900 \\
\hline-3.61594100 & -1.91559100 & 0.32168800 \\
\hline 4759110 & -3.37773300 & -0.25792800 \\
\hline
\end{tabular}




\begin{tabular}{|c|c|c|}
\hline-4.28375800 & -1.96943200 & -1.31965000 \\
\hline-2.01894300 & -4.21814600 & 0.01990700 \\
\hline-1.02278600 & -4.63365800 & -0.18071300 \\
\hline-2.73517500 & -5.05662800 & 0.03659900 \\
\hline-2.00268600 & -3.76916000 & 1.02270900 \\
\hline 4.41916200 & 1.35195200 & 1.04714400 \\
\hline 5.25225000 & 2.64216400 & 1.10601900 \\
\hline 4.67977900 & 3.52679000 & 1.41182800 \\
\hline 5.73593900 & 2.86893400 & 0.14725200 \\
\hline 6.05918300 & 2.50920700 & 1.84802800 \\
\hline 5.36946100 & 0.20489800 & 0.64079900 \\
\hline 5.83098100 & 0.36661600 & -0.34156800 \\
\hline 4.85131500 & -0.76333300 & 0.61350400 \\
\hline 6.18556900 & 0.12561000 & 1.37952500 \\
\hline 3.89213200 & 1.01985500 & 2.45305900 \\
\hline 4.73799500 & 0.97605600 & 3.16011600 \\
\hline 3.40903100 & 0.03384600 & 2.47423400 \\
\hline 3.17417500 & 1.75710000 & 2.83484200 \\
\hline 3.39388900 & 2.25952700 & -1.75668900 \\
\hline 3.48471400 & 3.77557700 & -1.53578100 \\
\hline 3.80404600 & 4.25932100 & -2.47612600 \\
\hline 4.20633000 & 4.05830900 & -0.76095000 \\
\hline 2.51052700 & 4.20315100 & -1.26162300 \\
\hline 4.71381000 & 1.72557800 & -2.33531500 \\
\hline 5.58633000 & 1.99998900 & -1.72725900 \\
\hline 4.87197300 & 2.15456900 & -3.33987900 \\
\hline 4.69889100 & 0.62945800 & -2.44344100 \\
\hline 2.29579500 & 2.00973900 & -2.80557700 \\
\hline 1.29763900 & 2.29512400 & -2.44731100 \\
\hline 2.26088800 & 0.96024800 & -3.12319900 \\
\hline 2.50774600 & 2.62169900 & -3.69919100 \\
\hline 1.07436300 & -1.86586900 & -1.04199900 \\
\hline 2.92918300 & 1.22248600 & -0.18032700 \\
\hline-4.88710800 & -0.38397100 & -1.64333600 \\
\hline-4.25396000 & .56900400 & -1.14470700 \\
\hline-4.79601700 & -2.38209800 & -0.04340700 \\
\hline-5.61584700 & -1.84238200 & 0.96779100 \\
\hline-3.84416900 & -4.19298300 & -0.74244900 \\
\hline-3.67948300 & -2.14864900 & -1.87457700 \\
\hline-4.47587900 & -3.75182700 & 0.03262600 \\
\hline-6.09377600 & -2.64252000 & 2.00507400 \\
\hline-5.76357000 & -4.00059200 & 2.06662700 \\
\hline 95048200 & -4.55209100 & 1.07212000 \\
\hline
\end{tabular}




\begin{tabular}{|c|c|c|c|}
\hline $\mathrm{H}$ & -6.72372500 & -2.19887800 & 2.78071400 \\
\hline $\mathrm{H}$ & -6.13574900 & -4.62288200 & 2.88427800 \\
\hline $\mathrm{H}$ & -4.68535600 & -5.61212800 & 1.10528000 \\
\hline $\mathrm{C}$ & -4.17069400 & 0.34760000 & -2.60433900 \\
\hline $\mathrm{H}$ & -3.53282500 & -0.20428100 & -3.30036000 \\
\hline $\mathrm{Ni}$ & -3.20957700 & 0.11281200 & -0.72622400 \\
\hline $\mathrm{C}$ & -3.47762900 & 1.72085300 & 0.85106400 \\
\hline $\mathrm{O}$ & -3.71531100 & 0.49854800 & 1.07776100 \\
\hline $\mathrm{C}$ & -3.65973900 & 2.82255800 & 1.88242000 \\
\hline $\mathrm{C}$ & -4.88296400 & 3.65171300 & 1.44058000 \\
\hline $\mathrm{C}$ & -2.40114300 & 3.70849500 & 1.88024700 \\
\hline $\mathrm{C}$ & -3.89752900 & 2.21570900 & 3.26893500 \\
\hline $\mathrm{H}$ & -5.79158400 & 3.02926800 & 1.40296600 \\
\hline $\mathrm{H}$ & -4.72320000 & 4.08612700 & 0.44240900 \\
\hline $\mathrm{H}$ & -5.06219300 & 4.47345300 & 2.15248700 \\
\hline $\mathrm{H}$ & -1.51392500 & 3.14119000 & 2.20029800 \\
\hline $\mathrm{H}$ & -2.53257300 & 4.55521700 & 2.57292400 \\
\hline $\mathrm{H}$ & -2.20087800 & 4.10370000 & 0.87413600 \\
\hline $\mathrm{H}$ & -3.03813700 & 1.60915400 & 3.59241000 \\
\hline $\mathrm{H}$ & -4.78229300 & 1.56261100 & 3.27292400 \\
\hline $\mathrm{H}$ & -4.05188300 & 3.01577100 & 4.01051900 \\
\hline $\mathrm{O}$ & -3.07924800 & 1.97899400 & -0.32710200 \\
\hline $\mathrm{H}$ & -5.85079800 & -0.77893000 & 0.95855200 \\
\hline $\mathrm{H}$ & -5.78277900 & 0.03058400 & -1.16881200 \\
\hline $\mathrm{C}$ & -4.56832400 & 1.72803700 & -3.03440800 \\
\hline $\mathrm{H}$ & -5.15050400 & 1.69971900 & -3.97385100 \\
\hline $\mathrm{H}$ & -3.68539000 & 2.35913200 & -3.22391800 \\
\hline $\mathrm{H}$ & -5.17326400 & 2.23377900 & -2.26761800 \\
\hline $\mathrm{C}$ & 5.65483400 & 0.83006700 & 0.89324900 \\
\hline $\mathrm{C}$ & 4.40371200 & 0.33301100 & 0.49041600 \\
\hline $\mathrm{C}$ & 3.35790300 & 1.26927800 & 0.30665800 \\
\hline $\mathrm{C}$ & 3.59305200 & 2.65382900 & 0.41222600 \\
\hline $\mathrm{C}$ & 4.86949400 & 3.09580900 & 0.77383800 \\
\hline $\mathrm{C}$ & 5.89449700 & 2.19227300 & 1.04312000 \\
\hline $\mathrm{C}$ & 1.45003400 & 2.97534700 & -0.77283500 \\
\hline $\mathrm{C}$ & 1.17534000 & 1.59814600 & -0.62769900 \\
\hline $\mathrm{C}$ & -0.02380400 & 1.04085400 & -1.13221700 \\
\hline $\mathrm{C}$ & -0.75422100 & 1.84700400 & -2.02851500 \\
\hline $\mathrm{C}$ & -0.37087200 & 3.13965300 & -2.36260700 \\
\hline $\mathrm{C}$ & 0.69468600 & 3.72199300 & -1.67961900 \\
\hline $\mathrm{H}$ & 6.46469200 & 0.12944000 & 1.08046600 \\
\hline $\mathrm{H}$ & 5.06204900 & 4.16419900 & 0.86772100 \\
\hline $\mathrm{H}$ & 6.87899200 & 2.54954000 & 1.35340900 \\
\hline $\mathrm{H}$ & -1.68223700 & 1.44533100 & -2.43152800 \\
\hline
\end{tabular}




\begin{tabular}{|c|c|c|c|}
\hline $\mathrm{H}$ & -0.95402700 & 3.71879100 & -3.08148300 \\
\hline $\mathrm{H}$ & 0.91899700 & 4.77680100 & -1.83498500 \\
\hline $\mathrm{C}$ & 2.43413300 & 3.61862000 & 0.20163700 \\
\hline $\mathrm{C}$ & 2.91119200 & 4.99521700 & -0.27968300 \\
\hline $\mathrm{H}$ & 2.06126300 & 5.68104400 & -0.39905000 \\
\hline $\mathrm{H}$ & 3.44640600 & 4.93054100 & -1.23909800 \\
\hline $\mathrm{H}$ & 3.57790600 & 5.46076200 & 0.45871100 \\
\hline $\mathrm{C}$ & 1.69740900 & 3.80293400 & 1.55551100 \\
\hline $\mathrm{H}$ & 2.37193300 & 4.25723500 & 2.29862700 \\
\hline $\mathrm{H}$ & 1.35080100 & 2.83990200 & 1.95697300 \\
\hline $\mathrm{H}$ & 0.81856700 & 4.45417300 & 1.42895000 \\
\hline $\mathrm{O}$ & 2.08444300 & 0.80174900 & 0.03653500 \\
\hline $\mathrm{C}$ & 4.79244800 & -2.36237800 & 1.70697600 \\
\hline $\mathrm{C}$ & 6.30200700 & -2.56135600 & 1.88705200 \\
\hline $\mathrm{H}$ & 6.85614200 & -1.61372000 & 1.93648000 \\
\hline $\mathrm{H}$ & 6.73863200 & -3.17611000 & 1.08753600 \\
\hline $\mathrm{H}$ & 6.48691000 & -3.09021700 & 2.83910200 \\
\hline $\mathrm{C}$ & 4.23635000 & -1.58318700 & 2.91118600 \\
\hline $\mathrm{H}$ & 4.76449600 & -0.63231700 & 3.06872000 \\
\hline $\mathrm{H}$ & 4.35162200 & -2.18907800 & 3.82665600 \\
\hline $\mathrm{H}$ & 3.16600300 & -1.35689700 & 2.79553300 \\
\hline $\mathrm{C}$ & 4.11512300 & -3.74683900 & 1.66858400 \\
\hline $\mathrm{H}$ & 3.02159900 & -3.65583700 & 1.59603600 \\
\hline $\mathrm{H}$ & 4.35108100 & -4.30801400 & 2.58963900 \\
\hline $\mathrm{H}$ & 4.45726800 & -4.35202500 & 0.81612600 \\
\hline $\mathrm{C}$ & 5.37336500 & -1.74277300 & -1.33870000 \\
\hline $\mathrm{C}$ & 4.72571500 & -0.98873100 & -2.51485300 \\
\hline $\mathrm{H}$ & 3.71618600 & -1.36036000 & -2.73406100 \\
\hline $\mathrm{H}$ & 5.33895000 & -1.12170500 & -3.42299600 \\
\hline $\mathrm{H}$ & 4.65613700 & 0.09277200 & -2.31778500 \\
\hline $\mathrm{C}$ & 5.35263500 & -3.24996600 & -1.64381200 \\
\hline $\mathrm{H}$ & 5.89106100 & -3.83396000 & -0.88242500 \\
\hline $\mathrm{H}$ & 5.84492400 & -3.44209800 & -2.61282600 \\
\hline $\mathrm{H}$ & 4.32369300 & -3.63785100 & -1.70889200 \\
\hline $\mathrm{C}$ & 6.82517900 & -1.26423300 & -1.21484700 \\
\hline $\mathrm{H}$ & 7.35764100 & -1.71161900 & -0.36659900 \\
\hline $\mathrm{H}$ & 6.89055000 & -0.17098100 & -1.12975500 \\
\hline $\mathrm{H}$ & 7.37398600 & -1.54759900 & -2.13064700 \\
\hline $\mathrm{C}$ & -0.53261300 & -1.05954100 & 1.08917200 \\
\hline $\mathrm{C}$ & -1.70734100 & -1.92627300 & 1.58380000 \\
\hline $\mathrm{H}$ & -1.87577600 & -2.80476600 & 0.94767700 \\
\hline $\mathrm{H}$ & -2.64194600 & -1.35841800 & 1.64341400 \\
\hline $\mathrm{H}$ & -1.46800600 & -2.29690500 & 2.59524200 \\
\hline $\mathrm{C}$ & -0.45717800 & 0.20631500 & 1.96049600 \\
\hline
\end{tabular}




\begin{tabular}{|c|c|c|c|}
\hline $\mathrm{H}$ & -0.47571400 & -0.08810600 & 3.02335700 \\
\hline $\mathrm{H}$ & -1.30846200 & 0.87436000 & 1.79223300 \\
\hline $\mathrm{H}$ & 0.46959800 & 0.76525300 & 1.78584300 \\
\hline $\mathrm{C}$ & 0.76345900 & -1.85139800 & 1.26046000 \\
\hline $\mathrm{H}$ & 0.92507000 & -2.02604500 & 2.33815900 \\
\hline $\mathrm{H}$ & 1.63564400 & -1.32376400 & 0.86761900 \\
\hline $\mathrm{H}$ & 0.72009300 & -2.83912900 & 0.78264200 \\
\hline $\mathrm{C}$ & -0.34831600 & -1.77743900 & -1.95458900 \\
\hline $\mathrm{C}$ & -0.82709200 & -3.18676500 & -1.58008000 \\
\hline $\mathrm{H}$ & -0.59374900 & -3.88012500 & -2.40607000 \\
\hline $\mathrm{H}$ & -1.91217300 & -3.21790600 & -1.41722200 \\
\hline $\mathrm{H}$ & -0.33584000 & -3.57525900 & -0.67962600 \\
\hline $\mathrm{C}$ & -0.96809600 & -1.40771700 & -3.31200200 \\
\hline $\mathrm{H}$ & -0.57121300 & -2.08715000 & -4.08522800 \\
\hline $\mathrm{H}$ & -0.72053000 & -0.38106900 & -3.61871400 \\
\hline $\mathrm{H}$ & -2.06005000 & -1.51406100 & -3.30539300 \\
\hline $\mathrm{C}$ & 1.17375600 & -1.74112800 & -2.09700600 \\
\hline $\mathrm{H}$ & 1.70626600 & -1.95688300 & -1.16518000 \\
\hline $\mathrm{H}$ & 1.51098500 & -0.75501900 & -2.44693800 \\
\hline $\mathrm{H}$ & 1.48954900 & -2.48757700 & -2.84735300 \\
\hline $\mathrm{P}$ & -0.98638400 & -0.50023700 & -0.68376700 \\
\hline $\mathrm{P}$ & 4.16111900 & -1.46831200 & 0.12441800 \\
\hline \multicolumn{4}{|l|}{ 6-f } \\
\hline $\mathrm{C}$ & -5.14716800 & -0.39803700 & -1.03740100 \\
\hline $\mathrm{C}$ & -4.33431800 & -1.56021600 & -1.24548100 \\
\hline $\mathrm{C}$ & -4.52155900 & -2.74413900 & -0.38253000 \\
\hline $\mathrm{C}$ & -4.82122600 & -2.61096000 & 0.98869100 \\
\hline $\mathrm{H}$ & -4.17575700 & -4.16839600 & -1.97162700 \\
\hline $\mathrm{H}$ & -4.06142200 & -1.79288300 & -2.28104900 \\
\hline $\mathrm{C}$ & -4.40272200 & -4.04364800 & -0.90944100 \\
\hline $\mathrm{C}$ & -4.99149900 & -3.73552700 & 1.79548800 \\
\hline $\mathrm{C}$ & -4.86316300 & -5.02134300 & 1.25806800 \\
\hline $\mathrm{C}$ & -4.56890600 & -5.16931900 & -0.10042200 \\
\hline $\mathrm{H}$ & -5.21611700 & -3.60645000 & 2.85746700 \\
\hline $\mathrm{H}$ & -4.99220700 & -5.90074500 & 1.89380600 \\
\hline $\mathrm{H}$ & -4.47037200 & -6.16786500 & -0.53437300 \\
\hline $\mathrm{C}$ & -4.85838100 & 0.77173600 & -1.72608100 \\
\hline $\mathrm{H}$ & -4.33934600 & 0.69028500 & -2.69126100 \\
\hline $\mathrm{Ni}$ & -3.22135800 & 0.03671300 & -0.63851900 \\
\hline $\mathrm{C}$ & -3.51970700 & 1.96170900 & 1.19341400 \\
\hline $\mathrm{O}$ & -4.25689700 & 1.11341400 & 1.71133800 \\
\hline $\mathrm{C}$ & -3.35319100 & 3.34774700 & 1.86286800 \\
\hline $\mathrm{C}$ & -4.70684500 & 4.07465400 & 1.76940700 \\
\hline $\mathrm{C}$ & -2.26063500 & 4.17865900 & 1.18064000 \\
\hline
\end{tabular}




\begin{tabular}{|c|c|c|c|}
\hline $\mathrm{C}$ & -3.00047900 & 3.12040600 & 3.34237100 \\
\hline $\mathrm{H}$ & -5.50428200 & 3.47037600 & 2.22717500 \\
\hline $\mathrm{H}$ & -4.98425800 & 4.27294400 & 0.72191500 \\
\hline $\mathrm{H}$ & -4.66133500 & 5.04371500 & 2.29389900 \\
\hline $\mathrm{H}$ & -1.28989900 & 3.66363100 & 1.21095700 \\
\hline $\mathrm{H}$ & -2.14939100 & 5.15283800 & 1.68620800 \\
\hline $\mathrm{H}$ & -2.49483900 & 4.36180900 & 0.12229600 \\
\hline $\mathrm{H}$ & -2.02816300 & 2.61397700 & 3.44760900 \\
\hline $\mathrm{H}$ & -3.76134000 & 2.49237500 & 3.82718900 \\
\hline $\mathrm{H}$ & -2.94153100 & 4.08183800 & 3.87886200 \\
\hline $\mathrm{O}$ & -2.85376100 & 1.77817000 & 0.10568800 \\
\hline $\mathrm{H}$ & -4.89502500 & -1.61390900 & 1.42856100 \\
\hline $\mathrm{H}$ & -5.81364900 & -0.34522900 & -0.17101300 \\
\hline $\mathrm{C}$ & -5.50541300 & 2.08904000 & -1.44176100 \\
\hline $\mathrm{H}$ & -6.29230700 & 2.30361600 & -2.18688100 \\
\hline $\mathrm{H}$ & -4.76762300 & 2.90331400 & -1.49844300 \\
\hline $\mathrm{H}$ & -5.96028400 & 2.10327000 & -0.44173200 \\
\hline $\mathrm{C}$ & 5.62164600 & 0.93519800 & 0.54787800 \\
\hline $\mathrm{C}$ & 4.35353800 & 0.39435900 & 0.27618100 \\
\hline $\mathrm{C}$ & 3.31188200 & 1.29939900 & -0.04189800 \\
\hline $\mathrm{C}$ & 3.56184700 & 2.67779500 & -0.18374000 \\
\hline C & 4.85365900 & 3.15572700 & 0.05956200 \\
\hline $\mathrm{C}$ & 5.87878600 & 2.29867500 & 0.44942900 \\
\hline $\mathrm{C}$ & 1.34447800 & 2.84881600 & -1.27485800 \\
\hline $\mathrm{C}$ & 1.08356400 & 1.51259100 & -0.90357200 \\
\hline $\mathrm{C}$ & -0.14967700 & 0.90247700 & -1.23043600 \\
\hline C & -0.96940400 & 1.57987800 & -2.15368000 \\
\hline $\mathrm{C}$ & -0.62801100 & 2.81406500 & -2.68974700 \\
\hline $\mathrm{C}$ & 0.50093500 & 3.46738700 & -2.20090000 \\
\hline $\mathrm{H}$ & 6.43124800 & 0.26858600 & 0.83399900 \\
\hline $\mathrm{H}$ & 5.05734000 & 4.22172000 & -0.03470400 \\
\hline $\mathrm{H}$ & 6.87559600 & 2.69042600 & 0.66371200 \\
\hline $\mathrm{H}$ & -1.92448400 & 1.12568600 & -2.42123700 \\
\hline $\mathrm{H}$ & -1.27910900 & 3.29834900 & -3.42019400 \\
\hline $\mathrm{H}$ & 0.70830900 & 4.48694500 & -2.52310200 \\
\hline $\mathrm{C}$ & 2.41214000 & 3.62352000 & -0.50650000 \\
\hline C & 2.89077800 & 4.86417900 & -1.27490800 \\
\hline $\mathrm{H}$ & 2.05522000 & 5.54984200 & -1.47066500 \\
\hline $\mathrm{H}$ & 3.35439500 & 4.59449600 & -2.23601100 \\
\hline $\mathrm{H}$ & 3.61992400 & 5.43670200 & -0.68605100 \\
\hline $\mathrm{C}$ & 1.77635400 & 4.08854300 & 0.83125200 \\
\hline $\mathrm{H}$ & 2.51818100 & 4.63432000 & 1.43575100 \\
\hline $\mathrm{H}$ & 1.41867500 & 3.23324200 & 1.42283700 \\
\hline $\mathrm{H}$ & 0.91807800 & 4.75101100 & 0.64091500 \\
\hline
\end{tabular}




\begin{tabular}{|c|c|c|c|}
\hline $\mathrm{O}$ & 2.02765200 & 0.80523100 & -0.19280400 \\
\hline $\mathrm{C}$ & 4.75994800 & -2.03289300 & 1.95931900 \\
\hline $\mathrm{C}$ & 6.27239000 & -2.22493300 & 2.12120500 \\
\hline $\mathrm{H}$ & 6.83980200 & -1.29701200 & 1.97000600 \\
\hline $\mathrm{H}$ & 6.67066200 & -2.98960400 & 1.43952800 \\
\hline $\mathrm{H}$ & 6.48502100 & -2.56644400 & 3.14985700 \\
\hline $\mathrm{C}$ & 4.25946500 & -1.03204000 & 3.01493100 \\
\hline $\mathrm{H}$ & 4.80882000 & -0.08110000 & 2.97592700 \\
\hline $\mathrm{H}$ & 4.39690800 & -1.46002200 & 4.02307500 \\
\hline $\mathrm{H}$ & 3.18942300 & -0.80622900 & 2.89310800 \\
\hline $\mathrm{C}$ & 4.06915600 & -3.38965700 & 2.20800300 \\
\hline $\mathrm{H}$ & 2.97354100 & -3.29667000 & 2.19070100 \\
\hline $\mathrm{H}$ & 4.36063500 & -3.78281400 & 3.19752400 \\
\hline $\mathrm{H}$ & 4.35237200 & -4.14182700 & 1.45700900 \\
\hline $\mathrm{C}$ & 5.24292300 & -2.00031800 & -1.16124900 \\
\hline $\mathrm{C}$ & 4.57383400 & -1.47156500 & -2.44339600 \\
\hline $\mathrm{H}$ & 3.56104000 & -1.87309500 & -2.57517600 \\
\hline $\mathrm{H}$ & 5.17124600 & -1.77080400 & -3.32205200 \\
\hline $\mathrm{H}$ & 4.50916400 & -0.37188000 & -2.44707200 \\
\hline $\mathrm{C}$ & 5.20300200 & -3.53762500 & -1.17777200 \\
\hline $\mathrm{H}$ & 5.75614500 & -3.97477400 & -0.33332100 \\
\hline $\mathrm{H}$ & 5.67007100 & -3.91281700 & -2.10475300 \\
\hline $\mathrm{H}$ & 4.17019200 & -3.91923700 & -1.14488600 \\
\hline $\mathrm{C}$ & 6.70047900 & -1.52307300 & -1.16482100 \\
\hline $\mathrm{H}$ & 7.24980900 & -1.79924700 & -0.25697200 \\
\hline $\mathrm{H}$ & 6.77337800 & -0.43546500 & -1.29945900 \\
\hline $\mathrm{H}$ & 7.22577600 & -1.98748400 & -2.01837300 \\
\hline $\mathrm{C}$ & -0.54015300 & -0.79105300 & 1.32789300 \\
\hline $\mathrm{C}$ & -1.77545700 & -1.43721400 & 1.98173500 \\
\hline $\mathrm{H}$ & -2.03980800 & -2.39821700 & 1.51998600 \\
\hline $\mathrm{H}$ & -2.65096900 & -0.77465200 & 1.93050500 \\
\hline $\mathrm{H}$ & -1.55812700 & -1.62928200 & 3.04621800 \\
\hline $\mathrm{C}$ & -0.33070300 & 0.60533400 & 1.93932400 \\
\hline $\mathrm{H}$ & -0.38573200 & 0.52219500 & 3.03714400 \\
\hline $\mathrm{H}$ & -1.09444100 & 1.31671500 & 1.60925400 \\
\hline $\mathrm{H}$ & 0.65590900 & 1.00780500 & 1.68276900 \\
\hline $\mathrm{C}$ & 0.69574600 & -1.64397200 & 1.60895400 \\
\hline $\mathrm{H}$ & 0.88523600 & -1.63090300 & 2.69568600 \\
\hline $\mathrm{H}$ & 1.58896300 & -1.26269000 & 1.10424200 \\
\hline $\mathrm{H}$ & 0.55991400 & -2.69442600 & 1.32079500 \\
\hline $\mathrm{C}$ & -0.44227300 & -2.01795200 & -1.59006000 \\
\hline $\mathrm{C}$ & -0.95475800 & -3.33853800 & -1.00211700 \\
\hline $\mathrm{H}$ & -0.78757700 & -4.15100700 & -1.72950300 \\
\hline $\mathrm{H}$ & -2.02839900 & -3.30591600 & -0.78437100 \\
\hline
\end{tabular}




\begin{tabular}{|c|c|c|c|}
\hline $\mathrm{H}$ & -0.42924700 & -3.61590900 & -0.07924500 \\
\hline $\mathrm{C}$ & -1.07544300 & -1.79682600 & -2.97477000 \\
\hline $\mathrm{H}$ & -0.79716900 & -2.63584200 & -3.63430200 \\
\hline $\mathrm{H}$ & -0.71166800 & -0.87215200 & -3.44639100 \\
\hline $\mathrm{H}$ & -2.17010300 & -1.75064400 & -2.92648100 \\
\hline $\mathrm{C}$ & 1.07615900 & -2.04875100 & -1.76069400 \\
\hline $\mathrm{H}$ & 1.62057300 & -2.16661100 & -0.81826300 \\
\hline $\mathrm{H}$ & 1.44006300 & -1.12759900 & -2.23736300 \\
\hline $\mathrm{H}$ & 1.34766400 & -2.89400700 & -2.41688500 \\
\hline $\mathrm{P}$ & -1.01454900 & -0.57270100 & -0.50217800 \\
\hline $\mathrm{P}$ & 4.07914900 & -1.43905900 & 0.25959200 \\
\hline \multicolumn{4}{|c|}{$7-\mathrm{a}$} \\
\hline $\mathrm{C}$ & 2.36569400 & -2.22607000 & 1.79128000 \\
\hline $\mathrm{C}$ & 3.24545100 & -2.11156600 & 0.64752000 \\
\hline $\mathrm{C}$ & 4.71097500 & -2.29157600 & 0.84459300 \\
\hline $\mathrm{C}$ & 5.42718200 & -1.68873800 & 1.89204900 \\
\hline $\mathrm{H}$ & 4.90957900 & -3.67702400 & -0.80501000 \\
\hline $\mathrm{H}$ & 2.91508700 & -2.74818200 & -0.17294900 \\
\hline $\mathrm{C}$ & 5.43132500 & -3.16172800 & 0.00225100 \\
\hline $\mathrm{C}$ & 6.78932000 & -1.92357700 & 2.08293900 \\
\hline $\mathrm{C}$ & 7.48800600 & -2.77417400 & 1.22182100 \\
\hline $\mathrm{C}$ & 6.79549200 & -3.39607300 & 0.17935100 \\
\hline $\mathrm{H}$ & 7.30954600 & -1.43015000 & 2.90847000 \\
\hline $\mathrm{H}$ & 8.55615000 & -2.95526200 & 1.36483000 \\
\hline $\mathrm{H}$ & 7.31823300 & -4.07628600 & -0.49831600 \\
\hline $\mathrm{C}$ & 1.01613300 & -2.31425200 & 1.62708400 \\
\hline $\mathrm{H}$ & 0.60583200 & -2.51158300 & 0.64546100 \\
\hline $\mathrm{H}$ & 4.91008800 & -1.01302500 & 2.56293800 \\
\hline $\mathrm{H}$ & 2.77173300 & -2.09093000 & 2.79637800 \\
\hline $\mathrm{C}$ & 0.01049000 & -2.33790000 & 2.73420100 \\
\hline $\mathrm{H}$ & -0.73281000 & -3.12945700 & 2.55002600 \\
\hline $\mathrm{H}$ & -0.53316800 & -1.37990000 & 2.74647700 \\
\hline $\mathrm{H}$ & 0.47854600 & -2.50691600 & 3.71630400 \\
\hline $\mathrm{O}$ & -1.95966300 & -1.32025700 & 0.34267200 \\
\hline $\mathrm{O}$ & 0.18089500 & -0.04489800 & 0.04904500 \\
\hline $\mathrm{P}$ & -1.27407400 & 0.17294000 & 0.56591700 \\
\hline B & -3.41199300 & -1.63016000 & 0.36647300 \\
\hline K & -2.76648800 & -0.47551300 & -2.25895500 \\
\hline K & -3.21266700 & -0.66589800 & 3.27768000 \\
\hline $\mathrm{O}$ & -1.36704000 & 0.50517400 & 2.06549100 \\
\hline $\mathrm{O}$ & -2.02681100 & 1.12532500 & -0.38180800 \\
\hline $\mathrm{O}$ & -3.70582900 & -2.52522400 & 1.53512400 \\
\hline B & -4.38240100 & -0.17935300 & 0.35061100 \\
\hline $\mathrm{O}$ & -3.75822000 & -2.48608700 & -0.81646200 \\
\hline
\end{tabular}




\begin{tabular}{|c|c|c|}
\hline $.0200+700$ & -5.07031000 & $1.07232+00$ \\
\hline-4.76219400 & 0.54565100 & 1.47313900 \\
\hline-4.90764500 & 0.39967100 & -0.79082500 \\
\hline-4.02233800 & -3.81072200 & -0.38397100 \\
\hline-4.33354100 & -4.78978800 & 2.01678300 \\
\hline-5.99770100 & -3.14182000 & 1.13136500 \\
\hline-5.24505700 & 1.85251300 & 1.07411300 \\
\hline-5.54546700 & 1.65790300 & -0.47750900 \\
\hline-5.05475600 & -4.44476600 & -1.31399500 \\
\hline-2.72275800 & -4.62605800 & -0.42600900 \\
\hline-4.85890100 & -5.68098300 & 1.63744400 \\
\hline-4.74020000 & -4.56373000 & 3.01639700 \\
\hline-3.26920200 & -5.03369500 & 2.13487700 \\
\hline-6.69494300 & -3.96210600 & 0.89994500 \\
\hline-6.23105800 & -2.77200700 & 2.14264400 \\
\hline-6.17740000 & -2.32150500 & 0.42154700 \\
\hline-6.47126700 & 2.18099100 & 1.91910000 \\
\hline-4.11710100 & 2.84580400 & 1.36443700 \\
\hline-4.90418900 & 2.72214600 & -1.36377300 \\
\hline-7.02749900 & 1.49986300 & -0.81235400 \\
\hline-4.61664200 & -4.59067200 & -2.31493400 \\
\hline-5.38140100 & -5.43115700 & -0.94611400 \\
\hline-5.93762400 & -3.80115100 & -1.42792200 \\
\hline-2.31746000 & -4.58768200 & -1.44805900 \\
\hline-2.88016400 & -5.68476500 & -0.16513100 \\
\hline-1.97241600 & -4.19585100 & 0.25073400 \\
\hline-6.17578000 & 2.26012800 & 2.97710600 \\
\hline-6.90983600 & 3.14633900 & 1.62311300 \\
\hline-7.24583000 & 1.40621900 & 1.84247600 \\
\hline-4.39538500 & 3.86991700 & 1.07241800 \\
\hline-3.91477500 & 2.85109900 & 2.44709200 \\
\hline-3.19425300 & 2.54203600 & 0.85099400 \\
\hline-5.13304800 & 2.50909600 & -2.42057100 \\
\hline-5.28980600 & 3.72722000 & -1.13433900 \\
\hline-3.81541000 & 2.70967200 & -1.23014000 \\
\hline-7.48768000 & 0.69143500 & -0.22658900 \\
\hline-7.59034400 & 2.42834300 & -0.63371100 \\
\hline-7.12825900 & 1.23904300 & -1.87716700 \\
\hline 0.45248200 & 3.15344800 & 2.27699100 \\
\hline 1.19483100 & 2.47905000 & 1.29042400 \\
\hline 0.82630700 & 2.76370500 & -0.03152200 \\
\hline-0.10592300 & 3.74529000 & -0.39121400 \\
\hline-0.79240600 & 4.40691600 & 0.62506900 \\
\hline 0.52721700 & 4.08923000 & 1.95580000 \\
\hline
\end{tabular}




\begin{tabular}{|c|c|c|}
\hline-0.19697000 & 2.72492800 & -2.62775900 \\
\hline 0.67503900 & 1.73896400 & -2.13263000 \\
\hline 0.85718100 & 0.48688200 & -2.75069000 \\
\hline 0.20553000 & 0.32223300 & -3.98881800 \\
\hline-0.66558500 & 1.28390400 & -4.50846600 \\
\hline-0.89115200 & 2.47250000 & -3.81122200 \\
\hline 0.60058600 & 2.93589500 & 3.32555200 \\
\hline-1.54527800 & 5.15746200 & 0.38540500 \\
\hline-1.09109400 & 4.57197100 & 2.75724800 \\
\hline 0.37432700 & -0.56414000 & -4.57947300 \\
\hline-1.15380400 & 1.10640500 & -5.47043600 \\
\hline-1.57778800 & 3.21697300 & -4.21472900 \\
\hline-0.25850900 & 4.05450200 & -1.87832100 \\
\hline 0.95334600 & 4.91500300 & -2.32438700 \\
\hline 0.89498100 & 5.13203700 & -3.40300400 \\
\hline 1.90459300 & 4.39936200 & -2.13137500 \\
\hline 0.96650900 & 5.86876600 & -1.77357600 \\
\hline-1.54502900 & 4.82911700 & -2.17873700 \\
\hline-1.55520700 & 5.78564200 & -1.63715400 \\
\hline-2.43837900 & 4.25805600 & -1.89049300 \\
\hline-1.61603700 & 5.07522600 & -3.24798400 \\
\hline 1.42233900 & 2.03169000 & -1.03274700 \\
\hline 2.54916200 & 1.21565500 & 1.58359000 \\
\hline 2.00087400 & -0.78151900 & -1.97186900 \\
\hline 2.54948600 & 1.19611400 & 3.53406300 \\
\hline 1.20860800 & 0.53935900 & 3.92930900 \\
\hline 1.29045100 & -0.54452000 & 3.81962200 \\
\hline 0.34217000 & 0.83900600 & 3.32508700 \\
\hline 0.99636200 & 0.74248100 & 4.99353300 \\
\hline 3.64541000 & 0.29536300 & 4.12808900 \\
\hline 4.66491700 & 0.60609800 & 3.86642800 \\
\hline 3.51849900 & -0.75489500 & 3.85071600 \\
\hline 3.56677800 & 0.33986900 & 5.22796600 \\
\hline 2.73006700 & 2.58956300 & 4.20424400 \\
\hline 3.73976100 & 2.69574500 & 4.62119900 \\
\hline 2.02817200 & 69210100 & 5.04792200 \\
\hline 2.56909500 & 3.44360100 & 3.54113000 \\
\hline 4.17100200 & 2.24427800 & 1.07541600 \\
\hline 3.82893900 & 3.39248400 & 0.11268400 \\
\hline 3.19463900 & 4.15199600 & 0.59048000 \\
\hline 3.32597500 & 3.05226700 & -0.79508900 \\
\hline 4.76928800 & 3.88470100 & -0.18926300 \\
\hline 5.08535800 & 1.23381600 & 0.37196400 \\
\hline 5.62619400 & 0.60922200 & 1.0926280 \\
\hline
\end{tabular}




\begin{tabular}{|c|c|c|c|}
\hline $\mathrm{H}$ & 5.83771900 & 1.75404900 & -0.24433400 \\
\hline $\mathrm{H}$ & 4.51357200 & 0.55809200 & -0.26901000 \\
\hline $\mathrm{C}$ & 4.98613700 & 2.88514900 & 2.20893100 \\
\hline $\mathrm{H}$ & 4.48327700 & 3.74939800 & 2.65774000 \\
\hline $\mathrm{H}$ & 5.93080500 & 3.25529400 & 1.77281300 \\
\hline $\mathrm{H}$ & 5.26053700 & 2.18348900 & 3.00387900 \\
\hline $\mathrm{C}$ & 3.56927600 & -0.37157600 & -3.04832500 \\
\hline $\mathrm{C}$ & 3.80384700 & 1.14510300 & -2.94354500 \\
\hline $\mathrm{H}$ & 3.78824200 & 1.49476900 & -1.90893100 \\
\hline $\mathrm{H}$ & 3.05527600 & 1.71655200 & -3.50868000 \\
\hline $\mathrm{H}$ & 4.79454600 & 1.38532800 & -3.36434100 \\
\hline $\mathrm{C}$ & 3.42397600 & -0.70996900 & -4.53757200 \\
\hline $\mathrm{H}$ & 4.32734400 & -0.35943400 & -5.06654100 \\
\hline $\mathrm{H}$ & 2.56546300 & -0.20229000 & -4.99774300 \\
\hline $\mathrm{H}$ & 3.34000400 & -1.78726400 & -4.72540800 \\
\hline $\mathrm{C}$ & 4.80020200 & -1.09384900 & -2.48849400 \\
\hline $\mathrm{H}$ & 5.70268300 & -0.73511200 & -3.01217600 \\
\hline $\mathrm{H}$ & 4.75411700 & -2.17817100 & -2.63140800 \\
\hline $\mathrm{H}$ & 4.94320900 & -0.90302800 & -1.42088200 \\
\hline $\mathrm{C}$ & 1.36179500 & -2.53930100 & -2.62727900 \\
\hline $\mathrm{C}$ & 0.69205300 & -2.66074900 & -4.01214900 \\
\hline $\mathrm{H}$ & 0.57810400 & -3.73744600 & -4.22290300 \\
\hline $\mathrm{H}$ & 1.27332100 & -2.23515500 & -4.83787900 \\
\hline $\mathrm{H}$ & -0.31999100 & -2.23813200 & -4.02655700 \\
\hline $\mathrm{C}$ & 2.53211400 & -3.54004100 & -2.65177400 \\
\hline $\mathrm{H}$ & 3.20878800 & -3.37944500 & -3.50041500 \\
\hline $\mathrm{H}$ & 2.11524000 & -4.55588900 & -2.75552700 \\
\hline $\mathrm{H}$ & 3.12841700 & -3.53684600 & -1.73514400 \\
\hline $\mathrm{C}$ & 0.31839300 & -3.04387300 & -1.61406800 \\
\hline $\mathrm{H}$ & -0.35502600 & -2.26318700 & -1.23346500 \\
\hline $\mathrm{H}$ & 0.80010800 & -3.52132400 & -0.75319400 \\
\hline $\mathrm{H}$ & -0.29353600 & -3.82656600 & -2.08731400 \\
\hline $\mathrm{Ni}$ & 1.97607500 & -0.63362400 & 0.32981100 \\
\hline \multicolumn{4}{|c|}{$7-b$} \\
\hline $\mathrm{C}$ & -2.00953500 & 2.12854800 & 2.36514700 \\
\hline $\mathrm{C}$ & -2.65804100 & 2.83591600 & 1.30546600 \\
\hline $\mathrm{C}$ & -4.13340200 & 2.87541000 & 1.20947700 \\
\hline $\mathrm{C}$ & -4.89479500 & 1.69338500 & 1.17566700 \\
\hline $\mathrm{H}$ & -4.23851600 & 5.03413200 & 1.13891900 \\
\hline $\mathrm{H}$ & -2.17269700 & 3.78030600 & 1.03435600 \\
\hline $\mathrm{C}$ & -4.81255100 & 4.10378100 & 1.12056700 \\
\hline $\mathrm{C}$ & -6.28443800 & 1.73695700 & 1.07344000 \\
\hline $\mathrm{C}$ & -6.94807200 & 2.96554400 & 0.98403000 \\
\hline $\mathrm{C}$ & -6.20415500 & 4.14896100 & 1.00486200 \\
\hline
\end{tabular}




\begin{tabular}{|c|c|c|c|}
\hline $\mathrm{H}$ & -6.85197000 & 0.80319400 & 1.04046300 \\
\hline $\mathrm{H}$ & -8.03635600 & 3.00002500 & 0.89110800 \\
\hline $\mathrm{H}$ & -6.71084200 & 5.11488400 & 0.93148200 \\
\hline $\mathrm{C}$ & -0.62436200 & 2.08026400 & 2.41141400 \\
\hline $\mathrm{H}$ & -0.05802500 & 2.83990800 & 1.86276400 \\
\hline $\mathrm{H}$ & -4.37788600 & 0.73267800 & 1.17948900 \\
\hline $\mathrm{H}$ & -2.58707200 & 1.48237600 & 3.03253900 \\
\hline $\mathrm{C}$ & 0.14812800 & 1.31334600 & 3.44234000 \\
\hline $\mathrm{H}$ & 1.02107900 & 1.90403600 & 3.76427100 \\
\hline $\mathrm{H}$ & 0.50870600 & 0.35548300 & 3.02709500 \\
\hline $\mathrm{H}$ & -0.47164900 & 1.09632100 & 4.32558300 \\
\hline $\mathrm{O}$ & 2.11447200 & 1.01830800 & 0.78838300 \\
\hline $\mathrm{O}$ & -0.15570200 & 0.13782400 & 0.14329300 \\
\hline $\mathrm{P}$ & 1.26535700 & -0.38045100 & 0.54746900 \\
\hline B & 3.57587600 & 1.21985500 & 0.70733700 \\
\hline K & 2.86696400 & 0.72798400 & -2.14680100 \\
\hline K & 3.23120500 & -0.35765100 & 3.25140100 \\
\hline $\mathrm{O}$ & 1.28628300 & -1.16344100 & 1.86941300 \\
\hline $\mathrm{O}$ & 1.91674900 & -1.09441500 & -0.65369600 \\
\hline $\mathrm{O}$ & 4.04748000 & 1.78568500 & 2.01960700 \\
\hline B & 4.45562500 & -0.23021500 & 0.31092200 \\
\hline $\mathrm{O}$ & 3.89615800 & 2.31493600 & -0.26983200 \\
\hline $\mathrm{C}$ & 4.89078100 & 2.89140100 & 1.75928000 \\
\hline $\mathrm{O}$ & 4.82936600 & -1.18620600 & 1.25131700 \\
\hline $\mathrm{O}$ & 4.96824200 & -0.56661900 & -0.93394300 \\
\hline $\mathrm{C}$ & 4.25735900 & 3.48625700 & 0.44640800 \\
\hline C & 4.86909800 & 3.83055300 & 2.96251600 \\
\hline $\mathrm{C}$ & 6.32515700 & 2.39103500 & 1.51942600 \\
\hline $\mathrm{C}$ & 5.30584200 & -2.36697400 & 0.55529600 \\
\hline $\mathrm{C}$ & 5.78439800 & -1.75771000 & -0.81745700 \\
\hline $\mathrm{C}$ & 5.21721100 & 4.31651900 & -0.40225600 \\
\hline $\mathrm{C}$ & 2.99589800 & 4.30608600 & 0.75280700 \\
\hline $\mathrm{H}$ & 5.40921300 & 4.76824700 & 2.75449200 \\
\hline $\mathrm{H}$ & 5.35863500 & 3.34746900 & 3.82415100 \\
\hline $\mathrm{H}$ & 3.83922500 & 4.07527300 & 3.25594700 \\
\hline $\mathrm{H}$ & 7.04894700 & 3.21629300 & 1.43037500 \\
\hline $\mathrm{H}$ & 6.62919900 & 1.75911100 & 2.36865200 \\
\hline $\mathrm{H}$ & 6.37884000 & 1.78191000 & 0.60567000 \\
\hline $\mathrm{C}$ & 6.41094600 & -3.00920200 & 1.38288000 \\
\hline C & 4.10794500 & -3.30924500 & 0.42311300 \\
\hline $\mathrm{C}$ & 5.52912200 & -2.64280700 & -2.02922200 \\
\hline $\mathrm{C}$ & 7.24066600 & -1.28954000 & -0.79627900 \\
\hline $\mathrm{H}$ & 4.68878500 & 4.71384100 & -1.28441100 \\
\hline $\mathrm{H}$ & 5.61679600 & 5.17533000 & 0.16128500 \\
\hline
\end{tabular}




\begin{tabular}{|c|c|c|c|}
\hline $\mathrm{H}$ & 6.05925700 & 3.71027900 & -0.76340200 \\
\hline $\mathrm{H}$ & 2.48504600 & 4.53487200 & -0.19293500 \\
\hline $\mathrm{H}$ & 3.22501300 & 5.26045400 & 1.25296200 \\
\hline $\mathrm{H}$ & 2.30516200 & 3.72481100 & 1.37863000 \\
\hline $\mathrm{H}$ & 5.99172200 & -3.39032100 & 2.32713300 \\
\hline $\mathrm{H}$ & 6.85599000 & -3.86330900 & 0.84858000 \\
\hline $\mathrm{H}$ & 7.20879100 & -2.29618600 & 1.62930500 \\
\hline $\mathrm{H}$ & 4.36474300 & -4.22594100 & -0.12897900 \\
\hline $\mathrm{H}$ & 3.77408300 & -3.60543500 & 1.42960200 \\
\hline $\mathrm{H}$ & 3.26340900 & -2.79361700 & -0.05675600 \\
\hline $\mathrm{H}$ & 5.88981100 & -2.13989900 & -2.94051400 \\
\hline $\mathrm{H}$ & 6.06591600 & -3.60030800 & -1.94055800 \\
\hline $\mathrm{H}$ & 4.45970100 & -2.85138700 & -2.15195500 \\
\hline $\mathrm{H}$ & 7.44042500 & -0.65002800 & 0.07582000 \\
\hline $\mathrm{H}$ & 7.94586700 & -2.13409200 & -0.78244800 \\
\hline $\mathrm{H}$ & 7.43590600 & -0.69395100 & -1.70105200 \\
\hline $\mathrm{C}$ & -1.46784500 & -4.05660200 & 1.36200500 \\
\hline $\mathrm{C}$ & -1.93306400 & -2.96049200 & 0.61753500 \\
\hline $\mathrm{C}$ & -1.25462100 & -2.66251500 & -0.58503300 \\
\hline $\mathrm{C}$ & -0.23279800 & -3.49616200 & -1.07389500 \\
\hline $\mathrm{C}$ & 0.21108100 & -4.55169500 & -0.27445000 \\
\hline $\mathrm{C}$ & -0.38404500 & -4.82323200 & 0.95298400 \\
\hline $\mathrm{C}$ & 0.01840800 & -1.92523400 & -2.99660000 \\
\hline $\mathrm{C}$ & -0.86507800 & -1.06771600 & -2.31116600 \\
\hline $\mathrm{C}$ & -1.01814500 & 0.28454800 & -2.71496600 \\
\hline $\mathrm{C}$ & -0.41242700 & 0.66196100 & -3.92636500 \\
\hline $\mathrm{C}$ & 0.40453600 & -0.19689300 & -4.65757300 \\
\hline $\mathrm{C}$ & 0.64191700 & -1.47731100 & -4.16408400 \\
\hline $\mathrm{H}$ & -1.96543100 & -4.31843300 & 2.28883800 \\
\hline $\mathrm{H}$ & 1.02242100 & -5.18879700 & -0.62426800 \\
\hline $\mathrm{H}$ & -0.02816700 & -5.65052300 & 1.57113900 \\
\hline $\mathrm{H}$ & -0.60360200 & 1.64452100 & -4.32905000 \\
\hline $\mathrm{H}$ & 0.84132500 & 0.13015200 & -5.60444400 \\
\hline $\mathrm{H}$ & 1.29949800 & -2.15137500 & -4.71337900 \\
\hline $\mathrm{C}$ & 0.26831700 & -3.34615700 & -2.50521400 \\
\hline $\mathrm{C}$ & -0.53824700 & -4.32797700 & -3.39228000 \\
\hline $\mathrm{H}$ & -0.21866500 & -4.25656000 & -4.44454600 \\
\hline $\mathrm{H}$ & -1.61579100 & -4.10593900 & -3.34806300 \\
\hline $\mathrm{H}$ & -0.39068200 & -5.36543100 & -3.05304900 \\
\hline $\mathrm{C}$ & 1.77161900 & -3.66704700 & -2.59583100 \\
\hline $\mathrm{H}$ & 1.98473400 & -4.69353200 & -2.26818800 \\
\hline $\mathrm{H}$ & 2.31488900 & -2.95849100 & -1.95843800 \\
\hline $\mathrm{H}$ & 2.13668600 & -3.59481400 & -3.62944200 \\
\hline $\mathrm{O}$ & -1.64490800 & -1.55322000 & -1.30303900 \\
\hline
\end{tabular}




\begin{tabular}{|c|c|c|c|}
\hline $\mathrm{P}$ & -3.42025900 & -2.00031800 & 1.17196500 \\
\hline $\mathrm{P}$ & -1.95058700 & 1.57409900 & -1.74866900 \\
\hline $\mathrm{C}$ & -3.26117600 & -1.94993200 & 3.09359600 \\
\hline $\mathrm{C}$ & -1.81869700 & -1.47953900 & 3.34367500 \\
\hline $\mathrm{H}$ & -1.52136900 & -0.69467500 & 2.63498800 \\
\hline $\mathrm{H}$ & -1.07977400 & -2.28049900 & 3.22231700 \\
\hline $\mathrm{H}$ & -1.72773200 & -1.07898500 & 4.36782200 \\
\hline $\mathrm{C}$ & -4.22587900 & -0.84470900 & 3.56645400 \\
\hline $\mathrm{H}$ & -5.27809100 & -1.08706700 & 3.36006800 \\
\hline $\mathrm{H}$ & -4.01499900 & 0.11975000 & 3.09423500 \\
\hline $\mathrm{H}$ & -4.12580000 & -0.71090600 & 4.65761200 \\
\hline $\mathrm{C}$ & -3.58176000 & -3.18992700 & 3.94193300 \\
\hline $\mathrm{H}$ & -4.63384200 & -3.49435700 & 3.84565300 \\
\hline $\mathrm{H}$ & -3.41676100 & -2.94000300 & 5.00514700 \\
\hline $\mathrm{H}$ & -2.95445000 & -4.06268700 & 3.72640700 \\
\hline $\mathrm{C}$ & -4.81651000 & -3.23135200 & 0.68332400 \\
\hline $\mathrm{C}$ & -4.90026400 & -3.15467200 & -0.85078400 \\
\hline $\mathrm{H}$ & -3.95351300 & -3.45122000 & -1.32778400 \\
\hline $\mathrm{H}$ & -5.15269500 & -2.14326400 & -1.19373200 \\
\hline $\mathrm{H}$ & -5.68460700 & -3.84119600 & -1.21473800 \\
\hline $\mathrm{C}$ & -6.13666800 & -2.71135100 & 1.27214300 \\
\hline $\mathrm{H}$ & -6.18260300 & -2.83179600 & 2.36427100 \\
\hline $\mathrm{H}$ & -6.98443900 & -3.27489800 & 0.84474700 \\
\hline $\mathrm{H}$ & -6.29502900 & -1.64656100 & 1.03777600 \\
\hline $\mathrm{C}$ & -4.61381800 & -4.69949400 & 1.07625700 \\
\hline $\mathrm{H}$ & -3.71958500 & -5.12690300 & 0.60199600 \\
\hline $\mathrm{H}$ & -5.48194900 & -5.29066800 & 0.73306400 \\
\hline $\mathrm{H}$ & -4.52877300 & -4.84506600 & 2.16009600 \\
\hline $\mathrm{C}$ & -3.66747700 & 1.45289300 & -2.60695500 \\
\hline $\mathrm{C}$ & -4.25037800 & 0.12599500 & -2.09950700 \\
\hline $\mathrm{H}$ & -4.33218600 & 0.10982800 & -1.00572400 \\
\hline $\mathrm{H}$ & -3.63847300 & -0.73442100 & -2.39608900 \\
\hline $\mathrm{H}$ & -5.25886900 & -0.01106800 & -2.52506000 \\
\hline $\mathrm{C}$ & -3.56937700 & 1.40104400 & -4.13751500 \\
\hline $\mathrm{H}$ & -4.58875700 & 1.30936400 & -4.55065400 \\
\hline $\mathrm{H}$ & -2.99048100 & 0.53943100 & -4.49439800 \\
\hline $\mathrm{H}$ & -3.13097100 & 2.31645300 & -4.56085300 \\
\hline $\mathrm{C}$ & -4.61927200 & 2.59342000 & -2.22231300 \\
\hline $\mathrm{H}$ & -5.64614500 & 2.30033900 & -2.49659300 \\
\hline $\mathrm{H}$ & -4.39434900 & 3.52131100 & -2.76250800 \\
\hline $\mathrm{H}$ & -4.61716200 & 2.80441100 & -1.15066100 \\
\hline $\mathrm{C}$ & -1.12626700 & 3.29255700 & -2.13737500 \\
\hline $\mathrm{C}$ & -0.70048300 & 3.69504300 & -3.56362200 \\
\hline $\mathrm{H}$ & -0.51375700 & 4.78226400 & -3.55915800 \\
\hline
\end{tabular}


$\begin{array}{lll}-2.09485400 & 4.39957100 & -1.67127300\end{array}$

$\begin{array}{lll}-1.50675000 & 5.29419800 & -1.40597100\end{array}$

$\begin{array}{lll}-2.69342700 \quad 4.12465100 & -0.80060300\end{array}$

$\begin{array}{lll}0.13389700 & 3.31948500 & -1.25361600\end{array}$

$\begin{array}{lll}0.74247200 & 2.41082900 & -1.34389400\end{array}$

$\begin{array}{lll}-0.11508500 & 3.40952700 & -0.19028700\end{array}$

$\begin{array}{lll}0.75386400 & 4.18817600 & -1.52676000\end{array}$

$\begin{array}{lll}-1.47167300 & 1.40002100 & 0.53451100\end{array}$

$\begin{array}{lll}-1.76851600 & 1.43725200 & 4.00458400\end{array}$

$\begin{array}{lll}-1.83270000 & 0.88168900 & 2.72184700\end{array}$

$\begin{array}{lll}-1.84213100 & 1.75818700 \quad 1.61532400\end{array}$

$\begin{array}{lll}-1.56806600 & 3.12117600 \quad 1.75291100\end{array}$

$-1.48426100 \quad 3.63129300 \quad 3.05720200$

$\begin{array}{lll}-1.63151100 & 2.81475300 \quad 4.17683300\end{array}$

$\begin{array}{lll}-1.78390300 & 3.32347400 & -0.72823700\end{array}$

$\begin{array}{lll}-2.37573200 & 2.05187100 & -0.68890200\end{array}$

$\begin{array}{lll}-3.29302900 & 1.64481200 & -1.69146200\end{array}$

$\begin{array}{lll}-3.31470600 & 2.39587300 & -2.87661900\end{array}$

$\begin{array}{lll}-2.52195500 & 3.53212700 & -3.03693400\end{array}$

$\begin{array}{lll}-1.81526100 & 4.02197500 & -1.94236700\end{array}$

$\begin{array}{lll}-1.77117900 & 0.78085500 \quad 4.87503100\end{array}$

$-1.28056200 \quad 4.69317100 \quad 3.20073900$

$\begin{array}{lll}-1.57768700 & 3.24275400 & 5.18011600\end{array}$

$\begin{array}{lll}-3.98633500 & 2.10900300 & -3.68169800\end{array}$

$\begin{array}{lll}-2.52433000 \quad 4.07716900 & -3.98356200\end{array}$

$\begin{array}{lll}-1.29815900 & 4.98002100 & -2.01708400\end{array}$

$\begin{array}{lll}-1.26564300 & 4.00124200 & 0.54044000\end{array}$

$\begin{array}{lll}0.25402400 & 4.25844900 & 0.44411300\end{array}$

$\begin{array}{lll}0.45353900 & 5.04421500 & -0.30350900\end{array}$

$\begin{array}{lll}0.80229300 & 3.36059600 & 0.13219300\end{array}$

$\begin{array}{lll}0.65132800 \quad 4.60440000 & 1.41078400\end{array}$

$\begin{array}{lll}-2.00572100 & 5.35235500 & 0.69122600\end{array}$

$\begin{array}{lll}-1.63583800 & 5.90760900 & 1.56520100\end{array}$

$\begin{array}{lll}-3.09045300 & 5.20428700 \quad 0.80843700\end{array}$

$-1.83943600 \quad 5.99226000 \quad-0.18627700$

$\begin{array}{lll}-2.10883400 & 1.19228300 & 0.37367100\end{array}$

$\begin{array}{lll}-3.07090300 & -1.81662400 & 2.93943300\end{array}$

$-3.54185900 \quad-1.48017700 \quad 4.36127600$

$\begin{array}{lll}-3.71573300 & -0.40276100 & 4.49192600\end{array}$

$-2.85356600 \quad-1.82352500 \quad 5.14205400$ 


\begin{tabular}{|c|c|c|}
\hline-4.50783200 & -1.98588700 & 4.53471600 \\
\hline-4.16345300 & -1.35695300 & 1.96619500 \\
\hline-5.05421500 & -1.99851600 & 2.07195400 \\
\hline-3.83995600 & -1.38718100 & 0.92161300 \\
\hline-4.46619300 & -0.32651600 & 2.18874000 \\
\hline-2.86297100 & -3.32999600 & 2.79416000 \\
\hline-2.52365700 & -3.59887900 & 1.79087800 \\
\hline-3.82248100 & -3.84487900 & 2.96698600 \\
\hline-2.14248300 & -3.73327100 & 3.51748400 \\
\hline 0.00796500 & -1.39102800 & 3.42885800 \\
\hline-0.30876400 & -2.04400500 & 4.78090900 \\
\hline 0.64629100 & -2.31161100 & 5.26574500 \\
\hline-0.89741300 & -2.96522400 & 4.70044700 \\
\hline-0.82816700 & -1.35432400 & 5.46091200 \\
\hline 0.91053500 & -0.17990800 & 3.71057300 \\
\hline 1.11979500 & 0.42140600 & 2.81920300 \\
\hline 1.85953500 & -0.55857800 & 4.13576200 \\
\hline 0.48539400 & 0.47900700 & 4.47723800 \\
\hline 0.76682100 & -2.38874000 & 2.52982500 \\
\hline 0.14065600 & -3.24523200 & 2.23650400 \\
\hline 1.63244400 & -2.80073600 & 3.08128600 \\
\hline 1.11744200 & -1.89743700 & 1.61045600 \\
\hline-5.39993600 & -0.21488000 & -2.89619800 \\
\hline-6.30328700 & 0.73251000 & -3.69925100 \\
\hline-5.81320100 & 1.66576400 & -4.00449800 \\
\hline-7.21317700 & 0.99491200 & -3.14332800 \\
\hline-6.62872700 & 0.21909100 & -4.62119600 \\
\hline-6.25449500 & -1.43674700 & -2.49540100 \\
\hline-7.10669900 & -1.16563300 & -1.85877100 \\
\hline-5.66275800 & -2.19258900 & -1.96286800 \\
\hline-6.66017600 & -1.91233800 & -3.40483000 \\
\hline-4.26712100 & -0.73775700 & -3.79804500 \\
\hline-4.70580800 & -1.18172600 & -4.70799500 \\
\hline-3.69221100 & -1.53260900 & -3.30317900 \\
\hline-3.56499500 & 0.04447800 & -4.11529300 \\
\hline-5.84415800 & 1.66205500 & -0.42128000 \\
\hline-6.20706600 & 2.90950600 & -1.23730900 \\
\hline-6.81226900 & 3.58592200 & -0.60794200 \\
\hline-6.79799300 & 2.68602100 & -2.13193900 \\
\hline-5.31203800 & 3.46735800 & -1.54700700 \\
\hline-7.11449500 & 0.88042100 & -0.05303400 \\
\hline-7.69635800 & 0.59140300 & -0.93939500 \\
\hline-7.76844500 & 1.51097100 & 0.57372700 \\
\hline 6.882058 & -0.03179100 & 0.51955700 \\
\hline
\end{tabular}


$-5.18025900 \quad 2.15385100 \quad 0.87674200$

$\begin{array}{lll}-4.35326200 & 2.84698400 & 0.67573200\end{array}$

$-4.79318000 \quad 1.33698800$

1.49599100

$\begin{array}{lll}-5.92429300 \quad 2.70607000 & 1.47551400\end{array}$

$\begin{array}{lll}-1.51221700 & -0.88702700 & 2.36035900\end{array}$

$\begin{array}{lll}-4.60874600 & 0.41266200 & -1.24720900\end{array}$

$\begin{array}{lll}-1.16338100 & -1.34449100 & -1.73371800\end{array}$

$\begin{array}{lll}-1.04187700 & -2.31414600 & -0.68673300\end{array}$

$\begin{array}{lll}-1.99486000 & -3.43043300 & -0.54796700\end{array}$

$\begin{array}{lll}-1.51339600 & -4.70511400 & -0.19013300\end{array}$

$\begin{array}{lll}-3.77513100 & -2.30808000 & -1.02446300\end{array}$

$\begin{array}{lll}-0.01488400 & -2.63271300 & -0.47411000\end{array}$

$\begin{array}{lll}-3.37682600 & -3.29106500 & -0.77126800\end{array}$

$\begin{array}{lll}-2.37764600 & -5.79216900 & -0.04631700\end{array}$

$\begin{array}{lll}-3.75000600 & -5.63275700 & -0.25986200\end{array}$

$\begin{array}{lll}-4.24169600 & -4.37466500 & -0.62727400\end{array}$

$\begin{array}{lll}-1.97691300 & -6.76961200 & 0.23528600\end{array}$

$\begin{array}{lll}-4.43047800 & -6.47974400 & -0.14263400\end{array}$

$\begin{array}{lll}-5.31347900 & -4.23455600 & -0.79247800\end{array}$

$\begin{array}{lll}-0.10256900 & -0.50572600 & -2.02658700\end{array}$

$\begin{array}{lll}0.89123800 & -0.79206700 & -1.66924500\end{array}$

$\begin{array}{lll}-0.44111900 & -4.83570500 & -0.02183000\end{array}$

$\begin{array}{lll}-2.13246300 & -1.17336500 & -2.20497400\end{array}$

$\begin{array}{lll}-0.17046800 & 0.66187900 & -2.95390000\end{array}$

$0.45334000 \quad 0.47007700 \quad-3.84948500$

$\begin{array}{lll}0.23432200 & 1.54992100 & -2.44395700\end{array}$

$\begin{array}{lll}-1.18993300 & 0.86898700 & -3.30009300\end{array}$

$2.47269200 \quad-0.58100900 \quad-0.15362000$

$\begin{array}{lll}0.45956900 & 0.65413000 & 0.74554700\end{array}$

$\begin{array}{lll}1.93106300 & 0.92705900 & 0.28445300\end{array}$

$3.80598200 \quad-0.92526100 \quad-0.71698300$

$\begin{array}{lll}3.88636600 & -0.88096400 & 2.19984200\end{array}$

$\begin{array}{lll}3.31465100 & 1.19356200 & -2.94618300\end{array}$

$1.99823600 \quad 1.84159400 \quad-0.95244300$

$\begin{array}{lll}2.81577300 & 1.35665900 & 1.47026200\end{array}$

$3.64770200 \quad-1.26068100 \quad-2.17068200$

$\begin{array}{lll}5.01147800 & 0.29965900 & -0.40855800\end{array}$

$\begin{array}{lll}4.26320000 & -2.21265900 & -0.11063800\end{array}$

$\begin{array}{lll}4.18082400 & -2.55164200 & -2.41734000\end{array}$

$\begin{array}{lll}5.34698700 & 1.36383400 & -1.23707800\end{array}$

$\begin{array}{lll}5.79253300 & 0.33317800 & 0.73689900\end{array}$

$\begin{array}{lll}4.00704700 & -3.26798700 & -1.02357800\end{array}$

$\begin{array}{lll}3.40650400 & -3.19982500 & -3.56249400\end{array}$

$\begin{array}{lll}5.66117400 & -2.40865000 & -2.80336600\end{array}$ 


\begin{tabular}{|c|c|c|}
\hline 6.09762300 & 2.33832000 & -0.46140700 \\
\hline 6.72777800 & 1.43616000 & 0.66530800 \\
\hline 4.99687700 & -4.40149300 & -0.76620200 \\
\hline 2.57269900 & -3.77811300 & -0.82273100 \\
\hline 3.70670600 & -4.24961700 & -3.71122400 \\
\hline 3.60417600 & -2.66031900 & -4.50359900 \\
\hline 2.32436000 & -3.16604700 & -3.37795600 \\
\hline 6.11719300 & -3.36760800 & -3.09529500 \\
\hline 5.74325200 & -1.71991200 & -3.65966900 \\
\hline 6.24103100 & -1.98562500 & -1.96995400 \\
\hline 7.10871000 & 3.01458200 & -1.37646900 \\
\hline 5.08588800 & 3.35179600 & 0.07625400 \\
\hline 6.79819000 & 2.09480900 & 2.03690500 \\
\hline 8.08473900 & 0.84362500 & 0.28348200 \\
\hline 4.78670800 & -4.86568100 & 0.21121500 \\
\hline 4.91852500 & -5.18883400 & -1.53339900 \\
\hline 6.03126600 & -4.03234600 & -0.74404600 \\
\hline 2.43578300 & -4.04600800 & 0.23675100 \\
\hline 2.34995000 & -4.66728300 & -1.43332800 \\
\hline 1.85222600 & -2.98867200 & -1.06451600 \\
\hline 6.58082700 & 3.62512700 & -2.12582000 \\
\hline 7.76606000 & 3.68759700 & -0.80402100 \\
\hline 7.73358400 & 2.28643600 & -1.91092300 \\
\hline 5.58339900 & 4.14598000 & 0.65395800 \\
\hline 4.56990400 & 3.82476400 & -0.77318400 \\
\hline 4.31674500 & 2.85860800 & 0.69190000 \\
\hline 7.24469400 & 1.39628500 & 2.76239700 \\
\hline 7.42792500 & 2.99791600 & 2.01204400 \\
\hline 5.79858200 & 2.37400900 & 2.39426200 \\
\hline 8.04667900 & 0.36271400 & -0.70493700 \\
\hline 8.87706800 & 1.60707700 & 0.27203600 \\
\hline 8.36008300 & 0.07436600 & 1.02093900 \\
\hline 0.91515100 & -0.53405800 & 0.15694700 \\
\hline 1.50865700 & -1.02147400 & 4.13241800 \\
\hline 1.70390900 & -0.66658000 & 2.79133900 \\
\hline 1.82914200 & -1.71639200 & 1.84763300 \\
\hline 1.46069400 & -3.02770500 & 2.17878100 \\
\hline 1.21381500 & -3.32345300 & 3.52557500 \\
\hline 1.30460200 & -2.34685900 & 4.51348000 \\
\hline 1.81051100 & -3.61225500 & -0.22987500 \\
\hline 2.47007900 & -2.37907500 & -0.35364300 \\
\hline 3.31337200 & -2.10744200 & -1.46311700 \\
\hline 3.27732100 & -3.01055500 & -2.53498500 \\
\hline
\end{tabular}




\begin{tabular}{|c|c|c|}
\hline 2.47458200 & -4.14979600 & -2.50854000 \\
\hline 1.78309800 & -4.46228900 & -1.34244700 \\
\hline 1.47097700 & -0.24043600 & 4.89022600 \\
\hline 0.93894700 & -4.34159400 & 3.80490000 \\
\hline 1.15246900 & -2.60307400 & 5.56407500 \\
\hline 3.91219200 & -2.83855700 & -3.39983100 \\
\hline 2.44015200 & -4.82058000 & -3.37006700 \\
\hline 1.22259900 & -5.39685700 & -1.28157700 \\
\hline 1.21028600 & -4.07436300 & 1.09535600 \\
\hline-0.31451200 & -4.28335500 & 0.93865200 \\
\hline-0.51330200 & -5.16023700 & 0.30073000 \\
\hline-0.79623200 & -3.41480900 & 0.47109000 \\
\hline-0.78607100 & -4.46198800 & 1.91682900 \\
\hline 1.88088700 & -5.41053700 & 1.49260000 \\
\hline 1.44049400 & -5.81657300 & 2.41525800 \\
\hline 2.96211200 & -5.27954500 & 1.65514700 \\
\hline 1.74253200 & -6.16943100 & 0.70930600 \\
\hline 2.35383200 & -1.41533700 & 0.61902700 \\
\hline 3.07364200 & 1.93450600 & 2.71272800 \\
\hline 3.62079600 & 1.57215700 & 4.09918600 \\
\hline 3.77288600 & 0.48962000 & 4.20997000 \\
\hline 2.98117200 & 1.92067200 & 4.91983000 \\
\hline 4.60459100 & 2.05717800 & 4.22545100 \\
\hline 4.04162000 & 1.38842700 & 1.65680900 \\
\hline 5.03445100 & 1.85585900 & 1.76978400 \\
\hline 3.68625800 & 1.57289300 & 0.63908800 \\
\hline 4.15756100 & 0.30397600 & 1.76227500 \\
\hline 2.96287700 & 3.45757500 & 2.57365500 \\
\hline 2.45639200 & 3.74921400 & 1.65050300 \\
\hline 3.97439300 & 3.89482900 & 2.54318300 \\
\hline 2.43537100 & 3.92287700 & 3.41666500 \\
\hline-0.00386300 & 1.76236400 & 3.33774300 \\
\hline 0.39506300 & 2.43414700 & 4.65827100 \\
\hline-0.52180200 & 2.79769800 & 5.15446400 \\
\hline 1.05317300 & 3.30071400 & 4.52543700 \\
\hline 0.87719900 & 1.73524700 & 5.35569600 \\
\hline-1.02262400 & 0.65921900 & 3.66243700 \\
\hline-1.24978800 & 0.00450500 & 2.81246500 \\
\hline-1.94704200 & 1.15427000 & 4.01673200 \\
\hline-0.69343600 & 0.01597100 & 4.48677100 \\
\hline-0.68800600 & 2.79700900 & 2.42071000 \\
\hline 0.01073500 & 3.56912600 & 2.06515300 \\
\hline-1.49223300 & 3.31111800 & 2.97725300 \\
\hline .13212500 & 2.30679800 & 1.5412910 \\
\hline
\end{tabular}




\begin{tabular}{|c|c|c|}
\hline 5.09049100 & -0.30432100 & -3.16704400 \\
\hline 6.02823800 & -1.25519600 & -3.92578200 \\
\hline 5.61372600 & -2.26231200 & -4.06436300 \\
\hline 7.00639000 & -1.35538800 & -3.43717100 \\
\hline 6.21394300 & -0.84234500 & -4.93309200 \\
\hline 5.80250000 & 1.05970000 & -3.04220400 \\
\hline 6.70871000 & 1.00752000 & -2.42377800 \\
\hline 5.14193200 & 1.82200900 & -2.60580700 \\
\hline 6.10357600 & 1.41277100 & -4.04355900 \\
\hline 3.82416200 & -0.07631300 & -4.00995200 \\
\hline 4.10863200 & 0.37804100 & -4.97467800 \\
\hline 3.12770300 & 0.61679700 & -3.52100300 \\
\hline 3.27633500 & -1.00176700 & -4.22815100 \\
\hline 5.99830300 & -1.74736100 & -0.54269700 \\
\hline 6.29511500 & -3.11155100 & -1.17969900 \\
\hline 7.11387000 & -3.59942600 & -0.62131500 \\
\hline 6.61132700 & -3.04426800 & -2.22659100 \\
\hline 5.42345700 & -3.77912400 & -1.12940400 \\
\hline 7.26211200 & -0.87395000 & -0.53606200 \\
\hline 7.68578300 & -0.73755000 & -1.54076900 \\
\hline 8.03979600 & -1.35485000 & 0.08218900 \\
\hline 7.06577900 & 0.12149000 & -0.10683200 \\
\hline 5.58897100 & -2.00502900 & 0.91798600 \\
\hline 4.68319600 & -2.62235100 & 0.99428100 \\
\hline 5.42030500 & -1.07420600 & 1.47370000 \\
\hline 6.39981900 & -2.55432500 & 1.42664500 \\
\hline 1.43688100 & 1.07772900 & 2.25359700 \\
\hline 4.55844500 & -0.73358900 & -1.35462900 \\
\hline 1.17672800 & 1.65460400 & -1.72781200 \\
\hline 1.04597400 & 2.74812900 & -0.84121400 \\
\hline 2.06627600 & 3.79771800 & -0.69812900 \\
\hline 3.43128700 & 3.58376100 & -0.96689100 \\
\hline 0.61419900 & 5.27459900 & -0.08387000 \\
\hline 0.02799400 & 3.11818600 & -0.67549700 \\
\hline 1.67160800 & 5.08619500 & -0.28869100 \\
\hline 4.36323900 & 4.60887700 & -0.81562500 \\
\hline 3.95700900 & 5.88040200 & -0.39443200 \\
\hline 2.60316500 & 6.11407000 & -0.13601900 \\
\hline 5.41846200 & 4.41129000 & -1.02206200 \\
\hline 4.68933900 & 6.68217900 & -0.27247200 \\
\hline 2.26996100 & 7.10386300 & 0.18688200 \\
\hline 0.12263900 & 0.75313400 & -1.88771500 \\
\hline-0.90355000 & 1.11251000 & -1.75389500 \\
\hline 3.77020000 & 2.59485700 & -1.27566100 \\
\hline
\end{tabular}




\begin{tabular}{|c|c|c|c|}
\hline $\mathrm{H}$ & 2.17233900 & 1.38760600 & -2.09094500 \\
\hline $\mathrm{C}$ & 0.28480100 & -0.54899300 & -2.61603800 \\
\hline $\mathrm{H}$ & -0.11420300 & -0.47582200 & -3.64816700 \\
\hline $\mathrm{H}$ & -0.25744000 & -1.34125800 & -2.07780700 \\
\hline $\mathrm{H}$ & 1.33687100 & -0.84529400 & -2.69559000 \\
\hline $\mathrm{O}$ & -2.48104700 & 0.59150300 & -0.09672000 \\
\hline $\mathrm{O}$ & -0.36097200 & -0.53193600 & 0.68970000 \\
\hline $\mathrm{P}$ & -1.85935300 & -0.88059300 & 0.34368800 \\
\hline B & -3.79332300 & 0.87482600 & -0.73927700 \\
\hline K & -3.99834400 & 0.80410900 & 2.18202600 \\
\hline K & -3.05948100 & -1.17537300 & -2.96674500 \\
\hline $\mathrm{O}$ & -1.96431600 & -1.84600400 & -0.84737900 \\
\hline $\mathrm{O}$ & -2.63409600 & -1.31008600 & 1.59938900 \\
\hline $\mathrm{O}$ & -3.55448700 & 1.24757900 & -2.17102500 \\
\hline B & -4.93958500 & -0.42059100 & -0.51512100 \\
\hline $\mathrm{O}$ & -4.36075800 & 2.12199700 & -0.14276400 \\
\hline $\mathrm{C}$ & -4.15491100 & 2.50540100 & -2.43642300 \\
\hline $\mathrm{O}$ & -5.20283700 & -1.47273900 & -1.38249400 \\
\hline $\mathrm{O}$ & -5.72456200 & -0.54203800 & 0.62225800 \\
\hline $\mathrm{C}$ & -4.12271400 & 3.20739000 & -1.02518100 \\
\hline $\mathrm{C}$ & -3.35096900 & 3.22149800 & -3.51888400 \\
\hline C & -5.59255700 & 2.27046200 & -2.92572900 \\
\hline C & -5.89914000 & -2.51473800 & -0.64265000 \\
\hline $\mathrm{C}$ & -6.60809000 & -1.68284500 & 0.48817300 \\
\hline $\mathrm{C}$ & -5.20487500 & 4.26490900 & -0.82219900 \\
\hline C & -2.74501100 & 3.81208500 & -0.72021600 \\
\hline $\mathrm{H}$ & -3.71027700 & 4.25139500 & -3.67493100 \\
\hline $\mathrm{H}$ & -3.45039000 & 2.68546400 & -4.47715700 \\
\hline $\mathrm{H}$ & -2.28303000 & 3.25554300 & -3.26484600 \\
\hline $\mathrm{H}$ & -6.08728900 & 3.19911600 & -3.25034300 \\
\hline $\mathrm{H}$ & -5.56811600 & 1.58229600 & -3.78597600 \\
\hline $\mathrm{H}$ & -6.20396200 & 1.80650900 & -2.13786600 \\
\hline $\mathrm{C}$ & -6.84349000 & -3.23799600 & -1.59139700 \\
\hline $\mathrm{C}$ & -4.83315400 & -3.46890700 & -0.10122400 \\
\hline C & -6.69570400 & -2.38704200 & 1.83548400 \\
\hline $\mathrm{C}$ & -7.97723300 & -1.14010400 & 0.07669800 \\
\hline $\mathrm{H}$ & -5.09307000 & 4.72786900 & 0.17172900 \\
\hline $\mathrm{H}$ & -5.13159800 & 5.06705500 & -1.57430400 \\
\hline $\mathrm{H}$ & -6.20961000 & 3.82396300 & -0.87319400 \\
\hline $\mathrm{H}$ & -2.70707900 & 4.09490800 & 0.34317800 \\
\hline $\mathrm{H}$ & -2.53175600 & 4.71041800 & -1.32050000 \\
\hline $\mathrm{H}$ & -1.96174800 & 3.06619300 & -0.89657200 \\
\hline $\mathrm{H}$ & -6.26126900 & -3.79097000 & -2.34513600 \\
\hline $\mathrm{H}$ & -7.46137900 & -3.96953300 & -1.04749800 \\
\hline
\end{tabular}




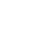

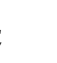

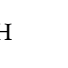

.

C

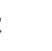

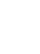

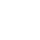

H

C

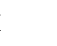

H

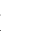

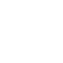

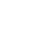

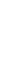

C

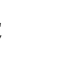

.

.

C

C

C

C

C

C

\begin{tabular}{|c|c|c|}
\hline 2.01344000 & 3.89208400 & -0.83702800 \\
\hline 1.01209600 & 4.03923500 & 0.20024400 \\
\hline 1.38076400 & 4.28538200 & 1.61856300 \\
\hline 2.13193700 & 3.36924400 & 2.38450800 \\
\hline 0.35016700 & 6.18513400 & 1.69273600 \\
\hline 0.16982600 & 4.66839300 & -0.11027000 \\
\hline 0.94928300 & 5.46558400 & 2.25752200 \\
\hline 2.46254700 & 3.64114700 & 3.71485600 \\
\hline 2.04271500 & 4.82762100 & 4.32520300 \\
\hline 1.27561000 & 5.73518400 & 3.58833000 \\
\hline 3.03595200 & 2.90655800 & 4.28852100 \\
\hline 2.29724300 & 5.03639200 & 5.36738100 \\
\hline 0.92824500 & 6.66186000 & 4.05336500 \\
\hline 1.62917100 & 3.79638300 & -2.16017600 \\
\hline 0.65353700 & 4.19599700 & -2.45345000 \\
\hline 2.37802700 & 2.40789500 & 1.93098700 \\
\hline 3.05700200 & 3.69371800 & -0.56488300 \\
\hline 2.52225400 & 3.29316100 & -3.24893200 \\
\hline 2.58919700 & 3.99970200 & -4.09463400 \\
\hline 2.14400500 & 2.33251900 & -3.64045600 \\
\hline 3.54060900 & 3.09909200 & -2.87552000 \\
\hline 6.24866400 & -3.14893100 & 0.15603500 \\
\hline 5.50100500 & -1.96820300 & 0.01151100 \\
\hline 4.14769600 & -1.99924800 & 0.42212800 \\
\hline 3.60335200 & -3.12588300 & 1.07022000 \\
\hline 4.40307800 & -4.26279400 & 1.22070000 \\
\hline 5.71084100 & -4.29275800 & 0.73950000 \\
\hline 1.81602400 & -1.62153600 & 1.86175400 \\
\hline 2.25839600 & -0.63573900 & 0.95381500 \\
\hline 1.58016700 & 0.59406100 & 0.83263600 \\
\hline 0.67751100 & 0.91041000 & 1.86999000 \\
\hline 0.43225800 & 0.05105300 & 2.93192700 \\
\hline
\end{tabular}


$-0.94224800 \quad-1.24612900$

2.88237400

$\mathrm{H}$

$\begin{array}{lll}-7.28252500 & -3.16589800 & -0.18107600\end{array}$

$\mathrm{H}$

$\begin{array}{lll}-3.99357000 & -5.14616300 & 1.71053500\end{array}$

$-6.31537200 \quad-5.19700900$

0.84051100

$\mathrm{H}$

$\begin{array}{lll}-0.12630800 & 1.84585000 & 1.81031000\end{array}$

$0.23882800 \quad 0.35716600$

3.73518900

$-0.62298000 \quad-1.97954100$

3.62253700

$\begin{array}{lll}-2.15579700 & -3.07539300 & 1.54338400\end{array}$

$\begin{array}{lll}-1.22562900 & -3.51313200 & 0.37788800\end{array}$

$\begin{array}{lll}-0.17266600 & -3.38678700 & 0.67317700\end{array}$

$\begin{array}{lll}-1.38711300 & -2.89300200 & -0.51453800\end{array}$

$\begin{array}{lll}-1.41532300 & -4.56612400 & 0.11195300\end{array}$

$\begin{array}{lll}-1.90709000 & -4.00574800 & 2.73720100\end{array}$

$\begin{array}{lll}-2.10383600 & -5.05335400 \quad 2.46957500\end{array}$

$\begin{array}{lll}-2.53855900 & -3.74392400 & 3.59968100\end{array}$

$\begin{array}{lll}-0.85466800 & -3.95938200 & 3.05056200\end{array}$

$\begin{array}{lll}-3.36210400 & -0.89685300 & 0.16914700\end{array}$

$\begin{array}{lll}-7.49531100 & -0.00853900 & 0.84338100\end{array}$

$\begin{array}{lll}-8.44825700 & -1.11899300 \quad 1.30171100\end{array}$

$\begin{array}{lll}-7.90458000 & -1.97396500 & 1.72650900\end{array}$

$\begin{array}{lll}-9.09879800 & -1.48640400 & 0.49853400\end{array}$

$\begin{array}{lll}-9.10385500 & -0.72565000 & 2.09924300\end{array}$

$\begin{array}{lll}-6.59947100 & 0.37451500 \quad 2.03618800\end{array}$

$\begin{array}{lll}-5.95357400 & -0.46167500 & 2.34735500\end{array}$

$\begin{array}{lll}-7.22908700 & 0.64716700 & 2.90098400\end{array}$

$\begin{array}{lll}-5.95483000 & 1.23362200 & 1.80507100\end{array}$

$\begin{array}{lll}-8.30520300 & 1.22583400 & 0.41429600\end{array}$

$\begin{array}{lll}-7.65124400 & 2.03725800 & 0.05734300\end{array}$

$\begin{array}{lll}-8.88026100 & 1.61258200 & 1.27344600\end{array}$

$\begin{array}{lll}-9.02612600 & 0.99064800 & -0.38283200\end{array}$

$\begin{array}{lll}-7.19344400 & -0.85792100 & -2.18726200\end{array}$

$\begin{array}{lll}-6.24243500 & -1.75969400 & -2.99292000\end{array}$

$\begin{array}{lll}-5.22345800 & -1.34534100 & -3.03458100\end{array}$

$\begin{array}{lll}-6.60891100 & -1.85095800 & -4.03025600\end{array}$

$\begin{array}{lll}-6.17444700 & -2.77147700 & -2.56916600\end{array}$

$\begin{array}{lll}-7.36268300 & 0.47211400 & -2.95001200\end{array}$

$\begin{array}{lll}-8.00870000 & 1.17955000 & -2.40919600\end{array}$

$\begin{array}{lll}-7.82687500 & 0.28426800 & -3.93402100\end{array}$

$\begin{array}{lll}-6.39457900 & 0.96562900 & -3.12073600\end{array}$

$\begin{array}{lll}-8.56660200 & -1.53048400 & -2.07890400\end{array}$

$\begin{array}{lll}-9.30084300 & -0.89393600 & -1.56526300\end{array}$

$\begin{array}{lll}-8.53151600 & -2.50049100 & -1.56441700\end{array}$

$\begin{array}{lll}-8.95830900 & -1.72144700 & -3.09400400\end{array}$

$\begin{array}{lll}-2.71324300 & 3.17019900 & -0.02514300\end{array}$ 
$\begin{array}{lll}-2.69058400 \quad 4.30012700 & -1.06503900\end{array}$

$\begin{array}{lll}-3.07539100 & 3.97576000 & -2.04194400\end{array}$

$\begin{array}{lll}-1.67519300 & 4.69604100 & -1.21623500\end{array}$

$-3.32726600 \quad 5.13344000 \quad-0.72109200$

$\begin{array}{lll}-2.24327000 & 3.72269700 & 1.33197200\end{array}$

$\begin{array}{lll}-2.84897100 \quad 4.61001700 & 1.58357100\end{array}$

$\begin{array}{lll}-1.19205000 & 4.02297900 & 1.33518800\end{array}$

$\begin{array}{lll}-2.38685000 & 2.98875700 & 2.13714400\end{array}$

$\begin{array}{lll}-4.13900600 & 2.64725400 & 0.18191300\end{array}$

$\begin{array}{lll}-4.73544300 & 3.41513800 & 0.70594100\end{array}$

$\begin{array}{lll}-4.14009800 \quad 1.74247000 & 0.80367700\end{array}$

$\begin{array}{lll}-4.66005400 & 2.40523900 & -0.74781600\end{array}$

$\begin{array}{lll}-2.01559400 & 1.04556000 & -2.23480900\end{array}$

$\begin{array}{lll}-3.51293800 & 1.08782500 & -2.54447100\end{array}$

$\begin{array}{lll}-3.68322100 & 0.54843500 & -3.49276500\end{array}$

$\begin{array}{lll}-3.88648900 & 2.11105000 & -2.69236200\end{array}$

$\begin{array}{lll}-4.11609600 & 0.60379300 & -1.76960800\end{array}$

$\begin{array}{lll}-1.27079300 & 1.84521300 & -3.32401100\end{array}$

$\begin{array}{lll}-1.60297100 & 1.48469700 & -4.31326900\end{array}$

$-0.18817800 \quad 1.70043000 \quad-3.24628500$

$\begin{array}{lll}-1.49512100 & 2.92141700 & -3.27777700\end{array}$

$\begin{array}{lll}-1.55524500 & -0.42439000 & -2.29159200\end{array}$

$\begin{array}{lll}-2.25448300 & -1.07382700 & -1.75451800\end{array}$

$\begin{array}{lll}-0.54328600 & -0.55350200 & -1.89602200\end{array}$

$\begin{array}{lll}-1.54656700 & -0.74392700 & -3.34865700\end{array}$

$\begin{array}{lll}-1.46123300 & 1.82534000 & -0.56634100\end{array}$

$-6.25890600 \quad-0.38162900 \quad-0.57612500$

$3.54044400 \quad 0.02468600 \quad-1.26594300$

$\begin{array}{lll}1.15298800 & 0.77500300 & -1.80352900\end{array}$

$\begin{array}{lll}2.02286600 & 0.05193500 & -0.71825900\end{array}$

$\begin{array}{lll}4.63618600 & -0.98554600 & -1.00164300\end{array}$

$\begin{array}{lll}2.66153700 & -3.41917400 & -1.25346200\end{array}$

$\begin{array}{lll}4.69720200 & 1.56556500 & 0.53357800\end{array}$

$1.96799000 \quad 1.16216900 \quad 0.40426200$

$\begin{array}{lll}1.59427400 & -1.35603600 & -0.32178700\end{array}$

$5.89538500 \quad-0.20663100 \quad-1.14580100$

$\begin{array}{lll}4.47549700 & -1.65164400 & 0.58806700\end{array}$

$\begin{array}{lll}4.70427100 & -2.01084600 & -2.06376700\end{array}$

$6.71003300 \quad-0.82076100 \quad-2.13535400$

$\begin{array}{lll}4.65652200 & -0.88227200 & 1.72896600\end{array}$

$4.14127400 \quad-2.95046300 \quad 0.93482900$

$5.65677300 \quad-1.57638300 \quad-3.03375000$

$\begin{array}{lll}7.50631100 & 0.25393100 & -2.86948900\end{array}$

$\begin{array}{lll}7.66839700 & -1.79590000 & -1.43620200\end{array}$ 


\begin{tabular}{|c|c|c|}
\hline 4.10820900 & -1.59921200 & 2.86853100 \\
\hline 4.17870900 & -3.09471000 & 2.37953000 \\
\hline 6.20829800 & -2.79709200 & -3.76446600 \\
\hline 4.96583400 & -0.64004100 & -4.03533700 \\
\hline 8.07520700 & -0.17148100 & -3.71192200 \\
\hline 8.22763900 & 0.72207400 & -2.18019400 \\
\hline 6.84701800 & 1.04303900 & -3.25556200 \\
\hline 8.39692400 & -2.24372000 & -2.12999300 \\
\hline 8.22582400 & -1.24915000 & -0.65963100 \\
\hline 7.10743500 & -2.60503300 & -0.94534600 \\
\hline 4.95255700 & -1.28859400 & 4.09569300 \\
\hline 2.67922600 & -1.09214400 & 3.04835600 \\
\hline 2.99633300 & -3.95463900 & 2.80670200 \\
\hline 5.49808000 & -3.78692800 & 2.71964100 \\
\hline 5.41937700 & -3.24929900 & -4.38752200 \\
\hline 7.04070400 & -2.52252200 & -4.43214500 \\
\hline 6.56317000 & -3.56193000 & -3.06010000 \\
\hline 4.09631100 & -1.16197700 & -4.46505900 \\
\hline 5.63323700 & -0.35198500 & -4.86269100 \\
\hline 4.59141600 & 0.26243800 & -3.53559900 \\
\hline 4.84186900 & -0.22569800 & 4.36098000 \\
\hline 4.62071200 & -1.88347600 & 4.96080300 \\
\hline 6.01889000 & -1.48576200 & 3.92279100 \\
\hline 2.19077300 & -1.55959400 & 3.91577800 \\
\hline 2.70456800 & -0.00629200 & 3.21615200 \\
\hline 2.07466100 & -1.26122100 & 2.14704100 \\
\hline 3.11863300 & -4.97815100 & 2.41860400 \\
\hline 2.93205000 & -4.01963500 & 3.90389000 \\
\hline 2.04922700 & -3.54986200 & 2.42628500 \\
\hline 6.35938800 & -3.17517200 & 2.41402600 \\
\hline 5.58249400 & -4.00063800 & 3.79545000 \\
\hline 5.55299700 & -4.74187000 & 2.17530300 \\
\hline 0.80697600 & 2.25522800 & -0.64710800 \\
\hline 1.86696500 & 4.35133900 & -0.73184200 \\
\hline 0.57933200 & 4.76347800 & -0.23674700 \\
\hline 0.45982300 & 5.10487800 & 1.19662500 \\
\hline 1.11560500 & 4.32880500 & 2.17839600 \\
\hline-0.84566400 & 6.79765300 & 0.87850000 \\
\hline-0.04382100 & 5.36761000 & -0.90798300 \\
\hline-0.32704000 & 6.18957700 & 1.62488700 \\
\hline 0.97714900 & 4.63368500 & 3.53271900 \\
\hline 0.18631300 & 5.71286400 & 3.94434800 \\
\hline 4643400 & 6.49018100 & 2.9815370 \\
\hline
\end{tabular}




\begin{tabular}{|c|c|c|c|}
\hline $\mathrm{H}$ & 1.48128600 & 4.01088100 & 4.27708500 \\
\hline $\mathrm{H}$ & 0.07473600 & 5.94329300 & 5.00688200 \\
\hline $\mathrm{H}$ & -1.08503400 & 7.33616700 & 3.28914400 \\
\hline $\mathrm{C}$ & 1.99615900 & 3.81288900 & -1.99968100 \\
\hline $\mathrm{H}$ & 1.23568400 & 4.05604000 & -2.75348700 \\
\hline $\mathrm{H}$ & 1.70211500 & 3.45576700 & 1.87146400 \\
\hline $\mathrm{H}$ & 2.69093800 & 4.21082400 & -0.02478300 \\
\hline $\mathrm{C}$ & 3.23362500 & 3.14077700 & -2.50366900 \\
\hline $\mathrm{H}$ & 3.58542700 & 3.60698000 & -3.43955800 \\
\hline $\mathrm{H}$ & 3.03710200 & 2.07432700 & -2.69076100 \\
\hline $\mathrm{H}$ & 4.04846800 & 3.19093300 & -1.76590700 \\
\hline $\mathrm{C}$ & -5.66992800 & -3.55005300 & 0.24563300 \\
\hline $\mathrm{C}$ & -5.05030400 & -2.30339800 & 0.05692300 \\
\hline $\mathrm{C}$ & -3.73001900 & -2.15028100 & 0.54362700 \\
\hline $\mathrm{C}$ & -3.10268200 & -3.16802800 & 1.28939900 \\
\hline $\mathrm{C}$ & -3.78213900 & -4.37523900 & 1.48154000 \\
\hline $\mathrm{C}$ & -5.04889400 & -4.58357900 & 0.94077300 \\
\hline $\mathrm{C}$ & -1.46324500 & -1.45451600 & 2.00004000 \\
\hline $\mathrm{C}$ & -1.99493200 & -0.57275800 & 1.03922900 \\
\hline $\mathrm{C}$ & -1.43687600 & 0.70911400 & 0.85300200 \\
\hline $\mathrm{C}$ & -0.53236700 & 1.15738000 & 1.83390500 \\
\hline $\mathrm{C}$ & -0.17373400 & 0.37740400 & 2.92324800 \\
\hline $\mathrm{C}$ & -0.59422000 & -0.94812800 & 2.96832200 \\
\hline $\mathrm{H}$ & -6.67106100 & -3.70964400 & -0.14783700 \\
\hline $\mathrm{H}$ & -3.30640100 & -5.17553800 & 2.04810800 \\
\hline $\mathrm{H}$ & -5.55669100 & -5.54118400 & 1.07627200 \\
\hline $\mathrm{H}$ & -0.07644400 & 2.13721300 & 1.71431500 \\
\hline $\mathrm{H}$ & 0.51736400 & 0.77133200 & 3.66958100 \\
\hline $\mathrm{H}$ & -0.20063700 & -1.60757300 & 3.74072600 \\
\hline $\mathrm{C}$ & -1.68275300 & -2.95047000 & 1.79399000 \\
\hline $\mathrm{C}$ & -0.69205400 & -3.39739800 & 0.68315200 \\
\hline $\mathrm{H}$ & 0.33115400 & -3.13727900 & 0.98784300 \\
\hline $\mathrm{H}$ & -0.91100900 & -2.87472900 & -0.25950200 \\
\hline $\mathrm{H}$ & -0.77187700 & -4.48310900 & 0.51062700 \\
\hline $\mathrm{C}$ & -1.38639600 & -3.76209500 & 3.06168700 \\
\hline $\mathrm{H}$ & -1.49576500 & -4.83966700 & 2.87796100 \\
\hline $\mathrm{H}$ & -2.05387400 & -3.48055600 & 3.89001800 \\
\hline $\mathrm{H}$ & -0.34754300 & -3.60944000 & 3.38423500 \\
\hline $\mathrm{O}$ & -3.05812500 & -0.98071700 & 0.25773500 \\
\hline $\mathrm{C}$ & -7.34771600 & -0.57964800 & 0.58406000 \\
\hline $\mathrm{C}$ & -8.20434100 & -1.77178100 & 1.02865000 \\
\hline $\mathrm{H}$ & -7.61106600 & -2.52083900 & 1.57037100 \\
\hline $\mathrm{H}$ & -8.71760900 & -2.26989300 & 0.19708200 \\
\hline $\mathrm{H}$ & -8.98224000 & -1.41316700 & 1.72613300 \\
\hline
\end{tabular}




\begin{tabular}{|c|c|c|}
\hline-6.62485400 & -0.02906800 & 1.82824400 \\
\hline-5.91051300 & -0.75877500 & 2.24143600 \\
\hline-7.36441500 & 0.19294600 & 2.61714900 \\
\hline-6.07725900 & 0.89854900 & 1.61261500 \\
\hline-8.25339400 & 0.52052800 & 0.00682900 \\
\hline-7.66814700 & 1.38085500 & -0.35445400 \\
\hline-8.94151900 & 0.88722400 & 0.78800600 \\
\hline-8.87047900 & 0.15180300 & -0.82599400 \\
\hline-6.66265800 & -1.55972400 & -2.34227300 \\
\hline-5.53386100 & -2.37160900 & -3.00065300 \\
\hline-4.57762900 & -1.82654400 & -2.98787200 \\
\hline-5.78954700 & -2.57336800 & -4.05546500 \\
\hline-5.37524200 & -3.33812700 & -2.50184300 \\
\hline-6.93689500 & -0.31508500 & -3.21024000 \\
\hline-7.71201300 & 0.33149500 & -2.77328100 \\
\hline-7.28819900 & -0.62408200 & -4.21038000 \\
\hline-6.03033400 & 0.29319500 & -3.34295100 \\
\hline-7.93806400 & -2.40856300 & -2.29223500 \\
\hline-8.79682500 & -1.84540900 & -1.90031000 \\
\hline-7.81919300 & -3.31983200 & -1.69084500 \\
\hline-8.20144700 & -2.72982600 & -3.31586500 \\
\hline-2.99274300 & 3.02824200 & -0.14484000 \\
\hline-3.04354500 & 4.15562400 & -1.18558200 \\
\hline-3.28454500 & 3.78028500 & -2.19092800 \\
\hline-2.08892000 & 4.69684800 & -1.24305200 \\
\hline-3.82816100 & 4.87945000 & -0.90691500 \\
\hline-2.68477300 & 3.61789400 & 1.24149000 \\
\hline-3.40088800 & 4.42951900 & 1.45366300 \\
\hline-1.67803000 & 4.03990900 & 1.30842700 \\
\hline-2.79248300 & 2.86255700 & 2.03271800 \\
\hline-4.34313700 & 2.31447100 & -0.05079400 \\
\hline-5.06190100 & 2.97239500 & 0.46777200 \\
\hline-4.26505500 & 1.38211900 & 0.52091200 \\
\hline-4.77103200 & 2.06302200 & -1.02479600 \\
\hline-1.80854600 & 0.97305200 & -2.23189800 \\
\hline-3.26933700 & 0.81714000 & -2.65851500 \\
\hline-3.29073500 & 0.23826000 & -3.59801300 \\
\hline-3.75346000 & 1.78106500 & -2.86849300 \\
\hline-3.86874300 & 0.27701600 & -1.91716600 \\
\hline-1.07066600 & 1.81316400 & -3.29494900 \\
\hline-1.27803000 & 1.38634600 & -4.29153200 \\
\hline 0.01349300 & 1.78840200 & -3.13108300 \\
\hline-1.41067600 & 2.86051000 & -3.31113100 \\
\hline & & \\
\hline
\end{tabular}




\begin{tabular}{|c|c|c|c|}
\hline $\mathrm{H}$ & 6.29332500 & -2.25520700 & 3.67836800 \\
\hline $\mathrm{H}$ & 2.51944000 & -1.73106500 & 4.15975700 \\
\hline $\mathrm{H}$ & 3.22579800 & -0.19727300 & 3.59552400 \\
\hline $\mathrm{H}$ & 2.28979100 & -1.17123900 & 2.45428500 \\
\hline $\mathrm{H}$ & 2.67883200 & -5.02614800 & 2.21285900 \\
\hline $\mathrm{H}$ & 2.85607600 & -4.26509500 & 3.81160500 \\
\hline $\mathrm{H}$ & 1.89440800 & -3.46059000 & 2.54231800 \\
\hline $\mathrm{H}$ & 6.16567100 & -3.76655100 & 1.96086100 \\
\hline $\mathrm{H}$ & 5.43309600 & -4.62580500 & 3.34627500 \\
\hline $\mathrm{H}$ & 5.08482100 & -5.14884000 & 1.67799200 \\
\hline $\mathrm{Ni}$ & 0.54567500 & 2.82326900 & -0.79811100 \\
\hline \multicolumn{4}{|c|}{ 8-a } \\
\hline $\mathrm{O}$ & 0.13111200 & -1.19910500 & 0.52119800 \\
\hline $\mathrm{P}$ & 0.08689800 & -0.62549900 & 2.11467400 \\
\hline B & -0.77899500 & -0.72717900 & -0.58784700 \\
\hline $\mathrm{Ni}$ & 1.94807200 & -0.90320600 & 1.03032700 \\
\hline K & 0.51173600 & 2.14593200 & -0.20179100 \\
\hline K & -2.26989600 & -2.59146100 & 1.07192900 \\
\hline $\mathrm{O}$ & -1.30563400 & -0.54672200 & 2.68064400 \\
\hline $\mathrm{O}$ & 1.02735900 & 0.59319800 & 1.77944100 \\
\hline $\mathrm{O}$ & 1.15127300 & -1.65883100 & 2.59309500 \\
\hline $\mathrm{O}$ & -1.32796400 & -1.91430800 & -1.29981600 \\
\hline B & -2.11159900 & 0.25371500 & -0.02980500 \\
\hline $\mathrm{O}$ & 0.02942500 & -0.02616900 & -1.61449200 \\
\hline $\mathrm{C}$ & 3.27565400 & -0.15022000 & -0.30534300 \\
\hline $\mathrm{C}$ & 3.28676900 & -2.34091900 & 0.65171300 \\
\hline $\mathrm{C}$ & 3.86052900 & -1.04264500 & 0.64761600 \\
\hline $\mathrm{O}$ & -2.07975800 & 1.63364300 & 0.09897700 \\
\hline $\mathrm{C}$ & -0.61441900 & -2.07823300 & -2.52068700 \\
\hline $\mathrm{O}$ & -3.38806200 & -0.22067700 & 0.22059000 \\
\hline $\mathrm{C}$ & -0.25802200 & -0.58941900 & -2.88802900 \\
\hline $\mathrm{C}$ & 3.52372300 & 1.29637500 & -0.33384800 \\
\hline $\mathrm{H}$ & 2.98684900 & -0.57430800 & -1.27318700 \\
\hline $\mathrm{C}$ & 3.58012000 & -3.35386400 & 1.72012500 \\
\hline $\mathrm{H}$ & 2.98011700 & -2.75660500 & -0.31757500 \\
\hline $\mathrm{H}$ & 4.55627100 & -0.70594600 & 1.42384200 \\
\hline $\mathrm{C}$ & -3.42315300 & 2.14162000 & 0.30287800 \\
\hline $\mathrm{C}$ & -1.50988000 & -2.78516100 & -3.53288000 \\
\hline $\mathrm{C}$ & 0.64440000 & -2.91310400 & -2.25546500 \\
\hline $\mathrm{C}$ & -4.21275500 & 0.85037900 & 0.75561100 \\
\hline $\mathrm{C}$ & 0.95950300 & -0.42944500 & -3.79272600 \\
\hline $\mathrm{C}$ & -1.45909900 & 0.15163300 & -3.49531100 \\
\hline $\mathrm{C}$ & 3.39392700 & 2.00891600 & -1.54627400 \\
\hline $\mathrm{C}$ & 3.81922000 & 2.03382400 & 0.83474600 \\
\hline
\end{tabular}




\begin{tabular}{|c|c|c|c|}
\hline $\mathrm{H}$ & 2.70029200 & -3.98936600 & 1.90570800 \\
\hline $\mathrm{H}$ & 4.41507400 & -4.01898600 & 1.43024500 \\
\hline $\mathrm{H}$ & 3.83882000 & -2.86979400 & 2.67358800 \\
\hline $\mathrm{C}$ & -3.36387300 & 3.24868600 & 1.34664300 \\
\hline $\mathrm{C}$ & -3.88492000 & 2.70220800 & -1.04293800 \\
\hline $\mathrm{H}$ & -1.69177100 & -3.82329600 & -3.21106600 \\
\hline $\mathrm{H}$ & -1.03934900 & -2.82262400 & -4.52856200 \\
\hline $\mathrm{H}$ & -2.48489100 & -2.28722100 & -3.62294200 \\
\hline $\mathrm{H}$ & 1.20163700 & -3.13964700 & -3.17766900 \\
\hline $\mathrm{H}$ & 0.34959500 & -3.86772400 & -1.79201200 \\
\hline $\mathrm{H}$ & 1.30319600 & -2.39155100 & -1.55096400 \\
\hline $\mathrm{C}$ & -5.60927200 & 0.72332800 & 0.16117700 \\
\hline $\mathrm{C}$ & -4.26182000 & 0.66881300 & 2.27252800 \\
\hline $\mathrm{H}$ & 0.81797400 & -0.95219200 & -4.75191700 \\
\hline $\mathrm{H}$ & 1.12471300 & 0.63711100 & -4.01567300 \\
\hline $\mathrm{H}$ & 1.86732900 & -0.81897500 & -3.31399300 \\
\hline $\mathrm{H}$ & -1.66816700 & -0.16236000 & -4.52953000 \\
\hline $\mathrm{H}$ & -1.24575900 & 1.23227800 & -3.50504300 \\
\hline $\mathrm{H}$ & -2.36433800 & -0.01254400 & -2.89288300 \\
\hline $\mathrm{C}$ & 3.54686500 & 3.39818800 & -1.59293800 \\
\hline $\mathrm{H}$ & 3.16339800 & 1.45495000 & -2.45952600 \\
\hline $\mathrm{C}$ & 3.97271900 & 3.41982800 & 0.78660800 \\
\hline $\mathrm{H}$ & 3.87888400 & 1.51431400 & 1.79227500 \\
\hline $\mathrm{H}$ & -4.37315600 & 3.60921300 & 1.59801800 \\
\hline $\mathrm{H}$ & -2.79358400 & 4.10379200 & 0.95086600 \\
\hline $\mathrm{H}$ & -2.87287300 & 2.90700000 & 2.26678200 \\
\hline $\mathrm{H}$ & -3.90521500 & 1.92281300 & -1.81799200 \\
\hline $\mathrm{H}$ & -4.88358600 & 3.15816300 & -0.97620200 \\
\hline $\mathrm{H}$ & -3.17740100 & 3.48015100 & -1.36896500 \\
\hline $\mathrm{H}$ & -6.24926100 & 1.56096200 & 0.47907900 \\
\hline $\mathrm{H}$ & -6.07747800 & -0.20854300 & 0.51358500 \\
\hline $\mathrm{H}$ & -5.58742000 & 0.69480400 & -0.93596200 \\
\hline $\mathrm{H}$ & -4.88248000 & 1.43989300 & 2.75214300 \\
\hline $\mathrm{H}$ & -4.71576600 & -0.30935100 & 2.49948700 \\
\hline $\mathrm{H}$ & -3.25333400 & 0.67415600 & 2.70638200 \\
\hline $\mathrm{C}$ & 3.83381200 & 4.11359000 & -0.42419500 \\
\hline $\mathrm{H}$ & 3.44428200 & 3.92333900 & -2.54656200 \\
\hline $\mathrm{H}$ & 4.19112700 & 3.96887800 & 1.70638200 \\
\hline $\mathrm{H}$ & 3.95260900 & 5.19934900 & -0.45496800 \\
\hline \multicolumn{4}{|c|}{$8-b$} \\
\hline $\mathrm{O}$ & -0.77564000 & -1.66545300 & 0.53203500 \\
\hline $\mathrm{P}$ & 0.40237000 & -1.02900000 & 1.43752700 \\
\hline B & -1.78285400 & -0.85572300 & -0.25366200 \\
\hline $\mathrm{Ni}$ & 2.76780000 & -1.29791900 & 0.60429400 \\
\hline
\end{tabular}




\begin{tabular}{|c|c|c|c|}
\hline K & 0.54237300 & 0.75658200 & -1.78374500 \\
\hline K & -2.71226800 & -1.20985500 & 2.49722800 \\
\hline $\mathrm{O}$ & -0.24529200 & -0.36110900 & 2.64178900 \\
\hline $\mathrm{O}$ & 1.26604000 & -0.08151500 & 0.48792700 \\
\hline $\mathrm{O}$ & 1.46230600 & -2.15166100 & 1.73288000 \\
\hline $\mathrm{O}$ & -3.09619000 & -1.53689100 & -0.14489000 \\
\hline B & -1.94886400 & 0.79560400 & 0.29176200 \\
\hline $\mathrm{O}$ & -1.44364100 & -0.94156800 & -1.70074300 \\
\hline $\mathrm{C}$ & 4.08909900 & -0.58569400 & -0.73019200 \\
\hline $\mathrm{C}$ & 4.40488300 & -2.42827500 & 0.80405600 \\
\hline $\mathrm{C}$ & 4.70025500 & -1.09016500 & 0.45042400 \\
\hline $\mathrm{O}$ & -1.27798600 & 1.87840000 & -0.25204900 \\
\hline $\mathrm{C}$ & -3.27579700 & -2.34639400 & -1.30293500 \\
\hline $\mathrm{O}$ & -2.81184200 & 1.22676000 & 1.28758100 \\
\hline $\mathrm{C}$ & -2.54423600 & -1.49632000 & -2.41005800 \\
\hline $\mathrm{C}$ & 3.95549400 & 0.83401700 & -1.09168300 \\
\hline $\mathrm{H}$ & 4.00624500 & -1.27821900 & -1.57943400 \\
\hline $\mathrm{C}$ & 4.74218000 & -3.01032300 & 2.14649700 \\
\hline $\mathrm{H}$ & 4.27556800 & -3.15506300 & -0.01099100 \\
\hline $\mathrm{H}$ & 5.14961000 & -0.40756000 & 1.18242900 \\
\hline $\mathrm{C}$ & -1.75311900 & 3.11366100 & 0.33589600 \\
\hline $\mathrm{C}$ & -4.77019600 & -2.54180800 & -1.54188400 \\
\hline $\mathrm{C}$ & -2.60196000 & -3.70902000 & -1.08444800 \\
\hline $\mathrm{C}$ & -2.50947300 & 2.60769600 & 1.62751200 \\
\hline $\mathrm{C}$ & -2.01863300 & -2.31000500 & -3.58943900 \\
\hline $\mathrm{C}$ & -3.42225800 & -0.34437600 & -2.92223200 \\
\hline $\mathrm{C}$ & 3.89240500 & 1.21002600 & -2.45063800 \\
\hline C & 3.75457100 & 1.83787400 & -0.12001100 \\
\hline $\mathrm{H}$ & 3.94579000 & -3.69557700 & 2.47748800 \\
\hline $\mathrm{H}$ & 5.68637100 & -3.58489200 & 2.12301700 \\
\hline $\mathrm{H}$ & 4.83940000 & -2.22550500 & 2.91294400 \\
\hline $\mathrm{C}$ & -0.54491000 & 4.00063200 & 0.60910400 \\
\hline $\mathrm{C}$ & -2.67240800 & 3.76800000 & -0.69593800 \\
\hline $\mathrm{H}$ & -5.19823600 & -3.16558400 & -0.74060200 \\
\hline $\mathrm{H}$ & -4.96190500 & -3.05133200 & -2.49989700 \\
\hline $\mathrm{H}$ & -5.30528600 & -1.58247400 & -1.53977800 \\
\hline $\mathrm{H}$ & -2.78774100 & -4.40320800 & -1.91916700 \\
\hline $\mathrm{H}$ & -3.01380400 & -4.16479900 & -0.16981300 \\
\hline $\mathrm{H}$ & -1.51992300 & -3.59252000 & -0.94038700 \\
\hline $\mathrm{C}$ & -3.81757800 & 3.33138600 & 1.91799100 \\
\hline C & -1.62759100 & 2.57695700 & 2.87615100 \\
\hline $\mathrm{H}$ & -2.83100600 & -2.84924900 & -4.10252400 \\
\hline $\mathrm{H}$ & -1.54608900 & -1.64100000 & -4.32771700 \\
\hline $\mathrm{H}$ & -1.26356900 & -3.03795100 & -3.26382500 \\
\hline
\end{tabular}




\begin{tabular}{|c|c|c|}
\hline-4.24433400 & -0.69329400 & -3.56628900 \\
\hline-2.79874300 & 0.34522200 & -3.51394300 \\
\hline-3.85224300 & 0.22169400 & -2.08300000 \\
\hline 3.63404600 & 2.53344700 & -2.82572800 \\
\hline 4.04417100 & 0.44723100 & -3.22009700 \\
\hline 3.50419400 & 3.15764300 & -0.49444500 \\
\hline 3.73983500 & 1.56537200 & 0.93627700 \\
\hline-0.83980700 & 4.92437400 & 1.13052100 \\
\hline-0.07349900 & 4.28983500 & -0.34351100 \\
\hline 0.20735500 & 3.47658900 & 1.21220200 \\
\hline-3.53671900 & 3.12894200 & -0.92731000 \\
\hline-3.04127000 & 4.74623000 & -0.35387000 \\
\hline-2.11018200 & 3.92454900 & -1.62940800 \\
\hline-3.64011500 & 4.40126100 & 2.10829200 \\
\hline-4.28413700 & 2.90428400 & 2.81898700 \\
\hline-4.53348200 & 3.23610800 & 1.09121400 \\
\hline-1.33993000 & 3.59017300 & 3.19315600 \\
\hline-2.19561900 & 2.12019200 & 3.70234800 \\
\hline-0.73373800 & 1.95713400 & 2.72133400 \\
\hline 3.43557200 & 3.51486400 & -1.84806600 \\
\hline 3.59304200 & 2.79838800 & -3.88577200 \\
\hline 3.33756300 & 3.91295800 & 0.27773200 \\
\hline 22982600 & 4.54855500 & -2.13625400 \\
\hline
\end{tabular}

\begin{tabular}{rrr}
-0.62349900 & -1.68315100 & -0.05169800 \\
0.20130600 & 1.72891400 & 0.38723500 \\
0.82884200 & -0.04652500 & -3.25072500 \\
0.65629300 & 1.58532400 & 1.68161900 \\
1.13118200 & 2.48654700 & -0.35071600 \\
0.00286800 & -0.34521300 & -2.01847200 \\
-3.24623800 & -0.38505100 & -1.99045000 \\
2.05872100 & 1.92517600 & 1.74605700 \\
2.23355200 & 2.87763000 & 0.50011800 \\
-1.00793600 & 0.96421600 & -1.78937100 \\
0.89064400 & 2.50646000 & -2.96001300 \\
0.72787100 & -0.46205400 & -0.62686900 \\
\hline-0.95619700 & -1.57381200 & -1.92932000 \\
\hline-1.74791300 & -0.17948100 & 0.29869200 \\
\hline 2.33831600 & 2.58268800 & 3.09171900 \\
2.84121500 & 0.61882800 & 1.62174900 \\
\hline 3.53297800 & 2.67246700 & -0.27153700 \\
2.03587900 & 4.35841200 & 0.82859600 \\
-1.27565100 & 1.19237400 & -0.32945000 \\
-2.70619500 & 0.13601700 & 1.33288900 \\
\hline & & \\
\hline
\end{tabular}




\begin{tabular}{|c|c|c|c|}
\hline $\mathrm{H}$ & 3.38516200 & 2.91866200 & 3.15665300 \\
\hline $\mathrm{H}$ & 2.16917200 & 1.85162100 & 3.89686800 \\
\hline $\mathrm{H}$ & 1.68083600 & 3.44287000 & 3.27593700 \\
\hline $\mathrm{H}$ & 2.53187100 & -0.05198300 & 2.43551800 \\
\hline $\mathrm{H}$ & 3.92598600 & 0.77902200 & 1.70648800 \\
\hline $\mathrm{H}$ & 2.61326100 & 0.10902600 & 0.67726400 \\
\hline $\mathrm{H}$ & 3.58736100 & 3.37950700 & -1.11724000 \\
\hline $\mathrm{H}$ & 4.40936000 & 2.86444800 & 0.36639000 \\
\hline $\mathrm{H}$ & 3.60694500 & 1.64999900 & -0.66473100 \\
\hline $\mathrm{H}$ & 2.86161200 & 4.76096300 & 1.43410800 \\
\hline $\mathrm{H}$ & 1.09202600 & 4.52248900 & 1.36850600 \\
\hline $\mathrm{H}$ & 1.99134800 & 4.93521400 & -0.10917000 \\
\hline $\mathrm{O}$ & -2.51417300 & 1.94867700 & -0.20621500 \\
\hline $\mathrm{C}$ & -3.93286400 & -0.76100400 & 1.15375600 \\
\hline C & -2.06371400 & -0.15204700 & 2.68766300 \\
\hline $\mathrm{C}$ & -3.05340300 & 1.68772600 & 1.07970600 \\
\hline $\mathrm{H}$ & -4.60838500 & -0.69657100 & 2.01922200 \\
\hline $\mathrm{H}$ & -3.60751700 & -1.79719500 & 1.05280500 \\
\hline $\mathrm{H}$ & -4.54041900 & -0.50195300 & 0.27459000 \\
\hline $\mathrm{H}$ & -1.85129300 & -1.22786300 & 2.76207300 \\
\hline $\mathrm{H}$ & -2.72600900 & 0.12785700 & 3.52115500 \\
\hline $\mathrm{H}$ & -1.10982300 & 0.37921900 & 2.78449600 \\
\hline $\mathrm{C}$ & -2.43234200 & 2.62913000 & 2.12517900 \\
\hline $\mathrm{C}$ & -4.55231100 & 1.99938600 & 1.02470300 \\
\hline $\mathrm{H}$ & -2.57897500 & 3.66588200 & 1.78414900 \\
\hline $\mathrm{H}$ & -2.92097900 & 2.52074000 & 3.10542600 \\
\hline $\mathrm{H}$ & -1.35894200 & 2.46325100 & 2.25700800 \\
\hline $\mathrm{H}$ & -4.68495000 & 3.07969800 & 0.86113600 \\
\hline $\mathrm{H}$ & -5.06150600 & 1.73330900 & 1.96306600 \\
\hline $\mathrm{H}$ & -5.05979100 & 1.48202300 & 0.19849500 \\
\hline $\mathrm{C}$ & -0.16132700 & -3.29222900 & 0.95883600 \\
\hline $\mathrm{C}$ & 0.78833300 & -3.14617100 & -0.07193600 \\
\hline $\mathrm{C}$ & 2.20961600 & -2.82063200 & 0.11617300 \\
\hline $\mathrm{C}$ & 2.84812200 & -2.87715400 & 1.36748800 \\
\hline $\mathrm{H}$ & 2.48993500 & -2.34331500 & -1.97284200 \\
\hline $\mathrm{H}$ & 0.54721200 & -3.57365900 & -1.05084600 \\
\hline C & 2.97482400 & -2.42411300 & -0.99798800 \\
\hline $\mathrm{C}$ & 4.20133600 & -2.55920200 & 1.50048000 \\
\hline $\mathrm{C}$ & 4.94655400 & -2.16507000 & 0.38573900 \\
\hline $\mathrm{C}$ & 4.32325800 & -2.09804200 & -0.86583400 \\
\hline $\mathrm{H}$ & 4.67587200 & -2.61539800 & 2.48388800 \\
\hline $\mathrm{H}$ & 6.00491100 & -1.91233500 & 0.49051200 \\
\hline $\mathrm{H}$ & 4.89244400 & -1.78425200 & -1.74517600 \\
\hline $\mathrm{C}$ & -1.50943800 & -3.44316300 & 0.54082900 \\
\hline
\end{tabular}




\begin{tabular}{|c|c|c|}
\hline-1.65241700 & -3.91352700 & -0.44228500 \\
\hline 2.28153800 & -3.17996600 & 2.25050600 \\
\hline 0.09913200 & -3.21654700 & 2.01976000 \\
\hline-2.57383100 & -3.81780400 & 1.54364400 \\
\hline-2.44789500 & -4.87427800 & 1.84711700 \\
\hline-3.59297300 & -3.73750900 & 1.13535900 \\
\hline-2.52858600 & -3.21430700 & 2.46334500 \\
\hline 0.00708100 & -1.65582400 & 0.38352900 \\
\hline-0.47938000 & 1.67934500 & 0.42618200 \\
\hline 1.30048700 & 0.74048500 & -2.91999300 \\
\hline-0.13989200 & 1.50419200 & 1.75374300 \\
\hline 0.23412200 & 2.76511500 & -0.09854200 \\
\hline 0.43571200 & -0.18211500 & -2.07119100 \\
\hline-2.45624500 & -1.49683000 & -2.44075900 \\
\hline 1.08131500 & 2.22419200 & 2.04017400 \\
\hline 1.08594400 & 3.34026900 & 0.92157500 \\
\hline-1.05509900 & 0.60318700 & -1.90246700 \\
\hline 0.09757100 & 2.91516600 & -2.71729200 \\
\hline 0.89373800 & -0.24597600 & -0.55968800 \\
\hline 0.08080300 & -1.56838800 & -2.62901100 \\
\hline-1.71427100 & -0.68529100 & 0.14237600 \\
\hline 1.00751300 & 2.75542500 & 3.46643400 \\
\hline 2.23335400 & 1.22626200 & 1.90457400 \\
\hline 2.45426200 & 3.60963100 & 0.30574900 \\
\hline 0.42939300 & 4.64915400 & 1.36104400 \\
\hline-1.58316700 & 0.74570400 & -0.53027200 \\
\hline-2.89731900 & -0.69245400 & 0.96540400 \\
\hline 1.88905700 & 3.37103100 & 3.70491000 \\
\hline 0.98757100 & 1.90966900 & 4.17062800 \\
\hline 0.10369000 & 3.35549100 & 3.63730900 \\
\hline 2.05963500 & 0.39721200 & 2.60743400 \\
\hline 3.20349400 & 1.68271900 & 2.15152900 \\
\hline 2.26796700 & 0.79720500 & 0.89526500 \\
\hline 2.37527800 & 4.40287000 & -0.45657900 \\
\hline 3.17007500 & 3.95763900 & 1.06649800 \\
\hline 2.85988200 & 2.70884900 & -0.17255100 \\
\hline 1.03653600 & 5.18402200 & 2.10650900 \\
\hline-0.56972500 & 4.47134500 & 1.78440400 \\
\hline 0.31144800 & 5.30694200 & 0.48538100 \\
\hline-3.00152600 & 1.06995600 & -0.64124700 \\
\hline-3.68890500 & -1.96142900 & 0.63298000 \\
\hline-2.49539100 & -0.71714200 & 2.43852600 \\
\hline 3.65924800 & 0.65850700 & 0.53976500 \\
\hline
\end{tabular}




\begin{tabular}{|c|c|c|c|}
\hline $\mathrm{H}$ & -4.52451500 & -2.11470400 & 1.33129800 \\
\hline $\mathrm{H}$ & -3.02240600 & -2.83044600 & 0.70163500 \\
\hline $\mathrm{H}$ & -4.12517400 & -1.94162200 & -0.37656500 \\
\hline $\mathrm{H}$ & -2.00373800 & -1.66726500 & 2.67302900 \\
\hline $\mathrm{H}$ & -3.37492500 & -0.62100200 & 3.09336200 \\
\hline $\mathrm{H}$ & -1.78771100 & 0.08908000 & 2.66113700 \\
\hline $\mathrm{C}$ & -3.56622600 & 1.77015600 & 1.60072200 \\
\hline $\mathrm{C}$ & -5.14052600 & 0.45605700 & 0.21069000 \\
\hline $\mathrm{H}$ & -3.96255700 & 2.69652400 & 1.15717600 \\
\hline $\mathrm{H}$ & -4.16100900 & 1.53914300 & 2.49710600 \\
\hline $\mathrm{H}$ & -2.53377200 & 1.95938900 & 1.91414500 \\
\hline $\mathrm{H}$ & -5.58176500 & 1.42549700 & -0.06711900 \\
\hline $\mathrm{H}$ & -5.70152800 & 0.06258900 & 1.07215000 \\
\hline $\mathrm{H}$ & -5.28784100 & -0.22432700 & -0.63964200 \\
\hline $\mathrm{C}$ & 0.98377100 & -3.18394900 & 1.25202200 \\
\hline $\mathrm{C}$ & 1.66691800 & -2.79064000 & 0.09018300 \\
\hline $\mathrm{C}$ & 2.99465800 & -2.16177700 & 0.04896300 \\
\hline C & 3.78225000 & -1.94189600 & 1.19263700 \\
\hline $\mathrm{H}$ & 2.86155300 & -1.84387000 & -2.08082400 \\
\hline $\mathrm{H}$ & 1.31658500 & -3.17353500 & -0.87461100 \\
\hline $\mathrm{C}$ & 3.47661700 & -1.70067600 & -1.19051600 \\
\hline $\mathrm{C}$ & 5.00782000 & -1.28020000 & 1.10114900 \\
\hline $\mathrm{C}$ & 5.47039300 & -0.82025100 & -0.13599900 \\
\hline $\mathrm{C}$ & 4.69810500 & -1.03633900 & -1.28215500 \\
\hline $\mathrm{H}$ & 5.60438700 & -1.11957900 & 2.00312200 \\
\hline $\mathrm{H}$ & 6.42737500 & -0.29661000 & -0.20573800 \\
\hline $\mathrm{H}$ & 5.04120300 & -0.67212800 & -2.25376300 \\
\hline $\mathrm{C}$ & -0.38332000 & -3.52913300 & 1.16966600 \\
\hline $\mathrm{H}$ & -0.71107100 & -4.01660900 & 0.23919400 \\
\hline $\mathrm{H}$ & 3.43623000 & -2.28610400 & 2.16954900 \\
\hline $\mathrm{H}$ & 1.42164200 & -3.00007700 & 2.23981900 \\
\hline $\mathrm{C}$ & -1.12152800 & -3.97701200 & 2.40590100 \\
\hline $\mathrm{H}$ & -0.95134800 & -5.05481300 & 2.58570300 \\
\hline $\mathrm{H}$ & -2.20986000 & -3.84066800 & 2.33005000 \\
\hline $\mathrm{H}$ & -0.77707000 & -3.43942900 & 3.30323900 \\
\hline \multicolumn{4}{|c|}{ 7-iso } \\
\hline $\mathrm{C}$ & 1.41544400 & -4.14989500 & 0.39684800 \\
\hline $\mathrm{C}$ & -0.00705000 & -4.06326400 & 0.23943100 \\
\hline $\mathrm{C}$ & -0.66282500 & -4.30194600 & -1.05847000 \\
\hline $\mathrm{C}$ & 0.00632200 & -4.13177000 & -2.28774000 \\
\hline $\mathrm{H}$ & -2.55913800 & -4.80712400 & -0.15750000 \\
\hline $\mathrm{H}$ & -0.60852400 & -4.38182400 & 1.09527500 \\
\hline $\mathrm{C}$ & -2.01786400 & -4.68355100 & -1.09781300 \\
\hline $\mathrm{C}$ & -0.65072700 & -4.33187100 & -3.50134700 \\
\hline
\end{tabular}




\begin{tabular}{|c|c|c|}
\hline-1.99811700 & -4.70652400 & -3.52193200 \\
\hline-2.67553000 & -4.88434300 & -2.31176500 \\
\hline-0.10908100 & -4.18903100 & -4.44011100 \\
\hline-2.51456200 & -4.85910500 & -4.47246200 \\
\hline-3.72753700 & -5.17793500 & -2.31368800 \\
\hline 1.98737900 & -3.76125000 & 1.59851700 \\
\hline 1.35025000 & -3.76640400 & 2.49435500 \\
\hline 1.05569700 & -3.82964200 & -2.29579400 \\
\hline 2.06945500 & -4.31272200 & -0.46822300 \\
\hline 3.45453800 & -3.68202900 & 1.86165100 \\
\hline 3.78285100 & -4.59528800 & 2.39153700 \\
\hline 3.68421100 & -2.82105000 & 2.50626300 \\
\hline 4.04128700 & -3.58388000 & 0.93808700 \\
\hline-7.21602100 & 1.66770100 & -0.90146200 \\
\hline-5.92210300 & 1.38866200 & -0.43393400 \\
\hline-5.58976800 & 0.03156800 & -0.20196500 \\
\hline-6.56045800 & -0.98258600 & -0.27608300 \\
\hline-7.84327900 & -0.64368600 & -0.72301300 \\
\hline-8.16710500 & 0.66490300 & -1.07026600 \\
\hline-4.93475800 & -2.43688800 & 0.92754000 \\
\hline-3.94524000 & -1.45555600 & 0.70852700 \\
\hline-2.60744100 & -1.67046500 & 1.11632100 \\
\hline-2.38196500 & -2.73632600 & 2.00632300 \\
\hline-3.39417400 & -3.60449500 & 2.39963300 \\
\hline-4.64887800 & -3.48768700 & 1.80444300 \\
\hline-7.48915100 & 2.69728800 & -1.12355000 \\
\hline-8.60091900 & -1.42171900 & -0.81611800 \\
\hline-9.16513200 & 0.90628900 & -1.44248100 \\
\hline-1.36871800 & -2.89300000 & 2.37244000 \\
\hline-3.18745800 & -4.40781100 & 3.10963000 \\
\hline-5.41009400 & -4.23788500 & 2.01632900 \\
\hline-6.20102900 & -2.42380900 & 0.07138400 \\
\hline-5.87686400 & -3.18364200 & -1.24073600 \\
\hline-5.61119400 & -4.22915900 & -1.02074200 \\
\hline-5.03046100 & -2.72073600 & -1.76871600 \\
\hline-6.74850800 & -3.17686300 & -1.91444100 \\
\hline-7.37148300 & -3.12838800 & 0.77828300 \\
\hline-8.26742000 & -3.14418300 & 0.14367800 \\
\hline-7.62749700 & -2.63105600 & 1.72597200 \\
\hline-7.13003400 & -4.17909500 & 0.98965700 \\
\hline-4.27467000 & -0.27573200 & 0.09024700 \\
\hline-5.63361700 & 3.49475100 & 1.55050600 \\
\hline-7.10585400 & 3.89981500 & 1.40838100 \\
\hline & 3.03363800 & 1.19999 \\
\hline
\end{tabular}




\begin{tabular}{|c|c|c|c|}
\hline $\mathrm{H}$ & -7.27449900 & 4.65270700 & 0.62952300 \\
\hline $\mathrm{H}$ & -7.45104800 & 4.33408700 & 2.36349400 \\
\hline $\mathrm{C}$ & -5.54689000 & 2.40717800 & 2.63709600 \\
\hline $\mathrm{H}$ & -6.08928200 & 1.49386400 & 2.34647300 \\
\hline $\mathrm{H}$ & -6.00536900 & 2.78084600 & 3.56893500 \\
\hline $\mathrm{H}$ & -4.50910300 & 2.13086500 & 2.86247000 \\
\hline $\mathrm{C}$ & -4.79772000 & 4.70677900 & 1.99355100 \\
\hline $\mathrm{H}$ & -3.73053300 & 4.45196400 & 2.08998900 \\
\hline $\mathrm{H}$ & -5.14745600 & 5.06173700 & 2.97845500 \\
\hline $\mathrm{H}$ & -4.88578700 & 5.55050200 & 1.29317700 \\
\hline $\mathrm{C}$ & -4.76853400 & 3.96404800 & -1.45386500 \\
\hline $\mathrm{C}$ & -4.75720000 & 3.12532800 & -2.74263400 \\
\hline $\mathrm{H}$ & -4.00418900 & 2.32490100 & -2.70525600 \\
\hline $\mathrm{H}$ & -4.51216200 & 3.77443800 & -3.60117500 \\
\hline $\mathrm{H}$ & -5.73176300 & 2.65881000 & -2.94201300 \\
\hline $\mathrm{C}$ & -3.42748200 & 4.71966300 & -1.35051300 \\
\hline $\mathrm{H}$ & -3.35863600 & 5.31436600 & -0.42722600 \\
\hline $\mathrm{H}$ & -3.32011000 & 5.41419500 & -2.20188200 \\
\hline $\mathrm{H}$ & -2.57224700 & 4.02819500 & -1.36619800 \\
\hline $\mathrm{C}$ & -5.90081100 & 4.99439400 & -1.53099400 \\
\hline $\mathrm{H}$ & -5.90726800 & 5.66947400 & -0.66416700 \\
\hline $\mathrm{H}$ & -6.89526200 & 4.53507100 & -1.61629300 \\
\hline $\mathrm{H}$ & -5.75822000 & 5.62124800 & -2.42912200 \\
\hline $\mathrm{C}$ & -0.83707400 & 0.54640900 & 1.90899700 \\
\hline $\mathrm{C}$ & 0.08859000 & 1.67229000 & 1.42902300 \\
\hline $\mathrm{H}$ & -0.42833600 & 2.35989700 & 0.74875800 \\
\hline $\mathrm{H}$ & 1.00400200 & 1.30141000 & 0.95842900 \\
\hline $\mathrm{H}$ & 0.39923700 & 2.26239200 & 2.30707400 \\
\hline $\mathrm{C}$ & -0.21767000 & -0.12595400 & 3.14895300 \\
\hline $\mathrm{H}$ & -0.21495000 & 0.60714500 & 3.97372100 \\
\hline $\mathrm{H}$ & 0.82260100 & -0.43918500 & 2.98975800 \\
\hline $\mathrm{H}$ & -0.81010800 & -0.98759800 & 3.49342500 \\
\hline $\mathrm{C}$ & -2.20161200 & 1.12611400 & 2.28771100 \\
\hline $\mathrm{H}$ & -2.04836000 & 1.93669500 & 3.02097100 \\
\hline $\mathrm{H}$ & -2.85053100 & 0.37290100 & 2.75770300 \\
\hline $\mathrm{H}$ & -2.73514300 & 1.55177700 & 1.42851900 \\
\hline $\mathrm{C}$ & -1.19293800 & -0.18509900 & -1.19541200 \\
\hline $\mathrm{C}$ & -1.73735700 & 1.23278900 & -1.36438600 \\
\hline $\mathrm{H}$ & -1.92504200 & 1.40669800 & -2.43736400 \\
\hline $\mathrm{H}$ & -1.01588600 & 1.99164500 & -1.03987800 \\
\hline $\mathrm{H}$ & -2.67576600 & 1.39254000 & -0.82469100 \\
\hline $\mathrm{C}$ & 0.23120700 & -0.23365100 & -1.77716700 \\
\hline $\mathrm{H}$ & 0.19852500 & 0.10981200 & -2.82502600 \\
\hline $\mathrm{H}$ & 0.60835200 & -1.26847200 & -1.76205600 \\
\hline
\end{tabular}




\begin{tabular}{|c|c|c|c|}
\hline $\mathrm{H}$ & 0.92553600 & 0.40900700 & -1.21995000 \\
\hline $\mathrm{C}$ & -2.07294000 & -1.16982200 & -1.98243800 \\
\hline $\mathrm{H}$ & -3.13706900 & -1.02594400 & -1.76527700 \\
\hline $\mathrm{H}$ & -1.81045100 & -2.21348900 & -1.78453000 \\
\hline $\mathrm{H}$ & -1.92606400 & -0.98996300 & -3.06005400 \\
\hline $\mathrm{P}$ & -1.03335700 & -0.82915100 & 0.59631200 \\
\hline $\mathrm{P}$ & -4.73784000 & 2.73754200 & 0.02792900 \\
\hline $\mathrm{Ni}$ & 0.74945400 & -2.25988100 & 0.71728300 \\
\hline B & 5.38336200 & 0.22471600 & 0.01461300 \\
\hline $\mathrm{O}$ & 4.52818500 & -0.87818000 & 0.73538700 \\
\hline $\mathrm{O}$ & 5.85549300 & -0.41584500 & -1.24468300 \\
\hline $\mathrm{O}$ & 6.63317100 & 0.46239900 & 0.78033600 \\
\hline $\mathrm{P}$ & 3.25046100 & -0.30102100 & 1.52713000 \\
\hline K & 3.34111900 & -0.88304600 & -1.72500700 \\
\hline C & 7.27256300 & -0.38710800 & -1.28865500 \\
\hline $\mathrm{C}$ & 7.66436800 & -0.35114800 & 0.23645200 \\
\hline $\mathrm{O}$ & 3.33513200 & 1.26672900 & 1.19698800 \\
\hline K & 5.48705700 & 1.93823200 & 2.56126300 \\
\hline $\mathrm{O}$ & 3.28443700 & -0.55624600 & 3.01755700 \\
\hline $\mathrm{O}$ & 1.99704300 & -0.84456400 & 0.74730100 \\
\hline $\mathrm{O}$ & 4.79283200 & 2.93397100 & 0.13619500 \\
\hline $\mathrm{C}$ & 7.77988500 & -1.61340500 & -2.04310500 \\
\hline $\mathrm{C}$ & 7.70769800 & 0.89278300 & -2.01785900 \\
\hline $\mathrm{C}$ & 9.01506900 & 0.29691600 & 0.52993400 \\
\hline $\mathrm{C}$ & 7.60809500 & -1.74321400 & 0.88274100 \\
\hline B & 4.26358900 & 1.57484400 & -0.05033600 \\
\hline $\mathrm{C}$ & 3.92179200 & 3.85379300 & -0.51384400 \\
\hline $\mathrm{H}$ & 8.87986700 & -1.67845000 & -2.01720300 \\
\hline $\mathrm{H}$ & 7.47428900 & -1.55531900 & -3.10072300 \\
\hline $\mathrm{H}$ & 7.36538600 & -2.53988700 & -1.62233600 \\
\hline $\mathrm{H}$ & 7.22237000 & 0.91830400 & -3.00573900 \\
\hline $\mathrm{H}$ & 8.79723300 & 0.94490700 & -2.17060100 \\
\hline $\mathrm{H}$ & 7.38286600 & 1.77989200 & -1.45582300 \\
\hline $\mathrm{H}$ & 9.22214100 & 0.25766800 & 1.61212300 \\
\hline $\mathrm{H}$ & 9.83622300 & -0.22926400 & 0.01654000 \\
\hline $\mathrm{H}$ & 9.02899900 & 1.35168200 & 0.22255600 \\
\hline $\mathrm{H}$ & 8.44706600 & -2.38438800 & 0.56803800 \\
\hline $\mathrm{H}$ & 6.65873500 & -2.24256700 & 0.65011600 \\
\hline $\mathrm{H}$ & 7.65880300 & -1.62681500 & 1.97685700 \\
\hline $\mathrm{O}$ & 3.36912200 & 1.68561900 & -1.23532100 \\
\hline $\mathrm{C}$ & 2.78685500 & 4.24848000 & 0.44375000 \\
\hline $\mathrm{C}$ & 4.71940100 & 5.09787600 & -0.89748900 \\
\hline $\mathrm{C}$ & 3.39643000 & 3.01101100 & -1.74230000 \\
\hline $\mathrm{H}$ & 2.13160400 & 5.02443600 & 0.01757900 \\
\hline
\end{tabular}




\begin{tabular}{|c|c|c|c|}
\hline $\mathrm{H}$ & 3.22776100 & 4.65474200 & 1.36884700 \\
\hline $\mathrm{H}$ & 2.18558000 & 3.37313000 & 0.71581400 \\
\hline $\mathrm{H}$ & 5.02627300 & 5.63854300 & 0.01266600 \\
\hline $\mathrm{H}$ & 4.11915700 & 5.78974700 & -1.51042200 \\
\hline $\mathrm{H}$ & 5.63098300 & 4.83593900 & -1.45161700 \\
\hline $\mathrm{C}$ & 4.37285100 & 3.04531200 & -2.92617500 \\
\hline $\mathrm{C}$ & 1.99670700 & 3.39818600 & -2.21793000 \\
\hline $\mathrm{H}$ & 4.05796000 & 2.29382100 & -3.66838200 \\
\hline $\mathrm{H}$ & 4.40185700 & 4.02493000 & -3.42860100 \\
\hline $\mathrm{H}$ & 5.38679100 & 2.78397300 & -2.59470800 \\
\hline $\mathrm{H}$ & 1.70553100 & 2.77006600 & -3.07551200 \\
\hline $\mathrm{H}$ & 1.95657400 & 4.44991900 & -2.54560400 \\
\hline $\mathrm{H}$ & 1.25031500 & 3.24906400 & -1.42686700 \\
\hline \multicolumn{4}{|c|}{ 8-iso } \\
\hline $\mathrm{Ni}$ & 2.94804400 & -1.43004500 & 0.40398800 \\
\hline B & -1.35112900 & 0.53233900 & 0.83601600 \\
\hline $\mathrm{O}$ & -0.08576200 & -0.10338600 & 1.57090200 \\
\hline $\mathrm{O}$ & -0.92420800 & 1.81448300 & 0.23987600 \\
\hline $\mathrm{O}$ & -2.29741500 & 0.92357300 & 1.89570800 \\
\hline $\mathrm{P}$ & 0.47583400 & -1.29431400 & 0.68439000 \\
\hline K & 0.26260000 & 1.06481900 & -1.93238800 \\
\hline $\mathrm{C}$ & -1.60379900 & 2.89651600 & 0.86352400 \\
\hline $\mathrm{C}$ & -2.05129000 & 2.28330500 & 2.24462000 \\
\hline $\mathrm{O}$ & -0.82833300 & -1.99344700 & 0.14786300 \\
\hline K & -2.46926300 & -1.60196800 & 2.45399700 \\
\hline $\mathrm{O}$ & 1.46781600 & -2.19961300 & 1.46792000 \\
\hline $\mathrm{O}$ & 1.37888200 & -0.74740700 & -0.49101600 \\
\hline $\mathrm{O}$ & -3.21989100 & -1.31895200 & -0.03131100 \\
\hline $\mathrm{C}$ & -0.65358600 & 4.08644500 & 0.97586200 \\
\hline $\mathrm{C}$ & -2.80602100 & 3.27121400 & -0.01411700 \\
\hline $\mathrm{C}$ & -3.33237700 & 2.88315900 & 2.81785900 \\
\hline $\mathrm{C}$ & -0.93688600 & 2.34909700 & 3.30004700 \\
\hline B & -1.88839900 & -0.76237400 & -0.25016200 \\
\hline $\mathrm{C}$ & -3.68017900 & -1.89115900 & -1.25655900 \\
\hline $\mathrm{H}$ & -1.10852000 & 4.91687700 & 1.53968300 \\
\hline $\mathrm{H}$ & -0.40507200 & 4.46375200 & -0.02998900 \\
\hline $\mathrm{H}$ & 0.28508600 & 3.79961100 & 1.46791100 \\
\hline $\mathrm{H}$ & -2.45191400 & 3.48200400 & -1.03599600 \\
\hline $\mathrm{H}$ & -3.33634200 & 4.16405100 & 0.35167600 \\
\hline $\mathrm{H}$ & -3.51599900 & 2.43436800 & -0.06912400 \\
\hline $\mathrm{H}$ & -3.55920200 & 2.41742800 & 3.79065900 \\
\hline $\mathrm{H}$ & -3.23060600 & 3.96770600 & 2.98378100 \\
\hline $\mathrm{H}$ & -4.18994200 & 2.70728000 & 2.15484400 \\
\hline $\mathrm{H}$ & -0.76539500 & 3.37682100 & 3.65717300 \\
\hline
\end{tabular}




\begin{tabular}{|c|c|c|c|}
\hline $\mathrm{H}$ & 0.00184900 & 1.93721000 & 2.90820300 \\
\hline $\mathrm{H}$ & -1.23361000 & 1.73771000 & 4.16769100 \\
\hline $\mathrm{O}$ & -1.78951600 & -0.56412000 & -1.69730800 \\
\hline $\mathrm{C}$ & -3.18720000 & -3.34135100 & -1.35914400 \\
\hline $\mathrm{C}$ & -5.20619000 & -1.86901200 & -1.26250600 \\
\hline $\mathrm{C}$ & -3.00861400 & -0.94970600 & -2.32793000 \\
\hline $\mathrm{H}$ & -3.58123500 & -3.84812300 & -2.25385000 \\
\hline $\mathrm{H}$ & -3.53957000 & -3.89901500 & -0.47617000 \\
\hline $\mathrm{H}$ & -2.09094700 & -3.38592900 & -1.36656300 \\
\hline $\mathrm{H}$ & -5.59186000 & -2.56793700 & -0.50272900 \\
\hline $\mathrm{H}$ & -5.60976200 & -2.18259100 & -2.23859700 \\
\hline $\mathrm{H}$ & -5.59553700 & -0.86996000 & -1.02536900 \\
\hline $\mathrm{C}$ & -3.82910800 & 0.32281700 & -2.57532700 \\
\hline $\mathrm{C}$ & -2.69220000 & -1.62871600 & -3.65800500 \\
\hline $\mathrm{H}$ & -3.22203700 & 1.03133800 & -3.16173700 \\
\hline $\mathrm{H}$ & -4.75567800 & 0.12728500 & -3.13690300 \\
\hline $\mathrm{H}$ & -4.08604000 & 0.80917900 & -1.62428400 \\
\hline $\mathrm{H}$ & -2.24746000 & -0.89845600 & -4.35413300 \\
\hline $\mathrm{H}$ & -3.60170400 & -2.02900100 & -4.13425700 \\
\hline $\mathrm{H}$ & -1.97317800 & -2.44801700 & -3.52617200 \\
\hline $\mathrm{C}$ & 4.78540800 & -0.82651400 & 0.69279800 \\
\hline $\mathrm{C}$ & 4.39392600 & -0.58707200 & -0.65638200 \\
\hline $\mathrm{C}$ & 4.02985700 & 0.73385700 & -1.19477600 \\
\hline $\mathrm{C}$ & 3.57554400 & 1.78217600 & -0.36596900 \\
\hline $\mathrm{H}$ & 4.34808700 & 0.13990800 & -3.24912500 \\
\hline $\mathrm{H}$ & 4.65995200 & -1.33904100 & -1.41076300 \\
\hline $\mathrm{C}$ & 4.01645100 & 0.94455600 & -2.58755900 \\
\hline $\mathrm{C}$ & 3.14706900 & 2.99432600 & -0.90771600 \\
\hline $\mathrm{C}$ & 3.14483700 & 3.19104300 & -2.29660100 \\
\hline $\mathrm{C}$ & 3.57973200 & 2.15588300 & -3.13378800 \\
\hline $\mathrm{H}$ & 2.79262100 & 3.78596800 & -0.24291500 \\
\hline $\mathrm{H}$ & 2.80758300 & 4.14075700 & -2.71949400 \\
\hline $\mathrm{H}$ & 3.58467100 & 2.29387400 & -4.21837900 \\
\hline $\mathrm{C}$ & 4.64903900 & -2.14024600 & 1.18846900 \\
\hline $\mathrm{H}$ & 4.83151400 & -2.97391300 & 0.49429700 \\
\hline $\mathrm{H}$ & 3.51507200 & 1.63086600 & 0.71306800 \\
\hline $\mathrm{H}$ & 4.90445800 & 0.00804000 & 1.39444500 \\
\hline $\mathrm{C}$ & 4.74662800 & -2.47609300 & 2.64814100 \\
\hline $\mathrm{H}$ & 5.75496900 & -2.83992100 & 2.91707100 \\
\hline $\mathrm{H}$ & 4.03029100 & -3.27121400 & 2.90888100 \\
\hline $\mathrm{H}$ & 4.51813200 & -1.60315700 & 3.27910100 \\
\hline \multicolumn{4}{|l|}{ S1 } \\
\hline $\mathrm{C}$ & -0.02755700 & 0.02733500 & -0.00009800 \\
\hline $\mathrm{O}$ & -0.62018100 & 1.14182600 & -0.00028200 \\
\hline
\end{tabular}




\begin{tabular}{|c|c|c|}
\hline 1.52607300 & 0.01067600 & 0.00017000 \\
\hline 1.99036400 & -0.74693400 & 1.25556900 \\
\hline 1.99080400 & -0.74720600 & -1.25490000 \\
\hline 2.09339500 & 1.43230500 & 0.00011600 \\
\hline 1.67132300 & -0.22582100 & 2.17401800 \\
\hline 1.56091000 & -1.75913500 & 1.27391000 \\
\hline 3.09033200 & -0.83072000 & 1.28180900 \\
\hline 1.67208500 & -0.22629500 & -2.17357600 \\
\hline 3.09078100 & -0.83099800 & -1.28073500 \\
\hline 1.56135500 & -1.75941100 & -1.27317100 \\
\hline 3.19688300 & 1.41105700 & 0.00031800 \\
\hline 1.75817900 & 1.99347700 & -0.88496800 \\
\hline 1.75785400 & 1.99367300 & 0.88495100 \\
\hline-0.60779000 & -1.09643300 & -0.00012700 \\
\hline-2.89339000 & 0.00073600 & -0.00023300 \\
\hline 2.72460800 & -0.45183200 & -0.03746800 \\
\hline 1.72828400 & 0.32781300 & -0.17570300 \\
\hline 4.13744400 & 0.17911200 & 0.10450700 \\
\hline 4.73463400 & -0.29939400 & 1.43866500 \\
\hline 4.99934500 & -0.33865300 & -1.05988300 \\
\hline 4.08308200 & 1.70970700 & 0.07491200 \\
\hline 4.13318100 & 0.05457900 & 2.29304200 \\
\hline 4.75914100 & -1.39776400 & 1.47544000 \\
\hline 5.76192600 & 0.08073800 & 1.56951200 \\
\hline 4.59244300 & -0.01159500 & -2.03158300 \\
\hline 6.03325700 & 0.03825100 & -0.98284400 \\
\hline 5.02576700 & -1.43777500 & -1.05734900 \\
\hline 5.09641600 & 2.13441400 & 0.17374600 \\
\hline 3.65368600 & 2.07814200 & -0.86983600 \\
\hline 3.47260600 & 2.10552100 & 0.90221400 \\
\hline 2.64564900 & -1.69895800 & -0.00713700 \\
\hline 0.00231100 & 2.07841000 & -0.17056200 \\
\hline-2.72429300 & -0.45037800 & -0.02646300 \\
\hline-1.72484600 & 0.33051600 & -0.13099900 \\
\hline-4.13883900 & 0.17949100 & 0.10230200 \\
\hline-4.74818100 & -0.30454100 & 1.42916300 \\
\hline-4.08465800 & 1.71019100 & 0.07965800 \\
\hline-4.98968200 & -0.33380000 & -1.07192300 \\
\hline-4.77351900 & -1.40310200 & 1.46105400 \\
\hline-4.15429800 & 0.04569700 & 2.29031200 \\
\hline-5.77652300 & 0.07530900 & 1.55232400 \\
\hline-3.64648600 & 2.08264100 & -0.85960600 \\
\hline-5.09905200 & 2.13422200 & 0.17017900 \\
\hline
\end{tabular}




\begin{tabular}{|c|c|c|c|}
\hline $\mathrm{H}$ & -3.48197300 & 2.10257400 & 0.91408000 \\
\hline $\mathrm{H}$ & -6.02464700 & 0.04170200 & -1.00243000 \\
\hline $\mathrm{H}$ & -4.57456700 & -0.00181900 & -2.03850400 \\
\hline $\mathrm{H}$ & -5.01470600 & -1.43289000 & -1.07481100 \\
\hline $\mathrm{O}$ & -2.64688200 & -1.69794900 & -0.01784000 \\
\hline K & -0.00026100 & -1.81418000 & -0.16798200 \\
\hline \multicolumn{4}{|c|}{ TS-S4 } \\
\hline $\mathrm{C}$ & -7.02706900 & 0.17671600 & 0.95563800 \\
\hline $\mathrm{C}$ & -5.75130200 & -0.17042500 & 0.47540600 \\
\hline $\mathrm{C}$ & -4.79308900 & 0.86221500 & 0.36047100 \\
\hline $\mathrm{C}$ & -5.13006200 & 2.19691100 & 0.66392200 \\
\hline $\mathrm{C}$ & -6.40489600 & 2.48713700 & 1.15545400 \\
\hline $\mathrm{C}$ & -7.35499300 & 1.48015500 & 1.31250400 \\
\hline $\mathrm{C}$ & -2.75182100 & 2.67134800 & 0.82264300 \\
\hline $\mathrm{C}$ & -2.45875000 & 1.35755600 & 0.38893300 \\
\hline $\mathrm{C}$ & -1.13393300 & 0.87192800 & 0.42677500 \\
\hline $\mathrm{C}$ & -0.19196100 & 1.66790100 & 1.11486000 \\
\hline $\mathrm{C}$ & -0.52049400 & 2.86715100 & 1.72859200 \\
\hline $\mathrm{C}$ & -1.79607300 & 3.39096600 & 1.53911400 \\
\hline $\mathrm{H}$ & -7.78028700 & -0.60111400 & 1.05698900 \\
\hline $\mathrm{H}$ & -6.66447300 & 3.51489600 & 1.40719500 \\
\hline $\mathrm{H}$ & -8.35097300 & 1.71383800 & 1.69533800 \\
\hline $\mathrm{H}$ & 0.84431000 & 1.32587600 & 1.14950500 \\
\hline $\mathrm{H}$ & 0.23939100 & 3.43265800 & 2.26985700 \\
\hline $\mathrm{H}$ & -2.02787000 & 4.38709500 & 1.91225100 \\
\hline $\mathrm{C}$ & -4.08582800 & 3.26571000 & 0.38959500 \\
\hline $\mathrm{C}$ & -4.03008600 & 3.52395900 & -1.13971300 \\
\hline $\mathrm{H}$ & -3.24770700 & 4.26224200 & -1.37443200 \\
\hline $\mathrm{H}$ & -3.80606700 & 2.60426800 & -1.69902400 \\
\hline $\mathrm{H}$ & -4.99701800 & 3.91277400 & -1.49609000 \\
\hline $\mathrm{C}$ & -4.39598600 & 4.59230700 & 1.08806300 \\
\hline $\mathrm{H}$ & -5.34372500 & 5.01165500 & 0.72168700 \\
\hline $\mathrm{H}$ & -4.46633500 & 4.47912500 & 2.18020100 \\
\hline $\mathrm{H}$ & -3.61658300 & 5.33513900 & 0.86775900 \\
\hline $\mathrm{O}$ & -3.50356100 & 0.56579500 & -0.06731500 \\
\hline $\mathrm{C}$ & -6.09393900 & -3.01654300 & 1.36874400 \\
\hline $\mathrm{C}$ & -7.59330700 & -3.33674200 & 1.34547200 \\
\hline $\mathrm{H}$ & -7.83754200 & -3.99118500 & 2.20113500 \\
\hline $\mathrm{H}$ & -8.22707000 & -2.44425500 & 1.43608200 \\
\hline $\mathrm{H}$ & -7.88792600 & -3.87571900 & 0.43403700 \\
\hline $\mathrm{C}$ & -5.72306100 & -2.36704400 & 2.71274500 \\
\hline $\mathrm{H}$ & -5.88542600 & -3.09179300 & 3.52927300 \\
\hline $\mathrm{H}$ & -4.66485900 & -2.06480600 & 2.74210400 \\
\hline $\mathrm{H}$ & -6.33039800 & -1.47708200 & 2.92762000 \\
\hline
\end{tabular}


$\begin{array}{lll}-5.62162500 & -5.03479900 & 2.04731700\end{array}$

$\begin{array}{lll}-5.49636400 & -4.84445700 & 0.28457300\end{array}$

$\begin{array}{lll}-6.35742100 & -2.06555400 & -1.63535500\end{array}$

$-6.23038500 \quad-3.52073500 \quad-2.11322900$

$\begin{array}{lll}-6.80750100 & -4.21492700 & -1.48471200\end{array}$

$\begin{array}{lll}-6.61866300 & -3.61140500 & -3.14250200\end{array}$

$\begin{array}{lll}-5.18152000 & -3.85711300 & -2.12093400\end{array}$

$\begin{array}{lll}-7.83305700 & -1.65198900 & -1.60456000\end{array}$

$\begin{array}{lll}-7.95361700 & -0.59140300 & -1.34326200\end{array}$

$\begin{array}{lll}-8.26896000 & -1.78957100 & -2.61025000\end{array}$

$\begin{array}{lll}-8.43136600 & -2.25016100 & -0.90609900\end{array}$

$\begin{array}{lll}-5.62173000 & -1.14835500 & -2.63043800\end{array}$

$\begin{array}{lll}-5.66350500 & -0.09278300 & -2.32017100\end{array}$

$\begin{array}{lll}-4.56548000 & -1.42671300 & -2.74339600\end{array}$

$-6.09896900 \quad-1.22054200 \quad-3.62312400$

$\begin{array}{lll}-0.52923900 & -1.80224900 & 1.29226800\end{array}$

$\begin{array}{lll}0.06587500 & -3.19179500 & 1.02850800\end{array}$

$0.03040500 \quad-3.77641800 \quad 1.96321000$

$\begin{array}{lll}-0.49860500 & -3.75830300 & 0.27699100\end{array}$

$\begin{array}{lll}1.10626100 & -3.13739900 & 0.69206900\end{array}$

$\begin{array}{lll}0.22757500 & -1.15499600 \quad 2.46171300\end{array}$

$\begin{array}{lll}0.19774900 & -1.84828100 & 3.31885500\end{array}$

$\begin{array}{lll}1.27764200 & -0.95058500 & 2.22985100\end{array}$

$\begin{array}{lll}-0.24851800 & -0.22246600 & 2.79146300\end{array}$

$\begin{array}{lll}-2.00308900 & -1.90989800 & 1.70741500\end{array}$

$-2.06032600 \quad-2.53843700 \quad 2.61284100$

$\begin{array}{lll}-2.42251900 & -0.92766600 & 1.96537900\end{array}$

$\begin{array}{lll}-2.64875400 & -2.35760000 & 0.94793400\end{array}$

$-1.10892000 \quad-1.44989400 \quad-1.77983400$

$\begin{array}{lll}-1.72089200 & -0.38732500 & -2.70957700\end{array}$

$\begin{array}{lll}-2.48234700 & 0.21584900 & -2.19888200\end{array}$

$\begin{array}{lll}-0.97389100 & 0.27719900 & -3.15210700\end{array}$

$\begin{array}{lll}-2.21815900 & -0.91006900 & -3.54350600\end{array}$

$-2.20036700 \quad-2.48155300 \quad-1.49664500$

$\begin{array}{lll}-2.53804800 & -2.89014000 & -2.46433500\end{array}$

$\begin{array}{lll}-1.85086400 & -3.33190800 & -0.90105500\end{array}$

$\begin{array}{lll}-3.07273100 & -2.04783300 & -1.00084600\end{array}$

$\begin{array}{lll}0.04574000 & -2.16496200 & -2.49910400\end{array}$

$\begin{array}{lll}-0.32710700 & -2.59976200 & -3.44196100\end{array}$

$\begin{array}{lll}0.86571900 & -1.47732200 & -2.73423900\end{array}$

$\begin{array}{lll}0.46533500 & -2.97138200 & -1.88638300\end{array}$

$0.91954000 \quad 1.55063100 \quad-1.95833500$ 
$\begin{array}{lll}-0.16914000 & 1.45754400 & -1.99555800\end{array}$

$\begin{array}{lll}-0.28763500 & -0.65775100 & -0.22957400\end{array}$

$\begin{array}{lll}-5.35033400 & -1.90581600 & -0.01153200\end{array}$

$\begin{array}{lll}1.54094500 \quad 1.20902700 & -3.31489600\end{array}$

$\begin{array}{lll}1.15447200 & 1.87968500 & -4.10756400\end{array}$

$2.63456000 \quad 1.32155800 \quad-3.29968100$

$\begin{array}{lll}1.32291000 & 0.18098500 & -3.63957000\end{array}$

$\begin{array}{lll}1.24138600 & 2.90222200 & -1.50141200\end{array}$

$2.29968100 \quad 3.17766600 \quad-1.49540500$

$\begin{array}{lll}0.33293400 & 3.82586800 & -1.10621400\end{array}$

$\begin{array}{lll}-0.72789700 & 3.56373900 & -1.17134200\end{array}$

$0.61192400 \quad 5.15351200 \quad-0.56641200$

$\begin{array}{lll}-0.45162000 & 6.06184100 & -0.37359700\end{array}$

$1.90537900 \quad 5.58418000 \quad-0.19459200$

$\begin{array}{lll}-0.23727600 & 7.33630000 & 0.15245500\end{array}$

$\begin{array}{lll}-1.46257600 & 5.75145700 & -0.64948800\end{array}$

$2.12067900 \quad 6.85776300 \quad 0.32771600$

$2.75037700 \quad 4.90152900 \quad-0.30361900$

$\begin{array}{lll}1.05198000 & 7.74554800 & 0.50647100\end{array}$

$\begin{array}{lll}-1.08330700 \quad 8.01595300 & 0.28689200\end{array}$

$3.13341800 \quad 7.16058000 \quad 0.60802200$

$\begin{array}{lll}1.22339400 & 8.74171300 \quad 0.92154600\end{array}$

$1.78081500 \quad 0.12172300 \quad-0.73759100$

$3.01148200 \quad-1.55422600 \quad-0.71948000$

$3.38707700 \quad 1.17628100 \quad-1.04799200$

$\begin{array}{lll}4.04764700 & -1.34054000 & -1.62170700\end{array}$

$2.70863600 \quad-2.90584700 \quad-0.68394200$

$4.33675500 \quad-2.53873700 \quad-2.36615600$

$3.62802300 \quad-3.66416300 \quad-1.51122800$

$\begin{array}{lll}4.35271000 & 0.73734400 & 0.90289200\end{array}$

$5.11645500 \quad-1.53150700 \quad 1.20957000$

$5.85221100 \quad-2.67713000 \quad-2.47514100$

$3.73415600 \quad-2.35346900 \quad-3.76068400$

$\begin{array}{lll}2.83097600 & -4.68024000 & -2.32246100\end{array}$

$\begin{array}{lll}4.58280800 & -4.39593600 & -0.57280900\end{array}$

$4.07954500 \quad-0.60345500 \quad 1.32897600$

$\begin{array}{lll}5.40503100 & -1.95787500 & 2.56772700\end{array}$

$6.13371200 \quad-3.62063300 \quad-2.96794800$

$6.25239000 \quad-1.84920100 \quad-3.08029800$

$6.32925300 \quad-2.63519000 \quad-1.48815100$

$4.15206900 \quad-1.44082000 \quad-4.21086900$

$3.96362300 \quad-3.20206600 \quad-4.42280500$

$2.64454900 \quad-2.23235600 \quad-3.71251700$

$2.32395800 \quad-5.37799700 \quad-1.63840200$ 


\begin{tabular}{|c|c|c|c|}
\hline $\mathrm{H}$ & 3.49787300 & -5.26892000 & -2.97160700 \\
\hline $\mathrm{H}$ & 2.06594700 & -4.20926100 & -2.95047100 \\
\hline $\mathrm{H}$ & 5.28841600 & -5.03269500 & -1.12693000 \\
\hline $\mathrm{H}$ & 5.14559900 & -3.67835000 & 0.03117900 \\
\hline $\mathrm{H}$ & 4.00114900 & -5.04103800 & 0.10296500 \\
\hline $\mathrm{O}$ & 3.40905800 & -0.73820400 & 2.52587300 \\
\hline $\mathrm{C}$ & 6.39921700 & -0.94976200 & 3.15536900 \\
\hline $\mathrm{C}$ & 6.03065700 & -3.34398700 & 2.56760000 \\
\hline $\mathrm{C}$ & 3.98410400 & -1.86820800 & 3.23085000 \\
\hline $\mathrm{H}$ & 6.71557200 & -1.23598900 & 4.16933600 \\
\hline $\mathrm{H}$ & 7.29324700 & -0.91742700 & 2.51493700 \\
\hline $\mathrm{H}$ & 5.97325600 & 0.06282800 & 3.19739200 \\
\hline $\mathrm{H}$ & 6.98694000 & -3.32083400 & 2.02310500 \\
\hline $\mathrm{H}$ & 6.23871000 & -3.66428400 & 3.60045000 \\
\hline $\mathrm{H}$ & 5.38829600 & -4.09506200 & 2.09727200 \\
\hline $\mathrm{C}$ & 3.14400700 & -3.11431700 & 2.95688200 \\
\hline $\mathrm{C}$ & 3.98763100 & -1.54365600 & 4.71788000 \\
\hline $\mathrm{H}$ & 2.11777000 & -2.94840000 & 3.30370300 \\
\hline $\mathrm{H}$ & 3.54402200 & -3.98528900 & 3.49627600 \\
\hline $\mathrm{H}$ & 3.11178900 & -3.33970800 & 1.88320400 \\
\hline $\mathrm{H}$ & 2.95232700 & -1.49280800 & 5.08787000 \\
\hline $\mathrm{H}$ & 4.51307000 & -2.32615200 & 5.28742000 \\
\hline $\mathrm{H}$ & 4.46528200 & -0.57649800 & 4.92221000 \\
\hline $\mathrm{C}$ & 4.32760300 & 1.30496700 & -0.27256900 \\
\hline $\mathrm{C}$ & 5.53844300 & 2.16308100 & -0.60552100 \\
\hline $\mathrm{C}$ & 6.76910000 & 1.23371600 & -0.58380900 \\
\hline $\mathrm{H}$ & 6.91409600 & 0.78625900 & 0.40906100 \\
\hline $\mathrm{H}$ & 6.65517700 & 0.41411300 & -1.30931400 \\
\hline $\mathrm{H}$ & 7.67100600 & 1.80885400 & -0.84545100 \\
\hline $\mathrm{C}$ & 5.37306300 & 2.79413300 & -1.99090200 \\
\hline $\mathrm{H}$ & 4.49419400 & 3.45393300 & -2.03455700 \\
\hline $\mathrm{H}$ & 6.26273900 & 3.39575500 & -2.23263600 \\
\hline $\mathrm{H}$ & 5.25411500 & 2.02481400 & -2.76745400 \\
\hline $\mathrm{C}$ & 5.67593200 & 3.25517900 & 0.47269000 \\
\hline $\mathrm{H}$ & 4.78775100 & 3.90675700 & 0.49462700 \\
\hline $\mathrm{H}$ & 5.80363700 & 2.81652300 & 1.47257100 \\
\hline $\mathrm{H}$ & 6.55351600 & 3.88358700 & 0.25460100 \\
\hline \multicolumn{4}{|c|}{ TS-S5 } \\
\hline $\mathrm{C}$ & 7.31695700 & 0.86441100 & -0.97564600 \\
\hline $\mathrm{C}$ & 6.09047500 & 0.37973500 & -0.48552400 \\
\hline $\mathrm{C}$ & 5.09614100 & 1.33140100 & -0.15941700 \\
\hline $\mathrm{C}$ & 5.36486200 & 2.71294000 & -0.25122700 \\
\hline C & 6.59407400 & 3.14235600 & -0.75637800 \\
\hline $\mathrm{C}$ & 7.56975800 & 2.22243300 & -1.13420700 \\
\hline
\end{tabular}


$2.96909200 \quad 3.09901500 \quad-0.22458300$

$\begin{array}{lll}2.74376400 & 1.71980800 & -0.02096000\end{array}$ 
$\begin{array}{lll}6.30976600 & 0.07667700 & 2.27122900\end{array}$

$\begin{array}{lll}5.28334300 & -1.33882400 & 2.59958400\end{array}$

$6.88534800 \quad-1.20272500 \quad 3.36280100$

$0.97364800 \quad-1.44415700 \quad-1.25053600$

$0.45413800 \quad-2.87707100 \quad-1.06973200$

$\begin{array}{lll}0.53775900 & -3.40750200 & -2.03295400\end{array}$

$\begin{array}{lll}1.04739400 & -3.43889900 & -0.33400600\end{array}$

$\begin{array}{lll}-0.59773400 & -2.90140700 & -0.76922800\end{array}$

$\begin{array}{lll}0.18341000 & -0.72183900 & -2.35712400\end{array}$

$0.14883500 \quad-1.37241600 \quad-3.24548500$

$\begin{array}{lll}-0.85089900 & -0.49298500 & -2.08638400\end{array}$

$\begin{array}{lll}0.67817700 & 0.21626300 & -2.64622400\end{array}$

$2.44401900 \quad-1.46259900 \quad-1.68311700$

$\begin{array}{lll}2.50476600 & -1.95113800 & -2.67083900\end{array}$

$\begin{array}{lll}2.84929800 & -0.44753100 & -1.79450300\end{array}$

$3.09853700 \quad-2.00819700 \quad-0.99897200$

$\begin{array}{lll}1.60052600 & -1.23881000 & 1.83437900\end{array}$

$\begin{array}{lll}2.01974500 & -0.12811200 & 2.81124400\end{array}$

$\begin{array}{lll}2.83091500 & 0.48252400 & 2.39465800\end{array}$

$\begin{array}{lll}1.19175100 & 0.53414800 & 3.08850500\end{array}$

$2.39532400 \quad-0.59496400 \quad 3.73789600$

$\begin{array}{lll}2.83221700 & -2.07354300 & 1.50118200\end{array}$

$3.25931200 \quad-2.45351600 \quad 2.44541200$

$2.59431200 \quad-2.95042200 \quad 0.88502300$

$3.60661100 \quad-1.49816400 \quad 0.98989600$

$\begin{array}{lll}0.56848300 & -2.15992600 & 2.49357500\end{array}$

$\begin{array}{lll}1.00954600 & -2.62410900 \quad 3.39208100\end{array}$

$\begin{array}{lll}-0.33244100 & -1.61369800 & 2.78941000\end{array}$

$\begin{array}{lll}0.24691000 & -2.95850300 & 1.81522500\end{array}$

$\begin{array}{lll}-1.10545800 & 1.47355200 & 2.18610000\end{array}$

$\begin{array}{lll}-0.04395800 & 1.75047100 & 2.17893300\end{array}$

$0.68435000 \quad-0.45738200 \quad 0.35538800$

$\begin{array}{lll}5.82441400 & -1.43310000 & -0.23379000\end{array}$

$\begin{array}{lll}-1.53140600 & 0.76650900 & 3.47295500\end{array}$

$\begin{array}{lll}-1.54136800 \quad 1.46087300 & 4.33683600\end{array}$

$\begin{array}{lll}-2.54959100 & 0.35593500 & 3.37368700\end{array}$

$\begin{array}{lll}-0.86830900 & -0.06418100 & 3.74857600\end{array}$

$\begin{array}{lll}-1.96019300 & 2.61769500 & 1.90155500\end{array}$

$\begin{array}{lll}-3.01513800 & 2.45378700 & 2.12828100\end{array}$

$\begin{array}{lll}-1.61641200 \quad 3.81038200 & 1.35620400\end{array}$

$\begin{array}{lll}-0.57266200 & 3.99371400 & 1.09341700\end{array}$

$\begin{array}{lll}-2.56116300 & 4.84521500 & 0.95455700\end{array}$

$\begin{array}{lll}-2.12106700 & 5.91943200 & 0.14282100\end{array}$ 
TS-S6

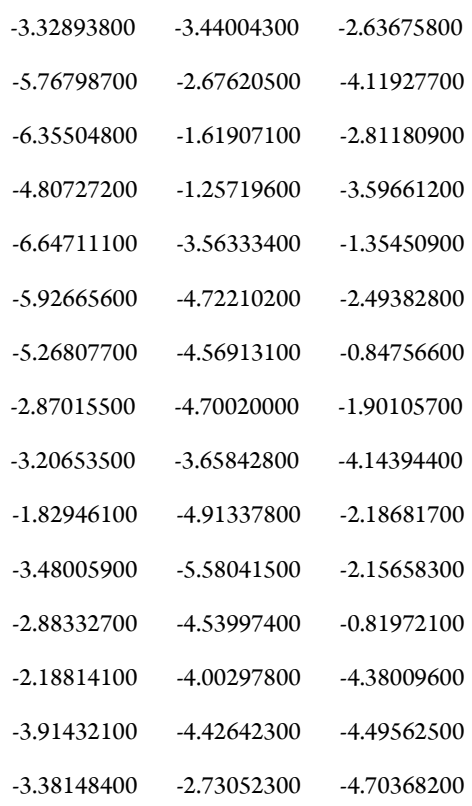


$-3.14991400$

$-0.81980600$

$-2.05471200$

$-3.67495700$

3.42354800

$-4.76694300$

0.73991800

3.17613200

$-3.41709600$

3.61018300

1.48433200

$-3.27733800$

4.01733300

$-0.04063100$

$-3.06099500$

1.69254600

0.17052800

$-3.21756300$

2.74097700

$-0.72166000$

$-3.07089800$

$2.20945600 \quad 1.48011700$

$-1.70949700$

0.18305200

$-2.25536800$

$-2.57532300$

$-1.06006200$

$-4.13440700$

$-3.11352300$

0.29933400

$-4.59528100$

$-3.56939800$

$0.21654000 \quad-5.59647100$

$-2.31218400$

$1.04932900-4.64417700$

$-3.86797600$

0.67431300

$-3.89076800$

$-1.43658900$

$-1.53330500 \quad-5.05903600$

$-1.00484200$

$-2.47792100$

$-4.69389900$

$-0.62875500$

$-0.79230300$

$-5.13292700$

$-1.82344900$

$-1.70542100$

$-6.07757000$

$-3.69231200$

$-2.11614700$

$-4.17716500$

$-3.29460500$

$-3.11725800$

$-3.95224500$

$-4.15122000 \quad-2.15269300 \quad-5.17856100$

$-4.47892500$

$-1.87467700 \quad-3.45176800$

$-1.99228700$

$4.78501900 \quad-0.33867600$

$-2.00081900$

$5.77279400 \quad 0.14473300$

$-1.90373300$

4.93768100

$-1.42253100$

$-1.10902800$

4.24016100

0.00015600

$-4.44141900$

4.83114700

$-0.60083600$

$-4.29740700$

$4.96130000 \quad-1.68398200$

$\begin{array}{lll}-4.46808200 & 5.83197500 & -0.14236200\end{array}$

$\begin{array}{lll}-5.41151900 \quad 4.35342100 & -0.44746200\end{array}$

$-4.84339600$

$3.76171400 \quad 2.02595700$

$\begin{array}{lll}-4.86813800 & 3.48566100 & 3.09358200\end{array}$

$\begin{array}{lll}-5.54630800 & 3.12915600 & 1.47294700\end{array}$

$\begin{array}{lll}-5.18444300 & 4.80499600 & 1.97420200\end{array}$

$\begin{array}{lll}-2.46329300 & 4.33408900 & 2.43126700\end{array}$

$\begin{array}{lll}-1.41585600 \quad 4.18193500 & 2.15333000\end{array}$

$\begin{array}{lll}-2.59387500 & 3.94462000 & 3.45258500\end{array}$

$\begin{array}{lll}-2.66824200 & 5.41529700 & 2.45337300\end{array}$

$-1.20763300$

$0.40587900 \quad-0.36851200$

$\begin{array}{lll}7.43660100 & 0.30655800 & 1.63119700\end{array}$

$\begin{array}{lll}6.32457200 & 0.26206700 & 0.76963300\end{array}$

$\begin{array}{lll}5.52468900 & 1.42668600 & 0.69969200\end{array}$

$\begin{array}{lll}5.92087800 & 2.61906100 & 1.33778500\end{array}$

C

C

$\begin{array}{lll}7.02852100 & 2.60653200 & 2.18753100\end{array}$ 


\begin{tabular}{|c|c|c|}
\hline 7.77234800 & 1.44115500 & 2.36198200 \\
\hline 3.67530400 & 3.48637600 & 1.00206200 \\
\hline 3.31654500 & 2.27649200 & 0.36845500 \\
\hline 1.95240800 & 1.96737800 & 0.12065800 \\
\hline 1.00222400 & 2.83511100 & 0.69415100 \\
\hline 1.34432300 & 3.98168700 & 1.39921300 \\
\hline 2.68575200 & 4.31979800 & 1.52669400 \\
\hline 8.06222000 & -0.57689900 & 1.72387200 \\
\hline 7.32431100 & 3.51711000 & 2.70752100 \\
\hline 8.63366800 & 1.42939600 & 3.03370100 \\
\hline-0.04719100 & 2.57236600 & 0.56294400 \\
\hline 0.57034600 & 4.62069200 & 1.82613200 \\
\hline 2.96404300 & 5.24354600 & 2.03193200 \\
\hline 5.14677300 & 3.87914400 & 0.98797800 \\
\hline 5.52470400 & 4.30459100 & -0.45625600 \\
\hline 4.94507000 & 5.19217700 & -0.75478700 \\
\hline 5.31718400 & 3.50489900 & -1.18112700 \\
\hline 6.59710300 & 4.54911400 & -0.51339900 \\
\hline 5.46248800 & 5.04509900 & 1.92855500 \\
\hline 6.52972500 & 5.30339900 & 1.87622200 \\
\hline 5.21259900 & 4.81342500 & 2.97482500 \\
\hline 4.90869700 & 5.94674400 & 1.63132500 \\
\hline 4.33551200 & 1.42112000 & -0.02184700 \\
\hline 6.27624100 & -2.74496500 & 0.81784700 \\
\hline 7.70257900 & -3.19290300 & 1.16260000 \\
\hline 7.65491900 & -4.09910500 & 1.79250100 \\
\hline 8.27089200 & -2.44344000 & 1.72966000 \\
\hline 8.28123000 & -3.45514700 & 0.26566600 \\
\hline 5.49131800 & -2.46681600 & 2.11079100 \\
\hline 5.40229100 & -3.39813400 & 2.69697200 \\
\hline 4.47229500 & -2.10911500 & 1.89834600 \\
\hline 5.98557100 & -1.71728300 & 2.74465700 \\
\hline 5.58237600 & -3.90590300 & 0.07441100 \\
\hline 4.52829200 & -3.68268400 & -0.14276400 \\
\hline 5.60876900 & -4.81616800 & 0.69832400 \\
\hline 6.07835300 & -4.14483500 & -0.87742700 \\
\hline 7.47630200 & -1.03479100 & -1.55417000 \\
\hline 7.48025900 & -2.29204700 & -2.43888800 \\
\hline 7.83358300 & -3.18007400 & -1.89440000 \\
\hline 8.15927900 & -2.14138000 & -3.29595600 \\
\hline 6.47929700 & -2.51326600 & -2.84219000 \\
\hline 8.88250100 & -0.79535800 & -0.99302000 \\
\hline 8.94690400 & 0.14990400 & -0.43662000 \\
\hline 9.60038100 & -0.72859900 & -1.83009600 \\
\hline
\end{tabular}


$9.22402800 \quad-1.60548700 \quad-0.33626900$

$\begin{array}{lll}7.08846800 & 0.17810000 & -2.42143100\end{array}$

$\begin{array}{lll}7.02354300 & 1.10213000 & -1.82561500\end{array}$

$\begin{array}{lll}6.12284100 & 0.02980500 & -2.92617700\end{array}$

$\begin{array}{lll}7.85525600 & 0.33984000 & -3.19896600\end{array}$

$2.11635700 \quad-0.67091100 \quad-1.51891700$

$3.32129000 \quad-0.40950600 \quad-2.42893100$

$\begin{array}{lll}3.88024200 & -1.35175800 & -2.53308600\end{array}$

$\begin{array}{lll}4.01043900 & 0.32267300 & -2.00038100\end{array}$

$\begin{array}{lll}3.02743300 & -0.09057500 & -3.43565600\end{array}$

$\begin{array}{lll}1.18650500 & -1.68927700 & -2.19700800\end{array}$

$0.82691500-1.33926600 \quad-3.17201000$

$0.30769500 \quad-1.92702800 \quad-1.58846100$

$\begin{array}{lll}1.75058100 & -2.62226300 & -2.36435300\end{array}$

$2.58644400 \quad-1.26736800 \quad-0.18932700$

$3.10365100 \quad-2.21480100 \quad-0.38821500$

$1.73614900-1.48059000 \quad 0.45486700$

$\begin{array}{lll}3.28487700 & -0.60851500 & 0.33411600\end{array}$

$\begin{array}{lll}1.06466400 & 2.03510800 & -2.61135600\end{array}$

$\begin{array}{lll}-0.02569400 & 3.08546800 & -2.37237000\end{array}$

$\begin{array}{lll}0.24189100 & 3.75658600 & -1.54898400\end{array}$

$\begin{array}{lll}-1.00286100 & 2.63130400 \quad-2.16295400\end{array}$

$\begin{array}{lll}-0.12501600 & 3.70998900 & -3.27689400\end{array}$

$\begin{array}{lll}2.40415600 & 2.76637600 & -2.79221200\end{array}$

$2.33605600 \quad 3.39883200 \quad-3.69422400$

$\begin{array}{lll}3.25380900 & 2.08800300 & -2.92927300\end{array}$

$\begin{array}{lll}2.62622000 & 3.43105600 & -1.94660300\end{array}$

$0.69981000 \quad 1.27673700 \quad-3.89358900$

$\begin{array}{lll}0.59887800 & 2.00661000 & -4.71470100\end{array}$

$\begin{array}{lll}-0.26072400 & 0.77268100 & -3.76782100\end{array}$

$\begin{array}{lll}1.45672900 & 0.54679600 & -4.20108000\end{array}$

$\begin{array}{lll}1.06356100 & 0.84631300 & -1.08224600\end{array}$

$6.01558900 \quad-1.21132200 \quad-0.31848400$

$\begin{array}{lll}-0.94053500 & 0.03976300 & 1.58170500\end{array}$

$\begin{array}{lll}-2.00594200 & -0.19038600 & 1.68615200\end{array}$

$\begin{array}{lll}-0.48081000 & 0.99880100 & 2.66590400\end{array}$

$\begin{array}{lll}-0.74096500 & 0.59152900 & 3.66328900\end{array}$

$\begin{array}{lll}-0.96746100 & 1.97547000 & 2.58574100\end{array}$

$0.60970100 \quad 1.15175200 \quad 2.65830500$

$\begin{array}{lll}-0.19053300 & -1.21349100 & 1.59891400\end{array}$

$\begin{array}{lll}-0.56512700 & -2.38479500 & 1.01725300\end{array}$

$0.77995100 \quad-1.18220600 \quad 2.11204200$

$\begin{array}{lll}-1.49608800 & -2.38329600 & 0.45252400\end{array}$

$0.21601800 \quad-3.62027300 \quad 0.97676400$ 


$\begin{array}{lll}-0.10960400 & -4.61506300 & 0.02727400 \\ 1.30321300 & -3.88076400 & 1.84475400 \\ 0.62020100 & -5.80072700 & -0.06203300 \\ -0.92393600 & -4.42286800 & -0.67523100 \\ 2.03274400 & -5.06608100 & 1.75260300 \\ 1.58055800 & -3.13965300 & 2.59853200 \\ 1.69794000 & -6.03511200 & 0.79868200 \\ 0.35150100 & -6.54471700 & -0.81658200 \\ 2.87250000 & -5.23580800 & 2.43152700 \\ 2.27181000 & -6.96205500 & 0.72790700\end{array}$

$\mathrm{B}_{2}$ pin $_{2}$

C

$\mathrm{H}$

C

H

C

$\mathrm{H}$

H

H

$\operatorname{pin}_{2}$

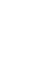

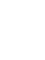

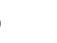

.

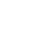

H

C

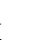

$\mathrm{H}$

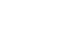

.

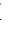

C

H

$$
\mathrm{H}
$$

C

C

B

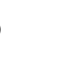

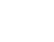

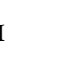

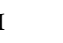

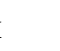

C

\begin{tabular}{|c|c|c|}
\hline-0.51175800 & 0.63856200 & 2.99177600 \\
\hline 0.51286000 & -0.55738700 & 3.00775400 \\
\hline 0.00013600 & 0.01155400 & 0.85305300 \\
\hline-0.46902900 & 1.05983700 & 1.60116500 \\
\hline 0.46959600 & -1.01611800 & 1.62906200 \\
\hline-0.13427900 & 1.81957600 & 3.87488600 \\
\hline-0.90125100 & 2.60458200 & 3.79234800 \\
\hline-0.07406000 & 1.51380000 & 4.93098400 \\
\hline 0.82817000 & 2.25785600 & 3.58075000 \\
\hline-1.95061000 & 0.20427600 & 3.27589700 \\
\hline-2.09392600 & -0.06439500 & 4.33297200 \\
\hline-2.62809500 & 1.03754700 & 3.03700300 \\
\hline-2.24109700 & -0.65488000 & 2.65360100 \\
\hline 1.95182100 & -0.11558300 & 3.27946800 \\
\hline 2.09554400 & 0.18158000 & 4.32883400 \\
\hline 2.62921700 & -0.95501400 & 3.06293700 \\
\hline 2.24206200 & 0.72642500 & 2.63405000 \\
\hline 0.13572900 & -1.71408100 & 3.92262900 \\
\hline 0.90267300 & -2.50103700 & 3.86105600 \\
\hline 0.07591700 & -1.37985300 & 4.97009300 \\
\hline-0.82683100 & -2.16015200 & 3.64082700 \\
\hline 0.59746700 & 0.47168600 & -3.00680200 \\
\hline-0.59856400 & -0.55286500 & -2.99272700 \\
\hline-0.00017700 & -0.01153200 & -0.85305300 \\
\hline-1.03836800 & -0.49113900 & -1.60858100 \\
\hline 1.03775000 & 0.44744600 & -1.62164500 \\
\hline-0.16048700 & -1.99539100 & -3.25124500 \\
\hline-0.99685800 & -2.66970600 & -3.01422100 \\
\hline 0.12227900 & -2.15314700 & -4.30257200 \\
\hline 0.69028900 & -2.27713100 & -2.61360600 \\
\hline-1.76769100 & -0.18778800 & -3.89662300 \\
\hline-1.44784400 & -0.14201100 & -4.94927300 \\
\hline-2.55371100 & -0.95371700 & -3.81405000 \\
\hline
\end{tabular}


TS-S7

C

$\begin{array}{rrr}-2.20988200 & 0.77851800 & -3.62160700 \\ 1.76628200 & 0.08227100 & -3.90089100 \\ 1.44607200 & 0.00801800 & -4.95180400 \\ 2.55232900 & 0.85015100 & -3.83935100 \\ 2.20857400 & -0.87623700 & -3.59997100 \\ 0.15929500 & 1.90669000 & -3.30412100 \\ 0.99574400 & 2.58716900 & -3.08572200 \\ -0.12383400 & 2.03593200 & -4.35923400 \\ -0.69126200 & 2.20558900 & -2.67404600\end{array}$

2.73379500

1.89872100

2.54141300

3.92722000

4.71204500

4.12275600

3.43918200

2.09620700

1.07034600

1.42383200

2.72879000

3.73647800

2.29984400

5.79447300

4.74571300

0.66779900

2.96694600

4.76460700

4.49737700

5.82645800

6.23126300

5.71665000

6.58280900

4.73274900

5.49615000

3.81485000

5.07424500

1.75443500

$-0.51880900$

$-0.2278530$

0.82835600

$-0.81208200$

$-0.53753500$

0.09653100

0.93397600

0.79639300

0.66602300

$0.69222000 \quad 0.80746300$

$\begin{array}{ll}1.84042700 & 0.95835700\end{array}$

$3.10421400 \quad 0.98705200$

$-1.66588700 \quad 1.36529900$

$-1.43068600 \quad 1.02630400$

$-2.36247600 \quad 1.26559500$

$-3.50891400 \quad 1.99618300$

$-3.72192900 \quad 2.43852900$

$-2.81782100 \quad 2.09877900$

$4.22282100 \quad 1.02704000$

$1.75162900 \quad 1.05108900$

$3.99580500 \quad 1.08853600$

$-4.25236900 \quad 2.23317500$

$-4.61428600 \quad 3.02168700$

$-3.02476200 \quad 2.39607000$

$\begin{array}{ll}-0.72813500 & 0.78071200\end{array}$

$-0.81653900 \quad 1.54034000$

\begin{tabular}{ll}
$-1.83761300 \quad 1.49848200$ \\
\hline
\end{tabular}

$-0.52765300 \quad 2.59684500$

$-0.16603800 \quad 1.07972500$

$-1.16427200 \quad-0.68633300$

$-0.53045500 \quad-1.15797900$

$-1.08159100 \quad-1.28243900$

$-2.21086800 \quad-0.71773700$

$\begin{array}{ll}-0.24388200 & 0.43149100\end{array}$

$3.91961700 \quad 0.46159700$

$5.05570400 \quad 1.45488200$

$5.15835100 \quad 1.73318200$

$4.94634100 \quad 2.37730700$

$6.01002700 \quad 0.99375900$

$4.28063100 \quad-0.90371000$ 
$1.19202200 \quad 4.34868400 \quad-0.87867900$

$\begin{array}{lll}-0.28906400 & 5.26524400 & -1.21849100\end{array}$

\section{$\begin{array}{lll}-0.17820600 & 3.56365800 & -1.68749300\end{array}$}

$\begin{array}{lll}-2.04942600 & 3.85497000 & 0.28989800\end{array}$

$\begin{array}{lll}-2.37166000 & 3.03442700 & -0.35816800\end{array}$

$\begin{array}{lll}-2.40524900 \quad 4.80768900 & -0.13933500\end{array}$

$\begin{array}{lll}-2.56166700 & 3.71413200 & 1.25056900\end{array}$

$\begin{array}{lll}-0.11922000 & 1.91489100 & 2.83358900\end{array}$

$0.48034600 \quad 0.53556900 \quad 3.13886700$

$\begin{array}{lll}0.10582600 & -0.22724400 & 2.45210300\end{array}$

$0.21466000 \quad 0.23570600 \quad 4.16589100$

$\begin{array}{lll}1.57743400 & 0.54535400 \quad 3.07609400\end{array}$

$\begin{array}{lll}-1.62192400 & 1.89891200 \quad 3.15805900\end{array}$

$\begin{array}{lll}-2.06142100 & 2.90568000 \quad 3.10633100\end{array}$

$\begin{array}{lll}-1.77920800 & 1.52812700 \quad 4.18548500\end{array}$

$\begin{array}{lll}-2.18445800 & 1.25524100 & 2.46449800\end{array}$

$0.61421900 \quad 2.92961100 \quad 3.71999800$

$0.14298300 \quad 3.91765800 \quad 3.73094600$

$1.66690400 \quad 3.04840900 \quad 3.42297900$

$0.61065100 \quad 2.55984100 \quad 4.76062100$

$\begin{array}{lll}-1.84276100 & -2.85512700 \quad 1.74200900\end{array}$

$\begin{array}{lll}-3.22032200 & -2.30396200 & 1.36752400\end{array}$

$\begin{array}{lll}-3.50134900 & -2.56207100 & 0.34187400\end{array}$

$\begin{array}{lll}-3.24777300 & -1.21130200 \quad 1.45319900\end{array}$

$\begin{array}{lll}-3.97686000 & -2.72229900 & 2.05327400\end{array}$

$\begin{array}{lll}-1.50676100 & -2.32812000 \quad 3.14515800\end{array}$

$\begin{array}{lll}-2.26894600 & -2.69777900 \quad 3.85219700\end{array}$

$-1.54128200 \quad-1.23247200 \quad 3.17328700$

$\begin{array}{lll}-0.52374400 & -2.65012200 & 3.51313000\end{array}$

$\begin{array}{lll}-1.97006700 & -4.38586800 & 1.77836500\end{array}$

$\begin{array}{lll}-2.66396000 & -4.65543200 & 2.59302200\end{array}$

$\begin{array}{lll}-1.03089400 & -4.91853800 \quad 1.96633400\end{array}$

$\begin{array}{lll}-2.40307400 & -4.77598400 & 0.84824400\end{array}$

$\begin{array}{lll}-0.35628300 & -3.31404200 & -1.02489800\end{array}$

$\begin{array}{lll}-1.60486000 & -3.20844000 & -1.91099000\end{array}$

$\begin{array}{lll}-1.45789500 & -3.80310500 & -2.82903700\end{array}$

$\begin{array}{lll}-1.81437100 & -2.16891900 & -2.19124400\end{array}$

$\begin{array}{lll}-2.50205300 & -3.59503700 & -1.40477100\end{array}$

$0.86712400 \quad-2.75765500 \quad-1.77546600$

$\begin{array}{lll}0.89727900 & -3.18605800 & -2.79098200\end{array}$

$1.80825800 \quad-3.02731400 \quad-1.27540300$

$\begin{array}{lll}0.83362900 & -1.66720800 & -1.86386200\end{array}$

$\begin{array}{lll}-0.06511600 & -4.78987900 & -0.72383800\end{array}$

$\begin{array}{lll}-0.94501700 & -5.34464400 & -0.38150100\end{array}$ 
$0.74391400 \quad-4.92060000 \quad 0.00803700$

$\begin{array}{lll}0.27149700 & -5.27046100 & -1.65902700\end{array}$

$-0.59643800$

0.04181700

$-0.21248900$

1.01162400

2.29867900

2.49396700

3.30056000

1.04922700

3.42135400

3.74563900

4.84208800

4.66792500

3.86674500

5.82002000

5.51384500

$-1.49065100$

$-1.37403200$

$-0.97823900$

$-0.28281100$

1.65182100

$-2.53718300$

$-2.15574300$

$-3.46577700$

$-2.79492500$

$-5.14208200$

$-4.93687300$

$-2.85335300$

$-3.79465500$

$-3.51050800$

$-5.56553300$

$-6.66205700$

$-5.31236500$

$-5.18764400$

$-5.39681800$

$-6.48475800$

$-4.89904700$

$-5.14649400$

$-5.92959400$

$-5.87343600$

$-6.99031200$

$-5.54842900$

$-5.81893700$
$2.10866700 \quad 0.46896100$

$2.12727900 \quad 0.92621400$

$0.47461100 \quad-2.60313700$

$1.02586300-2.36086800$

$0.65255800 \quad-2.93057700$

$-0.44873000 \quad-3.79409500$

$2.31358300 \quad-1.97275700$

$1.90877800 \quad-1.72546800$

$1.46357000-2.64804000$

$-0.71829700 \quad-4.34474800$

$0.10620300 \quad-4.06391200$

$1.20172700 \quad-3.21224700$

$-1.57885500 \quad-5.00835700$

$-0.10596100-4.50278000$

$1.85257300 \quad-2.97525800$

$1.00733700 \quad-2.11225400$

$2.03043200 \quad-1.74416100$

$-0.10876500-0.47089700$

$-0.37698500 \quad-3.28998900$

$-1.09444700 \quad-4.04510200$

$1.00774300 \quad-3.22661600$

$1.57378000 \quad-4.09603800$

$1.49864200 \quad-2.91235200$

$-0.00548100 \quad-3.56683500$

$-0.30515900 \quad-1.07170100$

$0.96160300-0.11878900$

$0.06031700-0.70408900$

$-0.75033100-1.33313500$

$1.03191300 \quad 0.05083200$

$0.81976000 \quad 1.26779400$

$0.74893200 \quad 1.20905300$

$1.70566000 \quad 1.86981200$

$-0.06228800 \quad 1.79908000$

$2.28697500 \quad-0.73097800$

$2.30125600-0.89595700$

$2.49128800-1.68755700$

$3.10933700 \quad-0.04441000$

$-1.45152400 \quad-0.43057300$

$-2.33315700 \quad-1.08704000$

$-1.18115100 \quad-0.31845200$

$-1.73980500 \quad 0.55244100$

$0.00883300 \quad-2.40964800$ 


\begin{tabular}{|c|c|c|c|}
\hline $\mathrm{H}$ & -6.86579700 & 0.31259300 & -2.25947100 \\
\hline $\mathrm{H}$ & -5.81582300 & -0.89892300 & -3.03222100 \\
\hline $\mathrm{H}$ & -5.31097500 & 0.79778500 & -2.97257800 \\
\hline \multicolumn{4}{|c|}{ TS-S8 } \\
\hline $\mathrm{C}$ & -4.73197100 & -0.94476300 & 2.99444900 \\
\hline $\mathrm{C}$ & -4.14082600 & -0.52038400 & 1.79502800 \\
\hline $\mathrm{C}$ & -3.86803500 & -1.50840900 & 0.81316300 \\
\hline $\mathrm{C}$ & -4.29436900 & -2.83668300 & 0.98593500 \\
\hline $\mathrm{C}$ & -4.87266100 & -3.20276500 & 2.20777000 \\
\hline $\mathrm{C}$ & -5.06769300 & -2.27601800 & 3.22474300 \\
\hline $\mathrm{C}$ & -3.45483500 & -3.30587900 & -1.32742500 \\
\hline $\mathrm{C}$ & -2.90543400 & -2.01020300 & -1.33028800 \\
\hline $\mathrm{C}$ & -2.04674500 & -1.58674000 & -2.38071200 \\
\hline $\mathrm{C}$ & -1.89679700 & -2.44909900 & -3.47660600 \\
\hline $\mathrm{C}$ & -2.49401800 & -3.70531600 & -3.52593300 \\
\hline $\mathrm{C}$ & -3.24430000 & -4.13234900 & -2.43839600 \\
\hline $\mathrm{H}$ & -4.95694800 & -0.20964600 & 3.76246700 \\
\hline $\mathrm{H}$ & -5.19425200 & -4.23463200 & 2.35494500 \\
\hline $\mathrm{H}$ & -5.51530900 & -2.57812500 & 4.17398300 \\
\hline $\mathrm{H}$ & -1.28495000 & -2.13260000 & -4.31534100 \\
\hline $\mathrm{H}$ & -2.35673700 & -4.34857900 & -4.39755300 \\
\hline $\mathrm{H}$ & -3.68567100 & -5.12999300 & -2.44881800 \\
\hline $\mathrm{C}$ & -4.24975500 & -3.85444500 & -0.14720300 \\
\hline $\mathrm{C}$ & -5.70399400 & -4.13640300 & -0.59786800 \\
\hline $\mathrm{H}$ & -5.72904000 & -4.87283600 & -1.41564600 \\
\hline $\mathrm{H}$ & -6.18702500 & -3.21348500 & -0.95359500 \\
\hline $\mathrm{H}$ & -6.30184800 & -4.53525500 & 0.23570000 \\
\hline $\mathrm{C}$ & -3.59501300 & -5.16613500 & 0.34341100 \\
\hline $\mathrm{H}$ & -4.17202300 & -5.61494000 & 1.16469500 \\
\hline $\mathrm{H}$ & -2.57323800 & -4.98076600 & 0.70408600 \\
\hline $\mathrm{H}$ & -3.54728000 & -5.91271900 & -0.46207100 \\
\hline $\mathrm{O}$ & -3.18876800 & -1.12018400 & -0.32272000 \\
\hline $\mathrm{C}$ & -3.36979300 & 2.06190700 & 3.15036900 \\
\hline $\mathrm{C}$ & -4.45173100 & 2.39709800 & 4.18827100 \\
\hline $\mathrm{H}$ & -4.99877800 & 1.51825800 & 4.55523800 \\
\hline $\mathrm{H}$ & -5.18420100 & 3.12429000 & 3.81307100 \\
\hline $\mathrm{H}$ & -3.96558600 & 2.85679600 & 5.06721200 \\
\hline $\mathrm{C}$ & -2.33998700 & 1.12894300 & 3.81485100 \\
\hline $\mathrm{H}$ & -2.82054500 & 0.27510500 & 4.31039800 \\
\hline $\mathrm{H}$ & -1.77802600 & 1.68737300 & 4.58309500 \\
\hline $\mathrm{H}$ & -1.61197200 & 0.71632800 & 3.10546500 \\
\hline $\mathrm{C}$ & -2.68654700 & 3.39129000 & 2.76018300 \\
\hline $\mathrm{H}$ & -1.92320400 & 3.26165900 & 1.98481400 \\
\hline $\mathrm{H}$ & -2.21318600 & 3.84461600 & 3.64857200 \\
\hline
\end{tabular}




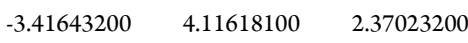

$-5.68745100$

$1.79794800 \quad 1.05501000$ 
$0.38113300 \quad-2.25230900 \quad 1.24111800$

$\begin{array}{lll}-0.12058100 & -3.25118000 & 0.38090500\end{array}$

$\begin{array}{lll}1.74226200 & -1.88705500 & 2.88155600\end{array}$

$\begin{array}{lll}-1.18412100 & -0.74177800 & 1.37103400\end{array}$

$\begin{array}{lll}1.34938900 & -2.63701300 & 2.19306200\end{array}$

$0.32684100 \quad-4.57253500 \quad 0.46184100$

$1.29068400 \quad-4.93830000 \quad 1.41360800$

$\begin{array}{lll}1.79814700 & -3.96034300 & 2.27950000\end{array}$

$\begin{array}{lll}-0.07850300 & -5.32007300 & -0.22498700\end{array}$

$\begin{array}{lll}1.63622500 & -5.97286900 & 1.48368600\end{array}$

$2.54748500 \quad-4.22731800 \quad 3.02886000$

$0.23816000 \quad 1.65311500 \quad 1.38357700$

$\begin{array}{lll}-0.81357600 & 1.64677100 & 1.68248500\end{array}$

$\begin{array}{lll}-0.17232400 & 0.44597100 & -0.44678700\end{array}$

$\begin{array}{lll}1.82013800 & 0.08436900 & 1.24086600\end{array}$

$\begin{array}{lll}-0.85569300 & -2.97535600 & -0.37263000\end{array}$

$\begin{array}{lll}1.03828500 & 2.43235200 & 2.45392000\end{array}$

$\begin{array}{lll}0.94470900 & 1.92665100 & 3.42777600\end{array}$

$0.67412900 \quad 3.46280500 \quad 2.57457900$

$2.10883500 \quad 2.47861000 \quad 2.20714600$

$\begin{array}{lll}1.33995700 & 4.50014800 & -0.90680300\end{array}$

$\begin{array}{lll}-0.14316200 & 4.86509600 & -0.47390300\end{array}$

$\begin{array}{lll}0.28813400 & 2.66303100 & 0.07873400\end{array}$

$\begin{array}{lll}1.51510500 & 3.16786400 & -0.35300900\end{array}$

$\begin{array}{lll}-0.71644800 & 3.57757300 & -0.15121100\end{array}$

$\begin{array}{lll}-0.97969900 & 5.52797700 & -1.56121100\end{array}$

$\begin{array}{lll}-0.56051200 & 6.50944300 & -1.82981000\end{array}$

$-2.00178600 \quad 5.68602600 \quad-1.18579600$

$\begin{array}{lll}-1.04656900 & 4.91817600 & -2.46789300\end{array}$

$\begin{array}{lll}-0.21978400 & 5.71529600 & 0.79767800\end{array}$

$\begin{array}{lll}0.14055300 & 6.73948500 & 0.62401700\end{array}$

$\begin{array}{lll}0.36166100 & 5.27910700 \quad 1.62002100\end{array}$

$\begin{array}{lll}-1.26858300 \quad 5.77242800 & 1.12279100\end{array}$

$1.54937000 \quad 4.42470100 \quad-2.41703100$

$\begin{array}{lll}2.54318300 & 4.00501800 \quad-2.63640400\end{array}$

$1.49966600 \quad 5.42418000 \quad-2.87266600$

$\begin{array}{lll}0.80447700 & 3.78639800 & -2.90145200\end{array}$

$2.40779800 \quad 5.40546200 \quad-0.29504000$

$\begin{array}{lll}2.23867600 & 6.45573700 & -0.57459700\end{array}$

$\begin{array}{lll}3.40509100 & 5.13539000 & -0.67924300\end{array}$

$2.42762100 \quad 5.33713400 \quad 0.80017100$

$5.04069800 \quad-1.78141900 \quad 1.23505600$

$5.74821300 \quad-1.51515300 \quad 2.44247000$

$5.12453200 \quad-0.58687300 \quad 0.40520500$ 
$5.21347500 \quad-2.41844800 \quad 3.54832100$

$\begin{array}{lll}7.23771000 & -1.80131600 & 2.20970100\end{array}$

$\begin{array}{lll}5.46110100 & 0.02462800 & 2.65825000\end{array}$

TS-S9 


\begin{tabular}{|c|c|c|}
\hline-3.27776200 & -0.05556100 & 0.15613000 \\
\hline-3.58110100 & 1.03644000 & -1.84308000 \\
\hline 4.32670000 & -2.38674500 & -3.03857000 \\
\hline 2.82467000 & -1.73325200 & -3.73037100 \\
\hline 4.03948100 & -0.62371100 & -3.05492300 \\
\hline 1.38049300 & -3.08062700 & -2.35919400 \\
\hline 2.69845100 & -3.89426200 & -1.53060400 \\
\hline 1.45232500 & -3.04230600 & -0.56902800 \\
\hline 4.91976700 & -2.58321300 & 1.19610900 \\
\hline 4.84434600 & -3.44462500 & -0.35930500 \\
\hline 3.41620000 & -3.39786800 & 0.71682300 \\
\hline 5.75855100 & -1.15955300 & -1.27178700 \\
\hline 4.74989100 & 0.30534900 & -1.09402700 \\
\hline 5.54157900 & -0.40013000 & 0.32485400 \\
\hline-4.38668300 & 0.73012600 & 0.35831900 \\
\hline-4.33505800 & 0.07960300 & -2.76771600 \\
\hline-2.77723200 & 2.03981400 & -2.65909800 \\
\hline-4.50085200 & 1.67845600 & -0.72413800 \\
\hline-5.03408700 & 0.61382500 & -3.42726400 \\
\hline-3.61344900 & -0.45525000 & -3.40534400 \\
\hline-4.89425900 & -0.67432200 & -2.19554300 \\
\hline-2.17557300 & 1.51477800 & -3.41885600 \\
\hline-3.44402700 & 2.73411200 & -3.19241300 \\
\hline-2.09518100 & 2.62570200 & -2.02948600 \\
\hline-3.98798600 & 3.02748300 & -0.21813100 \\
\hline-5.97000800 & 1.79798800 & -1.11028200 \\
\hline-4.54395900 & 3.29824000 & 0.69162300 \\
\hline-4.13794600 & 3.82380100 & -0.96197300 \\
\hline-2.92147600 & 2.99048400 & 0.03837700 \\
\hline-6.53298800 & 2.24713000 & -0.27818700 \\
\hline-6.09420700 & 2.44525100 & -1.99210600 \\
\hline-6.41468100 & 0.81777300 & -1.32508900 \\
\hline 0.67682000 & 1.88406700 & 1.42602700 \\
\hline 1.49419200 & 1.56191700 & 0.21817300 \\
\hline 2.89025300 & 2.11597600 & 0.21365800 \\
\hline 3.73956200 & 2.03415200 & 1.33101900 \\
\hline 2.75208900 & 2.77489000 & -1.83756000 \\
\hline 0.99032300 & 1.85936400 & -0.71315100 \\
\hline 3.39200400 & 2.71676200 & -0.95284300 \\
\hline 5.03152600 & 2.55686100 & 1.28863400 \\
\hline 5.51728600 & 3.15879400 & 0.12230500 \\
\hline 4.68961800 & 3.23274200 & -1.00088600 \\
\hline 5.67251500 & 2.48132700 & 2.17113600 \\
\hline 6.53248000 & 3.56168300 & 0.08951200 \\
\hline
\end{tabular}




\begin{tabular}{|c|c|c|c|}
\hline $\mathrm{H}$ & 5.05387600 & 3.69631200 & -1.92162600 \\
\hline $\mathrm{C}$ & -0.71832300 & 1.87410900 & 1.42783900 \\
\hline $\mathrm{H}$ & -1.23642600 & 1.91522300 & 0.46411300 \\
\hline $\mathrm{H}$ & 3.39129600 & 1.52974200 & 2.23367200 \\
\hline $\mathrm{H}$ & 1.21020800 & 2.12230800 & 2.35521100 \\
\hline $\mathrm{C}$ & -1.55264400 & 2.27497600 & 2.61686900 \\
\hline $\mathrm{H}$ & -1.86090500 & 3.33720500 & 2.57449200 \\
\hline $\mathrm{H}$ & -2.49240500 & 1.69765000 & 2.66868100 \\
\hline $\mathrm{H}$ & -1.00579800 & 2.14515200 & 3.56742100 \\
\hline \multicolumn{4}{|c|}{ TS-S10 } \\
\hline $\mathrm{C}$ & 5.33459700 & -0.48181000 & -0.28478700 \\
\hline $\mathrm{C}$ & 4.80558300 & -1.40468000 & 0.63324300 \\
\hline $\mathrm{C}$ & 4.50941500 & -2.82182600 & 0.34649600 \\
\hline $\mathrm{C}$ & 4.36733600 & -3.32875700 & -0.96129100 \\
\hline $\mathrm{H}$ & 4.48315600 & -3.35554000 & 2.44008900 \\
\hline $\mathrm{H}$ & 4.94619200 & -1.17923100 & 1.69661100 \\
\hline $\mathrm{C}$ & 4.37076500 & -3.72499800 & 1.41776000 \\
\hline $\mathrm{C}$ & 4.08039600 & -4.67471700 & -1.18231100 \\
\hline $\mathrm{C}$ & 3.93588200 & -5.55716800 & -0.10518500 \\
\hline $\mathrm{C}$ & 4.08962300 & -5.07398000 & 1.19682700 \\
\hline $\mathrm{H}$ & 3.96639500 & -5.03954500 & -2.20639200 \\
\hline $\mathrm{H}$ & 3.70934100 & -6.61125200 & -0.28171200 \\
\hline $\mathrm{H}$ & 3.98545500 & -5.75122500 & 2.04843000 \\
\hline $\mathrm{C}$ & 6.25738900 & 0.59868600 & 0.11782700 \\
\hline $\mathrm{H}$ & 5.92106600 & 1.63712300 & 0.12251800 \\
\hline $\mathrm{Ni}$ & 3.33732400 & -0.06361200 & -0.10277600 \\
\hline $\mathrm{C}$ & 3.36986900 & 2.39240100 & -0.58576700 \\
\hline $\mathrm{O}$ & 3.20236900 & 1.58989000 & -1.54968400 \\
\hline $\mathrm{C}$ & 3.24425300 & 3.90137100 & -0.83722100 \\
\hline $\mathrm{C}$ & 3.67232300 & 4.69382900 & 0.40220700 \\
\hline $\mathrm{C}$ & 1.76018900 & 4.17975400 & -1.14723400 \\
\hline $\mathrm{C}$ & 4.11369400 & 4.27883900 & -2.04656700 \\
\hline $\mathrm{H}$ & 4.71986300 & 4.48650600 & 0.66946800 \\
\hline $\mathrm{H}$ & 3.05125400 & 4.43264300 & 1.27062900 \\
\hline $\mathrm{H}$ & 3.57365400 & 5.77595400 & 0.21535900 \\
\hline $\mathrm{H}$ & 1.43849100 & 3.65244200 & -2.05702900 \\
\hline $\mathrm{H}$ & 1.59885800 & 5.25969500 & -1.30048900 \\
\hline $\mathrm{H}$ & 1.11698700 & 3.85175500 & -0.31758600 \\
\hline $\mathrm{H}$ & 3.82403500 & 3.69336900 & -2.93077200 \\
\hline $\mathrm{H}$ & 5.17997200 & 4.08327900 & -1.84599500 \\
\hline $\mathrm{H}$ & 4.00379500 & 5.35027100 & -2.28205300 \\
\hline $\mathrm{O}$ & 3.59552400 & 1.95682300 & 0.57939400 \\
\hline $\mathrm{H}$ & 4.46901900 & -2.65859100 & -1.81679400 \\
\hline $\mathrm{H}$ & 5.44289100 & -0.82371200 & -1.32936800 \\
\hline
\end{tabular}




\begin{tabular}{|c|c|c|}
\hline 7.70443000 & 0.28696200 & 0.31116300 \\
\hline 7.85038100 & -0.56806700 & 0.99813500 \\
\hline 8.26185500 & 1.14930300 & 0.70943900 \\
\hline 8.19392100 & -0.01027400 & -0.64117800 \\
\hline-5.72020100 & 0.89228100 & -0.40442300 \\
\hline-4.42421400 & 0.38088100 & -0.22343900 \\
\hline-3.38067700 & 1.31113500 & 0.00118400 \\
\hline-3.65081000 & 2.68294000 & 0.16465300 \\
\hline-4.96683300 & 3.13232900 & 0.01383900 \\
\hline-5.99814900 & 2.25150200 & -0.29997600 \\
\hline-1.37507700 & 2.88603400 & 1.11727100 \\
\hline-1.10386200 & 1.56464600 & 0.70478100 \\
\hline 0.16093300 & 0.97499900 & 0.94891800 \\
\hline 1.00577700 & 1.64236400 & 1.85769000 \\
\hline 0.65589500 & 2.85324000 & 2.44480600 \\
\hline-0.50343500 & 3.49852100 & 2.02206200 \\
\hline-6.53498000 & 0.20445200 & -0.61712100 \\
\hline-5.18684400 & 4.19376600 & 0.12294800 \\
\hline-7.01605700 & 2.62063300 & -0.44353000 \\
\hline 1.98344900 & 1.21795500 & 2.07217600 \\
\hline 1.32807000 & 3.32793500 & 3.16192100 \\
\hline-0.71616900 & 4.50457700 & 2.38119600 \\
\hline-2.50218100 & 3.64926700 & 0.42363900 \\
\hline-2.95626100 & 4.87067000 & 1.23647200 \\
\hline-2.12302900 & 5.57021900 & 1.38881500 \\
\hline-3.35411200 & 4.58081100 & 2.22083800 \\
\hline-3.73202300 & 5.43476900 & 0.70135600 \\
\hline-1.96477200 & 4.14461700 & -0.94531300 \\
\hline-2.76214400 & 4.66352600 & -1.50053200 \\
\hline-1.60831200 & 3.30795600 & -1.56358600 \\
\hline-1.12507900 & 4.84072200 & -0.80081300 \\
\hline-2.07663100 & 0.84902300 & 0.04381800 \\
\hline-4.92672500 & -2.12594400 & -1.76573000 \\
\hline-6.44089200 & -2.36262500 & -1.78354000 \\
\hline-7.01954000 & -1.44382200 & -1.61855700 \\
\hline-6.75373100 & -3.10659700 & -1.03779300 \\
\hline-6.73453400 & -2.75359700 & -2.77397000 \\
\hline-4.55212100 & -1.15764700 & -2.90087700 \\
\hline-5.12440900 & -0.22084300 & -2.85081200 \\
\hline-4.76576100 & -1.63171300 & -3.87462800 \\
\hline-3.48328900 & -0.89811500 & -2.88481400 \\
\hline-4.22194600 & -3.47381500 & -2.02222500 \\
\hline-3.13134200 & -3.35312100 & -2.09541200 \\
\hline .58056500 & -3.90944800 & -2.97111400 \\
\hline
\end{tabular}




\begin{tabular}{|c|c|c|}
\hline-4.42470400 & -4.20532800 & -1.22581700 \\
\hline-5.14246000 & -1.95211000 & 1.38608000 \\
\hline 4.38390500 & -1.32618400 & 2.57093700 \\
\hline-3.34551400 & -1.67697100 & 2.62576900 \\
\hline-4.88297700 & -1.60096600 & 3.51625700 \\
\hline-4.37040600 & -0.22636700 & 2.51142600 \\
\hline 5.04657300 & -3.48373000 & 1.48651300 \\
\hline-5.64566200 & -3.98706200 & 0.71301000 \\
\hline-5.42814800 & -3.81923700 & 2.46638000 \\
\hline-4.00566500 & -3.83266800 & 1.39541200 \\
\hline-6.61044700 & -1.51883000 & 1.48991400 \\
\hline-7.22829600 & -1.86892000 & 0.65412700 \\
\hline-6.70907700 & -0.42740200 & 1.56300500 \\
\hline-7.04187300 & -1.94162500 & 2.41470900 \\
\hline 0.38849000 & -0.74440200 & -1.61817800 \\
\hline 1.54126400 & -1.47087300 & -2.34027000 \\
\hline 1.77500400 & -2.44476900 & -1.88904300 \\
\hline 2.45621500 & -0.85772800 & -2.35911900 \\
\hline 1.25217700 & -1.65277300 & -3.38918200 \\
\hline 0.21811100 & 0.64411900 & -2.25788900 \\
\hline 0.06489900 & 0.51357000 & -3.34287700 \\
\hline 1.11300400 & 1.26461200 & -2.11852800 \\
\hline-0.66070500 & 1.16695100 & -1.86199800 \\
\hline-0.89826400 & -1.54879500 & -1.78967700 \\
\hline-1.16815200 & -1.55676400 & -2.85948800 \\
\hline-1.73395100 & -1.11990000 & -1.23026200 \\
\hline-0.78635800 & -2.59558200 & -1.47914200 \\
\hline 0.55743400 & -1.93255300 & 1.31261600 \\
\hline 0.95152000 & -3.25947300 & 0.65122800 \\
\hline 0.89291800 & -4.06816100 & 1.39774600 \\
\hline 1.97972600 & -3.23950700 & 0.27373000 \\
\hline 0.28158200 & -3.52932400 & -0.17463400 \\
\hline 1.40932400 & -1.73575700 & 2.57875200 \\
\hline 1.24511500 & -2.58567200 & 3.26226400 \\
\hline 1.13374500 & -0.81878400 & 3.12007200 \\
\hline 2.48124100 & -1.68757800 & 2.34323200 \\
\hline-0.92024500 & -1.93661400 & 1.69903300 \\
\hline-1.58969800 & -2.03132600 & 0.83577000 \\
\hline-1.19389100 & -1.01413700 & 2.23105200 \\
\hline-1.11867700 & -2.78474200 & 2.37774300 \\
\hline 1.00149800 & -0.48475100 & 0.16699900 \\
\hline & -1.44632600 & 然 \\
\hline
\end{tabular}

$\begin{array}{lll}-4.11748900 & -1.44632600 & -0.15793600\end{array}$

$5.29526500 \quad-0.55725800 \quad 0.13961100$ 
$4.27438300 \quad-3.90976300$

$4.44979000 \quad-1.60211000$

$4.36435700 \quad-4.07180900$

$4.59869900 \quad-4.49627400$

$4.45811900 \quad-5.59236300$

$4.34451900 \quad-5.37173000$

$4.68964300 \quad-4.65395700$

4.44050100

4.24082800

5.30211400

3.39085900

3.24925200

2.96171700

2.85240500

3.64316300

1.34718200

3.12911600

4.72541100

3.48392200

3.33436500

0.75781200

0.99726400

1.13751800

2.53111100

4.19118700

2.88564700

3.75681400

4.71034000

5.69716300

$-5.40843200$

$-4.18528000$

$-3.20523900$

$-3.51649300$

$-4.75498900$

$-5.68696200$

$-1.30997900$

$-0.98938000$

0.32136900

1.20759300

0.85892300

$-0.37944900$
$-6.60878200$

$-6.21763000$

0.71999700

0.06438200

2.89258300

1.66286600

3.89926300

5.19978700

4.18256300

3.30880000

5.02810500

5.61887900

5.94732000

3.25792400

4.87546800

4.63908600

2.40564800

3.03575900

4.04490400

3.29471000

$-2.35829400$

$-0.68663000$

0.67493800

0.20182000

1.16300100

2.53257900

2.94841100

2.03634500

2.88482800

1.55463800

1.05400600

1.86307500

3.13046800

3.64615500
2.07303700

1.81910100

0.99575100

$-1.74379900$

$-0.88594800$

0.48959600

$-2.82162800$

$-1.28647500$

1.17404200

0.69901700

0.16141900

$-0.50395700$

$-0.78133000$

$-1.61654900$

$-1.43533000$

$-1.45537600$

$-3.00671600$

$-1.55136100$

$-0.43232900$

$-2.18507500$

$-1.52532500$

$-2.23901900$

$-0.47736500$

$-3.18800300$

$-3.11982700$

$-3.79143200$

0.54127200

$-1.92636600$

$-0.87086000$

$-0.96662100$

$-0.46829300$

$-0.10825800$

$-0.07483900$

$-0.58070400$

$-1.06347700$

1.06222400

0.73680100

0.95502600

1.68448700

2.13754200

1.78186000 


\begin{tabular}{|c|c|c|c|}
\hline $\mathrm{H}$ & -6.16985300 & -0.04097600 & -1.26900100 \\
\hline $\mathrm{H}$ & -4.99425600 & 4.01308600 & -0.58684700 \\
\hline $\mathrm{H}$ & -6.64060200 & 2.37953900 & -1.47029200 \\
\hline $\mathrm{H}$ & 2.22262000 & 1.50704400 & 1.85421400 \\
\hline $\mathrm{H}$ & 1.58320500 & 3.73199300 & 2.68771900 \\
\hline $\mathrm{H}$ & -0.62585000 & 4.67573800 & 2.04561000 \\
\hline $\mathrm{C}$ & -2.58359300 & 3.55827000 & 0.55764700 \\
\hline $\mathrm{C}$ & -3.32816500 & 4.23935800 & 1.72947000 \\
\hline $\mathrm{H}$ & -2.70299200 & 5.00762200 & 2.20688200 \\
\hline $\mathrm{H}$ & -3.60977500 & 3.50023700 & 2.49477800 \\
\hline $\mathrm{H}$ & -4.24467700 & 4.73596200 & 1.37935300 \\
\hline $\mathrm{C}$ & -2.18697200 & 4.62181300 & -0.49393200 \\
\hline $\mathrm{H}$ & -3.07448700 & 5.15288400 & -0.87069500 \\
\hline $\mathrm{H}$ & -1.67764600 & 4.15443400 & -1.34892900 \\
\hline $\mathrm{H}$ & -1.50696700 & 5.36972800 & -0.06006200 \\
\hline $\mathrm{O}$ & -1.93966700 & 0.71246000 & 0.20654700 \\
\hline $\mathrm{C}$ & -4.64194100 & -2.54105600 & -1.62041100 \\
\hline $\mathrm{C}$ & -6.15342800 & -2.79420900 & -1.68015700 \\
\hline $\mathrm{H}$ & -6.74364100 & -1.86828700 & -1.71742100 \\
\hline $\mathrm{H}$ & -6.50921600 & -3.39340800 & -0.83062000 \\
\hline $\mathrm{H}$ & -6.38838900 & -3.36204200 & -2.59784400 \\
\hline $\mathrm{C}$ & -4.20444300 & -1.79383400 & -2.89167000 \\
\hline $\mathrm{H}$ & -4.77497300 & -0.86822700 & -3.04824500 \\
\hline $\mathrm{H}$ & -4.36712600 & -2.44139300 & -3.77068100 \\
\hline $\mathrm{H}$ & -3.13830600 & -1.52745300 & -2.86837400 \\
\hline $\mathrm{C}$ & -3.92170800 & -3.90485300 & -1.58592000 \\
\hline $\mathrm{H}$ & -2.82890000 & -3.78291500 & -1.61394900 \\
\hline $\mathrm{H}$ & -4.22052500 & -4.51146500 & -2.45858400 \\
\hline $\mathrm{H}$ & -4.16950300 & -4.48051000 & -0.68139000 \\
\hline $\mathrm{C}$ & -5.06256100 & -1.81007900 & 1.42420500 \\
\hline $\mathrm{C}$ & -4.36633200 & -1.01290500 & 2.54291000 \\
\hline $\mathrm{H}$ & -3.33697300 & -1.35453300 & 2.71425900 \\
\hline $\mathrm{H}$ & -4.92454100 & -1.13833700 & 3.48674700 \\
\hline $\mathrm{H}$ & -4.33620300 & 0.06505400 & 2.31956000 \\
\hline $\mathrm{C}$ & -5.03797700 & -3.30272500 & 1.79106800 \\
\hline $\mathrm{H}$ & -5.58920600 & -3.91791900 & 1.06445600 \\
\hline $\mathrm{H}$ & -5.51283300 & -3.45435700 & 2.77582900 \\
\hline $\mathrm{H}$ & -4.00786100 & -3.68808100 & 1.85390800 \\
\hline $\mathrm{C}$ & -6.51110800 & -1.31845700 & 1.32576000 \\
\hline $\mathrm{H}$ & -7.08883600 & -1.83270200 & 0.54860400 \\
\hline $\mathrm{H}$ & -6.56364300 & -0.23694300 & 1.13845100 \\
\hline $\mathrm{H}$ & -7.02072800 & -1.50396200 & 2.28811900 \\
\hline $\mathrm{C}$ & 0.57541700 & -0.87677300 & -1.44261800 \\
\hline $\mathrm{C}$ & 1.76924900 & -1.59409500 & -2.09574700 \\
\hline
\end{tabular}




\begin{tabular}{|c|c|c|c|}
\hline $\mathrm{H}$ & 2.02269100 & -2.53167500 & -1.58367100 \\
\hline $\mathrm{H}$ & 2.66461900 & -0.95472100 & -2.09973500 \\
\hline $\mathrm{H}$ & 1.52028900 & -1.84001700 & -3.14188400 \\
\hline $\mathrm{C}$ & 0.34806300 & 0.45898900 & -2.16778900 \\
\hline $\mathrm{H}$ & 0.25028000 & 0.26186800 & -3.24875600 \\
\hline $\mathrm{H}$ & 1.19418800 & 1.13881000 & -2.01124200 \\
\hline $\mathrm{H}$ & -0.57530600 & 0.94998500 & -1.83580400 \\
\hline $\mathrm{C}$ & -0.66869800 & -1.75039600 & -1.57184200 \\
\hline $\mathrm{H}$ & -0.91541400 & -1.84897400 & -2.64238500 \\
\hline $\mathrm{H}$ & -1.53443600 & -1.32026200 & -1.06121200 \\
\hline $\mathrm{H}$ & -0.51535000 & -2.76579600 & -1.18449800 \\
\hline $\mathrm{C}$ & 0.66953500 & -1.80117300 & 1.60358500 \\
\hline $\mathrm{C}$ & 1.03905700 & -3.19766900 & 1.08941700 \\
\hline $\mathrm{H}$ & 1.00158900 & -3.91600200 & 1.92555500 \\
\hline $\mathrm{H}$ & 2.05184100 & -3.23414600 & 0.67467600 \\
\hline $\mathrm{H}$ & 0.33833900 & -3.55398300 & 0.32421800 \\
\hline $\mathrm{C}$ & 1.49894100 & -1.48260400 & 2.85975500 \\
\hline $\mathrm{H}$ & 1.25135100 & -2.21054400 & 3.65038500 \\
\hline $\mathrm{H}$ & 1.27775100 & -0.47925500 & 3.25341600 \\
\hline $\mathrm{H}$ & 2.57660600 & -1.54433800 & 2.66349300 \\
\hline $\mathrm{C}$ & -0.81419600 & -1.74342200 & 1.96929200 \\
\hline $\mathrm{H}$ & -1.47660000 & -1.90320100 & 1.11032400 \\
\hline $\mathrm{H}$ & -1.07799500 & -0.77753500 & 2.42258300 \\
\hline $\mathrm{H}$ & -1.03094100 & -2.53047000 & 2.71276600 \\
\hline $\mathrm{P}$ & 1.15341800 & -0.48183700 & 0.32814600 \\
\hline $\mathrm{P}$ & -3.92094500 & -1.59598000 & -0.10630900 \\
\hline $\mathrm{C}$ & 5.17163800 & 1.05254100 & 2.15910100 \\
\hline $\mathrm{H}$ & 4.61096200 & 1.99041000 & 2.26372000 \\
\hline $\mathrm{H}$ & 6.17867600 & 1.21590300 & 2.58753900 \\
\hline $\mathrm{H}$ & 4.68621300 & 0.26675100 & 2.75427100 \\
\hline $\mathrm{H}$ & 5.72669200 & 1.52362200 & 0.09168100 \\
\hline \multicolumn{4}{|c|}{ TS-S12 } \\
\hline $\mathrm{Ni}$ & 1.37505800 & 0.21596600 & -0.61061000 \\
\hline $\mathrm{C}$ & 1.13028700 & -1.48480100 & -1.60248500 \\
\hline $\mathrm{C}$ & 2.31105500 & -0.70006300 & -1.97542200 \\
\hline C & -0.19167500 & -1.24335600 & -2.07880100 \\
\hline $\mathrm{H}$ & 1.27663400 & -2.45616900 & -1.11160900 \\
\hline K & 4.06646800 & 1.02241300 & 1.43091700 \\
\hline $\mathrm{C}$ & 3.64424500 & -1.02803800 & -1.45855900 \\
\hline $\mathrm{H}$ & 2.30156900 & -0.15723100 & -2.92981600 \\
\hline $\mathrm{C}$ & -0.96909400 & -2.42439900 & -2.61603100 \\
\hline $\mathrm{H}$ & -0.35747700 & -0.30608200 & -2.61915200 \\
\hline $\mathrm{P}$ & 0.98432300 & 1.85943500 & 1.38538400 \\
\hline $\mathrm{O}$ & 1.62693200 & 1.03625100 & 2.53485900 \\
\hline
\end{tabular}




\begin{tabular}{|c|c|c|c|}
\hline $\mathrm{O}$ & 2.01920000 & 1.89643300 & 0.17572300 \\
\hline $\mathrm{C}$ & 3.87716400 & -1.98348900 & -0.43364100 \\
\hline $\mathrm{C}$ & 4.76614400 & -0.27380100 & -1.88943000 \\
\hline $\mathrm{H}$ & -0.92407600 & -3.29218400 & -1.94208500 \\
\hline $\mathrm{H}$ & -0.46544800 & -2.72359000 & -3.55490300 \\
\hline $\mathrm{H}$ & -2.01412600 & -2.19298900 & -2.84505200 \\
\hline K & 0.39345600 & -1.24253500 & 2.35335700 \\
\hline $\mathrm{O}$ & 0.22694400 & 3.11213100 & 1.69257200 \\
\hline $\mathrm{C}$ & 5.13767800 & -2.13664200 & 0.15079300 \\
\hline $\mathrm{H}$ & 3.05281900 & -2.60599500 & -0.08005100 \\
\hline $\mathrm{C}$ & 6.02164500 & -0.42238400 & -1.29839000 \\
\hline $\mathrm{H}$ & 4.62579100 & 0.46398100 & -2.68394200 \\
\hline $\mathrm{O}$ & -1.12758300 & 1.61401800 & -1.34411800 \\
\hline $\mathrm{O}$ & -3.05404200 & -1.24101400 & -0.91120000 \\
\hline $\mathrm{C}$ & 6.22242100 & -1.34866400 & -0.26239900 \\
\hline $\mathrm{H}$ & 5.27709100 & -2.88511400 & 0.93671500 \\
\hline $\mathrm{H}$ & 6.85719800 & 0.18789200 & -1.65292600 \\
\hline $\mathrm{C}$ & -2.05539300 & 2.70584000 & -1.32675300 \\
\hline B & -1.24306200 & 0.86046900 & -0.15897800 \\
\hline $\mathrm{C}$ & -3.72112400 & -2.26294700 & -0.13354500 \\
\hline B & -1.77599100 & -1.02633700 & -0.45243800 \\
\hline $\mathrm{H}$ & 7.20762700 & -1.47203000 & 0.19331500 \\
\hline $\mathrm{C}$ & -1.24298000 & 3.99029200 & -1.13493000 \\
\hline $\mathrm{C}$ & -2.75939700 & 2.74466200 & -2.68291400 \\
\hline $\mathrm{C}$ & -3.02472400 & 2.36681200 & -0.09883800 \\
\hline $\mathrm{O}$ & -2.35422600 & 1.30128200 & 0.59056600 \\
\hline $\mathrm{O}$ & -0.04261600 & 0.64319600 & 0.65681700 \\
\hline $\mathrm{C}$ & -5.11558700 & -1.75774400 & 0.22189900 \\
\hline $\mathrm{C}$ & -3.84883400 & -3.52219300 & -0.99197200 \\
\hline $\mathrm{C}$ & -2.75693800 & -2.43630100 & 1.10750500 \\
\hline $\mathrm{O}$ & -1.50148400 & -1.90051300 & 0.60803900 \\
\hline $\mathrm{H}$ & -0.52286500 & 4.07255400 & -1.96407900 \\
\hline $\mathrm{H}$ & -1.88283500 & 4.88637600 & -1.14926700 \\
\hline $\mathrm{H}$ & -0.67519500 & 3.95829200 & -0.19572800 \\
\hline $\mathrm{H}$ & -3.25183700 & 1.79051000 & -2.91387300 \\
\hline $\mathrm{H}$ & -2.01403400 & 2.93779500 & -3.46991200 \\
\hline $\mathrm{H}$ & -3.51056200 & 3.54857800 & -2.72420100 \\
\hline $\mathrm{C}$ & -4.40600600 & 1.86396900 & -0.52414400 \\
\hline $\mathrm{C}$ & -3.17029700 & 3.51196800 & 0.90375100 \\
\hline $\mathrm{H}$ & -5.64248200 & -2.47816300 & 0.86665000 \\
\hline $\mathrm{H}$ & -5.70525900 & -1.63430900 & -0.69891300 \\
\hline $\mathrm{H}$ & -5.08090400 & -0.78740500 & 0.72970900 \\
\hline $\mathrm{H}$ & -4.44458100 & -4.29204400 & -0.47927000 \\
\hline $\mathrm{H}$ & -4.36329500 & -3.26423000 & -1.92959100 \\
\hline
\end{tabular}




\begin{tabular}{lrr}
-2.87499200 & -3.95537500 & -1.24875800 \\
-2.52843300 & -3.87614400 & 1.54816400 \\
-3.16093900 & -1.57020900 & 2.30463300 \\
-4.97183500 & 1.57386500 & 0.37467500 \\
-4.98081600 & 2.64581300 & -1.04280200 \\
-4.32879300 & 0.98209500 & -1.17185100 \\
-3.83921700 & 3.19578400 & 1.71993700 \\
-3.60763000 & 4.40703500 & 0.43460400 \\
-2.19482200 & 3.76125700 & 1.34058900 \\
-1.83957600 & -3.90360100 & 2.40860600 \\
-3.47099000 & -4.34444300 & 1.87019500 \\
\hline-2.09207700 & -4.48618700 & 0.74689700 \\
-2.40761400 & -1.66550500 & 3.10529800 \\
\hline-4.11853400 & -1.89352000 & 2.73674400 \\
-3.22691200 & -0.51106800 & 2.01905400
\end{tabular}

$-2.86805300 \quad-1.73201000$

$-2.97101600 \quad-0.75750500$

$-3.35173000 \quad-3.26568700$

$-0.49452300$

$-4.04004100$

0.18990400

$-0.12238200$

$-4.97659800$

$-0.04988200$

$-2.52731200 \quad-0.73706000$

$-4.27654200 \quad-2.35725300$

$-1.74371600 \quad-2.69224000$

$-4.75051700 \quad-0.92959000$

$-2.07599400 \quad 1.81560400$

$-5.06425900 \quad 0.52379600$

$-6.16497100 \quad-0.83073200$

$-3.03817900 \quad 1.52342200$

$-6.33002800 \quad 0.44190800$

$-6.82547100 \quad-0.69118100$

$-6.63140800 \quad-2.12874900$

$-3.62230700 \quad 2.69907300$

$-6.57596800 \quad 1.73660600$

$-7.42647500 \quad 0.29565300$

$-6.64469200-1.60355700$

$-7.91513300 \quad-0.56132200$

$-6.41729700 \quad 0.15757000$

$-6.09927900 \quad-2.28582000$

$-6.40510400 \quad-2.97545600$

$-7.71854300-2.14542100$

$-2.88836200 \quad 3.82444600$

$-7.57109900 \quad 1.73936200$
$-1.94504500$

$-0.54969000$

0.56483700

$-1.65596000$

0.10102700

$-3.30252400$

$-3.08037200$

$-2.07520400$

$-1.52930400$

1.07245400

$-0.16825400$

$-0.70128200$

0.89782000

0.79938000

$-0.03393400$

2.26693600

0.22568700

1.22663700

0.74568000

$-1.09106400$

2.85669600

2.17440700

2.83062200

$-0.72506300$

0.89663700

0.05607600

0.68555700

1.21466200 


\begin{tabular}{|c|c|c|c|}
\hline $\mathrm{H}$ & -6.52617100 & 2.58743700 & 0.04944000 \\
\hline $\mathrm{H}$ & -5.81297600 & 1.90625700 & 1.51235300 \\
\hline $\mathrm{H}$ & -7.28338900 & -0.59097500 & -1.72473100 \\
\hline $\mathrm{H}$ & -7.45387100 & 1.19670300 & -1.72638600 \\
\hline $\mathrm{H}$ & -8.41698100 & 0.20285600 & -0.62070200 \\
\hline $\mathrm{C}$ & -3.86431300 & 4.96129400 & 0.42166700 \\
\hline $\mathrm{C}$ & -1.84015200 & 4.23157900 & 1.72277900 \\
\hline $\mathrm{C}$ & -2.24136200 & 3.19365100 & -0.58819000 \\
\hline $\mathrm{H}$ & -4.27642900 & 5.32652500 & 1.37503300 \\
\hline $\mathrm{H}$ & -3.35901100 & 5.80522300 & -0.07380500 \\
\hline $\mathrm{H}$ & -4.70519000 & 4.63772200 & -0.20650900 \\
\hline $\mathrm{H}$ & -2.34193200 & 4.43684100 & 2.68022200 \\
\hline $\mathrm{H}$ & -1.30627900 & 5.14323500 & 1.41559400 \\
\hline $\mathrm{H}$ & -1.09562000 & 3.43575600 & 1.86997000 \\
\hline $\mathrm{C}$ & -0.89353800 & 3.78403400 & -0.96957700 \\
\hline $\mathrm{C}$ & -3.18380200 & 3.17293300 & -1.79734300 \\
\hline $\mathrm{H}$ & -0.52352000 & 3.33655600 & -1.90390900 \\
\hline $\mathrm{H}$ & -0.98787400 & 4.86973900 & -1.13434300 \\
\hline $\mathrm{H}$ & -0.14134600 & 3.58247200 & -0.19931300 \\
\hline $\mathrm{H}$ & -3.31367000 & 4.17778200 & -2.22666500 \\
\hline $\mathrm{H}$ & -2.74577600 & 2.52464800 & -2.57284000 \\
\hline $\mathrm{H}$ & -4.16571400 & 2.76231600 & -1.52438600 \\
\hline $\mathrm{C}$ & -2.72690000 & 0.41795400 & 2.67578200 \\
\hline $\mathrm{C}$ & -3.07076900 & 1.45291400 & 3.71839000 \\
\hline $\mathrm{C}$ & -1.41424000 & -0.13929600 & 2.73092500 \\
\hline $\mathrm{H}$ & -3.54754800 & -0.26096600 & 2.45431400 \\
\hline $\mathrm{H}$ & -2.26740300 & 2.19303400 & 3.84693900 \\
\hline $\mathrm{H}$ & -3.20178200 & 0.93179000 & 4.68553800 \\
\hline $\mathrm{H}$ & -4.00060700 & 1.98930700 & 3.49550000 \\
\hline $\mathrm{C}$ & -0.96422000 & -1.21538100 & 1.86692700 \\
\hline $\mathrm{H}$ & -0.83849900 & 0.10661700 & 3.63664400 \\
\hline $\mathrm{C}$ & -0.16968900 & -2.37981900 & 2.25760000 \\
\hline $\mathrm{H}$ & -1.59329600 & -1.38669400 & 0.99037500 \\
\hline $\mathrm{C}$ & 0.39489800 & -2.56400900 & 3.54368500 \\
\hline $\mathrm{C}$ & 0.08731900 & -3.38398600 & 1.28444200 \\
\hline $\mathrm{C}$ & 1.19282200 & -3.67497200 & 3.83063400 \\
\hline $\mathrm{H}$ & 0.20290200 & -1.82229500 & 4.32226500 \\
\hline $\mathrm{C}$ & 0.87031300 & -4.50253100 & 1.58361200 \\
\hline $\mathrm{H}$ & -0.32710600 & -3.25166300 & 0.27752900 \\
\hline $\mathrm{C}$ & 1.44114400 & -4.65512400 & 2.85689000 \\
\hline $\mathrm{H}$ & 1.61929100 & -3.78491900 & 4.83200500 \\
\hline $\mathrm{H}$ & 1.04427800 & -5.25807600 & 0.81157300 \\
\hline $\mathrm{H}$ & 2.05665500 & -5.52691000 & 3.09149600 \\
\hline $\mathrm{Ni}$ & 0.04971600 & 0.36869700 & 1.48435700 \\
\hline
\end{tabular}




\begin{tabular}{|c|c|c|c|}
\hline $\mathrm{O}$ & 0.99398300 & 2.12803600 & 1.17831300 \\
\hline $\mathrm{O}$ & 1.44770200 & -0.06118800 & 0.16963900 \\
\hline $\mathrm{P}$ & 1.91201500 & 1.46198200 & 0.10832400 \\
\hline K & 4.29803500 & 2.78922100 & -1.72051700 \\
\hline K & 2.91580200 & -1.84878800 & 1.24270600 \\
\hline $\mathrm{O}$ & 3.44139800 & 1.56310500 & 0.59484700 \\
\hline $\mathrm{O}$ & 1.82742200 & 2.00831900 & -1.32675900 \\
\hline $\mathrm{O}$ & 4.59296300 & 0.20711500 & -2.44237700 \\
\hline $\mathrm{O}$ & 4.93790300 & -0.21710000 & 1.43091600 \\
\hline B & 4.60469200 & 0.64410100 & 0.27690000 \\
\hline B & 4.35927300 & -0.29581500 & -1.16842200 \\
\hline $\mathrm{C}$ & 4.47612700 & -0.86771700 & -3.41212400 \\
\hline $\mathrm{C}$ & 5.97419400 & 0.41675900 & 2.17776900 \\
\hline $\mathrm{O}$ & 5.77650100 & 1.54954300 & 0.13813100 \\
\hline $\mathrm{O}$ & 3.94127800 & -1.61447800 & -1.25360800 \\
\hline $\mathrm{C}$ & 3.58335000 & -1.89926800 & -2.63495500 \\
\hline $\mathrm{C}$ & 3.85442800 & -0.31093300 & -4.68521200 \\
\hline $\mathrm{C}$ & 5.88934800 & -1.38134700 & -3.68508800 \\
\hline $\mathrm{C}$ & 5.34771300 & 1.40794700 & 3.16831600 \\
\hline $\mathrm{C}$ & 6.75401800 & -0.65021600 & 2.94065100 \\
\hline $\mathrm{C}$ & 6.79319800 & 1.13991400 & 1.04204800 \\
\hline $\mathrm{C}$ & 3.87383600 & -3.36223100 & -2.92717400 \\
\hline $\mathrm{C}$ & 2.09142900 & -1.59848700 & -2.78136200 \\
\hline $\mathrm{H}$ & 4.55243400 & 0.39395900 & -5.16354500 \\
\hline $\mathrm{H}$ & 3.64637200 & -1.11649100 & -5.40630700 \\
\hline $\mathrm{H}$ & 2.91723200 & 0.22272100 & -4.47964200 \\
\hline $\mathrm{H}$ & 5.89576400 & -2.15366000 & -4.46854700 \\
\hline $\mathrm{H}$ & 6.34544700 & -1.79975100 & -2.77615600 \\
\hline $\mathrm{H}$ & 6.51807300 & -0.54368500 & -4.02287700 \\
\hline $\mathrm{H}$ & 6.09848900 & 1.85405600 & 3.83957100 \\
\hline $\mathrm{H}$ & 4.61298300 & 0.86972600 & 3.78770300 \\
\hline $\mathrm{H}$ & 4.80885600 & 2.20546400 & 2.64082400 \\
\hline $\mathrm{H}$ & 6.11714900 & -1.08821600 & 3.72632700 \\
\hline $\mathrm{H}$ & 7.64234000 & -0.22310900 & 3.43328800 \\
\hline $\mathrm{H}$ & 7.07861400 & -1.46407800 & 2.27791400 \\
\hline $\mathrm{C}$ & 7.73385100 & 0.17230900 & 0.30746800 \\
\hline $\mathrm{C}$ & 7.57313300 & 2.36474400 & 1.51097200 \\
\hline $\mathrm{H}$ & 3.71012400 & -3.58832500 & -3.99225200 \\
\hline $\mathrm{H}$ & 3.19148400 & -3.99901000 & -2.34313700 \\
\hline $\mathrm{H}$ & 4.90450000 & -3.63475100 & -2.66356800 \\
\hline $\mathrm{H}$ & 1.52018400 & -2.18439500 & -2.04742300 \\
\hline $\mathrm{H}$ & 1.72132800 & -1.85467100 & -3.78447400 \\
\hline $\mathrm{H}$ & 1.91583500 & -0.53271500 & -2.58596200 \\
\hline $\mathrm{H}$ & 8.08619100 & 0.65582300 & -0.61691200 \\
\hline
\end{tabular}




\begin{tabular}{|c|c|c|c|}
\hline $\mathrm{H}$ & 8.61493500 & -0.10160900 & 0.90867900 \\
\hline $\mathrm{H}$ & 7.20359700 & -0.74959500 & 0.02618200 \\
\hline $\mathrm{H}$ & 8.13562000 & 2.79735200 & 0.66759300 \\
\hline $\mathrm{H}$ & 8.29826200 & 2.10200400 & 2.29800500 \\
\hline $\mathrm{H}$ & 6.89862500 & 3.13956600 & 1.89954900 \\
\hline \multicolumn{4}{|c|}{ S14 } \\
\hline $\mathrm{P}$ & -4.87168100 & -2.04763200 & 1.12892400 \\
\hline $\mathrm{O}$ & -4.00393500 & -0.88009100 & 2.16364600 \\
\hline K & -6.71294700 & -0.96990800 & 3.18905900 \\
\hline $\mathrm{K}$ & -1.59779100 & -0.99832000 & 0.69424500 \\
\hline B & -4.22123000 & 0.41206300 & 1.84114700 \\
\hline $\mathrm{K}$ & -4.49876400 & -0.41779900 & -1.54740700 \\
\hline $\mathrm{O}$ & -3.81066800 & -2.44149800 & 0.09697000 \\
\hline $\mathrm{O}$ & -5.95308300 & -1.07772600 & 0.56008900 \\
\hline $\mathrm{O}$ & -5.39619000 & -3.04125300 & 2.14445700 \\
\hline $\mathrm{O}$ & -4.93440000 & 1.27986500 & 2.64366400 \\
\hline $\mathrm{O}$ & -1.94048600 & -1.12268400 & -2.24156900 \\
\hline $\mathrm{O}$ & -3.73358800 & 1.02903700 & 0.69900100 \\
\hline C & -5.11643600 & 2.51675200 & 1.90228800 \\
\hline B & -1.60233500 & -0.21339300 & -3.23263200 \\
\hline C & -3.93354200 & 2.46536100 & 0.85507100 \\
\hline C & -5.04146100 & 3.68993500 & 2.86560200 \\
\hline C & -6.49935900 & 2.43119800 & 1.25345300 \\
\hline $\mathrm{O}$ & -0.98894400 & -0.81763800 & -4.29439700 \\
\hline C & -2.61452000 & 3.02812500 & 1.37177400 \\
\hline C & -4.27509100 & 3.07353600 & -0.49727800 \\
\hline $\mathrm{H}$ & -5.89007600 & 3.65553200 & 3.56623600 \\
\hline $\mathrm{H}$ & -5.09240700 & 4.64398500 & 2.31901300 \\
\hline $\mathrm{H}$ & -4.11382900 & 3.67230600 & 3.45152300 \\
\hline $\mathrm{H}$ & -6.57076500 & 1.55776200 & 0.58831000 \\
\hline $\mathrm{H}$ & -7.25980500 & 2.31985500 & 2.04318200 \\
\hline $\mathrm{H}$ & -6.74446100 & 3.34096200 & 0.68700200 \\
\hline C & -0.57115100 & -2.13663000 & -3.87139700 \\
\hline $\mathrm{H}$ & -2.63326700 & 4.12659800 & 1.42220100 \\
\hline $\mathrm{H}$ & -1.80638100 & 2.71235900 & 0.69780900 \\
\hline $\mathrm{H}$ & -2.36973400 & 2.63270400 & 2.36780900 \\
\hline $\mathrm{H}$ & -5.13858400 & 2.58564200 & -0.97270100 \\
\hline $\mathrm{H}$ & -3.41002200 & 2.99763600 & -1.16986800 \\
\hline $\mathrm{H}$ & -4.51389300 & 4.14203900 & -0.38824400 \\
\hline C & -0.63905700 & -3.08215900 & -5.06011600 \\
\hline $\mathrm{C}$ & 0.86778600 & -1.99220200 & -3.37107100 \\
\hline C & -1.58305400 & -2.46299200 & -2.70863700 \\
\hline $\mathrm{H}$ & 0.09923200 & -2.77779200 & -5.81765400 \\
\hline $\mathrm{H}$ & -0.40539300 & -4.11330300 & -4.75321700 \\
\hline
\end{tabular}




\begin{tabular}{|c|c|c|}
\hline-1.63044400 & -3.07190500 & -5.53230500 \\
\hline 1.45827500 & -1.49583200 & -4.15711800 \\
\hline 1.32290600 & -2.96895500 & -3.16695300 \\
\hline 0.89707900 & -1.39263300 & -2.44985100 \\
\hline-0.97845400 & -3.25266300 & -1.55541600 \\
\hline .87893100 & -3.12453600 & -3.17604400 \\
\hline-1.75840700 & -3.46402500 & -0.80890800 \\
\hline-0.58878700 & -4.21571900 & -1.91863200 \\
\hline-0.15484400 & -2.69960400 & -1.08670800 \\
\hline-2.70177400 & -4.14941900 & -3.53281400 \\
\hline-3.56642400 & -3.18618500 & -2.31836500 \\
\hline-3.35165900 & -2.55638100 & -3.99321500 \\
\hline-1.85186400 & 1.32545200 & -3.14833000 \\
\hline-0.45341600 & 1.82554000 & -2.76215700 \\
\hline-2.51384300 & 1.53871000 & -2.29161600 \\
\hline-0.31665100 & 2.99240900 & -1.90954500 \\
\hline 0.25951400 & 1.68448900 & -3.59023700 \\
\hline 0.94182800 & 3.74984500 & -1.81156600 \\
\hline-1.21150800 & 3.53904400 & -1.57811400 \\
\hline 2.04355600 & 3.54309600 & -2.67911200 \\
\hline 1.12423400 & 4.69271400 & -0.77186300 \\
\hline 3.26298400 & 4.19716800 & -2.48308600 \\
\hline 1.93184200 & 2.86480900 & -3.52940400 \\
\hline 2.33795900 & 5.34762200 & -0.58025600 \\
\hline 0.29608800 & 4.87391600 & -0.08139200 \\
\hline 3.42731100 & 5.09997000 & -1.42716300 \\
\hline 4.09312000 & 4.00643100 & -3.17055700 \\
\hline 2.44289500 & 6.05384000 & 0.24846900 \\
\hline 4.38196600 & 5.60773300 & -1.27132900 \\
\hline 0.07522000 & 1.38857700 & -0.96475600 \\
\hline 0.90245900 & -0.42101400 & -0.35093100 \\
\hline 0.23601500 & 1.49361100 & 1.05368100 \\
\hline 1.13083100 & 0.22210600 & 1.08963900 \\
\hline 3.02242300 & 0.29261600 & -1.71135500 \\
\hline 2.84746400 & -0.93092700 & 3.72017700 \\
\hline 2.66062400 & .70425800 & 1.19615300 \\
\hline 0.79658800 & -0.76866600 & 2.21804900 \\
\hline 3.87407900 & -2.23879200 & -0.44961500 \\
\hline 4.74484000 & 0.28921800 & 2.39425800 \\
\hline 4.01592300 & 0.03238700 & 1.13048500 \\
\hline 3.86630700 & -1.66269200 & 0.80874600 \\
\hline 3.89278100 & -3.68513800 & -0.29804900 \\
\hline 5.55706300 & 1.44854800 & 2.20657100 \\
\hline 4.80463700 & 0.75262600 & 0.1017340 \\
\hline
\end{tabular}




\begin{tabular}{|c|c|c|c|}
\hline $\mathrm{O}$ & 3.68522400 & -2.63550400 & 1.78439200 \\
\hline $\mathrm{C}$ & 3.27447400 & -3.86786800 & 1.13261500 \\
\hline $\mathrm{C}$ & 3.08556600 & -4.32034100 & -1.41773400 \\
\hline $\mathrm{C}$ & 5.35668400 & -4.11999400 & -0.38222800 \\
\hline $\mathrm{C}$ & 4.72041300 & 2.70982200 & 2.46716300 \\
\hline $\mathrm{C}$ & 6.72440400 & 1.39140900 & 3.18757700 \\
\hline $\mathrm{C}$ & 5.96881200 & 1.31361700 & 0.69240100 \\
\hline $\mathrm{C}$ & 3.81832200 & -5.04854500 & 1.92211700 \\
\hline $\mathrm{C}$ & 1.74387500 & -3.87503700 & 1.12303700 \\
\hline $\mathrm{H}$ & 3.56279400 & -4.11393600 & -2.38793300 \\
\hline $\mathrm{H}$ & 3.03902700 & -5.41306000 & -1.29008800 \\
\hline $\mathrm{H}$ & 2.06312500 & -3.92906500 & -1.44463000 \\
\hline $\mathrm{H}$ & 5.45994900 & -5.21462300 & -0.34298900 \\
\hline $\mathrm{H}$ & 5.95165000 & -3.68154000 & 0.43233200 \\
\hline $\mathrm{H}$ & 5.77985600 & -3.76843200 & -1.33525200 \\
\hline $\mathrm{H}$ & 5.33288400 & 3.62417800 & 2.42539200 \\
\hline $\mathrm{H}$ & 4.28304600 & 2.64387000 & 3.47668200 \\
\hline $\mathrm{H}$ & 3.89300000 & 2.79596600 & 1.75116900 \\
\hline $\mathrm{H}$ & 6.35476000 & 1.52193300 & 4.21774800 \\
\hline $\mathrm{H}$ & 7.45315500 & 2.19419200 & 2.99159800 \\
\hline $\mathrm{H}$ & 7.24444900 & 0.42505400 & 3.13746200 \\
\hline $\mathrm{C}$ & 7.12814700 & 0.32581400 & 0.49658700 \\
\hline $\mathrm{C}$ & 6.28963900 & 2.63739800 & 0.00377300 \\
\hline $\mathrm{H}$ & 3.60941900 & -5.99704400 & 1.40310000 \\
\hline $\mathrm{H}$ & 3.32978000 & -5.09392700 & 2.90789400 \\
\hline $\mathrm{H}$ & 4.90138900 & -4.96796400 & 2.08410200 \\
\hline $\mathrm{H}$ & 1.37470000 & -3.81125000 & 2.15688800 \\
\hline $\mathrm{H}$ & 1.34392400 & -4.79581200 & 0.67326600 \\
\hline $\mathrm{H}$ & 1.35339600 & -3.00097400 & 0.58514600 \\
\hline $\mathrm{H}$ & 7.21581300 & 0.09208300 & -0.57595300 \\
\hline $\mathrm{H}$ & 8.09301200 & 0.73201700 & 0.83823700 \\
\hline $\mathrm{H}$ & 6.93395800 & -0.61504400 & 1.03265900 \\
\hline $\mathrm{H}$ & 6.59220900 & 2.45211200 & -1.03988700 \\
\hline $\mathrm{H}$ & 7.12052000 & 3.16105900 & 0.50392900 \\
\hline $\mathrm{H}$ & 5.41302100 & 3.29790800 & -0.01637900 \\
\hline C & -2.43050900 & 1.97274100 & -4.41304400 \\
\hline $\mathrm{H}$ & -3.44147900 & 1.59474700 & -4.65523400 \\
\hline $\mathrm{H}$ & -1.79022200 & 1.76839800 & -5.28633600 \\
\hline $\mathrm{H}$ & -2.49721400 & 3.06806800 & -4.30121400 \\
\hline \multicolumn{4}{|c|}{ TS-S15 } \\
\hline $\mathrm{C}$ & 1.24826700 & 0.71641800 & 0.30751900 \\
\hline $\mathrm{C}$ & 0.71056600 & 1.30244800 & -0.89973500 \\
\hline $\mathrm{H}$ & 1.05721800 & 0.88766800 & -1.85522200 \\
\hline $\mathrm{C}$ & 1.66176000 & -0.66594500 & 0.35837800 \\
\hline
\end{tabular}




\begin{tabular}{|c|c|c|c|}
\hline $\mathrm{H}$ & 1.19741600 & -1.27601700 & -0.43526900 \\
\hline $\mathrm{H}$ & 1.41376800 & 1.32238900 & 1.20529600 \\
\hline $\mathrm{C}$ & 0.28453900 & 2.70976900 & -0.99070800 \\
\hline $\mathrm{C}$ & 0.08697900 & 3.30033300 & -2.25850600 \\
\hline $\mathrm{C}$ & 0.04171800 & 3.51376100 & 0.14529600 \\
\hline $\mathrm{C}$ & -0.32912900 & 4.62707000 & -2.38699000 \\
\hline $\mathrm{H}$ & 0.25833900 & 2.69438900 & -3.15351900 \\
\hline $\mathrm{C}$ & -0.36540800 & 4.84128800 & 0.01451500 \\
\hline $\mathrm{H}$ & 0.15322900 & 3.08501600 & 1.14265000 \\
\hline $\mathrm{C}$ & -0.55519000 & 5.40973100 & -1.25023300 \\
\hline $\mathrm{H}$ & -0.47776300 & 5.05345500 & -3.38306400 \\
\hline $\mathrm{H}$ & -0.54934500 & 5.43638900 & 0.91281800 \\
\hline $\mathrm{H}$ & -0.88070000 & 6.44811500 & -1.34730900 \\
\hline $\mathrm{O}$ & 5.92983000 & 2.41558300 & -0.48284100 \\
\hline $\mathrm{P}$ & 5.61021800 & 1.97944300 & 0.97079200 \\
\hline K & 7.58967400 & 0.53322100 & -0.81845700 \\
\hline K & 4.71652200 & 0.84278800 & 3.51584000 \\
\hline $\mathrm{O}$ & 4.42208300 & 0.75931500 & 0.77586200 \\
\hline $\mathrm{O}$ & 4.82900900 & 2.98323800 & 1.82175900 \\
\hline $\mathrm{O}$ & 6.78873800 & 1.26423400 & 1.67211800 \\
\hline $\mathrm{O}$ & 4.51606100 & -1.33223900 & 1.95007500 \\
\hline B & 4.55368200 & -0.64120100 & 0.67967200 \\
\hline K & 3.39475700 & 2.49449500 & -1.02941200 \\
\hline $\mathrm{C}$ & 5.24871400 & -2.54853400 & 1.89413500 \\
\hline $\mathrm{O}$ & 5.70246100 & -1.20214200 & -0.02875100 \\
\hline B & 3.16725700 & -1.07772500 & -0.69328100 \\
\hline $\mathrm{C}$ & 5.93476400 & -2.73385400 & 3.24992200 \\
\hline $\mathrm{C}$ & 4.28685300 & -3.72209300 & 1.66791700 \\
\hline $\mathrm{C}$ & 6.25170500 & -2.32249500 & 0.67571600 \\
\hline $\mathrm{H}$ & 6.54506300 & -3.64952000 & 3.27806500 \\
\hline $\mathrm{H}$ & 5.16450200 & -2.82604300 & 4.03225300 \\
\hline $\mathrm{H}$ & 6.58323200 & -1.88276800 & 3.50164500 \\
\hline $\mathrm{H}$ & 3.56334500 & -3.75549600 & 2.49603200 \\
\hline $\mathrm{H}$ & 4.82142800 & -4.68366900 & 1.65389900 \\
\hline $\mathrm{H}$ & 3.73271000 & -3.61295500 & 0.72756500 \\
\hline $\mathrm{C}$ & 6.33916500 & -3.50719500 & -0.28609300 \\
\hline $\mathrm{C}$ & 7.66398600 & -1.94049600 & 1.14128500 \\
\hline $\mathrm{H}$ & 7.03134900 & -3.26293200 & -1.10845300 \\
\hline $\mathrm{H}$ & 6.72661200 & -4.40697400 & 0.21662700 \\
\hline $\mathrm{H}$ & 5.36046600 & -3.73272700 & -0.72349400 \\
\hline $\mathrm{H}$ & 8.12213800 & -2.73036800 & 1.75330800 \\
\hline $\mathrm{H}$ & 8.32548000 & -1.82412900 & 0.26464500 \\
\hline $\mathrm{H}$ & 7.64329200 & -0.99788800 & 1.70690300 \\
\hline $\mathrm{O}$ & 3.28234300 & -0.16975700 & -1.77316600 \\
\hline
\end{tabular}




\begin{tabular}{|c|c|c|c|}
\hline $\mathrm{O}$ & 3.14000700 & -2.37983300 & -1.21588000 \\
\hline $\mathrm{C}$ & 2.93683100 & -2.31671800 & -2.64117400 \\
\hline $\mathrm{C}$ & 3.51205700 & -0.89509900 & -2.99412800 \\
\hline $\mathrm{C}$ & 2.79772700 & -0.18183000 & -4.13711600 \\
\hline & 3.25178700 & 0.80875100 & -4.30232700 \\
\hline $\mathrm{H}$ & 1.73066600 & -0.03480300 & -3.92676200 \\
\hline & 2.89233300 & -0.74966700 & -5.07601600 \\
\hline $\mathrm{C}$ & 5.02384700 & -0.90244700 & -3.24564600 \\
\hline $\mathrm{H}$ & 5.37358100 & 0.14176400 & -3.29351800 \\
\hline $\mathrm{H}$ & 5.28126400 & -1.39046500 & -4.19742900 \\
\hline $\mathrm{H}$ & 5.54144400 & -1.40617700 & -2.41933800 \\
\hline $\mathrm{C}$ & 1.43018800 & -2.42972300 & -2.90351900 \\
\hline $\mathrm{H}$ & 1.20452900 & -2.49812200 & -3.97830600 \\
\hline $\mathrm{H}$ & 0.87195700 & -1.57612100 & -2.49224500 \\
\hline $\mathrm{H}$ & 1.05267800 & -3.34001600 & -2.41417700 \\
\hline $\mathrm{C}$ & 3.66600700 & -3.48128800 & -3.29801800 \\
\hline $\mathrm{H}$ & 3.58696800 & -3.42893700 & -4.39540500 \\
\hline $\mathrm{H}$ & 3.21454100 & -4.43074500 & -2.97163200 \\
\hline $\mathrm{H}$ & 4.72826900 & -3.50251400 & -3.02392500 \\
\hline $\mathrm{C}$ & 1.57967200 & -1.35203800 & 1.70557500 \\
\hline $\mathrm{H}$ & 0.52431300 & -1.45485500 & 1.99939200 \\
\hline $\mathrm{H}$ & 2.10427300 & -0.77154100 & 2.47671800 \\
\hline $\mathrm{H}$ & 2.03506600 & -2.34592100 & 1.67627300 \\
\hline $\mathrm{N}$ & -0.54926700 & 0.06794700 & -0.14462400 \\
\hline B & -6.21345900 & -0.78380000 & 0.39730000 \\
\hline $\mathrm{O}$ & -1.65427900 & -1.25124200 & 0.87784600 \\
\hline $\mathrm{O}$ & -6.90430400 & -0.92255800 & -0.80110800 \\
\hline $\mathrm{O}$ & -6.47426700 & -1.88361200 & 1.21747900 \\
\hline & -2.90669300 & -0.75196300 & 0.07540400 \\
\hline K & -4.68928300 & 0.03803600 & -2.32959400 \\
\hline $\mathrm{C}$ & -7.48060400 & -2.24897600 & -0.88738200 \\
\hline C & -7.50874200 & -2.70346900 & 0.62355800 \\
\hline $\mathrm{O}$ & -3.79390100 & 0.06301200 & 1.14508800 \\
\hline K & -4.05844700 & -2.40411700 & 2.23507700 \\
\hline O & -3.80247900 & -1.86525300 & -0.50061700 \\
\hline $\mathrm{O}$ & -2.31193600 & 0.19328700 & -1.03376300 \\
\hline $\mathrm{O}$ & -5.16624500 & 1.53682700 & -0.31130500 \\
\hline C & -8.85255200 & -2.14199000 & -1.54129600 \\
\hline$C$ & -6.54374000 & -3.09187900 & -1.75668800 \\
\hline C & -7.15883400 & -4.16935600 & 0.85226100 \\
\hline$C$ & -8.81386200 & -2.35408100 & 1.34006000 \\
\hline & -5.20948100 & 0.57052600 & 0.81809400 \\
\hline & -5.04311300 & 2.84124000 & 0.24602500 \\
\hline & -9.35966600 & -3.11940600 & -1.55684200 \\
\hline
\end{tabular}




\begin{tabular}{|c|c|c|c|}
\hline $\mathrm{H}$ & -8.74035100 & -1.80504000 & -2.58350400 \\
\hline $\mathrm{H}$ & -9.49725800 & -1.41954900 & -1.02432600 \\
\hline $\mathrm{H}$ & -6.49786500 & -2.64724900 & -2.76504700 \\
\hline $\mathrm{H}$ & -6.91242600 & -4.12136100 & -1.87694000 \\
\hline $\mathrm{H}$ & -5.52659200 & -3.10239000 & -1.34076100 \\
\hline $\mathrm{H}$ & -7.18047200 & -4.39474700 & 1.93073200 \\
\hline $\mathrm{H}$ & -7.88870500 & -4.83283700 & 0.36312100 \\
\hline $\mathrm{H}$ & -6.16129600 & -4.41387400 & 0.46183700 \\
\hline $\mathrm{H}$ & -9.65367700 & -2.97392400 & 0.99255100 \\
\hline $\mathrm{H}$ & -9.07408200 & -1.29540500 & 1.19806700 \\
\hline $\mathrm{H}$ & -8.68452100 & -2.52358600 & 2.41977700 \\
\hline $\mathrm{O}$ & -5.64714500 & 1.35962700 & 1.96580200 \\
\hline $\mathrm{C}$ & -3.56478900 & 3.14207100 & 0.53285800 \\
\hline $\mathrm{C}$ & -5.57865900 & 3.85784500 & -0.75975500 \\
\hline $\mathrm{C}$ & -5.90107200 & 2.69467800 & 1.55863900 \\
\hline $\mathrm{H}$ & -3.41131000 & 4.18704700 & 0.84463300 \\
\hline $\mathrm{H}$ & -2.98069400 & 2.96256100 & -0.38047600 \\
\hline $\mathrm{H}$ & -3.16715100 & 2.47082800 & 1.30403700 \\
\hline $\mathrm{H}$ & -4.89799300 & 3.91917800 & -1.62520100 \\
\hline $\mathrm{H}$ & -5.64135400 & 4.86535300 & -0.31772100 \\
\hline $\mathrm{H}$ & -6.57477800 & 3.57567400 & -1.12845800 \\
\hline $\mathrm{C}$ & -7.40611600 & 2.83045900 & 1.27480300 \\
\hline $\mathrm{C}$ & -5.49980000 & 3.64968100 & 2.68041300 \\
\hline $\mathrm{H}$ & -7.96212100 & 2.48917600 & 2.16203100 \\
\hline $\mathrm{H}$ & -7.71025000 & 3.86641300 & 1.05505500 \\
\hline $\mathrm{H}$ & -7.69512600 & 2.19015200 & 0.42797800 \\
\hline $\mathrm{H}$ & -6.14704400 & 3.48872900 & 3.55776900 \\
\hline $\mathrm{H}$ & -5.60341400 & 4.70316600 & 2.37209100 \\
\hline $\mathrm{H}$ & -4.46212100 & 3.47513200 & 2.99476900 \\
\hline \multicolumn{4}{|c|}{ S16 } \\
\hline $\mathrm{C}$ & 3.31797600 & -2.33439200 & 0.56089000 \\
\hline $\mathrm{C}$ & 2.71489500 & -3.47985400 & -0.09541700 \\
\hline $\mathrm{H}$ & 2.91775200 & -4.48264100 & 0.31493200 \\
\hline $\mathrm{C}$ & 4.42360600 & -2.51322700 & 1.63508400 \\
\hline $\mathrm{H}$ & 5.02943900 & -3.39746800 & 1.36311500 \\
\hline $\mathrm{H}$ & 3.50297500 & -1.47224800 & -0.09789500 \\
\hline $\mathrm{C}$ & 2.34600100 & -3.49543100 & -1.52323900 \\
\hline $\mathrm{C}$ & 1.52393000 & -4.52783500 & -2.03584300 \\
\hline $\mathrm{C}$ & 2.73827000 & -2.48695400 & -2.43746400 \\
\hline $\mathrm{C}$ & 1.11023100 & -4.54732000 & -3.36585800 \\
\hline $\mathrm{H}$ & 1.19482100 & -5.31679700 & -1.35362400 \\
\hline $\mathrm{C}$ & 2.32025600 & -2.50416800 & -3.77289000 \\
\hline $\mathrm{H}$ & 3.38468100 & -1.67746600 & -2.09241000 \\
\hline $\mathrm{C}$ & 1.49700400 & -3.53009800 & -4.25037900 \\
\hline
\end{tabular}




\begin{tabular}{|c|c|c|c|}
\hline $\mathrm{H}$ & 0.47079400 & -5.36159000 & -3.71975600 \\
\hline $\mathrm{H}$ & 2.64802100 & -1.70466200 & -4.44492900 \\
\hline $\mathrm{H}$ & 1.16692700 & -3.54257300 & -5.29212200 \\
\hline $\mathrm{O}$ & 3.01747800 & 3.39334600 & -1.22563800 \\
\hline $\mathrm{P}$ & 2.14971400 & 2.14050000 & -1.15782800 \\
\hline K & 1.41255400 & 4.49338800 & -2.93776300 \\
\hline K & 0.44749500 & -0.47442300 & -1.64426400 \\
\hline $\mathrm{O}$ & 1.07535300 & 2.44211900 & 0.23426200 \\
\hline $\mathrm{O}$ & 2.77210300 & 0.81941700 & -0.70809900 \\
\hline $\mathrm{O}$ & 1.15439100 & 2.00205400 & -2.33090500 \\
\hline $\mathrm{O}$ & -0.98807400 & 3.25285400 & 1.11720200 \\
\hline B & 0.03708200 & 3.28003500 & 0.17358600 \\
\hline K & 2.37192700 & 0.45170300 & 1.79500000 \\
\hline $\mathrm{C}$ & -1.88894200 & 4.35698600 & 0.83945400 \\
\hline $\mathrm{O}$ & -0.20406000 & 4.25627500 & -0.78557300 \\
\hline B & 5.32996300 & -1.24549500 & 1.47425800 \\
\hline $\mathrm{C}$ & -3.31567600 & 3.92340100 & 1.14439600 \\
\hline $\mathrm{C}$ & -1.46853700 & 5.51841000 & 1.73863600 \\
\hline $\mathrm{C}$ & -1.60413100 & 4.62170300 & -0.68311500 \\
\hline $\mathrm{H}$ & -4.03135100 & 4.69201600 & 0.81530500 \\
\hline $\mathrm{H}$ & -3.44985800 & 3.80003100 & 2.23149000 \\
\hline $\mathrm{H}$ & -3.57054600 & 2.97480200 & 0.65616800 \\
\hline $\mathrm{H}$ & -1.48630200 & 5.18598900 & 2.78772500 \\
\hline $\mathrm{H}$ & -2.15052600 & 6.37566300 & 1.64120000 \\
\hline $\mathrm{H}$ & -0.44697800 & 5.85409900 & 1.50711100 \\
\hline $\mathrm{C}$ & -1.77032400 & 6.07133600 & -1.11411300 \\
\hline $\mathrm{C}$ & -2.38405700 & 3.68304500 & -1.60633200 \\
\hline $\mathrm{H}$ & -1.58796200 & 6.16767400 & -2.19660600 \\
\hline $\mathrm{H}$ & -2.79826100 & 6.41710800 & -0.92638100 \\
\hline $\mathrm{H}$ & -1.07503500 & 6.73818300 & -0.58709600 \\
\hline $\mathrm{H}$ & -3.45375300 & 3.93914700 & -1.62714400 \\
\hline $\mathrm{H}$ & -1.99408300 & 3.78584500 & -2.63076600 \\
\hline $\mathrm{H}$ & -2.27925700 & 2.62469700 & -1.32251400 \\
\hline $\mathrm{O}$ & 5.00285900 & -0.01794100 & 2.03249700 \\
\hline $\mathrm{O}$ & 6.43788300 & -1.15606300 & 0.67780900 \\
\hline $\mathrm{C}$ & 6.73500400 & 0.24918600 & 0.45548600 \\
\hline $\mathrm{C}$ & 6.04829300 & 0.93778900 & 1.69753000 \\
\hline $\mathrm{C}$ & 5.41453900 & 2.29187300 & 1.40322800 \\
\hline $\mathrm{H}$ & 4.92807500 & 2.68158100 & 2.31300200 \\
\hline $\mathrm{H}$ & 4.67256000 & 2.25490800 & 0.59522900 \\
\hline $\mathrm{H}$ & 6.18765700 & 3.02036700 & 1.11335100 \\
\hline $\mathrm{C}$ & 6.95794000 & 1.02929700 & 2.92274700 \\
\hline $\mathrm{H}$ & 6.35635300 & 1.32715900 & 3.79519700 \\
\hline $\mathrm{H}$ & 7.75416800 & 1.77543000 & 2.78415400 \\
\hline
\end{tabular}




\begin{tabular}{|c|c|c|c|}
\hline $\mathrm{H}$ & 7.42406800 & 0.05908900 & 3.14960500 \\
\hline $\mathrm{C}$ & 6.09076300 & 0.64255800 & -0.87472000 \\
\hline $\mathrm{H}$ & 6.36309000 & 1.66962400 & -1.16189700 \\
\hline $\mathrm{H}$ & 4.99252500 & 0.58255800 & -0.84112400 \\
\hline $\mathrm{H}$ & 6.45558400 & -0.04066600 & -1.65692700 \\
\hline $\mathrm{C}$ & 8.24663100 & 0.41796800 & 0.38932100 \\
\hline $\mathrm{H}$ & 8.51830200 & 1.48180600 & 0.30626400 \\
\hline $\mathrm{H}$ & 8.63446000 & -0.10042000 & -0.50071600 \\
\hline $\mathrm{H}$ & 8.74717700 & -0.00567800 & 1.27023600 \\
\hline $\mathrm{C}$ & 3.87606600 & -2.73374600 & 3.05319400 \\
\hline $\mathrm{H}$ & 3.32035100 & -1.85587300 & 3.42292000 \\
\hline $\mathrm{H}$ & 4.68351000 & -2.93580200 & 3.78023300 \\
\hline $\mathrm{H}$ & 3.17237000 & -3.58140300 & 3.07973300 \\
\hline $\mathrm{Ni}$ & 1.44636400 & -2.52504800 & 0.94413700 \\
\hline B & -3.92716200 & -0.55472500 & 0.55159800 \\
\hline $\mathrm{O}$ & 0.36436400 & -1.09252500 & 2.08968600 \\
\hline $\mathrm{O}$ & -4.94919000 & -1.48044100 & 0.71334500 \\
\hline $\mathrm{O}$ & -4.16144900 & 0.55067000 & 1.36518900 \\
\hline $\mathrm{P}$ & -0.92325300 & -1.74989700 & 1.49318500 \\
\hline K & -3.12780200 & -3.72352900 & 0.64024900 \\
\hline $\mathrm{C}$ & -5.79066300 & -1.09914800 & 1.83037800 \\
\hline $\mathrm{C}$ & -5.45713400 & 0.43569800 & 2.00164300 \\
\hline $\mathrm{O}$ & -1.29832300 & -0.74825400 & 0.24733700 \\
\hline K & -1.70726800 & 1.01964400 & 2.33248600 \\
\hline $\mathrm{O}$ & -2.15759600 & -1.76193700 & 2.40872700 \\
\hline $\mathrm{O}$ & -0.52282900 & -3.14055400 & 0.92359600 \\
\hline $\mathrm{O}$ & -2.75087200 & -2.05000800 & -1.28962200 \\
\hline $\mathrm{C}$ & -7.23962300 & -1.39513400 & 1.46523600 \\
\hline $\mathrm{C}$ & -5.35851800 & -1.94970600 & 3.02683800 \\
\hline $\mathrm{C}$ & -5.32581000 & 0.90047100 & 3.44791200 \\
\hline $\mathrm{C}$ & -6.40749000 & 1.36005600 & 1.23909000 \\
\hline B & -2.57773400 & -0.76883600 & -0.52822600 \\
\hline $\mathrm{C}$ & -3.04179800 & -1.79818300 & -2.65493900 \\
\hline $\mathrm{H}$ & -7.92403300 & -1.06133500 & 2.26054000 \\
\hline $\mathrm{H}$ & -7.37382000 & -2.48060000 & 1.34012700 \\
\hline $\mathrm{H}$ & -7.53181900 & -0.91115400 & 0.52420300 \\
\hline $\mathrm{H}$ & -5.53114000 & -3.01246100 & 2.78886300 \\
\hline $\mathrm{H}$ & -5.94928500 & -1.72091900 & 3.92613300 \\
\hline $\mathrm{H}$ & -4.28815000 & -1.81549000 & 3.23687500 \\
\hline $\mathrm{H}$ & -5.06123200 & 1.96944000 & 3.47116400 \\
\hline $\mathrm{H}$ & -6.27658000 & 0.78382700 & 3.99029000 \\
\hline $\mathrm{H}$ & -4.55334000 & 0.33695100 & 3.98877900 \\
\hline $\mathrm{H}$ & -7.41759400 & 1.36048200 & 1.67438800 \\
\hline $\mathrm{H}$ & -6.48142600 & 1.07257700 & 0.18058800 \\
\hline
\end{tabular}




\begin{tabular}{|c|c|c|c|}
\hline $\mathrm{H}$ & -6.01777100 & 2.38826600 & 1.27965000 \\
\hline $\mathrm{O}$ & -2.40650200 & 0.22285100 & -1.60796000 \\
\hline $\mathrm{C}$ & -1.89966300 & -2.36385500 & -3.50702300 \\
\hline $\mathrm{C}$ & -4.33186200 & -2.53448200 & -3.03403600 \\
\hline $\mathrm{C}$ & -3.16352000 & -0.21068200 & -2.72407500 \\
\hline $\mathrm{H}$ & -2.11150700 & -2.31252400 & -4.58605100 \\
\hline $\mathrm{H}$ & -1.74082400 & -3.41708000 & -3.23526200 \\
\hline $\mathrm{H}$ & -0.94589700 & -1.85218900 & -3.33593100 \\
\hline $\mathrm{H}$ & -4.15999800 & -3.62296500 & -2.99475100 \\
\hline $\mathrm{H}$ & -4.66012400 & -2.28981900 & -4.05691900 \\
\hline $\mathrm{H}$ & -5.14571200 & -2.29683600 & -2.33636000 \\
\hline $\mathrm{C}$ & -4.61418900 & 0.28033600 & -2.56719100 \\
\hline $\mathrm{C}$ & -2.57439900 & 0.40720300 & -3.99169400 \\
\hline $\mathrm{H}$ & -4.59958200 & 1.37077400 & -2.41741100 \\
\hline $\mathrm{H}$ & -5.22892500 & 0.06293000 & -3.45374800 \\
\hline $\mathrm{H}$ & -5.10444000 & -0.17322700 & -1.69507300 \\
\hline $\mathrm{H}$ & -2.69190900 & 1.50126300 & -3.95866400 \\
\hline $\mathrm{H}$ & -3.08699500 & 0.03681500 & -4.89332100 \\
\hline $\mathrm{H}$ & -1.50141000 & 0.19579200 & -4.09511500 \\
\hline \multicolumn{4}{|c|}{$\mathrm{BnPPh}_{2}$} \\
\hline$P$ & -0.51300100 & -0.61785200 & -0.88704500 \\
\hline $\mathrm{C}$ & -2.26899000 & -0.62518600 & -0.32156900 \\
\hline $\mathrm{C}$ & -2.78419700 & -1.54933300 & 0.60127800 \\
\hline $\mathrm{C}$ & -3.15594000 & 0.29079800 & -0.91912400 \\
\hline $\mathrm{C}$ & -4.14592900 & -1.55182900 & 0.92418300 \\
\hline $\mathrm{H}$ & -2.12894600 & -2.27826600 & 1.08090700 \\
\hline $\mathrm{C}$ & -4.51064200 & 0.29485900 & -0.58849500 \\
\hline $\mathrm{H}$ & -2.77614300 & 1.01481500 & -1.64555800 \\
\hline $\mathrm{C}$ & -5.01234000 & -0.62952700 & 0.33507300 \\
\hline $\mathrm{H}$ & -4.52764200 & -2.27885600 & 1.64575800 \\
\hline $\mathrm{H}$ & -5.18049200 & 1.02069700 & -1.05671700 \\
\hline $\mathrm{H}$ & -6.07462100 & -0.63016000 & 0.59127100 \\
\hline $\mathrm{C}$ & 0.01958400 & 1.08630000 & -0.40584200 \\
\hline $\mathrm{C}$ & 1.06344800 & 1.67088400 & -1.13904200 \\
\hline $\mathrm{C}$ & -0.51225300 & 1.78226300 & 0.69253000 \\
\hline $\mathrm{C}$ & 1.57648400 & 2.91960700 & -0.77637400 \\
\hline $\mathrm{H}$ & 1.48503300 & 1.13577700 & -1.99385800 \\
\hline $\mathrm{C}$ & -0.00510900 & 3.03295800 & 1.05068100 \\
\hline $\mathrm{H}$ & -1.32780800 & 1.34391500 & 1.27226600 \\
\hline $\mathrm{C}$ & 1.04242400 & 3.60305900 & 0.31852700 \\
\hline $\mathrm{H}$ & 2.39482900 & 3.35905000 & -1.35217800 \\
\hline $\mathrm{H}$ & -0.42748800 & 3.56530600 & 1.90697100 \\
\hline $\mathrm{H}$ & 1.43926800 & 4.58131200 & 0.60155300 \\
\hline $\mathrm{C}$ & 0.34566200 & -1.58948600 & 0.48247000 \\
\hline
\end{tabular}




\begin{tabular}{|c|c|c|}
\hline-0.01877800 & -1.24833200 & 1.46317900 \\
\hline 0.05516700 & -2.64404500 & 0.35342100 \\
\hline 1.83763800 & -1.43027700 & 0.37450000 \\
\hline 2.57145500 & -2.16636100 & -0.56988000 \\
\hline 2.51648200 & -0.48699400 & 1.16119200 \\
\hline 3.94691100 & -1.97354300 & -0.71567300 \\
\hline 2.05423500 & -2.89466600 & -1.20062900 \\
\hline 3.89221400 & -0.29222800 & 1.01757700 \\
\hline 1.95619600 & 0.10820800 & 1.88606300 \\
\hline 4.61309200 & -1.03490100 & 0.07845300 \\
\hline 4.50136500 & -2.55854800 & -1.45404400 \\
\hline 4.40164100 & 0.44770000 & 1.64008500 \\
\hline 5.68923800 & -0.88249700 & -0.03560000 \\
\hline 1.61042700 & 0.47031300 & -0.29868200 \\
\hline 2.74667000 & 0.92292100 & 1.12118700 \\
\hline 2.60305400 & -0.70619000 & -1.31422100 \\
\hline 3.95397800 & 1.75335500 & 0.80657400 \\
\hline 2.09026300 & 1.44537600 & 1.82960400 \\
\hline 3.01661300 & -0.03386100 & 1.59008300 \\
\hline 1.56244900 & 1.95102900 & -1.39557600 \\
\hline 0.36680200 & -0.21525700 & 0.34527000 \\
\hline 3.38059400 & -1.71072900 & -0.71377800 \\
\hline 2.44922100 & -0.72475900 & -2.71209200 \\
\hline 3.88010000 & 3.15281600 & 0.89365300 \\
\hline 5.15598500 & 1.17354500 & 0.36909900 \\
\hline 2.70888900 & 2.47251500 & -2.02258500 \\
\hline 0.32330600 & 2.56134500 & -1.63750900 \\
\hline-1.25655300 & 1.37601000 & 1.28340200 \\
\hline 0.37868700 & -2.36484700 & 0.13019900 \\
\hline 3.99348400 & -2.69866000 & -1.48805800 \\
\hline 3.50083800 & -1.74258400 & 0.36870600 \\
\hline 3.05492700 & -1.71777700 & -3.48458700 \\
\hline 1.84388900 & 0.03785200 & -3.20332600 \\
\hline 4.97002100 & 3.95230000 & 0.54519100 \\
\hline 2.94877100 & 3.61583200 & 1.22750100 \\
\hline 6.24677400 & 1.97146800 & 0.01575300 \\
\hline 5.23436300 & 0.08757100 & 0.29432300 \\
\hline 2.61125900 & 3.59146200 & -2.85060800 \\
\hline 3.67945300 & 1.99893300 & -1.87437400 \\
\hline 0.22470100 & 3.68224700 & -2.46692900 \\
\hline-0.57889100 & 2.15990700 & -1.18175900 \\
\hline .17552100 & 0.32113600 & 1.01058100 \\
\hline 56237600 & 1.56006700 & 2.56465900 \\
\hline
\end{tabular}


$\begin{array}{lll}-1.29813100 & 2.27824300 \quad 0.66955300\end{array}$

$\begin{array}{lll}-0.12865800 & -3.04745200 & -1.58397000\end{array}$

$-1.81338500$

$-3.31019400$

0.79457000

$0.99978000 \quad-3.10693700 \quad 1.10261200$

C

$\begin{array}{lll}3.83065200 & -2.70897000 & -2.87605600\end{array}$

4.59298900

2.91587500

$-3.46936200$

$-0.99795200$

3.51103500

$-0.75612900$

$-3.15843400$

$-2.55313600$

0.15527200

$-0.51794000$

$-1.02679500$

0.92637200

$-0.24951000$

$-2.09841500$

$-2.67317600$

1.49501400

1.57995000

4.30540700

7.01042200

1.30134400

$-4.02480300$

$-2.63701000$

0.89594200

0.13317100

0.22744700

$-1.07073700$

$-1.83590000$

$-1.07571500$

$-3.21059200$

$-1.43779800$

$-3.79413300$

$-2.49084900$

2.55541300

1.05513200

2.64991600

1.20702800

$-4.75558100$
$4.20273200 \quad-3.07075200$

$3.98911800 \quad-3.32671300$

$4.13432700 \quad-2.62922600$

$0.49350100 \quad-0.12276500$

$-0.28855200 \quad 1.84132300$

$2.75207000 \quad 2.80575600$

$0.56341600 \quad 3.56531300$

$-2.39188500 \quad-2.60760700$

$-2.83273600 \quad-1.81260800$

$-4.14005000 \quad-1.60043200$

$-3.17087300 \quad 2.16447800$

$\begin{array}{ll}-4.08492300 & 0.00388200\end{array}$

$-2.37125700 \quad 2.19099700$

$\begin{array}{ll}-4.34675000 & 0.79933400\end{array}$

$-3.48552500 \quad-3.48071800$

$3.98848800 \quad-0.17903800$

$5.07972100 \quad-3.71963300$

$-0.71784600 \quad-0.39318800$

$0.78626700 \quad-1.04108000$

$2.93281900 \quad 3.97392900$

$3.53952000 \quad 2.04714900$

$0.74290300 \quad 4.73090200$

$-0.36574600 \quad 3.41963100$

$-3.15721300 \quad-3.46013700$

$-0.99328300 \quad-2.72656000$

$-3.79368400 \quad 2.72931700$

$-2.57081900 \quad 2.79487200$

$\begin{array}{ll}-4.70267400 & 0.56894900\end{array}$

$-4.19923700 \quad-1.06375400$

$\begin{array}{ll}-2.85966100 & 2.95777700\end{array}$

$-1.39962700 \quad 2.42546000$

$-4.82960700 \quad 1.55699700$

$-4.94059100-0.03719400$

$-0.50335700 \quad-1.18736600$ 
H

$\mathrm{H}$

C

$\mathrm{H}$

$\mathrm{H}$

C

$\mathrm{H}$

C

$\mathrm{H}$

C

$\mathrm{H}$

$\mathrm{H}$

C

H

S18

$\mathrm{Ni}$

\begin{tabular}{|c|c|c|}
\hline-4.56754300 & -1.03267200 & 0.51191500 \\
\hline-3.39641500 & -1.55441300 & -0.72224000 \\
\hline 0.94606500 & 1.92510500 & 4.94349500 \\
\hline 1.44508000 & 3.86589200 & 4.12660600 \\
\hline 0.24780300 & -0.05078300 & 5.48297000 \\
\hline-2.67778300 & -2.54412300 & -4.39366600 \\
\hline-1.80717000 & -4.24841800 & -3.39296400 \\
\hline-1.91452700 & -0.37589100 & -3.65432600 \\
\hline-0.46765300 & -0.38412500 & -2.05424500 \\
\hline-4.06684500 & -4.56042900 & 1.93045500 \\
\hline-3.41348300 & -3.67735500 & 3.79689900 \\
\hline-4.45767600 & -5.29714100 & -0.06422400 \\
\hline 3.14218500 & -4.08703700 & 2.63555400 \\
\hline 2.92871000 & -2.27269100 & 3.80068400 \\
\hline 3.10247700 & -5.79171800 & 1.30384500 \\
\hline 1.53138400 & 2.06194000 & 5.85578700 \\
\hline-2.72338500 & -1.15107200 & -4.49166400 \\
\hline-3.30330800 & -3.16039300 & -5.04449200 \\
\hline-1.93961200 & 0.71476600 & -3.71556400 \\
\hline-4.94310100 & -5.04317000 & 2.36993400 \\
\hline 3.98013600 & -4.46705400 & 3.22516500 \\
\hline-3.38601600 & -0.67113900 & -5.21597700 \\
\hline-3.82023300 & 2.80333800 & -0.34683900 \\
\hline-4.74219700 & 3.88386500 & 0.22927300 \\
\hline-4.06947400 & 1.60832100 & 0.18879200 \\
\hline-2.95028700 & 3.01577300 & -1.16886900 \\
\hline-6.20861300 & 3.43422800 & 0.10821300 \\
\hline-4.52228100 & 5.19463600 & -0.53217400 \\
\hline-4.36585000 & 4.06109900 & 1.71433300 \\
\hline-6.48897600 & 3.27029700 & -0.94503400 \\
\hline-6.87501100 & 4.21013800 & 0.51818300 \\
\hline-6.38631000 & 2.49929400 & 0.65764300 \\
\hline-4.75786600 & 5.08110800 & -1.60134900 \\
\hline-5.17037800 & 5.98362600 & -0.11893300 \\
\hline-3.47767300 & 5.52997700 & -0.45642900 \\
\hline-4.51479900 & 3.12785600 & 2.27598800 \\
\hline-4.98974600 & 4.84682100 & 2.17003000 \\
\hline-3.31056400 & 4.35751200 & 1.82539100 \\
\hline 0.11891300 & -0.10071400 & -0.04834500 \\
\hline-2.07519000 & 0.31682600 & -0.77549200 \\
\hline-1.83698300 & 1.47493300 & -2.18380900 \\
\hline-3.33173000 & 1.16458700 & 0.28512600 \\
\hline & -0.98204200 & -1.52768500 \\
\hline
\end{tabular}


$-1.52310000$

$2.82467400 \quad-1.93724600$

$-3.47084300$

$0.69614500 \quad 1.60225900$

$-4.16629200$

$2.20489100 \quad-0.14793200$

$\begin{array}{lll}-4.03070200 & -0.47423600 & -2.07105900\end{array}$

$-2.61604600$

$-3.75912400$

$-1.44756600$

$-2.02464400$

$\begin{array}{lll}-1.18870400 & 3.68222400 & -2.98582900\end{array}$

$\begin{array}{lll}-1.53161300 & 3.20647400 & -0.91736700\end{array}$

$\begin{array}{lll}-4.40592800 & 1.26112100 \quad 2.47085300\end{array}$

$\begin{array}{lll}-2.84447300 & -0.12708300 & 1.94863500\end{array}$

$\begin{array}{lll}-5.09235200 & 2.78433500 & 0.72498900\end{array}$

$\begin{array}{lll}-4.09122000 & 2.57651400 & -1.17125300\end{array}$

$\begin{array}{lll}-2.93279100 & -2.86984100 & 0.11637000\end{array}$

$-5.06511500$

$-1.74289500 \quad 0.02531000$

$\begin{array}{lll}-1.14388600 & 3.20768300 & -4.30090100\end{array}$

$\begin{array}{lll}-1.41395300 & 1.48539500 & -5.58097500\end{array}$

$\begin{array}{lll}-0.94937400 & 4.72567300 & -2.76767400\end{array}$

$\begin{array}{lll}-5.21263600 & 2.31818600 & 2.03748000\end{array}$

$\begin{array}{lll}-4.50113900 & 0.87648600 \quad 3.48928000\end{array}$

$\begin{array}{lll}-5.72487300 & 3.60455400 & 0.37537500\end{array}$

$\begin{array}{lll}-3.40671600 & -3.68199900 & 1.14827300\end{array}$

$\begin{array}{lll}-1.91149900 & -2.98436100 & -0.24496100\end{array}$

$\begin{array}{lll}-5.53986700 & -2.55280700 \quad 1.06006400\end{array}$

$\begin{array}{lll}-5.71155600 & -0.97304500 & -0.40323000\end{array}$

$\begin{array}{lll}-0.86934000 & 3.87670000 & -5.11987700\end{array}$

$\begin{array}{lll}-5.93683000 & 2.77256000 & 2.71828600\end{array}$

$\begin{array}{lll}-4.71150800 & -3.52588700 & 1.62652500\end{array}$

$-2.75227300 \quad-4.44181800 \quad 1.58191800$

$\begin{array}{lll}-6.56018400 & -2.41877200 & 1.42850400\end{array}$

$\begin{array}{lll}-5.08067000 & -4.16015300 \quad 2.43637900\end{array}$

$\begin{array}{lll}1.05648600 & -1.49501500 & -1.15822400\end{array}$

$0.24840400 \quad-3.08280500 \quad-1.66416600$

$2.63604600 \quad-2.17792700 \quad-0.47123300$

$\begin{array}{lll}1.60245500 & -0.84586500 & -2.83340500\end{array}$

$\begin{array}{lll}-0.59301300 & -3.14871900 & -2.78759600\end{array}$

$0.35241600 \quad-4.21906900 \quad-0.84274400$

$\begin{array}{lll}2.91957600 & -1.95092500 & 0.88024300\end{array}$

$3.54541900 \quad-2.92310900 \quad-1.23994000$

$2.10795500 \quad-1.64466900 \quad-3.39459900$

$\begin{array}{lll}0.67306300 & -0.59650500 & -3.36440300\end{array}$

$2.46052900 \quad 0.38222100 \quad-2.71882300$ 
$-0.37354700 \quad-5.37781700 \quad-1.12785900$

$1.00694600 \quad-4.19432200 \quad 0.03012100$

$\begin{array}{lll}4.09378200 & -2.44449600 & 1.45650800\end{array}$

$2.21359600 \quad-1.37571100 \quad 1.47699400$

$\begin{array}{lll}4.72375700 & -3.40863400 & -0.67107100\end{array}$

$\begin{array}{lll}3.32921700 & -3.13728800 & -2.28916900\end{array}$

$\begin{array}{lll}1.86470500 & 1.63725800 & -2.51776500\end{array}$

$\begin{array}{lll}3.86045100 & 0.30393500 & -2.76488400\end{array}$

$\begin{array}{lll}-1.21503000 & -5.42697100 & -2.24299800\end{array}$

$\begin{array}{lll}-1.96646800 & -4.33692700 & -3.95375400\end{array}$

$\begin{array}{lll}-0.28159200 & -6.24798700 & -0.47258000\end{array}$

$5.00152100 \quad-3.16706700 \quad 0.68036700$

$\begin{array}{lll}4.29620600 & -2.25199300 \quad 2.51240000\end{array}$

$\begin{array}{lll}5.42747400 & -3.98138500 & -1.28093300\end{array}$

$2.64590100 \quad 2.78558200 \quad-2.38572900$

$\begin{array}{lll}0.77988500 & 1.71121000 \quad-2.46318700\end{array}$

$\begin{array}{lll}4.64492600 & 1.45289700 \quad-2.62528900\end{array}$

$4.34099200 \quad-0.66622500 \quad-2.90804100$

$\begin{array}{lll}-1.78723800 & -6.33121600 & -2.46328000\end{array}$

$5.92498900 \quad-3.54710800 \quad 1.12505900$

$\begin{array}{lll}4.04061500 & 2.69845800 & -2.43569500\end{array}$

$\begin{array}{lll}2.15791000 & 3.75172500 & -2.24149600\end{array}$

$5.73430100 \quad 1.37232600 \quad-2.66398100$

$\begin{array}{lll}4.65368600 & 3.59613100 & -2.32452000\end{array}$

$\begin{array}{lll}0.59149200 & 1.33580600 & 1.38930700\end{array}$

$0.03454000 \quad 3.09084500 \quad 1.24977800$

$\begin{array}{lll}2.39007900 & 1.61214500 & 1.74083900\end{array}$

$\begin{array}{lll}-0.05153800 & 0.83204200 & 3.07344300\end{array}$

$-1.24212200 \quad 3.49762800 \quad 1.67324800$

$\begin{array}{lll}0.84557600 & 4.02093200 & 0.57645200\end{array}$

$3.32688700 \quad 1.18174600 \quad 0.79493800$

$\begin{array}{lll}2.84832800 & 2.24245100 \quad 2.91018700\end{array}$

$0.34518700 \quad 1.47254700 \quad 3.87271300$

$\begin{array}{lll}-1.13990800 & 0.97879500 & 3.03809400\end{array}$

$\begin{array}{lll}0.26848100 & -0.62146600 & 3.29677200\end{array}$

$\begin{array}{lll}-1.69538500 & 4.79607100 & 1.42606300\end{array}$

$\begin{array}{lll}-1.90719800 \quad 2.79788200 & 2.17741600\end{array}$

$\begin{array}{lll}0.38850500 & 5.31552000 & 0.32226100\end{array}$

$1.84034400 \quad 3.72450700 \quad 0.24069800$

$4.69621800 \quad 1.36045400 \quad 1.01225400$

$2.97515600 \quad 0.69973500 \quad-0.11516700$

$4.21488800 \quad 2.41504100 \quad 3.13473900$ 
$2.13558300 \quad 2.61504500 \quad 3.64916500$

$1.22899600 \quad-1.03385800 \quad 4.22841000$

$-0.88558500$

5.70919200

0.74454300

$\begin{array}{lll}2.69360900 & 5.08773400 & 1.76106700\end{array}$

$1.03335000 \quad 6.02182300 \quad-0.20745000$

$\begin{array}{lll}5.14341800 & 1.96978400 & 2.18566300\end{array}$

$\begin{array}{lll}5.40800300 & 1.01277300 & 0.26103700\end{array}$

$4.55866500 \quad 2.90192300 \quad 4.05123000$

$\begin{array}{lll}-0.02231300 & -2.94310800 & 2.62253800\end{array}$

$\begin{array}{lll}-1.07007800 & -1.27956300 & 1.72654100\end{array}$

$\begin{array}{lll}1.55903400 & -2.38820200 & 4.36226800\end{array}$

$\begin{array}{lll}1.73044400 & -0.28702200 & 4.84899600\end{array}$

$\begin{array}{lll}-1.24387600 & 6.72229300 & 0.54582500\end{array}$

$\begin{array}{lll}6.21382800 & 2.10299900 \quad 2.36310500\end{array}$

$\begin{array}{lll}0.93249500 & -3.34634700 & 3.56247700\end{array}$

$\begin{array}{lll}-0.50987000 & -3.67968400 & 1.98245600\end{array}$

$\begin{array}{lll}2.31357800 & -2.69197100 & 5.09230400\end{array}$

$1.19357400 \quad-4.40263800 \quad 3.66277200$

$\begin{array}{lll}0.11562300 & -0.01959100 & 0.25021500\end{array}$

$\begin{array}{lll}2.05663600 & -0.24334500 & 1.46689300\end{array}$

$\begin{array}{lll}1.95547000 & 0.59841800 \quad 3.11575000\end{array}$

$\begin{array}{lll}3.74540000 & 0.35916000 & 0.92503900\end{array}$

$2.73755700 \quad-1.91602000 \quad 2.06466600$

$\begin{array}{lll}1.83612500 & -0.09349900 & 4.33093900\end{array}$

$\begin{array}{lll}1.92589800 & 2.00268100 & 3.15074100\end{array}$

$4.22941400 \quad-0.08595300 \quad-0.31522600$

$\begin{array}{lll}4.60884800 & 1.11262800 & 1.73394000\end{array}$

$\begin{array}{lll}3.38733300 & -1.69406000 & 2.92069000\end{array}$

$\begin{array}{lll}1.88948800 & -2.50338100 & 2.42386600\end{array}$

$\begin{array}{lll}3.51577400 & -2.69138900 & 1.04617300\end{array}$

$\begin{array}{lll}1.68508900 & 0.59918800 & 5.53672300\end{array}$

$1.84113800 \quad-1.18274100 \quad 4.34967300$

$\begin{array}{lll}1.78019900 & 2.69622500 & 4.35075500\end{array}$

$\begin{array}{lll}2.04213700 & 2.56001300 \quad 2.22075600\end{array}$

$\begin{array}{lll}5.51751200 & 0.22408200 & -0.74528000\end{array}$

$\begin{array}{lll}3.59287200 & -0.69053700 & -0.95458600\end{array}$

$5.89889600 \quad 1.43817700 \quad 1.30072100$

$\begin{array}{lll}4.28890500 & 1.44954100 & 2.71890800\end{array}$

$2.90289900 \quad-3.15469400 \quad-0.12551900$

$\begin{array}{lll}4.88391900 & -2.94040500 & 1.22383600\end{array}$

$\begin{array}{lll}1.65357800 & 1.99444200 & 5.55376900\end{array}$

$\begin{array}{lll}1.58653600 & 0.03862800 \quad 6.47009000\end{array}$ 
$\begin{array}{lll}6.35822700 & 0.99852100 & 0.05858600\end{array}$

5.61590300

$-2.91598300$

$-0.27839900$

5.38344900

$-3.64298400$

0.26329600

$\begin{array}{lll}1.52783400 & 2.53278200 & 6.49599300\end{array}$

$\begin{array}{lll}7.36523600 & 1.25398200 & -0.28081700\end{array}$

$4.99027600 \quad-4.10440200 \quad-0.89803000$

$3.13023500 \quad-4.20168000 \quad-2.00000100$

$6.68282900 \quad-3.82189000 \quad 0.41932900$

$5.56157000 \quad-4.65090700 \quad-1.65256900$

$\begin{array}{lll}-1.26498900 & -1.71624500 & 0.85812000\end{array}$

$\begin{array}{lll}-0.43206100 & -3.04638300 & 1.85508200\end{array}$

$\begin{array}{lll}-2.14626200 & -2.74436400 & -0.41766000\end{array}$

$\begin{array}{lll}-2.76691300 & -1.63558400 & 2.07828000\end{array}$

$\begin{array}{lll}-0.31301100 & -2.91617300 \quad 3.25213200\end{array}$

$0.14164800 \quad-4.17441300 \quad 1.24993000$

$\begin{array}{lll}-2.43346600 & -2.14752400 & -1.64886300\end{array}$

$\begin{array}{lll}-2.68985700 & -4.01150300 & -0.14588400\end{array}$

$\begin{array}{lll}-2.90812700 & -2.68995300 & 2.35330500\end{array}$

$\begin{array}{lll}-2.39095200 & -1.12005600 & 2.96625000\end{array}$

$-4.11118100 \quad-1.05197100 \quad 1.73387900$

$0.35959300 \quad-3.87603500 \quad 4.01064400$

$\begin{array}{lll}-0.73003400 & -2.04737400 & 3.76068300\end{array}$

$0.82379600 \quad-5.13160500 \quad 2.00460300$

$\begin{array}{lll}0.04780200 & -4.31253600 & 0.17376000\end{array}$

$\begin{array}{lll}-3.28868100 & -2.75840800 & -2.56777900\end{array}$

$\begin{array}{lll}-1.97901700 & -1.19107300 & -1.88788400\end{array}$

$-3.51731100 \quad-4.64418200 \quad-1.07605900$

$\begin{array}{lll}-2.48848600 & -4.50300400 & 0.80777000\end{array}$

$\begin{array}{lll}-4.55613300 & 0.10672600 & 2.39130600\end{array}$

$\begin{array}{lll}-5.00088100 & -1.68679400 & 0.84845300\end{array}$

$0.93824300 \quad-4.98702800 \quad 3.38918900$

$\begin{array}{lll}0.44062600 & -3.74812300 & 5.09315900\end{array}$

$\begin{array}{lll}1.27397200 & -5.99148200 & 1.50292000\end{array}$

$\begin{array}{lll}-3.83682700 & -4.00938400 & -2.28180400\end{array}$

$\begin{array}{lll}-3.50350800 & -2.26081100 & -3.51543000\end{array}$

$\begin{array}{lll}-3.93155900 & -5.63008700 & -0.84935700\end{array}$

$\begin{array}{lll}-5.83192400 & 0.62543400 \quad 2.16602800\end{array}$

$\begin{array}{lll}-3.89530300 & 0.60570300 & 3.09641800\end{array}$

$\begin{array}{lll}-6.27949100 & -1.17224700 \quad 0.62524400\end{array}$ 


\begin{tabular}{|c|c|c|}
\hline-4.69905000 & -2.59977400 & 0.33828900 \\
\hline 1.47367600 & -5.73406600 & 3.97971200 \\
\hline-4.49724100 & -4.49933100 & -3.00157700 \\
\hline-6.70176500 & -0.01035200 & 1.27837400 \\
\hline-6.14247000 & 1.53356200 & 2.68877500 \\
\hline-6.95144900 & -1.68757300 & -0.06632100 \\
\hline-7.70116200 & 0.39323900 & 1.09753500 \\
\hline-1.02292500 & 1.87089900 & 0.79956900 \\
\hline-1.63145400 & 1.91028900 & 2.56515100 \\
\hline-2.64951100 & 2.22650100 & -0.04256500 \\
\hline-0.30658800 & 3.61241500 & 0.84799800 \\
\hline-2.51640300 & 2.89302500 & 3.03925100 \\
\hline-1.19528000 & 0.92312800 & 3.45047400 \\
\hline-3.43860000 & 1.13841900 & -0.43817700 \\
\hline-3.12615500 & 3.51828600 & -0.32198400 \\
\hline-1.04349600 & 4.25405100 & 1.35545900 \\
\hline 0.52555500 & 3.54375800 & 1.56003200 \\
\hline 0.16385300 & 4.30770600 & -0.40725600 \\
\hline-2.94471700 & 2.88490500 & 4.36766800 \\
\hline-2.90245700 & 3.65692800 & 2.36234500 \\
\hline-1.61927400 & 0.90853300 & 4.78186400 \\
\hline-0.52285200 & 0.15862700 & 3.06975300 \\
\hline-4.65741000 & 1.31919400 & -1.09128600 \\
\hline-3.07913300 & 0.12994800 & -0.25677900 \\
\hline-4.34991600 & 3.70760400 & -0.97055700 \\
\hline-2.54272500 & 4.39843500 & -0.05565400 \\
\hline-0.18603400 & 3.89460800 & -1.69747600 \\
\hline 0.99647700 & 5.43194400 & -0.27401600 \\
\hline-2.49736000 & 1.88985700 & 5.24492700 \\
\hline-3.64098900 & 3.65136200 & 4.71764900 \\
\hline-1.26460500 & 0.12613100 & 5.45590100 \\
\hline-5.12090400 & 2.60932000 & -1.35844100 \\
\hline-5.23589700 & 0.44597100 & -1.39404100 \\
\hline-4.69431000 & 4.72319300 & -1.18210400 \\
\hline 0.29552300 & 4.56657300 & -2.82402200 \\
\hline-0.82104800 & 3.02431700 & -1.83775400 \\
\hline 1.47558200 & 6.11034100 & -1.39484800 \\
\hline 1.28409000 & 5.76859800 & 0.72657400 \\
\hline-2.83779500 & 1.87954800 & 6.28331700 \\
\hline-6.07382800 & 2.75841700 & -1.87245800 \\
\hline 1.13006200 & 5.67512100 & -2.67866900 \\
\hline 0.02283600 & 4.20339300 & -3.81660500 \\
\hline 2.13105400 & 6.97583400 & -1.26709100 \\
\hline 1.51509000 & 6.19579200 & -3.55887900 \\
\hline
\end{tabular}


$0.79161500 \quad 0.17238500 \quad-1.91544500$

$\begin{array}{lll}2.23984300 & 1.34139600 & -2.09227800\end{array}$

$-0.28628200$

1.48573900

3.16174500

2.42298000

$-1.66370200$

0.21407000

2.01509700

2.24362700

0.52814300

4.20538500

3.10248300

3.45943600

1.75173800

$-2.53171900$

$-2.05954400$

$-0.64322800$

1.28281000

0.32418100

$-0.13334400$

4.35580500

4.91235100

3.57702200

$-2.02301700$

$-3.60115000$

$-0.22861200$

$-0.47905300$

0.81317400

$-0.94633000$

0.00505800

5.17815400

$-2.69157200$

$-1.11624800$

$-0.61660100$

$-1.44466400$

$-1.75047300$

$-3.05789300$

$-5.19077300$

$-6.09110800$

$-4.82604300$

$0.82580800 \quad-2.49847500 \quad 0.60830500$

$\begin{array}{lll}-0.59609000 & -2.36817500 & 0.74056700\end{array}$

$\begin{array}{lll}-1.56415000 & -2.74900600 & -0.30462100\end{array}$

$-1.18936600 \quad-3.25656200 \quad-1.56729800$

$\begin{array}{lll}-3.26611700 & -2.16092400 & 0.89240000\end{array}$

$-1.02652100 \quad-2.35589500 \quad 1.75182400$ 
$\begin{array}{lll}-3.49869200 & -3.29366000 & -2.31657300\end{array}$

$\begin{array}{lll}-3.89218400 & -2.81314500 & -1.06213100\end{array}$

$\begin{array}{lll}-1.81227400 & -3.90731100 & -3.52485300\end{array}$

$-4.24072900 \quad-3.49786500 \quad-3.09223800$

$\begin{array}{lll}-4.95009800 & -2.63687100 & -0.84963500\end{array}$

$\begin{array}{lll}1.74047700 & -2.59695400 & 1.78756500\end{array}$

$\begin{array}{lll}1.29757200 & -2.09559200 & 2.66373700\end{array}$

$\begin{array}{lll}0.28352500 & -0.65342600 & 0.46647600\end{array}$

$\begin{array}{lll}3.09781000 & -0.66784000 & 1.01847500\end{array}$

$2.13911700 \quad 0.04518200 \quad 0.72593300$

$\begin{array}{lll}4.53657300 & -0.19758000 & 0.80559600\end{array}$

$\begin{array}{lll}4.52996000 & 1.26260600 & 0.33840500\end{array}$

$5.15806500 \quad-1.09803800 \quad-0.28201300$

$\begin{array}{lll}5.32033600 & -0.33866800 & 2.12259100\end{array}$

$4.07391200 \quad 1.92217200 \quad 1.09200800$

$3.96690200 \quad 1.38145100 \quad-0.59828300$

$5.56385600 \quad 1.60077200 \quad 0.16810900$

$5.18167800 \quad-2.15018500 \quad 0.03639600$

$\begin{array}{lll}6.19030900 & -0.77416800 & -0.48821900\end{array}$

$\begin{array}{lll}4.58687400 & -1.03267900 & -1.22150600\end{array}$

$\begin{array}{lll}6.35663800 & 0.00498000 & 1.97816800\end{array}$

$\begin{array}{lll}5.34638000 & -1.38324400 & 2.46343600\end{array}$

$\begin{array}{lll}4.86954600 & 0.27290800 & 2.92076900\end{array}$

$\begin{array}{lll}3.02334300 & -1.88418100 & 1.51291200\end{array}$

$1.24158400 \quad-2.95914900 \quad-0.29859300$

$\begin{array}{lll}-0.13668100 & -3.45399800 & -1.77868000\end{array}$

$\begin{array}{lll}2.18575400 & -3.99885900 & 2.16529700\end{array}$

$\begin{array}{lll}1.30373300 & -4.58622400 & 2.46165600\end{array}$

$2.90003800 \quad-3.98647200 \quad 3.00392300$

$\begin{array}{lll}2.65895900 & -4.49830200 & 1.30523000\end{array}$

$\begin{array}{lll}-1.14588900 & 0.90555100 & 0.09744300\end{array}$

$\begin{array}{lll}-2.82272600 & 0.75929100 & 0.82844500\end{array}$

$\begin{array}{lll}-0.71397600 & 2.66974800 & 0.41504400\end{array}$

$\begin{array}{lll}-1.44619100 & 0.94498800 & -1.75064000\end{array}$

$\begin{array}{lll}-4.00866900 & 0.79164900 & 0.08157700\end{array}$

$\begin{array}{lll}-2.89838000 & 0.52933200 & 2.21292400\end{array}$

$\begin{array}{lll}0.65048900 & 2.99613100 & 0.47479900\end{array}$

$\begin{array}{lll}-1.67006700 & 3.68883000 & 0.54492000\end{array}$

$\begin{array}{lll}-2.08131100 & 1.80024400 & -2.02372900\end{array}$

$\begin{array}{lll}-1.97277600 & 0.01091700 & -1.99933900\end{array}$

$\begin{array}{lll}-0.10197700 & 1.00151100 & -2.42449100\end{array}$

$\begin{array}{lll}-5.24519900 & 0.59811500 & 0.70653200\end{array}$ 


\begin{tabular}{rrr}
-3.97870300 & 0.95521800 & -0.99637100 \\
-4.13163200 & 0.34483000 & 2.83792000 \\
-1.97677500 & 0.48211700 & 2.79986000 \\
1.05084800 & 4.32423300 & 0.64496900 \\
1.39359500 & 2.20230600 & 0.38604500 \\
-1.26737300 & 5.01469000 & 0.72313800 \\
-2.73466100 & 3.44546100 & 0.50875800 \\
0.65939000 & -0.17368700 & -2.54770000 \\
0.47328600 & 2.21950200 & -2.81114900 \\
-5.31039800 & 0.37431200 & 2.08341500 \\
-6.16109700 & 0.61770300 & 0.11049000 \\
-4.17480000 & 0.16741000 & 3.91549500 \\
0.09383900 & 5.33528100 & 0.76994500 \\
2.11572700 & 4.56826300 & 0.68304800 \\
-2.01890800 & 5.80180200 & 0.82662200 \\
1.96042300 & -0.13052800 & -3.04934800 \\
0.21312600 & -1.12502500 & -2.25381400 \\
1.77829900 & 2.26483800 & -3.31295400 \\
-0.10028600 & 3.14301500 & -2.70180800 \\
-6.27684300 & 0.21951200 & 2.56945100 \\
0.40668900 & 6.37340600 & 0.90854100 \\
2.52747100 & 1.09088300 & -3.43015200 \\
2.53538700 & -1.05553500 & -3.14296900 \\
2.21201100 & 3.22393500 & -3.60718400 \\
3.54816400 & 1.12620400 & -3.81929400 \\
& & \\
\hline
\end{tabular}

TS-S21 


\begin{tabular}{|c|c|c|}
\hline-4.47352500 & -0.61408000 & -0.86700700 \\
\hline-4.60812300 & 0.86985900 & -0.50174100 \\
\hline-5.05532800 & -1.48818100 & 0.26339400 \\
\hline-5.20189900 & -0.90644100 & -2.18560900 \\
\hline-4.16096100 & 1.51183400 & -1.27691800 \\
\hline-4.11186100 & 1.09410300 & 0.45239300 \\
\hline-5.67291700 & 1.13848000 & -0.41231000 \\
\hline-4.96145500 & -2.55900500 & 0.02477200 \\
\hline-6.12449000 & -1.26206000 & 0.40592800 \\
\hline-4.53527200 & -1.29835300 & 1.21547800 \\
\hline-6.26901400 & -0.64810400 & -2.09261600 \\
\hline-5.12382900 & -1.96773800 & -2.45850000 \\
\hline-4.78070500 & -0.31326300 & -3.01312300 \\
\hline-2.72161300 & -2.00728700 & -1.70478700 \\
\hline-0.70644200 & -3.30453700 & 0.50067900 \\
\hline 0.66704100 & -3.07188500 & 2.07784100 \\
\hline-2.04058000 & -4.36667500 & -1.59763500 \\
\hline-1.32604400 & -5.19748900 & -1.72996300 \\
\hline-2.76441100 & -4.39311300 & -2.42331800 \\
\hline-2.57232900 & -4.52961300 & -0.64766000 \\
\hline 0.97744300 & 0.91345600 & -0.14586700 \\
\hline 2.58177800 & 0.88544300 & -1.03289600 \\
\hline 0.28876100 & 2.56396500 & -0.57599600 \\
\hline 1.43427800 & 1.16106500 & 1.64964500 \\
\hline 2.55324600 & 0.54200000 & -2.39549900 \\
\hline 3.81926600 & 1.12011700 & -0.41812300 \\
\hline-1.10318600 & 2.72851100 & -0.48953600 \\
\hline 1.09213800 & 3.65938900 & -0.92849500 \\
\hline 2.04557600 & 2.06809400 & 1.76241400 \\
\hline 2.03379600 & 0.28482200 & 1.94101600 \\
\hline 0.16326900 & 1.24579900 & 2.45053000 \\
\hline 3.73534600 & 0.44456400 & -3.12933700 \\
\hline 1.59313400 & 0.33920000 & -2.87799400 \\
\hline 5.00539400 & 1.01551700 & -1.15226700 \\
\hline 3.87292900 & 1.37125200 & 0.64173200 \\
\hline-1.67932300 & 3.97697600 & -0.73804200 \\
\hline-1.72723400 & 1.87291000 & -0.22761400 \\
\hline 0.51147100 & 4.90409700 & -1.18398700 \\
\hline 2.17528300 & 3.53986300 & -1.00598100 \\
\hline-0.55895700 & 0.07619000 & 2.73772100 \\
\hline-0.38975500 & 2.48293600 & 2.80883000 \\
\hline 4.96725500 & 0.67780600 & -2.50661500 \\
\hline 3.69808400 & 0.17770200 & -4.18844500 \\
\hline .96390700 & 1.19310000 & -0.65823000 \\
\hline
\end{tabular}




$\begin{array}{rrr}-0.87474800 & 5.06571000 & -1.08618700 \\ -2.76293600 & 4.09726700 & -0.66103400 \\ 1.14354100 & 5.75172000 & -1.46134900 \\ -1.80045800 & 0.14118400 & 3.36983200 \\ -0.13656100 & -0.88949700 & 2.45465400 \\ -1.63252700 & 2.55086900 & 3.44584600 \\ 0.15126300 & 3.40208100 & 2.57087600 \\ 5.89515900 & 0.59269900 & -3.07747300 \\ -1.32687600 & 6.04067800 & -1.28561200 \\ -2.34362200 & 1.38063000 & 3.72482100 \\ -2.34969400 & -0.77971000 & 3.58170300 \\ -2.04994400 & 3.52407500 & 3.71624600 \\ -3.31798300 & 1.43355100 & 4.21700900\end{array}$

H

H

C

$\mathrm{H}$

C

$\mathrm{H}$

$\mathrm{H}$

H

\begin{tabular}{|c|c|c|}
\hline 1.23891900 & -2.09065800 & 2.32168900 \\
\hline 1.81536000 & -0.80264600 & 2.10781300 \\
\hline-3.09669000 & -0.61142100 & 1.40259400 \\
\hline-3.49423900 & -1.44624400 & 0.34001800 \\
\hline-3.65227100 & 1.11924800 & 2.57339200 \\
\hline-1.59785000 & -0.04037300 & 2.86748500 \\
\hline 3.94260600 & 0.45757700 & 1.75368900 \\
\hline 4.69137500 & -1.22150000 & -0.33878600 \\
\hline 5.51919500 & -0.15152400 & 0.01864100 \\
\hline 5.13788200 & 0.68760900 & 1.06985800 \\
\hline-4.97080600 & -1.87795300 & -1.16633000 \\
\hline-6.45303900 & 0.02761300 & -0.51962700 \\
\hline-5.77423600 & 1.52752300 & 1.35987500 \\
\hline 0.05274800 & -2.17472200 & 2.83929400 \\
\hline 0.41294700 & -1.35979700 & 3.47962600 \\
\hline 2.62116400 & -0.97635100 & 1.03821400 \\
\hline 1.63816400 & -1.56708300 & 0.44446200 \\
\hline 4.02384600 & -1.37220200 & 0.52468100 \\
\hline 4.05385100 & -1.29911700 & -1.01040300 \\
\hline 4.29379200 & -2.81973100 & 0.97635300 \\
\hline 5.07831200 & -0.43234500 & 1.11662300 \\
\hline 3.83877300 & -0.28136000 & -1.36646600 \\
\hline 3.31091800 & -1.97555400 & -1.45564500 \\
\hline 5.05055000 & -1.58576600 & -1.38596900 \\
\hline 4.29104800 & -2.90113600 & 2.07562800 \\
\hline 5.28055500 & -3.15778100 & 0.61811300 \\
\hline 3.52943100 & -3.503555500 & 0.57835100 \\
\hline 6.08732200 & -0.72855100 & 0.78488500 \\
\hline 5.05151800 & -0.44870900 & 2.21555700 \\
\hline 90395100 & 0.60656300 & 0.79823000 \\
\hline
\end{tabular}


$2.47264500 \quad-0.17261300 \quad 1.96366900$ 
S23

$-3.04428200$

$-2.89669700$

1.33360600

$-3.54889900$

$-3.11215400-1.13100300$

$\mathrm{H}$

$\begin{array}{lll}-5.73397800 & -2.69708500 & 1.44543000\end{array}$

$\mathrm{H}$

$-3.52926800$

$-2.73022900 \quad 2.30576800$

C

$-5.36811300$

$-2.80228200$

0.42032800

$-4.45328400$

$-3.09827500 \quad-2.19115400$

$-5.82166600$

$-6.27544300$

$-2.92616400 \quad-1.95563300$

$\mathrm{H}$

$-4.08303000$

$-2.78284000$

$-0.64169500$

$\mathrm{H}$

$-6.52677700$

$-7.34253700$

$-0.88924300$

$-3.19892700$

$-3.21408500$

$\mathrm{H}$

C

$-1.27479700$

$-1.49017600$

$-2.48280400$

$-1.44763900$

0.53872100

$-2.90254900$

$-2.78998200$

$-2.65680000 \quad-0.43977900$

$-3.36419700 \quad 2.37727000$

3.35269800 


\begin{tabular}{|c|c|c|c|}
\hline $\mathrm{C}$ & -4.70911000 & 2.84728700 & 1.72031800 \\
\hline $\mathrm{C}$ & -0.58475600 & 4.24759600 & 1.84085600 \\
\hline $\mathrm{H}$ & -0.21864300 & 3.28331000 & 3.74868900 \\
\hline $\mathrm{H}$ & -1.05558700 & 4.91672800 & -0.16536100 \\
\hline $\mathrm{C}$ & -2.98405000 & 0.09596200 & -3.96128700 \\
\hline $\mathrm{H}$ & -1.18649000 & -1.10496600 & -3.90824100 \\
\hline $\mathrm{H}$ & -4.75051500 & 1.31945300 & -3.69214700 \\
\hline $\mathrm{C}$ & -7.05824700 & 2.52012200 & 0.24828000 \\
\hline $\mathrm{H}$ & -6.50442900 & 0.43534100 & 0.12671900 \\
\hline $\mathrm{C}$ & -5.53332600 & 3.94505700 & 1.46244300 \\
\hline $\mathrm{H}$ & -3.79930900 & 2.98260200 & 2.30635100 \\
\hline $\mathrm{H}$ & -0.16793100 & 5.20918500 & 2.15313300 \\
\hline $\mathrm{H}$ & -3.08216400 & 0.04904600 & -5.04912900 \\
\hline C & -6.70806500 & 3.78821300 & 0.72109300 \\
\hline $\mathrm{H}$ & -7.97982000 & 2.38219600 & -0.32298100 \\
\hline $\mathrm{H}$ & -5.25520100 & 4.92983000 & 1.84649500 \\
\hline $\mathrm{H}$ & -7.35132700 & 4.64835600 & 0.51952200 \\
\hline $\mathrm{O}$ & 2.54501400 & -0.83300500 & 1.00093000 \\
\hline $\mathrm{O}$ & 0.15810400 & -0.76398600 & 1.78196200 \\
\hline $\mathrm{P}$ & 1.01186600 & -0.71606600 & 0.45013600 \\
\hline B & 3.77244600 & -0.15677800 & 0.47963400 \\
\hline K & 2.23281200 & 2.49073000 & 0.68718400 \\
\hline K & 3.18022100 & -2.77248500 & -0.76776400 \\
\hline $\mathrm{O}$ & 0.74505600 & -1.96274300 & -0.42677600 \\
\hline $\mathrm{O}$ & 0.84928000 & 0.62744700 & -0.28304300 \\
\hline $\mathrm{O}$ & 4.86414600 & -1.17660100 & 0.57979500 \\
\hline B & 3.57094800 & 0.39389200 & -1.15313500 \\
\hline $\mathrm{O}$ & 4.21968900 & 0.93790200 & 1.38580200 \\
\hline $\mathrm{C}$ & 5.95093200 & -0.61904700 & 1.29878900 \\
\hline $\mathrm{O}$ & 3.49761700 & -0.46994600 & -2.23940100 \\
\hline $\mathrm{O}$ & 3.50546900 & 1.70999600 & -1.58429600 \\
\hline $\mathrm{C}$ & 5.23843400 & 0.42804900 & 2.23688500 \\
\hline $\mathrm{C}$ & 6.68929600 & -1.73223000 & 2.03716600 \\
\hline $\mathrm{C}$ & 6.90066300 & 0.06422100 & 0.30177100 \\
\hline C & 3.02848400 & 0.26994100 & -3.39686400 \\
\hline $\mathrm{C}$ & 3.43628500 & 1.74975100 & -3.03047800 \\
\hline $\mathrm{C}$ & 6.12976900 & 1.58114000 & 2.69066500 \\
\hline $\mathrm{C}$ & 4.59040700 & -0.24011000 & 3.45841700 \\
\hline $\mathrm{H}$ & 7.47619000 & -1.32872200 & 2.69482100 \\
\hline $\mathrm{H}$ & 7.17278800 & -2.40650400 & 1.31144900 \\
\hline $\mathrm{H}$ & 5.99778800 & -2.33180100 & 2.64455600 \\
\hline $\mathrm{H}$ & 7.82514300 & 0.42848900 & 0.77684600 \\
\hline $\mathrm{H}$ & 7.17905200 & -0.66378100 & -0.47637100 \\
\hline $\mathrm{H}$ & 6.40138000 & 0.91226800 & -0.18964000 \\
\hline
\end{tabular}


$\mathrm{P}(\text { o-Tol })_{3}$

P

C

C

$\mathrm{H}$

H

\begin{tabular}{|c|c|c|}
\hline 3.70816600 & -0.28760200 & -4.64014800 \\
\hline 1.51615900 & 0.06087300 & -3.46675100 \\
\hline 2.41169800 & 2.80343000 & -3.43246500 \\
\hline 4.82860700 & 2.13951200 & -3.52778500 \\
\hline 5.56531500 & 2.24978000 & 3.36118900 \\
\hline 7.00848100 & 1.21696500 & 3.24722600 \\
\hline 6.47880000 & 2.17832400 & 1.83703200 \\
\hline 3.92533100 & 0.48844200 & 3.94851700 \\
\hline 5.33642500 & -0.57237600 & 4.19793900 \\
\hline 3.97300000 & -1.09446000 & 3.15155300 \\
\hline 3.37057700 & -1.32151500 & -4.81264000 \\
\hline 3.44244400 & 0.30248700 & -5.53117700 \\
\hline 4.80182400 & -0.30067100 & -4.54175700 \\
\hline 1.07286800 & 0.57533900 & -4.33230100 \\
\hline 1.31610400 & -1.01623900 & -3.57025300 \\
\hline 1.03419700 & 0.39517400 & -2.53660200 \\
\hline 2.76785900 & 3.80190900 & -3.13275200 \\
\hline 2.26268900 & 2.81558800 & -4.52339700 \\
\hline 1.44206400 & 2.62202000 & -2.95042100 \\
\hline 5.57942500 & 1.39072500 & -3.23617000 \\
\hline 4.85676000 & 2.25830800 & -4.62131300 \\
\hline 5.11624300 & 3.09870900 & -3.07090200 \\
\hline-0.00012500 & 0.00068000 & 0.91051900 \\
\hline 1.26807600 & 1.07752500 & 0.10585200 \\
\hline 2.59352200 & 1.04808500 & 0.60816800 \\
\hline 3.54693900 & 1.90115100 & 0.03405300 \\
\hline 4.57127300 & 1.87996900 & 0.41622400 \\
\hline 3.21602600 & 2.77420100 & -1.00488700 \\
\hline 3.97939100 & 3.43149600 & -1.42886300 \\
\hline 1.90962800 & 2.80172300 & -1.49505600 \\
\hline 1.63736800 & 3.47729900 & -2.30981900 \\
\hline 0.94647900 & 1.95512600 & -0.94143300 \\
\hline-0.07229700 & 1.97450000 & -1.33223400 \\
\hline 2.99461100 & 0.10500400 & 1.71216300 \\
\hline 2.30266700 & 0.16872100 & 2.56769800 \\
\hline 4.01184900 & 0.32059400 & 2.07014600 \\
\hline 2.97301100 & -0.94323800 & 1.36955800 \\
\hline-1.56657300 & 0.55952100 & 0.10512700 \\
\hline-2.20666400 & 1.71990700 & 0.60891900 \\
\hline-3.42212700 & 2.11793500 & 0.03389000 \\
\hline-3.91826900 & 3.01381100 & 0.41733600 \\
\hline-4.00971800 & 1.39586800 & -1.00749200 \\
\hline-4.96067800 & 1.72730500 & -1.43214800 \\
\hline
\end{tabular}


$-3.82377200$

$0.25307300 \quad-1.49929600$

$-2.16257500$

$-1.66711800$

$-0.31953100 \quad-2.31608300$

$\begin{array}{lll}1.59355900 & 2.53760700 \quad 1.71550600\end{array}$

$\begin{array}{lll}-1.30226200 & 1.90518800 \quad 2.56987200\end{array}$

$\begin{array}{lll}-2.29098800 & 3.30838700 & 2.07451300\end{array}$

$\begin{array}{lll}-0.67571000 & 3.04589400 & 1.37506400\end{array}$

$0.29880600 \quad-1.63582400 \quad 0.10570000$

$\begin{array}{lll}-0.38844300 & -2.76947500 & 0.60833200\end{array}$

$\begin{array}{lll}-0.12595800 & -4.02148100 & 0.03385900\end{array}$

$\begin{array}{lll}-0.65556300 & -4.89841100 & 0.41631500\end{array}$

$0.79485400 \quad-4.17052200 \quad-1.00584600$

$0.98261400 \quad-5.16000600 \quad-1.43031600$

$\begin{array}{lll}1.47091600 & -3.05231300 & -1.49619700\end{array}$

$\begin{array}{lll}2.19152800 & -3.15364800 & -2.31157900\end{array}$

$1.21890900 \quad-1.79520200 \quad-0.94218200$

$\begin{array}{lll}1.74393900 & -0.92200800 & -1.33326800\end{array}$

$\begin{array}{lll}-1.40481300 & -2.64596700 & 1.71323400\end{array}$

$\begin{array}{lll}-1.00398900 & -2.07625700 & 2.56731700\end{array}$

$\begin{array}{lll}-1.72412900 & -3.63485100 \quad 2.07317700\end{array}$

$\begin{array}{lll}-2.30343300 & -2.10575100 \quad 1.37064700\end{array}$

$\begin{array}{lll}-0.06887600 & -0.13804200 & 0.17063300\end{array}$

$\begin{array}{lll}-1.98888100 & -0.40338000 & 0.68755500\end{array}$

$\begin{array}{lll}-1.39403800 & -1.65221300 & 0.34099600\end{array}$

$\begin{array}{lll}-2.26241800 & -0.10384200 & 2.10884400\end{array}$

$\begin{array}{lll}-2.70010200 & 0.05011100 & -0.01042500\end{array}$

$\begin{array}{lll}-1.87525900 & -2.38440000 & -0.88958200\end{array}$

$\begin{array}{lll}-1.19798900 & -2.32697100 \quad 1.18096500\end{array}$

$\begin{array}{lll}-3.47272100 & 0.50513100 & 2.48712900\end{array}$

$\begin{array}{lll}-1.34928400 & -0.44217000 & 3.13085400\end{array}$

$-1.20114500 \quad-3.71425700 \quad-1.15485800$

$\begin{array}{lll}-1.81579100 & -1.74864500 & -1.77580100\end{array}$

$\begin{array}{lll}-3.76358200 & 0.76730400 & 3.82738400\end{array}$

$\begin{array}{lll}-4.18771800 & 0.78437200 & 1.71013200\end{array}$

$-1.63607000 \quad-0.17399300 \quad 4.46869400$

$\begin{array}{lll}-0.39966100 & -0.91194000 & 2.86795100\end{array}$

$\begin{array}{lll}-1.64518300 & -4.20588600 \quad-2.03357000\end{array}$

$\begin{array}{lll}-1.31201100 & -4.39108200 & -0.29262000\end{array}$

$\begin{array}{lll}-0.13471400 & -3.56112800 & -1.35301100\end{array}$

$\begin{array}{lll}-2.84527800 & 0.43429600 & 4.82757900\end{array}$

$\begin{array}{lll}-4.71353200 & 1.23995300 \quad 4.09211200\end{array}$

$\begin{array}{lll}-0.90660800 & -0.44240400 \quad 5.23772300\end{array}$ 


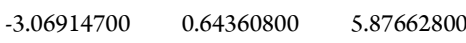

$-4.21986900$

$-1.92920900 \quad-1.29422500$

$-5.64052300$

$-2.32883600 \quad-0.87561100$

$-3.30339000$

$-2.70031900 \quad-0.70613500$

$\begin{array}{lll}-3.96190900 & -1.02037600 & -2.05528900\end{array}$

$-5.85759500$

$-6.64944000$

$-3.82217900$

$-1.17834100$

$-1.65071200$

$\begin{array}{lll}-5.77926800 & -2.07270000 & 0.63745100\end{array}$

$-5.72574900$

$-6.88145200$

$-4.03385000$

$-2.25206000$

$-5.15013300$

$-6.55978700$

$-0.89928700$

$\begin{array}{lll}-7.67607500 & -1.73520000 & -1.34674400\end{array}$

$\begin{array}{lll}-6.49120700 & -0.40407700 & -1.46204600\end{array}$

$\begin{array}{lll}-5.03953800 & -2.64954500 \quad 1.21035300\end{array}$

$\begin{array}{lll}-6.78744300 & -2.35962600 & 0.97779400\end{array}$

$\begin{array}{lll}-5.63187000 & -1.00808500 & 0.87504900\end{array}$

$\begin{array}{lll}-0.09540500 & 2.01226600 & -0.43290400\end{array}$

$\begin{array}{lll}0.80417100 & 3.41648700 & 0.40038900\end{array}$

$\begin{array}{lll}-1.75641900 & 2.84326300 & -0.56343600\end{array}$

$\begin{array}{lll}0.58475800 & 2.07087200 & -2.17070600\end{array}$

$\begin{array}{lll}1.46773500 & 3.28203400 & 1.63828900\end{array}$

$0.73472200 \quad 4.68540900 \quad-0.20717400$

$\begin{array}{lll}-2.44407100 \quad 3.23164800 & 0.61531500\end{array}$

$\begin{array}{lll}-2.35598200 & 3.08520700 & -1.80850400\end{array}$

$\begin{array}{lll}0.02005200 & 1.27576600 & -3.19962300\end{array}$

$\begin{array}{lll}1.75953100 & 2.79314700 \quad-2.45251200\end{array}$

$2.10114500 \quad 4.41518900 \quad 2.18365300$

$\begin{array}{lll}1.46702000 & 2.01943000 & 2.45478100\end{array}$

$\begin{array}{lll}1.35303500 & 5.79804200 & 0.35468600\end{array}$

$0.18083200 \quad 4.80062300 \quad-1.14068000$

$\begin{array}{lll}-3.73582500 & 3.76503700 & 0.49109900\end{array}$

$-1.82076000 \quad 3.17244900 \quad 1.98295300$

$\begin{array}{lll}-3.63750500 & 3.62976600 & -1.90999700\end{array}$

$\begin{array}{lll}-1.81165900 & 2.86302000 & -2.72328500\end{array}$

$0.62163800 \quad 1.28397000 \quad-4.46982000$

$\begin{array}{lll}-1.18051700 & 0.39139400 & -3.00260200\end{array}$

$2.35727100 \quad 2.76453500 \quad-3.71098000$

$2.22886600 \quad 3.38754100 \quad-1.67231400$

$\begin{array}{lll}2.62564200 & 4.31040800 \quad 3.13745900\end{array}$

$2.05546600 \quad 5.65665000 \quad 1.55647400$

$0.71679300 \quad 2.08733900 \quad 3.25996600$

$\begin{array}{lll}2.44270900 & 1.86450800 & 2.93294300\end{array}$

$1.21034800 \quad 1.13551800 \quad 1.85712700$ 


\begin{tabular}{lll}
2.83975800 & -2.27474500 & 5.04752400 \\
1.41987900 & -3.61473200 & 4.14633000 \\
4.64331400 & -1.10278200 & 2.77456500 \\
3.72392500 & -0.39923900 & 4.11750100 \\
3.43388700 & 0.13116400 & 2.44732000 \\
0.04717500 & -4.79591600 & 2.95034300 \\
6.93105300 & -0.51272000 & -0.11501500 \\
\hline 5.80641700 & 1.32008200 & -0.26291200 \\
4.45000600 & -2.80646200 & 0.98856900 \\
\hline 6.10880800 & -2.59313900 & 0.38307300 \\
\hline 4.77592200 & -2.91849600 & -0.74677300 \\
\hline 4.40744400 & 2.97299100 & -0.37978800 \\
\hline 2.16675700 & -3.96854400 & -4.86722200 \\
\hline 1.22369500 & -4.15130100 & 5.07771500 \\
\hline 6.69883000 & 1.94257500 & -0.36577700
\end{tabular}

S25

\begin{tabular}{|c|c|c|}
\hline-0.03662400 & -0.11416200 & 0.04720100 \\
\hline 1.83822400 & -1.33510500 & 0.03148800 \\
\hline 1.98599000 & -2.04752900 & -1.69580000 \\
\hline 2.09444000 & -2.87264600 & 1.09285400 \\
\hline 3.42725700 & -0.37347500 & 0.22683000 \\
\hline 3.13034700 & -2.50995600 & -2.39711100 \\
\hline 0.73624300 & -2.10079900 & -2.34335300 \\
\hline 1.46286200 & -3.03450900 & 2.35007200 \\
\hline 2.85720800 & -3.94594100 & 0.59965500 \\
\hline 4.11041400 & -0.14617900 & 1.44717600 \\
\hline 3.91210700 & 0.25764100 & -0.93326300 \\
\hline 2.95290000 & -2.98991900 & -3.70880400 \\
\hline 4.55089800 & -2.53421400 & -1.87559600 \\
\hline 0.57985500 & -2.59467600 & -3.63488600 \\
\hline-0.12763500 & -1.72467600 & -1.79666400 \\
\hline 1.66404000 & -4.22261700 & 3.07171400 \\
\hline 0.53291600 & -2.01610500 & 2.94021200 \\
\hline 3.04775200 & -5.12048600 & 1.32629900 \\
\hline 3.30248700 & -3.88541400 & -0.38654000 \\
\hline 5.26039000 & 0.66280100 & 1.43592500 \\
\hline 3.68720200 & -0.73093400 & 2.76721900 \\
\hline 5.06369700 & 1.03808400 & -0.92737400 \\
\hline 3.39147500 & 0.10526200 & -1.87557900 \\
\hline 3.83417100 & -3.34313100 & -4.25251800 \\
\hline 1.70600200 & -3.03911100 & -4.32896400 \\
\hline 4.63812100 & -2.32343200 & -0.80749300 \\
\hline 5.16650200 & -1.78643900 & -2.40032700 \\
\hline .0053200 & -3.51886400 & -2.0725550 \\
\hline
\end{tabular}


$-0.41294600 \quad-2.62789000 \quad-4.08841800$

$\begin{array}{lll}1.16645200 & -4.33236900 \quad 4.03960200\end{array}$

$\begin{array}{lll}2.45603300 & -5.25804200 & 2.58278800\end{array}$

$0.22875300 \quad-1.28532700 \quad 2.18146700$

$0.98967400 \quad-1.47239100 \quad 3.78260800$

$\begin{array}{lll}-0.37343600 & -2.50007800 & 3.32713500\end{array}$

$\begin{array}{lll}3.65171000 & -5.92679300 & 0.90277200\end{array}$

$\begin{array}{lll}5.78270200 & 0.83234400 & 2.38154900\end{array}$

$5.75060700 \quad 1.24261100 \quad 0.26850400$

$\begin{array}{lll}2.61193700 & -0.61898300 & 2.93166800\end{array}$

$\begin{array}{lll}4.20230400 & -0.22164400 \quad 3.59456100\end{array}$

$3.91646000 \quad-1.80521000 \quad 2.83527600$

$\begin{array}{lll}5.41267900 & 1.49227300 & -1.85527200\end{array}$

$1.61685200 \quad-3.42575000 \quad-5.34749600$

$2.59315700 \quad-6.17126000 \quad 3.16739900$

$\begin{array}{lll}6.65250100 & 1.85905000 & 0.29586800\end{array}$

$0.32379700 \quad 2.06831900 \quad 0.02720800$

$\begin{array}{lll}-1.00964900 & 3.21645100 & -0.60083700\end{array}$

$\begin{array}{lll}1.80715400 & 2.74175800 & -0.89072900\end{array}$

$0.63474400 \quad 2.68431400 \quad 1.75348300$

$\begin{array}{lll}-2.22241800 & 3.39161900 & 0.11375000\end{array}$

$\begin{array}{lll}-0.85122200 & 3.89078000 & -1.82294600\end{array}$

$\begin{array}{lll}2.04979500 & 2.39284800 & -2.24232500\end{array}$

$\begin{array}{lll}2.70766700 & 3.61386600 & -0.25611700\end{array}$

$\begin{array}{lll}0.56134100 & 4.01783100 & 2.22886100\end{array}$

$\begin{array}{lll}0.95851200 & 1.65732600 & 2.65374500\end{array}$

$\begin{array}{lll}-3.20697500 & 4.23592300 & -0.41896000\end{array}$

$\begin{array}{lll}-2.48163900 & 2.74132900 & 1.44219300\end{array}$

$\begin{array}{lll}-1.84882900 & 4.71593100 & -2.34364400\end{array}$

$0.08207500 \quad 3.79753700 \quad-2.37503100$

$3.10575200 \quad 3.01390700 \quad-2.92726700$

$\begin{array}{lll}1.23172600 & 1.35920500 & -2.96376400\end{array}$

$3.76710300 \quad 4.20335500 \quad-0.94492900$

$\begin{array}{lll}2.58942100 & 3.83644100 & 0.80263700\end{array}$

$\begin{array}{lll}0.79970100 & 4.24309200 \quad 3.59659100\end{array}$

$\begin{array}{lll}0.23246700 & 5.21226800 & 1.36902400\end{array}$

$\begin{array}{lll}1.19814300 & 1.90026300 & 4.00467300\end{array}$

$\begin{array}{lll}1.01898700 & 0.64649100 & 2.25181400\end{array}$

$\begin{array}{lll}-4.13760900 & 4.36368300 & 0.13911000\end{array}$

$\begin{array}{lll}-3.03634500 & 4.89587400 & -1.63471300\end{array}$

$\begin{array}{lll}-2.09489500 & 1.71924700 & 1.45465500\end{array}$

$\begin{array}{lll}-3.55711900 \quad 2.70188100 & 1.65773100\end{array}$

$\begin{array}{lll}-1.98786100 & 3.27878300 & 2.26689900\end{array}$

$\begin{array}{lll}-1.68689300 & 5.22506200 & -3.29726400\end{array}$ 
$3.27500100 \quad 2.74974900 \quad-3.97519600$

$\begin{array}{lll}3.95067800 & 3.92748800 & -2.30027300\end{array}$

$0.79316700 \quad 0.65210600 \quad-2.24826000$

$\begin{array}{lll}1.84799500 & 0.79146600 & -3.67601400\end{array}$

$\begin{array}{lll}0.40405700 & 1.81059600 & -3.53109900\end{array}$

$4.44610700 \quad 4.87789100 \quad-0.41774500$

$0.74349600 \quad 5.26873800 \quad 3.97181800$

$1.10843000 \quad 3.20893500 \quad 4.48116700$

$\begin{array}{lll}0.61574600 & 5.12062200 & 0.34730600\end{array}$

$\begin{array}{lll}0.65205500 & 6.12691900 & 1.81424900\end{array}$

$\begin{array}{lll}-0.85646100 & 5.35488500 & 1.28336700\end{array}$

$\begin{array}{lll}1.45348000 \quad 1.07592600 & 4.67474700\end{array}$

$\begin{array}{lll}-3.82760500 & 5.53993600 & -2.02634900\end{array}$

$4.76692500 \quad 4.39415400 \quad-2.85748600$

$\begin{array}{lll}1.28702400 & 3.42945800 & 5.53669600\end{array}$

$\begin{array}{lll}-2.14870600 & -0.84950900 & -0.02369000\end{array}$

$\begin{array}{lll}-2.54057500 & -2.38082600 & -1.02630600\end{array}$

$\begin{array}{lll}-3.45290100 & 0.30412200 & -0.69273600\end{array}$

$\begin{array}{lll}-2.81571700 & -1.18990700 & 1.67962000\end{array}$

$-1.90640800 \quad-3.61077900 \quad-0.72712200$

$\begin{array}{lll}-3.40232500 & -2.32672300 & -2.13537100\end{array}$

$\begin{array}{lll}-3.27319700 & 0.90822400 & -1.96100700\end{array}$

$\begin{array}{lll}-4.61457100 & 0.59929600 & 0.03717400\end{array}$

$\begin{array}{lll}-3.89649000 & -2.03784500 & 2.03350000\end{array}$

$\begin{array}{lll}-2.16775800 & -0.46242200 & 2.69205500\end{array}$

$\begin{array}{lll}-2.12917000 & -4.71773600 & -1.55977500\end{array}$

$\begin{array}{lll}-1.02850900 & -3.74958700 & 0.47531100\end{array}$

$\begin{array}{lll}-3.61922300 & -3.43821000 & -2.95049000\end{array}$

$\begin{array}{lll}-3.93925900 & -1.40661900 & -2.35893700\end{array}$

$\begin{array}{lll}-4.27330300 & 1.75307200 & -2.46126000\end{array}$

$\begin{array}{lll}-2.04561300 & 0.64452100 & -2.77936200\end{array}$

$\begin{array}{lll}-5.59853300 & 1.44758400 & -0.47366500\end{array}$

$\begin{array}{lll}-4.75564400 & 0.16776600 & 1.02743400\end{array}$

$\begin{array}{lll}-4.24351900 & -2.13359300 & 3.39326800\end{array}$

$\begin{array}{lll}-4.71646400 & -2.82149200 & 1.04132400\end{array}$

$\begin{array}{lll}-2.54434300 & -0.54817600 & 4.03024400\end{array}$

$\begin{array}{lll}-1.33322400 & 0.17824700 & 2.41193300\end{array}$

$\begin{array}{lll}-1.62344600 & -5.65786000 & -1.32310000\end{array}$

$\begin{array}{lll}-2.97275400 & -4.64302900 & -2.66731400\end{array}$

$\begin{array}{lll}-0.43367600 & -2.83762300 & 0.59372100\end{array}$

$\begin{array}{lll}-0.34962300 & -4.60856900 & 0.39342800\end{array}$

$\begin{array}{lll}-1.62080400 & -3.86402400 & 1.39810600\end{array}$

$\begin{array}{lll}-4.29915800 & -3.35931500 & -3.80258200\end{array}$

$\begin{array}{lll}-4.12356300 & 2.22125200 & -3.43726300\end{array}$ 


\begin{tabular}{|c|c|c|c|}
\hline $\mathrm{C}$ & -5.43287900 & 2.02057200 & -1.73534600 \\
\hline $\mathrm{H}$ & -1.17872200 & 0.53712200 & -2.11468000 \\
\hline $\mathrm{H}$ & -1.85354400 & 1.46377100 & -3.48414400 \\
\hline $\mathrm{H}$ & -2.12572800 & -0.29094000 & -3.35669100 \\
\hline $\mathrm{H}$ & -6.49318300 & 1.65750200 & 0.11808300 \\
\hline $\mathrm{H}$ & -5.07084000 & -2.79163900 & 3.67272500 \\
\hline $\mathrm{C}$ & -3.58609200 & -1.40505600 & 4.38549800 \\
\hline $\mathrm{H}$ & -4.98528500 & -2.22081400 & 0.16341300 \\
\hline $\mathrm{H}$ & -5.64502000 & -3.17560800 & 1.51261900 \\
\hline $\mathrm{H}$ & -4.17572800 & -3.70238600 & 0.66427100 \\
\hline $\mathrm{H}$ & -2.01568600 & 0.04187100 & 4.78260000 \\
\hline $\mathrm{H}$ & -3.12780200 & -5.51996800 & -3.30088500 \\
\hline $\mathrm{H}$ & -6.19465700 & 2.68824000 & -2.14549800 \\
\hline $\mathrm{H}$ & -3.89571200 & -1.50254900 & 5.42920100 \\
\hline \multicolumn{4}{|l|}{ S26 } \\
\hline $\mathrm{Ni}$ & 0.02909600 & -0.00070600 & -0.02896100 \\
\hline $\mathrm{P}$ & -0.08638000 & -1.69803400 & 1.51885700 \\
\hline $\mathrm{C}$ & -1.08536500 & -1.48695500 & 3.16405400 \\
\hline $\mathrm{C}$ & 1.52190500 & -2.25531800 & 2.33529100 \\
\hline $\mathrm{C}$ & -0.83215700 & -3.39649700 & 0.98343900 \\
\hline $\mathrm{C}$ & -0.63105600 & -1.13568000 & 4.46888200 \\
\hline $\mathrm{C}$ & -2.46367600 & -1.72227500 & 3.03608600 \\
\hline $\mathrm{C}$ & 1.88381000 & -3.50663800 & 2.91004100 \\
\hline $\mathrm{C}$ & 2.41434000 & -1.18934100 & 2.48375300 \\
\hline $\mathrm{C}$ & -0.57418200 & -3.98322400 & -0.28022900 \\
\hline $\mathrm{C}$ & -1.66933200 & -4.13220600 & 1.85068200 \\
\hline $\mathrm{C}$ & -1.57500000 & -1.06909500 & 5.51740100 \\
\hline $\mathrm{C}$ & 0.77617400 & -0.81424200 & 4.88981700 \\
\hline $\mathrm{C}$ & -3.37870400 & -1.67805400 & 4.08105900 \\
\hline $\mathrm{H}$ & -2.85683300 & -1.94297600 & 2.05771500 \\
\hline $\mathrm{C}$ & 3.09621900 & -3.58107500 & 3.61910900 \\
\hline $\mathrm{C}$ & 1.09853500 & -4.79184200 & 2.84726700 \\
\hline $\mathrm{C}$ & 3.59334900 & -1.27198500 & 3.21710100 \\
\hline $\mathrm{H}$ & 2.13785400 & -0.24787500 & 2.02098200 \\
\hline $\mathrm{C}$ & -1.20747200 & -5.19174500 & -0.63465500 \\
\hline $\mathrm{C}$ & 0.42016200 & -3.47037200 & -1.27207400 \\
\hline $\mathrm{C}$ & -2.28881300 & -5.32399100 & 1.49159200 \\
\hline $\mathrm{H}$ & -1.83051400 & -3.78722200 & 2.86494600 \\
\hline $\mathrm{H}$ & -1.20734200 & -0.79106900 & 6.50821000 \\
\hline $\mathrm{C}$ & -2.92824400 & -1.34511300 & 5.35441600 \\
\hline $\mathrm{H}$ & 1.41761000 & -1.70514900 & 4.89696500 \\
\hline $\mathrm{H}$ & 0.77039600 & -0.40058900 & 5.90695400 \\
\hline $\mathrm{H}$ & 1.24195000 & -0.07595700 & 4.23836100 \\
\hline $\mathrm{H}$ & -4.43412100 & -1.87861800 & 3.88147100 \\
\hline
\end{tabular}


$3.37836300 \quad-4.54278800 \quad 4.05509000$

$\begin{array}{lll}3.94084200 & -2.48606800 & 3.79482700\end{array}$

$\begin{array}{lll}0.15176400 & -4.72099400 & 3.39762300\end{array}$

$\begin{array}{lll}1.68338900 & -5.60341100 & 3.30353400\end{array}$

$\begin{array}{lll}0.85250800 & -5.09052400 & 1.82068500\end{array}$

$\begin{array}{lll}4.23060700 & -0.39522700 & 3.32794900\end{array}$

$\begin{array}{lll}-1.00332600 & -5.59978300 & -1.62733000\end{array}$

$\begin{array}{lll}-2.07098800 & -5.86034000 & 0.22138700\end{array}$

$0.59376800 \quad-2.39671100 \quad-1.15906500$

$\begin{array}{lll}0.10234300 & -3.67006600 & -2.29864400\end{array}$

$1.36508400 \quad-4.01530400 \quad-1.13560700$

$\begin{array}{lll}-2.92694000 & -5.83685300 & 2.21556300\end{array}$

$\begin{array}{lll}-3.61215700 & -1.28960300 & 6.20512700\end{array}$

$\begin{array}{lll}4.86630100 & -2.59210100 \quad 4.36608300\end{array}$

$\begin{array}{lll}-2.55502000 & -6.78933700 & -0.08993800\end{array}$

$\begin{array}{lll}2.15532200 & -0.04468300 & -0.90487700\end{array}$

$2.33012000 \quad-0.70583100 \quad-2.69784700$

$3.56645900 \quad-1.09177100 \quad-0.10399200$

$3.35669700 \quad 1.41844700 \quad-1.22044100$

$2.07000800 \quad 0.15283200 \quad-3.79964500$

$2.80608500 \quad-1.99136400 \quad-2.99119600$

$\begin{array}{lll}4.80211500 & -0.64493200 & 0.44974300\end{array}$

$3.34848700 \quad-2.47590800 \quad-0.08837000$

$\begin{array}{lll}4.50469200 & 1.41587400 & -2.07150200\end{array}$

$3.16724300 \quad 2.54432600 \quad-0.42066400$

$2.39793800 \quad-0.27100100 \quad-5.09729800$

$\begin{array}{lll}1.43834100 & 1.50665400 & -3.65825900\end{array}$

$3.11859500 \quad-2.40495900 \quad-4.28626200$

$\begin{array}{lll}3.00863600 & -2.69222200 & -2.19074800\end{array}$

$5.79557700 \quad-1.60514100 \quad 0.71948100$

$5.13140900 \quad 0.73954600 \quad 0.96312200$

$\begin{array}{lll}4.31999900 & -3.41776400 & 0.23788800\end{array}$

$2.35865900 \quad-2.83024600 \quad-0.32451900$

$5.26002000 \quad 2.59911200 \quad-2.16520600$

$\begin{array}{lll}5.08823600 & 0.23428400 & -2.81150100\end{array}$

$3.95930800 \quad 3.68992700 \quad-0.47744500$

$\begin{array}{lll}2.36880400 & 2.52804800 & 0.30053000\end{array}$

$2.20885900 \quad 0.41589400 \quad-5.92659900$

$2.94777600 \quad-1.52625300 \quad-5.35273500$

$\begin{array}{lll}0.61178000 & 1.46826700 & -2.94306900\end{array}$

$1.02124200 \quad 1.84470400 \quad-4.61696700$

$\begin{array}{lll}2.15430500 & 2.26241700 \quad-3.30765700\end{array}$

$3.51051400 \quad-3.41207500 \quad-4.44759200$

$6.75229800 \quad-1.24827100 \quad 1.11096100$ 
$\begin{array}{lll}5.86972500 & 0.64677500 & 1.77345200\end{array}$

$\begin{array}{lll}4.25104300 \quad 1.23915400 & 1.37788100\end{array}$

$\begin{array}{lll}4.07299000 & -4.48192800 & 0.22182600\end{array}$

$\begin{array}{lll}6.12396500 & 2.60085300 & -2.83560500\end{array}$

$\begin{array}{lll}4.99727700 & 3.73608800 & -1.39936100\end{array}$

$\begin{array}{lll}4.94042000 & -0.70636700 & -2.27657300\end{array}$

$6.17229200 \quad 0.38449900 \quad-2.92515500$

$\begin{array}{lll}4.66846400 & 0.11312400 & -3.81904000\end{array}$

$3.73921800 \quad 4.53176800 \quad 0.18128900$

$3.20633800-1.82053800 \quad-6.37285000$

$\begin{array}{lll}6.39425900 & -3.67949200 & 0.82043900\end{array}$

$5.62530700 \quad 4.62494700 \quad-1.50053300$

$\begin{array}{lll}-1.74347200 & -0.31711900 & -1.46000200\end{array}$

$\begin{array}{lll}-2.13111700 & 0.98088100 & -2.78507500\end{array}$

$\begin{array}{lll}-3.46863800 & -0.53122100 & -0.72670100\end{array}$

$\begin{array}{lll}-1.67995000 & -1.76782600 & -2.70850500\end{array}$

$\begin{array}{lll}-2.86506100 & 0.80807000 & -3.99198500\end{array}$

$\begin{array}{lll}-1.65248200 & 2.25840500 & -2.49835000\end{array}$

$\begin{array}{lll}-4.62974200 & 0.28481500 & -0.84957600\end{array}$

$\begin{array}{lll}-3.57378200 & -1.69959000 & 0.03596100\end{array}$

$\begin{array}{lll}-2.44452700 & -2.94800900 & -2.93194100\end{array}$

$\begin{array}{lll}-0.65596000 & -1.48516400 & -3.62712100\end{array}$

$-2.96220500 \quad 1.90045700 \quad-4.87102900$

$-3.65689600 \quad-0.41761300 \quad-4.37175100$

$\begin{array}{lll}-1.79417000 & 3.34784400 & -3.35460800\end{array}$

$\begin{array}{lll}-1.16471200 & 2.39869600 & -1.54698900\end{array}$

$\begin{array}{lll}-5.81338300 & -0.15759400 & -0.22680700\end{array}$

$\begin{array}{lll}-4.74176800 & 1.60564600 & -1.56892200\end{array}$

$\begin{array}{lll}-4.75491500 & -2.12542500 & 0.63751900\end{array}$

$\begin{array}{lll}-2.69018500 & -2.32534300 & 0.12696700\end{array}$

$-2.05657500 \quad-3.79670000 \quad-3.99014000$

$\begin{array}{lll}-3.71360300 & -3.38694100 & -2.24265400\end{array}$

$\begin{array}{lll}-0.33184100 & -2.30234700 & -4.70291100\end{array}$

$\begin{array}{lll}-0.10380000 & -0.56129800 & -3.50586400\end{array}$

$\begin{array}{lll}-3.51521100 & 1.76146500 & -5.80427400\end{array}$

$\begin{array}{lll}-2.43161300 & 3.15710000 & -4.57715800\end{array}$

$\begin{array}{lll}-3.04829000 & -1.16739500 & -4.89645800\end{array}$

$\begin{array}{lll}-4.47804000 & -0.12808900 & -5.04474300\end{array}$

$\begin{array}{lll}-4.09892900 & -0.90481200 & -3.49631900\end{array}$

$\begin{array}{lll}-1.39952900 & 4.32305200 & -3.06413800\end{array}$

$\begin{array}{lll}-6.70215500 & 0.47194000 & -0.31811000\end{array}$

$\begin{array}{lll}-5.89754800 & -1.34367900 & 0.50031000\end{array}$ 
$\begin{array}{lll}-3.89925600 & 2.27150000 \quad-1.36911000\end{array}$

$\begin{array}{lll}-5.65721500 & 2.11834800 & -1.24683900\end{array}$

$\begin{array}{lll}-4.80277100 & 1.47975900 & -2.65774500\end{array}$

$\begin{array}{lll}-4.77098600 & -3.06065800 & 1.20156700\end{array}$

$\begin{array}{lll}-2.63487800 & -4.71130100 & -4.14423200\end{array}$

$-1.02088400 \quad-3.50123900 \quad-4.86937900$

$-3.53513000 \quad-3.79131000 \quad-1.24052300$

$\begin{array}{lll}-4.18259400 & -4.18808000 & -2.83176400\end{array}$

$\begin{array}{lll}-4.44477400 & -2.57657400 & -2.14776500\end{array}$

$0.45732800 \quad-2.00364000 \quad-5.38884200$

$\begin{array}{lll}-2.54424500 & 3.98057600 & -5.28699400\end{array}$

$\begin{array}{lll}-6.84344100 & -1.64290600 & 0.95864100\end{array}$

$\begin{array}{lll}-0.77751100 & -4.18369000 & -5.68751400\end{array}$

$\begin{array}{lll}-0.45445600 & 2.02583300 & 0.94536800\end{array}$

$\begin{array}{lll}0.19611800 & 2.17509700 \quad 2.73096600\end{array}$

$\begin{array}{lll}-2.19957400 & 2.80002000 & 0.90594200\end{array}$

$0.35047700 \quad 3.62321700 \quad 0.28208300$

$\begin{array}{lll}-0.38789500 & 2.53167600 & 3.97237000\end{array}$

$\begin{array}{lll}1.59309300 & 1.98450400 \quad 2.71152900\end{array}$

$\begin{array}{lll}-3.30785200 & 2.14726000 & 1.48394500\end{array}$

$\begin{array}{lll}-2.44415700 & 4.03224800 & 0.26439500\end{array}$

$\begin{array}{lll}0.47200300 & 4.87703300 & 0.94313900\end{array}$

$\begin{array}{lll}0.71404200 & 3.56555900 & -1.06677500\end{array}$

$\begin{array}{lll}0.45192500 & 2.64982100 & 5.10012600\end{array}$

$\begin{array}{lll}-1.82645700 \quad 2.90696500 & 4.22506700\end{array}$

$2.41483000 \quad 2.16954600 \quad 3.81699600$

$\begin{array}{lll}2.05890700 & 1.69042300 & 1.77612500\end{array}$

$\begin{array}{lll}-4.56688000 & 2.76571100 \quad 1.49737800\end{array}$

$\begin{array}{lll}-3.14816500 & 0.81463200 & 2.11454100\end{array}$

$\begin{array}{lll}-3.70345200 & 4.63149700 & 0.26568700\end{array}$

$\begin{array}{lll}-1.64608800 & 4.55521500 \quad-0.25091700\end{array}$

$\begin{array}{lll}0.94117400 & 5.97818800 & 0.20241800\end{array}$

$0.07048700 \quad 5.15378200 \quad 2.36700000$

$\begin{array}{lll}1.13069500 & 4.67246500 & -1.80098400\end{array}$

$\begin{array}{lll}0.70274300 & 2.59535000 & -1.54076800\end{array}$

$\begin{array}{lll}-0.01283100 & 2.91852000 & 6.05281400\end{array}$

$\begin{array}{lll}1.83173800 & 2.49673200 & 5.04166300\end{array}$

$-2.36720400 \quad 3.19351100 \quad 3.32241900$

$-1.86640800 \quad 3.75795100 \quad 4.92163700$

$-2.37266200 \quad 2.07916900 \quad 4.70475400$

$3.49477600 \quad 2.03894600 \quad 3.72144200$

$\begin{array}{lll}-5.39802100 & 2.23619600 & 1.96976000\end{array}$

$\begin{array}{lll}-4.77300200 & 4.01032400 & 0.91106300\end{array}$

C $\quad-4.77300200 \quad 4.01032400 \quad 0.91106300$

$\begin{array}{llll}\mathrm{H} & -2.37487000 & 0.24806600 & 1.59077700\end{array}$ 
H

$\mathrm{H}$

$\mathrm{H}$

$\mathrm{H}$

C

$\mathrm{H}$

S27

$\mathrm{Ni}$

\begin{tabular}{|c|c|c|}
\hline-4.08244700 & 0.24594100 & 2.08594000 \\
\hline-2.83825900 & 0.89170100 & 3.16177000 \\
\hline-3.83933700 & 5.58961300 & -0.24177100 \\
\hline 1.03176700 & 6.94024100 & 0.71357900 \\
\hline 1.24954100 & 5.89924800 & -1.15574800 \\
\hline-0.92211100 & 4.74788500 & 2.59234300 \\
\hline 0.03439000 & 6.23864700 & 2.54251300 \\
\hline 0.77796500 & 4.72203200 & 3.08815900 \\
\hline 1.39231100 & 4.55844800 & -2.85463500 \\
\hline 2.44189200 & 2.62871500 & 5.93827400 \\
\hline-5.76115400 & 4.47670900 & 0.93083600 \\
\hline 1.58739600 & 6.78769900 & -1.69497200 \\
\hline 1.56188000 & 0.02185600 & 0.09001200 \\
\hline 1.85841400 & -0.54531800 & -2.00221800 \\
\hline 3.45453000 & 0.22566900 & -2.55232000 \\
\hline 0.50372700 & -0.10413100 & -3.19461100 \\
\hline 2.05265900 & -2.33638400 & -2.51571300 \\
\hline 3.60788300 & 1.43063200 & -3.28110400 \\
\hline 4.60244300 & -0.37487500 & -1.99700100 \\
\hline 0.52690400 & -0.31503300 & -4.59403200 \\
\hline-0.67264000 & 0.36695800 & -2.59196700 \\
\hline 0.99519300 & -3.22736900 & -2.19937800 \\
\hline 3.19452000 & -2.85217000 & -3.14917100 \\
\hline 4.90696600 & 1.94677300 & -3.44969100 \\
\hline 2.46909900 & 2.20552100 & -3.88658900 \\
\hline 5.87916900 & 0.14333800 & -2.19086300 \\
\hline 4.49266500 & -1.25780200 & -1.36993300 \\
\hline-0.62572800 & 0.00253900 & -5.33103200 \\
\hline 1.71320100 & -0.87777900 & -5.33300500 \\
\hline-1.81356100 & 0.66039400 & -3.33677700 \\
\hline-0.67373600 & 0.47648900 & -1.50454900 \\
\hline 1.15461400 & -4.59630000 & -2.46530400 \\
\hline-0.29968400 & -2.74351700 & -1.61982200 \\
\hline 3.33112900 & -4.21445600 & -3.41961300 \\
\hline 4.00116100 & -2.18258300 & -3.44222900 \\
\hline 5.02135400 & 2.87972800 & -4.00842000 \\
\hline 6.03638200 & 1.31793000 & -2.92902600 \\
\hline 2.15405400 & 1.77754400 & -4.85075700 \\
\hline 2.77325800 & 3.24391100 & -4.07198500 \\
\hline 1.58673200 & 2.22377300 & -3.24135500 \\
\hline 6.73979900 & -0.35822700 & -1.74200600 \\
\hline-0.60948100 & -0.14079200 & -6.41526100 \\
\hline 1.78414100 & 0.48444600 & -4.71998600 \\
\hline
\end{tabular}


$2.67077200 \quad-0.57817800 \quad-4.89087000$

$\begin{array}{lll}1.70261800 & -0.55404000 & -6.38478600\end{array}$

$\begin{array}{lll}1.69145600 & -1.97933200 & -5.32546600\end{array}$

$\begin{array}{lll}-2.72178800 & 1.00744600 & -2.84411900\end{array}$

$\begin{array}{lll}0.34389200 & -5.27730500 & -2.19249500\end{array}$

$2.31161300 \quad-5.09868200 \quad-3.06161100$

$\begin{array}{lll}-0.13044600 & -1.94702900 & -0.88432100\end{array}$

$\begin{array}{lll}-0.84778800 & -3.55905400 & -1.13248500\end{array}$

$\begin{array}{lll}-0.93602900 & -2.31733300 & -2.40708300\end{array}$

$\begin{array}{lll}4.23723500 & -4.58069500 & -3.90915300\end{array}$

$\begin{array}{lll}7.02724300 & 1.75229300 & -3.08383600\end{array}$

$\begin{array}{lll}-2.66795700 & 0.70896400 & -5.32142200\end{array}$

$\begin{array}{lll}2.41131100 & -6.17005800 & -3.25325100\end{array}$

$\begin{array}{lll}-4.54474400 & -0.42129100 & 0.22613000\end{array}$

$\begin{array}{lll}-6.07649600 & -1.20774500 & 0.89316700\end{array}$

$\begin{array}{lll}-4.55823700 & -0.94341800 & -1.54927500\end{array}$

$\begin{array}{lll}-4.76191000 & 1.41920200 & 0.16087800\end{array}$

$\begin{array}{lll}-6.24203600 & -1.26712400 & 2.29987900\end{array}$

$\begin{array}{lll}-7.00358600 & -1.85930000 & 0.06497500\end{array}$

$\begin{array}{lll}-5.31284300 & -0.38703100 & -2.61327900\end{array}$

$\begin{array}{lll}-3.70712000 & -2.03262900 & -1.80908700\end{array}$

$\begin{array}{lll}-5.84743300 & 2.22843100 & 0.57437200\end{array}$

$\begin{array}{lll}-3.60406000 & 2.04178600 \quad-0.34879000\end{array}$

$\begin{array}{lll}-7.35831000 & -1.93711800 & 2.81851300\end{array}$

$\begin{array}{lll}-5.24944400 & -0.62621700 \quad 3.23555600\end{array}$

$\begin{array}{lll}-8.10558800 & -2.52874200 & 0.60090900\end{array}$

$\begin{array}{lll}-6.86396600 & -1.84233300 & -1.01655300\end{array}$

$\begin{array}{lll}-5.16180300 & -0.94329500 & -3.89406900\end{array}$

$\begin{array}{lll}-6.27295000 & 0.75929800 & -2.43645100\end{array}$

$\begin{array}{lll}-3.59122100 & -2.58482200 & -3.08466200\end{array}$

$\begin{array}{lll}-3.11922900 & -2.44781100 & -0.98786800\end{array}$

$\begin{array}{lll}-5.71990200 & 3.62385600 & 0.43996500\end{array}$

$\begin{array}{lll}-7.14702000 \quad 1.69427400 & 1.12058000\end{array}$

$\begin{array}{lll}-3.51204700 & 3.42171500 & -0.50112200\end{array}$

$\begin{array}{lll}-2.74912000 & 1.41824200 & -0.61846400\end{array}$

$-7.49487600 \quad-1.97387400 \quad 3.90280400$

$\begin{array}{lll}-8.28891900 & -2.56113200 & 1.98405400\end{array}$

$-4.25882600 \quad-1.10219800 \quad 3.14598900$

$\begin{array}{lll}-5.57482200 & -0.71685000 & 4.28193000\end{array}$

$\begin{array}{lll}-5.10653900 & 0.44390800 \quad 3.01800500\end{array}$

$\begin{array}{lll}-8.81893500 & -3.02175800 & -0.06427300\end{array}$

$\begin{array}{lll}-5.73581900 & -0.51530400 & -4.72066000\end{array}$

$\begin{array}{lll}-4.31734200 & -2.02790900 & -4.13629300\end{array}$

$\begin{array}{lll}-6.89650300 & 0.63301500 & -1.54190700\end{array}$ 


\begin{tabular}{|c|c|c|c|}
\hline $\mathrm{H}$ & -6.93895900 & 0.84131500 & -3.30791800 \\
\hline $\mathrm{H}$ & -5.74582800 & 1.71910000 & -2.32384000 \\
\hline $\mathrm{H}$ & -2.92361600 & -3.43160500 & -3.25621700 \\
\hline $\mathrm{H}$ & -6.55690300 & 4.25267700 & 0.75579700 \\
\hline $\mathrm{C}$ & -4.58209200 & 4.22238400 & -0.09720900 \\
\hline $\mathrm{H}$ & -7.57745900 & 0.91006900 & 0.48356700 \\
\hline $\mathrm{H}$ & -7.88432600 & 2.50540500 & 1.20745400 \\
\hline $\mathrm{H}$ & -7.02732200 & 1.24898100 & 2.11893500 \\
\hline $\mathrm{H}$ & -2.60242500 & 3.86836400 & -0.90325900 \\
\hline $\mathrm{H}$ & -9.14887500 & -3.07881100 & 2.41632000 \\
\hline $\mathrm{H}$ & -4.22675700 & -2.43328500 & -5.14701400 \\
\hline $\mathrm{H}$ & -4.52689600 & 5.30970800 & -0.19077100 \\
\hline $\mathrm{P}$ & 1.80267100 & -1.53062000 & 1.62799100 \\
\hline $\mathrm{C}$ & 1.60235400 & -1.17029800 & 3.45289200 \\
\hline $\mathrm{C}$ & 3.52585700 & -2.17662000 & 1.41491700 \\
\hline $\mathrm{C}$ & 0.54500600 & -2.93576600 & 1.54123800 \\
\hline $\mathrm{C}$ & 1.72781200 & -2.10693100 & 4.50929000 \\
\hline $\mathrm{C}$ & 1.26904600 & 0.15283300 & 3.76133600 \\
\hline $\mathrm{C}$ & 4.65669900 & -1.76435000 & 2.16642100 \\
\hline $\mathrm{C}$ & 3.73095300 & -2.97241100 & 0.27449300 \\
\hline $\mathrm{C}$ & 0.65421600 & -4.33783200 & 1.36592500 \\
\hline $\mathrm{C}$ & -0.75211100 & -2.38901400 & 1.66682000 \\
\hline C & 1.49523900 & -1.66579300 & 5.82155700 \\
\hline $\mathrm{C}$ & 2.13243600 & -3.53890500 & 4.29563900 \\
\hline $\mathrm{C}$ & 1.04244400 & 0.57863700 & 5.06977300 \\
\hline $\mathrm{H}$ & 1.20739500 & 0.86348800 & 2.94538700 \\
\hline $\mathrm{C}$ & 5.92364800 & -2.22777500 & 1.76668400 \\
\hline $\mathrm{C}$ & 4.59977700 & -0.84173100 & 3.35665100 \\
\hline C & 4.99193200 & -3.43137500 & -0.09589600 \\
\hline $\mathrm{H}$ & 2.87684400 & -3.22633300 & -0.34687000 \\
\hline $\mathrm{C}$ & -0.53567200 & -5.09315000 & 1.27854200 \\
\hline $\mathrm{C}$ & 1.93179600 & -5.13723700 & 1.27917300 \\
\hline C & -1.91059700 & -3.15257900 & 1.59606000 \\
\hline $\mathrm{H}$ & -0.85873700 & -1.31550800 & 1.81526600 \\
\hline $\mathrm{H}$ & 1.59863600 & -2.38397200 & 6.63995000 \\
\hline $\mathrm{C}$ & 1.15023600 & -0.34299100 & 6.10971600 \\
\hline $\mathrm{H}$ & 1.30013900 & -4.13433300 & 3.89086600 \\
\hline $\mathrm{H}$ & 2.44506500 & -4.00118300 & 5.24331700 \\
\hline $\mathrm{H}$ & 2.96619700 & -3.62172200 & 3.58540100 \\
\hline $\mathrm{H}$ & 0.78842700 & 1.62275900 & 5.26485600 \\
\hline $\mathrm{H}$ & 6.79497700 & -1.91148400 & 2.34676800 \\
\hline $\mathrm{C}$ & 6.10306800 & -3.06153200 & 0.66406700 \\
\hline $\mathrm{H}$ & 3.94381900 & 0.01896300 & 3.18938000 \\
\hline $\mathrm{H}$ & 5.60494100 & -0.45945000 & 3.58542700 \\
\hline
\end{tabular}


$4.22462900 \quad-1.35046200 \quad 4.25694200$

$\begin{array}{lll}5.09845700 & -4.05547300 & -0.98492000\end{array}$

$\begin{array}{lll}-0.44415600 & -6.17364700 & 1.13555600\end{array}$

$\begin{array}{lll}-1.80234000 & -4.52902500 & 1.38304100\end{array}$

$\begin{array}{lll}2.24255500 & -5.27750400 & 0.23196600\end{array}$

$1.76927900 \quad-6.13964900 \quad 1.70363700$

$\begin{array}{lll}2.76925600 & -4.67932200 & 1.80872500\end{array}$

$\begin{array}{lll}-2.88388600 & -2.66514000 & 1.68159300\end{array}$

$0.97744300 \quad-0.03752200 \quad 7.14501600$

$\begin{array}{lll}7.10474400 & -3.40272300 & 0.39095100\end{array}$

$\begin{array}{lll}-2.69437000 & -5.15574700 & 1.30831800\end{array}$

$\begin{array}{lll}1.30005400 & 2.16560300 & 0.55983900\end{array}$

$\begin{array}{lll}1.24052200 & 3.46319200 & -0.78775100\end{array}$

$\begin{array}{lll}2.55751000 & 2.72295400 & 1.81489200\end{array}$

$\begin{array}{lll}-0.31097800 & 2.56263600 & 1.40844100\end{array}$

$2.17420700 \quad 4.47190700 \quad-1.12697300$

$0.05873500 \quad 3.36433400 \quad-1.54602000$

$\begin{array}{lll}3.89343000 & 2.26686200 & 1.69505400\end{array}$

$2.20019000 \quad 3.47141500 \quad 2.94829300$

$\begin{array}{lll}-0.83611400 \quad 3.84284200 & 1.72464900\end{array}$

$\begin{array}{lll}-1.02783700 & 1.42274300 & 1.79714800\end{array}$

$\begin{array}{lll}1.86901300 & 5.32376500 & -2.20575000\end{array}$

$\begin{array}{lll}3.45890600 & 4.73383200 & -0.38386700\end{array}$

$\begin{array}{lll}-0.22756600 & 4.21860600 & -2.60662500\end{array}$

$\begin{array}{lll}-0.66691900 & 2.59713200 & -1.28595300\end{array}$

$\begin{array}{lll}4.82714400 & 2.64039400 & 2.67155900\end{array}$

$\begin{array}{lll}4.31722800 & 1.37416000 \quad 0.56991600\end{array}$

$3.13983700 \quad 3.81995500 \quad 3.92009200$

$\begin{array}{lll}1.16522400 & 3.77308700 \quad 3.09535600\end{array}$

$\begin{array}{lll}-2.02451000 \quad 3.89918200 & 2.47221100\end{array}$

$\begin{array}{lll}-0.22002900 & 5.14733000 & 1.28465500\end{array}$

$\begin{array}{lll}-2.20312100 & 1.49770500 \quad 2.53965600\end{array}$

$\begin{array}{lll}-0.61350300 & 0.45970300 & 1.50028400\end{array}$

$\begin{array}{lll}2.58852300 & 6.10563900 & -2.46348900\end{array}$

$\begin{array}{lll}0.69135100 & 5.21409600 & -2.94260100\end{array}$

$3.28653600 \quad 4.93753600 \quad 0.68228000$

$\begin{array}{lll}3.97529500 & 5.60339800 & -0.81517400\end{array}$

$\begin{array}{lll}4.14900900 & 3.88180200 & -0.43792300\end{array}$

$-1.15669600 \quad 4.09620200 \quad-3.16787200$

$\begin{array}{lll}5.85838700 & 2.29306800 & 2.56769800\end{array}$

$4.46724000 \quad 3.41668800 \quad 3.77383900$

$\begin{array}{lll}3.61518700 & 0.52238200 & 0.47926300\end{array}$

$\begin{array}{lll}5.32741300 & 0.97442200 & 0.72384100\end{array}$

$4.29750000 \quad 1.88201000 \quad-0.40393100$ 


\begin{tabular}{|c|c|c|c|}
\hline $\mathrm{H}$ & 2.82816900 & 4.40205700 & 4.79088800 \\
\hline $\mathrm{H}$ & -2.43399500 & 4.88068100 & 2.72567200 \\
\hline $\mathrm{C}$ & -2.69798100 & 2.75117200 & 2.89186200 \\
\hline $\mathrm{H}$ & 0.87526200 & 5.13275700 & 1.29974700 \\
\hline $\mathrm{H}$ & -0.56002900 & 5.96941400 & 1.93218800 \\
\hline $\mathrm{H}$ & -0.51644500 & 5.39087200 & 0.25182200 \\
\hline $\mathrm{H}$ & -2.72676100 & 0.58555400 & 2.82292500 \\
\hline $\mathrm{H}$ & 0.49641500 & 5.89822600 & -3.77203700 \\
\hline $\mathrm{H}$ & 5.21562800 & 3.68562900 & 4.52343700 \\
\hline $\mathrm{H}$ & -3.62008800 & 2.84279600 & 3.47064100 \\
\hline \multicolumn{4}{|l|}{ S28 } \\
\hline $\mathrm{C}$ & -1.33615700 & -2.44807100 & -0.59520800 \\
\hline $\mathrm{C}$ & 0.00482600 & -2.33773400 & -1.08718500 \\
\hline $\mathrm{C}$ & 1.17298200 & -3.00746100 & -0.48341500 \\
\hline $\mathrm{C}$ & 1.08801300 & -3.86259400 & 0.63276200 \\
\hline $\mathrm{H}$ & 2.55622300 & -2.09679700 & -1.87246500 \\
\hline $\mathrm{H}$ & 0.13240800 & -2.15233000 & -2.16119600 \\
\hline $\mathrm{C}$ & 2.45700600 & -2.77324400 & -1.01936900 \\
\hline $\mathrm{C}$ & 2.22757300 & -4.45631900 & 1.17944600 \\
\hline $\mathrm{C}$ & 3.48902100 & -4.21637600 & 0.62869700 \\
\hline $\mathrm{C}$ & 3.59429600 & -3.36660000 & -0.47815600 \\
\hline $\mathrm{H}$ & 2.12625700 & -5.11829400 & 2.04422400 \\
\hline $\mathrm{H}$ & 4.38016400 & -4.67933000 & 1.05938800 \\
\hline $\mathrm{H}$ & 4.57361200 & -3.15489900 & -0.91458800 \\
\hline $\mathrm{C}$ & -2.52602700 & -2.33531300 & -1.49378100 \\
\hline $\mathrm{H}$ & -2.28426800 & -1.72836600 & -2.38140300 \\
\hline $\mathrm{Ni}$ & -0.66655100 & -0.66900000 & -0.29324500 \\
\hline $\mathrm{C}$ & -3.53134500 & -0.58734600 & -0.02557200 \\
\hline $\mathrm{O}$ & -2.47979000 & -0.01340200 & 0.25310600 \\
\hline $\mathrm{C}$ & -4.86784500 & -0.13633200 & 0.56540800 \\
\hline $\mathrm{C}$ & -4.64812600 & 1.10820200 & 1.43230200 \\
\hline $\mathrm{C}$ & -5.42102200 & -1.29263300 & 1.42194400 \\
\hline $\mathrm{C}$ & -5.83984500 & 0.18003200 & -0.58665000 \\
\hline $\mathrm{H}$ & -4.24288700 & 1.94193300 & 0.84114400 \\
\hline $\mathrm{H}$ & -3.94089100 & 0.91156100 & 2.25082300 \\
\hline $\mathrm{H}$ & -5.60602300 & 1.42873200 & 1.87036600 \\
\hline $\mathrm{H}$ & -5.59795800 & -2.19092300 & 0.81334200 \\
\hline $\mathrm{H}$ & -6.37492100 & -0.99157100 & 1.88277700 \\
\hline $\mathrm{H}$ & -4.72148600 & -1.55578200 & 2.23151400 \\
\hline $\mathrm{H}$ & -6.80525200 & 0.51669900 & -0.17745300 \\
\hline $\mathrm{H}$ & -6.01892000 & -0.70494400 & -1.21344600 \\
\hline $\mathrm{H}$ & -5.44645600 & 0.98395100 & -1.22965200 \\
\hline $\mathrm{O}$ & -3.65471100 & -1.62648900 & -0.82263100 \\
\hline $\mathrm{H}$ & -1.53016300 & -3.04231200 & 0.30848700 \\
\hline
\end{tabular}


$\begin{array}{lll}0.11328500 & -4.07618100 \quad 1.07532700\end{array}$

$-3.14196900 \quad-3.65499500 \quad-1.92604700$

$\begin{array}{lll}-2.40338700 & -4.21827100 & -2.51588200\end{array}$

$\begin{array}{lll}-4.04265600 & -3.50014600 & -2.54092800\end{array}$

$-3.41410500 \quad-4.25954500 \quad-1.04658300$

0.79402300

$0.92736300 \quad-0.04039200$

$\begin{array}{lll}0.24261900 & 2.24104100 & 1.14207400\end{array}$

$\begin{array}{lll}2.33024300 & 0.24655500 & 0.72386600\end{array}$

$\begin{array}{lll}1.42103900 & 1.99565600 & -1.41890400\end{array}$

$\begin{array}{lll}-0.88745900 & 3.02816800 & 0.80750000\end{array}$

$\begin{array}{lll}0.88374400 & 2.44416000 & 2.37314300\end{array}$

$\begin{array}{lll}2.22271300 & -0.63534500 & 1.83221400\end{array}$

$\begin{array}{lll}3.59459700 & 0.50639000 & 0.17396000\end{array}$

$\begin{array}{lll}1.18969200 & 1.68971500 & -2.77885200\end{array}$

$2.10097200 \quad 3.18137600 \quad-1.08553700$

$\begin{array}{lll}-1.35037700 & 3.96411700 & 1.74361000\end{array}$

$\begin{array}{lll}-1.56136100 & 2.92661900 & -0.53553300\end{array}$

$\begin{array}{lll}0.41008200 & 3.38635000 & 3.28903300\end{array}$

$\begin{array}{lll}1.77345600 & 1.86194600 & 2.61771000\end{array}$

$3.39628700 \quad-1.19259500 \quad 2.35663200$

$\begin{array}{lll}0.89852700 & -0.98603500 & 2.45204700\end{array}$

$\begin{array}{lll}4.74971000 & -0.06635400 & 0.71018200\end{array}$

$3.68385100 \quad 1.16384600 \quad-0.69065800$

$\begin{array}{lll}1.65810000 & 2.58827000 & -3.75424300\end{array}$

$\begin{array}{lll}0.46519800 & 0.44934200 & -3.22463700\end{array}$

$\begin{array}{lll}2.56544600 & 4.05487300 & -2.06635600\end{array}$

$2.26551000 \quad 3.42396700 \quad-0.03409600$

$\begin{array}{lll}-2.22680800 & 4.56825900 & 1.49258000\end{array}$

$\begin{array}{lll}-0.71890900 & 4.14434700 & 2.97592300\end{array}$

$\begin{array}{lll}-1.60893200 & 1.88638100 & -0.88421100\end{array}$

$\begin{array}{lll}-2.58477300 & 3.32773900 & -0.49684000\end{array}$

$\begin{array}{lll}-1.00392500 & 3.50045000 & -1.29454600\end{array}$

$0.92522800 \quad 3.52517500 \quad 4.24281100$

$3.31669300 \quad-1.87702100 \quad 3.20454600$

$\begin{array}{lll}4.65051400 & -0.91489600 & 1.81167400\end{array}$

$\begin{array}{llll}0.15877100 & -1.23145500 & 1.66657300\end{array}$

$\begin{array}{lll}0.99175700 & -1.84972000 & 3.12401000\end{array}$

$\begin{array}{lll}0.47496100 & -0.14589900 & 3.02564200\end{array}$

$5.72214200 \quad 0.15067200 \quad 0.26119800$

$\begin{array}{lll}1.47332200 & 2.36101500 & -4.80775400\end{array}$

$2.34044400 \quad 3.75433000 \quad-3.41258100$

$\begin{array}{lll}-0.34285300 & 0.18377100 & -2.52527500\end{array}$

$\begin{array}{lll}0.05074100 & 0.57547600 & -4.23572000\end{array}$

$1.14571900 \quad-0.41732500 \quad-3.24706900$ 


$\begin{array}{rrr}3.09375800 & 4.96771100 & -1.78081300 \\ -1.10571800 & 4.88020200 & 3.68548700 \\ 5.54465300 & -1.37518900 & 2.23925200 \\ 2.68973700 & 4.43171500 & -4.19603900\end{array}$

TS-S29

C

\begin{tabular}{|c|c|c|}
\hline 0.75005700 & -2.83697300 & -0.54025600 \\
\hline 0.51048700 & -2.29340100 & -1.01699600 \\
\hline 1.82164100 & -2.71945300 & -0.48727700 \\
\hline 1.97551300 & -3.54785400 & 0.64140200 \\
\hline 2.90620700 & -1.60149200 & -1.98501200 \\
\hline 0.55538700 & -2.06839900 & -2.09014300 \\
\hline 2.99626600 & -2.26215100 & -1.11875000 \\
\hline 3.24116500 & -3.91283500 & 1.10382300 \\
\hline 4.39287700 & -3.45709700 & 0.45690600 \\
\hline 4.25980300 & -2.62394700 & -0.65865300 \\
\hline 3.32688800 & -4.56281200 & 1.97907000 \\
\hline 5.38292800 & -3.74109200 & 0.82165500 \\
\hline 5.14906100 & -2.24576400 & -1.16886700 \\
\hline 1.90761900 & -2.94295600 & -1.38200300 \\
\hline 1.77691000 & -2.55011900 & -2.39551000 \\
\hline 0.51993500 & -0.90589300 & -0.14553700 \\
\hline 3.30384500 & -1.05764600 & 0.07420000 \\
\hline 2.30720100 & -0.60241900 & 0.67644900 \\
\hline 4.71279600 & -0.74067200 & 0.60778600 \\
\hline 4.62461900 & 0.15309200 & 1.84911200 \\
\hline 5.39460300 & -2.07502500 & 0.96047100 \\
\hline 5.50193300 & -0.02868600 & -0.50609800 \\
\hline 4.10576100 & 1.09708100 & 1.63116200 \\
\hline 4.07328400 & -0.34248700 & 2.66146000 \\
\hline 5.63778300 & 0.38912900 & 2.21255200 \\
\hline 5.48691700 & -2.71906700 & 0.07433200 \\
\hline 6.40411900 & -1.89054700 & 1.36180100 \\
\hline 4.82243100 & -2.62340600 & 1.72663200 \\
\hline 6.52497200 & 0.19527700 & -0.16346600 \\
\hline 5.56540300 & -0.65547100 & -1.40715900 \\
\hline 5.02238200 & 0.92299100 & -0.78558000 \\
\hline 3.27017100 & -1.78172400 & -0.98169400 \\
\hline 0.76527000 & -3.45732300 & 0.36288400 \\
\hline 1.09337000 & -3.92220700 & 1.16346000 \\
\hline 2.75298900 & -4.18229100 & -1.30423800 \\
\hline 2.16479400 & -5.02103700 & -1.71562800 \\
\hline 3.68199100 & -4.09148400 & -1.88263900 \\
\hline 3.00051600 & -4.42775500 & -0.26021900 \\
\hline 0.58163600 & 0.95944800 & -0.03169900 \\
\hline
\end{tabular}


$\begin{array}{lll}2.29601200 & 0.64639700 & 0.57011000\end{array}$

$\begin{array}{lll}0.60192900 & 2.55957100 \quad 2.32575600\end{array}$

$2.48639900 \quad-0.18096800 \quad 1.70896900$

$\begin{array}{lll}3.41667700 & 1.13291800 & -0.11953500\end{array}$

$\begin{array}{lll}0.37935000 & 1.72330400 & -2.79951800\end{array}$

$\begin{array}{lll}1.34949300 & 3.34692200 & -1.26684100\end{array}$

$\begin{array}{lll}-1.92732400 \quad 3.61205600 & 1.89048400\end{array}$

$\begin{array}{lll}-2.16457800 & 2.45512300 & -0.32416000\end{array}$

$0.05202800 \quad 3.44138700 \quad 3.25865200$

$\begin{array}{lll}1.60463800 & 2.16470000 & 2.49398900\end{array}$

$3.79699400 \quad-0.44454400 \quad 2.12861000$

$\begin{array}{lll}1.33516600 & -0.77925400 & 2.46949400\end{array}$

$\begin{array}{lll}4.71331000 & 0.84977800 & 0.31376300\end{array}$

$3.28191500 \quad 1.74044900 \quad-1.01369100$

$\begin{array}{lll}0.55253600 & 2.66749500 \quad-3.82863900\end{array}$

$\begin{array}{lll}-0.26385700 & 0.40648100 & -3.14177800\end{array}$

$1.51910900 \quad 4.26432500 \quad-2.30027700$

$\begin{array}{lll}1.64419300 & 3.62640600 & -0.25358100\end{array}$

$\begin{array}{lll}-2.92137200 & 4.03279000 & 1.71505900\end{array}$

$\begin{array}{lll}-1.22393000 \quad 3.96340200 & 3.04419500\end{array}$

$\begin{array}{lll}-2.04539000 & 1.41493100 & -0.65242100\end{array}$

$\begin{array}{lll}-3.23654200 & 2.65222700 & -0.17973500\end{array}$

$\begin{array}{lll}-1.81351900 \quad 3.10070700 & -1.14596300\end{array}$

$0.62280900 \quad 3.71692900 \quad 4.14886500$

$3.94718900 \quad-1.08381300 \quad 3.00176300$

$\begin{array}{lll}4.90427600 & 0.06500400 & 1.44967300\end{array}$

$\begin{array}{lll}0.61736000 & -1.25122800 \quad 1.77535000\end{array}$

$1.68314900 \quad-1.54248200 \quad 3.17863800$

$\begin{array}{lll}0.76869000 & -0.02020900 & 3.03180600\end{array}$

$5.56878100 \quad 1.24100800 \quad-0.24230800$

$0.22032700 \quad 2.40750700 \quad-4.83742100$

$\begin{array}{lll}1.11975200 & 3.91778500 & -3.59431900\end{array}$

$\begin{array}{lll}-0.83838700 & 0.00116800 & -2.29505800\end{array}$

$\begin{array}{lll}-0.93071000 & 0.51275600 & -4.01007600\end{array}$

$0.49531500 \quad-0.35006900 \quad-3.39883700$

$1.95167200 \quad 5.24638500 \quad-2.09495300$

$\begin{array}{lll}-1.67067700 & 4.64850400 \quad 3.76910000\end{array}$

$5.91291600 \quad-0.16849700 \quad 1.79914900$

$1.23714200 \quad 4.62696100 \quad-4.41761900$

$\begin{array}{lll}-0.25895500 & -3.12787200 & 0.72858400\end{array}$ 
$-3.02773800$

$-1.00547000$

$-3.40712300$

$-4.38607400$

$-5.23975400$

$-4.73992400$

$-4.75965600$

$-6.28352300$

$-5.39101600$

1.04788600

1.23651200

0.54648800

3.27724900

2.27728700

4.65817500

5.77692900

4.74257700

4.78317000

5.73641100

5.69228800

6.76348700

3.92831500

5.70380300

4.67581900

3.97124800

4.73998900

5.74624600

3.17925100

$-2.40791900$

$-0.50473700$

2.12092600

2.12467500

3.11165100

1.99033200

$-0.34052300$

$-1.19178600$

0.77397200

$-1.57713300$

$-1.15150100$

$-1.83546400$

1.79885100
$-0.67200800$

$-1.89726900$

1.16042700

$-2.39628800$

$-1.60437400$

$-0.98017100$

$-2.87691000$

$-1.46875400$

$-0.35083200$

$-3.18022700$

$-2.84293100$

$-1.28149800$

$-0.89266200$

$-0.92616700$

$-0.78125800$

$-0.92221700$

0.59761500

$-1.87987200$

$-1.90107900$

$-0.15258600$

$-0.82397700$

0.73591800

0.70659800

1.40786600

$-1.80400400$

$-2.88498600$

$-1.79260800$

$-0.92845300$

$-3.18682900$

$-3.65945100$

$-4.02548500$

$-5.03533100$

$-3.59010800$

$-4.13740400$

0.66792800

1.76113800

$1.90606000 \quad-0.90152500$

$0.28245200-1.41266500$

$1.55564800 \quad 2.52073500$

$2.90765900 \quad 0.61429200$

$2.52107000-0.14258100$ 
S31

$$
\begin{array}{lll}
-2.90553700 & 0.72320900 & -1.34495700
\end{array}
$$


$2.62671500 \quad 4.48470200 \quad 1.60004000$

$\begin{array}{lll}3.93104900 & 4.95760900 & 1.78232600\end{array}$

4.91259300

$4.08601200 \quad 2.26203800$

1.84996700

4.17999600

5.15468900

1.22133900

$\mathrm{H}$

5.93422300

1.18639300

1.90487900

1.28551600

0.95134000

$-0.22249200$

$-0.24961300$

$-0.71962300$

$-0.80931200$

$-2.27328600$

0.17122100

$-0.96875700$

$-3.67801200$

$-2.40923300$

$-3.08796800$

$-0.71527100$

$-1.25620100$

$-4.54744500$

$-3.99056600$

$-3.98186900$

$-5.42093900$

$-4.08945800$

$-4.19725700$

$-4.60481500$

$-5.72777000$

$-6.72031900$

$-4.04004100$

$-4.47509600$

$-5.46126700$

$-3.52466600$

$-6.31691100$

$-6.31567000$

$-4.80464700$

$-7.48920900$

$-7.12597600$

$-6.52267700$

$-4.98755500$

$-2.59788500$

$-3.66903600$

5.99673500

1.55242200

$4.44285100 \quad 2.41820400$

$-0.73137500 \quad 3.28734900$

$-1.56234300 \quad 3.28291400$

$2.79879000 \quad 1.70780600$

$1.38298700 \quad 3.36976100$

$-1.07964500 \quad 3.64980700$

$-1.74232600 \quad 4.53169300$

$-1.58957100 \quad 2.81129200$

$-0.17704800 \quad 3.87217300$

$-0.46010200 \quad 1.03970100$

$-0.78457100 \quad 0.56974200$

$0.29503200 \quad 0.40417900$

$\begin{array}{ll}-0.52393400 & 0.52893700\end{array}$

$-1.45623400 \quad-2.08876400$

$1.72708800 \quad 2.36054600$

$1.54561200 \quad 1.27093000$

$0.54704200-1.08941800$

$-0.44223500 \quad 1.74497600$

$0.76837100 \quad-0.58317700$

$-1.86693300-0.03937500$

$-1.55852200 \quad 1.76645300$

$2.09299500 \quad-0.17143900$

$0.67058600-1.94819400$

$-2.64798600 \quad 0.97190400$

$-1.93034500 \quad 3.21484200$

$-1.17880900 \quad 1.03902000$

$2.95918700 \quad-1.33431600$

$1.98027600 \quad-2.50018900$

$-3.72471400 \quad 0.31114400$

$-3.30747500 \quad 1.84355100$

$-2.85962100 \quad 3.27802200$

$-1.12867400 \quad 3.69093000$

$-2.06073700 \quad 3.79595700$

$-1.96453600 \quad 1.10610700$

$-0.26208200 \quad 1.49508100$

$-0.97048700 \quad-0.02288500$

$4.13046600 \quad-1.10491100$

$3.45433100-1.45641200$

$2.13665300 \quad-3.78388300$ 


\begin{tabular}{|c|c|c|}
\hline-5.97300100 & 2.01806500 & -2.80447600 \\
\hline-4.81446400 & -4.46058500 & -0.19437600 \\
\hline-6.06764200 & -4.26921200 & 1.05300400 \\
\hline-6.13332400 & -3.29351700 & -0.44313100 \\
\hline-2.83750800 & -3.86762500 & 1.18951600 \\
\hline-3.95077100 & -4.01195000 & 2.57555700 \\
\hline-2.93329700 & -2.54772800 & 2.37049700 \\
\hline-4.61575100 & 4.74649300 & -0.27127400 \\
\hline-5.04047000 & 4.77455300 & -1.99658200 \\
\hline-6.00308500 & 3.79780500 & -0.85210900 \\
\hline-2.47110200 & 4.12801100 & -2.31755600 \\
\hline-2.33785100 & 4.01766700 & -0.54662400 \\
\hline-1.90398400 & 2.60396300 & -1.52527800 \\
\hline-4.02014600 & 1.40904800 & -4.53283900 \\
\hline-3.79444600 & 3.14332400 & -4.21211100 \\
\hline-2.59990100 & 1.96343900 & -3.60544900 \\
\hline-6.56893200 & 1.87542300 & -1.89130400 \\
\hline-6.27393000 & 2.96501300 & -3.27701800 \\
\hline-6.21816700 & 1.19688100 & -3.49530000 \\
\hline 1.76443100 & -0.14339200 & 1.34693800 \\
\hline 2.94177400 & -0.75718600 & -0.50852100 \\
\hline 4.44344400 & -1.80323700 & -0.20760200 \\
\hline 1.84773800 & -1.86589300 & -1.51561600 \\
\hline 3.54982400 & 0.46330300 & -1.74968300 \\
\hline 5.59406200 & -1.24684600 & 0.40220200 \\
\hline 4.44974500 & -3.16427900 & -0.55800200 \\
\hline 1.20061200 & -2.98219600 & -0.93024900 \\
\hline 1.61949100 & -1.56907200 & -2.86787200 \\
\hline 2.85924700 & 1.68099400 & -1.95639000 \\
\hline 4.70297000 & 0.17739300 & -2.49987700 \\
\hline 6.68020100 & -2.09009700 & 0.68485600 \\
\hline 5.72986600 & 0.22290600 & 0.6839220 \\
\hline 5.54265900 & -3.98368200 & -0.27502100 \\
\hline 3.59354400 & -3.59425400 & -1.07429000 \\
\hline 0.37514300 & -3.77742400 & -1.74212300 \\
\hline 1.37125300 & -3.38629000 & 0.51078400 \\
\hline 0.78468500 & -2.36500900 & -3.65779700 \\
\hline 2.10266000 & -0.70224900 & -3.31840700 \\
\hline 3.38438100 & 2.58568500 & -2.89495200 \\
\hline 1.57837400 & 1.99721600 & -1.24999000 \\
\hline 5.19853500 & 1.08582300 & -3.43404400 \\
\hline 5.22227400 & -0.77052100 & -2.35065800 \\
\hline 7.56478100 & -1.65903600 & 1.16203900 \\
\hline 66183000 & -3.44650200 & 0.3625430 \\
\hline
\end{tabular}


H

$\mathrm{H}$

$\mathrm{H}$

$\mathrm{H}$

$\mathrm{H}$

C

\section{tBu-XPhos}

P

C

\begin{tabular}{|c|c|c|}
\hline 4.76390600 & 0.71970900 & 0.81143200 \\
\hline 6.33436300 & 0.39686400 & 1.58538900 \\
\hline 6.23516800 & 0.73105100 & -0.15449100 \\
\hline 5.51591800 & -5.03900800 & -0.55690400 \\
\hline-0.11431900 & -4.64798800 & -1.29536700 \\
\hline 0.16645700 & -3.48512100 & -3.09368800 \\
\hline 1.97029600 & -2.66749300 & 1.08023600 \\
\hline 0.38858000 & -3.44058900 & 0.99976900 \\
\hline 1.85386600 & -4.37440300 & 0.58717000 \\
\hline 0.63378900 & -2.11559900 & -4.71114300 \\
\hline 2.86396200 & 3.53427300 & -3.05051800 \\
\hline 4.53775500 & 2.30202900 & -3.62508000 \\
\hline 1.64066500 & 1.76216400 & -0.18088000 \\
\hline 1.31578600 & 3.06014600 & -1.34213600 \\
\hline 0.72937100 & 1.40481500 & -1.62910900 \\
\hline 6.09803700 & 0.84502400 & -4.00577200 \\
\hline 7.52261800 & -4.07747000 & 0.59767300 \\
\hline-0.47132600 & -4.13314800 & -3.70228200 \\
\hline 4.91987200 & 3.02984100 & -4.34559400 \\
\hline 1.74159100 & -0.55471200 & -0.67379100 \\
\hline 3.24338800 & -1.67906100 & -0.20978900 \\
\hline 4.66415700 & -1.13804000 & -0.41756900 \\
\hline 5.39240400 & -1.91775000 & -0.13154200 \\
\hline 4.88422900 & -0.25649400 & 0.19942100 \\
\hline 4.86503700 & -0.88104900 & -1.46596800 \\
\hline 3.06962000 & -2.93613900 & -1.08785100 \\
\hline 3.18253800 & -2.71945700 & -2.15939800 \\
\hline 2.07996800 & -3.39466100 & -0.94175000 \\
\hline 3.83464800 & -3.68577900 & -0.82015300 \\
\hline 3.08354200 & -2.11474200 & 1.25588600 \\
\hline 2.09400000 & -2.55380100 & 1.44216600 \\
\hline 3.22790100 & -1.28661100 & 1.96309700 \\
\hline 3.83435600 & -2.88943800 & 1.48898700 \\
\hline 2.25805400 & 0.59580800 & -2.11831700 \\
\hline 0.96309400 & 1.34078500 & -2.47611800 \\
\hline 1.12597600 & 1.97594200 & -3.36416800 \\
\hline 0.64926800 & 1.99604700 & -1.65458800 \\
\hline 0.13723900 & 0.64736900 & -2.69474400 \\
\hline 2.66646300 & -0.27505400 & -3.31598300 \\
\hline 2.75772100 & 0.35758300 & -4.21595700 \\
\hline 1.91359700 & -1.05021800 & -3.52972100 \\
\hline 3.63678100 & -0.76969500 & -3.16765000 \\
\hline .34220600 & 1.63951900 & -1.8258990 \\
\hline
\end{tabular}


$\begin{array}{lll}4.30604500 & 1.19378800 & -1.55082100\end{array}$

$\begin{array}{lll}3.03849600 & 2.32617600 & -1.02266900\end{array}$

$\begin{array}{lll}3.51117500 & 2.25198100 & -2.72976700\end{array}$

$\begin{array}{lll}1.56606600 & 0.63666300 & 0.74982800\end{array}$

$\begin{array}{lll}2.68315300 & 1.20298200 & 1.39250700\end{array}$

$\begin{array}{lll}2.56200000 & 1.99550800 & 2.53390300\end{array}$

$3.45498300 \quad 2.41361600 \quad 3.00527700$

$\begin{array}{lll}1.29688400 & 2.23519300 \quad 3.07143000\end{array}$

$1.18175100 \quad 2.83528700 \quad 3.97748900$

$\begin{array}{lll}0.17421900 & 1.70284500 & 2.43933400\end{array}$

$\begin{array}{lll}0.27534500 & 0.92425100 & 1.27254100\end{array}$

$\begin{array}{lll}-1.00699900 & 0.44470600 & 0.64916100\end{array}$

$\begin{array}{lll}-1.44277600 & -0.88803800 & 0.84930500\end{array}$

$\begin{array}{lll}-0.67008400 & -1.83869600 & 1.75161800\end{array}$

$0.35881200 \quad-1.46480200 \quad 1.81681500$

$\begin{array}{lll}-0.60321200 & -3.27084900 & 1.21261500\end{array}$

$\begin{array}{lll}-1.58612100 & -3.76845700 & 1.23548300\end{array}$

$\begin{array}{lll}0.07819400 & -3.88180800 \quad 1.82652300\end{array}$

$\begin{array}{lll}-0.23855700 & -3.28569800 & 0.17441900\end{array}$

$-1.24702500 \quad-1.80653200 \quad 3.17594900$

$\begin{array}{lll}-1.22750800 & -0.78685000 & 3.59080600\end{array}$

$\begin{array}{lll}-0.66597500 & -2.45790700 \quad 3.85008100\end{array}$

$-2.29383500 \quad-2.15280400 \quad 3.18666000$

$\begin{array}{lll}-2.64378400 & -1.31172400 & 0.27394200\end{array}$

$\begin{array}{lll}-2.96753600 & -2.34221400 & 0.42930000\end{array}$

$\begin{array}{lll}-3.44382800 & -0.45360200 & -0.48871900\end{array}$

$\begin{array}{lll}-4.72838900 & -0.94236800 & -1.13801900\end{array}$

$\begin{array}{lll}-5.20380800 & -0.06543500 & -1.60982000\end{array}$

$\begin{array}{lll}-4.43496400 & -1.96346500 & -2.24754600\end{array}$

$\begin{array}{lll}-3.75463100 & -1.54424300 & -3.00549100\end{array}$

$\begin{array}{lll}-5.36379100 & -2.27330000 & -2.75482700\end{array}$

$\begin{array}{lll}-3.95832600 & -2.86940900 & -1.83808300\end{array}$

$-5.71719200 \quad-1.51013400 \quad-0.11081900$

$\begin{array}{lll}-5.31681800 & -2.41447700 & 0.37596100\end{array}$

$\begin{array}{lll}-6.66759300 & -1.78941600 & -0.59477800\end{array}$

$-5.93950400 \quad-0.77561000 \quad 0.67934900$

$\begin{array}{lll}-3.01883500 & 0.86683000 & -0.64263300\end{array}$

$\begin{array}{lll}-1.82298800 & 1.34110800 & -0.08303800\end{array}$

$\begin{array}{lll}-1.47988800 & 2.82055900 & -0.24200900\end{array}$

$\begin{array}{lll}-0.40043400 & 2.93437300 & -0.06081800\end{array}$

$\begin{array}{lll}-1.78377500 & 3.37213200 & -1.64345600\end{array}$

$\begin{array}{lll}-1.38812900 & 2.72605100 & -2.43933200\end{array}$

$\begin{array}{lll}-1.33752700 & 4.37268000 & -1.76270800\end{array}$

$-2.86710800 \quad 3.48216500 \quad-1.81089900$ 


$\begin{array}{rrr}-2.21690300 & 3.66943100 & 0.80851600 \\ -3.30836700 & 3.57872600 & 0.68244000 \\ -1.95203400 & 4.73492300 & 0.70529000 \\ -1.97445400 & 3.35986300 & 1.83432300 \\ 3.67937700 & 1.01377200 & 0.99736500 \\ -0.81682700 & 1.88669400 & 2.85731900 \\ -3.64477000 & 1.55179500 & -1.21868800\end{array}$

$1.04908900 \quad-1.85070500 \quad-0.08674800$

$0.38898300 \quad-3.35733700 \quad 0.91374400$

$\begin{array}{lll}0.68272500 & -4.75704500 & 0.35303600\end{array}$

$0.21720700 \quad-5.50149200 \quad 1.02141100$

$\begin{array}{lll}1.75284000 & -4.99707800 & 0.30994500\end{array}$

$\begin{array}{lll}0.25487400 & -4.91260100 & -0.64456400\end{array}$

$\begin{array}{lll}-1.13673800 & -3.18057800 & 0.98964500\end{array}$

$\begin{array}{lll}-1.61942500 & -3.20885800 & 0.00474000\end{array}$

$\begin{array}{lll}-1.40910800 & -2.22931500 \quad 1.46064400\end{array}$

$\begin{array}{lll}-1.57383700 & -3.99198200 & 1.59492200\end{array}$

$\begin{array}{lll}0.94335700 & -3.27398200 & 2.34348000\end{array}$

$0.68766000 \quad-2.32019000 \quad 2.81765200$

$\begin{array}{lll}2.03368700 & -3.40243700 & 2.38673800\end{array}$

$0.48709700 \quad-4.07344600 \quad 2.95119400$

$\begin{array}{lll}1.09310000 & -2.30924100 & -1.95005800\end{array}$

$\begin{array}{lll}1.47016100 & -0.99576900 & -2.65021500\end{array}$

$1.46702500 \quad-1.13989400 \quad-3.74369000$

$\begin{array}{lll}2.47817500 & -0.67097400 & -2.35849900\end{array}$

$0.76819100 \quad-0.18961700 \quad-2.41317000$

$-0.30119600 \quad-2.76278400 \quad-2.40402000$

$\begin{array}{lll}-0.34698200 & -2.74907000 & -3.50554700\end{array}$

$\begin{array}{lll}-1.09016900 & -2.10116700 \quad-2.03770400\end{array}$

$\begin{array}{lll}-0.53052200 & -3.78642000 & -2.07811700\end{array}$

$\begin{array}{lll}2.12663500 & -3.36886300 & -2.35481900\end{array}$

$1.97946700 \quad-4.33319400 \quad-1.85460600$

$\begin{array}{lll}3.15578800 & -3.03402300 & -2.16939900\end{array}$

$\begin{array}{lll}2.03286100 & -3.54641800 & -3.44026400\end{array}$

$2.87125300 \quad-1.74386000 \quad 0.26900800$

$\begin{array}{lll}3.69292100 & -2.87927100 & 0.36792600\end{array}$

$\begin{array}{lll}5.06806600 & -2.77665500 & 0.58140400\end{array}$

$\begin{array}{lll}5.67522500 & -3.68192400 & 0.65769700\end{array}$

$\begin{array}{lll}5.65683500 & -1.51596900 & 0.68812000\end{array}$

$6.73260500 \quad-1.41797700 \quad 0.85315300$

$\begin{array}{lll}4.85871300 & -0.37685000 & 0.59702500\end{array}$

$\begin{array}{lll}3.47070800 & -0.46382200 & 0.39476700\end{array}$

$\begin{array}{lll}2.64092800 & 0.77340200 & 0.40232900\end{array}$ 
$1.63030500 \quad 0.85256200 \quad 1.44792300$

$\begin{array}{lll}1.91202600 & 0.17988000 & 2.79714200\end{array}$

0.68862700

$\begin{array}{lll}0.36722200 & 1.04215200 \quad 4.08649700\end{array}$

0.92313500

$-0.16722400$

3.02927000

3.94911400

3.27557000

2.71863400

0.70828000

0.07260900

0.97740200

0.29731100

0.69363700

$-1.22794300$

$-1.54281200$

$-1.62766300$

$-1.70177400$

0.70258700

0.32544700

0.28916900

1.79777000

1.98872500

2.79350100

3.79856300

4.18359900

3.15678700

2.24824100

3.86464300

2.87847900

4.99479800

4.67051900

5.73106700

5.50867100

3.26060600

5.31046500

$-0.03050500$

$-1.88238700$

$-1.75354800$

$-2.72363700$

$-1.94383300$

$-1.68149700$

$0.52921600 \quad-1.98269300$ 
S33

\begin{tabular}{|c|c|c|}
\hline-2.21490700 & -0.99722900 & -0.52797500 \\
\hline-4.12822300 & 0.42688000 & 1.24919800 \\
\hline-2.47936800 & -0.57362300 & 1.99992000 \\
\hline-2.69333400 & 1.39865800 & 2.86467800 \\
\hline-0.92777600 & 1.67935600 & -2.29124500 \\
\hline-2.37615700 & -0.09559800 & -3.03473700 \\
\hline-4.68170700 & -0.72866800 & 0.87053700 \\
\hline-3.04275000 & 2.39833700 & 2.56522700 \\
\hline-3.34776700 & 1.03463700 & 3.67115100 \\
\hline-1.67589800 & 1.49027700 & 3.26267100 \\
\hline-0.86983500 & 2.17863100 & -3.59154800 \\
\hline-0.34917000 & 2.16277700 & -1.50443300 \\
\hline-2.97436000 & -0.98547200 & -2.82343000 \\
\hline-2.31744200 & 0.40075800 & -4.33947700 \\
\hline-4.13677400 & -1.80776800 & 0.96565100 \\
\hline-6.08141900 & -0.51149700 & 0.28406600 \\
\hline-1.56162000 & 1.54040000 & -4.62761900 \\
\hline-0.26516700 & 3.06526000 & -3.79961000 \\
\hline-2.86771100 & -0.10721100 & -5.13612100 \\
\hline-6.70933000 & -1.87260400 & -0.03139400 \\
\hline-6.94874000 & 0.25477400 & 1.29851500 \\
\hline-5.92608800 & 0.31572400 & -1.00739600 \\
\hline-1.51036600 & 1.92681600 & -5.64851000 \\
\hline-6.09595200 & -2.43839500 & -0.74785800 \\
\hline-6.80959200 & -2.48743100 & 0.87590500 \\
\hline-7.71166000 & -1.73393500 & -0.46639400 \\
\hline-6.51866300 & 1.23999900 & 1.52722700 \\
\hline-7.96130900 & 0.40479000 & 0.89066600 \\
\hline-7.04465100 & -0.30483000 & 2.24323800 \\
\hline-6.91247400 & 0.47763700 & -1.47117400 \\
\hline-5.47247200 & 1.29560900 & -0.80180600 \\
\hline-5.28791500 & -0.20308800 & -1.73973900 \\
\hline 2.20221200 & 3.83387300 & -1.01518800 \\
\hline 0.66452500 & 1.10116200 & -0.30598100 \\
\hline 1.55556900 & 0.54881100 & 1.2855820 \\
\hline 1.60507800 & 0.65256400 & -1.91012700 \\
\hline 0.82969700 & 3.00162100 & -0.07216300 \\
\hline 0.57677300 & 0.62001000 & 2.3043400 \\
\hline 2.87655500 & 0.17232600 & 1.68630300 \\
\hline 2.97045100 & 1.29132700 & -2.18032500 \\
\hline 0.64172800 & 0.94152000 & -3.07912300 \\
\hline 1.76701700 & -0.86281800 & -1.82924800 \\
\hline 0.52640100 & 3.68146400 & -1.4094100 \\
\hline
\end{tabular}


$2.21569700 \quad 3.42695500 \quad 0.40154400$

$\begin{array}{lll}-0.20289300 & 3.44826800 & 0.97129800\end{array}$

$\begin{array}{lll}-0.44603400 & 0.85415000 & 2.00722500\end{array}$ 


\begin{tabular}{|c|c|c|}
\hline 3.27725600 & -3.15807200 & -1.51896900 \\
\hline 2.82873300 & -4.35988000 & -0.28767300 \\
\hline 4.53339900 & -4.06874200 & -0.65172600 \\
\hline 3.49601300 & -4.04090700 & 2.11977900 \\
\hline 4.05364300 & -2.46279900 & 2.71405000 \\
\hline 5.14654600 & -3.47893800 & 1.74582300 \\
\hline 7.02264300 & 1.85021700 & 2.57736100 \\
\hline 5.66430100 & 1.12409500 & 3.46260200 \\
\hline 5.94894400 & 2.87743700 & 3.56220600 \\
\hline 6.58656500 & 3.57128500 & 0.78311400 \\
\hline 5.25508300 & 4.41283800 & 1.59236100 \\
\hline 5.00020500 & 3.67895400 & -0.00762200 \\
\hline 8.17933400 & 0.45757200 & -1.67554000 \\
\hline 7.08931500 & -0.55724500 & -3.21984100 \\
\hline 8.49401400 & -1.64772000 & -1.41576100 \\
\hline 6.47993000 & -1.46297800 & -3.37445400 \\
\hline 7.93757800 & -0.59875900 & -3.92297400 \\
\hline 6.46729800 & 0.31187200 & -3.48575600 \\
\hline 7.96733400 & -2.61011100 & -1.52296400 \\
\hline 8.85767100 & -1.57843900 & -0.37831500 \\
\hline 9.37092200 & -1.67670900 & -2.08316600 \\
\hline 1.49803700 & 0.25857100 & -0.57142200 \\
\hline 1.88495000 & -2.05098500 & -0.20206800 \\
\hline-3.35800800 & 0.61136600 & -1.13406600 \\
\hline-2.76637800 & 1.92367100 & -1.26560500 \\
\hline-1.44485500 & -3.31951200 & -1.61955000 \\
\hline-1.26755400 & -2.77827800 & 1.47817200 \\
\hline-3.71800400 & -2.23322200 & 0.11268000 \\
\hline-3.90706200 & 0.29962500 & 0.18077100 \\
\hline-3.97116000 & -0.05379400 & -2.37782700 \\
\hline-2.47180000 & 2.22969600 & -2.27204300 \\
\hline-3.27274700 & 2.98140700 & -0.41523700 \\
\hline-2.40065100 & -4.49899800 & -1.84380800 \\
\hline-0.04366500 & -3.90892700 & -1.39136400 \\
\hline-1.43426000 & -2.50285300 & -2.92095000 \\
\hline-1.89056600 & -1.86231600 & 2.54312300 \\
\hline 0.25842700 & -2.64363300 & 1.53707600 \\
\hline-1.65103500 & -4.22891000 & 1.79473400 \\
\hline-4.35834600 & -3.47582200 & 0.27189800 \\
\hline-4.47301600 & -1.06154600 & 0.36045300 \\
\hline-4.00202900 & 1.27160000 & 1.17821200 \\
\hline-3.94002300 & -1.14479900 & -2.24353500 \\
\hline-3.24945200 & 0.28418100 & -3.68564000 \\
\hline 4500920 & 0.34782800 & -2.50572500 \\
\hline
\end{tabular}




\begin{tabular}{|c|c|c|c|}
\hline $\mathrm{C}$ & -3.55164600 & 4.39031800 & -0.93331300 \\
\hline $\mathrm{C}$ & -3.79975600 & 2.63526400 & 0.80657400 \\
\hline $\mathrm{H}$ & -2.03979000 & -5.07033000 & -2.71693500 \\
\hline $\mathrm{H}$ & -3.42592400 & -4.17693000 & -2.06790700 \\
\hline $\mathrm{H}$ & -2.42385200 & -5.19787300 & -0.99653200 \\
\hline $\mathrm{H}$ & -0.03512300 & -4.64916800 & -0.58088900 \\
\hline $\mathrm{H}$ & 0.71150900 & -3.15151800 & -1.16955800 \\
\hline $\mathrm{H}$ & 0.27607400 & -4.42883700 & -2.31032400 \\
\hline $\mathrm{H}$ & -0.81287700 & -1.60352500 & -2.84564300 \\
\hline $\mathrm{H}$ & -2.44734900 & -2.19219600 & -3.20105200 \\
\hline $\mathrm{H}$ & -1.03893800 & -3.12321100 & -3.74347700 \\
\hline $\mathrm{H}$ & -1.44828300 & -2.08347100 & 3.52852100 \\
\hline $\mathrm{H}$ & -2.97700500 & -2.00289500 & 2.61916100 \\
\hline $\mathrm{H}$ & -1.69995300 & -0.80784700 & 2.31532700 \\
\hline $\mathrm{H}$ & 0.59898600 & -2.64680300 & 2.58502500 \\
\hline $\mathrm{H}$ & 0.58997400 & -1.70294100 & 1.08932000 \\
\hline $\mathrm{H}$ & 0.76080400 & -3.47178800 & 1.02769500 \\
\hline $\mathrm{H}$ & -1.34093400 & -4.93500000 & 1.01179400 \\
\hline $\mathrm{H}$ & -2.72727200 & -4.35050400 & 1.96896000 \\
\hline $\mathrm{H}$ & -1.14024600 & -4.53222300 & 2.72567500 \\
\hline $\mathrm{C}$ & -5.69886600 & -3.57935500 & 0.64385400 \\
\hline $\mathrm{H}$ & -3.80643900 & -4.39528600 & 0.12240700 \\
\hline $\mathrm{C}$ & -5.82549500 & -1.18169600 & 0.73244300 \\
\hline $\mathrm{C}$ & -4.35656500 & 0.96224400 & 2.62720400 \\
\hline $\mathrm{H}$ & -3.43244100 & 1.32752300 & -3.98733200 \\
\hline $\mathrm{H}$ & -3.61598100 & -0.35665500 & -4.50353400 \\
\hline $\mathrm{H}$ & -2.16380000 & 0.14703800 & -3.61155400 \\
\hline $\mathrm{H}$ & -6.03713700 & 0.03591900 & -1.63098300 \\
\hline $\mathrm{H}$ & -5.90683700 & -0.11303800 & -3.39812600 \\
\hline $\mathrm{H}$ & -5.54569800 & 1.44107900 & -2.60145300 \\
\hline $\mathrm{H}$ & -3.80500600 & 4.99934500 & -0.04789200 \\
\hline $\mathrm{C}$ & -2.43291600 & 5.11758400 & -1.67576600 \\
\hline $\mathrm{C}$ & -4.81250200 & 4.34016800 & -1.81968300 \\
\hline $\mathrm{H}$ & -4.14880000 & 3.42217200 & 1.48110700 \\
\hline $\mathrm{H}$ & -6.15559600 & -4.56613800 & 0.75395800 \\
\hline $\mathrm{C}$ & -6.44111400 & -2.42155800 & 0.87804900 \\
\hline $\mathrm{H}$ & -6.39962600 & -0.26863200 & 0.89316200 \\
\hline $\mathrm{H}$ & -4.45858800 & -0.12796400 & 2.72685800 \\
\hline $\mathrm{C}$ & -3.22172700 & 1.40401100 & 3.56617900 \\
\hline $\mathrm{C}$ & -5.68445800 & 1.59401100 & 3.07283200 \\
\hline $\mathrm{H}$ & -1.57281000 & 5.30986400 & -1.02421800 \\
\hline $\mathrm{H}$ & -2.79786200 & 6.09099700 & -2.04312000 \\
\hline $\mathrm{H}$ & -2.08248500 & 4.55070100 & -2.55235900 \\
\hline $\mathrm{H}$ & -4.61975200 & 3.76613700 & -2.74065400 \\
\hline
\end{tabular}




\begin{tabular}{|c|c|c|c|}
\hline $\mathrm{H}$ & -5.12669200 & 5.35516400 & -2.11707900 \\
\hline $\mathrm{H}$ & -5.65154200 & 3.85950300 & -1.29422200 \\
\hline $\mathrm{H}$ & -7.49345100 & -2.48189700 & 1.16675100 \\
\hline $\mathrm{H}$ & -2.26352100 & 0.96161200 & 3.26135000 \\
\hline $\mathrm{H}$ & -3.42422000 & 1.09766800 & 4.60633700 \\
\hline $\mathrm{H}$ & -3.09675900 & 2.49876100 & 3.55555100 \\
\hline $\mathrm{H}$ & -5.64071900 & 2.69455600 & 3.03644300 \\
\hline $\mathrm{H}$ & -5.92333900 & 1.30939100 & 4.11128800 \\
\hline $\mathrm{H}$ & -6.52332000 & 1.27621600 & 2.43490200 \\
\hline \multicolumn{4}{|l|}{ S34 } \\
\hline $\mathrm{C}$ & -4.23010600 & 0.22710100 & 0.40508300 \\
\hline $\mathrm{C}$ & -4.04309200 & -1.06518100 & -0.20809600 \\
\hline $\mathrm{C}$ & -4.26323200 & -2.33930400 & 0.49803000 \\
\hline $\mathrm{C}$ & -4.24910100 & -2.45351700 & 1.90564100 \\
\hline $\mathrm{H}$ & -4.48274700 & -3.47154700 & -1.32992400 \\
\hline $\mathrm{H}$ & -4.25271400 & -1.14146300 & -1.28442600 \\
\hline $\mathrm{C}$ & -4.45335100 & -3.52768800 & -0.23826500 \\
\hline $\mathrm{C}$ & -4.40297500 & -3.68743500 & 2.53594400 \\
\hline $\mathrm{C}$ & -4.57343000 & -4.85671100 & 1.78546100 \\
\hline $\mathrm{C}$ & -4.60025800 & -4.76490000 & 0.39065200 \\
\hline $\mathrm{H}$ & -4.38208200 & -3.73911400 & 3.62820900 \\
\hline $\mathrm{H}$ & -4.68806800 & -5.82345600 & 2.28176600 \\
\hline $\mathrm{H}$ & -4.74131000 & -5.66492500 & -0.21450800 \\
\hline $\mathrm{C}$ & -4.64846400 & 1.40095000 & -0.41846700 \\
\hline $\mathrm{H}$ & -4.40277000 & 1.22828400 & -1.47856900 \\
\hline $\mathrm{Ni}$ & -2.37413700 & -0.05948100 & 0.08438100 \\
\hline $\mathrm{C}$ & -2.68139500 & 2.75176300 & 0.37072800 \\
\hline $\mathrm{O}$ & -1.90494000 & 1.81517900 & 0.55696300 \\
\hline $\mathrm{C}$ & -2.27144000 & 4.19862300 & 0.65422000 \\
\hline $\mathrm{C}$ & -0.78996100 & 4.23984400 & 1.04038300 \\
\hline $\mathrm{C}$ & -3.14420700 & 4.72203500 & 1.81162200 \\
\hline $\mathrm{C}$ & -2.50929700 & 5.04195500 & -0.61219200 \\
\hline $\mathrm{H}$ & -0.15684100 & 3.86400200 & 0.22466900 \\
\hline $\mathrm{H}$ & -0.59259200 & 3.62493000 & 1.92889800 \\
\hline $\mathrm{H}$ & -0.49257600 & 5.27657200 & 1.26132100 \\
\hline $\mathrm{H}$ & -4.21117000 & 4.70348000 & 1.54638900 \\
\hline $\mathrm{H}$ & -2.86432300 & 5.76013100 & 2.05080800 \\
\hline $\mathrm{H}$ & -3.00403100 & 4.11495500 & 2.72044000 \\
\hline $\mathrm{H}$ & -2.20181300 & 6.08355800 & -0.42902300 \\
\hline $\mathrm{H}$ & -3.56898600 & 5.03933000 & -0.90350400 \\
\hline $\mathrm{H}$ & -1.91606500 & 4.65880200 & -1.45779200 \\
\hline $\mathrm{O}$ & -3.91755300 & 2.66065800 & -0.06698600 \\
\hline $\mathrm{H}$ & -4.56333900 & 0.27878400 & 1.45176500 \\
\hline $\mathrm{H}$ & -4.09537800 & -1.56059100 & 2.51511400 \\
\hline
\end{tabular}


$\begin{array}{lll}-6.10940500 & 1.79678200 & -0.28818500\end{array}$

$\begin{array}{lll}-6.73833600 & 0.96103000 & -0.63010400\end{array}$

$\begin{array}{lll}-6.34589900 & 2.68756800 & -0.89142000\end{array}$

$\begin{array}{lll}-6.35888900 & 2.00700800 \quad 0.76394200\end{array}$

$\begin{array}{lll}-0.43641200 & -0.88491600 & -0.58784200\end{array}$

$\begin{array}{lll}0.58459100 & 0.55828100 & -1.27275800\end{array}$

$\begin{array}{lll}-0.52357800 & -2.03403700 & -2.12626700\end{array}$

$\begin{array}{lll}0.34770500 & -1.80920700 & 0.87756600\end{array}$

$\begin{array}{lll}-0.30292000 & 1.34877400 & -2.04352800\end{array}$

$\begin{array}{lll}1.94660300 & 0.97773300 & -1.23614100\end{array}$

$\begin{array}{lll}0.82377200 & -2.22509100 & -2.82140400\end{array}$

$-1.08953200 \quad-3.39503200 \quad-1.69700900$

$\begin{array}{lll}-1.50984500 & -1.41387500 & -3.13130700\end{array}$

$\begin{array}{lll}-0.74456700 & -2.72383600 & 1.46683300\end{array}$

$1.61923400 \quad-2.61361000 \quad 0.60461800$

$\begin{array}{lll}0.61646500 & -0.72515900 & 1.92834900\end{array}$

$\begin{array}{lll}-1.36552900 & 1.10683700 & -1.99941500\end{array}$

$\begin{array}{lll}0.10376400 & 2.41634100 \quad-2.83749900\end{array}$

$\begin{array}{lll}2.33839000 & 2.05781500 & -2.05719800\end{array}$

$3.05101900 \quad 0.49357900 \quad-0.33182200$

$\begin{array}{lll}0.71039400 & -2.92806800 & -3.66515000\end{array}$

$\begin{array}{lll}1.19886300 & -1.27708600 & -3.23059600\end{array}$

$1.58217000 \quad-2.63681700 \quad-2.14703500$

$\begin{array}{lll}-1.31896000 & -3.98748700 & -2.59888700\end{array}$

$-0.37301000 \quad-3.97572600 \quad-1.10158500$

$\begin{array}{lll}-2.01760200 & -3.29007800 & -1.12056500\end{array}$

$\begin{array}{lll}-2.47541000 & -1.18451900 & -2.65944300\end{array}$

$-1.11773300 \quad-0.49444400 \quad-3.58658800$

$\begin{array}{lll}-1.68684300 & -2.13300000 & -3.94909400\end{array}$

$\begin{array}{lll}-1.68461000 & -2.17276100 & 1.59906000\end{array}$

$\begin{array}{lll}-0.96109300 & -3.60622600 & 0.85564600\end{array}$

$\begin{array}{lll}-0.41111100 & -3.07934000 & 2.45625200\end{array}$

$1.98532700 \quad-3.04996900 \quad 1.55029800$

$\begin{array}{lll}1.43811800 & -3.44838100 & -0.08720400\end{array}$

$\begin{array}{lll}2.42466500 & -1.99105100 & 0.20100600\end{array}$

$0.91692200 \quad-1.19845100 \quad 2.87809900$

$\begin{array}{lll}1.42433400 & -0.06416600 & 1.61856300\end{array}$

$\begin{array}{lll}-0.28320000 & -0.11939200 & 2.11558800\end{array}$

$\begin{array}{lll}-0.63401600 & 2.97292000 & -3.42079100\end{array}$

$\begin{array}{lll}1.45371800 & 2.76218600 & -2.86765400\end{array}$

$3.38724300 \quad 2.35770100 \quad-2.03013800$

$4.02725900 \quad-0.42508000 \quad-0.78461400$

$\begin{array}{lll}3.20957400 & 1.10271400 & 0.94108800\end{array}$

$\begin{array}{lll}1.81167900 & 3.58889900 & -3.48589500\end{array}$ 
C

C

\section{TS-S35}

C

\begin{tabular}{|c|c|c|}
\hline 5.06049600 & -0.81081000 & 0.07867600 \\
\hline 4.02951500 & -0.95571400 & -2.21167100 \\
\hline 4.25994300 & 0.68269700 & 1.76713100 \\
\hline 2.31087800 & 2.25039400 & 1.40368500 \\
\hline 5.79335100 & -1.54010700 & -0.27074400 \\
\hline 5.18149900 & -0.28924100 & 1.36882900 \\
\hline 3.03143300 & -0.77697100 & -2.62668000 \\
\hline 4.31740900 & -2.46015600 & -2.30465500 \\
\hline 5.02939800 & -0.17124800 & -3.07699900 \\
\hline 4.37026000 & 1.12668700 & 2.75818500 \\
\hline 1.32984300 & 2.12120300 & 0.92473000 \\
\hline 2.88412600 & 3.59985900 & 0.93695100 \\
\hline 2.08347700 & 2.29875800 & 2.92023000 \\
\hline 6.26480300 & -0.77102400 & 2.31945800 \\
\hline 3.70010200 & -3.04141600 & -1.60432900 \\
\hline 4.11292000 & -2.82611000 & -3.32347500 \\
\hline 5.37266800 & -2.68810600 & -2.08478200 \\
\hline 5.01591400 & -0.53789700 & -4.11688400 \\
\hline 4.79503600 & 0.90305000 & -3.09570600 \\
\hline 6.05626600 & -0.28327300 & -2.69164300 \\
\hline 3.89022900 & 3.75906000 & 1.35875700 \\
\hline 2.96384600 & 3.65646700 & -0.15646900 \\
\hline 2.24177500 & 4.43014800 & 1.27175800 \\
\hline 2.99119000 & 2.61161500 & 3.46062100 \\
\hline 1.30198200 & 3.03745700 & 3.15637000 \\
\hline 1.76495600 & 1.32938500 & 3.32892000 \\
\hline 6.22530500 & -0.11896400 & 3.20870600 \\
\hline 5.97912300 & -2.20735600 & 2.78457200 \\
\hline 7.67123500 & -0.65224300 & 1.71841900 \\
\hline 6.00675000 & -2.91077200 & 1.93596300 \\
\hline 6.72735500 & -2.54084200 & 3.52263500 \\
\hline 4.98280400 & -2.28534800 & 3.24771700 \\
\hline 7.79567300 & -1.31425900 & 0.84602200 \\
\hline 7.88183700 & 0.37792300 & 1.39018800 \\
\hline 8.43676300 & -0.93752600 & 2.45846300 \\
\hline 4.36550200 & 0.18171500 & 0.36545400 \\
\hline 4.00643100 & -1.09156700 & -0.25425700 \\
\hline 4.25523000 & -2.37402600 & 0.43368800 \\
\hline 4.25244700 & -2.50180600 & 1.83939000 \\
\hline-4.49468700 & -3.47788400 & -1.40862700 \\
\hline-4.19928000 & -1.16204900 & -1.33429000 \\
\hline 46915700 & -3.54748300 & -0.31788300 \\
\hline 8300 & -3.73811700 & 2.45583700 \\
\hline
\end{tabular}




\begin{tabular}{|c|c|c|}
\hline-4.63315800 & -4.89381000 & 1.69038000 \\
\hline-4.64821200 & -4.78792400 & 0.29661400 \\
\hline-4.42857000 & -3.80256800 & 3.54736800 \\
\hline-4.77417700 & -5.86287000 & 2.17524300 \\
\hline-4.80699300 & -5.67798300 & -0.31850400 \\
\hline-4.75290800 & 1.31783700 & -0.41899700 \\
\hline-4.72057800 & 1.16279300 & -1.50222300 \\
\hline-2.42869800 & -0.02033800 & 0.11048500 \\
\hline-2.50942700 & 2.75333000 & 0.28712400 \\
\hline-1.88548500 & 1.77612700 & 0.75512500 \\
\hline-2.06943800 & 4.17786000 & 0.66996900 \\
\hline-0.59604000 & 4.17755700 & 1.08652100 \\
\hline-2.96122400 & 4.59515300 & 1.85712800 \\
\hline-2.28318900 & 5.14073000 & -0.50687200 \\
\hline 0.04758800 & 3.86192400 & 0.2528680 \\
\hline-0.42139700 & 3.49201400 & 1.92560600 \\
\hline-0.29111000 & 5.19135600 & 1.39130100 \\
\hline-4.02463100 & 4.59942500 & 1.57176900 \\
\hline-2.69358000 & 5.60987600 & 2.19385900 \\
\hline-2.83489700 & 3.90890200 & 2.70960100 \\
\hline-1.99167600 & 6.16238100 & -0.21430300 \\
\hline-3.33413700 & 5.15551400 & -0.82619700 \\
\hline-1.66771800 & 4.84887900 & -1.37217700 \\
\hline-3.53632700 & 2.68265400 & -0.47475900 \\
\hline-4.69194900 & 0.19649400 & 1.41254800 \\
\hline-4.08011100 & -1.61962300 & 2.45937700 \\
\hline-5.90263800 & 2.16473500 & 0.04897800 \\
\hline-6.82787600 & 1.57220700 & -0.05817900 \\
\hline-6.00964100 & 3.08770200 & -0.53650200 \\
\hline-5.79449600 & 2.42310800 & 1.11361500 \\
\hline-0.49198800 & -0.82568600 & -0.58763600 \\
\hline 0.54075500 & 0.63942700 & -1.19889300 \\
\hline-0.59750200 & -1.89526000 & -2.17964600 \\
\hline 0.28380800 & -1.81910400 & 0.83374900 \\
\hline-0.33853500 & 1.48112100 & -1.92214800 \\
\hline 1.91190600 & 1.03043100 & -1.15138300 \\
\hline 0.75152100 & -2.06407300 & -2.87676100 \\
\hline-1.17445600 & -3.27136500 & -1.82049800 \\
\hline-1.56862200 & -1.21401600 & -3.15899200 \\
\hline-0.81191400 & -2.75023700 & 1.38544100 \\
\hline 1.54720700 & -2.62288100 & 0.52085100 \\
\hline 0.56480300 & -0.78113800 & 1.92702600 \\
\hline-1.40464200 & 1.25561400 & -1.89003500 \\
\hline 0.080152 & 2.58399400 & -2.0593100 \\
\hline
\end{tabular}


$2.31364900 \quad 2.14795300 \quad-1.91530700$

$\begin{array}{lll}3.02263100 & 0.47335500 & -0.29606800\end{array}$

$\begin{array}{lll}0.63646600 & -2.73070100 & -3.74899000\end{array}$

$1.13158800 \quad-1.10184000 \quad-3.24498000$

$\begin{array}{lll}1.50498200 & -2.50748000 & -2.21772400\end{array}$

$-1.41242500 \quad-3.81296500 \quad-2.75161400$

$\begin{array}{lll}-0.45996100 & -3.88855300 & -1.26044600\end{array}$

$\begin{array}{lll}-2.09833700 & -3.19112300 & -1.23480100\end{array}$

$\begin{array}{lll}-2.54117900 & -1.00908800 & -2.69223900\end{array}$

$\begin{array}{lll}-1.16791400 & -0.27123800 & -3.55437400\end{array}$

$\begin{array}{lll}-1.73440800 & -1.88464800 & -4.01909300\end{array}$

$\begin{array}{lll}-1.73572100 & -2.19128800 & 1.57689200\end{array}$

$\begin{array}{lll}-1.06052800 & -3.58522100 & 0.72205900\end{array}$

$\begin{array}{lll}-0.46338600 & -3.17646200 & 2.34099600\end{array}$

$\begin{array}{lll}1.91612400 & -3.09345100 & 1.44858900\end{array}$

$\begin{array}{lll}1.35229600 & -3.43240700 & -0.19648400\end{array}$

$2.35547200 \quad-1.99466800 \quad 0.13243000$

$\begin{array}{lll}0.83853100 & -1.29703900 & 2.86238300\end{array}$

$\begin{array}{lll}1.39504700 & -0.13325800 & 1.65124000\end{array}$

$\begin{array}{lll}-0.31830500 & -0.15461700 & 2.12340500\end{array}$

$\begin{array}{lll}-0.65346000 & 3.18141700 & -3.20544900\end{array}$

$\begin{array}{lll}1.43473100 & 2.91038100 & -2.67820800\end{array}$

$\begin{array}{lll}3.36769700 & 2.42799400 & -1.88286300\end{array}$

$3.97724900 \quad-0.42949300 \quad-0.82181700$

$3.21585200 \quad 0.99960300 \quad 1.00883200$

$\begin{array}{lll}1.80209600 & 3.76458000 & -3.25189700\end{array}$

$\begin{array}{lll}5.01470100 & -0.88876200 & -0.00052100\end{array}$

$3.95825600 \quad-0.86425000 \quad-2.28086700$

$\begin{array}{lll}4.26724100 & 0.50652100 & 1.79157600\end{array}$

$\begin{array}{lll}2.35982600 & 2.14466600 & 1.54919700\end{array}$

$5.72876600 \quad-1.60508000 \quad-0.41002700$

$\begin{array}{lll}5.16292700 & -0.45641500 & 1.31920900\end{array}$

$2.96001200 \quad-0.64333700 \quad-2.67372600$

$\begin{array}{lll}4.22374400 & -2.36335000 & -2.47500000\end{array}$

$\begin{array}{lll}4.96260200 & -0.03852800 & -3.10164300\end{array}$

$\begin{array}{lll}4.40080800 & 0.88708800 & 2.80589700\end{array}$

$\begin{array}{lll}1.37237800 & 2.07658500 & 1.07286400\end{array}$

$2.97446700 \quad 3.49964700 \quad 1.15621300$

$2.14552000 \quad 2.11365900 \quad 3.06791300$

$\begin{array}{lll}6.24457500 & -1.02347000 & 2.22336100\end{array}$

$3.60851300 \quad-2.98106500 \quad-1.80452500$

$\begin{array}{lll}4.00103800 & -2.66088800 & -3.51215800\end{array}$

$\begin{array}{lll}5.27858900 & -2.61899400 & -2.28472500\end{array}$

$4.93593700 \quad-0.33587500 \quad-4.16317900$ 


\begin{tabular}{|c|c|c|c|}
\hline $\mathrm{H}$ & 4.74351800 & 1.03783800 & -3.04804200 \\
\hline $\mathrm{H}$ & 5.99062500 & -0.18955100 & -2.73324200 \\
\hline $\mathrm{H}$ & 3.99028100 & 3.59944700 & 1.57303500 \\
\hline $\mathrm{H}$ & 3.04410000 & 3.62065400 & 0.06725900 \\
\hline $\mathrm{H}$ & 2.36477900 & 4.32891700 & 1.54877700 \\
\hline $\mathrm{H}$ & 3.06601300 & 2.36902000 & 3.61702300 \\
\hline $\mathrm{H}$ & 1.38733500 & 2.86029400 & 3.35108900 \\
\hline $\mathrm{H}$ & 1.80121300 & 1.13293000 & 3.42507900 \\
\hline $\mathrm{H}$ & 6.25591400 & -0.40563000 & 3.13756000 \\
\hline $\mathrm{C}$ & 5.89913900 & -2.46165500 & 2.64038600 \\
\hline $\mathrm{C}$ & 7.64137700 & -0.94930200 & 1.59427200 \\
\hline $\mathrm{H}$ & 5.87049800 & -3.13052900 & 1.76423100 \\
\hline $\mathrm{H}$ & 6.64742400 & -2.86064400 & 3.34518800 \\
\hline $\mathrm{H}$ & 4.91176100 & -2.50885200 & 3.12624200 \\
\hline $\mathrm{H}$ & 7.71565200 & -1.58564400 & 0.69740800 \\
\hline $\mathrm{H}$ & 7.89358200 & 0.08075200 & 1.29639400 \\
\hline $\mathrm{H}$ & 8.40805700 & -1.29595000 & 2.30624600 \\
\hline \multicolumn{4}{|l|}{ S36 } \\
\hline $\mathrm{C}$ & -4.01785800 & 0.22192600 & 1.81696800 \\
\hline $\mathrm{C}$ & -4.21398300 & -0.44490600 & 0.58263400 \\
\hline $\mathrm{C}$ & -4.58041900 & -1.86548000 & 0.45842900 \\
\hline $\mathrm{C}$ & -4.43060300 & -2.80034100 & 1.50216900 \\
\hline $\mathrm{H}$ & -5.27681200 & -1.61347900 & -1.57119800 \\
\hline $\mathrm{H}$ & -4.59318400 & 0.17251900 & -0.24226900 \\
\hline $\mathrm{C}$ & -5.12971000 & -2.32458800 & -0.75512200 \\
\hline $\mathrm{C}$ & -4.77824900 & -4.13901300 & 1.32375600 \\
\hline $\mathrm{C}$ & -5.29658500 & -4.58182000 & 0.10204500 \\
\hline $\mathrm{C}$ & -5.47996700 & -3.66236800 & -0.93463200 \\
\hline $\mathrm{H}$ & -4.64415900 & -4.84437200 & 2.14792500 \\
\hline $\mathrm{H}$ & -5.56510300 & -5.63190400 & -0.03553500 \\
\hline $\mathrm{H}$ & -5.89819000 & -3.98897400 & -1.89011700 \\
\hline $\mathrm{C}$ & -3.77089300 & 1.59392400 & 1.83699200 \\
\hline $\mathrm{H}$ & -4.20001400 & 2.21110600 & 1.04197000 \\
\hline $\mathrm{Ni}$ & -2.42277100 & 0.59522000 & 0.58374500 \\
\hline $\mathrm{C}$ & -1.90118600 & 2.98803300 & -0.26838300 \\
\hline $\mathrm{O}$ & -1.32779400 & 2.21563400 & 0.58836200 \\
\hline $\mathrm{C}$ & -1.38852700 & 4.44157600 & -0.35461600 \\
\hline $\mathrm{C}$ & -1.75078700 & 5.03800100 & -1.71991600 \\
\hline $\mathrm{C}$ & 0.12637100 & 4.49690600 & -0.13662700 \\
\hline $\mathrm{C}$ & -2.10290800 & 5.23359700 & 0.75860400 \\
\hline $\mathrm{H}$ & -2.83218700 & 4.97574700 & -1.90326200 \\
\hline $\mathrm{H}$ & -1.24244700 & 4.49907100 & -2.53381300 \\
\hline $\mathrm{H}$ & -1.44286200 & 6.09555900 & -1.76925400 \\
\hline $\mathrm{H}$ & 0.38993100 & 4.08630800 & 0.84602400 \\
\hline
\end{tabular}


$0.48436900 \quad 5.53846000 \quad-0.18908900$

$\begin{array}{lll}0.65889600 & 3.91438600 & -0.90252300\end{array}$

$\begin{array}{lll}-1.83102800 & 4.85531500 & 1.75559000\end{array}$

$-3.19774700$

$-1.81945000$

$-2.86327800$

$-4.02905900$

$-3.84097400$

$-3.26779700$

$-4.03861300$

$-2.39752400$

$-2.95563600$

$-0.75651200$

0.41410900

$-1.16756700$

0.01467800

$-0.38130900$

1.82546900

0.05496700

$-1.74375700$

$-2.23498500$

$-1.09904400$

1.15681900

0.49061400

$-1.46378300$

0.14531700

2.33265600

2.90592700

$-0.24721600$

0.47314400

0.84548500

$-2.11452900$

$-0.98408300$

$-2.58561500$

$-3.06076500$

$-1.81476600$

$-2.65173300$

$-1.93666800$

$-1.48699500$

$-0.68458400$

1.55924500

$\begin{array}{lll}0.81816100 & -3.38024200 & 0.40850300\end{array}$

$\begin{array}{lll}1.98338000 & -2.03413900 & 0.41347400\end{array}$

$0.87180000 \quad-0.74354400 \quad 3.05664800$ 
H

\begin{tabular}{|c|c|c|}
\hline 1.29827800 & 0.20557700 & 1.62518400 \\
\hline 0.32725300 & 0.35554300 & 2.31725400 \\
\hline 0.52990900 & 2.49365400 & -3.88027600 \\
\hline 1.52738600 & 2.10405200 & -3.30916900 \\
\hline 3.41426800 & 1.58686600 & -2.43012000 \\
\hline 3.69547800 & -1.00916100 & -0.80097100 \\
\hline 3.25513600 & 0.85740000 & 0.71445300 \\
\hline 1.97577200 & 2.72917900 & -4.08495500 \\
\hline 4.72010100 & -1.43218200 & 0.05432400 \\
\hline 3.50380500 & -1.73843100 & -2.12521300 \\
\hline 4.28432000 & 0.38960100 & 1.54159300 \\
\hline 2.60225300 & 2.20318700 & 1.02274700 \\
\hline 5.30508200 & -2.31125800 & -0.22161800 \\
\hline 5.01371400 & -0.76499800 & 1.24631100 \\
\hline 2.52254600 & -1.45074800 & -2.51916000 \\
\hline 3.52626700 & -3.26730400 & -1.99090600 \\
\hline 4.55710700 & -1.28300200 & -3.14828900 \\
\hline 4.53730500 & 0.94589800 & 2.44634500 \\
\hline 1.58643900 & 2.18967100 & 0.60432700 \\
\hline 3.38537200 & 3.33050500 & 0.32660400 \\
\hline 2.48000900 & 2.52338200 & 2.51772200 \\
\hline 6.07864300 & -1.27912000 & 2.20056100 \\
\hline 2.85633000 & -3.62149900 & -1.19380700 \\
\hline 3.20974900 & -3.73797600 & -2.93542100 \\
\hline 4.53699900 & -3.64347400 & -1.76539900 \\
\hline 4.40711500 & -1.79195500 & -4.11490100 \\
\hline 4.50714000 & -0.19915700 & -3.32672200 \\
\hline 5.57516300 & -1.51742200 & -2.79616200 \\
\hline 4.42967700 & 3.34819300 & 0.67971100 \\
\hline 3.40070400 & 3.20665000 & -0.76432100 \\
\hline 2.93605500 & 4.31097600 & 0.54814700 \\
\hline 3.45876500 & 2.74553300 & 2.97234500 \\
\hline 1.85268000 & 3.41810300 & 2.65466200 \\
\hline 2.02084400 & 1.70320800 & 3.08764700 \\
\hline 6.20869300 & -0.51019000 & 2.98124700 \\
\hline 5.61083000 & -2.56820400 & 2.89371000 \\
\hline 7.43603000 & -1.47980100 & 1.51472900 \\
\hline 5.46437500 & -3.38019700 & 2.16218700 \\
\hline 6.35283500 & -2.91169300 & 3.63349100 \\
\hline 4.65328600 & -2.41440900 & 3.41610000 \\
\hline 7.38800400 & -2.26786300 & 0.74549200 \\
\hline 7.77872500 & -0.55440600 & 1.02540300 \\
\hline 20205100 & -1.78224000 & 2.24738800 \\
\hline
\end{tabular}


$0.69138800 \quad-3.01865700 \quad-1.15113700$

$\begin{array}{lll}-0.63133100 & -2.57701300 & -1.48236200\end{array}$

$\begin{array}{lll}-0.87831500 & -1.93466900 & -2.78748900\end{array}$

$0.01470500 \quad-0.98652800 \quad-3.32849400$

$\begin{array}{lll}-2.72243600 & -3.00416100 & -3.14106000\end{array}$

$-1.46009700$

$-2.00922100$

$-0.19844500$

$-1.31113800$

$-2.21816700$

0.49881000

$-1.47725200$

$-3.09628900$

0.96278900

0.14851700

0.83598400

1.53003300

2.34418200

2.58332800

2.43300500

3.09705500

3.74566200

1.40643300

2.29514600

5.07815000

4.59407000

3.67108000

1.69107800

2.50588600

5.78386400

4.93021300

5.93601200

7.05793900

4.47226300

5.24304700

6.81731700

7.46016200

8.07641200

4.13013400

5.06144700

8.07226100

$\begin{array}{lll}6.11608200 & -2.68370300 & 1.81318000\end{array}$

$\begin{array}{lll}8.39586200 & -3.08708100 & -0.35760300\end{array}$

$\begin{array}{lll}7.62364800 & -2.59761600 & -1.88376500\end{array}$ 
$\begin{array}{lll}6.67350400 & -3.46067800 & -0.65272800\end{array}$

$\begin{array}{lll}9.10950500 & -0.55813500 & -0.32670500\end{array}$

$8.03212500 \quad-0.14374700 \quad-1.68579000$

$\begin{array}{lll}7.83203400 & 0.67352400 & -0.11652000\end{array}$

$\begin{array}{lll}4.41034000 & 3.84949600 & -2.80741600\end{array}$

$2.64139000 \quad 3.35892500 \quad-1.09199600$

$\begin{array}{lll}4.44350200 & 4.90342100 & 0.57172700\end{array}$

$6.44983500 \quad 4.21933400 \quad-0.76844000$

$\begin{array}{lll}7.82366000 & -1.23380200 & 3.27234600\end{array}$

$\begin{array}{lll}8.83904200 & -1.93223700 & 1.99130800\end{array}$

$\begin{array}{lll}8.50658000 & -0.17680500 & 2.01532700\end{array}$

$5.76571600 \quad-2.55158500 \quad 2.84891300$

$\begin{array}{lll}6.78949600 & -3.55513400 & 1.78963300\end{array}$

$5.23546700 \quad-2.88891600 \quad 1.19115900$

$3.71399000 \quad 3.41476300 \quad-3.54137000$

$\begin{array}{lll}4.25715700 & 4.93994800 & -2.80951500\end{array}$

$5.43419900 \quad 3.63863600 \quad-3.14428800$

$2.29299800 \quad 4.40296000 \quad-1.10237200$

$\begin{array}{lll}2.06552300 & 2.79365200 & -1.83855300\end{array}$

$2.42987600 \quad 2.89982600 \quad-0.11614600$

$\begin{array}{lll}5.15544800 & 5.21625600 & 1.35192800\end{array}$

$\begin{array}{lll}4.20693400 & 5.78894200 & -0.03847800\end{array}$

$3.52121800 \quad 4.56157300 \quad 1.05954700$

$\begin{array}{lll}6.90676500 & 3.43361800 & -1.38759100\end{array}$

$6.41772300 \quad 5.15195500 \quad-1.35132000$

$\begin{array}{lll}7.10161600 & 4.38192900 & 0.10342700\end{array}$

$\begin{array}{lll}0.09990900 & -1.62569900 & 0.17976600\end{array}$

$\begin{array}{lll}-1.72632400 & -0.40955000 & 0.99489500\end{array}$

$\begin{array}{lll}-3.20773700 & -1.56728400 & 0.69959800\end{array}$ 
$\begin{array}{lll}-0.41332400 & 0.74703200 & -1.36136000\end{array}$

$-2.10281900$

$0.33385100 \quad-1.75636600$

$-2.93808700$

3.12532200

$-0.13348000$

$-3.92063000$

$1.66861800 \quad-0.31189200$

$\begin{array}{lll}-3.49204200 & 2.24679000 & 1.30117600\end{array}$

$\begin{array}{lll}-0.97210800 & 2.58544100 \quad 1.78829400\end{array}$

$0.27224000 \quad 1.68442000 \quad 0.88006300$

$\begin{array}{lll}-0.54918100 & 3.05826100 & 0.13531200\end{array}$

$0.39368500 \quad-0.47617400 \quad 3.19044700$

$\begin{array}{lll}-0.06389300 & 1.21118800 & 3.04866200\end{array}$

$\begin{array}{lll}-0.46524400 & 0.34483800 & 4.54574600\end{array}$

$\begin{array}{lll}-2.99643900 & 0.75118900 & 4.42514200\end{array}$

$-2.52151100 \quad 1.85200000 \quad 3.11975300$

$\begin{array}{lll}-3.78651100 & 0.63988600 & 2.83959300\end{array}$

$\begin{array}{lll}-2.01491900 & -1.42486700 \quad 4.66770900\end{array}$

$\begin{array}{lll}-3.09906500 & -1.90623800 & 3.34520900\end{array}$

$\begin{array}{lll}-1.36673600 & -2.32779800 \quad 3.28681700\end{array}$

$\begin{array}{lll}-3.09678600 & -5.01222800 & 0.95100100\end{array}$

$\begin{array}{lll}-4.86079400 & -3.88197300 & 0.37896500\end{array}$

$\begin{array}{lll}-6.39698100 & -2.47273700 & -0.11758400\end{array}$

$\begin{array}{lll}-6.14882300 & 0.34895200 & 1.23639200\end{array}$

$\begin{array}{lll}-5.56586500 & 0.35486700 & -1.14070600\end{array}$

$\begin{array}{lll}-5.50695300 & -4.75119600 & 0.23511900\end{array}$

$\begin{array}{lll}-6.97377900 & 1.45723600 & 1.00857100\end{array}$

$\begin{array}{lll}-6.15946600 & -0.30430200 & 2.61692200\end{array}$

$\begin{array}{lll}-6.42708100 & 1.44484700 & -1.32118400\end{array}$

$\begin{array}{lll}-4.83170400 & -0.21986600 & -2.34690700\end{array}$

$\begin{array}{lll}-7.53124900 \quad 1.87902000 & 1.84587900\end{array}$

$\begin{array}{lll}-7.12409900 & 2.02582900 & -0.26003300\end{array}$

$\begin{array}{lll}-5.21447400 & -0.85414000 & 2.73472700\end{array}$

$\begin{array}{lll}-7.29942200 & -1.33276800 & 2.72203900\end{array}$

$\begin{array}{lll}-6.27483100 & 0.70010800 \quad 3.77305100\end{array}$

$\begin{array}{lll}-6.54717500 & 1.86978700 & -2.31944600\end{array}$

$\begin{array}{lll}-3.92694200 & -0.72259900 & -1.97445300\end{array}$

$\begin{array}{lll}-4.38790700 & 0.85555300 & -3.34765800\end{array}$

$-5.68226900 \quad-1.28183500 \quad-3.06194200$

$\begin{array}{lll}-8.00151200 & 3.24763500 & -0.47891400\end{array}$

$\begin{array}{lll}-7.20439500 & -2.12851100 \quad 1.97148800\end{array}$

$\begin{array}{lll}-7.30236900 & -1.80685400 \quad 3.71760300\end{array}$

$\begin{array}{lll}-8.27709800 & -0.84470600 & 2.57570200\end{array}$

$\begin{array}{lll}-6.05595300 & 0.20236400 \quad 4.73110800\end{array}$

$\begin{array}{lll}-5.58074900 & 1.54559800 \quad 3.66388500\end{array}$

$\begin{array}{lll}-7.29338800 & 1.11272400 \quad 3.85085300\end{array}$

$\begin{array}{lll}-5.23973100 & 1.25252100 & -3.92329700\end{array}$ 
$\begin{array}{lll}-3.89574700 & 1.70201800 \quad-2.84822000\end{array}$

$-3.67539800$

$0.42838800 \quad-4.06769900$

$-6.63458400$

$-0.84826100 \quad-3.40995800$

$-5.14842000$

$-1.67451800 \quad-3.94173600$

$\begin{array}{lll}-5.91808500 & -2.12961100 & -2.40343200\end{array}$

dppb

$-8.02904300 \quad 3.43168000 \quad-1.56646900$

$\begin{array}{lll}-9.44495800 & 3.02172400 & -0.00960300\end{array}$

$\begin{array}{lll}-7.39050000 & 4.49040100 & 0.18577500\end{array}$

$\begin{array}{lll}-9.49561200 & 2.86720600 & 1.08067600\end{array}$

$\begin{array}{lll}-10.07417200 & 3.89509900 & -0.24755100\end{array}$

$\begin{array}{lll}-9.89067700 & 2.13850300 & -0.49401300\end{array}$

$\begin{array}{lll}-7.34002000 & 4.37019000 & 1.28059000\end{array}$

$\begin{array}{lll}-6.36655100 & 4.67327400 & -0.17634400\end{array}$

$\begin{array}{lll}-7.99434600 & 5.38855400 & -0.02549200\end{array}$

$\begin{array}{lll}1.81363000 & -0.04321500 & 0.00726100\end{array}$

$\begin{array}{lll}-1.90292600 & -0.13915700 & 0.38250100\end{array}$

$\begin{array}{lll}1.27894300 & -0.41571700 & 1.76235200\end{array}$

$0.43472600 \quad-1.11671400 \quad 1.68422800$

$2.08357600 \quad-0.94929100 \quad 2.29256400$

$\begin{array}{lll}0.87265200 & 0.83971000 & 2.54075000\end{array}$

$\begin{array}{lll}0.61962800 & 0.53664600 & 3.57290400\end{array}$

$\begin{array}{lll}1.74773000 & 1.50609800 \quad 2.62904600\end{array}$

$\begin{array}{lll}-0.29773900 & 1.64957500 & 1.96667400\end{array}$

$\begin{array}{lll}-0.03734800 & 2.03798800 & 0.96743600\end{array}$

$\begin{array}{lll}-0.42803400 & 2.53905600 \quad 2.60504900\end{array}$

$\begin{array}{lll}-1.64529900 \quad 0.91063700 & 1.91068300\end{array}$

$\begin{array}{lll}-2.47461000 & 1.63326600 & 1.97316100\end{array}$

$\begin{array}{lll}-1.73780100 & 0.24271800 & 2.78412700\end{array}$

$\begin{array}{lll}3.43999200 & 0.78139300 & 0.31082700\end{array}$

$3.69867500 \quad 1.98020900 \quad-0.37152600$

$2.94913600 \quad 2.37246100 \quad-1.06437000$

$4.89667800 \quad 2.67430500 \quad-0.17129800$

$\begin{array}{lll}5.08290500 & 3.60653900 & -0.71063000\end{array}$

$\begin{array}{lll}5.85069500 & 2.17624200 & 0.71851700\end{array}$

$6.78705800 \quad 2.71683800 \quad 0.87848000$

$\begin{array}{lll}5.60464500 & 0.98188500 & 1.40603500\end{array}$

$\begin{array}{lll}6.34959700 & 0.58800100 & 2.10234200\end{array}$

$\begin{array}{lll}4.40908100 & 0.29083900 & 1.20373400\end{array}$

$\begin{array}{lll}4.23319000 & -0.64511400 & 1.73910500\end{array}$

$2.29865300 \quad-1.73469900 \quad-0.55058600$

$3.57073400 \quad-2.04288100 \quad-1.06000600$

$\begin{array}{lll}4.35148600 & -1.28032900 & -1.07555100\end{array}$

$3.85232500 \quad-3.32288400 \quad-1.54760000$ 
$4.85001000 \quad-3.54404500 \quad-1.93621500$

$2.86943400 \quad-4.31543700 \quad-1.53678800$

$3.09261400 \quad-5.31641200 \quad-1.91434600$

$1.59455600 \quad-4.01521000 \quad-1.04539400$

$0.81418900 \quad-4.78080700 \quad-1.04119800$

$\begin{array}{lll}1.30816800 & -2.73624800 & -0.56539600\end{array}$

$0.29999100 \quad-2.50837700 \quad-0.20917200$

$\begin{array}{lll}-2.12216900 & 1.18595200 & -0.89538000\end{array}$

$\begin{array}{lll}-1.20678100 & 1.26036500 & -1.95564100\end{array}$

$\begin{array}{lll}-0.38505600 & 0.54322600 & -2.00597800\end{array}$

$\begin{array}{lll}-1.33010600 & 2.25527300 & -2.93178200\end{array}$

$\begin{array}{lll}-0.60892900 & 2.30265500 & -3.75185500\end{array}$

$\begin{array}{lll}-2.37188300 & 3.18241100 & -2.85929100\end{array}$

$\begin{array}{lll}-2.47009600 & 3.95869300 & -3.62256400\end{array}$

$\begin{array}{lll}-3.29427200 & 3.11192900 & -1.80849000\end{array}$

$\begin{array}{lll}-4.11521000 & 3.83154100 & -1.75122200\end{array}$

$\begin{array}{lll}-3.17082000 & 2.11967500 & -0.83506100\end{array}$

$\begin{array}{lll}-3.90327800 & 2.06294100 & -0.02573100\end{array}$

$\begin{array}{lll}-3.66106100 & -0.67168000 & 0.58895100\end{array}$

$\begin{array}{lll}-4.29652800 & -1.24638800 & -0.52860300\end{array}$

$\begin{array}{lll}-3.75808900 & -1.31397100 & -1.47834300\end{array}$

$-5.60503800 \quad-1.72017100 \quad-0.44476400$

$\begin{array}{lll}-6.08207200 & -2.15459400 & -1.32716000\end{array}$

$\begin{array}{lll}-6.30472400 & -1.64360700 & 0.76538500\end{array}$

$\begin{array}{lll}-7.32909700 & -2.01799900 & 0.83373800\end{array}$

$\begin{array}{lll}-5.68254500 & -1.08703900 & 1.88380600\end{array}$

$\begin{array}{lll}-6.21956100 & -1.02233300 & 2.83385500\end{array}$

$-4.37187000 \quad-0.60284900 \quad 1.79749300$

$-3.91360400 \quad-0.16662000 \quad 2.68627200$

$\begin{array}{lll}-1.54777400 & 1.51001500 \quad 0.93236900\end{array}$

$\begin{array}{lll}0.81687700 & -0.93446000 & 1.74469700\end{array}$

$-1.25754300 \quad 2.28612100 \quad 2.61510300$

$\begin{array}{lll}-0.22836400 & 2.66568600 & 2.59951600\end{array}$

$\begin{array}{lll}-1.91298700 & 3.16734600 & 2.70247900\end{array}$

$\begin{array}{lll}-1.47735200 & 1.38963800 \quad 3.84006000\end{array}$

$\begin{array}{lll}-1.09373800 & 1.94009900 \quad 4.71785300\end{array}$

$\begin{array}{lll}-2.56121700 \quad 1.27398900 & 4.00473900\end{array}$

$\begin{array}{lll}-0.86133500 & -0.01423900 & 3.81541000\end{array}$

$\begin{array}{lll}-1.46284400 & -0.64621500 \quad 3.14577400\end{array}$

$\begin{array}{lll}-0.99768400 & -0.46047600 \quad 4.81344700\end{array}$

$0.62700400 \quad-0.11014000 \quad 3.42283200$

$\begin{array}{lll}1.20209800 & -0.67876500 & 4.16767600\end{array}$

$\begin{array}{lll}1.09446400 & 0.88342800 & 3.37740500\end{array}$ 
$\begin{array}{lll}-4.87104600 & -2.06425500 & 1.73283800\end{array}$

$\begin{array}{lll}-5.83401300 & -0.12430400 & 1.75774600\end{array}$

$\begin{array}{lll}-6.82826900 & -0.52613800 & 1.96934300\end{array}$

$\begin{array}{lll}-5.65250000 & 1.25527300 & 1.60892600\end{array}$

$\begin{array}{lll}-6.50460500 & 1.93308200 & 1.70846200\end{array}$

$\begin{array}{lll}-4.38248900 & 1.77230300 & 1.33969000\end{array}$

$\begin{array}{lll}-4.25096800 & 2.85151200 & 1.23176100\end{array}$

$\begin{array}{lll}-1.82886500 & 3.04244600 & -0.06424700\end{array}$

$\begin{array}{lll}-2.65630000 & 2.99962100 & -1.20121400\end{array}$

$\begin{array}{lll}-3.23862200 & 2.10104100 \quad-1.40361100\end{array}$

$\begin{array}{lll}-2.75437500 & 4.08885200 & -2.06687600\end{array}$

$\begin{array}{lll}-3.40709000 & 4.02805700 & -2.94172800\end{array}$

$-2.01987300 \quad 5.25375700 \quad-1.81659900$

$\begin{array}{lll}-2.08829400 & 6.10645600 & -2.49657000\end{array}$

$\begin{array}{lll}-1.20556200 & 5.31656800 & -0.68428200\end{array}$

$\begin{array}{lll}-0.62857900 & 6.21998400 & -0.47170500\end{array}$

$\begin{array}{lll}-1.11319600 & 4.22547900 \quad 0.18558300\end{array}$

$\begin{array}{lll}-0.45916600 & 4.30335400 & 1.05292900\end{array}$

$\begin{array}{lll}0.10891200 & -2.58697500 & 2.23632300\end{array}$

$\begin{array}{lll}-0.93774800 & -3.12146300 & 1.48028300\end{array}$

$\begin{array}{lll}-1.27384500 & -2.57172500 \quad 0.60429900\end{array}$

$\begin{array}{lll}-1.54320900 & -4.33131300 & 1.83132400\end{array}$

$\begin{array}{lll}-2.36251100 & -4.72457300 & 1.22459900\end{array}$

$-1.09966400 \quad-5.02799800 \quad 2.95669800$

$\begin{array}{lll}-1.56790300 & -5.97442600 & 3.23901300\end{array}$

$\begin{array}{lll}-0.05037100 & -4.50715200 & 3.72394200\end{array}$

$0.30303700 \quad-5.04822400 \quad 4.60566600$

$\begin{array}{lll}0.54700900 & -3.29675600 & 3.36773800\end{array}$

$\begin{array}{lll}1.36984500 & -2.90900500 \quad 3.97257100\end{array}$

$\begin{array}{lll}2.62629400 & -1.34654600 & 1.74610600\end{array}$

$\begin{array}{lll}3.10392700 & -2.63786600 & 1.46201900\end{array}$

$2.40180300 \quad-3.46396900 \quad 1.34289100$

$\begin{array}{lll}4.47348400 & -2.88705100 & 1.33735900\end{array}$

$\begin{array}{lll}4.81818300 & -3.90003600 & 1.11246800\end{array}$

$5.39765100 \quad-1.85236300 \quad 1.50037400$

$\begin{array}{lll}6.46798700 & -2.04595800 \quad 1.39654400\end{array}$

$\begin{array}{lll}4.93780700 & -0.56462600 & 1.78905100\end{array}$

$\begin{array}{lll}5.64621100 & 0.25897300 & 1.90690000\end{array}$

$\begin{array}{lll}3.56994800 & -0.31626000 & 1.90354300\end{array}$

$3.23756900 \quad 0.70293400 \quad 2.09686200$ 


\begin{tabular}{|c|c|c|}
\hline 1.42452100 & 1.58728800 & -0.85683900 \\
\hline 0.85891700 & -0.94875200 & -1.63795500 \\
\hline 0.85617200 & 2.40063800 & -2.44535900 \\
\hline-0.21517100 & 2.59435300 & -2.29001600 \\
\hline 1.32798200 & 3.38497600 & -2.55658900 \\
\hline 1.08778100 & 1.62736500 & -3.74660000 \\
\hline 0.58719600 & 2.19388700 & -4.55244900 \\
\hline 2.16369900 & 1.63867400 & -3.98649200 \\
\hline 0.60548400 & 0.17507800 & -3.78073000 \\
\hline 1.30587400 & -0.44600300 & -3.20363800 \\
\hline 0.69172700 & -0.19352300 & -4.81539700 \\
\hline-0.83288200 & -0.05059400 & -3.28118000 \\
\hline-1.42896900 & -0.61611800 & -4.01167900 \\
\hline-1.35603300 & 0.90524400 & -3.13028100 \\
\hline 3.10419100 & 0.95281300 & -1.34309300 \\
\hline 3.26370400 & -0.43578300 & -1.43895000 \\
\hline 2.41650300 & -1.07292700 & -1.19673600 \\
\hline 4.48378600 & -0.99622400 & -1.81908700 \\
\hline 4.58120000 & -2.08212600 & -1.88446200 \\
\hline 5.57511000 & -0.16843900 & -2.09376800 \\
\hline 6.53632000 & -0.60178800 & -2.38202500 \\
\hline 5.43443300 & 1.21909200 & -1.98673700 \\
\hline 6.28603200 & 1.87317200 & -2.19264200 \\
\hline 4.20768300 & 1.77611400 & -1.61476000 \\
\hline 4.11905500 & 2.86026500 & -1.52767800 \\
\hline 1.94203500 & 3.07959700 & 0.1369490 \\
\hline 1.98607800 & 2.91668700 & 1.5276460 \\
\hline 1.67060500 & 1.95968700 & 1.9346690 \\
\hline 2.37466000 & 3.95158700 & 2.37992600 \\
\hline 2.39367900 & 3.78848400 & 3.4606820 \\
\hline 2.71855800 & 5.19684800 & 1.8473560 \\
\hline 3.01361600 & 6.01785500 & 2.5054370 \\
\hline 2.67655200 & 5.38249900 & 0.4626230 \\
\hline 2.94020500 & 6.35229000 & 0.0325580 \\
\hline 2.29614900 & 4.33551300 & -0.38220400 \\
\hline 2.27242800 & .52356800 & -1.45572900 \\
\hline-0.03541300 & .53497400 & -2.16809800 \\
\hline 0.94938600 & 3.09691300 & -1.35022500 \\
\hline 1.19695900 & -2.59650800 & -0.41725600 \\
\hline 1.60592400 & -4.27691400 & -1.71145800 \\
\hline 2.37251800 & -4.69373000 & -1.05358500 \\
\hline 1.28105000 & -4.91313700 & -2.91079600 \\
\hline 1.79238500 & -5.83408700 & -3.20245000 \\
\hline $27+252$ & -4.36499700 & -3.7392290 \\
\hline
\end{tabular}




\begin{tabular}{rrr}
0.03225700 & -4.85944000 & -4.67836100 \\
-0.35882900 & -3.18800600 & -3.36977000 \\
-1.13549900 & -2.78216200 & -4.02172600 \\
-2.62285800 & -1.53593300 & -1.65970100 \\
-2.97904300 & -2.85308600 & -1.32320100 \\
-2.20273900 & -3.59149600 & -1.12025600 \\
-4.31980700 & -3.24306800 & -1.26774700 \\
-4.57051000 & -4.27390400 & -1.00310100 \\
-5.33515600 & -2.32777400 & -1.55818700 \\
-6.38352200 & -2.63192500 & -1.51036300 \\
-4.99501500 & -1.02037100 & -1.91443400 \\
\hline-5.77654700 & -0.29289600 & -2.14699600 \\
\hline-3.65528100 & -0.63346500 & -1.96241800 \\
\hline-3.41034200 & 0.39049500 & -2.24140600 \\
-0.03242300 & 0.18889700 & 0.05911000
\end{tabular}

$0.09713200 \quad-1.46953800 \quad 0.99709600$

$\begin{array}{lll}-1.17796900 & -0.83724900 & 0.86359300\end{array}$

$\begin{array}{lll}-1.87126700 & -0.16408700 & 1.97551400\end{array}$

$\begin{array}{lll}-1.20575000 & 0.25884300 & 3.14654600\end{array}$

$\begin{array}{lll}-3.78570900 & -0.19412700 & 0.97570400\end{array}$

$\begin{array}{lll}-1.85241800 & -1.18787900 & 0.07709800\end{array}$

$\begin{array}{lll}-3.25123400 & 0.10666900 & 1.88032200\end{array}$

$\begin{array}{lll}-1.89366100 & 0.90596500 & 4.17354300\end{array}$

$-3.26595000 \quad 1.15542800 \quad 4.06491900$

$\begin{array}{lll}-3.93862600 & 0.75116500 & 2.90734700\end{array}$

$\begin{array}{llr}-1.35082400 & 1.21838700 \quad 5.07036200\end{array}$

$\begin{array}{lll}-3.80287500 & 1.66421200 \quad 4.86931700\end{array}$

$\begin{array}{lll}-5.00806400 & 0.94841000 & 2.79888700\end{array}$

$\begin{array}{lll}0.45924700 & -2.61502300 & 0.08330300\end{array}$

$-1.50943000 \quad-3.83103100 \quad-0.60696300$

$\begin{array}{lll}-1.62312400 & -3.15978400 & -1.61376800\end{array}$

$-2.51971300 \quad-4.89502500 \quad-0.16361300$

$\begin{array}{lll}-3.55581300 & -5.09986600 & -1.27310900\end{array}$

$\begin{array}{lll}-1.78721800 & -6.21489800 & 0.13399900\end{array}$

$\begin{array}{lll}-3.20440200 & -4.37276300 & 1.11567000\end{array}$

$\begin{array}{lll}-4.08140400 & -4.16246400 & -1.50584700\end{array}$

$\begin{array}{lll}-3.08267300 & -5.45425200 & -2.20167500\end{array}$

$\begin{array}{lll}-4.30096900 & -5.84775700 & -0.95918300\end{array}$

$\begin{array}{lll}-1.04730100 & -6.08891300 & 0.93661500\end{array}$

$\begin{array}{lll}-2.51162300 & -6.98393400 & 0.44705300\end{array}$

$\begin{array}{lll}-1.26225100 & -6.58962300 & -0.75984000\end{array}$

$\begin{array}{lll}-3.94912200 & -5.10395300 & 1.46941500\end{array}$

$-2.47253000 \quad-4.20400600 \quad 1.91844800$ 
$\begin{array}{lll}-3.72565800 & -3.42054400 & 0.92906600\end{array}$

$\begin{array}{lll}-0.50552700 & -3.71343500 & 0.26186000\end{array}$

$\begin{array}{lll}-0.13457400 & 0.07733500 & 3.25076800\end{array}$

$\begin{array}{lll}0.53221700 & -1.55443600 & 2.00082700\end{array}$

$\begin{array}{lll}0.37569600 & -2.31933200 & -0.97004300\end{array}$

$\begin{array}{lll}1.82834300 & -3.20236200 & 0.35774500\end{array}$

$\begin{array}{lll}1.89717800 & -3.56268100 & 1.39612300\end{array}$

$2.04311500 \quad-4.04159700 \quad-0.32118100$

$\begin{array}{lll}2.59607400 & -2.43247600 & 0.21230700\end{array}$

$\begin{array}{lll}0.30722800 & 0.32902800 & 0.15768900\end{array}$

$\begin{array}{lll}2.40369700 & 0.78886300 & 0.24462800\end{array}$

$\begin{array}{lll}-0.69644100 & 1.91648500 & -0.91306500\end{array}$

$\begin{array}{lll}2.87392400 & 2.59559400 & 0.21009600\end{array}$

$\begin{array}{lll}3.53809300 & 0.06604100 & -1.00555700\end{array}$

$\begin{array}{lll}3.10916700 & 0.28940500 & 1.87083600\end{array}$

$\begin{array}{lll}0.29451800 & 3.42402800 & -1.36456700\end{array}$

$\begin{array}{lll}-1.29887200 & 1.32587200 & -2.55246500\end{array}$

$\begin{array}{lll}-2.17376700 & 2.68492600 & -0.13463300\end{array}$

$\begin{array}{lll}2.15192700 & 3.09182800 & 0.87833200\end{array}$

$3.86336800 \quad 2.71809000 \quad 0.67824800$

$2.87580500 \quad 3.25544700 \quad-1.17422700$

$2.98259300 \quad-0.71136700 \quad-2.03267900$

$\begin{array}{lll}4.92326200 & 0.29999500 & -0.99897100\end{array}$

$\begin{array}{lll}4.02441200 & -0.76086000 & 2.03297400\end{array}$

$\begin{array}{lll}2.60857900 & 0.93039600 & 3.01941600\end{array}$

$\begin{array}{lll}1.62644400 & 3.04954200 & -2.04062600\end{array}$

$\begin{array}{lll}-0.31304900 & 4.09090600 & -1.99413900\end{array}$

$0.45803400 \quad 3.97583900 \quad-0.42579500$

$\begin{array}{lll}-1.47189900 & -0.05589700 & -2.73137500\end{array}$

$\begin{array}{lll}-1.59191600 & 2.18720300 & -3.62291600\end{array}$

$\begin{array}{lll}-3.45541300 & 2.65989400 & -0.70157700\end{array}$

$\begin{array}{lll}-2.00081900 & 3.27415500 & 1.13016200\end{array}$

$3.03654200 \quad 4.33799800 \quad-1.02511900$

$\begin{array}{lll}3.74919000 & 2.89245600 & -1.73989900\end{array}$

$\begin{array}{lll}1.90460200 & -0.88763700 & -2.04672000\end{array}$

$3.79560500 \quad-1.25464100 \quad-3.03140600$

$\begin{array}{lll}5.73610800 & -0.24336600 & -1.99561600\end{array}$

$\begin{array}{lll}5.37123800 & 0.90536600 & -0.20727200\end{array}$

$\begin{array}{lll}4.42590900 & -1.27702000 & 1.15987300\end{array}$

$\begin{array}{lll}4.42913300 & -1.16048600 & 3.31085700\end{array}$

$3.01886700 \quad 0.53604000 \quad 4.29341600$

$\begin{array}{lll}1.87680600 & 1.73616300 & 2.91861000\end{array}$

$\begin{array}{lll}1.59218400 & 2.00057800 & -2.37991500\end{array}$

$\begin{array}{lll}1.74836300 & 3.64380900 & -2.95991200\end{array}$ 
$-1.93638600$

$-0.56799500 \quad-3.94519200$

$\begin{array}{lll}2.04946400 & 1.67574900 & -4.83955800\end{array}$

$-1.47175500 \quad 3.26647800 \quad-3.51183900$

$\begin{array}{lll}-3.61348200 & 2.18728200 & -1.67251600\end{array}$

$\begin{array}{lll}-4.53766100 & 3.23449300 & -0.02681600\end{array}$

$\begin{array}{lll}-3.07860600 & 3.85355300 & 1.79723000\end{array}$

$\begin{array}{lll}-1.01768000 & 3.25890800 & 1.60790700\end{array}$

$3.34990200-1.85931000 \quad-3.82487100$

$\begin{array}{lll}5.17307500 & -1.02290600 & -3.01257000\end{array}$

$6.81305000 \quad-0.05782000 \quad-1.98072400$

$5.13985300 \quad-1.98401800 \quad 3.41760700$

$3.93033000 \quad-0.51476700 \quad 4.44410600$

$2.61877500 \quad 1.04605800 \quad 5.17339700$

$\begin{array}{lll}-2.06817800 & -1.64737400 & -4.04706700\end{array}$

$\begin{array}{lll}-2.22406300 & 0.29703900 & -5.00355500\end{array}$

$\begin{array}{lll}-2.27312600 & 2.35789800 & -5.66387900\end{array}$

$\begin{array}{lll}-5.53222300 & 3.20699900 & -0.47959700\end{array}$

$\begin{array}{lll}-4.35181400 & 3.83820000 & 1.21832600\end{array}$

$\begin{array}{lll}-2.92960600 \quad 4.29893100 & 2.78342100\end{array}$

$\begin{array}{lll}5.81073700 & -1.44751000 & -3.79195100\end{array}$

$4.24663900 \quad-0.82955900 \quad 5.44150400$

$\begin{array}{lll}-2.58368700 & -0.09912000 & -5.95670100\end{array}$

$\begin{array}{lll}-5.19943800 & 4.28291700 & 1.74553300\end{array}$

$\begin{array}{lll}-3.54819500 & -0.30218200 & 2.17836900\end{array}$

$\begin{array}{lll}-2.19885200 & 0.17296600 & 2.33287700\end{array}$

$\begin{array}{lll}-1.85269200 & 1.55885800 \quad 2.69554300\end{array}$

$\begin{array}{lll}-2.74738600 & 2.64036900 & 2.54323000\end{array}$

$0.15702200 \quad 1.04112100 \quad 3.30087700$

$\begin{array}{lll}-1.42983700 & -0.55452000 & 2.62911000\end{array}$

$\begin{array}{lll}-0.55527400 & 1.85910000 \quad 3.16152900\end{array}$

$\begin{array}{lll}-2.36261800 \quad 3.94627400 & 2.84108800\end{array}$

$-1.06653600 \quad 4.22525700 \quad 3.29162500$

$\begin{array}{lll}-0.16424200 & 3.16861900 \quad 3.44830700\end{array}$

$\begin{array}{lll}-3.07920700 \quad 4.76057200 & 2.70417400\end{array}$

$\begin{array}{lll}-0.76570100 & 5.25117700 \quad 3.51733000\end{array}$

$\begin{array}{lll}0.85151600 \quad 3.36296100 & 3.80385000\end{array}$

$\begin{array}{lll}-3.88535100 & -1.74928700 \quad 2.32600200\end{array}$

$\begin{array}{lll}-2.99461100 & -2.37148900 \quad 2.14111100\end{array}$

$\begin{array}{lll}-4.92041300 & -1.82414400 & 0.04597300\end{array}$

$\begin{array}{lll}-4.12992200 & -1.05682500 & -0.50600700\end{array}$

$\begin{array}{lll}-6.07396100 & -2.46576900 & -0.72780300\end{array}$

$\begin{array}{lll}-5.85568700 & -3.99181800 & -0.73205500\end{array}$ 
$-4.89904700 \quad-4.25489100 \quad-1.21189000$

$\begin{array}{lll}-6.66347500 & -4.48302000 & -1.29697900\end{array}$

$\begin{array}{lll}-6.22595400 & -0.83749600 & -2.18159400\end{array}$

$\begin{array}{lll}-6.91424100 & -2.39107000 & -2.72444300\end{array}$

$\begin{array}{lll}-5.14691600 & -2.15149000 & -2.68734700\end{array}$

$\begin{array}{lll}-8.24133200 & -2.56478000 & -0.56975400\end{array}$

$\begin{array}{lll}-7.55375700 & -1.03366800 & 0.02699700\end{array}$

$\begin{array}{lll}-7.41187100 & -2.51434400 \quad 1.00968900\end{array}$

$-4.88884000 \quad-2.19566100 \quad 1.30529400$

$\begin{array}{lll}-4.38431400 & 0.37454600 & 2.40544400\end{array}$

$\begin{array}{lll}-3.75289900 & 2.45604900 \quad 2.16054400\end{array}$

$\begin{array}{lll}-4.52880500 & -2.14596200 & 3.64308600\end{array}$

$\begin{array}{lll}-3.82146100 & -1.94328500 & 4.46133800\end{array}$

$-4.79330800 \quad-3.21509000 \quad 3.66140900$

$\begin{array}{lll}-5.44001500 & -1.55329800 \quad 3.82087400\end{array}$

$\begin{array}{lll}-2.69495200 & -0.09484000 & 0.46943300\end{array}$

$\begin{array}{lll}-1.16130100 & 0.63658500 & -0.83805900\end{array}$

$\begin{array}{lll}0.55830900 & 0.71011100 & -0.15379500\end{array}$

$\begin{array}{lll}-0.87584700 & -0.15390000 & -2.47842500\end{array}$

$\begin{array}{lll}-1.51071600 & 2.38670100 & -1.27556600\end{array}$

$\begin{array}{lll}0.49618700 & 1.30368300 & 0.77124100\end{array}$

$1.20930100 \quad 1.25528000 \quad-0.85501100$

$\begin{array}{lll}1.09931100 & -0.68508900 & 0.15937900\end{array}$

$\begin{array}{lll}-1.54289100 & -1.35596800 & -2.75267800\end{array}$

$\begin{array}{lll}-0.00219100 & 0.37935900 & -3.44013700\end{array}$

$\begin{array}{lll}-2.31500900 & 2.68689200 & -2.38855000\end{array}$

$\begin{array}{lll}-1.09655900 & 3.43407800 & -0.43689200\end{array}$

$1.24985600 \quad-1.24307200 \quad-0.78093400$

$\begin{array}{lll}0.33403600 & -1.24173300 & 0.72681500\end{array}$

$2.40380600 \quad-0.67920800 \quad 0.96368200$

$\begin{array}{lll}-2.22719900 & -1.76064300 & -2.00447000\end{array}$

$\begin{array}{lll}-1.33865300 & -2.01953100 & -3.96691700\end{array}$

$0.20167000 \quad-0.28170800 \quad-4.65232500$

$\begin{array}{lll}0.51432200 & 1.32236600 & -3.24322200\end{array}$

$\begin{array}{lll}-2.65878400 & 1.88182800 & -3.04227500\end{array}$

$\begin{array}{lll}-2.67637100 & 4.00578200 & -2.66866900\end{array}$

$\begin{array}{lll}-1.45972900 & 4.75329000 & -0.71950800\end{array}$

$\begin{array}{lll}-0.50169300 & 3.22811000 & 0.45385400\end{array}$

$\begin{array}{lll}2.22843600 & -0.20435300 & 1.94532800\end{array}$

$2.69125900 \quad-1.72298200 \quad 1.17337700$

$\begin{array}{lll}3.55772700 & 0.03471500 & 0.25673000\end{array}$ 
TS-S41

\begin{tabular}{|c|c|c|}
\hline-1.86406500 & -2.95602100 & -4.17133300 \\
\hline-0.46613100 & -1.48364700 & -4.91707800 \\
\hline 0.88231100 & 0.14096300 & -5.39593600 \\
\hline-3.29779000 & 4.22329100 & -3.54131400 \\
\hline-2.24728800 & 5.04486600 & -1.83609000 \\
\hline-1.12887000 & 5.55451100 & -0.05429600 \\
\hline 5.14071200 & -0.04559900 & 1.24185600 \\
\hline 3.71571100 & -0.38712700 & -0.75085000 \\
\hline 3.32077900 & 1.10362600 & 0.13310200 \\
\hline-0.30605000 & -1.99963300 & -5.86722900 \\
\hline-2.53070900 & 6.07740600 & -2.05507500 \\
\hline 5.51634000 & -1.85349600 & 1.09294000 \\
\hline 6.36705100 & 0.65170200 & 0.04980500 \\
\hline 5.45041300 & -2.64468600 & 2.24980400 \\
\hline 5.82428100 & -2.46865200 & -0.13265800 \\
\hline 7.73335500 & 0.42280200 & 0.30067300 \\
\hline 6.01535300 & 1.45884200 & -1.04392000 \\
\hline 5.21918800 & -2.17409800 & 3.20933300 \\
\hline 5.67687500 & -4.02384800 & 2.18522300 \\
\hline 6.05371500 & -3.84373300 & -0.19788500 \\
\hline 5.89117300 & -1.86508100 & -1.04117500 \\
\hline 8.02919300 & -0.19951600 & 1.14998300 \\
\hline 8.71475200 & 0.96877200 & -0.52601300 \\
\hline 7.00100600 & 2.01405600 & -1.86781500 \\
\hline 4.96671800 & 1.66159100 & -1.26739100 \\
\hline 5.62127200 & -4.62786600 & 3.09449500 \\
\hline 5.97910800 & -4.62484800 & 0.96142200 \\
\hline 6.29310400 & -4.31011300 & -1.15713600 \\
\hline 9.76967800 & 0.77145900 & -0.31833800 \\
\hline 8.35189500 & 1.76943600 & -1.61522100 \\
\hline 6.70624000 & 2.63827300 & -2.71548800 \\
\hline 6.16057000 & -5.70135800 & 0.90885300 \\
\hline 9.12032100 & 2.20071700 & -2.26150600 \\
\hline-3.86324600 & 1.05501300 & 1.83134100 \\
\hline-2.47636400 & 1.43760000 & 2.07042100 \\
\hline-1.96474700 & 2.80445100 & 1.85076700 \\
\hline-2.43613200 & 3.62723600 & 0.80498200 \\
\hline-0.51156400 & 2.67387700 & 3.44420000 \\
\hline-1.97061000 & 0.92168700 & 2.90024300 \\
\hline-0.89633000 & 3.29235300 & 2.62882600 \\
\hline-1.86086700 & 4.87109500 & 0.55042200 \\
\hline-0.79089900 & 5.33408100 & 1.32695900 \\
\hline-0.31375100 & 4.53534600 & 2.36998600 \\
\hline
\end{tabular}




\begin{tabular}{|c|c|c|}
\hline-2.24054300 & 5.47964900 & -0.27469300 \\
\hline-0.33607600 & 6.30581200 & 1.11962900 \\
\hline 0.51883800 & 4.88157600 & 2.98868500 \\
\hline-4.46547800 & -0.08049700 & 2.47827800 \\
\hline-3.79730300 & -0.62162400 & 3.15703900 \\
\hline-4.55796200 & -1.60004100 & 0.17527900 \\
\hline-3.77296800 & -0.89342300 & -0.49285400 \\
\hline-5.36378900 & -2.70179900 & -0.53616800 \\
\hline-5.05913600 & -4.04569000 & 0.15016000 \\
\hline-4.97774700 & -2.76881900 & -2.01763600 \\
\hline-6.85808900 & -2.36212200 & -0.38898100 \\
\hline-5.33000400 & -4.01577400 & 1.21510400 \\
\hline-3.98775500 & -4.29413700 & 0.07568700 \\
\hline-5.62822500 & -4.85618000 & -0.33289200 \\
\hline-5.18580000 & -1.81848800 & -2.53045800 \\
\hline-5.55325000 & -3.56389200 & -2.51821500 \\
\hline-3.90706000 & -2.98757000 & -2.14370200 \\
\hline-7.47250900 & -3.12688500 & -0.89081100 \\
\hline-7.09000600 & -1.38732100 & -0.84823000 \\
\hline-7.15278400 & -2.32065900 & 0.66951200 \\
\hline-4.76999900 & -1.51078900 & 1.43596900 \\
\hline-4.55844600 & 1.79249500 & 1.41036900 \\
\hline-3.24127900 & 3.26761400 & 0.16097800 \\
\hline-5.87674900 & 0.04534000 & 2.98303400 \\
\hline-5.87672500 & 0.76294500 & 3.82130000 \\
\hline-6.28021100 & -0.91139300 & 3.34139000 \\
\hline-6.54011400 & 0.44375300 & 2.19994500 \\
\hline-2.59599000 & 0.34840900 & 0.49928900 \\
\hline-0.77509500 & 0.42415400 & -0.62368800 \\
\hline 0.70360100 & 1.25308700 & 0.09945500 \\
\hline-0.09275100 & -1.17794500 & -1.22169100 \\
\hline-1.10560100 & 1.33873400 & -2.18035500 \\
\hline 0.39582900 & 2.26785200 & 0.39401200 \\
\hline 1.48299900 & 1.33716800 & -0.67315700 \\
\hline 1.21058100 & 0.46927400 & 1.30833800 \\
\hline-0.60875300 & -2.35956300 & -0.67007700 \\
\hline 0.92181900 & -1.25659000 & -2.18779100 \\
\hline-2.08289200 & 0.81078500 & -3.04394000 \\
\hline-0.50059300 & 2.56209600 & -2.50120900 \\
\hline 1.43089400 & -0.56430600 & 0.99831100 \\
\hline 0.40086200 & 0.39065800 & 2.05116900 \\
\hline 2.44776400 & 1.06574600 & 1.98567300 \\
\hline-1.41010700 & -2.29000300 & 0.06881800 \\
\hline-0.11204900 & -3.60409500 & -1.06905000 \\
\hline
\end{tabular}


$\begin{array}{lll}1.41608200 & -2.49923500 & -2.58874600\end{array}$

$\begin{array}{lll}1.32779000 & -0.34469500 & -2.63054000\end{array}$

2.57905300

$-2.42883700$

$-0.85654600$

0.24604100

2.17501400

2.75689900

3.65208700

$-0.52037400$

0.90133200

2.21110400

$-3.18576300$

$-1.81632200$

$-0.37825900$

4.04554900

3.46156200

4.53813200

1.29173500

$-2.09162600$

5.82977500

4.15281300

6.34881900

6.68053200

3.29940600

5.01588700

5.71360600

7.68187900

8.01163000

6.29849000

2.62637700

3.30175100

5.01916000

5.69342400

8.06699800

8.51945000

8.65758400

2.62895900

4.16068300

5.69361600

9.56204200

4.16474700

$-0.12773500 \quad-2.78445600$

$1.48457000 \quad-4.21456200$

$3.24014800-3.67221500$

$3.00004300-1.83767600$

$2.01842900 \quad 2.47224300$

$0.38158800 \quad 2.79254800$

$1.32972400 \quad 1.07088900$

$-4.51906900 \quad-0.63234300$

$-3.67447400-2.02925800$

$-2.55106900 \quad-3.33655400$

$1.06180300 \quad-4.88014600$

$2.70288900-4.53232000$

$4.19358600 \quad-3.91080800$

$-0.07169300 \quad-0.11546900$

$2.21345800 \quad 0.43965400$

$1.56233600 \quad 1.68280900$

$-4.64560400 \quad-2.34374000$

$3.23320600-5.44739100$

$0.24318800 \quad-0.46336600$

$-1.50768400 \quad 1.04413200$

$1.54639200 \quad-0.55181300$

$-0.83679300 \quad-0.76379300$

$-2.59820400 \quad 0.81724700$

$-1.53792800 \quad 2.15272100$

$2.40823700 \quad-0.33709200$

$1.76180300 \quad-0.91495300$

$-0.62053100-1.12264100$

$-1.85904000 \quad-0.70611600$

$\begin{array}{ll}-2.58478200 & -0.04229800\end{array}$

$-3.69660900 \quad 1.68316500$

$-2.63400000 \quad 3.01710200$

$-0.70051500 \quad 2.33793600$

$2.78329800 \quad-0.97277700$

$0.68074600-1.19885600$

$-1.47447900 \quad-1.34312700$

$-4.53669900 \quad 1.49320200$

$-3.71550200 \quad 2.78419200$

$-2.64681500 \quad 3.87729300$

$0.84986500 \quad-1.47905100$

$-4.57233200 \quad 3.46283700$

$-3.44157900 \quad 2.36193800 \quad 1.97680300$ 


\begin{tabular}{|c|c|c|}
\hline-2.02237900 & 2.21898000 & 1.91388800 \\
\hline-1.16205000 & 3.16489600 & 1.17738100 \\
\hline-1.56846900 & 3.74266900 & -0.04149600 \\
\hline 0.45888500 & 3.02696800 & 2.59877700 \\
\hline-1.53685500 & 1.74390700 & 2.77702100 \\
\hline 0.12461300 & 3.46902500 & 1.65683600 \\
\hline-0.71713700 & 4.58729100 & -0.75298300 \\
\hline 0.56415900 & 4.87315400 & -0.26775000 \\
\hline 0.97946100 & 4.31185200 & 0.94355300 \\
\hline-1.05033700 & 5.01028900 & -1.70387700 \\
\hline 1.23435000 & 5.52716500 & -0.83070800 \\
\hline 1.97743500 & 4.52785700 & 1.33321800 \\
\hline-4.20076300 & 1.33376700 & 2.53354100 \\
\hline-3.73686100 & 0.69411300 & 3.29451600 \\
\hline-2.94090000 & 0.67099200 & 1.01958600 \\
\hline-4.20953000 & -1.69481700 & 1.09759800 \\
\hline-4.17501800 & -0.64229300 & 0.35042100 \\
\hline-5.15829800 & -2.80870900 & 0.60112000 \\
\hline-4.96129100 & -4.07425600 & 1.44053400 \\
\hline-4.86509700 & -3.10610000 & -0.87874600 \\
\hline-6.60323100 & -2.29681800 & 0.74818000 \\
\hline-5.12901300 & -3.87186500 & 2.50804500 \\
\hline-3.93685100 & -4.46275400 & 1.33668800 \\
\hline-5.66171700 & -4.86255800 & 1.11826300 \\
\hline-5.02710500 & -2.21664300 & -1.50364100 \\
\hline-5.52620700 & -3.90956000 & -1.24480700 \\
\hline-3.82460900 & -3.43249500 & -1.02217200 \\
\hline-6.75213100 & -1.37220800 & 0.17119000 \\
\hline-6.84626300 & -2.08773400 & 1.80288500 \\
\hline-7.31944300 & -3.05194800 & 0.38327300 \\
\hline-3.55626000 & -1.81549400 & 2.13871300 \\
\hline-2.54636800 & 3.49308300 & -0.45724000 \\
\hline-3.93819000 & 3.13064900 & 1.37221900 \\
\hline-5.69265400 & 1.25860500 & 2.43578600 \\
\hline-6.16838500 & 1.66951200 & 3.34456900 \\
\hline-6.02554300 & 0.21463200 & 2.34269600 \\
\hline-6.07392500 & 1.81694500 & 1.56715100 \\
\hline-1.46636500 & 0.15950700 & -0.54591100 \\
\hline 0.30934000 & 0.64544100 & -0.43666400 \\
\hline-1.34781000 & -1.62859000 & -0.94082800 \\
\hline-2.07486700 & 0.92801300 & -2.09349900 \\
\hline 0.35557900 & 1.74371600 & -0.45123700 \\
\hline 0.80894500 & 0.27550100 & -1.34509900 \\
\hline 0.99103700 & 0.10815900 & 0.82256500 \\
\hline
\end{tabular}


$-1.29477800$

$-2.09725300 \quad-2.26224400$

$-3.44249300$

$0.78427900-2.39507000$

$-1.25681700$

0.98435500

0.40396200

2.43158600

$-1.35032800$

$-1.09794700$

$-1.14561000$

$-1.37747700$

$-4.08149100$

$-3.97213700$

$-1.79541800$

$-0.19802600$

2.43451900

2.81386600

3.37282500

$-1.03365700$

$-1.04258600$

$-1.11349900$

$-5.03439000$

$-3.15147300$

$-1.14940400$

5.10782600

3.37727400

3.03457600

$-0.92907900$

$-3.57017500$

5.59594900

6.04372300

5.72469600

5.80301200

7.44970800

5.43865600

5.57464300

6.04168500

6.12472400

5.71785300

7.94019200

8.22539300

6.21755500

4.35163900

6.13690100
$1.67719900 \quad-2.95155700$

$-0.99449300 \quad 0.80939300$

$0.40882300 \quad 1.70507000$

$0.60351400 \quad 0.98847700$

$-2.19834000 \quad 1.14812900$

$-3.90824200-0.14255400$

$-3.46231500 \quad-2.51929000$

$-1.39558800 \quad-3.09458500$

$0.20923600-1.72032700$

$1.37454100-3.54318600$

$2.27190500 \quad-4.09695500$

$1.81353600 \quad-2.73065300$

$1.70549300 \quad 1.03612800$

$0.24878900 \quad 1.95984800$

$0.13970800 \quad-0.12483800$

$-4.61261600 \quad 0.69018700$

$-4.36979000-1.46069900$

$-3.81843500 \quad-3.55199200$

$1.25549300-3.77069900$

$2.12143400 \quad-4.39571400$

$2.85671900 \quad-4.75651500$

$0.78203200 \quad 0.11896600$

$-0.96158800 \quad-0.19362100$

$0.53009400-1.09855100$

$-5.43757600 \quad-1.66349500$

$2.58766300 \quad-5.29104000$

$-0.17957500 \quad 1.62473100$

$-0.12953200-1.18556600$

$0.51139500 \quad 2.83945600$

$-1.56939400 \quad 1.60965400$

$-0.09268500 \quad-1.12489700$

$-0.79584700 \quad-2.26283100$

$1.59437100 \quad 2.85938000$

$-0.17118300 \quad 4.01855300$

$-2.25064000 \quad 2.78473200$

$-2.12116500 \quad 0.67029400$

$0.42670500 \quad-0.29658600$

$-0.71895600 \quad-2.09980900$

$-1.41537600 \quad-3.24685200$

$\begin{array}{ll}-0.84267200 & -2.34389200\end{array}$

$0.37964300 \quad 4.95780800$ 


$\begin{array}{lll}6.24296100 & -1.55306800 & 3.99243300 \\ 6.28485000 & -3.33168500 & 2.75976900 \\ 9.31568200 & -0.68647200 & -2.02901700 \\ 7.61076800 & -1.38296000 & -3.16786100 \\ 5.72764500 & -1.93105100 & -4.07698300 \\ 6.49555900 & -2.08808000 & 4.91137200 \\ 8.21741500 & -1.87077900 & -3.93478100\end{array}$

H

$\mathrm{H}$

C

$\mathrm{H}$

H

H

C

.

H

.

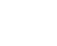

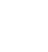

.

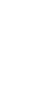

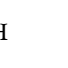

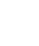

i

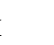

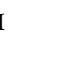

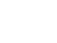

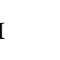

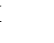

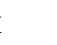

O

P

B




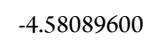

$-7.08743200$

$-5.95027100$

$-5.83900300$

$-4.78384300$

$-3.50315600$

$-6.45091400$

$-7.22834000$

$-5.51462700$

$-7.46700300$

$-7.90788100$

$-6.80266600$

$-7.26421300$

$-4.76103900$

$-5.05988200$

$-7.18774900$

$-3.81162500$

$-5.23438300$

$-5.42646000$

$-2.54386000$

$-3.73308000$

$-3.36921900$

$-7.25147200$

$-7.40675900$

$-8.12680000$

$-4.76786500$

$-4.81192100$

$-3.81442300$

$-5.02787100$

$-5.54104800$

$-4.02793700$

$-7.75691500$

$-7.79962100$

$-7.01434600$

0.96668600

2.81031100

0.74791800

0.55662200

3.06245000

3.24536200

3.35281100

0.51647100

0.86750800
$-4.60866000$

$-3.56867700$

1.73999300

$1.03932900-2.34324700$

$-5.14559500-1.30566500$

$-4.63111800 \quad 0.78540400$

$-5.64765500 \quad 1.39490900$

$-4.25269900 \quad 2.18069500$

$-4.60356200 \quad 2.50354900$

$-4.54267200 \quad-0.75868100$

$-3.04547500 \quad 0.10429700$

$-2.96997900 \quad-1.28955100$

$2.46696800-0.68853200$

$2.64835100 \quad-0.62181800$

$1.83002900-3.38614600$

$0.59161800 \quad-2.90662900$

$-5.40570900-1.75574800$

$-6.07771200 \quad-0.92802600$

$-4.74378600 \quad-2.10108000$

$-4.68803800 \quad 0.24691100$

$-5.63278700 \quad 1.18240100$

$-3.92674200 \quad 1.61658200$

$2.91874400 \quad 0.31557000$

$3.28004200-1.41757700$

$1.78965500-0.74538600$

$3.55469300-1.24625800$

$2.95381900 \quad 0.43300000$

$2.10528700 \quad-0.74421700$

$1.26904300-4.33364700$

$2.79963300 \quad-3.58860300$

$2.01484000 \quad-3.06000600$

$0.01638100 \quad-2.16193000$

$1.44502500 \quad-3.23523900$

$-0.06168600 \quad-3.77530100$

$0.98461300-0.09859200$

$1.03349200 \quad 0.04920000$

$-0.43127200 \quad-1.25647900$

$2.45739500-1.10986200$

$1.99083800 \quad 0.53085900$

$1.04296400 \quad-0.96166500$

$\begin{array}{ll}-0.13204600 & 0.87777800\end{array}$

$-1.71528000 \quad-0.73170500$

$-0.26712900 \quad-2.64659700$ 


\begin{tabular}{|c|c|c|c|}
\hline $\mathrm{H}$ & 11.32731300 & -1.40113200 & -2.65299300 \\
\hline $\mathrm{C}$ & 9.56116000 & -1.03657400 & -3.85154400 \\
\hline $\mathrm{H}$ & 7.64956700 & -0.60706400 & -4.76480600 \\
\hline $\mathrm{H}$ & 8.08911900 & -5.00236600 & 3.15280400 \\
\hline $\mathrm{H}$ & 10.05928300 & -1.25136800 & -4.80018100 \\
\hline \multicolumn{4}{|c|}{ TS-S44 } \\
\hline $\mathrm{P}$ & 1.50480400 & 0.33409000 & -0.36966100 \\
\hline $\mathrm{C}$ & 2.84692200 & 0.48266600 & 0.92538700 \\
\hline $\mathrm{C}$ & 2.19244900 & -0.81218200 & -1.63681500 \\
\hline $\mathrm{C}$ & 4.10952800 & 1.20634000 & 0.55917000 \\
\hline $\mathrm{H}$ & 2.35110700 & 0.97687500 & 1.76975700 \\
\hline $\mathrm{H}$ & 3.05011900 & -0.54989300 & 1.24061500 \\
\hline $\mathrm{C}$ & 1.46543700 & 1.90923800 & -1.31512600 \\
\hline $\mathrm{Ni}$ & -0.41530300 & -0.28212500 & 0.48409600 \\
\hline $\mathrm{C}$ & 2.98235800 & -1.91942500 & -1.29057200 \\
\hline $\mathrm{C}$ & 1.80177300 & -0.65679200 & -2.97944400 \\
\hline $\mathrm{C}$ & 4.21885600 & 2.58520400 & 0.79954000 \\
\hline $\mathrm{C}$ & 5.18208800 & 0.54754000 & -0.06271800 \\
\hline $\mathrm{C}$ & 2.61291300 & 2.41419600 & -1.95684200 \\
\hline $\mathrm{C}$ & 0.24379500 & 2.57625700 & -1.48308500 \\
\hline $\mathrm{C}$ & -0.92016200 & 1.37604500 & 1.55550600 \\
\hline $\mathrm{P}$ & -0.56093200 & -2.43904600 & 0.07757700 \\
\hline $\mathrm{C}$ & 3.37737300 & -2.84379600 & -2.26102100 \\
\hline $\mathrm{H}$ & 3.28555900 & -2.08111500 & -0.25695600 \\
\hline $\mathrm{C}$ & 2.18987700 & -1.58551500 & -3.94617400 \\
\hline $\mathrm{H}$ & 1.18359800 & 0.19267500 & -3.27208500 \\
\hline $\mathrm{C}$ & 5.36122600 & 3.29032300 & 0.41776700 \\
\hline $\mathrm{H}$ & 3.38970700 & 3.10697200 & 1.28071800 \\
\hline $\mathrm{C}$ & 6.32436900 & 1.25230900 & -0.45026100 \\
\hline $\mathrm{H}$ & 5.11949300 & -0.52491700 & -0.25440300 \\
\hline $\mathrm{C}$ & 2.53889100 & 3.58538000 & -2.71037700 \\
\hline $\mathrm{H}$ & 3.56237700 & 1.88524000 & -1.88550700 \\
\hline $\mathrm{C}$ & 0.17128700 & 3.74521200 & -2.24744000 \\
\hline $\mathrm{H}$ & -0.68186300 & 2.20346400 & -1.04813800 \\
\hline $\mathrm{C}$ & -2.01722000 & 0.45311100 & 1.67377900 \\
\hline $\mathrm{C}$ & 0.01713600 & 1.64311900 & 2.65509200 \\
\hline $\mathrm{H}$ & -1.07626100 & 2.22023300 & 0.88085800 \\
\hline $\mathrm{C}$ & -0.68537400 & -2.94177300 & -1.70333300 \\
\hline $\mathrm{C}$ & -1.92627800 & -3.31858200 & 0.94020500 \\
\hline $\mathrm{C}$ & 0.90211900 & -3.33442200 & 0.73546600 \\
\hline $\mathrm{C}$ & 2.98028700 & -2.68318100 & -3.59061400 \\
\hline $\mathrm{H}$ & 3.99212700 & -3.69820100 & -1.96898500 \\
\hline $\mathrm{H}$ & 1.87049600 & -1.44979900 & -4.98215500 \\
\hline $\mathrm{C}$ & 6.41668400 & 2.62762300 & -0.21561600 \\
\hline
\end{tabular}




\begin{tabular}{|c|c|c|c|}
\hline $\mathrm{H}$ & 5.42472700 & 4.36417600 & 0.61046400 \\
\hline $\mathrm{H}$ & 7.14750300 & 0.72318100 & -0.93720600 \\
\hline $\mathrm{C}$ & 1.31920800 & 4.25841200 & -2.85250600 \\
\hline $\mathrm{H}$ & 3.43932800 & 3.97031800 & -3.19548100 \\
\hline $\mathrm{H}$ & -0.80006300 & 4.23026200 & -2.35906300 \\
\hline $\mathrm{C}$ & -2.93161300 & 0.29843500 & 0.62126000 \\
\hline $\mathrm{H}$ & -2.20932400 & -0.07260200 & 2.61446700 \\
\hline $\mathrm{C}$ & 0.73388100 & 2.85661000 & 2.68272300 \\
\hline $\mathrm{C}$ & 0.29749400 & 0.69130300 & 3.65893500 \\
\hline C & -1.76080500 & -2.16445000 & -2.42975300 \\
\hline $\mathrm{H}$ & 0.30684000 & -2.72469800 & -2.12735300 \\
\hline $\mathrm{H}$ & -0.85205400 & -4.02434000 & -1.79626200 \\
\hline $\mathrm{C}$ & -2.00572400 & -3.16150500 & 2.33484300 \\
\hline $\mathrm{C}$ & -2.90356600 & -4.08024200 & 0.28728900 \\
\hline $\mathrm{C}$ & 1.56799400 & -2.77152000 & 1.83582800 \\
\hline $\mathrm{C}$ & 1.37849100 & -4.53426500 & 0.18974600 \\
\hline $\mathrm{H}$ & 3.28607600 & -3.40912500 & -4.34781600 \\
\hline $\mathrm{H}$ & 7.30991500 & 3.17871800 & -0.51996600 \\
\hline $\mathrm{H}$ & 1.26610200 & 5.17378300 & -3.44765400 \\
\hline $\mathrm{C}$ & -4.04245800 & -0.68926200 & 0.61895200 \\
\hline $\mathrm{H}$ & -2.70073500 & 0.78344400 & -0.32540200 \\
\hline C & 1.69507300 & 3.10523400 & 3.66319800 \\
\hline $\mathrm{H}$ & 0.53235100 & 3.60465200 & 1.91194300 \\
\hline $\mathrm{C}$ & 1.26107600 & 0.93939200 & 4.63680600 \\
\hline $\mathrm{H}$ & -0.24076800 & -0.25865900 & 3.66675400 \\
\hline $\mathrm{C}$ & -2.79703300 & -2.82279400 & -3.10817200 \\
\hline $\mathrm{C}$ & -1.76610100 & -0.76048600 & -2.41388000 \\
\hline $\mathrm{C}$ & -3.03550100 & -3.75755100 & 3.06040400 \\
\hline $\mathrm{H}$ & -1.25508300 & -2.56234900 & 2.85553100 \\
\hline $\mathrm{C}$ & -3.94381200 & -4.66872200 & 1.01474000 \\
\hline $\mathrm{H}$ & -2.87736200 & -4.20554800 & -0.79393100 \\
\hline $\mathrm{C}$ & 2.69663900 & -3.39145300 & 2.37630300 \\
\hline $\mathrm{H}$ & 1.21088800 & -1.83023100 & 2.25975800 \\
\hline $\mathrm{C}$ & 2.51393100 & -5.14865200 & 0.72317600 \\
\hline $\mathrm{H}$ & 0.87505600 & -4.99028200 & -0.66446100 \\
\hline $\mathrm{H}$ & -4.97293800 & -0.17009300 & 0.34936700 \\
\hline $\mathrm{H}$ & -4.17220400 & -1.18889400 & 1.58900800 \\
\hline $\mathrm{H}$ & -3.86485000 & -1.45525300 & -0.15227600 \\
\hline $\mathrm{C}$ & 1.97015200 & 2.14582900 & 4.64334300 \\
\hline $\mathrm{H}$ & 2.23711700 & 4.05431600 & 3.66120100 \\
\hline $\mathrm{H}$ & 1.46316000 & 0.18321800 & 5.40006500 \\
\hline $\mathrm{C}$ & -3.81670400 & -2.09861500 & -3.73386500 \\
\hline $\mathrm{H}$ & -2.80882200 & -3.91587700 & -3.14473100 \\
\hline C & -2.79095900 & -0.03066100 & -3.01733000 \\
\hline
\end{tabular}




\begin{tabular}{|c|c|c|c|}
\hline $\mathrm{H}$ & -0.97208300 & -0.22756600 & -1.88643300 \\
\hline $\mathrm{C}$ & -4.01330000 & -4.51057200 & 2.39962200 \\
\hline $\mathrm{H}$ & -3.08183800 & -3.62765900 & 4.14433900 \\
\hline $\mathrm{H}$ & -4.70556300 & -5.25066400 & 0.49017600 \\
\hline $\mathrm{C}$ & 3.17736600 & -4.57798200 & 1.81425100 \\
\hline $\mathrm{H}$ & 3.20691000 & -2.93960300 & 3.23038300 \\
\hline $\mathrm{H}$ & 2.88422700 & -6.07763600 & 0.28267400 \\
\hline $\mathrm{H}$ & 2.72712800 & 2.33759900 & 5.40731000 \\
\hline $\mathrm{C}$ & -3.82276300 & -0.70197000 & -3.68064200 \\
\hline $\mathrm{H}$ & -4.61716200 & -2.63123700 & -4.25365400 \\
\hline $\mathrm{H}$ & -2.79595600 & 1.05799700 & -2.92698300 \\
\hline $\mathrm{H}$ & -4.82794000 & -4.96915100 & 2.96508400 \\
\hline $\mathrm{H}$ & 4.06677100 & -5.05964600 & 2.22757600 \\
\hline $\mathrm{H}$ & -4.63307600 & -0.13701300 & -4.14764300 \\
\hline $\mathrm{C}$ & -3.61740900 & 3.02469000 & -0.05337900 \\
\hline $\mathrm{C}$ & -3.99535100 & 4.51816000 & 0.19233200 \\
\hline $\mathrm{O}$ & -4.12555600 & 2.17438300 & 0.73311000 \\
\hline $\mathrm{O}$ & -2.80739700 & 2.78417600 & -0.98045600 \\
\hline $\mathrm{C}$ & -5.13841800 & 4.64500900 & 1.20332500 \\
\hline $\mathrm{C}$ & -4.39555800 & 5.15895800 & -1.14385200 \\
\hline $\mathrm{C}$ & -2.73267200 & 5.20585300 & 0.74305500 \\
\hline $\mathrm{H}$ & -6.04485400 & 4.13312400 & 0.84468900 \\
\hline $\mathrm{H}$ & -5.38860200 & 5.70614700 & 1.37630900 \\
\hline $\mathrm{H}$ & -4.86717200 & 4.18882000 & 2.16607800 \\
\hline $\mathrm{H}$ & -5.30385700 & 4.68657100 & -1.55423300 \\
\hline $\mathrm{H}$ & -4.60401800 & 6.23548600 & -1.01849300 \\
\hline $\mathrm{H}$ & -3.59360400 & 5.04102100 & -1.88626200 \\
\hline $\mathrm{H}$ & -2.41479600 & 4.74197600 & 1.69158400 \\
\hline $\mathrm{H}$ & -2.91979400 & 6.27602200 & 0.93777000 \\
\hline $\mathrm{H}$ & -1.89934700 & 5.12464200 & 0.02904000 \\
\hline \multicolumn{4}{|c|}{ S45 } \\
\hline $\mathrm{C}$ & 4.29751100 & -0.91897900 & 0.99000700 \\
\hline $\mathrm{C}$ & 3.97179400 & -0.39216300 & -0.30556800 \\
\hline $\mathrm{C}$ & 4.03090500 & -1.15417500 & -1.56409900 \\
\hline $\mathrm{C}$ & 4.07106400 & -2.56489800 & -1.61136700 \\
\hline $\mathrm{H}$ & 3.96032500 & 0.62590100 & -2.78609100 \\
\hline $\mathrm{H}$ & 4.07463600 & 0.69084200 & -0.44404100 \\
\hline $\mathrm{C}$ & 3.98820000 & -0.46717800 & -2.79492400 \\
\hline $\mathrm{C}$ & 4.05268100 & -3.24763900 & -2.82664900 \\
\hline $\mathrm{C}$ & 3.99639500 & -2.54719700 & -4.03774100 \\
\hline $\mathrm{C}$ & 3.96915300 & -1.14984800 & -4.01197200 \\
\hline $\mathrm{H}$ & 4.07763200 & -4.34087500 & -2.83012900 \\
\hline $\mathrm{H}$ & 3.97708300 & -3.08541300 & -4.98845600 \\
\hline $\mathrm{H}$ & 3.93098300 & -0.58565700 & -4.94782600 \\
\hline
\end{tabular}




\begin{tabular}{|c|c|c|}
\hline 4.85686500 & -0.00657800 & 2.04430700 \\
\hline 2.38968700 & -0.85532500 & 0.69791100 \\
\hline 6.67658400 & 1.22730100 & 1.04076300 \\
\hline 5.93525900 & 2.11470500 & 0.67541600 \\
\hline 8.15978500 & 1.13038800 & 0.66337300 \\
\hline 9.01048600 & 0.90974100 & 1.92574100 \\
\hline 8.30610800 & -0.07363200 & -0.28944500 \\
\hline 8.58374400 & 2.42011400 & -0.04575800 \\
\hline 8.89834400 & 1.74729100 & 2.63351800 \\
\hline 8.72271000 & -0.01529500 & 2.44458600 \\
\hline 10.07626300 & 0.83796200 & 1.65463300 \\
\hline 7.67175000 & 0.04680900 & -1.18220100 \\
\hline 9.35232700 & -0.16687600 & -0.62305000 \\
\hline 8.01399800 & -1.01011100 & 0.20715800 \\
\hline 9.64315700 & 2.35843100 & -0.34132000 \\
\hline 7.98087600 & 2.59468300 & -0.94859100 \\
\hline 8.45960100 & 3.29584100 & 0.60958400 \\
\hline 6.29475500 & 0.19305700 & 1.79516300 \\
\hline 4.70711700 & -1.93909300 & 1.05223600 \\
\hline 4.10392300 & -3.13325100 & -0.67979400 \\
\hline 0.88467000 & 1.70966000 & -1.04932700 \\
\hline-0.22395200 & 1.02181200 & -0.51418400 \\
\hline-1.46037400 & 1.70360000 & -0.48199100 \\
\hline-1.55077800 & 3.05051400 & -0.88995700 \\
\hline-0.43041000 & 3.67482600 & -1.44570400 \\
\hline 0.78567900 & 3.00427100 & -1.54472300 \\
\hline-3.99114800 & 2.83338100 & -0.86698500 \\
\hline-3.83807100 & 1.50191000 & -0.43355300 \\
\hline-4.93925300 & 0.61810700 & -0.38387500 \\
\hline-6.15913200 & 1.07625800 & -0.91241500 \\
\hline-6.29582300 & 2.35000100 & -1.45531700 \\
\hline-5.21718800 & 3.23193400 & -1.40599800 \\
\hline 1.84327400 & 1.19004400 & -1.06179700 \\
\hline-0.50077200 & 4.70864400 & -1.78200100 \\
\hline 1.66116900 & 3.49868300 & -1.97064500 \\
\hline-7.02518800 & 0.41814300 & -0.89051400 \\
\hline-7.25098600 & 2.66942800 & -1.87798300 \\
\hline-5.34152600 & 4.25105700 & -1.77089300 \\
\hline-2.84529200 & 3.80010100 & -0.60285900 \\
\hline-2.86431900 & 4.15890200 & 0.90762400 \\
\hline-3.81046200 & 4.65739500 & 1.17105200 \\
\hline-2.76574900 & 3.26103300 & 1.53522900 \\
\hline-2.02919500 & 4.83478700 & 1.14977100 \\
\hline .97076100 & 5.09621800 & -1.40924100 \\
\hline
\end{tabular}




\begin{tabular}{|c|c|c|c|}
\hline $\mathrm{H}$ & -2.14969700 & 5.78535100 & -1.16812000 \\
\hline $\mathrm{H}$ & -2.96097900 & 4.90951900 & -2.49372600 \\
\hline $\mathrm{H}$ & -3.90141500 & 5.62294400 & -1.15573000 \\
\hline $\mathrm{O}$ & -2.59200000 & 1.05921300 & -0.01499400 \\
\hline $\mathrm{P}$ & 0.28247300 & -0.66383000 & 0.04976200 \\
\hline $\mathrm{P}$ & -4.78840800 & -1.01427000 & 0.47076500 \\
\hline C & -0.35684100 & -1.04099600 & 1.80814200 \\
\hline $\mathrm{C}$ & -0.53386100 & 0.30355000 & 2.52709300 \\
\hline $\mathrm{H}$ & 0.35210300 & 0.94665900 & 2.40618000 \\
\hline $\mathrm{H}$ & -1.41156200 & 0.84442400 & 2.15052900 \\
\hline $\mathrm{H}$ & -0.67700800 & 0.12776700 & 3.60697700 \\
\hline $\mathrm{C}$ & 0.85556200 & -1.77869900 & 2.41779000 \\
\hline $\mathrm{H}$ & 0.69735100 & -1.96766600 & 3.49409800 \\
\hline $\mathrm{H}$ & 1.04968800 & -2.74988300 & 1.93987500 \\
\hline $\mathrm{H}$ & 1.78973300 & -1.15091100 & 2.39676800 \\
\hline $\mathrm{C}$ & -0.33220400 & -1.85481400 & -1.27995800 \\
\hline $\mathrm{C}$ & 0.40414000 & -1.44086600 & -2.56586200 \\
\hline $\mathrm{H}$ & 0.10449200 & -0.43841800 & -2.90587800 \\
\hline $\mathrm{H}$ & 1.49338700 & -1.44668200 & -2.43288500 \\
\hline $\mathrm{H}$ & 0.15752200 & -2.15448300 & -3.36981700 \\
\hline $\mathrm{C}$ & 0.12985400 & -3.26584200 & -0.88629300 \\
\hline $\mathrm{H}$ & -0.38219000 & -3.64000200 & 0.01121000 \\
\hline $\mathrm{H}$ & -0.08841900 & -3.96710700 & -1.70914900 \\
\hline $\mathrm{H}$ & 1.21538600 & -3.29027300 & -0.70589400 \\
\hline $\mathrm{C}$ & -1.83991100 & -1.80928100 & -1.51902500 \\
\hline $\mathrm{H}$ & -2.16091500 & -0.81312100 & -1.85491300 \\
\hline $\mathrm{H}$ & -2.10617800 & -2.53036500 & -2.31137900 \\
\hline $\mathrm{H}$ & -2.42177700 & -2.05418800 & -0.62379300 \\
\hline $\mathrm{C}$ & -5.61674600 & -0.58818900 & 2.14837500 \\
\hline $\mathrm{C}$ & -4.62782700 & 0.36940600 & 2.84048900 \\
\hline $\mathrm{H}$ & -3.62580800 & -0.07000500 & 2.94013200 \\
\hline $\mathrm{H}$ & -4.53078100 & 1.32078400 & 2.29571400 \\
\hline $\mathrm{H}$ & -4.99443500 & 0.60706500 & 3.85401700 \\
\hline $\mathrm{C}$ & -6.98894900 & 0.09230300 & 2.10365300 \\
\hline $\mathrm{H}$ & -7.75945700 & -0.52969100 & 1.63202000 \\
\hline $\mathrm{H}$ & -7.32446000 & 0.30373700 & 3.13462500 \\
\hline $\mathrm{H}$ & -6.94936000 & 1.05403000 & 1.57301000 \\
\hline $\mathrm{C}$ & -5.69795900 & -1.88795700 & 2.96441300 \\
\hline $\mathrm{H}$ & -5.98628200 & -1.65828700 & 4.00469000 \\
\hline $\mathrm{H}$ & -6.44964400 & -2.58319900 & 2.56329300 \\
\hline $\mathrm{H}$ & -4.72903300 & -2.41103200 & 2.99370400 \\
\hline $\mathrm{C}$ & -5.87211500 & -2.24778600 & -0.52775900 \\
\hline $\mathrm{C}$ & -5.31651700 & -3.63400300 & -0.13777900 \\
\hline $\mathrm{H}$ & -5.82998100 & -4.41998000 & -0.71832100 \\
\hline
\end{tabular}




\begin{tabular}{llc}
-4.23834700 & -3.70883100 & -0.34509400 \\
-5.46876900 & -3.85786400 & 0.92837000 \\
-5.56922500 & -2.01857300 & -2.01858800 \\
-6.03315800 & -1.10127200 & -2.40509100 \\
-4.48841300 & -1.95281700 & -2.21169900 \\
-5.96101600 & -2.86676900 & -2.60611900 \\
-7.39025900 & -2.25816100 & -0.30777600 \\
\hline-7.65837500 & -2.51245200 & 0.72710500 \\
-7.87244900 & -1.30407700 & -0.56082700 \\
-7.84370400 & -3.02840000 & -0.95664100 \\
-1.60935700 & -1.89472200 & 1.96508700 \\
\hline-1.48598400 & -2.89951900 & 1.53620100 \\
\hline-1.82753200 & -2.02752400 & 3.03965600 \\
\hline-2.48552900 & -1.43549700 & 1.49370600 \\
\hline 4.38879100 & 0.98486200 & 1.98083600 \\
\hline 4.75839200 & -0.55405200 & 3.45618400 \\
5.24722300 & -1.53917600 & 3.52821100 \\
\hline 3.70252100 & -0.67520400 & 3.74085500 \\
5.23904100 & 0.12351100 & 4.17867600 \\
\hline & & \\
\hline
\end{tabular}

S46

C 


\begin{tabular}{|c|c|c|c|}
\hline $\mathrm{H}$ & -6.56684500 & -1.93329800 & -1.82523700 \\
\hline $\mathrm{H}$ & -4.95560700 & -1.16211200 & -1.83561900 \\
\hline $\mathrm{H}$ & -8.22825500 & -1.39895000 & 0.04459600 \\
\hline $\mathrm{H}$ & -7.01453900 & -1.87269200 & 1.26467100 \\
\hline $\mathrm{H}$ & -7.77607600 & -0.27264500 & 1.35254000 \\
\hline $\mathrm{O}$ & -4.16839000 & 0.59551400 & -0.09786600 \\
\hline $\mathrm{H}$ & -1.87113200 & 1.44492100 & -1.29237800 \\
\hline $\mathrm{H}$ & -1.12820300 & 0.56041500 & -3.08551000 \\
\hline $\mathrm{Ni}$ & 0.00846900 & 0.73825000 & 0.32398600 \\
\hline $\mathrm{P}$ & 1.92668200 & -0.11757100 & 0.89516500 \\
\hline C & 3.24541600 & -0.17869800 & -0.37431300 \\
\hline $\mathrm{C}$ & 2.03965200 & -1.71710800 & 1.77875500 \\
\hline $\mathrm{C}$ & 2.58811100 & 1.17573800 & 2.08184100 \\
\hline $\mathrm{C}$ & 2.89818300 & 0.07882100 & -1.70745900 \\
\hline $\mathrm{C}$ & 4.58947300 & -0.42647800 & -0.04723900 \\
\hline $\mathrm{C}$ & 1.37837400 & -1.84365400 & 3.01355900 \\
\hline $\mathrm{C}$ & 2.63900500 & -2.85168800 & 1.20955600 \\
\hline $\mathrm{H}$ & 2.03905600 & 1.05905600 & 3.02978400 \\
\hline $\mathrm{H}$ & 3.66063100 & 1.05847800 & 2.29032800 \\
\hline $\mathrm{C}$ & 2.26651700 & 2.49158300 & 1.41820600 \\
\hline $\mathrm{C}$ & 3.88011300 & 0.08558200 & -2.70241100 \\
\hline $\mathrm{H}$ & 1.85506500 & 0.26472300 & -1.96648300 \\
\hline $\mathrm{C}$ & 5.56845900 & -0.41876600 & -1.04132000 \\
\hline $\mathrm{H}$ & 4.87157100 & -0.63384700 & 0.98816900 \\
\hline $\mathrm{C}$ & 1.34240500 & -3.07008600 & 3.67721600 \\
\hline $\mathrm{H}$ & 0.87426900 & -0.97895800 & 3.45428100 \\
\hline $\mathrm{C}$ & 2.59205600 & -4.08223100 & 1.87190100 \\
\hline $\mathrm{H}$ & 3.13863900 & -2.77782100 & 0.24251200 \\
\hline C & 0.92785500 & 2.75957900 & 1.05160300 \\
\hline $\mathrm{C}$ & 3.25908600 & 3.38388100 & 1.00488300 \\
\hline C & 5.21384900 & -0.16188000 & -2.37138000 \\
\hline $\mathrm{H}$ & 3.59600400 & 0.28137600 & -3.73878800 \\
\hline $\mathrm{H}$ & 6.61172400 & -0.61345400 & -0.78010400 \\
\hline C & 1.95008000 & -4.19430900 & 3.10729400 \\
\hline $\mathrm{H}$ & 0.82959100 & -3.15170800 & 4.63873700 \\
\hline $\mathrm{H}$ & 3.06091100 & -4.95813800 & 1.41648600 \\
\hline C & 0.59397200 & 3.90655400 & 0.32775800 \\
\hline $\mathrm{H}$ & 0.09784900 & 2.13871400 & 1.47605000 \\
\hline $\mathrm{C}$ & 2.92753700 & 4.528333300 & 0.26742500 \\
\hline $\mathrm{H}$ & 4.30420800 & 3.17484600 & 1.24589200 \\
\hline $\mathrm{H}$ & 5.98165200 & -0.15665800 & -3.14922600 \\
\hline $\mathrm{H}$ & 1.91638600 & -5.15661000 & 3.62375900 \\
\hline C & 1.59987800 & 4.79115000 & -0.07615300 \\
\hline $\mathrm{H}$ & -0.45029600 & 4.09683300 & 0.07196000 \\
\hline
\end{tabular}




\begin{tabular}{|c|c|c|c|}
\hline $\mathrm{H}$ & 3.71848000 & 5.21321700 & -0.04823600 \\
\hline $\mathrm{H}$ & 1.34716900 & 5.68130200 & -0.65690300 \\
\hline $\mathrm{H}$ & -2.81188800 & 0.37280500 & 1.44729600 \\
\hline $\mathrm{C}$ & -3.11203000 & 2.46146800 & 1.01759400 \\
\hline $\mathrm{H}$ & -3.98780700 & 2.55746600 & 1.67679300 \\
\hline $\mathrm{H}$ & -3.25134300 & 3.12616300 & 0.14957900 \\
\hline $\mathrm{H}$ & -2.22679000 & 2.80124700 & 1.57591800 \\
\hline \multicolumn{4}{|c|}{$(t \mathrm{Bu}-\mathrm{XantPhos}) \mathrm{AuCl}$} \\
\hline $\mathrm{C}$ & 2.28880000 & 1.28617600 & 0.23641200 \\
\hline $\mathrm{C}$ & 3.45952600 & 1.95824100 & 0.63394500 \\
\hline $\mathrm{H}$ & 4.34355200 & 1.38132100 & 0.89404500 \\
\hline $\mathrm{C}$ & 3.52009500 & 3.34651700 & 0.71489500 \\
\hline $\mathrm{H}$ & 4.44166900 & 3.83380700 & 1.03994100 \\
\hline $\mathrm{C}$ & 2.40755300 & 4.11347300 & 0.36401700 \\
\hline $\mathrm{H}$ & 2.46942300 & 5.20065400 & 0.40941000 \\
\hline $\mathrm{C}$ & 1.22981000 & 3.49757600 & -0.06177600 \\
\hline $\mathrm{C}$ & 1.17961400 & 2.09184200 & -0.09625600 \\
\hline $\mathrm{C}$ & 0.00072300 & 4.25088200 & -0.57098500 \\
\hline $\mathrm{C}$ & -1.22854000 & 3.49799800 & -0.06157300 \\
\hline $\mathrm{C}$ & -2.40598000 & 4.11429900 & 0.36446100 \\
\hline $\mathrm{H}$ & -2.46744900 & 5.20149900 & 0.40994500 \\
\hline $\mathrm{C}$ & -3.51874500 & 3.34772600 & 0.71546900 \\
\hline $\mathrm{H}$ & -4.44008700 & 3.83534100 & 1.04068400 \\
\hline $\mathrm{C}$ & -3.45870600 & 1.95943900 & 0.63441200 \\
\hline $\mathrm{H}$ & -4.34292900 & 1.38283600 & 0.89448600 \\
\hline $\mathrm{C}$ & -2.28828300 & 1.28696200 & 0.23668200 \\
\hline $\mathrm{C}$ & -1.17884300 & 2.09223600 & -0.09610000 \\
\hline $\mathrm{C}$ & 0.00058000 & 4.18425600 & -2.12228300 \\
\hline $\mathrm{H}$ & 0.00034200 & 3.14359600 & -2.47582700 \\
\hline $\mathrm{H}$ & 0.89570300 & 4.68416800 & -2.52350300 \\
\hline $\mathrm{H}$ & -0.89440900 & 4.68452500 & -2.52335100 \\
\hline $\mathrm{C}$ & 0.00103100 & 5.72065800 & -0.14057000 \\
\hline $\mathrm{H}$ & 0.00115600 & 5.82617100 & 0.95461700 \\
\hline $\mathrm{H}$ & -0.87976000 & 6.24222000 & -0.54062400 \\
\hline $\mathrm{H}$ & 0.88193400 & 6.24190400 & -0.54079300 \\
\hline $\mathrm{C}$ & 3.28577800 & -0.98891500 & -1.34700700 \\
\hline $\mathrm{C}$ & 2.40955000 & -0.60028700 & -2.55506200 \\
\hline $\mathrm{H}$ & 2.99205500 & -0.75049100 & -3.47944100 \\
\hline $\mathrm{H}$ & 2.10672200 & 0.45864900 & -2.52062100 \\
\hline $\mathrm{H}$ & 1.51244800 & -1.23373900 & -2.61010200 \\
\hline $\mathrm{C}$ & 4.62035000 & -0.23733300 & -1.43040400 \\
\hline $\mathrm{H}$ & 5.15503700 & -0.57242000 & -2.33503700 \\
\hline $\mathrm{H}$ & 5.27705300 & -0.43420300 & -0.57248800 \\
\hline $\mathrm{H}$ & 4.47619600 & 0.84839500 & -1.51853600 \\
\hline
\end{tabular}


$3.51847300 \quad-2.50853500 \quad-1.37404700$

$\begin{array}{lll}4.00032500 & -2.77113100 & -2.33076100\end{array}$

$\begin{array}{lll}2.56901800 & -3.06410500 & -1.32068000\end{array}$

$\begin{array}{lll}4.19140100 & -2.83978600 & -0.56970900\end{array}$

$2.97474000 \quad-1.18357400 \quad 1.81228200$

$4.50596400 \quad-1.17042700 \quad 1.92123200$

$\begin{array}{lll}4.79574800 & -1.55664400 & 2.91301700\end{array}$

$\begin{array}{lll}4.93406000 & -0.16358800 & 1.83125600\end{array}$

$4.97807100 \quad-1.81602600 \quad 1.16857400$

$\begin{array}{lll}2.47997100 & -2.63474900 & 1.98122000\end{array}$

$\begin{array}{lll}2.86769500 & -3.03998400 & 2.93079400\end{array}$

$2.81411800 \quad-3.29051400 \quad 1.16686600$

$\begin{array}{lll}1.38159700 & -2.68126000 & 2.00831900\end{array}$

$\begin{array}{lll}2.35727100 & -0.32573500 & 2.93037300\end{array}$

$\begin{array}{lll}2.58047400 & -0.78731500 & 3.90656500\end{array}$

$\begin{array}{lll}1.26261600 & -0.26594700 \quad 2.82530900\end{array}$

$2.75485900 \quad 0.69853600 \quad 2.93890400$

$-2.97557000 \quad-1.18302600 \quad 1.81191800$

$\begin{array}{lll}-2.35832800 & -0.32556700 & 2.93041000\end{array}$

$\begin{array}{lll}-2.58180100 & -0.78736500 & 3.90643700\end{array}$

$\begin{array}{lll}-2.75581300 & 0.69874000 & 2.93910700\end{array}$

$\begin{array}{lll}-1.26364900 & -0.26584100 & 2.82565800\end{array}$

$\begin{array}{lll}-2.48104100 & -2.63431200 & 1.98058200\end{array}$

$\begin{array}{lll}-2.86967200 & -3.04002700 & 2.92958100\end{array}$

$\begin{array}{lll}-1.38270800 & -2.68092300 \quad 2.00869400\end{array}$

$\begin{array}{lll}-2.81447900 & -3.28962700 & 1.16558300\end{array}$

$-4.50684600 \quad-1.16984100 \quad 1.92034500$

$-4.79695200 \quad-1.55621300 \quad 2.91197900$

$\begin{array}{lll}-4.97870700 & -1.81532100 & 1.16742800\end{array}$

$\begin{array}{lll}-4.93490100 & -0.16297500 & 1.83039300\end{array}$

$\begin{array}{lll}-3.28581700 & -0.98754700 & -1.34728300\end{array}$

$\begin{array}{lll}-2.40925000 & -0.59899100 & -2.55514800\end{array}$

$\begin{array}{lll}-2.99157400 & -0.74914300 & -3.47964700\end{array}$

$\begin{array}{lll}-1.51223000 & -1.23257600 & -2.60999300\end{array}$

$\begin{array}{lll}-2.10631500 & 0.45992800 & -2.52064000\end{array}$

$\begin{array}{lll}-3.51897800 & -2.50708000 & -1.37469900\end{array}$

$\begin{array}{lll}-4.00085100 & -2.76931800 & -2.33150400\end{array}$

$\begin{array}{lll}-4.19204000 & -2.83832200 & -0.57048000\end{array}$

$\begin{array}{lll}-2.56968200 & -3.06292300 & -1.32143800\end{array}$

$\begin{array}{lll}-4.62011800 & -0.23549300 & -1.43076600\end{array}$

$\begin{array}{lll}-5.15480000 & -0.57031800 & -2.33549300\end{array}$

$\begin{array}{lll}-4.47557400 & 0.85019100 & -1.51876300\end{array}$

$\begin{array}{lll}-5.27700800 & -0.43220900 & -0.57295400\end{array}$

$0.00026000 \quad 1.49896200 \quad-0.47700200$ 


\begin{tabular}{|c|c|c|c|}
\hline $\mathrm{Cl}$ & -0.00059200 & -3.39530400 & -1.67251200 \\
\hline $\mathrm{P}$ & 2.22471600 & -0.56323100 & 0.17334600 \\
\hline $\mathrm{P}$ & -2.22491200 & -0.56247500 & 0.17337300 \\
\hline $\mathrm{Au}$ & -0.00022400 & -1.35796700 & -0.08348700 \\
\hline \multicolumn{4}{|c|}{ ( $t \mathrm{Bu}-\mathrm{Xant}$ Phos) $\mathrm{RhCl}$} \\
\hline $\mathrm{Rh}$ & -0.00000400 & -1.02409200 & -0.04641600 \\
\hline$P$ & 2.26663300 & -0.78786500 & 0.00576300 \\
\hline $\mathrm{O}$ & 0.00000500 & 1.15555700 & -0.04305900 \\
\hline $\mathrm{P}$ & -2.26663900 & -0.78784600 & 0.00574700 \\
\hline $\mathrm{C}$ & 1.21733100 & 1.83930200 & -0.04637200 \\
\hline $\mathrm{C}$ & 2.39631100 & 1.05912100 & -0.03418200 \\
\hline $\mathrm{C}$ & 3.62433000 & 1.73342000 & -0.00786000 \\
\hline $\mathrm{C}$ & 3.69089500 & 3.12489900 & -0.01017700 \\
\hline $\mathrm{C}$ & 2.51185300 & 3.86020900 & -0.03540700 \\
\hline $\mathrm{C}$ & 1.25426200 & 3.23924300 & -0.04946000 \\
\hline $\mathrm{C}$ & -1.21731400 & 1.83931200 & -0.04637400 \\
\hline $\mathrm{C}$ & -2.39630100 & 1.05914100 & -0.03418500 \\
\hline $\mathrm{C}$ & -3.62431400 & 1.73345000 & -0.00781400 \\
\hline $\mathrm{C}$ & -3.69086700 & 3.12493000 & -0.01012000 \\
\hline $\mathrm{C}$ & -2.51181900 & 3.86023000 & -0.03537500 \\
\hline $\mathrm{C}$ & -1.25423400 & 3.23925300 & -0.04945200 \\
\hline $\mathrm{C}$ & 0.00001700 & 4.10455900 & -0.06734500 \\
\hline $\mathrm{H}$ & 4.54569800 & 1.15835700 & 0.01871400 \\
\hline $\mathrm{H}$ & 4.65778800 & 3.63166700 & 0.00856100 \\
\hline $\mathrm{H}$ & 2.56323200 & 4.95001600 & -0.03969600 \\
\hline $\mathrm{H}$ & -4.54568500 & 1.15839400 & 0.01879300 \\
\hline $\mathrm{H}$ & -4.65775600 & 3.63170500 & 0.00865000 \\
\hline $\mathrm{H}$ & -2.56319000 & 4.95003700 & -0.03965300 \\
\hline $\mathrm{C}$ & 0.00002400 & 5.01946500 & 1.18117400 \\
\hline $\mathrm{C}$ & 0.00001800 & 4.96461400 & -1.35427100 \\
\hline $\mathrm{H}$ & 0.00002500 & 4.41655900 & 2.10122800 \\
\hline $\mathrm{H}$ & 0.88711900 & 5.66894100 & 1.19594300 \\
\hline $\mathrm{H}$ & -0.88706800 & 5.66894500 & 1.19594800 \\
\hline $\mathrm{H}$ & 0.88833700 & 5.61170200 & -1.39626200 \\
\hline $\mathrm{H}$ & 0.00001300 & 4.32265900 & -2.24747100 \\
\hline $\mathrm{H}$ & -0.88829500 & 5.61171100 & -1.39625700 \\
\hline $\mathrm{C}$ & 3.22661300 & -1.39470000 & -1.52427800 \\
\hline $\mathrm{C}$ & 3.10433200 & -1.28023600 & 1.65072800 \\
\hline $\mathrm{C}$ & -3.10436100 & -1.28019500 & 1.65070400 \\
\hline $\mathrm{C}$ & -3.22660900 & -1.39469400 & -1.52429900 \\
\hline $\mathrm{C}$ & 2.71570500 & -2.73777900 & 1.95829700 \\
\hline $\mathrm{H}$ & 3.18262200 & -3.44981200 & 1.26590900 \\
\hline $\mathrm{H}$ & 3.05735100 & -2.98716100 & 2.97725200 \\
\hline $\mathrm{H}$ & 1.63009700 & -2.88829400 & 1.89589200 \\
\hline
\end{tabular}




\begin{tabular}{|c|c|c|c|}
\hline $\begin{array}{c}\mathrm{Cl} \\
(t \mathrm{Bu}-\end{array}$ & -0.00001000 & -3.36755400 & -0.21811300 \\
\hline $\mathrm{Rh}$ & 0.00000500 & -1.22448000 & 0.08864800 \\
\hline $\mathrm{P}$ & -2.24010200 & -0.96447800 & 0.01714000 \\
\hline $\mathrm{O}$ & -0.00000700 & 1.02015100 & 0.07732100 \\
\hline $\mathrm{P}$ & 2.24010800 & -0.96445100 & 0.01713000 \\
\hline $\mathrm{C}$ & -1.21340900 & 1.68707100 & 0.01391000 \\
\hline $\mathrm{C}$ & -2.38441700 & 0.89192300 & -0.01672900 \\
\hline $\mathrm{C}$ & -3.61402800 & 1.55694300 & -0.10348200 \\
\hline $\mathrm{C}$ & -3.68997700 & 2.94860000 & -0.14082700 \\
\hline $\mathrm{C}$ & -2.51844400 & 3.69686000 & -0.09487400 \\
\hline $\mathrm{C}$ & -1.25777100 & 3.08658600 & -0.02311800 \\
\hline $\mathrm{C}$ & 1.21338800 & 1.68708600 & 0.01391600 \\
\hline $\mathrm{C}$ & 2.38440500 & 0.89195100 & -0.01671900 \\
\hline $\mathrm{C}$ & 3.61401000 & 1.55698500 & -0.10343500 \\
\hline $\mathrm{C}$ & 3.68994400 & 2.94864300 & -0.14077200 \\
\hline $\mathrm{C}$ & 2.51840200 & 3.69689000 & -0.09483900 \\
\hline $\mathrm{C}$ & 1.25773500 & 3.08660100 & -0.02310500 \\
\hline $\mathrm{C}$ & -0.00002400 & 3.95074800 & 0.03774700 \\
\hline $\mathrm{H}$ & -4.52991700 & 0.97320000 & -0.14664200 \\
\hline $\mathrm{H}$ & -4.65966100 & 3.44650300 & -0.20521800 \\
\hline $\mathrm{H}$ & -2.58089000 & 4.78591500 & -0.11648000 \\
\hline $\mathrm{H}$ & 4.52990700 & 0.97325200 & -0.14657600 \\
\hline $\mathrm{H}$ & 4.65962400 & 3.44655700 & -0.20513900 \\
\hline $\mathrm{H}$ & 2.58083600 & 4.78594500 & -0.11643600 \\
\hline $\mathrm{C}$ & -0.00002500 & 4.93705700 & -1.15365700 \\
\hline $\mathrm{C}$ & -0.00003500 & 4.73687800 & 1.37148900 \\
\hline $\mathrm{H}$ & -0.00001500 & 4.39061500 & -2.10829000 \\
\hline $\mathrm{H}$ & -0.88487800 & 5.58877200 & -1.13033500 \\
\hline $\mathrm{H}$ & 0.88481700 & 5.58878700 & -1.13032500 \\
\hline $\mathrm{H}$ & -0.89076900 & 5.37852200 & 1.44876100 \\
\hline $\mathrm{H}$ & -0.00003500 & 4.04563800 & 2.22719200 \\
\hline $\mathrm{H}$ & 0.89069000 & 5.37853300 & 1.44877000 \\
\hline $\mathrm{C}$ & -3.21433400 & -1.48114500 & 1.57552700 \\
\hline $\mathrm{C}$ & -3.09606500 & -1.53248100 & -1.59809600 \\
\hline $\mathrm{C}$ & 3.09607500 & -1.53245400 & -1.59810400 \\
\hline $\mathrm{C}$ & 3.21435900 & -1.48111900 & 1.57549900 \\
\hline $\mathrm{C}$ & -2.63523600 & -2.97880800 & -1.85731300 \\
\hline $\mathrm{H}$ & -3.02135800 & -3.68123900 & -1.10615700 \\
\hline $\mathrm{H}$ & -3.00547600 & -3.30559300 & -2.84426900 \\
\hline $\mathrm{H}$ & -1.53866700 & -3.04352400 & -1.84327600 \\
\hline $\mathrm{C}$ & -4.62749500 & -1.47499300 & -1.63556300 \\
\hline $\mathrm{H}$ & -5.02042200 & -0.46050600 & -1.47903000 \\
\hline $\mathrm{H}$ & -4.97648600 & -1.80386900 & -2.62972100 \\
\hline
\end{tabular}




\begin{tabular}{|c|c|c|}
\hline-5.08493200 & -2.14585600 & -0.89460700 \\
\hline-2.51012200 & -0.63813100 & -2.70500200 \\
\hline-2.79335500 & -1.04294600 & -3.69116600 \\
\hline-2.87985400 & 0.39545400 & -2.64138500 \\
\hline-1.41003300 & -0.61746500 & -2.64229700 \\
\hline-2.32311800 & -1.02349100 & 2.74486900 \\
\hline-2.21645400 & 0.07256300 & 2.76339700 \\
\hline-2.77872900 & -1.33664200 & 3.70016200 \\
\hline-1.31564500 & -1.45841300 & 2.66471000 \\
\hline-4.61825700 & -0.89388700 & 1.77446600 \\
\hline-4.58748200 & 0.19326100 & 1.92901500 \\
\hline-5.29757900 & -1.10978900 & 0.93977900 \\
\hline-5.06356300 & -1.33641800 & 2.68229100 \\
\hline-3.28491000 & -3.01712600 & 1.57317100 \\
\hline-4.00075800 & -3.39454300 & 0.82791500 \\
\hline-2.29848400 & -3.45919700 & 1.36748500 \\
\hline-3.62293500 & -3.36913900 & 2.56255700 \\
\hline 3.28489800 & -3.01710200 & 1.57315200 \\
\hline 3.62295900 & -3.36911700 & 2.56252400 \\
\hline 2.29845100 & -3.45915000 & 1.36751400 \\
\hline 4.00070100 & -3.39454300 & 0.82786400 \\
\hline 4.61830200 & -0.89389000 & 1.77438200 \\
\hline 4.58755800 & 0.19326300 & 1.92890200 \\
\hline 5.06362500 & -1.33640900 & 2.68220500 \\
\hline 5.29759300 & -1.10983200 & 0.93967900 \\
\hline 2.32318700 & -1.02342800 & 2.74485900 \\
\hline 2.77881900 & -1.33657400 & 3.70014400 \\
\hline 2.21654600 & 0.07262900 & 2.76337300 \\
\hline 1.31570200 & -1.45833000 & 2.66473700 \\
\hline 4.62749900 & -1.47482600 & -1.63561400 \\
\hline 5.02033700 & -0.46029300 & -1.47916300 \\
\hline 5.08501600 & -2.14559500 & -0.89462100 \\
\hline 4.97649600 & -1.80374100 & -2.62975700 \\
\hline 2.51001500 & -0.63823100 & -2.70505200 \\
\hline 1.40992700 & -0.61765600 & -2.64231200 \\
\hline 2.87965900 & 0.39538900 & -2.64152200 \\
\hline 2.79324900 & -1.04309100 & -3.69119800 \\
\hline 2.63538000 & -2.97884200 & -1.85721500 \\
\hline 1.53881800 & -3.04366100 & -1.84315800 \\
\hline 3.00563900 & -3.30566100 & -2.84415300 \\
\hline 3.02157900 & -3.68118300 & -1.10601300 \\
\hline 0.00001500 & -2.78569700 & 0.11168000 \\
\hline & 101758 & 1.49364200 \\
\hline
\end{tabular}


$-4.10263500$

$-0.57200700$

0.12634600

$\begin{array}{lll}-6.20204300 & 0.68742600 & 0.26024300\end{array}$

$\begin{array}{lll}-6.49919200 & 0.11598300 & 1.50061900\end{array}$

$\begin{array}{lll}-5.61162200 & -0.82035600 & 2.04274100\end{array}$

$\begin{array}{lll}-6.89449100 & 1.40274300 & -0.19263400\end{array}$

$\begin{array}{lll}-7.41521800 & 0.38606500 & 2.03197100\end{array}$

$-5.83015700 \quad-1.28746100 \quad 3.00710900$

$\begin{array}{lll}-1.72240100 & -0.63154100 & -2.84615300\end{array}$

$-1.32070100 \quad-1.64476000 \quad-2.74932000$

$\begin{array}{lll}0.51261900 & 0.33876700 & -2.82856600\end{array}$

$0.71407800 \quad-0.00711400 \quad-1.67575100$

$\begin{array}{lll}1.04222800 & 1.91974900 & -4.74018400\end{array}$

$2.28741500 \quad-0.24789500 \quad-4.40796400$

$\begin{array}{lll}0.32562300 & 1.42102300 & -5.40715700\end{array}$

$1.86196700 \quad 2.32488800 \quad-5.35418800$

$\begin{array}{lll}0.52789800 & 2.76683900 & -4.26191600\end{array}$

$3.10300700 \quad 0.11302900 \quad-5.05467300$

$1.56875000 \quad-0.79554100 \quad-5.03729900$

$\begin{array}{lll}2.71557100 & -0.95044300 & -3.67634700\end{array}$

$\begin{array}{lll}-0.63659400 & 0.16077100 & -3.46039000\end{array}$

$\begin{array}{lll}-4.83555200 & 0.77159300 & -1.40082000\end{array}$

$\begin{array}{lll}-2.65536400 & 0.87080600 & -1.65506700\end{array}$

$\begin{array}{lll}-2.82228500 & -0.61619000 & -3.89354000\end{array}$

$\begin{array}{lll}-3.65382600 & -1.25284200 & -3.55825500\end{array}$

$\begin{array}{lll}-2.45856600 & -0.98866700 & -4.86446700\end{array}$

$\begin{array}{lll}-3.20853100 & 0.40577300 & -4.03439800\end{array}$

$\begin{array}{lll}1.61303500 & 0.94798000 & -3.69820600\end{array}$

$2.62995200 \quad 1.65523100 \quad-2.79202000$

$3.45873700 \quad 2.05210200 \quad-3.39879500$

$\begin{array}{lll}3.04333900 & 0.96574300 & -2.04405700\end{array}$

$2.17068600 \quad 2.49420700 \quad-2.24867300$

$2.91725600 \quad-3.06038900 \quad-0.02496900$

$2.01352400 \quad-2.05076100 \quad 0.35172500$

$\begin{array}{lll}2.60017200 & -0.83953500 & 0.75853200\end{array}$

$3.99016700 \quad-0.62381200 \quad 0.80634400$

$4.83511000 \quad-1.64892600 \quad 0.37975700$

$\begin{array}{lll}4.29676500 & -2.86628500 & -0.03445500\end{array}$

$\begin{array}{lll}3.51960000 & 1.78589600 & 1.00142900\end{array}$

$2.15551900 \quad 1.46429300 \quad 0.93808200$ 
$1.14251800 \quad 2.41137500 \quad 0.70678700$

$\begin{array}{lll}1.57237500 & 3.73800700 & 0.54123900\end{array}$

$2.92382500 \quad 4.08489600 \quad 0.55002900$

$3.89684200 \quad 3.11223600$

0.77438200

$\mathrm{H}$

$\begin{array}{lll}2.55273400 & -4.03179600 & -0.32887000\end{array}$

$5.91618900 \quad-1.51085200 \quad 0.39305800$

$\begin{array}{lll}4.95438800 & -3.67864500 & -0.35241600\end{array}$

$\begin{array}{lll}0.84609800 & 4.52586300 & 0.40125400\end{array}$

$3.21559400 \quad 5.12696900 \quad 0.39885500$

$\begin{array}{lll}4.94898800 & 3.39608500 & 0.80213700\end{array}$

$\begin{array}{lll}4.48664700 & 0.68264500 & 1.42297000\end{array}$

$5.93363900 \quad 0.99828000 \quad 1.03139600$

$\begin{array}{lll}6.27484400 & 1.92450100 & 1.51485800\end{array}$

$6.04708500 \quad 1.11300900 \quad-0.05742800$

$\begin{array}{lll}6.61318800 & 0.20312000 & 1.36929100\end{array}$

$\begin{array}{lll}4.40744700 & 0.54330300 & 2.96657000\end{array}$

$5.05860900 \quad-0.27533700 \quad 3.31167600$

$\begin{array}{lll}3.38086700 & 0.32544200 & 3.29565500\end{array}$

$\begin{array}{lll}4.72841300 & 1.47836500 \quad 3.45220800\end{array}$

$1.76605700 \quad 0.16883100 \quad 1.15003700$

$\begin{array}{lll}-0.08304200 & -3.01100700 & 2.12527500\end{array}$

$\begin{array}{lll}1.01164400 & -3.98874400 & 2.57304400\end{array}$

$2.00138100 \quad-3.51102100 \quad 2.58598200$

$\begin{array}{lll}1.07683700 & -4.89178600 & 1.95803200\end{array}$

$0.79198600 \quad-4.31518800 \quad 3.60501100$

$\begin{array}{lll}-0.06395200 & -1.83452000 & 3.10313300\end{array}$

$-0.21627500 \quad-2.20178900 \quad 4.13267600$

$\begin{array}{lll}-0.85740400 & -1.11436600 & 2.87914000\end{array}$

$\begin{array}{lll}0.89922500 & -1.30858400 & 3.07732000\end{array}$

$\begin{array}{lll}-1.46216400 & -3.67862100 & 2.20814900\end{array}$

$-2.24586600 \quad-3.01901800 \quad 1.81299400$

$\begin{array}{lll}-1.70714300 & -3.89207600 \quad 3.26281400\end{array}$

$\begin{array}{lll}-1.51017100 \quad-4.63077700 & 1.66429300\end{array}$

$\begin{array}{lll}-0.09146700 & -3.74097000 & -0.88371200\end{array}$

$\begin{array}{lll}-1.58255000 & -3.93046400 & -1.23205700\end{array}$

$\begin{array}{lll}-1.69227700 & -4.88271200 & -1.77720700\end{array}$

$\begin{array}{lll}-1.97184900 & -3.15257000 & -1.89235200\end{array}$

$\begin{array}{lll}-2.22971200 & -3.98634400 & -0.34866800\end{array}$

$0.35432000 \quad-5.14221000 \quad-0.40983800$

$0.27847500 \quad-5.82768400 \quad-1.27159400$

$\begin{array}{lll}-0.31128100 & -5.53906000 & 0.36727100\end{array}$

$1.37712100 \quad-5.22248200 \quad-0.03357700$

$\begin{array}{lll}0.64286300 & -3.39669300 & -2.19674200\end{array}$

$1.72468800 \quad-3.56174700 \quad-2.14234700$ 
$0.49538600 \quad-2.35082000 \quad-2.48630000$

$\begin{array}{lll}0.25323100 & -4.03252400 & -3.00989900\end{array}$

S48

$\begin{array}{lll}-1.51815100 & 3.24166600 & -0.36601500\end{array}$

$\begin{array}{lll}-1.32773700 & 4.74920600 & -0.05610800\end{array}$

$\begin{array}{lll}-0.48199800 & 5.18454600 & -0.60328700\end{array}$

$\begin{array}{lll}-1.22158000 \quad 4.99676100 & 1.00274800\end{array}$

$\begin{array}{lll}-2.22226300 & 5.28082800 & -0.42378300\end{array}$

$\begin{array}{lll}-3.02207900 & 2.95821200 & -0.20023000\end{array}$

$\begin{array}{lll}-3.58101500 & 3.36150800 & -1.06179100\end{array}$

$\begin{array}{lll}-3.42279100 & 3.44263400 & 0.70152900\end{array}$

$\begin{array}{lll}-3.25374800 & 1.89623100 & -0.11874700\end{array}$

$-1.07777500 \quad 3.06909300 \quad-1.82961300$

$\begin{array}{lll}-1.57175300 & 3.83205700 & -2.45625200\end{array}$

$\begin{array}{lll}-1.33211200 & 2.09373100 & -2.24264100\end{array}$

$0.00828200 \quad 3.21295500 \quad-1.93039000$

$\begin{array}{lll}-1.14706800 & 2.06337500 \quad 2.48517200\end{array}$

$\begin{array}{lll}-1.45663300 \quad 3.49630000 & 2.92730800\end{array}$

$\begin{array}{lll}-1.66894900 \quad 3.48924000 & 4.01131100\end{array}$

$\begin{array}{lll}-2.33865800 & 3.91953300 \quad 2.43130600\end{array}$

$\begin{array}{lll}-0.60011500 & 4.16887400 \quad 2.77243600\end{array}$

$\begin{array}{lll}-2.38769300 & 1.18652400 \quad 2.70717900\end{array}$

$\begin{array}{lll}-3.27235600 & 1.58497300 \quad 2.19358100\end{array}$

$\begin{array}{lll}-2.62577300 \quad 1.12475500 & 3.78313500\end{array}$

$\begin{array}{lll}-2.23214300 & 0.17125600 & 2.32338800\end{array}$

$0.01222000 \quad 1.57441200 \quad 3.36972500$

$\begin{array}{lll}0.83501200 & 2.30363800 \quad 3.38668200\end{array}$

$\begin{array}{lll}0.41992300 & 0.61818800 & 3.03922600\end{array}$

$\begin{array}{lll}-0.34796900 & 1.45199500 \quad 4.40559600\end{array}$

$\begin{array}{lll}-0.62600900 & 1.81511600 \quad 0.62875400\end{array}$

$\begin{array}{lll}0.13932800 & -2.22185600 & 0.36497500\end{array}$

$\begin{array}{lll}-0.99341900 & -0.29921700 & -0.23193500\end{array}$

\begin{tabular}{lll}
2.14990600 & 1.52437300 & -1.59081900 \\
2.76640400 & 1.75562700 & -0.31015600 \\
3.94980700 & 0.99574500 & 0.15828400 \\
4.79004700 & 0.27975400 & -0.71795200 \\
3.71031000 & 1.58562000 & 2.22030200 \\
2.81278400 & 2.79454400 & 0.03705600 \\
4.32549500 & 1.02124400 & 1.51757400 \\
5.91651300 & -0.40646800 & -0.25659200 \\
6.25402300 & -0.38638400 & 1.09882000 \\
5.45159200 & 0.34500500 & 1.98284100 \\
6.54111700 & -0.95438500 & -0.96795900 \\
\hline 7.13371100 & -0.92386400 & 1.46137100
\end{tabular}


$\begin{array}{lll}1.67069800 & 2.65848800 & -2.44153200\end{array}$

$\begin{array}{lll}1.45116700 & 3.54103700 & -1.82002700\end{array}$

$\begin{array}{lll}-0.67592600 & 1.23294700 & -1.49642600\end{array}$

$\begin{array}{lll}-1.37704200 & 1.06324000 & -4.92448200\end{array}$

$\begin{array}{lll}-2.37623300 & 3.06629700 & -3.75287900\end{array}$

$\begin{array}{lll}-0.60882600 & 1.69678700 & -5.38944200\end{array}$

$\begin{array}{lll}-2.23706700 \quad 1.00010800 & -5.60971800\end{array}$

$\begin{array}{lll}-0.96177500 & 0.05006300 & -4.80673500\end{array}$

$\begin{array}{lll}-3.25757600 & 3.05068900 & -4.41342000\end{array}$

$\begin{array}{lll}-1.61940900 \quad 3.72637500 & -4.20129900\end{array}$

$\begin{array}{lll}-2.68423900 & 3.49841000 & -2.78718100\end{array}$

$\begin{array}{lll}0.38818600 & 2.35915900 & -3.14868900\end{array}$

$\begin{array}{lll}4.56931000 & 0.28453400 & -1.78614200\end{array}$

$\begin{array}{lll}2.47370600 & 0.65601600 \quad-2.17797700\end{array}$

$2.61041000 \quad 3.04933900 \quad-3.56955400$

$\begin{array}{lll}3.56708100 & 3.38171100 & -3.13928300\end{array}$

$2.19536100 \quad 3.86466300 \quad-4.18329300$

$2.80841200 \quad 2.18358200 \quad-4.22133600$

$\begin{array}{lll}-1.83170900 & 1.63520300 & -3.57030900\end{array}$

$\begin{array}{lll}-2.90352900 & 0.73661500 & -2.94453500\end{array}$

$\begin{array}{lll}-3.77327700 & 0.66887900 & -3.61622900\end{array}$

$\begin{array}{lll}-3.24161500 & 1.12693200 & -1.97484300\end{array}$

$\begin{array}{lll}-2.51501400 & -0.27781800 \quad-2.77619200\end{array}$

$\begin{array}{lll}-3.24662800 & 2.15103900 \quad 1.61471200\end{array}$

$\begin{array}{lll}-2.19921300 & 1.27425900 & 1.30223400\end{array}$

$\begin{array}{lll}-2.56278100 & -0.01611300 & 0.83381800\end{array}$

$\begin{array}{lll}-3.90986500 & -0.41072000 & 0.73551900\end{array}$

$\begin{array}{lll}-4.90824100 & 0.52793900 & 1.02420500\end{array}$

$\begin{array}{lll}-4.58717900 \quad 1.80652700 & 1.45712600\end{array}$

$\begin{array}{lll}-3.09588400 & -2.72978900 & 0.25801200\end{array}$

$\begin{array}{lll}-1.78924700 & -2.21211900 & 0.28670500\end{array}$

$\begin{array}{lll}-0.66085400 & -3.04573900 & 0.10105400\end{array}$

$\begin{array}{lll}-0.88513500 & -4.42410300 & -0.02824400\end{array}$

$-2.16876400 \quad-4.96504700 \quad-0.02074300$

$\begin{array}{lll}-3.26063700 & -4.11176200 & 0.09945800\end{array}$

$\begin{array}{lll}-3.02227900 & 3.13741400 & 1.99991500\end{array}$

$\begin{array}{lll}-5.95601000 & 0.24274500 & 0.91900200\end{array}$

$\begin{array}{lll}-5.37155300 & 2.52894800 \quad 1.69299200\end{array}$

$\begin{array}{lll}-0.03427100 & -5.09025600 & -0.14773900\end{array}$

$-2.31626200 \quad-6.04212400 \quad-0.12631700$

$\begin{array}{lll}-4.26878600 & -4.52728600 & 0.06907900\end{array}$

$-4.32203200 \quad-1.82754000 \quad 0.34095000$ 


\begin{tabular}{|c|c|c|c|}
\hline $\mathrm{C}$ & -5.01814800 & -1.80108600 & -1.04051000 \\
\hline $\mathrm{H}$ & -5.34547300 & -2.81118800 & -1.33187800 \\
\hline $\mathrm{H}$ & -4.33466300 & -1.42738200 & -1.81554800 \\
\hline $\mathrm{H}$ & -5.90476900 & -1.14823400 & -1.02233000 \\
\hline $\mathrm{C}$ & -5.30496000 & -2.38136100 & 1.39914100 \\
\hline $\mathrm{H}$ & -6.20655400 & -1.75719700 & 1.47219500 \\
\hline $\mathrm{H}$ & -4.83164700 & -2.41752900 & 2.39199300 \\
\hline $\mathrm{H}$ & -5.63845700 & -3.39636100 & 1.14072000 \\
\hline $\mathrm{O}$ & -1.55283400 & -0.87689400 & 0.49937400 \\
\hline $\mathrm{C}$ & -0.08792500 & 1.14066200 & 3.30142900 \\
\hline $\mathrm{C}$ & -1.24564200 & 1.33546500 & 4.28732000 \\
\hline $\mathrm{H}$ & -2.16229200 & 0.83448100 & 3.94484500 \\
\hline $\mathrm{H}$ & -1.48173300 & 2.39213100 & 4.46749500 \\
\hline $\mathrm{H}$ & -0.97006900 & 0.89091700 & 5.25982900 \\
\hline $\mathrm{C}$ & 0.17751100 & -0.36261900 & 3.13098100 \\
\hline $\mathrm{H}$ & 0.51040200 & -0.78741900 & 4.09317500 \\
\hline $\mathrm{H}$ & 0.95534500 & -0.54240400 & 2.37602500 \\
\hline $\mathrm{H}$ & -0.72247900 & -0.90756900 & 2.81749300 \\
\hline C & 1.19243300 & 1.78433900 & 3.84790600 \\
\hline $\mathrm{H}$ & 2.01519600 & 1.68843300 & 3.12733000 \\
\hline $\mathrm{H}$ & 1.49587700 & 1.26631200 & 4.77328300 \\
\hline $\mathrm{H}$ & 1.06369400 & 2.84707300 & 4.09259300 \\
\hline $\mathrm{C}$ & -0.46223400 & 3.72854500 & 1.58130000 \\
\hline $\mathrm{C}$ & 0.99339700 & 4.21612300 & 1.44645100 \\
\hline $\mathrm{H}$ & 1.01285500 & 5.31529600 & 1.53897500 \\
\hline $\mathrm{H}$ & 1.40075000 & 3.95317900 & 0.46613200 \\
\hline $\mathrm{H}$ & 1.66363200 & 3.80384200 & 2.21030800 \\
\hline $\mathrm{C}$ & -1.03748400 & 4.40960900 & 2.83641300 \\
\hline $\mathrm{H}$ & -1.05438400 & 5.50027300 & 2.66776300 \\
\hline $\mathrm{H}$ & -0.40405800 & 4.23412100 & 3.71649500 \\
\hline $\mathrm{H}$ & -2.05757200 & 4.11285300 & 3.10538100 \\
\hline $\mathrm{C}$ & -1.18562200 & 4.21407600 & 0.30915500 \\
\hline $\mathrm{H}$ & -2.26723100 & 4.03608500 & 0.31441600 \\
\hline $\mathrm{H}$ & -0.76964200 & 3.71736200 & -0.57847500 \\
\hline $\mathrm{H}$ & -1.02401300 & 5.29933100 & 0.19339800 \\
\hline $\mathrm{C}$ & 1.38483300 & -2.67435700 & -1.90393800 \\
\hline $\mathrm{C}$ & 1.10256600 & -4.07537100 & -2.45862500 \\
\hline $\mathrm{H}$ & 0.03762300 & -4.33776700 & -2.39561400 \\
\hline $\mathrm{H}$ & 1.68460100 & -4.86293900 & -1.96383500 \\
\hline $\mathrm{H}$ & 1.37579400 & -4.09353000 & -3.52915800 \\
\hline $\mathrm{C}$ & 2.85183600 & -2.30384300 & -2.15793300 \\
\hline $\mathrm{H}$ & 3.04164900 & -2.23402100 & -3.24340600 \\
\hline $\mathrm{H}$ & 3.54613400 & -3.05263100 & -1.75059600 \\
\hline $\mathrm{H}$ & 3.10439000 & -1.33794100 & -1.70635000 \\
\hline
\end{tabular}




\begin{tabular}{|c|c|c|}
\hline 0.47939700 & -1.67455000 & -2.64308400 \\
\hline 0.70405200 & -1.69371000 & -3.72387500 \\
\hline 0.63241400 & -0.65727900 & -2.26770200 \\
\hline-0.58513400 & -1.92604900 & -2.51634000 \\
\hline 2.13716400 & -3.44121800 & 1.05749100 \\
\hline 2.65114700 & -4.76420300 & 0.47484000 \\
\hline 3.28418700 & -5.26563600 & 1.22852400 \\
\hline 3.27322800 & -4.61509500 & -0.41805000 \\
\hline 1.84509200 & -5.46565700 & 0.21870400 \\
\hline 3.34832000 & -2.55301800 & 1.39921200 \\
\hline 3.92038000 & -2.26904100 & 0.50607700 \\
\hline 4.03375900 & -3.08940100 & 2.07820600 \\
\hline 3.03939300 & -1.62113300 & 1.89238800 \\
\hline 1.36654400 & -3.73712300 & 2.35453900 \\
\hline 0.55888100 & -4.46690900 & 2.20404700 \\
\hline 0.92114000 & -2.83059900 & 2.78608000 \\
\hline 2.06136400 & -4.15433400 & 3.10426200 \\
\hline 1.03380100 & -2.30854300 & -0.04773900 \\
\hline 0.41496500 & 1.78958000 & 1.53092000 \\
\hline 0.85215300 & 1.24886000 & -0.21403900 \\
\hline 0.77096200 & -0.36921800 & -2.29950600 \\
\hline 1.87407600 & 0.56309300 & -2.17164300 \\
\hline 3.25962500 & 0.21846300 & -1.81704500 \\
\hline 3.59551100 & -0.90166100 & -1.02612800 \\
\hline 4.09456100 & 1.93107400 & -2.83918300 \\
\hline 1.83647900 & 1.43134800 & -2.84774800 \\
\hline 4.32188200 & 1.05176400 & -2.22953600 \\
\hline 4.91510500 & -1.17180700 & -0.67143800 \\
\hline 5.95523200 & -0.32993800 & -1.08690700 \\
\hline 5.64509300 & 0.78518700 & -1.87081600 \\
\hline 5.13526200 & -2.04269100 & -0.04695100 \\
\hline 6.98898200 & -0.53955000 & -0.80103100 \\
\hline 6.44111000 & 1.45634900 & -2.20623200 \\
\hline-0.14799100 & -0.15920300 & -3.47620700 \\
\hline 0.14302300 & 0.76989400 & -3.99287700 \\
\hline-1.95129200 & 1.12790900 & -2.48788800 \\
\hline-1.23461700 & 1.69914400 & -1.67990400 \\
\hline-3.34000800 & 1.64224900 & -2.88599000 \\
\hline-3.68701000 & 2.87007500 & -2.03803700 \\
\hline-4.38748200 & 0.52964000 & -2.71806600 \\
\hline-3.25589400 & 2.04637200 & -4.37317600 \\
\hline-3.01071000 & 3.70875300 & -2.25938400 \\
\hline 3.59450800 & 2.65877500 & -0.96464700 \\
\hline
\end{tabular}




\begin{tabular}{|c|c|c|}
\hline-4.71867400 & 3.19533000 & -2.24458600 \\
\hline-4.10729400 & -0.36510900 & -3.29146600 \\
\hline-5.36830700 & 0.88072600 & -3.07726400 \\
\hline-4.49198600 & 0.23548400 & -1.66665100 \\
\hline .20707400 & 2.50341100 & -4.68990300 \\
\hline-3.06077100 & 1.17385500 & -5.01285900 \\
\hline-2.45489200 & 2.78436400 & -4.54238900 \\
\hline-1.58976800 & 0.05588100 & -3.17266400 \\
\hline 2.79236600 & -1.53700200 & -0.65310400 \\
\hline 0.97080600 & -1.42554900 & -2.10196700 \\
\hline-0.15868900 & -1.31727500 & -4.45645600 \\
\hline-0.79922500 & -1.10921800 & -5.32799000 \\
\hline-0.52999700 & -2.22855300 & -3.96259200 \\
\hline 0.86610300 & -1.51045800 & -4.80733500 \\
\hline 0.54875600 & 0.70457400 & -0.72343700 \\
\hline-0.79839900 & -0.01467700 & 0.73539400 \\
\hline 1.14512500 & 2.19460700 & 0.96532200 \\
\hline-1.25142100 & 0.94027000 & 1.64839300 \\
\hline-1.62825600 & -1.08929000 & 0.40508400 \\
\hline-0.33232700 & 1.94402400 & 2.03913500 \\
\hline 2.65155900 & 1.83694800 & 2.06319900 \\
\hline 1.07467400 & 4.03514900 & 0.45228400 \\
\hline-2.55813400 & 0.90238500 & 2.15091200 \\
\hline-3.01740300 & -0.98262300 & 0.56129500 \\
\hline-1.03234200 & -2.29428400 & -0.04533500 \\
\hline-0.71160900 & 2.81705200 & 3.06561900 \\
\hline 2.61463300 & 0.31680600 & 2.26937200 \\
\hline 3.91607900 & 2.20892100 & 1.27608600 \\
\hline 2.65160900 & 2.51668100 & 3.43732500 \\
\hline 1.61904200 & 5.06148700 & 1.45175300 \\
\hline-0.39316900 & 4.36198500 & 0.12566000 \\
\hline 1.88250400 & 4.11745100 & -0.85763300 \\
\hline-2.89345500 & 1.81420000 & 3.16480600 \\
\hline-3.62163900 & -0.05153700 & 1.61356000 \\
\hline-3.82116900 & -1.92522800 & -0.09640900 \\
\hline-1.87687600 & -3.19353900 & -0.71056700 \\
\hline 0.65133100 & -2.75591500 & 0.61506500 \\
\hline-1.97768500 & 2.74333000 & 3.64673600 \\
\hline-0.01835300 & 3.59092400 & 3.39154600 \\
\hline 2.56799800 & -0.22364800 & 1.31611600 \\
\hline 1.73891100 & 0.02805100 & 2.86545000 \\
\hline 3.51722900 & -0.00964100 & 2.81368600 \\
\hline 4.04137100 & 3.29718600 & 1.18111800 \\
\hline 3.91116200 & 1.76980600 & 0.27005500 \\
\hline
\end{tabular}


$4.80340000 \quad 1.81938400 \quad 1.80258000$

$2.66552900 \quad 3.61181200 \quad 3.37485100$

$3.55727100 \quad 2.20881100$

3.98881900

$\begin{array}{lll}1.78600800 & 2.21204800 \quad 4.04347100\end{array}$

$\begin{array}{lll}2.69176400 & 4.92822900 & 1.64792800\end{array}$

$\begin{array}{lll}1.08565500 & 5.04190200 & 2.41253600\end{array}$

$\begin{array}{lll}1.48944000 & 6.07437900 & 1.03180900\end{array}$

$\begin{array}{lll}-0.84583400 & 3.59445900 & -0.51697000\end{array}$

$\begin{array}{lll}-0.43427200 & 5.32533300 & -0.41144900\end{array}$

$\begin{array}{lll}-1.00873300 & 4.45999000 & 1.03062100\end{array}$

$\begin{array}{lll}2.94037300 & 3.85389300 & -0.71948300\end{array}$

$1.84127000 \quad 5.14779000 \quad-1.25113600$

$\begin{array}{lll}1.46577500 & 3.43723200 & -1.61279100\end{array}$

$\begin{array}{lll}-3.90217200 & 1.79501400 & 3.58040700\end{array}$

$\begin{array}{lll}-4.81424400 & 0.75814700 & 1.06822800\end{array}$

$\begin{array}{lll}-4.12350200 & -0.95859400 & 2.76776900\end{array}$

$-3.25270200 \quad-2.97654000 \quad-0.80743900$

$\begin{array}{lll}-4.90723200 & -1.84435700 & -0.02577500\end{array}$

$-1.46754200 \quad-4.11356800 \quad-1.11820900$

$0.03958100 \quad-3.37205100 \quad 2.35671800$

$\begin{array}{lll}1.23588200 & -4.32164900 & -0.36412200\end{array}$

$\begin{array}{lll}-2.26020400 \quad 3.43282400 & 4.44511700\end{array}$

$\begin{array}{lll}-5.58386400 & 0.09509800 & 0.64681700\end{array}$

$\begin{array}{lll}-4.49823000 & 1.45926000 & 0.28709300\end{array}$

$\begin{array}{lll}-5.29018500 & 1.33963300 & 1.87122000\end{array}$

$\begin{array}{lll}-4.56031100 & -0.35758600 & 3.57944600\end{array}$

$\begin{array}{lll}-3.29963300 & -1.55352600 & 3.18700600\end{array}$

$\begin{array}{lll}-4.89721700 & -1.65305300 \quad 2.40813300\end{array}$

$\begin{array}{lll}-3.88364500 & -3.68278600 & -1.35129400\end{array}$

$\begin{array}{lll}-1.24199200 & -4.21566100 & 2.35747800\end{array}$

$1.18290000 \quad-4.14099800 \quad 3.03686900$

$\begin{array}{lll}-0.22866700 & -2.11166300 \quad 3.19416200\end{array}$

$0.50527300 \quad-5.65431500 \quad-0.13261800$

$\begin{array}{lll}2.71413100 & -4.51735900 & 0.03839600\end{array}$

$\begin{array}{lll}1.23043200 & -3.99333600 & -1.86760300\end{array}$

$-1.48320500 \quad-4.50451300 \quad 3.39607600$

$\begin{array}{lll}-1.15224600 & -5.13937000 & 1.77422100\end{array}$

$\begin{array}{lll}-2.10091600 & -3.65307400 & 1.96712700\end{array}$

$\begin{array}{lll}1.35710900 & -5.12716000 & 2.58681000\end{array}$

$\begin{array}{lll}0.92758800 & -4.30882000 & 4.09737600\end{array}$

$2.12766200 \quad-3.57567500 \quad 3.01069200$

$\begin{array}{lll}-1.05239700 & -1.50952600 & 2.79305100\end{array}$

$0.65815500 \quad-1.47059100 \quad 3.26959700$

$\begin{array}{lll}-0.51882200 & -2.40861500 \quad 4.21674400\end{array}$ 
$0.58567900 \quad-5.99747800 \quad 0.90672300$

$0.97416500 \quad-6.43011400 \quad-0.76308600$

$\begin{array}{lll}-0.55999900 & -5.62728200 & -0.39533000\end{array}$ 
$1.40983500 \quad 1.12893900 \quad-4.83617900$

$\begin{array}{lll}0.72516600 & 1.94612800 & -3.40389200\end{array}$

0.36160700

$-0.40673600$

0.68389100

$-0.54239100$

1.34899200

1.12960200

0.74371300

$-2.12696800$

0.16863400

2.55523700

2.45938700

0.18037200

1.31327800

$-2.70341100$

$-3.11931400$

$-1.92870800$

0.12585300

1.61614500

$-0.59464900$

3.09931800

3.30057600

2.92243000

0.69088100

$-1.60104000$

2.47344000

0.86263600

$-2.83495200$

$-2.04910200$

$-3.68416000$

$-2.83011500$

$-3.22426300$

$-4.11387000$

$-1.48520700$

2.91181300

$-1.30320200$

$-0.90285400$

0.67420200

$0.60190500 \quad-5.63805400$

$1.64224800-2.86155800$

$2.03150600 \quad-4.51507200$

$2.26910300 \quad-3.15719400$

$-1.65858100 \quad-4.57339200$
$-4.64000700$

0.74449500

1.35485600

0.21981100

2.38196200

2.40979300

1.14516500

2.28355400

0.39837100

$-0.43201100$

3.55497000

2.34002400

1.74956600

3.88582200

2.36488300

0.67653100

$-0.01280500$

3.44376000

1.55846600

$-0.38799200$

$-1.19359200$

0.06376600

4.09695500

4.04092400

1.30731900

2.85661200

2.84508600

1.89462000

0.67261100

2.20717800

4.02721200

4.38825300

4.40696700

2.67731500

3.22873100

2.09880500

$-0.17832800$

0.33949700

1.46768700

0.20354300 
$-0.14678200 \quad-5.40824100 \quad-0.21223400$

$\begin{array}{lll}-0.51238900 & -3.82145800 & -0.92580000\end{array}$

$\begin{array}{lll}4.03654300 & 0.37089500 & 3.84141700\end{array}$

$4.67300400 \quad 1.15317400 \quad 1.08471600$

$\begin{array}{lll}3.52162700 & 2.85273100 & 2.53737000\end{array}$

$2.06071700 \quad 3.97188400 \quad-1.23207700$

$3.96814200 \quad 3.58141800 \quad-0.31356300$

$\begin{array}{lll}0.01224500 & 4.34612400 & -1.74309800\end{array}$

$\begin{array}{lll}-1.50729200 & 3.44779400 & 1.71352300\end{array}$

$\begin{array}{lll}-2.68294500 & 3.42408200 & -1.18441000\end{array}$

$2.90686900 \quad-1.49513800 \quad 5.00920300$

$\begin{array}{lll}5.21546600 & 1.93100900 & 0.52688900\end{array}$

$\begin{array}{lll}4.56389100 & 0.27849700 & 0.43499500\end{array}$

$\begin{array}{lll}5.29717600 & 0.86465600 & 1.94356300\end{array}$

$\begin{array}{lll}4.09871300 & 2.53252800 \quad 3.41756100\end{array}$

$\begin{array}{lll}2.56130900 \quad 3.26013200 & 2.88601900\end{array}$

$\begin{array}{lll}4.07997200 & 3.66454400 \quad 2.04869200\end{array}$

$\begin{array}{lll}2.43886900 & 4.78284300 & -1.85821400\end{array}$

$\begin{array}{lll}-0.86548700 & 4.83955900 & 1.62818600\end{array}$

$\begin{array}{lll}-2.93395600 \quad 3.55667300 & 2.27479400\end{array}$

$\begin{array}{lll}-0.66296900 & 2.62583600 & 2.70245000\end{array}$

$\begin{array}{lll}-2.62542400 & 4.95761400 & -1.13652300\end{array}$

$\begin{array}{lll}-4.13756900 & 2.99420000 \quad-0.89732600\end{array}$

$\begin{array}{lll}-2.34886000 & 2.94724200 & -2.60837200\end{array}$

$\begin{array}{lll}-0.76329900 & 5.24981700 \quad 2.64866800\end{array}$

$\begin{array}{lll}-1.45874000 & 5.55661700 \quad 1.05087900\end{array}$

$0.14284000 \quad 4.80017600 \quad 1.19178400$

$\begin{array}{lll}-3.56344100 \quad 4.23668500 & 1.68459800\end{array}$

$\begin{array}{lll}-2.89553700 \quad 3.95985800 & 3.30132400\end{array}$

$\begin{array}{lll}-3.43328100 & 2.57578800 & 2.31972600\end{array}$

$\begin{array}{lll}0.40389300 & 2.65682100 \quad 2.44936700\end{array}$

$\begin{array}{lll}-0.97025000 & 1.57598600 & 2.75331500\end{array}$

$-0.76440100 \quad 3.05800900 \quad 3.71220200$

$\begin{array}{lll}-3.02771700 & 5.35481500 & -0.19531900\end{array}$

$\begin{array}{lll}-3.25287400 & 5.36362200 & -1.94921400\end{array}$

$\begin{array}{lll}-1.61479000 & 5.36382000 & -1.27050000\end{array}$

$\begin{array}{lll}-4.80532900 & 3.44060900 & -1.65401200\end{array}$

$\begin{array}{lll}-4.49217000 & 3.32361000 & 0.08781600\end{array}$

$\begin{array}{lll}-4.25759700 & 1.90388700 & -0.95109100\end{array}$

$\begin{array}{lll}-3.00987800 & 3.46533100 & -3.32379500\end{array}$

$\begin{array}{lll}-2.52754300 & 1.87074800 & -2.72833000\end{array}$

$\begin{array}{lll}-1.31264300 & 3.15165300 & -2.90359700\end{array}$

3-base complex

C

$-3.53714600 \quad 4.06786000 \quad-1.30188200$ 


\begin{tabular}{|c|c|c|}
\hline-3.49976600 & 2.78769900 & -0.72654100 \\
\hline-4.51980600 & 2.50267300 & 0.19968800 \\
\hline-5.46052300 & 3.44038700 & 0.64772800 \\
\hline-5.46308300 & 4.69663200 & 0.03281700 \\
\hline-4.52281400 & 4.99557600 & -0.95500600 \\
\hline-6.74218500 & 1.59144600 & 1.65801700 \\
\hline-5.81650200 & 0.73728300 & 1.04237400 \\
\hline-6.10832700 & -0.58721700 & 0.67420000 \\
\hline-7.34482000 & -1.09679900 & 1.10565700 \\
\hline-8.25313100 & -0.29897300 & 1.80270300 \\
\hline-7.97136500 & 1.04818200 & 2.04296200 \\
\hline-2.77890400 & 4.36144500 & -2.02008800 \\
\hline-6.18654300 & 5.45437000 & 0.33482100 \\
\hline-4.52977600 & 5.97761100 & -1.43336800 \\
\hline-7.62899500 & -2.11854800 & 0.86630400 \\
\hline-9.20771900 & -0.72126900 & 2.12476900 \\
\hline-8.71698900 & 1.67432400 & 2.53389300 \\
\hline-6.32659300 & 3.05057800 & 1.85407400 \\
\hline-5.44778800 & 3.14651900 & 3.12784100 \\
\hline-6.03148700 & 2.85547700 & 4.01536100 \\
\hline-4.57406500 & 2.48329500 & 3.06234000 \\
\hline-5.08536200 & 4.17689900 & 3.26763900 \\
\hline-7.53712900 & 3.97465500 & 2.01510500 \\
\hline-7.21373000 & 5.01496800 & 2.16296200 \\
\hline-8.19963100 & 3.93902600 & 1.13691900 \\
\hline-8.12430800 & 3.69993500 & 2.90310800 \\
\hline-4.57687700 & 1.22990100 & 0.71611700 \\
\hline-1.32000600 & 1.70158800 & -2.61097800 \\
\hline-1.04786600 & 3.09273500 & -3.20353600 \\
\hline-1.96807900 & 3.58665900 & -3.54355100 \\
\hline-0.50856800 & 3.75632000 & -2.52054700 \\
\hline-0.41115500 & 2.96692000 & -4.09595000 \\
\hline-2.26768900 & 0.95845800 & -3.56404100 \\
\hline-1.81278800 & 0.91335700 & -4.56853500 \\
\hline-2.44579400 & -0.06914800 & -3.22542600 \\
\hline-3.23875200 & 1.46441600 & -3.66130900 \\
\hline 0.02049500 & 0.94606400 & -2.54593200 \\
\hline-0.07950200 & -0.01779200 & -2.03191800 \\
\hline 0.34749000 & 0.75003900 & -3.58367900 \\
\hline 0.79805400 & 1.55331100 & -2.06104600 \\
\hline-0.97275900 & 2.40320200 & 0.46061900 \\
\hline-0.45766800 & 3.78455800 & 0.03454400 \\
\hline-0.04288600 & 4.28541200 & 0.92727500 \\
\hline & & \\
\hline
\end{tabular}




\begin{tabular}{|c|c|c|c|}
\hline $\mathrm{H}$ & -1.24840700 & 4.43811200 & -0.35659900 \\
\hline $\mathrm{C}$ & -1.81902800 & 2.54518700 & 1.74065500 \\
\hline $\mathrm{H}$ & -2.34348100 & 1.61147000 & 1.99315300 \\
\hline $\mathrm{H}$ & -1.14740400 & 2.79102100 & 2.58005700 \\
\hline $\mathrm{H}$ & -2.56052200 & 3.35096700 & 1.66856200 \\
\hline $\mathrm{C}$ & 0.24316900 & 1.54421300 & 0.82419900 \\
\hline $\mathrm{H}$ & 0.87064100 & 1.29712100 & -0.03638700 \\
\hline $\mathrm{H}$ & 0.86719200 & 2.15594300 & 1.49940100 \\
\hline $\mathrm{H}$ & -0.04177400 & 0.64167800 & 1.36912900 \\
\hline $\mathrm{C}$ & -5.22975200 & -3.27605900 & -0.36593700 \\
\hline $\mathrm{C}$ & -6.53812700 & -3.87736900 & -0.90316100 \\
\hline $\mathrm{H}$ & -7.44602600 & -3.42106400 & -0.49123300 \\
\hline $\mathrm{H}$ & -6.59792000 & -3.82501000 & -1.99820900 \\
\hline $\mathrm{H}$ & -6.56161100 & -4.94723500 & -0.63277900 \\
\hline $\mathrm{C}$ & -4.07846100 & -3.97479200 & -1.11073500 \\
\hline $\mathrm{H}$ & -4.10776200 & -3.79252200 & -2.19278700 \\
\hline $\mathrm{H}$ & -3.09867900 & -3.65606400 & -0.74760700 \\
\hline $\mathrm{H}$ & -4.15855400 & -5.06453800 & -0.95901100 \\
\hline $\mathrm{C}$ & -5.10468000 & -3.60357000 & 1.13053900 \\
\hline $\mathrm{H}$ & -5.00270800 & -4.69446400 & 1.25859600 \\
\hline $\mathrm{H}$ & -4.22052700 & -3.13096100 & 1.56753500 \\
\hline $\mathrm{H}$ & -5.97873500 & -3.27872400 & 1.71036700 \\
\hline $\mathrm{C}$ & -5.86233000 & -0.82225500 & -2.17677500 \\
\hline $\mathrm{C}$ & -7.37738800 & -1.05550100 & -2.23977100 \\
\hline $\mathrm{H}$ & -7.77004800 & -0.55864400 & -3.14418700 \\
\hline $\mathrm{H}$ & -7.65701600 & -2.11163100 & -2.30751400 \\
\hline $\mathrm{H}$ & -7.89651400 & -0.61294100 & -1.37832000 \\
\hline $\mathrm{C}$ & -5.17417500 & -1.52212700 & -3.35742100 \\
\hline $\mathrm{H}$ & -5.39521700 & -2.59770300 & -3.38835800 \\
\hline $\mathrm{H}$ & -5.53024700 & -1.08531100 & -4.30571600 \\
\hline $\mathrm{H}$ & -4.08311100 & -1.39488000 & -3.32691400 \\
\hline $\mathrm{C}$ & -5.63102800 & 0.68899400 & -2.28253900 \\
\hline $\mathrm{H}$ & -6.23973800 & 1.24576400 & -1.55646900 \\
\hline $\mathrm{H}$ & -4.58301600 & 0.94295100 & -2.11107800 \\
\hline $\mathrm{H}$ & -5.91687800 & 1.04139900 & -3.28753600 \\
\hline $\mathrm{C}$ & -1.92252100 & -1.96594100 & 0.92292000 \\
\hline $\mathrm{C}$ & -0.96186500 & -1.50090300 & -0.03556500 \\
\hline $\mathrm{C}$ & -0.52435000 & -2.34494600 & -1.18543600 \\
\hline $\mathrm{C}$ & -1.31444100 & -2.53097400 & -2.33353900 \\
\hline $\mathrm{H}$ & 1.37643000 & -2.82766700 & -0.28550000 \\
\hline $\mathrm{H}$ & -0.13416700 & -0.90618100 & 0.35315900 \\
\hline $\mathrm{C}$ & 0.73966700 & -2.96579500 & -1.16186300 \\
\hline $\mathrm{C}$ & -0.87102700 & -3.29408700 & -3.41516700 \\
\hline $\mathrm{C}$ & 0.38403400 & -3.91077500 & -3.37247500 \\
\hline
\end{tabular}


$\begin{array}{lll}-1.50888700 & -3.40683800 & -4.29598800\end{array}$

$0.73163500 \quad-4.52023900 \quad-4.21062000$

$2.15781100 \quad-4.24421800 \quad-2.17819200$

$\begin{array}{lll}-1.75522200 & -1.49927400 & 2.34588800\end{array}$

$\begin{array}{lll}-0.82220700 & -2.43055300 \quad 3.03388000\end{array}$

$0.46004900 \quad-2.13445600 \quad 3.14708600$

$\begin{array}{lll}1.28186800 & -3.30825300 & 3.69250300\end{array}$

$0.94235200 \quad-1.05391100 \quad 2.84528500$

$\begin{array}{lll}0.72607800 & -3.70925400 & 5.07127300\end{array}$

$\begin{array}{lll}1.14327600 & -4.48364100 & 2.70502500\end{array}$

$2.75181400 \quad-2.88910800 \quad 3.81258600$

$\begin{array}{lll}0.79648200 & -2.87423800 & 5.78710500\end{array}$

$\begin{array}{lll}-0.32807600 & -4.01304200 \quad 5.00211800\end{array}$

$1.30486900 \quad-4.55355700 \quad 5.47823800$

$\begin{array}{lll}1.48189000 & -4.19962000 & 1.69687900\end{array}$

$1.75849500 \quad-5.33259700 \quad 3.04274900$

$\begin{array}{lll}0.09909900 & -4.81712300 & 2.62697300\end{array}$

$3.35097400 \quad-3.72984200 \quad 4.19482800$

$\begin{array}{lll}3.16975800 & -2.59472500 & 2.83924800\end{array}$

$\begin{array}{lll}2.87444700 & -2.04456000 & 4.50805500\end{array}$

$\begin{array}{lll}-2.28925200 & -2.04628400 & -2.36641600\end{array}$

$\begin{array}{lll}-2.71485000 & -0.59106000 & -0.25783800\end{array}$

$-2.11184500 \quad 1.54118700 \quad-0.86511600$

$-4.95855500 \quad-1.37463400 \quad-0.55518400$

$\begin{array}{lll}-2.28862500 & -2.99492600 & 0.84979900\end{array}$

$\begin{array}{lll}-2.99923600 & -1.46632500 \quad 3.21301700\end{array}$

$-3.41853800 \quad-2.47371200 \quad 3.35068500$

$\begin{array}{lll}-3.76544600 & -0.83325600 & 2.74338400\end{array}$

$\begin{array}{lll}-2.76770000 & -1.05696200 \quad 4.20889000\end{array}$

$\begin{array}{lll}-1.27739000 & -0.51375400 \quad 2.36055400\end{array}$

$\begin{array}{lll}3.00112700 & 0.36232300 & 1.88480400\end{array}$

$3.40230500 \quad 0.38342900 \quad-0.75353600$

$5.29815700 \quad 1.61511900 \quad 1.61101100$

$\begin{array}{lll}4.79155700 & -0.16351500 & -0.37115500\end{array}$

$\begin{array}{lll}4.63226600 & -1.26482800 & 0.61518000\end{array}$

$\begin{array}{lll}3.56653700 & 1.88803100 & -1.45468300\end{array}$

$\begin{array}{lll}2.95939200 & -0.84058400 & -2.98230600\end{array}$

$\begin{array}{lll}5.57432000 & 1.30658500 & 0.18847500\end{array}$

$\begin{array}{lll}6.30279000 & 2.49923400 & 2.08522200\end{array}$

$\begin{array}{lll}5.32022000 & -0.91311900 & -1.59038100\end{array}$

$5.25999300 \quad-2.45989800 \quad 0.19223900$

$\begin{array}{lll}4.90711800 & 2.39852600 & -0.72249200\end{array}$

$3.90284800 \quad 1.59848300 \quad-2.94004700$ 


\begin{tabular}{|c|c|c|c|}
\hline $\mathrm{O}$ & 2.35716000 & 2.73660000 & -1.15038700 \\
\hline K & 6.55642300 & 1.37194500 & -2.54361000 \\
\hline $\mathrm{O}$ & 7.04083900 & 1.54017900 & 0.05958100 \\
\hline $\mathrm{C}$ & 7.57775000 & 2.00659100 & 1.29034600 \\
\hline C & 6.40867100 & 2.35122200 & 3.60279500 \\
\hline C & 5.91782300 & 3.94764900 & 1.74657400 \\
\hline $\mathrm{C}$ & 6.03903400 & -2.04673600 & -1.13203800 \\
\hline $\mathrm{C}$ & 4.17130000 & -3.50310900 & -0.07695100 \\
\hline $\mathrm{C}$ & 6.16855600 & -2.96938500 & 1.31394000 \\
\hline $\mathrm{C}$ & 8.26451200 & 0.82623600 & 1.99228600 \\
\hline $\mathrm{C}$ & 8.60463000 & 3.10219400 & 1.00913700 \\
\hline $\mathrm{H}$ & 5.49166400 & 2.73790000 & 4.07713700 \\
\hline $\mathrm{H}$ & 6.52229900 & 1.29926500 & 3.89888000 \\
\hline $\mathrm{H}$ & 7.25826700 & 2.92380300 & 4.00898500 \\
\hline $\mathrm{H}$ & 5.84865800 & 4.09237200 & 0.66176600 \\
\hline $\mathrm{H}$ & 4.91940100 & 4.15114600 & 2.16503500 \\
\hline $\mathrm{H}$ & 6.62381300 & 4.67656500 & 2.17619500 \\
\hline $\mathrm{C}$ & 6.02649700 & -3.11385900 & -2.22877400 \\
\hline C & 7.49591700 & -1.64051900 & -0.86148800 \\
\hline $\mathrm{H}$ & 3.55316300 & -3.61524100 & 0.82484100 \\
\hline $\mathrm{H}$ & 3.51475100 & -3.17093300 & -0.88852100 \\
\hline $\mathrm{H}$ & 4.57624800 & -4.49400800 & -0.33498600 \\
\hline $\mathrm{H}$ & 5.55544900 & -3.23688200 & 2.18887700 \\
\hline $\mathrm{H}$ & 6.73288900 & -3.86653300 & 1.01185600 \\
\hline $\mathrm{H}$ & 6.87933200 & -2.19482000 & 1.62964500 \\
\hline $\mathrm{H}$ & 7.53084800 & 0.04035500 & 2.22148900 \\
\hline $\mathrm{H}$ & 9.02497100 & 0.39958200 & 1.32014600 \\
\hline $\mathrm{H}$ & 8.76738300 & 1.12323900 & 2.92583600 \\
\hline $\mathrm{H}$ & 8.99504900 & 3.54368200 & 1.94078800 \\
\hline $\mathrm{H}$ & 9.45974600 & 2.67854200 & 0.45691900 \\
\hline $\mathrm{H}$ & 8.17214300 & 3.90571300 & 0.39754600 \\
\hline $\mathrm{H}$ & 6.55622200 & -2.73503300 & -3.11787200 \\
\hline $\mathrm{H}$ & 6.53667900 & -4.03459400 & -1.90446600 \\
\hline $\mathrm{H}$ & 5.00659100 & -3.38570800 & -2.53685600 \\
\hline $\mathrm{H}$ & 7.95702100 & -1.30854600 & -1.80820200 \\
\hline $\mathrm{H}$ & 7.54358600 & -0.80333800 & -0.15571800 \\
\hline $\mathrm{H}$ & 8.10528600 & -2.47524200 & -0.48492600 \\
\hline \multicolumn{4}{|c|}{ TS-S51 } \\
\hline $\mathrm{C}$ & -3.44144900 & 4.16205400 & -1.29172300 \\
\hline $\mathrm{C}$ & -3.38958200 & 2.84380400 & -0.81009400 \\
\hline $\mathrm{C}$ & -4.38020800 & 2.47324400 & 0.11665100 \\
\hline $\mathrm{C}$ & -5.25543800 & 3.39142000 & 0.70980100 \\
\hline $\mathrm{C}$ & -5.27261000 & 4.69324500 & 0.19729500 \\
\hline $\mathrm{C}$ & -4.40099100 & 5.06322800 & -0.82874800 \\
\hline
\end{tabular}


$-5.70744700$

$0.67275900 \quad 0.91728500$

$-6.11451600$

$-0.60684600$

0.50507400

$-8.18250800$

$-0.24251100$

1.75246300

$-7.77390300$

1.04938500

2.08258000

$-2.70435900$

4.50228700

$-2.01468100$

$-5.95538100$

$5.43343100 \quad 0.61442100$

$-4.42956500$

6.07905100

$-1.22879900$

$-7.73512600$

$-2.02681500$

0.65832000

$-9.15259800$

$-0.61313800$

2.09075400

$-8.43299200$

1.68823700

2.66999200

$-6.01816100$

2.93659100

1.95622400

$-5.01670400$

2.86376600

3.13859100

$-5.53160300$

2.51511200

4.04723000

$-4.19132200$

2.16985800

2.92953800

$-4.58456300$

3.85631400

3.33835600

$-7.14670000$

$3.90191300 \quad 2.32821600$

$-6.74312900$

4.90331400

2.53414600

$-7.89733200$

3.98704500

1.52802700

$-7.65312000$

$3.57350400 \quad 3.24656900$

$-4.45542500$

1.13761200

0.51392100

$-1.26972200$

$1.70054000 \quad-2.72624900$

$-0.99192400$

$3.05631900 \quad-3.38845200$

$-1.91421900$

3.62342400

$-3.57687900$

$-0.29136400$

$3.67462700 \quad-2.81872900$

$-0.52902400$

$2.86893000 \quad-4.37238800$

$-2.32212700$

$0.96277800 \quad-3.56498900$

$-1.93874600$

$0.82819000 \quad-4.59050800$

$-2.54286700$

$-0.02906100-3.15248900$

$-3.26179600$

$1.52903700 \quad-3.63182500$

0.03725400

$0.88602800 \quad-2.70104900$

$-0.08129600$

$-0.05756900 \quad-2.15125000$

$\begin{array}{lll}0.30578800 & 0.64078100 & -3.74461600\end{array}$

$\begin{array}{lll}0.86576900 & 1.46869800 & -2.27069900\end{array}$

$\begin{array}{lll}-0.76269600 & 2.63099800 & 0.27511900\end{array}$

$\begin{array}{lll}-0.18385200 & 3.93701700 & -0.28086300\end{array}$

$0.27481200 \quad 4.49122900 \quad 0.55663300$

$0.62120100 \quad 3.73671300 \quad-0.99921300$

$\begin{array}{lll}-0.94979900 & 4.59318200 & -0.71554700\end{array}$

$\begin{array}{lll}-1.59279800 & 2.92562200 & 1.53770300\end{array}$

$\begin{array}{lll}-2.14381800 & 2.03482900 & 1.87563600\end{array}$

$\begin{array}{lll}-0.90495300 & 3.21512000 & 2.34905300\end{array}$

$\begin{array}{lll}-2.30663500 & 3.74763500 & 1.39717400\end{array}$ 
$\begin{array}{lll}0.97604100 & 1.34580600 & -0.15195800\end{array}$

$\begin{array}{lll}1.08582500 & 2.34114000 & 1.29239800\end{array}$

$\begin{array}{lll}0.05888500 & 0.89346600 & 1.33367600\end{array}$

$\begin{array}{lll}-5.40279800 & -3.37869900 & -0.39716200\end{array}$

$-6.69358000 \quad-3.98462900 \quad-0.96687500$

$\begin{array}{lll}-7.60950800 & -3.49456100 & -0.61217400\end{array}$

$\begin{array}{lll}-6.70295500 & -3.96815200 & -2.06539100\end{array}$

$-6.75036800 \quad-5.04313100 \quad-0.65888100$

$-4.21891800 \quad-4.12775100 \quad-1.03653900$

$\begin{array}{lll}-4.15909900 & -3.96399600 & -2.12163200\end{array}$

$\begin{array}{lll}-3.25755900 & -3.82977800 & -0.60265500\end{array}$

$\begin{array}{lll}-4.34071600 & -5.21172900 & -0.87204600\end{array}$

$\begin{array}{lll}-5.35413800 & -3.61541000 & 1.12267300\end{array}$

$\begin{array}{lll}-5.22975200 & -4.69416100 \quad 1.31637700\end{array}$

$\begin{array}{lll}-4.51200500 & -3.09570400 & 1.59555400\end{array}$

$-6.26944800 \quad-3.29029700 \quad 1.63321100$

$\begin{array}{lll}-5.96726400 & -0.95057600 & -2.33827200\end{array}$

$\begin{array}{lll}-7.48277900 & -1.16808700 & -2.41717100\end{array}$

$-7.86938400 \quad-0.65345700 \quad-3.31435900$

$\begin{array}{lll}-7.76222100 & -2.22368900 & -2.50500000\end{array}$

$\begin{array}{lll}-8.00520500 & -0.73934100 & -1.55004800\end{array}$

$-5.27295700 \quad-1.65457600 \quad-3.51411600$

$\begin{array}{lll}-5.44547400 & -2.74087900 & -3.50544200\end{array}$

$\begin{array}{lll}-5.66815200 & -1.26506700 \quad-4.46761000\end{array}$

$\begin{array}{lll}-4.18750700 & -1.47959200 & -3.51094600\end{array}$

$\begin{array}{lll}-5.70318600 & 0.56193800 & -2.41628400\end{array}$

$-6.33519100 \quad 1.11997600 \quad-1.71015200$

$\begin{array}{lll}-4.65795700 & 0.80296900 & -2.19695000\end{array}$

$\begin{array}{lll}-5.93683600 & 0.92952200 & -3.42923900\end{array}$

$\begin{array}{lll}-2.10989300 & -1.70396100 & 1.14075500\end{array}$

$\begin{array}{lll}-1.17649500 & -1.29243400 & 0.11297200\end{array}$

$\begin{array}{lll}-0.78197800 & -2.20880200 & -0.99611600\end{array}$

$\begin{array}{lll}-1.59288900 & -2.42879300 & -2.12061600\end{array}$

$\begin{array}{lll}1.11257900 & -2.70114200 & -0.08525000\end{array}$

$\begin{array}{lll}-0.30891000 & -0.75240000 & 0.49817900\end{array}$

$\begin{array}{lll}0.46240100 & -2.86555800 & -0.94728700\end{array}$

$-1.18608900 \quad-3.26035400 \quad-3.16540900$

$0.04714200 \quad-3.91591100 \quad-3.09934500$

$\begin{array}{lll}0.86487700 & -3.72239100 & -1.97841100\end{array}$

$\begin{array}{lll}-1.83728500 & -3.40082700 & -4.03228900\end{array}$

$0.36508400 \quad-4.58152000 \quad-3.90603500$

$\begin{array}{lll}1.81841200 & -4.25122200 & -1.90307600\end{array}$

$\begin{array}{lll}-2.11929700 & -1.05090700 \quad 2.38850300\end{array}$ 
O

C

\begin{tabular}{|c|c|c|}
\hline-0.84219200 & -2.07779200 & 3.58122000 \\
\hline 0.39393200 & -1.82844100 & 3.42295400 \\
\hline 1.34731700 & -2.89956400 & 4.02824200 \\
\hline 0.86876900 & -0.84547000 & 2.81843200 \\
\hline 0.95911500 & -3.14664600 & 5.49437400 \\
\hline 1.14690600 & -4.18917300 & 3.21145700 \\
\hline 2.80960300 & -2.45057300 & 3.94221000 \\
\hline 1.09568800 & -2.23458100 & 6.09915800 \\
\hline-0.09502200 & -3.44766700 & 5.57112300 \\
\hline 1.58564800 & -3.94018000 & 5.93524800 \\
\hline 1.37535000 & -4.02341600 & 2.14611900 \\
\hline 1.80855600 & -4.99187000 & 3.57818200 \\
\hline 0.10504000 & -4.53427900 & 3.27963300 \\
\hline 3.46904800 & -3.21001400 & 4.39348400 \\
\hline 3.13289400 & -2.31126000 & 2.90112800 \\
\hline 2.97337000 & -1.50604100 & 4.48543500 \\
\hline-2.55445800 & -1.92154400 & -2.16516700 \\
\hline 2.72233000 & -0.15238100 & -0.05639500 \\
\hline 1.93778300 & 1.69878200 & -0.94195500 \\
\hline 5.07453000 & -1.51023500 & -0.72934100 \\
\hline-2.66217900 & -2.63883100 & 1.04392500 \\
\hline-3.26807100 & -1.16911200 & 3.34144600 \\
\hline-3.58577100 & -2.21587300 & 3.45162200 \\
\hline-4.13092100 & -0.59435200 & 2.96576900 \\
\hline-2.99952300 & -0.78360200 & 4.33382800 \\
\hline-1.50826400 & -0.15248600 & 2.47500200 \\
\hline 2.98498900 & 0.34043800 & 1.81714500 \\
\hline 3.42004400 & 0.24917800 & -0.84744800 \\
\hline 5.30857000 & 1.57216500 & 1.45929000 \\
\hline 4.79788400 & -0.30847600 & -0.41145800 \\
\hline 4.61933100 & -1.33486600 & 0.64566300 \\
\hline 3.64256800 & 1.69267200 & -1.65075900 \\
\hline 2.97901100 & -1.10798900 & -2.99392400 \\
\hline 5.60220300 & 1.17585900 & 0.06441700 \\
\hline 6.33135700 & 2.44825300 & 1.90654800 \\
\hline 5.31474200 & -1.14836100 & -1.57917500 \\
\hline 5.11439100 & -2.60142300 & 0.25955600 \\
\hline 4.97651500 & 2.22590700 & -0.92574400 \\
\hline 4.00200000 & 1.28827900 & -3.10459400 \\
\hline 2.44935600 & 2.59540500 & -1.45060700 \\
\hline 6.63904500 & 1.02082700 & -2.63613000 \\
\hline 7.07591000 & 1.36600900 & -0.05280500 \\
\hline 7.60455500 & 1.87920600 & 1.16233400 \\
\hline 6.40696600 & 2.37609900 & 3.43141400 \\
\hline
\end{tabular}




\section{TS-S52}

\begin{tabular}{|c|c|c|}
\hline 5.99456000 & 3.88819000 & 1.48718400 \\
\hline 5.95879500 & -2.29484400 & -1.04662200 \\
\hline 3.92313900 & -3.52301700 & -0.02218300 \\
\hline 5.93508900 & -3.18922500 & 1.40906100 \\
\hline 8.24334300 & 0.71555600 & 1.93498400 \\
\hline 8.66908200 & 2.92844400 & 0.84606000 \\
\hline 5.49488500 & 2.81484400 & 3.86832300 \\
\hline 6.48123900 & 1.33784600 & 3.78287700 \\
\hline 7.26754700 & 2.94228200 & 3.82331200 \\
\hline 5.94272900 & 3.97844400 & 0.39524900 \\
\hline 4.99780400 & 4.14220000 & 1.88138900 \\
\hline 6.71664600 & 4.61804300 & 1.88757200 \\
\hline 5.93054700 & -3.41082100 & -2.09216000 \\
\hline 7.42122200 & -1.94551800 & -0.73327500 \\
\hline 3.27191400 & -3.54655200 & 0.86315700 \\
\hline 3.32712200 & -3.13713800 & -0.85730500 \\
\hline 4.22119200 & -4.55779300 & -0.25291900 \\
\hline 5.27683600 & -3.38648400 & 2.26901600 \\
\hline 6.41316000 & -4.14095700 & 1.12536200 \\
\hline 6.71237400 & -2.48800000 & 1.73998400 \\
\hline 7.48227700 & -0.03486900 & 2.19235600 \\
\hline 8.99869300 & 0.23205000 & 1.29634500 \\
\hline 8.74304000 & 1.04394800 & 2.85980300 \\
\hline 9.05562100 & 3.40546100 & 1.76167500 \\
\hline 9.52115300 & 2.45265400 & 0.33291600 \\
\hline 8.27212800 & 3.71176300 & 0.18620200 \\
\hline 6.50923400 & -3.10420200 & -2.97867700 \\
\hline 6.38047100 & -4.33887900 & -1.70536800 \\
\hline 4.90862300 & -3.64732100 & -2.42338900 \\
\hline 7.93008800 & -1.65614500 & -1.66928700 \\
\hline 7.47877000 & -1.09395100 & -0.04551600 \\
\hline 7.97928000 & -2.79550800 & -0.31358800 \\
\hline 1.22922400 & 0.74489900 & 1.87116400 \\
\hline 0.20713800 & 0.37007100 & 0.97226400 \\
\hline 0.95222700 & -0.20472800 & 1.53267600 \\
\hline 0.97520200 & -0.61078300 & 2.88513100 \\
\hline 0.05896900 & -0.20287900 & 3.73132400 \\
\hline 1.14467700 & 0.52087400 & 3.23853600 \\
\hline-3.32928300 & -1.30718000 & 2.59378300 \\
\hline-3.28068600 & -0.71440500 & 1.31695000 \\
\hline 4.46220900 & -0.43364200 & 0.59010400 \\
\hline 5.68123900 & -0.84837900 & 1.15330600 \\
\hline 74824100 & -1.45069400 & 2.40560800 \\
\hline
\end{tabular}




\begin{tabular}{|c|c|c|}
\hline-4.57435100 & -1.65504700 & 3.12659300 \\
\hline 2.13050200 & 1.22353100 & 1.46308200 \\
\hline 0.03802000 & -0.48100000 & 4.78449200 \\
\hline 1.94773700 & 0.84846800 & 3.90133500 \\
\hline-6.60210300 & -0.68750600 & 0.59908700 \\
\hline-6.71146500 & -1.75474300 & 2.82139400 \\
\hline-4.62815400 & -2.11673200 & 4.11198200 \\
\hline-2.02489700 & -1.63452600 & 3.30978500 \\
\hline-2.19948500 & -1.69133100 & 4.83304300 \\
\hline-2.92008200 & -2.46983500 & 5.11791500 \\
\hline-2.54592400 & -0.72986800 & 5.24150000 \\
\hline-1.25337100 & -1.95826900 & 5.32333000 \\
\hline-1.53770100 & -3.02479400 & 2.81951900 \\
\hline-0.57039100 & -3.27627900 & 3.28199600 \\
\hline-1.40375000 & -3.04119600 & 1.72807200 \\
\hline-2.27154400 & -3.80244000 & 3.08437400 \\
\hline-2.06025700 & -0.41538300 & 0.73987400 \\
\hline 0.23894300 & 2.24008200 & -1.34499300 \\
\hline-1.26384000 & 2.45497000 & -1.18292200 \\
\hline-1.57497500 & 2.27032700 & -0.14546100 \\
\hline-1.87024300 & 1.80772000 & -1.82484900 \\
\hline-1.51488800 & 3.50262600 & -1.42614600 \\
\hline 0.98357400 & 3.25126300 & -0.45893900 \\
\hline 0.80972000 & 4.27302700 & -0.83668600 \\
\hline 2.06412000 & 3.06017200 & -0.45346200 \\
\hline 0.63472900 & 3.21782800 & 0.58379100 \\
\hline 0.67828400 & 2.44258000 & -2.80148000 \\
\hline 1.75636600 & 2.25399300 & -2.92574800 \\
\hline 0.48217900 & 3.48531200 & -3.10402100 \\
\hline 0.13127500 & 1.79018100 & -3.49640200 \\
\hline 0.15382700 & -0.87180000 & -1.85960400 \\
\hline 1.17878600 & -1.04314500 & -2.99778100 \\
\hline 1.23514700 & -0.15388600 & -3.64211900 \\
\hline 0.87063700 & -1.89115600 & -3.63353100 \\
\hline 2.18571700 & -1.25196700 & -2.61494700 \\
\hline-1.23693500 & -0.67103900 & -2.45662800 \\
\hline-1.99155400 & -0.45693100 & -1.69309200 \\
\hline-1.53409700 & -1.59110800 & -2.98923400 \\
\hline-1.25569500 & 0.14621300 & -3.19204000 \\
\hline 0.17128400 & -2.13910200 & -0.98721800 \\
\hline 1.10906100 & -2.23177100 & -0.42182400 \\
\hline 0.06900200 & -3.02664300 & -1.63401100 \\
\hline-0.66604400 & -2.14751500 & -0.27804600 \\
\hline 45350000 & 2.04664100 & -0.64786700 \\
\hline
\end{tabular}




\begin{tabular}{|c|c|c|}
\hline-4.56799000 & 2.88580800 & 0.29202900 \\
\hline-3.61997800 & 3.16930200 & -0.18315500 \\
\hline-4.33456200 & 2.34621700 & 1.22384500 \\
\hline-5.09691800 & 3.81416400 & 0.56937800 \\
\hline-6.82674800 & 1.88896400 & 0.01713700 \\
\hline-7.30766000 & 2.88113600 & 0.08293900 \\
\hline-6.74211900 & 1.50766600 & 1.04367100 \\
\hline-7.50662800 & 1.23513600 & -0.54251600 \\
\hline-5.61210600 & 2.79647000 & -1.98094300 \\
\hline-4.65105800 & 2.89539200 & -2.51014400 \\
\hline-5.99681200 & 3.81381200 & -1.79346900 \\
\hline-6.32450900 & 2.29658900 & -2.65386000 \\
\hline-5.33372400 & -0.68024000 & -2.23962000 \\
\hline-4.82215900 & -2.10516900 & -1.96589500 \\
\hline-5.12547100 & -2.77123600 & -2.79227000 \\
\hline-5.23188700 & -2.51792300 & -1.03330500 \\
\hline-3.72461700 & -2.14183000 & -1.89522600 \\
\hline-6.86661500 & -0.68353600 & -2.22341300 \\
\hline-7.28333900 & -0.99289600 & -1.25506700 \\
\hline-7.23611600 & -1.40360600 & -2.97530400 \\
\hline-7.28789700 & 0.29772900 & -2.48400200 \\
\hline-4.85992800 & -0.25395500 & -3.64359300 \\
\hline-3.76510600 & -0.30444700 & -3.73355000 \\
\hline-5.16938200 & 0.77191000 & -3.89131400 \\
\hline-5.29453400 & -0.92437300 & -4.40555600 \\
\hline 4.40116900 & 0.48299300 & -1.02165400 \\
\hline 0.83267400 & 0.52916400 & -0.77559000 \\
\hline 4.54097000 & 0.97872300 & -1.41177100 \\
\hline 4.81707400 & 1.09410500 & -0.01147900 \\
\hline 4.69052000 & 2.33365400 & 0.76521200 \\
\hline 4.48121900 & 3.59941800 & 0.17931000 \\
\hline 4.88924400 & 1.29506600 & 2.65193400 \\
\hline 5.45317100 & 0.33145500 & 0.45136600 \\
\hline 4.72214700 & 2.26570400 & 2.17629700 \\
\hline 4.28575200 & 4.73523400 & 0.96597700 \\
\hline 4.29671600 & 4.64374800 & 2.36153200 \\
\hline 4.52301200 & 3.39927000 & 2.96180200 \\
\hline 4.12302700 & 5.70288400 & 0.48370100 \\
\hline 4.13814500 & 5.53360900 & 2.97539000 \\
\hline 4.54326500 & 3.31361400 & 4.05165500 \\
\hline 5.02705300 & -0.12981300 & -2.18414100 \\
\hline 4.65548600 & -0.16324500 & -3.21178700 \\
\hline 3.88987500 & -2.28611200 & -0.67207700 \\
\hline 43432800 & -1.58891400 & 0.25457200 \\
\hline
\end{tabular}




\begin{tabular}{lll}
4.05776200 & -3.80913700 & -0.46389000 \\
3.41321000 & -4.54973000 & -1.64739400 \\
3.40119600 & -4.24051700 & 0.85142500 \\
\hline 5.56835300 & -4.10488900 & -0.42262800 \\
\hline 3.86464100 & -4.23653700 & -2.59914000 \\
\hline 2.33227000 & -4.34486600 & -1.70172400 \\
\hline 3.54677200 & -5.63830500 & -1.53672500 \\
\hline 3.84509100 & -3.71787000 & 1.71071900 \\
\hline 3.52846900 & -5.32544100 & 0.99963200 \\
\hline 2.32322000 & -4.01885300 & 0.85273100 \\
\hline 5.74409800 & -5.18153400 & -0.26339500 \\
\hline 6.05840200 & -3.55858200 & 0.39996900 \\
\hline 6.05409100 & -3.81292100 & -1.36513700 \\
\hline 4.24334100 & -1.86129900 & -1.83095300 \\
\hline 4.15201500 & 1.81814400 & -1.99674600 \\
4.47691500 & 3.69293400 & -0.90838400 \\
\hline 6.43015500 & -0.63190800 & -2.01666400 \\
\hline 7.11342900 & 0.16417800 & -2.36369800 \\
6.61649900 & -1.53528300 & -2.61106400 \\
6.67403900 & -0.83383300 & -0.96430300 \\
\hline 3.01859200 & 0.39966100 & -0.28155400 \\
\hline
\end{tabular}

TS-S53

\begin{tabular}{rrr}
-0.97926500 & 1.77048900 & 0.74226800 \\
0.24375400 & 2.39896200 & 1.15477500 \\
0.78609700 & 3.59051900 & 0.47167900 \\
0.70618800 & 3.74997100 & -0.92592700 \\
1.52060100 & 4.48795500 & 2.29455600 \\
0.45232400 & 2.38464500 & 2.22910200 \\
1.45002200 & 4.58831400 & 1.20884900 \\
1.26470300 & 4.86021800 & -1.55750200 \\
1.92695300 & 5.84063800 & -0.81013600 \\
2.01416200 & 5.69907100 & 0.57770900 \\
\hline 1.19198300 & 4.95599800 & -2.64403000 \\
\hline 2.36996300 & 6.70781700 & -1.30580200 \\
\hline 2.52413600 & 6.45983500 & 1.17444400 \\
\hline-1.55373600 & 0.70160800 & 1.42860500 \\
\hline-2.27243300 & 0.06128100 & 0.91573400 \\
\hline 0.66648000 & 0.61929100 & 0.42811300 \\
-3.63381900 & 1.52512400 & 2.08760200 \\
-4.50738500 & 0.66378600 & 1.76809600 \\
-5.99033100 & 1.15270400 & 1.89116500 \\
-4.29169300 & -0.50142300 & 1.37545800 \\
-6.15462900 & 2.41743900 & 1.03472100 \\
\hline .25226400 & 1.49528600 & 3.36668300 \\
\hline
\end{tabular}


$\begin{array}{lll}-5.97962800 & 2.19856300 & -0.03202400\end{array}$

$\begin{array}{lll}-5.42875400 & 3.18359500 & 1.34280300\end{array}$

$\begin{array}{lll}-7.17268300 & 2.83504200 & 1.12881100\end{array}$

$\begin{array}{lll}-6.14249300 & 0.60248800 \quad 4.00558100\end{array}$

$\begin{array}{lll}-7.27396100 & 1.88871500 \quad 3.51127800\end{array}$

$\begin{array}{lll}-5.53248900 & 2.24975700 & 3.71677300\end{array}$

$\begin{array}{lll}-8.00762800 & 0.40255400 & 1.52747800\end{array}$

$\begin{array}{lll}-6.83039400 & -0.85791600 & 2.00263500\end{array}$

$\begin{array}{lll}-6.78681200 & -0.18989100 & 0.36507500\end{array}$

$\begin{array}{lll}-1.43346500 & 2.07569600 & -0.19948100\end{array}$

$0.22087300 \quad 2.97853600 \quad-1.52413300$

$\begin{array}{lll}-1.17804600 & 0.24736800 & 2.80529300\end{array}$

$\begin{array}{lll}-2.03845700 & -0.26148700 \quad 3.25738000\end{array}$

$\begin{array}{lll}-0.87261400 & 1.08198000 & 3.45259300\end{array}$

$\begin{array}{lll}-0.34456100 & -0.47447000 & 2.77433000\end{array}$

$4.09269500 \quad-2.31390800 \quad 0.31885900$

$\begin{array}{lll}2.92005100 & -1.54041500 & 0.27995400\end{array}$

$\begin{array}{lll}1.70089800 & -2.23574700 & 0.39107000\end{array}$

$\begin{array}{lll}1.62207700 & -3.58816100 & 0.74944800\end{array}$

$2.81668200 \quad-4.31579500 \quad 0.77997600$

$\begin{array}{lll}4.03955900 & -3.69282100 & 0.51982300\end{array}$

$\begin{array}{lll}-0.80166100 & -3.53938000 & 0.30696800\end{array}$

$\begin{array}{lll}-0.60963700 & -2.25905900 & -0.22718300\end{array}$

$-1.46705100 \quad-1.69249500 \quad-1.18825200$

$\begin{array}{lll}-2.63280000 & -2.41308600 & -1.49069900\end{array}$

$\begin{array}{lll}-2.90033900 & -3.64813100 & -0.90533600\end{array}$

$\begin{array}{lll}-1.97242300 & -4.22284400 & -0.03971000\end{array}$

$\begin{array}{lll}5.06218300 & -1.83324100 & 0.21608700\end{array}$

$\begin{array}{lll}2.79992700 & -5.37722100 & 1.02675100\end{array}$

$4.96223600 \quad-4.27691600 \quad 0.53019500$

$\begin{array}{lll}-3.33634000 & -2.01147800 & -2.21509500\end{array}$

$-3.82276700 \quad-4.17849000 \quad-1.15021300$

$\begin{array}{lll}-2.16621000 & -5.21215300 & 0.37375100\end{array}$

$0.26909800 \quad-4.12072900 \quad 1.22814500$

$0.03502000 \quad-3.59152400 \quad 2.66632500$

$\begin{array}{lll}-0.94049100 & -3.93611400 & 3.04227600\end{array}$

$\begin{array}{lll}0.03161800 & -2.49409300 \quad 2.69403800\end{array}$

$\begin{array}{lll}0.82471900 & -3.95092700 & 3.34428400\end{array}$

$\begin{array}{lll}0.23418000 & -5.65248600 & 1.25660900\end{array}$

$1.00928900 \quad-6.04444800 \quad 1.93022000$

$\begin{array}{lll}0.38785100 & -6.08565400 & 0.25668800\end{array}$

$\begin{array}{lll}-0.72688300 & -6.01325700 & 1.64744400\end{array}$

$0.52166800 \quad-1.53446800 \quad 0.17790700$ 
$3.94441500 \quad 1.13991400 \quad-0.79428700$

$\begin{array}{lll}5.26734600 & 0.42985800 & -1.10619800\end{array}$

5.10293300

$-0.56258200-1.54777600$

5.91776600

$0.32460600-0.22891100$

$\mathrm{H}$

$\begin{array}{lll}5.81802900 & 1.02844000 & -1.85172400\end{array}$

3.11020000

$1.18607200 \quad-2.08332300$

$\begin{array}{lll}3.62971000 & 1.80643500 & -2.83249900\end{array}$

$\begin{array}{lll}2.11477000 & 1.61533600 & -1.91210100\end{array}$

$2.98602800 \quad 0.18479300 \quad-2.51252100$

$\begin{array}{lll}4.21690500 & 2.58091300 & -0.33497900\end{array}$

$\begin{array}{lll}3.29310900 & 3.11151800 & -0.07301400\end{array}$

$\begin{array}{lll}4.68871400 & 3.13385700 & -1.16352400\end{array}$

$\begin{array}{lll}4.90393200 & 2.62411700 & 0.52104400\end{array}$

$\begin{array}{lll}3.59710200 & 0.45722300 & 2.26761800\end{array}$

$\begin{array}{lll}5.11604400 & 0.25991200 & 2.32961800\end{array}$

$\begin{array}{lll}5.43504800 & 0.29644200 & 3.38511000\end{array}$

$5.66062000 \quad 1.05256000 \quad 1.79876500$

$\begin{array}{lll}5.43206500 & -0.71303600 & 1.93236900\end{array}$

$2.90566100 \quad-0.60305000 \quad 3.14226900$

$\begin{array}{lll}1.81078200 & -0.54848100 \quad 3.04659400\end{array}$

$\begin{array}{lll}3.15401100 & -0.41233800 & 4.19935400\end{array}$

$3.22446200 \quad-1.62602000 \quad 2.90131500$

$3.24292600 \quad 1.83861900 \quad 2.84245800$

$\begin{array}{lll}3.56226200 & 2.67099700 & 2.20530700\end{array}$

$3.74157400 \quad 1.95467800 \quad 3.81914200$

$\begin{array}{lll}2.16468400 & 1.93181500 \quad 3.01233000\end{array}$

$\begin{array}{lll}-2.52025000 & 0.68820900 & -2.74291200\end{array}$

$\begin{array}{lll}-3.20821600 & 0.09224600 & -3.98067700\end{array}$

$\begin{array}{lll}-3.53830000 & -0.94637600 & -3.84664500\end{array}$

$\begin{array}{lll}-2.56705600 & 0.13494100 & -4.87136200\end{array}$

$\begin{array}{lll}-4.10877400 & 0.69031000 & -4.20290800\end{array}$

$\begin{array}{lll}-2.07378900 & 2.12076100 & -3.10179100\end{array}$

$\begin{array}{lll}-1.28757500 & 2.13972000 & -3.87072900\end{array}$

$\begin{array}{lll}-1.70235400 & 2.66459800 & -2.22282300\end{array}$

$\begin{array}{lll}-2.93592900 & 2.68612100 & -3.49392100\end{array}$

$\begin{array}{lll}-3.54289800 & 0.78698500 & -1.59653500\end{array}$

$\begin{array}{lll}-4.41388000 & 1.35949300 & -1.95812700\end{array}$

$\begin{array}{lll}-3.15455600 & 1.32420400 & -0.72433900\end{array}$

$\begin{array}{lll}-3.90865200 & -0.17974100 & -1.23388900\end{array}$

$\begin{array}{lll}-0.05010900 & -1.07834900 & -3.59026300\end{array}$

$\begin{array}{lll}-0.86313100 & -2.11929000 & -4.36978800\end{array}$

$\begin{array}{lll}-0.18264800 & -2.67381400 & -5.03999200\end{array}$

$\begin{array}{lll}-1.64327800 & -1.67631800 & -4.99840600\end{array}$

$\begin{array}{lll}-1.33165000 & -2.85639000 & -3.70217600\end{array}$ 


$\begin{array}{lrrr}\mathrm{C} & 0.46183800 & 0.01215600 & -4.54426600 \\ \mathrm{H} & -0.36211000 & 0.51815200 & -5.06814300 \\ \mathrm{H} & 1.11014900 & -0.44029700 & -5.31436500 \\ \mathrm{H} & 1.05096900 & 0.77679500 & -4.01621800 \\ \mathrm{C} & 1.14713400 & -1.81103300 & -2.95776100 \\ \mathrm{H} & 0.83077900 & -2.72240900 & -2.43075400 \\ \mathrm{H} & 1.68829200 & -1.18145600 & -2.24361100 \\ \mathrm{H} & 1.85410500 & -2.11719900 & -3.74724100 \\ \mathrm{P} & 2.84024500 & 0.29667100 & 0.50837000 \\ \mathrm{P} & -0.91992400 & -0.18803600 & -2.11735300\end{array}$

\section{Cartesian coordinates of the structures (optimization in acetonitrile)} TS5/TS22

\begin{tabular}{|c|c|c|c|}
\hline $\mathrm{P}$ & 0.96144800 & -0.72204400 & 0.11121600 \\
\hline $\mathrm{C}$ & 0.46092100 & -2.47430600 & -0.40449300 \\
\hline $\mathrm{C}$ & 0.28553300 & -0.22515900 & 1.82530600 \\
\hline $\mathrm{C}$ & -1.03436900 & -2.62725300 & -0.67630500 \\
\hline $\mathrm{C}$ & 0.93428200 & -3.45986100 & 0.67218700 \\
\hline $\mathrm{C}$ & 1.23056200 & -2.74519900 & -1.70953900 \\
\hline $\mathrm{C}$ & 0.23346600 & 0.37930500 & -1.19992100 \\
\hline $\mathrm{Ni}$ & 3.12342400 & -0.24607800 & 0.18543000 \\
\hline $\mathrm{C}$ & -1.01210900 & -0.89507800 & 2.26963000 \\
\hline $\mathrm{C}$ & 0.10495100 & 1.30275500 & 1.82772300 \\
\hline $\mathrm{C}$ & 1.40360700 & -0.58059600 & 2.82599000 \\
\hline $\mathrm{H}$ & -1.36596500 & -1.92440100 & -1.45337600 \\
\hline $\mathrm{H}$ & -1.23838100 & -3.64983900 & -1.04004600 \\
\hline $\mathrm{H}$ & -1.65807900 & -2.45197700 & 0.20655300 \\
\hline $\mathrm{H}$ & 0.36225500 & -3.36064200 & 1.60479100 \\
\hline $\mathrm{H}$ & 0.80164200 & -4.49216400 & 0.30655000 \\
\hline $\mathrm{H}$ & 1.99920000 & -3.32132300 & 0.90209200 \\
\hline $\mathrm{H}$ & 0.87324600 & -2.10433200 & -2.52966500 \\
\hline $\mathrm{H}$ & 1.07636300 & -3.79459700 & -2.01222200 \\
\hline $\mathrm{H}$ & 2.30720300 & -2.57716500 & -1.58674200 \\
\hline $\mathrm{C}$ & -1.02399700 & 1.02620400 & -1.29085600 \\
\hline $\mathrm{C}$ & 1.20653500 & 0.72289700 & -2.15687500 \\
\hline $\mathrm{C}$ & 4.37038500 & -1.67414300 & 0.57477500 \\
\hline $\mathrm{C}$ & 5.05542200 & -0.38311900 & 0.50833400 \\
\hline $\mathrm{H}$ & -0.89670700 & -1.97971500 & 2.40287800 \\
\hline $\mathrm{H}$ & -1.31023900 & -0.47839300 & 3.24700300 \\
\hline $\mathrm{H}$ & -1.83014700 & -0.72434100 & 1.56359000 \\
\hline $\mathrm{H}$ & 0.97386400 & 1.81438100 & 1.39446100 \\
\hline $\mathrm{H}$ & -0.01066400 & 1.64832600 & 2.86884200 \\
\hline $\mathrm{H}$ & -0.79157200 & 1.60975100 & 1.27442100 \\
\hline $\mathrm{H}$ & 2.31916200 & -0.00370900 & 2.62698900 \\
\hline $\mathrm{H}$ & 1.06107600 & -0.33985200 & 3.84705100 \\
\hline
\end{tabular}


$\begin{array}{lll}1.65997700 & -1.64952600 & 2.80078600\end{array}$

$\begin{array}{lll}-1.20464100 & 2.12416400 & -2.15352500\end{array}$ 
$3.02896700 \quad 1.73485300 \quad 0.07409000$

$-5.68829600$

2.58671000

$-0.13879400$

$-4.78843100$

4.13537200

$-1.32722500$

$-6.37830200$

0.82722800

0.87046300

$-5.31605700$

$-2.01464800 \quad-0.72993900$

$-5.12730100$

1.37049600

2.35490900

$4.97685700 \quad-5.51956700 \quad-3.18061200$

$2.36231900 \quad 4.34699400 \quad-0.03730500$

$\begin{array}{lll}2.90350800 \quad 4.30201000 & 2.42022500\end{array}$

$\begin{array}{lll}4.75191600 & 4.59125500 & 0.72893400\end{array}$

$\begin{array}{lll}-6.61583300 & 3.13937600 & 0.02664400\end{array}$

$-6.68984500 \quad-1.39116700 \quad-1.00017300$

$\begin{array}{lll}-5.46990800 & -3.50653700 & -0.39431600\end{array}$

$\begin{array}{lll}-4.46945100 & -1.88204100 & -2.00952900\end{array}$

$\begin{array}{lll}-6.65771700 & -1.46449300 & 2.38868100\end{array}$

$\begin{array}{lll}-4.67034900 & -0.18206500 & 3.21783100\end{array}$

$\begin{array}{lll}-4.54924100 & -2.66312100 & 2.96814400\end{array}$

$\begin{array}{lll}1.39328700 & 3.85408300 & 0.13138300\end{array}$

$\begin{array}{lll}2.20809000 & 5.43673700 \quad 0.01218400\end{array}$

$2.69271100 \quad 4.08989300 \quad-1.05420500$

$\begin{array}{lll}1.94550900 & 3.80948400 & 2.65116500\end{array}$

$\begin{array}{lll}2.75144800 & 5.39134500 & 2.48451300\end{array}$

$3.63438300 \quad 4.00628900 \quad 3.18658900$

$\begin{array}{lll}4.64342900 & 5.68752700 & 0.75110800\end{array}$

$5.13442500 \quad 4.30649400 \quad-0.26482700$

$5.50071000 \quad 4.30179400 \quad 1.48084200$

$\begin{array}{lll}-6.60284000 & -0.34947300 & -1.33983200\end{array}$

$\begin{array}{lll}-7.18965500 & -1.95649400 & -1.80692400\end{array}$

$\begin{array}{lll}-7.35012300 & -1.41250000 & -0.12510400\end{array}$

$\begin{array}{lll}-4.49745600 & -3.97442700 & -0.17187400\end{array}$

$\begin{array}{lll}-5.90823200 & -4.03748100 & -1.25703600\end{array}$

$\begin{array}{lll}-6.13370000 & -3.66973800 & 0.46689000\end{array}$

$\begin{array}{lll}-4.26958000 & -0.82885300 & -2.26318500\end{array}$

$\begin{array}{lll}-5.01559600 & -2.32882900 & -2.85831600\end{array}$

$\begin{array}{lll}-3.50535400 & -2.40202600 & -1.92047300\end{array}$

$\begin{array}{lll}-7.03030100 & -2.34726500 & 1.85080200\end{array}$

$\begin{array}{lll}-6.98657800 & -1.55912600 & 3.43885400\end{array}$

$\begin{array}{lll}-7.15254900 & -0.57595400 & 1.97483800\end{array}$

$\begin{array}{lll}-3.58213800 & -0.02907600 \quad 3.16580200\end{array}$

$\begin{array}{lll}-4.93068400 & -0.37675300 & 4.27274800\end{array}$

$\begin{array}{lll}-5.15785600 & 0.75666100 & 2.92108000\end{array}$

$\begin{array}{lll}-3.45252800 & -2.61579300 \quad 3.04946600\end{array}$

$\begin{array}{lll}-4.96036700 & -2.81216500 \quad 3.98178900\end{array}$

$-4.80460500 \quad-3.55154300 \quad 2.37145700$ 
TS19/TS23/TS-Conformation-B

C

C

$$
-2.01872100
$$$$
-1.42599400
$$ 
$1.82416100 \quad-4.57532300 \quad 3.24634000$

$1.19635100 \quad-3.26254200 \quad 4.26161500$

$0.08674100 \quad-4.22903900 \quad 3.25376100$

$\begin{array}{lll}1.26363800 & -3.58179000 & 0.78794400\end{array}$

$\begin{array}{lll}1.34110600 & -2.90256200 & -0.07555000\end{array}$

$2.11371500 \quad-4.28245700 \quad 0.74176400$

$0.33866000 \quad-4.16556300 \quad 0.68527800$

$\begin{array}{lll}2.73998400 & -2.21235400 & 2.24618400\end{array}$

$2.85803300 \quad-1.57407200 \quad 3.12956300$

$\begin{array}{lll}3.46178200 & -3.04146400 & 2.33438300\end{array}$

$\begin{array}{lll}3.01032500 & -1.63072200 & 1.35893300\end{array}$

$\begin{array}{lll}-2.09736700 & 3.86810100 & -0.55734000\end{array}$

$\begin{array}{lll}-3.31601200 & 4.61933500 & -1.10988100\end{array}$

$\begin{array}{lll}-3.72668500 & 4.18075900 & -2.02845700\end{array}$

$\begin{array}{lll}-4.12478600 & 4.68440300 & -0.36987900\end{array}$

$\begin{array}{lll}-3.01203400 & 5.65332300 & -1.35008800\end{array}$

$\begin{array}{lll}-1.66555300 \quad 4.57400000 & 0.74550100\end{array}$

$\begin{array}{lll}-2.46505700 & 4.57725800 & 1.49886500\end{array}$

$\begin{array}{lll}-0.78200500 & 4.10153900 & 1.19553300\end{array}$

$\begin{array}{lll}-1.41245900 & 5.62507600 & 0.52463100\end{array}$

$\begin{array}{lll}-0.93225500 & 3.96447400 & -1.55550100\end{array}$

$\begin{array}{lll}-0.68696700 & 5.02655400 & -1.72564600\end{array}$

$\begin{array}{lll}-0.02886400 & 3.47624100 & -1.16555100\end{array}$

$\begin{array}{lll}-1.16930500 & 3.51468500 & -2.52941700\end{array}$

$\begin{array}{lll}-4.06697400 & 1.68622100 & 0.54895900\end{array}$

$\begin{array}{lll}-5.20042800 & 1.96405100 & -0.44578800\end{array}$

$\begin{array}{lll}-6.16037200 & 1.68272800 & 0.02239800\end{array}$

$\begin{array}{lll}-5.27309200 & 3.01889100 & -0.73222400\end{array}$

$\begin{array}{lll}-5.09680500 & 1.36335100 & -1.36039000\end{array}$

$\begin{array}{lll}-4.27062700 & 2.52245100 & 1.82200700\end{array}$

$\begin{array}{lll}-4.34103000 \quad 3.59677400 & 1.60086000\end{array}$

$\begin{array}{lll}-5.21457600 & 2.22298300 & 2.30883400\end{array}$

$\begin{array}{lll}-3.45708500 & 2.37104900 \quad 2.54811600\end{array}$

$\begin{array}{lll}-4.13418600 & 0.19355200 & 0.91699700\end{array}$

$\begin{array}{lll}-4.09410800 & -0.45040100 & 0.02698100\end{array}$

$\begin{array}{lll}-3.32954100 & -0.11451300 & 1.59614700\end{array}$

$\begin{array}{lll}-5.09429800 & -0.00964200 & 1.42094500\end{array}$

$\begin{array}{lll}1.57183400 & 1.00402600 & -1.02561000\end{array}$

$2.18445200 \quad 0.62969800 \quad 0.23692800$

$\begin{array}{lll}2.48542900 & 1.61580300 \quad 1.28981100\end{array}$

$\begin{array}{lll}1.65817000 \quad 2.73397700 & 1.51647300\end{array}$

$\begin{array}{lll}4.25039900 & 0.57846300 & 1.98442700\end{array}$

$2.93013800 \quad-0.16764200 \quad 0.16275600$

$3.59504500 \quad 1.43905600 \quad 2.14002100$ 
$\begin{array}{lll}4.02694300 & -5.53143500 & -1.77404000\end{array}$

$\begin{array}{lll}3.87513300 & -6.89254500 & 0.31596800\end{array}$

$\begin{array}{lll}4.07481100 & -5.75981100 & 2.53532900\end{array}$

$5.42817700 \quad 0.56572100 \quad-0.21228300$

$\begin{array}{lll}5.44955300 & 0.98477700 & 0.79947800\end{array}$

$3.40710400 \quad 0.02130500 \quad-0.06795300$

$3.23074200 \quad 2.83661900 \quad-0.34002700$

$\begin{array}{lll}2.89323000 & 1.69605400 & -0.84056600\end{array}$

$2.79306400 \quad 4.05488200 \quad-1.19491000$

$\begin{array}{lll}3.54205600 & 5.30930100 & -0.73277200\end{array}$

$1.28038600 \quad 4.25301200 \quad-0.98844700$

$3.08211900 \quad 3.79168600 \quad-2.68050500$

$\begin{array}{lll}4.62919900 & 5.20265300 & -0.87734100\end{array}$

$3.36687900 \quad 5.50247900 \quad 0.33520900$

$3.20754500 \quad 6.18934900 \quad-1.30675700$

$\begin{array}{lll}0.71509300 & 3.36392900 & -1.30069000\end{array}$

$0.92214200 \quad 5.11312000 \quad-1.57836800$

$\begin{array}{lll}1.04871400 & 4.44612500 & 0.06866600\end{array}$

$2.52060700 \quad 2.92101800 \quad-3.04711600$

$4.15370500 \quad 3.59982700 \quad-2.85212000$

$\begin{array}{lll}2.79504500 & 4.66706200 & -3.28705400\end{array}$

$3.82904300 \quad 2.99870500 \quad 0.72600500$

$4.33074600 \quad-3.08619200 \quad-1.65228400$

$5.43527700 \quad-1.25610300 \quad-1.34738600$

$5.99003500 \quad 1.43152400 \quad-1.29871000$

$\begin{array}{lll}7.09201900 & 1.47494300 & -1.22291500\end{array}$

$5.62552800 \quad 2.46391300 \quad-1.21366000$

$5.73688500 \quad 1.04880300 \quad-2.29934800$

$\begin{array}{lll}-5.36233600 & 0.88502200 & -0.88664900\end{array}$

$\begin{array}{lll}-4.14756400 & 0.32816800 & -0.45700700\end{array}$

$\begin{array}{lll}-3.15678500 & 1.21430600 & 0.03944100\end{array}$

$\begin{array}{lll}-3.45539000 & 2.56590100 \quad 0.28158800\end{array}$

$-4.68767600 \quad 3.06805200 \quad-0.15815700$

$\begin{array}{lll}-5.62484000 & 2.24948900 & -0.78077900\end{array}$

$\begin{array}{lll}-1.23649500 & 2.73003300 & 1.43484700\end{array}$

$\begin{array}{lll}-0.92781600 & 1.46474200 & 0.90239000\end{array}$

$\begin{array}{lll}0.38657700 & 0.93828900 & 1.01259300\end{array}$

$\begin{array}{lll}1.29712400 & 1.63385900 & 1.82561800\end{array}$

$\begin{array}{lll}0.96064600 & 2.81636800 & 2.47647300\end{array}$

$\begin{array}{lll}-0.28877200 & 3.37502600 & 2.24073400\end{array}$

$\begin{array}{lll}-6.13011200 & 0.23019200 & -1.29291400\end{array}$

$\begin{array}{lll}-4.91931900 \quad 4.12216200 & 0.00383100\end{array}$

$\begin{array}{lll}-6.57281300 & 2.66037100 & -1.13513900\end{array}$

$2.31496400 \quad 1.25509000 \quad 1.91257300$ 
$1.69480200 \quad 3.32779900 \quad 3.10112400$

$-0.53271000$

$4.34844800 \quad 2.66918300$

C

$-2.52855200$

3.46503000

1.08934300

$-3.26593000$

3.87759100

2.38490800

$-2.64155000 \quad 4.53969700 \quad 3.00262200$

$-3.52609100$

2.98993200

2.98219400

$-4.19392300$

$4.41940100 \quad 2.14978500$

$-2.16990200$

4.72809800

0.27106600

$-3.07319000$

5.30682700

0.02661600

$-1.66997200$

$4.45416100 \quad-0.66966700$

$-1.49666200$

5.38499300

0.84123500

$-1.89425700$

$0.70947900 \quad 0.28006100$

$-4.65418800$

$-2.21342600-1.97894400$

$-6.17109200$

$-6.73458100$

$-2.41151600$

$-2.08805600$

$-6.55188100$

$-1.47371900$

$-1.99280500$

$-6.40496400$

$-4.17882200$

$-3.12074100-1.34041300$

$-4.73362000$

$-4.33704000$

$-3.10812000$

$-3.97601100$

$-2.87941900$

$-4.29910900$

$-4.24366400$

$-5.08757400$

$-4.38332800$

$-3.37043400$

$-4.96353300$

$-4.31031000$

$-5.10332300$

$-5.65794400$

$-5.59529700$

$-4.08291900$

$-6.52214600$

$-7.09596100$

$-6.54550500$

$-7.04977200$

0.57807900

$1.76467800-1.43738200-2.30518600$

$2.04027600 \quad-2.40529900 \quad-1.86327900$

$\begin{array}{lll}2.65455700 & -0.78886800 & -2.29511700\end{array}$

$\begin{array}{lll}1.48213900 & -1.61510900 & -3.35664600\end{array}$

$\begin{array}{lll}0.30140700 & 0.59372900 & -2.18097400\end{array}$ 
H

$\mathrm{H}$

$\mathrm{H}$

C

$\mathrm{H}$

$\mathrm{H}$

24/Conformation-B

C

C

C

\begin{tabular}{|c|c|c|}
\hline 0.15419600 & 0.47064000 & -3.26760300 \\
\hline 1.14145400 & 1.27802100 & -2.01357700 \\
\hline-0.61376600 & 1.04064600 & -1.77075600 \\
\hline-0.65698400 & -1.66631400 & -1.74260100 \\
\hline-0.93488400 & -1.66445400 & -2.80983900 \\
\hline-1.51291400 & -1.29645900 & -1.17087700 \\
\hline-0.47849200 & -2.71067800 & -1.45914300 \\
\hline 0.68230200 & -1.94371600 & 1.40140600 \\
\hline 0.99355400 & -3.31479100 & 0.78408300 \\
\hline 0.98608100 & -4.07703200 & 1.58003600 \\
\hline 1.97596400 & -3.34664200 & 0.30487000 \\
\hline 0.23735400 & -3.60809800 & 0.04560300 \\
\hline 1.52759100 & -1.73467700 & 2.67051500 \\
\hline 1.31608800 & -2.55105700 & 3.38102300 \\
\hline 1.27880900 & -0.78678200 & 3.17047600 \\
\hline 2.60298700 & -1.73546700 & 2.45896400 \\
\hline-0.79700000 & -1.88265300 & 1.79060200 \\
\hline-1.46982800 & -1.96058900 & 0.92844700 \\
\hline-1.03884100 & -0.95391500 & 2.32587800 \\
\hline-1.01735800 & -2.72449700 & 2.46999200 \\
\hline 1.18677700 & -0.53851800 & 0.22274200 \\
\hline .92815400 & -1.50999700 & -0.34123800 \\
\hline 2.89798400 & 3.38859300 & -0.92187400 \\
\hline 2.02730300 & 2.34605500 & -0.56244300 \\
\hline 2.13372200 & 1.16135100 & -1.31097800 \\
\hline 2.82855700 & 1.07046700 & -2.52062600 \\
\hline 3.66743100 & 2.14044100 & -2.85420700 \\
\hline 3.74545800 & 3.26603300 & -2.02626900 \\
\hline 2.35922500 & -1.34942500 & -2.58275900 \\
\hline 1.89586600 & -1.21605100 & -1.26748400 \\
\hline 1.89692200 & -2.27375400 & -0.33800600 \\
\hline 2.20423600 & -3.54675100 & -0.84564900 \\
\hline 2.55987500 & -3.73320000 & -2.18237200 \\
\hline 2.68158100 & -2.63444400 & -3.03402600 \\
\hline 2.89185200 & 4.31950600 & -0.35790200 \\
\hline 4.24938200 & 2.11011300 & -3.77592500 \\
\hline 4.42119000 & 4.08434900 & -2.28500200 \\
\hline 2.20628100 & -4.40607200 & -0.17914900 \\
\hline 2.79050900 & -4.73623600 & -2.54846700 \\
\hline 3.02372600 & -2.78076000 & -4.05852700 \\
\hline 2.46072400 & -0.09342700 & -3.44584400 \\
\hline 1.05718700 & 0.21265500 & -4.03484100 \\
\hline 0.73918500 & -0.60880600 & -4.6951910 \\
\hline
\end{tabular}


H

$\mathrm{H}$

C

H

$\mathrm{H}$

$\mathrm{H}$

O

\begin{tabular}{|c|c|c|}
\hline 0.30370200 & 0.32526900 & -3.24201900 \\
\hline 1.08392100 & 1.14609900 & -4.61824100 \\
\hline 3.46040600 & -0.25676400 & -4.59322800 \\
\hline 3.51229500 & 0.66432200 & -5.19094000 \\
\hline 4.47052300 & -0.48993700 & -4.22347000 \\
\hline 3.14446900 & -1.05960800 & -5.27380000 \\
\hline 1.45498900 & 0.04026900 & -0.84791500 \\
\hline 0.89066400 & 3.03842500 & 2.18174900 \\
\hline 2.02712300 & 4.06277500 & 2.26745600 \\
\hline 2.97145000 & 3.64969500 & 1.88472300 \\
\hline 1.80544400 & 4.98916100 & 1.72159900 \\
\hline 2.19004000 & 4.33022300 & 3.32603500 \\
\hline 1.31595400 & 1.75872200 & 2.91784200 \\
\hline 1.59143200 & 2.00791500 & 3.95680900 \\
\hline 0.50428500 & 1.01835900 & 2.94403600 \\
\hline 2.18603800 & 1.28862200 & 2.44389800 \\
\hline 0.37516500 & 3.58054500 & 2.86129800 \\
\hline-1.22425800 & 2.88899900 & 2.75235600 \\
\hline-0.17710900 & 3.69712200 & 3.94017200 \\
\hline-0.66927300 & 4.56405600 & 2.47063700 \\
\hline-0.46307300 & 3.85012500 & -0.54987300 \\
\hline 0.09689900 & 5.26809200 & -0.40221300 \\
\hline-0.49511500 & 5.95235400 & -1.03471600 \\
\hline 0.02502300 & 5.63492800 & 0.63102400 \\
\hline 1.14180600 & 5.34604600 & -0.72958400 \\
\hline-0.41181200 & 3.42049500 & -2.02768800 \\
\hline-0.74454900 & 2.37755500 & -2.15141300 \\
\hline-1.08812100 & 4.06459900 & -2.61408500 \\
\hline 0.59607700 & 3.51091700 & -2.45564500 \\
\hline-1.93637400 & 3.83894400 & -0.10920100 \\
\hline-2.07225200 & 4.16473900 & 0.92889300 \\
\hline-2.50609300 & 4.53055400 & -0.75271400 \\
\hline-2.37116700 & 2.83880300 & -0.21508100 \\
\hline 1.15142200 & -3.43231500 & 2.37523000 \\
\hline 2.12190400 & -4.60712400 & 2.55809700 \\
\hline 2.50958500 & -5.01060400 & 1.61375600 \\
\hline 2.97678800 & -4.33291600 & 3.19035600 \\
\hline 1.58925700 & -5.42813000 & 3.06962900 \\
\hline 0.73610500 & -2.94661500 & 3.78003300 \\
\hline 1.59981000 & -2.62819300 & 4.37977100 \\
\hline 0.03270500 & -2.10458200 & 3.73009100 \\
\hline 0.24158500 & -3.77134900 & 4.32129600 \\
\hline-0.11471600 & -3.91725700 & 1.64954700 \\
\hline .57422000 & -4.73402900 & 2.23183700 \\
\hline
\end{tabular}


$0.09358300 \quad-4.30064900 \quad 0.64118500$

$3.64103600 \quad-1.59625900 \quad 1.81346200$ 
$-6.89147800$

$-1.26961500$

$-0.35437700$

$-3.23619700$

$\mathrm{Ni}$

$-0.36466700$

\subsection{0}

1.75693400

$-1.59163900$

$-2.06492700$

$-1.30117500$

$-1.68064900$

$-1.35917700$

$-0.24539000$

4.55583000

4.37875500

4.28626500

4.39182300

4.66299200

4.30985300

4.10775600

4.02152800

4.13091100

4.02694300

3.87513300

4.07481100

5.42817700

5.44955300

3.40710400

3.23074200

2.89323000

2.79306400

3.54205600

1.28038600

3.08211900

4.62919900

3.36687900

3.20754500

0.71509300

0.92214200

1.0487140

2.52060700

4.15370500

2.79504500

3.82904300
2.44390700

0.02500400

0.42145800

1.46456500

0.33260900

$-2.33791000$

$-2.32789800$

$-3.33380200$

$-1.70831400$

$-2.08610100 \quad-2.41376900$

$-0.80276200 \quad 0.36801500$

$-1.54615100 \quad-0.62735100$

$-3.00602100 \quad-0.51498400$

$-3.66472500 \quad 0.72814000$

$-3.30401500 \quad-2.65525100$

$-1.19151500 \quad-1.66085300$

$-3.79221100-1.68114500$

$-5.04656600 \quad 0.79754000$

$-5.81107500 \quad-0.37252700$

$-5.17532000 \quad-1.61318300$

$-5.53143500 \quad 1.77404000$

$-6.89254500-0.31596800$

$-5.75981100 \quad-2.53532900$

$0.56572100 \quad 0.21228300$

$0.98477700 \quad-0.79947800$

$0.02130500 \quad 0.06795300$

$2.83661900 \quad 0.34002700$

$1.69605400 \quad 0.84056600$

$4.05488200 \quad 1.19491000$

$5.30930100 \quad 0.73277200$

$4.25301200 \quad 0.98844700$

$3.79168600 \quad 2.68050500$

$5.20265300 \quad 0.87734100$

$5.50247900-0.33520900$

$6.18934900 \quad 1.30675700$

$3.36392900 \quad 1.30069000$

$5.11312000 \quad 1.57836800$

$4.44612500 \quad-0.06866600$

$2.92101800 \quad 3.04711600$

$3.59982700 \quad 2.85212000$

$4.66706200 \quad 3.28705400$

$2.99870500 \quad-0.72600500$ 
$4.33074600 \quad-3.08619200 \quad 1.65228400$

$5.43527700 \quad-1.25610300 \quad 1.34738600$

$5.99003500 \quad 1.43152400 \quad 1.29871000$

$7.09201900 \quad 1.47494300 \quad 1.22291500$

$\begin{array}{lll}5.62552800 & 2.46391300 & 1.21366000\end{array}$

$5.73688500 \quad 1.04880300 \quad 2.29934800$

$\begin{array}{lll}-5.36233600 & 0.88502200 & 0.88664900\end{array}$

$\begin{array}{lll}-4.14756400 & 0.32816800 & 0.45700700\end{array}$

$\begin{array}{lll}-3.15678500 & 1.21430600 & -0.03944100\end{array}$

$\begin{array}{lll}-3.45539000 & 2.56590100 & -0.28158800\end{array}$

$\begin{array}{lll}-4.68767600 & 3.06805200 & 0.15815700\end{array}$

$\begin{array}{lll}-5.62484000 & 2.24948900 & 0.78077900\end{array}$

$\begin{array}{lll}-1.23649500 & 2.73003300 & -1.43484700\end{array}$

$\begin{array}{lll}-0.92781600 & 1.46474200 & -0.90239000\end{array}$

$\begin{array}{lll}0.38657700 & 0.93828900 & -1.01259300\end{array}$

$\begin{array}{lll}1.29712400 & 1.63385900 & -1.82561800\end{array}$

$0.96064600 \quad 2.81636800 \quad-2.47647300$

$\begin{array}{lll}-0.28877200 & 3.37502600 & -2.24073400\end{array}$

$\begin{array}{lll}-6.13011200 & 0.23019200 & 1.29291400\end{array}$

$\begin{array}{lll}-4.91931900 & 4.12216200 & -0.00383100\end{array}$

$\begin{array}{lll}-6.57281300 \quad 2.66037100 & 1.13513900\end{array}$

$\begin{array}{lll}2.31496400 & 1.25509000 & -1.91257300\end{array}$

$\begin{array}{lll}1.69480200 & 3.32779900 & -3.10112400\end{array}$

$\begin{array}{lll}-0.53271000 & 4.34844800 & -2.66918300\end{array}$

$\begin{array}{lll}-2.52855200 & 3.46503000 & -1.08934300\end{array}$

$\begin{array}{lll}-3.26593000 & 3.87759100 & -2.38490800\end{array}$

$\begin{array}{lll}-2.64155000 & 4.53969700 & -3.00262200\end{array}$

$\begin{array}{lll}-3.52609100 & 2.98993200 \quad-2.98219400\end{array}$

$\begin{array}{lll}-4.19392300 & 4.41940100 & -2.14978500\end{array}$

$\begin{array}{lll}-2.16990200 & 4.72809800 & -0.27106600\end{array}$

$\begin{array}{lll}-3.07319000 & 5.30682700 & -0.02661600\end{array}$

$\begin{array}{lll}-1.66997200 & 4.45416100 \quad 0.66966700\end{array}$

$\begin{array}{lll}-1.49666200 & 5.38499300 & -0.84123500\end{array}$

$\begin{array}{lll}-1.89425700 & 0.70947900 & -0.28006100\end{array}$

$\begin{array}{lll}-4.65418800 & -2.21342600 & 1.97894400\end{array}$

$\begin{array}{lll}-6.17109200 & -2.41151600 \quad 2.08805600\end{array}$

$\begin{array}{lll}-6.73458100 & -1.47371900 & 1.99280500\end{array}$

$\begin{array}{lll}-6.55188100 & -3.12074100 & 1.34041300\end{array}$

$\begin{array}{lll}-6.40496400 & -2.83002100 \quad 3.08313900\end{array}$

$\begin{array}{lll}-4.17882200 & -1.30422400 & 3.12533300\end{array}$

$\begin{array}{lll}-4.73362000 & -0.35662600 & 3.15835600\end{array}$

$\begin{array}{lll}-4.33704000 & -1.81972800 \quad 4.08854300\end{array}$

$-3.10812000 \quad-1.06581600 \quad 3.04668300$

$\begin{array}{lll}-3.97601100 & -3.59031900 \quad 2.13882300\end{array}$ 


\begin{tabular}{|c|c|c|}
\hline-0.15606100 & -0.74941800 & 0.46041900 \\
\hline 0.11733100 & 0.47135200 & -0.25187400 \\
\hline 0.37371400 & 1.77768600 & 0.38009900 \\
\hline 0.44562400 & 1.96694400 & 1.77853100 \\
\hline 0.54077900 & 2.79773400 & -1.52015300 \\
\hline-0.25967900 & 0.54786600 & -1.27981600 \\
\hline 0.59449900 & 2.91240300 & -0.43346500 \\
\hline 0.73615700 & 3.21748500 & 2.32780000 \\
\hline 0.96111300 & 4.32655800 & 1.50344300 \\
\hline 0.88416200 & 4.16242600 & 0.11488300 \\
\hline 0.78409500 & 3.32803700 & 3.41504900 \\
\hline 1.18792800 & 5.30400400 & 1.93648500 \\
\hline 1.04983800 & 5.01738600 & -0.54702500 \\
\hline-1.07792700 & -1.77104000 & -0.14433200 \\
\hline-2.46970000 & -1.35829900 & 0.12070000 \\
\hline-3.11567700 & -0.62949600 & -0.79100300 \\
\hline-4.41856300 & -0.04863000 & -0.22906500 \\
\hline-2.70441000 & -0.42404800 & -1.91513700 \\
\hline-5.23886500 & -1.15175200 & 0.45996500 \\
\hline-4.01913900 & 1.03171200 & 0.79743900 \\
\hline-5.22655900 & 0.58021400 & -1.36807300 \\
\hline-5.50055900 & -1.95308200 & -0.25003800 \\
\hline-4.68247200 & -1.60149800 & 1.29423700 \\
\hline-6.17611900 & -0.72922300 & 0.85632500 \\
\hline-3.40495200 & 1.81697800 & 0.32787100 \\
\hline-4.92178200 & 1.50639200 & 1.21453400 \\
\hline-3.44017500 & 0.59717300 & 1.62563300 \\
\hline-6.15089200 & 1.02784200 & -0.97033200 \\
\hline-4.65198900 & 1.36744100 & -1.87782100 \\
\hline-5.50611200 & -0.17303200 & -2.12114000 \\
\hline 0.27277600 & 1.12038700 & 2.44624300 \\
\hline 1.67811700 & -0.69450100 & -0.15607300 \\
\hline-0.25301400 & -0.70691300 & 1.55606600 \\
\hline-0.94200000 & -3.16496300 & 0.43720900 \\
\hline-1.06639800 & -3.14702400 & 1.53238600 \\
\hline 0.05727900 & -3.56392600 & 0.21195700 \\
\hline-1.69613700 & -3.84583900 & 0.01254500 \\
\hline-0.96146300 & -1.79647900 & -1.23570500 \\
\hline 2.55926500 & -2.26274700 & 0.36640400 \\
\hline 3.10779500 & -3.23222000 & 0.69070200 \\
\hline 3.78372500 & -4.44904000 & 1.10038800 \\
\hline 3.05066300 & -5.25942500 & 1.23556500 \\
\hline 31100300 & -4.28049600 & 2.05237000 \\
\hline
\end{tabular}




\begin{tabular}{|c|c|c|c|}
\hline $\mathrm{H}$ & 4.51599200 & -4.75001900 & 0.33531600 \\
\hline $\mathrm{N}$ & 3.04707200 & 0.28742300 & -1.00177200 \\
\hline $\mathrm{C}$ & 3.84048000 & 0.98290900 & -1.48323400 \\
\hline $\mathrm{C}$ & 4.82279700 & 1.86896100 & -2.07909500 \\
\hline $\mathrm{H}$ & 4.51226200 & 2.91611800 & -1.93966700 \\
\hline $\mathrm{H}$ & 5.80502900 & 1.71781500 & -1.60522200 \\
\hline $\mathrm{H}$ & 4.90971900 & 1.66131200 & -3.15696100 \\
\hline \multicolumn{4}{|c|}{ TS26 } \\
\hline $\mathrm{C}$ & -0.25824800 & -1.51939100 & 0.26800300 \\
\hline $\mathrm{C}$ & -1.42666300 & -0.83646400 & 0.82507400 \\
\hline $\mathrm{C}$ & -2.74602600 & -0.87115300 & 0.16688700 \\
\hline $\mathrm{C}$ & -2.90799600 & -1.11745400 & -1.21515100 \\
\hline $\mathrm{H}$ & -3.81594000 & -0.40003900 & 1.98656200 \\
\hline $\mathrm{H}$ & -1.49567300 & -0.79750800 & 1.92248200 \\
\hline $\mathrm{C}$ & -3.91091400 & -0.59429700 & 0.91416700 \\
\hline $\mathrm{C}$ & -4.16917400 & -1.09374900 & -1.81175500 \\
\hline $\mathrm{C}$ & -5.31298200 & -0.81448300 & -1.05298600 \\
\hline $\mathrm{C}$ & -5.17251100 & -0.56240300 & 0.31611500 \\
\hline $\mathrm{H}$ & -4.26057400 & -1.29021200 & -2.88380600 \\
\hline $\mathrm{H}$ & -6.29916900 & -0.79321000 & -1.52344800 \\
\hline $\mathrm{H}$ & -6.05389000 & -0.34333300 & 0.92555400 \\
\hline $\mathrm{C}$ & 0.84456600 & -1.92197700 & 1.09172100 \\
\hline $\mathrm{H}$ & 0.77344700 & -1.62581500 & 2.14325200 \\
\hline $\mathrm{C}$ & 2.56948400 & -0.01740400 & -0.03609300 \\
\hline $\mathrm{O}$ & 1.66227100 & 0.69791900 & -0.52088400 \\
\hline $\mathrm{C}$ & 4.02476900 & 0.21595000 & -0.48599800 \\
\hline $\mathrm{C}$ & 4.90182300 & 0.42758800 & 0.76026600 \\
\hline $\mathrm{C}$ & 4.11269100 & 1.43921200 & -1.40370900 \\
\hline $\mathrm{C}$ & 4.48177600 & -1.04655600 & -1.24057800 \\
\hline $\mathrm{H}$ & 4.85212100 & -0.44201400 & 1.43120600 \\
\hline $\mathrm{H}$ & 4.57809700 & 1.31645600 & 1.32628100 \\
\hline $\mathrm{H}$ & 5.95191500 & 0.57844200 & 0.46234500 \\
\hline $\mathrm{H}$ & 3.50015600 & 1.30680500 & -2.30755900 \\
\hline $\mathrm{H}$ & 5.15776700 & 1.59843400 & -1.71406800 \\
\hline $\mathrm{H}$ & 3.76361600 & 2.34871300 & -0.89168600 \\
\hline $\mathrm{H}$ & 5.51739300 & -0.92035700 & -1.59519400 \\
\hline $\mathrm{H}$ & 3.84225100 & -1.23711100 & -2.11792200 \\
\hline $\mathrm{H}$ & 4.44370000 & -1.93197800 & -0.58919500 \\
\hline $\mathrm{O}$ & 2.41225500 & -0.95808300 & 0.81359200 \\
\hline $\mathrm{H}$ & -0.34664100 & -2.04293600 & -0.69245200 \\
\hline $\mathrm{H}$ & -2.03052600 & -1.31780000 & -1.83351600 \\
\hline $\mathrm{C}$ & 1.48355300 & -3.25918700 & 0.86254200 \\
\hline $\mathrm{H}$ & 0.76345400 & -4.03092500 & 1.18734200 \\
\hline $\mathrm{H}$ & 2.41016100 & -3.38653400 & 1.43786500 \\
\hline
\end{tabular}


$1.69168400 \quad-3.42256800 \quad-0.20591000$

$-0.16822400$

0.42761800

0.15045400

$\begin{array}{lll}-0.81296800 & 2.14810800 \quad 0.31453600\end{array}$

$-1.25156400$

3.21887300

0.39888800

C

$\begin{array}{lll}-1.80863900 & 4.55402100 & 0.50582700\end{array}$

$\begin{array}{lll}-1.00190600 \quad 5.29022000 & 0.64538900\end{array}$

$\begin{array}{lll}-2.36343400 & 4.80119400 & -0.41283100\end{array}$

$\begin{array}{lll}-2.49684400 & 4.60659300 & 1.36385500\end{array}$

27

H

H

C

C

C

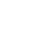

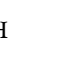

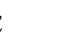

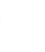

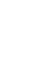

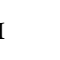

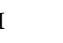

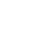

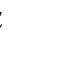

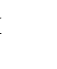

C

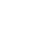

C

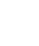

H

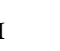

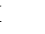

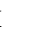

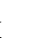

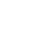

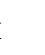

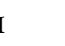

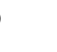

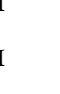

$0.90293900 \quad-2.18725100 \quad 0.02645100$

$\begin{array}{lll}1.64404300 & -1.24656700 & -0.73485700\end{array}$

$2.93736000 \quad-0.67829200 \quad-0.31745500$

$\begin{array}{lll}3.31711200 & -0.59011400 & 1.03717700\end{array}$

$\begin{array}{lll}3.52449800 & -0.19593300 & -2.34062000\end{array}$

$1.45284800 \quad-1.20874300 \quad-1.81533300$

$3.80927200 \quad-0.14719900 \quad-1.28607100$

$\begin{array}{lll}4.53187600 & -0.01057100 & 1.40324600\end{array}$

$\begin{array}{lll}5.39377400 & 0.50611300 & 0.42738500\end{array}$

$\begin{array}{lll}5.02431400 & 0.43727300 & -0.91957300\end{array}$

$\begin{array}{lll}4.80529500 & 0.04878800 & 2.46006500\end{array}$

$\begin{array}{lll}6.34273100 & 0.96430400 & 0.71693900\end{array}$

$\begin{array}{lll}5.68570000 & 0.84133600 & -1.69055100\end{array}$

$\begin{array}{lll}-0.43593200 & -2.41399000 & -0.33417200\end{array}$

$\begin{array}{lll}-0.70229600 & -2.32423200 & -1.39594200\end{array}$

$\begin{array}{lll}-0.07263400 & -0.44566900 & -0.03341000\end{array}$

$\begin{array}{lll}-2.62858200 & 0.22412900 & -0.32980800\end{array}$

$\begin{array}{lll}-1.82858200 & -0.03996500 & 0.64426900\end{array}$

$\begin{array}{lll}-4.06124500 & 0.61700400 & 0.09908000\end{array}$

$\begin{array}{lll}-4.92350900 & 0.90656000 & -1.13272600\end{array}$

$\begin{array}{lll}-3.96942500 & 1.87184700 & 0.98500400\end{array}$

$\begin{array}{lll}-4.66765100 & -0.54487800 & 0.90469400\end{array}$

$\begin{array}{lll}-4.98924500 & 0.02458800 & -1.78784300\end{array}$

$-4.50153000 \quad 1.72974300 \quad-1.72901600$

$\begin{array}{lll}-5.94546000 & 1.18817000 & -0.82896700\end{array}$

$\begin{array}{lll}-3.35344600 & 1.67776000 & 1.87517200\end{array}$

$\begin{array}{lll}-4.97265500 & 2.18812900 \quad 1.31643700\end{array}$

$\begin{array}{lll}-3.51453100 & 2.71214300 & 0.43425000\end{array}$

$\begin{array}{lll}-4.05515200 & -0.77047900 \quad 1.78958600\end{array}$

$\begin{array}{lll}-4.73229200 & -1.45973600 & 0.29246400\end{array}$

$\begin{array}{lll}-5.68710400 & -0.29219700 & 1.24138600\end{array}$

$\begin{array}{lll}-2.30427700 & 0.18030000 & -1.52286400\end{array}$

$\begin{array}{lll}2.64180100 & -0.95823400 & 1.81256800\end{array}$

$\begin{array}{lll}1.26138700 & -2.51303400 & 1.01000600\end{array}$

$-1.39602500 \quad-3.19047800 \quad 0.51555200$ 


$\begin{array}{rrr}-1.44629700 & -4.24537700 & 0.19064300 \\ -2.41265700 & -2.77661100 & 0.43450900 \\ -1.10380400 & -3.17455600 & 1.57694300 \\ 0.54583000 & 1.33998000 & 0.04058900 \\ 0.97610700 & 2.41100300 & 0.09315900 \\ 1.53560100 & 3.74567500 & 0.16039700 \\ 1.06010100 & 4.38743800 & -0.59701500 \\ 1.35890700 & 4.17276800 & 1.15936900 \\ 2.61883300 & 3.69857300 & -0.02983500\end{array}$

S28

$-0.09813100$

0.03718500

0.17544600

0.59154600

$-0.39371600$

$-0.47436200$

$-0.07085800$

0.74782000

0.49966700

0.08958300

1.06955500

0.62426200

$-0.11018300$

$-0.48364600$

$-2.63911100$

$-3.03089000$

$-4.31931500$

$-2.45017800$

$-5.35884700$

$-3.88887000$

$-4.90694000$

$-5.68552300$

$-4.93614300$

$-6.25331700$

$-3.14294500$

$-4.75371200$

$-3.43885600$

$-5.79600800$

$-4.16834200$

$-5.21071200$

0.80230600

1.68431100

$-0.00231400$

$-0.37759800$
0.87917200

0.02983500

0.54015800

1.85552600

$-1.35087300$

$-0.93927300$

$-0.32670100$

2.28218700

1.40757600

0.09818700

3.30791600

1.74411000

$-0.59638700$

0.34857900

0.40646900

$-0.62038300$

$-0.40913800$

$-1.12259500 \quad 0.38843400$

$0.91598400 \quad 0.39803000$

$0.11191100 \quad-1.75397100$

$-2.01700600 \quad-0.16878500$

$-1.46032200 \quad 1.34531700$

$-0.51003900 \quad 0.59480200$

$1.51094100-0.15419300$

$1.56848000 \quad 0.60812300$

$0.62020100 \quad 1.35827400$

$0.74813600-1.60382100$

$0.67635100-2.34094400$

$-0.75936900 \quad-2.35679600$

$1.28727200 \quad 2.55225500$

$\begin{array}{ll}-0.61688500 & -0.12862500\end{array}$

$-0.64019300 \quad 1.96709300$

1.11637600 


$$
\begin{array}{ccc}
-0.45811700 & -3.07059700 & 2.20094300 \\
0.59964200 & -3.71463000 & 0.91935000 \\
-1.16045200 & -3.94445200 & 0.80340000 \\
-0.62022100 & -2.03229800 & -0.73148300 \\
2.85430500 & -2.09449500 & 0.14222200 \\
3.55490000 & -2.99922400 & 0.31870500 \\
4.43020700 & -4.13220000 & 0.54464300 \\
3.83215200 & -5.01823100 & 0.80729600 \\
5.12306600 & -3.90598800 & 1.36976700 \\
5.01049900 & -4.34313600 & -0.36679800 \\
\hline 2.75986100 & 0.59270300 & -1.07835500 \\
\hline 3.38305400 & 1.40399200 & -1.62031200 \\
\hline 4.15150100 & 2.43233700 & -2.29316900 \\
\hline 3.67114900 & 3.41101700 & -2.13966200 \\
5.17433100 & 2.46163000 & -1.88741500 \\
4.19751200 & 2.21704500 & -3.37186900
\end{array}
$$


H

$\mathrm{H}$

$\mathrm{H}$

$\mathrm{H}$

$\mathrm{O}$

$\mathrm{H}$

$\mathrm{H}$

\begin{tabular}{|c|c|c|}
\hline-3.51453100 & 2.71214300 & -0.43425000 \\
\hline-4.05515200 & -0.77047900 & -1.78958600 \\
\hline-4.73229200 & -1.45973600 & -0.29246400 \\
\hline-5.68710400 & -0.29219700 & -1.24138600 \\
\hline-2.30427700 & 0.18030000 & 1.52286400 \\
\hline 2.64180100 & -0.95823400 & -1.81256800 \\
\hline 1.26138700 & -2.51303400 & -1.01000600 \\
\hline-1.39602500 & -3.19047800 & -0.51555200 \\
\hline-1.44629700 & -4.24537700 & -0.19064300 \\
\hline-2.41265700 & -2.77661100 & -0.43450900 \\
\hline-1.10380400 & -3.17455600 & -1.57694300 \\
\hline 0.54583000 & 1.33998000 & -0.04058900 \\
\hline 0.97610700 & 2.41100300 & -0.09315900 \\
\hline 1.53560100 & 3.74567500 & -0.16039700 \\
\hline 1.06010100 & 4.38743800 & 0.59701500 \\
\hline 1.35890700 & 4.17276800 & -1.15936900 \\
\hline 2.61883300 & 3.69857300 & 0.02983500 \\
\hline-2.06596000 & -1.12082400 & 0.00869700 \\
\hline 0.01309600 & 0.94084000 & 0.38574600 \\
\hline 2.11334800 & -1.14096000 & 0.18114200 \\
\hline-1.18065400 & 1.54717700 & 0.07833800 \\
\hline-2.31132400 & 0.71775500 & -0.06864700 \\
\hline-3.53795900 & 1.35688400 & -0.31805500 \\
\hline-3.61809400 & 2.74174100 & -0.46970400 \\
\hline-2.46851900 & 3.52801300 & -0.35652400 \\
\hline-1.23126100 & 2.94358800 & -0.06523700 \\
\hline 1.18133300 & 1.50561400 & -0.06426700 \\
\hline 2.28084500 & 0.65164400 & -0.28514100 \\
\hline 3.42601800 & 1.23186300 & -0.85950600 \\
\hline 3.47804700 & 2.59286900 & -1.16306300 \\
\hline 2.39196400 & 3.42031800 & -0.86408400 \\
\hline 1.22894800 & 2.89231300 & -0.29473600 \\
\hline 0.04994300 & 3.73880100 & 0.19035400 \\
\hline-4.44533700 & 0.76246300 & -0.40702700 \\
\hline-4.58250700 & 3.21246100 & -0.67500100 \\
\hline-2.54490700 & 4.60919300 & -0.47520900 \\
\hline 4.28687600 & 0.60788100 & -1.08849900 \\
\hline 4.37579000 & 3.01569500 & -1.62032800 \\
\hline 2.46073600 & 4.48880300 & -1.07019800 \\
\hline 0.01376800 & 5.11886400 & -0.47359300 \\
\hline 0.20013000 & 3.92559100 & 1.72317000 \\
\hline-0.09780200 & 5.04238000 & -1.56606600 \\
\hline-0.82009400 & 5.71669900 & -0.08023300 \\
\hline
\end{tabular}


$0.93393000 \quad 5.67922400 \quad-0.25595200$

$\begin{array}{lll}-0.64641800 & 4.50833000 & 2.11942400\end{array}$

$\begin{array}{lll}0.22315600 & 2.95628800 \quad 2.24367400\end{array}$

$\begin{array}{lll}1.13507400 \quad 4.46087000 & 1.95237900\end{array}$

$\begin{array}{lll}-2.59815700 & -1.47749000 & 1.81329900\end{array}$

$\begin{array}{lll}-3.32467500 & -1.86866700 & -1.23076700\end{array}$

$\begin{array}{lll}2.31662600 & -2.03540500 & -1.50034800\end{array}$

$\begin{array}{lll}3.61002500 & -1.44771300 & 1.33106200\end{array}$

$-2.79880000 \quad-3.29510400 \quad-1.49901200$

$\begin{array}{lll}-2.81333300 & -3.92252300 & -0.59633700\end{array}$

$\begin{array}{lll}-3.43471000 & -3.78587900 & -2.25610100\end{array}$

$\begin{array}{lll}-1.76691700 & -3.27968700 & -1.88329100\end{array}$

$\begin{array}{lll}-4.80169600 & -1.96484600 & -0.82838700\end{array}$

$\begin{array}{lll}-5.25277600 & -0.98923500 & -0.60275700\end{array}$

$\begin{array}{lll}-5.37564600 & -2.40356100 & -1.66399300\end{array}$

$\begin{array}{lll}-4.94672400 & -2.61821200 & 0.04338000\end{array}$

$\begin{array}{lll}-3.18714400 & -1.07087000 & -2.53990700\end{array}$

$\begin{array}{lll}-3.67093800 & -1.62917300 & -3.35992800\end{array}$

$\begin{array}{lll}-3.66207000 & -0.08203900 & -2.48238400\end{array}$

$\begin{array}{lll}-2.12996300 & -0.92346000 & -2.81674900\end{array}$

$\begin{array}{lll}-1.45705900 & -0.91168400 & 2.67974900\end{array}$

$\begin{array}{lll}-1.38172700 & 0.18282200 & 2.59392800\end{array}$

$\begin{array}{lll}-1.64980600 & -1.15124800 \quad 3.74000400\end{array}$

$\begin{array}{lll}-0.48175500 & -1.33879500 & 2.40138800\end{array}$

$\begin{array}{lll}-3.91655300 & -0.83616800 & 2.25785100\end{array}$

$\begin{array}{lll}-3.88639400 & 0.25923500 & 2.16458900\end{array}$

$\begin{array}{lll}-4.77727300 & -1.20514600 \quad 1.68662900\end{array}$

$-4.09773800 \quad-1.07143900 \quad 3.32204600$

$\begin{array}{lll}-2.65394400 & -2.99960700 & 2.00859300\end{array}$

$\begin{array}{lll}-3.50157400 & -3.45456500 & 1.47651700\end{array}$

$-1.72774900 \quad-3.48651200 \quad 1.66289100$

$\begin{array}{lll}-2.77393900 & -3.23016000 \quad 3.08133300\end{array}$

$3.67501800 \quad-2.96336200 \quad 1.58304500$

$\begin{array}{lll}4.35384400 & -3.16772000 & 2.42899600\end{array}$

$\begin{array}{lll}2.68696600 & -3.37896300 & 1.83861200\end{array}$

$4.06226400 \quad-3.50855800 \quad 0.70950500$

$\begin{array}{lll}4.99834100 & -0.94178400 & 0.91980800\end{array}$

$\begin{array}{lll}5.04340300 & 0.15534100 & 0.89838000\end{array}$

$5.73756100 \quad-1.28042800 \quad 1.66758300$

$5.32098200 \quad-1.32317000 \quad-0.05745900$

$3.20783200 \quad-0.74204200 \quad 2.64092200$

$3.99466500 \quad-0.88895000 \quad 3.40119800$

$\begin{array}{lll}3.08348800 & 0.34317200 & 2.49438800\end{array}$

$2.26467500 \quad-1.13955000 \quad 3.04775000$ 


\begin{tabular}{lll}
3.73149200 & -2.14008400 & -2.07732600 \\
4.18949600 & -1.15441100 & -2.24093700 \\
4.39662800 & -2.73023100 & -1.43075400 \\
3.69007400 & -2.64856200 & -3.05751200 \\
1.41008300 & -1.27989000 & -2.48889600 \\
0.40460200 & -1.11262200 & -2.07031600 \\
1.82978300 & -0.30277900 & -2.76819900 \\
1.29555900 & -1.87524900 & -3.41124000 \\
1.73825600 & -3.45035100 & -1.30639700 \\
0.70620600 & -3.41237200 & -0.92365100 \\
1.72203600 & -3.98067700 & -2.27453800 \\
\hline 2.33775600 & -4.05275800 & -0.60905700
\end{tabular}

30

$\mathrm{H}$

$\mathrm{H}$

$\mathrm{H}$

C

H

$\mathrm{H}$

$\mathrm{H}$

C

$\mathrm{H}$

H

H

C

.

.

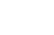

.

.

.

.

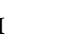

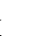

.

$\mathrm{Ni}$

.

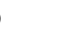

C

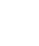

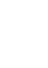

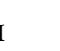

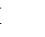

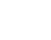

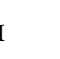

H

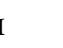

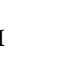

H

\begin{tabular}{|c|c|c|}
\hline 4.29541100 & -0.94836800 & 0.95513000 \\
\hline 3.96142000 & -0.40051800 & -0.33264500 \\
\hline 4.01626400 & -1.14440400 & -1.60180200 \\
\hline 4.06798100 & -2.55521400 & -1.66828000 \\
\hline 3.92012500 & 0.65140900 & -2.80149800 \\
\hline 4.06467100 & 0.68427100 & -0.45933300 \\
\hline 3.95997700 & -0.44130000 & -2.82445600 \\
\hline 4.04656500 & -3.22214600 & -2.89320400 \\
\hline 3.97703700 & -2.50620200 & -4.09577500 \\
\hline 3.93800600 & -1.10851800 & -4.05088600 \\
\hline 4.07898100 & -4.31518600 & -2.91097100 \\
\hline 3.95540200 & -3.03226400 & -5.05337000 \\
\hline 3.88850600 & -0.53217400 & -4.97900800 \\
\hline 4.85115700 & -0.05389600 & 2.02466000 \\
\hline 2.38777900 & -0.87554300 & 0.67839200 \\
\hline 6.68348200 & 1.21911600 & 1.07895500 \\
\hline 5.94191900 & 2.11841800 & 0.73624100 \\
\hline 8.17354600 & 1.14769700 & 0.72538400 \\
\hline 9.00226800 & 0.88360600 & 1.99402000 \\
\hline 8.34857500 & -0.01593400 & -0.27166800 \\
\hline 8.60395700 & 2.46656100 & 0.07646700 \\
\hline 8.86507300 & 1.69002400 & 2.73274500 \\
\hline 8.71656000 & -0.06664600 & 2.46668000 \\
\hline 10.07293600 & 0.83564700 & 1.73839500 \\
\hline 7.73296600 & 0.13880400 & -1.17265000 \\
\hline 9.40255200 & -0.08733200 & -0.58471600 \\
\hline 8.05442600 & -0.97403600 & 0.18131800 \\
\hline 9.66967500 & 2.41980900 & -0.19754000 \\
\hline 8.02154400 & 2.67282200 & -0.83361200 \\
\hline 8.46308800 & 3.31430300 & 0.76481800 \\
\hline 29363700 & 0.15525800 & 1.78208800 \\
\hline
\end{tabular}


$4.70284900 \quad-1.97053400 \quad 1.00079400$

$\begin{array}{lll}4.10744800 & -3.13639900 & -0.74478400\end{array}$

$\begin{array}{lll}0.87843100 & 1.70097700 & -1.08940900\end{array}$

$\begin{array}{lll}-0.22555600 & 1.01707000 & -0.53837700\end{array}$

$\begin{array}{lll}-1.46096300 & 1.70115000 & -0.49620500\end{array}$

$\begin{array}{lll}-1.55258500 & 3.04834700 & -0.90567800\end{array}$

$\begin{array}{lll}-0.43713400 & 3.66978500 & -1.47516500\end{array}$

$\begin{array}{lll}0.77669300 & 2.99509600 & -1.58747300\end{array}$

$\begin{array}{lll}-3.99206900 & 2.83586100 & -0.85854600\end{array}$

$\begin{array}{lll}-3.83824500 & 1.50411100 & -0.42630700\end{array}$

$\begin{array}{lll}-4.94020900 & 0.62158400 & -0.36613600\end{array}$

$\begin{array}{lll}-6.16497300 & 1.08221400 & -0.88276500\end{array}$

$\begin{array}{lll}-6.30396900 & 2.35739700 & -1.42370300\end{array}$

$\begin{array}{lll}-5.22294000 & 3.23807600 & -1.38512500\end{array}$

$1.83638000 \quad 1.18007000 \quad-1.11479000$

$\begin{array}{lll}-0.50882000 & 4.70354100 & -1.81163100\end{array}$

$\begin{array}{lll}1.64732400 \quad 3.48643000 & -2.02740100\end{array}$

$\begin{array}{lll}-7.03293800 & 0.42684700 & -0.85259200\end{array}$

$\begin{array}{lll}-7.26325500 & 2.67925400 & -1.83548300\end{array}$

$\begin{array}{lll}-5.34900000 \quad 4.25851300 & -1.74606300\end{array}$

$\begin{array}{lll}-2.84226800 & 3.80123600 & -0.60743500\end{array}$

$\begin{array}{lll}-2.84506400 \quad 4.16192100 & 0.90262100\end{array}$

$\begin{array}{lll}-3.78733900 & 4.66503500 & 1.17085100\end{array}$

$\begin{array}{lll}-2.74385100 & 3.26497000 & 1.53174800\end{array}$

$\begin{array}{lll}-2.00607000 \quad 4.83755500 & 1.13213100\end{array}$

$\begin{array}{lll}-2.97346200 & 5.09595500 & -1.41476800\end{array}$

$\begin{array}{lll}-2.14892700 & 5.78335400 & -1.18102900\end{array}$

$\begin{array}{lll}-2.97278200 & 4.90603900 & -2.49888200\end{array}$

$\begin{array}{lll}-3.90320600 & 5.62042000 & -1.15417900\end{array}$

$\begin{array}{lll}-2.58760700 & 1.05858800 & -0.02051900\end{array}$

$0.27772700 \quad-0.66755900 \quad 0.03083100$

$\begin{array}{lll}-4.78889800 & -1.01070500 & 0.49000800\end{array}$

$\begin{array}{lll}-0.34412000 & -1.03723100 & 1.79520100\end{array}$

$\begin{array}{lll}-0.51098500 & 0.30894400 & 2.51317400\end{array}$

$\begin{array}{lll}0.37800300 & 0.94660600 & 2.38432200\end{array}$

$\begin{array}{lll}-1.39076600 & 0.85279600 & 2.14496800\end{array}$

$\begin{array}{lll}-0.64411600 & 0.13146100 & 3.59379800\end{array}$

$\begin{array}{lll}0.87435200 & -1.77601500 & 2.39084900\end{array}$

$0.72427700 \quad-1.96908900 \quad 3.46717400$

$\begin{array}{lll}1.06491300 & -2.74421100 & 1.90535700\end{array}$

$1.80550700 \quad-1.14278700 \quad 2.37492400$

$\begin{array}{lll}-0.34061600 & -1.86323300 & -1.29281300\end{array}$

$\begin{array}{lll}0.38196600 & -1.45099600 & -2.58707800\end{array}$

$0.07504300 \quad-0.45103500 \quad-2.92864200$ 
$1.47260200 \quad-1.45131300 \quad-2.46222600$

$\begin{array}{lll}0.12952500 & -2.16991400 & -3.38462100\end{array}$

$\begin{array}{lll}0.12998600 & -3.27108800 & -0.89776000\end{array}$

$\begin{array}{lll}-0.37243100 & -3.63986500 & 0.00702200\end{array}$

$\begin{array}{lll}-0.09382800 & -3.97501700 & -1.71697100\end{array}$

$1.21662100 \quad-3.28944900 \quad-0.72180500$

$\begin{array}{lll}-1.85009100 & -1.82545600 & -1.52178800\end{array}$

$\begin{array}{lll}-2.18028700 & -0.82848700 & -1.84681900\end{array}$

$\begin{array}{lll}-2.11667800 & -2.54308100 & -2.31698200\end{array}$

$\begin{array}{lll}-2.42237600 & -2.08311500 & -0.62415700\end{array}$

$\begin{array}{lll}-5.60140800 & -0.57957000 & 2.17254700\end{array}$

$\begin{array}{lll}-4.60680300 & 0.37681300 & 2.85835400\end{array}$

$\begin{array}{lll}-3.60729700 & -0.06838500 & 2.96102100\end{array}$

$\begin{array}{lll}-4.50431700 & 1.32538700 \quad 2.30900300\end{array}$

$\begin{array}{lll}-4.97367000 & 0.61999500 \quad 3.87055400\end{array}$

$\begin{array}{lll}-6.97327200 & 0.10187000 & 2.13465500\end{array}$

$\begin{array}{lll}-7.74343800 & -0.51745500 & 1.65931300\end{array}$

$\begin{array}{lll}-7.30402800 & 0.30653500 & 3.16861300\end{array}$

$\begin{array}{lll}-6.93385300 & 1.06684800 & 1.60952100\end{array}$

$\begin{array}{lll}-5.68040100 & -1.87879100 & 2.98977100\end{array}$

$\begin{array}{lll}-5.96603400 & -1.64545900 \quad 4.03004500\end{array}$

$\begin{array}{lll}-6.43209400 & -2.57352200 & 2.58818400\end{array}$

$\begin{array}{lll}-4.71058700 & -2.40080900 \quad 3.01718900\end{array}$

$\begin{array}{lll}-5.87990100 & -2.24365100 & -0.49992700\end{array}$

$-5.31893700 \quad-3.62944000 \quad-0.11639800$

$\begin{array}{lll}-5.84120500 & -4.41390300 & -0.69119800\end{array}$

$\begin{array}{lll}-4.24326800 & -3.70454600 & -0.33826300\end{array}$

$\begin{array}{lll}-5.46002000 & -3.85079700 & 0.95191100\end{array}$

$\begin{array}{lll}-5.59298100 & -2.01273300 & -1.99361100\end{array}$

$\begin{array}{lll}-6.06823200 & -1.09783800 & -2.37275000\end{array}$

$-4.51400400 \quad-1.94362500 \quad-2.19830100$

$\begin{array}{lll}-5.98871100 & -2.86317800 & -2.57529700\end{array}$

$\begin{array}{lll}-7.39528000 & -2.25514300 & -0.26359500\end{array}$

$\begin{array}{lll}-7.64890700 & -2.50814600 & 0.77522200\end{array}$

$\begin{array}{lll}-7.87868400 & -1.30084900 & -0.51292800\end{array}$

$\begin{array}{lll}-7.85199600 & -3.02768400 & -0.90763800\end{array}$

$\begin{array}{lll}-1.59484600 & -1.89010300 & 1.96661900\end{array}$

$\begin{array}{lll}-1.47590100 & -2.89229500 & 1.53057400\end{array}$

$\begin{array}{lll}-1.79567700 & -2.02402000 & 3.04416300\end{array}$

$\begin{array}{lll}-2.47622200 & -1.42551600 \quad 1.51065900\end{array}$

$\begin{array}{lll}4.38242100 & 0.93767600 & 1.97819700\end{array}$

$\begin{array}{lll}4.75051100 & -0.62475900 & 3.42672700\end{array}$

$5.23999300 \quad-1.61081800 \quad 3.48330800$

$3.69344200 \quad-0.75103200 \quad 3.70366300$ 
31

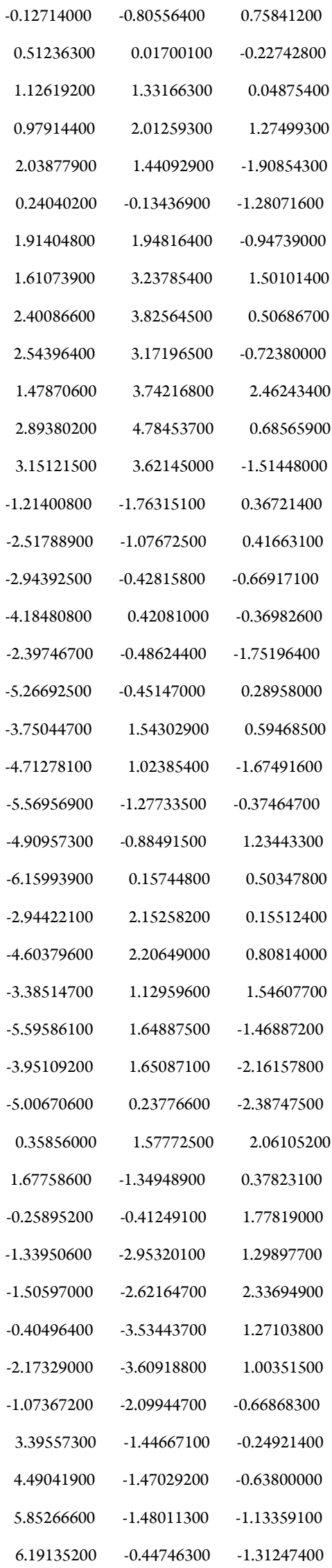




\begin{tabular}{|c|c|c|c|}
\hline $\mathrm{H}$ & 6.51796700 & -1.95117500 & -0.39324400 \\
\hline $\mathrm{H}$ & 5.90824000 & -2.04560800 & -2.07693900 \\
\hline \multicolumn{4}{|c|}{$\mathrm{MeCN}$} \\
\hline $\mathrm{C}$ & 0.00000000 & 0.00000000 & -1.17677200 \\
\hline $\mathrm{H}$ & 0.00000000 & 1.03421500 & -1.55330500 \\
\hline $\mathrm{H}$ & -0.89565600 & -0.51710700 & -1.55330500 \\
\hline $\mathrm{H}$ & 0.89565600 & -0.51710700 & -1.55330500 \\
\hline $\mathrm{C}$ & 0.00000000 & 0.00000000 & 0.27742100 \\
\hline $\mathrm{N}$ & 0.00000000 & 0.00000000 & 1.43657400 \\
\hline \multicolumn{4}{|c|}{ Conformation-A } \\
\hline $\mathrm{C}$ & 1.90381100 & 1.21749500 & 0.98860400 \\
\hline $\mathrm{C}$ & 1.65064300 & -0.15744600 & 1.25743700 \\
\hline $\mathrm{C}$ & 0.98450200 & -0.60924100 & 2.50770100 \\
\hline $\mathrm{C}$ & 1.03132200 & 0.14962000 & 3.69661700 \\
\hline $\mathrm{H}$ & 0.19808600 & -2.43368000 & 1.65689300 \\
\hline $\mathrm{H}$ & 2.35446600 & -0.88536200 & 0.85155500 \\
\hline $\mathrm{C}$ & 0.27078400 & -1.82585100 & 2.55621200 \\
\hline $\mathrm{C}$ & 0.37301000 & -0.26585200 & 4.85736100 \\
\hline $\mathrm{C}$ & -0.34395100 & -1.46658100 & 4.87567400 \\
\hline $\mathrm{C}$ & -0.38180100 & -2.24852800 & 3.71465100 \\
\hline $\mathrm{H}$ & 0.42675600 & 0.35309200 & 5.75760900 \\
\hline $\mathrm{H}$ & -0.86249900 & -1.79061400 & 5.78161300 \\
\hline $\mathrm{H}$ & -0.93816700 & -3.18987600 & 3.70481400 \\
\hline $\mathrm{C}$ & 3.12676800 & 1.61632300 & 0.19046000 \\
\hline $\mathrm{C}$ & 5.23830000 & 0.48429400 & -0.14878000 \\
\hline $\mathrm{O}$ & 5.27718300 & 0.82373200 & -1.31415600 \\
\hline $\mathrm{C}$ & 6.28764500 & -0.39608500 & 0.54040800 \\
\hline $\mathrm{C}$ & 7.31766800 & -0.85692400 & -0.49491000 \\
\hline $\mathrm{C}$ & 6.97350800 & 0.44405100 & 1.63515100 \\
\hline $\mathrm{C}$ & 5.58890300 & -1.61316200 & 1.17419900 \\
\hline $\mathrm{H}$ & 6.84481600 & -1.45494100 & -1.28894600 \\
\hline $\mathrm{H}$ & 7.81652700 & -0.00004800 & -0.97173200 \\
\hline $\mathrm{H}$ & 8.08634500 & -1.47796000 & -0.00863000 \\
\hline $\mathrm{H}$ & 6.24769100 & 0.78223100 & 2.38895000 \\
\hline $\mathrm{H}$ & 7.74500200 & -0.15769300 & 2.14165300 \\
\hline $\mathrm{H}$ & 7.46286200 & 1.33289700 & 1.20463500 \\
\hline $\mathrm{H}$ & 6.33665600 & -2.26271800 & 1.65670900 \\
\hline $\mathrm{H}$ & 4.85779800 & -1.30298300 & 1.93403700 \\
\hline $\mathrm{H}$ & 5.05959100 & -2.20862600 & 0.41424600 \\
\hline $\mathrm{O}$ & 4.26870000 & 0.83844400 & 0.69639100 \\
\hline $\mathrm{C}$ & -1.29675300 & -3.90572200 & -0.75975800 \\
\hline $\mathrm{C}$ & -1.12362100 & -2.52271200 & -0.58397100 \\
\hline $\mathrm{C}$ & -2.12667800 & -1.84870400 & 0.13328500 \\
\hline C & -3.31564000 & -2.45409900 & 0.55892200 \\
\hline
\end{tabular}


$\begin{array}{lll}-2.83311600 & 1.57720800 & -0.41621600\end{array}$

$\begin{array}{lll}-3.97936800 & 2.30064300 & -0.78612200\end{array}$

$\begin{array}{lll}-5.25987700 & 1.77876400 & -0.58865800\end{array}$

$\begin{array}{lll}-5.43481700 & 0.53906500 & 0.03757800\end{array}$

$-0.55283500 \quad-4.48143300 \quad-1.30800200$

$\begin{array}{lll}-4.35882500 & -4.34540200 & 0.67553300\end{array}$

$\begin{array}{lll}-2.54673600 & -5.63246000 & -0.42299400\end{array}$

$\begin{array}{lll}-3.87453600 & 3.29194100 & -1.22346000\end{array}$

$\begin{array}{lll}-6.13310700 & 2.35882200 & -0.89622600\end{array}$

$\begin{array}{lll}-6.44326400 & 0.17152900 & 0.23102000\end{array}$

$-4.36668100 \quad-1.54182400 \quad 1.19817300$

$\begin{array}{lll}-5.76040000 & -2.17220100 & 1.18854200\end{array}$

$\begin{array}{lll}-6.49263700 & -1.49514300 \quad 1.65148800\end{array}$

$\begin{array}{lll}-6.09798000 & -2.40143900 & 0.16630100\end{array}$

$\begin{array}{lll}-5.76899400 & -3.10325300 & 1.77322400\end{array}$

$-3.94724600 \quad-1.25488600 \quad 2.66346100$

$\begin{array}{lll}-3.94377900 & -2.18953400 \quad 3.24560400\end{array}$

$\begin{array}{lll}-2.93972100 & -0.81852100 & 2.71396500\end{array}$

$\begin{array}{lll}-4.65456000 & -0.55213200 & 3.13110900\end{array}$

$\begin{array}{lll}-1.96221600 & -0.50060900 & 0.36877000\end{array}$

$0.15688200 \quad-1.46643700 \quad-1.41053900$

$\begin{array}{lll}-1.08858600 & 2.23483400 & -0.41521700\end{array}$

$\begin{array}{lll}1.74519100 & -2.54894900 & -1.59511400\end{array}$

$2.01251400 \quad-3.32332400 \quad-0.29247100$

$\begin{array}{lll}2.04039000 & -2.68096900 & 0.59232900\end{array}$

$1.26716000-4.11001600 \quad-0.11498400$

$2.99719000 \quad-3.81375900 \quad-0.37414800$

$2.89220500 \quad-1.55107000 \quad-1.83081100$

$2.77325600 \quad-1.00244900 \quad-2.77559600$

$2.94913900 \quad-0.81354100 \quad-1.02733700$

$\begin{array}{lll}3.85231200 & -2.09074800 & -1.87564600\end{array}$

$\begin{array}{lll}1.76620900 & -3.56555100 & -2.74532700\end{array}$

$\begin{array}{lll}1.73957000 & -3.08032700 & -3.72864200\end{array}$

$2.71587000 \quad-4.12521300 \quad-2.68809300$

$0.95160100 \quad-4.29971100 \quad-2.69340400$

$\begin{array}{lll}-0.66518000 & -1.33089800 & -3.15339800\end{array}$

$\begin{array}{lll}-1.98568700 & -0.58968600 & -2.90494500\end{array}$

$\begin{array}{lll}-2.73086100 & -1.24794200 & -2.43503300\end{array}$

$\begin{array}{lll}-1.84353000 & 0.27611600 & -2.25650300\end{array}$

$\begin{array}{lll}-2.40455900 & -0.24081600 & -3.86282400\end{array}$ 
TS-Conformation-A

\begin{tabular}{|c|c|c|}
\hline 0.27337400 & -0.47624200 & -4.01871200 \\
\hline 1.16914500 & -1.03800600 & -4.32086600 \\
\hline-0.24636900 & -0.15975500 & -4.93890000 \\
\hline 0.60554400 & 0.42780600 & -3.48604900 \\
\hline-1.02617100 & -2.62887600 & -3.88879500 \\
\hline-1.57920600 & -3.32764200 & -3.24498300 \\
\hline-1.69034800 & -2.37335500 & -4.73295700 \\
\hline-0.16178100 & -3.15081600 & -4.30893500 \\
\hline-0.92495400 & 3.44793800 & -1.89991300 \\
\hline-1.46534000 & 2.76804600 & -3.16575700 \\
\hline-0.94269300 & 1.83079900 & -3.37886800 \\
\hline-2.54282300 & 2.56055800 & -3.10696500 \\
\hline-1.29945700 & 3.44029200 & -4.02463500 \\
\hline-1.58960200 & 4.82633200 & -1.77641700 \\
\hline-1.38629300 & 5.39154300 & -2.70244600 \\
\hline-2.68062500 & 4.76730900 & -1.66892300 \\
\hline-1.18907400 & 5.41818200 & -0.94451700 \\
\hline 0.59034600 & 3.65334000 & -2.07880700 \\
\hline 0.77545000 & 4.24690800 & -2.99017500 \\
\hline 1.02933300 & 4.19749900 & -1.23231200 \\
\hline 1.12029700 & 2.69364300 & -2.17922500 \\
\hline-1.25929700 & 3.28417700 & 1.20714400 \\
\hline-2.52433600 & 4.14553700 & 1.33041900 \\
\hline-2.47216900 & 4.69498800 & 2.28669000 \\
\hline-2.62877000 & 4.88852200 & 0.53274700 \\
\hline-3.43706500 & 3.53625200 & 1.35812600 \\
\hline-0.02502200 & 4.18353100 & 1.36621600 \\
\hline-0.04260300 & 5.03497900 & 0.67229200 \\
\hline-0.00393200 & 4.59422800 & 2.39018400 \\
\hline 0.90776500 & 3.63451500 & 1.20703100 \\
\hline-1.30021400 & 2.25264800 & 2.34466700 \\
\hline-2.23527700 & 1.67291200 & 2.33131600 \\
\hline-0.46985400 & 1.54497700 & 2.28470200 \\
\hline-1.24492900 & 2.77062600 & 3.31712700 \\
\hline 0.39060800 & 0.51653800 & -0.13924900 \\
\hline 1.59554600 & 1.08409500 & 3.71060000 \\
\hline 1.63649400 & 1.94266700 & 1.76170900 \\
\hline 3.02931900 & 1.34035600 & -0.86871300 \\
\hline 3.48899700 & 3.08734300 & 0.29434400 \\
\hline 3.65606700 & 3.37089200 & 1.34563400 \\
\hline 4.40106700 & 3.30483700 & -0.28101100 \\
\hline 2.67751500 & 3.70847500 & -0.10583900 \\
\hline
\end{tabular}


$-1.82439700$

$-1.57219000$

$-1.52170300$

$-1.45597800$

$-2.48111400$

$-1.40656600$

$-1.28566100$

$-1.10081400$

$-1.17086600$

$-1.25417200$

$-0.91118600$

$-1.03646400$

$-2.58915400$

$-5.10637600$

$-4.71881800$

$-6.15194500$

$-6.95539800$

$-7.09652600$

$-5.33936300$

$-6.28976900$

$-7.53740200$

$-7.66290600$

$-6.52760700$

$-7.80488200$

$-7.68618300$

$-6.00723400$

$-4.72772800$

$-4.65873800$

$-4.68202100$

0.70378000

0.74681800

1.56190200

2.40218100

2.33367500

1.46666700

3.79405400

2.81991300

3.03682900

4.34759700

5.36364000

5.08431200

0.09061400

2.95969900

1.41278900
$-0.05441700$

$-2.73286500-0.46328000$

$\begin{array}{ll}-0.49326200 & 0.23672700\end{array}$

$-2.33215000 \quad-1.47192400$

$-1.34141800 \quad-4.06839300$

$-2.70981500 \quad-3.84687000$

$-3.19984200 \quad-2.53802500$

$-0.94206400 \quad-5.08573500$

$-3.38649800 \quad-4.68375700$

$-4.26703300 \quad-2.34258700$

$2.11126600 \quad 0.34813800$

$0.67202900 \quad 0.08015900$

$-0.05850500 \quad 1.01129900$

$0.10285100 \quad-0.92923900$

$-1.03395700 \quad-0.29072700$

$1.21161400 \quad-1.40950300$

$-0.43844800 \quad-2.12149300$

$-1.83080000 \quad 0.06985800$

$-0.67331500 \quad 0.57356000$

$-1.46934900-1.01710400$

$2.04030100-1.85373500$

$0.82609300-2.16278600$

$1.62182200-0.57236000$

$-0.84380800 \quad-2.90091800$

$0.35766300 \quad-2.57356000$

$-1.24478800 \quad-1.80515200$

$1.84122900-0.18149500$

$-3.73957800 \quad 1.33478000$

$-2.37883500 \quad 0.99156500$

$-2.02643800 \quad-0.09869800$

$-2.92951400 \quad-0.75993700$

$-4.27504600 \quad-0.38161100$

$-4.67991400 \quad 0.63789700$

$-0.98562600 \quad-1.34105200$

$-0.15646700 \quad-0.76781900$

$1.19189700 \quad-0.43740900$

$1.67332600-0.59296500$

$0.84801200-1.08048000$

$-0.46413500 \quad-1.47906700$

$-4.07140800 \quad 2.17121800$

$-5.01574300 \quad-0.88017300$

$-5.73451000 \quad 0.91766500$ 
$4.57814700 \quad 2.70663200 \quad-0.33669300$

$\begin{array}{lll}6.37780100 & 1.24118800 & -1.18106500\end{array}$

$5.87900800 \quad-1.07629800 \quad-1.90678100$

$3.32926000 \quad-2.36108100 \quad-1.83829400$

$\begin{array}{lll}4.50385300 & -3.29462100 & -2.13741000\end{array}$

$5.15193000 \quad-2.86270800 \quad-2.91338100$

$5.11369700 \quad-3.48378700 \quad-1.24081800$

$\begin{array}{lll}4.14235700 & -4.25908100 & -2.52124300\end{array}$

$2.50660000 \quad-2.14705400 \quad-3.13522700$

$\begin{array}{lll}2.13278900 & -3.11220200 & -3.50938900\end{array}$

$\begin{array}{lll}1.64210700 & -1.49229100 & -2.95955500\end{array}$

$3.13679500 \quad-1.68799000 \quad-3.91301400$

$\begin{array}{lll}1.58530800 & -0.70359400 & -0.49626800\end{array}$

$0.01696100 \quad-0.98923300 \quad 1.97055300$

$\begin{array}{lll}1.55716000 & 2.23599700 \quad-0.03359600\end{array}$

$\begin{array}{lll}-1.55538900 & -1.63778200 & 2.88511800\end{array}$

$\begin{array}{lll}-2.42857400 & -2.52387800 & 1.98061300\end{array}$

$\begin{array}{lll}-2.78897700 & -2.00430100 \quad 1.08930100\end{array}$

$\begin{array}{lll}-1.92134100 & -3.44915400 \quad 1.67670900\end{array}$

$\begin{array}{lll}-3.32499200 & -2.81236900 \quad 2.55457800\end{array}$

$\begin{array}{lll}-2.36560400 & -0.38263200 & 3.26901900\end{array}$

$\begin{array}{lll}-1.74467100 & 0.39971400 & 3.72833000\end{array}$

$\begin{array}{lll}-2.90612300 & 0.02912900 & 2.40813200\end{array}$

$\begin{array}{lll}-3.13316400 & -0.66928400 \quad 4.00833400\end{array}$

$-1.27732700 \quad-2.44072600 \quad 4.16554300$

$\begin{array}{lll}-0.82115300 & -1.82867100 \quad 4.95230800\end{array}$

$\begin{array}{lll}-2.24501000 \quad-2.79784800 & 4.55624800\end{array}$

$\begin{array}{lll}-0.64740800 & -3.32334600 & 3.99578700\end{array}$

$\begin{array}{lll}1.41857900 & -0.79422000 & 3.29187000\end{array}$

$\begin{array}{lll}2.67074200 & -0.41025000 & 2.49517300\end{array}$

$3.07927300 \quad-1.27123900 \quad 1.94777200$

$\begin{array}{lll}2.46542600 & 0.38327900 & 1.77778000\end{array}$

$3.45173400 \quad-0.05409500 \quad 3.18560400$

$\begin{array}{lll}1.00855300 & 0.35948700 & 4.21911600\end{array}$

$\begin{array}{lll}0.18066000 & 0.07664600 \quad 4.88438100\end{array}$

$1.86207800 \quad 0.64747000 \quad 4.85571100$

$\begin{array}{lll}0.69514500 & 1.24685400 & 3.64927000\end{array}$

$\begin{array}{lll}1.80233300 & -2.03427200 & 4.11544500\end{array}$

$1.92385200 \quad-2.92672200 \quad 3.48474700$

$\begin{array}{lll}2.77937800 & -1.83878700 \quad 4.59004100\end{array}$

$1.09760500 \quad-2.26586500 \quad 4.91828000$

$\begin{array}{lll}2.07711100 & 3.58938800 & 1.22275000\end{array}$

$\begin{array}{lll}2.93894700 & 2.96952700 & 2.33152000\end{array}$

$2.40931900 \quad 2.17592100 \quad 2.86512600$ 
H

$\mathrm{H}$

$\mathrm{H}$

$\mathrm{H}$

\section{TS-S54}

C

\begin{tabular}{|c|c|c|}
\hline 3.88790600 & 2.56426300 & 1.95401900 \\
\hline 3.17845700 & 3.75649500 & 3.06620100 \\
\hline 2.82278900 & 4.80618400 & 0.65897100 \\
\hline 3.04057400 & 5.49323700 & 1.49440800 \\
\hline 3.78308200 & 4.54120400 & 0.19717500 \\
\hline 2.22461900 & 5.36489400 & -0.07151400 \\
\hline 0.75793700 & 4.07438800 & 1.85038100 \\
\hline 0.97914700 & 4.79650200 & 2.65411500 \\
\hline 0.12155600 & 4.58208700 & 1.11271800 \\
\hline 0.18366200 & 3.24222900 & 2.28516000 \\
\hline 1.34371200 & 3.03330700 & -1.78158700 \\
\hline 2.58168100 & 3.70774300 & -2.38947900 \\
\hline 2.32288900 & 4.02063800 & -3.41599400 \\
\hline 2.90344300 & 4.60319000 & -1.84875700 \\
\hline 3.43339800 & 3.01984400 & -2.46928300 \\
\hline 0.19637300 & 4.04800400 & -1.71271500 \\
\hline 0.46807100 & 4.93611500 & -1.12467600 \\
\hline-0.05633200 & 4.38729800 & -2.73138400 \\
\hline-0.70242600 & 3.61017700 & -1.27145700 \\
\hline 0.96732200 & 1.86628400 & -2.70861700 \\
\hline 1.83915100 & 1.23502500 & -2.93331400 \\
\hline 0.19610300 & 1.22709300 & -2.27285300 \\
\hline 0.58544100 & 2.26297400 & -3.66380000 \\
\hline 0.24870900 & 0.74876700 & 0.39250900 \\
\hline-1.69191300 & 0.58049100 & -3.20656700 \\
\hline-1.78134000 & 1.86357400 & -1.61590600 \\
\hline-2.78266300 & 1.60056000 & 1.28923800 \\
\hline-2.71479500 & 3.59565100 & 0.35763500 \\
\hline-2.82780500 & 4.00106500 & -0.65763200 \\
\hline-3.58063100 & 3.89815300 & 0.96057600 \\
\hline-1.81713000 & 4.04606100 & 0.80818200 \\
\hline
\end{tabular}

\begin{tabular}{lll}
-0.00747300 & 2.53724200 & -1.17862500 \\
0.72055500 & 1.49902100 & -0.55714700 \\
2.09169700 & 1.73797800 & -0.31615500 \\
2.64060400 & 3.02666400 & -0.50479000 \\
1.87767700 & 4.01609800 & -1.12793400 \\
0.56048600 & 3.76086500 & -1.50787300 \\
4.87370400 & 2.08206100 & -0.25044700 \\
4.28049400 & 0.81139800 & -0.11933500 \\
5.05009800 & -0.36981900 & -0.21192200 \\
6.40932900 & -0.23004900 & -0.54718200 \\
6.99068600 & 1.01521500 & -0.76886900 \\
\hline 6.22455900 & 2.16815400 & -0.59907100
\end{tabular}




\begin{tabular}{|c|c|c|}
\hline-1.06600600 & 2.36605600 & -1.38425200 \\
\hline 2.30613800 & 5.00263900 & -1.30155800 \\
\hline-0.03473600 & 4.52847600 & -2.00700200 \\
\hline 7.02774000 & -1.12086800 & -0.63242200 \\
\hline 8.04563700 & 1.08926500 & -1.04272600 \\
\hline 6.69090500 & 3.14521900 & -0.72258600 \\
\hline 4.02082900 & 3.29739100 & 0.07959800 \\
\hline 4.63413400 & 4.60448300 & -0.42909300 \\
\hline 5.61462400 & 4.77663300 & 0.03624100 \\
\hline 4.76396800 & 4.60084700 & -1.52183500 \\
\hline 4.00055100 & 5.46010600 & -0.15705600 \\
\hline 3.87833300 & 3.39179600 & 1.62239300 \\
\hline 3.22604300 & 4.23791500 & 1.89077700 \\
\hline 3.44150100 & 2.47545200 & 2.04611000 \\
\hline 4.86537600 & 3.54739400 & 2.08538300 \\
\hline 2.91527300 & 0.71976900 & 0.12676000 \\
\hline-0.21740700 & -1.09578000 & -1.60096500 \\
\hline 1.22485100 & -1.55413600 & -1.83344100 \\
\hline 1.90160600 & -0.69806500 & -1.96881000 \\
\hline 1.62196700 & -2.16287100 & -1.01598600 \\
\hline 1.26246500 & -2.15863600 & -2.75603300 \\
\hline-0.67478400 & -0.31283500 & -2.84463000 \\
\hline-0.72721600 & -1.00471700 & -3.70144400 \\
\hline-1.66441500 & 0.13667900 & -2.71240200 \\
\hline 0.03644300 & 0.48507900 & -3.10287700 \\
\hline-1.14777500 & -2.29763200 & -1.37927300 \\
\hline-2.16930200 & -1.97992000 & -1.13631600 \\
\hline-1.19144700 & -2.90087600 & -2.30151200 \\
\hline-0.79389300 & -2.95259100 & -0.57174400 \\
\hline 0.03172400 & -0.72378300 & 1.53884600 \\
\hline 0.94057200 & -1.94851700 & 1.47140000 \\
\hline 1.12149400 & -2.30432100 & 2.50012800 \\
\hline 0.48265000 & -2.78055600 & 0.92011400 \\
\hline 1.91185200 & -1.72670300 & 1.01861300 \\
\hline 0.68089300 & 0.33529000 & 2.44431400 \\
\hline 0.08596500 & 1.25598600 & 2.50162000 \\
\hline 0.75800500 & -0.07408000 & 3.46557900 \\
\hline 1.69316600 & 0.58777400 & 2.10659300 \\
\hline-1.31731800 & -1.14533500 & 2.15315700 \\
\hline-1.86144500 & -1.85278400 & 1.51096600 \\
\hline-1.13068200 & -1.64210400 & 3.12042200 \\
\hline-1.96852100 & -0.27795500 & 2.33582900 \\
\hline 5.02531900 & -3.25092000 & -1.08082100 \\
\hline 6.43843000 & -3.80765400 & -0.86932300 \\
\hline
\end{tabular}


$\begin{array}{lll}7.21215600 & -3.02825900 & -0.86976400\end{array}$

$\begin{array}{lll}6.52038200 & -4.37490100 & 0.06860800\end{array}$

$6.67708700 \quad-4.50324300 \quad-1.69352900$

$4.03253400 \quad-4.43252000 \quad-1.06274300$

$\begin{array}{lll}4.00375000 & -4.93223300 & -0.08311700\end{array}$

$3.01037300 \quad-4.10510000 \quad-1.30645300$

$\begin{array}{lll}4.33625900 & -5.18398700 & -1.81210500\end{array}$

$\begin{array}{lll}4.93508300 & -2.57318900 & -2.45863800\end{array}$

$5.07669800 \quad-3.33038000 \quad-3.24911300$

$3.95178100 \quad-2.10395000 \quad-2.61973800$

$\begin{array}{lll}5.70425700 & -1.79998100 & -2.59286300\end{array}$

$5.07855100 \quad-2.30478900 \quad 1.93368600$

$\begin{array}{lll}6.59724100 & -2.15108300 & 2.07263400\end{array}$

$\begin{array}{lll}6.88363000 & -2.34585900 \quad 3.12168900\end{array}$

$\begin{array}{lll}7.15720100 & -2.85287300 & 1.44311800\end{array}$

$6.92734300 \quad-1.13066900 \quad 1.83215500$

$\begin{array}{lll}4.65080000 & -3.70900600 & 2.38907300\end{array}$

$\begin{array}{lll}5.16585500 & -4.49842400 & 1.82224500\end{array}$

$\begin{array}{lll}4.90225400 & -3.84712600 \quad 3.45486600\end{array}$

$3.56496200 \quad-3.85961300 \quad 2.27816100$

$\begin{array}{lll}4.40730500 & -1.25758800 & 2.84271800\end{array}$

$\begin{array}{lll}4.66917100 & -0.23022800 & 2.54493000\end{array}$

$3.31272600 \quad-1.34592700 \quad 2.83802300$

$\begin{array}{lll}4.75349700 & -1.39936700 \quad 3.88122700\end{array}$

$\begin{array}{lll}-4.41606900 & 1.11897000 & 0.26085400\end{array}$

$\begin{array}{lll}-3.88325800 & 0.05277000 & -0.55911300\end{array}$

$\begin{array}{lll}-4.04053800 & 0.10017000 & -2.02560400\end{array}$

$-3.95811300 \quad 1.31045700 \quad-2.74694200$

$\begin{array}{lll}-4.31988900 & -2.03337400 & -2.22243200\end{array}$

$\begin{array}{lll}-3.97902800 & -0.95410300 & -0.13738100\end{array}$

$\begin{array}{lll}-4.24706300 & -1.08439900 & -2.75944400\end{array}$

$\begin{array}{lll}-4.06819700 \quad 1.33089100 & -4.13744900\end{array}$

$-4.25994000 \quad 0.14126000 \quad-4.85167200$

$-4.35141600 \quad-1.06666500 \quad-4.15251400$

$\begin{array}{lll}-3.99288500 & 2.28195300 & -4.67173900\end{array}$

$\begin{array}{lll}-4.33793900 & 0.15732200 & -5.94162900\end{array}$

$\begin{array}{lll}-4.50618700 & -2.00309300 & -4.69539600\end{array}$

$\begin{array}{lll}-4.57699400 & 0.95331500 & 1.64447800\end{array}$

$\begin{array}{lll}-6.46826200 & -0.01281500 \quad 1.78748100\end{array}$

$\begin{array}{lll}-6.33104600 & -1.26931900 & 1.61745400\end{array}$

$\begin{array}{lll}-7.59518200 & -1.98960900 & 1.05440300\end{array}$

$\begin{array}{lll}-5.29412900 & -1.92743700 & 1.81873400\end{array}$

$\begin{array}{lll}-8.82237500 & -1.60155900 & 1.89236300\end{array}$

$-7.78209300 \quad-1.50019600 \quad-0.39334200$ 


\begin{tabular}{|c|c|c|c|}
\hline $\mathrm{C}$ & -7.40548700 & -3.50847600 & 1.07017000 \\
\hline $\mathrm{H}$ & -8.71352000 & -1.93994400 & 2.93678800 \\
\hline $\mathrm{H}$ & -8.95306600 & -0.50989900 & 1.90456200 \\
\hline $\mathrm{H}$ & -9.73920300 & -2.06037100 & 1.48411400 \\
\hline $\mathrm{H}$ & -6.91024400 & -1.75853400 & -1.01718400 \\
\hline $\mathrm{H}$ & -8.67381600 & -1.96061100 & -0.85222000 \\
\hline $\mathrm{H}$ & -7.90220200 & -0.40677200 & -0.42130600 \\
\hline $\mathrm{H}$ & -8.28997300 & -4.01554900 & 0.64810800 \\
\hline $\mathrm{H}$ & -6.52300800 & -3.80243800 & 0.48325600 \\
\hline $\mathrm{H}$ & -7.25622100 & -3.88099400 & 2.09604100 \\
\hline $\mathrm{H}$ & -3.78041900 & 2.24478100 & -2.20931400 \\
\hline $\mathrm{N}$ & -2.41948200 & 1.03940200 & 0.31100500 \\
\hline $\mathrm{P}$ & -0.41528300 & 0.08866900 & -0.13374800 \\
\hline $\mathrm{P}$ & 4.31033600 & -2.01350300 & 0.20010400 \\
\hline $\mathrm{H}$ & -4.83961900 & 2.01792100 & -0.20173800 \\
\hline $\mathrm{C}$ & -4.90061300 & 2.08695100 & 2.55970200 \\
\hline $\mathrm{H}$ & -5.46750900 & 2.87745100 & 2.04424600 \\
\hline $\mathrm{H}$ & -3.96779400 & 2.53398000 & 2.94310300 \\
\hline $\mathrm{H}$ & -5.48128800 & 1.73158800 & 3.42121200 \\
\hline $\mathrm{H}$ & -4.18044300 & 0.04210700 & 2.09174800 \\
\hline $\mathrm{N}$ & -1.77349600 & 2.50492400 & 1.29545600 \\
\hline $\mathrm{C}$ & -1.31667300 & 3.44811900 & 1.78771900 \\
\hline $\mathrm{C}$ & -0.68026400 & 4.61213300 & 2.36915300 \\
\hline $\mathrm{H}$ & -0.30697200 & 5.26199600 & 1.56219700 \\
\hline $\mathrm{H}$ & 0.16947200 & 4.29190300 & 2.99221800 \\
\hline $\mathrm{H}$ & -1.39538700 & 5.17398200 & 2.98855900 \\
\hline \multicolumn{4}{|c|}{ TS-S55 } \\
\hline $\mathrm{C}$ & -0.09466800 & -0.69619500 & 1.10984900 \\
\hline $\mathrm{C}$ & 0.50655100 & 0.07976500 & 0.05024100 \\
\hline $\mathrm{C}$ & 1.22833700 & 1.34211000 & 0.28594300 \\
\hline $\mathrm{C}$ & 1.90702500 & 1.60582600 & 1.49390000 \\
\hline $\mathrm{H}$ & 0.76508400 & 2.12508100 & -1.67383500 \\
\hline $\mathrm{H}$ & 0.01499300 & 0.01714800 & -0.92943800 \\
\hline $\mathrm{C}$ & 1.28230800 & 2.31609600 & -0.72959600 \\
\hline $\mathrm{C}$ & 2.59805100 & 2.80281100 & 1.68053300 \\
\hline $\mathrm{C}$ & 2.63739800 & 3.76416700 & 0.66235100 \\
\hline $\mathrm{C}$ & 1.97687700 & 3.51389500 & -0.54481200 \\
\hline $\mathrm{H}$ & 3.11727300 & 2.98640400 & 2.62495700 \\
\hline $\mathrm{H}$ & 3.18206000 & 4.70013500 & 0.80967300 \\
\hline $\mathrm{H}$ & 2.00175700 & 4.25606800 & -1.34713700 \\
\hline $\mathrm{C}$ & -0.86057800 & -1.82838900 & 0.78586800 \\
\hline $\mathrm{O}$ & -2.83053800 & -1.08736000 & 0.63705800 \\
\hline $\mathrm{C}$ & -2.99584600 & -0.53996500 & -0.50436300 \\
\hline $\mathrm{C}$ & -4.08214800 & 0.57820400 & -0.53797300 \\
\hline
\end{tabular}




\begin{tabular}{|c|c|c|}
\hline-2.34228800 & -0.78462800 & -1.53379100 \\
\hline-5.34710000 & 0.10478600 & 0.19134500 \\
\hline-3.47987700 & 1.78931500 & 0.19862100 \\
\hline-4.41932900 & 0.96159600 & -1.98131500 \\
\hline-5.79763700 & -0.76419200 & -0.31761700 \\
\hline-5.11125500 & -0.19516000 & 1.22223200 \\
\hline-6.10534200 & 0.90583000 & 0.22293200 \\
\hline-2.55592500 & 2.13304600 & -0.29600500 \\
\hline-4.19006100 & 2.63354800 & 0.21576600 \\
\hline-3.22736700 & 1.52695500 & 1.23737600 \\
\hline-5.16498400 & 1.77449200 & -2.00297800 \\
\hline-3.52183100 & 1.29715700 & -2.52055900 \\
\hline-4.83506300 & 0.10405500 & -2.53454400 \\
\hline 1.90372300 & 0.85521100 & 2.28721700 \\
\hline 1.52313700 & -1.50754900 & 0.32878100 \\
\hline-0.02423300 & -0.38137900 & 2.15730800 \\
\hline-1.16014100 & -2.89268400 & 1.78994400 \\
\hline-1.28766500 & -2.47260700 & 2.79943000 \\
\hline-0.31778700 & -3.60656500 & 1.82680400 \\
\hline-2.06495500 & -3.45063200 & 1.51449300 \\
\hline-0.95720700 & -2.08360800 & -0.26954600 \\
\hline 3.08528500 & -1.53541800 & -0.64877600 \\
\hline 4.07360700 & -1.49633200 & -1.25321000 \\
\hline 5.30475300 & -1.43415800 & -2.01301500 \\
\hline 5.59124700 & -0.38192600 & -2.16570700 \\
\hline 6.10735800 & -1.95140000 & -1.46496300 \\
\hline 5.16427900 & -1.91754200 & -2.99228600 \\
\hline
\end{tabular}

(R)-1 
(S) -2

C

\begin{tabular}{|c|c|c|}
\hline-0.99763600 & 2.56608400 & -0.87706800 \\
\hline-1.99305900 & 2.94371500 & -0.59929300 \\
\hline-0.99434400 & 2.32698300 & -1.95237700 \\
\hline-0.26045900 & 3.36295700 & -0.69433200 \\
\hline-1.63099100 & 0.29330700 & -0.31590700 \\
\hline-2.76485100 & 0.29210700 & 0.40112300 \\
\hline-3.00862100 & 1.11176500 & 1.25978100 \\
\hline-3.68296700 & -0.86580300 & 0.00056900 \\
\hline-4.01017400 & -0.74267300 & -1.49955200 \\
\hline-4.69433600 & -1.55248500 & -1.79891400 \\
\hline-3.10103000 & -0.81304200 & -2.11369200 \\
\hline-4.50206700 & 0.21842100 & -1.72060100 \\
\hline-4.96890600 & -0.79662700 & 0.82970000 \\
\hline-5.63399100 & -1.63042500 & 0.55597200 \\
\hline-5.50821700 & 0.14644000 & 0.65339100 \\
\hline-4.75423200 & -0.86560200 & 1.90667700 \\
\hline-2.94514100 & -2.19088100 & 0.27206300 \\
\hline-3.59470000 & -3.04027700 & 0.00725400 \\
\hline-2.67862800 & -2.28455200 & 1.33726200 \\
\hline-2.02217600 & -2.26171100 & -0.32128600 \\
\hline-0.69002000 & 1.57669400 & 1.01929200 \\
\hline-1.86100000 & 0.83842600 & 0.76555500 \\
\hline-0.90032300 & 1.43062000 & 0.03102300 \\
\hline-3.08677900 & 0.19484600 & 0.26973400 \\
\hline-1.73967000 & 0.82051400 & 1.85542700 \\
\hline 0.37585900 & 2.01013000 & 0.58018100 \\
\hline-0.99811600 & 1.46601800 & -1.06119900 \\
\hline-3.38055300 & 0.04108000 & -1.10213900 \\
\hline-4.02075300 & -0.30046400 & 1.20180000 \\
\hline 0.73936200 & 3.35314600 & -0.06965300 \\
\hline 0.26900800 & 2.13174100 & 1.67154600 \\
\hline-4.56049200 & -0.57510100 & -1.51718500 \\
\hline-2.67568200 & 0.40337200 & -1.85360800 \\
\hline-5.20238200 & -0.91888800 & 0.78620500 \\
\hline-3.80987100 & -0.19473500 & 2.26959000 \\
\hline-0.04374600 & 4.10990900 & 0.10416700 \\
\hline 1.68631100 & 3.74685500 & 0.33368600 \\
\hline 0.86442400 & 3.24582900 & -1.15947000 \\
\hline-5.47962300 & -1.05886500 & -0.57671600 \\
\hline-4.76550700 & -0.68298800 & -2.58563100 \\
\hline-5.90922000 & -1.29375100 & 1.53114500 \\
\hline-6.40215700 & -1.54312800 & -0.90643400 \\
\hline 1.46077200 & 0.88877000 & 0.3289680 \\
\hline
\end{tabular}


$\begin{array}{lll}1.81470100 & -0.03986000 \quad 1.26817000\end{array}$

$\begin{array}{lll}2.07736500 & 0.69902000 & -0.87625600\end{array}$

$\begin{array}{lll}2.55283000 & -1.09333700 & 0.58424600\end{array}$

$\begin{array}{lll}1.54544600 & -2.19981500 & 0.27151400\end{array}$

$3.11109000 \quad-1.20434000 \quad-1.96236500$

$\begin{array}{lll}4.41819000 & 0.34861300 & -0.49732600\end{array}$

$3.18999900 \quad-2.11368600 \quad 2.37509300$

$\begin{array}{lll}4.27440200 & -2.34633300 & 0.98106900\end{array}$

$\begin{array}{lll}4.28416000 & -0.80077800 & 1.87645400\end{array}$

$\begin{array}{lll}1.07600800 & -2.53023400 & 1.21041700\end{array}$

$\begin{array}{lll}2.03223100 & -3.06808300 & -0.19562000\end{array}$

$0.75035900 \quad-1.84013100 \quad-0.39849600$

$3.49036700 \quad-0.60824200 \quad-2.80656700$

$\begin{array}{lll}3.78427800 & -2.06371500 & -1.82210200\end{array}$

$2.11390200 \quad-1.57823000 \quad-2.22955900$

$5.24161300 \quad-0.37720900 \quad-0.43123900$

$\begin{array}{lll}4.61138300 & 1.01172700 & -1.35410400\end{array}$

$4.41835300 \quad 0.96215600 \quad 0.41597100$

$1.05446800 \quad 0.03274100 \quad-0.00239100$

$1.60896000 \quad-1.09161300 \quad-0.07032700$

$\begin{array}{lll}-0.52550300 & 0.00892900 & -0.00732600\end{array}$

$\begin{array}{lll}-1.00011000 & -0.78464000 & -1.23302300\end{array}$

$\begin{array}{lll}-0.98337600 & -0.71268500 & 1.27019700\end{array}$

$\begin{array}{lll}-1.10853000 & 1.42347700 & -0.04238300\end{array}$

$\begin{array}{lll}-0.70855500 & -0.28065700 & -2.17128000\end{array}$

$\begin{array}{lll}-0.53875500 & -1.78316600 & -1.23413600\end{array}$

$\begin{array}{lll}-2.09895700 & -0.90174300 & -1.24512800\end{array}$

$\begin{array}{lll}-0.66782000 & -0.16038400 & 2.17267000\end{array}$

$\begin{array}{lll}-2.08278700 & -0.81751500 & 1.31016800\end{array}$

$\begin{array}{lll}-0.53536000 & -1.71682900 \quad 1.31885800\end{array}$

$\begin{array}{lll}-2.21358300 & 1.40249700 & -0.02542900\end{array}$

$\begin{array}{lll}-0.75950000 & 2.01488400 & 0.81746000\end{array}$

$\begin{array}{lll}-0.79129700 & 1.95999300 & -0.95067900\end{array}$

$1.61139900 \quad 1.15218100 \quad 0.08246500$

$\begin{array}{rrr}-1.00012800 & -0.13718900 & 0.25714600 \\ -0.32574300 & -2.03399700 & 0.07319200 \\ -1.64197400 & -2.03962300 & 0.56534300 \\ 0.62941800 & -1.24052400 & 0.74745300 \\ -0.13331100 & -2.39368200 & -0.94367300 \\ -2.78673500 & -2.66319600 & -0.17501800\end{array}$


$\mathrm{B}_{2} \mathrm{Pin}_{2}-\mathrm{K}_{3} \mathrm{PO}_{4}$

\begin{tabular}{|c|c|c|}
\hline-1.77820000 & -1.92440500 & 1.65070600 \\
\hline 1.95551600 & -0.87770100 & 0.21892400 \\
\hline 0.52991800 & -1.15000900 & 1.83721700 \\
\hline-3.73218900 & -2.14458600 & 0.04510700 \\
\hline-2.91503400 & -3.71501800 & 0.13691200 \\
\hline-2.62406400 & -2.64749100 & -1.26330800 \\
\hline 2.91655700 & -0.34944700 & 1.10050500 \\
\hline 2.28539500 & -0.98147300 & -1.14633500 \\
\hline 4.17181500 & 0.05235400 & 0.63887600 \\
\hline 2.66835300 & -0.25305400 & 2.16073100 \\
\hline 3.54058800 & -0.58348600 & -1.60684800 \\
\hline 1.55127400 & -1.36347800 & -1.85870000 \\
\hline 4.49001300 & -0.06442400 & -0.71788200 \\
\hline 4.90397400 & 0.45853300 & 1.34139000 \\
\hline 3.77870900 & -0.67178200 & -2.66990500 \\
\hline 5.47080900 & 0.25021400 & -1.08284300 \\
\hline-0.05269700 & 1.50270000 & 0.22177200 \\
\hline 0.59361300 & 2.45973800 & 0.19842600 \\
\hline 1.42352500 & 3.64516400 & 0.17094900 \\
\hline 1.19518700 & 4.27702200 & 1.04289300 \\
\hline 2.48290400 & 3.34769400 & 0.20189300 \\
\hline 1.22864600 & 4.21347600 & -0.75133500 \\
\hline-2.70971700 & 0.57626800 & -0.11047600 \\
\hline-3.74357300 & 1.03262800 & -0.35020100 \\
\hline-5.03846600 & 1.60383900 & -0.65482300 \\
\hline-5.25645900 & 1.46882300 & -1.72553100 \\
\hline-5.81450400 & 1.09880900 & -0.05965900 \\
\hline-5.03522700 & 2.67832400 & -0.41642900 \\
\hline 0.07148800 & -0.89245800 & -2.67200800 \\
\hline-0.99049500 & -1.74036800 & -0.33056600 \\
\hline 1.17643500 & -2.92156400 & -0.97854600 \\
\hline 0.34170700 & -2.45735600 & 0.24025700 \\
\hline 0.39731700 & -0.56533400 & 2.93322900 \\
\hline-1.04759800 & -0.19454600 & -0.07762000 \\
\hline 1.14762900 & -1.16622500 & 0.87897800 \\
\hline 0.03948800 & -3.43847800 & 1.35331500 \\
\hline-1.71176600 & 0.38407400 & -1.27799900 \\
\hline-2.01063500 & 0.08571500 & 1.01839600 \\
\hline 0.62882100 & 0.18096800 & 0.29507400 \\
\hline-2.94993300 & 0.98290500 & -0.91825700 \\
\hline-3.30021200 & 0.28763000 & 0.45270300 \\
\hline 1.59423600 & 0.51676900 & -0.82649300 \\
\hline 0.85517300 & 1.25291400 & 1.28149600 \\
\hline
\end{tabular}


$(\mathrm{KOPiv})_{4}$

C

O

\begin{tabular}{|c|c|c|}
\hline-2.72677200 & 2.49280800 & -0.75033400 \\
\hline-3.97159900 & 0.74108000 & -2.02755400 \\
\hline-4.13121100 & 1.14501300 & 1.40470200 \\
\hline-3.99449900 & -1.06974100 & 0.26063800 \\
\hline 2.06440700 & 1.84923900 & -0.63680700 \\
\hline 1.99643100 & 2.02171900 & 0.93146700 \\
\hline-2.02207200 & 2.69092300 & 0.06973600 \\
\hline-2.29226600 & 2.89483800 & -1.67852100 \\
\hline-3.66303700 & 3.03639700 & -0.54990100 \\
\hline-4.96862800 & 1.11699800 & -1.74595100 \\
\hline-3.66112000 & 1.27070500 & -2.94322800 \\
\hline-4.05665900 & -0.32743300 & -2.26891100 \\
\hline-4.35499900 & 0.57448000 & 2.32114600 \\
\hline-3.59405200 & 2.05695100 & 1.69959600 \\
\hline-5.09171800 & 1.43544300 & 0.94953100 \\
\hline-3.43389200 & -1.70012300 & -0.44101600 \\
\hline-4.02012500 & -1.59165800 & 1.23098700 \\
\hline-5.03213300 & -0.95919500 & -0.09207100 \\
\hline 3.46652500 & 1.98743600 & -1.22668200 \\
\hline 1.11141600 & 2.80129800 & -1.36999400 \\
\hline 3.22941700 & 1.45389600 & 1.65233600 \\
\hline 1.78431400 & 3.46425300 & 1.38713500 \\
\hline 3.43605300 & 1.80658700 & -2.31314600 \\
\hline 3.86430200 & 3.00154700 & -1.06527700 \\
\hline 4.18514500 & 1.28211000 & -0.78290300 \\
\hline 1.08497000 & 2.52440900 & -2.43656500 \\
\hline 0.09333500 & 2.70381500 & -0.97322500 \\
\hline 1.42861200 & 3.85275600 & -1.30237300 \\
\hline 3.03378300 & 1.45401700 & 2.73613100 \\
\hline 3.42640900 & 0.41093300 & 1.36795700 \\
\hline 4.13945500 & 2.04606900 & 1.47158400 \\
\hline 1.77093800 & 3.50981200 & 2.48819200 \\
\hline 2.59441300 & 4.12145500 & 1.03267000 \\
\hline 0.82606200 & 3.85982300 & 1.0241780 \\
\hline 3.32610600 & 1.53543700 & -0.59777400 \\
\hline-1.51267100 & -2.27633500 & 1.96844900 \\
\hline-1.36780700 & 1.32110300 & 1.13183400 \\
\hline-2.96888200 & -2.74117800 & 2.22613000 \\
\hline-3.59816100 & -3.16067900 & 0.88504000 \\
\hline-2.99638200 & -3.91844600 & 3.20475700 \\
\hline-3.75552900 & -1.55362000 & 2.81130200 \\
\hline-3.69054500 & -2.30088100 & 0.20335100 \\
\hline-3.00034500 & -3.94328900 & 0.38796100 \\
\hline
\end{tabular}


$\begin{array}{lll}-4.61223700 & -3.56327100 & 1.04284200\end{array}$

$\begin{array}{lll}-2.54720500 & -3.64247300 \quad 4.17044900\end{array}$

$-4.03395100$

$-4.24435400$

3.38636400

$-2.43060400$

$-4.78414100$

$-4.77584000$

2.80954400

$\mathrm{H}$

$-3.27881000$

$-3.82661000$

$-0.56831700$

1.38227500

1.28530300

0.27667000

2.69966300

3.45164000

2.60645500

3.44656200

3.62613900

2.89387200

4.43808200

3.61423200

2.07109600

2.06400100

4.44299600

2.89290000

3.59295300

1.24084200

$-1.52627700$

$-1.35859200$

$-0.36736100$

$-2.78781000$

$-3.46692800$

$-3.58112900$

$-2.73375300$

$-2.88114800$

$-3.58667800$

$-4.47146200$

$-3.07411900$

$-4.58463000$

$-3.71870700$

$-3.75171400$

$-2.24491100$

$-2.16450500$

$-1.28326000$

1.48115600

1.58462700

$-1.85762400 \quad 3.06676900$

$-1.17130200 \quad 3.72933500$

$-0.72807900 \quad 2.08581400$

$-2.82899800 \quad 2.56897400$

$-1.37482300 \quad 1.43779800$

$2.19860400 \quad 2.15902200$

$2.73433600 \quad 2.66274100$

$2.70965400 \quad 2.53588000$

$3.08569500 \quad 1.24635500$

$3.92835200 \quad 3.45824900$

$1.56745000 \quad 3.24922000$

$2.19953500 \quad 0.61652500$

$3.83569500 \quad 0.65991600$

$3.51597300 \quad 1.48515000$

$4.28330500 \quad 3.73082900$

$4.75634700 \quad 2.96874600$

$3.68442300 \quad 4.38368700$

$1.90390000 \quad 3.58045800$

$1.22320100 \quad 4.13847400$

$0.70732400 \quad 2.57727200$

$1.22610900 \quad 1.33082300$

$1.27660700 \quad 1.31947100$

$2.22142200 \quad-2.09800500$

$2.81207700 \quad-2.57441000$

$2.68860300 \quad-2.47531900$

$1.55259400-3.26282400$

$2.96181600 \quad-1.18506500$

$3.95653300-3.33210200$

$1.28022900-4.15642500$

$0.65248700 \quad-2.63959400$

$1.86038000 \quad-3.59757500$

$3.71131400-0.55359400$

$3.35135200-1.42281400$

$2.03885900-0.60060800$

$4.28010900 \quad-3.60578300$

$4.78088800 \quad-2.79039300$

$3.78485100-4.25753000$

$1.22433700 \quad-1.30206400$

$1.36860400-1.28552400$

$-2.17522200 \quad-2.02496800$ 


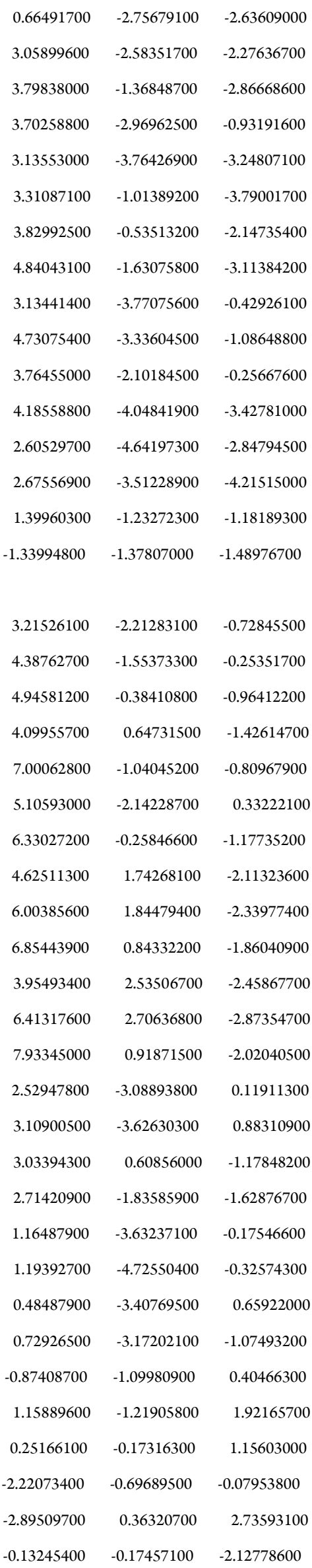


O

O

O

\begin{tabular}{|c|c|c|}
\hline 1.08413800 & 0.49809300 & 0.03540100 \\
\hline-0.47616700 & 0.77535000 & 2.12458100 \\
\hline-2.40343700 & -1.36980300 & -1.40849600 \\
\hline-2.41070000 & 1.02534700 & -0.24252700 \\
\hline-3.30503800 & -1.28715100 & 0.76321300 \\
\hline-3.65712000 & -2.03154200 & -1.41930300 \\
\hline-1.79488000 & 1.77541400 & -1.23547900 \\
\hline-3.21026700 & 1.84514100 & 0.54059000 \\
\hline-3.84621600 & -2.42015500 & 0.09518200 \\
\hline-3.59202400 & -3.21963300 & -2.37515800 \\
\hline-4.73732500 & -1.04585300 & -1.89415400 \\
\hline-1.91311300 & 3.18370200 & -0.89403700 \\
\hline-3.21418100 & 3.18971700 & -0.01171200 \\
\hline-5.29915500 & -2.60745000 & 0.52361300 \\
\hline-3.03187800 & -3.66673600 & 0.47176300 \\
\hline-4.51080800 & -3.82568000 & -2.32447700 \\
\hline-3.48248000 & -2.86108200 & -3.41182000 \\
\hline-2.73268800 & -3.86621900 & -2.15002300 \\
\hline-5.71868400 & -1.52755100 & -2.02561800 \\
\hline-4.43042500 & -0.62487600 & -2.86477700 \\
\hline-4.84947700 & -0.21467100 & -1.18249400 \\
\hline-2.00584600 & 3.99649300 & -2.17620800 \\
\hline-0.65484000 & 3.55540000 & -0.10781300 \\
\hline-3.20251700 & 4.18722900 & 1.13743900 \\
\hline-4.49760100 & 3.33008400 & -0.82954300 \\
\hline-5.34326000 & -2.90394400 & 1.58453200 \\
\hline-5.79164600 & -3.39772800 & -0.06558900 \\
\hline-5.87365600 & -1.67761800 & 0.40985700 \\
\hline-3.01001000 & -3.75725900 & 1.56964000 \\
\hline-3.46996400 & -4.59087800 & 0.06286000 \\
\hline-1.99546800 & -3.57385700 & 0.12046600 \\
\hline-1.05386600 & 3.93003500 & -2.72581400 \\
\hline-2.19181100 & 5.05784100 & -1.94997700 \\
\hline-2.80631500 & 3.63305100 & -2.83443800 \\
\hline-0.65970800 & 4.61815600 & 0.17680900 \\
\hline 0.22626300 & 3.37002600 & -0.73946900 \\
\hline-0.55049000 & 2.92402200 & 0.78640300 \\
\hline-4.14786300 & 4.12069400 & 1.69861100 \\
\hline-3.10657300 & 5.21643700 & 0.75850700 \\
\hline-2.37480800 & 3.99243500 & 1.83256000 \\
\hline-4.51503600 & 2.62184000 & -1.67097800 \\
\hline-4.61533200 & 4.34930900 & -1.22620000 \\
\hline-5.36035000 & 3.11176200 & -0.18168100 \\
\hline & & \\
\hline
\end{tabular}




\begin{tabular}{|c|c|c|c|}
\hline $\mathrm{N}$ & 3.58750100 & 0.19252200 & 1.94777300 \\
\hline $\mathrm{C}$ & 4.00069700 & 1.12263200 & 2.49421900 \\
\hline $\mathrm{C}$ & 4.51219200 & 2.29845100 & 3.17054000 \\
\hline $\mathrm{H}$ & 4.39332900 & 2.18470900 & 4.25887300 \\
\hline $\mathrm{H}$ & 3.95306100 & 3.18642600 & 2.83742100 \\
\hline $\mathrm{H}$ & 5.57858700 & 2.43159100 & 2.93304100 \\
\hline \multicolumn{4}{|l|}{ S73 } \\
\hline $\mathrm{C}$ & 4.46534600 & -2.37863600 & -0.66656200 \\
\hline $\mathrm{C}$ & 4.88647000 & -3.00231300 & -1.96669200 \\
\hline $\mathrm{C}$ & 4.73224400 & -1.02571100 & -0.34941800 \\
\hline $\mathrm{H}$ & 4.30880000 & -3.07647100 & 0.16913300 \\
\hline $\mathrm{H}$ & 4.98169700 & -2.24676100 & -2.76253800 \\
\hline $\mathrm{H}$ & 5.85601200 & -3.52573700 & -1.87641800 \\
\hline $\mathrm{H}$ & 4.14567100 & -3.74746900 & -2.29894300 \\
\hline $\mathrm{C}$ & 4.06808100 & -0.48531000 & 0.78401700 \\
\hline $\mathrm{H}$ & 5.20245300 & -0.36627000 & -1.08955400 \\
\hline $\mathrm{C}$ & 3.90838100 & 0.94708800 & 1.07950600 \\
\hline $\mathrm{H}$ & 3.94274800 & -1.14516300 & 1.65338300 \\
\hline $\mathrm{C}$ & 3.81361600 & 1.91804400 & 0.06024000 \\
\hline $\mathrm{C}$ & 3.71708900 & 1.36830800 & 2.41278600 \\
\hline $\mathrm{C}$ & 3.53621400 & 3.25090400 & 0.36405900 \\
\hline $\mathrm{H}$ & 3.91722300 & 1.61261800 & -0.98269800 \\
\hline $\mathrm{C}$ & 3.43344000 & 2.70496600 & 2.71717800 \\
\hline $\mathrm{H}$ & 3.78448900 & 0.63024700 & 3.21788600 \\
\hline $\mathrm{C}$ & 3.33670100 & 3.65279100 & 1.69229300 \\
\hline $\mathrm{H}$ & 3.45408700 & 3.98234200 & -0.44419400 \\
\hline $\mathrm{H}$ & 3.28533800 & 3.00384400 & 3.75832500 \\
\hline $\mathrm{H}$ & 3.10585300 & 4.69537300 & 1.92387100 \\
\hline $\mathrm{Ni}$ & 2.80522200 & -1.26708100 & -0.57033700 \\
\hline $\mathrm{O}$ & 1.25825000 & -0.09639800 & -0.50231800 \\
\hline $\mathrm{P}$ & 0.45465900 & -1.05688000 & -1.48624400 \\
\hline K & 0.53591000 & 0.67968500 & 1.83001600 \\
\hline $\mathrm{O}$ & -0.74934700 & -1.72949400 & -0.64799900 \\
\hline $\mathrm{O}$ & -0.14084100 & -0.39868800 & -2.72403300 \\
\hline $\mathrm{O}$ & 1.54791000 & -2.16222700 & -1.74144000 \\
\hline B & -1.73515300 & -0.93529600 & 0.18617100 \\
\hline $\mathrm{O}$ & -1.42344200 & -1.03789400 & 1.63188300 \\
\hline K & -2.68479100 & -1.14976300 & -2.59737000 \\
\hline $\mathrm{O}$ & -3.02942700 & -1.65076800 & 0.03500000 \\
\hline B & -1.90115600 & 0.74121700 & -0.28057000 \\
\hline $\mathrm{C}$ & -2.26673300 & -2.03075200 & 2.21205200 \\
\hline $\mathrm{O}$ & -2.77535000 & 1.18908300 & -1.25892900 \\
\hline $\mathrm{C}$ & -3.56397200 & -1.91460000 & 1.32535500 \\
\hline $\mathrm{O}$ & -1.26844300 & 1.81787400 & 0.31447600 \\
\hline
\end{tabular}




\begin{tabular}{|c|c|c|}
\hline-2.47497100 & -1.70332400 & 3.68776100 \\
\hline-1.59325200 & -3.40310000 & 2.07732200 \\
\hline-2.53202200 & 2.60120600 & -1.51663300 \\
\hline-4.40787300 & -3.18423200 & 1.27028600 \\
\hline-4.44070100 & -0.72670700 & 1.74991500 \\
\hline-1.81998100 & 3.06359500 & -0.18793400 \\
\hline-1.52673100 & -1.82644200 & 4.23637900 \\
\hline-3.21660800 & -2.37733800 & 4.14536400 \\
\hline-2.81295200 & -0.66693200 & 3.82618900 \\
\hline-2.15800100 & -4.19518000 & 2.59336900 \\
\hline-0.58993200 & -3.35001100 & 2.52962100 \\
\hline-1.47207900 & -3.67705300 & 1.02074800 \\
\hline-3.86366900 & 3.28359700 & -1.79250400 \\
\hline-1.62900200 & 2.67556600 & -2.74781400 \\
\hline-4.74947800 & -3.48089300 & 2.27480500 \\
\hline-5.30091800 & -3.01296600 & 0.64744700 \\
\hline-3.84479000 & -4.01873100 & 0.83009600 \\
\hline-4.96904500 & -0.91333000 & 2.69753200 \\
\hline-5.19431200 & -0.54406300 & 0.96787100 \\
\hline-3.83753700 & 0.18656600 & 1.86214100 \\
\hline-0.67445700 & 4.04718600 & -0.38362700 \\
\hline-2.78856800 & 3.57582300 & 0.87721600 \\
\hline-4.29152000 & 2.89531100 & -2.72975200 \\
\hline-3.72503000 & 4.36952500 & -1.90781000 \\
\hline-4.58868200 & 3.10568200 & -0.98743200 \\
\hline-1.38937600 & 3.71645300 & -3.00911800 \\
\hline-2.15709400 & 2.22614400 & -3.60377300 \\
\hline-0.70275400 & 2.10450700 & -2.59697600 \\
\hline-0.23127000 & 4.29916500 & 0.59262400 \\
\hline-1.03491700 & 4.98022600 & -0.84299000 \\
\hline 0.11631000 & 3.62460800 & -1.01728500 \\
\hline-2.24524300 & 3.69345700 & 1.82748800 \\
\hline-3.21313300 & 4.55234000 & 0.60252400 \\
\hline .61386000 & 2.86751300 & 1.04156800 \\
\hline
\end{tabular}

S74

$\begin{array}{rrr}1.09844100 & -0.46158700 & 0.77473300 \\ -1.52986400 & 1.27409200 & 0.38326100 \\ 1.48410300 & -0.08972500 & -1.07295400 \\ -1.97568500 & 2.25684300 & -0.49899800 \\ -0.62502900 & 1.81928400 & 1.27307700 \\ 0.22171600 & -0.89647900 & -1.66237700 \\ -2.86146800 & 0.95297800 & -2.67992400 \\ -1.10315300 & 3.41746900 & -0.38495800 \\ -0.54547900 & 3.25539000 & 1.07498700\end{array}$




\begin{tabular}{|c|c|c|}
\hline-0.53988300 & -1.09391800 & -0.17735700 \\
\hline 3.21979400 & -1.89254000 & -1.87012800 \\
\hline-0.61056200 & -0.06616800 & -2.62490000 \\
\hline 0.68937500 & -2.27127000 & -2.14782300 \\
\hline-2.95272700 & -0.71325600 & -0.73653500 \\
\hline-1.92101700 & 4.67673700 & -0.62616400 \\
\hline-0.02084400 & 3.26526900 & -1.45626100 \\
\hline 0.90010900 & 3.69619500 & 1.25510500 \\
\hline-1.434688800 & 3.90259900 & 2.13660800 \\
\hline-1.90711100 & -0.41348000 & 0.25587400 \\
\hline-3.64702300 & -1.88641500 & -0.32050200 \\
\hline-1.30761500 & 5.57511500 & -0.45630300 \\
\hline-2.26952400 & 4.69949200 & -1.67090500 \\
\hline-2.80152500 & 4.72463000 & 0.02826700 \\
\hline-0.49695100 & 3.23440400 & -2.44884500 \\
\hline 0.67244000 & 4.11915800 & -1.44629000 \\
\hline 0.54389600 & 2.33091000 & -1.32501800 \\
\hline 1.21062800 & 3.53493600 & 2.29880200 \\
\hline 1.00933400 & 4.76830100 & 1.03006300 \\
\hline 1.57930900 & 3.12995900 & 0.60546800 \\
\hline-1.37428900 & 5.00039100 & 2.10178700 \\
\hline-2.48672300 & 3.60524100 & 2.01344100 \\
\hline-1.10072200 & 3.57113500 & 3.13163100 \\
\hline-2.34322600 & -1.10519200 & 1.46447600 \\
\hline-2.89352100 & -3.12384100 & -0.82967500 \\
\hline-5.05111600 & -1.86785900 & -0.91955000 \\
\hline-3.59368300 & -1.74428300 & 1.24381800 \\
\hline-3.43077200 & -4.05766600 & -0.60239100 \\
\hline-2.78830400 & -3.04675700 & -1.92356700 \\
\hline-1.88197000 & -3.16962300 & -0.40715100 \\
\hline-4.99231700 & -1.99101400 & -2.01376500 \\
\hline-5.66362700 & -2.69368700 & -0.52409800 \\
\hline-5.56902100 & -0.92090800 & -0.71232200 \\
\hline-4.70076800 & -0.82234200 & 1.77652500 \\
\hline-3.62854700 & -3.07199300 & 1.99761800 \\
\hline-4.47899600 & -0.57763100 & 2.82717500 \\
\hline-5.69773800 & -1.28828900 & 1.73552300 \\
\hline-4.72995900 & 0.11931200 & 1.20775900 \\
\hline-3.61381800 & -2.88899800 & 3.08425600 \\
\hline-4.54460100 & -3.63944300 & 1.76607400 \\
\hline-2.75881500 & -3.69565100 & 1.74997100 \\
\hline 2.15173800 & -0.81138200 & 2.42893500 \\
\hline 2.75220600 & 0.21532600 & 1.65125800 \\
\hline & & \\
\hline
\end{tabular}




\begin{tabular}{rrr}
4.83957400 & -1.08388300 & 1.01720700 \\
3.78377100 & 1.96338400 & -0.08368800 \\
2.44552700 & 1.25147600 & 1.83425300 \\
4.40637300 & 1.07037400 & 0.00857500 \\
5.97851600 & -1.22707600 & 0.21564100 \\
6.32956300 & -0.22900100 & -0.70351000 \\
\hline 5.53862100 & 0.92501600 & -0.79566800 \\
\hline 6.59431100 & -2.12552600 & 0.30994600 \\
\hline 7.21417500 & -0.34515000 & -1.33419100 \\
\hline 5.80416200 & 1.71416600 & -1.50390600 \\
0.80606400 & -0.68252600 & 2.79998500 \\
\hline 0.39871000 & 0.32439500 & 2.95055000 \\
\hline 4.58417700 & -1.87830400 & 1.72148100 \\
\hline 2.62352200 & -1.79822200 & 2.49832800 \\
\hline 0.03407300 & -1.80934200 & 3.42128700 \\
0.01605900 & -1.71649900 & 4.52325700 \\
\hline 1.00260200 & -1.78913000 & 3.05608200 \\
\hline 0.47724200 & -2.78772500 & 3.17326700 \\
\hline & & \\
\hline
\end{tabular}

.17326700

TS-S75

\begin{tabular}{|c|c|c|c|}
\hline $\mathrm{Ni}$ & 0.86889600 & 0.15400400 & 0.84132900 \\
\hline B & -1.11914800 & 0.91403900 & 0.02264000 \\
\hline $\mathrm{O}$ & 1.52816500 & -0.59086100 & -0.79314900 \\
\hline $\mathrm{O}$ & -0.93024400 & 1.31600800 & -1.30369800 \\
\hline $\mathrm{O}$ & -1.64372100 & 1.96656500 & 0.74891800 \\
\hline $\mathrm{P}$ & 0.71274400 & -1.96607100 & -0.97096100 \\
\hline K & -1.84247900 & -0.50805200 & -3.00176600 \\
\hline $\mathrm{C}$ & -1.07006900 & 2.75363300 & -1.39342600 \\
\hline $\mathrm{C}$ & -1.96010800 & 3.06820600 & -0.13811700 \\
\hline $\mathrm{O}$ & -0.34804200 & -1.86098200 & 0.27825700 \\
\hline K & 3.78857100 & -1.91295900 & -1.27812100 \\
\hline $\mathrm{O}$ & -0.01173700 & -2.01371200 & -2.31682600 \\
\hline $\mathrm{O}$ & 1.65311800 & -3.14315600 & -0.68745200 \\
\hline $\mathrm{O}$ & -2.52800400 & -1.00206900 & -0.56355900 \\
\hline $\mathrm{C}$ & -1.69846300 & 3.10745700 & -2.73570800 \\
\hline $\mathrm{C}$ & 0.33742100 & 3.34458500 & -1.30725400 \\
\hline $\mathrm{C}$ & -1.62519100 & 4.37477200 & 0.56784900 \\
\hline C & -3.45848100 & 2.99941100 & -0.43652400 \\
\hline B & -1.42997900 & -0.85428100 & 0.43373300 \\
\hline $\mathrm{C}$ & -3.58441500 & -1.75032400 & 0.03941500 \\
\hline $\mathrm{H}$ & -1.88001100 & 4.19070700 & -2.80556400 \\
\hline $\mathrm{H}$ & -1.01261000 & 2.83130500 & -3.55256700 \\
\hline $\mathrm{H}$ & -2.65798700 & 2.59537800 & -2.89564900 \\
\hline $\mathrm{H}$ & 0.96034900 & 2.89268000 & -2.09385800 \\
\hline $\mathrm{H}$ & 0.33088000 & 4.43481400 & -1.4508230 \\
\hline
\end{tabular}


$0.80311500 \quad 3.11870200 \quad-0.33872900$

$\begin{array}{lll}-2.28453600 & 4.50348400 & 1.43995900\end{array}$

$\begin{array}{lll}-1.77932700 & 5.23179900 & -0.10578700\end{array}$

$\begin{array}{lll}-0.58580600 & 4.39066000 & 0.92155100\end{array}$

$\begin{array}{lll}-3.78191100 & 3.83988100 & -1.06799100\end{array}$

$\begin{array}{lll}-3.72424900 & 2.05889500 & -0.94055300\end{array}$

$\begin{array}{lll}-4.01645500 & 3.04936500 & 0.50978800\end{array}$

$\begin{array}{lll}-2.07803600 & -1.08686000 & 1.71159600\end{array}$

$\begin{array}{lll}-3.30533300 & -3.24397800 & -0.17984800\end{array}$

$\begin{array}{lll}-4.90107500 & -1.37174800 & -0.63351500\end{array}$

$\begin{array}{lll}-3.46925200 & -1.32553000 & 1.55073800\end{array}$

$\begin{array}{lll}-4.12208800 & -3.87790300 & 0.19871100\end{array}$

$\begin{array}{lll}-3.20331900 & -3.43075000 & -1.26101100\end{array}$

$\begin{array}{lll}-2.36408200 & -3.53972300 & 0.30219400\end{array}$

$\begin{array}{lll}-4.90436900 & -1.72295800 & -1.67897600\end{array}$

$\begin{array}{lll}-5.75840000 & -1.83989000 & -0.12429900\end{array}$

$\begin{array}{lll}-5.05074400 & -0.28350000 & -0.63947600\end{array}$

$\begin{array}{lll}-4.23249800 & -0.02926900 & 1.85241800\end{array}$

$\begin{array}{lll}-3.90454000 & -2.40732000 & 2.53821100\end{array}$

$\begin{array}{lll}-3.96479800 & 0.31254400 & 2.86459200\end{array}$

$\begin{array}{lll}-5.32399600 & -0.16990100 & 1.81939300\end{array}$

$\begin{array}{lll}-3.95012900 & 0.76200700 & 1.14908400\end{array}$

$\begin{array}{lll}-3.80456900 & -2.03057200 & 3.56886100\end{array}$

$\begin{array}{lll}-4.95813600 & -2.69048500 & 2.38371000\end{array}$

$\begin{array}{lll}-3.28197100 & -3.30780000 & 2.44839900\end{array}$

$\begin{array}{lll}1.85346500 & 0.69694300 & 2.50483800\end{array}$

$\begin{array}{lll}2.50387800 \quad 1.20911300 & 1.35379900\end{array}$

$3.84972000 \quad 0.82797500 \quad 0.90231800$

$\begin{array}{lll}4.63088000 & -0.16010600 & 1.54119500\end{array}$

$\begin{array}{lll}3.79134000 & 2.22051300 & -0.75160800\end{array}$

$\begin{array}{lll}2.17473500 & 2.18908200 & 0.98840400\end{array}$

$\begin{array}{lll}4.38583700 & 1.45798100 & -0.24147600\end{array}$

$5.89796700 \quad-0.49942600 \quad 1.05403100$

$6.41341400 \quad 0.13141500 \quad-0.08828500$

$\begin{array}{lll}5.64793900 & 1.11320500 & -0.73387900\end{array}$

$6.48632100 \quad-1.26389200 \quad 1.56828900$

$\begin{array}{lll}7.40181600 & -0.13763300 & -0.46848400\end{array}$

$6.03750300 \quad 1.61256600 \quad-1.62475200$

$0.51030200 \quad 1.03109300 \quad 2.71117800$

$\begin{array}{lll}0.15065400 & 1.99245500 & 2.33394900\end{array}$

$\begin{array}{lll}4.24724500 & -0.67261800 & 2.42526700\end{array}$

$2.29700600 \quad-0.12487600 \quad 3.07819200$

$\begin{array}{lll}-0.32492800 & 0.39934600 & 3.78322700\end{array}$

$\begin{array}{lll}-0.46386400 & 1.09546100 \quad 4.63044900\end{array}$ 
$\begin{array}{lll}0.14320100 & -0.52092100 & 4.16845800\end{array}$

$0.01169200 \quad-1.04504300 \quad-1.63578700$

$\begin{array}{lll}-1.63986400 & -2.78038200 & 0.60669300\end{array}$

$2.43448700 \quad 0.95275800 \quad-1.95854700$

$2.51003700 \quad-2.97133100 \quad 0.69133400$

$\begin{array}{lll}0.63995200 & -1.77759800 & -0.19176400\end{array}$

$\begin{array}{lll}-3.39245000 & -0.80046600 & 0.71779900\end{array}$

$\begin{array}{lll}-1.30242800 & -1.79345000 & -1.78735300\end{array}$

$\begin{array}{lll}1.11856700 & -1.21633800 \quad-2.66934700\end{array}$

$\begin{array}{lll}-0.10592400 & 0.47400300 & -1.24620600\end{array}$

$\begin{array}{lll}3.95222000 & -2.78965000 & 0.66667100\end{array}$

$1.94948400 \quad-1.84618000 \quad 0.14310000$

$-4.72204800 \quad-1.05820100 \quad 0.17417300$

$\begin{array}{lll}-2.82961400 & 0.31152500 & 0.07322000\end{array}$

$\begin{array}{lll}4.56464400 & -3.49270800 & 1.86727100\end{array}$

$\begin{array}{lll}4.45566800 & -3.41446800 & -0.63502200\end{array}$

$\begin{array}{lll}4.08465900 & -1.22119200 \quad 0.68153600\end{array}$

$2.86112100 \quad-0.81612900 \quad-0.00131600$

$\begin{array}{lll}-5.63311400 & -1.49958800 & 1.31065800\end{array}$

$\begin{array}{lll}-4.59260000 & -2.17760500 & -0.86228400\end{array}$

$\begin{array}{lll}-5.08842800 & 0.33023600 & -0.46737000\end{array}$

$\begin{array}{lll}-3.78620400 & 0.90042300 & -0.74132700\end{array}$

$\begin{array}{lll}-1.11408900 & 1.16636500 & 0.20873300\end{array}$

$\begin{array}{lll}5.64461200 & -3.28839100 & 1.92307900\end{array}$

$\begin{array}{lll}4.42869800 & -4.58058200 & 1.76809700\end{array}$

$4.09671100 \quad-3.17463400 \quad 2.80816900$

$\begin{array}{lll}4.14182500 & -4.46857400 & -0.66871900\end{array}$

$5.55269100 \quad-3.37889100 \quad-0.70112400$

$4.03401600 \quad-2.90506300 \quad-1.51485100$

$\begin{array}{lll}5.27624800 & -0.66548900 & -0.08295500\end{array}$

$\begin{array}{lll}4.02964100 & -0.63721300 & 2.09146500\end{array}$

$\begin{array}{lll}-6.66617200 & -1.62850800 \quad 0.95202200\end{array}$

$\begin{array}{lll}-5.29173600 & -2.46885300 & 1.70774100\end{array}$

$\begin{array}{lll}-5.63715100 & -0.77651900 \quad 2.13659200\end{array}$

$\begin{array}{lll}-5.55797200 & -2.39278700 & -1.34177000\end{array}$

$\begin{array}{lll}-4.28004900 & -3.11217100 & -0.36615700\end{array}$

$-3.84940600 \quad-1.92446100 \quad-1.63110200$

$\begin{array}{lll}-5.79709400 & 1.27511400 & 0.50576400\end{array}$

$\begin{array}{lll}-5.86991600 & 0.24654500 & -1.77171300\end{array}$

$\begin{array}{lll}-1.95983600 & 2.06455900 \quad 1.76770700\end{array}$

$\begin{array}{lll}5.28669300 & 0.43309100 & -0.00886000\end{array}$

$6.21761200 \quad-1.03926300 \quad 0.34709800$ 


\begin{tabular}{|c|c|c|c|}
\hline $\mathrm{H}$ & 5.24691700 & -0.94417100 & -1.14532500 \\
\hline $\mathrm{H}$ & 4.95103200 & -0.85643200 & 2.64968300 \\
\hline $\mathrm{H}$ & 3.91995800 & 0.45286800 & 2.02550600 \\
\hline $\mathrm{H}$ & 3.17426700 & -1.03547400 & 2.65714100 \\
\hline $\mathrm{H}$ & -5.83614900 & 2.27987600 & 0.05815400 \\
\hline $\mathrm{H}$ & -6.82760700 & 0.95059000 & 0.71346400 \\
\hline $\mathrm{H}$ & -5.25428600 & 1.34980400 & 1.45888200 \\
\hline $\mathrm{H}$ & -6.07339700 & 1.26244700 & -2.14433200 \\
\hline $\mathrm{H}$ & -6.83600200 & -0.25914600 & -1.61933300 \\
\hline $\mathrm{H}$ & -5.30864000 & -0.29223800 & -2.54665100 \\
\hline $\mathrm{C}$ & -2.50050300 & 1.37795900 & 3.00402600 \\
\hline $\mathrm{C}$ & -0.56349100 & 2.40075400 & 1.71771900 \\
\hline $\mathrm{H}$ & -2.64764600 & 2.81188800 & 1.34362700 \\
\hline $\mathrm{H}$ & -1.80654900 & 0.60112700 & 3.36514600 \\
\hline $\mathrm{H}$ & -2.65629800 & 2.09438300 & 3.83285300 \\
\hline $\mathrm{H}$ & -3.46319700 & 0.88352300 & 2.80953100 \\
\hline $\mathrm{C}$ & -0.01139300 & 3.08370800 & 0.63365900 \\
\hline $\mathrm{H}$ & 0.10503300 & 1.94839400 & 2.46205300 \\
\hline $\mathrm{C}$ & 1.40190400 & 3.32729900 & 0.36730500 \\
\hline $\mathrm{H}$ & -0.69525100 & 3.65273900 & -0.00787600 \\
\hline $\mathrm{C}$ & 2.45783100 & 2.78125000 & 1.13722000 \\
\hline $\mathrm{C}$ & 1.76069900 & 4.11478000 & -0.75637100 \\
\hline $\mathrm{C}$ & 3.79254100 & 3.01016400 & 0.79624900 \\
\hline $\mathrm{H}$ & 2.22894200 & 2.15524300 & 2.00200600 \\
\hline $\mathrm{C}$ & 3.09757300 & 4.33639600 & -1.09941100 \\
\hline $\mathrm{H}$ & 0.96565500 & 4.54732100 & -1.37044800 \\
\hline $\mathrm{C}$ & 4.12768700 & 3.78295000 & -0.32582200 \\
\hline $\mathrm{H}$ & 4.58516200 & 2.57443300 & 1.40960600 \\
\hline $\mathrm{H}$ & 3.33663200 & 4.94461200 & -1.97620300 \\
\hline $\mathrm{H}$ & 5.17426600 & 3.95287600 & -0.58920200 \\
\hline 14 & & & \\
\hline $\mathrm{O}$ & 0.82259900 & -1.13139100 & 0.26398800 \\
\hline $\mathrm{C}$ & 2.15844800 & -0.65144000 & 0.44400000 \\
\hline B & -0.04807700 & -0.00001500 & -0.00002800 \\
\hline $\mathrm{C}$ & 3.14110100 & -1.72623900 & -0.00499200 \\
\hline $\mathrm{C}$ & 2.36596200 & -0.34079500 & 1.93057200 \\
\hline $\mathrm{C}$ & 2.15846000 & 0.65138700 & -0.44402800 \\
\hline $\mathrm{O}$ & -1.06919100 & 0.33481300 & 1.06602000 \\
\hline $\mathrm{O}$ & 0.82261500 & 1.13135200 & -0.26403100 \\
\hline $\mathrm{H}$ & 3.08742700 & -2.59085200 & 0.67600100 \\
\hline $\mathrm{H}$ & 4.17532000 & -1.34832800 & 0.01330600 \\
\hline $\mathrm{H}$ & 2.91532600 & -2.07926800 & -1.02057600 \\
\hline $\mathrm{H}$ & 3.40329200 & -0.04588600 & 2.14954300 \\
\hline $\mathrm{H}$ & 2.13512500 & -1.24339900 & 2.51770700 \\
\hline
\end{tabular}


$\begin{array}{lll}2.36599200 & 0.34076400 & -1.93060400\end{array}$

$\begin{array}{lll}3.14111500 & 1.72616700 & 0.00499700\end{array}$

$-2.29519900$

$-1.03914100$

1.69409600

3.40335100

2.13505300

3.08743500

4.17533400

2.91534900

$-3.04433900$

$-1.03902600$

$-3.04424700$

$-1.06918800$

$-1.28880800$

$-2.21744100$

$-2.13192000$

0.03418100

0.12987900

$-0.31637800$

$-1.48156500$

3.56666800

3.82625700

1.58493500

2.22457700

2.47838400

4.62723000

5.62813300

4.75556500

4.12283800

4.44269500

5.49582200

3.89043200

4.39775300

3.51768000

3.13122900

4.51458000

$2.84631500 \quad 2.40043200$

$4.51107800 \quad-0.51801500$

$\begin{array}{lll}5.55307800 & -0.18529200 & 1.69857300\end{array}$

$\begin{array}{lll}4.25258100 & -0.42630500 & 2.89166500\end{array}$

$\begin{array}{lll}4.44552700 & -1.57928200 & 1.55078300\end{array}$ 
TS-S78

\begin{tabular}{rrr}
1.00159100 & -3.14450400 & 0.08904400 \\
0.77526900 & -4.20885900 & -0.10790800 \\
2.03817500 & -2.96929700 & -0.23477600 \\
0.96472700 & -2.99037200 & 1.17840400 \\
-3.46645300 & -0.71772400 & -0.20503600 \\
-4.40486200 & -0.07829000 & -1.04314800 \\
-3.76513100 & -0.78638700 & 1.17412700 \\
-5.58922100 & 0.45795200 & -0.53378800 \\
-4.19210300 & -0.00469500 & -2.11343600 \\
-4.95091800 & -0.25534700 & 1.68133800 \\
\hline-3.05407300 & -1.25105700 & 1.86057600 \\
\hline-5.87342500 & 0.37107400 & 0.83316500 \\
\hline-6.29599000 & 0.94647500 & -1.21022000 \\
\hline-5.15535100 & -0.32405100 & 2.75340000 \\
\hline-6.79937100 & 0.78974800 & 1.23508500 \\
-0.81932700 & 1.46663800 & -0.32542700 \\
-1.19864300 & 2.56116900 & -0.29332300 \\
-1.67276700 & 3.93051000 & -0.25148900 \\
-2.70636000 & 3.95856500 & 0.12650200 \\
-1.64986500 & 4.36051600 & -1.26495100 \\
\hline .02805000 & 4.53209900 & 0.40780000 \\
\hline & & \\
\hline
\end{tabular}

$1.30453700 \quad-1.57818400 \quad-0.15200200$

$\begin{array}{lll}2.21446000 & -0.91360300 & 0.67257000\end{array}$

$1.99250900 \quad-0.83970400 \quad 1.74402300$

$\begin{array}{lll}-0.10709200 & -1.84918700 & 0.20622400\end{array}$

$\begin{array}{lll}-0.23039300 & -2.06462600 & 1.27853400\end{array}$

$0.41415500 \quad 0.13915000 \quad 0.00655000$

$\begin{array}{lll}1.61532500 & -1.86047200 & -1.16604700\end{array}$

$\begin{array}{lll}-3.49411400 & 0.05428100 & -0.72961900\end{array}$

$\begin{array}{lll}-3.59441500 & -0.31384400 & 0.79951500\end{array}$

$\begin{array}{lll}-1.41867000 & -0.52421800 & 0.05843900\end{array}$

$\begin{array}{lll}-2.19783900 & -0.47906400 & -1.09037600\end{array}$

$\begin{array}{lll}-2.20242100 & -0.37070000 & 1.19581200\end{array}$

$\begin{array}{lll}-4.30789600 & 0.72302400 & 1.65752200\end{array}$

$\begin{array}{lll}-5.34642700 & 0.86075600 & 1.31915800\end{array}$

$\begin{array}{lll}-4.33391400 & 0.38364600 & 2.70474100\end{array}$

$\begin{array}{lll}-3.79716100 & 1.69461400 & 1.62889800\end{array}$

$-4.19616800 \quad-1.69789200 \quad 1.04922100$

$\begin{array}{lll}-5.27909700 & -1.71079700 \quad 0.85585400\end{array}$

$\begin{array}{lll}-3.72113400 & -2.46456400 & 0.42084000\end{array}$

$\begin{array}{lll}-4.03150200 & -1.97185400 \quad 2.10253900\end{array}$

$\begin{array}{lll}-3.45070400 & 1.56451900 & -0.97831700\end{array}$

$\begin{array}{lll}-3.15861600 & 1.74325600 & -2.02437300\end{array}$ 
$\begin{array}{lll}-2.71179400 & 2.05794300 & -0.33099700\end{array}$

$-4.55478000$

$-5.56336000$

$-0.58914800-1.61207900$

$-4.40839700$

$-0.27547300-1.30118900$

$-4.50122400$

$-0.73185000$

$-0.27492600 \quad-2.65727700$

$\mathrm{H}$

$\begin{array}{lll}-0.19257200 & -3.90343000 & -0.49535700\end{array}$

$\begin{array}{lll}-1.78940600 & -3.12475900 & -0.40638600\end{array}$

$\begin{array}{lll}-0.67603400 & -2.70434100 & -1.72237700\end{array}$

$\begin{array}{lll}3.59766800 & -0.58074400 & 0.31236100\end{array}$

$\begin{array}{lll}4.49292800 & -0.15691900 & 1.31875000\end{array}$

$\begin{array}{lll}4.09288900 & -0.64631100 & -1.01023800\end{array}$

$5.81538300 \quad 0.18028500 \quad 1.02397700$

$\begin{array}{lll}4.13532400 & -0.09437700 & 2.35055900\end{array}$

$\begin{array}{lll}5.41536500 & -0.31548800 & -1.30294500\end{array}$

$3.42884400 \quad-0.94890500 \quad-1.82287200$

$\begin{array}{lll}6.28922000 & 0.10172600 & -0.28988000\end{array}$

$\begin{array}{lll}6.48180000 & 0.50478000 & 1.82802800\end{array}$

$5.76833300 \quad-0.37699300 \quad-2.33619400$

$\begin{array}{lll}7.32369900 & 0.36487500 & -0.52398700\end{array}$

$0.21923800 \quad 1.93695300 \quad-0.03091300$

$\begin{array}{lll}0.10010500 & 3.09179900 & -0.08957700\end{array}$

$\begin{array}{lll}-0.06551500 & 4.53073300 & -0.16187000\end{array}$

$0.79675800 \quad 4.99029200 \quad-0.66995800$

$\begin{array}{lll}-0.14046500 & 4.94555900 & 0.85568800\end{array}$

$\begin{array}{lll}-0.98488200 & 4.77857600 & -0.71559500\end{array}$

$\begin{array}{lll}0.61531100 & -1.78477400 & 0.05702600\end{array}$

$\begin{array}{lll}1.60014500 & -1.06674000 & 0.79973300\end{array}$

$1.50050800 \quad-1.02893300 \quad 1.89405800$

$\begin{array}{lll}-0.66668600 & -2.35354500 & 0.66819900\end{array}$

$\begin{array}{lll}-0.56229600 & -2.39973500 & 1.76775600\end{array}$

$0.29528600 \quad 0.11086100 \quad-0.06696100$

$0.89448700 \quad-2.22218500 \quad-0.91272000$

$\begin{array}{lll}-2.88601800 & 0.32593600 & -0.90328700\end{array}$

$\begin{array}{lll}-3.42535700 & 0.30111900 & 0.57518000\end{array}$

$\begin{array}{lll}-1.75451100 & -1.25428500 & 0.35550700\end{array}$

$\begin{array}{lll}-1.63868500 & -0.43479000 & -0.77786000\end{array}$

$\begin{array}{lll}-2.85656000 & -0.93834700 & 1.09388100\end{array}$

$\begin{array}{lll}-2.86251000 \quad 1.43350600 & 1.43263500\end{array}$

$-3.29386500 \quad 2.40468600 \quad 1.15047400$

$\begin{array}{lll}-3.10718900 & 1.23854900 \quad 2.48756800\end{array}$

$-1.76870300 \quad 1.49011700 \quad 1.33566100$ 


\begin{tabular}{|c|c|c|c|}
\hline $\mathrm{C}$ & -4.93916800 & 0.23601700 & 0.69478600 \\
\hline $\mathrm{H}$ & -5.39826900 & 1.11947100 & 0.22558000 \\
\hline $\mathrm{H}$ & -5.34756600 & -0.66641000 & 0.22116600 \\
\hline $\mathrm{H}$ & -5.22640400 & 0.22608300 & 1.75728300 \\
\hline $\mathrm{C}$ & -2.56938600 & 1.70537700 & -1.45079500 \\
\hline $\mathrm{H}$ & -2.20014800 & 1.61938400 & -2.48418700 \\
\hline $\mathrm{H}$ & -3.47759600 & 2.32651100 & -1.46297300 \\
\hline $\mathrm{H}$ & -1.80077200 & 2.21018200 & -0.85463500 \\
\hline $\mathrm{C}$ & -3.76144600 & -0.45688700 & -1.87817800 \\
\hline $\mathrm{H}$ & -4.70401800 & 0.07211500 & -2.07927500 \\
\hline $\mathrm{H}$ & -3.22156400 & -0.57297400 & -2.82976100 \\
\hline $\mathrm{H}$ & -3.99747000 & -1.45963100 & -1.49200900 \\
\hline $\mathrm{C}$ & -1.02942900 & -3.75299900 & 0.15004000 \\
\hline $\mathrm{H}$ & -0.23019400 & -4.48067800 & 0.37442500 \\
\hline $\mathrm{H}$ & -1.96428300 & -4.12617900 & 0.60049900 \\
\hline $\mathrm{H}$ & -1.16934900 & -3.74566800 & -0.94433700 \\
\hline $\mathrm{C}$ & 2.96166500 & -0.77600500 & 0.31761300 \\
\hline $\mathrm{C}$ & 3.93235500 & -0.27971300 & 1.21577700 \\
\hline $\mathrm{C}$ & 3.35920400 & -0.94015000 & -1.03016100 \\
\hline $\mathrm{C}$ & 5.22400600 & 0.04439500 & 0.79471400 \\
\hline $\mathrm{H}$ & 3.65697000 & -0.14381200 & 2.26588500 \\
\hline $\mathrm{C}$ & 4.65069100 & -0.62210400 & -1.44949900 \\
\hline $\mathrm{H}$ & 2.63788600 & -1.30596900 & -1.76370600 \\
\hline $\mathrm{C}$ & 5.59713100 & -0.12477600 & -0.54304300 \\
\hline $\mathrm{H}$ & 5.94700800 & 0.42901300 & 1.51995600 \\
\hline $\mathrm{H}$ & 4.92261500 & -0.75997300 & -2.50008100 \\
\hline $\mathrm{H}$ & 6.60719200 & 0.12683800 & -0.87591500 \\
\hline $\mathrm{N}$ & 0.63919200 & 1.91633000 & 0.07634400 \\
\hline $\mathrm{C}$ & 0.96539500 & 3.03099900 & 0.14131600 \\
\hline $\mathrm{C}$ & 1.37503000 & 4.42045100 & 0.22367500 \\
\hline $\mathrm{H}$ & 2.34085000 & 4.56018200 & -0.28699600 \\
\hline $\mathrm{H}$ & 1.48613600 & 4.71734500 & 1.27854500 \\
\hline $\mathrm{H}$ & 0.62258500 & 5.06912700 & -0.25183900 \\
\hline
\end{tabular}

\section{Cartesian coordinates of the structures (optimization in DMF)} TS5/TS22

$\begin{array}{lrrr}\text { C } & -1.14753500 & 0.67334800 & 2.18195700 \\ \text { C } & -0.20611900 & 0.37938500 & 1.17489300 \\ \text { C } & 1.02006400 & 1.08180300 & 1.22078900 \\ \text { C } & 1.18117600 & 2.19883500 & 2.06854700 \\ \text { C } & 0.22294700 & 2.43290500 & 3.05959100 \\ \text { C } & -0.91704800 & 1.63591700 & 3.15783000 \\ \text { C } & 3.48019300 & 2.48617700 & 1.17389400 \\ \text { C } & 3.31476300 & 1.24015600 & 0.53982000 \\ \text { C } & 4.42113900 & 0.53330100 & 0.00758300\end{array}$


$\begin{array}{lll}-2.10370700 & 0.14516100 & 2.15923000\end{array}$

$0.34735500 \quad 3.27102500 \quad 3.74463700$

$\begin{array}{lll}-1.65666500 & 1.81855500 \quad 3.94047100\end{array}$

$6.53282100 \quad 0.66844900 \quad-0.40060300$

$\begin{array}{lll}6.82907900 & 2.90433900 & 0.60737800\end{array}$

$4.89533700 \quad 4.03708900 \quad 1.66377600$

$\begin{array}{lll}2.27217700 & 3.21808300 & 1.74702300\end{array}$

$\begin{array}{lll}2.65161900 & 4.06097000 & 2.97406500\end{array}$

$3.40479500 \quad 4.81647600 \quad 2.71451300$

$\begin{array}{lll}3.05064300 & 3.43564800 & 3.78751100\end{array}$

$\begin{array}{lll}1.78093800 & 4.61302800 \quad 3.35262600\end{array}$

$\begin{array}{lll}1.70974700 & 4.16399900 & 0.65275700\end{array}$

$\begin{array}{lll}0.81421600 & 4.68706400 \quad 1.02265000\end{array}$

$1.43034100 \quad 3.60578500 \quad-0.25351200$

$\begin{array}{lll}2.46741000 & 4.91363900 & 0.37433300\end{array}$

$\begin{array}{lll}2.05498200 & 0.68525300 & 0.40549300\end{array}$

$\begin{array}{lll}-0.43957900 & -2.47673000 & 0.39360300\end{array}$

$\begin{array}{lll}1.05574300 & -2.60658500 & 0.67150600\end{array}$

$\begin{array}{lll}1.37201200 & -1.90010400 \quad 1.45193700\end{array}$

$1.67901700 \quad-2.41683300 \quad-0.20827600$

$\begin{array}{lll}1.27607000 & -3.62651600 \quad 1.03286300\end{array}$

$\begin{array}{lll}-1.21155800 & -2.76317800 & 1.69359800\end{array}$

$\begin{array}{lll}-1.04579500 & -3.81228900 & 1.99135100\end{array}$

$\begin{array}{lll}-2.28959600 & -2.60799000 & 1.56750900\end{array}$

$\begin{array}{lll}-0.86582300 & -2.12385600 & 2.51982200\end{array}$

$\begin{array}{lll}-0.89204500 & -3.46720100 & -0.68720400\end{array}$

$\begin{array}{lll}-1.95423300 & -3.33648200 & -0.93341700\end{array}$

$\begin{array}{lll}-0.75927200 & -4.49774300 & -0.31662900\end{array}$

$\begin{array}{lll}-0.30668500 & -3.36860500 & -1.61152100\end{array}$

$\begin{array}{lll}-0.30074600 & -0.24700200 & -1.85058500\end{array}$

$\begin{array}{lll}-1.41394200 & -0.65428400 & -2.83660200\end{array}$

$\begin{array}{lll}-1.64181300 & -1.72865800 & -2.79043200\end{array}$

$\begin{array}{lll}-1.08460900 & -0.42339200 & -3.86421600\end{array}$

$\begin{array}{lll}-2.34360200 & -0.09845400 & -2.64191300\end{array}$

$\begin{array}{lll}1.01675400 & -0.88300800 & -2.28827300\end{array}$

$1.82886100 \quad-0.67912800 \quad-1.58398800$

$\begin{array}{lll}1.30251500 & -0.46874500 & -3.27052000\end{array}$

$0.93355000 \quad-1.97200100 \quad-2.40893900$

$\begin{array}{lll}-0.17030500 & 1.28573300 & -1.88366400\end{array}$

$\begin{array}{lll}-1.04812400 & 1.77663500 & -1.44473800\end{array}$

$-0.08357900 \quad 1.61625900 \quad-2.93245300$ 
$0.72461100 \quad 1.63040100 \quad-1.35126700$

$\begin{array}{lll}5.28960600 & -2.16736400 & 0.70013300\end{array}$

$\begin{array}{lll}4.45544700 & -2.09796900 & 1.99253700\end{array}$

$\begin{array}{lll}3.46467600 & -2.55520300 & 1.86766300\end{array}$

$\begin{array}{lll}4.31371000 & -1.06039600 & 2.33500200\end{array}$

$\begin{array}{lll}4.97819000 & -2.64410500 & 2.79687800\end{array}$

$6.70350700 \quad-1.67135700 \quad 1.02472800$

$\begin{array}{lll}7.16681600 & -2.36572600 & 1.74824500\end{array}$

$6.69035400 \quad-0.67875800 \quad 1.49605500$

$\begin{array}{lll}7.35862800 & -1.62762800 & 0.14668900\end{array}$

$\begin{array}{lll}5.34739600 & -3.62951600 & 0.22872400\end{array}$

$4.34764700 \quad-4.01120000 \quad-0.03397000$

$\begin{array}{lll}5.74702000 & -4.26501500 \quad 1.03803300\end{array}$

$6.00369800 \quad-3.75597600 \quad-0.64479500$

$\begin{array}{lll}5.13577400 & -1.21396400 & -2.30975000\end{array}$

$\begin{array}{lll}4.70570400 & 0.06773600 & -3.04355300\end{array}$

$4.97139100 \quad-0.01605800 \quad-4.11181900$

$\begin{array}{lll}5.20600200 & 0.96066000 & -2.64308100\end{array}$

$\begin{array}{lll}3.61907500 & 0.23243300 & -2.98258000\end{array}$

$6.66364600 \quad-1.33067800 \quad-2.34696000$

$\begin{array}{lll}7.16752100 & -0.49380500 & -1.84490400\end{array}$

$6.99798900-1.32589600 \quad-3.39982000$

$7.01864500 \quad-2.26827600 \quad-1.89704000$

$\begin{array}{lll}4.53394400 & -2.42210500 & -3.05534900\end{array}$

$3.43806200 \quad-2.34768000 \quad-3.12512800$

$\begin{array}{lll}4.77673300 & -3.37481600 & -2.56160200\end{array}$

$4.93787200 \quad-2.46671700 \quad-4.08194900$

$4.25203300 \quad-1.21090100 \quad-0.60043000$

$-0.95139700 \quad-0.72796800 \quad-0.12316600$

$\begin{array}{lll}-5.04726900 & -0.39980100 & -0.47676000\end{array}$

$\begin{array}{lll}-4.35659600 & -1.68467100 & -0.58492700\end{array}$

$\begin{array}{lll}-4.57483700 & -2.75339600 & 0.40813700\end{array}$

$\begin{array}{lll}-4.89286900 & -2.48239100 & 1.75829900\end{array}$

$\begin{array}{lll}-4.15860600 & -4.34722300 & -0.99186900\end{array}$

$\begin{array}{lll}-4.22012900 & -2.07392000 & -1.60410400\end{array}$

$-4.39608700 \quad-4.10532800 \quad 0.04751400$

$\begin{array}{lll}-5.01413400 & -3.50882100 & 2.69483700\end{array}$

$\begin{array}{lll}-4.81449000 & -4.84379300 & 2.31989000\end{array}$

$\begin{array}{lll}-4.50533700 & -5.13322500 & 0.98672600\end{array}$

$\begin{array}{lll}-5.25521700 & -3.26424500 \quad 3.73324100\end{array}$

$\begin{array}{lll}-4.90082800 & -5.64597700 \quad 3.05707400\end{array}$

$-4.35144600 \quad-6.16971000 \quad 0.67332000$

$\begin{array}{lll}-5.34984800 & 0.39996400 & -1.63198800\end{array}$

$\begin{array}{lll}-4.98353700 & -0.00681600 & -2.58016600\end{array}$ 


\begin{tabular}{rrr}
-3.60721000 & 2.39560800 & -0.87912700 \\
-3.02714900 & 1.71361500 & -0.00648600 \\
-3.39571100 & 3.91969700 & -0.89156600 \\
-3.00537900 & 4.35953800 & -2.31281500 \\
-2.29053500 & 4.30633800 & 0.09775600 \\
-4.73095300 & 4.57068900 & -0.48574400 \\
-3.78877000 & 4.09728900 & -3.03805700 \\
-2.06745600 & 3.87718000 & -2.63195800 \\
\hline-2.85450900 & 5.45054800 & -2.34147900 \\
-2.54558500 & 4.00932200 & 1.12511100 \\
-2.13648700 & 5.39714800 & 0.07893900 \\
\hline-1.33860200 & 3.82043600 & -0.16068900 \\
\hline-4.63039600 & 5.66803600 & -0.47818800 \\
\hline-5.03850200 & 4.25042400 & 0.52314500 \\
\hline-5.53206500 & 4.30149400 & -1.19016100 \\
\hline-4.38392500 & 1.93811000 & -1.78829400 \\
-5.66094000 & -0.19480700 & 0.40970700 \\
-6.01790900 & -1.44744300 & 2.08369700 \\
-6.69741500 & 1.05758700 & -1.72227100 \\
-7.45305000 & 0.26432000 & -1.85825200 \\
\hline-11311600 & -0.26084500 & -0.16516900 \\
\hline
\end{tabular}

TS20/TS23/TS-Conformation-A

\begin{tabular}{|c|c|c|c|}
\hline $\mathrm{C}$ & -1.95436300 & 1.37672700 & -0.67923800 \\
\hline $\mathrm{C}$ & -1.82691800 & -0.04655000 & -0.51117900 \\
\hline $\mathrm{C}$ & -1.57554200 & -0.95379900 & -1.65411400 \\
\hline C & -1.51068600 & -0.50208100 & -2.98930100 \\
\hline $\mathrm{H}$ & -1.48933400 & -2.72796000 & -0.42590000 \\
\hline $\mathrm{H}$ & -2.48928900 & -0.47034000 & 0.24141300 \\
\hline $\mathrm{C}$ & -1.42604200 & -2.34022900 & -1.43876100 \\
\hline $\mathrm{C}$ & -1.27526100 & -1.38433200 & -4.04635700 \\
\hline C & -1.10506400 & -2.75137700 & -3.80582200 \\
\hline $\mathrm{C}$ & -1.19003600 & -3.22376700 & -2.49157300 \\
\hline $\mathrm{H}$ & -1.23214700 & -0.99855800 & -5.06852600 \\
\hline $\mathrm{H}$ & -0.91552800 & -3.44051000 & -4.63253500 \\
\hline $\mathrm{H}$ & -1.06784100 & -4.28965000 & -2.28141000 \\
\hline $\mathrm{C}$ & -2.56466300 & 2.13600600 & 0.32299800 \\
\hline $\mathrm{C}$ & -5.09813900 & 0.70149300 & 0.06894200 \\
\hline $\mathrm{O}$ & -4.71151800 & -0.01373800 & 1.01296600 \\
\hline $\mathrm{C}$ & -6.13360100 & 0.10837300 & -0.93889200 \\
\hline $\mathrm{C}$ & -6.91124800 & -1.04607700 & -0.30093900 \\
\hline $\mathrm{C}$ & -7.10297300 & 1.19754800 & -1.41417600 \\
\hline $\mathrm{C}$ & -5.31399800 & -0.41303000 & -2.13452700 \\
\hline
\end{tabular}


$\begin{array}{lll}-6.22868400 & -1.83053700 \quad 0.05508300\end{array}$

$-7.49678300$

$-0.70021900 \quad 0.56695100$

$-7.61318800$

$-6.55192700$

$-1.49379000$

$-1.02557100$

$-7.80419400$

$2.04029100-1.85449300$

$-7.70077300$

$-5.97481700$

$-4.71824800$

$0.39649100 \quad-2.58391900$

$-4.61764900$

$-1.20787200 \quad-1.82310500$

$\begin{array}{lll}-4.68381100 & 1.86949800 & -0.20679500\end{array}$

$\begin{array}{lll}0.66843000 & -3.72758500 & 1.36151100\end{array}$

$0.72409000 \quad-2.36949700 \quad 1.01013500$

$\begin{array}{lll}1.54054600 & -2.03125900 & -0.08357500\end{array}$

$2.37101900 \quad-2.94601200 \quad-0.74092400$

$2.28951800 \quad-4.28861100 \quad-0.35512400$

$1.42044900 \quad-4.67911100 \quad 0.66811000$

$3.78079100 \quad-1.01873200 \quad-1.33407700$

$2.81500800 \quad-0.17735200 \quad-0.76488600$

$3.04421600 \quad 1.17107400 \quad-0.44316500$

$4.35885500 \quad 1.63998100 \quad-0.60302300$

$\begin{array}{lll}5.36683000 & 0.80232200 & -1.08617500\end{array}$

$5.07548300 \quad-0.50962700 \quad-1.47642000$

$\begin{array}{lll}0.05368100 & -4.04884300 \quad 2.20093700\end{array}$

$2.90706100 \quad-5.03853100 \quad-0.85051400$

$1.35669100 \quad-5.73162500 \quad 0.95368400$

$\begin{array}{lll}4.59923100 & 2.67280800 & -0.35366900\end{array}$

$6.38441300 \quad 1.18571500 \quad-1.19013100$

$5.86461000 \quad-1.13109700 \quad-1.90106100$

$\begin{array}{lll}3.30249000 & -2.39246600 & -1.82312000\end{array}$

$\begin{array}{lll}4.46756300 & -3.33890200 & -2.11798000\end{array}$

$5.11901100 \quad-2.91861300 \quad-2.89756700$

$\begin{array}{lll}5.07689300 & -3.52891600 & -1.22116700\end{array}$

$4.09665500 \quad-4.30223500 \quad-2.49573600$

$\begin{array}{lll}2.48104900 & -2.17759800 & -3.12047800\end{array}$

$2.09621800 \quad-3.14076900 \quad-3.48852100$

$1.62399600 \quad-1.51210200 \quad-2.94887100$

$3.11546000-1.73047900 \quad-3.90186500$

$1.57567300 \quad-0.71111800 \quad-0.48895100$

$\begin{array}{lll}0.00775200 & -0.96637200 & 1.97933700\end{array}$

$\begin{array}{lll}1.57282100 & 2.22967000 & -0.04844700\end{array}$

$\begin{array}{lll}-1.56902700 & -1.58936700 & 2.90357300\end{array}$

$\begin{array}{lll}-2.45172900 & -2.48028000 & 2.01338700\end{array}$

$-2.81086000 \quad-1.97007800 \quad 1.11616900$

$\begin{array}{lll}-1.95345300 & -3.41443800 & 1.72196300\end{array}$ 
$\begin{array}{lll}-1.73660500 & 0.46657700 & 3.70746100\end{array}$

$-2.91629500$

$0.07600200 \quad 2.40837100$

$\begin{array}{lll}-3.12767600 & -0.58792300 & 4.02475200\end{array}$

$-1.29531900$

$-2.37774600$ 4.19386000

$\begin{array}{lll}-0.83177200 & -1.76019700 & 4.97203400\end{array}$

$\begin{array}{lll}-2.26522100 & -2.72078200 & 4.59180100\end{array}$

$\begin{array}{lll}-0.67431400 & -3.26863600 & 4.03440700\end{array}$

$\begin{array}{lll}1.41307300 & -0.77215800 & 3.29709500\end{array}$

$\begin{array}{lll}2.66749200 & -0.40394200 & 2.49686100\end{array}$

$3.06887600 \quad-1.27170000 \quad 1.95472800$

$\begin{array}{lll}2.46853700 & 0.38696500 & 1.77493600\end{array}$

$3.45229000 \quad-0.05009800 \quad 3.18421900$

$\begin{array}{lll}1.01394400 & 0.39139600 & 4.21657800\end{array}$

$\begin{array}{lll}0.18310600 & 0.12126600 & 4.88350500\end{array}$

$\begin{array}{lll}1.86980100 & 0.67502400 & 4.85206800\end{array}$

$\begin{array}{lll}0.70973700 & 1.27842800 & 3.64126600\end{array}$

$\begin{array}{lll}1.78738100 & -2.00966900 & 4.12876900\end{array}$

$1.89804100 \quad-2.90840900 \quad 3.50494600$

$\begin{array}{lll}2.76808100 & -1.82067500 & 4.59853000\end{array}$

$\begin{array}{lll}1.08380400 & -2.22840300 & 4.93626100\end{array}$

$\begin{array}{lll}2.10170600 & 3.59007300 & 1.19436900\end{array}$

$\begin{array}{lll}2.95981600 & 2.97475300 & 2.30849100\end{array}$

$\begin{array}{lll}2.42495200 & 2.19063800 \quad 2.85098300\end{array}$

$\begin{array}{lll}3.90552900 & 2.55859700 & 1.93459000\end{array}$

$3.20621100 \quad 3.76691500 \quad 3.03533300$

$2.85494500 \quad 4.79585300 \quad 0.61785100$

$\begin{array}{lll}3.07983000 & 5.48939600 & 1.44606900\end{array}$

$\begin{array}{lll}3.81214500 & 4.51947500 & 0.15628100\end{array}$

$2.25897700 \quad 5.35213400 \quad-0.11635700$

$\begin{array}{lll}0.78568300 & 4.08954400 & 1.81728100\end{array}$

$1.01116600 \quad 4.81750100 \quad 2.61458600$

$\begin{array}{lll}0.15306800 & 4.59501300 & 1.07478800\end{array}$

$\begin{array}{lll}0.20507900 & 3.26556700 & 2.25947400\end{array}$

$1.36392100 \quad 3.00782100 \quad-1.80512400$

$\begin{array}{lll}2.60355200 & 3.67253400 & -2.41984600\end{array}$

$2.34995300 \quad 3.96322100 \quad-3.45422800$

$2.91889000 \quad 4.58073600 \quad-1.89651800$

$\begin{array}{lll}3.45841500 & 2.98650600 & -2.48078500\end{array}$

$0.22014800 \quad 4.02667100 \quad-1.74770400$

$\begin{array}{lll}0.49502400 & 4.92032100 & -1.16942800\end{array}$

$\begin{array}{lll}-0.03199300 & 4.35606800 & -2.76981100\end{array}$

$\begin{array}{rrr}-0.67974200 & 3.59689600 & -1.30137800\end{array}$ 
C

H

$\mathrm{H}$

$\mathrm{H}$

$\mathrm{Ni}$

$\mathrm{H}$

$\mathrm{H}$

$\mathrm{H}$

C

$\mathrm{H}$

$\mathrm{H}$

H

TS-Conformation-B

C

C

C

C

C

C

C

C

$\begin{array}{rrr}0.98529200 & 1.82988400 & -2.71791800 \\ 1.85941900 & 1.20356900 & -2.94736600 \\ 0.22443900 & 1.18760200 & -2.26812500 \\ 0.58933500 & 2.21490200 & -3.67211400 \\ -0.24724300 & 0.75844900 & 0.39046300 \\ -1.66866000 & 0.55241900 & -3.21293100 \\ -1.76809900 & 1.85592700 & -1.64146300 \\ -2.77009200 & 1.63565600 & 1.26720300 \\ -2.68466900 & 3.62035000 & 0.31524000 \\ -2.78652700 & 4.01658700 & -0.70471500 \\ -3.55640600 & 3.93050800 & 0.90557100 \\ -1.79136000 & 4.07377100 & 0.77183000\end{array}$

$-2.24643000$

$-1.61984500$

$-2.11427800$

$-3.01475300$

$-3.60669700$

$-3.25927800$

$-3.29231600$

$-2.50491400$

$-2.58285100$

$-3.45305300$

$-4.20996000$

$-4.14397100$

$-1.92243100$

$-4.33043000$

$-3.73947500$

$-3.56462600$

$-4.87590600$

$-4.76364100$

$-3.16648300$

$-1.86300000$

$-1.92926900$

$-0.98026700$

$-1.71311700$

$-4.35064900$

$-4.22362700$

$-5.30627900$

$-4.41110900$

$-1.64578000$

0.09460400

$-0.63986600$

$-1.72336900$

$-3.57194000$

1.45865600

$-2.40382200 \quad 0.99123000$

$-1.86377600 \quad-0.20984400$

$-2.54246400-1.03992300$

$-3.70690500 \quad-0.53963500$

$-4.19070000 \quad 0.72448300$

$-0.51065900 \quad-2.37073200$

$0.15051200 \quad-1.41743100$

$1.53452700-1.18457400$

$2.26429100 \quad-2.01298300$

$1.64219500-3.00465900$

$0.25851100-3.16993500$

$-4.02328200 \quad 2.39258800$

$-4.25273400-1.14515200$

$-5.09096800 \quad 1.11442900$

$3.33574500-1.86519600$

$2.23700600 \quad-3.63392200$

$-0.22354600 \quad-3.92580900$

$-2.02696900 \quad-2.47164700$

$-2.36487300-3.24305700$

$-1.98433300 \quad-4.27411900$

$-1.91287200 \quad-2.76884600$

$-3.45506300 \quad-3.27890100$

$-2.66803900-3.19799400$

$-3.75886800 \quad-3.24765700$

$-2.45151200 \quad-2.69698500$

$-2.30641300 \quad-4.23398900$

$-0.61790500-0.62634300$

$-1.62258900 \quad 3.43467200$

$-2.71773800 \quad 4.21723700$

$-2.68704000 \quad 4.03677700$ 
$\begin{array}{lll}-0.27384200 & -3.72742800 & 3.99485400\end{array}$

$\begin{array}{lll}-0.48528900 & -2.53936300 & 5.29535400\end{array}$

$\begin{array}{lll}-0.54643000 & -0.27167400 \quad 3.78384100\end{array}$

$\begin{array}{lll}-0.41068000 & -0.07265000 & 4.86042300\end{array}$

$\begin{array}{lll}-0.08601500 & 0.55142600 & 3.22238900\end{array}$

$\begin{array}{lll}-1.62366600 & -0.27160600 & 3.57970000\end{array}$

$\begin{array}{lll}1.57060700 & -1.57503900 \quad 3.86035900\end{array}$

$\begin{array}{lll}2.13428000 & -0.81303000 & 3.30465500\end{array}$

$1.61933400 \quad-1.30890800 \quad 4.92940500$

$\begin{array}{lll}2.07391700 & -2.54324100 & 3.73798800\end{array}$

$\begin{array}{lll}1.12739800 & -3.23823800 & 0.93252500\end{array}$

$0.98607700 \quad-4.50047900 \quad 1.79213100$

$\begin{array}{lll}1.53915600 & -5.32121500 \quad 1.30402000\end{array}$

$\begin{array}{lll}1.41670200 & -4.36793600 & 2.79425900\end{array}$

$\begin{array}{lll}-0.05545800 & -4.82923100 & 1.89846600\end{array}$

$\begin{array}{lll}0.68603400 & -3.55366900 & -0.50817500\end{array}$

$0.63025700 \quad-2.63973300 \quad-1.11931200$

$\begin{array}{lll}1.43371000 & -4.21840800 & -0.97196000\end{array}$

$\begin{array}{lll}-0.28706200 & -4.06141900 \quad-0.55164900\end{array}$

$\begin{array}{lll}2.61401500 & -2.82919200 & 0.88082500\end{array}$

$\begin{array}{lll}2.94799800 & -2.26773700 \quad 1.76101900\end{array}$

$3.22322900 \quad-3.74735100 \quad 0.82522900$

$\begin{array}{lll}2.84494800 & -2.24532900 & -0.01861400\end{array}$

$\begin{array}{lll}-1.30748600 & 4.08177000 & -0.21615900\end{array}$

$\begin{array}{lll}-2.45171500 & 5.09380400 & -0.06772400\end{array}$

$\begin{array}{lll}-3.34330500 & 4.83801300 & -0.65429400\end{array}$

$\begin{array}{lll}-2.75376200 & 5.21707500 & 0.98121200\end{array}$

$\begin{array}{lll}-2.09678200 & 6.07740000 & -0.42205100\end{array}$

$\begin{array}{lll}-0.16284200 & 4.52658900 & 0.71462000\end{array}$

$\begin{array}{lll}-0.42043300 & 4.42199400 \quad 1.77862900\end{array}$

$\begin{array}{lll}0.75572600 & 3.95573000 & 0.52737600\end{array}$

$\begin{array}{lll}0.06208100 & 5.59088300 & 0.52972900\end{array}$

$\begin{array}{lll}-0.77361400 & 4.12083200 & -1.65783800\end{array}$

$-0.32841200 \quad 5.11300600 \quad-1.84537500$

$\begin{array}{lll}0.00976100 & 3.37164800 & -1.82556300\end{array}$

$\begin{array}{lll}-1.55751600 & 3.96293900 & -2.40956400\end{array}$

$\begin{array}{lll}-3.08582700 & 2.17333000 & 1.56073500\end{array}$

$\begin{array}{lll}-4.41579100 \quad 2.85038600 & 1.21118800\end{array}$

$\begin{array}{lll}-5.16814700 \quad 2.56266800 & 1.96693000\end{array}$

$\begin{array}{lll}-4.35362900 & 3.94376700 & 1.21512100\end{array}$

$\begin{array}{lll}-4.79932100 & 2.52556300 \quad 0.23277600\end{array}$

$\begin{array}{lll}-2.52272300 & 2.75850500 \quad 2.86483200\end{array}$

$\begin{array}{lll}-2.37956600 & 3.84671100 & 2.79192200\end{array}$

$-3.22743400 \quad 2.57055600 \quad 3.69308400$ 
Conformation-A

C $\begin{array}{lll}1.89997600 & 1.21593000 & 0.99848300\end{array}$

$\begin{array}{lll}1.64640700 & -0.15982800 & 1.26182700\end{array}$

$\begin{array}{lll}0.96961800 & -0.61647500 & 2.50413300\end{array}$

$0.99748200 \quad 0.14222200 \quad 3.69372000$

$\begin{array}{lll}0.20651300 & -2.44671900 & 1.64474300\end{array}$

$\begin{array}{lll}2.35257700 & -0.88601200 & 0.85709400\end{array}$

$\begin{array}{lll}0.26295300 & -1.83739500 & 2.54403000\end{array}$

$\begin{array}{lll}0.32527400 & -0.27597900 & 4.84537300\end{array}$

$\begin{array}{lll}-0.38650700 & -1.47975400 & 4.85409400\end{array}$

$\begin{array}{lll}-0.40369500 & -2.26259400 & 3.69325100\end{array}$

$\begin{array}{lll}0.36392800 & 0.34337500 & 5.74615900\end{array}$

$\begin{array}{lll}-0.91645300 & -1.80575100 & 5.75273900\end{array}$

$\begin{array}{lll}-0.95488600 & -3.20690400 \quad 3.67620300\end{array}$

$\begin{array}{lll}3.12507600 & 1.61846200 & 0.20523200\end{array}$

$5.23937100 \quad 0.49275500 \quad-0.13271300$

$\begin{array}{lll}5.28516900 & 0.84284500 & -1.29420400\end{array}$

$\begin{array}{lll}6.28351900 & -0.39597000 & 0.55454500\end{array}$

$7.31424600 \quad-0.85597100 \quad-0.48019200$

$\begin{array}{lll}6.97086900 & 0.43551700 & 1.65470700\end{array}$

$5.57937500 \quad-1.61353900 \quad 1.18084600$

$6.84141400 \quad-1.44854900 \quad-1.27830200$

$\begin{array}{lll}7.81791700 & 0.00071700 & -0.95218100\end{array}$

$8.07943800 \quad-1.48237300 \quad 0.00488600$

$\begin{array}{lll}6.24593100 & 0.77191300 & 2.41014100\end{array}$

$\begin{array}{lll}7.74021600 & -0.17117800 & 2.15869500\end{array}$

$7.46360200 \quad 1.32548400 \quad 1.23022500$

$\begin{array}{lll}6.32355400 & -2.26780000 & 1.66272100\end{array}$

$\begin{array}{lll}4.84683200 & -1.30514800 \quad 1.93996300\end{array}$

$5.05055000 \quad-2.20468500 \quad 0.41714000$

$\begin{array}{lll}4.26651600 & 0.84155300 & 0.71189900\end{array}$

$\begin{array}{lll}-1.28399500 & -3.90389400 & -0.76917100\end{array}$

$\begin{array}{lll}-1.11350000 & -2.52097000 & -0.59109600\end{array}$

$\begin{array}{lll}-2.11703700 & -1.85008900 & 0.12824400\end{array}$

$\begin{array}{lll}-3.30415500 & -2.45854700 & 0.55433500\end{array}$

$\begin{array}{lll}-3.43960400 & -3.83516700 & 0.34475000\end{array}$

$-2.42054600 \quad-4.55678300 \quad-0.28501900$

$\begin{array}{lll}-4.31721600 & -0.22485400 & 0.41925600\end{array}$

$\begin{array}{lll}-3.04852500 & 0.29734500 & 0.12534900\end{array}$

$\begin{array}{lll}-2.83151800 & 1.57435400 & -0.41765100\end{array}$

$\begin{array}{lll}-3.97955000 & 2.29522300 & -0.78677700\end{array}$

$\begin{array}{lll}-5.25859800 & 1.76994400 & -0.58912000\end{array}$

$\begin{array}{lll}-5.43032200 & 0.52962400 & 0.03657900\end{array}$ 
$\begin{array}{lll}-0.53965200 & -4.47727300 & -1.31936500\end{array}$

$-4.34308700$

$-4.35245300 \quad 0.66938500$

$-2.53002200$

$-5.63364700$

$-0.43348700$

$-3.87760000$

3.28710200

$-1.22365800$

$-6.13341700$

$2.34797500 \quad-0.89609400$

$-6.43792900$

$-4.35622800$

0.15979000

0.23018500

$\begin{array}{lll}-5.74831500 & -2.18256500 & 1.18784100\end{array}$

$\begin{array}{lll}-6.48179500 & -1.50760100 \quad 1.65194900\end{array}$

$\begin{array}{lll}-6.08703300 & -2.41256000 & 0.16609700\end{array}$

$\begin{array}{lll}-5.75441300 & -3.11377800 & 1.77237200\end{array}$

$\begin{array}{lll}-3.93516100 & -1.26161800 \quad 2.66025800\end{array}$

$\begin{array}{lll}-3.92829100 & -2.19624200 & 3.24243800\end{array}$

$\begin{array}{lll}-2.92880300 & -0.82252900 & 2.71005600\end{array}$

$\begin{array}{lll}-4.64361300 & -0.56100100 \quad 3.12953600\end{array}$

$\begin{array}{lll}-1.95531400 & -0.50202100 & 0.36503000\end{array}$

$0.16481300 \quad-1.46091200 \quad-1.41551000$

$\begin{array}{lll}-1.08873100 & 2.23609600 & -0.41433700\end{array}$

$\begin{array}{lll}1.75576600 & -2.53950400 & -1.60006300\end{array}$

$2.02304100 \quad-3.31858800 \quad-0.30032900$

$\begin{array}{lll}2.05055400 & -2.68025500 & 0.58740400\end{array}$

$1.27872200 \quad-4.10701400 \quad-0.12598700$

$3.00805300 \quad-3.80819300 \quad-0.38342200$

$2.90030800 \quad-1.53762100 \quad-1.82944300$

$2.78136800 \quad-0.98484800 \quad-2.77186200$

$2.95346600 \quad-0.80337000 \quad-1.02266400$

$3.86231000 \quad-2.07381900 \quad-1.87543900$

$\begin{array}{lll}1.78066200 & -3.55181000 & -2.75374900\end{array}$

$\begin{array}{lll}1.75282600 & -3.06380100 & -3.73566500\end{array}$

$2.73209700 \quad-4.10877000 \quad-2.69853300$

$0.96873900 \quad-4.28926000 \quad-2.70434100$

$\begin{array}{lll}-0.65713600 & -1.32545000 & -3.15839200\end{array}$

$\begin{array}{lll}-1.97911000 & -0.58725600 & -2.90873800\end{array}$

$\begin{array}{lll}-2.72340500 & -1.24768600 & -2.44031200\end{array}$

$\begin{array}{lll}-1.83878900 & 0.27767600 & -2.25874900\end{array}$

$\begin{array}{lll}-2.39900500 & -0.23738100 & -3.86584000\end{array}$

$\begin{array}{lll}0.27959800 & -0.46793600 & -4.02267500\end{array}$

$\begin{array}{lll}1.17589700 & -1.02771300 \quad-4.32712600\end{array}$

$\begin{array}{lll}-0.24083500 & -0.15021600 & -4.94209800\end{array}$

$\begin{array}{lll}0.61140700 & 0.43562500 & -3.48892100\end{array}$

$-1.01552400 \quad-2.62310000 \quad-3.89533300$

$\begin{array}{lll}-1.56863100 & -3.32348300 & -3.25323900\end{array}$

$\begin{array}{lll}-1.67913700 & -2.36799700 & -4.74014700\end{array}$

$-0.15027100 \quad-3.14381900 \quad-4.31537500$ 


\begin{tabular}{|c|c|c|c|}
\hline $\mathrm{C}$ & -0.92539600 & 3.44965700 & -1.89852600 \\
\hline $\mathrm{C}$ & -1.46385100 & 2.76959400 & -3.16492000 \\
\hline $\mathrm{H}$ & -0.93932700 & 1.83360900 & -3.37889700 \\
\hline $\mathrm{H}$ & -2.54106800 & 2.56013000 & -3.10717100 \\
\hline $\mathrm{H}$ & -1.29905000 & 3.44246400 & -4.02359100 \\
\hline $\mathrm{C}$ & -1.59196200 & 4.82693700 & -1.77522800 \\
\hline $\mathrm{H}$ & -1.38924400 & 5.39281100 & -2.70104200 \\
\hline $\mathrm{H}$ & -2.68304000 & 4.76666800 & -1.66843700 \\
\hline $\mathrm{H}$ & -1.19260100 & 5.41974700 & -0.94338800 \\
\hline $\mathrm{C}$ & 0.58970000 & 3.65719100 & -2.07574600 \\
\hline $\mathrm{H}$ & 0.77522400 & 4.25218900 & -2.98617100 \\
\hline $\mathrm{H}$ & 1.02744800 & 4.20116900 & -1.22842800 \\
\hline $\mathrm{H}$ & 1.12140500 & 2.69854700 & -2.17732500 \\
\hline $\mathrm{C}$ & -1.26522400 & 3.28511000 & 1.20773900 \\
\hline $\mathrm{C}$ & -2.52985300 & 4.14720600 & 1.32781300 \\
\hline $\mathrm{H}$ & -2.48135900 & 4.69433700 & 2.28568400 \\
\hline $\mathrm{H}$ & -2.63069900 & 4.89269300 & 0.53200300 \\
\hline $\mathrm{H}$ & -3.44361900 & 3.53916500 & 1.35067600 \\
\hline $\mathrm{C}$ & -0.03074300 & 4.18311200 & 1.37078300 \\
\hline $\mathrm{H}$ & -0.04450600 & 5.03455300 & 0.67666000 \\
\hline $\mathrm{H}$ & -0.01235700 & 4.59450800 & 2.39457900 \\
\hline $\mathrm{H}$ & 0.90206600 & 3.63298600 & 1.21542300 \\
\hline $\mathrm{C}$ & -1.31014200 & 2.25301300 & 2.34443600 \\
\hline $\mathrm{H}$ & -2.24770700 & 1.67724100 & 2.33141500 \\
\hline $\mathrm{H}$ & -0.48325400 & 1.54155200 & 2.28327500 \\
\hline $\mathrm{H}$ & -1.25192400 & 2.76948200 & 3.31756900 \\
\hline $\mathrm{Ni}$ & 0.39032500 & 0.51905500 & -0.13807300 \\
\hline $\mathrm{H}$ & 1.55741000 & 1.07911200 & 3.71566200 \\
\hline $\mathrm{H}$ & 1.62861800 & 1.93895300 & 1.77222000 \\
\hline $\mathrm{H}$ & 3.03101900 & 1.34513500 & -0.85500100 \\
\hline $\mathrm{C}$ & 3.48232700 & 3.09036400 & 0.31233000 \\
\hline $\mathrm{H}$ & 3.64180900 & 3.37499900 & 1.36455100 \\
\hline $\mathrm{H}$ & 4.39752600 & 3.31083400 & -0.25679800 \\
\hline $\mathrm{H}$ & 2.67216600 & 3.70973100 & -0.09343800 \\
\hline \multicolumn{4}{|c|}{ 24/Conformation-B } \\
\hline $\mathrm{C}$ & 2.89449100 & 3.39750700 & -0.89629300 \\
\hline $\mathrm{C}$ & 2.02467800 & 2.35162200 & -0.54466700 \\
\hline $\mathrm{C}$ & 2.13735900 & 1.17015400 & -1.29733000 \\
\hline $\mathrm{C}$ & 2.83798600 & 1.08530400 & -2.50395800 \\
\hline $\mathrm{C}$ & 3.67590600 & 2.15831500 & -2.82966100 \\
\hline $\mathrm{C}$ & 3.74729700 & 3.28085000 & -1.99715800 \\
\hline $\mathrm{C}$ & 2.37302300 & -1.33507300 & -2.57794400 \\
\hline $\mathrm{C}$ & 1.90233100 & -1.20778700 & -1.26482200 \\
\hline $\mathrm{C}$ & 1.89782100 & -2.26942900 & -0.34002500 \\
\hline
\end{tabular}


$2.20834700 \quad-3.53999200 \quad-0.85146700$

$2.57212600 \quad-3.72024900 \quad-2.18682100$ 
$\begin{array}{lll}1.14123500 & -3.43973600 & 2.36462900\end{array}$

$2.11247800 \quad-4.61388900 \quad 2.54624800$ 


\begin{tabular}{|c|c|c|}
\hline 4.25764400 & 0.28731600 & -2.44687700 \\
\hline 6.02163500 & -2.78787800 & -2.16620400 \\
\hline 5.88691200 & -1.39365900 & -0.06788600 \\
\hline 6.91709900 & -0.43509700 & -2.15694300 \\
\hline 94918800 & -2.79690300 & -3.26607300 \\
\hline 5.26427800 & -3.47748800 & -1.76809000 \\
\hline 7.01749600 & -3.16928000 & -1.88874600 \\
\hline 5.74162500 & -0.38576600 & 0.35292300 \\
\hline 6.86891200 & -1.76490500 & 0.26695500 \\
\hline 5.10770700 & -2.04989300 & 0.34706600 \\
\hline 7.91272100 & -0.80072500 & -1.85984000 \\
\hline 6.79485600 & 0.58782900 & -1.77208800 \\
\hline 6.88425700 & -0.38635800 & -3.25666100 \\
\hline 1.27784300 & -1.17733700 & 2.42943800 \\
\hline .36540700 & 0.46592800 & 0.02347000 \\
\hline 0.45088100 & 2.47596900 & 0.43250500 \\
\hline 1.74809200 & -1.85528500 & 1.46311600 \\
\hline 1.58558200 & -1.81234000 & 0.31443900 \\
\hline 2.04488500 & -0.35148700 & -2.35638100 \\
\hline 1.27573500 & -2.37133200 & -2.34357800 \\
\hline 1.64894400 & -2.61147700 & -3.35140600 \\
\hline 1.33276400 & -3.28106300 & -1.72459300 \\
\hline-0.22073300 & -2.08255300 & -2.42480700 \\
\hline 5.24454600 & -0.80773300 & -0.35934000 \\
\hline 4.55283200 & -1.55237600 & 0.63251000 \\
\hline 4.37066200 & -3.01105400 & 0.51489800 \\
\hline 4.27798000 & -3.66485400 & -0.73074500 \\
\hline 4.37697300 & -3.31713200 & 2.65421300 \\
\hline 4.65942900 & -1.20133400 & 1.66728900 \\
\hline 4.29463800 & -3.80105000 & 1.67799100 \\
\hline 4.09175600 & -5.04529000 & -0.80549700 \\
\hline 3.99778700 & -5.81336000 & 0.36149400 \\
\hline 4.10794500 & -5.18274900 & 1.60456700 \\
\hline 4.01079600 & -5.52623100 & -1.78395900 \\
\hline 3.84507100 & -6.89374800 & 0.30065200 \\
\hline 4.04616600 & -5.77021200 & 2.52448700 \\
\hline 5.43003800 & 0.56024200 & -0.20033800 \\
\hline 5.44884100 & 0.97756400 & 0.81221200 \\
\hline 3.40823600 & 0.01754000 & -0.06218300 \\
\hline 3.23367100 & 2.83388800 & -0.33902400 \\
\hline 2.89622800 & 1.69087700 & -0.83532500 \\
\hline 2.79712800 & 4.04705300 & -1.20260100 \\
\hline 3.56252800 & 5.29936000 & -0.76261400 \\
\hline
\end{tabular}




\begin{tabular}{|c|c|c|}
\hline-1.49145400 & 5.38731700 & 0.83664600 \\
\hline-1.89254400 & 0.71152200 & 0.28123800 \\
\hline-4.65304400 & -2.20851900 & -1.97913900 \\
\hline-6.16984300 & -2.40630100 & -2.08841500 \\
\hline-6.73372500 & -1.46882000 & -1.99218800 \\
\hline-6.55106400 & -3.11676100 & -1.34210600 \\
\hline-6.40408200 & -2.82339700 & -3.08405500 \\
\hline-4.17769600 & -1.29836400 & -3.12460200 \\
\hline-4.73318400 & -0.35111700 & -3.15801400 \\
\hline-4.33456600 & -1.81316600 & -4.08846100 \\
\hline-3.10723800 & -1.05873400 & -3.04556600 \\
\hline-3.97531100 & -3.58527500 & -2.14034000 \\
\hline-2.87862700 & -3.49826500 & -2.16337100 \\
\hline-4.29895900 & -4.05074900 & -3.08753500 \\
\hline-4.24225500 & -4.27346500 & -1.32420500 \\
\hline-5.08626800 & -1.89801000 & 1.13858900 \\
\hline-4.38161900 & -1.28127300 & 2.36086000 \\
\hline-3.37048700 & -1.68686900 & 2.50166900 \\
\hline-4.96386500 & -1.50524500 & 3.27142600 \\
\hline-4.30427600 & -0.18535600 & 2.27977400 \\
\hline-5.10403600 & -3.42667800 & 1.29725900 \\
\hline-5.66036900 & -3.92029500 & 0.48714400 \\
\hline-5.59533000 & -3.69610100 & 2.24831800 \\
\hline-4.08438500 & -3.84379700 & 1.31731000 \\
\hline-6.52007000 & -1.35956500 & 1.09517300 \\
\hline-7.09430600 & -1.73643900 & 0.24071900 \\
\hline-6.54267000 & -0.26106000 & 1.06974900 \\
\hline-7.04894400 & -1.67379700 & 2.01269100 \\
\hline 0.57983400 & -0.78753900 & -1.56628800 \\
\hline 1.76602600 & -1.43802300 & -2.30257900 \\
\hline 2.03935300 & -2.40674400 & -1.86098100 \\
\hline 2.65706200 & -0.79110200 & -2.29172200 \\
\hline 1.48470000 & -1.61524400 & -3.35451200 \\
\hline 0.30662600 & 0.59570600 & -2.17897700 \\
\hline 0.16331000 & 0.47457000 & -3.26641200 \\
\hline 1.14665100 & 1.27903400 & -2.00746800 \\
\hline-0.61009500 & 1.04268300 & -1.77218500 \\
\hline-0.65650000 & -1.66286500 & -1.74327000 \\
\hline-0.93323800 & -1.65973000 & -2.81084200 \\
\hline-1.51237400 & -1.29197000 & -1.17217500 \\
\hline-0.48023500 & -2.70789300 & -1.46075100 \\
\hline 0.67840000 & -1.94276900 & 1.40269200 \\
\hline 0.98566000 & -3.31466800 & 0.78520900 \\
\hline 0.98080500 & -4.07669900 & 1.58141100 \\
\hline
\end{tabular}




$\begin{array}{rrr}1.96571000 & -3.34854500 & 0.30129800 \\ 0.22578000 & -3.60791700 & 0.05039000 \\ 1.52413700 & -1.73565900 & 2.67168300 \\ 1.31109000 & -2.55123600 & 3.38271800 \\ 1.27757100 & -0.78718400 & 3.17184800 \\ 2.59963600 & -1.73845200 & 2.46071100 \\ -0.80057400 & -1.87764700 & 1.79232400 \\ -1.47464500 & -1.95124800 & 0.93073400 \\ -1.03909700 & -0.94953100 & 2.33016600 \\ -1.02341300 & -2.72034100 & 2.46994300 \\ 1.18700000 & -0.53886700 & 0.22461600 \\ -3.92630700 & -1.50692600 & -0.34109300\end{array}$

21

$\mathrm{H}$$$
\text { C }
$$

$\mathrm{H}$

H

H

C

$\mathrm{H}$

H

$\mathrm{H}$

$\mathrm{H}$

H

\begin{tabular}{|c|c|c|}
\hline 5.24454600 & -0.80773300 & 0.35934000 \\
\hline 4.55283200 & -1.55237600 & -0.63251000 \\
\hline 4.37066200 & -3.01105400 & -0.51489800 \\
\hline 4.27798000 & -3.66485400 & 0.73074500 \\
\hline 4.37697300 & -3.31713200 & -2.65421300 \\
\hline 4.65942900 & -1.20133400 & -1.66728900 \\
\hline 4.29463800 & -3.80105000 & -1.67799100 \\
\hline 4.09175600 & -5.04529000 & 0.80549700 \\
\hline 3.99778700 & -5.81336000 & -0.36149400 \\
\hline 4.10794500 & -5.18274900 & -1.60456700 \\
\hline 4.01079600 & -5.52623100 & 1.78395900 \\
\hline 3.84507100 & -6.89374800 & -0.30065200 \\
\hline 4.04616600 & -5.77021200 & -2.52448700 \\
\hline 5.43003800 & 0.56024200 & 0.20033800 \\
\hline 5.44884100 & 0.97756400 & -0.81221200 \\
\hline 3.40823600 & 0.01754000 & 0.06218300 \\
\hline 3.23367100 & 2.83388800 & 0.33902400 \\
\hline 2.89622800 & 1.69087700 & 0.83532500 \\
\hline 2.79712800 & 4.04705300 & 1.20260100 \\
\hline 3.56252800 & 5.29936000 & 0.76261400 \\
\hline 1.28871300 & 4.26086600 & 0.98082200 \\
\hline 3.06651900 & 3.76662900 & 2.68846800 \\
\hline 4.64659500 & 5.18307000 & 0.92279600 \\
\hline 3.40590600 & 5.50197600 & -0.30634800 \\
\hline 3.22652200 & 6.17776700 & 1.33841400 \\
\hline 0.71279000 & 3.36950900 & 1.26638900 \\
\hline 0.92838400 & 5.11081900 & 1.58423200 \\
\hline 1.07207300 & 4.47855100 & -0.07477300 \\
\hline 2.48951500 & 2.90077000 & 3.04211600 \\
\hline 4.13337600 & 3.55786400 & 2.86984600 \\
\hline 2.78564800 & 4.64046600 & 3.30031300 \\
\hline
\end{tabular}


$3.82891600 \quad 3.00198300 \quad-0.72703200$

$\begin{array}{lll}4.32853000 & -3.08357200 & 1.65285200\end{array}$

$5.43863500 \quad-1.25925200 \quad 1.33909400$

$5.99701600 \quad 1.42712800 \quad 1.28302300$

$\begin{array}{lll}7.09885300 & 1.46875100 & 1.20283300\end{array}$

$5.63390600 \quad 2.45994200 \quad 1.19741100$

$\begin{array}{lll}5.74777300 & 1.04664700 \quad 2.28550500\end{array}$

$\begin{array}{lll}-5.36017300 & 0.88836100 & 0.88543100\end{array}$

$\begin{array}{lll}-4.14553500 & 0.33119500 & 0.45609800\end{array}$

$\begin{array}{lll}-3.15452500 & 1.21699200 & -0.04027900\end{array}$

$\begin{array}{lll}-3.45237100 & 2.56883100 & -0.28182800\end{array}$

$\begin{array}{lll}-4.68459800 \quad 3.07127400 & 0.15755500\end{array}$

$\begin{array}{lll}-5.62214900 & 2.25284800 \quad 0.77949200\end{array}$

$\begin{array}{lll}-1.23276900 & 2.73280700 & -1.43333000\end{array}$

$\begin{array}{lll}-0.92509600 & 1.46683900 & -0.90220100\end{array}$

$\begin{array}{lll}0.38886600 & 0.93946300 & -1.01278700\end{array}$

$1.30029000 \quad 1.63562700 \quad-1.82407400$

$\begin{array}{lll}0.96492600 & 2.81924800 \quad-2.47321600\end{array}$

$\begin{array}{lll}-0.28416000 & 3.37829100 & -2.23776200\end{array}$

$\begin{array}{lll}-6.12849100 & 0.23413700 & 1.29165000\end{array}$

$\begin{array}{lll}-4.91603800 & 4.12552800 & -0.00385500\end{array}$

$\begin{array}{lll}-6.57014500 \quad 2.66397000 & 1.13358700\end{array}$

$2.31823700 \quad 1.25710200 \quad-1.91097100$

$\begin{array}{lll}1.70011500 & 3.33103900 & -3.09623600\end{array}$

$\begin{array}{lll}-0.52703600 & 4.35239300 & -2.66531200\end{array}$

$\begin{array}{lll}-2.52452300 & 3.46816900 & -1.08798800\end{array}$

$\begin{array}{lll}-3.26058700 & 3.88230400 & -2.38355100\end{array}$

$\begin{array}{lll}-2.63590900 & 4.54505200 & -3.00035300\end{array}$

$\begin{array}{lll}-3.52044100 & 2.99546300 & -2.98228700\end{array}$

$\begin{array}{lll}-4.18887900 & 4.42402600 & -2.14920500\end{array}$

$\begin{array}{lll}-2.16573700 \quad 4.72998800 & -0.26815900\end{array}$

$\begin{array}{lll}-3.06859000 & 5.30956400 & -0.02388900\end{array}$

$\begin{array}{lll}-1.66723200 & 4.45495500 & 0.67306800\end{array}$

$\begin{array}{lll}-1.49145400 & 5.38731700 & -0.83664600\end{array}$

$\begin{array}{lll}-1.89254400 & 0.71152200 & -0.28123800\end{array}$

$-4.65304400 \quad-2.20851900 \quad 1.97913900$

$\begin{array}{lll}-6.16984300 & -2.40630100 & 2.08841500\end{array}$

$\begin{array}{lll}-6.73372500 & -1.46882000 & 1.99218800\end{array}$

$-6.55106400 \quad-3.11676100 \quad 1.34210600$

$\begin{array}{lll}-6.40408200 & -2.82339700 \quad 3.08405500\end{array}$

$\begin{array}{lll}-4.17769600 & -1.29836400 & 3.12460200\end{array}$

$\begin{array}{lll}-4.73318400 & -0.35111700 \quad 3.15801400\end{array}$

$\begin{array}{lll}-4.33456600 & -1.81316600 \quad 4.08846100\end{array}$

$-3.10723800 \quad-1.05873400 \quad 3.04556600$ 
$-2.87862700$

$-4.29895900$

$-4.24225500$

$-5.08626800$

$-4.38161900$

$-3.37048700$

$-4.96386500$

$-4.30427600$

$-5.10403600$

$-5.66036900$

$-5.59533000$

$-4.08438500$

$-6.52007000$

$-7.09430600$

$-6.54267000$

$-7.04894400$

0.57983400

1.76602600

2.03935300

2.65706200

1.48470000

0.30662600

0.16331000

1.14665100

$-0.61009500$

$-0.65650000$

2.14034000

$-3.49826500$

2.16337100

$-4.05074900$

3.08753500

$-4.27346500 \quad 1.32420500$

$-1.89801000-1.13858900$

$-1.28127300$

$-2.36086000$

$-1.68686900 \quad-2.50166900$

$-1.50524500 \quad-3.27142600$

$-0.18535600 \quad-2.27977400$

$-3.42667800-1.29725900$

$-3.92029500 \quad-0.48714400$

$-3.69610100 \quad-2.24831800$

$-3.84379700 \quad-1.31731000$

$-1.35956500 \quad-1.09517300$

$-1.73643900 \quad-0.24071900$

$-0.26106000-1.06974900$

$-1.67379700 \quad-2.01269100$

$-0.78753900 \quad 1.56628800$

$-1.43802300 \quad 2.30257900$

$-2.40674400 \quad 1.86098100$

$-0.79110200 \quad 2.29172200$

$-1.61524400 \quad 3.35451200$

$0.59570600 \quad 2.17897700$

$0.47457000 \quad 3.26641200$

$1.27903400 \quad 2.00746800$

$1.04268300 \quad 1.77218500$

$-0.93323800$

$-1.66286500$

1.74327000

$-1.51237400$

$-1.65973000 \quad 2.81084200$

$\begin{array}{lll}-0.48023500 & -2.70789300 & 1.46075100\end{array}$

$0.67840000 \quad-1.94276900 \quad-1.40269200$

$0.98566000 \quad-3.31466800 \quad-0.78520900$

$0.98080500 \quad-4.07669900 \quad-1.58141100$

$\begin{array}{lll}1.96571000 & -3.34854500 & -0.30129800\end{array}$

$0.22578000 \quad-3.60791700 \quad-0.05039000$

$\begin{array}{lll}1.52413700 & -1.73565900 & -2.67168300\end{array}$

$\begin{array}{lll}1.31109000 & -2.55123600 & -3.38271800\end{array}$

$1.27757100 \quad-0.78718400 \quad-3.17184800$

$\begin{array}{lll}2.59963600 & -1.73845200 & -2.46071100\end{array}$

$\begin{array}{lll}-0.80057400 & -1.87764700 & -1.79232400\end{array}$

$\begin{array}{lll}-1.47464500 & -1.95124800 & -0.93073400\end{array}$

$\begin{array}{lll}-1.03909700 & -0.94953100 & -2.33016600\end{array}$

$\begin{array}{lll}-1.02341300 & -2.72034100 & -2.46994300\end{array}$

$1.18700000 \quad-0.53886700 \quad-0.22461600$ 


\begin{tabular}{|c|c|c|c|}
\hline $\mathrm{P}$ & -3.92630700 & -1.50692600 & 0.34109300 \\
\hline \multicolumn{4}{|c|}{ tBu-XantPhos } \\
\hline $\mathrm{P}$ & -2.08370800 & -1.13690600 & -0.01797200 \\
\hline $\mathrm{O}$ & -0.00087200 & 0.93118400 & 0.36708000 \\
\hline $\mathrm{P}$ & 2.10920600 & -1.14916800 & 0.13823500 \\
\hline $\mathrm{C}$ & -1.19774200 & 1.53356200 & 0.06340800 \\
\hline $\mathrm{C}$ & -2.32710400 & 0.70262100 & -0.08540800 \\
\hline $\mathrm{C}$ & -3.55526900 & 1.34077100 & -0.33025900 \\
\hline $\mathrm{C}$ & -3.63861100 & 2.72577500 & -0.47656100 \\
\hline $\mathrm{C}$ & -2.49051700 & 3.51384500 & -0.36256400 \\
\hline $\mathrm{C}$ & -1.25205000 & 2.93048900 & -0.07536000 \\
\hline $\mathrm{C}$ & 1.16205600 & 1.49704200 & -0.09344200 \\
\hline $\mathrm{C}$ & 2.26137100 & 0.64636700 & -0.32411800 \\
\hline $\mathrm{C}$ & 3.40630600 & 1.23409800 & -0.89175700 \\
\hline $\mathrm{C}$ & 3.45457900 & 2.59604900 & -1.18949300 \\
\hline $\mathrm{C}$ & 2.36620300 & 3.41916600 & -0.88648700 \\
\hline $\mathrm{C}$ & 1.20661000 & 2.88547200 & -0.31658200 \\
\hline $\mathrm{C}$ & 0.02849000 & 3.72674100 & 0.17908900 \\
\hline $\mathrm{H}$ & -4.46184100 & 0.74534800 & -0.42015000 \\
\hline $\mathrm{H}$ & -4.60436200 & 3.19517000 & -0.67872400 \\
\hline $\mathrm{H}$ & -2.56928700 & 4.59524700 & -0.47773000 \\
\hline $\mathrm{H}$ & 4.27214600 & 0.61534300 & -1.11727400 \\
\hline $\mathrm{H}$ & 4.35196200 & 3.02374500 & -1.64294700 \\
\hline $\mathrm{H}$ & 2.43239300 & 4.48886100 & -1.08722000 \\
\hline $\mathrm{C}$ & -0.01327000 & 5.11056200 & -0.47606800 \\
\hline $\mathrm{C}$ & 0.18566600 & 3.90441900 & 1.71208100 \\
\hline $\mathrm{H}$ & -0.12958600 & 5.04087400 & -1.56854000 \\
\hline $\mathrm{H}$ & -0.84630400 & 5.70483800 & -0.07551100 \\
\hline $\mathrm{H}$ & 0.90667600 & 5.67165100 & -0.25923700 \\
\hline $\mathrm{H}$ & -0.65994700 & 4.48312800 & 2.11629200 \\
\hline $\mathrm{H}$ & 0.21301900 & 2.93238000 & 2.22732500 \\
\hline $\mathrm{H}$ & 1.12067700 & 4.43999800 & 1.94047900 \\
\hline $\mathrm{C}$ & -2.61986800 & -1.49901700 & 1.78562200 \\
\hline $\mathrm{C}$ & -3.34748100 & -1.87595900 & -1.25887800 \\
\hline $\mathrm{C}$ & 2.52836800 & -2.06529700 & -1.49197200 \\
\hline $\mathrm{C}$ & 3.50238700 & -1.35880600 & 1.43464900 \\
\hline $\mathrm{C}$ & -2.82198300 & -3.30034300 & -1.53768500 \\
\hline $\mathrm{H}$ & -2.83303600 & -3.93357500 & -0.63887500 \\
\hline $\mathrm{H}$ & -3.45990900 & -3.78746000 & -2.29549900 \\
\hline $\mathrm{H}$ & -1.79126000 & -3.28267000 & -1.92545400 \\
\hline $\mathrm{C}$ & -4.82346500 & -1.97670600 & -0.85406100 \\
\hline $\mathrm{H}$ & -5.27594300 & -1.00368700 & -0.62013400 \\
\hline $\mathrm{H}$ & -5.39853100 & -2.40929900 & -1.69215400 \\
\hline $\mathrm{H}$ & -4.96701600 & -2.63749000 & 0.01234500 \\
\hline
\end{tabular}




\begin{tabular}{|c|c|c|c|}
\hline $\mathrm{C}$ & -3.21574800 & -1.06908600 & -2.56270800 \\
\hline $\mathrm{H}$ & -3.69418500 & -1.62569100 & -3.38703300 \\
\hline $\mathrm{H}$ & -3.69907800 & -0.08461500 & -2.49944600 \\
\hline $\mathrm{H}$ & -2.16003000 & -0.90992800 & -2.83864700 \\
\hline $\mathrm{C}$ & -1.48273100 & -0.93682400 & 2.65917100 \\
\hline $\mathrm{H}$ & -1.39923100 & 0.15685400 & 2.57064800 \\
\hline $\mathrm{H}$ & -1.68625600 & -1.17083000 & 3.71871600 \\
\hline $\mathrm{H}$ & -0.50738700 & -1.37123400 & 2.39364300 \\
\hline $\mathrm{C}$ & -3.93877600 & -0.85774400 & 2.22864800 \\
\hline $\mathrm{H}$ & -3.90640400 & 0.23824500 & 2.14305800 \\
\hline $\mathrm{H}$ & -4.79857600 & -1.22113200 & 1.65266400 \\
\hline $\mathrm{H}$ & -4.12449200 & -1.09919900 & 3.29070300 \\
\hline $\mathrm{C}$ & -2.67837200 & -3.02145300 & 1.97609700 \\
\hline $\mathrm{H}$ & -3.52549200 & -3.47394600 & 1.44104000 \\
\hline $\mathrm{H}$ & -1.75227500 & -3.50922600 & 1.63143100 \\
\hline $\mathrm{H}$ & -2.80127500 & -3.25555100 & 3.04779700 \\
\hline $\mathrm{C}$ & 3.62555100 & -2.86262800 & 1.72636000 \\
\hline $\mathrm{H}$ & 4.24643600 & -3.01576600 & 2.62603800 \\
\hline $\mathrm{H}$ & 2.64318800 & -3.32498000 & 1.91468400 \\
\hline $\mathrm{H}$ & 4.10589300 & -3.40235000 & 0.89719200 \\
\hline $\mathrm{C}$ & 4.88859100 & -0.78071400 & 1.12707000 \\
\hline $\mathrm{H}$ & 4.86740100 & 0.31534300 & 1.05468200 \\
\hline $\mathrm{H}$ & 5.57634200 & -1.03723400 & 1.95277900 \\
\hline $\mathrm{H}$ & 5.32263600 & -1.18049600 & 0.20161200 \\
\hline $\mathrm{C}$ & 2.94783800 & -0.65151400 & 2.68575200 \\
\hline $\mathrm{H}$ & 3.66616900 & -0.74772700 & 3.51856900 \\
\hline $\mathrm{H}$ & 2.78682700 & 0.42422800 & 2.50781300 \\
\hline $\mathrm{H}$ & 1.99106500 & -1.08832900 & 3.01212300 \\
\hline $\mathrm{C}$ & 3.99765900 & -2.14219600 & -1.91645900 \\
\hline $\mathrm{H}$ & 4.44651000 & -1.14965400 & -2.05814300 \\
\hline $\mathrm{H}$ & 4.60672300 & -2.69776800 & -1.18929200 \\
\hline $\mathrm{H}$ & 4.07160000 & -2.67557100 & -2.88128700 \\
\hline $\mathrm{C}$ & 1.70631500 & -1.36676000 & -2.58952600 \\
\hline $\mathrm{H}$ & 0.65695800 & -1.22305600 & -2.28310500 \\
\hline $\mathrm{H}$ & 2.12044800 & -0.38252400 & -2.85183900 \\
\hline $\mathrm{H}$ & 1.70662200 & -1.98797500 & -3.50190000 \\
\hline $\mathrm{C}$ & 1.97753500 & -3.49403000 & -1.31910900 \\
\hline $\mathrm{H}$ & 0.90794500 & -3.48154000 & -1.05652900 \\
\hline $\mathrm{H}$ & 2.08999300 & -4.05276500 & -2.26463100 \\
\hline $\mathrm{H}$ & 2.51137300 & -4.05376500 & -0.53761400 \\
\hline \multicolumn{4}{|c|}{ DMF } \\
\hline $\mathrm{N}$ & -0.34178000 & -0.01977700 & 0.00009400 \\
\hline $\mathrm{C}$ & -1.58129200 & -0.76659500 & -0.00004100 \\
\hline $\mathrm{H}$ & -2.18536600 & -0.52853000 & -0.89297400 \\
\hline
\end{tabular}




$\begin{array}{rrr}-1.36770200 & -1.84504700 & 0.00004800 \\ -2.18542700 & -0.52840600 & 0.89280200 \\ -0.43190400 & 1.42579700 & 0.00000100 \\ 0.58296900 & 1.84229900 & -0.00051900 \\ -0.97262700 & 1.78379800 & -0.89325300 \\ -0.97201900 & 1.78387400 & 0.89354000 \\ 0.86308400 & -0.64099400 & 0.00007300 \\ 0.75511000 & -1.75113800 & -0.00019000 \\ 1.95477400 & -0.09095700 & -0.00003900\end{array}$

S56

$\mathrm{H}$

C

$\mathrm{H}$

$\mathrm{H}$

H

C

H

O

.

C

C

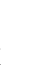

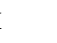

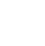

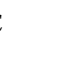

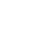

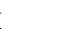

.

.

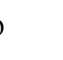

.

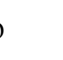

.

C

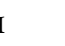

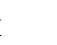

.

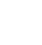

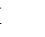

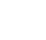

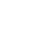

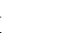

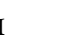

i

C

\begin{tabular}{|c|c|c|}
\hline-0.87902400 & 0.59694000 & -0.17902900 \\
\hline-0.52924400 & -0.72823900 & 0.31003000 \\
\hline 0.09515000 & -1.77287100 & -0.50311800 \\
\hline 0.51289600 & -1.57885400 & -1.84378900 \\
\hline 0.10903800 & -3.20737600 & 1.11797800 \\
\hline-1.03087600 & -1.09477400 & 1.21573400 \\
\hline 0.41389000 & -3.02276700 & 0.08360100 \\
\hline 1.23041200 & -2.55791900 & -2.53312100 \\
\hline 1.55325100 & -3.77723800 & -1.92301400 \\
\hline 1.13011000 & -3.99997400 & -0.60601600 \\
\hline 1.54333700 & -2.36581600 & -3.56391200 \\
\hline 2.12015200 & -4.53897100 & -2.46391200 \\
\hline 1.36547000 & -4.94628600 & -0.10981200 \\
\hline-1.94247700 & 1.37418100 & 0.53936600 \\
\hline-3.27582100 & 0.97090000 & 0.04153800 \\
\hline-3.95963000 & 0.03702100 & 0.70212200 \\
\hline-5.19291500 & -0.42400000 & -0.08648000 \\
\hline-3.63620800 & -0.41105700 & 1.78385700 \\
\hline-6.03582800 & 0.79502700 & -0.49819800 \\
\hline-4.68765600 & -1.16364300 & -1.34115600 \\
\hline-6.02481500 & -1.37204500 & 0.78197000 \\
\hline-6.38303700 & 1.35402000 & 0.38621400 \\
\hline-5.45998700 & 1.48297500 & -1.13318600 \\
\hline-6.92420800 & 0.46521100 & -1.06057400 \\
\hline-4.04759700 & -2.01809600 & -1.06716000 \\
\hline-5.54192100 & -1.54889700 & -1.92104100 \\
\hline-4.10243100 & -0.49484100 & -1.98916100 \\
\hline-6.90515200 & -1.72161300 & 0.21979300 \\
\hline-5.43842600 & -2.25091100 & 1.08814600 \\
\hline-6.37793700 & -0.87003700 & 1.69596900 \\
\hline 0.28651100 & -0.63527500 & -2.34461000 \\
\hline 0.87051800 & 0.55626800 & 0.56160200 \\
\hline-0.92943600 & 0.76108600 & -1.26973700 \\
\hline .86038900 & 2.87502500 & 0.33334100 \\
\hline
\end{tabular}




\begin{tabular}{|c|c|c|}
\hline-1.88168400 & 3.12905500 & -0.73932200 \\
\hline-0.91631400 & 3.24847700 & 0.75573700 \\
\hline-2.69647900 & 3.39389600 & 0.82860100 \\
\hline-1.92915100 & 1.13780300 & 1.61124900 \\
\hline 2.39399700 & -0.48555100 & 1.34402400 \\
\hline 3.15454300 & -1.02546300 & 0.52393800 \\
\hline 3.86280400 & -2.13360700 & 0.76330800 \\
\hline 3.30219400 & -0.61350800 & -0.49193800 \\
\hline 4.74842000 & -2.69357400 & -0.23980600 \\
\hline 3.75456500 & -2.83951700 & 2.02671500 \\
\hline 5.78766900 & -2.71439600 & 0.12863200 \\
\hline 4.70637400 & -2.08724300 & -1.15449500 \\
\hline 4.44393000 & -3.72400400 & -0.48427200 \\
\hline 2.99193000 & -2.35392000 & 2.64604300 \\
\hline 4.72028300 & -2.82832300 & 2.55919100 \\
\hline 3.46831600 & -3.88830900 & 1.84710200 \\
\hline 1.89736900 & 2.21299800 & 0.43690500 \\
\hline 1.72973000 & 3.08531400 & -0.43454800 \\
\hline 2.50648100 & 4.16220000 & -0.57293800 \\
\hline 0.90509700 & 3.02265500 & -1.16660900 \\
\hline 2.23648900 & 5.15913300 & -1.59312000 \\
\hline 3.63987900 & 4.39784600 & 0.30450300 \\
\hline 2.04410200 & 6.14048000 & -1.12879100 \\
\hline 1.35572000 & 4.86470600 & -2.17961200 \\
\hline 3.10062600 & 5.25930400 & -2.27018700 \\
\hline 3.75465800 & 3.54556300 & 0.98376500 \\
\hline 3.48258700 & 5.31692500 & 0.89308900 \\
\hline .55831100 & 4.52015800 & -0.2920960 \\
\hline
\end{tabular}

TS-S57

C 
$3.92390000 \quad-0.52151600 \quad-0.42731000$

$4.00217300 \quad-0.58418600 \quad-1.96389100$

$\begin{array}{lll}4.84197600 & -1.58199800 & 0.18765400\end{array}$

$\begin{array}{lll}4.33373500 & 0.87856200 & 0.06220800\end{array}$

$\begin{array}{lll}3.35450700 & 0.17715500 & -2.42250600\end{array}$

$\begin{array}{lll}3.68850500 & -1.57302700 & -2.33670600\end{array}$

$5.03783400 \quad-0.40830300 \quad-2.29676100$

$\begin{array}{lll}4.79299200 & -1.56737100 & 1.28688300\end{array}$

$5.88483500 \quad-1.39020200 \quad-0.11215600$

$\begin{array}{lll}4.56879100 & -2.59338900 & -0.14750300\end{array}$

$\begin{array}{lll}5.38325800 & 1.07913800 & -0.20638000\end{array}$

$\begin{array}{lll}4.24208800 & 0.95858200 & 1.15784100\end{array}$

$3.70634500 \quad 1.65924900 \quad-0.39196200$

$\begin{array}{lll}2.20146400 & -1.77920900 & 0.66997000\end{array}$

$\begin{array}{lll}-0.67734800 & -2.47289300 & -0.76976700\end{array}$

$\begin{array}{lll}-2.25799800 & -1.46357800 & -1.86548200\end{array}$

$\begin{array}{lll}1.00936400 & -3.93704500 & 0.73088100\end{array}$

$0.20141400 \quad-4.61273500 \quad 1.06222300$

$1.92328400 \quad-4.19238700 \quad 1.28431800$

$1.17478300 \quad-4.11159500 \quad-0.34342000$

$\begin{array}{lll}-0.22034800 & -0.08132400 & 0.17998900\end{array}$

$\begin{array}{lll}-0.58604300 & 1.82243500 & 0.41515000\end{array}$

$0.21532400 \quad 2.71211300 \quad 0.06843300$

$\begin{array}{lll}-0.02646000 & 4.01786400 & 0.18360400\end{array}$

$\begin{array}{lll}1.19883300 & 2.45750200 \quad-0.36432100\end{array}$

$0.95541500 \quad 5.00246900 \quad-0.23453900$

$\begin{array}{lll}-1.27631600 & 4.51435200 & 0.73350700\end{array}$

$\begin{array}{lll}0.53861300 & 5.64814800 & -1.02483500\end{array}$

$1.84966300 \quad 4.49592600 \quad-0.62173600$

$\begin{array}{lll}1.24546900 & 5.63937300 & 0.61720300\end{array}$

$\begin{array}{lll}-1.92515700 \quad 3.66626500 & 0.97981900\end{array}$

$\begin{array}{lll}-1.78074900 & 5.16239100 & -0.00146200\end{array}$

$\begin{array}{lll}-1.08229400 & 5.10536100 & 1.64363600\end{array}$

$\begin{array}{lll}-0.53295400 & -2.67421200 & 0.27361100\end{array}$

$\begin{array}{lll}-1.29013200 & -1.63672500 & 0.87112100\end{array}$

$\begin{array}{lll}-2.58917300 & -1.13829600 & 0.39744600\end{array}$

$\begin{array}{lll}-3.10861300 & -1.44202300 & -0.87683900\end{array}$

$\begin{array}{lll}-2.92120700 \quad 0.00937000 & 2.19841000\end{array}$

$\begin{array}{lll}-1.06004000 & -1.38191000 & 1.91438700\end{array}$

$\begin{array}{lll}-3.31791400 & -0.25130100 & 1.21339700\end{array}$

$-4.31692800 \quad-0.89274200 \quad-1.30647900$ 


\begin{tabular}{|c|c|c|c|}
\hline $\mathrm{C}$ & -5.03321500 & -0.01735100 & -0.48083900 \\
\hline $\mathrm{C}$ & -4.52469000 & 0.30292800 & 0.78192000 \\
\hline $\mathrm{H}$ & -4.70056800 & -1.14318000 & -2.29911100 \\
\hline $\mathrm{H}$ & -5.97637200 & 0.41584200 & -0.82287100 \\
\hline $\mathrm{H}$ & -5.06903800 & 0.99094700 & 1.43409800 \\
\hline $\mathrm{C}$ & 0.82637200 & -2.76728100 & 0.65076000 \\
\hline $\mathrm{H}$ & 1.08070300 & -2.52691300 & 1.69315100 \\
\hline $\mathrm{Ni}$ & 0.37934300 & -0.94276900 & -0.01784500 \\
\hline $\mathrm{C}$ & 2.64808700 & 0.31650600 & 0.19294400 \\
\hline $\mathrm{O}$ & 2.14464400 & -0.49510500 & -0.66598000 \\
\hline $\mathrm{C}$ & 4.02084600 & 0.91302200 & -0.18652300 \\
\hline $\mathrm{C}$ & 4.58017800 & 1.74473100 & 0.97114700 \\
\hline $\mathrm{C}$ & 3.81171200 & 1.80771700 & -1.42204800 \\
\hline $\mathrm{C}$ & 4.98818900 & -0.23093000 & -0.53063600 \\
\hline $\mathrm{H}$ & 4.72725700 & 1.12735300 & 1.87089800 \\
\hline $\mathrm{H}$ & 3.89471000 & 2.56179100 & 1.24057000 \\
\hline $\mathrm{H}$ & 5.55272800 & 2.18420500 & 0.69363600 \\
\hline $\mathrm{H}$ & 3.41718700 & 1.22406400 & -2.26699300 \\
\hline $\mathrm{H}$ & 4.76345700 & 2.27036700 & -1.73280900 \\
\hline $\mathrm{H}$ & 3.09566600 & 2.61839200 & -1.20654200 \\
\hline $\mathrm{H}$ & 4.60087600 & -0.83460700 & -1.36379100 \\
\hline $\mathrm{H}$ & 5.13378900 & -0.90023800 & 0.33381200 \\
\hline $\mathrm{H}$ & 5.97523300 & 0.16866900 & -0.81799700 \\
\hline $\mathrm{O}$ & 2.08212600 & 0.63329700 & 1.25226200 \\
\hline $\mathrm{H}$ & -2.55359800 & -2.10216100 & -1.54665700 \\
\hline $\mathrm{H}$ & -0.89210700 & -3.19649600 & -0.62081300 \\
\hline $\mathrm{C}$ & 1.81658100 & -3.63289300 & -0.07176900 \\
\hline $\mathrm{H}$ & 1.93568700 & -4.61257000 & 0.42575800 \\
\hline $\mathrm{H}$ & 2.80684800 & -3.15153500 & -0.09178400 \\
\hline $\mathrm{H}$ & 1.50972400 & -3.81611600 & -1.11356300 \\
\hline $\mathrm{O}$ & -0.42000900 & 0.75003600 & -0.62756300 \\
\hline $\mathrm{C}$ & -0.67674900 & 1.65270900 & 0.19401100 \\
\hline $\mathrm{N}$ & -1.59329600 & 2.59660900 & -0.00611400 \\
\hline $\mathrm{H}$ & -0.12809100 & 1.72077000 & 1.14875100 \\
\hline $\mathrm{C}$ & -1.82440100 & 3.63949600 & 0.97713300 \\
\hline $\mathrm{C}$ & -2.40965500 & 2.61928300 & -1.20778500 \\
\hline $\mathrm{H}$ & -1.65534000 & 4.63254700 & 0.52999600 \\
\hline $\mathrm{H}$ & -1.13955200 & 3.50892300 & 1.82557100 \\
\hline $\mathrm{H}$ & -2.86336200 & 3.59288700 & 1.34218200 \\
\hline $\mathrm{H}$ & -2.21117400 & 1.71706000 & -1.79646200 \\
\hline $\mathrm{H}$ & -2.17664600 & 3.51214400 & -1.81119300 \\
\hline $\mathrm{H}$ & -3.47462800 & 2.65001300 & -0.93134200 \\
\hline
\end{tabular}

TS-S59

C

$\begin{array}{lll}-0.78830900 & 0.69179700 & -0.59829200\end{array}$ 
$\begin{array}{lll}-1.22806600 & -0.85309300 & 0.87249900\end{array}$

$\begin{array}{lll}-0.11882100 & -3.05222900 & -0.09760000\end{array}$

$\begin{array}{lll}1.26075300 & -2.78620900 & -2.50028300\end{array}$

$1.19305800 \quad-4.04104400 \quad-1.88242300$

$\begin{array}{lll}0.49565300 & -4.16420900 & -0.67496400\end{array}$

$\begin{array}{lll}1.80292600 & -2.67149000 & -3.44322800\end{array}$

$\begin{array}{lll}1.67872500 & -4.90920600 & -2.33469400\end{array}$

$0.43246700 \quad-5.13576300 \quad-0.17680300$

$\begin{array}{lll}-1.41936100 & 1.72462000 & 0.13201500\end{array}$

$\begin{array}{lll}-3.37925600 & 1.46861300 & -0.21140700\end{array}$

$\begin{array}{lll}-3.90600100 & 0.65004800 & 0.62371600\end{array}$

$\begin{array}{lll}-5.17984700 & -0.07197200 & 0.09217700\end{array}$

$\begin{array}{lll}-3.46438200 & 0.37072300 & 1.74733300\end{array}$

$\begin{array}{lll}-6.11991300 & 0.94058500 & -0.57651100\end{array}$

$\begin{array}{lll}-4.69710100 & -1.09859500 & -0.94942700\end{array}$

$\begin{array}{lll}-5.90484100 & -0.78997300 & 1.23318000\end{array}$

$\begin{array}{lll}-6.47994800 & 1.68896800 & 0.14975000\end{array}$

$\begin{array}{lll}-5.60234800 & 1.47853700 & -1.38346500\end{array}$

$\begin{array}{lll}-7.00302000 & 0.43390000 & -1.00194600\end{array}$

$\begin{array}{lll}-4.01056300 & -1.83211300 & -0.49451400\end{array}$

$-5.54844600 \quad-1.65329000 \quad-1.37941000$

$\begin{array}{lll}-4.15907300 & -0.59918600 & -1.76945400\end{array}$

$\begin{array}{lll}-6.78812800 & -1.33070800 \quad 0.85279600\end{array}$

$-5.24043800 \quad-1.51177700 \quad 1.72950700$

$\begin{array}{lll}-6.24657600 & -0.07597400 & 1.99966700\end{array}$

$\begin{array}{lll}0.73787300 & -0.70174300 & -2.41108600\end{array}$

$\begin{array}{lll}0.85288500 & 0.57248900 & 0.47002200\end{array}$

$\begin{array}{lll}-0.65283000 & 0.84106100 & -1.67705800\end{array}$

$\begin{array}{lll}-1.34255600 & 3.15469800 & -0.30354700\end{array}$

$\begin{array}{lll}-1.32886300 & 3.24466000 & -1.40091600\end{array}$

$\begin{array}{lll}-0.42041700 & 3.61751000 \quad 0.08600300\end{array}$

$\begin{array}{lll}-2.19419100 \quad 3.72688700 & 0.08833500\end{array}$

$\begin{array}{lll}-1.59334500 & 1.54296300 & 1.19279400\end{array}$

$2.09480000 \quad-0.67458300 \quad 1.38035200$

$2.86482000 \quad-1.33537700 \quad 0.65783600$

$3.32309200 \quad-2.54946200 \quad 0.96354700$

$3.22713700 \quad-0.94702500 \quad-0.31109600$

$\begin{array}{lll}4.26304500 & -3.24189800 & 0.10057300\end{array}$

$\begin{array}{lll}2.91111800 & -3.22808900 & 2.18005500\end{array}$

$5.20871600 \quad-3.43098800 \quad 0.63504600$ 


\begin{tabular}{|c|c|c|c|}
\hline $\mathrm{H}$ & 4.47242800 & -2.63433300 & -0.78973500 \\
\hline $\mathrm{H}$ & 3.84204600 & -4.20834100 & -0.21885100 \\
\hline $\mathrm{H}$ & 2.11280400 & -2.65303100 & 2.66232800 \\
\hline $\mathrm{H}$ & 3.76275900 & -3.32501500 & 2.87400000 \\
\hline $\mathrm{H}$ & 2.54275200 & -4.23643000 & 1.93546900 \\
\hline $\mathrm{O}$ & 2.06580700 & 2.11855800 & 0.54973500 \\
\hline $\mathrm{C}$ & 2.23091000 & 2.90550100 & -0.40276600 \\
\hline $\mathrm{N}$ & 3.11283900 & 3.90402200 & -0.40185000 \\
\hline $\mathrm{H}$ & 1.63993000 & 2.82058900 & -1.33214800 \\
\hline $\mathrm{C}$ & 3.24231800 & 4.79616700 & -1.54094000 \\
\hline $\mathrm{C}$ & 3.97267800 & 4.15856900 & 0.74175000 \\
\hline $\mathrm{H}$ & 3.02193200 & 5.83348700 & -1.24031500 \\
\hline $\mathrm{H}$ & 2.54182400 & 4.49789900 & -2.33240100 \\
\hline $\mathrm{H}$ & 4.26946700 & 4.76010700 & -1.93873100 \\
\hline $\mathrm{H}$ & 3.79280000 & 3.39683000 & 1.50859900 \\
\hline $\mathrm{H}$ & 3.76198100 & 5.15684100 & 1.15904500 \\
\hline $\mathrm{H}$ & 5.02945100 & 4.12659100 & 0.43130300 \\
\hline \multicolumn{4}{|l|}{$S 60$} \\
\hline $\mathrm{C}$ & -0.53295400 & -2.67421200 & -0.27361100 \\
\hline $\mathrm{C}$ & -1.29013200 & -1.63672500 & -0.87112100 \\
\hline $\mathrm{C}$ & -2.58917300 & -1.13829600 & -0.39744600 \\
\hline C & -3.10861300 & -1.44202300 & 0.87683900 \\
\hline $\mathrm{H}$ & -2.92120700 & 0.00937000 & -2.19841000 \\
\hline $\mathrm{H}$ & -1.06004000 & -1.38191000 & -1.91438700 \\
\hline $\mathrm{C}$ & -3.31791400 & -0.25130100 & -1.21339700 \\
\hline $\mathrm{C}$ & -4.31692800 & -0.89274200 & 1.30647900 \\
\hline $\mathrm{C}$ & -5.03321500 & -0.01735100 & 0.48083900 \\
\hline $\mathrm{C}$ & -4.52469000 & 0.30292800 & -0.78192000 \\
\hline $\mathrm{H}$ & -4.70056800 & -1.14318000 & 2.29911100 \\
\hline $\mathrm{H}$ & -5.97637200 & 0.41584200 & 0.82287100 \\
\hline $\mathrm{H}$ & -5.06903800 & 0.99094700 & -1.43409800 \\
\hline $\mathrm{C}$ & 0.82637200 & -2.76728100 & -0.65076000 \\
\hline $\mathrm{H}$ & 1.08070300 & -2.52691300 & -1.69315100 \\
\hline $\mathrm{Ni}$ & 0.37934300 & -0.94276900 & 0.01784500 \\
\hline $\mathrm{C}$ & 2.64808700 & 0.31650600 & -0.19294400 \\
\hline $\mathrm{O}$ & 2.14464400 & -0.49510500 & 0.66598000 \\
\hline $\mathrm{C}$ & 4.02084600 & 0.91302200 & 0.18652300 \\
\hline $\mathrm{C}$ & 4.58017800 & 1.74473100 & -0.97114700 \\
\hline $\mathrm{C}$ & 3.81171200 & 1.80771700 & 1.42204800 \\
\hline $\mathrm{C}$ & 4.98818900 & -0.23093000 & 0.53063600 \\
\hline $\mathrm{H}$ & 4.72725700 & 1.12735300 & -1.87089800 \\
\hline $\mathrm{H}$ & 3.89471000 & 2.56179100 & -1.24057000 \\
\hline $\mathrm{H}$ & 5.55272800 & 2.18420500 & -0.69363600 \\
\hline $\mathrm{H}$ & 3.41718700 & 1.22406400 & 2.26699300 \\
\hline
\end{tabular}




\begin{tabular}{|c|c|c|c|}
\hline & 4.76345700 & 2.27036700 & 1.73280900 \\
\hline & 3.09566600 & 2.61839200 & 1.20654200 \\
\hline & 4.60087600 & -0.83460700 & 1.36379100 \\
\hline & 5.13378900 & -0.90023800 & -0.33381200 \\
\hline & 5.97523300 & 0.16866900 & 0.81799700 \\
\hline & 2.08212600 & 0.63329700 & -1.25226200 \\
\hline & -2.55359800 & -2.10216100 & 1.54665700 \\
\hline & -0.89210700 & -3.19649600 & 0.62081300 \\
\hline & 1.81658100 & -3.63289300 & 0.07176900 \\
\hline & 1.93568700 & -4.61257000 & -0.42575800 \\
\hline & 2.80684800 & -3.15153500 & 0.09178400 \\
\hline & 1.50972400 & -3.81611600 & 1.11356300 \\
\hline & -0.42000900 & 0.75003600 & 0.62756300 \\
\hline & -0.67674900 & 1.65270900 & -0.19401100 \\
\hline & -1.59329600 & 2.59660900 & 0.00611400 \\
\hline & -0.12809100 & 1.72077000 & -1.14875100 \\
\hline & -1.82440100 & 3.63949600 & -0.97713300 \\
\hline & -2.40965500 & 2.61928300 & 1.20778500 \\
\hline & -1.65534000 & 4.63254700 & -0.52999600 \\
\hline & -1.13955200 & 3.50892300 & -1.82557100 \\
\hline & -2.86336200 & 3.59288700 & -1.34218200 \\
\hline & -2.21117400 & 1.71706000 & 1.79646200 \\
\hline & -2.17664600 & 3.51214400 & 1.81119300 \\
\hline & -3.47462800 & 2.65001300 & 0.93134200 \\
\hline
\end{tabular}

\section{Cartesian coordinates of the structures (optimization in EtOAc)}

\section{TS5/TS22}

\begin{tabular}{|c|c|c|c|}
\hline C & -1.14946000 & 0.66072300 & 2.17519200 \\
\hline $\mathrm{C}$ & -0.20436200 & 0.37747800 & 1.16773700 \\
\hline $\mathrm{C}$ & 1.01798200 & 1.08446300 & 1.22420300 \\
\hline $\mathrm{C}$ & 1.17068000 & 2.19353700 & 2.08425000 \\
\hline C & 0.21239500 & 2.41392400 & 3.07707600 \\
\hline $\mathrm{C}$ & -0.92505200 & 1.61215500 & 3.16254600 \\
\hline $\mathrm{C}$ & 3.49468200 & 2.45409200 & 1.27916800 \\
\hline $\mathrm{C}$ & 3.32992800 & 1.22761200 & 0.60619600 \\
\hline $\mathrm{C}$ & 4.44307700 & 0.51168300 & 0.10302500 \\
\hline $\mathrm{C}$ & 5.70742000 & 1.11412300 & 0.21960900 \\
\hline $\mathrm{C}$ & 5.88669300 & 2.33642400 & 0.85961300 \\
\hline $\mathrm{C}$ & 4.78236200 & 2.98577000 & 1.40597600 \\
\hline $\mathrm{H}$ & -2.10402500 & 0.13023700 & 2.14416200 \\
\hline $\mathrm{H}$ & 0.33424700 & 3.24329700 & 3.77313400 \\
\hline $\mathrm{H}$ & -1.66835100 & 1.78312100 & 3.94422600 \\
\hline $\mathrm{H}$ & 6.57443300 & 0.60469300 & -0.19264800 \\
\hline $\mathrm{H}$ & 6.88304900 & 2.77688400 & 0.94105100 \\
\hline $\mathrm{H}$ & 4.92341400 & 3.93606700 & 1.91997300 \\
\hline
\end{tabular}


$2.26532800 \quad 3.20903500 \quad 1.76764600$

$\begin{array}{lll}2.58326400 & 4.11465800 & 2.96474800\end{array}$

$3.33405600 \quad 4.86960300$

2.69545800

$2.95985000 \quad 3.53890400 \quad 3.82393100$

$\begin{array}{lll}1.68947700 & 4.66991400 \quad 3.28016000\end{array}$

$1.74794700 \quad 4.09962300 \quad 0.60564300$

$\begin{array}{lll}0.83389600 & 4.63253200 & 0.91042700\end{array}$

$\begin{array}{lll}1.51125600 & 3.49991300 & -0.28545400\end{array}$

$2.51346700 \quad 4.84046700 \quad 0.32516500$

$\begin{array}{lll}2.06213900 & 0.71028900 & 0.40590500\end{array}$

$\begin{array}{lll}-0.43028900 & -2.47675100 & 0.37239500\end{array}$

$1.06919100 \quad-2.61230100 \quad 0.62432500$

$\begin{array}{lll}1.40548400 & -1.89959900 & 1.39047100\end{array}$

$\begin{array}{lll}1.67809900 & -2.43862400 & -0.26883300\end{array}$

$\begin{array}{lll}1.28829200 & -3.62964000 & 0.99361100\end{array}$

$\begin{array}{lll}-1.18254300 & -2.76317300 & 1.68377300\end{array}$

$\begin{array}{lll}-1.01782700 & -3.81392000 & 1.97580900\end{array}$

$\begin{array}{lll}-2.26194300 & -2.60532200 & 1.57432900\end{array}$

$\begin{array}{lll}-0.82020100 & -2.12783900 & 2.50577400\end{array}$

$-0.90518400 \quad-3.46318000 \quad-0.70252800$

$\begin{array}{lll}-1.97339000 & -3.33272700 & -0.91994000\end{array}$

$\begin{array}{lll}-0.76207600 & -4.49525200 & -0.34040000\end{array}$

$\begin{array}{lll}-0.34176500 & -3.35924500 & -1.64003800\end{array}$

$\begin{array}{lll}-0.30100000 & -0.23984300 & -1.86082100\end{array}$

$\begin{array}{lll}-1.41862100 & -0.63786000 & -2.84569600\end{array}$

$\begin{array}{lll}-1.65372000 & -1.71070900 & -2.80150200\end{array}$

$\begin{array}{lll}-1.09168200 & -0.40596900 & -3.87392500\end{array}$

$\begin{array}{lll}-2.34427200 & -0.07738300 & -2.64588700\end{array}$

$\begin{array}{lll}1.01377400 & -0.87790900 & -2.30330300\end{array}$

$\begin{array}{lll}1.82689000 & -0.68285600 & -1.59725900\end{array}$

$1.30162500 \quad-0.45820900 \quad-3.28271400$

$\begin{array}{lll}0.92728600 & -1.96581800 & -2.43153500\end{array}$

$\begin{array}{lll}-0.16358900 & 1.29215400 & -1.88639700\end{array}$

$\begin{array}{lll}-1.03368300 & 1.78466400 & -1.43385500\end{array}$

$\begin{array}{lll}-0.08483700 & 1.62961800 & -2.93369500\end{array}$

$0.73885900 \quad 1.62550500 \quad-1.36021300$

$\begin{array}{lll}5.30005300 & -2.25263400 & 0.58705200\end{array}$

$\begin{array}{lll}4.47192300 & -2.27884200 & 1.88502500\end{array}$

$3.48054600 \quad-2.72414200 \quad 1.73024400$

$\begin{array}{lll}4.33204000 & -1.26794200 & 2.30030400\end{array}$

$\begin{array}{lll}4.99687300 & -2.88167600 \quad 2.64627000\end{array}$

$\begin{array}{lll}6.72267900 & -1.80539300 & 0.94677900\end{array}$

$\begin{array}{lll}7.18320300 & -2.57220500 & 1.59492200\end{array}$

$6.72437000 \quad-0.86440900 \quad 1.51363800$ 
$\begin{array}{lll}7.37256800 & -1.68402700 & 0.07190400\end{array}$

$\begin{array}{lll}5.34137800 & -3.67154600 & -0.00455100\end{array}$

$\begin{array}{lll}4.33997800 & -4.01556800 & -0.30884500\end{array}$

$\begin{array}{lll}5.72336200 & -4.37886800 & 0.75186800\end{array}$

$\begin{array}{lll}6.00646600 & -3.73570400 & -0.87865300\end{array}$

5.12793000 $-1.05721100 \quad-2.33570100$

$\begin{array}{lll}4.67567900 & 0.27266700 & -2.96220500\end{array}$

$\begin{array}{lll}4.93774200 & 0.28235500 & -4.03455800\end{array}$

$\begin{array}{lll}5.16234400 & 1.13680200 & -2.48835300\end{array}$

$\begin{array}{lll}3.58700100 & 0.41457300 & -2.88387200\end{array}$

$6.65678200 \quad-1.15071400 \quad-2.39480700$

$7.15612500 \quad-0.34177900 \quad-1.84403300$

$6.98143000 \quad-1.07052800 \quad-3.44774500$

$\begin{array}{lll}7.02839700 & -2.11208400 & -2.01386100\end{array}$

$4.53446300 \quad-2.21309400 \quad-3.16593200$

$\begin{array}{lll}3.43733200 & -2.15143300 & -3.21650000\end{array}$

$4.79643100 \quad-3.19723500 \quad-2.75043200$

$\begin{array}{lll}4.92596300 & -2.17297200 & -4.19749200\end{array}$

$\begin{array}{lll}4.25881300 & -1.18540100 \quad-0.62261000\end{array}$

$\begin{array}{lll}-0.94805900 & -0.72743900 & -0.13406500\end{array}$

$\begin{array}{lll}-5.04539600 & -0.41275300 & -0.46835400\end{array}$

$\begin{array}{lll}-4.34765300 & -1.69479300 & -0.56963600\end{array}$

$\begin{array}{lll}-4.56191500 & -2.75654700 & 0.43258100\end{array}$

$\begin{array}{lll}-4.85917800 & -2.47322600 & 1.78438800\end{array}$

$-4.18202400 \quad-4.36357100 \quad-0.96194200$

$\begin{array}{lll}-4.21593600 & -2.09202900 & -1.58651700\end{array}$

$\begin{array}{lll}-4.40266800 & -4.11199800 & 0.07886300\end{array}$

$\begin{array}{lll}-4.98039500 & -3.49158900 & 2.72912100\end{array}$

$\begin{array}{lll}-4.80091100 & -4.83073100 & 2.36035500\end{array}$

$\begin{array}{lll}-4.51192300 & -5.13239800 & 1.02592900\end{array}$

$\begin{array}{lll}-5.20513500 & -3.23759500 \quad 3.76887800\end{array}$

$\begin{array}{lll}-4.88693800 & -5.62705400 \quad 3.10385700\end{array}$

$\begin{array}{lll}-4.37386400 & -6.17258900 & 0.71759800\end{array}$

$\begin{array}{lll}-5.35643000 & 0.37849000 & -1.62671200\end{array}$

$\begin{array}{lll}-4.98268600 & -0.02670100 & -2.57287200\end{array}$

$\begin{array}{lll}-3.62697000 & 2.39043000 \quad-0.88613000\end{array}$

$\begin{array}{lll}-3.02334700 & 1.71227800 & -0.02758600\end{array}$

$\begin{array}{lll}-3.42792300 & 3.91663800 & -0.89992700\end{array}$

$\begin{array}{lll}-3.02224100 & 4.35133600 & -2.31900200\end{array}$

$\begin{array}{lll}-2.33905300 & 4.31353800 & 0.10354800\end{array}$

$\begin{array}{lll}-4.77239400 & 4.56151600 & -0.51570200\end{array}$

$\begin{array}{lll}-3.79272300 & 4.07468100 & -3.05266400\end{array}$

$\begin{array}{lll}-2.07494700 & 3.87741100 & -2.62237500\end{array}$

$-2.88243900 \quad 5.44370200 \quad-2.35414200$ 


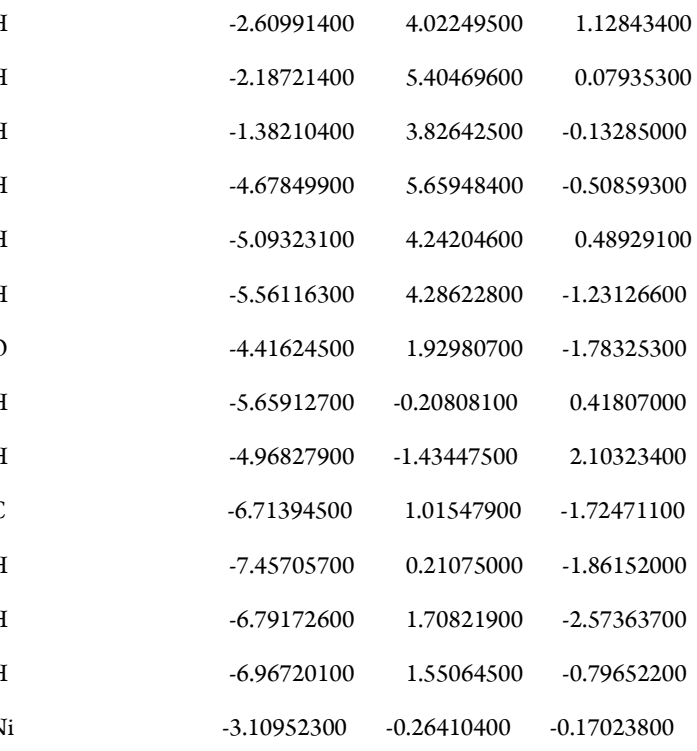

TS19/TS23/TS-Conformation-B

\begin{tabular}{|c|c|c|c|}
\hline $\mathrm{C}$ & -2.14708500 & -3.46487900 & 1.78563000 \\
\hline $\mathrm{C}$ & -1.55177500 & -2.33177500 & 1.20431700 \\
\hline $\mathrm{C}$ & -2.08135300 & -1.91133300 & -0.03020800 \\
\hline $\mathrm{C}$ & -2.98473600 & -2.67796400 & -0.77703300 \\
\hline $\mathrm{C}$ & -3.54298900 & -3.80364400 & -0.16257500 \\
\hline $\mathrm{C}$ & -3.16058900 & -4.16589600 & 1.13124100 \\
\hline $\mathrm{C}$ & -3.33444800 & -0.77745200 & -2.27487200 \\
\hline $\mathrm{C}$ & -2.53873700 & -0.02072600 & -1.40257800 \\
\hline $\mathrm{C}$ & -2.63864900 & 1.37689900 & -1.29288000 \\
\hline $\mathrm{C}$ & -3.54046200 & 2.01392400 & -2.16230800 \\
\hline $\mathrm{C}$ & -4.30784800 & 1.29352400 & -3.07577800 \\
\hline $\mathrm{C}$ & -4.21853600 & -0.09777400 & -3.11833500 \\
\hline $\mathrm{H}$ & -1.80003500 & -3.82530000 & 2.75016400 \\
\hline $\mathrm{H}$ & -4.26818300 & -4.41569800 & -0.69907800 \\
\hline $\mathrm{H}$ & -3.61484200 & -5.03600000 & 1.61046800 \\
\hline $\mathrm{H}$ & -3.66732900 & 3.09202600 & -2.10813200 \\
\hline $\mathrm{H}$ & -4.99861400 & 1.81800000 & -3.73975400 \\
\hline $\mathrm{H}$ & -4.84517100 & -0.65663000 & -3.81320600 \\
\hline $\mathrm{C}$ & -3.17966200 & -2.29406200 & -2.24466800 \\
\hline $\mathrm{C}$ & -1.88807000 & -2.67374700 & -3.01638800 \\
\hline $\mathrm{H}$ & -1.98387700 & -2.38316600 & -4.07401400 \\
\hline $\mathrm{H}$ & -1.00471800 & -2.16666600 & -2.60367000 \\
\hline $\mathrm{H}$ & -1.71568000 & -3.75978000 & -2.96361900 \\
\hline $\mathrm{C}$ & -4.36723100 & -3.01899400 & -2.88108500 \\
\hline $\mathrm{H}$ & -4.21639500 & -4.10726800 & -2.84454500 \\
\hline $\mathrm{H}$ & -5.31447300 & -2.78142500 & -2.37372900 \\
\hline $\mathrm{H}$ & -4.46378600 & -2.74889100 & -3.94198600 \\
\hline $\mathrm{O}$ & -1.64882800 & -0.69934800 & -0.56604400 \\
\hline $\mathrm{C}$ & 0.18193300 & -1.31954500 & 3.54897500 \\
\hline
\end{tabular}




\begin{tabular}{|c|c|c|}
\hline & & \\
\hline-1.60190300 & -2.37126000 & 4.26851700 \\
\hline-0.12826300 & -3.38135200 & 4.28476900 \\
\hline-0.34713800 & -2.09729000 & 5.48618500 \\
\hline-0.48074200 & 0.04142200 & 3.80636800 \\
\hline-0.33030000 & 0.32565200 & 4.86146700 \\
\hline-0.04494500 & 0.82632000 & 3.17507900 \\
\hline-1.56115300 & 0.00482300 & 3.62250000 \\
\hline 1.66283300 & -1.21170700 & 3.94575700 \\
\hline 2.20119100 & -0.47730500 & 3.33149000 \\
\hline 1.72140300 & -0.86967800 & 4.99246800 \\
\hline 2.18546800 & -2.17560100 & 3.88403800 \\
\hline 1.21445700 & -3.09519400 & 1.15545100 \\
\hline 1.09733300 & -4.30049900 & 2.09712100 \\
\hline 1.66671300 & -5.14061600 & 1.66409000 \\
\hline 1.52486100 & -4.09551800 & 3.08835900 \\
\hline 0.06258300 & -4.64369100 & 2.22337000 \\
\hline 0.76995600 & -3.51281000 & -0.25809900 \\
\hline 0.69614700 & -2.64152700 & -0.92600200 \\
\hline 1.52826300 & -4.18946800 & -0.68470100 \\
\hline-0.19372000 & -4.03999700 & -0.26170500 \\
\hline 2.69415500 & -2.66686200 & 1.06438300 \\
\hline 3.02570600 & -2.04074500 & 1.90032800 \\
\hline 3.31695300 & -3.57738400 & 1.06940200 \\
\hline 2.91161300 & -2.14919800 & 0.12217100 \\
\hline-1.40267200 & 4.02666300 & -0.58529900 \\
\hline-2.56707200 & 5.02417600 & -0.50940700 \\
\hline-3.46454100 & 4.70166400 & -1.05241900 \\
\hline-2.85366300 & 5.23801200 & 0.52933100 \\
\hline-2.24022700 & 5.97832600 & -0.95822200 \\
\hline-0.25626100 & 4.58456900 & 0.28095600 \\
\hline-0.49550500 & 4.57333900 & 1.35418600 \\
\hline 0.67647800 & 4.02583900 & 0.13366900 \\
\hline-0.06327300 & 5.63269000 & -0.00439900 \\
\hline-0.89014600 & 3.94041900 & -2.03244500 \\
\hline-0.46475300 & 4.91671100 & -2.32059900 \\
\hline-0.09655100 & 3.19096300 & -2.13888000 \\
\hline-1.68018000 & 3.69655700 & -2.75417300 \\
\hline-3.09394800 & 2.24784900 & 1.39733700 \\
\hline-4.44946100 & 2.85543600 & 1.01791700 \\
\hline-5.17781700 & 2.62242200 & 1.81472200 \\
\hline-4.41778900 & 3.94570800 & 0.91738900 \\
\hline-4.84487500 & 2.43006000 & 0.08397200 \\
\hline .519 & 2.96653700 & 2.62824900 \\
\hline
\end{tabular}


$\begin{array}{lll}-2.39939500 & 4.04561400 \quad 2.45112000\end{array}$

$-3.20565300 \quad 2.84659000 \quad 3.48448300$

$\begin{array}{lll}-1.54219000 & 2.55667200 & 2.92060500\end{array}$

$-3.32206000$

$0.76077800 \quad 1.71784100$

$-3.91859500$

$0.26781600 \quad 0.93627000$

$-2.37721700$

0.21542400

1.81351800

$-3.87567700$

$0.66427600 \quad 2.66655600$

$\begin{array}{lll}1.49454100 & 1.13090300 & -0.93550100\end{array}$

2.08042300

$0.55929500 \quad 0.25906300$

$\begin{array}{lll}2.60097800 & 1.35988000 & 1.38842900\end{array}$

$\begin{array}{lll}1.83392800 & 2.35237000 & 2.02565600\end{array}$

4.49353300

0.31911000

1.43032000

2.70022400

$-0.31498600$

0.06058200

3.88356600

1.09136400

1.90490300

2.32781800

$3.05678400 \quad 3.12375700$

3.60628300

$2.78064900 \quad 3.62233300$

4.38137200

1.79272300

3.00600600

1.70519200

3.81790800

3.60228500

$3.99290300 \quad 3.32737800 \quad 4.48617600$

5.38075500

3.38649700

1.37306000

$0.32356300 \quad-2.08162500$

3.21075100

$0.42451000-3.00526900$

3.99172000

$-0.48742200 \quad-2.57451400$

$5.51595100 \quad-0.21315600 \quad-2.75287800$

$3.64976000-1.53116600 \quad-1.99101300$

$\begin{array}{lll}5.77592800 & 0.91271300 & -3.75815900\end{array}$

$6.03874300 \quad 0.19601400 \quad-1.36301100$

$6.21050500 \quad-1.50266400 \quad-3.20933800$

$\begin{array}{lll}5.38082200 & 0.65641500 & -4.75418200\end{array}$

$5.28915400 \quad 1.84611000 \quad-3.44197400$

$6.85893700 \quad 1.09981300 \quad-3.85932800$

$5.87325000 \quad-0.61133700 \quad-0.63342700$

$\begin{array}{lll}7.11974200 & 0.41518200 & -1.39796800\end{array}$

$\begin{array}{lll}5.52063700 & 1.09706800 & -0.99654800\end{array}$

$\begin{array}{lll}7.30436000 & -1.36597500 & -3.25767200\end{array}$

$5.98755100 \quad-2.32478500 \quad-2.51470300$

$5.86461600 \quad-1.80696800 \quad-4.21173100$

$\begin{array}{lll}0.82450700 & 2.54133000 & 1.66595900\end{array}$

$\begin{array}{lll}0.21643400 & 0.06731700 & 0.25509900\end{array}$

$0.09401800 \quad-1.60475600 \quad 1.66065200$

$\begin{array}{lll}-1.71592800 & 2.23877000 & 0.05999600\end{array}$

$\begin{array}{lll}1.32919800 & 2.20718500 \quad-1.01934500\end{array}$

$\begin{array}{lll}0.57703200 & 0.72388900 & -3.28296700\end{array}$

C

$0.81790700 \quad 1.74934900 \quad-3.59847900$ 
H

$\mathrm{H}$

$\mathrm{H}$

TS-Conformation-A

C

C

C

C

$\mathrm{H}$

$\mathrm{H}$

C $\begin{array}{rrr}-0.50049400 & 0.68930400 & -3.05463700 \\ 0.77259500 & 0.04682200 & -4.12519700 \\ 1.56211000 & -0.74149100 & -1.94825200\end{array}$

$\begin{array}{lll}-1.93127300 & 1.40755800 & -0.74358200\end{array}$

$\begin{array}{lll}-1.82504300 & -0.01250800 & -0.54694500\end{array}$

$\begin{array}{lll}-1.57550100 & -0.94810000 & -1.66559700\end{array}$

$\begin{array}{lll}-1.46301300 & -0.52716200 & -3.00701500\end{array}$

$\begin{array}{lll}-1.58276800 & -2.69556900 & -0.39912400\end{array}$

$\begin{array}{lll}-2.49859300 & -0.40691300 & 0.21129600\end{array}$

$\begin{array}{lll}-1.47799000 & -2.33287900 & -1.41752700\end{array}$

$\begin{array}{lll}-1.23326800 & -1.43906100 & -4.03914000\end{array}$

$\begin{array}{lll}-1.11462100 & -2.80505500 & -3.76560700\end{array}$

$-1.24592300 \quad-3.24609200 \quad-2.44492000$

$-1.15489500 \quad-1.07752500 \quad-5.06796800$

$\begin{array}{lll}-0.93054700 & -3.51775900 & -4.57335200\end{array}$

$\begin{array}{lll}-1.16538000 & -4.31045700 & -2.20929600\end{array}$

$\begin{array}{lll}-2.47426100 & 2.19885900 & 0.26532400\end{array}$

$\begin{array}{lll}-5.07285100 & 0.79171900 & 0.02936900\end{array}$

$\begin{array}{lll}-4.64943500 & 0.07606900 & 0.96187800\end{array}$

$\begin{array}{lll}-6.11057100 & 0.15424300 & -0.95110300\end{array}$

$\begin{array}{lll}-7.07027700 & -0.75941200 & -0.18053200\end{array}$

$\begin{array}{lll}-6.89710800 & 1.23301300 & -1.70299600\end{array}$

$\begin{array}{lll}-5.28867200 & -0.67922500 & -1.95267400\end{array}$

$\begin{array}{lll}-6.50942700 & -1.50974900 & 0.39386000\end{array}$

$\begin{array}{lll}-7.68075300 & -0.18215700 & 0.53459800\end{array}$

$\begin{array}{lll}-7.76011900 & -1.27974200 & -0.86761600\end{array}$

$\begin{array}{lll}-6.21782900 & 1.90143700 & -2.25033200\end{array}$

$\begin{array}{lll}-7.60015200 & 0.77724700 & -2.42193000\end{array}$

$\begin{array}{lll}-7.48184200 & 1.85745100 & -1.00756200\end{array}$

$-5.94472900 \quad-1.14811100 \quad-2.70689100$

$\begin{array}{lll}-4.55630500 & -0.05042800 & -2.48323800\end{array}$

$\begin{array}{lll}-4.73064600 & -1.47759400 & -1.44032800\end{array}$

$\begin{array}{lll}-4.69311300 & 1.96627400 & -0.24535100\end{array}$

$\begin{array}{lll}0.55252300 & -3.70791700 & 1.39943600\end{array}$

$\begin{array}{lll}0.64907800 & -2.35534800 \quad 1.03695400\end{array}$

$\begin{array}{lll}1.48758400 & -2.04868200 & -0.04909300\end{array}$

$2.30174500 \quad-2.99087900 \quad-0.68718300$

$2.18060700 \quad-4.32663400 \quad-0.28904900$

$\begin{array}{lll}1.28842700 & -4.68523300 & 0.72557500\end{array}$

$3.76366100-1.10517800 \quad-1.29119800$

$2.81390400 \quad-0.23386500 \quad-0.74015600$

$3.07275500 \quad 1.11382000 \quad-0.43679400$

$\begin{array}{lll}4.39868300 & 1.54967200 & -0.59521700\end{array}$ 
$\begin{array}{lll}-0.08392600 & -4.00376800 & 2.23194500\end{array}$

$2.78467300 \quad-5.09690200 \quad-0.76951200$

$\begin{array}{lll}1.19313000 & -5.73306000 & 1.01927800\end{array}$

$\begin{array}{lll}4.66176200 & 2.58027800 & -0.35948900\end{array}$

$\begin{array}{lll}6.41696800 & 1.04045000 & -1.16143000\end{array}$

$\begin{array}{lll}5.84809300 & -1.27321400 & -1.84121600\end{array}$

$3.25696200 \quad-2.47369400 \quad-1.76642000$

$\begin{array}{lll}4.40219100 & -3.45159300 & -2.03696000\end{array}$

$5.06909100 \quad-3.06094000 \quad-2.81898500$

$5.00043700 \quad-3.64225200 \quad-1.13287900$

$\begin{array}{lll}4.01230800 & -4.41188000 & -2.40318500\end{array}$

$2.45256100 \quad-2.25663100 \quad-3.07416700$

$\begin{array}{lll}2.05254300 & -3.21571600 & -3.43598100\end{array}$

$\begin{array}{lll}1.60444000 & -1.57671200 & -2.91727500\end{array}$

$3.10231100-1.82877000 \quad-3.85380200$

$\begin{array}{lll}1.56107900 & -0.73309300 & -0.46520100\end{array}$

$\begin{array}{lll}-0.04137000 & -0.92563000 & 1.98506300\end{array}$

$\begin{array}{lll}1.62360700 & 2.21116100 \quad-0.06827600\end{array}$

$\begin{array}{lll}-1.63802000 & -1.49869200 & 2.90855600\end{array}$

$\begin{array}{lll}-2.54232600 & -2.37249800 & 2.02230800\end{array}$

$\begin{array}{lll}-2.90753800 & -1.85182300 & 1.13370200\end{array}$

$\begin{array}{lll}-2.06210700 & -3.31356500 & 1.72300300\end{array}$

$\begin{array}{lll}-3.43919400 & -2.63195000 & 2.60849800\end{array}$

$\begin{array}{lll}-2.40331800 & -0.20676800 & 3.26348700\end{array}$

$\begin{array}{lll}-1.75418000 & 0.56819700 & 3.69591800\end{array}$

$\begin{array}{lll}-2.94307100 & 0.19507500 & 2.39649200\end{array}$

$\begin{array}{lll}-3.17320500 & -0.44733700 & 4.01628900\end{array}$

$\begin{array}{lll}-1.38680200 & -2.28381400 & 4.20599100\end{array}$

$\begin{array}{lll}-0.91150300 & -1.67157700 \quad 4.98129700\end{array}$

$\begin{array}{lll}-2.36543600 & -2.60062900 \quad 4.60367000\end{array}$

$-0.78585300 \quad-3.19036900 \quad 4.05730700$

$\begin{array}{lll}1.36177500 & -0.75154400 & 3.31076300\end{array}$

$\begin{array}{lll}2.63001100 & -0.41645300 & 2.51818800\end{array}$

$3.01570700 \quad-1.29544500 \quad 1.98329100$

$\begin{array}{lll}2.45596900 & 0.37525400 & 1.79082600\end{array}$

$3.41815500 \quad-0.07779600 \quad 3.20943500$

$\begin{array}{lll}0.98330400 & 0.42693500 & 4.21984600\end{array}$

$\begin{array}{lll}0.13930000 & 0.18272900 & 4.88003000\end{array}$

$1.83937500 \quad 0.69346100 \quad 4.86244500$

$0.70695200 \quad 1.31771000 \quad 3.63635200$

$1.70440900 \quad-1.99133600 \quad 4.15323000$

$\begin{array}{lll}1.80235000 & -2.89539500 \quad 3.53515500\end{array}$ 
H

$\mathrm{H}$

C

C

$\mathrm{H}$

\begin{tabular}{|c|c|c|}
\hline 2.68528000 & -1.82030200 & 4.62935500 \\
\hline 0.99130800 & -2.19170200 & 4.95701300 \\
\hline 2.17607600 & 3.57529900 & 1.16004800 \\
\hline 3.01500800 & 2.95620500 & 2.28705900 \\
\hline 2.45937600 & 2.19432100 & 2.84037400 \\
\hline 3.95002200 & 2.50839600 & 1.92317800 \\
\hline 3.28015200 & 3.75251000 & 3.00254300 \\
\hline 2.95944500 & 4.75554500 & 0.57056400 \\
\hline 3.19756100 & 5.45602600 & 1.38901700 \\
\hline 3.91173200 & 4.45091000 & 0.11658900 \\
\hline 2.38052400 & 5.31517400 & -0.17461900 \\
\hline 0.86861100 & 4.11386100 & 1.76927200 \\
\hline 1.10574700 & 4.84856700 & 2.55680800 \\
\hline 0.25026500 & 4.62209300 & 1.01668800 \\
\hline 0.26613200 & 3.31040100 & 2.21991600 \\
\hline 1.44498000 & 2.97050800 & -1.83635100 \\
\hline 2.70064200 & 3.60350400 & -2.45228100 \\
\hline 2.46235000 & 3.87492000 & -3.49535400 \\
\hline 3.02330100 & 4.51921600 & -1.94667100 \\
\hline 3.54573200 & 2.90382000 & -2.48971100 \\
\hline 0.31908900 & 4.00936100 & -1.79870000 \\
\hline 0.60275500 & 4.90311400 & -1.22460600 \\
\hline 0.08144300 & 4.33617100 & -2.82499700 \\
\hline 0.59155400 & 3.59718100 & -1.35768600 \\
\hline 1.04989900 & 1.78664200 & -2.73462900 \\
\hline 1.91349800 & 1.14211500 & -2.95195300 \\
\hline 0.27682000 & 1.16243200 & -2.27983000 \\
\hline 0.66462100 & 2.16467100 & -3.69594000 \\
\hline .23812500 & 0.78572700 & 0.37065700 \\
\hline 1.58252900 & 0.52633600 & -3.25721200 \\
\hline 1.73425300 & 1.86746500 & -1.71332500 \\
\hline 2.71015900 & 1.70962400 & 1.20839300 \\
\hline 2.58865200 & 3.68288700 & 0.23513200 \\
\hline 2.65770300 & 4.06927600 & -0.79106500 \\
\hline-3.48431100 & 3.99256700 & 0.78838200 \\
\hline 1.71604200 & 4.14515900 & 0.72231400 \\
\hline 1.90802800 & 1.21127300 & 0.98065800 \\
\hline 1.65087700 & -0.16036300 & 1.25466600 \\
\hline 0.98106500 & -0.60523000 & 2.50602300 \\
\hline 1.04157700 & 0.15200700 & 3.69438200 \\
\hline 0.17188200 & -2.41966100 & 1.65672600 \\
\hline 2.35641800 & -0.89032700 & 0.85568000 \\
\hline .25420300 & -1.81281400 & 2.55566600 \\
\hline
\end{tabular}


$\begin{array}{lll}-0.34813700 & -1.44818700 \quad 4.87631000\end{array}$

$\begin{array}{lll}-0.39922300 & -2.22867900 & 3.71559900\end{array}$

$0.44716300 \quad 0.36078400 \quad 5.75743600$

$\begin{array}{lll}-0.86714000 & -1.76678700 \quad 5.78396700\end{array}$

$\begin{array}{lll}-0.96611300 & -3.16366600 & 3.70655100\end{array}$

$3.13183600 \quad 1.60404000 \quad 0.18082500$

$\begin{array}{lll}5.24204500 & 0.47123800 & -0.15478900\end{array}$

$5.27907300 \quad 0.79971900 \quad-1.32233000$

$\begin{array}{lll}6.29592400 & -0.39817200 & 0.54302100\end{array}$

$\begin{array}{lll}7.32823000 & -0.86305900 & -0.48827700\end{array}$

$\begin{array}{lll}6.97740300 & 0.45563300 & 1.62997400\end{array}$

$\begin{array}{lll}5.60284000 & -1.61188400 & 1.18917800\end{array}$

$6.85870500 \quad-1.46869100 \quad-1.27832800$

$\begin{array}{lll}7.82227800 & -0.00837200 & -0.97363600\end{array}$

$\begin{array}{lll}8.10037900 & -1.47682700 & 0.00208500\end{array}$

$\begin{array}{lll}6.25006300 & 0.79604400 & 2.38109200\end{array}$

$\begin{array}{lll}7.75353200 & -0.13556700 & 2.14210900\end{array}$

$\begin{array}{lll}7.46128700 & 1.34384200 & 1.19185900\end{array}$

$6.35194800 \quad-2.25257900 \quad 1.68162900$

$\begin{array}{lll}4.86648800 & -1.29807500 & 1.94223000\end{array}$

$\begin{array}{lll}5.07983900 & -2.22026400 & 0.43488700\end{array}$

$\begin{array}{lll}4.27192400 & 0.82812100 \quad 0.68974100\end{array}$

$\begin{array}{lll}-1.31271500 & -3.90385200 & -0.75173100\end{array}$

$\begin{array}{lll}-1.13445800 & -2.52142400 & -0.57820000\end{array}$

$\begin{array}{lll}-2.13509000 & -1.84281300 & 0.13781500\end{array}$

$\begin{array}{lll}-3.32597600 & -2.44364900 & 0.56443000\end{array}$

$-3.46918300 \quad-3.81958400 \quad 0.35756400$

$\begin{array}{lll}-2.45361100 & -4.54903700 & -0.26836700\end{array}$

$\begin{array}{lll}-4.32561900 & -0.20418900 & 0.42536700\end{array}$

$\begin{array}{lll}-3.05385000 & 0.30934100 & 0.12955300\end{array}$

$\begin{array}{lll}-2.82910600 & 1.58339000 & -0.41720000\end{array}$

$\begin{array}{lll}-3.97314800 & 2.30933500 & -0.78791000\end{array}$

$\begin{array}{lll}-5.25525500 & 1.79308700 & -0.58841700\end{array}$

$\begin{array}{lll}-5.43398800 & 0.55588100 & 0.04078400\end{array}$

$-0.57032300 \quad-4.48335200 \quad-1.29814800$

$\begin{array}{lll}-4.37632400 & -4.33077300 \quad 0.68159900\end{array}$

$\begin{array}{lll}-2.56896300 & -5.62565900 & -0.41381700\end{array}$

$\begin{array}{lll}-3.86562100 & 3.29918200 & -1.22780700\end{array}$

$\begin{array}{lll}-6.12659000 & 2.37542300 & -0.89703300\end{array}$

$\begin{array}{lll}-6.44376600 & 0.19260500 & 0.23522300\end{array}$

$-4.37181400 \quad-1.52642100 \quad 1.20489000$

$\begin{array}{lll}-5.76806400 & -2.15133300 & 1.19916200\end{array}$

$\begin{array}{lll}-6.49709400 & -1.47220200 \quad 1.66437400\end{array}$ 
$\begin{array}{lll}-6.11003400 & -2.38028200 & 0.17829200\end{array}$

$\begin{array}{lll}-5.78010900 & -3.08170700 \quad 1.78505000\end{array}$

$-3.94676300 \quad-1.23910800$

2.66856200

$-3.95256400$

3.25422400

$\begin{array}{lll}-2.93371500 & -0.81597600 & 2.71744500\end{array}$

$-4.64481200$

$-1.96565900$

$-0.52665300$

3.13560000

0.14960800

0.36994500

$-1.08087700$

$-1.46929800 \quad-1.40522400$

$2.02530500 \quad-2.68644600 \quad 0.60211600$

$1.24750600 \quad-4.11439500 \quad-0.09920500$

$2.97827500 \quad-3.82710700 \quad-0.35803800$

$2.88512700 \quad-1.56825800 \quad-1.82305300$

$2.76955400 \quad-1.01546700 \quad-2.76565200$

$2.94764600 \quad-0.83189700 \quad-1.01904400$

$\begin{array}{lll}3.84299000 & -2.11153800 & -1.87213900\end{array}$

$\begin{array}{lll}1.75150500 & -3.57977100 & -2.73398600\end{array}$

$1.72725500 \quad-3.09792500 \quad-3.71910200$

$2.69875800 \quad-4.14345500 \quad-2.67646600$

$0.93491800 \quad-4.31189600 \quad-2.68259000$

$\begin{array}{lll}-0.67220800 & -1.33981800 & -3.15019600\end{array}$

$\begin{array}{lll}-1.98876500 & -0.59082700 & -2.90388000\end{array}$

$\begin{array}{lll}-2.73769600 & -1.24356300 & -2.43248200\end{array}$

$\begin{array}{lll}-1.84218100 & 0.27568700 & -2.25747000\end{array}$

$\begin{array}{lll}-2.40725200 & -0.24179300 & -3.86192200\end{array}$

$0.27015700 \quad-0.49422100 \quad-4.02013600$

$\begin{array}{lll}1.16117400 & -1.06235000 & -4.32460300\end{array}$

$\begin{array}{lll}-0.24823900 & -0.17581300 & -4.94048100\end{array}$

$\begin{array}{lll}0.61135700 & 0.40824700 & -3.49079200\end{array}$

$\begin{array}{lll}-1.04115500 & -2.63873700 & -3.88006500\end{array}$

$\begin{array}{lll}-1.60299700 & -3.32917600 & -3.23490400\end{array}$

$\begin{array}{lll}-1.70089900 & -2.38349600 & -4.72780600\end{array}$

$\begin{array}{lll}-0.18056000 & -3.17061500 & -4.29582200\end{array}$

$\begin{array}{lll}-0.91698600 & 3.44449200 & -1.90477000\end{array}$

$\begin{array}{lll}-1.45396700 & 2.76063300 & -3.17001200\end{array}$

$\begin{array}{lll}-0.92736600 & 1.82495900 & -3.38028900\end{array}$

$\begin{array}{lll}-2.53031800 & 2.54690400 & -3.11234700\end{array}$

$\begin{array}{lll}-1.29061400 & 3.43060400 & -4.03124000\end{array}$

$\begin{array}{lll}-1.58283500 & 4.82272500 & -1.78569000\end{array}$

$\begin{array}{lll}-1.37618300 & 5.38916300 & -2.71031000\end{array}$

$\begin{array}{lll}-2.67458900 & 4.76547200 & -1.68354000\end{array}$

$-1.18717600 \quad 5.41562900 \quad-0.95221100$ 
Conformation-B

\begin{tabular}{|c|c|c|}
\hline 0.59828100 & 3.65160800 & -2.08206200 \\
\hline 0.78710300 & 4.22972800 & -3.00257000 \\
\hline 1.03329100 & 4.21149500 & -1.24386900 \\
\hline 1.13162800 & 2.69218400 & -2.16171300 \\
\hline-1.24572100 & 3.28521400 & 1.20171400 \\
\hline-2.50749800 & 4.15094500 & 1.32809900 \\
\hline-2.45399800 & 4.69729800 & 2.28610000 \\
\hline-2.60972400 & 4.89853900 & 0.53417500 \\
\hline-3.42278300 & 3.54550800 & 1.35420100 \\
\hline-0.00803000 & 4.18086400 & 1.35434500 \\
\hline-0.02971000 & 5.03694700 & 0.66590500 \\
\hline 0.02520700 & 4.58694000 & 2.37982700 \\
\hline 0.92172100 & 3.63035800 & 1.18324900 \\
\hline-1.28724000 & 2.25481000 & 2.34032900 \\
\hline-2.22648500 & 1.68218800 & 2.33313600 \\
\hline-0.46469600 & 1.53858400 & 2.27789600 \\
\hline-1.22288500 & 2.77166500 & 3.31277800 \\
\hline 0.39261800 & 0.51120400 & -0.14838600 \\
\hline 1.61950000 & 1.07804500 & 3.70653400 \\
\hline 1.64029000 & 1.94006600 & 1.75007300 \\
\hline 3.03377900 & 1.32499000 & -0.87756600 \\
\hline 3.49608900 & 3.07516900 & 0.27782600 \\
\hline 3.66336500 & 3.36395900 & 1.32766400 \\
\hline 4.40840700 & 3.28838400 & -0.29851000 \\
\hline 2.68631000 & 3.69564700 & -0.12655500 \\
\hline 3.02994800 & 3.25105500 & -1.06019200 \\
\hline 2.11929700 & 2.25859000 & -0.66174800 \\
\hline 2.19501500 & 1.03491600 & -1.34921500 \\
\hline 2.90207100 & 0.86081600 & -2.54270600 \\
\hline 3.78278700 & 1.88278300 & -2.91542000 \\
\hline 3.88829500 & 3.04501300 & -2.14334600 \\
\hline 2.34785500 & -1.54062100 & -2.49414000 \\
\hline 1.87013300 & -1.32703600 & -1.19460300 \\
\hline 1.82143300 & -2.33770700 & -0.21541900 \\
\hline 2.09447000 & -3.64284800 & -0.65637800 \\
\hline 2.46608300 & -3.90611900 & -1.97537000 \\
\hline 2.63622100 & -2.85511200 & -2.87698800 \\
\hline 3.04584500 & 4.20896900 & -0.54352400 \\
\hline 4.37541300 & 1.78638700 & -3.82568500 \\
\hline 4.59506800 & 3.82619100 & -2.43218800 \\
\hline 2.05554900 & -4.46904600 & 0.05006100 \\
\hline 2.66942800 & -4.93279700 & -2.28825400 \\
\hline 2.98990000 & -3.06220800 & -3.88702600 \\
\hline
\end{tabular}


$0.04641600 \quad-3.56649700 \quad 4.48226900$

$-0.28326800$

$-3.81603800$

1.80975800

$-0.78167500$

$-4.58792000$

2.42066000

$-1.00067200$

$-2.99603600$

1.67061100

$-0.07500900 \quad-4.25307100$

0.82350800

3.55203100

$-1.62725100$

1.93318800

$\begin{array}{lll}4.45571900 & -2.81387300 & 1.57795700\end{array}$

$5.50822000-2.52559300$

1.74942600

$4.25899700 \quad-3.70212900$

2.18800800

$\begin{array}{lll}4.36547400 & -3.09443300 & 0.51893900\end{array}$

$3.69244600 \quad-1.29212300 \quad 3.42628400$

$3.46819700 \quad-2.15963900 \quad 4.06339900$

$\begin{array}{lll}4.73119900 & -0.98741200 & 3.64125000\end{array}$

$3.03076100 \quad-0.46498300 \quad 3.72664200$

$\begin{array}{lll}4.02878100 & -0.42412300 & 1.09998200\end{array}$

$\begin{array}{lll}4.08286200 & -0.66661700 & 0.02927100\end{array}$

$3.38511900 \quad 0.45666100 \quad 1.21286200$

$\begin{array}{lll}5.04609800 & -0.14070800 & 1.41950500\end{array}$

$\begin{array}{lll}-1.72627700 & -0.84704400 & -0.26245300\end{array}$

$-2.24477100$

$-2.58130600$

$-1.95511400$

$-4.02451600$

$-2.84171400$

$-3.51999000$

$-2.24570900$

$-3.17379900$

$-3.80977000$

$-1.73446600$

$-3.39713100$

$-4.54004500$

$-2.13942900$

$-3.55040200$

$-4.51669600$

$-5.90016600$

$-4.32756500$

$-6.08293800$

$-5.96415800$

$-6.98215900$

$-6.00936500-2.80331900$

$-5.32184300$

$-7.07714600$

$-5.81456200$

$-6.95019400$

$-1.81969400$
0.27102700

1.68652800

2.73132700

1.26582000

$-0.41368600$

2.05287700

4.06834600

4.41324000

3.39315800

4.85232900

5.46211800

3.64231500

$-1.64467700$

$-1.60473200$

$-1.90805900$

$-1.53133400$

$-2.39510200$

$-2.11053000$

0.00899900

$-2.07233500$

$-3.21037800$

$-1.72231400$

$-1.84045700$

0.44729100

0.33282600 


\begin{tabular}{|c|c|c|c|}
\hline $\mathrm{H}$ & -5.19055300 & -2.11540000 & 0.41724100 \\
\hline $\mathrm{H}$ & -7.97981000 & -0.82918700 & -1.78749400 \\
\hline $\mathrm{H}$ & -6.86291200 & 0.55849400 & -1.67232600 \\
\hline $\mathrm{H}$ & -6.94134900 & -0.39250800 & -3.17063800 \\
\hline $\mathrm{H}$ & -1.20155600 & -0.84393700 & 2.47726800 \\
\hline $\mathrm{Ni}$ & -0.34979800 & 0.49856800 & -0.02640400 \\
\hline $\mathrm{P}$ & 0.53884600 & 2.48838900 & 0.28979600 \\
\hline $\mathrm{P}$ & 1.66318000 & -1.82575400 & 1.56318800 \\
\hline $\mathrm{H}$ & -1.64858600 & -1.70235700 & 0.42057700 \\
\hline $\mathrm{H}$ & -2.11327000 & -0.41083800 & -2.33429000 \\
\hline $\mathrm{C}$ & -1.35734500 & -2.43427400 & -2.20991500 \\
\hline $\mathrm{H}$ & -1.74957000 & -2.73324300 & -3.19460500 \\
\hline $\mathrm{H}$ & -1.41302700 & -3.30301200 & -1.53493500 \\
\hline $\mathrm{H}$ & -0.30061300 & -2.16373100 & -2.32405900 \\
\hline \multicolumn{4}{|l|}{6} \\
\hline $\mathrm{C}$ & 5.24201400 & -0.81142200 & -0.37053200 \\
\hline $\mathrm{C}$ & 4.55031400 & -1.55335100 & 0.62387600 \\
\hline $\mathrm{C}$ & 4.37004400 & -3.01294100 & 0.51346300 \\
\hline $\mathrm{C}$ & 4.27901100 & -3.67376000 & -0.72833700 \\
\hline $\mathrm{H}$ & 4.37648100 & -3.30908300 & 2.65401700 \\
\hline $\mathrm{H}$ & 4.66337000 & -1.19992700 & 1.65729300 \\
\hline $\mathrm{C}$ & 4.29426100 & -3.79740700 & 1.68001100 \\
\hline $\mathrm{C}$ & 4.09441200 & -5.05458100 & -0.79629100 \\
\hline $\mathrm{C}$ & 4.00028300 & -5.81645600 & 0.37427400 \\
\hline $\mathrm{C}$ & 4.10900300 & -5.17930100 & 1.61374100 \\
\hline $\mathrm{H}$ & 4.01535200 & -5.54067200 & -1.77229400 \\
\hline $\mathrm{H}$ & 3.84889100 & -6.89725200 & 0.31903400 \\
\hline $\mathrm{H}$ & 4.04764000 & -5.76190100 & 2.53670500 \\
\hline $\mathrm{C}$ & 5.43142000 & 0.55580400 & -0.21438600 \\
\hline $\mathrm{H}$ & 5.45023400 & 0.97669700 & 0.79649900 \\
\hline $\mathrm{Ni}$ & 3.40595100 & 0.01624200 & -0.07249700 \\
\hline $\mathrm{C}$ & 3.23754200 & 2.82452600 & -0.32774100 \\
\hline $\mathrm{O}$ & 2.89560900 & 1.69069700 & -0.84500500 \\
\hline $\mathrm{C}$ & 2.80464600 & 4.05505300 & -1.16698200 \\
\hline $\mathrm{C}$ & 3.49125900 & 5.31548800 & -0.63107200 \\
\hline $\mathrm{C}$ & 1.27866500 & 4.20652100 & -1.03620000 \\
\hline $\mathrm{C}$ & 3.17817200 & 3.84031800 & -2.64205200 \\
\hline $\mathrm{H}$ & 4.58756600 & 5.23731300 & -0.70193100 \\
\hline $\mathrm{H}$ & 3.24185700 & 5.48099900 & 0.42684100 \\
\hline $\mathrm{H}$ & 3.17373300 & 6.20025700 & -1.20778100 \\
\hline $\mathrm{H}$ & 0.75416800 & 3.32310700 & -1.42466100 \\
\hline $\mathrm{H}$ & 0.93156300 & 5.08861100 & -1.59999200 \\
\hline $\mathrm{H}$ & 0.98456900 & 4.33630900 & 0.01458000 \\
\hline $\mathrm{H}$ & 2.67788800 & 2.95211600 & -3.05280000 \\
\hline
\end{tabular}


H

H

O

$\mathrm{H}$

H

C

H

H

$\mathrm{H}$

C

C

\begin{tabular}{|c|c|c|}
\hline 4.26464200 & 3.70275400 & -2.76572600 \\
\hline 2.87991900 & 4.71481600 & -3.24463000 \\
\hline 3.83933000 & 2.96792900 & 0.73764100 \\
\hline 4.33087800 & -3.09776600 & -1.65369200 \\
\hline 5.43568600 & -1.26619000 & -1.34894200 \\
\hline 5.99810900 & 1.42200400 & -1.29761000 \\
\hline 7.10103000 & 1.44908200 & -1.22815400 \\
\hline 5.64862800 & 2.45811000 & -1.19949600 \\
\hline 5.73240200 & 1.05384400 & -2.30042300 \\
\hline 5.36980100 & 0.88310900 & -0.88087100 \\
\hline-4.15228500 & 0.32981200 & -0.45570100 \\
\hline-3.15919200 & 1.21926000 & 0.02986800 \\
\hline-3.45797200 & 2.57175500 & 0.26625300 \\
\hline-4.69257300 & 3.07012100 & -0.17038400 \\
\hline-5.63289500 & 2.24769500 & -0.78218600 \\
\hline-1.23668000 & 2.74047900 & 1.41441200 \\
\hline-0.92841300 & 1.47355200 & 0.88654900 \\
\hline 0.38512000 & 0.94613900 & 1.00016700 \\
\hline 1.29498200 & 1.64372900 & 1.81150500 \\
\hline 0.95994000 & 2.82954800 & 2.45647000 \\
\hline-0.28846200 & 3.38783100 & 2.21760300 \\
\hline-6.13959800 & 0.22509100 & -1.27806900 \\
\hline-4.92350900 & 4.12541300 & -0.01496500 \\
\hline-6.58289400 & 2.65603600 & -1.13399700 \\
\hline 2.31341200 & 1.26743900 & 1.90050300 \\
\hline 1.69661400 & 3.34308600 & 3.07600600 \\
\hline-0.53008500 & 4.36433400 & 2.64025000 \\
\hline-2.52663900 & 3.47646800 & 1.06259800 \\
\hline-3.26097500 & 3.90605100 & 2.35446000 \\
\hline-2.63645000 & 4.57571700 & 2.96383200 \\
\hline-3.52032900 & 3.02667400 & 2.96400400 \\
\hline-4.18994600 & 4.44498700 & 2.11642500 \\
\hline-2.16382500 & 4.72795600 & 0.22851200 \\
\hline-3.06425100 & 5.30826500 & -0.02336300 \\
\hline-1.66588200 & 4.44046000 & -0.70898300 \\
\hline-1.48612000 & 5.38943300 & 0.78817000 \\
\hline-1.89561400 & 0.71699700 & 0.26578700 \\
\hline-4.65338300 & -2.21783400 & -1.96707200 \\
\hline-6.16981700 & -2.42037400 & -2.07425000 \\
\hline-6.73667800 & -1.48372700 & -1.98676200 \\
\hline-6.55029800 & -3.12506400 & -1.32211500 \\
\hline-6.40478800 & -2.84640000 & -3.06590500 \\
\hline-4.18204400 & -1.31084200 & -3.11685600 \\
\hline .73753800 & -0.36378000 & -3.15186700 \\
\hline
\end{tabular}




\begin{tabular}{|c|c|c|}
\hline-4.33956500 & -1.82837800 & -4.07914700 \\
\hline-3.11223500 & -1.06859400 & -3.04009900 \\
\hline-3.97178400 & -3.59349300 & -2.12301000 \\
\hline-2.87562700 & -3.50298000 & -2.14348100 \\
\hline-4.29227200 & -4.06386400 & -3.06888300 \\
\hline-4.23672400 & -4.28000200 & -1.30490900 \\
\hline-5.08174800 & -1.89721800 & 1.14984900 \\
\hline-4.37786500 & -1.27036100 & 2.36754600 \\
\hline-3.36265300 & -1.66614900 & 2.50476600 \\
\hline-4.95421600 & -1.49553900 & 3.28156000 \\
\hline-4.31048900 & -0.17434700 & 2.28141900 \\
\hline-5.09035400 & -3.42529600 & 1.31584300 \\
\hline-5.64733700 & -3.92644200 & 0.51065400 \\
\hline-5.57675100 & -3.69391900 & 2.26963100 \\
\hline-4.06836500 & -3.83630500 & 1.33365400 \\
\hline-6.51871300 & -1.36656700 & 1.10765400 \\
\hline-7.09435800 & -1.75170400 & 0.25769200 \\
\hline-6.54689000 & -0.26845000 & 1.07614300 \\
\hline-7.04402300 & -1.67775600 & 2.02824400 \\
\hline 0.57864800 & -0.79623700 & -1.57078800 \\
\hline 1.76337900 & -1.45465700 & -2.30259500 \\
\hline 2.03799200 & -2.41941000 & -1.85318900 \\
\hline 2.65302600 & -0.80577700 & -2.30039500 \\
\hline 1.48076400 & -1.64168500 & -3.35247600 \\
\hline 0.31043600 & 0.58299200 & -2.19409500 \\
\hline 0.16306100 & 0.45410900 & -3.28006600 \\
\hline 1.15467800 & 1.26311500 & -2.03075900 \\
\hline-0.60241500 & 1.03774700 & -1.78771500 \\
\hline-0.66003900 & -1.66985200 & -1.74030300 \\
\hline-0.93963500 & -1.67182000 & -2.80715700 \\
\hline-1.51383300 & -1.29545700 & -1.16854700 \\
\hline-0.48582300 & -2.71379100 & -1.45290100 \\
\hline 0.67649300 & -1.93504000 & 1.40239400 \\
\hline 0.98471300 & -3.30942500 & 0.79101800 \\
\hline 0.97832100 & -4.06885900 & 1.58950400 \\
\hline 1.96653300 & -3.34769100 & 0.31122900 \\
\hline 0.22629400 & -3.60582500 & 0.05608300 \\
\hline 1.52185400 & -1.72258200 & 2.67083900 \\
\hline 1.30846700 & -2.53438000 & 3.38603700 \\
\hline 1.27639200 & -0.77150600 & 3.16634100 \\
\hline 2.59721400 & -1.72868000 & 2.45914900 \\
\hline-0.80285000 & -1.86832000 & 1.79065500 \\
\hline-1.47697700 & -1.94873000 & 0.92957000 \\
\hline 04 & 100 & .3223930 \\
\hline
\end{tabular}


21

C

C

$$
\begin{array}{ccc}
-1.02563800 & -2.70682100 & 2.47354900 \\
1.18465200 & -0.53513900 & 0.21896600 \\
-3.92483200 & -1.50655900 & -0.33326200
\end{array}
$$




\begin{tabular}{|c|c|c|}
\hline-3.15919200 & 1.21926000 & -0.02986800 \\
\hline-3.45797200 & 2.57175500 & -0.26625300 \\
\hline-4.69257300 & 3.07012100 & 0.17038400 \\
\hline-5.63289500 & 2.24769500 & 0.78218600 \\
\hline-1.23668000 & 2.74047900 & -1.41441200 \\
\hline-0.92841300 & 1.47355200 & -0.88654900 \\
\hline 0.38512000 & 0.94613900 & -1.00016700 \\
\hline 1.29498200 & 1.64372900 & -1.81150500 \\
\hline 0.95994000 & 2.82954800 & -2.45647000 \\
\hline-0.28846200 & 3.38783100 & -2.21760300 \\
\hline-6.13959800 & 0.22509100 & 1.27806900 \\
\hline-4.92350900 & 4.12541300 & 0.01496500 \\
\hline-6.58289400 & 2.65603600 & 1.13399700 \\
\hline 2.31341200 & 1.26743900 & -1.90050300 \\
\hline 1.69661400 & 3.34308600 & -3.07600600 \\
\hline-0.53008500 & 4.36433400 & -2.64025000 \\
\hline-2.52663900 & 3.47646800 & -1.06259800 \\
\hline-3.26097500 & 3.90605100 & -2.35446000 \\
\hline-2.63645000 & 4.57571700 & -2.96383200 \\
\hline-3.52032900 & 3.02667400 & -2.96400400 \\
\hline-4.18994600 & 4.44498700 & -2.11642500 \\
\hline-2.16382500 & 4.72795600 & -0.22851200 \\
\hline-3.06425100 & 5.30826500 & 0.02336300 \\
\hline-1.66588200 & 4.44046000 & 0.70898300 \\
\hline-1.48612000 & 5.38943300 & -0.78817000 \\
\hline-1.89561400 & 0.71699700 & -0.26578700 \\
\hline-4.65338300 & 2.21783400 & 1.96707200 \\
\hline-6.16981700 & -2.42037400 & 2.07425000 \\
\hline-6.73667800 & -1.48372700 & 1.98676200 \\
\hline-6.55029800 & -3.12506400 & 1.32211500 \\
\hline-6.40478800 & -2.84640000 & 3.06590500 \\
\hline-4.18204400 & -1.31084200 & 3.11685600 \\
\hline-4.73753800 & -0.36378000 & 3.15186700 \\
\hline-4.33956500 & -1.82837800 & 4.07914700 \\
\hline-3.11223500 & -1.06859400 & 3.04009900 \\
\hline-3.97178400 & -3.59349300 & 2.12301000 \\
\hline-2.87562700 & -3.50298000 & 2.14348100 \\
\hline-4.29227200 & -4.06386400 & 3.06888300 \\
\hline-4.23672400 & -4.28000200 & 1.30490900 \\
\hline-5.08174800 & -1.89721800 & -1.14984900 \\
\hline-4.37786500 & -1.27036100 & -2.36754600 \\
\hline-3.36265300 & -1.66614900 & -2.50476600 \\
\hline-4.95421600 & -1.49553900 & -3.28156000 \\
\hline-4.31048900 & -0.17434700 & -2.2814190 \\
\hline
\end{tabular}




\begin{tabular}{|c|c|c|c|}
\hline $\mathrm{C}$ & -5.09035400 & -3.42529600 & -1.31584300 \\
\hline $\mathrm{H}$ & -5.64733700 & -3.92644200 & -0.51065400 \\
\hline $\mathrm{H}$ & -5.57675100 & -3.69391900 & -2.26963100 \\
\hline $\mathrm{H}$ & -4.06836500 & -3.83630500 & -1.33365400 \\
\hline $\mathrm{C}$ & -6.51871300 & -1.36656700 & -1.10765400 \\
\hline $\mathrm{H}$ & -7.09435800 & -1.75170400 & -0.25769200 \\
\hline $\mathrm{H}$ & -6.54689000 & -0.26845000 & -1.07614300 \\
\hline $\mathrm{H}$ & -7.04402300 & -1.67775600 & -2.02824400 \\
\hline $\mathrm{C}$ & 0.57864800 & -0.79623700 & 1.57078800 \\
\hline $\mathrm{C}$ & 1.76337900 & -1.45465700 & 2.30259500 \\
\hline $\mathrm{H}$ & 2.03799200 & -2.41941000 & 1.85318900 \\
\hline $\mathrm{H}$ & 2.65302600 & -0.80577700 & 2.30039500 \\
\hline $\mathrm{H}$ & 1.48076400 & -1.64168500 & 3.35247600 \\
\hline $\mathrm{C}$ & 0.31043600 & 0.58299200 & 2.19409500 \\
\hline $\mathrm{H}$ & 0.16306100 & 0.45410900 & 3.28006600 \\
\hline $\mathrm{H}$ & 1.15467800 & 1.26311500 & 2.03075900 \\
\hline $\mathrm{H}$ & -0.60241500 & 1.03774700 & 1.78771500 \\
\hline $\mathrm{C}$ & -0.66003900 & -1.66985200 & 1.74030300 \\
\hline $\mathrm{H}$ & -0.93963500 & -1.67182000 & 2.80715700 \\
\hline $\mathrm{H}$ & -1.51383300 & -1.29545700 & 1.16854700 \\
\hline $\mathrm{H}$ & -0.48582300 & -2.71379100 & 1.45290100 \\
\hline $\mathrm{C}$ & 0.67649300 & -1.93504000 & -1.40239400 \\
\hline $\mathrm{C}$ & 0.98471300 & -3.30942500 & -0.79101800 \\
\hline $\mathrm{H}$ & 0.97832100 & -4.06885900 & -1.58950400 \\
\hline $\mathrm{H}$ & 1.96653300 & -3.34769100 & -0.31122900 \\
\hline $\mathrm{H}$ & 0.22629400 & -3.60582500 & -0.05608300 \\
\hline $\mathrm{C}$ & 1.52185400 & -1.72258200 & -2.67083900 \\
\hline $\mathrm{H}$ & 1.30846700 & -2.53438000 & -3.38603700 \\
\hline $\mathrm{H}$ & 1.27639200 & -0.77150600 & -3.16634100 \\
\hline $\mathrm{H}$ & 2.59721400 & -1.72868000 & -2.45914900 \\
\hline $\mathrm{C}$ & -0.80285000 & -1.86832000 & -1.79065500 \\
\hline $\mathrm{H}$ & -1.47697700 & -1.94873000 & -0.92957000 \\
\hline $\mathrm{H}$ & -1.04209400 & -0.93696400 & -2.32239300 \\
\hline $\mathrm{H}$ & -1.02563800 & -2.70682100 & -2.47354900 \\
\hline $\mathrm{P}$ & 1.18465200 & -0.53513900 & -0.21896600 \\
\hline $\mathrm{P}$ & -3.92483200 & -1.50655900 & 0.33326200 \\
\hline \multicolumn{4}{|c|}{ tBu-XantPhos } \\
\hline $\mathrm{P}$ & -2.04451300 & -1.11169400 & -0.02870900 \\
\hline $\mathrm{O}$ & 0.00879600 & 0.95343100 & 0.40009400 \\
\hline $\mathrm{P}$ & 2.07973900 & -1.13511200 & 0.16268800 \\
\hline $\mathrm{C}$ & -1.19122400 & 1.55804500 & 0.11847700 \\
\hline $\mathrm{C}$ & -2.32001200 & 0.72543300 & -0.01867800 \\
\hline $\mathrm{C}$ & -3.55399100 & 1.36536600 & -0.22527100 \\
\hline $\mathrm{C}$ & -3.64087900 & 2.75196600 & -0.35611700 \\
\hline
\end{tabular}


$-1.24507800$

$2.95438900 \quad-0.01760100$

$1.15909400 \quad 1.50672500 \quad-0.10276000$

2.24182500

3.36641900

$0.64374400-0.36012300$

3.41359400

2.34630200

$2.57221400 \quad-1.29769700$

$1.20432000 \quad 2.89203000 \quad-0.34068400$

$\begin{array}{lll}0.04867400 & 3.74330800 & 0.19268200\end{array}$

$\begin{array}{lll}-4.46233300 & 0.77089600 & -0.30238200\end{array}$

$\begin{array}{lll}-4.61165200 & 3.22261500 & -0.52940600\end{array}$

$\begin{array}{lll}-2.57088900 & 4.62111800 \quad-0.38388100\end{array}$

$\begin{array}{lll}4.21662000 & 0.58397300 & -1.23723100\end{array}$

$\begin{array}{lll}4.29510300 & 2.98691100 & -1.79249200\end{array}$

$2.41463900 \quad 4.47641900 \quad-1.17319100$

$\begin{array}{lll}-0.00688800 & 5.12509400 & -0.46563600\end{array}$

$0.25846100 \quad 3.92377900 \quad 1.71920400$

$\begin{array}{lll}-0.16186900 & 5.05257800 & -1.55297700\end{array}$

$\begin{array}{lll}-0.82138600 & 5.72674700 & -0.03833900\end{array}$

$0.92356800 \quad 5.68118900 \quad-0.28295300$

$\begin{array}{lll}-0.57048000 & 4.50649400 \quad 2.15128600\end{array}$

$\begin{array}{lll}0.29828300 & 2.95237700 & 2.23437000\end{array}$

$1.20323000 \quad 4.45481200 \quad 1.91588600$

$\begin{array}{lll}-2.48457100 & -1.55481600 & 1.78286300\end{array}$

$-3.36193600 \quad-1.82408000 \quad-1.22964100$

$\begin{array}{lll}2.33460100 & -2.10059400 & -1.47180200\end{array}$

$\begin{array}{lll}3.56943800 & -1.35453700 \quad 1.34663300\end{array}$

$-2.84299100 \quad-3.23716000 \quad-1.57217400$

$\begin{array}{lll}-2.82086800 & -3.90027900 & -0.69591900\end{array}$

$\begin{array}{lll}-3.50410000 & -3.70221900 & -2.32408900\end{array}$

$\begin{array}{lll}-1.82559600 & -3.20137400 & -1.99137400\end{array}$

$\begin{array}{lll}-4.81819900 & -1.94205000 & -0.76253500\end{array}$

$\begin{array}{lll}-5.26183100 & -0.97810400 & -0.47883900\end{array}$

$\begin{array}{lll}-5.43212300 & -2.35282500 & -1.58405600\end{array}$

$\begin{array}{lll}-4.92198900 & -2.62654600 & 0.09125500\end{array}$

$\begin{array}{lll}-3.28842000 & -0.98340600 & -2.51722100\end{array}$

$\begin{array}{lll}-3.80420300 & -1.51766100 & -3.33385300\end{array}$

$\begin{array}{lll}-3.76458500 & 0.00021500 & -2.40787200\end{array}$

$\begin{array}{lll}-2.24568300 & -0.81809300 & -2.83486100\end{array}$

$\begin{array}{lll}-1.30198600 & -1.02783200 & 2.61765600\end{array}$

$\begin{array}{lll}-1.23385500 & 0.06970800 & 2.58486100\end{array}$

$\begin{array}{lll}-1.43970400 & -1.32224600 \quad 3.67291200\end{array}$

$\begin{array}{lll}-0.34131300 & -1.43213300 & 2.26522600\end{array}$

$\begin{array}{lll}-3.78062100 & -0.93867900 & 2.32159800\end{array}$ 


\begin{tabular}{|c|c|c|c|}
\hline $\mathrm{H}$ & -3.76046100 & 0.15956500 & 2.26555300 \\
\hline $\mathrm{H}$ & -4.67240700 & -1.29192000 & 1.78802600 \\
\hline $\mathrm{H}$ & -3.90333400 & -1.21144200 & 3.38525300 \\
\hline $\mathrm{C}$ & -2.52887900 & -3.08410500 & 1.91342400 \\
\hline $\mathrm{H}$ & -3.39772100 & -3.52154900 & 1.40101400 \\
\hline $\mathrm{H}$ & -1.61736100 & -3.55088800 & 1.50778800 \\
\hline $\mathrm{H}$ & -2.60064100 & -3.36241800 & 2.97927300 \\
\hline $\mathrm{C}$ & 3.67802700 & -2.85474000 & 1.66308500 \\
\hline $\mathrm{H}$ & 4.35195800 & -3.00506600 & 2.52432300 \\
\hline $\mathrm{H}$ & 2.69992400 & -3.28994500 & 1.92341000 \\
\hline $\mathrm{H}$ & 4.09261800 & -3.42318300 & 0.81747400 \\
\hline $\mathrm{C}$ & 4.94578500 & -0.82038500 & 0.93224900 \\
\hline $\mathrm{H}$ & 4.95057500 & 0.27472900 & 0.84734800 \\
\hline $\mathrm{H}$ & 5.68502900 & -1.08699000 & 1.70885000 \\
\hline $\mathrm{H}$ & 5.30148100 & -1.24398900 & -0.01605100 \\
\hline $\mathrm{C}$ & 3.12458300 & -0.60747900 & 2.61933800 \\
\hline $\mathrm{H}$ & 3.90238300 & -0.69608200 & 3.39799300 \\
\hline $\mathrm{H}$ & 2.96815400 & 0.46597000 & 2.42464700 \\
\hline $\mathrm{H}$ & 2.18704300 & -1.01632400 & 3.02716600 \\
\hline $\mathrm{C}$ & 3.75658200 & -2.19675800 & -2.03286300 \\
\hline $\mathrm{H}$ & 4.18558100 & -1.21040800 & -2.25892800 \\
\hline $\mathrm{H}$ & 4.43674000 & -2.72249100 & -1.34700200 \\
\hline $\mathrm{H}$ & 3.74113100 & -2.76747300 & -2.97905000 \\
\hline $\mathrm{C}$ & 1.41376600 & -1.42046100 & -2.50082000 \\
\hline $\mathrm{H}$ & 0.39655400 & -1.28094000 & -2.10005000 \\
\hline $\mathrm{H}$ & 1.79659400 & -0.43680900 & -2.80943000 \\
\hline $\mathrm{H}$ & 1.33770800 & -2.05265800 & -3.40267300 \\
\hline $\mathrm{C}$ & 1.79411400 & -3.51939500 & -1.20980200 \\
\hline $\mathrm{H}$ & 0.76179800 & -3.48847600 & -0.82851700 \\
\hline $\mathrm{H}$ & 1.79364100 & -4.09812300 & -2.15015600 \\
\hline $\mathrm{H}$ & 2.40763000 & -4.07069600 & -0.48295600 \\
\hline \multicolumn{4}{|c|}{ EtOAc } \\
\hline $\mathrm{C}$ & 2.13252100 & -0.89047100 & -0.00000300 \\
\hline $\mathrm{H}$ & 2.03729600 & -1.53677800 & 0.88667800 \\
\hline $\mathrm{H}$ & 3.11277600 & -0.39855300 & -0.00019800 \\
\hline $\mathrm{H}$ & 2.03706400 & -1.53706900 & -0.88644500 \\
\hline $\mathrm{C}$ & 1.03915800 & 0.14449200 & -0.00000600 \\
\hline $\mathrm{O}$ & 1.20576100 & 1.34266100 & -0.00000700 \\
\hline $\mathrm{O}$ & -0.17506800 & -0.42991400 & 0.00000700 \\
\hline $\mathrm{C}$ & -1.31494100 & 0.44982100 & 0.00001500 \\
\hline $\mathrm{H}$ & -1.26423600 & 1.10162200 & -0.88719400 \\
\hline $\mathrm{H}$ & -1.26424700 & 1.10159100 & 0.88724600 \\
\hline $\mathrm{C}$ & -2.56363600 & -0.40182000 & -0.00001000 \\
\hline $\mathrm{H}$ & -3.45509600 & 0.24491700 & -0.00000200 \\
\hline
\end{tabular}




\begin{tabular}{|c|c|c|}
\hline-2.60384600 & -1.04489800 & -0.89341400 \\
\hline-2.60386000 & -1.04494200 & 0.89336100 \\
\hline 0.90578500 & 0.76765600 & 0.36893600 \\
\hline 0.75953500 & -0.54849800 & -0.22539800 \\
\hline 0.45237600 & -1.77104400 & 0.52935300 \\
\hline 0.05320200 & -1.76834700 & 1.88802100 \\
\hline 0.79749900 & -3.06986800 & -1.16617000 \\
\hline 1.27873900 & -0.73341900 & -1.17534400 \\
\hline 0.48058100 & -3.02685900 & -0.12027200 \\
\hline-0.31420300 & -2.94431000 & 2.54212600 \\
\hline-0.29587800 & -4.17451300 & 1.87167000 \\
\hline 0.11092900 & -4.20328400 & 0.53320400 \\
\hline-0.61966800 & -2.90107100 & 3.59180000 \\
\hline-0.58818000 & -5.09347600 & 2.38566500 \\
\hline 0.14334300 & -5.15310400 & -0.00828100 \\
\hline 1.78807500 & 1.78447300 & -0.29356300 \\
\hline 3.19024200 & 1.56559400 & 0.11575800 \\
\hline 3.97153300 & 0.79611600 & -0.64302800 \\
\hline 5.29584600 & 0.47149400 & 0.06109100 \\
\hline 3.66494600 & 0.38393500 & -1.74294400 \\
\hline 5.96548400 & 1.77016900 & 0.54170700 \\
\hline 4.96249000 & -0.42813800 & 1.26834300 \\
\hline 6.21700400 & -0.27169400 & -0.91098800 \\
\hline 6.18682200 & 2.44059400 & -0.30488800 \\
\hline 5.32177800 & 2.31341600 & 1.24780500 \\
\hline 6.91689400 & 1.53858300 & 1.04750500 \\
\hline 4.44812900 & -1.34807200 & 0.94687800 \\
\hline 5.88845700 & -0.71845600 & 1.79057400 \\
\hline 4.30874400 & 0.09306500 & 1.98263900 \\
\hline 7.16360300 & -0.53012300 & -0.41029700 \\
\hline 5.75037400 & -1.20037100 & -1.27057900 \\
\hline 6.45142000 & 0.34650400 & -1.79133500 \\
\hline 0.02403700 & -0.82468900 & 2.43607700 \\
\hline 0.82796600 & 0.52557700 & -0.34552500 \\
\hline 0.95657000 & 0.84553700 & 1.46802400 \\
\hline 1.46164700 & 3.21507300 & 0.09112300 \\
\hline 1.52063400 & 3.34834000 & 1.18405100 \\
\hline 0.43515400 & 3.44881100 & -0.22587900 \\
\hline 2.15470700 & 3.92808100 & -0.38270700 \\
\hline 1.76039500 & 1.66392200 & -1.38444500 \\
\hline-2.00759200 & 2.09437600 & -0.13450700 \\
\hline-2.82253400 & 2.21147400 & 0.77847200 \\
\hline-2.97864200 & 1.21441800 & 1.88185000 \\
\hline
\end{tabular}


O

TS-S62

C

C

C

\begin{tabular}{|c|c|c|}
\hline-3.62960200 & 3.25325300 & 0.86072200 \\
\hline-3.16673600 & 0.22525200 & 1.43982300 \\
\hline-2.02478200 & 1.14969100 & 2.42835700 \\
\hline-3.78807700 & 1.48891100 & 2.56936000 \\
\hline 3.53464600 & 4.26794400 & -0.16955300 \\
\hline-2.51241700 & 4.67679600 & -0.16176000 \\
\hline-3.69667800 & 3.78796000 & -1.14698000 \\
\hline-4.57153700 & 5.32513800 & 0.12452700 \\
\hline-4.52011000 & 6.11154500 & -0.64436400 \\
\hline-4.39587200 & 5.79077700 & 1.10684400 \\
\hline-5.58664000 & 4.89837500 & 0.11460700 \\
\hline-2.30778500 & -0.67993500 & -0.91588500 \\
\hline-2.17772200 & -1.59820100 & -1.72346600 \\
\hline-1.42551300 & -1.48333200 & -3.01024800 \\
\hline-2.68374600 & -2.79643300 & -1.50091900 \\
\hline-1.52570000 & -0.46533700 & -3.40920100 \\
\hline-0.35863600 & -1.65975900 & -2.80008500 \\
\hline-1.76663000 & -2.22787300 & -3.74136200 \\
\hline-3.27291700 & -3.04405800 & -0.19977400 \\
\hline-2.53463900 & -2.77007700 & 0.56731100 \\
\hline-4.14993000 & -2.38841000 & -0.08451300 \\
\hline-3.64419300 & -4.50526200 & -0.13011600 \\
\hline-4.08535600 & -4.72283000 & 0.85497900 \\
\hline-2.75528100 & -5.14156400 & -0.25590500 \\
\hline-4.38083200 & -4.76865200 & -0.90541300 \\
\hline-0.06339100 & -2.09766900 & 0.26737800 \\
\hline-1.27681800 & -1.48756700 & 0.83609700 \\
\hline-2.59616200 & -1.66792600 & 0.20713900 \\
\hline-2.75326300 & -1.88687100 & -1.18107600 \\
\hline-3.68588700 & -1.39998700 & 2.05596200 \\
\hline-1.33173100 & -1.43475600 & 1.93468400 \\
\hline-3.77415500 & -1.56803300 & 0.97847700 \\
\hline-4.01692500 & -2.00753600 & -1.75829900 \\
\hline-5.17318400 & -1.90305900 & -0.97462400 \\
\hline-5.04002900 & -1.67811400 & 0.39949200 \\
\hline-4.10201000 & -2.17728900 & -2.83556800 \\
\hline-6.16257700 & -1.99373600 & -1.43000700 \\
\hline-5.93087000 & -1.59418300 & 1.02874400 \\
\hline 1.09334100 & -2.36149200 & 1.07501100 \\
\hline 1.02868500 & -2.00775200 & 2.10938900 \\
\hline 2.61124100 & -0.36914700 & -0.17704900 \\
\hline 1.63265700 & 0.24722800 & -0.65870700 \\
\hline 4.031 & 0.00484700 & -0.64221 \\
\hline
\end{tabular}


$\begin{array}{lll}3.99042700 & 1.22588400 & -1.56645000\end{array}$

$\begin{array}{lll}4.93780600 & -0.56174700 & 1.26744400\end{array}$

$4.49522000 \quad 1.16117300 \quad 1.16171300$

$5.92431200 \quad 0.55795600 \quad 0.28564500$

$\begin{array}{lll}3.37401400 & 1.03482800 & -2.45665600\end{array}$

$\begin{array}{lll}5.01045000 & 1.47815100 & -1.89801800\end{array}$

$\begin{array}{lll}3.57132200 & 2.10486400 \quad-1.05338300\end{array}$

$\begin{array}{lll}5.62042800 & -0.98839900 & -1.75812700\end{array}$

$3.98084500 \quad-1.46212100 \quad-2.27218400$

$4.65126400 \quad-2.09424700 \quad-0.74530200$

$\begin{array}{lll}2.56081900 & -1.31252500 & 0.68309600\end{array}$

$\begin{array}{lll}-0.14469000 & -2.70155500 & -0.64700600\end{array}$

$\begin{array}{lll}-1.86726000 & -1.94258000 & -1.81722300\end{array}$

$\begin{array}{lll}1.82539800 & -3.66126300 & 0.91130100\end{array}$

$1.17365100 \quad-4.46211100 \quad 1.30266600$

$\begin{array}{lll}2.77432800 & -3.68337200 & 1.46383400\end{array}$

$2.01932000 \quad-3.87529000 \quad-0.15089700$

$\begin{array}{lll}-0.13047800 & -0.19229900 & 0.04905500\end{array}$

$\begin{array}{lll}-0.83357900 & 1.63038700 & 0.05819600\end{array}$

$\begin{array}{lll}-0.38439600 & 2.57790000 & 0.70055300\end{array}$

$\begin{array}{lll}0.73181000 & 2.46091400 \quad 1.68865600\end{array}$

$\begin{array}{lll}-0.85888900 & 3.79935600 & 0.55006100\end{array}$

$\begin{array}{lll}0.55723600 & 1.58032700 \quad 2.32363900\end{array}$

$\begin{array}{lll}1.66496900 & 2.28466400 \quad 1.13134500\end{array}$

$\begin{array}{lll}0.83314000 & 3.36755300 & 2.29763200\end{array}$

$\begin{array}{lll}-1.92345300 & 3.99710000 & -0.41536100\end{array}$

$\begin{array}{lll}-1.55487900 & 3.68646800 & -1.40522100\end{array}$

$\begin{array}{lll}-2.76195200 & 3.33642000 & -0.14744300\end{array}$

$\begin{array}{lll}-2.31169700 & 5.45547700 & -0.38393200\end{array}$

$\begin{array}{lll}-3.11902400 & 5.63381700 & -1.11104400\end{array}$

$\begin{array}{lll}-1.45882300 & 6.09917600 & -0.65017900\end{array}$

$\begin{array}{lll}-2.67492300 & 5.74798300 & 0.61364300\end{array}$

$\begin{array}{lll}-0.21735200 & -2.66146500 & -0.18864600 \\ -1.09328200 & -1.98497800 & 0.69822600 \\ -2.43514700 & -1.50162400 & 0.34019600 \\ -2.79988500 & -1.21965000 & -0.99235800 \\ -3.09767300 & -1.41084100 & 2.39483900 \\ -0.91112600 & -2.09149700 & 1.77549400 \\ -3.36690800 & -1.21066100 & 1.35437400 \\ -4.04443000 & -0.66729400 & -1.29409600 \\ -4.96015200 & -0.38344900 & -0.27358200\end{array}$




\begin{tabular}{|c|c|c|c|}
\hline $\mathrm{C}$ & -4.61514000 & -0.66197600 & 1.05258400 \\
\hline $\mathrm{H}$ & -4.30034000 & -0.44810000 & -2.33401900 \\
\hline $\mathrm{H}$ & -5.93272200 & 0.05412000 & -0.51138900 \\
\hline $\mathrm{H}$ & -5.31989100 & -0.44344500 & 1.85907300 \\
\hline $\mathrm{C}$ & 1.14746100 & -2.71299800 & 0.16522700 \\
\hline $\mathrm{H}$ & 1.39701900 & -2.76858900 & 1.23357900 \\
\hline $\mathrm{Ni}$ & 0.46564100 & -0.83456900 & 0.12579800 \\
\hline $\mathrm{C}$ & 2.82979000 & 0.27739100 & 0.48094900 \\
\hline $\mathrm{O}$ & 2.07279500 & -0.00337000 & -0.52625600 \\
\hline $\mathrm{C}$ & 4.13506100 & 1.02265900 & 0.12424300 \\
\hline $\mathrm{C}$ & 4.96479600 & 1.26979900 & 1.38711000 \\
\hline $\mathrm{C}$ & 3.75637600 & 2.36465400 & -0.52759600 \\
\hline $\mathrm{C}$ & 4.93285500 & 0.16767500 & -0.87514800 \\
\hline $\mathrm{H}$ & 5.22762900 & 0.32243700 & 1.88139400 \\
\hline $\mathrm{H}$ & 4.40730400 & 1.87641100 & 2.11638400 \\
\hline $\mathrm{H}$ & 5.89853400 & 1.80022500 & 1.13616900 \\
\hline $\mathrm{H}$ & 3.18835600 & 2.20696000 & -1.45615700 \\
\hline $\mathrm{H}$ & 4.66162000 & 2.94561300 & -0.77127100 \\
\hline $\mathrm{H}$ & 3.13669100 & 2.97405700 & 0.15112600 \\
\hline $\mathrm{H}$ & 4.33680000 & -0.03803700 & -1.77585800 \\
\hline $\mathrm{H}$ & 5.22078400 & -0.79868100 & -0.42880900 \\
\hline $\mathrm{H}$ & 5.85780700 & 0.68659400 & -1.17808200 \\
\hline $\mathrm{O}$ & 2.54762000 & -0.01147800 & 1.64831200 \\
\hline $\mathrm{H}$ & -2.08902000 & -1.41046700 & -1.79926800 \\
\hline $\mathrm{H}$ & -0.52581200 & -2.89701200 & -1.21423500 \\
\hline $\mathrm{C}$ & 2.21800400 & -3.18098300 & -0.77514600 \\
\hline $\mathrm{H}$ & 2.42733100 & -4.25693600 & -0.63430600 \\
\hline $\mathrm{H}$ & 3.15924600 & -2.63955300 & -0.59558900 \\
\hline $\mathrm{H}$ & 1.93341400 & -3.02388600 & -1.82712800 \\
\hline $\mathrm{O}$ & -0.53518800 & 0.85624600 & 0.28018900 \\
\hline $\mathrm{C}$ & -0.88889800 & 1.54336400 & -0.67904000 \\
\hline $\mathrm{C}$ & -0.32978600 & 1.41343100 & -2.05821800 \\
\hline $\mathrm{O}$ & -1.83600600 & 2.44783500 & -0.55813900 \\
\hline $\mathrm{H}$ & -0.51093800 & 2.32125900 & -2.64708800 \\
\hline $\mathrm{H}$ & 0.74034800 & 1.17783400 & -1.98933500 \\
\hline $\mathrm{H}$ & -0.83859000 & 0.56824800 & -2.55079200 \\
\hline $\mathrm{C}$ & -2.53069700 & 2.54331900 & 0.71453700 \\
\hline $\mathrm{H}$ & -2.79562700 & 1.52729300 & 1.03769300 \\
\hline $\mathrm{H}$ & -1.83375400 & 2.96836900 & 1.45343900 \\
\hline $\mathrm{C}$ & -3.74881000 & 3.41033500 & 0.51152600 \\
\hline $\mathrm{H}$ & -4.29294400 & 3.50139800 & 1.46444900 \\
\hline $\mathrm{H}$ & -4.42805200 & 2.96365000 & -0.23099400 \\
\hline $\mathrm{H}$ & -3.47124700 & 4.42135800 & 0.17461900 \\
\hline
\end{tabular}


$\begin{array}{lll}-0.53451900 \quad 1.80814700 & 0.76415900\end{array}$

$0.09350100 \quad 1.95054600 \quad 2.02118800$

$\begin{array}{lll}-1.31761600 & 2.90554800 & -0.92507000\end{array}$

$\begin{array}{lll}-1.40816300 & 0.53604700 & -0.76249300\end{array}$

$\begin{array}{lll}-0.82637200 & 2.98741700 & 0.04818800\end{array}$

$\begin{array}{lll}0.41205900 & 3.20903500 & 2.53172600\end{array}$

$0.12070400 \quad 4.36819000 \quad 1.80173700$

$\begin{array}{lll}-0.50277100 & 4.24735900 & 0.55572400\end{array}$

$\begin{array}{lll}0.89775800 & 3.28748900 & 3.50835400\end{array}$

$\begin{array}{lll}0.37584500 & 5.35289800 & 2.20081600\end{array}$

$\begin{array}{lll}-0.74170200 & 5.14185700 & -0.02586400\end{array}$

$\begin{array}{lll}-1.22675100 & -1.93444400 & 0.24119900\end{array}$

$\begin{array}{lll}-3.31514700 & -1.99321500 & 0.40079800\end{array}$

$\begin{array}{lll}-3.83763900 & -1.28456900 & -0.52419800\end{array}$

$\begin{array}{lll}-5.27375300 & -0.76481800 & -0.20548200\end{array}$

$\begin{array}{lll}-3.29296000 & -0.95087300 & -1.59013200\end{array}$

$\begin{array}{lll}-6.13369500 & -1.91156800 & 0.34402600\end{array}$

$\begin{array}{lll}-5.11443300 & 0.32574000 & 0.86979400\end{array}$

$\begin{array}{lll}-5.92223700 & -0.17189200 & -1.45894300\end{array}$

$\begin{array}{lll}-6.26634700 & -2.70580400 & -0.41018600\end{array}$

$\begin{array}{lll}-5.65770000 & -2.36399400 & 1.22553000\end{array}$

$\begin{array}{lll}-7.13646800 & -1.55020000 & 0.63071500\end{array}$

$\begin{array}{lll}-4.48539300 & 1.15381300 & 0.50255300\end{array}$

$\begin{array}{lll}-6.09419600 & 0.74499200 & 1.15691400\end{array}$

$\begin{array}{lll}-4.63402600 & -0.08544400 & 1.77039500\end{array}$

$\begin{array}{lll}-6.92725000 & 0.22228300 & -1.22934400\end{array}$

$\begin{array}{lll}-5.31125800 & 0.64497500 & -1.86899500\end{array}$

$\begin{array}{lll}-6.02617500 & -0.93131100 & -2.25028300\end{array}$

$0.34563800 \quad 1.06071200 \quad 2.60146200$

$0.79765300 \quad-0.53804200 \quad-0.12831300$

$\begin{array}{lll}-0.77822800 & -0.75709100 & 1.98928500\end{array}$

$\begin{array}{lll}-0.98215000 & -3.27640100 & 0.85312600\end{array}$

$\begin{array}{lll}-1.07680900 & -3.24201100 & 1.94970500\end{array}$

$0.03880800 \quad-3.61717600 \quad 0.61083900$

$\begin{array}{lll}-1.69251700 & -4.01590100 & 0.46071300\end{array}$

$\begin{array}{lll}-1.38473900 & -1.88633400 & -0.83621000\end{array}$

$2.14498600 \quad-2.00678100 \quad-0.11599400$

$\begin{array}{lll}3.10430600 & -2.03249500 & 0.65676000\end{array}$

$3.32724700 \quad-1.01518300 \quad 1.72958800$

$\begin{array}{lll}4.01587300 & -2.97869200 & 0.59558500\end{array}$

$\begin{array}{lll}3.37753600 & -0.01897100 & 1.26556300\end{array}$

$2.45552200 \quad-1.02092100 \quad 2.40260600$ 
$4.24238000 \quad-1.21716800 \quad 2.29891400$

$3.86994300 \quad-4.00583500 \quad-0.42081600$

$2.91513000 \quad-4.52644600 \quad-0.25020400$

$3.81547800 \quad-3.51400500 \quad-1.40381600$

$5.05359600 \quad-4.93572800 \quad-0.31090200$

$4.96655900 \quad-5.72688500 \quad-1.07159800$

$5.09281100 \quad-5.41302900 \quad 0.68058400$

$\begin{array}{lll}5.99874600 & -4.39659800 & -0.47992700\end{array}$

$\begin{array}{lll}2.04727500 & 0.81469600 & -0.87088800\end{array}$

$\begin{array}{lll}1.76464800 & 1.62343700 & -1.75763900\end{array}$

$\begin{array}{lll}0.89049300 & 1.31059300 & -2.92763700\end{array}$

$2.20423700 \quad 2.86201400 \quad-1.72511800$

$\begin{array}{lll}1.01454500 & 0.25826800 & -3.21542700\end{array}$

$\begin{array}{lll}-0.15800300 & 1.45731400 & -2.62173100\end{array}$

$\begin{array}{lll}1.10253300 & 1.97728400 & -3.77339700\end{array}$

$\begin{array}{lll}2.91767300 & 3.29859600 & -0.53751800\end{array}$

$2.31344700 \quad 3.03068800 \quad 0.34043900$

$\begin{array}{lll}3.86869700 & 2.74628100 & -0.48492200\end{array}$

$3.13008700 \quad 4.78831300 \quad-0.64743700$

$3.66194000 \quad 5.14607100 \quad 0.24777000$

$\begin{array}{lll}2.16736000 & 5.31755700 & -0.70918400\end{array}$

$\begin{array}{lll}3.73266400 & 5.04338500 & -1.53321000\end{array}$

$\begin{array}{lll}-0.21735200 & -2.66146500 & 0.18864600\end{array}$

$\begin{array}{lll}-1.09328200 & -1.98497800 & -0.69822600\end{array}$

$\begin{array}{lll}-2.43514700 & -1.50162400 & -0.34019600\end{array}$

$\begin{array}{lll}-2.79988500 & -1.21965000 & 0.99235800\end{array}$

$-3.09767300 \quad-1.41084100 \quad-2.39483900$

$\begin{array}{lll}-0.91112600 & -2.09149700 & -1.77549400\end{array}$

$\begin{array}{lll}-3.36690800 & -1.21066100 & -1.35437400\end{array}$

$\begin{array}{lll}-4.04443000 & -0.66729400 & 1.29409600\end{array}$

$\begin{array}{lll}-4.96015200 & -0.38344900 & 0.27358200\end{array}$

$\begin{array}{lll}-4.61514000 & -0.66197600 & -1.05258400\end{array}$

$\begin{array}{lll}-4.30034000 & -0.44810000 & 2.33401900\end{array}$

$\begin{array}{lll}-5.93272200 & 0.05412000 & 0.51138900\end{array}$

$\begin{array}{lll}-5.31989100 & -0.44344500 & -1.85907300\end{array}$

$\begin{array}{lll}1.14746100 & -2.71299800 & -0.16522700\end{array}$

$1.39701900 \quad-2.76858900 \quad-1.23357900$

$0.46564100 \quad-0.83456900 \quad-0.12579800$

$\begin{array}{lll}2.82979000 & 0.27739100 & -0.48094900\end{array}$

$2.07279500 \quad-0.00337000 \quad 0.52625600$

$4.13506100 \quad 1.02265900 \quad-0.12424300$

$\begin{array}{lll}4.96479600 & 1.26979900 & -1.38711000\end{array}$

$3.75637600 \quad 2.36465400 \quad 0.52759600$ 


\begin{tabular}{|c|c|c|}
\hline 4.93285500 & 0.16767500 & 0.87514800 \\
\hline 5.22762900 & 0.32243700 & -1.88139400 \\
\hline 4.40730400 & 1.87641100 & -2.11638400 \\
\hline 5.89853400 & 1.80022500 & -1.13616900 \\
\hline 3.18835600 & 2.20696000 & 1.45615700 \\
\hline 4.66162000 & 2.94561300 & 0.77127100 \\
\hline 3.13669100 & 2.97405700 & -0.15112600 \\
\hline 4.33680000 & -0.03803700 & 1.77585800 \\
\hline 5.22078400 & -0.79868100 & 0.42880900 \\
\hline 5.85780700 & 0.68659400 & 1.17808200 \\
\hline 2.54762000 & -0.01147800 & -1.64831200 \\
\hline 2.08902000 & -1.41046700 & 1.79926800 \\
\hline 0.52581200 & -2.89701200 & 1.21423500 \\
\hline 2.21800400 & -3.18098300 & 0.77514600 \\
\hline 2.42733100 & -4.25693600 & 0.63430600 \\
\hline 3.15924600 & -2.63955300 & 0.59558900 \\
\hline 1.93341400 & -3.02388600 & 1.82712800 \\
\hline 0.53518800 & 0.85624600 & -0.28018900 \\
\hline 0.88889800 & 1.54336400 & 0.67904000 \\
\hline 0.32978600 & 1.41343100 & 2.05821800 \\
\hline 1.83600600 & 2.44783500 & 0.55813900 \\
\hline 0.51093800 & 2.32125900 & 2.64708800 \\
\hline 0.74034800 & 1.17783400 & 1.98933500 \\
\hline 0.83859000 & 0.56824800 & 2.55079200 \\
\hline 2.53069700 & 2.54331900 & -0.71453700 \\
\hline 2.79562700 & 1.52729300 & -1.03769300 \\
\hline 1.83375400 & 2.96836900 & -1.45343900 \\
\hline 3.74881000 & 3.41033500 & -0.51152600 \\
\hline 4.29294400 & 3.50139800 & -1.46444900 \\
\hline 4.42805200 & 2.96365000 & 0.23099400 \\
\hline 47124700 & 4.42135800 & -0.17461900 \\
\hline
\end{tabular}

Cartesian coordinates of the structures (optimization in THF) TS5/TS22

$\begin{array}{llll}\text { C } & -1.14961800 & 0.65938400 & 2.17690100 \\ \text { C } & -0.20510500 & 0.37634900 & 1.16880100 \\ \text { C } & 1.01726200 & 1.08344100 & 1.22448300 \\ \text { C } & 1.17029200 & 2.19284100 & 2.08412700 \\ \text { C } & 0.21250700 & 2.41325000 & 3.07749400 \\ \text { C } & -0.92472700 & 1.61112000 & 3.16396000 \\ \text { C } & 3.49281400 & 2.45548200 & 1.27440000 \\ \text { C } & 3.32826800 & 1.22806000 & 0.60317600 \\ \text { C } & 4.44121200 & 0.51272200 & 0.09864700 \\ \text { C } & 5.70500200 & 1.11714400 & 0.21101700 \\ \text { C } & 5.88388100 & 2.34109700 & 0.84811800\end{array}$


$\begin{array}{lll}4.78002600 & 2.98957300 \quad 1.39656300\end{array}$

$-2.10411200$

$0.12868500 \quad 2.14660200$

0.33451800

3.24298300

3.77309600

$-1.66740700$

$1.78205300 \quad 3.94624800$

$\begin{array}{lll}6.57185300 & 0.60811100 & -0.20213000\end{array}$

$\begin{array}{lll}6.87971900 & 2.78334100 & 0.92602900\end{array}$

$\begin{array}{lll}4.92095800 & 3.94091700 & 1.90865700\end{array}$

$\begin{array}{lll}2.26405300 \quad 3.20903400 & 1.76662000\end{array}$

$2.58433000 \quad 4.11284300 \quad 2.96447500$

$\begin{array}{lll}3.33445400 & 4.86822300 & 2.69466500\end{array}$

$2.96282000 \quad 3.53571700 \quad 3.82192100$

$1.69106900 \quad 4.66732200 \quad 3.28266200$

$\begin{array}{lll}1.74368400 & 4.10119100 & 0.60724500\end{array}$

$0.83032000 \quad 4.63343900 \quad 0.91520000$

$\begin{array}{lll}1.50507600 & 3.50289200 & -0.28434400\end{array}$

$\begin{array}{lll}2.50840500 & 4.84273900 & 0.32648900\end{array}$

$2.06075300 \quad 0.70867700 \quad 0.40602100$

$\begin{array}{lll}-0.43202600 & -2.47770000 & 0.37380300\end{array}$

$\begin{array}{lll}1.06710600 & -2.61308000 & 0.62783800\end{array}$

$1.40219000 \quad-1.90074900 \quad 1.39488800$

$\begin{array}{lll}1.67696200 & -2.43858800 & -0.26450900\end{array}$

$\begin{array}{lll}1.28603300 & -3.63059500 & 0.99670300\end{array}$

$\begin{array}{lll}-1.18587800 & -2.76449700 & 1.68417100\end{array}$

$\begin{array}{lll}-1.02138200 & -3.81533800 & 1.97604800\end{array}$

$\begin{array}{lll}-2.26512000 & -2.60615200 & 1.57360000\end{array}$

$\begin{array}{lll}-0.82456400 & -2.12944100 & 2.50685600\end{array}$

$\begin{array}{lll}-0.90492500 & -3.46425900 & -0.70185500\end{array}$

$-1.97266400 \quad-3.33402100 \quad-0.92181400$

$\begin{array}{lll}-0.76234600 & -4.49623700 & -0.33921000\end{array}$

$\begin{array}{lll}-0.33971700 & -3.36034200 & -1.63826000\end{array}$

$\begin{array}{lll}-0.30192100 & -0.24115800 & -1.85994700\end{array}$

$\begin{array}{lll}-1.41893900 & -0.64060500 & -2.84488200\end{array}$

$\begin{array}{lll}-1.65294300 & -1.71368400 & -2.80036300\end{array}$

$\begin{array}{lll}-1.09166600 & -0.40886000 & -3.87302100\end{array}$

$\begin{array}{lll}-2.34517700 & -0.08079800 & -2.64582100\end{array}$

$1.01338600 \quad-0.87846300 \quad-2.30185700$

$\begin{array}{lll}1.82631700 & -0.68160700 & -1.59614300\end{array}$

$1.30063500 \quad-0.45942500 \quad-3.28170400$

$0.92782100 \quad-1.96658200 \quad-2.42885500$

$\begin{array}{lll}-0.16568100 & 1.29090800 & -1.88637300\end{array}$

$\begin{array}{lll}-1.03668800 & 1.78314700 & -1.43539800\end{array}$

$\begin{array}{lll}-0.08607800 & 1.62755300 & -2.93384900\end{array}$

$\begin{array}{lll}0.73586000 & 1.62565400 & -1.35948500\end{array}$

$5.29940900 \quad-2.24871700 \quad 0.59277400$ 


\begin{tabular}{lrr}
-3.42010200 & 3.91653100 & -0.90076900 \\
-3.01649700 & 4.35072500 & -2.32047700 \\
-2.32838000 & 4.31172600 & 0.10022100 \\
-4.76268600 & 4.56359000 & -0.51362000 \\
-3.78923100 & 4.07642800 & -3.05265100 \\
-2.07101100 & 3.87455200 & -2.62598900 \\
-2.87417300 & 5.44275600 & -2.35529900 \\
-2.59732700 & 4.02130200 & 1.12580300 \\
-2.17498200 & 5.40262600 & 0.07563200 \\
-1.37270200 & 3.82340500 & -0.13887300 \\
-4.66697700 & 5.66139600 & -0.50736000 \\
\hline-5.08157800 & 4.24503000 & 0.49227100 \\
\hline-5.55363300 & 4.28920100 & -1.22718400 \\
-4.40909200 & 1.93045500 & -1.78534700 \\
\hline-5.65952800 & -0.20433200 & 0.41681900 \\
-4.97527300 & -1.43355400 & 2.10078000 \\
-6.71041200 & 1.02451600 & -1.72424600 \\
-7.45656500 & 0.22244800 & -1.86019300 \\
-6.78679100 & 1.71721000 & -2.57332800 \\
-6.96069800 & 1.56072800 & -0.79584500 \\
-3.11040800 & -0.26381700 & -0.16938300 \\
\hline
\end{tabular}

TS19/TS23/TS-Conformation-B

\begin{tabular}{|c|c|c|c|}
\hline $\mathrm{C}$ & -2.18393500 & -3.47005500 & 1.73589200 \\
\hline $\mathrm{C}$ & -1.57804400 & -2.33492900 & 1.17000700 \\
\hline $\mathrm{C}$ & -2.10122600 & -1.89449600 & -0.06013800 \\
\hline $\mathrm{C}$ & -3.01046400 & -2.64341200 & -0.81781500 \\
\hline $\mathrm{C}$ & -3.57952500 & -3.77198500 & -0.21873200 \\
\hline $\mathrm{C}$ & -3.20222400 & -4.15406800 & 1.07092300 \\
\hline $\mathrm{C}$ & -3.34235900 & -0.72024500 & -2.29017400 \\
\hline $\mathrm{C}$ & -2.54030300 & 0.01730100 & -1.40744800 \\
\hline $\mathrm{C}$ & -2.62774800 & 1.41391200 & -1.27772500 \\
\hline $\mathrm{C}$ & -3.52432600 & 2.07159800 & -2.13720500 \\
\hline $\mathrm{C}$ & -4.29799200 & 1.37065400 & -3.06056700 \\
\hline $\mathrm{C}$ & -4.22068200 & -0.02072200 & -3.12343800 \\
\hline $\mathrm{H}$ & -1.84180600 & -3.84514000 & 2.69651900 \\
\hline $\mathrm{H}$ & -4.30936500 & -4.37051300 & -0.76418800 \\
\hline $\mathrm{H}$ & -3.66475800 & -5.02619400 & 1.53840600 \\
\hline $\mathrm{H}$ & -3.64237200 & 3.14984500 & -2.06702800 \\
\hline $\mathrm{H}$ & -4.98463500 & 1.91040400 & -3.71654500 \\
\hline $\mathrm{H}$ & -4.85244200 & -0.56346100 & -3.82646100 \\
\hline $\mathrm{C}$ & -3.20026400 & -2.23837300 & -2.28046900 \\
\hline $\mathrm{C}$ & -1.91072600 & -2.61831100 & -3.05545800 \\
\hline $\mathrm{H}$ & -2.00334400 & -2.31385900 & -4.10946600 \\
\hline $\mathrm{H}$ & -1.02389400 & -2.12322900 & -2.63552800 \\
\hline
\end{tabular}




\begin{tabular}{|c|c|c|}
\hline 1.74706500 & -3.70627800 & -3.01609600 \\
\hline-4.39274600 & -2.94485300 & -2.92809000 \\
\hline-4.25096400 & -4.03471000 & -2.90564500 \\
\hline-5.33865600 & -2.70616000 & -2.41880400 \\
\hline-4.48541100 & -2.65957300 & -3.98535100 \\
\hline-1.65659900 & -0.68026400 & -0.58028300 \\
\hline 0.16042400 & -1.37131700 & 3.53246700 \\
\hline-0.54589800 & -2.42304200 & 4.39660400 \\
\hline-1.63286300 & -2.41720200 & 4.23652200 \\
\hline-0.16903500 & -3.44087800 & 4.23684200 \\
\hline-0.37531400 & -2.17287900 & 5.45800000 \\
\hline-0.49066400 & -0.00853900 & 3.80865500 \\
\hline-0.33771800 & 0.26000900 & 4.86754200 \\
\hline-0.04823900 & 0.78099900 & 3.18789700 \\
\hline-1.57136000 & -0.03323700 & 3.62465400 \\
\hline 1.64185400 & -1.28108900 & 3.93159900 \\
\hline 2.18666900 & -0.54343600 & 3.32692300 \\
\hline 1.70237000 & -0.95328300 & 4.98273100 \\
\hline 2.15666200 & -2.24825300 & 3.85757500 \\
\hline 1.18076500 & -3.12171300 & 1.11609000 \\
\hline 1.05257800 & -4.33806300 & 2.04198800 \\
\hline 1.61214100 & -5.17830800 & 1.59651500 \\
\hline 1.48434700 & -4.15059900 & 3.03484100 \\
\hline 0.01456800 & -4.67165800 & 2.16658200 \\
\hline 0.73407000 & -3.51716000 & -0.30315400 \\
\hline 0.66714900 & -2.63652700 & -0.95961000 \\
\hline 1.48700900 & -4.19479200 & -0.73785000 \\
\hline-0.23380000 & -4.03645800 & -0.31441500 \\
\hline 2.66454300 & -2.70601400 & 1.03227700 \\
\hline 2.99978700 & -2.09095500 & 1.87496000 \\
\hline 3.27905200 & -3.62219700 & 1.02992000 \\
\hline 2.88914100 & -2.18048200 & 0.09609000 \\
\hline-1.35964700 & 4.03743200 & -0.53055900 \\
\hline-2.51173800 & 5.04748400 & -0.43837300 \\
\hline-3.41244300 & 4.74356800 & -0.98666700 \\
\hline 9602600 & 5.24754300 & 0.60369300 \\
\hline-2.17341400 & 6.00472000 & -0.87186700 \\
\hline-0.20541900 & 4.56667500 & 0.34273900 \\
\hline-0.44561900 & 4.54587700 & 1.41557200 \\
\hline 0.71804900 & 3.99459500 & 0.18876100 \\
\hline 0.00409600 & 5.61505600 & 0.07020900 \\
\hline-0.85065200 & 3.96654200 & -1.97977300 \\
\hline & & \\
\hline & 3.20710900 & -2.10012900 \\
\hline
\end{tabular}




\begin{tabular}{|c|c|c|c|}
\hline $\mathrm{H}$ & -1.64593800 & 3.74650400 & -2.70343200 \\
\hline $\mathrm{C}$ & -3.07413700 & 2.25129800 & 1.42471800 \\
\hline $\mathrm{C}$ & -4.42229200 & 2.87966400 & 1.05312000 \\
\hline $\mathrm{H}$ & -5.15303700 & 2.64510400 & 1.84730000 \\
\hline $\mathrm{H}$ & -4.37776500 & 3.97064700 & 0.96633900 \\
\hline $\mathrm{H}$ & -4.82303400 & 2.47082100 & 0.11406000 \\
\hline $\mathrm{C}$ & -2.49270700 & 2.94646400 & 2.66568500 \\
\hline $\mathrm{H}$ & -2.35965300 & 4.02638000 & 2.50320600 \\
\hline $\mathrm{H}$ & -3.18059500 & 2.82244600 & 3.51961300 \\
\hline $\mathrm{H}$ & -1.52006300 & 2.52126900 & 2.95296400 \\
\hline $\mathrm{C}$ & -3.31984000 & 0.76274400 & 1.72421500 \\
\hline $\mathrm{H}$ & -3.92025200 & 0.28767400 & 0.93455300 \\
\hline $\mathrm{H}$ & -2.38151900 & 0.20532300 & 1.81402100 \\
\hline $\mathrm{H}$ & -3.87636900 & 0.65946900 & 2.67050200 \\
\hline $\mathrm{C}$ & 1.50491700 & 1.12461900 & -0.91754100 \\
\hline $\mathrm{C}$ & 2.08471000 & 0.53263900 & 0.27019600 \\
\hline $\mathrm{C}$ & 2.61476500 & 1.31212200 & 1.41000100 \\
\hline $\mathrm{C}$ & 1.85589200 & 2.29839600 & 2.06634100 \\
\hline $\mathrm{H}$ & 4.50129100 & 0.25934900 & 1.42860200 \\
\hline $\mathrm{H}$ & 2.69527100 & -0.34541100 & 0.06020100 \\
\hline $\mathrm{C}$ & 3.89772700 & 1.02789300 & 1.91720800 \\
\hline $\mathrm{C}$ & 2.35811600 & 2.98259700 & 3.17342000 \\
\hline $\mathrm{C}$ & 3.63709900 & 2.69149800 & 3.66222400 \\
\hline $\mathrm{C}$ & 4.40397400 & 1.70883900 & 3.02734400 \\
\hline $\mathrm{H}$ & 1.74153800 & 3.73925000 & 3.66661800 \\
\hline $\mathrm{H}$ & 4.03029100 & 3.22231800 & 4.53299300 \\
\hline $\mathrm{H}$ & 5.40339000 & 1.46886200 & 3.40035600 \\
\hline $\mathrm{C}$ & 1.38293500 & 0.33263700 & -2.07525000 \\
\hline $\mathrm{O}$ & 3.21354900 & 0.44221800 & -2.99403400 \\
\hline $\mathrm{C}$ & 3.99781700 & -0.47460300 & -2.57825100 \\
\hline $\mathrm{C}$ & 5.52049900 & -0.19260400 & -2.75550400 \\
\hline $\mathrm{O}$ & 3.65931300 & -1.52738600 & -2.01004900 \\
\hline $\mathrm{C}$ & 5.77517100 & 0.94492700 & -3.74895300 \\
\hline $\mathrm{C}$ & 6.04288800 & 0.20384000 & -1.36179900 \\
\hline $\mathrm{C}$ & 6.22006500 & -1.47400500 & -3.22660800 \\
\hline $\mathrm{H}$ & 5.38003100 & 0.69775700 & -4.74730500 \\
\hline $\mathrm{H}$ & 5.28566400 & 1.87328300 & -3.42230800 \\
\hline $\mathrm{H}$ & 6.85737700 & 1.13679400 & -3.84930900 \\
\hline $\mathrm{H}$ & 5.88194500 & -0.61189100 & -0.64055100 \\
\hline $\mathrm{H}$ & 7.12278700 & 0.42832900 & -1.39560700 \\
\hline $\mathrm{H}$ & 5.52108900 & 1.09843900 & -0.98486000 \\
\hline $\mathrm{H}$ & 7.31311600 & -1.33158000 & -3.27525600 \\
\hline $\mathrm{H}$ & 6.00250600 & -2.30461700 & -2.54039500 \\
\hline $\mathrm{H}$ & 5.87395500 & -1.76925800 & -4.23163200 \\
\hline
\end{tabular}


$\mathrm{H}$

$\mathrm{Ni}$

P

P

$\mathrm{H}$

C

H

$\mathrm{H}$

$\mathrm{H}$

$\mathrm{H}$

TS-Conformation-A

C

C

C

C

H

$\mathrm{H}$

C

C

C

C

$\mathrm{H}$

$\begin{array}{rrr}0.84579700 & 2.49761900 & 1.71438000 \\ 0.21453900 & 0.06426600 & 0.26052100 \\ 0.07259400 & -1.62810200 & 1.63978700 \\ -1.69591400 & 2.24453200 & 0.08796100 \\ 1.34922000 & 2.20326000 & -0.98938500 \\ 0.58601100 & 0.75045500 & -3.27010900 \\ 0.82896200 & 1.77918200 & -3.57310700 \\ -0.49094900 & 0.71618600 & -3.03936800 \\ 0.77704600 & 0.08322300 & -4.12123100 \\ 1.56253700 & -0.73547500 & -1.95340000\end{array}$

$-1.96351700$

$-1.82487400$

$-1.57096300$

$-1.51412600$

$-1.47134700$

$-2.48200500$

$-1.41190700$

$-1.27806000$

$-1.09820800$

$-1.17444700$

$-1.24374400$

$-0.90901100$

$-1.04525300$

$-2.52120400$

$-5.09389400$

$-4.66890100$

$-6.13231700$

$-6.79062600$

$-7.19904000$

$-5.34104600$

$-6.03740600$

$-7.33802900$

$-7.50738200$

$-6.72788800$

$-7.90121700$

$-7.78814300$

$-6.00670100$

$-4.84430600$

$-4.56178800$

$-4.72187500$

$0.65807700 \quad-3.72351800 \quad 1.38117200$

$\begin{array}{lll}0.71616800 & -2.36756800 & 1.02245800\end{array}$

$\begin{array}{lll}1.53699500 & -2.03578000 & -0.06960800\end{array}$
$1.37628200 \quad-0.68911300$

$-0.04507900 \quad-0.52415600$

$-0.94954800-1.66802500$

$-0.49676600 \quad-3.00276900$

$-2.72214300 \quad-0.43954100$

$-0.47146900 \quad 0.23119800$

$-2.33431100-1.45255300$

$-1.37714200 \quad-4.06051900$

$-2.74275200 \quad-3.82007000$

$-3.21599800-2.50604800$

$-0.99172200 \quad-5.08306800$

$-3.43078600 \quad-4.64777400$

$-4.28097800 \quad-2.29625000$

$2.13610900 \quad 0.33716800$

$0.68558500 \quad 0.07572300$

$-0.07544300 \quad 0.96854000$

$0.12100000 \quad-0.94685200$

$-1.15241200 \quad-0.40977100$

$1.18021900-1.25220600$

$-0.20276700 \quad-2.22800800$

$-1.92243300-0.19054400$

$-0.95382800 \quad 0.52600000$

$-1.56229300-1.14267700$

$2.10966300 \quad-1.60159400$

$0.82524900 \quad-2.02671100$

$1.42265000 \quad-0.35130800$

$-0.59827100 \quad-3.01494000$

$0.69936500 \quad-2.61667100$

$\begin{array}{ll}-0.95648100 & -2.03309900\end{array}$

$1.87805000-0.13464100$

$-3.72351800-1.38117200$ 
$2.36700000 \quad-2.95557100 \quad-0.72028200$

$2.28384000 \quad-4.29541200 \quad-0.32604900$

$1.41215700 \quad-4.67960200 \quad 0.69700600$

$3.78037700 \quad-1.03534700 \quad-1.32519700$

$2.81701000 \quad-0.18863900 \quad-0.75938900$

3.05108400

$1.16086900-0.44530800$

$\begin{array}{lll}4.36678200 & 1.62506600 & -0.61001500\end{array}$

$\begin{array}{lll}5.37190400 & 0.78264500 & -1.08971900\end{array}$

$5.07605100 \quad-0.53049800 \quad-1.47163000$

$\begin{array}{lll}0.03792500 & -4.03891000 & 2.21875000\end{array}$

$2.90188700 \quad-5.04872900 \quad-0.81553200$

$\begin{array}{lll}1.34674400 & -5.73032200 & 0.98852900\end{array}$

$\begin{array}{lll}4.61051100 & 2.65853400 & -0.36665100\end{array}$

$6.39035900 \quad 1.16257700 \quad-1.19707700$

$5.86297800 \quad-1.15679500 \quad-1.89313600$

$3.29835600 \quad-2.41034100 \quad-1.80652000$

$\begin{array}{lll}4.46127100 & -3.36099600 & -2.09717500\end{array}$

$\begin{array}{lll}5.11197200 & -2.94848300 & -2.88154600\end{array}$

$5.07224900 \quad-3.54671500 \quad-1.20062200$

$\begin{array}{lll}4.08801700 & -4.32600900 & -2.46825700\end{array}$

$2.47554900 \quad-2.20062700 \quad-3.10405800$

$2.09177700 \quad-3.16518000 \quad-3.46904100$

$1.61587900 \quad-1.53854500 \quad-2.93385800$

$3.10825300 \quad-1.75399200 \quad-3.88713700$

$1.57652400 \quad-0.71686400 \quad-0.47988000$

$\begin{array}{lll}-0.00791000 & -0.96127800 & 1.98035500\end{array}$

$\begin{array}{lll}1.58472900 & 2.22616300 & -0.05204500\end{array}$

$\begin{array}{lll}-1.59030300 & -1.58361400 & 2.89454000\end{array}$

$\begin{array}{lll}-2.46440300 & -2.47816900 & 1.99878300\end{array}$

$\begin{array}{lll}-2.82388200 & -1.96922600 & 1.10107600\end{array}$

$\begin{array}{lll}-1.96178500 & -3.41091800 \quad 1.71006500\end{array}$

$\begin{array}{lll}-3.36532300 & -2.75188700 & 2.57230500\end{array}$

$\begin{array}{lll}-2.39428300 & -0.31606700 \quad 3.25311600\end{array}$

$\begin{array}{lll}-1.76651300 & 0.48179200 & 3.67539100\end{array}$

$\begin{array}{lll}-2.95623800 & 0.06460200 & 2.39061800\end{array}$

$\begin{array}{lll}-3.14743100 & -0.57859900 \quad 4.01551000\end{array}$

$\begin{array}{lll}-1.32343900 & -2.36783600 & 4.18913900\end{array}$

$\begin{array}{lll}-0.86853500 & -1.74638800 & 4.96940300\end{array}$

$\begin{array}{lll}-2.29504300 & -2.71318400 \quad 4.58034700\end{array}$

$\begin{array}{lll}-0.69765600 & -3.25724200 & 4.03950400\end{array}$

$\begin{array}{lll}1.38979700 & -0.75795300 & 3.30529400\end{array}$

$\begin{array}{lll}2.65002300 & -0.39833000 & 2.51013900\end{array}$

$3.05291500 \quad-1.27081100 \quad 1.97713500$

$\begin{array}{lll}2.45782000 & 0.38731300 \quad 1.78050000\end{array}$ 
H

C

$\mathrm{H}$

$\mathrm{H}$

$\mathrm{H}$

C

$\mathrm{H}$

$\mathrm{H}$

$\mathrm{H}$

C

\begin{tabular}{|c|c|c|}
\hline 3.43186300 & -0.04114800 & 3.19912300 \\
\hline 0.98613700 & 0.41370800 & 4.21255600 \\
\hline 0.14701300 & 0.15267000 & 4.87278600 \\
\hline 1.83602800 & 0.69898700 & 4.85528700 \\
\hline 0.69131200 & 1.29765100 & 3.62760300 \\
\hline 1.75815500 & -1.98840800 & 4.15026500 \\
\hline 1.87363900 & -2.89207900 & 3.53460800 \\
\hline 2.73567100 & -1.79632800 & 4.62524900 \\
\hline 1.04925700 & -2.20050500 & 4.95484300 \\
\hline 2.12432300 & 3.58929100 & 1.18426600 \\
\hline 2.98390600 & 2.97579200 & 2.29873500 \\
\hline 2.44640500 & 2.20036600 & 2.85104800 \\
\hline 3.92352100 & 2.54745300 & 1.92340600 \\
\hline 3.24145100 & 3.77126100 & 3.01796600 \\
\hline 2.88057100 & 4.78942800 & 0.59963300 \\
\hline 3.11042600 & 5.48711200 & 1.42283900 \\
\hline 3.83576200 & 4.50834600 & 0.13673000 \\
\hline 2.28542800 & 5.34401200 & -0.13633700 \\
\hline 0.81326800 & 4.09886700 & 1.80980000 \\
\hline 1.04480400 & 4.82888900 & 2.60335400 \\
\hline 0.18035500 & 4.60479100 & 1.06791600 \\
\hline 0.22879500 & 3.28048100 & 2.25711900 \\
\hline 1.37280300 & 3.00138200 & -1.80953400 \\
\hline 2.61303400 & 3.65656400 & -2.43348200 \\
\hline 2.35579600 & 3.94616000 & -3.46714400 \\
\hline 2.93773100 & 4.56450900 & -1.91536600 \\
\hline 3.46241900 & 2.96409400 & -2.49790800 \\
\hline 0.23374800 & 4.02521300 & -1.74697500 \\
\hline 0.51657100 & 4.92136000 & -1.17625300 \\
\hline-0.02779100 & 4.35109100 & -2.76773100 \\
\hline-0.66316600 & 3.60019700 & -1.28972500 \\
\hline 0.98177900 & 1.82319900 & -2.71651900 \\
\hline 1.84796200 & 1.18457500 & -2.94140500 \\
\hline 0.21260700 & 1.19216100 & -2.26509700 \\
\hline 0.59227300 & 2.20740900 & -3.67366700 \\
\hline .24440000 & 0.76350300 & 0.38620200 \\
\hline-1.68362000 & 0.55605900 & -3.22532000 \\
\hline 1.78837700 & 1.85991400 & -1.65124100 \\
\hline-2.72614900 & 1.62943600 & 1.27796900 \\
\hline 2.65894100 & 3.61853500 & 0.33206400 \\
\hline-2.75242400 & 4.01788200 & -0.68737100 \\
\hline-3.54756900 & 3.90820800 & 0.90702600 \\
\hline 1.78312800 & 4.08606600 & 0.80810200 \\
\hline
\end{tabular}

3/Conformation-A 
$1.90629100 \quad 1.21242000 \quad 0.98453100$

$\begin{array}{lll}1.64982700 & -0.15991600 & 1.25700500\end{array}$

$\begin{array}{lll}0.97839800 & -0.60658500 & 2.50666000\end{array}$

$\begin{array}{lll}1.03236600 & 0.15137900 & 3.69504500\end{array}$

$\begin{array}{lll}0.17801900 & -2.42423000 \quad 1.65567700\end{array}$

$2.35559500 \quad-0.88929400 \quad 0.85735300$

$\begin{array}{lll}0.25531000 & -1.81669300 & 2.55461400\end{array}$

$\begin{array}{lll}0.37054200 & -0.25847500 & 4.85527300\end{array}$

$\begin{array}{lll}-0.35642800 & -1.45269800 & 4.87309200\end{array}$

$\begin{array}{lll}-0.40089200 & -2.23397800 \quad 3.71250700\end{array}$

$\begin{array}{lll}0.43002800 & 0.35938000 & 5.75591800\end{array}$

$\begin{array}{lll}-0.87772000 & -1.77242000 & 5.77902700\end{array}$

$\begin{array}{lll}-0.96492800 & -3.17067800 & 3.70200200\end{array}$

$3.13022400 \quad 1.60687800 \quad 0.18570300$

$5.24097500 \quad 0.47505100 \quad-0.15091300$

$\begin{array}{lll}5.27954000 & 0.80767300 & -1.31735100\end{array}$

$6.29312100 \quad-0.39804500 \quad 0.54475900$

$\begin{array}{lll}7.32451900 & -0.86264300 & -0.48747800\end{array}$

$\begin{array}{lll}6.97641000 & 0.45193900 & 1.63350800\end{array}$

$5.59804300 \quad-1.61204500 \quad 1.18809700$

$\begin{array}{lll}6.85385500 & -1.46603500 & -1.27860000\end{array}$

$\begin{array}{lll}7.82031000 & -0.00785000 & -0.97090300\end{array}$

$8.09546200 \quad-1.47877000 \quad 0.00176700$

$\begin{array}{lll}6.24992700 & 0.79205700 & 2.38561800\end{array}$

$\begin{array}{lll}7.75139400 & -0.14210100 & 2.14403500\end{array}$

$\begin{array}{lll}7.46191800 & 1.34020700 \quad 1.19732100\end{array}$

$6.34634100 \quad-2.25519600 \quad 1.67853900$

$4.86281000 \quad-1.29874600 \quad 1.94246800$

$\begin{array}{lll}5.07345100 & -2.21751100 & 0.43259100\end{array}$

$\begin{array}{lll}4.27056600 & 0.83043100 & 0.69378400\end{array}$

$-1.30722500 \quad-3.90404200 \quad-0.75459500$

$\begin{array}{lll}-1.13041300 & -2.52150100 & -0.58029600\end{array}$

$\begin{array}{lll}-2.13183300 & -1.84421100 & 0.13588000\end{array}$

$\begin{array}{lll}-3.32218300 & -2.44639200 & 0.56205200\end{array}$

$\begin{array}{lll}-3.46396000 & -3.82245600 & 0.35473200\end{array}$

$-2.44753900 \quad-4.55056600 \quad-0.27147900$

$\begin{array}{lll}-4.32421300 & -0.20798700 & 0.42322000\end{array}$

$\begin{array}{lll}-3.05291200 & 0.30708500 & 0.12805400\end{array}$

$\begin{array}{lll}-2.82942300 & 1.58160600 & -0.41812400\end{array}$

$\begin{array}{lll}-3.97409000 & 2.30652900 & -0.78907400\end{array}$

$\begin{array}{lll}-5.25570700 & 1.78866000 & -0.59020700\end{array}$

$\begin{array}{lll}-5.43334100 & 0.55099400 & 0.03854200\end{array}$

$\begin{array}{lll}-0.56427800 & -4.48248100 & -1.30138000\end{array}$

$\begin{array}{lll}-4.37058800 & -4.33466400 & 0.67858800\end{array}$ 
$\begin{array}{lll}-3.86742000 & 3.29666600 & -1.22857700\end{array}$

$\begin{array}{lll}-6.12758500 & 2.37013300 & -0.89890500\end{array}$

$-6.44275800$

0.18651200

0.23263000

C

$\begin{array}{lll}-4.36929300 & -1.53049800 & 1.20232200\end{array}$

$-5.76478500$

$-6.49468900$

2.15692900

1.19601400

$-1.47845400$

1.66077200

$-6.10597100 \quad-2.38620000$

0.17495900

$-5.77587200$

$-3.94520900$

$-3.08737500$

1.78178100

$-3.94902700$

2.66618600

$-2.93325600$

3.25129500

$-4.64513300$

2.71540900

$-1.96388100$

3.13339900

0.15283000

0.36870400

$-1.08217700$

$-1.40654600$

1.73779900

2.00088200

2.02978200

1.25325100

2.98384400

2.88844600

2.77253000

2.94891000

3.84709200

1.75774200

1.73313300

2.70576800

$0.94202800 \quad-4.30811100 \quad-2.68547700$

$\begin{array}{lll}-0.66848300 & -1.33752300 & -3.15138000\end{array}$

$\begin{array}{lll}-1.98603500 & -0.59038200 & -2.90488600\end{array}$

$\begin{array}{lll}-2.73428900 & -1.24443300 & -2.43417300\end{array}$

$\begin{array}{lll}-1.84070000 & 0.27589300 & -2.25786800\end{array}$

$\begin{array}{lll}-2.40448100 & -0.24124400 & -3.86290200\end{array}$

$\begin{array}{lll}0.27316700 & -0.48993400 & -4.02011600\end{array}$

$1.16500600 \quad-1.05681500 \quad-4.32450300$

$\begin{array}{lll}-0.24541400 & -0.17149400 & -4.94033900\end{array}$

$\begin{array}{lll}0.61282700 & 0.41262900 & -3.48987800\end{array}$

$\begin{array}{lll}-1.03543000 & -2.63623200 & -3.88252100\end{array}$

$\begin{array}{lll}-1.59601700 & -3.32832200 & -3.23802100\end{array}$

$\begin{array}{lll}-1.69558800 & -2.38096800 & -4.72994000\end{array}$

$\begin{array}{lll}-0.17393400 & -3.16623500 & -4.29876200\end{array}$

$\begin{array}{lll}-0.91777700 & 3.44508500 & -1.90395000\end{array}$

$\begin{array}{lll}-1.45422300 & 2.76166800 & -3.16958600\end{array}$ 
$\begin{array}{lll}-0.92774600 & 1.82596500 & -3.38004900\end{array}$

$\begin{array}{lll}-2.53073800 & 2.54856100 & -3.11251000\end{array}$

Conformation-B

C

$\begin{array}{lll}-1.29017600 \quad 3.43206700 & -4.03033800\end{array}$

$\begin{array}{lll}-1.58400600 & 4.82305000 & -1.78461100\end{array}$

$\begin{array}{lll}-1.37769800 & 5.38927200 & -2.70943400\end{array}$

$\begin{array}{lll}-2.67568700 & 4.76512000 & -1.68212800\end{array}$

$\begin{array}{lll}-1.18814200 & 5.41609700 & -0.95132100\end{array}$

$\begin{array}{lll}0.59752200 & 3.65238900 & -2.08043900\end{array}$

$0.78621500 \quad 4.23300400 \quad-2.99940500$

$\begin{array}{lll}1.03241000 & 4.20999300 & -1.24064800\end{array}$

$\begin{array}{lll}1.13073600 & 2.69307100 & -2.16300900\end{array}$

$\begin{array}{lll}-1.24961100 \quad 3.28526000 & 1.20225900\end{array}$

$\begin{array}{lll}-2.51184800 \quad 4.15045300 & 1.32700900\end{array}$

$\begin{array}{lll}-2.45954100 \quad 4.69697200 & 2.28498700\end{array}$

$\begin{array}{lll}-2.61340300 \quad 4.89765000 & 0.53267400\end{array}$

$\begin{array}{lll}-3.42692200 \quad 3.54463300 & 1.35222100\end{array}$

$\begin{array}{lll}-0.01250800 \quad 4.18129800 & 1.35705700\end{array}$

$\begin{array}{lll}-0.03264400 & 5.03655200 & 0.66758700\end{array}$

$\begin{array}{lll}0.01783300 & 4.58830400 & 2.38226900\end{array}$

$0.91782300 \quad 3.63084000 \quad 1.18892400$

$\begin{array}{lll}-1.29219100 & 2.25469800 & 2.34063700\end{array}$

$\begin{array}{lll}-2.23123300 \quad 1.68166400 & 2.33228100\end{array}$

$\begin{array}{lll}-0.46905100 & 1.53909400 & 2.27864000\end{array}$

$\begin{array}{lll}-1.22901700 \quad 2.77158100 & 3.31316200\end{array}$

$0.39239900 \quad 0.51255300 \quad-0.14613900$

$\begin{array}{lll}1.60674000 & 1.07960400 \quad 3.70884500\end{array}$

$\begin{array}{lll}1.63768600 & 1.94043700 \quad 1.75440600\end{array}$

$3.03259200 \quad 1.32911800 \quad-0.87306400$

$\begin{array}{lll}3.49356500 & 3.07802900 & 0.28483400\end{array}$

$3.65982000 \quad 3.36572100 \quad 1.33513700$

$\begin{array}{lll}4.40627800 & 3.29251200 & -0.29043200\end{array}$

$\begin{array}{lll}2.68371000 & 3.69857400 & -0.11936000\end{array}$

$\begin{array}{lll}3.01095800 & 3.27072700 & -1.04636700 \\ 2.10660100 & 2.27118200 & -0.65118900 \\ 2.18722200 & 1.05153700 & -1.34526900 \\ 2.89209200 & 0.88762000 & -2.54143200 \\ 3.76620200 & 1.91643700 & -2.91099500 \\ 3.86756200 & 3.07502800 & -2.13285100 \\ 2.35025600 & -1.51701000 & -2.50474200 \\ 1.87436300 & -1.31317100 & -1.20300600 \\ 1.83153300 & -2.32976100 & -0.22966900 \\ 2.10965400 & -3.63108300 & -0.67861600 \\ 2.47998500 & -3.88496700 & -1.99986800\end{array}$




\begin{tabular}{|c|c|c|}
\hline-5.95410100 & -1.44275900 & 0.00372000 \\
\hline-6.97387500 & -0.46089200 & -2.08034800 \\
\hline-6.00253400 & -2.80989000 & -3.21017000 \\
\hline-5.31521600 & -3.50388900 & -1.71956600 \\
\hline-7.07017500 & -3.20261400 & -1.83821600 \\
\hline-5.80403400 & -0.44132000 & 0.43775100 \\
\hline-6.93990700 & -1.81196800 & 0.32988600 \\
\hline-5.18037100 & -2.10786200 & 0.41401700 \\
\hline-7.97123900 & -0.82985300 & -1.79298800 \\
\hline-6.85408500 & 0.55804300 & -1.68425300 \\
\hline-6.93432200 & -0.39894800 & -3.17898500 \\
\hline-1.19539800 & -0.86980900 & 2.47462300 \\
\hline-0.35130200 & 0.49461000 & -0.02094700 \\
\hline 0.52746000 & 2.48851000 & $0.3046140 c$ \\
\hline 1.67409400 & -1.82916200 & 1.55210100 \\
\hline-1.63945700 & -1.71613100 & 0.41244500 \\
\hline-2.10457500 & -0.40921400 & -2.33528800 \\
\hline-1.34714200 & -2.43226600 & -2.22075200 \\
\hline-1.73809200 & -2.72683300 & -3.20726300 \\
\hline-1.40167300 & -3.30448500 & -1.55011900 \\
\hline-0.29087500 & -2.15909000 & -2.33309000 \\
\hline 5.24163600 & -0.81221800 & -0.37087000 \\
\hline 4.55057300 & -1.55403300 & 0.62390300 \\
\hline 4.36936000 & -3.01346600 & 0.51297200 \\
\hline 4.27783100 & -3.67377100 & -0.72909600 \\
\hline 4.37544700 & -3.30993400 & 2.65347000 \\
\hline 4.66319400 & -1.20067800 & 1.65734700 \\
\hline 4.29311800 & -3.79812900 & 1.67941700 \\
\hline 4.09224300 & -5.05447600 & -0.79736000 \\
\hline 3.99766500 & -5.81664700 & 0.37305400 \\
\hline 4.10692100 & -5.17993500 & 1.61276800 \\
\hline 4.01265800 & -5.54025900 & -1.77347300 \\
\hline 3.84547200 & -6.89731500 & 0.31748700 \\
\hline 4.04513700 & -5.76277300 & $2.5355500 c$ \\
\hline 5.43121200 & 0.55510300 & -0.21462800 \\
\hline 5.45120200 & 0.97542300 & 0.7964870 \\
\hline 3.40634900 & 0.01629500 & -0.07140200 \\
\hline 3.23756300 & 2.82591500 & -0.32765400 \\
\hline 2.89565700 & 1.69142600 & $-0.8427050 c$ \\
\hline 2.80346400 & 4.05486500 & -1.16873400 \\
\hline 3.49721100 & 5.31513600 & -0.64180200 \\
\hline 1.27876800 & 4.21132600 & -1.02903900 \\
\hline 3.16706600 & 3.83420100 & -2.6453430 \\
\hline
\end{tabular}


$\begin{array}{lll}4.59279300 & 5.23396700 \quad-0.72091600\end{array}$

$3.25601800 \quad 5.48465300 \quad 0.41737600$

$3.17768700 \quad 6.19897900 \quad-1.21881600$

$0.74956100 \quad 3.32734000 \quad-1.40990300$

$0.93047000 \quad 5.09143400 \quad-1.59516200$

$0.99146800 \quad 4.34740400 \quad 0.02287100$

$\begin{array}{lll}2.65852400 & 2.94846600 & -3.05127500\end{array}$

$\begin{array}{lll}4.25188600 & 3.68916200 & -2.77477000\end{array}$

$2.87093600 \quad 4.70919400 \quad-3.24824000$

$\begin{array}{lll}3.84007200 & 2.97173400 & 0.73717100\end{array}$

$\begin{array}{lll}4.32992800 & -3.09748300 & -1.65424800\end{array}$

$5.43403000 \quad-1.26668200 \quad-1.34964600$

$5.99708300 \quad 1.42100900 \quad-1.29849900$

$\begin{array}{lll}7.09988500 & 1.45072000 & -1.22811300\end{array}$

$5.64523400 \quad 2.45656800 \quad-1.20276000$

$\begin{array}{lll}5.73345600 & 1.05027700 & -2.30092400\end{array}$

$\begin{array}{lll}-5.36820000 & 0.88355700 & -0.88227200\end{array}$

$\begin{array}{lll}-4.15105900 & 0.32993800 & -0.45626200\end{array}$

$\begin{array}{lll}-3.15823200 & 1.21916900 & 0.03012900\end{array}$

$\begin{array}{lll}-3.45669800 & 2.57172900 & 0.26641100\end{array}$

$\begin{array}{lll}-4.69094700 & 3.07051500 & -0.17089300\end{array}$

$\begin{array}{lll}-5.63098300 & 2.24824700 & -0.78346900\end{array}$

$\begin{array}{lll}-1.23605000 & 2.73983900 & 1.41576600\end{array}$

$\begin{array}{lll}-0.92780300 & 1.47288800 & 0.88783000\end{array}$

$0.38568200 \quad 0.94538200 \quad 1.00159100$

$1.29535800 \quad 1.64248900 \quad 1.81365400$

$\begin{array}{lll}0.95998800 & 2.82794400 & 2.45912500\end{array}$

$\begin{array}{lll}0.28820200 & 3.38671700 & 2.21977700\end{array}$

$\begin{array}{lll}-6.13795500 & 0.22587700 & -1.28010500\end{array}$

$\begin{array}{lll}-4.92181000 & 4.12579400 & -0.01535500\end{array}$

$\begin{array}{lll}-6.58070200 & 2.65678900 & -1.13579800\end{array}$

$\begin{array}{lll}2.31355000 & 1.26554800 & 1.90288700\end{array}$

$1.69592500 \quad 3.34109200 \quad 3.07990000$

$\begin{array}{lll}-0.53001800 & 4.36302800 & 2.64275500\end{array}$

$\begin{array}{lll}-2.52563600 & 3.47612300 & 1.06339900\end{array}$

$\begin{array}{lll}-3.26049200 & 3.90585400 & 2.35484800\end{array}$

$\begin{array}{lll}-2.63583400 & 4.57523400 & 2.96438500\end{array}$

$\begin{array}{lll}-3.52056600 & 3.02648200 & 2.96412200\end{array}$

$\begin{array}{lll}-4.18897300 & 4.44528500 \quad 2.11608000\end{array}$

$\begin{array}{lll}-2.16215800 & 4.72775500 & 0.22984000\end{array}$

$\begin{array}{lll}-3.06250300 & 5.30819700 & -0.02194100\end{array}$

$-1.66403100 \quad 4.44040900 \quad-0.70763200$

$\begin{array}{lll}-1.48467600 & 5.38878200 & 0.79027900\end{array}$

$\begin{array}{lll}-1.89483000 & 0.71651600 & 0.26688700\end{array}$ 


\begin{tabular}{|c|c|c|}
\hline-4.65331600 & -2.21760100 & -1.96744000 \\
\hline-6.16981300 & -2.41951600 & -2.07466500 \\
\hline-6.73617700 & -1.48264400 & -1.98657800 \\
\hline-6.55031800 & -3.12449800 & -1.32283100 \\
\hline-6.40464400 & -2.84497100 & -3.06658700 \\
\hline-4.18153500 & -1.31100800 & -3.11729900 \\
\hline-4.73693300 & -0.36388900 & -3.15250900 \\
\hline-4.33942100 & -1.82880000 & -4.07939000 \\
\hline-3.11154700 & -1.06937500 & -3.04070000 \\
\hline-3.97224500 & -3.59351300 & -2.12316100 \\
\hline-2.87601800 & -3.50339600 & -2.14433500 \\
\hline-4.29339500 & -4.06393800 & -3.06877600 \\
\hline-4.23728400 & -4.27954700 & -1.30469400 \\
\hline-5.08186100 & -1.89613000 & 1.14951700 \\
\hline-4.37753300 & -1.26992600 & 2.36724200 \\
\hline-3.36306000 & -1.66742700 & 2.50522000 \\
\hline-4.95476700 & -1.49405400 & 3.28095100 \\
\hline-4.30834600 & -0.17401600 & 2.28098800 \\
\hline-5.09198800 & -3.42416100 & 1.31568300 \\
\hline-5.64917100 & -3.92475800 & 0.51032500 \\
\hline-5.57880400 & -3.69198100 & 2.26947200 \\
\hline-4.07037600 & -3.83615100 & 1.33384100 \\
\hline-6.51832200 & -1.36421900 & 1.10706800 \\
\hline-7.09395600 & -1.74860200 & 0.25679600 \\
\hline-6.54557100 & -0.26606000 & 1.07579900 \\
\hline-7.04394200 & -1.67523700 & 2.02752900 \\
\hline 0.57919700 & -0.79541800 & -1.57022200 \\
\hline 1.76386100 & -1.45400200 & -2.30192400 \\
\hline 2.03729700 & -2.41936500 & -1.85311600 \\
\hline 2.65435200 & -0.80624600 & -2.29829600 \\
\hline 1.48171600 & -1.63977200 & -3.35213900 \\
\hline 0.31153700 & 0.58423000 & -2.19288200 \\
\hline 0.16437400 & 0.45579400 & -3.27892600 \\
\hline 1.15576300 & 1.26424700 & -2.02892600 \\
\hline-0.60133600 & 1.03906200 & -1.78655400 \\
\hline-0.65977700 & -1.66842300 & -1.74079700 \\
\hline-0.93834400 & -1.67027600 & -2.80791700 \\
\hline-1.51385700 & -1.29334800 & -1.16993500 \\
\hline-0.48629300 & -2.71239800 & -1.45306900 \\
\hline 0.67663300 & -1.93578000 & 1.40254900 \\
\hline 0.98411000 & -3.30999300 & 0.79042900 \\
\hline 0.97720900 & -4.06966200 & 1.58869100 \\
\hline 1.96574600 & -3.34815700 & 0.31024400 \\
\hline 0.22547700 & -3.60545400 & 0.05531500 \\
\hline
\end{tabular}


$\mathrm{H}$

$\mathrm{H}$

$\mathrm{H}$

C

H

$\mathrm{H}$

H

P

H

$\mathrm{Ni}$

O

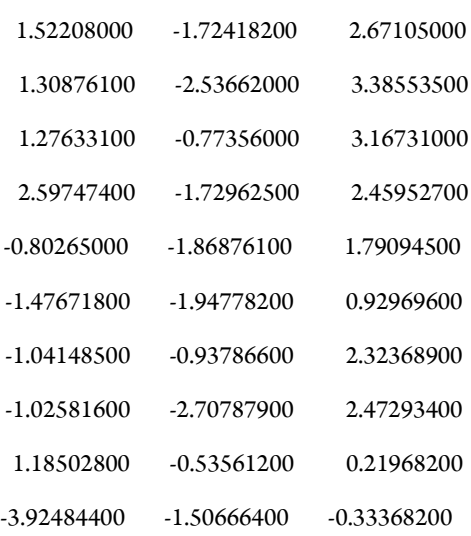

$\begin{array}{rrr}1.52208000 & -1.72418200 & 2.67105000 \\ 1.30876100 & -2.53662000 & 3.38553500 \\ 1.27633100 & -0.77356000 & 3.16731000 \\ 2.59747400 & -1.72962500 & 2.45952700 \\ -0.80265000 & -1.86876100 & 1.79094500 \\ -1.47671800 & -1.94778200 & 0.92969600 \\ -1.04148500 & -0.93786600 & 2.32368900 \\ -1.02581600 & -2.70787900 & 2.47293400 \\ 1.18502800 & -0.53561200 & 0.21968200 \\ -3.92484400 & -1.50666400 & -0.33368200\end{array}$


$5.43403000 \quad-1.26668200 \quad 1.34964600$

$\begin{array}{lll}5.99708300 & 1.42100900 & 1.29849900\end{array}$

$\begin{array}{lll}7.09988500 & 1.45072000 & 1.22811300\end{array}$

$5.64523400 \quad 2.45656800 \quad 1.20276000$

$\begin{array}{lll}5.73345600 & 1.05027700 & 2.30092400\end{array}$

$\begin{array}{lll}-5.36820000 & 0.88355700 & 0.88227200\end{array}$

$\begin{array}{lll}-4.15105900 & 0.32993800 & 0.45626200\end{array}$

$\begin{array}{lll}-3.15823200 & 1.21916900 & -0.03012900\end{array}$

$\begin{array}{lll}-3.45669800 & 2.57172900 & -0.26641100\end{array}$

$\begin{array}{lll}-4.69094700 & 3.07051500 & 0.17089300\end{array}$

$\begin{array}{lll}-5.63098300 & 2.24824700 & 0.78346900\end{array}$

$\begin{array}{lll}-1.23605000 & 2.73983900 & -1.41576600\end{array}$

$\begin{array}{lll}-0.92780300 & 1.47288800 & -0.88783000\end{array}$

$\begin{array}{lll}0.38568200 & 0.94538200 & -1.00159100\end{array}$

$1.29535800 \quad 1.64248900 \quad-1.81365400$

$\begin{array}{lll}0.95998800 & 2.82794400 & -2.45912500\end{array}$

$\begin{array}{lll}-0.28820200 & 3.38671700 & -2.21977700\end{array}$

$\begin{array}{lll}-6.13795500 & 0.22587700 & 1.28010500\end{array}$

$\begin{array}{lll}-4.92181000 & 4.12579400 & 0.01535500\end{array}$

$\begin{array}{lll}-6.58070200 & 2.65678900 & 1.13579800\end{array}$

$\begin{array}{lll}2.31355000 & 1.26554800 & -1.90288700\end{array}$

$\begin{array}{lll}1.69592500 & 3.34109200 & -3.07990000\end{array}$

$\begin{array}{lll}-0.53001800 & 4.36302800 & -2.64275500\end{array}$

$\begin{array}{lll}-2.52563600 & 3.47612300 & -1.06339900\end{array}$

$\begin{array}{lll}-3.26049200 & 3.90585400 & -2.35484800\end{array}$

$\begin{array}{lll}-2.63583400 & 4.57523400 & -2.96438500\end{array}$

$\begin{array}{lll}-3.52056600 & 3.02648200 & -2.96412200\end{array}$

$\begin{array}{lll}-4.18897300 & 4.44528500 \quad-2.11608000\end{array}$

$\begin{array}{lll}-2.16215800 & 4.72775500 & -0.22984000\end{array}$

$\begin{array}{lll}-3.06250300 & 5.30819700 & 0.02194100\end{array}$

$\begin{array}{lll}-1.66403100 & 4.44040900 \quad 0.70763200\end{array}$

$\begin{array}{lll}-1.48467600 & 5.38878200 & -0.79027900\end{array}$

$\begin{array}{lll}-1.89483000 & 0.71651600 & -0.26688700\end{array}$

$\begin{array}{lll}-4.65331600 & -2.21760100 & 1.96744000\end{array}$

$\begin{array}{lll}-6.16981300 & -2.41951600 & 2.07466500\end{array}$

$\begin{array}{lll}-6.73617700 & -1.48264400 \quad 1.98657800\end{array}$

$\begin{array}{lll}-6.55031800 & -3.12449800 \quad 1.32283100\end{array}$

$\begin{array}{lll}-6.40464400 & -2.84497100 \quad 3.06658700\end{array}$

$-4.18153500 \quad-1.31100800 \quad 3.11729900$

$\begin{array}{lll}-4.73693300 & -0.36388900 & 3.15250900\end{array}$

$\begin{array}{lll}-4.33942100 & -1.82880000 \quad 4.07939000\end{array}$

$-3.11154700 \quad-1.06937500 \quad 3.04070000$

$\begin{array}{lll}-3.97224500 & -3.59351300 & 2.12316100\end{array}$

$\begin{array}{lll}-2.87601800 & -3.50339600 & 2.14433500\end{array}$ 
H

$\mathrm{H}$

C

\begin{tabular}{|c|c|c|}
\hline-4.29339500 & -4.06393800 & 3.06877600 \\
\hline-4.23728400 & -4.27954700 & 1.30469400 \\
\hline-5.08186100 & -1.89613000 & -1.14951700 \\
\hline-4.37753300 & -1.26992600 & -2.36724200 \\
\hline-3.36306000 & -1.66742700 & -2.50522000 \\
\hline-4.95476700 & -1.49405400 & -3.28095100 \\
\hline-4.30834600 & -0.17401600 & -2.28098800 \\
\hline-5.09198800 & -3.42416100 & -1.31568300 \\
\hline-5.64917100 & -3.92475800 & -0.51032500 \\
\hline-5.57880400 & -3.69198100 & -2.26947200 \\
\hline-4.07037600 & -3.83615100 & -1.33384100 \\
\hline-6.51832200 & -1.36421900 & -1.10706800 \\
\hline-7.09395600 & -1.74860200 & -0.25679600 \\
\hline-6.54557100 & -0.26606000 & -1.07579900 \\
\hline-7.04394200 & -1.67523700 & -2.02752900 \\
\hline 0.57919700 & -0.79541800 & 1.57022200 \\
\hline 1.76386100 & -1.45400200 & 2.30192400 \\
\hline 2.03729700 & -2.41936500 & 1.85311600 \\
\hline 2.65435200 & -0.80624600 & 2.29829600 \\
\hline 1.48171600 & -1.63977200 & 3.35213900 \\
\hline 0.31153700 & 0.58423000 & 2.19288200 \\
\hline 0.16437400 & 0.45579400 & 3.27892600 \\
\hline 1.15576300 & 1.26424700 & 2.02892600 \\
\hline-0.60133600 & 1.03906200 & 1.78655400 \\
\hline-0.65977700 & -1.66842300 & 1.74079700 \\
\hline-0.93834400 & -1.67027600 & 2.80791700 \\
\hline-1.51385700 & -1.29334800 & 1.16993500 \\
\hline-0.48629300 & -2.71239800 & 1.45306900 \\
\hline 0.67663300 & -1.93578000 & -1.40254900 \\
\hline 0.98411000 & -3.30999300 & -0.79042900 \\
\hline 0.97720900 & -4.06966200 & -1.58869100 \\
\hline 1.96574600 & -3.34815700 & -0.31024400 \\
\hline 0.22547700 & -3.60545400 & -0.05531500 \\
\hline 1.52208000 & -1.72418200 & -2.67105000 \\
\hline 1.30876100 & -2.53662000 & -3.38553500 \\
\hline 1.27633100 & -0.77356000 & -3.16731000 \\
\hline 2.59747400 & -1.72962500 & -2.45952700 \\
\hline-0.80265000 & -1.86876100 & -1.79094500 \\
\hline-1.47671800 & -1.94778200 & -0.92969600 \\
\hline-1.04148500 & -0.93786600 & -2.32368900 \\
\hline-1.02581600 & -2.70787900 & -2.47293400 \\
\hline 1.18502800 & -0.53561200 & -0.21968200 \\
\hline 3.92484400 & -1.50666400 & 0.33368200 \\
\hline
\end{tabular}




\begin{tabular}{|c|c|c|}
\hline-2.03966800 & -1.11049200 & 0.01963500 \\
\hline 0.02426400 & 0.95259300 & 0.4066660 \\
\hline 2.09966900 & -1.13589200 & 0.20984400 \\
\hline-1.16920000 & 1.55747300 & 0.09834100 \\
\hline-2.29840100 & 0.72682800 & -0.04870700 \\
\hline-3.52546200 & 1.36510300 & -0.29668400 \\
\hline-3.60658800 & 2.74976900 & -0.44915100 \\
\hline-2.45724400 & 3.53667400 & -0.33967100 \\
\hline-1.21967600 & 2.95320900 & -0.04888800 \\
\hline 1.18887300 & 1.51082100 & -0.05697400 \\
\hline 2.28051500 & 0.64953100 & -0.28557900 \\
\hline 3.41703600 & 1.22044600 & -0.88504300 \\
\hline 3.47067400 & 2.57972900 & -1.19716400 \\
\hline 2.39460100 & 3.41506000 & -0.88514400 \\
\hline 1.23773600 & 2.89556700 & -0.29619800 \\
\hline 0.06500600 & 3.74553100 & 0.1991230 \\
\hline-4.43261300 & 0.76994200 & -0.38482600 \\
\hline-4.57152000 & 3.21982400 & -0.65367200 \\
\hline-2.53420700 & 4.61744900 & -0.46189900 \\
\hline 4.26917400 & 0.59063700 & -1.12960400 \\
\hline 4.36237900 & 2.99478200 & -1.67304500 \\
\hline 2.46539800 & 4.48199000 & -1.09907100 \\
\hline 0.02721900 & 5.12617700 & -0.4628960 \\
\hline 0.22632300 & 3.92968300 & 1.7310590 \\
\hline-0.09329200 & 5.05178900 & -1.55449500 \\
\hline-0.80176000 & 5.72652600 & -0.06254300 \\
\hline 0.95015900 & 5.68477000 & -0.2518910 \\
\hline-0.61659200 & 4.51220300 & 2.1354630 \\
\hline 0.25154700 & 2.95958900 & 2.2496580 \\
\hline 1.16370500 & 4.46258700 & 1.9559780 \\
\hline-2.57887500 & 1.47734200 & 1.82045100 \\
\hline-3.29715500 & -1.85456900 & 1.22625900 \\
\hline 2.19776700 & -2.03940700 & -1.47726200 \\
\hline 3.65508300 & -1.46922100 & 1.27637100 \\
\hline-2.76233600 & -3.27554300 & 1.50532800 \\
\hline-2.76884400 & -3.90920300 & -0.60683400 \\
\hline-3.39580900 & -3.76749900 & -2.26393100 \\
\hline-1.73146900 & -3.25047500 & -1.89054000 \\
\hline-4.77426800 & -1.96331600 & -0.82795600 \\
\hline-5.23126500 & -0.99305500 & -0.59081800 \\
\hline-5.34616600 & -2.39437600 & -1.66913600 \\
\hline-4.91900100 & -2.62829900 & 0.03516700 \\
\hline-3.16397300 & -1.04598800 & -2.52925000 \\
\hline 3.63937600 & -1.60211800 & -3.35584100 \\
\hline
\end{tabular}


$\begin{array}{lll}1.31307500 & -0.77180300 & -1.07817500\end{array}$

$\begin{array}{lll}-1.31304900 & -0.77180900 & -1.07819200\end{array}$

$\begin{array}{lll}0.76082100 & -0.57631500 & -0.38600700\end{array}$

$\begin{array}{lll}0.49435200 & 0.69548900 & 0.26990500\end{array}$

$\begin{array}{lll}0.11228300 & 1.92607900 & -0.43243200\end{array}$

$\begin{array}{lll}-0.30554400 & 1.95100000 & -1.78630300\end{array}$

$\begin{array}{lll}0.41020400 & 3.17916600 & 1.30581800\end{array}$

$\begin{array}{lll}0.96557300 & 0.87106200 & 1.24663200\end{array}$

$\begin{array}{lll}0.07673000 & 3.15679000 & 0.26420100\end{array}$

$\begin{array}{lll}-0.75476700 & 3.12614500 & -2.38887600\end{array}$

$\begin{array}{lll}-0.80374200 & 4.32849700 & -1.67063400\end{array}$

$\begin{array}{lll}0.37744900 & 4.33171700 & -0.33757100\end{array}$

$\begin{array}{lll}-1.07340100 \quad 3.10428200 & -3.43533900\end{array}$

$\begin{array}{lll}-1.16013900 & 5.24638200 & -2.14472500\end{array}$

$\begin{array}{lll}-0.39412400 & 5.26108100 & 0.23942400\end{array}$

$\begin{array}{lll}1.67400700 & -1.56525700 & 0.27669900\end{array}$

$3.07671200 \quad-1.27126600 \quad-0.08042200$

$3.80421400 \quad-0.50289900 \quad 0.73071100$

$\begin{array}{lll}5.14481900 & -0.11970800 & 0.08927300\end{array}$

$\begin{array}{lll}3.44437600 & -0.13584000 & 1.83047600\end{array}$

$5.87870900 \quad-1.38801300 \quad-0.37920700$

$\begin{array}{lll}4.83599300 & 0.78710600 & -1.11861000\end{array}$

$\begin{array}{lll}5.99697700 & 0.63730700 & 1.11208600\end{array}$

$6.08480100 \quad-2.06360600 \quad 0.46714700$

$5.28604600 \quad-1.94145000 \quad-1.12127800$

$6.84324700 \quad-1.11704700 \quad-0.83843100$

$\begin{array}{lll}4.27674100 & 1.68425800 & -0.80771500\end{array}$

$\begin{array}{lll}5.77422400 & 1.11639800 & -1.59369000\end{array}$

$\begin{array}{lll}4.23381700 & 0.25567400 & -1.86974100\end{array}$

$\begin{array}{lll}6.95567300 & 0.93433400 & 0.65800300\end{array}$

$5.48431000 \quad 1.54459500 \quad 1.46369800$

$6.21236100 \quad 0.01289800 \quad 1.99279800$

$\begin{array}{lll}-0.28778200 & 1.02805800 & -2.36936700\end{array}$

$\begin{array}{lll}-1.01529600 & -0.48148100 & 0.24905300\end{array}$

$0.88009600 \quad-0.58667500 \quad-1.48310400$

$\begin{array}{lll}1.42975100 & -3.00146000 & -0.14847400\end{array}$

$1.52202500 \quad-3.10771400 \quad-1.24194000$ 
$0.40949500 \quad-3.29232600 \quad 0.14045300$

$\begin{array}{lll}2.14592500 & -3.69015500 & 0.32721800\end{array}$

TS-S67

$\begin{array}{lll}1.60606300 & -1.47517300 \quad 1.36883300\end{array}$

$\begin{array}{lll}-2.65739100 & 0.40984000 & 0.99591400\end{array}$

$\begin{array}{lll}-3.63063100 & 0.77900800 & -0.00679000\end{array}$

$\begin{array}{lll}-2.58717000 & 1.42088800 \quad 2.02973000\end{array}$

$\begin{array}{lll}-4.49734400 & 1.83294600 & 0.66572800\end{array}$

$\begin{array}{lll}-4.17105200 & -0.12921100 & -0.30897100\end{array}$

$\begin{array}{lll}-3.10553300 & 1.19186300 & -0.88451000\end{array}$

$\begin{array}{lll}-3.47151000 & 2.56242100 & 1.54029000\end{array}$

$\begin{array}{lll}-1.53657000 & 1.70774600 & 2.16736100\end{array}$

$\begin{array}{lll}-2.96192300 & 0.97343500 & 2.96555400\end{array}$

$\begin{array}{lll}-4.99329500 & 2.48913900 & -0.06383100\end{array}$

$\begin{array}{lll}-5.27205100 & 1.35577900 & 1.28824300\end{array}$

$\begin{array}{lll}-2.87907900 & 3.25840500 \quad 0.92612700\end{array}$

$\begin{array}{lll}-3.92202900 \quad 3.12592300 & 2.36994100\end{array}$

$\begin{array}{lll}-2.08235600 & -2.09608900 & -0.18699200\end{array}$

$\begin{array}{lll}-2.97208000 & -2.76244900 & 0.73137500\end{array}$

$\begin{array}{lll}-2.15590200 & -2.67187400 & -1.50509000\end{array}$

$-3.86146800 \quad-3.63013300 \quad-0.15148500$

$\begin{array}{lll}-3.51062800 & -1.99565900 & 1.30503000\end{array}$

$\begin{array}{lll}-2.36710800 & -3.36616700 & 1.42968400\end{array}$

$-2.92449700 \quad-3.97457800 \quad-1.31619800$

$\begin{array}{lll}-1.13186100 & -2.80289600 & -1.88099000\end{array}$

$\begin{array}{lll}-2.68885300 & -1.96920400 \quad-2.16889800\end{array}$

$\begin{array}{lll}-4.24085000 & -4.51445200 & 0.38030800\end{array}$

$\begin{array}{lll}-4.72512700 & -3.04932500 & -0.51432500\end{array}$

$\begin{array}{lll}-2.23569300 & -4.78458900 & -1.02626800\end{array}$

$\begin{array}{lll}-3.45526100 & -4.28276500 & -2.22835000\end{array}$

\begin{tabular}{lll}
-0.10448800 & -1.87013800 & 0.20986000 \\
-1.29734600 & -1.25573200 & 0.81463300 \\
-2.61743400 & -1.30101700 & 0.16659800 \\
-2.78577400 & -1.47195100 & -1.22723100 \\
-3.68910900 & -0.94632100 & 2.01140100 \\
-1.36047100 & -1.28268400 & 1.91370500 \\
-3.78564600 & -1.08248500 & 0.93016500 \\
-4.04933900 & -1.42782200 & -1.81699900 \\
-5.19384600 & -1.20299000 & -1.04148200 \\
\hline-5.04926400 & -1.02929400 & 0.33930500 \\
\hline-4.14220400 & -1.56454700 & -2.89831100 \\
\hline-6.18182700 & -1.16416900 & -1.50694900 \\
\hline-5.93030200 & -0.85488000 & 0.96373100 \\
\hline 1.02891800 & -2.25364900 & 1.00324000
\end{tabular}


$0.96701500 \quad-1.98414000 \quad 2.06283500$

$\begin{array}{lll}2.65169000 & -0.22836400 & -0.05109500\end{array}$

$\begin{array}{lll}1.70821100 & 0.47740500 & -0.47651900\end{array}$

$4.09304700 \quad 0.12532700 \quad-0.46459400$

$\begin{array}{lll}4.94934900 & 0.27779200 & 0.80454900\end{array}$

$4.11475200 \quad 1.42630900 \quad-1.27306000$

$4.62691300 \quad-1.03821500 \quad-1.32086100$

$\begin{array}{lll}4.94719400 & -0.64853200 & 1.39647200\end{array}$

$\begin{array}{lll}4.57062500 & 1.09284400 & 1.44296800\end{array}$

$\begin{array}{lll}5.99039400 & 0.51595400 & 0.53297600\end{array}$

$\begin{array}{lll}3.50556100 & 1.34196600 & -2.18470100\end{array}$

$5.14909200 \quad 1.66515900 \quad-1.56824100$

$\begin{array}{lll}3.72278900 & 2.27044300 & -0.68585200\end{array}$

$5.65821700 \quad-0.82671100 \quad-1.64644800$

$\begin{array}{lll}4.00931200 & -1.18253900 \quad-2.22239200\end{array}$

$4.62860600 \quad-1.97925300 \quad-0.75171600$

$2.54824800 \quad-1.25165800 \quad 0.70692200$

$\begin{array}{lll}-0.19536400 & -2.38677100 & -0.75537300\end{array}$

$\begin{array}{lll}-1.90900500 & -1.62542800 & -1.85980700\end{array}$

$1.70011700 \quad-3.57023000 \quad 0.74060700$

$\begin{array}{lll}1.00517500 & -4.36699000 & 1.05922800\end{array}$

$\begin{array}{lll}2.63900500 & -3.68391600 & 1.29899700\end{array}$

$1.89855400 \quad-3.70730100 \quad-0.33344900$

$\begin{array}{lll}-0.07729000 & 0.04861800 & 0.16035500\end{array}$

$\begin{array}{lll}-0.72166500 & 1.88896800 & 0.31416000\end{array}$

$\begin{array}{lll}-2.12175700 & 2.22091100 \quad 0.44597400\end{array}$

$\begin{array}{lll}0.07930700 & 3.06732300 & 0.07982900\end{array}$

$\begin{array}{lll}-2.21444900 \quad 3.68734500 & 0.04028100\end{array}$

$\begin{array}{lll}-2.42008100 & 2.05433200 & 1.49472800\end{array}$

$\begin{array}{lll}-2.70377300 & 1.54423900 & -0.19405900\end{array}$

$\begin{array}{lll}-0.83682700 & 4.22798600 & 0.44253400\end{array}$

$\begin{array}{lll}0.38020400 & 3.08805900 & -0.98094100\end{array}$

$0.98416000 \quad 2.99444000 \quad 0.69880000$

$\begin{array}{lll}-2.35437800 & 3.77297600 & -1.04949500\end{array}$

$\begin{array}{lll}-3.04916400 & 4.20372500 & 0.53558600\end{array}$

$\begin{array}{lll}-0.56143400 & 5.15479000 \quad-0.08104600\end{array}$

$\begin{array}{lll}-0.80054700 & 4.41961700 \quad 1.52740600\end{array}$

$\begin{array}{lll}-0.82355800 & 2.52047800 \quad 0.12160200\end{array}$

$\begin{array}{lll}-1.55023800 & 1.62893000 & -0.70955300\end{array}$

$\begin{array}{lll}-2.79927400 & 0.95932500 & -0.31569200\end{array}$

$\begin{array}{lll}-3.10289600 & 0.67958100 & 1.03214400\end{array}$

$\begin{array}{lll}-3.46806300 & 0.70166400 & -2.35443300\end{array}$

$\begin{array}{lll}-1.37654200 & 1.68935400 & -1.79216700\end{array}$ 
$\begin{array}{lll}-6.05531000 & -0.99598600 & 0.65462100\end{array}$

$\begin{array}{lll}-5.53397600 & -0.53359400 & -1.74448400\end{array}$

$\begin{array}{lll}0.51858400 & 2.77827900 & -0.23596800\end{array}$

$0.76182400 \quad 2.80529800 \quad-1.30794900$

$\begin{array}{lll}0.17016000 & 0.82838400 & -0.02464900\end{array}$

$\begin{array}{lll}2.56336500 & -0.14372500 & -0.33421600\end{array}$

$\begin{array}{lll}1.91338400 & 0.35459800 & 0.65896900\end{array}$

$3.99645700 \quad-0.62078600 \quad-0.01541500$

$4.67931300 \quad-1.13774000 \quad-1.28475400$

$3.90870800 \quad-1.74501000 \quad 1.03203800$

$\begin{array}{lll}4.78623500 & 0.56579000 & 0.56382000\end{array}$

$4.74326100 \quad-0.35024100 \quad-2.05084000$

$\begin{array}{lll}4.12050400 & -1.97778600 & -1.72357200\end{array}$

$5.70171600 \quad-1.48258000 \quad-1.05740900$

$3.40756400 \quad-1.39223100 \quad 1.94516600$

$\begin{array}{lll}4.91662300 & -2.10052100 & 1.30390200\end{array}$

$3.34323300 \quad-2.60733500 \quad 0.64162700$

$4.31023800 \quad 0.94316800 \quad 1.48018000$

$\begin{array}{lll}4.84020500 & 1.39638500 & -0.15947300\end{array}$

$5.81865900 \quad 0.26273500 \quad 0.80595300$

$2.08673600 \quad-0.24620400 \quad-1.47436300$

$\begin{array}{lll}-2.40558300 & 0.98412500 & 1.81555800\end{array}$

$-1.18939700 \quad 2.78390300 \quad 1.12121400$

$1.48104100 \quad 3.49729400 \quad 0.66239900$

$\begin{array}{lll}1.52234900 & 4.57511000 \quad 0.42207700\end{array}$

$2.49799200 \quad 3.09361600 \quad 0.54256900$

$\begin{array}{lll}1.20096400 \quad 3.39496800 & 1.72234800\end{array}$

$\begin{array}{lll}-0.53297300 & -1.01674700 & 0.10095800\end{array}$

$\begin{array}{lll}-0.80177600 & -1.79647600 & -1.07573800\end{array}$

$\begin{array}{lll}-0.17779900 & -1.93975500 & 1.13922400\end{array}$

$\begin{array}{lll}-1.53544200 & -3.03693100 & -0.55213000\end{array}$

$0.15840700 \quad-2.04094100 \quad-1.55727300$

$\begin{array}{lll}-1.39647900 & -1.17455900 & -1.75613200\end{array}$

$\begin{array}{lll}-1.16853600 & -3.09096300 & 0.95480100\end{array}$

$\begin{array}{lll}-0.26049100 & -1.41294300 \quad 2.09853600\end{array}$

$0.86633300 \quad-2.26434900 \quad 0.99778400$

$\begin{array}{lll}-2.62144000 & -2.92913800 & -0.68237400\end{array}$

$\begin{array}{lll}-1.21608800 & -3.93792400 & -1.09490400\end{array}$

$\begin{array}{lll}-2.06239900 & -2.92519200 & 1.57305100\end{array}$ 
TS-S69

\begin{tabular}{|c|c|c|}
\hline 0.73417300 & -0.56263400 & -0.84224700 \\
\hline 0.53762300 & 0.64785500 & -0.06661200 \\
\hline 0.17303300 & 1.94319000 & -0.66100700 \\
\hline 0.51038100 & 2.04863800 & -1.89325700 \\
\hline 0.95969700 & 3.08449300 & 0.99662200 \\
\hline 1.13016400 & 0.72407100 & 0.85497200 \\
\hline 0.42532300 & 3.13819500 & 0.04424200 \\
\hline 0.92119900 & 3.28642600 & -2.38903700 \\
\hline 0.66970500 & 4.46068500 & -1.66869000 \\
\hline 0.00839600 & 4.37696600 & -0.44791400 \\
\hline-1.44791100 & 3.33605200 & -3.34617500 \\
\hline-0.99620200 & 5.42877800 & -2.05655900 \\
\hline 0.21855200 & 5.28471700 & 0.12446100 \\
\hline 1.18679100 & -1.73347800 & -0.20227400 \\
\hline 3.25404500 & -1.62782200 & -0.33615000 \\
\hline 3.72297800 & -0.89874700 & 0.60375200 \\
\hline 5.12208400 & -0.28164900 & 0.29714700 \\
\hline 3.15675400 & -0.63036400 & 1.67553400 \\
\hline 6.09196900 & -1.39919400 & -0.11458200 \\
\hline 4.93366400 & 0.69913600 & -0.87351300 \\
\hline 5.66326400 & 0.46152600 & 1.52086800 \\
\hline 6.24348400 & -2.11718100 & 0.70930100 \\
\hline 5.69696600 & -1.95504000 & -0.97703600 \\
\hline 7.07885300 & -0.98556600 & -0.38510000 \\
\hline 4.21721400 & 1.49563600 & -0.61079000 \\
\hline 5.88959800 & 1.17871900 & -1.14612800 \\
\hline 4.54078300 & 0.17448600 & -1.75686500 \\
\hline 6.64851900 & 0.90763400 & 1.30111900 \\
\hline 4.97937400 & 1.26555700 & 1.83028900 \\
\hline 5.77839900 & -0.21740700 & 2.38015600 \\
\hline 0.73199600 & 1.14487600 & -2.46502600 \\
\hline 0.96504400 & -0.52632400 & 0.13283600 \\
\hline 0.67318000 & -0.55896800 & -1.93762400 \\
\hline 1.04839900 & -3.08540400 & -0.82601700 \\
\hline 1.11746100 & -3.03522800 & -1.92379000 \\
\hline 0.06536000 & -3.51097500 & -0.56322900 \\
\hline 1.82562100 & -3.76554100 & -0.45322300 \\
\hline 1.31446200 & -1.69115000 & 0.87954400 \\
\hline-2.37335800 & 0.49936400 & 1.09948900 \\
\hline 3.46405300 & 0.99242400 & 0.28431400 \\
\hline 2.05817000 & 1.44396500 & 2.15634000 \\
\hline 4.11531 & 2.07567900 & 1.1302290 \\
\hline
\end{tabular}


$\begin{array}{lll}-4.12204700 \quad 0.14428800 & 0.04785000\end{array}$

$-3.05186700$

$1.40435400 \quad-0.65171000$

$-2.90615300$

$2.67419200 \quad 1.85847100$

$-2.32472400$

$0.97146500 \quad 3.11582600$

$-4.65931900$

2.80731900

0.51604300

$-4.82169800$

$1.62967900 \quad 1.84912200$

$-2.35911600$

$-3.17389400$

3.35648000

1.18930100

$-2.17494500$

3.22361400

2.77218700

$-3.00098500$

$-0.12178000$

$-2.66420900$

0.91998400

$-4.16765100$

$-1.43161400$

$-3.29672400$

$-2.39167000$

0.18392700

$-3.54825900$

$-1.76582200$

$-3.19634100$

$-4.56416300$

$-4.98801800$

$-2.94768200$

$-4.29310900$

$-0.82355800$

$-1.55023800$

$-2.79927400$

$-3.10289600$

$-3.46806300$

$-1.37654200$

$-3.69290800$

$-4.26531400$

$-5.14838700$

$-4.85475600$

$-4.47959200$

$-6.05531000$

$-5.53397600$

0.51858400

0.76182400

0.17016000

2.56336500

1.91338400

3.99645700

$3.90870800 \quad-1.74501000 \quad-1.03203800$ 


\begin{tabular}{|c|c|c|c|}
\hline $\mathrm{C}$ & 4.78623500 & 0.56579000 & -0.56382000 \\
\hline $\mathrm{H}$ & 4.74326100 & -0.35024100 & 2.05084000 \\
\hline $\mathrm{H}$ & 4.12050400 & -1.97778600 & 1.72357200 \\
\hline $\mathrm{H}$ & 5.70171600 & -1.48258000 & 1.05740900 \\
\hline $\mathrm{H}$ & 3.40756400 & -1.39223100 & -1.94516600 \\
\hline $\mathrm{H}$ & 4.91662300 & -2.10052100 & -1.30390200 \\
\hline $\mathrm{H}$ & 3.34323300 & -2.60733500 & -0.64162700 \\
\hline $\mathrm{H}$ & 4.31023800 & 0.94316800 & -1.48018000 \\
\hline $\mathrm{H}$ & 4.84020500 & 1.39638500 & 0.15947300 \\
\hline $\mathrm{H}$ & 5.81865900 & 0.26273500 & -0.80595300 \\
\hline $\mathrm{O}$ & 2.08673600 & -0.24620400 & 1.47436300 \\
\hline $\mathrm{H}$ & -2.40558300 & 0.98412500 & -1.81555800 \\
\hline $\mathrm{H}$ & -1.18939700 & 2.78390300 & -1.12121400 \\
\hline $\mathrm{C}$ & 1.48104100 & 3.49729400 & -0.66239900 \\
\hline $\mathrm{H}$ & 1.52234900 & 4.57511000 & -0.42207700 \\
\hline $\mathrm{H}$ & 2.49799200 & 3.09361600 & -0.54256900 \\
\hline $\mathrm{H}$ & 1.20096400 & 3.39496800 & -1.72234800 \\
\hline $\mathrm{O}$ & -0.53297300 & -1.01674700 & -0.10095800 \\
\hline $\mathrm{C}$ & -0.80177600 & -1.79647600 & 1.07573800 \\
\hline $\mathrm{C}$ & -0.17779900 & -1.93975500 & -1.13922400 \\
\hline $\mathrm{C}$ & -1.53544200 & -3.03693100 & 0.55213000 \\
\hline $\mathrm{H}$ & 0.15840700 & -2.04094100 & 1.55727300 \\
\hline $\mathrm{H}$ & -1.39647900 & -1.17455900 & 1.75613200 \\
\hline $\mathrm{C}$ & -1.16853600 & -3.09096300 & -0.95480100 \\
\hline $\mathrm{H}$ & -0.26049100 & -1.41294300 & -2.09853600 \\
\hline $\mathrm{H}$ & 0.86633300 & -2.26434900 & -0.99778400 \\
\hline $\mathrm{H}$ & -2.62144000 & -2.92913800 & 0.68237400 \\
\hline $\mathrm{H}$ & -1.21608800 & -3.93792400 & 1.09490400 \\
\hline $\mathrm{H}$ & -2.06239900 & -2.92519200 & -1.57305100 \\
\hline $\mathrm{H}$ & -0.72309500 & -4.05120500 & -1.25110400 \\
\hline
\end{tabular}

Cartesian coordinates of the structures (optimization in hexane) TS22

$\begin{array}{llll}\text { C } & -1.14991600 & 0.67032500 & 2.16656400 \\ \text { C } & -0.20097000 & 0.38402300 & 1.16413500 \\ \text { C } & 1.02171700 & 1.08931800 & 1.22499900 \\ \text { C } & 1.17402500 & 2.19648200 & 2.08680000 \\ \text { C } & 0.21176100 & 2.41866600 & 3.07509800 \\ \text { C } & -0.92799800 & 1.62098400 & 3.15443700 \\ \text { C } & 3.50353300 & 2.44944000 & 1.29509700 \\ \text { C } & 3.33634500 & 1.22647200 & 0.61631500 \\ \text { C } & 4.44922400 & 0.50846900 & 0.11588800 \\ \text { C } & 5.71555000 & 1.10370900 & 0.24380700 \\ \text { C } & 5.89759400 & 2.32091000 & 0.89164400 \\ \text { C } & 4.79291700 & 2.97303400 & 1.43292100\end{array}$




\begin{tabular}{|c|c|c|}
\hline-2.10561600 & 0.14265300 & 2.13004300 \\
\hline 0.33298400 & 3.24655400 & 3.77282300 \\
\hline-1.67530200 & 1.79431100 & 3.93140500 \\
\hline 6.58176400 & 0.59142800 & -0.16642000 \\
\hline 6.89550500 & 2.75562900 & 0.98215800 \\
\hline 4.93540200 & 3.92004800 & 1.95224900 \\
\hline 2.27359600 & 3.20859100 & 1.77562900 \\
\hline 2.58725700 & 4.11445700 & 2.97393700 \\
\hline 3.34179000 & 4.86773300 & 2.71012500 \\
\hline 2.95644900 & 3.53910000 & 3.83632200 \\
\hline 1.69417000 & 4.67416500 & 3.28342800 \\
\hline 1.76623200 & 4.09994400 & 0.60956100 \\
\hline 0.85061800 & 4.63478100 & 0.90604500 \\
\hline 1.53398800 & 3.49937000 & -0.28178700 \\
\hline 2.53448900 & 4.83864400 & 0.33102600 \\
\hline 2.06822900 & 0.71612800 & 0.40714000 \\
\hline-0.41988300 & -2.47049700 & 0.36252900 \\
\hline 1.08130000 & -2.60701800 & 0.60357100 \\
\hline 1.42288500 & -1.89264200 & 1.36559800 \\
\hline 1.68643200 & -2.43686400 & -0.29283200 \\
\hline 1.30133100 & -3.62350700 & 0.97468800 \\
\hline 1.16380600 & -2.75640300 & 1.67877600 \\
\hline-0.99847600 & -3.80688400 & 1.97067900 \\
\hline-2.24394500 & -2.60035800 & 1.57631800 \\
\hline-0.79559200 & -2.12087000 & 2.49782100 \\
\hline 0.90499200 & -3.45464600 & -0.70997500 \\
\hline-1.97527800 & -3.32301900 & -0.91514500 \\
\hline-0.75991300 & -4.48770500 & -0.35203600 \\
\hline-0.35028600 & -3.35035000 & -1.65270000 \\
\hline 0.29588800 & -0.22760300 & -1.86403200 \\
\hline-1.41898500 & -0.61178200 & -2.84832200 \\
\hline-1.66438900 & -1.68239900 & -2.80762700 \\
\hline-1.09376600 & -0.37898100 & -3.87689600 \\
\hline-2.33907300 & -0.04443000 & -2.64323600 \\
\hline 1.01344100 & -0.87279300 & -2.31266000 \\
\hline 1.82880800 & -0.69246900 & -1.60504000 \\
\hline 1.30499500 & -0.44803500 & -3.28888200 \\
\hline 0.91814800 & -1.95873200 & -2.45099000 \\
\hline-0.14696600 & 1.30352700 & -1.88172200 \\
\hline-1.00893900 & 1.79947000 & -1.41724400 \\
\hline-0.07455700 & 1.64771300 & -2.92731200 \\
\hline 0.76333400 & 1.62454300 & -1.36165400 \\
\hline 5.29453900 & -2.26615100 & 0.56877700 \\
\hline 4.46949100 & -2.29940700 & 1.86854500 \\
\hline
\end{tabular}


$3.47344900 \quad-2.73198300 \quad 1.71082600$

$\begin{array}{lll}4.34113800 & -1.29209200 & 2.29538600\end{array}$

$\begin{array}{lll}4.98943600 & -2.91603400 & 2.62199800\end{array}$

$6.72134500 \quad-1.83357600 \quad 0.93106200$

$\begin{array}{lll}7.17678100 & -2.60833500 & 1.57312700\end{array}$

$6.73124600 \quad-0.89724700 \quad 1.50511100$

$\begin{array}{lll}7.37300800 & -1.71221500 & 0.05741200\end{array}$

$\begin{array}{lll}5.32373900 & -3.67914600 & -0.03782200\end{array}$

$4.31975200 \quad-4.01069500 \quad-0.34657100$

$\begin{array}{lll}5.69891300 & -4.39891700 & 0.71001000\end{array}$

$5.98901900 \quad-3.74131900 \quad-0.91202100$

$5.12522900 \quad-1.03420700 \quad-2.33912800$

$\begin{array}{lll}4.66970500 & 0.30165000 & -2.95085800\end{array}$

$\begin{array}{lll}4.93416400 & 0.32670300 & -4.02227800\end{array}$

$\begin{array}{lll}5.14989900 & 1.16214300 & -2.46430100\end{array}$

$\begin{array}{lll}3.58050000 & 0.43802300 & -2.87382600\end{array}$

$6.65450200 \quad-1.12247100 \quad-2.40077500$

$\begin{array}{lll}7.15232200 & -0.31843000 & -1.84153100\end{array}$

$6.97995400 \quad-1.02904400 \quad-3.45223200$

$7.03114100 \quad-2.08658900 \quad-2.03184300$

$\begin{array}{lll}4.53395100 & -2.18277100 & -3.18097200\end{array}$

$\begin{array}{lll}3.43678300 & -2.12500500 & -3.22658600\end{array}$

$4.79985800 \quad-3.17089400 \quad-2.77799800$

$\begin{array}{lll}4.92153300 & -2.13067000 & -4.21338600\end{array}$

$4.25521700 \quad-1.17959800 \quad-0.62684600$

$\begin{array}{lll}-0.94294700 & -0.72168000 & -0.13821900\end{array}$

$\begin{array}{lll}-5.04096300 & -0.42754400 & -0.45875500\end{array}$

$\begin{array}{lll}-4.33234400 & -1.70407600 & -0.55717200\end{array}$

$\begin{array}{lll}-4.54602800 & -2.76652900 & 0.44546000\end{array}$

$\begin{array}{lll}-4.83106300 & -2.48173800 & 1.79868000\end{array}$

$\begin{array}{lll}-4.19013900 & -4.37453800 & -0.95299400\end{array}$

$\begin{array}{lll}-4.20090400 & -2.10252800 & -1.57380100\end{array}$

$\begin{array}{lll}-4.40058900 & -4.12195900 & 0.08962100\end{array}$

$\begin{array}{lll}-4.95608800 & -3.49922800 & 2.74298400\end{array}$

$\begin{array}{lll}-4.79184200 & -4.83886100 & 2.37154300\end{array}$

$-4.51372400 \quad-5.14199700 \quad 1.03597000$

$\begin{array}{lll}-5.17183400 & -3.24439200 \quad 3.78423000\end{array}$

$-4.88105800 \quad-5.63501500 \quad 3.11457500$

$\begin{array}{lll}-4.38776500 & -6.18302600 & 0.72595100\end{array}$

$\begin{array}{lll}-5.36210300 & 0.35811400 & -1.61686800\end{array}$

$\begin{array}{lll}-4.97919300 & -0.04053600 & -2.56233500\end{array}$

$\begin{array}{lll}-3.64736900 & 2.38826800 & -0.88734700\end{array}$

$\begin{array}{lll}-3.01650300 & 1.71293200 & -0.04743200\end{array}$

$-3.46063900 \quad 3.91660600 \quad-0.90110900$ 


\begin{tabular}{|c|c|c|c|}
\hline $\mathrm{C}$ & -3.03859700 & 4.34573700 & -2.31763100 \\
\hline $\mathrm{C}$ & -2.38872400 & 4.32317800 & 0.11673900 \\
\hline $\mathrm{C}$ & -4.81392900 & 4.55559800 & -0.53979400 \\
\hline $\mathrm{H}$ & -3.79534700 & 4.05401700 & -3.05957300 \\
\hline $\mathrm{H}$ & -2.08221000 & 3.87999600 & -2.60416500 \\
\hline $\mathrm{H}$ & -2.90927800 & 5.43917200 & -2.36022300 \\
\hline $\mathrm{H}$ & -2.67118900 & 4.02949200 & 1.13757300 \\
\hline $\mathrm{H}$ & -2.24433600 & 5.41545300 & 0.09382200 \\
\hline $\mathrm{H}$ & -1.42521300 & 3.84176900 & -0.10345300 \\
\hline $\mathrm{H}$ & -4.72743900 & 5.65416700 & -0.53578000 \\
\hline $\mathrm{H}$ & -5.14773200 & 4.23928900 & 0.46187800 \\
\hline $\mathrm{H}$ & -5.59003900 & 4.27182100 & -1.26532600 \\
\hline $\mathrm{O}$ & -4.45239000 & 1.92670400 & -1.76975000 \\
\hline $\mathrm{H}$ & -5.65477400 & -0.22776600 & 0.42845400 \\
\hline $\mathrm{H}$ & -4.92849200 & -1.44199900 & 2.11781500 \\
\hline $\mathrm{C}$ & -6.72996100 & 0.97300900 & -1.72106900 \\
\hline $\mathrm{H}$ & -7.46017700 & 0.15735100 & -1.86113300 \\
\hline $\mathrm{H}$ & -6.81354500 & 1.66688500 & -2.56838900 \\
\hline $\mathrm{H}$ & -6.99534200 & 1.50361100 & -0.79383700 \\
\hline $\mathrm{Ni}$ & -3.10361900 & -0.26489700 & -0.17320300 \\
\hline \multicolumn{4}{|c|}{ TS23/TS-Conformation-B } \\
\hline $\mathrm{C}$ & -1.71730200 & -3.15929000 & 2.52696600 \\
\hline $\mathrm{C}$ & -1.25553800 & -2.11257700 & 1.71037800 \\
\hline $\mathrm{C}$ & -1.81893100 & -2.03200100 & 0.42332900 \\
\hline $\mathrm{C}$ & -2.61023200 & -3.04484400 & -0.13266100 \\
\hline $\mathrm{C}$ & -3.03680600 & -4.07145800 & 0.71543300 \\
\hline $\mathrm{C}$ & -2.63134500 & -4.09980400 & 2.05174000 \\
\hline $\mathrm{C}$ & -3.15904000 & -1.56859700 & -2.00513800 \\
\hline $\mathrm{C}$ & -2.48557500 & -0.54776100 & -1.31932600 \\
\hline $\mathrm{C}$ & -2.76153700 & 0.81719100 & -1.51493700 \\
\hline $\mathrm{C}$ & -3.70668000 & 1.13196800 & -2.50635300 \\
\hline $\mathrm{C}$ & -4.35381400 & 0.14071100 & -3.24135500 \\
\hline $\mathrm{C}$ & -4.09605800 & -1.20374400 & -2.97628300 \\
\hline $\mathrm{H}$ & -1.33918300 & -3.26177000 & 3.54037600 \\
\hline $\mathrm{H}$ & -3.67479600 & -4.86712200 & 0.33063400 \\
\hline $\mathrm{H}$ & -2.98338100 & -4.89594500 & 2.71122500 \\
\hline $\mathrm{H}$ & -3.96229700 & 2.17204200 & -2.69366000 \\
\hline $\mathrm{H}$ & -5.08164100 & 0.41789700 & -4.00692500 \\
\hline $\mathrm{H}$ & -4.63097500 & -1.97422300 & -3.53083200 \\
\hline $\mathrm{C}$ & -2.81796800 & -3.01144700 & -1.64766000 \\
\hline $\mathrm{C}$ & -1.46993900 & -3.38079600 & -2.32289200 \\
\hline $\mathrm{H}$ & -1.57164200 & -3.33692000 & -3.41811400 \\
\hline $\mathrm{H}$ & -0.66739400 & -2.68970200 & -2.03070100 \\
\hline $\mathrm{H}$ & -1.16268900 & -4.39850100 & -2.03751200 \\
\hline
\end{tabular}




\begin{tabular}{|c|c|c|}
\hline-3.89149700 & -3.99982800 & -2.10864100 \\
\hline-3.61317500 & -5.02714100 & -1.83328700 \\
\hline-4.87492400 & -3.77606800 & -1.66825200 \\
\hline-3.99046500 & -3.98527500 & -3.20322600 \\
\hline-1.53753700 & -0.91445000 & -0.36063700 \\
\hline 0.28388300 & -0.36619800 & 3.73329300 \\
\hline-0.32235000 & -1.24255300 & 4.83623000 \\
\hline-1.39234000 & -1.42741700 & 4.66718500 \\
\hline 0.18882500 & -2.20579600 & 4.95375600 \\
\hline-0.23565200 & -0.70754600 & 5.79745500 \\
\hline-0.53912900 & 0.92702200 & 3.64706000 \\
\hline-0.46220500 & 1.47211100 & 4.60271600 \\
\hline-0.17173800 & 1.58478300 & 2.84941400 \\
\hline-1.60029800 & 0.71739500 & 3.47000600 \\
\hline 1.72763200 & 0.01612600 & 4.09894800 \\
\hline 2.19662900 & 0.63642600 & 3.32332700 \\
\hline 1.71017900 & 0.61103100 & 5.02711200 \\
\hline 2.36434700 & -0.85951500 & 4.28238000 \\
\hline 1.59319500 & -2.53429600 & 1.85956800 \\
\hline 1.60251800 & -3.46952000 & 3.07633100 \\
\hline 2.29458000 & -4.30326200 & 2.86968600 \\
\hline 1.96426500 & -2.97008400 & 3.98583400 \\
\hline 0.62084800 & -3.91501000 & 3.28289700 \\
\hline 1.24470100 & -3.34852800 & 0.60053300 \\
\hline 1.12838200 & -2.69737100 & -0.27773600 \\
\hline 2.08049400 & -4.03114900 & 0.37811700 \\
\hline 0.33554900 & -3.95271500 & 0.72457400 \\
\hline 3.00816000 & -1.95229700 & 1.65578600 \\
\hline 3.22717400 & -1.08973800 & 2.29467500 \\
\hline 3.74312700 & -2.73706600 & 1.90102600 \\
\hline 3.18360700 & -1.68283400 & 0.60743800 \\
\hline-1.88470900 & 3.69661200 & -1.38519800 \\
\hline-3.16291400 & 4.53357000 & -1.53548700 \\
\hline-3.99806900 & 3.98948400 & -1.99479700 \\
\hline-3.50678100 & 4.93383400 & -0.57187400 \\
\hline-2.94309400 & 5.39968100 & -2.18358200 \\
\hline-0.84276300 & 4.56029600 & -0.64638000 \\
\hline-1.11490300 & 4.74673400 & 0.40296700 \\
\hline 0.15242700 & 4.09845200 & -0.66266500 \\
\hline-0.76079100 & 5.54056600 & -1.14559900 \\
\hline-1.31319300 & 3.37358100 & -2.77600000 \\
\hline-0.99360500 & 4.31144100 & -3.26087300 \\
\hline-0.43515400 & 2.71993900 & -2.71271700 \\
\hline-2.04123300 & 2.88904800 & -3.43895300 \\
\hline
\end{tabular}




\begin{tabular}{|c|c|c|c|}
\hline $\mathrm{Ni}$ & 0.24042300 & 0.16860400 & 0.20474800 \\
\hline $\mathrm{P}$ & 0.29169400 & -1.11606000 & 1.97482600 \\
\hline $\mathrm{P}$ & -1.99181000 & 2.06027200 & -0.37941400 \\
\hline $\mathrm{H}$ & 1.13672700 & 2.14549200 & -1.50943100 \\
\hline $\mathrm{C}$ & 0.44686900 & 0.17123100 & -3.39592100 \\
\hline $\mathrm{H}$ & 0.64849600 & 1.11369800 & -3.92546600 \\
\hline $\mathrm{H}$ & -0.63260600 & 0.13134000 & -3.17798900 \\
\hline $\mathrm{H}$ & 0.69585200 & -0.65898100 & -4.06919200 \\
\hline $\mathrm{H}$ & 1.52379700 & -0.91934900 & -1.79054500 \\
\hline \multicolumn{4}{|c|}{ TS-Conformation-A } \\
\hline $\mathrm{C}$ & -1.97253700 & 1.44224500 & -0.75596700 \\
\hline $\mathrm{C}$ & -1.85282000 & 0.02148300 & -0.59296400 \\
\hline $\mathrm{C}$ & -1.58852800 & -0.89400300 & -1.72000200 \\
\hline $\mathrm{C}$ & -1.46502500 & -0.45641100 & -3.05482700 \\
\hline $\mathrm{H}$ & -1.60340600 & -2.65124300 & -0.46868200 \\
\hline $\mathrm{H}$ & -2.51190000 & -0.38602800 & 0.17213900 \\
\hline $\mathrm{C}$ & -1.48173300 & -2.27951000 & -1.48201100 \\
\hline $\mathrm{C}$ & -1.21433200 & -1.35523500 & -4.09217500 \\
\hline $\mathrm{C}$ & -1.08434300 & -2.72191700 & -3.82849200 \\
\hline $\mathrm{C}$ & -1.22763700 & -3.17907400 & -2.51506300 \\
\hline $\mathrm{H}$ & -1.13123600 & -0.98473100 & -5.11707600 \\
\hline $\mathrm{H}$ & -0.88607400 & -3.42465300 & -4.64137600 \\
\hline $\mathrm{H}$ & -1.14303100 & -4.24508900 & -2.29032800 \\
\hline $\mathrm{C}$ & -2.32413900 & 2.21249300 & 0.33886900 \\
\hline $\mathrm{C}$ & -5.09302700 & 0.68842300 & 0.10944400 \\
\hline $\mathrm{O}$ & -4.54481400 & -0.17059200 & 0.84606700 \\
\hline $\mathrm{C}$ & -6.04446900 & 0.17404300 & -1.02324100 \\
\hline $\mathrm{C}$ & -6.20507400 & -1.34697800 & -0.96925500 \\
\hline $\mathrm{C}$ & -7.40820000 & 0.86138500 & -0.86161300 \\
\hline $\mathrm{C}$ & -5.41652300 & 0.58718200 & -2.36504000 \\
\hline $\mathrm{H}$ & -5.23751700 & -1.85178000 & -1.10945000 \\
\hline $\mathrm{H}$ & -6.60015900 & -1.66820900 & 0.00644200 \\
\hline $\mathrm{H}$ & -6.89525200 & -1.69807700 & -1.75723500 \\
\hline $\mathrm{H}$ & -7.28102800 & 1.95361400 & -0.84597000 \\
\hline $\mathrm{H}$ & -8.09319100 & 0.59457800 & -1.68596200 \\
\hline $\mathrm{H}$ & -7.89028300 & 0.56542100 & 0.08559500 \\
\hline $\mathrm{H}$ & -6.05665500 & 0.29118000 & -3.21503200 \\
\hline $\mathrm{H}$ & -5.27039400 & 1.67701900 & -2.39587300 \\
\hline $\mathrm{H}$ & -4.43263900 & 0.10935300 & -2.50495900 \\
\hline $\mathrm{O}$ & -4.92740900 & 1.93062500 & 0.16328700 \\
\hline $\mathrm{C}$ & 0.51102700 & -3.71620700 & 1.38963400 \\
\hline $\mathrm{C}$ & 0.61911100 & -2.36252500 & 1.03474600 \\
\hline $\mathrm{C}$ & 1.47891800 & -2.05512300 & -0.03378400 \\
\hline $\mathrm{C}$ & 2.29881000 & -2.99974600 & -0.66112300 \\
\hline
\end{tabular}


$2.16549000 \quad-4.33582400 \quad-0.26955200$

$\begin{array}{lll}1.25499600 & -4.69445600 & 0.72768400\end{array}$

$3.78546900 \quad-1.12452400 \quad-1.22803900$

$2.83282800 \quad-0.24771200 \quad-0.69092600$

$\begin{array}{lll}3.09908200 & 1.09764700 & -0.38239200\end{array}$

$\begin{array}{lll}4.43193800 & 1.52068700 & -0.50971300\end{array}$

$\begin{array}{lll}5.42515100 & 0.64513700 & -0.95219600\end{array}$

$\begin{array}{lll}5.09945100 & -0.65931500 & -1.33757500\end{array}$

$\begin{array}{lll}-0.14289400 & -4.01064500 & 2.20852800\end{array}$

$2.77551100 \quad-5.10637200 \quad-0.74154800$

$\begin{array}{lll}1.15017200 & -5.74246900 & 1.01658700\end{array}$

$\begin{array}{lll}4.69795100 & 2.54934300 & -0.26898900\end{array}$

$\begin{array}{lll}6.45756700 & 0.99249900 & -1.03010200\end{array}$

$5.87884400 \quad-1.31097100 \quad-1.73339400$

$3.27446500 \quad-2.48445800 \quad-1.72237200$

$\begin{array}{lll}4.41608400 & -3.46987800 & -1.98214200\end{array}$

$5.09793500 \quad-3.08029400 \quad-2.75176700$

$\begin{array}{lll}4.99947800 & -3.67345200 & -1.07134400\end{array}$

$4.02540300 \quad-4.42411300 \quad-2.36299000$

$2.49190000 \quad-2.25289800 \quad-3.04127200$

$2.09750000 \quad-3.20746500 \quad-3.42018100$

$1.63859400 \quad-1.57814500 \quad-2.89232500$

$3.15322700 \quad-1.81637600 \quad-3.80614000$

$\begin{array}{lll}1.57033900 & -0.73628300 & -0.44169600\end{array}$

$\begin{array}{lll}-0.09407700 & -0.94207000 & 1.97862100\end{array}$

$\begin{array}{lll}1.64985300 & 2.20710700 \quad-0.06439800\end{array}$

$\begin{array}{lll}-1.70243500 & -1.52710000 & 2.86874700\end{array}$

$-2.58604100 \quad-2.39608200 \quad 1.95616800$

$\begin{array}{lll}-2.95755600 & -1.86343000 & 1.07740600\end{array}$

$\begin{array}{lll}-2.09689400 & -3.33147500 & 1.65251400\end{array}$

$\begin{array}{lll}-3.49007400 & -2.66628900 & 2.52538200\end{array}$

$\begin{array}{lll}-2.48046100 & -0.23978600 & 3.22197600\end{array}$

$\begin{array}{lll}-1.83494700 & 0.55639900 \quad 3.62039600\end{array}$

$\begin{array}{lll}-3.05933200 & 0.12661800 & 2.36136100\end{array}$

$\begin{array}{lll}-3.22355300 & -0.47793400 & 4.00117600\end{array}$

$-1.46353400 \quad-2.32375800 \quad 4.16209700$

$\begin{array}{lll}-1.00277300 & -1.71794700 \quad 4.95132000\end{array}$

$\begin{array}{lll}-2.44581800 & -2.64893000 & 4.54218300\end{array}$

$\begin{array}{lll}-0.85657700 & -3.22719800 \quad 4.01612800\end{array}$

$\begin{array}{lll}1.28814700 & -0.76097800 & 3.32482200\end{array}$

$\begin{array}{lll}2.56331800 & -0.39639400 & 2.55748500\end{array}$

$2.96895800 \quad-1.25943300 \quad 2.01197000$

$\begin{array}{lll}2.38827900 & 0.40600900 & 1.84195600\end{array}$

$3.33826600 \quad-0.06064400 \quad 3.26497300$ 
Conformation-A

\begin{tabular}{|c|c|c|}
\hline 0.88352400 & 0.40245400 & 4.24232200 \\
\hline 0.02827500 & 0.14825600 & 4.88336200 \\
\hline 1.72519500 & 0.66602000 & 4.90485700 \\
\hline 0.61362100 & 1.29972500 & 3.66550200 \\
\hline 1.63421200 & -2.00709700 & 4.15651200 \\
\hline 1.76274900 & -2.89926000 & 3.52700600 \\
\hline 2.60053700 & -1.82769700 & 4.65832000 \\
\hline 0.90671900 & -2.23348900 & 4.94002000 \\
\hline 2.17293400 & 3.59528700 & 1.14446900 \\
\hline 2.95590800 & 2.99175800 & 2.31916300 \\
\hline 2.36678400 & 2.24727500 & 2.86283200 \\
\hline 3.89954500 & 2.52403500 & 2.00625300 \\
\hline 3.20060800 & 3.79812100 & 3.03045600 \\
\hline 2.99643000 & 4.74746900 & 0.55313800 \\
\hline 3.22429700 & 5.46372800 & 1.36045300 \\
\hline 3.95635200 & 4.41686300 & 0.13424900 \\
\hline 2.45211600 & 5.30068000 & -0.22251300 \\
\hline 0.84832000 & 4.16340600 & 1.68674400 \\
\hline 1.06074400 & 4.94739800 & 2.43257000 \\
\hline 0.23809600 & 4.61418600 & 0.89311400 \\
\hline 0.24352900 & 3.38378600 & 2.17337400 \\
\hline 1.50538800 & 2.92551600 & -1.85244700 \\
\hline 2.78028000 & 3.52302100 & -2.46371100 \\
\hline 2.56072700 & 3.78386300 & -3.51331200 \\
\hline 3.11664200 & 4.44000600 & -1.96880500 \\
\hline 3.61106000 & 2.80570600 & -2.48073100 \\
\hline 0.39198500 & 3.97779600 & -1.86147500 \\
\hline 0.67561000 & 4.88901700 & -1.31602000 \\
\hline 0.16754900 & 4.27246100 & -2.90019400 \\
\hline-0.52644000 & 3.58653300 & -1.41614200 \\
\hline 1.10026100 & 1.72562300 & -2.72365100 \\
\hline 1.94923400 & 1.05156600 & -2.90332800 \\
\hline 0.30048000 & 1.13579600 & -2.26836700 \\
\hline 0.74650600 & 2.08245500 & -3.70473800 \\
\hline 0.24043700 & 0.80893000 & 0.36689900 \\
\hline-1.59440000 & 0.59811500 & -3.29540500 \\
\hline-1.79136700 & 1.93069100 & -1.71566000 \\
\hline-2.60313800 & 1.68338700 & 1.24969200 \\
\hline-2.52257300 & 3.68401200 & 0.35801000 \\
\hline-2.20430400 & 4.18468600 & -0.56557000 \\
\hline-3.60771900 & 3.82885400 & 0.48042200 \\
\hline-2.02539500 & 4.15253400 & 1.22112200 \\
\hline & 1.210982 & 0.9611440 \\
\hline
\end{tabular}


$\begin{array}{lll}-3.85022200 & 3.30865500 & -1.24007600\end{array}$

$\begin{array}{lll}-6.11735200 & 2.40222600 & -0.91203500\end{array}$

\section{$\begin{array}{lll}-6.45190900 & 0.22581800 & 0.22611800\end{array}$}

$\begin{array}{lll}-4.39320100 & -1.50277400 & 1.20913400\end{array}$

$\begin{array}{lll}-5.79382900 & -2.11841700 & 1.20329000\end{array}$

$\begin{array}{lll}-6.51913900 & -1.43518100 & 1.66844000\end{array}$

$\begin{array}{lll}-6.13793900 & -2.34698200 & 0.18315200\end{array}$

$\begin{array}{lll}-5.81368100 & -3.04753000 & 1.79104300\end{array}$

$\begin{array}{lll}-3.96795800 & -1.21377200 & 2.67262800\end{array}$

$\begin{array}{lll}-3.98671000 & -2.14270500 & 3.26347600\end{array}$

$\begin{array}{lll}-2.94916900 & -0.80535700 & 2.72229600\end{array}$

$\begin{array}{lll}-4.65674400 & -0.48965300 & 3.13527400\end{array}$

$\begin{array}{lll}-1.97980300 & -0.49057600 & 0.37704000\end{array}$

$0.13658800 \quad-1.48021300 \quad-1.38568600$

$\begin{array}{lll}-1.07178300 & 2.22228300 & -0.42732500\end{array}$

$\begin{array}{lll}1.71247100 & -2.58471200 & -1.55582400\end{array}$

$1.96639200 \quad-3.34009800 \quad-0.23915000$

$\begin{array}{lll}1.99301900 & -2.68347800 & 0.63503900\end{array}$

$1.21477600 \quad-4.11779200 \quad-0.04910700$

$2.94738800 \quad-3.84053400 \quad-0.30312500$

$2.87163200 \quad-1.60355900 \quad-1.80359900$

$2.76493500 \quad-1.06225600 \quad-2.75357100$

$\begin{array}{lll}2.93725500 & -0.85569600 & -1.01090800\end{array}$

$\begin{array}{lll}3.82705000 & -2.15158900 & -1.84334100\end{array}$

$\begin{array}{lll}1.72739800 & -3.61757200 & -2.69177900\end{array}$

$\begin{array}{lll}1.71207700 & -3.14755300 & -3.68281200\end{array}$

$\begin{array}{lll}2.66909900 & -4.18913900 & -2.62503900\end{array}$

$0.90503200 \quad-4.34298500 \quad-2.63896400$

$\begin{array}{lll}-0.67814800 & -1.35789400 & -3.13593000\end{array}$

$\begin{array}{lll}-1.99272600 & -0.60273400 & -2.89811400\end{array}$

$\begin{array}{lll}-2.74634700 & -1.25085600 & -2.42838700\end{array}$

$\begin{array}{lll}-1.84549700 & 0.26451600 & -2.25288100\end{array}$

$\begin{array}{lll}-2.40685600 & -0.25429100 & -3.85823100\end{array}$

$0.27160700 \quad-0.51937000 \quad-4.00474900$

$\begin{array}{lll}1.16221100 & -1.09069100 & -4.30419300\end{array}$

$-0.23956900 \quad-0.20106400 \quad-4.92908400$

$\begin{array}{lll}0.61513000 & 0.38245000 & -3.47612800\end{array}$

$\begin{array}{lll}-1.04986000 & -2.65801300 & -3.86239100\end{array}$

$-1.61844600 \quad-3.34273900 \quad-3.21724500$

$\begin{array}{lll}-1.70460900 & -2.40460600 & -4.71438800\end{array}$

$-0.19065900 \quad-3.19620500 \quad-4.27278100$

$\begin{array}{lll}-0.90594500 & 3.43116200 & -1.91876700\end{array}$

$\begin{array}{lll}-1.44707600 & 2.74340200 & -3.18040200\end{array}$

$\begin{array}{lll}-0.92180000 & 1.80662100 & -3.38839300\end{array}$ 


\begin{tabular}{|c|c|c|c|}
\hline $\mathrm{H}$ & -2.52281600 & 2.52829800 & -3.11942600 \\
\hline $\mathrm{H}$ & -1.28647700 & 3.40876500 & -4.04565300 \\
\hline $\mathrm{C}$ & -1.56481400 & 4.81359300 & -1.80688800 \\
\hline $\mathrm{H}$ & -1.35047700 & 5.37887100 & -2.73039200 \\
\hline $\mathrm{H}$ & -2.65757500 & 4.76462900 & -1.71141400 \\
\hline $\mathrm{H}$ & -1.17225200 & 5.40621400 & -0.97196300 \\
\hline $\mathrm{C}$ & 0.60998300 & 3.63128400 & -2.09972100 \\
\hline $\mathrm{H}$ & 0.80343800 & 4.18678100 & -3.03295600 \\
\hline $\mathrm{H}$ & 1.04615600 & 4.20972300 & -1.27497400 \\
\hline $\mathrm{H}$ & 1.14132700 & 2.66937800 & -2.15368400 \\
\hline $\mathrm{C}$ & -1.22728800 & 3.28530000 & 1.18922500 \\
\hline $\mathrm{C}$ & -2.48743500 & 4.15342900 & 1.31791500 \\
\hline $\mathrm{H}$ & -2.42923300 & 4.70506200 & 2.27242700 \\
\hline $\mathrm{H}$ & -2.59384000 & 4.89812300 & 0.52157100 \\
\hline $\mathrm{H}$ & -3.40329600 & 3.54959500 & 1.35172500 \\
\hline $\mathrm{C}$ & 0.01261800 & 4.17983700 & 1.33089500 \\
\hline $\mathrm{H}$ & -0.01547500 & 5.03897000 & 0.64632600 \\
\hline $\mathrm{H}$ & 0.05866700 & 4.58377800 & 2.35653900 \\
\hline $\mathrm{H}$ & 0.94005100 & 3.62942300 & 1.14820400 \\
\hline $\mathrm{C}$ & -1.26559200 & 2.25989800 & 2.33274100 \\
\hline $\mathrm{H}$ & -2.20362100 & 1.68576700 & 2.32845100 \\
\hline $\mathrm{H}$ & -0.44287600 & 1.54344600 & 2.27700100 \\
\hline $\mathrm{H}$ & -1.20321600 & 2.78047200 & 3.30312200 \\
\hline $\mathrm{Ni}$ & 0.39587200 & 0.50158600 & -0.15876000 \\
\hline $\mathrm{H}$ & 1.70455100 & 1.08010300 & 3.69707200 \\
\hline $\mathrm{H}$ & 1.65012700 & 1.94753700 & 1.72313300 \\
\hline $\mathrm{H}$ & 3.03974900 & 1.30973900 & -0.90156900 \\
\hline $\mathrm{C}$ & 3.51445300 & 3.06236900 & 0.24361100 \\
\hline $\mathrm{H}$ & 3.68930200 & 3.35444300 & 1.29115900 \\
\hline $\mathrm{H}$ & 4.42462100 & 3.26628900 & -0.33909800 \\
\hline $\mathrm{H}$ & 2.70725700 & 3.68656300 & -0.15991000 \\
\hline \multicolumn{4}{|c|}{ Conformation-B } \\
\hline $\mathrm{C}$ & 3.05527300 & 3.21908600 & -1.09400800 \\
\hline $\mathrm{C}$ & 2.13614700 & 2.23785500 & -0.68811600 \\
\hline $\mathrm{C}$ & 2.21239300 & 1.00361100 & -1.35690000 \\
\hline $\mathrm{C}$ & 2.92859200 & 0.80976100 & -2.54222000 \\
\hline $\mathrm{C}$ & 3.81740200 & 1.82178200 & -2.92183000 \\
\hline $\mathrm{C}$ & 3.92195000 & 2.99429400 & -2.16632400 \\
\hline $\mathrm{C}$ & 2.36501400 & -1.58845400 & -2.46357900 \\
\hline $\mathrm{C}$ & 1.87719200 & -1.35359700 & -1.17128000 \\
\hline $\mathrm{C}$ & 1.81702500 & -2.35020200 & -0.17859700 \\
\hline $\mathrm{C}$ & 2.08983800 & -3.66195300 & -0.59875400 \\
\hline $\mathrm{C}$ & 2.47284000 & -3.94607500 & -1.90963900 \\
\hline $\mathrm{C}$ & 2.65336600 & -2.90884900 & -2.82431200 \\
\hline
\end{tabular}


$2.27147200 \quad-4.98614800 \quad 1.94283500$

$2.70238800 \quad-4.25092700 \quad 3.50134800$

$1.27986700 \quad-5.29541300 \quad 3.37681500$

$0.50497600 \quad-2.74294300 \quad 3.92994400$

$\begin{array}{lll}1.35443500 & -2.39221400 & 4.53185000\end{array}$

$\begin{array}{lll}-0.18919100 & -1.90129000 \quad 3.80948100\end{array}$

$-0.01189100 \quad-3.52558100 \quad 4.51117300$

$\begin{array}{lll}-0.31678700 & -3.79536300 & 1.83099400\end{array}$

$\begin{array}{lll}-0.83382200 & -4.55228400 & 2.44489700\end{array}$

$\begin{array}{lll}-1.02361400 & -2.97138700 \quad 1.66521700\end{array}$

$\begin{array}{lll}-0.09747900 & -4.24911700 & 0.85500300\end{array}$

$3.52398800 \quad-1.62064700 \quad 1.98202100$

$\begin{array}{lll}4.42909700 & -2.81505400 & 1.65695400\end{array}$

$5.48112900 \quad-2.52541100 \quad 1.82843200$

$\begin{array}{lll}4.22921400 & -3.69071300 & 2.28399000\end{array}$

$\begin{array}{lll}4.34524900 & -3.11775800 & 0.60366000\end{array}$

$3.64750900 \quad-1.26150800 \quad 3.47125000$

$3.41434600 \quad-2.11755600 \quad 4.12056200$

$\begin{array}{lll}4.68398800 & -0.95608400 & 3.69549900\end{array}$

$2.98434500 \quad-0.42876400 \quad 3.75112000$

$\begin{array}{lll}4.01442400 & -0.43252900 & 1.13499200\end{array}$

$\begin{array}{lll}4.08890800 & -0.69598100 & 0.07070600\end{array}$

$\begin{array}{lll}3.36602200 & 0.44802600 & 1.21687500\end{array}$

$\begin{array}{lll}5.02470300 & -0.13859900 & 1.46676000\end{array}$

$\begin{array}{lll}-1.73819300 & -0.83263400 & -0.25645400\end{array}$

$\begin{array}{lll}-2.24353800 & 0.41907600 & 0.25285200\end{array}$

$\begin{array}{lll}-2.57736900 & 0.67496100 & 1.66379500\end{array}$

$\begin{array}{lll}-1.94642900 & -0.01145600 & 2.72266200\end{array}$

$\begin{array}{lll}-4.02414100 & 2.21477800 & 1.20940200\end{array}$

$\begin{array}{lll}-2.83145600 & 1.02397600 & -0.44624200\end{array}$

$\begin{array}{lll}-3.51517200 & 1.66985800 \quad 2.00867200\end{array}$

$\begin{array}{lll}-2.23235700 & 0.28397300 & 4.05355100\end{array}$

$-3.16017000 \quad 1.28206600 \quad 4.37691100$

$\begin{array}{lll}-3.80073400 & 1.97077500 & 3.34266400\end{array}$

$\begin{array}{lll}-1.71759000 & -0.26116300 \quad 4.84993400\end{array}$

$\begin{array}{lll}-3.38040900 \quad 1.51798700 & 5.42091200\end{array}$

$\begin{array}{lll}-4.53170900 & 2.75009300 & 3.57577100\end{array}$

$\begin{array}{lll}-2.15387800 & -1.27305600 & -1.63147100\end{array}$

$\begin{array}{lll}-3.56038100 & -1.72665200 & -1.58514500\end{array}$

$\begin{array}{lll}-4.52925800 & -0.85946600 & -1.87914600\end{array}$

$-5.91201700 \quad-1.42184700 \quad-1.52173600$

$\begin{array}{lll}-4.34256900 & 0.24555100 & -2.34451200\end{array}$

$\begin{array}{lll}-6.08477000 & -2.82000400 & -2.13924900\end{array}$

$\begin{array}{lll}-5.98241300 & -1.51477400 & 0.01623500\end{array}$ 


\begin{tabular}{|c|c|c|c|}
\hline C & -6.99343700 & -0.47101000 & -2.04380900 \\
\hline $\mathrm{H}$ & -6.01124900 & -2.78231400 & -3.23839700 \\
\hline $\mathrm{H}$ & -5.31761100 & -3.51472100 & -1.77011200 \\
\hline $\mathrm{H}$ & -7.07596800 & -3.22659500 & -1.88087000 \\
\hline $\mathrm{H}$ & -5.84300500 & -0.52665300 & 0.48220900 \\
\hline $\mathrm{H}$ & -6.96634800 & -1.90271000 & 0.32638900 \\
\hline $\mathrm{H}$ & -5.20381400 & -2.18427100 & 0.40916800 \\
\hline $\mathrm{H}$ & -7.99239900 & -0.84925700 & -1.77415700 \\
\hline $\mathrm{H}$ & -6.87620300 & 0.53611000 & -1.61855800 \\
\hline $\mathrm{H}$ & -6.94592700 & -0.37451800 & -3.13936900 \\
\hline $\mathrm{H}$ & -1.19267100 & -0.76077300 & 2.48154900 \\
\hline $\mathrm{Ni}$ & -0.34625900 & 0.50224500 & -0.03837900 \\
\hline $\mathrm{P}$ & 0.54785700 & 2.48555800 & 0.25024200 \\
\hline $\mathrm{P}$ & 1.63748800 & -1.81340900 & 1.59085500 \\
\hline $\mathrm{H}$ & -1.66974400 & -1.67561700 & 0.44223300 \\
\hline $\mathrm{H}$ & -2.12903700 & -0.42862200 & -2.33321400 \\
\hline $\mathrm{C}$ & -1.37169900 & -2.45163200 & -2.17871700 \\
\hline $\mathrm{H}$ & -1.76041300 & -2.76177100 & -3.16115500 \\
\hline $\mathrm{H}$ & -1.43649300 & -3.31184900 & -1.49410100 \\
\hline $\mathrm{H}$ & -0.31262600 & -2.18863200 & -2.28839500 \\
\hline
\end{tabular}

\section{Cartesian coordinates of the structures (optimization in benzene)} TS22

\begin{tabular}{|c|c|c|c|}
\hline $\mathrm{C}$ & -1.14933300 & 0.66700600 & 2.16808600 \\
\hline $\mathrm{C}$ & -0.20131900 & 0.38234500 & 1.16419700 \\
\hline $\mathrm{C}$ & 1.02110100 & 1.08828500 & 1.22451800 \\
\hline $\mathrm{C}$ & 1.17327400 & 2.19514900 & 2.08690000 \\
\hline $\mathrm{C}$ & 0.21207900 & 2.41580000 & 3.07657300 \\
\hline $\mathrm{C}$ & -0.92692000 & 1.61690200 & 3.15667700 \\
\hline $\mathrm{C}$ & 3.50212600 & 2.44961100 & 1.29461000 \\
\hline $\mathrm{C}$ & 3.33556600 & 1.22672100 & 0.61551200 \\
\hline $\mathrm{C}$ & 4.44873100 & 0.50911800 & 0.11509800 \\
\hline $\mathrm{C}$ & 5.71482100 & 1.10496500 & 0.24328100 \\
\hline $\mathrm{C}$ & 5.89622600 & 2.32204300 & 0.89165200 \\
\hline $\mathrm{C}$ & 4.79129700 & 2.97366800 & 1.43313100 \\
\hline $\mathrm{H}$ & -2.10465700 & 0.13851000 & 2.13262600 \\
\hline $\mathrm{H}$ & 0.33341900 & 3.24325000 & 3.77486100 \\
\hline $\mathrm{H}$ & -1.67331100 & 1.78876700 & 3.93494100 \\
\hline $\mathrm{H}$ & 6.58147800 & 0.59351200 & -0.16706200 \\
\hline $\mathrm{H}$ & 6.89404000 & 2.75705200 & 0.98248900 \\
\hline $\mathrm{H}$ & 4.93344400 & 3.92051300 & 1.95294400 \\
\hline $\mathrm{C}$ & 2.27182800 & 3.20814500 & 1.77518100 \\
\hline $\mathrm{C}$ & 2.58493100 & 4.11486900 & 2.97281500 \\
\hline $\mathrm{H}$ & 3.33834100 & 4.86897300 & 2.70808200 \\
\hline $\mathrm{H}$ & 2.95545200 & 3.54056500 & 3.83542100 \\
\hline
\end{tabular}


$1.69125000 \quad 4.67353500 \quad 3.28259700$

$1.76347400 \quad 4.09861300$

0.60899100

$0.84803700 \quad 4.63360100$

0.90588600

$1.53098700 \quad 3.49799000$

$-0.28234600$

$2.53135600 \quad 4.83758400$

0.32998000

2.06735800

0.71602400

0.40622800

$-0.42164700 \quad-2.47196300$

0.36312400

$\begin{array}{lll}1.07930000 & -2.60857300 & 0.60545400\end{array}$

$1.42051500 \quad-1.89491200 \quad 1.36835000$

$\begin{array}{lll}1.68470300 & -2.43784600 & -0.29066500\end{array}$

$\begin{array}{lll}1.29916800 & -3.62539400 & 0.97581500\end{array}$

$-1.16662200 \quad-2.75815300 \quad 1.67860900$

$\begin{array}{lll}-1.00248400 & -3.80904100 & 1.96995900\end{array}$

$\begin{array}{lll}-2.24657300 & -2.60089400 & 1.57565100\end{array}$

$\begin{array}{lll}-0.79857800 & -2.12375200 & 2.49866900\end{array}$

$\begin{array}{lll}-0.90544200 & -3.45614700 & -0.70983100\end{array}$

$\begin{array}{lll}-1.97524200 & -3.32406000 & -0.91744500\end{array}$

$\begin{array}{lll}-0.76166700 & -4.48926300 & -0.35132600\end{array}$

$\begin{array}{lll}-0.34893200 & -3.35258000 & -1.65159400\end{array}$

$\begin{array}{lll}-0.29692600 & -0.22983700 & -1.86426500\end{array}$

$\begin{array}{lll}-1.41890000 & -0.61674400 & -2.84860000\end{array}$

$\begin{array}{lll}-1.66242800 & -1.68779900 & -2.80745600\end{array}$

$\begin{array}{lll}-1.09361600 & -0.38426900 & -3.87726900\end{array}$

$\begin{array}{lll}-2.34014600 & -0.05070900 & -2.64481400\end{array}$

$\begin{array}{lll}1.01356100 & -0.87329300 & -2.31189100\end{array}$

$\begin{array}{lll}1.82843500 & -0.69078900 & -1.60430400\end{array}$

$\begin{array}{lll}1.30459900 & -0.44878400 & -3.28837000\end{array}$

$0.91996100 \quad-1.95950000 \quad-2.44936600$

$\begin{array}{lll}-0.15064900 & 1.30143900 & -1.88314000\end{array}$

$\begin{array}{lll}-1.01407100 & 1.79644200 & -1.42036700\end{array}$

$\begin{array}{lll}-0.07760500 & 1.64491100 & -2.92895100\end{array}$

$\begin{array}{lll}0.75835800 & 1.62501800 & -1.36233600\end{array}$

$\begin{array}{lll}5.29677300 & -2.26483000 & 0.56853700\end{array}$

$\begin{array}{lll}4.47057000 & -2.30042100 & 1.86743100\end{array}$

$\begin{array}{lll}3.47605800 & -2.73650900 & 1.70907900\end{array}$

$4.33830100 \quad-1.29356100 \quad 2.29435200$

$\begin{array}{lll}4.99170300 & -2.91525200 & 2.62161300\end{array}$

$\begin{array}{lll}6.72241200 & -1.82988900 & 0.93218800\end{array}$

$\begin{array}{lll}7.17912000 & -2.60481800 & 1.57324700\end{array}$

$\begin{array}{lll}6.73041900 & -0.89450100 & 1.50790300\end{array}$

$\begin{array}{lll}7.37433400 & -1.70574100 & 0.05909300\end{array}$

$5.32928000 \quad-3.67743600 \quad-0.03862400$

$\begin{array}{lll}4.32633800 & -4.01118500 & -0.34860400\end{array}$

$5.70487300 \quad-4.39678800 \quad 0.70949500$ 
$5.99578000 \quad-3.73812100 \quad-0.91201500$

$\begin{array}{lll}5.12678800 & -1.03336200 & -2.33968100\end{array}$

$\begin{array}{lll}4.67093700 & 0.30227700 & -2.95140200\end{array}$ 


$\begin{array}{lrrr}\mathrm{H} & -5.14019100 & 4.23635600 & 0.46755300 \\ \mathrm{H} & -5.58785500 & 4.27411800 & -1.25811300 \\ \mathrm{O} & -4.44730100 & 1.92610000 & -1.77233900 \\ \mathrm{H} & -5.65460700 & -0.22388400 & 0.42945500 \\ \mathrm{H} & -4.93530300 & -1.44140200 & 2.11713700 \\ \mathrm{C} & -6.72917500 & 0.98053800 & -1.71696500 \\ \mathrm{H} & -7.46221200 & 0.16676300 & -1.85383600 \\ \mathrm{H} & -6.81394800 & 1.67309600 & -2.56531800 \\ \mathrm{H} & -6.99055100 & 1.51311500 & -0.78966100 \\ \mathrm{Ni} & -3.10482700 & -0.26492100 & -0.17291000\end{array}$

TS23/TS-Conformation-B

\begin{tabular}{|c|c|c|c|}
\hline $\mathrm{C}$ & -1.79303300 & -3.23495900 & 2.39380400 \\
\hline $\mathrm{C}$ & -1.30880700 & -2.16569100 & 1.62063600 \\
\hline $\mathrm{C}$ & -1.86362500 & -2.02361000 & 0.33567900 \\
\hline $\mathrm{C}$ & -2.67245500 & -2.99824400 & -0.26202800 \\
\hline $\mathrm{C}$ & -3.12176600 & -4.04944800 & 0.54292700 \\
\hline $\mathrm{C}$ & -2.72208400 & -4.13888200 & 1.87842600 \\
\hline $\mathrm{C}$ & -3.18736200 & -1.43784200 & -2.07422300 \\
\hline $\mathrm{C}$ & -2.49480900 & -0.45891200 & -1.34758700 \\
\hline $\mathrm{C}$ & -2.74220100 & 0.91787100 & -1.48973700 \\
\hline $\mathrm{C}$ & -3.68027500 & 1.29176800 & -2.46709900 \\
\hline $\mathrm{C}$ & -4.34674300 & 0.34369000 & -3.24087800 \\
\hline $\mathrm{C}$ & -4.11566500 & -1.01538400 & -3.03042500 \\
\hline $\mathrm{H}$ & -1.42097600 & -3.38263800 & 3.40388400 \\
\hline $\mathrm{H}$ & -3.77386200 & -4.81624200 & 0.12439300 \\
\hline $\mathrm{H}$ & -3.09178000 & -4.95372700 & 2.50463400 \\
\hline $\mathrm{H}$ & -3.91303100 & 2.34399600 & -2.61183000 \\
\hline $\mathrm{H}$ & -5.06840600 & 0.66570500 & -3.99472800 \\
\hline $\mathrm{H}$ & -4.66471300 & -1.75184300 & -3.61671100 \\
\hline $\mathrm{C}$ & -2.87514200 & -2.90023200 & -1.77479300 \\
\hline $\mathrm{C}$ & -1.53274700 & -3.26880100 & -2.46143500 \\
\hline $\mathrm{H}$ & -1.63111600 & -3.18092800 & -3.55436600 \\
\hline $\mathrm{H}$ & -0.71738400 & -2.60531900 & -2.14162600 \\
\hline $\mathrm{H}$ & -1.24606400 & -4.30268600 & -2.21523800 \\
\hline $\mathrm{C}$ & -3.96593300 & -3.84839200 & -2.27719900 \\
\hline $\mathrm{H}$ & -3.70589800 & -4.89130600 & -2.04589000 \\
\hline $\mathrm{H}$ & -4.94549000 & -3.62607500 & -1.82752500 \\
\hline $\mathrm{H}$ & -4.06389200 & -3.78499300 & -3.37011600 \\
\hline $\mathrm{O}$ & -1.55628700 & -0.88228800 & -0.40299700 \\
\hline $\mathrm{C}$ & 0.25189200 & -0.53769700 & 3.72370300 \\
\hline $\mathrm{C}$ & -0.37983000 & -1.44738300 & 4.78493400 \\
\hline $\mathrm{H}$ & -1.45361500 & -1.59732700 & 4.60561500 \\
\hline $\mathrm{H}$ & 0.10608500 & -2.42780600 & 4.86356200 \\
\hline $\mathrm{H}$ & -0.28343600 & -0.95713000 & 5.76892400 \\
\hline
\end{tabular}




\begin{tabular}{|c|c|c|}
\hline-0.54235000 & 0.77549600 & 3.68926800 \\
\hline-0.47078500 & 1.27152500 & 4.67184600 \\
\hline-0.14654100 & 1.46333400 & 2.93154300 \\
\hline-1.60447300 & 0.59811400 & 3.48487600 \\
\hline 1.70040700 & -0.20136800 & 4.11438300 \\
\hline 2.18859700 & 0.43916200 & 3.36753000 \\
\hline 1.68833400 & 0.35689100 & 5.06517100 \\
\hline 2.31783100 & -1.09632400 & 4.26791700 \\
\hline 1.53116000 & -2.64126800 & 1.76295500 \\
\hline 1.51588500 & -3.62797900 & 2.93796200 \\
\hline 2.19046200 & -4.46737000 & 2.69817100 \\
\hline 1.88432300 & -3.17653100 & 3.86955900 \\
\hline 0.52375000 & -4.05956300 & 3.12225200 \\
\hline 1.17674600 & -3.39326200 & 0.46745200 \\
\hline 1.06507300 & -2.70012000 & -0.37891500 \\
\hline 2.00550400 & -4.07373600 & 0.21398600 \\
\hline 0.26137700 & -3.99339400 & 0.56254500 \\
\hline 2.95767400 & -2.07566400 & 1.59378700 \\
\hline 3.18308100 & -1.23795900 & 2.26291100 \\
\hline 3.67796600 & -2.87948800 & 1.82058500 \\
\hline 3.14975400 & -1.77261600 & 0.55751700 \\
\hline-1.78888800 & 3.76554500 & -1.25804700 \\
\hline-3.04410700 & 4.64132400 & -1.37584500 \\
\hline-3.89174900 & 4.13719200 & -1.85766900 \\
\hline-3.37939100 & 5.01111900 & -0.39732500 \\
\hline-2.80045300 & 5.52640000 & -1.98897800 \\
\hline-0.72156200 & 4.57177500 & -0.49270400 \\
\hline-0.99150900 & 4.73754100 & 0.56073200 \\
\hline 0.25666200 & 4.07546200 & -0.51894100 \\
\hline-0.60386900 & 5.56251400 & -0.96340700 \\
\hline-1.23192900 & 3.47580400 & -2.66187100 \\
\hline-0.89310500 & 4.42191900 & -3.11687700 \\
\hline-0.36932600 & 2.80005800 & -2.62759400 \\
\hline-1.97537100 & 3.03156800 & -3.33593500 \\
\hline-3.35564100 & 2.20559200 & 0.99195400 \\
\hline-4.75104900 & 2.60761200 & 0.49942600 \\
\hline-5.47753600 & 2.43947500 & 1.31398800 \\
\hline-4.82128400 & 3.66447300 & 0.21885100 \\
\hline-5.07768900 & 1.99680800 & -0.35464400 \\
\hline-2.88307400 & 3.17662700 & 2.08484200 \\
\hline-2.85903200 & 4.21540200 & 1.72331500 \\
\hline-3.57612200 & 3.14383600 & 2.94294700 \\
\hline-1.87955500 & 2.92103400 & 2.45430700 \\
\hline $3.4349 / 70$ & 0.77887600 & 1.55943500 \\
\hline
\end{tabular}


H

H

$\mathrm{H}$

\begin{tabular}{|c|c|c|}
\hline-3.99100700 & 0.10901500 & 0.87161600 \\
\hline-2.46745700 & 0.34434300 & 1.74582700 \\
\hline-4.01304000 & 0.79043600 & 2.51045800 \\
\hline 1.40504300 & 1.12791000 & -1.15954900 \\
\hline 2.04572400 & 0.80493300 & 0.09715900 \\
\hline 2.50297100 & 1.82349700 & 1.06543100 \\
\hline 1.65452300 & 2.84252400 & 1.53490900 \\
\hline 4.47851700 & 0.97052400 & 1.24690600 \\
\hline 2.72769300 & -0.04274500 & 0.02432800 \\
\hline 3.80457900 & 1.75552300 & 1.59753100 \\
\hline 2.08698500 & 3.76279200 & 2.48927200 \\
\hline 3.38424300 & 3.68259200 & 3.00754700 \\
\hline 4.24057200 & 2.67352200 & 2.55575800 \\
\hline 1.40321800 & 4.54052200 & 2.84057700 \\
\hline 3.72406800 & 4.39931900 & 3.75933800 \\
\hline 5.25670400 & 2.60032000 & 2.95234200 \\
\hline 1.26895300 & 0.11985500 & -2.12441500 \\
\hline 3.15164000 & 0.03542700 & -3.11477000 \\
\hline 3.92562500 & -0.74933700 & -2.48277300 \\
\hline 5.45171500 & -0.57041400 & -2.75048100 \\
\hline 3.58229900 & -1.60546600 & -1.64307300 \\
\hline 5.70818500 & 0.29744000 & -3.98583800 \\
\hline 6.02705200 & 0.11791900 & -1.49861900 \\
\hline 6.09587400 & -1.95186900 & -2.92397100 \\
\hline 5.27776100 & -0.16097800 & -4.89015800 \\
\hline 5.24880500 & 1.28986400 & -3.87415700 \\
\hline 6.79180000 & 0.42848700 & -4.15132100 \\
\hline 5.86832800 & -0.50646400 & -0.60613800 \\
\hline 7.11083800 & 0.29539500 & -1.60866600 \\
\hline 5.53987600 & 1.09159800 & -1.32583800 \\
\hline 7.19146100 & -1.86763000 & -3.02772700 \\
\hline 5.86956200 & -2.59014500 & -2.05830700 \\
\hline 5.71044800 & -2.45891500 & -3.82451400 \\
\hline 0.63266000 & 2.87993700 & 1.16117500 \\
\hline 0.23468600 & 0.15246900 & 0.22189700 \\
\hline 0.25323700 & -1.20730500 & 1.93345500 \\
\hline-1.94404400 & 2.09859100 & -0.30828300 \\
\hline 1.17234700 & 2.16391400 & -1.41761500 \\
\hline 0.47550200 & 0.27169700 & -3.38199600 \\
\hline 0.69941500 & 1.22536000 & -3.88172300 \\
\hline-0.60468700 & 0.25157700 & -3.16531200 \\
\hline 0.70350400 & -0.54290500 & -4.08153700 \\
\hline 1.52598900 & -0.89232700 & -1.81279300 \\
\hline
\end{tabular}

TS-Conformation-A 
$-1.83479500$

$0.00810400 \quad-0.56546400$

$-1.46696100$

$-0.52048800 \quad-3.02161200$

$\begin{array}{lll}-1.62760300 & -2.66829000 & -0.39844900\end{array}$

$-1.24163500 \quad-1.44157500 \quad-4.04545200$

$-1.13710100 \quad-2.80606600 \quad-3.76030600$

$\begin{array}{lll}-1.27918500 & -3.23742900 & -2.43806900\end{array}$

$\begin{array}{lll}-1.15796300 & -1.08939100 & -5.07685500\end{array}$

$\begin{array}{lll}-0.95858700 & -3.52624200 & -4.56251000\end{array}$

$\begin{array}{lll}-1.21310000 & -4.30091300 & -2.19533000\end{array}$

$\begin{array}{lll}-2.35609700 & 2.22523000 & 0.29330300\end{array}$

$\begin{array}{lll}-5.06409300 & 0.73761100 & 0.07108300\end{array}$

$\begin{array}{lll}-4.56144900 & -0.07489300 & 0.88399600\end{array}$

$\begin{array}{lll}-6.06803800 & 0.18316100 & -0.99403700\end{array}$

$\begin{array}{lll}-6.32376500 & -1.31192500 & -0.79073200\end{array}$

$\begin{array}{lll}-7.38260400 & 0.96937000 & -0.88109300\end{array}$

$\begin{array}{lll}-5.44023200 & 0.42252900 & -2.37741700\end{array}$

$\begin{array}{lll}-5.39304600 & -1.88965800 & -0.89464900\end{array}$

$\begin{array}{lll}-6.72051800 & -1.51382300 & 0.21576300\end{array}$

$\begin{array}{lll}-7.04934200 & -1.69062200 & -1.53257500\end{array}$

$\begin{array}{lll}-7.18948100 & 2.04773900 & -0.97825600\end{array}$

$\begin{array}{lll}-8.10038300 & 0.66608700 & -1.66380300\end{array}$

$\begin{array}{lll}-7.86270800 & 0.80125000 & 0.09806400\end{array}$

$\begin{array}{lll}-6.10979200 & 0.07773900 & -3.18517800\end{array}$

$\begin{array}{lll}-5.23497400 & 1.49326000 & -2.52314300\end{array}$

$\begin{array}{lll}-4.48624000 & -0.12108600 & -2.47734800\end{array}$

$\begin{array}{lll}-4.81574800 & 1.96851900 & -0.00594300\end{array}$

$0.49937600 \quad-3.69511700 \quad 1.43623000$

$\begin{array}{lll}0.61273400 & -2.34723300 & 1.06197100\end{array}$

$1.46131700 \quad-2.05971200 \quad-0.02126900$

$\begin{array}{lll}2.26593900 & -3.01797700 & -0.64739400\end{array}$

$2.12789400 \quad-4.34791100 \quad-0.23653500$

$1.22794900 \quad-4.68702500 \quad 0.77709600$

$\begin{array}{lll}3.75488600 & -1.15921500 & -1.26567900\end{array}$

$2.81655800 \quad-0.26974000 \quad-0.72380900$

$3.09532700 \quad 1.07758500 \quad-0.43571800$

$\begin{array}{lll}4.42706100 & 1.49283500 & -0.59801800\end{array}$

$5.40678300 \quad 0.60720700 \quad-1.05021800$

$\begin{array}{lll}5.06828200 & -0.70169500 & -1.40860200\end{array}$

$\begin{array}{lll}-0.14628900 & -3.97446600 & 2.26710800\end{array}$

$2.72539000 \quad-5.12927100 \quad-0.70684800$ 
$1.11957300 \quad-5.73060100 \quad 1.08063700$

$\begin{array}{lll}4.70366400 & 2.52277000 & -0.37549900\end{array}$

$6.43862600 \quad 0.94928600 \quad-1.15531800$

$5.83714000 \quad-1.36249900 \quad-1.80986000$

$\begin{array}{lll}3.22935900 & -2.52420100 & -1.73005300\end{array}$

$\begin{array}{lll}4.36133400 & -3.52031900 & -1.99091500\end{array}$

$\begin{array}{lll}5.03310200 & -3.14820300 & -2.77786600\end{array}$

$\begin{array}{lll}4.95795100 & -3.71171000 & -1.08600400\end{array}$

$3.95960000 \quad-4.47875100 \quad-2.34909800$

$2.42939300 \quad-2.30783800 \quad-3.04078800$

$2.01908100 \quad-3.26447700 \quad-3.39693200$

$\begin{array}{lll}1.58644600 & -1.62032000 & -2.89097300\end{array}$

$\begin{array}{lll}3.08380300 & -1.89200800 & -3.82302900\end{array}$

$\begin{array}{lll}1.55614700 & -0.74714500 & -0.44636000\end{array}$

$\begin{array}{lll}-0.07574200 & -0.90656500 & 1.99365400\end{array}$

$\begin{array}{lll}1.66239900 & 2.19964100 & -0.08487600\end{array}$

$\begin{array}{lll}-1.68207600 & -1.46074200 & 2.91042700\end{array}$

$\begin{array}{lll}-2.58471100 & -2.33909200 & 2.02595300\end{array}$

$-2.96409200 \quad-1.81947800 \quad 1.14271200$

$-2.10508500 \quad-3.28161000 \quad 1.72974600$

$\begin{array}{lll}-3.48106300 & -2.59643000 & 2.61333800\end{array}$

$\begin{array}{lll}-2.44445300 & -0.16046000 & 3.24671300\end{array}$

$\begin{array}{lll}-1.78984300 & 0.63092100 & 3.63988400\end{array}$

$\begin{array}{lll}-3.01209300 & 0.20705800 & 2.38018500\end{array}$

$\begin{array}{lll}-3.19475000 & -0.38063600 & 4.02448800\end{array}$

$\begin{array}{lll}-1.43776300 & -2.23417300 & 4.21648700\end{array}$

$-0.96202600 \quad-1.61776400 \quad 4.98838700$

$-2.41887700 \quad-2.54185000 \quad 4.61418300$

$\begin{array}{lll}-0.84162400 & -3.14623500 & 4.08068500\end{array}$

$\begin{array}{lll}1.32265500 & -0.72503200 & 3.32405000\end{array}$

$\begin{array}{lll}2.59587000 & -0.39782200 & 2.53671200\end{array}$

$2.98103000 \quad-1.27911600 \quad 2.00568500$

$\begin{array}{lll}2.42801400 & 0.39275600 & 1.80653800\end{array}$

$\begin{array}{lll}3.38349400 & -0.06120900 & 3.22959000\end{array}$

$0.94455400 \quad 0.46167600 \quad 4.22252300$

$0.08992200 \quad 0.23220200 \quad 4.87406200$

$\begin{array}{lll}1.79475000 & 0.72383200 & 4.87470400\end{array}$

$\begin{array}{lll}0.68486000 & 1.35211000 \quad 3.63077300\end{array}$

$1.65810200 \quad-1.95978100 \quad 4.17677700$

$\begin{array}{lll}1.76340700 & -2.86698900 & 3.56467700\end{array}$

$2.63416300 \quad-1.78671200 \quad 4.66182700$

$0.93804000 \quad-2.15741400 \quad 4.97487300$

$2.23378400 \quad 3.57809100 \quad 1.11714600$

$3.05286800 \quad 2.96619100 \quad 2.26287100$ 
H

H

$\mathrm{H}$

C

H

$\mathrm{H}$

H

C

Conformation-A

C

C

C

C

$\mathrm{H}$

$\mathrm{H}$

C

C

C

C

$\mathrm{H}$

\begin{tabular}{|c|c|c|}
\hline 2.47711300 & 2.22899500 & 2.82962300 \\
\hline 3.97919000 & 2.48795200 & 1.91598200 \\
\hline 3.33246400 & 3.77077800 & 2.96330900 \\
\hline 3.04421000 & 4.72831300 & 0.50486600 \\
\hline 3.30122400 & 5.43940000 & 1.30807100 \\
\hline 3.98828200 & 4.39431700 & 0.05418400 \\
\hline 2.47835300 & 5.28802500 & -0.25028300 \\
\hline 0.93325500 & 4.15499500 & 1.70650300 \\
\hline 1.17888300 & 4.91663900 & 2.46522400 \\
\hline 0.31545100 & 4.63875200 & 0.93799500 \\
\hline 0.32371400 & 3.37622400 & 2.18926000 \\
\hline 1.48655300 & 2.93008600 & -1.86466000 \\
\hline 2.74775500 & 3.52910800 & -2.50211000 \\
\hline 2.50777200 & 3.78347800 & -3.54892900 \\
\hline 3.09000200 & 4.45019800 & -2.01923100 \\
\hline 3.58078500 & 2.81465900 & -2.53141200 \\
\hline 0.37547500 & 3.98438500 & -1.83704600 \\
\hline 0.68016300 & 4.89292600 & -1.29814700 \\
\hline 0.11805900 & 4.28322800 & -2.86694700 \\
\hline-0.52907200 & 3.59575200 & -1.36280400 \\
\hline 1.06332500 & 1.73551600 & -2.73514200 \\
\hline 1.91085900 & 1.06713300 & -2.94222400 \\
\hline 0.27918100 & 1.13773000 & -2.26390500 \\
\hline 0.68212300 & 2.09901200 & -3.70350100 \\
\hline-0.22943800 & 0.80939600 & 0.36322500 \\
\hline-1.57831300 & 0.53173700 & -3.28019900 \\
\hline-1.78042000 & 1.88744100 & -1.73706800 \\
\hline-2.59907100 & 1.73089600 & 1.23269600 \\
\hline-2.52102500 & 3.70263300 & 0.26334600 \\
\hline-2.43556200 & 4.11985200 & -0.74889300 \\
\hline-3.52582300 & 3.92722600 & 0.64664100 \\
\hline-1.79062200 & 4.20051200 & 0.92044200 \\
\hline 1.91569900 & 1.20885300 & 0.96325800 \\
\hline 1.65619000 & -0.15842600 & 1.24866700 \\
\hline 0.99242000 & -0.59070300 & 2.50872900 \\
\hline 1.08697100 & 0.16466700 & 3.69504400 \\
\hline 0.13385800 & -2.38684200 & 1.67039600 \\
\hline 2.36223700 & -0.89151900 & 0.85627600 \\
\hline 0.24207000 & -1.78227700 & 2.56805100 \\
\hline 0.43820300 & -0.23089600 & 4.86683700 \\
\hline-0.31536600 & -1.40756700 & 4.89712800 \\
\hline-0.40065500 & -2.18607800 & 3.73796800 \\
\hline 0.52983500 & 0.38336900 & 5.7670570 \\
\hline
\end{tabular}


$\begin{array}{lll}-0.82523600 & -1.71676900 \quad 5.81306200\end{array}$

$\begin{array}{lll}-0.98487400 & -3.11022500 \quad 3.73780000\end{array}$

C

$3.13938300 \quad 1.59215500 \quad 0.15835800$

$5.24313100 \quad 0.45035300 \quad-0.17533000$

$\begin{array}{lll}5.27055400 & 0.75699200 & -1.34795700\end{array}$

$6.30542300 \quad-0.40136300 \quad 0.53190400$

$\begin{array}{lll}7.33652100 & -0.87374900 & -0.49724100\end{array}$

$\begin{array}{lll}6.98398800 & 0.47322800 & 1.60397600\end{array}$

$5.62136800 \quad-1.60886700 \quad 1.19896600$

$6.86781700 \quad-1.49134700 \quad-1.27821900$

$\begin{array}{lll}7.82182800 & -0.02276900 & -0.99736600\end{array}$

$8.11519700 \quad-1.47631400 \quad-0.00316600$

$\begin{array}{lll}6.25605300 & 0.82065300 & 2.35111100\end{array}$

$\begin{array}{lll}7.76570900 & -0.10376800 & 2.12393500\end{array}$

$\begin{array}{lll}7.46122400 & 1.35773100 & 1.15144600\end{array}$

$\begin{array}{lll}6.37411100 & -2.23780500 \quad 1.70102100\end{array}$

$4.88307000 \quad-1.28795200 \quad 1.94697700$

$\begin{array}{lll}5.10185700 & -2.23336400 & 0.45543200\end{array}$

$\begin{array}{lll}4.27652100 & 0.81733800 & 0.67037700\end{array}$

$\begin{array}{lll}-1.33873200 & -3.90421500 & -0.73590400\end{array}$

$\begin{array}{lll}-1.15362900 & -2.52241100 & -0.56589000\end{array}$

$-2.15231000 \quad-1.83729600 \quad 0.14656600$

$\begin{array}{lll}-3.34669800 & -2.43157700 & 0.57278200\end{array}$

$\begin{array}{lll}-3.49645600 & -3.80672900 & 0.36768100\end{array}$

$-2.48372000 \quad-4.54267100 \quad-0.25442000$

$\begin{array}{lll}-4.33459500 & -0.18691900 & 0.42912000\end{array}$

$\begin{array}{lll}-3.05987400 & 0.31878300 & 0.13269800\end{array}$

$\begin{array}{lll}-2.82762100 & 1.58984300 & -0.41774900\end{array}$

$\begin{array}{lll}-3.96787700 & 2.32040200 & -0.79015500\end{array}$

$\begin{array}{lll}-5.25268200 & 1.81230000 & -0.59008000\end{array}$

$\begin{array}{lll}-5.43824700 & 0.57797700 & 0.04173000\end{array}$

$\begin{array}{lll}-0.59733300 & -4.48858700 & -1.27833600\end{array}$

$\begin{array}{lll}-4.40700700 & -4.31258000 & 0.69034300\end{array}$

$\begin{array}{lll}-2.60411800 & -5.61891400 & -0.39762400\end{array}$

$\begin{array}{lll}-3.85505600 & 3.30849800 & -1.23219000\end{array}$

$\begin{array}{lll}-6.12048400 & 2.39860100 & -0.90068500\end{array}$

$\begin{array}{lll}-6.45006400 & 0.22040900 & 0.23554500\end{array}$

$-4.38725900 \quad-1.50713200 \quad 1.21181300$

$\begin{array}{lll}-5.78705500 & -2.12441300 & 1.20830300\end{array}$

$\begin{array}{lll}-6.51235800 & -1.44223800 \quad 1.67506900\end{array}$

$\begin{array}{lll}-6.13292300 & -2.35287000 & 0.18869100\end{array}$

$\begin{array}{lll}-5.80474900 & -3.05390800 \quad 1.79556900\end{array}$

$\begin{array}{lll}-3.95947900 & -1.21909300 & 2.67462700\end{array}$

$\begin{array}{lll}-3.97482000 & -2.14880800 \quad 3.26441400\end{array}$ 
$\begin{array}{lll}-2.94163200 & -0.80803600 & 2.72285500\end{array}$

$-4.64899800$

$-0.49725400 \quad 3.13984300$

$\begin{array}{lll}-1.97657100 & -0.49115300 & 0.37547700\end{array}$

$\begin{array}{lll}0.13733200 & -1.47725300 & -1.39277100\end{array}$

$\begin{array}{lll}-1.07417800 & 2.22515800 & -0.42452300\end{array}$

$\begin{array}{lll}1.71425300 & -2.57973400 & -1.56726100\end{array}$

$1.97067900 \quad-3.34038300 \quad-0.25427000$

$\begin{array}{lll}2.00011200 & -2.68749700 & 0.62268500\end{array}$

$1.21926400 \quad-4.11863100 \quad-0.06563800$

$2.95120600 \quad-3.84122000 \quad-0.32275700$

$2.87251300 \quad-1.59687900 \quad-1.81196100$

$2.76251900 \quad-1.04903200 \quad-2.75791800$

$\begin{array}{lll}2.94081200 & -0.85469000 & -1.01409000\end{array}$

$3.82766300 \quad-2.14495200 \quad-1.85860600$

$\begin{array}{lll}1.72836700 & -3.60804000 & -2.70718600\end{array}$

$\begin{array}{lll}1.71063400 & -3.13427800 & -3.69642000\end{array}$

$2.67095500 \quad-4.17873300 \quad-2.64436400$

$\begin{array}{lll}0.90705100 & -4.33476300 & -2.65513400\end{array}$

$\begin{array}{lll}-0.68062600 & -1.35262300 & -3.14118700\end{array}$

$\begin{array}{lll}-1.99449500 & -0.59738900 & -2.89981100\end{array}$

$\begin{array}{lll}-2.74750800 & -1.24585000 & -2.42944600\end{array}$

$\begin{array}{lll}-1.84589300 & 0.26922100 & -2.25400700\end{array}$

$-2.41048500 \quad-0.24777100 \quad-3.85872200$

$\begin{array}{lll}0.26728000 & -0.51363800 & -4.01141400\end{array}$

$1.15701600 \quad-1.08505500 \quad-4.31342700$

$\begin{array}{lll}-0.24603400 & -0.19478700 & -4.93443300\end{array}$

$\begin{array}{lll}0.61216600 & 0.38808900 & -3.48345500\end{array}$

$\begin{array}{lll}-1.05406400 & -2.65185900 & -3.86809400\end{array}$

$\begin{array}{lll}-1.62178300 & -3.33724600 & -3.22280800\end{array}$

$\begin{array}{lll}-1.71034500 & -2.39729500 & -4.71865200\end{array}$

$-0.19585200 \quad-3.19000500 \quad-4.28075200$

$\begin{array}{lll}-0.91046100 & 3.43636300 & -1.91379400\end{array}$

$\begin{array}{lll}-1.45056600 & 2.74968400 & -3.17630500\end{array}$

$\begin{array}{lll}-0.92369300 & 1.81408500 & -3.38597900\end{array}$

$\begin{array}{lll}-2.52609700 & 2.53313200 & -3.11574800\end{array}$

$\begin{array}{lll}-1.29107100 \quad 3.41669900 & -4.04054000\end{array}$

$\begin{array}{lll}-1.57196700 & 4.81710200 & -1.79889800\end{array}$

$\begin{array}{lll}-1.36070900 & 5.38368700 & -2.72236900\end{array}$

$\begin{array}{lll}-2.66443600 & 4.76577100 & -1.70113100\end{array}$

$\begin{array}{lll}-1.17850500 & 5.40981300 & -0.96437700\end{array}$

$\begin{array}{lll}0.60497400 & 3.63963300 & -2.09464500\end{array}$

$0.79671700 \quad 4.19995900 \quad-3.02541200$

$\begin{array}{lll}1.04079400 & 4.21500700 \quad-1.26750600\end{array}$

$\begin{array}{lll}1.13805600 & 2.67893300 \quad-2.15417400\end{array}$ 


\begin{tabular}{|c|c|c|c|}
\hline $\mathrm{C}$ & -1.22919000 & 3.28474400 & 1.19419400 \\
\hline $\mathrm{C}$ & -2.48909800 & 4.15253600 & 1.32553100 \\
\hline $\mathrm{H}$ & -2.43079500 & 4.70119800 & 2.28184100 \\
\hline $\mathrm{H}$ & -2.59535100 & 4.89985100 & 0.53160800 \\
\hline $\mathrm{H}$ & -3.40526500 & 3.54895500 & 1.35767500 \\
\hline $\mathrm{C}$ & 0.01075200 & 4.17887800 & 1.33692300 \\
\hline $\mathrm{H}$ & -0.01771400 & 5.03926200 & 0.65392400 \\
\hline $\mathrm{H}$ & 0.05743500 & 4.58094000 & 2.36336000 \\
\hline $\mathrm{H}$ & 0.93814500 & 3.62881800 & 1.15274500 \\
\hline $\mathrm{C}$ & -1.26672600 & 2.25689900 & 2.33540500 \\
\hline $\mathrm{H}$ & -2.20610300 & 1.68484100 & 2.33270700 \\
\hline $\mathrm{H}$ & -0.44570900 & 1.53888400 & 2.27481900 \\
\hline $\mathrm{H}$ & -1.20002200 & 2.77485000 & 3.30697300 \\
\hline $\mathrm{Ni}$ & 0.39435400 & 0.50346200 & -0.15778900 \\
\hline $\mathrm{H}$ & 1.68578800 & 1.07727900 & 3.69711800 \\
\hline $\mathrm{H}$ & 1.65020900 & 1.94343000 & 1.72777900 \\
\hline $\mathrm{H}$ & 3.03869200 & 1.30812800 & -0.89859200 \\
\hline $\mathrm{C}$ & 3.51014600 & 3.06247700 & 0.24551600 \\
\hline $\mathrm{H}$ & 3.68323500 & 3.35616600 & 1.29296500 \\
\hline $\mathrm{H}$ & 4.42078900 & 3.26790900 & -0.33604900 \\
\hline $\mathrm{H}$ & 2.70240800 & 3.68490000 & -0.15970600 \\
\hline \multicolumn{4}{|c|}{ Conformation-B } \\
\hline $\mathrm{C}$ & 3.07392000 & 3.20486300 & -1.09570600 \\
\hline $\mathrm{C}$ & 2.14944300 & 2.22894700 & -0.68900500 \\
\hline $\mathrm{C}$ & 2.21875200 & 0.99355300 & -1.35637700 \\
\hline $\mathrm{C}$ & 2.93431600 & 0.79443600 & -2.54117500 \\
\hline $\mathrm{C}$ & 3.82904700 & 1.80096500 & -2.92155100 \\
\hline $\mathrm{C}$ & 3.93981000 & 2.97379400 & -2.16734600 \\
\hline $\mathrm{C}$ & 2.35627200 & -1.60009900 & -2.46097800 \\
\hline $\mathrm{C}$ & 1.86929400 & -1.36176700 & -1.16894800 \\
\hline $\mathrm{C}$ & 1.80254000 & -2.35779000 & -0.17595100 \\
\hline $\mathrm{C}$ & 2.06803700 & -3.67131000 & -0.59547800 \\
\hline $\mathrm{C}$ & 2.44994800 & -3.95810700 & -1.90612200 \\
\hline $\mathrm{C}$ & 2.63690300 & -2.92233300 & -2.82117500 \\
\hline $\mathrm{H}$ & 3.09516200 & 4.17031100 & -0.59363200 \\
\hline $\mathrm{H}$ & 4.42936100 & 1.68355300 & -3.82419500 \\
\hline $\mathrm{H}$ & 4.65731800 & 3.74289400 & -2.46168900 \\
\hline $\mathrm{H}$ & 2.01231600 & -4.48625000 & 0.12270600 \\
\hline $\mathrm{H}$ & 2.64660600 & -4.99101700 & -2.20187300 \\
\hline $\mathrm{H}$ & 2.99767200 & -3.14760800 & -3.82468200 \\
\hline $\mathrm{C}$ & 2.52558900 & -0.40681500 & -3.39889100 \\
\hline $\mathrm{C}$ & 1.14367300 & -0.07548400 & -4.02425300 \\
\hline $\mathrm{H}$ & 0.78852000 & -0.92144300 & -4.63258600 \\
\hline $\mathrm{H}$ & 0.39096000 & 0.12415500 & -3.24831500 \\
\hline
\end{tabular}


$\begin{array}{lll}1.21543800 & 0.81633100 & -4.66597100\end{array}$

$\begin{array}{lll}3.53005000 & -0.68468700 & -4.51971300\end{array}$

$\begin{array}{lll}3.63077000 & 0.19426300 & -5.17225800\end{array}$

$\begin{array}{lll}4.52474900 & -0.94123800 & -4.12463600\end{array}$

$3.18741400 \quad-1.51057600 \quad-5.15890300$

$1.47738700 \quad-0.06331800 \quad-0.84271900$

$\begin{array}{lll}0.99185500 & 3.14229200 & 1.97887300\end{array}$

$2.15562400 \quad 4.13895200 \quad 2.02552100$

$3.09405400 \quad 3.68021700 \quad 1.68178300$

$\begin{array}{lll}1.97054800 & 5.04245700 & 1.42990800\end{array}$

$\begin{array}{lll}2.31385500 & 4.46118000 & 3.06949700\end{array}$

$\begin{array}{lll}1.37285600 & 1.89886900 & 2.79624000\end{array}$

$\begin{array}{lll}1.61676900 & 2.19850900 & 3.82996800\end{array}$

$\begin{array}{lll}0.55367700 & 1.16948400 & 2.83204400\end{array}$

$\begin{array}{lll}2.25371800 & 1.39747700 & 2.37787200\end{array}$

$\begin{array}{lll}-0.27104700 & 3.75710900 & 2.60039400\end{array}$

$\begin{array}{lll}-1.13723700 & 3.08441700 & 2.51901800\end{array}$

$\begin{array}{lll}-0.09252100 & 3.93561600 & 3.67405500\end{array}$

$\begin{array}{lll}-0.53100100 & 4.72303200 & 2.14559800\end{array}$

$\begin{array}{lll}-0.26818200 & 3.84790800 & -0.81965500\end{array}$

$\begin{array}{lll}0.33923100 & 5.25101500 & -0.72100100\end{array}$

$\begin{array}{lll}-0.20037100 & 5.92487000 & -1.40902200\end{array}$

$\begin{array}{lll}0.24104800 & 5.67557500 & 0.28794500\end{array}$

$\begin{array}{lll}1.39785000 & 5.27838000 & -1.01155400\end{array}$

$\begin{array}{lll}-0.19111200 & 3.34758500 & -2.27332800\end{array}$

$\begin{array}{lll}-0.55870400 & 2.31271800 & -2.35439800\end{array}$

$\begin{array}{lll}-0.83161000 & 3.98082100 & -2.90957200\end{array}$

$0.82943000 \quad 3.38494200 \quad-2.67875200$

$\begin{array}{lll}-1.75506000 & 3.91255200 & -0.43040000\end{array}$

$\begin{array}{lll}-1.91586800 & 4.25336000 & 0.59924100\end{array}$

$-2.26964800 \quad 4.62194500 \quad-1.10040500$

$\begin{array}{lll}-2.23305900 & 2.93373000 & -0.54427100\end{array}$

$\begin{array}{lll}0.92585700 & -3.32387000 & 2.57114900\end{array}$

$\begin{array}{lll}1.83557500 & -4.52745800 & 2.85188800\end{array}$

$\begin{array}{lll}2.24444700 & -4.99518000 & 1.94703300\end{array}$

$2.67658000 \quad-4.26175300 \quad 3.50627200$

$\begin{array}{lll}1.24994400 & -5.30044100 & 3.37990900\end{array}$

$\begin{array}{lll}0.48617200 & -2.74440900 & 3.93249000\end{array}$

$\begin{array}{lll}1.33669200 & -2.39672200 & 4.53470800\end{array}$

$\begin{array}{lll}-0.20505000 & -1.90026800 & 3.81189200\end{array}$

$\begin{array}{lll}-0.03376800 & -3.52499500 & 4.51385500\end{array}$

$\begin{array}{lll}-0.33919200 & -3.79363400 & 1.83365800\end{array}$

$\begin{array}{lll}-0.85959700 & -4.54836500 & 2.44752800\end{array}$

$\begin{array}{lll}-1.04273100 & -2.96674500 \quad 1.66795000\end{array}$ 
$\begin{array}{lll}-0.12160200 & -4.24879100 & 0.85790300\end{array}$

$\begin{array}{lll}3.51192200 & -1.63720600 & 1.98607700\end{array}$

$\begin{array}{lll}4.41136000 & -2.83613700 & 1.66244000\end{array}$

$\begin{array}{lll}4.20616900 & -3.71087500 & 2.28907400\end{array}$

$\begin{array}{lll}4.32816500 & -3.13839700 & 0.60889700\end{array}$

$3.63568700 \quad-1.27822400 \quad 3.47521100$

$\begin{array}{lll}3.39927600 & -2.13357500 & 4.12430100\end{array}$

$\begin{array}{lll}4.67306900 & -0.97618700 & 3.70014200\end{array}$

$\begin{array}{lll}2.97511500 & -0.44321100 & 3.75476500\end{array}$

$\begin{array}{lll}4.00849100 & -0.45204500 & 1.13866800\end{array}$

$4.08451300 \quad-0.71713700 \quad 0.07486200$

$\begin{array}{lll}3.36301100 & 0.43086100 & 1.21805600\end{array}$

$\begin{array}{lll}5.01910400 & -0.16116100 & 1.47227600\end{array}$

$\begin{array}{lll}-1.73957900 & -0.82257300 & -0.25706900\end{array}$

$\begin{array}{lll}-2.24145000 & 0.43126400 & 0.25134600\end{array}$

$\begin{array}{lll}-2.57485700 & 0.68969200 & 1.66177900\end{array}$

$\begin{array}{lll}-1.94863900 & 0.00016200 & 2.72158700\end{array}$

$\begin{array}{lll}-4.01193600 & 2.23847300 & 1.20583300\end{array}$

$\begin{array}{lll}-2.82776200 & 1.03751900 & -0.44804300\end{array}$

$\begin{array}{lll}-3.50684400 & 1.69062600 & 2.00563300\end{array}$

$\begin{array}{lll}-2.23326100 & 0.29852100 & 4.05215500\end{array}$

$\begin{array}{lll}-3.15520500 & 1.30253700 & 4.37446000\end{array}$

$\begin{array}{lll}-3.79120200 & 1.99429900 & 3.33931200\end{array}$

$\begin{array}{lll}-1.72188100 & -0.24908200 & 4.84907000\end{array}$

$\begin{array}{lll}-3.37429300 & 1.54073700 & 5.41825300\end{array}$

$\begin{array}{lll}-4.51741100 & 2.77843400 & 3.57144900\end{array}$

$\begin{array}{lll}-2.15780700 & -1.26291700 & -1.63114400\end{array}$

$\begin{array}{lll}-3.56586200 & -1.71412700 & -1.58186200\end{array}$

$\begin{array}{lll}-4.53395000 & -0.84871200 & -1.88185700\end{array}$

$-5.91722500 \quad-1.40724200 \quad-1.52008200$

$\begin{array}{lll}-4.34668300 & 0.25260800 & -2.35648900\end{array}$

$\begin{array}{lll}-6.09133800 & -2.80943600 & -2.12785300\end{array}$

$\begin{array}{lll}-5.98807400 & -1.48910400 & 0.01837400\end{array}$

$\begin{array}{lll}-6.99841000 & -0.45985800 & -2.04868900\end{array}$

$\begin{array}{lll}-6.01540100 & -2.77994500 & -3.22714700\end{array}$

$\begin{array}{lll}-5.32670200 & -3.50349200 & -1.75214600\end{array}$

$\begin{array}{lll}-7.08387000 & -3.21233200 & -1.86868900\end{array}$

$\begin{array}{lll}-5.84726500 & -0.49788800 & 0.47747500\end{array}$

$\begin{array}{lll}-6.97270600 & -1.87317500 & 0.33118500\end{array}$

$\begin{array}{lll}-5.21098300 & -2.15719800 & 0.41680000\end{array}$

$\begin{array}{lll}-7.99742700 & -0.83608600 & -1.77633300\end{array}$

$\begin{array}{lll}-6.88183900 & 0.55025600 & -1.63019700\end{array}$

$\begin{array}{lll}-6.95159300 & -0.37112700 & -3.14500800\end{array}$ 


$\begin{array}{lrrr}\mathrm{H} & -1.19944900 & -0.75410000 & 2.48166700 \\ \mathrm{Ni} & -0.34350400 & 0.50750900 & -0.03962900 \\ \mathrm{P} & 0.56271700 & 2.48723300 & 0.24839200 \\ \mathrm{P} & 1.62496700 & -1.82091700 & 1.59375600 \\ \mathrm{H} & -1.67219100 & -1.66506400 & 0.44232500 \\ \mathrm{H} & -2.13298500 & -0.41913600 & -2.33351200 \\ \mathrm{C} & -1.37868200 & -2.44275500 & -2.17961600 \\ \mathrm{H} & -1.76971300 & -2.75251700 & -3.16135900 \\ \mathrm{H} & -1.44263300 & -3.30294800 & -1.49482900 \\ \mathrm{H} & -0.31965000 & -2.18093700 & -2.29220500\end{array}$

\section{Cartesian coordinates of the structures (optimization in DCM)}

TS22

\begin{tabular}{|c|c|c|c|}
\hline $\mathrm{C}$ & 1.26230200 & -3.77430300 & 1.68105000 \\
\hline C & 0.88167600 & -2.50312300 & 1.22044700 \\
\hline C & 1.86969200 & -1.48662400 & 1.23269900 \\
\hline C & 3.21329100 & -1.78550500 & 1.52964900 \\
\hline $\mathrm{C}$ & 3.53521900 & -3.07416400 & 1.97051900 \\
\hline $\mathrm{C}$ & 2.56352000 & -4.06236900 & 2.08257700 \\
\hline $\mathrm{C}$ & 3.77200700 & 0.53538700 & 0.77798100 \\
\hline C & 2.39072100 & 0.78551000 & 0.71409200 \\
\hline $\mathrm{C}$ & 1.88671800 & 2.07487800 & 0.41131100 \\
\hline C & 2.81635800 & 3.04303400 & 0.00566600 \\
\hline $\mathrm{C}$ & 4.18442700 & 2.78219500 & -0.05022800 \\
\hline $\mathrm{C}$ & 4.65496100 & 1.54513500 & 0.37460600 \\
\hline $\mathrm{H}$ & 0.53004200 & -4.57329700 & 1.70591500 \\
\hline $\mathrm{H}$ & 4.56986000 & -3.30382600 & 2.23094400 \\
\hline $\mathrm{H}$ & 2.82150800 & -5.05981800 & 2.44499100 \\
\hline $\mathrm{H}$ & 2.47518400 & 4.03983700 & -0.25025600 \\
\hline $\mathrm{H}$ & 4.87914900 & 3.55827700 & -0.37922700 \\
\hline $\mathrm{H}$ & 5.72939400 & 1.35952700 & 0.40466200 \\
\hline $\mathrm{C}$ & 4.32859500 & -0.76016500 & 1.35727400 \\
\hline $\mathrm{C}$ & 5.40715200 & -1.34700600 & 0.41821600 \\
\hline $\mathrm{H}$ & 6.24363800 & -0.64599700 & 0.28642900 \\
\hline $\mathrm{H}$ & 4.98208900 & -1.56844000 & -0.57234000 \\
\hline $\mathrm{H}$ & 5.82553700 & -2.27658400 & 0.82999300 \\
\hline $\mathrm{C}$ & 4.95723000 & -0.44367200 & 2.73573500 \\
\hline $\mathrm{H}$ & 5.37151400 & -1.35415800 & 3.19591400 \\
\hline $\mathrm{H}$ & 4.20335200 & -0.02490200 & 3.41998700 \\
\hline $\mathrm{H}$ & 5.77277800 & 0.28864000 & 2.63370100 \\
\hline $\mathrm{O}$ & 1.47658000 & -0.20585200 & 0.96706500 \\
\hline $\mathrm{C}$ & -2.01117700 & -2.69531800 & 1.81918900 \\
\hline $\mathrm{C}$ & -1.66579200 & -3.84941300 & 2.76887600 \\
\hline $\mathrm{H}$ & -0.74472300 & -3.65954600 & 3.33576500 \\
\hline $\mathrm{H}$ & -2.48449700 & -3.94795700 & 3.50281800 \\
\hline
\end{tabular}


$1.46352900 \quad 3.85570100 \quad 2.93143300$

$\begin{array}{lll}1.47224300 & 4.81329500 & 2.39499900\end{array}$

$\begin{array}{lll}2.40896900 & 3.33740000 & 2.71739500\end{array}$

$\begin{array}{lll}1.44820100 & 4.08178300 \quad 4.01276800\end{array}$

$\begin{array}{lll}-2.37208000 & 0.67786200 & -1.85230300\end{array}$

$-3.13092200$

$-4.03504600$

$-0.21970400 \quad-0.97883000$

$-3.63591000$

0.05667700

$-5.67663700$

$-3.59417500$

$-5.33968200$

$-4.50376800$

$-5.79678600$

$-6.20700900$

$-4.16350700$

$-6.47306100$

$-7.21198100$

$-2.18621300$

$-2.60720100$

0.50530900

1.89730900

1.95162700

3.04111500

1.99197500

1.13642100

1.86284000

2.91145000

3.02860300

4.00938700

2.97009300

2.94974300

1.94073400

1.17411900

$-0.47851900$

$-2.28858600$

$-2.61522600$

$-2.30497000$

$-3.36315000$

$-1.99949700$

$-1.70927300$

0.53518600

$-1.22246000$

$-0.78106400$

0.09861000

$-2.30485300$

$2.47295600 \quad 0.72730100$ 
TS23/TS-Conformation-A

\begin{tabular}{|c|c|c|c|}
\hline $\mathrm{C}$ & -1.95664300 & 1.37813100 & -0.67526600 \\
\hline $\mathrm{C}$ & -1.81822700 & -0.04579500 & -0.52721600 \\
\hline $\mathrm{C}$ & -1.56632700 & -0.93608300 & -1.68334300 \\
\hline $\mathrm{C}$ & -1.52154200 & -0.46710300 & -3.01310300 \\
\hline $\mathrm{H}$ & -1.44409200 & -2.72245600 & -0.47707400 \\
\hline $\mathrm{H}$ & -2.47680100 & -0.48228400 & 0.22117700 \\
\hline $\mathrm{C}$ & -1.39709000 & -2.32250800 & -1.48602300 \\
\hline $\mathrm{C}$ & -1.28755900 & -1.33319400 & -4.08337700 \\
\hline $\mathrm{C}$ & -1.09869300 & -2.70071700 & -3.86104900 \\
\hline $\mathrm{C}$ & -1.16312000 & -3.19018700 & -2.55208500 \\
\hline $\mathrm{H}$ & -1.26220200 & -0.93494500 & -5.10128400 \\
\hline $\mathrm{H}$ & -0.91140400 & -3.37768000 & -4.69826400 \\
\hline $\mathrm{H}$ & -1.02618900 & -4.25687000 & -2.35615000 \\
\hline $\mathrm{C}$ & -2.55834100 & 2.12150200 & 0.34210000 \\
\hline $\mathrm{C}$ & -5.10088400 & 0.67047600 & 0.07332800 \\
\hline $\mathrm{O}$ & -4.68182100 & -0.07393300 & 0.98123200 \\
\hline $\mathrm{C}$ & -6.16252000 & 0.10817100 & -0.92368600 \\
\hline $\mathrm{C}$ & -6.82057200 & -1.15529700 & -0.36242800 \\
\hline $\mathrm{C}$ & -7.22685700 & 1.17467000 & -1.21428000 \\
\hline $\mathrm{C}$ & -5.40033300 & -0.23098300 & -2.21870400 \\
\hline $\mathrm{H}$ & -6.07075800 & -1.93180200 & -0.15363200 \\
\hline $\mathrm{H}$ & -7.34649000 & -0.94420000 & 0.58276500 \\
\hline $\mathrm{H}$ & -7.55596100 & -1.56305300 & -1.07738800 \\
\hline $\mathrm{H}$ & -6.75735800 & 2.09638600 & -1.58600300 \\
\hline $\mathrm{H}$ & -7.94905200 & 0.81715200 & -1.96853900 \\
\hline $\mathrm{H}$ & -7.79261300 & 1.43084600 & -0.30239700 \\
\hline $\mathrm{H}$ & -6.08499400 & -0.63350600 & -2.98513400 \\
\hline $\mathrm{H}$ & -4.91115900 & 0.66573400 & -2.62881600 \\
\hline $\mathrm{H}$ & -4.61827600 & -0.98434400 & -2.03273100 \\
\hline $\mathrm{O}$ & -4.70406800 & 1.85111700 & -0.17049100 \\
\hline C & 0.68500600 & -3.73737000 & 1.34412200 \\
\hline $\mathrm{C}$ & 0.73529400 & -2.37780600 & 0.99761800 \\
\hline $\mathrm{C}$ & 1.55770100 & -2.03121700 & -0.08874000 \\
\hline $\mathrm{C}$ & 2.39732200 & -2.93960400 & -0.74340700 \\
\hline $\mathrm{C}$ & 2.32242500 & -4.28339200 & -0.36067300 \\
\hline $\mathrm{C}$ & 1.44877300 & -4.68245600 & 0.65519100 \\
\hline $\mathrm{C}$ & 3.79790200 & -1.00326300 & -1.32789000 \\
\hline $\mathrm{C}$ & 2.82564100 & -0.16842600 & -0.75935700 \\
\hline $\mathrm{C}$ & 3.04811400 & 1.18013700 & -0.43277400 \\
\hline $\mathrm{C}$ & 4.36132700 & 1.65517200 & -0.58709500 \\
\hline $\mathrm{C}$ & 5.37495000 & 0.82439600 & -1.06955700 \\
\hline $\mathrm{C}$ & 5.09051700 & -0.48760300 & -1.46432900 \\
\hline $\mathrm{H}$ & 0.06382300 & -4.06408400 & 2.17658900 \\
\hline
\end{tabular}


$2.94830200 \quad-5.02784400 \quad-0.85375400$

$\begin{array}{lll}1.38942200 & -5.73604000 & 0.93754600\end{array}$

$\begin{array}{lll}4.59605800 & 2.68816800 & -0.33340400\end{array}$

$6.39105000 \quad 1.21274400 \quad-1.16891600$

$5.88363300 \quad-1.10473600 \quad-1.88778000$

$3.32891100 \quad-2.37855200 \quad-1.82164200$

$\begin{array}{lll}4.50096800 & -3.31745800 & -2.11462400\end{array}$

$\begin{array}{lll}5.15182400 & -2.89242500 & -2.89211900\end{array}$

$5.10918400 \quad-3.50536100 \quad-1.21665800$

$\begin{array}{lll}4.13712500 & -4.28233200 & -2.49524700\end{array}$

$2.50945300 \quad-2.16631400 \quad-3.12109400$

$2.13793700 \quad-3.13198300 \quad-3.49585400$

$\begin{array}{lll}1.64209600 & -1.51494000 & -2.94815600\end{array}$

$3.14060100 \quad-1.70624700 \quad-3.89758500$

$1.58836600 \quad-0.70902100 \quad-0.48876600$

$\begin{array}{lll}0.00206200 & -0.98470600 & 1.96856100\end{array}$

$\begin{array}{lll}1.57259900 & 2.23163700 \quad-0.03438600\end{array}$

$\begin{array}{lll}-1.57784700 & -1.62905300 & 2.87300200\end{array}$

$\begin{array}{lll}-2.44791100 & -2.51096000 & 1.96101900\end{array}$

$\begin{array}{lll}-2.80473000 & -1.98846600 & 1.07011200\end{array}$

$\begin{array}{lll}-1.94143500 & -3.43680000 & 1.65725300\end{array}$

$\begin{array}{lll}-3.34877200 & -2.79789700 & 2.52830100\end{array}$

$\begin{array}{lll}-2.38721400 & -0.37204600 & 3.25344000\end{array}$

$\begin{array}{lll}-1.76654100 & 0.41209200 & 3.71020000\end{array}$

$\begin{array}{lll}-2.93022100 & 0.03519900 & 2.39174500\end{array}$

$\begin{array}{lll}-3.15510900 \quad-0.65561200 & 3.99331400\end{array}$

$\begin{array}{lll}-1.30943300 & -2.43406400 \quad 4.15445900\end{array}$

$-0.85892100 \quad-1.82328800 \quad 4.94553500$

$\begin{array}{lll}-2.28000200 & -2.79100400 & 4.53778900\end{array}$

$\begin{array}{lll}-0.67869500 & -3.31731400 & 3.99035100\end{array}$

$1.39628600 \quad-0.79007200 \quad 3.29876000$

$\begin{array}{lll}2.65544400 & -0.41354900 & 2.50947100\end{array}$

$3.06503700 \quad-1.27808900 \quad 1.96881200$

$\begin{array}{lll}2.45862700 & 0.37744600 & 1.78680000\end{array}$

$\begin{array}{lll}3.43330500 & -0.05738500 & 3.20345200\end{array}$

$0.98420900 \quad 0.36788200 \quad 4.21978500$

$\begin{array}{lll}0.14846000 & 0.09175600 & 4.87804600\end{array}$

$\begin{array}{lll}1.83284800 & 0.65309600 & 4.86416000\end{array}$

$\begin{array}{lll}0.68067100 & 1.25569100 \quad 3.64532600\end{array}$

$\begin{array}{lll}1.77052500 & -2.02843000 & 4.12951100\end{array}$

$\begin{array}{lll}1.89325900 & -2.92352500 & 3.50291400\end{array}$

$2.74544100 \quad-1.83550300 \quad 4.60947300$

$\begin{array}{lll}1.06050000 & -2.25472600 \quad 4.92918800\end{array}$

$2.09761100 \quad 3.58629700 \quad 1.21915900$ 
TS-Conformation-B

C

\begin{tabular}{|c|c|c|}
\hline 2.95347200 & 2.96537100 & 2.33214000 \\
\hline 2.41794000 & 2.17754800 & 2.86849100 \\
\hline 3.89988700 & 2.55087600 & 1.95835800 \\
\hline 3.19777800 & 3.75320700 & 3.06427200 \\
\hline 2.85121700 & 4.79743700 & 0.65337100 \\
\hline 3.07080500 & 5.48688300 & 1.48629000 \\
\hline 3.81157900 & 4.52704100 & 0.19480600 \\
\hline 2.25863900 & 5.35699400 & -0.08092100 \\
\hline 0.77997900 & 4.08159300 & 1.84235700 \\
\hline 1.00283600 & 4.80611200 & 2.64341300 \\
\hline 0.14710900 & 4.58943100 & 1.10180300 \\
\hline 0.19971400 & 3.25500700 & 2.27965100 \\
\hline 1.36387200 & 3.02788200 & -1.78345600 \\
\hline 2.60613800 & 3.69192200 & -2.39422900 \\
\hline 2.34867100 & 4.00638900 & -3.42053500 \\
\hline 2.93641200 & 4.58550800 & -1.85540200 \\
\hline 3.45169700 & 2.99664300 & -2.47548100 \\
\hline 0.22314400 & 4.04996100 & -1.71386100 \\
\hline 0.50279500 & 4.94010600 & -1.13255300 \\
\hline-0.03417800 & 4.38604600 & -2.73237100 \\
\hline-0.67566200 & 3.61944600 & -1.26515100 \\
\hline 0.97747900 & 1.86184000 & -2.70748900 \\
\hline 1.84239500 & 1.21978100 & -2.92764600 \\
\hline 0.19826300 & 1.23218400 & -2.27212900 \\
\hline 0.60306200 & 2.25881000 & -3.66550700 \\
\hline 0.24362800 & 0.75366100 & 0.38714700 \\
\hline-1.69813200 & 0.58755600 & -3.22137800 \\
\hline-1.77680700 & 1.87231200 & -1.63107700 \\
\hline-2.76092900 & 1.60737300 & 1.27927700 \\
\hline-2.69530900 & 3.60448000 & 0.35032500 \\
\hline-2.79665000 & 4.01083400 & -0.66573500 \\
\hline-3.57518000 & 3.89574100 & 0.93804400 \\
\hline-1.81115600 & 4.06392800 & 0.81867200 \\
\hline-2.38202300 & -3.63319400 & 1.14736900 \\
\hline-1.71060800 & -2.45459300 & 0.77693000 \\
\hline-2.20694400 & -1.78055500 & -0.35425200 \\
\hline-3.15767500 & -2.33549100 & -1.22014300 \\
\hline-3.79294200 & -3.51413300 & -0.81600800 \\
\hline-3.43985400 & -4.13245400 & 0.38621900 \\
\hline-3.35832200 & -0.17630900 & -2.35457600 \\
\hline-2.52187100 & 0.35586500 & -1.36286600 \\
\hline-2.52388500 & 1.71696900 & -1.00926900 \\
\hline 3.36221000 & 2.56020100 & -1.75919000 \\
\hline
\end{tabular}




\begin{tabular}{|c|c|c|}
\hline-4.16461300 & 2.06878100 & -2.78760700 \\
\hline-4.17748100 & 0.70296400 & -3.06989900 \\
\hline-2.06099000 & -4.18583600 & 2.02619400 \\
\hline-4.55567200 & -3.96630900 & -1.45008800 \\
\hline-3.95354700 & -5.04307200 & 0.70235600 \\
\hline-3.40899100 & 3.62024000 & -1.52283600 \\
\hline-4.80276100 & 2.75118300 & -3.35347000 \\
\hline-4.83161200 & 0.31958800 & -3.85246600 \\
\hline-3.31744200 & -1.68204700 & -2.59343600 \\
\hline-2.05435500 & -2.01629600 & -3.43121800 \\
\hline-2.11677300 & -1.52765400 & -4.41584600 \\
\hline-1.13599900 & -1.67051200 & -2.93610000 \\
\hline-1.97323400 & -3.10384200 & -3.58224300 \\
\hline-4.55388100 & -2.19005200 & -3.33847000 \\
\hline-4.49123000 & -3.27725000 & -3.48732000 \\
\hline-5.48303700 & -1.96611300 & -2.79286700 \\
\hline-4.61877600 & -1.73623300 & -4.33730300 \\
\hline-1.69024500 & -0.52294500 & -0.66280600 \\
\hline 0.07020900 & -1.97903300 & 3.25031000 \\
\hline-0.67547100 & -3.12882600 & 3.93857100 \\
\hline-1.76123400 & -3.05584100 & 3.78668500 \\
\hline-0.33892800 & -4.12019900 & 3.61146100 \\
\hline-0.49512300 & -3.06147600 & 5.02525500 \\
\hline-0.53257100 & -0.65556100 & 3.74369400 \\
\hline-0.39454700 & -0.58072900 & 4.83554800 \\
\hline-0.04390300 & 0.20996100 & 3.27806300 \\
\hline-1.60864500 & -0.60092500 & 3.53995400 \\
\hline 1.55539900 & -2.00444900 & 3.64658500 \\
\hline 2.12764200 & -1.21738600 & 3.13613900 \\
\hline 1.63516300 & -1.82195000 & 4.73114500 \\
\hline 2.02688400 & -2.97409100 & 3.43841900 \\
\hline 1.00788600 & -3.37682700 & 0.58138300 \\
\hline 0.81228100 & -4.72068300 & 1.29381100 \\
\hline 1.32871700 & -5.50404700 & 0.71308400 \\
\hline 1.24902000 & -4.72254400 & 2.30205300 \\
\hline-0.24268100 & 5.01406400 & 1.36604700 \\
\hline 0.55107400 & -3.50465100 & -0.88325500 \\
\hline 0.54934800 & -2.52459000 & -1.38463900 \\
\hline 1.26195600 & -4.15143900 & -1.42337900 \\
\hline-0.44878800 & 3.94904300 & -0.97855400 \\
\hline 2.51159700 & -3.03425000 & 0.56181700 \\
\hline 2.88698000 & -2.62925400 & 1.50846000 \\
\hline 3.07113600 & -3.96268800 & 0.35686200 \\
\hline & & \\
\hline
\end{tabular}




\begin{tabular}{|c|c|c|}
\hline-1.12285900 & 4.11603900 & 0.15572100 \\
\hline-2.21874100 & 5.15768800 & 0.41985700 \\
\hline-3.13300100 & 4.99143300 & -0.16387900 \\
\hline-2.49360100 & 5.19946600 & 1.48251700 \\
\hline-1.82916500 & 6.15340800 & 0.14497200 \\
\hline 0.05612400 & 4.42746700 & 1.09818100 \\
\hline-0.18658700 & 4.23539200 & 2.15341400 \\
\hline 0.94811800 & 3.84199800 & 0.84048000 \\
\hline 0.32044400 & 5.49479900 & 1.00723200 \\
\hline-0.61305200 & 4.26715100 & -1.28743700 \\
\hline-0.13004700 & 5.25360900 & -1.39199500 \\
\hline 0.13507200 & 3.50673900 & -1.54159600 \\
\hline-1.41550200 & 4.21038000 & -2.03345700 \\
\hline-2.96908100 & 2.14259400 & 1.78921300 \\
\hline-4.27221400 & 2.90367500 & 1.51940600 \\
\hline-5.02950000 & 2.57909000 & 2.25491200 \\
\hline-4.16485000 & 3.98867500 & 1.62258500 \\
\hline-4.67683200 & 2.68443900 & 0.52057600 \\
\hline-2.36672600 & 2.58804900 & 3.13099100 \\
\hline-2.16835700 & 3.67000500 & 3.14676600 \\
\hline-3.07426700 & 2.36879300 & 3.94906600 \\
\hline-1.42452000 & 2.06490200 & 3.35218000 \\
\hline-3.30920000 & 0.64284100 & 1.83821300 \\
\hline-3.93107400 & 0.34420000 & 0.98158700 \\
\hline-2.40871700 & 0.01981900 & 1.83282100 \\
\hline-3.87942700 & 0.42005800 & 2.75540000 \\
\hline 1.53065500 & 1.13273100 & -0.75272600 \\
\hline 2.09536500 & 0.32721000 & 0.31099300 \\
\hline 2.66530300 & 0.88190800 & 1.55770600 \\
\hline 1.95742400 & 1.78002800 & 2.37738500 \\
\hline 4.49601100 & -0.25217400 & 1.38046300 \\
\hline 2.67260900 & -0.52258800 & -0.05242600 \\
\hline 3.93489000 & 0.45615700 & 1.99566300 \\
\hline 2.49685600 & 2.24501200 & 3.57719700 \\
\hline 3.76188700 & 1.81561700 & 3.99612500 \\
\hline 4.47792800 & 0.91696600 & 3.19797700 \\
\hline 1.91989300 & 2.93696000 & 4.19713300 \\
\hline 4.18314200 & 2.17361400 & 4.93901700 \\
\hline 5.46510400 & 0.56985900 & 3.51518900 \\
\hline 1.40513700 & 0.56568100 & -2.03909100 \\
\hline 3.21510900 & 0.78965900 & -2.90445100 \\
\hline 4.01977700 & -0.15341400 & -2.58345400 \\
\hline 5.52492100 & 0.22659100 & -2.69687200 \\
\hline & & \\
\hline
\end{tabular}


$5.79034100 \quad 1.35004600 \quad-1.67872100$ 
$7.82167500 \quad-0.00371700 \quad-0.99433500$

$\begin{array}{lll}8.11367600 & -1.46609400 & -0.01378800\end{array}$

$\begin{array}{lll}6.25881600 & 0.80608400 & 2.36294500\end{array}$

$\begin{array}{lll}7.76633700 & -0.11914600 & 2.12344600\end{array}$

$\begin{array}{lll}7.46312600 & 1.35420300 & 1.16654100\end{array}$

$\begin{array}{lll}6.37820500 & -2.24793900 \quad 1.67368900\end{array}$

$\begin{array}{lll}4.89020000 & -1.29997800 & 1.94272300\end{array}$

$\begin{array}{lll}5.09670100 & -2.22500600 & 0.43558900\end{array}$

$4.27351700 \quad 0.81689500 \quad 0.68029600$

$\begin{array}{lll}-1.32712300 & -3.90359000 & -0.74783600\end{array}$

$\begin{array}{lll}-1.14507700 & -2.52146700 & -0.57430500\end{array}$

$\begin{array}{lll}-2.14480000 & -1.83977300 & 0.14036000\end{array}$

$\begin{array}{lll}-3.33849500 & -2.43706700 & 0.56456500\end{array}$

$\begin{array}{lll}-3.48547200 & -3.81276600 & 0.35777800\end{array}$

$\begin{array}{lll}-2.47076000 & -4.54540600 & -0.26611000\end{array}$

$\begin{array}{lll}-4.33127800 & -0.19439800 & 0.42281100\end{array}$

$\begin{array}{lll}-3.05711900 \quad 0.31558200 & 0.13055600\end{array}$

$\begin{array}{lll}-2.82753000 & 1.58893100 & -0.41594600\end{array}$

$\begin{array}{lll}-3.96866600 & 2.31846100 & -0.78917400\end{array}$

$\begin{array}{lll}-5.25285100 & 1.80589400 & -0.59291800\end{array}$

$\begin{array}{lll}-5.43667800 & 0.56884200 & 0.03546700\end{array}$

$\begin{array}{lll}-0.58563500 & -4.48521100 & -1.29313300\end{array}$

$\begin{array}{lll}-4.39486700 & -4.32085300 & 0.68031200\end{array}$

$\begin{array}{lll}-2.58897400 & -5.62171000 & -0.41158400\end{array}$

$\begin{array}{lll}-3.85677200 & 3.30793900 & -1.22864700\end{array}$

$\begin{array}{lll}-6.12170600 & 2.39088800 & -0.90347700\end{array}$

$\begin{array}{lll}-6.44781400 & 0.20803200 & 0.22731700\end{array}$

$-4.38406800 \quad-1.51692900 \quad 1.20166600$

$\begin{array}{lll}-5.78217600 & -2.13795800 \quad 1.19122200\end{array}$

$\begin{array}{lll}-6.51052300 & -1.45625900 & 1.65364000\end{array}$

$\begin{array}{lll}-6.12115100 & -2.36591600 \quad 0.16915300\end{array}$

$\begin{array}{lll}-5.79816700 & -3.06829700 \quad 1.77697600\end{array}$

$\begin{array}{lll}-3.96370200 & -1.23100800 \quad 2.66695400\end{array}$

$\begin{array}{lll}-3.97229400 & -2.16394300 & 3.25180900\end{array}$

$\begin{array}{lll}-2.95070200 & -0.80793800 & 2.71816800\end{array}$

$\begin{array}{lll}-4.66282300 & -0.51831500 \quad 3.13194300\end{array}$

$\begin{array}{lll}-1.97170100 & -0.49289200 & 0.37436600\end{array}$

$0.14280100 \quad-1.47428700 \quad-1.40212800$

$\begin{array}{lll}-1.07795800 & 2.23338300 & -0.41760700\end{array}$

$\begin{array}{lll}1.72360100 & -2.56967300 & -1.58299100\end{array}$

$1.98473700 \quad-3.34024100 \quad-0.27681700$

$2.01592900 \quad-2.69399700 \quad 0.60504200$

$1.23439300 \quad-4.12111900 \quad-0.09505200$

$2.96614800 \quad-3.83795600 \quad-0.35446300$ 
$2.87880100 \quad-1.58176100 \quad-1.82180500$

$2.76295100 \quad-1.02858900 \quad-2.76416600$

$2.94646700 \quad-0.84654300 \quad-1.01713200$

$3.83400700 \quad-2.12966300 \quad-1.87262600$

$\begin{array}{lll}1.73820600 & -3.59043100 & -2.72978600\end{array}$

$1.71660100 \quad-3.10894200 \quad-3.71510700$

$\begin{array}{lll}2.68319100 & -4.15769100 & -2.67041600\end{array}$

$0.91821300 \quad-4.31853100 \quad-2.67773800$

$\begin{array}{lll}-0.67758100 & -1.34131700 & -3.14721400\end{array}$

$\begin{array}{lll}-1.99262900 & -0.58942800 & -2.90182400\end{array}$

$-2.74286200 \quad-1.24066500 \quad-2.43049700$

$\begin{array}{lll}-1.84460500 & 0.27703400 & -2.25560900\end{array}$

$\begin{array}{lll}-2.40917500 & -0.23996000 & -3.86052800\end{array}$

$0.26752400 \quad-0.49765500 \quad-4.01622600$

$\begin{array}{lll}1.15811500 & -1.06774000 & -4.31803500\end{array}$

$\begin{array}{lll}-0.24950700 & -0.17956100 & -4.93741400\end{array}$

$0.60865900 \quad 0.40491700 \quad-3.48703400$

$\begin{array}{lll}-1.04861800 & -2.63923800 & -3.87794600\end{array}$

$\begin{array}{lll}-1.60979600 & -3.33000900 & -3.23263800\end{array}$

$\begin{array}{lll}-1.70905700 & -2.38201900 & -4.72451300\end{array}$

$\begin{array}{lll}-0.18856700 & -3.17095200 & -4.29487000\end{array}$

$\begin{array}{lll}-0.91007100 & 3.44429700 & -1.90464200\end{array}$

$\begin{array}{lll}-1.44934400 & 2.76147800 & -3.16949800\end{array}$

$\begin{array}{lll}-0.92703000 & 1.82307600 & -3.37822000\end{array}$

$\begin{array}{lll}-2.52681700 & 2.55360400 & -3.11217600\end{array}$

$\begin{array}{lll}-1.28181100 & 3.43025100 & -4.03080900\end{array}$

$\begin{array}{lll}-1.57161400 & 4.82473500 & -1.78624500\end{array}$

$\begin{array}{lll}-1.36350300 & 5.38905000 & -2.71180500\end{array}$

$\begin{array}{lll}-2.66336100 & 4.77033000 & -1.68315200\end{array}$

$\begin{array}{lll}-1.17323000 & 5.41683700 & -0.95353000\end{array}$

$0.60585700 \quad 3.64632700 \quad-2.08226400$

$\begin{array}{lll}0.79545000 & 4.22580500 & -3.00172300\end{array}$

$1.04292900 \quad 4.20274200 \quad-1.24287400$

$\begin{array}{lll}1.13543100 & 2.68501100 & -2.16480500\end{array}$

$\begin{array}{lll}-1.23919700 & 3.28817100 & 1.20280100\end{array}$

$\begin{array}{lll}-2.50010700 \quad 4.15537300 & 1.32843100\end{array}$

$\begin{array}{lll}-2.44418000 & 4.70496200 & 2.28441600\end{array}$

$\begin{array}{lll}-2.60284600 & 4.89958900 & 0.53147800\end{array}$

$\begin{array}{lll}-3.41557100 & 3.55038400 & 1.35804200\end{array}$

$\begin{array}{lll}-0.00042600 & 4.18252400 & 1.35479500\end{array}$

$\begin{array}{lll}-0.01958100 & 5.03592300 & 0.66312200\end{array}$

$\begin{array}{lll}0.03070300 & 4.59149700 \quad 2.37918700\end{array}$

$0.92881600 \quad 3.62997800 \quad 1.18743100$

$\begin{array}{lll}-1.28092700 & 2.25913100 \quad 2.34260700\end{array}$ 
H

$\mathrm{H}$

$\mathrm{H}$

$\mathrm{Ni}$

$\mathrm{H}$

$\mathrm{H}$

$\mathrm{H}$

C

$\mathrm{H}$

$\mathrm{H}$

Conformation-B

C

C

C

C

C

C

C

C

C

C

\begin{tabular}{|c|c|c|}
\hline-2.21739800 & 1.68197300 & 2.33233400 \\
\hline-0.45386500 & 1.54756200 & 2.28403400 \\
\hline-1.22286200 & 2.77840600 & 3.31420100 \\
\hline 0.39235400 & 0.50879500 & -0.14358100 \\
\hline 1.63435000 & 1.07575200 & 3.70734700 \\
\hline 1.64902400 & 1.93664700 & 1.75045600 \\
\hline 3.02992300 & 1.31689500 & -0.88242100 \\
\hline 3.50330200 & 3.06596800 & 0.27240300 \\
\hline 3.67854200 & 3.35124000 & 1.32190100 \\
\hline 4.41271400 & 3.27690200 & -0.30952400 \\
\hline 2.69285800 & 3.69007000 & -0.12483500 \\
\hline 2.98655800 & 3.27474800 & -1.09377000 \\
\hline 2.09046200 & 2.27430200 & -0.68247400 \\
\hline 2.16457700 & 1.05194200 & -1.37246500 \\
\hline 2.85181600 & 0.88561100 & -2.57855500 \\
\hline 3.71767700 & 1.91563500 & -2.96462500 \\
\hline 3.82782700 & 3.07725100 & -2.19197500 \\
\hline 2.31945500 & -1.52082400 & -2.52361400 \\
\hline 1.86356400 & -1.31352600 & -1.21516300 \\
\hline 1.84133400 & -2.32669700 & -0.23721900 \\
\hline 2.11717500 & -3.62883800 & -0.68566000 \\
\hline 2.46687600 & -3.88658200 & -2.01184000 \\
\hline 2.61185400 & -2.83236600 & -2.91420700 \\
\hline 3.00355800 & 4.23146300 & -0.57488300 \\
\hline 4.29493900 & 1.82568000 & -3.88535000 \\
\hline 4.52367000 & 3.86426000 & -2.49132100 \\
\hline 2.09825100 & -4.45665500 & 0.01953900 \\
\hline 2.67334200 & -4.91090200 & -2.33033100 \\
\hline 2.94895000 & -3.03433600 & -3.93092500 \\
\hline 2.44587900 & -0.30984000 & -3.44567600 \\
\hline 1.04440500 & 0.00497000 & -4.03536700 \\
\hline 0.69644600 & -0.83765000 & -4.65251100 \\
\hline 0.30379100 & 0.17471000 & -3.24052000 \\
\hline 1.08743400 & 0.90912100 & -4.66231400 \\
\hline 3.42984700 & -0.55189900 & -4.59263600 \\
\hline 3.50172800 & 0.34063600 & -5.23026100 \\
\hline 4.43663400 & -0.79769600 & -4.22200100 \\
\hline 3.08574900 & -1.37346400 & -5.23644500 \\
\hline 1.45693700 & -0.02720000 & -0.85585300 \\
\hline 0.97375700 & 3.11706900 & 2.02739400 \\
\hline 2.12513200 & 4.12784700 & 2.06888500 \\
\hline 3.06251100 & 3.68528400 & 1.70196100 \\
\hline 1.91721100 & 5.03509800 & 1.48683400 \\
\hline
\end{tabular}


$2.29560700 \quad 4.43650100 \quad 3.11503600$

$\begin{array}{lll}1.38399500 & 1.86310400 \quad 2.81416000\end{array}$

$\begin{array}{lll}1.65749300 & 2.14933200 & 3.84421900\end{array}$

$\begin{array}{lll}0.56698600 & 1.13128000 & 2.86421700\end{array}$

$\begin{array}{lll}2.25204200 & 1.36819500 \quad 2.36254100\end{array}$

$\begin{array}{lll}-0.28463200 & 3.70315100 & 2.68410100\end{array}$

$\begin{array}{lll}-1.14206200 & 3.01887200 & 2.60195600\end{array}$

$\begin{array}{lll}-0.08685800 & 3.86110300 \quad 3.75771500\end{array}$

$\begin{array}{lll}-0.56504600 & 4.67385400 \quad 2.25299000\end{array}$

$\begin{array}{lll}-0.35754500 & 3.85131100 & -0.73347300\end{array}$

$0.22780300 \quad 5.26308800 \quad-0.62578000$

$\begin{array}{lll}-0.34110600 & 5.93688300 & -1.28996200\end{array}$

$\begin{array}{lll}0.14711200 & 5.66718200 & 0.39297800\end{array}$

$\begin{array}{lll}1.27869800 & 5.31206700 & -0.93999600\end{array}$

$\begin{array}{lll}-0.30151500 & 3.37333800 & -2.19594400\end{array}$

$\begin{array}{lll}-0.64332400 & 2.33008700 & -2.28595300\end{array}$

$\begin{array}{lll}-0.96955600 & 4.00216400 \quad-2.80784700\end{array}$

$\begin{array}{lll}0.70942700 & 3.44088900 & -2.62078300\end{array}$

$\begin{array}{lll}-1.83593800 & 3.88390400 & -0.30969200\end{array}$

$\begin{array}{lll}-1.97669200 & 4.21657700 & 0.72559700\end{array}$

$\begin{array}{lll}-2.37820300 & 4.58810900 & -0.96321400\end{array}$

$\begin{array}{lll}-2.29813300 & 2.89646600 & -0.41768500\end{array}$

$\begin{array}{lll}1.06446500 & -3.34179900 & 2.52761800\end{array}$

$\begin{array}{lll}2.00334500 & -4.53174700 & 2.76845900\end{array}$

$2.38298100 \quad-4.98867200 \quad 1.84534000$

$2.86397200 \quad-4.25310200 \quad 3.39096300$

$\begin{array}{lll}1.44844800 & -5.31469900 & 3.31491800\end{array}$

$0.65931100 \quad-2.78061300 \quad 3.90732500$

$\begin{array}{lll}1.52709200 & -2.44120200 & 4.48943800\end{array}$

$\begin{array}{lll}-0.03510400 & -1.93497800 & 3.81580300\end{array}$

$0.15641100 \quad-3.57080800 \quad 4.49085200$

$\begin{array}{lll}-0.21410000 & -3.82967900 & 1.82540800\end{array}$

$\begin{array}{lll}-0.69601000 & -4.60202800 \quad 2.44874900\end{array}$

$\begin{array}{lll}-0.94057900 & -3.01652200 & 1.69217600\end{array}$

$\begin{array}{lll}-0.01621400 & -4.27144700 \quad 0.83910300\end{array}$

$3.60213400 \quad-1.60245400 \quad 1.88384700$

$\begin{array}{lll}4.51035200 & -2.78046300 & 1.51157700\end{array}$

$5.56289300 \quad-2.48196500 \quad 1.66412000$

$\begin{array}{lll}4.33278600 & -3.67042100 & 2.12505200\end{array}$

$\begin{array}{lll}4.40300300 & -3.06161000 & 0.45436800\end{array}$

$3.76558000 \quad-1.26746400 \quad 3.37476200$

$\begin{array}{lll}3.55924000 & -2.13689400 & 4.01532700\end{array}$

$\begin{array}{lll}4.80557900 & -0.95445500 & 3.57090000\end{array}$

$3.10290100 \quad-0.44524300 \quad 3.68631700$ 
$\begin{array}{lll}4.05380900 & -0.39322000 & 1.04544200\end{array}$

$\begin{array}{lll}4.08668000 & -0.63063900 & -0.02726300\end{array}$

$3.40710700 \quad 0.48288500 \quad 1.17646400$

$5.07579100 \quad-0.10547300 \quad 1.34547400$

$\begin{array}{lll}-1.72342600 & -0.86297800 & -0.23105800\end{array}$

$\begin{array}{lll}-2.24283000 & 0.37049100 & 0.31538700\end{array}$

$\begin{array}{lll}-2.56417400 & 0.58249600 & 1.73650900\end{array}$

$\begin{array}{lll}-1.92719500 & -0.13891100 & 2.76956100\end{array}$

$\begin{array}{lll}-4.01307500 \quad 2.13862200 & 1.34279900\end{array}$

$\begin{array}{lll}-2.85467900 & 0.98252700 & -0.35721400\end{array}$

$\begin{array}{lll}-3.50093300 & 1.56553300 \quad 2.12031100\end{array}$

$-2.20621600 \quad 0.11281000 \quad 4.11165100$

$\begin{array}{lll}-3.13299700 & 1.09975100 \quad 4.47377200\end{array}$

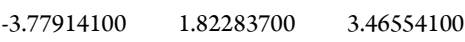

$\begin{array}{lll}-1.68682600 & -0.45989600 & 4.88548400\end{array}$

$\begin{array}{lll}-3.34735700 & 1.30096000 & 5.52641900\end{array}$

$\begin{array}{lll}-4.50819800 \quad 2.59486700 & 3.72791900\end{array}$

$\begin{array}{lll}-2.15209000 & -1.27605300 & -1.60988700\end{array}$

$\begin{array}{lll}-3.56630500 & -1.72169100 & -1.56043000\end{array}$

$-4.53132200 \quad-0.85875000 \quad-1.86904300$

$\begin{array}{lll}-5.91814700 & -1.41377700 & -1.51902400\end{array}$

$\begin{array}{lll}-4.33907200 & 0.24343100 & -2.34490700\end{array}$

$\begin{array}{lll}-6.09172100 & -2.81288800 & -2.13436000\end{array}$

$\begin{array}{lll}-6.00122200 & -1.50111700 & 0.01854300\end{array}$

$\begin{array}{lll}-6.99381100 & -0.46226300 & -2.05159000\end{array}$

$\begin{array}{lll}-6.00459900 & -2.77773000 & -3.23262100\end{array}$

$\begin{array}{lll}-5.33425700 & -3.51213900 & -1.75320700\end{array}$

$\begin{array}{lll}-7.08845500 & -3.21123600 & -1.88539900\end{array}$

$\begin{array}{lll}-5.85987700 & -0.51127000 \quad 0.48106200\end{array}$

$\begin{array}{lll}-6.99033300 & -1.88169600 & 0.32052800\end{array}$

$-5.23100500 \quad-2.17555400 \quad 0.42030800$

$\begin{array}{lll}-7.99454200 & -0.84053900 & -1.78946700\end{array}$

$\begin{array}{lll}-6.88158000 & 0.54501200 & -1.62431700\end{array}$

$\begin{array}{lll}-6.93781200 & -0.36910900 & -3.14727400\end{array}$

$\begin{array}{lll}-1.17515000 & -0.88174400 \quad 2.50341900\end{array}$

$\begin{array}{lll}-0.35437600 & 0.49397400 & -0.01367800\end{array}$

$\begin{array}{lll}0.52395700 & 2.49232100 & 0.29319800\end{array}$

$\begin{array}{lll}1.71004700 \quad-1.82003800 & 1.54492600\end{array}$

$\begin{array}{lll}-1.63136600 & -1.72075700 & 0.44744100\end{array}$

$\begin{array}{lll}-2.12882500 & -0.42147900 & -2.29842600\end{array}$

$\begin{array}{lll}-1.38213300 & -2.44875500 & -2.18535700\end{array}$

$-1.78018300 \quad-2.73773200 \quad-3.17064900$

$\begin{array}{lll}-1.44337100 & -3.32098100 & -1.51527400\end{array}$

$-0.32348700 \quad-2.18589500 \quad-2.30103400$ 


\section{Cartesian coordinates of the structures (optimization in heptane)}

\section{Tss}

C

C

C

C

2.16702700

1.16442800

1.22512600

2.08686800

3.07531900

3.15485900

1.29482800

0.61616200

0.11571900

0.24352700

0.89127200

1.43256000

2.13067500

3.77297600

3.93195800

$-0.16669500$

0.98171600

1.95181700

1.77537200

2.97340800

2.70928400

3.83587300

3.28288500

0.60920900

0.90577800

$-0.28198800$

0.33041900

0.40714100

0.36247100

0.60357400

1.36549900

$-0.29286900$

0.97482100

1.67863200

1.97036600

1.57616900

2.49779400

$-0.71016800$

$-0.91543000$

$-0.35231800$

$-1.65284500$

$-1.86383900$ 


\begin{tabular}{|c|c|c|}
\hline-1.41923800 & -0.61196400 & -2.84810400 \\
\hline-1.66471400 & -1.68256200 & -2.80733000 \\
\hline-1.09395100 & -0.37926800 & -3.87667600 \\
\hline-2.33929500 & -0.04451800 & -2.64312400 \\
\hline 1.01319300 & -0.87292000 & -2.31254400 \\
\hline 1.82856300 & -0.69263300 & -1.60492300 \\
\hline 1.30472500 & -0.44804100 & -3.28871600 \\
\hline 0.91791000 & -1.95884500 & -2.45097900 \\
\hline-0.14724400 & 1.30333900 & -1.88149500 \\
\hline-1.00914900 & 1.79926800 & -1.41690200 \\
\hline-0.07499800 & 1.64754000 & -2.92708600 \\
\hline 0.76311200 & 1.62439300 & -1.36155100 \\
\hline 5.29403400 & -2.26610600 & 0.56890200 \\
\hline 4.46900200 & -2.29923600 & 1.86866700 \\
\hline 3.47304800 & -2.73205700 & 1.71104500 \\
\hline 4.34045200 & -1.29186200 & 2.29530900 \\
\hline 4.98908000 & -2.91561100 & 2.62223000 \\
\hline 6.72082500 & -1.83346900 & 0.93109100 \\
\hline 7.17629800 & -2.60818600 & 1.57317700 \\
\hline 6.73071600 & -0.89710700 & 1.50508800 \\
\hline 7.37242800 & -1.71212100 & 0.05739900 \\
\hline 5.32319700 & -3.67914300 & -0.03755200 \\
\hline 4.31918900 & -4.01065900 & -0.34628200 \\
\hline 5.69831300 & -4.39884200 & 0.71037500 \\
\hline 5.98849100 & -3.74141700 & -0.91173000 \\
\hline 5.12466800 & -1.03457100 & -2.33910200 \\
\hline 4.66919000 & 0.30121700 & -2.95097600 \\
\hline 4.93355300 & 0.32606600 & -4.02242100 \\
\hline 5.14953300 & 1.16173400 & -2.46460800 \\
\hline 3.58000300 & 0.43770700 & -2.87385500 \\
\hline 6.65391600 & -1.12293100 & -2.40079900 \\
\hline 7.15180100 & -0.31883800 & -1.84169000 \\
\hline 6.97928800 & -1.02966500 & 29300 \\
\hline 7.03049600 & -2.08702600 & -2.03175600 \\
\hline 4.53328700 & -2.18319400 & -3.18076800 \\
\hline 3.43611800 & -2.12535800 & -3.22638000 \\
\hline 4.79914400 & -3.17128100 & -2.77767000 \\
\hline 4.92085900 & -2.13123500 & -4.21319000 \\
\hline 4.25476100 & -1.17970200 & -0.62680200 \\
\hline 0.94313100 & -0.72199100 & -0.13805700 \\
\hline-5.04099900 & -0.42679700 & -0.45894700 \\
\hline-4.33286700 & -1.70355400 & -0.55760500 \\
\hline-4.54645600 & -2.76601100 & 0.4449980 \\
\hline .83159100 & 2.48125500 & 1.7982170 \\
\hline
\end{tabular}




\begin{tabular}{|c|c|c|c|}
\hline $\mathrm{H}$ & -4.18997900 & -4.37394900 & -0.95337900 \\
\hline $\mathrm{H}$ & -4.20143400 & -2.10188100 & -1.57427700 \\
\hline $\mathrm{C}$ & -4.40056800 & -4.12142000 & 0.08921500 \\
\hline $\mathrm{C}$ & -4.95624200 & -3.49875300 & 2.74256500 \\
\hline $\mathrm{C}$ & -4.79152600 & -4.83835100 & 2.37118700 \\
\hline C & -4.51332900 & -5.14144900 & 1.03561500 \\
\hline $\mathrm{H}$ & -5.17204800 & -3.24394600 & 3.78379900 \\
\hline $\mathrm{H}$ & -4.88043400 & -5.63449700 & 3.11425600 \\
\hline $\mathrm{H}$ & -4.38699100 & -6.18244100 & 0.72564700 \\
\hline $\mathrm{C}$ & -5.36161500 & 0.35933300 & -1.61692900 \\
\hline $\mathrm{H}$ & -4.97924700 & -0.03957000 & -2.56249900 \\
\hline $\mathrm{C}$ & -3.64594100 & 2.38835700 & -0.88707500 \\
\hline $\mathrm{O}$ & -3.01621600 & 1.71290600 & -0.04640300 \\
\hline $\mathrm{C}$ & -3.45837000 & 3.91654900 & -0.90109100 \\
\hline C & -3.03698100 & 4.34551600 & -2.31782200 \\
\hline $\mathrm{C}$ & -2.38563200 & 4.32259000 & 0.11608400 \\
\hline C & -4.81115800 & 4.55611000 & -0.53893400 \\
\hline $\mathrm{H}$ & -3.79438300 & 4.05436900 & -3.05931500 \\
\hline $\mathrm{H}$ & -2.08107900 & 3.87918100 & -2.60500700 \\
\hline $\mathrm{H}$ & -2.90699600 & 5.43886700 & -2.36037400 \\
\hline $\mathrm{H}$ & -2.66752000 & 4.02894100 & 1.13708200 \\
\hline $\mathrm{H}$ & -2.24079700 & 5.41479900 & 0.09313700 \\
\hline $\mathrm{H}$ & -1.42246700 & 3.84081600 & -0.10483500 \\
\hline $\mathrm{H}$ & -4.72426600 & 5.65464500 & -0.53525800 \\
\hline $\mathrm{H}$ & -5.14433300 & 4.24014800 & 0.46305100 \\
\hline $\mathrm{H}$ & -5.58790100 & 4.27243500 & -1.26383800 \\
\hline $\mathrm{O}$ & -4.45052200 & 1.92694100 & -1.76997900 \\
\hline $\mathrm{H}$ & -5.65478200 & -0.22691100 & 0.42825600 \\
\hline $\mathrm{H}$ & -4.92934100 & -1.44153900 & 2.11731200 \\
\hline $\mathrm{C}$ & -6.72895600 & 0.97540900 & -1.72069600 \\
\hline $\mathrm{H}$ & -7.45993900 & 0.16041200 & -1.86055900 \\
\hline $\mathrm{H}$ & -6.81220800 & 1.66936400 & -2.56797800 \\
\hline $\mathrm{H}$ & -6.99357300 & 1.50622000 & -0.79336600 \\
\hline $\mathrm{Ni}$ & -3.10367900 & -0.26491300 & -0.17289800 \\
\hline \multicolumn{4}{|c|}{ TS20/TS-Conformation-A } \\
\hline $\mathrm{C}$ & -1.94824600 & 1.45581500 & -0.76457800 \\
\hline $\mathrm{C}$ & -1.84350200 & 0.03538700 & -0.59000500 \\
\hline $\mathrm{C}$ & -1.59074100 & -0.89130300 & -1.71116400 \\
\hline $\mathrm{C}$ & -1.46148900 & -0.46461900 & -3.04894800 \\
\hline $\mathrm{H}$ & -1.63094200 & -2.64013800 & -0.44890100 \\
\hline $\mathrm{H}$ & -2.50936100 & -0.35999700 & 0.17521400 \\
\hline $\mathrm{C}$ & -1.50423300 & -2.27670000 & -1.46461600 \\
\hline $\mathrm{C}$ & -1.22376600 & -1.37369800 & -4.08046300 \\
\hline $\mathrm{C}$ & -1.11323900 & -2.74038500 & -3.80816200 \\
\hline
\end{tabular}


$\begin{array}{lll}-1.13572300 & -1.01091800 & -5.10773600\end{array}$

$\begin{array}{lll}-0.92515100 & -3.45117200 & -4.61645400\end{array}$

$\begin{array}{lll}-1.19401100 & -4.25230800 & -2.25997300\end{array}$

$\begin{array}{lll}-2.32662200 & 2.23784700 & 0.31380200\end{array}$

$\begin{array}{lll}-5.07069800 & 0.73572200 & 0.07311900\end{array}$

$\begin{array}{lll}-4.54361700 & -0.08549800 & 0.86419300\end{array}$

$\begin{array}{lll}-6.06658500 & 0.18015800 & -0.99913800\end{array}$

$\begin{array}{lll}-6.29741600 & -1.32194300 \quad-0.81902100\end{array}$

$\begin{array}{lll}-7.39350300 & 0.94322000 & -0.87383500\end{array}$

$\begin{array}{lll}-5.44392500 & 0.45193000 & -2.37902400\end{array}$

$\begin{array}{lll}-5.35696500 & -1.88210200 & -0.93040800\end{array}$

$\begin{array}{lll}-6.69085600 & -1.54513800 & 0.18426100\end{array}$

$\begin{array}{lll}-7.01632000 & -1.70139500 & -1.56697600\end{array}$

$\begin{array}{lll}-7.21631000 & 2.02583200 & -0.95095300\end{array}$

$\begin{array}{lll}-8.10562600 & 0.64256000 & -1.66269200\end{array}$

$\begin{array}{lll}-7.87120400 & 0.74986800 & 0.10171900\end{array}$

$\begin{array}{lll}-6.11026400 & 0.11305700 & -3.19192800\end{array}$

$\begin{array}{lll}-5.25308000 & 1.52784800 & -2.50505800\end{array}$

$\begin{array}{lll}-4.48319200 & -0.07756700 & -2.49001600\end{array}$

$\begin{array}{lll}-4.85028800 & 1.97125300 & 0.02462100\end{array}$

$\begin{array}{lll}0.47787900 & -3.70815100 & 1.40308200\end{array}$

$\begin{array}{lll}0.59914200 & -2.35689900 & 1.04346200\end{array}$

$\begin{array}{lll}1.45972100 & -2.06194300 & -0.02799700\end{array}$

$2.26896500 \quad-3.01671600 \quad-0.65366800$

$\begin{array}{lll}2.12301500 & -4.35000400 & -0.25713900\end{array}$

$\begin{array}{lll}1.21068800 & -4.69606200 & 0.74289400\end{array}$

$3.77171800 \quad-1.15751700 \quad-1.23218900$

$\begin{array}{lll}2.82873700 & -0.26993900 & -0.69569600\end{array}$

$\begin{array}{lll}3.10766000 & 1.07411500 & -0.39283600\end{array}$

$\begin{array}{lll}4.44379000 & 1.48472200 & -0.52638200\end{array}$

$\begin{array}{lll}5.42767200 & 0.59876500 & -0.96907400\end{array}$

$\begin{array}{lll}5.08936500 & -0.70438100 & -1.34803500\end{array}$

$\begin{array}{lll}-0.17762200 & -3.99322000 & 2.22406600\end{array}$

$2.72430900 \quad-5.12824200 \quad-0.72771400$

$1.09576600 \quad-5.74202700 \quad 1.03536800$

$\begin{array}{lll}4.72021600 & 2.51159100 & -0.28983900\end{array}$

$\begin{array}{lll}6.46281500 & 0.93677100 & -1.05183900\end{array}$

$\begin{array}{lll}5.86180300 & -1.36434700 & -1.74373300\end{array}$

$\begin{array}{lll}3.24684000 & -2.51481900 & -1.71931800\end{array}$

$\begin{array}{lll}4.37849200 & -3.51194000 & -1.97796800\end{array}$

$\begin{array}{lll}5.06138500 & -3.13230200 & -2.75163200\end{array}$

$\begin{array}{lll}4.96288500 & -3.71663800 & -1.06806500\end{array}$

$3.97790400 \quad-4.46433000 \quad-2.35309800$ 
$\begin{array}{lll}1.61734200 & -1.59692400 & -2.88918300\end{array}$

$3.12696300 \quad-1.85552000 \quad-3.80581800$

$\begin{array}{lll}1.56283400 & -0.74575500 & -0.44072900\end{array}$

$\begin{array}{lll}-0.09564100 & -0.92422000 & 1.98318600\end{array}$

$\begin{array}{lll}1.66890600 & 2.19693700 & -0.07140700\end{array}$

$\begin{array}{lll}-1.70727600 & -1.48850000 & 2.88227900\end{array}$

$\begin{array}{lll}-2.60514200 & -2.34966400 \quad 1.97629800\end{array}$

$\begin{array}{lll}-2.97599700 \quad-1.81426300 & 1.09893000\end{array}$

$\begin{array}{lll}-2.12662600 & -3.28972700 \quad 1.67041600\end{array}$

$\begin{array}{lll}-3.50811100 & -2.61133300 \quad 2.55123400\end{array}$

$\begin{array}{lll}-2.46903800 & -0.19196100 \quad 3.23520000\end{array}$

$\begin{array}{lll}-1.81555900 & 0.59159700 & 3.64545700\end{array}$

$\begin{array}{lll}-3.03233600 & 0.18902100 & 2.37132700\end{array}$

$\begin{array}{lll}-3.22407600 & -0.42381300 & 4.00481000\end{array}$

$\begin{array}{lll}-1.47273700 & -2.28391500 & 4.17713200\end{array}$

$\begin{array}{lll}-1.00195300 & -1.68083800 \quad 4.96249500\end{array}$

$\begin{array}{lll}-2.45704300 & -2.59687100 \quad 4.56231600\end{array}$

$-0.87642200 \quad-3.19437200 \quad 4.03134600$

$\begin{array}{lll}1.29256100 & -0.75507000 & 3.32558200\end{array}$

$\begin{array}{lll}2.56935000 & -0.40629300 & 2.55378600\end{array}$

$2.96425300 \quad-1.27517500 \quad 2.00969700$

$\begin{array}{lll}2.40081900 & 0.39589400 & 1.83654400\end{array}$

$3.34973500 \quad-0.07677100 \quad 3.25822100$

$\begin{array}{lll}0.90245700 & 0.41516900 & 4.24054800\end{array}$

$\begin{array}{lll}0.04709800 & 0.17072700 & 4.88527300\end{array}$

$1.74873200 \quad 0.67321500 \quad 4.89939000$

$\begin{array}{lll}0.63880700 & 1.31277900 \quad 3.66136000\end{array}$

$\begin{array}{lll}1.62812000 & -2.00225700 \quad 4.15990100\end{array}$

$\begin{array}{lll}1.74713400 & -2.89716400 & 3.53240900\end{array}$

$\begin{array}{lll}2.59706900 & -1.83073400 & 4.65943400\end{array}$

$0.90016200 \quad-2.21986800 \quad 4.94545700$

$\begin{array}{lll}2.21230300 \quad 3.58253700 & 1.13216900\end{array}$

$\begin{array}{lll}2.99484200 & 2.97397200 & 2.30457800\end{array}$

$2.40055000 \quad 2.23744600 \quad 2.85336600$

$\begin{array}{lll}3.93166900 & 2.49513600 \quad 1.98810000\end{array}$

$\begin{array}{lll}3.25185000 & 3.77969800 & 3.01225200\end{array}$

$\begin{array}{lll}3.04452700 & 4.72509400 & 0.53438600\end{array}$

$\begin{array}{lll}3.28495700 & 5.43985200 & 1.33941300\end{array}$

$3.99808400 \quad 4.38361800 \quad 0.10982300$

$\begin{array}{lll}2.50142900 & 5.28318300 & -0.23863700\end{array}$

$\begin{array}{lll}0.89666100 & 4.16603700 & 1.67987800\end{array}$

$\begin{array}{lll}1.12144200 & 4.94615000 & 2.42617000\end{array}$ 


\begin{tabular}{|c|c|c|c|}
\hline $\mathrm{H}$ & 0.29022700 & 4.62600300 & 0.88852500 \\
\hline $\mathrm{H}$ & 0.28341600 & 3.39331900 & 2.16722500 \\
\hline C & 1.52605200 & 2.91527600 & -1.85959700 \\
\hline $\mathrm{C}$ & 2.80314300 & 3.50147600 & -2.47718000 \\
\hline $\mathrm{H}$ & 2.58166000 & 3.76039000 & -3.52686400 \\
\hline $\mathrm{H}$ & 3.14795800 & 4.41768700 & -1.98677100 \\
\hline $\mathrm{H}$ & 3.62867400 & 2.77810400 & -2.49488600 \\
\hline C & 0.42165200 & 3.97698700 & -1.86400700 \\
\hline $\mathrm{H}$ & 0.71839900 & 4.88768100 & -1.32464000 \\
\hline $\mathrm{H}$ & 0.19079300 & 4.26967700 & -2.90189700 \\
\hline $\mathrm{H}$ & -0.49621200 & 3.59607300 & -1.40884900 \\
\hline $\mathrm{C}$ & 1.10840200 & 1.71769900 & -2.72829800 \\
\hline $\mathrm{H}$ & 1.95247300 & 1.03875200 & -2.91292400 \\
\hline $\mathrm{H}$ & 0.30831400 & 1.13246000 & -2.26788800 \\
\hline $\mathrm{H}$ & 0.75075900 & 2.07651600 & -3.70725900 \\
\hline $\mathrm{Ni}$ & -0.23199100 & 0.81482500 & 0.36472800 \\
\hline $\mathrm{H}$ & -1.57739100 & 0.58980400 & -3.29665200 \\
\hline $\mathrm{H}$ & -1.75878100 & 1.93450000 & -1.72740300 \\
\hline $\mathrm{H}$ & -2.60394400 & 1.72066100 & 1.23153600 \\
\hline C & -2.49667200 & 3.71376800 & 0.32007600 \\
\hline $\mathrm{H}$ & -2.22384800 & 4.18934100 & -0.63097700 \\
\hline $\mathrm{H}$ & -3.56630400 & 3.89381100 & 0.50558400 \\
\hline $\mathrm{H}$ & -1.93204200 & 4.18439000 & 1.13989800 \\
\hline
\end{tabular}

TS19/TS-Conformation-B

\begin{tabular}{|c|c|c|c|}
\hline $\mathrm{C}$ & -1.72153500 & -3.16332500 & 2.51982600 \\
\hline $\mathrm{C}$ & -1.25857600 & -2.11530100 & 1.70559900 \\
\hline $\mathrm{C}$ & -1.82167800 & -2.03123400 & 0.41867200 \\
\hline C & -2.61410300 & -3.04192600 & -0.13962900 \\
\hline $\mathrm{C}$ & -3.04184400 & -4.06999600 & 0.70609300 \\
\hline $\mathrm{C}$ & -2.63651200 & -4.10180300 & 2.04237400 \\
\hline $\mathrm{C}$ & -3.16127100 & -1.56076100 & -2.00864200 \\
\hline $\mathrm{C}$ & -2.48649600 & -0.54233000 & -1.32057600 \\
\hline $\mathrm{C}$ & -2.76070500 & 0.82338800 & -1.51304800 \\
\hline $\mathrm{C}$ & -3.70566700 & 1.14170300 & -2.50349300 \\
\hline $\mathrm{C}$ & -4.35420000 & 0.15291600 & -3.24060400 \\
\hline $\mathrm{C}$ & -4.09802100 & -1.19249200 & -2.97875200 \\
\hline $\mathrm{H}$ & -1.34360200 & -3.26839300 & 3.53304200 \\
\hline $\mathrm{H}$ & -3.68068200 & -4.86407700 & 0.31942200 \\
\hline $\mathrm{H}$ & -2.98946300 & -4.89905000 & 2.70002100 \\
\hline $\mathrm{H}$ & -3.95991500 & 2.18256400 & -2.68829700 \\
\hline $\mathrm{H}$ & -5.08185800 & 0.43275300 & -4.00536100 \\
\hline $\mathrm{H}$ & -4.63395700 & -1.96102000 & -3.53502600 \\
\hline $\mathrm{C}$ & -2.82172700 & -3.00479000 & -1.65454400 \\
\hline $\mathrm{C}$ & -1.47402300 & -3.37394700 & -2.33051500 \\
\hline
\end{tabular}




\begin{tabular}{|c|c|c|c|}
\hline $\mathrm{H}$ & -0.98762600 & 4.31788100 & -3.25221700 \\
\hline $\mathrm{H}$ & -0.43166300 & 2.72426500 & -2.70766500 \\
\hline $\mathrm{H}$ & -2.03789700 & 2.89771300 & -3.43254500 \\
\hline $\mathrm{C}$ & -3.40023000 & 2.17583000 & 0.92556600 \\
\hline $\mathrm{C}$ & -4.80733000 & 2.51855200 & 0.42126300 \\
\hline $\mathrm{H}$ & -5.52669900 & 2.36851900 & 1.24553400 \\
\hline $\mathrm{H}$ & -4.90597200 & 3.55936200 & 0.09308400 \\
\hline $\mathrm{H}$ & -5.12017500 & 1.86151400 & -0.40313400 \\
\hline C & -2.95597800 & 3.19963500 & 1.98134200 \\
\hline $\mathrm{H}$ & -2.96487900 & 4.22472500 & 1.58262200 \\
\hline $\mathrm{H}$ & -3.64636500 & 3.17661200 & 2.84182200 \\
\hline $\mathrm{H}$ & -1.94441500 & 2.98915700 & 2.35707000 \\
\hline $\mathrm{C}$ & -3.45665500 & 0.76875800 & 1.54570400 \\
\hline $\mathrm{H}$ & -3.97252200 & 0.05843500 & 0.88364700 \\
\hline $\mathrm{H}$ & -2.45664100 & 0.37081400 & 1.74738200 \\
\hline $\mathrm{H}$ & -4.01512100 & 0.79879300 & 2.49605500 \\
\hline C & 1.38719400 & 1.12215100 & -1.21411000 \\
\hline $\mathrm{C}$ & 2.03937000 & 0.84972000 & 0.04839000 \\
\hline C & 2.48408900 & 1.90707700 & 0.97961800 \\
\hline $\mathrm{C}$ & 1.62174200 & 2.92928100 & 1.41530200 \\
\hline $\mathrm{H}$ & 4.47179600 & 1.08900800 & 1.18467200 \\
\hline $\mathrm{H}$ & 2.73342200 & 0.01007500 & -0.00146900 \\
\hline $\mathrm{C}$ & 3.78763100 & 1.87603300 & 1.50997700 \\
\hline C & 2.04256700 & 3.88794900 & 2.33644300 \\
\hline $\mathrm{C}$ & 3.34187400 & 3.84419600 & 2.85387200 \\
\hline $\mathrm{C}$ & 4.21197600 & 2.83280400 & 2.43479600 \\
\hline $\mathrm{H}$ & 1.34847900 & 4.66754800 & 2.66223000 \\
\hline $\mathrm{H}$ & 3.67278000 & 4.59107500 & 3.57974600 \\
\hline $\mathrm{H}$ & 5.22983500 & 2.78822100 & 2.83101100 \\
\hline $\mathrm{C}$ & 1.24413600 & 0.07756300 & -2.13559500 \\
\hline $\mathrm{O}$ & 3.14247300 & -0.04454200 & -3.13927600 \\
\hline $\mathrm{C}$ & 3.91107200 & -0.80015200 & -2.46937000 \\
\hline $\mathrm{C}$ & 5.43958400 & -0.64097200 & -2.73725900 \\
\hline $\mathrm{O}$ & 3.56225400 & -1.61689000 & -1.59158600 \\
\hline $\mathrm{C}$ & 5.70573600 & 0.19655100 & -3.99128400 \\
\hline $\mathrm{C}$ & 6.02070200 & 0.06869800 & -1.50020500 \\
\hline C & 6.06987100 & -2.03271900 & -2.87879600 \\
\hline $\mathrm{H}$ & 5.27044100 & -0.27719600 & -4.88506700 \\
\hline $\mathrm{H}$ & 5.25573000 & 1.19553300 & -3.90182300 \\
\hline $\mathrm{H}$ & 6.79078100 & 0.31291800 & -4.15842400 \\
\hline $\mathrm{H}$ & 5.84989400 & -0.53257400 & -0.59430500 \\
\hline $\mathrm{H}$ & 7.10716100 & 0.22854200 & -1.61081400 \\
\hline $\mathrm{H}$ & 5.54670300 & 1.05299000 & -1.35260800 \\
\hline $\mathrm{H}$ & 7.16636100 & -1.96187400 & -2.98295300 \\
\hline
\end{tabular}




$\begin{array}{lrrr}\mathrm{H} & 5.83587800 & -2.64857800 & -1.99903100 \\ \mathrm{H} & 5.68013300 & -2.55566500 & -3.76823800 \\ \mathrm{H} & 0.59860200 & 2.93843500 & 1.04279000 \\ \mathrm{Ni} & 0.24009800 & 0.16793700 & 0.20554600 \\ \mathrm{P} & 0.28964500 & -1.12094000 & 1.97243500 \\ \mathrm{P} & -1.98870900 & 2.06263500 & -0.37490000 \\ \mathrm{H} & 1.13927200 & 2.14582800 & -1.50534900 \\ \mathrm{C} & 0.44734700 & 0.17632900 & -3.39581900 \\ \mathrm{H} & 0.64961900 & 1.11976100 & -3.92339000 \\ \mathrm{H} & -0.63204800 & 0.13710300 & -3.17740500 \\ \mathrm{H} & 0.69503300 & -0.65270900 & -4.07102300 \\ \mathrm{H} & 1.52226700 & -0.91897300 & -1.79255500\end{array}$

Cartesian coordinates of the structures (optimization in 1,2-dichloroethane) TS5

\begin{tabular}{|c|c|c|c|}
\hline $\mathrm{C}$ & -1.14534100 & 0.63640000 & 2.17851000 \\
\hline $\mathrm{C}$ & -0.20061800 & 0.36096100 & 1.16805500 \\
\hline $\mathrm{C}$ & 1.01962700 & 1.07180800 & 1.22669400 \\
\hline $\mathrm{C}$ & 1.16924300 & 2.17793300 & 2.09156900 \\
\hline $\mathrm{C}$ & 0.21158200 & 2.39057200 & 3.08667600 \\
\hline $\mathrm{C}$ & -0.92314200 & 1.58413200 & 3.16994200 \\
\hline $\mathrm{C}$ & 3.49201800 & 2.44949500 & 1.28704200 \\
\hline $\mathrm{C}$ & 3.33119000 & 1.22497800 & 0.60981000 \\
\hline $\mathrm{C}$ & 4.44653300 & 0.51262500 & 0.10652200 \\
\hline $\mathrm{C}$ & 5.70951900 & 1.11776200 & 0.22618900 \\
\hline $\mathrm{C}$ & 5.88478400 & 2.33923800 & 0.86927400 \\
\hline $\mathrm{C}$ & 4.77821500 & 2.98447100 & 1.41650800 \\
\hline $\mathrm{H}$ & -2.09888900 & 0.10406500 & 2.14534000 \\
\hline $\mathrm{H}$ & 0.33109500 & 3.21771500 & 3.78576100 \\
\hline $\mathrm{H}$ & -1.66601200 & 1.74902400 & 3.95332400 \\
\hline $\mathrm{H}$ & 6.57829300 & 0.61127600 & -0.18602400 \\
\hline $\mathrm{H}$ & 6.87988900 & 2.78206400 & 0.95270200 \\
\hline $\mathrm{H}$ & 4.91587500 & 3.93379500 & 1.93319900 \\
\hline $\mathrm{C}$ & 2.26026100 & 3.19854000 & 1.77847400 \\
\hline $\mathrm{C}$ & 2.57543600 & 4.10067200 & 2.97894100 \\
\hline $\mathrm{H}$ & 3.32415500 & 4.85830300 & 2.71174500 \\
\hline $\mathrm{H}$ & 2.95383300 & 3.52284300 & 3.83594800 \\
\hline $\mathrm{H}$ & 1.67970100 & 4.65157600 & 3.29630000 \\
\hline $\mathrm{C}$ & 1.73961200 & 4.09166800 & 0.61989900 \\
\hline $\mathrm{H}$ & 0.82485500 & 4.62133000 & 0.92800700 \\
\hline $\mathrm{H}$ & 1.50399400 & 3.49422200 & -0.27301800 \\
\hline $\mathrm{H}$ & 2.50329800 & 4.83555300 & 0.34271700 \\
\hline $\mathrm{O}$ & 2.06428100 & 0.70586800 & 0.40578000 \\
\hline $\mathrm{C}$ & -0.44403800 & -2.49139300 & 0.35843700 \\
\hline $\mathrm{C}$ & 1.05481300 & -2.64579500 & 0.60346600 \\
\hline
\end{tabular}




\begin{tabular}{|c|c|c|c|}
\hline $\mathrm{H}$ & 1.40768000 & -1.92748500 & 1.35685900 \\
\hline $\mathrm{H}$ & 1.65817100 & -2.49658100 & -0.29813200 \\
\hline $\mathrm{H}$ & 1.26131300 & -3.66093800 & 0.98551300 \\
\hline $\mathrm{C}$ & -1.19310300 & -2.77537800 & 1.67218500 \\
\hline $\mathrm{H}$ & -1.04194600 & -3.83042300 & 1.95592600 \\
\hline $\mathrm{H}$ & -2.27060400 & -2.59999400 & 1.56953500 \\
\hline $\mathrm{H}$ & -0.81695000 & -2.15087600 & 2.49619700 \\
\hline $\mathrm{C}$ & -0.93230700 & -3.46933500 & -0.71842500 \\
\hline $\mathrm{H}$ & -2.00131800 & -3.33252400 & -0.92797900 \\
\hline $\mathrm{H}$ & -0.79084900 & -4.50391800 & -0.36286600 \\
\hline $\mathrm{H}$ & -0.37527000 & -3.36184600 & -1.65933600 \\
\hline $\mathrm{C}$ & -0.29880300 & -0.24398800 & -1.86447300 \\
\hline $\mathrm{C}$ & -1.41740500 & -0.63449100 & -2.85130600 \\
\hline $\mathrm{H}$ & -1.65664500 & -1.70652700 & -2.81025300 \\
\hline $\mathrm{H}$ & -1.08777000 & -0.40110300 & -3.87829100 \\
\hline $\mathrm{H}$ & -2.34053100 & -0.07015000 & -2.65071400 \\
\hline $\mathrm{C}$ & 1.01368100 & -0.88693300 & -2.30681800 \\
\hline $\mathrm{H}$ & 1.82625900 & -0.69907200 & -1.59799100 \\
\hline $\mathrm{H}$ & 1.30632600 & -0.46423100 & -3.28343300 \\
\hline $\mathrm{H}$ & 0.92073200 & -1.97383500 & -2.43976600 \\
\hline $\mathrm{C}$ & -0.15596900 & 1.28760800 & -1.88526500 \\
\hline $\mathrm{H}$ & -1.02707600 & 1.78148700 & -1.43596700 \\
\hline $\mathrm{H}$ & -0.07208000 & 1.62674100 & -2.93157700 \\
\hline $\mathrm{H}$ & 0.74527400 & 1.61721700 & -1.35456600 \\
\hline $\mathrm{C}$ & 5.30574000 & -2.25251400 & 0.58776300 \\
\hline $\mathrm{C}$ & 4.47110000 & -2.28599900 & 1.88137700 \\
\hline $\mathrm{H}$ & 3.48085400 & -2.73139400 & 1.71841100 \\
\hline $\mathrm{H}$ & 4.32785300 & -1.27718600 & 2.30067200 \\
\hline $\mathrm{H}$ & 4.99276800 & -2.89226000 & 2.64211200 \\
\hline $\mathrm{C}$ & 6.72532200 & -1.80157600 & 0.95439800 \\
\hline $\mathrm{H}$ & 7.18569800 & -2.56904800 & 1.60180700 \\
\hline $\mathrm{H}$ & 6.72156900 & -0.86212000 & 1.52377500 \\
\hline $\mathrm{H}$ & 7.37707900 & -1.67498800 & 0.08167600 \\
\hline $\mathrm{C}$ & 5.35450100 & -3.66928600 & -0.00844600 \\
\hline $\mathrm{H}$ & 4.35534000 & -4.01662100 & -0.31641400 \\
\hline $\mathrm{H}$ & 5.73738900 & -4.37679600 & 0.74729100 \\
\hline $\mathrm{H}$ & 6.02205100 & -3.72727200 & -0.88100800 \\
\hline $\mathrm{C}$ & 5.13678500 & -1.05282500 & -2.33375600 \\
\hline $\mathrm{C}$ & 4.68242400 & 0.27658100 & -2.95966200 \\
\hline $\mathrm{H}$ & 4.94609600 & 0.28726000 & -4.03155800 \\
\hline $\mathrm{H}$ & 5.16725500 & 1.14099400 & -2.48444400 \\
\hline $\mathrm{H}$ & 3.59321700 & 0.41580600 & -2.88253400 \\
\hline $\mathrm{C}$ & 6.66592400 & -1.14337600 & -2.38950300 \\
\hline $\mathrm{H}$ & 7.16200800 & -0.33413400 & -1.83631500 \\
\hline
\end{tabular}




\begin{tabular}{|c|c|c|c|}
\hline $\mathrm{H}$ & 6.99219800 & -1.06171100 & -3.44179200 \\
\hline $\mathrm{H}$ & 7.03758300 & -2.10447500 & -2.00803100 \\
\hline $\mathrm{C}$ & 4.54696900 & -2.20919100 & -3.16591100 \\
\hline $\mathrm{H}$ & 3.44981600 & -2.14896500 & -3.21952200 \\
\hline $\mathrm{H}$ & 4.81003600 & -3.19288800 & -2.75019900 \\
\hline $\mathrm{H}$ & 4.94120100 & -2.16714200 & -4.19630100 \\
\hline $\mathrm{P}$ & 4.26713200 & -1.18372800 & -0.62188600 \\
\hline $\mathrm{P}$ & -0.94787500 & -0.73491300 & -0.14023000 \\
\hline C & -5.04333700 & -0.39614300 & -0.47120400 \\
\hline C & -4.35491500 & -1.68340100 & -0.57000600 \\
\hline $\mathrm{C}$ & -4.57758800 & -2.74192500 & 0.43384300 \\
\hline $\mathrm{C}$ & -4.87329200 & -2.45417000 & 1.78530100 \\
\hline $\mathrm{H}$ & -4.20963900 & -4.35391500 & -0.95824700 \\
\hline $\mathrm{H}$ & -4.22559000 & -2.08327200 & -1.58611600 \\
\hline C & -4.42974500 & -4.09942200 & 0.08191700 \\
\hline $\mathrm{C}$ & -5.00459900 & -3.47039200 & 2.73130800 \\
\hline $\mathrm{C}$ & -4.83716800 & -4.81183900 & 2.36423600 \\
\hline C & -4.54937100 & -5.11778600 & 1.03025200 \\
\hline $\mathrm{H}$ & -5.22807700 & -3.21311200 & 3.77049700 \\
\hline $\mathrm{H}$ & -4.93143300 & -5.60645000 & 3.10853700 \\
\hline $\mathrm{H}$ & -4.42037800 & -6.15952500 & 0.72333000 \\
\hline C & -5.34702500 & 0.39388300 & -1.63272400 \\
\hline $\mathrm{H}$ & -4.97581000 & -0.01761300 & -2.57705600 \\
\hline $\mathrm{C}$ & -3.61084300 & 2.39670100 & -0.88811300 \\
\hline $\mathrm{O}$ & -3.01570700 & 1.71760800 & -0.02397400 \\
\hline C & -3.41331500 & 3.92265800 & -0.90138000 \\
\hline C & -2.99560600 & 4.35622700 & -2.31736500 \\
\hline C & -2.33400000 & 4.32252400 & 0.11093800 \\
\hline $\mathrm{C}$ & -4.76182800 & 4.56631400 & -0.52913800 \\
\hline $\mathrm{H}$ & -3.75905000 & 4.07685900 & -3.05746500 \\
\hline $\mathrm{H}$ & -2.04453400 & 3.88372700 & -2.61079400 \\
\hline $\mathrm{H}$ & -2.85840000 & 5.44883800 & -2.35237500 \\
\hline $\mathrm{H}$ & -2.61596400 & 4.03645000 & 1.13444100 \\
\hline $\mathrm{H}$ & -2.18134700 & 5.41337000 & 0.08418300 \\
\hline $\mathrm{H}$ & -1.37521300 & 3.83406100 & -0.11514600 \\
\hline $\mathrm{H}$ & -4.66735000 & 5.66413900 & -0.51908500 \\
\hline $\mathrm{H}$ & -5.09205500 & 4.24426700 & 0.47193700 \\
\hline $\mathrm{H}$ & -5.54369600 & 4.29289000 & -1.25294500 \\
\hline $\mathrm{O}$ & -4.39140800 & 1.93432100 & -1.79223800 \\
\hline $\mathrm{H}$ & -5.65664700 & -0.18480500 & 0.41398000 \\
\hline $\mathrm{H}$ & -4.97352300 & -1.41405800 & 2.10254700 \\
\hline $\mathrm{C}$ & -6.69830100 & 1.04337000 & -1.73345800 \\
\hline $\mathrm{H}$ & -7.44884100 & 0.24525400 & -1.86822800 \\
\hline $\mathrm{H}$ & -6.76892700 & 1.73325000 & -2.58538000 \\
\hline
\end{tabular}


TS20/TS-Conformation-A

C

C

C

C

$\mathrm{H}$

$\mathrm{H}$

C

C

C

C

$\mathrm{H}$

$\mathrm{H}$

$\mathrm{H}$

C

C

O

C

C

C

C

$\mathrm{H}$

\begin{tabular}{|c|c|c|}
\hline-1.95710000 & 1.37822200 & -0.67094200 \\
\hline 1.82017600 & -0.04582300 & -0.52199500 \\
\hline 1.56899500 & -0.93709300 & -1.67752700 \\
\hline 1.52342000 & -0.46892500 & -3.00756600 \\
\hline-1.44854500 & -2.72308300 & -0.47033500 \\
\hline-2.47828200 & -0.48189100 & 0.22702400 \\
\hline-1.40084600 & -2.32358600 & -1.47944600 \\
\hline 1.28933500 & -1.33574700 & -4.07724300 \\
\hline-1.10126800 & -2.70325400 & -3.85414200 \\
\hline-1.16669500 & -3.19194800 & -2.54493100 \\
\hline-1.26304900 & -0.93802100 & -5.09530800 \\
\hline-0.91367400 & -3.38071700 & -4.69084400 \\
\hline-1.03037300 & -4.25858000 & -2.34838000 \\
\hline-2.56483100 & 2.12234300 & 0.34337000 \\
\hline-5.09751900 & 0.67183900 & 0.07516800 \\
\hline-4.68738700 & -0.06644800 & 0.99159300 \\
\hline-6.15045400 & 0.10540700 & -0.92805200 \\
\hline-6.80825800 & -1.15945300 & -0.36999400 \\
\hline-7.21610200 & 1.16843900 & -1.22627900 \\
\hline-5.37907800 & -0.23291800 & -2.21776500 \\
\hline-6.05757300 & -1.93383500 & -0.15633500 \\
\hline-7.34072300 & -0.94944100 & 0.57172100 \\
\hline-7.53787000 & -1.56966900 & -1.08935500 \\
\hline-6.74741000 & 2.09098100 & -1.59698700 \\
\hline-7.93295000 & 0.80767500 & -1.98401100 \\
\hline-7.78780300 & 1.42426300 & -0.31802200 \\
\hline-6.05815900 & -0.63666900 & -2.98842500 \\
\hline-4.88829700 & 0.66417800 & -2.62497000 \\
\hline-4.59744000 & -0.98531700 & -2.02646900 \\
\hline-4.69716500 & 1.85111400 & -0.17270300 \\
\hline 0.68539300 & -3.73672000 & 1.34576500 \\
\hline 0.73512000 & -2.37722900 & 0.99888000 \\
\hline 1.55525000 & -2.03105800 & -0.08930600 \\
\hline 2.39336600 & -2.93958000 & -0.74562300 \\
\hline 2.31906600 & -4.28330700 & -0.36264700 \\
\hline 1.44749200 & -4.68198100 & 0.65518000 \\
\hline 3.79340600 & -1.00356800 & -1.33218300 \\
\hline 2.82231300 & -0.16859200 & -0.76203000 \\
\hline 3.04540900 & 1.17981300 & -0.43548800 \\
\hline 4.35849100 & 1.65461400 & -0.59149300 \\
\hline .37117000 & 0.82360500 & -1.07563400 \\
\hline
\end{tabular}


$\begin{array}{lll}0.06616800 & -4.06331400 & 2.17975000\end{array}$

$1.38859900 \quad-5.73548800 \quad 0.93783400$

$\begin{array}{lll}4.59379100 & 2.68751000 & -0.33788700\end{array}$

$6.38719800 \quad 1.21173200 \quad-1.17635700$

$5.87828400 \quad-1.10541500 \quad-1.89526800$

$\begin{array}{lll}3.32283700 & -2.37841200 & -1.82557000\end{array}$

$\begin{array}{lll}4.49367900 & -3.31763500 & -2.12172200\end{array}$

$\begin{array}{lll}5.14305000 & -2.89225500 & -2.90024900\end{array}$

$5.10369900 \quad-3.50641200 \quad-1.22518200$

$4.12848500 \quad-4.28207200 \quad-2.50213300$

$\begin{array}{lll}2.50060700 & -2.16499000 & -3.12300600\end{array}$

$2.12648600 \quad-3.13007500 \quad-3.49666900$

$1.63499300 \quad-1.51173300 \quad-2.94827200$

$3.13083000 \quad-1.70631500 \quad-3.90104500$

$1.58532000 \quad-0.70907600 \quad-0.48983700$

$\begin{array}{lll}0.00448900 & -0.98327500 & 1.97058000\end{array}$

$\begin{array}{lll}1.57014000 & 2.23116400 & -0.03570500\end{array}$

$\begin{array}{lll}-1.57377500 & -1.62616400 \quad 2.87860900\end{array}$

$\begin{array}{lll}-2.44604700 & -2.50862300 & 1.96938900\end{array}$

$\begin{array}{lll}-2.80330900 & -1.98735500 & 1.07792600\end{array}$

$\begin{array}{lll}-1.94079000 & -3.43536700 \quad 1.66639400\end{array}$

$\begin{array}{lll}-3.34623100 & -2.79402400 & 2.53855300\end{array}$

$\begin{array}{lll}-2.38169200 & -0.36846800 \quad 3.25936700\end{array}$

$\begin{array}{lll}-1.76004100 & 0.41489700 & 3.71606900\end{array}$

$\begin{array}{lll}-2.92396500 & 0.03981900 & 2.39780800\end{array}$

$-3.14972900 \quad-0.65135800 \quad 3.99936900$

$\begin{array}{lll}-1.30342100 & -2.43007900 \quad 4.16024400\end{array}$

$\begin{array}{lll}-0.85091700 & -1.81889700 \quad 4.94986000\end{array}$

$\begin{array}{lll}-2.27346500 & -2.78602700 & 4.54584000\end{array}$

$\begin{array}{lll}-0.67364400 & -3.31386700 \quad 3.99546400\end{array}$

$\begin{array}{lll}1.40109300 & -0.78847500 & 3.29790600\end{array}$

$\begin{array}{lll}2.65864500 & -0.41216200 & 2.50604200\end{array}$

$\begin{array}{lll}3.06706600 & -1.27678000 & 1.96456700\end{array}$

$\begin{array}{lll}2.46029100 & 0.37880100 \quad 1.78380300\end{array}$

$\begin{array}{lll}3.43787600 & -0.05591400 & 3.19842600\end{array}$

$\begin{array}{lll}0.99069900 & 0.36978500 & 4.21922300\end{array}$

$0.15685300 \quad 0.09345200 \quad 4.87980700$

$\begin{array}{lll}1.84084200 & 0.65589500 \quad 4.86118500\end{array}$

$0.68511000 \quad 1.25704200 \quad 3.64497400$

$\begin{array}{lll}1.77679800 & -2.02659800 \quad 4.12818500\end{array}$

$\begin{array}{lll}1.89848100 & -2.92182400 & 3.50154200\end{array}$

$2.75249900 \quad-1.83344000 \quad 4.60643300$ 


\begin{tabular}{|c|c|c|c|}
\hline $\mathrm{H}$ & 1.06813300 & -2.25281000 & 4.92909200 \\
\hline C & 2.09565200 & 3.58615400 & 1.21671300 \\
\hline C & 2.95267300 & 2.96586300 & 2.32908400 \\
\hline $\mathrm{H}$ & 2.41766900 & 2.17844400 & 2.86654300 \\
\hline $\mathrm{H}$ & 3.89872900 & 2.55126400 & 1.95448800 \\
\hline $\mathrm{H}$ & 3.19772500 & 3.75420000 & 3.06041500 \\
\hline C & 2.84830900 & 4.79728300 & 0.64991300 \\
\hline $\mathrm{H}$ & 3.06845500 & 5.48692700 & 1.48251600 \\
\hline $\mathrm{H}$ & 3.80827300 & 4.52686100 & 0.19053900 \\
\hline $\mathrm{H}$ & 2.25478800 & 5.35647300 & -0.08389900 \\
\hline C & 0.77824700 & 4.08084100 & 1.84077300 \\
\hline $\mathrm{H}$ & 1.00142500 & 4.80505900 & 2.64200400 \\
\hline $\mathrm{H}$ & 0.14494600 & 4.58887500 & 1.10070100 \\
\hline $\mathrm{H}$ & 0.19844500 & 3.25384500 & 2.27798000 \\
\hline C & 1.35993700 & 3.02626900 & -1.78501700 \\
\hline C & 2.60135400 & 3.69092900 & -2.39670500 \\
\hline $\mathrm{H}$ & 2.34330400 & 4.00381600 & -3.42334500 \\
\hline $\mathrm{H}$ & 2.93061300 & 4.58555000 & -1.85897700 \\
\hline $\mathrm{H}$ & 3.44779700 & 2.99664100 & -2.47723000 \\
\hline C & 0.21880500 & 4.04783900 & -1.71552600 \\
\hline $\mathrm{H}$ & 0.49828800 & 4.93815100 & -1.13439800 \\
\hline $\mathrm{H}$ & -0.03856300 & 4.38355400 & -2.73414300 \\
\hline $\mathrm{H}$ & -0.67986900 & 3.61725200 & -1.26670100 \\
\hline C & 0.97403200 & 1.85945700 & -2.70831600 \\
\hline $\mathrm{H}$ & 1.83987700 & 1.21922400 & -2.93023600 \\
\hline $\mathrm{H}$ & 0.19699600 & 1.22819200 & -2.27143300 \\
\hline $\mathrm{H}$ & 0.59683100 & 2.25573600 & -3.66551400 \\
\hline $\mathrm{Ni}$ & -0.24428200 & 0.75365700 & 0.38929900 \\
\hline $\mathrm{H}$ & -1.69904700 & 0.58575200 & -3.21648600 \\
\hline $\mathrm{H}$ & -1.77727800 & 1.87111500 & -1.62740800 \\
\hline $\mathrm{H}$ & -2.76497300 & 1.61027700 & 1.28222500 \\
\hline C & -2.69846400 & 3.60567500 & 0.35007900 \\
\hline $\mathrm{H}$ & -2.80256800 & 4.01057400 & -0.66628100 \\
\hline $\mathrm{H}$ & -3.57484200 & 3.90075100 & 0.94112100 \\
\hline $\mathrm{H}$ & -1.81085500 & 4.06318900 & 0.81369600 \\
\hline \multicolumn{4}{|c|}{ TS19/TS-Conformation-B } \\
\hline C & -2.37875500 & -3.63701100 & 1.14056800 \\
\hline $\mathrm{C}$ & -1.70831000 & -2.45676400 & 0.77358100 \\
\hline $\mathrm{C}$ & -2.20422700 & -1.78081400 & -0.35660500 \\
\hline $\mathrm{C}$ & -3.15286600 & -2.33502000 & -1.22514700 \\
\hline C & -3.78714600 & -3.51539200 & -0.82465900 \\
\hline $\mathrm{C}$ & -3.43492600 & -4.13580200 & 0.37678500 \\
\hline $\mathrm{C}$ & -3.35532300 & -0.17352400 & -2.35437400 \\
\hline $\mathrm{C}$ & -2.52071700 & 0.35748800 & -1.360564 \\
\hline
\end{tabular}




\begin{tabular}{|c|c|c|}
\hline-2.52471200 & 1.71767300 & -1.00374200 \\
\hline-3.36418900 & 2.56140900 & -1.75182800 \\
\hline-4.16531500 & 2.07116100 & -2.78182900 \\
\hline-4.17558100 & 0.70608700 & -3.06793600 \\
\hline-2.05816400 & -4.19141300 & 2.01847200 \\
\hline-4.54829600 & -3.96729700 & -1.46082800 \\
\hline-3.94785600 & -5.04777800 & 0.69016200 \\
\hline-3.41297000 & 3.62076500 & -1.51281200 \\
\hline-4.80444400 & 2.75391900 & -3.34611000 \\
\hline-4.82843800 & 0.32361100 & -3.85200700 \\
\hline-3.31079400 & -1.67844800 & -2.59709400 \\
\hline-2.04481200 & -2.00759300 & -3.43248000 \\
\hline-2.10658700 & -1.51740300 & -4.41636800 \\
\hline-1.12845800 & -1.66005700 & -2.93478700 \\
\hline-1.96039100 & -3.09463500 & -3.58525000 \\
\hline-4.54412400 & -2.18717300 & -3.34644000 \\
\hline-4.47832800 & -3.27372400 & -3.49856400 \\
\hline-5.47501300 & -1.96732200 & -2.80216800 \\
\hline-4.60793100 & -1.73009200 & -4.34384300 \\
\hline-1.68874900 & -0.52196900 & -0.66186300 \\
\hline 0.07237000 & -1.98351400 & 3.24754100 \\
\hline-0.67379700 & -3.13414900 & 3.93360100 \\
\hline-1.75938600 & -3.06100600 & 3.78056800 \\
\hline-0.33686800 & -4.12499300 & 3.60532600 \\
\hline-0.49452000 & -3.06817200 & 5.02053800 \\
\hline-0.53010400 & -0.66059000 & 3.74272600 \\
\hline-0.39127200 & -0.58709200 & 4.83455400 \\
\hline-0.04198500 & 0.20565600 & 3.27773900 \\
\hline-1.60634300 & -0.60583800 & 3.53995900 \\
\hline 1.55742000 & -2.00980300 & 3.64408800 \\
\hline 2.12984000 & -1.22150900 & 3.13566400 \\
\hline 1.63671900 & -1.82984900 & 4.72910400 \\
\hline 2.02902000 & -2.97894700 & 3.43387200 \\
\hline 1.01018800 & -3.37743300 & 0.57687000 \\
\hline 0.81588000 & -4.72222800 & 1.28775600 \\
\hline 1.33261900 & -5.50451500 & 0.70585400 \\
\hline 1.25301600 & -4.72488300 & 2.29582000 \\
\hline-0.23883400 & -5.01639500 & 1.36024600 \\
\hline 0.55313600 & -3.50379500 & -0.88780900 \\
\hline 0.55117100 & -2.52325500 & -1.38832900 \\
\hline 1.26391100 & -4.15025200 & -1.42851300 \\
\hline-0.44671200 & -3.94814700 & -0.98351700 \\
\hline 2.51361700 & -3.03377200 & 0.55729100 \\
\hline 2.88893600 & -2.62908200 & 1.50410000 \\
\hline
\end{tabular}

.

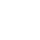

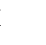

H

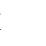

.

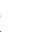

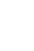

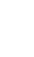

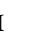

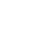

C

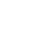

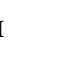

H

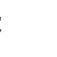

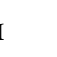

H

.

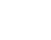

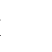

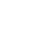

C

.

$\mathrm{H}$

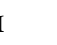

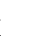

H

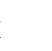




\begin{tabular}{|c|c|c|c|}
\hline $\mathrm{H}$ & 3.07383500 & -3.96161700 & 0.35153300 \\
\hline $\mathrm{H}$ & 2.76334000 & -2.33505000 & -0.24947500 \\
\hline $\mathrm{C}$ & -1.12571800 & 4.11522500 & 0.16590800 \\
\hline $\mathrm{C}$ & -2.22232300 & 5.15510700 & 0.43338400 \\
\hline $\mathrm{H}$ & -3.13657000 & 4.98951200 & -0.15055800 \\
\hline $\mathrm{H}$ & -2.49670800 & 5.19362100 & 1.49627800 \\
\hline $\mathrm{H}$ & -1.83370100 & 6.15189100 & 0.16102000 \\
\hline $\mathrm{C}$ & 0.05385900 & 4.42531400 & 1.10800000 \\
\hline $\mathrm{H}$ & -0.18689500 & 4.22838500 & 2.16280300 \\
\hline $\mathrm{H}$ & 0.94664200 & 3.84271700 & 0.84641600 \\
\hline $\mathrm{H}$ & 0.31583400 & 5.49354300 & 1.02091300 \\
\hline $\mathrm{C}$ & -0.61736200 & 4.27018700 & -1.27728500 \\
\hline $\mathrm{H}$ & -0.13596500 & 5.25762900 & -1.37998800 \\
\hline $\mathrm{H}$ & 0.13171500 & 3.51143600 & -1.53367900 \\
\hline $\mathrm{H}$ & -1.42040900 & 4.21399000 & -2.02269800 \\
\hline $\mathrm{C}$ & -2.96955600 & 2.13678100 & 1.79565100 \\
\hline $\mathrm{C}$ & -4.27332400 & 2.89744000 & 1.52804100 \\
\hline $\mathrm{H}$ & -5.03017800 & 2.57011500 & 2.26276900 \\
\hline $\mathrm{H}$ & -4.16691400 & 3.98224500 & 1.63419200 \\
\hline $\mathrm{H}$ & -4.67787400 & 2.68063900 & 0.52864500 \\
\hline $\mathrm{C}$ & -2.36711300 & 2.57963100 & 3.13819000 \\
\hline $\mathrm{H}$ & -2.16936100 & 3.66166600 & 3.15602800 \\
\hline $\mathrm{H}$ & -3.07437800 & 2.35823200 & 3.95591700 \\
\hline $\mathrm{H}$ & -1.42449200 & 2.05665000 & 3.35806100 \\
\hline $\mathrm{C}$ & -3.30847200 & 0.63666400 & 1.84103500 \\
\hline $\mathrm{H}$ & -3.93033200 & 0.33970300 & 0.98379100 \\
\hline $\mathrm{H}$ & -2.40755500 & 0.01431100 & 1.83384000 \\
\hline $\mathrm{H}$ & -3.87823300 & 0.41121200 & 2.75784600 \\
\hline $\mathrm{C}$ & 1.52715200 & 1.13403800 & -0.75397800 \\
\hline $\mathrm{C}$ & 2.09624900 & 0.32678300 & 0.30618900 \\
\hline $\mathrm{C}$ & 2.66686200 & 0.87837500 & 1.55380900 \\
\hline $\mathrm{C}$ & 1.96010400 & 1.77617200 & 2.37494800 \\
\hline $\mathrm{H}$ & 4.49534500 & -0.25927900 & 1.37593900 \\
\hline $\mathrm{H}$ & 2.67385200 & -0.52148900 & -0.06020700 \\
\hline $\mathrm{C}$ & 3.93525900 & 0.44919000 & 1.99190900 \\
\hline $\mathrm{C}$ & 2.49955100 & 2.23745700 & 3.57612400 \\
\hline $\mathrm{C}$ & 3.76339800 & 1.80462100 & 3.99519600 \\
\hline $\mathrm{C}$ & 4.47822300 & 0.90622200 & 3.19571000 \\
\hline $\mathrm{H}$ & 1.92338700 & 2.92913000 & 4.19706400 \\
\hline $\mathrm{H}$ & 4.18454400 & 2.15965300 & 4.93922500 \\
\hline $\mathrm{H}$ & 5.46433200 & 0.55628800 & 3.51305900 \\
\hline $\mathrm{C}$ & 1.40250500 & 0.57119200 & -2.04306900 \\
\hline $\mathrm{O}$ & 3.20657000 & 0.79842400 & -2.90455000 \\
\hline $\mathrm{C}$ & 4.01238600 & -0.14616800 & -2.58897600 \\
\hline
\end{tabular}




\begin{tabular}{|c|c|c|c|}
\hline $\mathrm{C}$ & 5.51665200 & 0.23784600 & -2.69509900 \\
\hline $\mathrm{O}$ & 3.68870200 & -1.27694500 & -2.19380700 \\
\hline $\mathrm{C}$ & 5.80457900 & 0.76238000 & -4.11058500 \\
\hline $\mathrm{C}$ & 5.77705900 & 1.35146400 & -1.66499500 \\
\hline $\mathrm{C}$ & 6.40186400 & -0.97356600 & -2.39116000 \\
\hline $\mathrm{H}$ & 5.62236900 & -0.01863200 & -4.86797100 \\
\hline $\mathrm{H}$ & 5.15716700 & 1.61929900 & -4.34682000 \\
\hline $\mathrm{H}$ & 6.85623000 & 1.08281000 & -4.20317400 \\
\hline $\mathrm{H}$ & 5.55188500 & 1.00739600 & -0.64210900 \\
\hline $\mathrm{H}$ & 6.83299500 & 1.67004700 & -1.69102700 \\
\hline $\mathrm{H}$ & 5.14271500 & 2.22631200 & -1.86975900 \\
\hline $\mathrm{H}$ & 7.46840500 & -0.70093300 & -2.46291700 \\
\hline $\mathrm{H}$ & 6.21169300 & -1.35968100 & -1.37857800 \\
\hline $\mathrm{H}$ & 6.20921000 & -1.79576900 & -3.09761000 \\
\hline $\mathrm{H}$ & 0.95999900 & 2.08108100 & 2.07212400 \\
\hline $\mathrm{Ni}$ & 0.21338300 & -0.05863400 & 0.24462600 \\
\hline $\mathrm{P}$ & -0.02025100 & -1.93375200 & 1.34181100 \\
\hline $\mathrm{P}$ & -1.56859800 & 2.26464500 & 0.48494100 \\
\hline $\mathrm{H}$ & 1.40647900 & 2.21230800 & -0.63047200 \\
\hline $\mathrm{C}$ & 0.61428100 & 1.21977400 & -3.13699300 \\
\hline $\mathrm{H}$ & 0.86955100 & 2.28469600 & -3.23597500 \\
\hline $\mathrm{H}$ & -0.46303200 & 1.15336700 & -2.91650400 \\
\hline $\mathrm{H}$ & 0.79925700 & 0.72537400 & -4.10017000 \\
\hline $\mathrm{H}$ & 1.52704100 & -0.50955500 & -2.10924300 \\
\hline
\end{tabular}

\section{Cartesian coordinates of the structures (optimization in $N, N$-dimethylacetamide)}

\section{TS5}

$\begin{array}{llll}\text { C } & -1.14758400 & 0.67303600 & 2.18172300 \\ \text { C } & -0.20616600 & 0.37932000 & 1.17458700 \\ \text { C } & 1.01998400 & 1.08181300 & 1.22062300 \\ \text { C } & 1.18101000 & 2.19868500 & 2.06862700 \\ \text { C } & 0.22277900 & 2.43248600 & 3.05972500 \\ \text { C } & -0.91715600 & 1.63540600 & 3.15779400 \\ \text { C } & 3.48019200 & 2.48609500 & 1.17453600 \\ \text { C } & 3.31484200 & 1.24020800 & 0.54016000 \\ \text { C } & 4.42133800 & 0.53338800 & 0.00811100 \\ \text { C } & 5.67198500 & 1.17280700 & 0.03220200 \\ \text { C } & 5.84566400 & 2.42893400 & 0.60629600 \\ \text { C } & 4.75538200 & 3.06308200 & 1.19652900 \\ \text { H } & -2.10372600 & 0.14479100 & 2.15897900 \\ \text { H } & 0.34710500 & 3.27043300 & 3.74500900 \\ \text { H } & -1.65678300 & 1.81781400 & 3.94050000 \\ \text { H } & 6.53315600 & 0.66849900 & -0.39936800 \\ \mathrm{H} & 6.82930200 & 2.90408700 & 0.60923400 \\ \mathrm{H} & & 4.03677900 & 1.66523100\end{array}$


$3.21798900 \quad 1.74740200$

$\begin{array}{lll}2.65119100 & 4.06081500 \quad 2.97456900\end{array}$

3.40438200

3.05009000

4.81640500

2.71527000

1.78044500

1.70988100

0.81424400

1.43075900

2.46756900

2.05501500

$-0.43961300$

1.05570800

1.37187500

1.67900800

$1.27612300 \quad-3.62652300$

$-1.21161600 \quad-2.76318400$

$-1.04603600-3.81232700$

$-2.28964200 \quad-2.60786800$

$-0.86582000 \quad-2.12397400$

$-0.89195400 \quad-3.46741700$

$-1.95407000 \quad-3.33666100$

$-0.75941100 \quad-4.49794900$

$-0.30637200 \quad-3.36910900$

$-0.30079800$

$-1.41381400$

$-1.64139600$

$-1.08455200$

$-2.34366300$

1.01687300

1.82888900

1.30261200

0.93388500

$-0.17074400$

$-1.04883800$

$-0.08380400$

0.72392900

5.29038800

4.45606900

$3.46555300 \quad-2.55684200$

$4.31368600 \quad-1.06175600$

$4.97897800 \quad-2.64525900$

$6.70411800-1.67128000$

$7.16772100 \quad-2.36609700$

$6.69070500 \quad-0.67910100$
3.78809900

3.35302300

0.65305200

1.02270100

$-0.25338200$

0.37483900

0.40533700

0.39361200

0.67159100

1.45217100

$-0.20814900$

1.03280800

1.69360300

1.99139300

1.56758300

2.51990000

$-0.68711300$

$-0.93363300$

$-0.31639100$

$-1.61133000$

$-1.85088800$

$-2.83692100$

$-2.79075100$

$-3.86457300$

$-2.64237100$

$-2.28836300$

$-1.58406000$

$-3.27067800$

$-2.40888600$

$-1.88415300$

$-1.44556000$

$-2.93295300$

$-1.35156300$

0.69948600

1.99183700

1.86673200

2.33478200

2.79606700

1.02450900

1.74742600

1.49673400 


\begin{tabular}{|c|c|c|c|}
\hline $\mathrm{H}$ & 7.35928700 & -1.62657100 & 0.14654100 \\
\hline $\mathrm{C}$ & 5.34873400 & -3.62938200 & 0.22729800 \\
\hline $\mathrm{H}$ & 4.34920300 & -4.01136400 & -0.03581800 \\
\hline $\mathrm{H}$ & 5.74837700 & -4.26523600 & 1.03633500 \\
\hline $\mathrm{H}$ & 6.00530300 & -3.75524000 & -0.64611700 \\
\hline $\mathrm{C}$ & 5.13634900 & -1.21252400 & -2.30999600 \\
\hline $\mathrm{C}$ & 4.70581400 & 0.06941100 & -3.04311200 \\
\hline $\mathrm{H}$ & 4.97129700 & -0.01374200 & -4.11149200 \\
\hline $\mathrm{H}$ & 5.20594400 & 0.96232000 & -2.64236400 \\
\hline $\mathrm{H}$ & 3.61915000 & 0.23386000 & -2.98190900 \\
\hline $\mathrm{C}$ & 6.66426100 & -1.32866600 & -2.34730700 \\
\hline $\mathrm{H}$ & 7.16794400 & -0.49183100 & -1.84497300 \\
\hline $\mathrm{H}$ & 6.99860700 & -1.32330500 & -3.40017800 \\
\hline $\mathrm{H}$ & 7.01966500 & -2.26634400 & -1.89786400 \\
\hline $\mathrm{C}$ & 4.53497500 & -2.42048200 & -3.05624300 \\
\hline $\mathrm{H}$ & 3.43905500 & -2.34649500 & -3.12601900 \\
\hline $\mathrm{H}$ & 4.77811400 & -3.37342100 & -2.56308600 \\
\hline $\mathrm{H}$ & 4.93887100 & -2.46443100 & -4.08289600 \\
\hline $\mathrm{P}$ & 4.25259400 & -1.21060600 & -0.60063500 \\
\hline $\mathrm{P}$ & -0.95147500 & -0.72809600 & -0.12342600 \\
\hline $\mathrm{C}$ & -5.04745600 & -0.39998600 & -0.47647300 \\
\hline $\mathrm{C}$ & -4.35679700 & -1.68483800 & -0.58482600 \\
\hline $\mathrm{C}$ & -4.57474000 & -2.75357400 & 0.40829600 \\
\hline $\mathrm{C}$ & -4.89243800 & -2.48262200 & 1.75854700 \\
\hline $\mathrm{H}$ & -4.15886300 & -4.34743100 & -0.99184100 \\
\hline $\mathrm{H}$ & -4.22072700 & -2.07410900 & -1.60405100 \\
\hline $\mathrm{C}$ & -4.39598800 & -4.10549600 & 0.04762300 \\
\hline $\mathrm{C}$ & -5.01335500 & -3.50906900 & 2.69510800 \\
\hline $\mathrm{C}$ & -4.81367400 & -4.84401400 & 2.32011200 \\
\hline $\mathrm{C}$ & -4.50487200 & -5.13340100 & 0.98686400 \\
\hline $\mathrm{H}$ & -5.25418700 & -3.26451200 & 3.73359100 \\
\hline $\mathrm{H}$ & -4.89972600 & -5.64621900 & 3.05732700 \\
\hline $\mathrm{H}$ & -4.35098000 & -6.16988400 & 0.67339800 \\
\hline $\mathrm{C}$ & -5.35054200 & 0.39967900 & -1.63163400 \\
\hline $\mathrm{H}$ & -4.98460100 & -0.00711500 & -2.57995300 \\
\hline $\mathrm{C}$ & -3.60793500 & 2.39552800 & -0.87930900 \\
\hline $\mathrm{O}$ & -3.02766900 & 1.71357200 & -0.00675900 \\
\hline $\mathrm{C}$ & -3.39667100 & 3.91968100 & -0.89165200 \\
\hline $\mathrm{C}$ & -3.00704900 & 4.35987700 & -2.31297900 \\
\hline $\mathrm{C}$ & -2.29109500 & 4.30639100 & 0.09719900 \\
\hline $\mathrm{C}$ & -4.73181300 & 4.57040000 & -0.48506800 \\
\hline $\mathrm{H}$ & -3.79068800 & 4.09775400 & -3.03801200 \\
\hline $\mathrm{H}$ & -2.06919400 & 3.87775000 & -2.63272000 \\
\hline $\mathrm{H}$ & -2.85631400 & 5.45092000 & -2.34153700 \\
\hline
\end{tabular}




\begin{tabular}{|c|c|c|c|}
\hline $\mathrm{H}$ & -2.54569200 & 4.00958800 & 1.12473700 \\
\hline $\mathrm{H}$ & -2.13697300 & 5.39719900 & 0.07823200 \\
\hline $\mathrm{H}$ & -1.33925800 & 3.82045600 & -0.16155600 \\
\hline $\mathrm{H}$ & -4.63135700 & 5.66776500 & -0.47692700 \\
\hline $\mathrm{H}$ & -5.03909700 & 4.24961000 & 0.52375100 \\
\hline $\mathrm{H}$ & -5.53320100 & 4.30163700 & -1.18934900 \\
\hline $\mathrm{O}$ & -4.38475600 & 1.93795000 & -1.78834700 \\
\hline $\mathrm{H}$ & -5.66084000 & -0.19496800 & 0.41019600 \\
\hline $\mathrm{H}$ & -5.01748200 & -1.44770100 & 2.08405800 \\
\hline $\mathrm{C}$ & -6.69824700 & 1.05705700 & -1.72145200 \\
\hline $\mathrm{H}$ & -7.45377400 & 0.26359200 & -1.85704500 \\
\hline $\mathrm{H}$ & -6.77060800 & 1.75166500 & -2.56932900 \\
\hline $\mathrm{H}$ & -6.93948200 & 1.59380500 & -0.79088500 \\
\hline $\mathrm{Ni}$ & -3.11326200 & -0.26090300 & -0.16545700 \\
\hline \multicolumn{4}{|c|}{ TS20/TS-Conformation-A } \\
\hline $\mathrm{C}$ & -1.95422500 & 1.37689900 & -0.67884100 \\
\hline $\mathrm{C}$ & -1.82692100 & -0.04641700 & -0.51095300 \\
\hline $\mathrm{C}$ & -1.57561000 & -0.95353600 & -1.65401500 \\
\hline $\mathrm{C}$ & -1.51075500 & -0.50165800 & -2.98915000 \\
\hline $\mathrm{H}$ & -1.48948700 & -2.72790800 & -0.42606900 \\
\hline $\mathrm{H}$ & -2.48932300 & -0.47026200 & 0.24159200 \\
\hline $\mathrm{C}$ & -1.42611200 & -2.33999900 & -1.43885700 \\
\hline C & -1.27529000 & -1.38377300 & -4.04631000 \\
\hline $\mathrm{C}$ & -1.10504100 & -2.75083700 & -3.80595400 \\
\hline $\mathrm{C}$ & -1.19004400 & -3.22339500 & -2.49177200 \\
\hline $\mathrm{H}$ & -1.23217800 & -0.99786000 & -5.06844300 \\
\hline $\mathrm{H}$ & -0.91546300 & -3.43986600 & -4.63276400 \\
\hline $\mathrm{H}$ & -1.06784000 & -4.28931700 & -2.28173700 \\
\hline $\mathrm{C}$ & -2.56499700 & 2.13605300 & 0.32324300 \\
\hline $\mathrm{C}$ & -5.09851200 & 0.70182200 & 0.06896000 \\
\hline $\mathrm{O}$ & -4.71256700 & -0.01276200 & 1.01373500 \\
\hline $\mathrm{C}$ & -6.13390100 & 0.10839300 & -0.93881200 \\
\hline $\mathrm{C}$ & -6.91194400 & -1.04555800 & -0.30043400 \\
\hline $\mathrm{C}$ & -7.10302800 & 1.19751700 & -1.41473600 \\
\hline $\mathrm{C}$ & -5.31434400 & -0.41379700 & -2.13412700 \\
\hline $\mathrm{H}$ & -6.22975800 & -1.83021800 & 0.05590300 \\
\hline $\mathrm{H}$ & -7.49742300 & -0.69923700 & 0.56732300 \\
\hline $\mathrm{H}$ & -7.61404600 & -1.49333800 & -1.02488700 \\
\hline $\mathrm{H}$ & -6.55193700 & 2.03981900 & -1.85585900 \\
\hline $\mathrm{H}$ & -7.80455100 & 0.80033400 & -2.16867500 \\
\hline $\mathrm{H}$ & -7.70058700 & 1.59263400 & -0.57572800 \\
\hline $\mathrm{H}$ & -5.97519100 & -0.82926400 & -2.91453700 \\
\hline $\mathrm{H}$ & -4.71830600 & 0.39528700 & -2.58393600 \\
\hline $\mathrm{H}$ & -4.61828700 & -1.20878400 & -1.82238300 \\
\hline
\end{tabular}




\begin{tabular}{|c|c|c|}
\hline-4.68373200 & 1.86954900 & -0.20740300 \\
\hline 0.66821500 & -3.72783900 & 1.36053700 \\
\hline 0.72404900 & -2.36964600 & 1.00961100 \\
\hline 1.54046400 & -2.03117500 & -0.08406300 \\
\hline 2.37082300 & -2.94581900 & -0.74171400 \\
\hline 2.28909400 & -4.28854000 & -0.35639000 \\
\hline 1.42000100 & -4.67924600 & 0.66673400 \\
\hline 3.78075600 & -1.01844200 & -1.33427100 \\
\hline 2.81498900 & -0.17713900 & -0.76492700 \\
\hline 3.04422300 & 1.17122700 & -0.44294000 \\
\hline 4.35889500 & 1.64009500 & -0.60261100 \\
\hline 5.36685400 & 0.80248300 & -1.08585900 \\
\hline 5.07547700 & -0.50935500 & -1.47642200 \\
\hline 0.05353100 & -4.04933700 & 2.19992100 \\
\hline 2.90647800 & -5.03843400 & -0.85203600 \\
\hline 1.35607600 & -5.73186400 & 0.95194100 \\
\hline 4.59937100 & 2.67284400 & -0.35302600 \\
\hline 6.38447900 & 1.18585100 & -1.18965500 \\
\hline 5.86464800 & -1.13072400 & -1.90114900 \\
\hline 3.30246200 & -2.39206200 & -1.82366700 \\
\hline 4.46755300 & -3.33847300 & -2.11853700 \\
\hline 5.11917500 & -2.91809500 & -2.89794500 \\
\hline 5.07676900 & -3.52873600 & -1.22168200 \\
\hline 4.09674200 & -4.30173600 & -2.49659200 \\
\hline 2.48122400 & -2.17692600 & -3.12109700 \\
\hline 2.09654900 & -3.14002900 & -3.48951800 \\
\hline 1.62407700 & -1.51155000 & -2.94948900 \\
\hline 3.11567200 & -1.72952300 & -3.90231100 \\
\hline 1.57564300 & -0.71092600 & -0.48908200 \\
\hline 0.00802500 & -0.96673600 & 1.97935800 \\
\hline 1.57282900 & 2.22982100 & -0.04818200 \\
\hline-1.56873800 & -1.58978800 & 2.90364400 \\
\hline-2.45158500 & -2.48051600 & 2.01344600 \\
\hline-2.81033500 & -1.97039300 & 1.11603100 \\
\hline-1.95364900 & -3.41494100 & 1.72227700 \\
\hline-3.34966000 & -2.75248600 & 2.59286000 \\
\hline-2.36658300 & -0.32165200 & 3.27110900 \\
\hline-1.73605000 & 0.46622800 & 3.70753500 \\
\hline-2.91600000 & 0.07572500 & 2.40873900 \\
\hline-3.12710700 & -0.58813600 & 4.02517600 \\
\hline-1.29502300 & -2.37836800 & 4.19379900 \\
\hline-0.83127300 & -1.76106200 & 4.97205700 \\
\hline-2.26492200 & -2.72130000 & 4.59187200 \\
\hline-0.67 & -3.26940400 & 4.03417500 \\
\hline
\end{tabular}




\begin{tabular}{|c|c|c|}
\hline 1.41359000 & -0.77325500 & 3.29702000 \\
\hline 2.66783400 & -0.40449300 & 2.49676300 \\
\hline 3.06928600 & -1.27192600 & 1.95414200 \\
\hline 2.46866800 & 0.38674300 & 1.77525000 \\
\hline 3.45276500 & -0.05090400 & 3.18411800 \\
\hline 1.01466400 & 0.38969900 & 4.21733500 \\
\hline 0.18388500 & 0.11926100 & 4.88421900 \\
\hline 1.87058900 & 0.67281900 & 4.85298300 \\
\hline 0.71049200 & 1.27721300 & 3.64275200 \\
\hline 1.78803600 & -2.01128900 & 4.12783800 \\
\hline 1.89885000 & -2.90962500 & 3.50344500 \\
\hline 2.76872300 & -1.82254500 & 4.59776300 \\
\hline 1.08453200 & -2.23069300 & 4.93522500 \\
\hline 2.10168800 & 3.59016000 & 1.19473600 \\
\hline 2.95938900 & 2.97455900 & 2.30899800 \\
\hline 2.42425000 & 2.19041700 & 2.85120700 \\
\hline 3.90520000 & 2.55835900 & 1.93536600 \\
\hline 3.20569600 & 3.76651600 & 3.03611200 \\
\hline 2.85527100 & 4.79581300 & 0.61840600 \\
\hline 3.08027100 & 5.48927200 & 1.44668200 \\
\hline 3.81247700 & 4.51934200 & 0.15689400 \\
\hline 2.25950800 & 5.35236300 & -0.11577700 \\
\hline 0.78565800 & 4.08992900 & 1.81738900 \\
\hline 1.01110900 & 4.81778900 & 2.61480900 \\
\hline 0.15333100 & 4.59566700 & 1.07482900 \\
\hline 0.20469400 & 3.26613900 & 2.25945900 \\
\hline 1.36398400 & 3.00810300 & -1.80482700 \\
\hline 2.60362900 & 3.67283200 & -2.41948800 \\
\hline 2.35016200 & 3.96345400 & -3.45393500 \\
\hline 2.91889300 & 4.58112700 & -1.89625300 \\
\hline 3.45858900 & 2.98689900 & -2.48035300 \\
\hline 0.22024900 & 4.02698100 & -1.74732900 \\
\hline 0.49520600 & 4.92069600 & -1.16918300 \\
\hline-0.03207800 & 4.35636400 & -2.76940900 \\
\hline-0.67962100 & 3.59729800 & -1.30086200 \\
\hline 0.98532400 & 1.83022800 & -2.71768900 \\
\hline 1.85948600 & 1.20406600 & -2.94745500 \\
\hline 0.22465500 & 1.18778000 & -2.26781700 \\
\hline 0.58910700 & 2.21525100 & -3.67178600 \\
\hline 0.24720400 & 0.75841600 & 0.39064700 \\
\hline-1.66878900 & 0.55285600 & -3.21270100 \\
\hline-1.76786900 & 1.85617700 & -1.64101300 \\
\hline-2.77060200 & 1.63563800 & 1.26738200 \\
\hline 68484600 & 3.62040500 & 0.31569700 \\
\hline
\end{tabular}




\begin{tabular}{|c|c|c|c|}
\hline $\mathrm{H}$ & -2.78691500 & 4.01686900 & -0.70415900 \\
\hline $\mathrm{H}$ & -3.55634500 & 3.93070500 & 0.90633400 \\
\hline $\mathrm{H}$ & -1.79137500 & 4.07366300 & 0.77215000 \\
\hline \multicolumn{4}{|c|}{ TS19/TS-Conformation-B } \\
\hline $\mathrm{C}$ & -2.35802800 & -3.68014700 & 1.04538300 \\
\hline $\mathrm{C}$ & -1.69188900 & -2.48552900 & 0.71907000 \\
\hline $\mathrm{C}$ & -2.17163200 & -1.78854400 & -0.40520600 \\
\hline $\mathrm{C}$ & -3.09215800 & -2.33336100 & -1.30857800 \\
\hline $\mathrm{C}$ & -3.72254900 & -3.52900300 & -0.94941400 \\
\hline $\mathrm{C}$ & -3.39182100 & -4.17146200 & 0.24678600 \\
\hline $\mathrm{C}$ & -3.29809500 & -0.15008800 & -2.39058400 \\
\hline $\mathrm{C}$ & -2.49377600 & 0.36979600 & -1.36639500 \\
\hline $\mathrm{C}$ & -2.52482300 & 1.72128500 & -0.97952300 \\
\hline $\mathrm{C}$ & -3.36342900 & 2.56912400 & -1.72426000 \\
\hline $\mathrm{C}$ & -4.13585700 & 2.09059900 & -2.78141900 \\
\hline $\mathrm{C}$ & -4.11758200 & 0.73294100 & -3.10060800 \\
\hline $\mathrm{H}$ & -2.05049900 & -4.25306400 & 1.91634000 \\
\hline $\mathrm{H}$ & -4.46313100 & -3.97594500 & -1.61294100 \\
\hline $\mathrm{H}$ & -3.90194400 & -5.09553100 & 0.52797500 \\
\hline $\mathrm{H}$ & -3.43559100 & 3.62149900 & -1.46116500 \\
\hline $\mathrm{H}$ & -4.77519000 & 2.77640800 & -3.34194300 \\
\hline $\mathrm{H}$ & -4.74706800 & 0.35922200 & -3.90778300 \\
\hline $\mathrm{C}$ & -3.22194000 & -1.64715900 & -2.66865300 \\
\hline $\mathrm{C}$ & -1.92648200 & -1.93357500 & -3.47396400 \\
\hline $\mathrm{H}$ & -1.97177700 & -1.42450700 & -4.44923500 \\
\hline $\mathrm{H}$ & -1.03130300 & -1.57743700 & -2.94450200 \\
\hline $\mathrm{H}$ & -1.81568800 & -3.01536800 & -3.64633700 \\
\hline C & -4.42358900 & -2.15811900 & -3.46524700 \\
\hline $\mathrm{H}$ & -4.33315300 & -3.23904500 & -3.64318700 \\
\hline $\mathrm{H}$ & -5.37325200 & -1.96949900 & -2.94202800 \\
\hline $\mathrm{H}$ & -4.46822900 & -1.67525500 & -4.45152700 \\
\hline $\mathrm{O}$ & -1.66498600 & -0.51613400 & -0.66971200 \\
\hline $\mathrm{C}$ & 0.05381300 & -2.03440200 & 3.22221600 \\
\hline $\mathrm{C}$ & -0.70355900 & -3.19259100 & 3.88192200 \\
\hline $\mathrm{H}$ & -1.78572200 & -3.12076000 & 3.70531100 \\
\hline $\mathrm{H}$ & -0.35666300 & -4.17912900 & 3.55119800 \\
\hline $\mathrm{H}$ & -0.54776800 & -3.13568000 & 4.97307200 \\
\hline $\mathrm{C}$ & -0.55746400 & -0.71667900 & 3.72050900 \\
\hline $\mathrm{H}$ & -0.43033700 & -0.65123700 & 4.81433800 \\
\hline $\mathrm{H}$ & -0.06883700 & 0.15596200 & 3.26764000 \\
\hline $\mathrm{H}$ & -1.63183800 & -0.66453400 & 3.50784300 \\
\hline $\mathrm{C}$ & 1.53223400 & -2.06505400 & 3.64198900 \\
\hline $\mathrm{H}$ & 2.11307900 & -1.26998500 & 3.15391800 \\
\hline $\mathrm{H}$ & 1.59317700 & -1.89994900 & 4.73063000 \\
\hline
\end{tabular}




\begin{tabular}{|c|c|c|c|}
\hline $\mathrm{H}$ & 2.00822800 & -3.03105500 & 3.42710100 \\
\hline $\mathrm{C}$ & 1.03124200 & -3.39936100 & 0.55032900 \\
\hline $\mathrm{C}$ & 0.84584600 & -4.74991400 & 1.25212500 \\
\hline $\mathrm{H}$ & 1.37421300 & -5.52376500 & 0.66917700 \\
\hline $\mathrm{H}$ & 1.27559900 & -4.75389900 & 2.26328800 \\
\hline $\mathrm{H}$ & -0.20653900 & -5.05438800 & 1.31623800 \\
\hline $\mathrm{C}$ & 0.58520300 & -3.52190500 & -0.91792900 \\
\hline $\mathrm{H}$ & 0.57993500 & -2.53962500 & -1.41527900 \\
\hline $\mathrm{H}$ & 1.30323000 & -4.16326700 & -1.45566600 \\
\hline $\mathrm{H}$ & -0.41112900 & -3.97216300 & -1.02281200 \\
\hline $\mathrm{C}$ & 2.53023100 & -3.03777100 & 0.54385200 \\
\hline $\mathrm{H}$ & 2.89423700 & -2.64051600 & 1.49850800 \\
\hline $\mathrm{H}$ & 3.10357800 & -3.95593300 & 0.33022700 \\
\hline $\mathrm{H}$ & 2.77413600 & -2.32399900 & -0.25200900 \\
\hline $\mathrm{C}$ & -1.17802200 & 4.11384700 & 0.25943300 \\
\hline $\mathrm{C}$ & -2.28886300 & 5.13649500 & 0.53213900 \\
\hline $\mathrm{H}$ & -3.19041700 & 4.97607300 & -0.07265500 \\
\hline $\mathrm{H}$ & -2.58173400 & 5.14720200 & 1.59072200 \\
\hline $\mathrm{H}$ & -1.90512500 & 6.14303200 & 0.28970700 \\
\hline $\mathrm{C}$ & -0.01489700 & 4.41452100 & 1.22472800 \\
\hline $\mathrm{H}$ & -0.26312300 & 4.17703200 & 2.26951700 \\
\hline $\mathrm{H}$ & 0.89088500 & 3.85683600 & 0.95269600 \\
\hline $\mathrm{H}$ & 0.22854900 & 5.48967000 & 1.17616600 \\
\hline $\mathrm{C}$ & -0.65153100 & 4.30308200 & -1.17296500 \\
\hline $\mathrm{H}$ & -0.17477900 & 5.29542800 & -1.24808900 \\
\hline $\mathrm{H}$ & 0.10522200 & 3.55391000 & -1.43609900 \\
\hline $\mathrm{H}$ & -1.44512600 & 4.25959900 & -1.92944100 \\
\hline $\mathrm{C}$ & -3.02456100 & 2.07995900 & 1.81788500 \\
\hline $\mathrm{C}$ & -4.33078700 & 2.83425100 & 1.54494100 \\
\hline $\mathrm{H}$ & -5.09695200 & 2.48263500 & 2.25872300 \\
\hline $\mathrm{H}$ & -4.23706200 & 3.91731700 & 1.67799900 \\
\hline $\mathrm{H}$ & -4.71631900 & 2.63737600 & 0.53384000 \\
\hline $\mathrm{C}$ & -2.44933400 & 2.49798000 & 3.17993000 \\
\hline $\mathrm{H}$ & -2.25715500 & 3.58033900 & 3.22209200 \\
\hline $\mathrm{H}$ & -3.17051300 & 2.25688400 & 3.97985700 \\
\hline $\mathrm{H}$ & -1.50792300 & 1.97546600 & 3.40641100 \\
\hline $\mathrm{C}$ & -3.34978500 & 0.57604000 & 1.82251700 \\
\hline $\mathrm{H}$ & -3.95178700 & 0.29331200 & 0.94630200 \\
\hline $\mathrm{H}$ & -2.44381100 & -0.03895900 & 1.81999800 \\
\hline $\mathrm{H}$ & -3.93568100 & 0.32469300 & 2.72236300 \\
\hline $\mathrm{C}$ & 1.51625500 & 1.15477600 & -0.72673600 \\
\hline $\mathrm{C}$ & 2.10108100 & 0.32660900 & 0.30944300 \\
\hline $\mathrm{C}$ & 2.65299000 & 0.85043500 & 1.57636900 \\
\hline $\mathrm{C}$ & 1.93700400 & 1.74051700 & 2.39888800 \\
\hline
\end{tabular}




\begin{tabular}{|c|c|c|c|}
\hline $\mathrm{H}$ & 4.47549800 & -0.30156100 & 1.41888800 \\
\hline $\mathrm{H}$ & 2.69416500 & -0.50105300 & -0.08040200 \\
\hline $\mathrm{C}$ & 3.90817300 & 0.40219100 & 2.03367500 \\
\hline $\mathrm{C}$ & 2.45486500 & 2.17502600 & 3.61926800 \\
\hline C & 3.70559900 & 1.72276900 & 4.05761600 \\
\hline $\mathrm{C}$ & 4.42906800 & 0.83212500 & 3.25724200 \\
\hline $\mathrm{H}$ & 1.87121200 & 2.86047800 & 4.24020300 \\
\hline $\mathrm{H}$ & 4.10933600 & 2.05651900 & 5.01695800 \\
\hline $\mathrm{H}$ & 5.40470300 & 0.46667200 & 3.58942100 \\
\hline C & 1.39699800 & 0.62700700 & -2.03062800 \\
\hline $\mathrm{O}$ & 3.20301000 & 0.90331400 & -2.88277700 \\
\hline $\mathrm{C}$ & 4.01379800 & -0.04787700 & -2.60280600 \\
\hline $\mathrm{C}$ & 5.51616800 & 0.35644900 & -2.66637500 \\
\hline $\mathrm{O}$ & 3.69827900 & -1.19929500 & -2.26558800 \\
\hline $\mathrm{C}$ & 5.81046500 & 1.02425800 & -4.01771800 \\
\hline $\mathrm{C}$ & 5.76073600 & 1.36182900 & -1.52681900 \\
\hline $\mathrm{C}$ & 6.41138500 & -0.87102900 & -2.47945100 \\
\hline $\mathrm{H}$ & 5.64041400 & 0.32403700 & -4.85314000 \\
\hline $\mathrm{H}$ & 5.15697500 & 1.89525900 & -4.16994200 \\
\hline $\mathrm{H}$ & 6.86015100 & 1.36032000 & -4.06894700 \\
\hline $\mathrm{H}$ & 5.52979000 & 0.91497200 & -0.54579100 \\
\hline $\mathrm{H}$ & 6.81468200 & 1.68783600 & -1.51031000 \\
\hline $\mathrm{H}$ & 5.12336800 & 2.25030900 & -1.64753200 \\
\hline $\mathrm{H}$ & 7.47578500 & -0.58256100 & -2.51338200 \\
\hline $\mathrm{H}$ & 6.21704000 & -1.36144900 & -1.51415200 \\
\hline $\mathrm{H}$ & 6.23439400 & -1.61823000 & -3.26897800 \\
\hline $\mathrm{H}$ & 0.94571400 & 2.06000100 & 2.08275500 \\
\hline $\mathrm{Ni}$ & 0.22523300 & -0.08503900 & 0.23430300 \\
\hline $\mathrm{P}$ & -0.01327200 & -1.96913100 & 1.31740200 \\
\hline $\mathrm{P}$ & -1.60265900 & 2.25294200 & 0.53542600 \\
\hline $\mathrm{H}$ & 1.37534300 & 2.22665300 & -0.57187300 \\
\hline $\mathrm{C}$ & 0.59953700 & 1.29706800 & -3.10443100 \\
\hline $\mathrm{H}$ & 0.84348800 & 2.36696900 & -3.17500200 \\
\hline $\mathrm{H}$ & -0.47670400 & 1.21467500 & -2.88455300 \\
\hline $\mathrm{H}$ & 0.78587500 & 0.83052800 & -4.08118600 \\
\hline $\mathrm{H}$ & 1.53845100 & -0.44936800 & -2.12906300 \\
\hline
\end{tabular}

